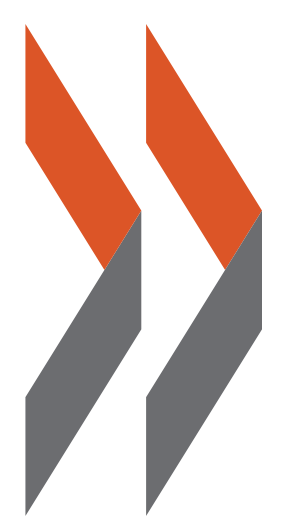

PISA 2012 Ergebnisse:

Exzellenz durch

Chancengerechtigkeit

ALLEN SCHÜLERINNEN UND SCHÜLERN DIE VORAUSSETZUNGEN ZUM ERFOLG SICHERN

BAND II

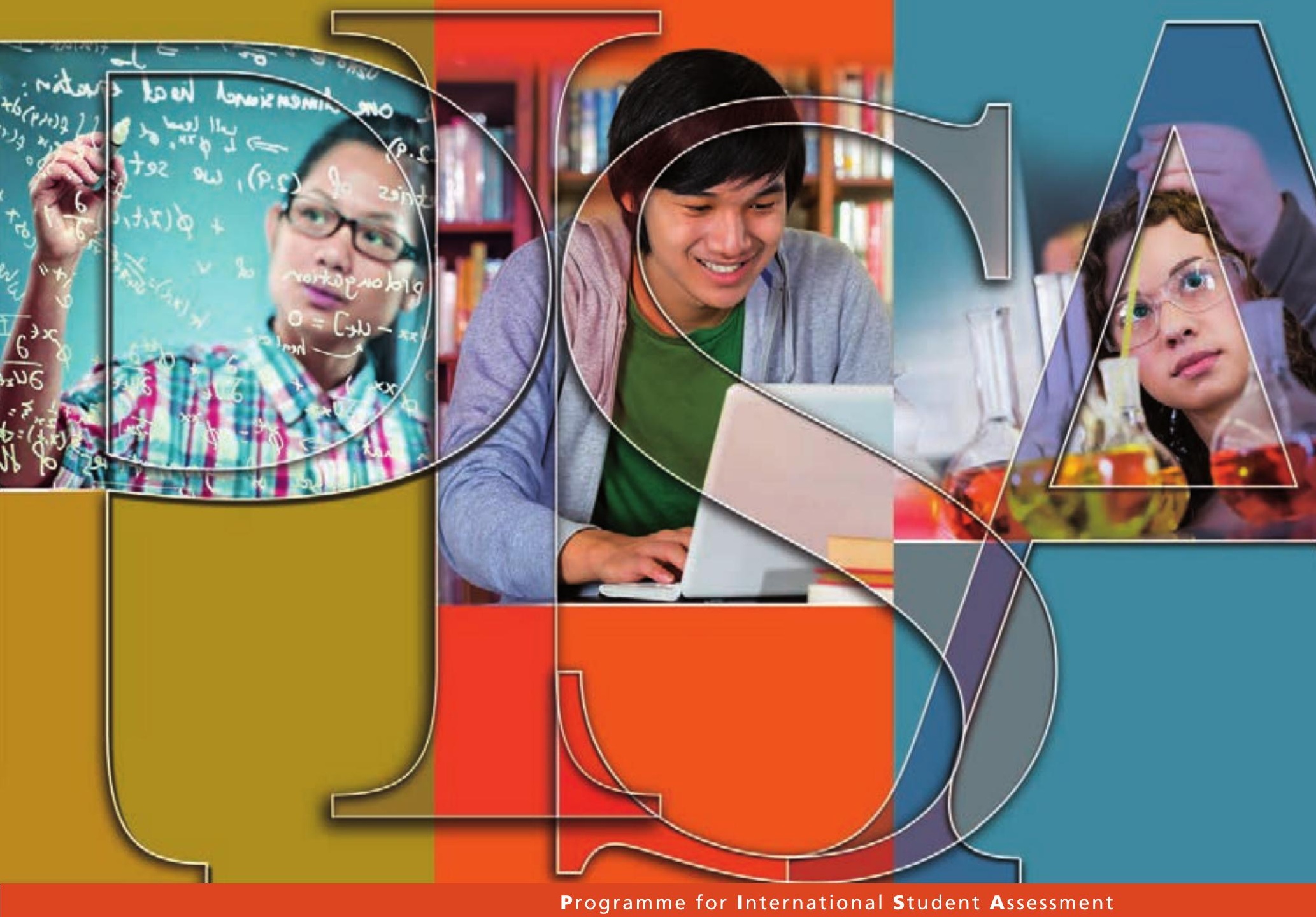

(1) $)$ OECD

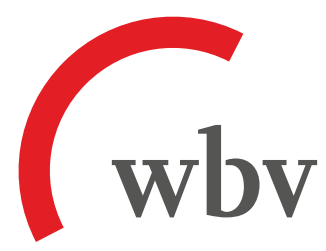





\title{
PISA 2012 Ergebnisse: Exzellenz durch Chancengerechtigkeit
}

\author{
ALLEN SCHÜLERINNEN UND SCHÜLERN \\ DIE VORAUSSETZUNGEN ZUM ERFOLG SICHERN \\ (BAND II)
}

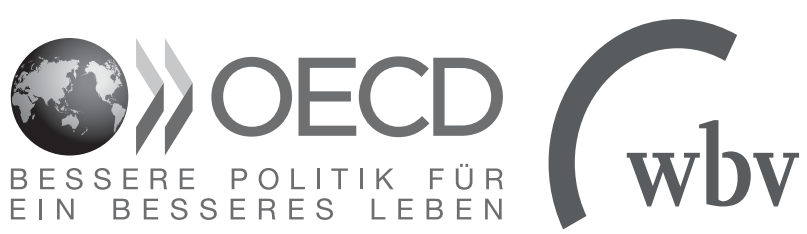


Das vorliegende Dokument wird unter der Verantwortung des Generalsekretärs der OECD veröffentlicht. Die darin zum Ausdruck gebrachten Meinungen und Argumente spiegeln nicht zwangsläufig die offizielle Einstellung der OECD-Mitgliedstaaten wider.

Dieses Dokument und die darin enthaltenen Karten berühren nicht den völkerrechtlichen Status und die Souveränität über Territorien, den Verlauf der internationalen Grenzen und Grenzlinien sowie den Namen von Territorien, Städten und Gebieten.

Bitte zitieren Sie diese Publikation wie folgt:

OECD (2014), PISA 2012 Ergebnisse: Exzellenz durch Chancengerechtigkeit (Band II): Allen Schülerinnen und Schülern die Voraussetzungen zum Erfolg sichern, PISA, W. Bertelsmann Verlag, Germany.

http://dx.doi.org/10.1787/9789264207486-de

ISBN 978-3-7639-5470-4 (Print)

ISBN 978-92-64-20748-6 (PDF)

Publikationsreihe: PISA

ISSN 1990-8547 (Print)

ISSN 1996-3793 (Online)

Die statistischen Daten für Israel wurden von den zuständigen israelischen Stellen bereitgestellt, die für sie verantwortlich zeichnen. Die Verwendung dieser Daten durch die OECD erfolgt unbeschadet des völkerrechtlichen Status der Golanhöhen, von Ost-Jerusalem und der israelischen Siedlungen im Westjordanland.

Originaltitel: PISA 2012 Results: Excellence through Equity (Volume II): Giving Every Student the Chance to Succeed - Résultats du PISA 2012 : L'équité au service de l'excellence (Volume II) : Offrir à chaque élève la possibilité de réussir Übersetzung durch den Deutschen Übersetzungsdienst der OECD.

@ O OECD 2014. Übersetzung durch den Deutschen Übersetzungsdienst der OECD.

๑) 2014 W. Bertelsmann Verlag für die deutsche Ausgabe. Veröffentlicht mit Genehmigung der OECD.

Korrigenda zu OECD-Veröffentlichungen sind verfügbar unter: www.oecd.org/publishing/corrigenda

Diese Publikation ist frei verfügbar zum Download unter wbv-open-access.de

Diese Publikation ist unter folgender Creative-Commons-Lizenz veröffentlicht:

http://creativecommons.org/licenses/by-nc-nd/3.0/de/

(c) $(1) \Theta \Theta$

Verlag: W. Bertelsmann Verlag, Bielefeld, wbv.de

Foto(s):

( Flying Colours Ltd /Getty Images, @ Jacobs Stock Photography /Kzenon, @ khoa vu /Flickr /Getty Images, @ Mel Curtis /Corbis, @ Shutterstock /Kzenon, @ Simon Jarratt /Corbis

Die OECD gestattet das Kopieren, Herunterladen und Abdrucken von OECD-Inhalten für den eigenen Gebrauch sowie das Einfügen von Auszügen aus OECD-Veröffentlichungen, -Datenbanken und -Multimediaprodukten in eigene Dokumente, Präsentationen, Blogs, Websites und Lehrmaterialien, vorausgesetzt die Quelle und der Urheberrechtsinhaber werden in geeigneter Weise genannt. Sämtliche Anfragen bezüglich Verwendung für öffentliche oder kommerzielle Zwecke bzw. Übersetzungsrechte sind zu richten an: rights@oecd.org. Die Genehmigung zur Kopie von Teilen dieser Publikation für den öffentlichen oder kommerziellen Gebrauch ist direkt einzuholen beim Copyright Clearance Center (CCC) unter info@copyright.com oder beim Centre français d'exploitation du droit de copie (CFC) unter contact@cfcopies.com. 


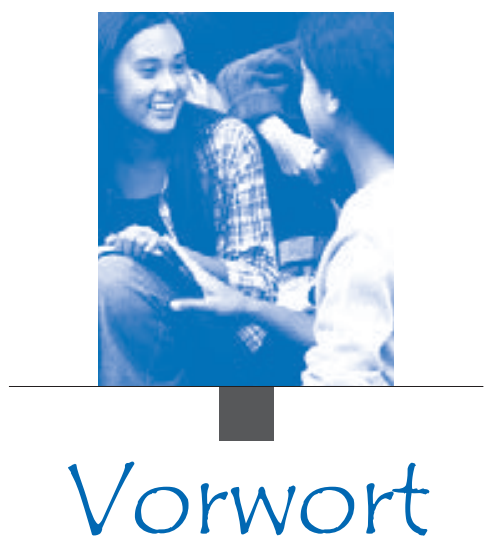

Es ist ein zentrales Anliegen der Politikverantwortlichen in aller Welt, Bürgerinnen und Bürger mit den notwendigen Fähigkeiten und Fertigkeiten auszustatten, damit diese ihr volles Potenzial entfalten, an der zunehmend vernetzten globalen Wirtschaft teilhaben und letztlich bessere Arbeitsplätze für ein besseres Leben nutzen können. Die Ergebnisse der kürzlich durchgeführten OECD-Erhebung über die Fähigkeiten und Fertigkeiten Erwachsener zeigen, dass hochqualifizierte Erwachsene im Vergleich zu geringqualifizierten mit doppelt so hoher Wahrscheinlichkeit in einem Beschäftigungsverhältnis stehen und ihr Einkommen mit fast dreimal höherer Wahrscheinlichkeit über dem Medianeinkommen liegt. Mit anderen Worten: Für Menschen mit geringem Kompetenzniveau ist der Zugang zu besser bezahlten und attraktiveren Arbeitsplätzen erheblich eingeschränkt. Hochqualifizierte Menschen sind zudem mit größerer Wahrscheinlichkeit ehrenamtlich tätig, betrachten sich eher als Akteure denn als Objekte politischer Prozesse und bringen anderen mit größerer Wahrscheinlichkeit Vertrauen entgegen. Die Gerechtigkeit, Integrität und Inklusivität des öffentlichen Handelns werden durch die Kompetenzen der Bürger bestimmt.

Die anhaltende Wirtschaftskrise hat die Dringlichkeit - sowohl im Bildungssystem als auch am Arbeitsplatz -, in den Kompetenzerwerb und die Kompetenzentwicklung der Bürgerinnen und Bürger zu investieren, weiter erhöht. In einer Zeit, in der die Lage der öffentlichen Haushalte angespannt ist und wenig Raum für zusätzliche geldpolitische und fiskalische Impulse bleibt, sind Strukturreformen zur Produktivitätssteigerung, etwa im Bereich der Bildung und der Kompetenzentwicklung, eine grundlegende Voraussetzung für künftiges Wachstum. Tatsächlich sind Investitionen in diesen Bereichen unerlässlich, um die Konjunkturerholung zu stützen und seit langem bestehende Probleme, wie Jugendarbeitslosigkeit und geschlechtsspezifische Ungleichheiten, anzugehen.

In diesem Zusammenhang blicken mehr und mehr Länder über die eigenen Landesgrenzen hinaus, um Informationen über die erfolgreichsten und effizientesten Politiken und Praktiken einzuholen. In einer globalen Wirtschaft bemisst sich Erfolg nämlich nicht mehr ausschließlich an nationalen Standards, sondern auch an jenen Bildungssystemen, die die besten Ergebnisse und die raschesten Verbesserungen erzielen. Im Lauf des letzten Jahrzehnts ist die Internationale OECDSchulleistungsstudie PISA zum weltweit wichtigsten Maßstab für die Beurteilung der Qualität, der Chancengerechtigkeit und der Effizienz von Schulsystemen avanciert. Doch die von PISA ermittelte Faktengrundlage geht weit über statistisches Benchmarking hinaus. Durch die Bestimmung der Merkmale leistungsstarker Bildungssysteme ermöglicht PISA Regierungen und Bildungsexperten, wirksame Maßnahmen zu identifizieren, die dann an den jeweiligen lokalen Kontext angepasst werden können.

Die Ergebnisse der PISA-Erhebung 2012, die zu einem Zeitpunkt durchgeführt wurde, als viele der 65 teilnehmenden Länder und Volkswirtschaften mit den Auswirkungen der Krise zu kämpfen hatten, machen deutlich, dass bei den Bildungsergebnissen sowohl innerhalb als auch zwischen den einzelnen Ländern große Unterschiede bestehen. Durch die Nutzung der Daten früherer PISA-Erhebungsrunden war es möglich, die Entwicklung der Schülerleistungen im Zeitverlauf bzw. in den einzelnen Erhebungsbereichen zu untersuchen. In 40 der 64 Länder und Volkswirtschaften, für die vergleichbare Daten vorhanden waren, wurden die Durchschnittsergebnisse in mindestens einem Bereich verbessert. Leistungsstarke Länder und Volkswirtschaften wie Shanghai (China) und Singapur konnten ihren Vorsprung weiter ausbauen, während in Ländern wie Brasilien, Mexiko, Tunesien und der Türkei eine deutliche Anhebung des niedrigen Leistungsniveaus erreicht werden konnte. 
Einige Bildungssysteme haben bewiesen, dass es möglich ist, hohe und ausgewogene Lernerträge zu gewährleisten und zugleich rasche Verbesserungen zu erzielen. Unter den 13 Ländern und Volkswirtschaften, in denen sich die Mathematikleistungen zwischen 2003 und 2012 deutlich verbesserten, gab es drei, die in diesem Zeitraum darüber hinaus Fortschritte im Hinblick auf die Chancengerechtigkeit in der Bildung erzielten, und neun weitere konnten ihr hohes Niveau an Chancengerechtigkeit beibehalten und zugleich ihre Leistung verbessern - das beweist, dass Chancengerechtigkeit im Bildungswesen nicht auf Kosten des Leistungsniveaus gehen muss.

Die Ergebnisse von PISA 2012 zeigen jedoch auch, dass bei den Mathematikleistungen große länderspezifische Unterschiede bestehen. 245 Punkte - das Äquivalent von knapp sechs Schuljahren - trennen die besten und schlechtesten Durchschnittsergebnisse der Länder, die am PISA-Mathematiktest 2012 teilgenommen haben. Noch deutlicher fallen die Unterschiede bei den Mathematikleistungen innerhalb der einzelnen Länder aus, liegen zwischen den leistungsstärksten und den leistungsschwächsten Schülerinnen und Schülern eines Landes doch häufig mehr als 300 Punkte - was mehr als sieben Schuljahren entspricht. Fest steht, dass es in sämtlichen Ländern und Volkswirtschaften hervorragende Schülerinnen und Schüler gibt, doch nur in wenigen ist es allen Schülerinnen und Schülern möglich, Spitzenleistungen zu erzielen.

Der Bericht legt ferner besorgniserregende geschlechtsspezifische Unterschiede bei der Einstellung der Schülerinnen und Schüler zum Fach Mathematik offen: Auch wenn Mädchen ebenso gute Mathematikleistungen erbringen wie Jungen, verfügen sie eigenen Angaben zufolge über weniger Ausdauer und Lernmotivation im Bereich Mathematik, haben weniger Vertrauen in ihre Mathematikkompetenz und größere Angst vor dem Fach. Schneiden Mädchen beim Mathematiktest im Durchschnitt bereits schlechter ab als Jungen, ist der Leistungsrückstand der Mädchen unter den leistungsstärksten Schülerinnen und Schülern sogar noch stärker ausgeprägt. Diese Ergebnisse haben nicht nur gravierende Konsequenzen im Hinblick auf die Hochschulbildung, in der junge Frauen in Studienfächern wie Naturwissenschaften, Technik, Ingenieurwissenschaften und Mathematik bereits heute unterrepräsentiert sind, sondern auch für die Zeit nach dem Studium, wenn diese jungen Frauen in den Arbeitsmarkt eintreten. Das bestätigt die Erkenntnisse der OECDGender-Initiative, die einige der Faktoren identifizierte, die Ursache geschlechtsspezifischer Ungleichheiten in Bildung, Beschäftigung und Unternehmertum sind bzw. diese verstärken. Eine Förderung der positiven Einstellung der Mädchen zum und ihrer Investitionen in Mathematiklernen würde maßgeblich zur Verringerung dieses Abstands beitragen.

Aus der PISA-Erhebung 2012 geht zudem hervor, dass die leistungsstärksten Schulsysteme Bildungsressourcen gerechter auf begünstigte und benachteiligte Schulen verteilen und den einzelnen Schulen im Hinblick auf Lehrplangestaltung und Beurteilungsmethoden ein größeres Maß an Autonomie einräumen. Kennzeichnend für erfolgreiche Schulsysteme ist außerdem die Überzeugung, dass alle Schülerinnen und Schüler ein hohes Leistungsniveau erreichen können, sowie die Bereitschaft, sämtliche Akteure des Bildungssystems einzubeziehen - einschließlich der Schülerinnen und Schüler, etwa durch Einholen ihrer Meinungen zu den Lehrmethoden.

PISA ist nicht nur ein präziser Indikator jener Kompetenzen von Schülerinnen und Schülern, die ihnen im späteren Leben eine volle Teilhabe an der Gesellschaft ermöglichen, sondern auch ein schlagkräftiges Instrument, das Länder und Volkswirtschaften für die Optimierung ihrer Bildungspolitik nutzen können. Eine spezifische, für jeden Kontext ideal geeignete Kombination von Politiken und Praktiken gibt es nicht. Verbesserungen sind jedoch in allen Ländern möglich, selbst in den leistungsstärksten. Deshalb legt die OECD alle drei Jahre einen Bericht zum Stand der Bildung rund um den Globus vor, um Informationen über die besten Politiken und Praktiken bereitzustellen und den Ländern eine zeitnahe und gezielte Unterstützung anzubieten, damit diese in der Lage sind, allen Schülerinnen und Schülern die bestmögliche Bildung zur Verfügung zu stellen. Wir haben keine Zeit zu verlieren, denn hohe Jugendarbeitslosigkeit, wachsende soziale Ungleichheit, beträchtliche geschlechtsspezifische Ungleichheiten und das dringende Erfordernis, das Wirtschaftswachstum anzukurbeln, sind Faktoren, die die Realität zahlreicher Länder kennzeichnen. Die OECD steht bereit, um die Politikverantwortlichen bei diesem schwierigen und entscheidenden Unterfangen zu unterstützen.

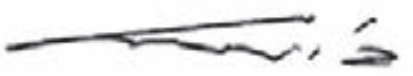

Angel Gurría

Generalsekretär der OECD 


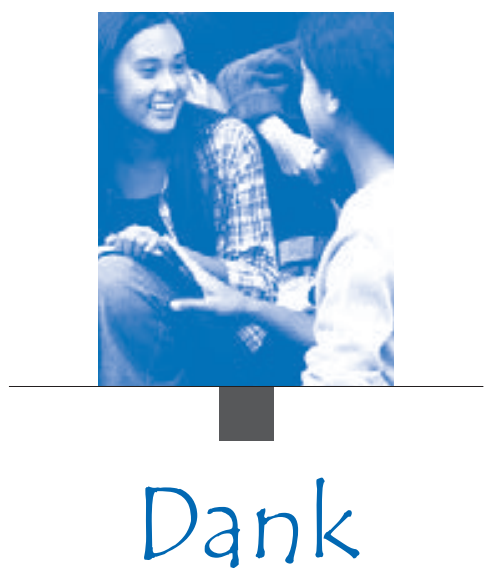

Dieser Bericht ist das Ergebnis eines Kooperationsprojekts der PISA-Teilnehmerländer, der im PISA-Konsortium vertretenen Experten und Einrichtungen sowie des OECD-Sekretariats. Verfasst wurde er von Andreas Schleicher, Francesco Avvisati, Francesca Borgonovi, Miyako Ikeda, Hiromichi Katayama, Flore-Anne Messy, Chiara Monticone, Guillermo Montt, Sophie Vayssettes und Pablo Zoido aus der OECD-Direktion für Bildung und Kompetenzen und der OECD-Direktion für Finanzfragen. Simone Bloem und Giannina Rech sorgten für Unterstützung im Bereich Statistik, und Marilyn Achiron übernahm die redaktionelle Koordination. Analytische und redaktionelle Unterstützung kam darüber hinaus von Adele Atkinson, Jonas Bertling, Marika Boiron, Célia Braga-Schich, Tracey Burns, Michael Davidson, Cassandra Davis, Elizabeth Del Bourgo, John A. Dossey, Joachim Funke, Samuel Greiff, Tue Halgreen, Ben Jensen, Eckhard Klieme, André Laboul, Henry Levin, Juliette Mendelovits, Tadakazu Miki, Christian Monseur, Simon Normandeau, Mathilde Overduin, Elodie Pools, Dara Ramalingam, William H. Schmidt (dessen Studie zum Thema Lernmöglichkeiten mit dem Forschungsstipendium Thomas J. Alexander gefördert wurde), Kaye Stacey, Lazar Stankov, Ross Turner, Elisabeth Villoutreix und Allan Wigfield. Für die Erhebung der Systemdaten waren Bonifacio Agapin, Estelle Herbaut und Jean Yip vom NESLI-Team der OECD zuständig. Band II stützt sich zudem auf von Jaap Scheerens und Douglas Willms im Zusammenhang mit PISA 2000 durchgeführte Analysen. Für die administrative Seite waren Claire Chetcuti, Juliet Evans, Jennah Huxley und Diana Tramontano zuständig.

Der Australian Council for Educational Research (ACER) wurde von der OECD beauftragt, die Entwicklung der Rahmenkonzepte in den Bereichen Mathematik, Problemlösen und finanzielle Allgemeinbildung für PISA 2012 zu leiten. Darüber hinaus beauftragte die OECD die Einrichtung Achieve, mit dem ACER das Mathematik-Rahmenkonzept auszuarbeiten. Kaye Stacey führte den Vorsitz in der Expertengruppe, die die Ausarbeitung des Rahmenkonzepts und der Erhebungsinstrumente im Bereich Mathematik leitete, Joachim Funke war mit der Leitung der entsprechenden Expertengruppe im Bereich Problemlösen betraut, und Annamaria Lusardi stand der Expertengruppe im Bereich finanzielle Allgemeinbildung vor. Die PISA-Erhebungsinstrumente und das Datenmaterial für den Bericht wurden vom PISA-Konsortium unter der Leitung von Raymond Adams vom ACER vorbereitet.

Die Orientierungen für die Gestaltung des Berichts insgesamt kamen vom PISA-Verwaltungsrat unter dem Vorsitz von Lorna Bertrand (Vereinigtes Königreich) und dem stellvertretenden Vorsitz von Benó Csapó (Ungarn), Daniel McGrath (Vereinigte Staaten) und Ryo Watanabe (Japan). In Anhang C sind die Mitglieder der verschiedenen PISA-Organe wie auch die Fachleute und Consultants aufgeführt, die an diesem Bericht und der PISA-Erhebung allgemein mitgewirkt haben. 



\section{Inhaltsverzeichnis}

ZUSAMMENFASSUNG

HINWEISE FÜR DEN LESER

WAS IST PISA?

KAPITEL 1 DEFINITION UND MESSUNG DER BILDUNGSGERECHTIGKEIT.

Wie im Rahmen von PISA die Bildungsgerechtigkeit untersucht wird

- Quantität und Qualität der Bildungsressourcen

- Unterrichtsinhalt und Unterrichtspraxis

- Bessere Leistungen mit mehr Bildungsgerechtigkeit verbinden ………....................................................................................................... 30

Untersuchung der Frage der Bildungsgerechtigkeit in dieser Berichtsreihe ………............................................................. 31

KAPITEL 2 VERTEILUNGSGERECHTIGKEIT DER BILDUNGSERTRÄGE _.................................................................. 33

Leistungen und sozioökonomischer Status im Vergleich der Schulsysteme …................................................................... 35

- Leistungsunterschiede im Zusammenhang mit dem sozioökonomischen Status .......................................................................... 39

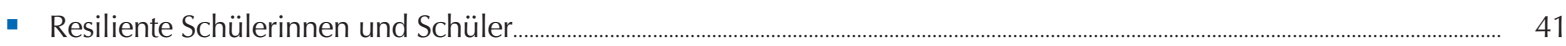

- Durchschnittsergebnisse nach Berücksichtigung des sozioökonomischen Status ...................................................................... 41

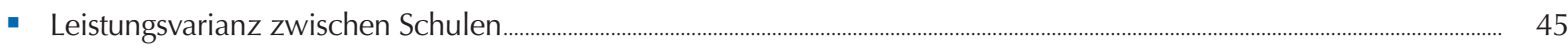

- Leistungsunterschiede zwischen Schulen und sozioökonomische Disparitäten ................................................................. 49

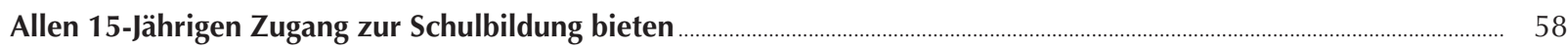

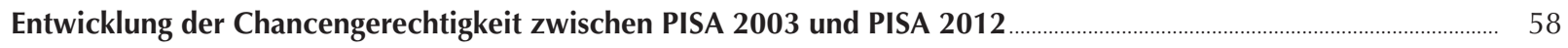

- Entwicklung des Profils und der Verteilung der Schülerinnen und Schüler in den Schulen ............................................... 61

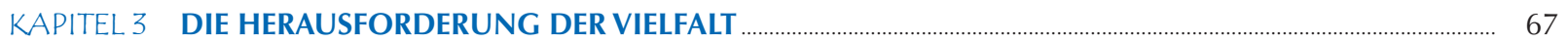

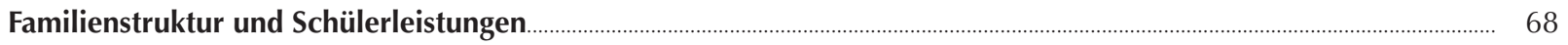

Erwerbsstatus der Eltern: Zielausrichtung der Bildungspolitik durch sozialpolitische Maßnahmen für Arbeitslose.. 71

Standort der Schulen und Leistungsunterschiede zwischen verschiedenen geografischen Gebieten ............................ 71

Bildungsgerechtigkeit für Schülerinnen und Schüler mit Migrationshintergrund .................................................................... 76

- Auswirkungen anderer sozialpolitischer Maßnahmen auf das Profil der Schülerinnen und Schüler mit

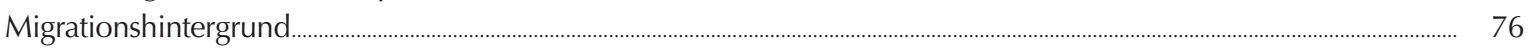

- Ein hohes Leistungsniveau in einer vielfältigen Schülerpopulation ......................................................................................... 77

- Ein höheres Leistungsniveau in einer zunehmend vielfältigen Schülerpopulation ................................................................... 79

- Sprachminderheiten unter den Schülerinnen und Schülern mit Migrationshintergrund ...................................................... 84

- Schülerinnen und Schüler der ersten und zweiten Generation.............................................................................................................. 85

- „Nachteil des späten Zuwanderungsalters" ................................................................................................................................................ 86

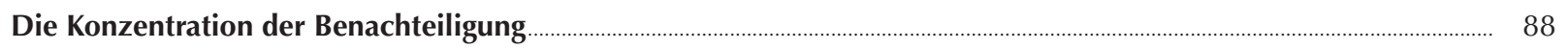

Leistung, Migrationsstatus und Herkunftsland ……...................................................................................................................... 90

KAPITEL 4 CHANCENGERECHTIGKEIT BEI DEN LERNMÖGLICHKEITEN UND

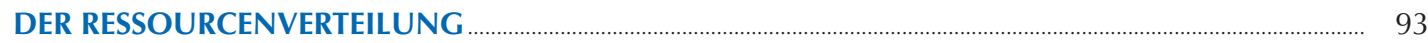

Disparitäten beim Kontakt mit reiner Mathematik, beim sozioökonomischen Hintergrund und bei den Leistungen.... 97

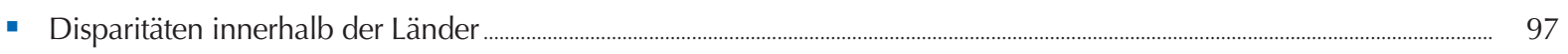

EXZELLENZ DURCH CHANCENGERECHTIGKEIT: ALLEN SCHÜLERINNEN UND SCHÜLERN DIE VORAUSSETZUNGEN ZUM ERFOLG SICHERN - BAND II 
- Unterschiede beim Kontakt mit Mathematik und durchschnittliche Mathematikleistungen in verschiedenen Schulsystemen.

- Zwischenschulische Unterschiede bei den Lernmöglichkeiten, dem sozioökonomischen Hintergrund und den Leistungen

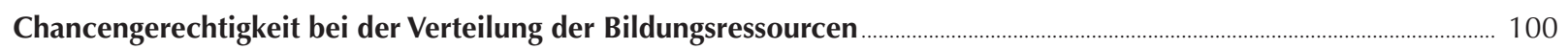

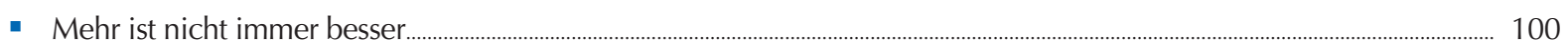

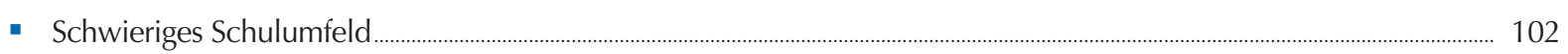

- Lernmöglichkeiten außerhalb der Schule und Erwartungen der Eltern an die Schulen............................................................ 102

Lernmöglichkeiten, Ressourcen, Leistungen und soziö̈konomischer Status ......................................................................... 105

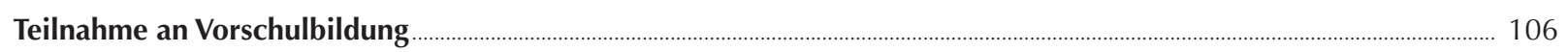

KAPITEL 5 POLITIKIMPLIKATIONEN DER FRAGE DER BILDUNGSGERECHTIGKEIT _............................................. 111

Zusammenhangsmuster zwischen Schülerleistungen und sozioökonomischem Hintergrund ........................................... 113

Überproportional hoher Anteil leistungsschwacher Schüler ............................................................................................................. 120

Unterschiedliche Steigung und Stärke der sozioökonomischen Gradiente ................................................................................. 120

- Allgemein ausgerichtete Maßnahmen für Länder, in denen die Leistungsunterschiede gering sind und ein schwacher Zusammenhang zwischen Leistung und sozioökonomischem Status besteht....................................................... 121

- Maßnahmen, die bei der sozioökonomischen Benachteiligung ansetzen, für Länder mit geringen Leistungsunterschieden und einem starken Zusammenhang zwischen Leistung und sozioökonomischem Status....

- Maßnahmen, die sowohl bei der Leistung als auch bei der sozioökonomischen Benachteiligung ansetzen, für Länder mit großen Leistungsunterschieden und einem starken Zusammenhang zwischen Leistung und sozioökonomischem Status.

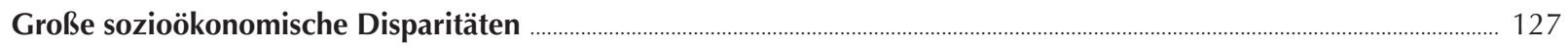

Gezielte Ausrichtung von Maßnahmen auf leistungsschwache und sozioökonomisch benachteiligte Schulen ........... 129

Gezielte Ausrichtung von Maßnahmen auf bestimmte Schülerinnen und Schüler innerhalb von Schulen ................... 131

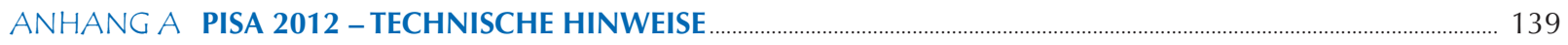

Anhang A1 Konstruktion der auf den Kontextfragebogen für Schüler, Schulen und Eltern beruhenden Mathematikskalen

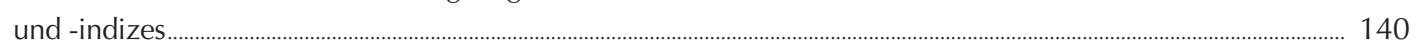

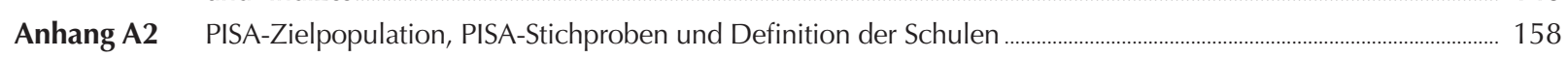

Anhang A3 Technische Hinweise zu den in diesem Band enthaltenen Analysen ………...................................................................... 171

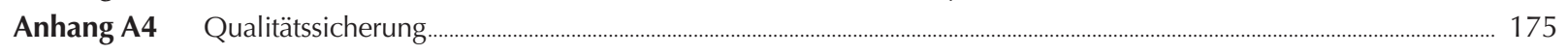

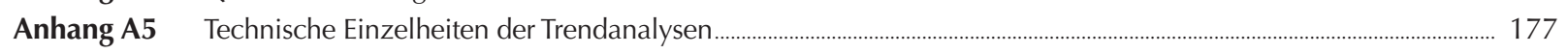

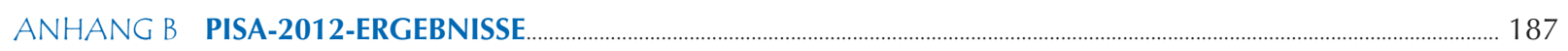

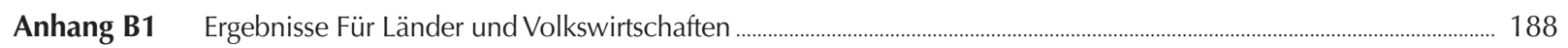

ANHANG C ENTWICKLUNG UND UMSETZUNG VON PISA - EIN KOOPERATIONSPROJEKT .............................. 295 


\section{KÄSTEN}

Kasten II.1.1 Wie sind die PISA-Ergebnisse zu interpretieren?

Kasten II.2.

Was ist der sozioökonomische Status und wie wird er gemessen?

Kasten II.2.2

Standort, Regionen und Varianz der Schülerleistungen.

Kasten II.2.3 Welche Merkmale weisen Schülerinnen und Schüler auf, die sozioökonomisch begünstigte bzw. benachteiligte Schulen besuchen?

Kasten II.2.4 Verbesserung der PISA-Ergebnisse: Mexiko

Kasten II.2

Vergleich der Indizes zwischen PISA 2003 und PISA 2012

Kasten II.3.1 Populationsrelevanz oder zuschreibbares Risiko.......

Kasten II.3.2 Verbesserung der PISA-Ergebnisse: Deutschland

Kasten II.3.3 Sprachminderheiten unter den Schülerinnen und Schülern ohne Migrationshintergrund ........................................................ $\quad$ 8

Kasten II.5.1 Ein Rahmen für Maßnahmen zur Verbesserung der Schülerleistungen und der Bildungsgerechtigkeit

\section{ABBILDUNGEN}

Überblick über die PISA-Teilnehmerländer und -volkswirtschaften

Abbildung II.1.1 Wahrscheinlichkeit positiver sozialer und wirtschaftlicher Ergebnisse unter Erwachsenen mit hoher Lesekompetenz ..

Abbildung II.1.2 Schülerleistungen und Bildungsgerechtigkeit

Abbildung II.2.1 Sozioökonomischer Status und Leistungen der Schüler, OECD-Länder.

Abbildung II.2.2 Vergleich der Mathematikleistungen und der Bildungsgerechtigkeit der Länder/Volkswirtschaften.

Abbildung II.2.3 Anteil der durch Merkmale des sozioökonomischen Status erklärten Varianz der Mathematikleistungen ......

Abbildung II.2.4 Prozentsatz resilienter Schüler.....

Abbildung II.2.5 Durchschnittsergebnisse in Mathematik, vor und nach Berücksichtigung des sozioökonomischen Profils der Länder/Volkswirtschaften.

Abbildung II.2.6 Durchschnittliche Mathematikleistungen, nach nationalen Quartilen des sozioökonomischen Status..

Abbildung II.2.a Durchschnittliche Mathematikleistungen in den Ländern und Regionen.

Abbildung II.2.7 Gesamtvarianz der Mathematikleistungen sowie Varianz zwischen und innerhalb von Schulen .....

Abbildung II.2.8 Leistungsvarianz zwischen und innerhalb von Schulen, erklärt durch den sozioökonomischen Status der Schüler

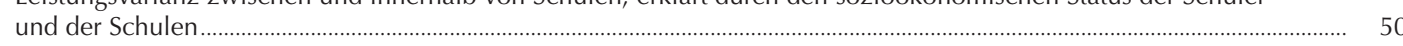

Abbildung II.2.9 Leistungsunterschiede innerhalb und zwischen Schulen zwischen verschiedenen sozioökonomischen Gruppen............... 51

Abbildung II.2.10 Mathematikleistungen der Schüler an sozioökonomisch begünstigten, durchschnittlichen und benachteiligten Schulen ..

Abbildung II.2.11 Verteilung der Schüler in Bezug auf die Leistungen und sozioökonomischen Profile der Schulen ......

Abbildung II.2.12 Veränderung der Stärke der sozioökonomischen Gradiente zwischen 2003 und 2012 und annualisierte Mathematikergebnisse

Abbildung II.2.13 Veränderung der Steigung der sozioökonomischen Gradiente zwischen 2003 und 2012 und annualisierte

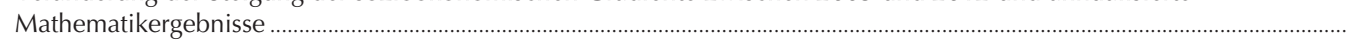

Abbildung II.2.14 Veränderung der Schülerresilienz gegenüber dem sozioökonomischen Status zwischen 2003 und 2012

Abbildung II.2.15 Veränderungen der sozialen Inklusion zwischen 2003 und 2012

Abbildung II.3.1 Leistungsunterschied im Bereich Mathematik, nach Familientyp

Abbildung II.3.2 Leistungsunterschied im Bereich Mathematik, nach Erwerbsstatus der Eltern .......................................................

Abbildung II.3.3 Durchschnittsergebnisse im Bereich Mathematik, nach Schulstandort, nach Berücksichtigung des sozioökomischen Status..... 75

Abbildung II.3.4 Leistungsunterschied in Mathematik zwischen Schülern mit und ohne Migrationshintergrund.................................... 78

Abbildung II.3.5 Veränderung der Mathematikleistungen der Schüler mit Migrationshintergrund zwischen 2003 und 2012 .................... 80

Abbildung II.3.6 Veränderung des Anteils der Schüler mit Migrationshintergrund zwischen 2003 und 2012 _................................... 81

Abbildung II.3.7 Leistungsunterschied im Bereich Mathematik, nach Migrationsstatus und sprachlichem Hintergrund .......................... 86

Abbildung II.3.8 Prozentsatz der Schüler mit Mathematikleistungen unter bzw. über dem Grundkompetenzniveau (Stufe 2), nach Migrationshintergrund 
Abbildung II.3.9 Anteil der Schüler mit Migrationshintergrund in sozioökonomisch benachteiligten, durchschnittlichen und begünstigten

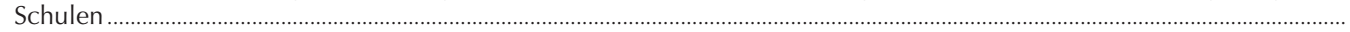

Abbildung II.4.1 Überblick über die PISA-Messgrößen der Chancengerechtigkeit beim Kontakt mit reiner Mathematik.

Abbildung II.4.2 Umfang der Leistungsunterschiede, die mit dem Kontakt der Schüler mit reiner Mathematik zusammenhängen, nach sozioökonomischem Profil der Schulen

Abbildung II.4.3 Zusammenhang zwischen den Mathematikleistungen und der Varianz des Kontakts der Schüler mit reiner Mathematik....... 98

Abbildung II.4.4 Zwischenschulische Unterschiede beim Kontakt mit reiner Mathematik, beim sozioökonomischen Status und bei den Leistungen.

Abbildung II.4.5 Überblick über die PISA-Messgrößen der Chancengerechtigkeit bei der Verteilung der Bildungsressourcen ......................... 101

Abbildung II.4.6 Anzahl und Qualität der Lehrkräfte, nach sozioökonomischem Profil der Schulen .................................................................. 103

Abbildung II.4.7 Unterschiede bei der Lehrerqualität, erklärt durch das sozioökonomische Profil der Schüler und der Schulen .................... 104

Abbildung II.4.8 Unterschiede bei der Schuldisziplin, nach sozioökonomischem Profil der Schulen ...................................................................... 104

Abbildung II.4.9 Unterschiede bei der Schuldisziplin, erklärt durch das sozioökonomische Profil der Schüler und der Schulen......

Abbildung Il.4.10 Leistungsunterschiede, die mit Unterschieden beim Kontakt mit reiner Mathematik und bei den Ressourcen zusammenhängen

Abbildung II.4.11 Leistungsunterschiede im Bereich Mathematik, nach Teilnahme an Vorschulbildung ............................................................... 107

Abbildung II.4.12 Vorschulbildung, Mathematikleistungen und soziö̈konomischer Status der Schüler .............................................................. 108

Abbildung II.5.1a Überblick über die PISA-Messgrößen der Bildungsgerechtigkeit .................................................................................................. 115

Abbildung II.5.1b Überblick über die PISA-Messgrößen der Bildungsgerechtigkeit (Forts.) ….............................................................................. 116

Abbildung II.5.2 Zusammenhang zwischen den Leistungen der Schulen und ihrem sozioökonomischen Profil in Ländern mit einem hohen Anteil von Schülern, die unter Stufe 2 abschneiden: Indonesien, Kasachstan, Peru und Tunesien............................... 121

Abbildung II.5.3 Zusammenhang zwischen den Leistungen der Schulen und ihrem sozioökonomischen Profil in Ländern mit hohen Leistungen sowie flachen und schwachen Gradienten: Kanada und Vietnam

Abbildung II.5.4 Zusammenhang zwischen den Leistungen der Schulen und ihrem sozioökonomischen Profil in Ländern mit durchschnittlichen oder niedrigen Leistungen sowie flachen und schwachen Gradienten: Brasilien, Italien, Mexiko und die Vereinigten Staaten

Abbildung II.5.5 Zusammenhang zwischen den Leistungen der Schulen und ihrem sozioökonomischen Profil in Ländern mit durchschnittlichen oder niedrigen Leistungen sowie flachen und starken Gradienten: Chile, Griechenland, Malaysia und die Türkei ..... 124

Abbildung II.5.6 Zusammenhang zwischen den Leistungen der Schulen und ihrem sozioökonomischen Profil in Ländern mit hohen

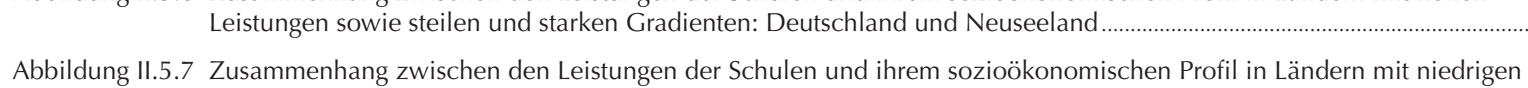

Leistungen sowie steilen und starken Gradienten: Ungarn, Israel, Singapur und die Slowakische Republik ......................... 126

Abbildung II.5.8 Zusammenhang zwischen den Leistungen der Schulen und ihrem sozioökonomischen Profil in Ländern mit starken sozioökonomischen Disparitäten und durchschnittlicher Bildungsgerechtigkeit: Bulgarien, Kolumbien, Costa Rica und Irland

Abbildung II.5.9 Zusammenhang zwischen den Leistungen der Schulen und ihrem sozioökonomischen Profil in Ländern mit hoher Bildungsgerechtigkeit, aber starken sozioökonomischen Disparitäten: Hongkong (China), Jordanien, Katar und Thailand .... 128

Abbildung II.5.10 Zusammenhang zwischen den Leistungen der Schulen und ihrem sozioökonomischen Profil in Ländern mit starken zwischenschulischen Gradienten: Island und Uruguay

Abbildung II.5.11 Zusammenhang zwischen den Leistungen der Schulen und ihrem sozioökonomischen Profil in Ländern mit schwachen zwischenschulischen Gradienten: Norwegen und Portugal.

Abbildung II.5.12 Zusammenhang zwischen den Leistungen der Schulen und ihrem sozioökonomischen Profil in Ländern mit starken innerschulischen Leistungsunterschieden: Dänemark, Finnland, Spanien und die Schweiz

Abbildung II.5.13 Zusammenhang zwischen den Leistungen der Schulen und ihrem sozioökonomischen Profil in allen anderen Ländern und Volkswirtschaften

Abbildung A3.1 Bezeichnungen in einer zweidimensionalen Tabelle.

Abbildung A5.1 Annualisierte Veränderung der Mathematikleistungen seit PISA 2003 und beobachtete Leistungsdifferenz zwischen PISA 2012 und PISA 2003.

Abbildung A5.2 Annualisierte Veränderung der Leistungen in Lesekompetenz seit PISA 2000 und beobachtete Leistungsdifferenz zwischen PISA 2012 und PISA 2000

Abbildung A5.3 Annualisierte Veränderung der Leistungen in Naturwissenschaften seit PISA 2006 und beobachtete Leistungsdifferenz zwischen PISA 2012 und PISA 2006. 


\section{TABELLEN}

Tabelle II.A Überblick über die Bildungsgerechtigkeit in PISA 2012 und Veränderung seit PISA 2003...

Tabelle A1.1 Bildungsabschluss der Eltern, umgerechnet in Bildungsjahre

Tabelle A1.2 Mehrebenenmodell zur Schätzung von Klasseneffekten im Bereich Mathematik nach Berücksichtigung mehrerer Hintergrundvariablen .....

Tabelle A1.3 Rotationsverfahren beim Schülerfragebogen .

Tabelle A2.1 PISA-Zielpopulationen und -Stichproben

Tabelle A2.2

Ausschlüsse

Tabelle A2.3

Beteiligungsquoten.

Tabelle A2.4a Prozentsatz der Schüler in den einzelnen Klassenstufen ...

Tabelle A2.4b

Prozentsatz der Schüler in den einzelnen Klassenstufen, nach Geschlecht

Tabelle A5. 1

Linking-Fehler bei Leistungsvergleichen zwischen PISA 2012 und früheren Erhebungen

Tabelle A5.2

Linking-Fehler bei Vergleichen der Kompetenzstufen zwischen PISA 2012 und früheren Erhebungen...

Tabelle A5.3

Linking-Fehler bei Vergleichen der annualisierten und kurvilinearen Veränderung zwischen PISA 2012 und früheren Erhebungen...

Tabelle II.2.1 Zusammenhang zwischen den Leistungen in Mathematik, Lesekompetenz und Naturwissenschaften und dem sozioökonomischen Status

Tabelle II.2.2 Merkmale des sozioökonomischen Status, nach Quartilen des sozioökonomischen Status innerhalb der

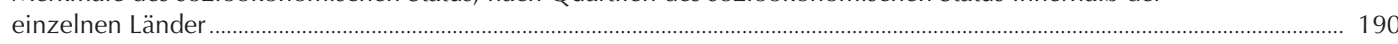

Tabelle II.2.3 Merkmale des soziö̈konomischen Status im Ländervergleich................................................................................................... 197

Tabelle II.2.4a Sozioökonomischer Status der Schüler und Mathematikleistungen ............................................................................................ 199

Tabelle II.2.4b Veränderung beim sozioökonomischen Status und bei den Mathematikleistungen der Schüler zwischen 2003 und 2012 .... 200

Tabelle II.2.5 Schülerleistungen und ausgewählte Merkmale des sozioökonomischen Status im Ländervergleich ......................................... 203

Tabelle II.2.6 Zusammenhang zwischen Mathematikleistungen und Merkmalen des sozioökonomischen Status ........................................ 207

Tabelle II.2.7a Prozentsatz der resilienten Schüler und der Leistungsschwachen unter den sozioökonomisch benachteiligten Schülern,

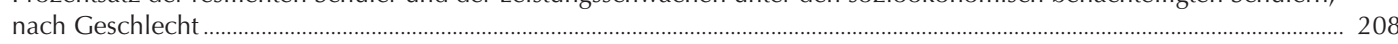

Tabelle II.2.7b Veränderung beim Prozentsatz der resilienten Schüler unter den sozioökonomisch benachteiligten Schülern zwischen

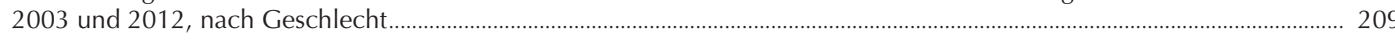

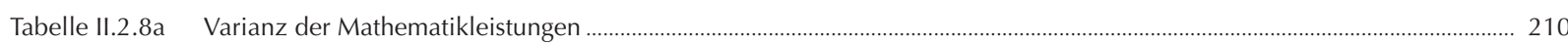

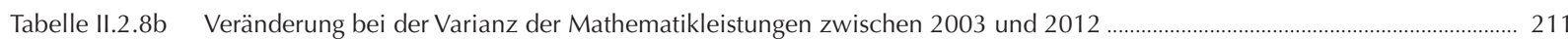

Tabelle II.2.9a Zusammenhang zwischen den Mathematikleistungen und dem sozioökonomischen Status, zwischen und innerhalb

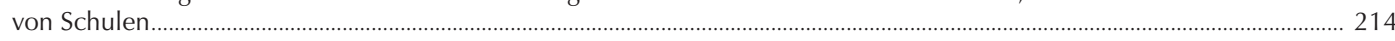

Tabelle II.2.9b Von 2003 bis 2012 festzustellende Veränderung des Zusammenhangs zwischen den Mathematikleistungen und dem sozioökonomischen Status, zwischen und innerhalb von Schulen ............................................................................................ 216

Tabelle II.2.10 Bildungsabschluss und Beruf der Eltern sowie Ausstattung des Elternhauses der Schüler, nach dem sozioökonomischen Profil der Schulen

Tabelle II.2.11 Leistungen und sozioökonomisches Profil der Schulen ......................................................................................................... 222

Tabelle II.2.12 Veränderung der Bildungsteilnahme 15-Jähriger zwischen 2003 und 2012 ............................................................................. 224

Tabelle II.2.13a Soziö̈konomischer Status der Schüler ...................................................................................................................................... 226

Tabelle II.2.13b Veränderung beim sozioökonomischen Status der Schüler zwischen 2003 und 2012 …......................................................... 228

Tabelle II.3.1 Schülerleistungen in Mathematik und Familientyp............................................................................................................................. 231

Tabelle II.3.2 Schülerleistungen in Mathematik, Erwerbsstatus und sozioökonomischer Status der Eltern..................................................... 233

Tabelle II.3.3a Zusammenhang zwischen den Schülerleistungen in Mathematik und dem Schulstandort ....................................................... 236

Tabelle II.3.3b Von 2003 bis 2012 festzustellende Veränderung des Zusammenhangs zwischen Mathematikleistungen und

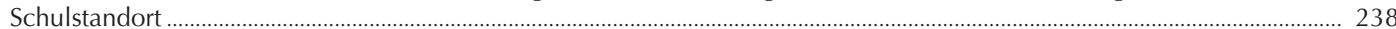

Tabelle II.3.4a Schülerleistungen in Mathematik und Migrationshintergrund ….......................................................................................... 241

Tabelle II.3.4b Von 2003 bis 2012 festzustellende Veränderung des Zusammenhangs zwischen Mathematikleistungen und Migrationshintergrund 
Tabelle II.3.5 Mathematikleistungen, Migrationshintergrund und zu Hause gesprochene Sprache.............................................................2 246

Tabelle II.3.6a Mathematikleistungen und Migrationshintergrund für Schüler der 1. und 2. Generation........................................................ 252

Tabelle Il.3.6b Von 2003 bis 2012 beobachtete Veränderung des Zusammenhangs zwischen Mathematikleistungen und Migrationshintergrund für Schüler der 1. und 2. Generation ............................................................................................... 253

Tabelle II.3.7 Kompetenzstufen im Bereich Mathematik, nach Migrationshintergrund für Schüler der 1. und 2. Generation ..................... 259

Tabelle II.3.8 Mathematikleistungen, Schüler der 1. Generation und Zuwanderungsalter ................................................................................ 260

Tabelle II.3.9 Konzentration der Schüler mit Migrationshintergrund in der Schule ............................................................................................... 262

Tabelle II.3.10 Konzentration von Schülern, die zu Hause nicht die Testsprache sprechen .............................................................................. 264

Tabelle II.3.11 Aufnahmeland/-volkswirtschaft, Herkunftsland und Mathematikleistungen …….................................................................. 266

Tabelle II.4.1 Chancengerechtigkeit bei den Lernmöglichkeiten: Reine Mathematik ................................................................................ 268

Tabelle II.4.2 Mathematikleistungen und Schülerpopulation, nach sozioökonomischem Profil der Schulen............................................. 271

Tabelle II.4.3 Ungleichheit beim Zugang zu Unterrichtsinhalten: Reine Mathematik ......................................................................................... 275

Tabelle II.4.4 Korrelation zwischen den Schülerleistungen und ausgewählten Schüler- und Schulmerkmalen .............................................. 277

Tabelle II.4.5 Korrelation zwischen dem sozioökonomischen Status der Schüler und ausgewählten Schüler- und Schulmerkmalen........ 278

Tabelle II.4.6 Korrelation zwischen dem sozioökonomischen Profil der Schulen und ausgewählten Schüler- und Schulmerkmalen........ 279

Tabelle II.4.7 Korrelation zwischen der Varianz des sozioökonomischen Status der Schüler innerhalb einer Schule und ausgewählten Schüler- und Schulmerkmalen........................................................................................................................................ 280

Tabelle II.4.8 Ungleichheit beim Zugang zu Bildungsressourcen: Schüler/Lehrer-Quote …………............................................................. 281

Tabelle II.4.9 Ungleichheit beim Zugang zu Bildungsressourcen: Prozentsatz der Lehrkräfte mit Hochschulabschluss............................. 283

Tabelle II.4.10 Ungleichheit beim Zugang zu Bildungsressourcen: Schuldisziplin........................................................................................... 285

Tabelle II.4.11 Effekt des sozioökonomischen Status nach Berücksichtigung der Schülermerkmale und der Bildungsressourcen

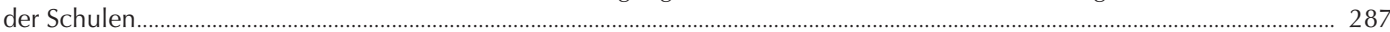

Tabelle II.4.12 Teilnahme an Vorschulbildung, Mathematikleistungen und sozioökonomischer Status der Schüler ........................................ 290

Tabelle II.4.13 Zusammenhang zwischen Leistungen, Teilnahme an Vorschulbildung und sozioökonomischem Status ............................... 293

Tabelle II.4.14 Zusammenhang zwischen Leistungen, Teilnahme an Vorschulbildung und Migrationshintergrund.................................... 294

\section{Dieser Bericht enthält 《lstan ermöglicht, Dateien im Excel-Format herunterzuladen.}

Suchen Sie die StatLinks rechts unter den in diesem Bericht wiedergegebenen Tabellen oder Abbildungen. Um die entsprechende Datei im Excel-Format herunterzuladen, genügt es, den jeweiligen Link, beginnend mit $\boldsymbol{h t t p : / / d x . d o i . o r g , ~ i n ~ d e n ~ I n t e r n e t b r o w s e r ~ e i n z u g e b e n . ~}$ Wenn Sie die elektronische PDF-Version online lesen, dann brauchen Sie nur den Link anzuklicken. Sie finden StatLinks in weiteren OECD-Publikationen. 


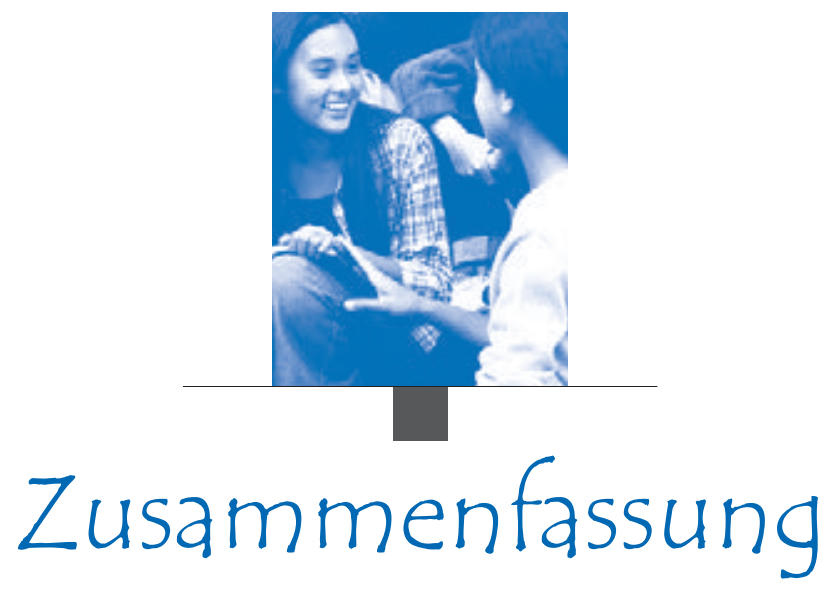

In PISA bedeutet Bildungsgerechtigkeit, dass allen Schülerinnen und Schülern, unabhängig von ihrem Geschlecht, ihrem familiären Hintergrund oder ihrem sozioökonomischen Status, die gleichen Bildungschancen geboten werden. Nach dieser Definition heißt Bildungsgerechtigkeit nicht, dass alle die gleichen Ergebnisse erzielen sollten. Vielmehr ist sie so zu verstehen, dass der sozioökonomische Status der Schülerinnen und Schüler oder die Tatsache, dass sie einen Migrationshintergrund haben, kaum oder gar keinen Einfluss auf ihre Leistungen hat und dass allen Schülerinnen und Schülern unabhängig von ihrer Herkunft Zugang zu qualitativ hochwertigen Bildungsressourcen und Lernmöglichkeiten geboten wird.

Unter den 39 Ländern und Volkswirtschaften, die an PISA 2003 und an PISA 2012 teilgenommen haben, gelang es Mexiko, der Türkei und Deutschland, in diesem Zeitraum sowohl ihre Leistungen im Bereich Mathematik als auch die Chancengerechtigkeit in der Bildung zu steigern.

Diesen drei Ländern gelang es, sowohl die Chancengerechtigkeit als auch die Schülerleistungen zu erhöhen, entweder weil sich der Zusammenhang zwischen sozioökonomischem Hintergrund und Mathematikleistungen verringert hat oder weil der durchschnittliche Leistungsabstand zwischen sozioökonomisch benachteiligten und sozioökonomisch begünstigten Schülern abgenommen hat. Zehn weitere Länder und Volkswirtschaften erzielten 2012 bessere Ergebnisse als 2003, ohne dass sich der Grad der Bildungsgerechtigkeit verändert hätte.

Australien, Kanada, Estland, Finnland, Hongkong (China), Japan, Korea, Liechtenstein, Macau (China) und die Niederlande erzielen zugleich hohe Leistungen und eine gerechte Verteilung der Bildungschancen, wie sie im Rahmen von PISA 2012 gemessen wurden.

Die Chancengerechtigkeit in der Bildung entspricht in zehn Ländern und Volkswirtschaften dem Durchschnitt, in drei der 23 Länder und Volkswirtschaften, deren Leistungen in Mathematik den OECD-Durchschnitt übertreffen, liegt sie darunter. In allen Ländern und Volkswirtschaften, die an PISA 2012 teilnahmen, hat der sozioökonomische Status der Schülerinnen und Schüler starke Auswirkungen auf ihre Leistungen. Im OECD-Raum erklären sich 15\% der Varianz der Schülerleistungen in Mathematik aus Unterschieden im sozioökonomischen Hintergrund der Schülerinnen und Schüler. Unter den am besten abschneidenden Ländern reicht der Anteil von 3\% in Macau (China) bis zu 20\% in Belgien. Dagegen kann in Bulgarien, Chile, Frankreich, Ungarn, Peru, der Slowakischen Republik und Uruguay über 20\% des Unterschieds bei den Schülerleistungen dem sozioökonomischen Hintergrund der Schülerinnen und Schüler zugeschrieben werden.

Im OECD-Raum erzielten sozioökonomisch bessergestellte Schülerinnen und Schüler im Bereich Mathematik durchschnittlich 39 Punkte mehr - was einem Leistungsvorsprung von fast einem Schuljahr entspricht - als Schülerinnen und Schüler aus weniger begünstigten Verhältnissen.

Unter den 23 leistungsstärksten Ländern und Volkswirtschaften sind die durch den sozioökonomischen Status bedingten Leistungsunterschiede in Kanada, Estland, Finnland, Hongkong (China), Macau (China) und Vietnam unterdurchschnittlich stark, sie entsprechen in 12 Ländern und Volkswirtschaften etwa dem Durchschnitt und liegen in 5 darüber. Auffällige Leistungsunterschiede werden auch unter Schülerinnen und Schülern in sozioökonomisch begünstigten Schulen und in benachteiligten Schulen beobachtet: Im Durchschnitt der OECD-Länder schneiden Schülerinnen und Schüler, die in sozioökonomischer Hinsicht begünstigte Schulen besuchen, in Mathematik um mehr als 104 Punkte besser ab als Schüler in sozioökonomisch benachteiligten Schulen. 
In den OECD-Ländern sind rd. 6\% der gesamten Schülerpopulation "resilient", was bedeutet, dass sie im Vergleich zu Schülerinnen und Schülern aus anderen Ländern trotz eines ungünstigen sozioökonomischen Hintergrunds über Erwarten gut abschneiden.

In Hongkong (China), Macau (China), Shanghai (China), Singapur und Vietnam sind mindestens 13\% der gesamten Schülerpopulation resilient und gehören zu den 25\% der Schülerinnen und Schüler aller teilnehmenden Länder und Volkswirtschaften, die nach Berücksichtigung des sozioökonomischen Status die höchsten Ergebnisse erzielen. Zwischen 2003 und 2012 nahm der Anteil der resilienten Schülerinnen und Schüler in Deutschland, Italien, Mexiko, Polen, Tunesien und der Türkei zu.

In den OECD-Ländern ist der Anteil der Schülerinnen und Schüler mit Migrationshintergrund zwischen 2003 und 2012 von 9\% auf 12\% gestiegen, während sich der Leistungsabstand in Mathematik zwischen Schülerinnen und Schülern mit und ohne Migrationshintergrund im selben Zeitraum um 10 Punkte verringert hat.

Meistens sind Schülerinnen und Schüler mit Migrationshintergrund im Vergleich zu Schülern ohne Migrationshintergrund in sozioökonomischer Hinsicht benachteiligt, und selbst bei einem Vergleich von Schülerinnen und Schülern mit ähnlichem sozioökonomischem Status schneiden Schüler mit Migrationshintergrund in Mathematik schlechter ab als jene ohne Migrationshintergrund. 2012 erreichten sie vor Berücksichtigung des sozioökonomischen Status in Mathematik im Durchschnitt 37 Punkte weniger als Schüler ohne Migrationshintergrund, nach Berücksichtigung des sozioökonomischen Status waren es durchschnittlich 23 Punkte weniger. In Kanada, Irland, Israel, Neuseeland und dem Vereinigten Königreich schneiden Schülerinnen und Schüler mit und ohne Migrationshintergrund indessen gleich gut ab.

\section{Zwischen der Konzentration der zugewanderten Schülerinnen und Schüler in einer Schule und schlechten} Leistungen besteht nicht automatisch ein Zusammenhang.

Im Allgemeinen sind Schülerinnen und Schüler mit Migrationshintergrund und jene, die die Testsprache zu Hause nicht sprechen, tendenziell in benachteiligten Schulen konzentriert. In den Vereinigten Staaten beispielsweise sind $40 \%$ der Schülerinnen und Schüler in sozioökonomisch benachteiligten Schulen Schüler mit Migrationshintergrund, in sozioökonomisch begünstigten Schulen sind es indessen 13\%. Im OECD-Raum erzielen Schüler, die eine Schule besuchen, in der mehr als jeder vierte Schüler ein Schüler mit Migrationshintergrund ist, tendenziell schlechtere Ergebnisse als Schüler in Schulen, in denen es keine Schüler mit Migrationshintergrund gibt. Jedoch reduziert sich der 19-Punkte-Unterschied zwischen den beiden Gruppen nach Berücksichtigung des sozioökonomischen Status der Schüler und Schulen um mehr als die Hälfte - auf 7 Punkte. Belgien, Estland Griechenland, Mexiko und Portugal sind die einzigen Länder, in denen die Leistungsunterschiede zwischen diesen beiden Gruppen nach Berücksichtigung des sozioökonomischen Status 20 Punkte oder mehr betragen.

Im OECD-Durchschnitt erzielten Schülerinnen und Schüler, die eigenen Angaben zufolge über ein Jahr an Vorschulbildung teilgenommen hatten, im Bereich Mathematik 53 Punkte mehr - was einem Leistungsvorsprung von über einem Schuljahr entspricht - als Schülerinnen und Schüler, die nicht an Vorschulbildung teilgenommen hatten.

In allen außer zwei Ländern mit verfügbaren Daten schneiden Schülerinnen und Schüler, die über ein Jahr an Vorschulbildung teilgenommen hatten, vor Berücksichtigung des sozioökonomischen Status besser ab als Schüler, die keine Vorschule besucht hatten. Dieses Ergebnis bleibt auch nach Berücksichtigung des sozioökonomischen Status unverändert, außer in Kroatien, Estland, Irland, Korea, Lettland, Slowenien und den Vereinigten Staaten.

Im OECD-Raum wird sozioökonomisch benachteiligten Schulen mindestens eine gleich hohe, wenn nicht höhere Zahl an Mathematiklehrern im Verhältnis zur Schülerzahl zugeteilt wie sozioökonomisch begünstigten Schulen; sozioökonomisch benachteiligte Schulen haben aber tendenziell große Schwierigkeiten, qualifizierte Lehrkräfte anzuwerben.

In den Niederlanden ist der Anteil der qualifizierten Lehrkräfte in sozioökonomisch begünstigten Schulen (52\%) dreimal höher als der Anteil der qualifizierten Lehrkräfte in sozioökonomisch benachteiligten Schulen (14\%), während die Schüler/ Lehrer-Quote in sozioökonomisch begünstigten Schulen um 28\% höher ist als in sozioökonomisch benachteiligten Schulen (18 bzw. 14 Schüler je Lehrkraft). 
Tabelle II.A [1/2]

ÜBERBLICK ÜBER DIE BILDUNGSGERECHTIGKEIT IN PISA 2012 UND VERÄNDERUNG SEIT PISA 2003

Länder/Volkswirtschaften mit über dem OECD-Durchschnitt liegenden Durchschnittsergebnissen in Mathematik

Länder/Volkswirtschaften, in denen der Zusammenhang zwischen Mathematikleistungen und sozioökonomischem Status schwächer als im OECD-Durchschnitt ist Länder/Volkswirtschaften mit unter dem OECD-Durchschnitt liegenden Leistungsunterschieden zwischen den verschiedenen sozioökonomischen Gruppen

Länder/Volkswirtschaften mit Durchschnittsergebnissen in Mathematik, die sich statistisch nicht signifikant vom OECD-Durchschnitt unterscheiden Länder/Volkswirtschaften, in denen sich der Zusammenhang zwischen Mathematikleistungen und sozioökon. Status statistisch nicht signifikant vom OECD-Durchschnitt unterscheidet Länder/Volkswirtschaften mit Leistungsunterschieden innerhalb der sozioökonomischen Verteilung, die statistisch nicht signifikant vom OECD-Durchschnitt abweichen

Länder/Volkswirtschaften mit unter dem OECD-Durchschnitt liegenden Durchschnittsergebnissen in Mathematik irtschaften, in denen der Zusammenhang zwischen Mathematikleistungen und sozioökonomischem Status stärker als im OECD-Durchschnitt ist änder/Volkswirtschaften mit über dem OECD-Durchschnitt liegenden Leistungsunterschieden innerhalb der sozioökonomischen Verteilung

\begin{tabular}{|c|c|c|c|c|}
\hline & $\begin{array}{c}\text { Mittelwert } \\
\text { in Mathematik }\end{array}$ & $\begin{array}{c}\text { Stärke des Zusammenhangs } \\
\text { zwischen Mathematikleistungen } \\
\text { und sozioökonomischem Status }\end{array}$ & $\begin{array}{c}\text { Leistungsunterschiede } \\
\text { zwischen den verschiedenen } \\
\text { sozioökonomischen Gruppen }\end{array}$ & Prozentsatz der resilienten Schüler \\
\hline & Mittelwert & $\begin{array}{l}\text { Prozentsatz der erklärten Varianz } \\
\text { der Mathematikleistungen }\end{array}$ & $\begin{array}{l}1 \text { ESCS1-Einheit entsprechende } \\
\text { Punktzahldifferenz in Mathematik }\end{array}$ & $\begin{array}{c}\text { Prozentsatz der sozioökonomisch } \\
\text { benachteiligten Schüler, die zu den } \\
25 \% \text { der Schüler aller teilnehmenden } \\
\text { Länder Nolkswirtschaften gehören, } \\
\text { die nach Berücksichtigung des ESCS1 } \\
\text { die höchsten Ergebnisse erzielen }\end{array}$ \\
\hline OECD-Durchschnitt & 494 & 14.8 & 39 & 6.4 \\
\hline Macau (China) & 538 & 2.6 & 17 & 16.9 \\
\hline Hongkong (China) & 561 & 7.5 & 27 & 18.1 \\
\hline Liechtenstein & 535 & 7.6 & 28 & 10.1 \\
\hline Estland & 521 & 8.6 & 29 & 9.5 \\
\hline Finnland & 519 & 9.4 & 33 & 8.1 \\
\hline Kanada & 518 & 9.4 & 31 & 8.3 \\
\hline Japan & 536 & 9.8 & 41 & 11.3 \\
\hline Korea & 554 & 10.1 & 42 & 12.7 \\
\hline Niederlande & 523 & 11.5 & 40 & 8.6 \\
\hline Australien & 504 & 12.3 & 42 & 6.3 \\
\hline Schweiz & 531 & 12.8 & 38 & 9.9 \\
\hline Singapur & 573 & 14.4 & 44 & 15.1 \\
\hline Irland & 501 & 14.6 & 38 & 6.3 \\
\hline Vietnam & 511 & 14.6 & 29 & 16.9 \\
\hline Shanghai (China) & 613 & 15.1 & 41 & 19.2 \\
\hline Slowenien & 501 & 15.6 & 42 & 5.9 \\
\hline Österreich & 506 & 15.8 & 43 & 6.1 \\
\hline Dänemark & 500 & 16.5 & 39 & 4.9 \\
\hline Polen & 518 & 16.6 & 41 & 8.4 \\
\hline Deutschland & 514 & 16.9 & 43 & 7.5 \\
\hline Chinesisch Taipeh & 560 & 17.9 & 58 & 12.3 \\
\hline Neuseeland & 500 & 18.4 & 52 & 5.3 \\
\hline Belgien & 515 & 19.6 & 49 & 7.1 \\
\hline Norwegen & 489 & 7.4 & 32 & 5.3 \\
\hline Island & 493 & 7.7 & 31 & 5.2 \\
\hline Ver. Königreich & 494 & 12.5 & 41 & 5.8 \\
\hline Lettland & 491 & 14.7 & 35 & 6.4 \\
\hline Tschech. Rep. & 499 & 16.2 & 51 & 5.9 \\
\hline Portugal & 487 & 19.6 & 35 & 7.7 \\
\hline Frankreich & 495 & 22.5 & 57 & 5.4 \\
\hline Katar & 376 & 5.6 & 27 & 0.4 \\
\hline Kasachstan & 432 & 8.0 & 27 & 2.1 \\
\hline Jordanien & 386 & 8.4 & 22 & 0.9 \\
\hline Indonesien & 375 & $\frac{0.4}{9.6}$ & 20 & 2.5 \\
\hline Ver. Arab. Emirate & 434 & 9.8 & 33 & 1.2 \\
\hline Thailand & 427 & 9.9 & 22 & 6.3 \\
\hline Italien & 485 & 10.1 & 30 & 6.4 \\
\hline Mexiko & 413 & 10.4 & 19 & 3.9 \\
\hline Schweden & 478 & 10.6 & 36 & 4.3 \\
\hline Russ. Föderation & 482 & 11.4 & 38 & 5.2 \\
\hline Serbien & 449 & 11.7 & 34 & 3.6 \\
\hline Kroatien & 471 & 12.0 & 36 & 5.1 \\
\hline Tunesien & 388 & 12.4 & 22 & 2.9 \\
\hline Montenegro & 410 & 12.7 & 33 & 1.3 \\
\hline Malaysia & 421 & 13.4 & $\frac{53}{30}$ & 2.7 \\
\hline Litauen & 479 & 13.8 & 36 & 5.6 \\
\hline Zypern* & 440 & 14.1 & 38 & 1.9 \\
\hline Türkei & 448 & 14.5 & 32 & 7.2 \\
\hline Ver. Staaten & 481 & 14.8 & 35 & 5.2 \\
\hline Argentinien & 388 & 15.1 & 26 & 1.1 \\
\hline Kolumbien & 376 & 15.4 & 25 & 1.5 \\
\hline Griechenland & 453 & 15.5 & 34 & 3.2 \\
\hline Brasilien & 391 & 15.7 & 26 & 1.7 \\
\hline Spanien & 484 & 15.8 & 34 & 6.4 \\
\hline Israel & 466 & 17.2 & 51 & 3.1 \\
\hline Luxemburg & 490 & 18.3 & 37 & 6.1 \\
\hline Costa Rica & 407 & 18.9 & 24 & 1.9 \\
\hline Rumänien & 445 & 19.3 & 38 & 2.8 \\
\hline Bulgarien & 439 & 22.3 & 42 & 2.1 \\
\hline Uruguay & 409 & 22.8 & 37 & 2.1 \\
\hline Ungarn & 477 & 23.1 & 47 & 4.1 \\
\hline Chile & 423 & 23.1 & 34 & 1.7 \\
\hline Peru & 368 & 23.4 & 33 & 0.5 \\
\hline Slowak. Rep. & 482 & 24.6 & 54 & 3.9 \\
\hline
\end{tabular}

Anmerkung: Länder/Volkswirtschaften, die zwischen PISA 2003 und PISA 2012 (2012-2003) eine statistisch signifikanteVeränderung aufweisen, sind durch Fettdruck gekennzeichnet. 1. ESCS bezieht sich auf den PISA-Index des wirtschaftlichen, sozialen und kulturellen Status.

Die Länder und Volkswirtschaften sind in drei Gruppen dargestellt: Länder/Volkswirtschaften, deren Durchschnittsergebnisse über dem OECD-Durchschnitt liegen, Länder/Volkswirtschaften Durchschnitt liegen. Innerhalb jeder dieser Gruppen sind die Länder/Volkswirtschaften in absteigender Reihenfolge der in PISA 2012 beobachteten Stärke des Zusammenhangs zwischen Leistung und sozioökonomischem Status angeordnet.

$*$ Vgl. Hinweise für den Leser.

Quelle: OECD, PISA-2012-Datenbank, Tabelle II.2.1, II.2.7a, II.2.7b, II.2.8b und II.2.9b. 


\section{Tabelle II.A [2/2]}

\section{ÜBERBLICK ÜBER DIE BILDUNGSGERECHTIGKEIT IN PISA 2012 UND VERÄNDERUNG SEIT PISA 2003}

\section{Länder/Nolkswirtschaften mit über dem OECD-Durchschnitt liegenden Durchschnittsergebnissen in Mathematik}

Länder/Volkswirtschaften, in denen der Zusammenhang zwischen Mathematikleistungen und sozioökonomischem Status schwächer als im OECD-Durchschnitt ist Länder/Volkswirtschaften mit unter dem OECD-Durchschnitt liegenden Leistungsunterschieden zwischen den verschiedenen sozioökonomischen Gruppen

Länder/Volkswirtschaften mit Durchschnittsergebnissen in Mathematik, die sich statistisch nicht signifikant vom OECD-Durchschnitt unterscheiden Länder/Nolkswirtschaften, in denen sich der Zusammenhang zwischen Mathematikleistungen und sozioökon. Status statistisch nicht signifikant vom OECD-Durchschnitt unterscheidet Länder/Volkswirtschaften mit Leistungsunterschieden innerhalb der sozioökonomischen Verteilung, die statistisch nicht signifikant vom OECD-Durchschnitt abweichen Länder/Volkswirtschaften mit unter dem OECD-Durchschnitt liegenden Durchschnittsergebnissen in Mathematik Volkswirtschaften, in denen der Zusammenhang zwischen Mathematikleistungen und sozioökonomischem Status stärker als im OECD-Durchschnitt is
Länder/Volkswirtschaften mit über dem OECD-Durchschnitt liegenden Leistungsunterschieden innerhalb der sozioökonomischen Verteilung

\begin{tabular}{|c|c|c|}
\hline & $\begin{array}{l}\text { Leistungstrends im Bereich } \\
\text { Mathematik }\end{array}$ & $\begin{array}{l}\text { Trends bei der Stärke des } \\
\text { Zusammenhangs zwischen } \\
\text { Mathematikleistungen und } \\
\text { sozioökonomischem Status } \\
\end{array}$ \\
\hline & $\begin{array}{l}\text { Veränderung des Mittelwerts in } \\
\text { Mathematik zwischen PISA 2003 } \\
\text { und PISA } 2012 \text { (2012-2003) }\end{array}$ & \begin{tabular}{|c|} 
Veränderung des Prozentsatzes der \\
durch den ESCS1 der Schüler erklärten \\
Varianz der Mathematikleistungen \\
zwischen PISA 2003 und \\
PISA 2012 (2012-2003)
\end{tabular} \\
\hline OECD-Durchschnitt & -3 & -2.0 \\
\hline
\end{tabular}

\begin{tabular}{c|c}
$\begin{array}{c}\text { Trends bei der Steigung der } \\
\text { sozioökonomischen Gradiente im } \\
\text { Bereich Mathematik }\end{array}$ & $\begin{array}{c}\text { Trends beim Prozentsatz der } \\
\text { resilienten Schüler }\end{array}$ \\
\hline $\begin{array}{c}\text { Veränderung der 1 ESCS1-Einheit } \\
\text { entsprechenden Punktzahldifferenz } \\
\text { in Mathematik zwischen PISA 2003 } \\
\text { und PISA 2012 (2012-2003) }\end{array}$ & $\begin{array}{c}\text { Veränderung des Prozentsatzes der } \\
\text { resilienten Schüler zwischen PISA } \\
2003 \text { und PISA 2012 (2012-2003) }\end{array}$ \\
\hline
\end{tabular}

\begin{tabular}{|c|c|c|c|c|}
\hline Macau (China) & 11 & 0.8 & 5 & -2.5 \\
\hline Hongkong (China) & 11 & -0.4 & -3 & 1.1 \\
\hline Liechtenstein & -1 & -14.9 & -19 & C \\
\hline Estland & $\mathrm{m}$ & $\mathrm{m}$ & $\mathrm{m}$ & $\mathrm{m}$ \\
\hline Finnland & -26 & -1.1 & 5 & -3.3 \\
\hline Kanada & -14 & -0.8 & 1 & -1.6 \\
\hline Japan & 2 & -2.0 & -2 & 0.5 \\
\hline Korea & 12 & -4.4 & 5 & 0.6 \\
\hline Niederlande & -15 & -6.8 & 0 & -1.7 \\
\hline Australien & -20 & -1.6 & 2 & -1.9 \\
\hline Schweiz & 4 & -5.2 & -3 & 0.8 \\
\hline Singapur & $\mathrm{m}$ & $\mathrm{m}$ & $\mathrm{m}$ & $\mathrm{m}$ \\
\hline Irland & -1 & -1.1 & 2 & -0.2 \\
\hline Vietnam & $\mathrm{m}$ & $\mathrm{m}$ & $\mathrm{m}$ & $\mathrm{m}$ \\
\hline Shanghai (China) & $\mathrm{m}$ & $\mathrm{m}$ & $\mathrm{m}$ & $\mathrm{m}$ \\
\hline Slowenien & $\mathrm{m}$ & $\mathrm{m}$ & $\mathrm{m}$ & $\mathrm{m}$ \\
\hline Österreich & 0 & 0.8 & 2 & -0.6 \\
\hline Dänemark & -14 & -0.8 & 1 & -1.7 \\
\hline Polen & 27 & 0.2 & 1 & 2.5 \\
\hline Deutschland & 11 & -6.9 & -1 & 1.3 \\
\hline Chinesisch Taipeh & $\mathrm{m}$ & $\mathrm{m}$ & $\mathrm{m}$ & $\mathrm{m}$ \\
\hline Neuseeland & -24 & 1.8 & 8 & -2.9 \\
\hline Belgien & -15 & -3.4 & -2 & -1.2 \\
\hline Norwegen & -6 & -4.7 & -8 & 1.1 \\
\hline Island & -22 & 0.6 & 5 & -1.7 \\
\hline Ver. Königreich & $\mathrm{m}$ & $\mathrm{m}$ & $\mathrm{m}$ & $\mathrm{m}$ \\
\hline Lettland & 7 & 2.8 & 1 & 0.4 \\
\hline Tschech. Rep. & -17 & -23 & 5 & -0.7 \\
\hline Portugal & 21 & 1.1 & 7 & -0.1 \\
\hline Frankreich & -16 & 2.2 & 14 & -2.5 \\
\hline Katar & $\mathrm{m}$ & $\mathrm{m}$ & $\mathrm{m}$ & $\mathrm{m}$ \\
\hline Kasachstan & $\mathrm{m}$ & $\mathrm{m}$ & $\mathrm{m}$ & $\mathrm{m}$ \\
\hline Jordanien & $\mathrm{m}$ & $\mathrm{m}$ & $\mathrm{m}$ & $\mathrm{m}$ \\
\hline Indonesien & 15 & 2.4 & -1 & 0.7 \\
\hline Ver. Arab. Emirate & $\mathrm{m}$ & $\mathrm{m}$ & $\mathrm{m}$ & $\mathrm{m}$ \\
\hline Thailand & 10 & -1.5 & -1 & -1.5 \\
\hline Italien & 20 & -2.2 & -1 & 1.8 \\
\hline Mexiko & 28 & -6.8 & -11 & 2.5 \\
\hline Schweden & -31 & -3.7 & -1 & -2.9 \\
\hline Russ. Föderation & 14 & 0.8 & 7 & -1.2 \\
\hline Serbien & $\mathrm{m}$ & $\mathrm{m}$ & $\mathrm{m}$ & $\mathrm{m}$ \\
\hline Kroatien & $\mathrm{m}$ & $\mathrm{m}$ & $\mathrm{m}$ & $\mathrm{m}$ \\
\hline Tunesien & 29 & -1.4 & -3 & 1.5 \\
\hline Montenegro & $\mathrm{m}$ & $\mathrm{m}$ & $\mathrm{m}$ & $\mathrm{m}$ \\
\hline Malaysia & $\mathrm{m}$ & $\mathrm{m}$ & $\mathrm{m}$ & $\mathrm{m}$ \\
\hline Litauen & $\mathrm{m}$ & $\mathrm{m}$ & $\mathrm{m}$ & $\mathrm{m}$ \\
\hline Zypern* & $\mathrm{m}$ & $\mathrm{m}$ & $\mathrm{m}$ & $\mathrm{m}$ \\
\hline Türkei & 25 & -10.4 & -18 & 4.4 \\
\hline Ver. Staten & -2 & -4.2 & -7 & 0.9 \\
\hline Argentinien & $\mathrm{m}$ & $\mathrm{m}$ & $\mathrm{m}$ & $\mathrm{m}$ \\
\hline Kolumbien & $\mathrm{m}$ & $\mathrm{m}$ & $\mathrm{m}$ & $\mathrm{m}$ \\
\hline Griechenland & 8 & -0.5 & -2 & 0.4 \\
\hline Brasilien & 35 & 0.7 & -5 & -0.2 \\
\hline Spanien & -1 & 3.2 & 6 & -2.1 \\
\hline Israel & $\mathrm{m}$ & $\mathrm{m}$ & $\mathrm{m}$ & $\mathrm{m}$ \\
\hline Luxemburg & -3 & 1.7 & 2 & -0.1 \\
\hline Costa Rica & $\mathrm{m}$ & $\mathrm{m}$ & $\mathrm{m}$ & $\mathrm{m}$ \\
\hline Rumänien & $\mathrm{m}$ & $\mathrm{m}$ & $\mathrm{m}$ & $\mathrm{m}$ \\
\hline Bulgarien & $\mathrm{m}$ & $\mathrm{m}$ & $\mathrm{m}$ & $\mathrm{m}$ \\
\hline Uruguay & -13 & 6.9 & 3 & -1.5 \\
\hline Ungarn & -13 & -2.6 & -3 & 0.1 \\
\hline Chile & $\mathrm{m}$ & $\mathrm{m}$ & $\mathrm{m}$ & $\mathrm{m}$ \\
\hline Peru & $\mathrm{m}$ & $\mathrm{m}$ & $\mathrm{m}$ & $\mathrm{m}$ \\
\hline Slowak. Rep. & -17 & 1.0 & 6 & -0.6 \\
\hline
\end{tabular}

Anmerkung: Länder/Volkswirtschaften, die zwischen PISA 2003 und PISA 2012 (2012-2003) eine statistisch signifikanteVeränderung aufweisen, sind durch Fettdruck gekennzeichnet. 1. ESCS bezieht sich auf den PISA-Index des wirtschaftlichen, sozialen und kulturellen Status.

Die Länder und Volkswirtschaften sind in drei Gruppen dargestellt: Länder/Volkswirtschaften, deren Durchschnittsergebnisse über dem OECD-Durchschnitt liegen, Länder/Volkswirtschaften, deren Durchschnittsergebnisse sich statistisch nicht signifikant vom OECD-Durchschnitt unterscheiden, und Länder/Volkswirtschaften, deren Durchschnittsergebnisse unter dem OECDDurchschnitt liegen. Innerhalb jeder dieser Gruppen sind die Länder/Volkswirtschaften in absteigender Reihenfolge der in PISA 2012 beobachteten Stärke des Zusammenhangs zwischen Leistung und sozioökonomischem Status angeordnet.

$* \mathrm{Vgl}$. Hinweise für den Leser.

Quelle: OECD, PISA-2012-Datenbank, Tabelle II.2.1, II.2.7a, II.2.7b, II.2.8b und II.2.9b.

StatLink Ainst http://dx.doi.org/10.1787/888932964889 


\section{Hinweise für den Leser}

\section{Die den Abbildungen zu Grunde liegenden Daten}

Die Daten, auf die sich dieser Band bezieht, sind Anhang B zu entnehmen, zusätzliche Informationen, darunter einige Tabellen, finden sich auf der PISA-Website unter www.pisa.oecd.org.

Fehlende Daten werden durch vier Symbole ausgedrückt:

a Die Kategorie ist für das betreffende Land nicht anwendbar. Es gibt diesbezüglich keine Daten.

c Die Zahl der Beobachtungen reicht nicht aus, um verlässliche Schätzungen zu liefern (d.h. es gibt weniger als 30 Schüler bzw. weniger als 5 Schulen mit validen Daten).

m Es stehen keine Daten zur Verfügung. Entsprechende Daten wurden von dem betreffenden Land nicht übermittelt oder wurden zwar erhoben, später jedoch aus technischen Gründen aus der Publikation herausgenommen.

w Die Daten wurden auf Ersuchen des betreffenden Landes zurückgezogen oder nicht erhoben.

\section{Erfassung der einzelnen Länder}

Diese Publikation enthält Daten über 65 Länder und Volkswirtschaften, wozu alle 34 OECD-Länder und 31 Partnerländer und -volkswirtschaften zählen (vgl. die Karte im Abschnitt Was ist PISA?).

Die statistischen Daten für Israel wurden von den zuständigen israelischen Stellen bereitgestellt, die für sie verantwortlich zeichnen. Die Verwendung dieser Daten durch die OECD erfolgt unbeschadet des völkerrechtlichen Status der Golanhöhen, von Ost-Jerusalem und der israelischen Siedlungen im Westjordanland.

Bei den statistischen Daten zu Zypern wurden zwei Anmerkungen hinzugefügt:

1. Anmerkung der Türkei: Die Informationen in diesem Bericht unter der Überschrift „Zypern“ beziehen sich auf den südlichen Teil der Insel. Es existiert keine den türkischen und den griechischen Bevölkerungsteil der Insel gemeinsam vertretende Instanz. Die Türkei erkennt die Türkische Republik Nordzypern (TRNZ) an. Bis im Rahmen der Vereinten Nationen eine dauerhafte und gerechte Lösung gefunden ist, wird sich die Türkei ihre Stellungnahme zur „Zypernfrage“ vorbehalten.

2. Anmerkung aller in der OECD vertretenen EU-Mitgliedstaaten und der Europäischen Union: Die Republik Zypern wird von allen Mitgliedern der Vereinten Nationen mit Ausnahme der Türkei anerkannt. Die Informationen in diesem Bericht beziehen sich auf das Gebiet, das sich de facto unter der Kontrolle der Regierung der Republik Zypern befindet.

\section{Berechnung der internationalen Durchschnittswerte}

Für die meisten Indikatoren in diesem Bericht wurde ein OECD-Durchschnitt berechnet, der dem arithmetischen Mittel der jeweiligen Länderschätzungen entspricht. Der OECD-Durchschnitt dient dem Vergleich der Leistungen in verschiedenen Schulsystemen. Bei einigen Ländern sind für bestimmte Indikatoren möglicherweise keine Daten verfügbar bzw. treffen einzelne Kategorien u.U. nicht zu. Der Leser sollte daher beachten, dass sich der Begriff „OECD-Durchschnitt” auf die in die jeweiligen Vergleiche einbezogenen OECD-Länder bezieht.

\section{Runden von Zahlen}

Wegen des Auf- und Abrundens einiger Zahlen in den Tabellen stimmt die Summe der Zahlen möglicherweise nicht immer mit der Gesamtsumme überein. Summen, Differenzen und Durchschnittswerte werden stets auf der Grundlage der exakten Zahlenwerte berechnet und erst danach auf- bzw. abgerundet.

Alle Standardfehler in dieser Publikation wurden bis auf zwei Dezimalstellen auf- oder abgerundet. Wenn der Wert 0,0 bzw. 0,00 angegeben ist, bedeutet dies nicht, dass der Standardfehler bei null liegt, sondern dass er geringer ist als 0,05 bzw. 0,005.

\section{Darstellung der Schülerdaten}

Der Bericht verwendet den Begriff „15-Jährige“ als Kurzform für die Zielpopulation von PISA. PISA betrifft Schülerinnen und Schüler, die zum Zeitpunkt der Erhebung zwischen 15 Jahre und 3 Monate und 16 Jahre und 2 Monate alt sind, eine Schule besuchen und mindestens sechs Jahre formaler Bildung abgeschlossen haben, ganz gleich in welcher Art von Bildungseinrichtung sie eingeschrieben sind und unabhängig davon, ob es sich um eine Ganztags- oder Halbtagsschule, eine allgemein- oder berufsbildende Einrichtung, eine öffentliche oder private Schule oder eine Auslandsschule im betreffenden Teilnehmerland handelt.

\section{Darstellung der Schuldaten}

Die Leiterinnen und Leiter der Schulen, in denen Schülerinnen und Schüler am Test teilnahmen, haben durch Ausfüllen eines Schulfragebogens Informationen über die Merkmale ihrer jeweiligen Schule geliefert. Bei der Darstellung der Antworten der Schulleiterinnen und Schulleiter in dieser Publikation wurde eine Gewichtung in der Weise vorgenommen, dass ihre Zahl im richtigen Verhältnis zur Anzahl der 15-Jährigen in der betreffenden Schule steht. 


\section{Fokussierung auf statistisch signifikante Unterschiede}

In diesem Band werden nur statistisch signifikante Unterschiede oder Veränderungen erörtert. Diese sind in den Abbildungen mit dunkleren Farbtönen und in den Tabellen mit Fettdruck gekennzeichnet. Wegen weiterer Informationen vgl. Anhang A3.

\section{Im Bericht verwendete Abkürzungen}

\begin{tabular}{ll|ll}
\hline ESCS & PISA-Index des wirtschaftlichen, sozialen und kulturellen Status & KKP & Kaufkraftparitäten \\
\hline BIP & Bruttoinlandsprodukt & S.D. & Standardabweichung \\
\hline ISCED & Internationale Standardklassifikation des Bildungswesens & S.E. $\quad$ Standardfehler \\
\hline ISCO & Internationale Standardklassifikation der Berufe & $\begin{array}{l}\text { STEM Naturwissenschaften, Technik, } \\
\text { Ingenieurwissenschaften und } \\
\text { Mathematik }\end{array}$ \\
\hline
\end{tabular}

\section{Weiterführende Dokumentation}

Wegen näherer Einzelheiten zu den in PISA verwendeten Erhebungsinstrumenten und Methoden vgl. PISA 2012 Technical Report (OECD, erscheint demnächst). Es ist zu beachten, dass die Nummerierung der Tabellen nicht fortlaufend ist, sondern Sprünge enthält, da einige Tabellen nur online erscheinen und in dieser Publikation nicht enthalten sind. Die Online-Datentabellen können auf der PISA-Website (www.pisa.oecd.org) konsultiert werden.

Dieser Bericht verwendet den StatLinks-Service für OECD-Veröffentlichungen. Unter jeder Tabelle und Abbildung befindet sich ein URL-Link, der zu einer Datei im Excel ${ }^{\mathrm{TM}}$-Format führt, die die zu Grunde liegenden Daten enthält. Diese URL sind stabil und bleiben im Zeitverlauf unverändert. Darüber hinaus können Leser der E-Books diese Links direkt anklicken, die Excel-Datei öffnet sich dann in einem neuen Fenster, wenn der Internetbrowser aktiviert ist. 


\section{Was ist PISA?}

„Was sollten die Bürger wissen und was sollten sie können?“ Das ist die Frage, die der Erhebung zu Grunde liegt, die weltweit im Dreijahresturnus unter 15-jährigen Schülerinnen und Schülern durchgeführt wird und als Internationale Schulleistungsstudie PISA bekannt ist. In der PISA-Studie wird evaluiert, inwieweit Schülerinnen und Schüler gegen Ende ihrer Pflichtschulzeit wichtige Kenntnisse und Fähigkeiten erworben haben, die für eine volle Teilhabe am Leben moderner Gesellschaften unerlässlich sind. In der Erhebung, die sich auf Lesekompetenz, Mathematik, Naturwissenschaften und Problemlösung konzentriert, wird nicht nur geprüft, ob die Schülerinnen und Schüler das Gelernte wiedergeben können, sondern es wird auch untersucht, wie gut sie aus dem Gelernten extrapolieren und ihr Wissen in ungewohnten Situationen - sowohl im schulischen als auch im außerschulischen Kontext - anwenden können. Diesem Ansatz liegt die Feststellung zu Grunde, dass in modernen Gesellschaften nicht Wissen an sich entscheidend ist, sondern die Fähigkeit, dieses Wissen anzuwenden.

PISA ist ein kontinuierliches Programm, das Erkenntnisse für die Bildungspolitik und -praxis bietet und hilft, die Trends im Erwerb von Kenntnissen und Fähigkeiten in den einzelnen Ländern und Volkswirtschaften sowie in verschiedenen Bevölkerungsgruppen innerhalb der Länder zu beobachten. Die PISA-Ergebnisse verdeutlichen, was im Bildungsbereich möglich ist, indem sie aufzeigen, was die Schülerinnen und Schüler in den Bildungssystemen können, die am besten abschneiden und die schnellsten Verbesserungen erzielen. Sie ermöglichen es den politischen Entscheidungsträgern in aller Welt, die Kenntnisse und Fähigkeiten der Schülerinnen und Schüler ihres Landes im Vergleich zu denen anderer Länder zu beurteilen, politischeVorgaben festzulegen, die sich an in anderen Bildungssystemen erreichten messbaren Zielen orientieren, und aus in anderen Ländern angewandten Grundsätzen und Vorgehensweisen zu lernen. PISA kann zwar keine Kausalzusammenhänge zwischen Grundsätzen bzw. Verfahren und Schülerleistungen aufdecken, die Studie kann den Pädagogen, politischen Entscheidungsträgern und der interessierten Öffentlichkeit jedoch zeigen, wo die Gemeinsamkeiten und Unterschiede zwischen den Bildungssystemen liegen - und was dies für die Schülerinnen und Schüler bedeutet.

\section{Ein Test, an dem die ganze Welt teilnehmen kann}

PISA wird heute in zahlreichen Weltregionen als Erhebungsinstrument eingesetzt. Die erste Erhebung umfasste 43 Länder und Volkswirtschaften (32 im Jahr 2000 und 11 im Jahr 2002), in der zweiten Erhebung (2003) waren es 41, in der dritten Erhebung (2006) betrug die Teilnehmerzahl 57, und in der vierten Erhebung belief sich die Zahl auf 75 (65 im Jahr 2009 und 10 im Jahr 2010). An PISA 2012 haben bisher 65 Länder und Volkswirtschaften teilgenommen.

Neben den OECD-Mitgliedsländern wurde oder wird die Erhebung in folgenden Ländern und Volkswirtschaften durchgeführt:

Ost-, Süd- und Südostasien: Himachal Pradesh (Indien), Hongkong (China), Indonesien, Macau (China), Malaysia, Shanghai (China), Singapur, Chinesisch Taipeh, Tamil Nadu (Indien), Thailand und Vietnam.

Mittel-, Süd- und Osteuropa: Albanien, Aserbaidschan, Bulgarien, Georgien, Kasachstan, Kirgisistan, Kroatien, Lettland, Liechtenstein, Litauen, die ehemalige jugoslawische Republik Mazedonien, Malta, Moldau, Montenegro, Rumänien, die Russische Föderation und Serbien.

Naher Osten: Jordanien, Katar und Vereinigte Arabische Emirate.

Mittel- und Südamerika: Argentinien, Brasilien, Costa Rica, Kolumbien, Niederländische Antillen, Panama, Peru, Trinidad und Tobago, Uruguay und Miranda (Venezuela).

Afrika: Mauritius und Tunesien.

EXZELLENZ DURCH CHANCENGERECHTIGKEIT: ALLEN SCHÜLERINNEN UND SCHÜLERN DIE VORAUSSETZUNGEN ZUM ERFOLG SICHERN - BAND ॥ 
Die Entscheidungen über Umfang und Art der Leistungsmessung und der Hintergrundbefragung in PISA werden von den Teilnehmerstaaten auf der Grundlage von Empfehlungen führender Experten getroffen. Es werden erhebliche Ressourcen und Anstrengungen darauf verwendet, bei dem Erhebungsmaterial kulturelle und sprachliche Breite sowie Ausgewogenheit zu gewährleisten. Da die Gestaltung und Übersetzung der Tests sowie die Stichprobenauswahl und Datenerhebung einer strengen Qualitätskontrolle unterliegen, wird den PISA-Ergebnissen ein hoher Grad an Validität und Reliabilität beigemessen.

\section{Überblick über die PISA-Teilnehmerländer und -volkswirtschaften}

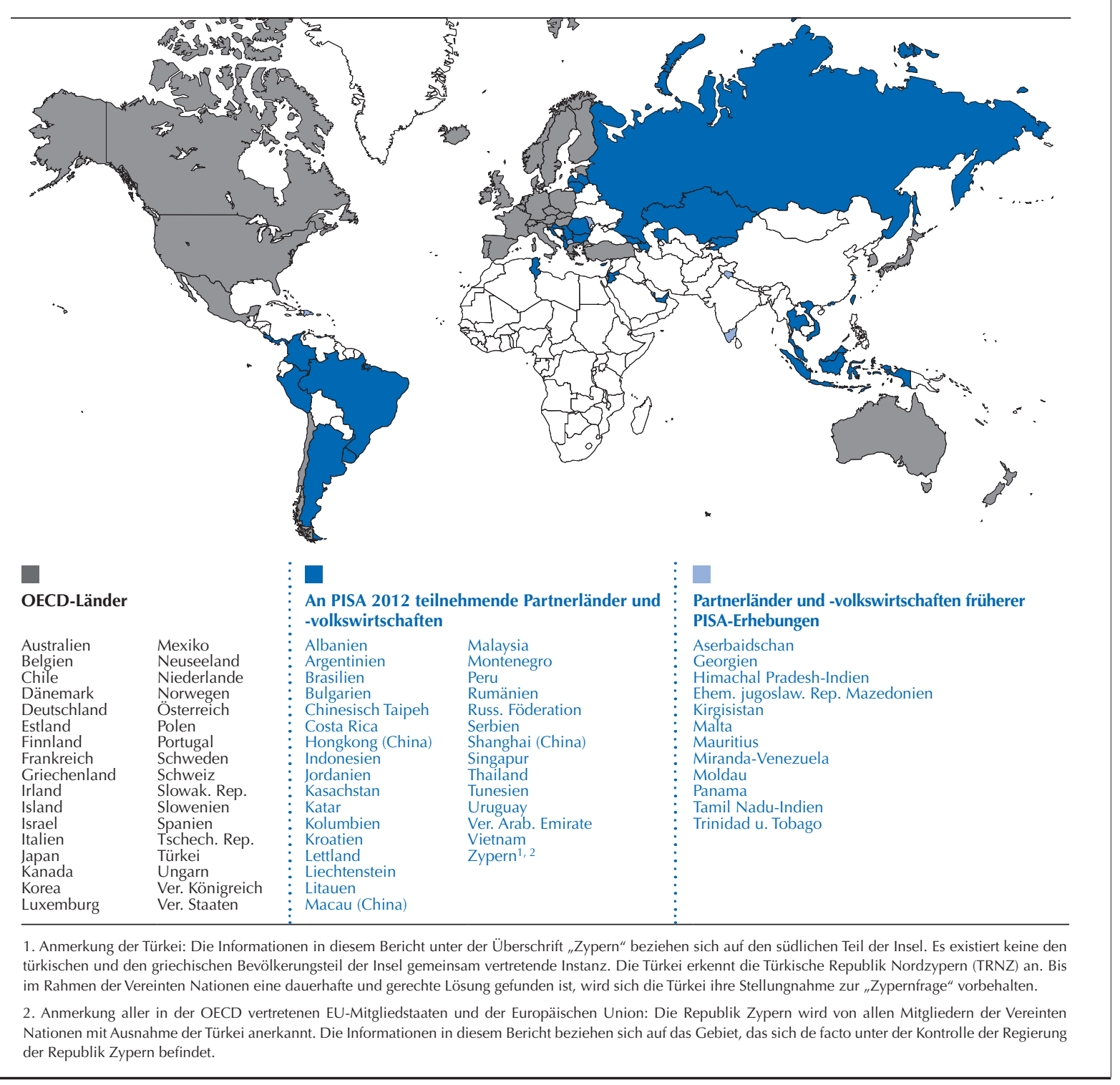

Zu den Besonderheiten von PISA gehören:

- Politikorientierung, wobei Daten über die Lernergebnisse der Schülerinnen und Schüler mit Informationen über deren Hintergrund und Lerneinstellung sowie über wichtige Faktoren, die ihr Lernen innerhalb und außerhalb der Schule beeinflussen, verknüpft werden, um die Leistungsunterschiede aufzuzeigen und zu untersuchen, wodurch sich leistungsstarke Schüler, Schulen und Bildungssysteme auszeichnen; 


\section{Hauptmerkmale von PISA 2012}

\section{Inhalt}

- Der Schwerpunkt der PISA-Erhebung 2012 lag auf Mathematik, wobei Lesekompetenz, Naturwissenschaften und Problemlösen die Nebenkomponenten der Erhebung bildeten. PISA 2012 umfasste darüber hinaus zum ersten Mal eine Beurteilung der finanziellen Allgemeinbildung junger Menschen, die für die Länder fakultativ war.

- PISA beurteilt nicht nur, ob die Schülerinnen und Schüler das Gelernte wiedergeben können, sondern auch, ob sie aus dem Gelernten extrapolieren und ihr Wissen in neuen Situationen anwenden können. Das Hauptaugenmerk gilt der Beherrschung von Prozessen, dem Verständnis von Konzepten sowie der Fähigkeit, mit verschiedenen Arten von Situationen umzugehen.

\section{Die Schülerinnen und Schüler}

- Etwa 510000 Schülerinnen und Schüler absolvierten stellvertretend für die rd. 28 Millionen 15-Jährigen in den Schulen der 65 teilnehmenden Länder und Volkswirtschaften die Testrunde 2012.

\section{Erhebung}

- Es wurden papiergestützte Tests verwendet, wobei die Testdauer für alle Schülerinnen und Schüler insgesamt zwei Stunden betrug. In einer Reihe von Ländern und Volkswirtschaften waren weitere 40 Minuten für einen computergestützten Test in Mathematik, Lesekompetenz und Problemlösen vorgesehen.

- Bei den Aufgaben handelte es sich um eine Mischung aus Multiple-Choice-Aufgaben und Fragen, bei denen die Schülerinnen und Schüler eigene Antworten formulieren mussten. Die Items waren in Aufgabengruppen organisiert, die sich jeweils auf eine in Text- bzw. Bildmaterial dargestellte reale Lebenssituation bezogen. Insgesamt enthielt der Aufgabenkatalog Items für eine Testdauer von rd. 390 Minuten, wobei die einzelnen Schülerinnen und Schüler unterschiedliche Kombinationen von Items bearbeiteten.

- Die Schülerinnen und Schüler beantworteten einen Hintergrundfragebogen, dessen Bearbeitung etwa 30 Minuten in Anspruch nahm und der Fragen über sie selbst, ihr Zuhause sowie ihre Schul- und Lernerfahrungen enthielt. Den Schulleitungen wurde ein in 30 Minuten auszufüllender Fragebogen unterbreitet, der sich auf das Schulsystem und das Lernumfeld bezog. In einigen Ländern und Volkswirtschaften wurden fakultative Fragebogen an die Eltern verteilt, in denen sie gebeten wurden, Auskunft über Fragen zu geben, die ihre Einstellung und ihr Engagement in Bezug auf die Schule ihres Kindes, ihre Unterstützung für das Lernen zu Hause und die beruflichen Erwartungen ihres Kindes, insbesondere im Bereich Mathematik, betrafen. Die Länder und Volkswirtschaften hatten die Wahl zwischen zwei weiteren fakultativen Fragebogen für die Schülerinnen und Schüler: In einem Fragebogen wurden die Schülerinnen und Schüler gefragt, wie vertraut sie mit Informationsund Kommunikationstechnologien sind und wie gut sie damit umgehen können. Der zweite Fragebogen befasste sich mit ihrer bisherigen Schulzeit, einschließlich etwaiger Unterbrechungen, sowie der Frage, ob und wie sie sich auf ihre spätere Berufslaufbahn vorbereiten.

- ein innovatives Konzept der "Grundkompetenzen“ bzw. der "Grundbildung“ (literacy), das sich auf die Fähigkeit der Schülerinnen und Schüler bezieht, im Hinblick auf die Identifizierung, Interpretation und Lösung von Problemen in einer Vielzahl von Situationen Kenntnisse und Fähigkeiten in wichtigen Fächern zu nutzen, analytisch vorzugehen, logisch zu denken und effizient zu kommunizieren;

- Relevanz für das lebenslange Lernen, weil bei PISA auch Informationen über die Lernmotivation, die Selbsteinschätzung und die Lernstrategien der Schülerinnen und Schüler erhoben werden;

- Regelmäßigkeit, dank der die Länder und Volkswirtschaften ihre Fortschritte bei der Verwirklichung entscheidender Lernziele beobachten können; sowie

- große Reichweite, was in PISA 2012 durch dieTeilnahme von allen 34 OECD-Mitgliedsländern sowie 31 Partnerländern und -volkswirtschaften verdeutlicht wird. 


\section{WELCHE SCHÜLERINNEN UND SCHÜLER NEHMEN AN PISA TEIL?}

Auf Grund länderspezifischer Unterschiede im Hinblick auf die Art und die Verbreitung von Vorschuleinrichtungen, das reguläre Einschulungsalter, die Struktur des Bildungssystems sowie die Wiederholungsquoten sind die Klassenstufen häufig kein guter Indikator für den Stand der kognitiven Entwicklung der Schülerinnen und Schüler. Um die Schülerleistungen auf internationaler Ebene besser vergleichen zu können, zielt PISA auf eine bestimmte Altersgruppe von Schülerinnen und Schülern ab. Die PISA-Studie erfasst Schülerinnen und Schüler, die zum Zeitpunkt der Erhebung zwischen 15 Jahren und 3 Monaten und 16 Jahren und 2 Monaten alt sind und die mindestens sechs Jahre formaler Bildung abgeschlossen haben. Sie können Bildungseinrichtungen jeder Art besuchen: eine Ganztags- oder Halbtagsschule, einen allgemeinbildenden oder berufsorientierten Bildungsgang, eine öffentliche oder private Schule oder eine Auslandsschule innerhalb des Landes oder der Volkswirtschaft. (Wegen einer operationalen Definition dieser Zielpopulation vgl. Anhang A2). Dank der Wahl dieser Altersgruppe können bei PISA die Kenntnisse und Fähigkeiten von Personen, die im gleichen Jahr geboren sind und im Alter von 15 Jahren noch zur Schule gehen, trotz ihrer unterschiedlichen Bildungsbiografie innerhalb und außerhalb der Schule länderübergreifend und im Zeitverlauf auf einer einheitlichen Basis verglichen werden.

Die Population der teilnehmenden Schülerinnen und Schüler wird nach strengen technischen Standards definiert, und das Gleiche gilt für die von der Teilnahme ausgeschlossenen Schüler (vgl. Anhang A2). Die Gesamtausschlussrate für ein Land musste unter 5\% liegen, um sicherzustellen, dass unter normalen Umständen etwaige Verzerrungen bei den Mittelwerten für die einzelnen Länder innerhalb einer Spanne von plus/minus 5 Skalenpunkten bleiben, d.h. in der Regel in der Größenordnung von 2 Standardfehlern der Stichprobe. Der Ausschluss konnte auf Ebene der teilnehmenden Schulen oder der teilnehmenden Schüler innerhalb der Schulen erfolgen (vgl. Anhang A2, Tabelle A2.1 und A2.2).

Es gibt mehrere mögliche Gründe für den Ausschluss einer Schule bzw. einer Schülerin oder eines Schülers aus der PISA-Erhebung. Ausschlüsse auf Schulebene können sich dadurch erklären, dass die betreffenden Schulen in einer abgelegenen Region liegen und daher schwer erreichbar sind, dass sie zu klein sind oder wegen organisatorischer oder operationeller Faktoren nicht berücksichtigt werden können. Zu Ausschlüssen auf Schülerebene kann es im Fall kognitiver Behinderungen oder einer unzureichenden Kenntnis der Testsprache kommen.

In 28 der 65 an PISA 2012 teilnehmenden Länder und Volkswirtschaften belief sich die Ausschlussrate auf Schulebene auf weniger als 1\%, und in allen Ländern lag sie unter 4\%. Bei Berücksichtigung des Ausschlusses von Schülerinnen und Schülern innerhalb der Schulen gemäß den international festgelegten Ausschlusskriterien erhöht sich die Ausschlussrate geringfügig. Dennoch liegt die Gesamtausschlussquote in 30 teilnehmenden Ländern und Volkswirtschaften unter $2 \%$, in 57 Teilnehmerländern unter 5\% und in sämtlichen Ländern und Volkswirtschaften mit Ausnahme Luxemburgs (8,4\%) unter 7\%. In 11 der 34 OECD-Länder belief sich die Ausschlussrate auf Schulebene auf weniger als 1\%, und in 31 OECDLändern lag sie unter 3\%. Bei zusätzlicher Berücksichtigung der Zahl der ausgeschlossenen Schüler innerhalb der Schulen lagen 11 OECD-Länder unter 2\% und 26 OECD-Länder unter 5\%.

(Wegen genauerer Informationen zu den Beschränkungen in Bezug auf den Umfang der Ausschlüsse in PISA 2012 vgl. Anhang A2).

\section{WELCHE ART VON ERGEBNISSEN BIETET DER TEST?}

Die PISA-Erhebung bietet drei Hauptarten von Ergebnissen:

- Basisindikatoren, die ein Referenzprofil der Kenntnisse und Fähigkeiten der Schülerinnen und Schüler ergeben;

- Indikatoren, die zeigen, wie die Fähigkeiten mit wichtigen demografischen, sozialen, wirtschaftlichen und bildungsbezogenen Variablen zusammenhängen;

- Trendindikatoren, die Veränderungen in Bezug auf die Schülerleistungen und den Zusammenhang zwischen den Variablen bzw. Ergebnissen auf Schülerebene und auf Schulebene verdeutlichen;

Die Indikatoren können zwar wichtige Sachverhalte deutlich machen, sie bieten jedoch keine direkten Antworten auf Grundsatzfragen. Daher hat PISA außerdem einen politikorientierten Analyseplan entwickelt, in dem die Indikatoren als Basis für eine Grundsatzdiskussion verwendet werden.

\section{WO SIND DIE ERGEBNISSE ZU FINDEN?}

Dies ist der zweite von sechs Bänden, in denen die Ergebnisse von PISA 2012 vorgestellt werden. Zunächst wird das Konzept der Chancengerechtigkeit in der Bildung definiert und dargelegt, wie Chancengerechtigkeit in PISA gemessen wird. Kapitel 2 
legt das Augenmerk auf den Zusammenhang zwischen dem sozioökonomischen Status und den Schülerleistungen in Mathematik, in Kapitel 3 werden verschiedene Aspekte des sozioökonomischen Hintergrunds der Schülerinnen und Schüler untersucht, die Einfluss auf die Bildungserträge haben, wie die Familienstruktur und der Migrationshintergrund, und in Kapitel 4 wird der enge Zusammenhang untersucht, der zwischen den Bildungsressourcen, wie Lernmöglichkeiten, Qualität und Anzahl der Lehrkräfte, Schuldisziplin, sozioökonomischer Status und Mathematikleistungen besteht. Sofern vergleichbare Daten verfügbar sind, werden die Entwicklungen dieser Variablen zwischen 2003 und 2012 untersucht, und im gesamten Kapitel finden sich Fallstudien zu Politikreformen von Ländern, die ihre Ergebnisse im Verlauf ihrer PISATeilnahme verbessert haben. Im abschließenden Kapitel werden die Politikimplikationen der PISA-Ergebnisse untersucht.

Die anderen fünf Bände umfassen die folgenden Themenbereiche:

Band I, Was Schülerinnen und Schüler wissen und können: Schülerleistungen in Lesekompetenz, Mathematik und Naturwissenschaften gibt einen Überblick über die Schülerleistungen bei der PISA-Erhebung 2012. Er beschreibt die Definitions-, Messungs- und Darstellungsmethoden für die Schülerleistungen und führt anschließend die Ergebnisse des Tests auf, die zeigen, wozu die Schülerinnen und Schüler im Bereich Mathematik in der Lage sind. Nach einer Darstellung der Gesamtergebnisse im Bereich Mathematik wird untersucht, inwieweit diese Ergebnisse auf Subskalen verschiedener Aspekte der mathematischen Grundbildung variieren. In Anbetracht der Tatsache, dass bei jedem Vergleich der Ergebnisse der Bildungssysteme den sozialen und wirtschaftlichen Rahmenbedingungen der Länder und den von diesen für die Bildung bereitgestellten Ressourcen Rechnung getragen werden muss, werden die Ergebnisse in diesem Band auch im wirtschaftlichen und sozialen Kontext der einzelnen Länder dargestellt. Außerdem wird in diesem Band der Zusammenhang zwischen der Häufigkeit und Intensität des Schülerkontakts mit fachlichen Inhalten in der Schule, also den „Lernmöglichkeiten“, und den Schülerleistungen aufgezeigt. Abschließend werden die Ergebnisse der Schülerinnen und Schüler in Lesekompetenz und Naturwissenschaften beschrieben. Bei Vorliegen vergleichbarer Daten werden die Trends bei den Schülerleistungen in Mathematik zwischen 2003 und 2012, in Lesekompetenz zwischen 2000 und 2012 und in Naturwissenschaften zwischen 2006 und 2012 analysiert. Über den gesamten Band hinweg werden die Politikreformen von Ländern, die ihre Ergebnisse im Verlauf ihrer Teilnahme an PISA verbessert haben, in Fallstudien eingehender untersucht.

Band III, Lernbereitschaft: Engagement, Motivation und Selbsteinschätzung von Schülerinnen und Schülern (Ready to Learn: Student Engagement, Drive and Self-Beliefs - nicht auf Deutsch verfügbar), untersucht das schulische Engagement, die Einsatz- und Leistungsbereitschaft sowie das Vertrauen der Schülerinnen und Schüler in die eigenen Lernfähigkeiten im Bereich der Mathematik. Der Band zeigt, welche Schülerinnen und Schüler besonders anfällig dafür sind, ein schwaches Engagement und eine negative motivationale Orientierung gegenüber der Schule im Allgemeinen und gegenüber Mathematik im Besonderen zu entwickeln, und er verdeutlicht, inwieweit Engagement, Einsatzbereitschaft, Motivation und Selbsteinschätzung mit den Mathematikleistungen in Zusammenhang stehen. Der Band zeigt, welche Rolle die Schulen spielen können, um das Wohlergehen der Schülerinnen und Schüler zu fördern, und welche Rolle die Eltern spielen können, um bei ihren Kindern die Freude am Lernen und die motivationale Orientierung gegenüber dem Lernen zu fördern. Wenn vergleichbare Daten vorliegen, wird untersucht, wie sich das Engagement, die Einsatzbereitschaft, die Motivation und die Selbsteinschätzung der Schülerinnen und Schüler von 2003 bis 2012 verändert haben und wie sich die motivationalen Orientierungen in diesem Zeitraum in bestimmten Untergruppen, insbesondere sozioökonomisch privilegierte und benachteiligte Schülerinnen und Schüler, Jungen und Mädchen sowie Schülerinnen und Schüler auf unterschiedlichen Kompetenzstufen in Mathematik, verändert haben. Über den gesamten Band hinweg werden die Politikreformen von Ländern, die ihre Ergebnisse im Verlauf ihrer Teilnahme an PISA verbessert haben, in Fallstudien eingehender untersucht.

Band IV, Was macht Schulen erfolgreich? Lernumfeld und schulische Organisation in PISA (What Makes Schools Successful? Resources, Policies and Practices - nicht auf Deutsch verfügbar), untersucht den Zusammenhang zwischen den Schülerleistungen und verschiedenen Merkmalen der Schulen und der betreffenden Schulsysteme. Darin wird erörtert, wie 15-jährige Schülerinnen und Schüler ausgewählt und in verschiedene Schulen, Programme und Bildungsniveaus eingestuft werden und wie die personellen, finanziellen und zeitlichen Ressourcen sowie die Lehr- und Sachmittel auf die verschiedenen Schulen verteilt werden. Der Band untersucht darüber hinaus, wie die Schulsysteme ein ausgewogenes Verhältnis zwischen Autonomie und Zusammenarbeit schaffen und wie das Lernumfeld in der Schule die Schülerleistungen beeinflusst. Wenn vergleichbare Daten vorliegen, werden die Trends dieser Variablen für den Zeitraum 2003-2012 untersucht, und über den gesamten Band verteilt finden sich Fallstudien, in denen die Politikreformen von Ländern untersucht werden, die ihre Ergebnisse im Verlauf ihrer Teilnahme an PISA verbessert haben. 
Band V, Lernen für das Leben: Schülerleistungen im Bereich Problemlösekompetenz (Skills for Life: Student Performance in Problem Solving - nicht auf Deutsch verfügbar), befasst sich mit der in PISA 2012 vorgenommenen Evaluierung der Schülerleistungen im Bereich Problemlösen. Darin wird die Fähigkeit der Schülerinnen und Schüler gemessen, auf nichtroutinemäßige Situationen zu reagieren, um ihr Potenzial als konstruktive und reflektierende Bürger zu verwirklichen. Er zeigt die Beweggründe für die Evaluierung der Problemlösefähigkeiten und beschreibt die Leistung innerhalb der Länder und Volkswirtschaften im Vergleich. Der Band verdeutlicht darüber hinaus die relativen Stärken und Schwächen der einzelnen Schulsysteme und untersucht, wie sie mit den einzelnen Schülermerkmalen, wie Geschlecht, Migrationshintergrund und sozioökonomischer Status, zusammenhängen. Der Band untersucht außerdem die Rolle der Bildung bei der Förderung der Problemlösefähigkeiten.

Band VI, Schülerinnen und Schüler und ihr Bezug zum Geld: Finanzielle Allgemeinbildung für das 21. Jahrhundert (Students and Money: Financial Literacy Skills for the 21st Century - nicht auf Deutsch verfügbar), untersucht die Leistungen von 15-jährigen Schülerinnen und Schülern im Bereich der finanziellen Allgemeinbildung in den 18 Ländern und Volkswirtschaften, die an dieser optionalen Evaluierung teilgenommen haben. Erörtert wird darüber hinaus der Zusammenhang zwischen der finanziellen Allgemeinbildung und dem Hintergrund der Schülerinnen und Schüler und ihrer Familien sowie der Mathematik- und Lesekompetenz der Schülerinnen und Schüler. Der Band untersucht außerdem den Zugang der Schülerinnen und Schüler zu Geld sowie ihre Erfahrungen in Finanzfragen. Er bietet ferner einen Überblick über den aktuellen Stand der Vermittlung von Finanzwissen in den Schulen und verweist auf die einschlägigen Fallstudien.

Die in PISA 2012 für die Evaluierung der Leistungen in Mathematik, Lesekompetenz und Naturwissenschaften verwendeten Rahmenstrukturen werden in PISA 2012 Assessment and Analytical Framework: Mathematics, Reading, Science, Problem Solving and Financial Literacy (OECD, 2013) ausführlich beschrieben. Sie werden in diesem Band zusammengefasst.

Technische Anhänge am Ende dieses Berichts erläutern die Konstruktion der Fragebogenindizes und befassen sich mit Fragen der Stichprobenauswahl, der Qualitätssicherungsverfahren, der Reliabilität des Kodierungsprozesses und den zur Entwicklung der Erhebungsinstrumente eingesetzten Methoden. Viele der in den technischen Anhängen behandelten Fragen werden im PISA 2012 Technical Report (OECD, erscheint demnächst) eingehender erörtert.

Alle in der Analyse zitierten Datentabellen sind in Anhang B1 am Ende des jeweiligen Bandes beigefügt, und eine Reihe zusätzlicher Datentabellen ist online verfügbar (www.pisa.oecd.org). In jedem Band findet sich auch eine Anleitung für den Leser, die Hinweise darauf gibt, wie die Tabellen und Abbildungen zu diesem Bericht zu interpretieren sind. Die Daten aus Regionen innerhalb der Teilnehmerländer sind in Anhang B2 aufgeführt.

\section{Literaturverzeichnis}

OECD (erscheint demnächst), PISA 2012 Technical Report, PISA, OECD Publishing.

OECD (2013), PISA 2012 Assessment and Analytical Framework: Mathematics, Reading, Science, Problem Solving and Financial Literacy, PISA, OECD Publishing, http://dx.doi.org/10.1787/9789264190511-en. 


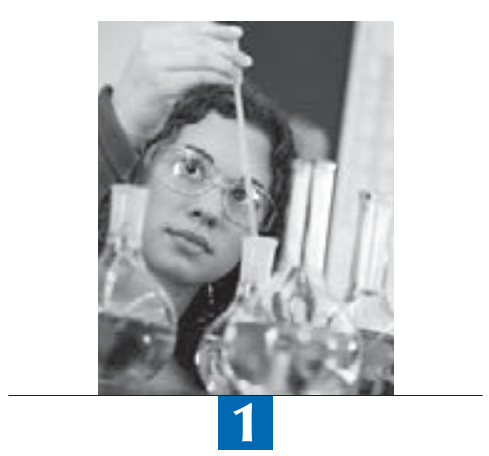

\section{Definition und Messung der Bildungsgerechtigkeit}

In diesem Kapitel wird erörtert, wie Bildungsgerechtigkeit in PISA definiert und gemessen wird, wobei für alle PISA-Teilnehmerländer und -volkswirtschaften Schülergruppen identifiziert werden, die am stärksten gefährdet sind, wenn das Bildungssystem nicht allen Schülerinnen und Schülern die gleichen Erfolgschancen bietet. 
Durch die Messung der Kenntnisse und Fähigkeiten 15-Jähriger gibt PISA Aufschluss darüber, wie die teilnehmenden Länder und Volkswirtschaften am Aufbau ihres künftigen Pools an Talenten arbeiten. Die neue Erhebung über die Fähigkeiten und Fertigkeiten Erwachsener (PIAAC) zeigt, dass zwischen den Ergebnissen der Länder bei den verschiedenen PISAErhebungsrunden und den Leistungen der entsprechenden Alterskohorten in den Bereichen Lesekompetenz und alltagsmathematische Kompetenz, die im OECD Skills Outlook 2013 (OECD, 2013) beschrieben sind, eine enge Korrelation besteht. Durch die Analyse der PISA-Ergebnisse im Kontext der verschiedenen sozialen Merkmale der Schüler und der Schulen - z.B. sozioökonomischer Status, Geschlecht und Migrationshintergrund - wird zudem deutlich, inwieweit es den Teilnehmerländern und -volkswirtschaften gelingt, Verteilungsgerechtigkeit bei den Bildungsmöglichkeiten und den Bildungserträgen zu gewährleisten, was auch ein Anhaltspunkt für die soziale Gerechtigkeit in der Gesellschaft insgesamt ist.

\section{Ergebnisse der Datenanalyse}

- Unter den 39 Ländern und Volkswirtschaften, die an PISA 2003 und an PISA 2012 teilgenommen haben, gelang es Mexiko, der Türkei und Deutschland, in diesem Zeitraum sowohl ihre Leistungen im Bereich Mathematik als auch die Chancengerechtigkeit in der Bildung zu steigern.

- Australien, Kanada, Estland, Finnland, Hongkong (China), Japan, Korea, Liechtenstein und Macau (China) erzielen zugleich hohe Leistungen und eine gerechte Verteilung der Bildungserträge, wie sie im Rahmen von PISA 2012 gemessen wurden.

Was Menschen wissen und wie sie dieses Wissen einsetzen können, hat große Auswirkungen auf ihre Chancen im Leben (Abb. II.1.1). Die Erhebung über die Fähigkeiten und Fertigkeiten Erwachsener (PIAAC) zeigt z.B., dass diejenigen, die die besten Ergebnisse im Bereich Lesekompetenz erzielen, mit dreimal größerer Wahrscheinlichkeit hohe Löhne beziehen als diejenigen mit den niedrigsten Lesekompetenzniveaus, und dass Personen mit geringen Lesekompetenzen auch ein mehr als doppelt so hohes Arbeitslosigkeitsrisiko haben (OECD, 2013).

- Abbildung II.1.1

\section{Wahrscheinlichkeit positiver sozialer und wirtschaftlicher Ergebnisse unter Erwachsenen mit hoher Lesekompetenz}

Für Erwachsene, die die Kompetenzstufen 4 oder 5 in Lesekompetenz erreichen, besteht im Vergleich zu Erwachsenen, die maximal Kompetenzstufe 1 erreichen, eine erhöhte Wahrscheinlichkeit (Odds Ratio, bereinigt), dass sie laut eigenen Angaben ein höheres Einkommen beziehen, anderen größeres Vertrauen entgegenbringen und über politisches Effektivitätsbewusstsein verfügen, sich einer guten Gesundheit erfreuen, sich ehrenamtlich engagieren und einer bezahlten Beschäftigung nachgehen

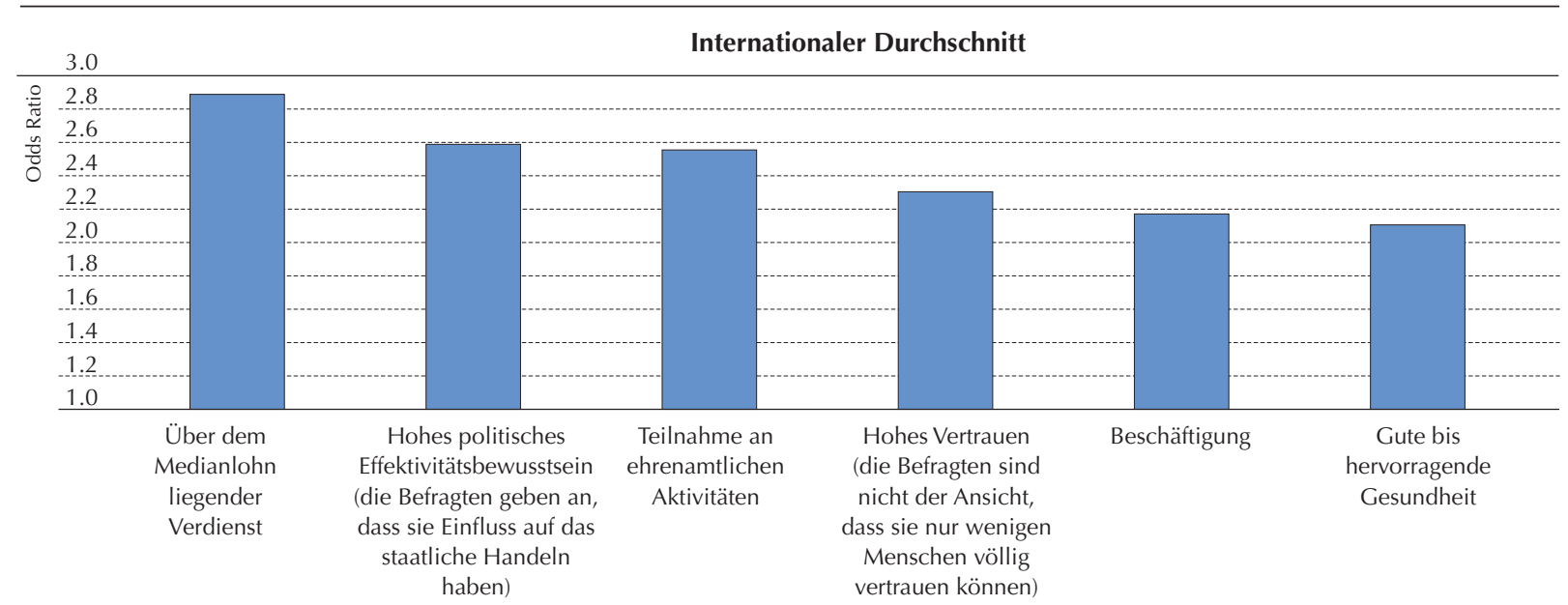

Anmerkung: Die Odds Ratios sind um Alter, Geschlecht, Bildungsniveau, Migrationsstatus und sprachlichen Hintergrund bereinigt.

Als hoher Verdienst gilt ein Stundenlohn, der über dem Median des jeweiligen Landes liegt.

Quelle: Auf Grundlage von Daten aus der Erhebung über die Fähigkeiten und Fertigkeiten Erwachsener (PIAAC), 2012.

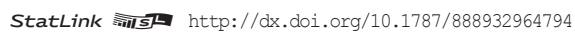


Die Kompetenzverteilung in der Bevölkerung hat auch bedeutende Auswirkungen auf die Verteilung der wirtschaftlichen und sozialen Merkmale in der Gesellschaft. PIAAC zeigt beispielsweise, dass eine stärkere Ungleichheit bei den Leseund alltagsmathematischen Kompetenzen mit einer stärkeren Einkommensungleichheit assoziiert ist. Wenn ein großer Teil der Erwachsenen über ein geringes Niveau an Lese- und alltagsmathematischer Kompetenz verfügt, kann dies die Einführung sowie Verbreitung produktivitätssteigernder Technologien und Verfahren der Arbeitsorganisation behindern, was wiederum einem Anstieg des Lebensstandards entgegenwirkt. Anders ausgedrückt bestimmt die Bildung von heute die Wirtschaft von morgen.

Und das Kompetenzniveau wirkt sich nicht nur auf Verdienste, Beschäftigungschancen, Wirtschaftswachstum und Wohlstand aus: In allen Ländern geben Personen mit geringerem Lesekompetenzniveau im Vergleich zu Personen mit gutem Lesekompetenzniveau mit größerer Wahrscheinlichkeit an, sich in schlechter gesundheitlicher Verfassung zu befinden, sind eher der Überzeugung, kaum Einfluss auf das politische Geschehen zu haben, und bringen anderen Menschen mit geringerer Wahrscheinlichkeit Vertrauen entgegen. Ungleichheit in der Kompetenzverteilung innerhalb der Gesellschaft drückt sich folglich auch in allgemeineren Formen sozialer Ungleichheit aus.

- Abbildung II.1.2 "

\section{Schülerleistungen und Bildungsgerechtigkeit}

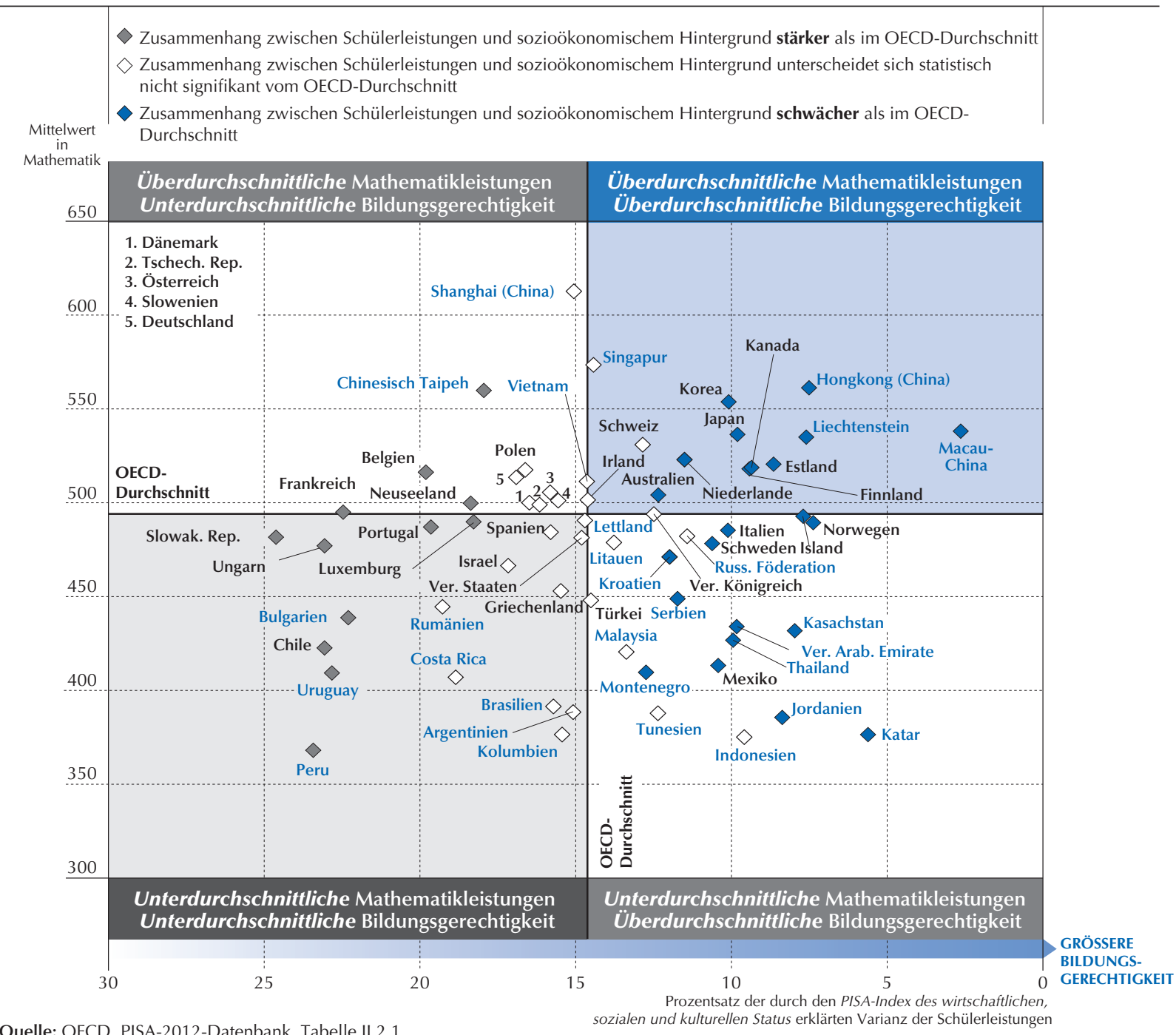

sozialen und kulturellen Status erklärten Varianz der Schülerleistungen

StatLink 光IS工 http://dx.doi.org/10.1787/888932964794 
In PISA bedeutet Bildungsgerechtigkeit ${ }^{1}$, dass allen Schülerinnen und Schülern, unabhängig von ihrem Geschlecht, ihrem familiären Hintergrund oder ihrem sozioökonomischen Status, die gleichen Bildungschancen geboten werden. Je stärker sich beispielsweise der sozioökonomische Hintergrund eines Schülers auf seine Leistung auswirkt, umso weniger gerecht ist das Schulsystem. Nach dieser Definition heißt Bildungsgerechtigkeit nicht, dass alle die gleichen Ergebnisse erzielen sollten, noch ist damit gemeint, dass allen Schülern die gleichen Bildungsinhalte vermittelt und die gleichen Bildungsressourcen zur Verfügung gestellt werden sollten.

Die PISA-Studie kommt durchgehend zu dem Schluss, dass hohe Leistungen und eine gerechtere Verteilung der Bildungserträge nicht unvereinbar sind: Keines dieser beiden Ziele muss zu Gunsten des anderen aufgegeben werden. In 20 der 23 Länder und Volkswirtschaften, die in PISA 2012 im Bereich Mathematik höhere Ergebnisse erzielten als der OECD-Durchschnitt, entspricht der Zusammenhang zwischen den Schülerleistungen und dem sozioökonomischen Hintergrund (Anteil der Leistungsvarianz, der sich aus Unterschieden beim sozioökonomischen Status erklärt) dem OECD-Durchschnitt oder ist sogar geringer ausgeprägt. In Australien, Kanada, Estland, Finnland, Hongkong (China), Japan, Korea, Liechtenstein, Macau (China) und den Niederlanden erzielen die Schulsysteme hohe Leistungen im Bereich Mathematik², während der Zusammenhang zwischen den Leistungen und dem sozioökonomischen Hintergrund der Schülerinnen und Schüler schwächer ist als im Durchschnitt. In Österreich, Dänemark, Deutschland, Irland, Polen, Slowenien, Shanghai (China), Singapur, der Schweiz und Vietnam erzielen die Schulsysteme hohe Mathematikleistungen, ohne dass die Ungleichheit bei den Bildungserträgen deshalb größer wäre (Abb. II.1.2).

Noch ermutigender ist, dass die Trenddaten von 2003 bis 2012 zeigen, dass unter den 39 Ländern und Volkswirtschaften, die an beiden PISA-Erhebungen teilnahmen, 13 ihre durchschnittlichen Ergebnisse in Mathematik verbessern konnten. Innerhalb dieser Gruppe von Ländern und Volkswirtschaften gelang es Mexiko, der Türkei und Deutschland zudem, die Bildungsgerechtigkeit zu erhöhen, entweder weil sich der Zusammenhang zwischen sozioökonomischem Hintergrund und Mathematikleistungen verringert hat oder weil der durchschnittliche Leistungsabstand zwischen sozioökonomisch benachteiligten und sozioökonomisch begünstigten Schülern abgenommen hat. Zehn weitere Länder und Volkswirtschaften erzielten in PISA 2012 bessere Ergebnisse als in PISA 2003, ohne dass sich der Grad der Bildungsgerechtigkeit verändert hätte.

Die Leistungsunterschiede zwischen sozioökonomisch benachteiligten und sozioökonomisch begünstigten Schülern, zwischen Schülern mit und ohne Migrationshintergrund oder zwischen Schülern in ländlichen oder städtischen Gebieten sind ein Anhaltspunkt für den Grad der Bildungsgerechtigkeit, den ein Schulsystem gewährleistet. An ihnen zeigt sich häufig, wie verschiedene Merkmale der Schüler bzw. ihres Lernumfelds mit den Schülerleistungen zusammenhängen. Kasten II.1.1 liefert Einzelheiten dazu, wie Leistungsunterschiede in PISA zu interpretieren sind. Durch die Beobachtung der Entwicklung solcher Disparitäten im Zeitverlauf können die Schulsysteme sehen, ob und wie die Ungleichheiten bei den Bildungschancen und Bildungserträgen zu- oder abnehmen.

\section{Kasten II.1.1 Wie sind die PISA-Ergebnisse zu interpretieren?}

Mathematik war 2003 erstmals Haupttestbereich der PISA-Studie. Die Mathematikgesamtskala wurde damals standardisiert, indem der Mittelwert bei 500 Punkten und die Standardabweichung bei 100 Punkten angesetzt wurde. Dies bedeutet, dass im Durchschnitt der OECD-Länder, die an PISA 2003 teilgenommen haben, der typische Schüler 500 Punkte erzielte und etwa zwei Drittel der Schüler Werte zwischen 400 und 600 Punkten erreichten. Fast 40\% der Schülerinnen und Schüler erzielten zwischen 450 und 550 Punkten. Ein Leistungsabstand von 100 Punkten entspricht somit einem großen Leistungsunterschied. Die Testergebnisse lassen sich am besten analysieren, wenn sie mit einem bestimmten Standard, z.B. der Durchschnittsleistung der OECD-Länder oder den Beschreibungen der verschiedenen Kompetenzstufen auf den PISA-Skalen, verglichen werden. In PISA gibt es weder ein Spitzen-/Höchstergebnis noch ein Mindestergebnis; ein Schüler, eine Schule oder ein Schulsystem kann den PISA-Test weder "bestehen" noch bei ihm "durchfallen".

Eine Kompetenzstufe in Mathematik erstreckt sich über rd. 70 Punkte, was bedeutet, dass zwischen Schülerinnen und Schülern auf ein und demselben Kompetenzniveau große Fähigkeits- und Wissensunterschiede bestehen können. Eine Differenz von 70 Punkten entspricht im OECD-Durchschnitt etwa zwei Schuljahren. Der durchschnittliche Leistungsunterschied zwischen zwei Schülern, die zwei verschiedene aufeinanderfolgende Schuljahre besuchen 
(z.B. Klasse 9 und 10), beträgt im OECD-Durchschnitt rd. 41 Punkte. Wie bereits Band I zu entnehmen war, beläuft sich die Differenz bei den Mathematikleistungen zwischen dem Mittelwert des Landes mit den höchsten und dem des Landes mit den niedrigsten Ergebnissen in PISA 2012 auf 245 Punkte (vgl. Tabelle II.2.1). Der durchschnittliche Leistungsunterschied in Mathematik zwischen dem obersten und dem untersten Quartil der Schüler im OECDRaum beträgt 128 Punkte (vgl. Tabelle I.2.3a).

Die Leistungsunterschiede in Mathematik, die mit sozioökonomischen Merkmalen der Schüler oder der Schulen zusammenhängen, sind allerdings zumeist deutlich geringer als eine Kompetenzstufe. Im typischen OECD-Land erzielen die Jungen in Mathematik durchschnittlich 11 Punkte mehr als die Mädchen (vgl. Tabelle I.2.3a), während Schüler ohne Migrationshintergrund durchschnittlich 34 Punkte mehr erreichen als Schüler mit Migrationshintergrund (vgl. Tabelle II.3.4a). Sozioökonomisch begünstigte Schüler (d.h. Schüler im oberen Quartil der sozioökonomischen Verteilung ihres Landes) erzielen durchschnittlich 90 Punkte mehr als sozioökonomisch benachteiligte Schüler (im unteren Quartil der Verteilung), und Schüler in städtischen Räumen erreichen durchschnittlich 31 Punkte mehr als Schüler in ländlichen Gebieten (vgl. Tabelle II.3.3a).

\section{WIE IM RAHMEN VON PISA DIE BILDUNGSGERECHTIGKEIT UNTERSUCHT WIRD Quantität und Qualität der Bildungsressourcen}

Erfolgreiche Bildungssysteme - sowohl im Hinblick auf Bildungsqualität als auch auf Bildungsgerechtigkeit - sorgen dafür, dass die besten Ressourcen dort zur Verfügung stehen, wo mit ihnen der stärkste Effekt erzielt werden kann. PISA liefert Informationen darüber, wie Schulsysteme die Bildungsressourcen verteilen und ob diese Verteilung an den Merkmalen der Schüler oder der Schulen, wie z.B. sozioökonomischer Status, Migrationshintergrund oder Schulstandort, orientiert ist.

Im Rahmen der PISA-Erhebung werden Schülern und Schulleitungen Fragebogen unterbreitet, mit denen Informationen über Quantität und Qualität der zur Verfügung stehenden Bildungsressourcen gesammelt werden. Die Schulleitungen werden beispielsweise zur Qualität der schulischen Infrastruktur und zum Angebot an qualifizierten Lehrkräften befragt. Die Schüler werden gefragt, wie viel Unterrichtszeit auf die verschiedenen Fächer entfällt und wie viel Zeit sie für außerschulisches Lernen aufwenden.

PISA misst die Gerechtigkeit der Verteilung der Ressourcen innerhalb der Schulsysteme, indem die Antworten auf die Fragebogen analysiert und mit den Testergebnissen verglichen werden. Dieser Band gibt Einblick in einige schulpolitische Maßnahmen und Vorgehensweisen, darunter auch die Frage der Ressourcenallokation. In Band IV wird dann eingehender untersucht, wie sich bestimmte Maßnahmen und Vorgehensweisen auf Ebene der Schulen und der Schulsysteme - einschließlich der Allokation der Bildungsressourcen - auf die Leistungen der Schüler und der Schulen auswirken und inwieweit sich darin der Grad der Bildungsgerechtigkeit ausdrückt, die ein Schulsystem gewährleistet.

\section{Unterrichtsinhalt und Unterrichtspraxis}

Weder die Quantität noch die Qualität der Bildungsressourcen können einen Effekt auf den Lernerfolg der Schülerinnen und Schüler haben, wenn ihnen im Unterricht nicht die Inhalte vermittelt werden, die sie benötigen, um voll am Leben der Gesellschaft teilzuhaben. Aus diesem Grund werden bei PISA Mathematikaufgaben gestellt, denen 15-Jährige auch in ihrem Mathematikunterricht begegnen sollten, wobei die relative Vertrautheit mit solchen Aufgaben gemessen wird, die die Schüler im Verlauf ihrer Schulzeit erworben haben. In diesem Band wird untersucht, wie sich die Vertrautheit und die Häufigkeit des Kontakts mit mathematischen Konzepten und Verfahren - anders ausgedrückt die "MathematikLernmöglichkeiten" - von Schüler zu Schüler, von Schule zu Schule und von Schulsystem zu Schulsystem unterscheiden und wie sich diese Unterschiede auf die Gerechtigkeit der Verteilung der Bildungserträge auswirken.

PISA beurteilt die Bildungsgerechtigkeit auch, indem das Lernumfeld in den Schulen untersucht wird. Daten zu Fragen wie den Lehrer-Schüler-Beziehungen, zur Arbeitshaltung der Lehrkräfte und zur Unterrichtsdisziplin, die mit Hilfe der Schüler- und der Schulleiterfragebogen erhoben wurden, werden miteinander verknüpft und mit den Schülerleistungen verglichen. Große Unterschiede bei der Qualität des Lernumfelds innerhalb und zwischen den Schulen können ein Anhaltspunkt für Ungleichheiten bei den Bildungschancen sein. 


\section{Bessere Leistungen mit mehr Bildungsgerechtigkeit verbinden}

Die Länder und Volkswirtschaften, die an PISA teilnehmen, machen deutlich, dass Exzellenz und Bildungsgerechtigkeit unter ganz unterschiedlichen Umfeldbedingungen möglich sind.

\section{Nationaleinkommen}

Ein hohes Einkommen ist weder eine Voraussetzung noch eine Garantie für hohe Leistungen und eine große Bildungsgerechtigkeit. Wie bereits in Band I erörtert, erklärt das unterschiedliche Pro-Kopf-BIP der Länder rd. 12\% der zwischen den OECD-Ländern zu beobachtenden Varianz der durchschnittlichen Schülerleistungen; in der Gruppe der Partnerländer und -volkswirtschaften erklärt es $21 \%$ der Leistungsvarianz (vgl. Abb. I.2.1) ${ }^{3}$. Länder und Volkswirtschaften mit vergleichbarem Wohlstandsniveau können in PISA sehr unterschiedliche Durchschnittsergebnisse haben. Kanada und Polen erzielten z.B. beide 518 Punkte im Mathematiktest, doch das Pro-Kopf-BIP von Polen ist nur halb so hoch wie das von Kanada. Das Pro-Kopf-BIP von Japan und Frankreich entspricht in etwa dem OECD-Durchschnitt (35 000 US-\$), die durchschnittlichen Mathematikleistungen der japanischen Schüler liegen mit 536 Punkten jedoch weit über dem OECD-Durchschnitt, wohingegen die der französischen Schüler in etwa dem OECD-Durchschnitt entsprechen. Außerdem weisen einige Länder mit sehr unterschiedlichem Pro-Kopf-BIP ein ähnliches Leistungsniveau auf: In Lettland ebenso wie in Luxemburg liegen die Schülerleistungen leicht unter dem OECD-Durchschnitt, das Pro-Kopf-BIP von Lettland beträgt jedoch weniger als 17000 US-\$, während es sich in Luxemburg auf über 84000 US-\$ beläuft (vgl. Tabelle I.2.27).

Länder und Volkswirtschaften wie Estland, Hongkong (China), Polen, Shanghai (China), Singapur, Slowenien, Chinesisch Taipeh und Vietnam zeigen, dass das Bild von einer Welt, die sich klar in fortgeschrittene Länder mit hohem Bildungsniveau und Schwellenländer mit geringem Bildungsniveau unterteilen lässt, längst nicht mehr der Realität entspricht. Einige aufstrebende Volkswirtschaften, vor allem in Ostasien, verzeichnen einen rapiden Anstieg des Bildungsniveaus und der Bildungsqualität ihrer Bevölkerung. Wie in Band IV erläutert wird, kann es auf den ersten Blick den Anschein haben, dass Hocheinkommensländer und -volkswirtschaften - hier definiert als Länder und Volkswirtschaften mit einem Pro-Kopf-BIP von über 20000 US-\$ -, Länder also, die mehr für Bildung ausgeben können und das auch tun, bei PISA bessere Ergebnisse erzielen. Effektiv haben Hocheinkommensländer und -volkswirtschaften einen durchschnittlichen Leistungsvorsprung in Mathematik von fast 70 Punkten gegenüber Ländern, deren Pro-Kopf-BIP unter 20000 US-\$ liegt. Doch während dieser Zusammenhang zwischen höherem Einkommen und besseren Leistungen in der Gruppe der Länder, die unter dieser Einkommensgrenze angesiedelt sind, deutlich erkennbar ist, lässt sich in der Gruppe der Länder mit höherem Einkommen kein solcher Zusammenhang feststellen.

In Bezug auf die Bildungsgerechtigkeit stellt sich die Situation ähnlich dar. Bei Betrachtung aller teilnehmenden Länder und Volkswirtschaften ist nur ein schwacher Zusammenhang zwischen Pro-Kopf-BIP und Bildungsgerechtigkeit zu erkennen. Insgesamt besteht offenbar keine Korrelation zwischen dem Pro-Kopf-BIP und der Stärke des Zusammenhangs zwischen den Leistungen und dem sozioökonomischen Hintergrund der Schüler. In der Gruppe der Länder, deren ProKopf-BIP unter 20000 US-\$ liegt, besteht eine positive Korrelation zwischen einem höheren Pro-Kopf-BIP und stärkeren Leistungsunterschieden zwischen sozioökonomisch begünstigten und sozioökonomisch benachteiligten Schülern und Schulen - mit anderen Worten: Die Verteilungsgerechtigkeit der Bildungserträge ist geringer. In der Gruppe der Hocheinkommensländer ist ein solcher Zusammenhang jedoch nicht mehr zu erkennen.

Wie in Band IV untersucht wird, besteht insgesamt kein Zusammenhang zwischen den Bildungsausgaben und den durchschnittlichen Schülerleistungen. Die PISA-Ergebnisse zeigen, dass wenn einmal ein gewisses Ausgabenniveau erreicht ist, darüber hinausgehende Ausgaben kein Prädiktor für höhere Leistungen mehr sind (vgl. Abb. IV.1.8). Ausgaben in Höhe von bis zu 50000 US-\$ je Schüler im Alter von 6-15 Jahren korrelieren positiv mit höheren Durchschnittsleistungen, aber auch mit Leistungsunterschieden zwischen Schülern mit unterschiedlichem sozioökonomischem Status. Dieses Ergebnis zeigt, wie wichtig es ist, dass Länder, die ihre Bildungsausgaben ausgehend von einem relativ niedrigen Niveau anzuheben versuchen, darauf achten, dass sie auch wirksame Maßnahmen zur Sicherung der Bildungsgerechtigkeit einrichten.

Wie die Länder ihre begrenzten Ressourcen ausgeben, ist ebenso wichtig, wenn nicht sogar wichtiger als die Frage, wie viel sie ausgeben. Wie in Band IV untersucht wird, besteht im Allgemeinen ein Zusammenhang zwischen Unterschieden bei der Allokation der Bildungsressourcen und Unterschieden bei den Schülerleistungen (vgl. Tabelle IV.1.20). Insbesondere ist festzustellen, dass eine gerechtere Verteilung der Bildungsressourcen mit höheren Mathematikergebnissen assoziiert ist. Wie in Abbildung IV.1.11 aufgezeigt wird, erklären sich 30\% der im OECD-Raum zu beobachtenden Leistungsvarianz in Mathematik - selbst nach Berücksichtigung des Pro-Kopf-BIP - aus Unterschieden bei derVerteilung der Bildungsressourcen zwischen sozioökonomisch begünstigten und sozioökonomisch benachteiligten Schulen. 


\section{Sozioökonomische Heterogenität}

Auch in sozioökonomisch heterogenen Schülerpopulationen kann zugleich ein hohes Leistungsniveau und ein hohes $\mathrm{Maß}$ an Bildungsgerechtigkeit erreicht werden. Unter den 23 Ländern und Volkswirtschaften, deren Mittelwerte über dem OECD-Durchschnitt liegen, können Hongkong (China) sowie Macau (China) ein überdurchschnittliches Maß an Bildungsgerechtigkeit vorweisen, obwohl ihre Schülerpopulation in sozioökonomischer Hinsicht überdurchschnittlich heterogen ist (was am Abstand zwischen dem 5. und dem 95. Perzentil der sozioökonomischen Verteilung gemessen wird, vgl. Abb. II.5.1a); in Finnland, das ebenfalls zu dieser Ländergruppe gehört, entspricht die sozioökonomische Heterogenität dem Durchschnitt. In der Gruppe der Länder/Volkswirtschaften mit hohen Leistungen und einem durchschnittlichen Grad an Bildungsgerechtigkeit (gemessen an der Stärke des Zusammenhangs zwischen den Leistungen und dem sozioökonomischen Hintergrund der Schüler) ist die sozioökonomische Heterogenität der Schülerpopulation in Shanghai (China) ebenso wie in Singapur überdurchschnittlich stark ausgeprägt, wohingegen sie in den Niederlanden, der Schweiz, Dänemark und Deutschland dem Durchschnitt entspricht.

Desgleichen ist auch beim prozentualen Anteil der besonders leistungsschwachen im Verhältnis zu dem der besonders leistungsstarken Schüler, beim Leistungsabstand zwischen den oberen und den unteren 25\% der Schüler oder einfach bei der Leistungsvarianz, wenn überhaupt, nur ein schwacher Zusammenhang mit der Bildungsgerechtigkeit festzustellen. Länder und Volkswirtschaften mit höherem Leistungsniveau weisen in der Tendenz eine stärkere Leistungsvarianz auf (vgl. Abb. I.2.24), diese Unterschiede korrelieren jedoch nur schwach mit sozioökonomischen Unterschieden. In Shanghai (China), Singapur und Chinesisch Taipeh z.B. bewegen sich sowohl die durchschnittlichen Leistungen als auch die Gesamtvarianz der Schülerleistungen auf hohem Niveau (vgl. Tabelle II.2.8a).

\section{Schülerinnen und Schüler mit Migrationshintergrund}

Kanada, Hongkong (China) und Macau (China) verbinden ein hohes Leistungsniveau mit einem überdurchschnittlichen Grad an Bildungsgerechtigkeit - und dabei haben über 30\% der Schülerinnen und Schüler in diesen Ländern und Volkswirtschaften einen Migrationshintergrund. Die Leistungsunterschiede zwischen Schülern mit und ohne Migrationshintergrund sind in Kanada und Hongkong (China) effektiv relativ gering. In Spanien und Griechenland sind demgegenüber relativ große Leistungsunterschiede zwischen Schülern mit und ohne Migrationshintergrund festzustellen, und das obwohl der Anteil der Schüler mit Migrationshintergrund dort zwar immer noch erheblich, aber doch geringer ist (vgl. Tabelle II.3.4a).

\section{UNTERSUCHUNG DER FRAGE DER BILDUNGSGERECHTIGKEIT IN DIESER BERICHTSREIHE}

In Kapitel 2 dieses Bandes wird die Verteilungsgerechtigkeit der Bildungserträge und insbesondere der Zusammenhang zwischen Leistung und sozioökonomischem Hintergrund analysiert, sowohl auf Ebene der Schüler als auch der Schulen. In Kapitel 3 wird der Zusammenhang zwischen einer Reihe von Schüler- und Schulmerkmalen einerseits und den Schülerleistungen andererseits untersucht. Dabei richtet sich der Blick auf Merkmale wie Familienstruktur, Migrationshintergrund, im Elternhaus gesprochene Sprache und Schulstandort, wobei analysiert wird, ob diese Merkmale Auswirkungen auf die Leistungen der Schüler haben und wenn ja, wie diese Auswirkungen geartet sind. In Kapitel 4 wird beschrieben, wie die Häufigkeit des Kontakts und die Vertrautheit mit reiner Mathematik, die Lernmöglichkeiten und die Lernressourcen auf verschiedene Schülergruppen verteilt sind. Kapitel 5 schließt mit einer Erörterung der Politikoptionen sowie der Konsequenzen, die die Länder aus der Evidenz und den Analysen ziehen können, die in diesem Band vorgestellt werden.

Dieser Band ist nicht der einzige in der Reihe PISA 2012 Ergebnisse, der sich mit der Frage der Bildungsgerechtigkeit auseinandersetzt. In Band I wurde bereits auf die Leistungsunterschiede zwischen Jungen und Mädchen als einem wichtigen Aspekt der Verteilungsgerechtigkeit eingegangen. Dabei wurde die Klischeevorstellung widerlegt, dass Mädchen in Mathematik systematisch weniger gute Leistungen erzielen als Jungen, und erörtert, wie komplex diese Thematik in Wirklichkeit ist. Da Mathematikkompetenzen für Mädchen und Jungen gleichermaßen unerlässlich sind, um auf ihrem weiteren Bildungs- und Berufsweg erfolgreich zu sein, sind Ungleichheiten zwischen Jungen und Mädchen hier nicht nur unfair, sondern schaden letztlich auch Gesellschaft und Wirtschaft insgesamt.

Band III befasst sich mit Unterschieden in Bezug auf Einstellungen, Verhaltensweisen und Lernansätzen je nach Geschlecht, sozioökonomischem Hintergrund, Familienstruktur und Schulstandort. Auch diese Unterschiede sind mit Ungleichheiten beim Erwerb von Kenntnissen und Kompetenzen assoziiert.

Band IV untersucht, wie die von den Schulen und Schulsystemen gewählten Maßnahmen und Vorgehensweisen mit Leistungsniveau und Bildungsgerechtigkeit in Zusammenhang stehen. Einige dieser Maßnahmen werden zwar schon in diesem Band angesprochen, in Band IV werden sie jedoch eingehender erörtert. 


\section{Anmerkungen}

1. Diese Definition gründet sich auf frühere PISA-Erhebungsrunden sowie den Rahmen zur Messung der Bildungsgerechtigkeit von Bildung auf einen Blick 2011: OECD-Indikatoren (OECD, 2011). Der konzeptuelle Rahmen dieses Kapitels stützt sich insbesondere stark auf Levin (2010).

2. In diesem Band liegt das Hauptaugenmerk auf den Mathematikergebnissen. Der Großteil der in diesem Band vorgestellten Analysen lässt sich jedoch auf alle anderen Erhebungsbereiche von PISA 2012 übertragen. Die Ergebnisse dürften unabhängig vom Bereich weitgehend identisch sein, auf einigen Gebieten könnten jedoch erhebliche Unterschiede festzustellen sein.

3. Das Pro-Kopf-BIP wird hier in KKP gemessen, d.h. in äquivalenten Einheiten, sog. Kaufkraftparitäten.

\section{Literaturverzeichnis}

Levin, H. (2010), "A Guiding Framework for Measuring Educational Equity", INES Network for the Collection and the Adjudication of System-Level Descriptive Information on Educational Structures, Policies and Practices, EDU/EDPC/INES/NESLI(2010)6, März 2010.

OECD (2013), OECD Skills Outlook 2013: First Results from the Survey of Adult Skills, OECD Publishing, http://dx.doi.org/10.1787/ 9789264204256-en.

OECD (2011), Bildung auf einen Blick 2011: OECD-Indikatoren, W. Bertelsmann Verlag, Bielefeld. 


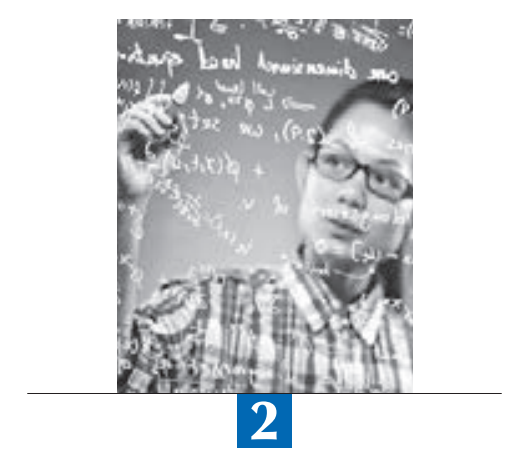

\section{Verteilungsgerechtigkeit der Bildungserträge}

Im vorliegenden Kapitel liegt das Augenmerk auf dem Zusammenhang zwischen den Schülerleistungen in Mathematik und dem sozioökonomischen Status. Dabei werden die Länderunterschiede im Hinblick auf diesen Zusammenhang untersucht und die Entwicklungen in Bezug auf die Bildungsgerechtigkeit vor dem Hintergrund des sozioökonomischen Status zwischen 2003 und 2012 erörtert, wobei insbesondere auf die Länder und Volkswirtschaften eingegangen wird, die sowohl ihre Ergebnisse als auch die Bildungsgerechtigkeit ihrer Schulsysteme verbessert haben. 
Im OECD-Raum lassen sich 14,8\% der Leistungsunterschiede zwischen den Schülerinnen und Schülern durch Disparitäten beim sozioökonomischen Hintergrund der Schülerinnen und Schüler erklären, unabhängig davon, ob Mathematik, Lesekompetenz oder Naturwissenschaften betrachtet werden ${ }^{1}$ (Tabelle II.2.1). In Ländern und Volkswirtschaften, in denen dieser Zusammenhang stark ist, erzielen Schülerinnen und Schüler aus sozioökonomisch benachteiligten Familien mit geringerer Wahrscheinlichkeit hohe Leistungen. Rund 39 Punkte, was einem Schuljahr entspricht, liegen zwischen den Mathematikleistungen von Schülerinnen und Schülern, die als sozioökonomisch begünstigt betrachtet werden, und Schülerinnen und Schülern, deren sozioökonomischer Status nahe am OECD-Durchschnitt liegt (Tabelle II.2.1), wobei die Differenz bei den Leistungen in Lesekompetenz und Naturwissenschaften sehr ähnlich ist².

In den OECD-Ländern verfügen die Eltern sozioökonomisch begünstigter Schülerinnen und Schüler (Schülerinnen und Schüler, die im obersten Quartil der sozioökonomischen Verteilung bzw. eine Standardabweichung über dem Durchschnitt auf dem PISA-Index des wirtschaftlichen, sozialen und kulturellen Status liegen) über ein hohes Bildungsniveau (95\% haben einen Tertiärabschluss erworben) und sind als Fach- oder Führungskräfte tätig (97\%). Hingegen verfügen die Eltern sozioökonomisch benachteiligter Schülerinnen und Schüler (Schülerinnen und Schüler, die im untersten Quartil der sozioökonomischen Verteilung bzw. eine Standardabweichung unter dem Durchschnitt auf dem PISA-Index des wirtschaftlichen, sozialen und kulturellen Status liegen) über ein deutlich niedrigeres Bildungsniveau, und sehr wenige (6\%) sind als Fach- oder Führungskräfte tätig. Sozioökonomisch begünstigte Schülerinnen und Schüler verfügen eigenen Angaben zufolge darüber hinaus über deutlich mehr Bücher in ihrem Elternhaus als ihre sozioökonomisch benachteiligten Mitschülerinnen und Mitschüler (im Durchschnitt 282 verglichen mit 69), ebenso wie Kunstwerke, klassische Literatur und Gedichtbände (Tabelle II.2.2). Sozioökonomisch benachteiligte Schülerinnen und Schüler besitzen zwar weniger Bücher, Kulturgüter und bestimmte Bildungsressourcen in ihrem Elternhaus, die große Mehrheit verfügt dort jedoch über Zugang zu einem Schreibtisch, einen ruhigen Platz zum Lernen, ein Wörterbuch, einen Computer und einen Internetanschluss (Tabelle II.2.2). Eine ausführlichere Definition der im Rahmen von PISA gemessenen sozioökonomischen Begünstigung bzw. Benachteiligung findet sich in Kasten II.2.1 weiter unten.

Die großen Leistungsunterschiede, die mit dem sozioökonomischen Status bzw. mit dem Migrations- oder sprachlichen Hintergrund der Schülerinnen und Schüler sowie der Schulen zusammenhängen, deuten darauf hin, dass die Lernmöglichkeiten im jeweiligen Schulsystem nicht ausgewogen verteilt sind bzw. dass nicht alle Schülerinnen und Schüler Zugang zu einem qualitativ hochwertigen Unterricht sowie zu den materiellen, finanziellen und Humanressourcen haben, die ihnen dabei helfen könnten, in der Schule und darüber hinaus Erfolg zu haben.

\section{Ergebnisse der Datenanalyse}

- In den OECD-Ländern sind rd. 6\% der Schülerinnen und Schüler - nahezu eine Million - „resilient“, was bedeutet, dass sie im Vergleich zu Schülerinnen und Schülern aus anderen Ländern trotz eines ungünstigen sozioökonomischen Hintergrunds über Erwarten gut abschneiden. In Korea, Hongkong (China), Macau (China), Shanghai (China), Singapur und Vietnam sind mindestens 13\% der Schülerinnen und Schüler resilient und gehören zu den 25\% der Schülerinnen und Schüler aller teilnehmenden Länder und Volkswirtschaften, die die höchsten Ergebnisse erzielen.

- Im OECD-Raum erzielten sozioökonomisch bessergestellte Schülerinnen und Schüler im Bereich Mathematik durchschnittlich 39 Punkte mehr als Schülerinnen und Schüler aus weniger begünstigten Verhältnissen, was einem Leistungsvorsprung von fast einem Schuljahr entspricht.

PISA misst lediglich die Leistungen 15-jähriger Schülerinnen und Schüler und ist nicht in der Lage, die Leistungen 15-Jähriger zu messen, die an keinem Bildungsprogramm teilnehmen. In den meisten OECD-Ländern gibt es sehr wenige 15-Jährige, die keine Schule besuchen, in einigen Partnerländern sieht die Lage jedoch anders aus. In diesen Ländern ist der Effekt des sozialen Hintergrunds auf die Lernergebnisse 15-Jähriger wahrscheinlich unterzeichnet.

In diesem Kapitel wird untersucht, inwiefern die Varianz der Schülerleistungen mit dem sozioökonomischen Status zusammenhängt und wie dieser Zusammenhang durch die Art und Weise geprägt wird, wie die Schülerleistungen und der sozioökonomische Status innerhalb der Länder variieren ${ }^{3}$. Die Chancengerechtigkeit im Bildungswesen wird im Kontext der Durchschnittsergebnisse in Mathematik analysiert. 


\section{LEISTUNGEN UND SOZIOÖKONOMISCHER STATUS IM VERGLEICH DER SCHULSYSTEME}

Auch wenn viele sozioökonomisch benachteiligte Schülerinnen und Schüler in der Schule Erfolg haben und viele von ihnen im Rahmen der PISA-Erhebung hohe Leistungen erzielten, ist der sozioökonomische Status in vielen Ländern dennoch ein starker Prädiktor für die Schülerleistungen, der in den meisten an PISA teilnehmenden Ländern und Volkswirtschaften mit erheblichen Leistungsunterschieden im Zusammenhang steht. Sozioökonomisch begünstigte Schülerinnen und Schüler schneiden in der Tendenz besser ab als ihre sozioökonomisch benachteiligten Mitschülerinnen und Mitschüler, wobei der Leistungsabstand größer ist als bei allen anderen Vergleichen zwischen zwei Schülergruppen, und für Schulen gilt dies analog.

Gleichwohl ist der sozioökonomische Status kein Schicksal: Zahlreichen Ländern und Volkswirtschaften, deren Durchschnittsergebnisse in PISA sich verbessert haben, ist es gelungen, den Zusammenhang zwischen dem sozioökonomischen Status und den Leistungen abzuschwächen. Manchmal führt dies zu einerVerringerung des Leistungsabstands zwischen sozioökonomisch begünstigten bzw. benachteiligten Schülerinnen und Schülern. Abbildung II.2.1 zeigt die sozioökonomische Gradiente, eine Darstellung des Zusammenhangs zwischen sozioökonomischem Status und Leistung. In diesem Bericht steht die Stärke dieses Zusammenhangs im Mittelpunkt, der die wichtigste Messgröße der Bildungsgerechtigkeit in PISA ist. Wenn dieser Zusammenhang schwach ist, ist der sozioökonomische Status der Schülerinnen und Schüler kein Prädiktor für ihre Leistungen. Eine einzelne Messgröße kann zwar die Bildungsgerechtigkeit nicht in ihrer Komplexität erfassen, sie kann jedoch eine nützliche Bezugsgröße für den Vergleich der Schulsysteme bieten.

Bildungserfolg kann definiert werden als eine Kombination aus einem hohen Leistungsniveau und einem hohen $\mathrm{Maß}$ an Bildungsgerechtigkeit. Wie aus Abbildung II.2.2 hervorgeht, ist der Zusammenhang zwischen Leistung und sozioökonomischem Status in zehn der 23 Länder und Volkswirtschaften, deren Schulsysteme in PISA 2012 über dem OECD-Durchschnitt abgeschnitten haben, unterdurchschnittlich stark: in Australien, Kanada, Estland, Finnland, Hongkong (China), Japan, Korea, Liechtenstein, Macau (China) und den Niederlanden ${ }^{4}$. In zehn der 23 Länder und Volkswirtschaften (Österreich, Dänemark, Deutschland, Irland, Polen, Shanghai-China, Singapur, Slowenien, die Schweiz und Vietnam) ist dieser Zusammenhang in etwa durchschnittlich stark. Nur in zwei leistungsstarken Ländern und einer leistungsstarken Volkswirtschaft - Belgien, Neuseeland und Chinesisch Taipeh - ist der Zusammenhang zwischen Leistung und sozioökonomischem Status überdurchschnittlich stark (Abb. II.2.2).

\section{Kasten II.2.1 Was ist der sozioökonomische Status und wie wird er gemessen?}

Der sozioökonomische Status ist ein breit angelegtes Konzept, in dem eine Vielzahl von Aspekten in Bezug auf die Schülerinnen und Schüler, die Schulen oder die Schulsysteme zusammengefasst ist. Der sozioökonomische Status der Schülerinnen und Schüler wird anhand eines Index geschätzt, des PISA-Index des wirtschaftlichen, sozialen und kulturellen Status, der auf Indikatoren wie dem Bildungsabschluss und dem Beruf der Eltern, dem Umfang und der Art der Ausstattung des Elternhauses, die als Hilfsindikatoren für den Wohlstand des Haushalts betrachtet werden, sowie den im Elternhaus verfügbaren Bildungsressourcen beruht. Der Index ist so konzipiert, dass die internationale Vergleichbarkeit gewährleistet ist (vgl. PISA 2012 Technical Report [OECD, erscheint demnächst]). Die Schülerinnen und Schüler werden als sozioökonomisch begünstigt betrachtet, wenn sie zu den 25\% der Schülerinnen und Schüler mit dem höchsten PISA-Index des wirtschaftlichen, sozialen und kulturellen Status in ihrem jeweiligen Land bzw. in ihrer jeweiligen Volkswirtschaft zählen; sozioökonomisch benachteiligte Schülerinnen und Schüler sind diejenigen, die zu den 25\% der Schülerinnen und Schüler mit dem niedrigsten PISA-Index des wirtschaftlichen, sozialen und kulturellen Status gehören.

Die PISA-Studie kommt durchgehend zu dem Schluss, dass der sozioökonomische Status auf Schulsystem-, Schulsowie Schülerebene mit dem Leistungsniveau zusammenhängt. Diese Muster sind zum Teil auf die inhärenten Vorteile zurückzuführen, die die Ressourcen bieten, mit denen ein relativ hoher sozioökonomischer Status verbunden ist. Sie spiegeln jedoch auch andere Aspekte wider, die zwar mit dem sozioökonomischen Status zusammenhängen, aber nicht durch den entsprechenden PISA-Index gemessen wurden. So hängt ein höherer sozioökonomischer Status auf Schulsystemebene beispielsweise mit größerem Wohlstand und höheren Bildungsausgaben zusammen. Auf Schulebene steht ein höherer sozioökonomischer Status mit einer Reihe von Merkmalen der betreffenden Gemeinde im Zusammenhang, die sich u.U. auf die Schülerleistungen auswirken, beispielsweise mit einem sicheren Umfeld sowie der Verfügbarkeit qualitativ hochwertiger Bildungsressourcen wie öffentlichen Bibliotheken oder Museen. Auf Schülerebene kann der sozioökonomische Hintergrund mit der Einstellung der Eltern zur Bildung im Allgemeinen und mit ihrem Engagement für die Bildung ihrer Kinder im Besonderen zusammenhängen.

EXZELLENZ DURCH CHANCENGERECHTIGKEIT: ALLEN SCHÜLERINNEN UND SCHÜLERN DIE VORAUSSETZUNGEN ZUM ERFOLG SICHERN - BAND II 
Im typischen OECD-Land verfügt die Mehrheit der Eltern (52\%) über einen Tertiärabschluss (ISCED 5 bzw. 6), ein geringer Anteil hat als höchsten Bildungsabschluss einen Sekundarstufe-II-Abschluss (ISCED 2) erzielt (12\%), und der Rest verfügt über andere Abschlüsse des postsekundären Bereichs (ISCED 3 oder 4). Im Durchschnitt sind 4\% der Eltern als Hilfsarbeitskraft (im Rahmen der ISCO-Berufshauptgruppe 9), 16\% als angelernte Fachkraft (im Rahmen der ISCOBerufshauptgruppen 6, 7 oder 8), 26\% als angelernte Bürokraft oder in einem Dienstleistungsberuf (im Rahmen der ISCO-Berufshauptgruppen 4 oder 5) und 54\% als Fach- oder Führungskräfte (im Rahmen der ISCO-Berufshauptgruppen 1, 2 oder 3) tätig6. Im Durchschnitt verfügen über 90\% der Schülerinnen und Schüler in ihrem Elternhaus über einen Schreibtisch, einen ruhigen Platz zum Lernen, ein Wörterbuch, einen Internetanschluss und einen DVDPlayer. Gedichtbände zählen zu den am seltensten im Haushalt vorhandenen Gütern: Unter 50\% der Schülerinnen und Schüler machten Angaben über das Vorhandensein von Gedichtbänden in ihrem Elternhaus. Klassische Literatur und Bildungssoftware sind ebenfalls relativ selten, gefolgt von technischen Nachschlagewerken und Kunstwerken. Im durchschnittlichen Haushalt sind über 155 Bücher vorhanden (Tabelle II.2.3). Hinter diesem allgemeinen Profil verbergen sich beträchtliche Unterschiede zwischen den einzelnen Ländern. Beispielsweise haben die Eltern in Island im Durchschnitt über 16 Jahre in Bildung verbracht, wohingegen Eltern in der Türkei im Durchschnitt weniger als neun Jahre in Bildung verbracht haben. Im Durchschnitt standen Schülerinnen und Schülern in Ungarn, Korea und Luxemburg eigenen Angaben zufolge über 220 Bücher in ihrem Elternhaus zur Verfügung, Schülerinnen und Schülern in Brasilien, Kolumbien und Tunesien hingegen weniger als 45 Bücher (Tabelle II.2.3).

In den OECD-Ländern verfügen die Eltern sozioökonomisch begünstigter Schülerinnen und Schüler über ein hohes Bildungsniveau: Die überwiegende Mehrheit (95\%) hat einen Tertiärabschluss erworben, und nahezu alle (97\%) sind als Fach- oder Führungskraft tätig. Im Hinblick auf die Ausstattung des Elternhauses ist die Zahl der Bücher eines der klarsten Unterscheidungsmerkmale sozioökonomisch bessergestellter Schülerinnen und Schüler gegenüber ihren Mitschülerinnen und Mitschülern. Sozioökonomisch begünstigte Schülerinnen und Schüler verfügen eigenen Angaben zufolge in ihrem Elternhaus im Durchschnitt über 282 Bücher, verglichen mit durchschnittlich 69 Büchern im Elternhaus ihrer sozioökonomisch benachteiligten Mitschülerinnen und Mitschüler. Auch andere Kulturgüter, z.B. Kunstwerke, klassische Literatur und Gedichtbände, kennzeichnen sozioökonomisch bessergestellte Schülerinnen und Schüler: Mindestens sieben von zehn sozioökonomisch begünstigten Schülerinnen und Schülern verfügen in ihrem Elternhaus über diese Güter. Zudem gaben sozioökonomisch begünstigte Schülerinnen und Schüler häufiger als sozioökonomisch benachteiligte Schülerinnen und Schüler an, dass technische Nachschlagewerke, Bildungssoftware sowie ein Geschirrspüler in ihrem Elternhaus vorhanden sind (Tabelle II.2.2).

Dagegen ist das Bildungsniveau der Eltern sozioökonomisch benachteiligter Schülerinnen und Schüler deutlich niedriger. Im OECD-Raum verfügen die meisten Eltern sozioökonomisch benachteiligter Schülerinnen und Schüler (nahezu 55\%) über einen Abschluss des postsekundären Bereichs, 35\% haben allenfalls einen Sekundarstufe-Il-Abschluss als höchsten Bildungsabschluss erzielt, und nur $10 \%$ verfügen über einen Tertiärabschluss. Sehr wenige sozioökonomisch benachteiligte Schülerinnen und Schüler haben einen Elternteil, der als Fach- oder Führungskraft arbeitet (6\%); die meisten Eltern dieser Schülerinnen und Schüler sind als angelernte Bürokraft oder in einem Dienstleistungsberuf tätig (41\%). Rund 39\% sind als Hilfsarbeitskraft tätig, und $13 \%$ arbeiten als angelernte Fachkraft. Sozioökonomisch benachteiligte Schülerinnen und Schüler besitzen zwar weniger Bücher, Kulturgüter und Bildungsressourcen in ihrem Elternhaus, mindestens $84 \%$ verfügen dort jedoch über Zugang zu einem Schreibtisch, einen ruhigen Platz zum Lernen, ein Wörterbuch, einen Computer und einen Internetanschluss. Die meisten (73\%) verfügen eigenen Angaben zufolge im Elternhaus zudem über Lehrbücher (Tabelle II.2.2).

Einige andere materielle Messgrößen des Wohlstands, z.B. die Zahl der Fernsehgeräte und der Mobiltelefone in einem Haushalt, sind unter sozioökonomisch begünstigten und benachteiligten Schülerinnen und Schülern vergleichbar; es bestehen jedoch ausgeprägte Unterschiede im Hinblick auf die Zahl der Computer und Autos sowie die Zimmer mit Badewanne oder Dusche. So verfügen sozioökonomisch benachteiligte Schülerinnen und Schüler beispielsweise über weniger als zwei Computer in ihrem Elternhaus, sozioökonomisch begünstigte Schülerinnen und Schüler hingegen über mehr als drei (Tabelle II.2.2).

Hinter dem OECD-Durchschnitt verbergen sich indessen in Bezug auf jedes dieser Merkmale bedeutende Unterschiede zwischen den einzelnen Ländern. Zur Veranschaulichung werden in dem vorliegenden PISA-Band einige Merkmale unter verschiedenen Gesichtspunkten betrachtet, bei der Analyse der beim Vergleich zu Tage tretenden Unterschiede ist jedoch Vorsicht geboten, da sie notwendigerweise lediglich Teil komplexerer Zusammenhänge sind (Tabelle II.2.2). 
- Abbildung II.2.1

Sozioökonomischer Status und Leistungen der Schüler, OECD-Länder

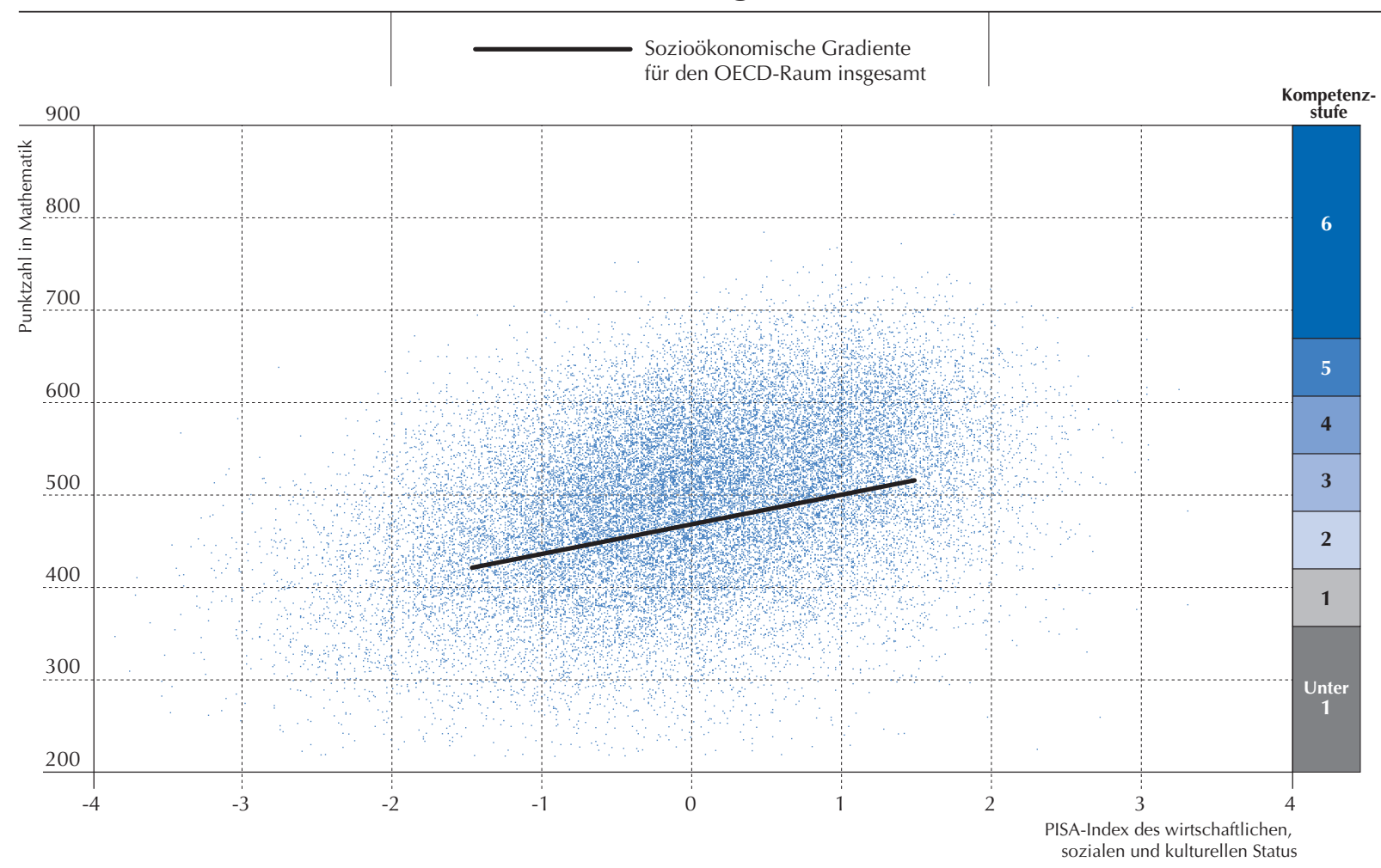

Anmerkung: Jeder Punkt steht für einen nach dem Zufallsprinzip aus zehn Schülern des OECD-Raums ausgewählten Schüler. Quelle: OECD, PISA-2012-Datenbank.

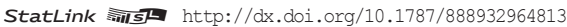

\section{Zur Interpretation dieser Abbildung:}

Jeder Punkt in Abbildung II.2.1 steht für einen nach dem Zufallsprinzip aus einem OECD-Land ausgewählten Schüler (10\% aller Schüler des OECD-Raums sind in der Abbildung dargestellt). Auf der x-Achse ist der sozioökonomische Status der Schülerinnen und Schüler angegeben (gemessen am PISA-Index des wirtschaftlichen, sozialen und kulturellen Status). Die y-Achse gibt die Mathematikergebnisse der Schülerinnen und Schüler in PISA 2012 wieder. Die dunkle Linie inmitten der Punkte zeigt den Zusammenhang zwischen den Leistungen der Schülerinnen und Schüler und ihrem sozioökonomischen Status, die sogenannte sozioökonomische Gradiente. Die Linie stellt die typische Leistung eines Schülers bzw. einer Schülerin in Anbetracht seines bzw. ihres sozioökonomischen Status dar. Die Stärke der sozioökonomischen Gradiente bezieht sich darauf, inwieweit der sozioökonomische Status ein Prädiktor für die Leistungen ist. Wenn die Punkte sehr nahe an der dunklen Linie angesiedelt sind, entsprechen die Mathematikleistungen der Schülerinnen und Schüler denen, die in Anbetracht ihres sozioökonomischen Status zu erwarten wären, womit die sozioökonomische Gradiente als stark gilt. Wenn die Punkte weit von der Linie entfernt sind, entsprechen die tatsächlichen Leistungen der Schülerinnen und Schüler nicht denen, die ihr sozioökonomischer Status erwarten ließe, womit die sozioökonomische Gradiente als schwach gilt. Die Stärke der sozioökonomischen Gradiente wird gemessen durch den Anteil der Leistungsvarianz, der sich aus Unterschieden beim sozioökonomischen Status erklärt.

Die Steigung der sozioökonomischen Gradiente bezieht sich auf die Auswirkungen des sozioökonomischen Status auf die Leistungen bzw. den durchschnittlichen Leistungsunterschied zwischen zwei Schülern, deren sozioökonomischer Status sich auf dem PISA-Index des wirtschaftlichen, sozialen und kulturellen Status um eine Einheit unterscheidet. Insofern handelt es sich um eine summarische Messgröße der zwischen den verschiedenen sozioökonomischen Gruppen zu beobachtenden Leistungsunterschiede. Eine flache parallel zur x-Achse verlaufende Gerade bedeutet, dass nur geringe Leistungsunterschiede im Zusammenhang mit dem sozioökonomischen Status bestehen; mit anderen Worten schließen sozioökonomisch begünstigte und sozioökonomisch benachteiligte Schüler gleich gut ab. Eine steile Gerade signalisiert indessen, dass die durch den sozioökonomischen Status bedingten Leistungsunterschiede groß sind.

In Kapitel 5 dieses Bands wird die sozioökonomische Gradiente für alle Länder und Volkswirtschaften vorgestellt, die an PISA 2012 teilgenommen haben. Die meisten Länder weisen entweder eine steile, starke sozioökonomische Gradiente oder eine flache, schwache Gradiente auf. Wie jedoch aus Abbildung II.2.2 ersichtlich, sind auch zwei andere Kombinationen möglich. Australien ist das einzige Land, in dem die mit dem sozioökonomischen Status verbundenen Leistungsunterschiede verhältnismäßig groß sind (größer als im OECD-Durchschnitt), die Stärke des Zusammenhangs zwischen Leistung und sozioökonomischem Status aber verhältnismäßig schwach ist (schwächer als im OECD-Durchschnitt); daher lässt sich für dieses Land eine steile, schwache sozioökonomische Gradiente feststellen. In Portugal, Chile, Costa Rica und Peru ist der Zusammenhang zwischen Leistung und sozioökonomischem Status stark, die Leistungsunterschiede im Zusammenhang mit dem sozioökonomischen Status sind jedoch gering, folglich sind diese Länder durch flache, starke sozioökonomische Gradienten gekennzeichnet. 
Abbildung II.2.2

\title{
Vergleich der Mathematikleistungen und der Bildungsgerechtigkeit der Länder/Volkswirtschaften
}

\author{
Länder/Volkswirtschaften mit über dem OECD-Durchschnitt liegenden Durchschnittsergebnissen in Mathematik \\ Länder/Volkswirtschaften, in denen der Zusammenhang zwischen Mathematikleistungen und sozioökonomischem Status schwächer als im OECD-Durchschnitt ist \\ Länder/Nolkswirtschaften mit unter dem OECD-Durchschnitt liegenden Leistungsunterschieden zwischen den verschiedenen sozioökonomischen Gruppen \\ Länder/Volkswirtschaften mit Durchschnittsergebnissen in Mathematik, die sich statistisch nicht signifikant vom OECD-Durchschnitt unterscheiden \\ Länder/Volkswirtschaften, in denen sich der Zusammenhang zwischen Mathematikleistungen und sozioökon. Status statistisch nicht signifikant vom OECD-Durchschnitt unterscheidet \\ Länder/Volkswirtschaften mit Leistungsunterschieden innerhalb der sozioökonomischen Verteilung, die sich statistisch nicht signifikant vom OECD-Durchschnitt unterscheiden \\ Länder/Volkswirtschaften mit unter dem OECD-Durchschnitt liegenden Durchschnittsergebnissen in Mathematik \\ Länder/Volkswirtschaften, in denen der Zusammenhang zwischen Mathematikleistungen und sozioökonomischem Status stärker als im OECD-Durchschnitt ist \\ Länder/Volkswirtschaften mit über dem OECD-Durchschnitt liegenden Leistungsunterschieden innerhalb der sozioökonomischen Verteilung
}

\begin{tabular}{|c|c|c|}
\hline & $\begin{array}{c}\text { Mittelwert } \\
\text { in Mathematik }\end{array}$ & $\begin{array}{l}\text { Stärke des Zusammenhangs } \\
\text { zwischen Mathematikleistungen und } \\
\text { sozioökonomischem Status }\end{array}$ \\
\hline & Mittelwert & $\begin{array}{l}\text { Prozentsatz der erklärten Varianz } \\
\text { der Mathematikleistungen }\end{array}$ \\
\hline OECD-Durchschnitt & 494 & 14.8 \\
\hline
\end{tabular}

\begin{tabular}{|c}
$\begin{array}{c}\text { Leistungsunterschiede zwischen } \\
\text { den verschiedenen } \\
\text { sozioökonomischen Gruppen }\end{array}$ \\
\hline $\begin{array}{c}\text { Punktzahldifferenz bei den Mathematik- } \\
\text { leistungen, die einem Anstieg um eine Einheit } \\
\text { auf dem PISA-Index des wirtschaftlichen, sozialen } \\
\text { und kulturellen Status entspricht }\end{array}$ \\
\hline 39 \\
\hline
\end{tabular}

\begin{tabular}{|c|}
\hline $\begin{array}{l}\text { Macau (China) } \\
\text { Hongkong (China) }\end{array}$ \\
\hline Liechtenstein \\
\hline Estland \\
\hline Finnland \\
\hline Kanada \\
\hline Japan \\
\hline Korea \\
\hline Niederlande \\
\hline Australien \\
\hline Schweiz \\
\hline Singapur \\
\hline Irland \\
\hline Vietnam \\
\hline Shanghai (China) \\
\hline Slowenien \\
\hline Österreich \\
\hline Dänemark \\
\hline Polen \\
\hline Deutschland \\
\hline Chinesisch Taipeh \\
\hline Neuseeland \\
\hline Belgien \\
\hline Norwegen \\
\hline Island \\
\hline Ver. Königreich \\
\hline Lettland \\
\hline Tschech. Rep. \\
\hline Portugal \\
\hline Frankreich \\
\hline Katar \\
\hline Kasachstan \\
\hline Jordanien \\
\hline Indonesien \\
\hline Ver. Arab. Emirate \\
\hline Thailand \\
\hline Italien \\
\hline Mexiko \\
\hline Schweden \\
\hline Russ. Föderation \\
\hline Serbien \\
\hline Kroatien \\
\hline Tunesien \\
\hline Montenegro \\
\hline Malaysia \\
\hline Litauen \\
\hline Türkei \\
\hline Ver. Staaten \\
\hline Argentinien \\
\hline Kolumbien \\
\hline Griechenland \\
\hline Brasilien \\
\hline Spanien \\
\hline Israel \\
\hline Luxemburg \\
\hline Costa Rica \\
\hline Rumänien \\
\hline Bulgarien \\
\hline Uruguay \\
\hline Ungarn \\
\hline Chile \\
\hline Peru \\
\hline
\end{tabular}

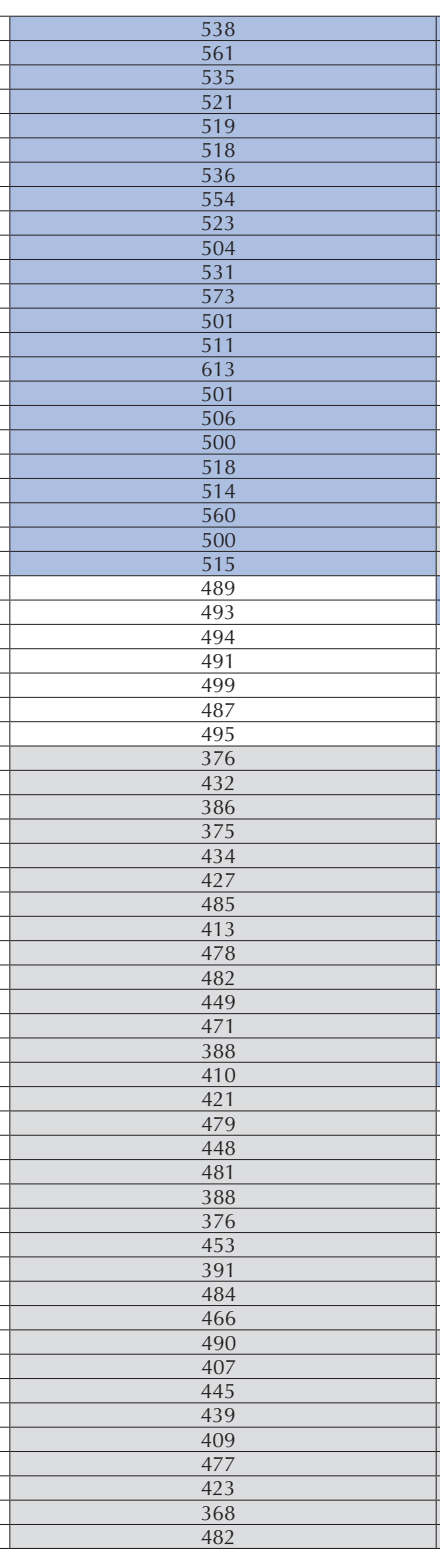
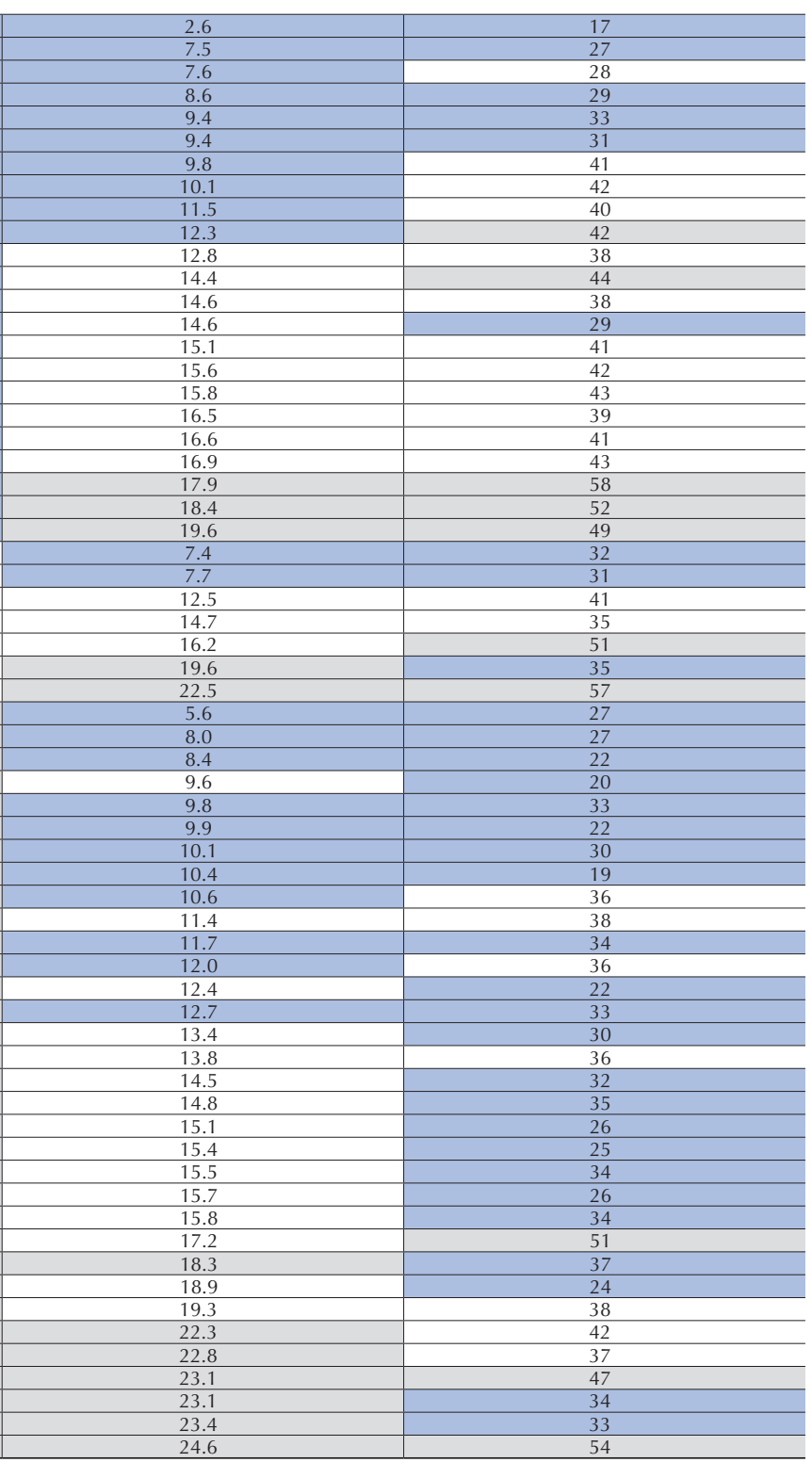

Anmerkung: Die Länder und Volkswirtschaften sind in drei Gruppen dargestellt: Länder/Volkswirtschaften, deren Durchschnittsergebnisse über dem OECD-Durchschnitt liegen, Länder/Volkswirtschaften, deren Durchschnittsergebnisse sich statistisch nicht signifikant vom OECD-Durchschnitt unterscheiden, und Länder/Volkswirtschaften, deren Durchschnittsergebnisse unter dem OECD-Durchschnitt liegen. Innerhalb jeder dieser Gruppen sind die Länder/Volkswirtschaften in absteigender Reihenfolge der Stärke des Zusammenhangs zwischen Leistung und sozioökonomischem Status angeordnet. 
Wie weiter unten erörtert, verzeichneten die Türkei, Mexiko und Deutschland seit 2003 eine Verbesserung der Gesamtleistungen in Mathematik sowie eine gerechtere Verteilung der Bildungserträge. Zudem blieb die Bildungsgerechtigkeit mit einer Ausnahme in den anderen Ländern und Volkswirtschaften, deren Ergebnisse sich gegenüber PISA 2003 verbessert haben, stabil oder erhöhte sich, was zeigt, dass Verbesserungen der Mathematikleistungen nicht zulasten der Chancengerechtigkeit im Schulsystem gehen müssen. Kasten II.2.4 bietet ausführlichere Informationen über die Verbesserung der PISA-Ergebnisse Mexikos, einschließlich der bildungspolitischen Maßnahmen und Programme, die das Land in den vergangenen zehn Jahren eingeführt hat. In Kasten II.3.2 wird ferner umrissen, wie Deutschland seine Ergebnisse verbessert hat, und Kasten I.2.5 (in Band I der vorliegenden PISA-Reihe) befasst sich mit der Verbesserung der Ergebnisse der Türkei in PISA 5 .

\section{Leistungsunterschiede im Zusammenhang mit dem sozioökonomischen Status}

Im Durchschnitt beträgt der Leistungsunterschied zwischen sozioökonomisch begünstigten (oberstes Quartil des sozioökonomischen Status) und sozioökonomisch benachteiligten (unterstes Quartil des sozioökonomischen Status) Schülerinnen und Schülern 90 Punkte, was über zwei Schuljahren sowie über einer PISA-Kompetenzstufe entspricht. Bei sozioökonomisch benachteiligten Schülerinnen und Schülern ist die Wahrscheinlichkeit im Durchschnitt mehr als doppelt so hoch wie bei Schülerinnen und Schülern, die nicht als sozioökonomisch benachteiligt betrachtet werden, im untersten Quartil der Leistungsverteilung zu liegen (Tabelle II.2.4a).

Die OECD-Länder, in denen die Bildungserträge, gemessen an der Stärke des Zusammenhangs zwischen Leistung und sozioökonomischem Status, gerechter verteilt sind, verzeichnen geringere Leistungsunterschiede zwischen Schülerinnen und Schülern verschiedener sozioökonomischer Gruppen, gemessen an der Steigung der sozioökonomischen Gradiente. Die Korrelation zwischen der Steigung und der Stärke der sozioökonomischen Gradiente beträgt in den OECDLändern 0,62 und in allen teilnehmenden Ländern und Volkswirtschaften 0,58. Kanada, Estland, Finnland, Hongkong (China) und Macau (China) verbinden hohe Leistungen mit einem schwachen Zusammenhang zwischen Leistung und sozioökonomischem Status sowie relativ geringen Leistungsunterschieden zwischen den einzelnen sozioökonomischen Gruppen. Unter den leistungsstarken Ländern und Volkswirtschaften verfügen lediglich Belgien, Neuseeland und Chinesisch Taipeh über Schulsysteme, in denen die Leistungsunterschiede überdurchschnittlich ausfallen, ebenso wie die Stärke des Zusammenhangs zwischen sozioökonomischem Status und Leistung. Unter den Ländern, deren Ergebnisse am oder unter dem OECD-Durchschnitt liegen, ist in Frankreich, Ungarn und der Slowakischen Republik dasselbe Muster zu beobachten. Australien ist das einzige Land, in dem die Leistungsvarianz in Mathematik zwischen sozioökonomisch begünstigten bzw. benachteiligten Schülerinnen und Schülern groß, die Stärke des Zusammenhangs zwischen Leistung und sozioökonomischem Status jedoch unterdurchschnittlich ist. Chile, Costa Rica, Peru und Portugal sind die einzigen Länder, in denen der Leistungsabstand trotz eines starken Zusammenhangs zwischen sozioökonomischem Status und Leistung relativ gering ist (Abb. II.2.2).

Welches Merkmal des sozioökonomischen Status bei der Erklärung dieser Differenzen eine größere Rolle spielt, unterscheidet sich von einem Land zum anderen. So schneiden Schülerinnen und Schüler, deren Eltern ein hohes Bildungsniveau aufweisen, in den OECD-Ländern beispielsweise um 77 Punkte besser ab als Schülerinnen und Schüler, deren Eltern ein niedriges Bildungsniveau aufweisen, was einer ganzen Kompetenzstufe entspricht ${ }^{7}$. Ein ähnlicher Abstand (85 Punkte) ist unter Schülerinnen und Schülern, deren Eltern als Hilfsarbeitskräfte tätig sind, und Schülerinnen und Schülern, deren Eltern als Fach- oder Führungskräfte arbeiten, zu beobachten. In Estland ist der mit dem Bildungsniveau bzw. dem Beruf der Eltern verbundene Leistungsabstand mit zwischen 53 und 56 Punkten nicht ganz so groß. In Italien und Spanien jedoch ist der beobachtete Leistungsabstand zwischen Schülerinnen und Schülern, deren Eltern in unterschiedlichen Berufen tätig sind, mit rd. 82 Punkten größer als der Leistungsabstand von rd. 48 bzw. 57 Punkten zwischen Schülerinnen und Schülern, deren Eltern ein unterschiedliches Bildungsniveau aufweisen (Tabelle II.2.5).

In Abbildung II.2.3 wird für jedes Land zusammengefasst, inwieweit verschiedene Merkmale des sozioökonomischen Status mit den Leistungen der Schülerinnen und Schüler im Bereich Mathematik in Beziehung stehen. Da diese Merkmale in der Regel miteinander zusammenhängen - z.B. haben Eltern mit einem höheren Bildungsniveau wahrscheinlich auch eine höhere berufliche Stellung -, ist der Einfluss dieser verschiedenen Aspekte in der Grafik zusammen dargestellt und wird der Teil der Varianz der Schülerleistungen, der sich jeweils aus den einzelnen Merkmalen erklärt, nach Ausklammerung des Einflusses der anderen Merkmale aufgezeigt. Das letzte Segment zeigt den Teil der Varianz der Schülerleistungen, der durch das Zusammenwirken der verschiedenen Faktoren erklärt werden kann (Tabelle II.2.6). 
- Abbildung II.2.3 "

\section{Anteil der durch Merkmale des sozioökonomischen Status erklärten Varianz der Mathematikleistungen}

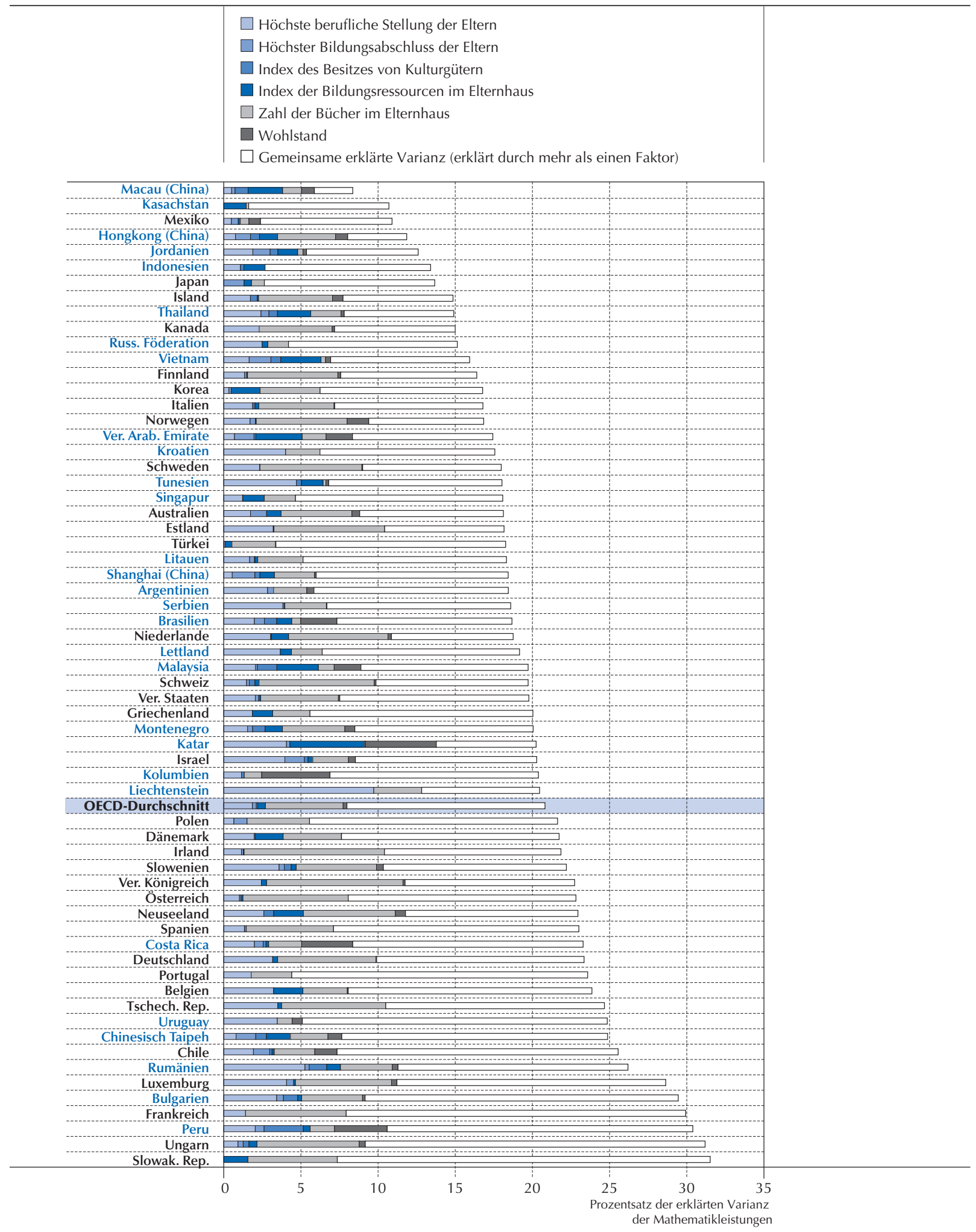

Die Länder und Volkswirtschaften sind in aufsteigender Reihenfolge nach der Summe der erklärten Gesamtvarianz der Mathematikleistungen nach Merkmalen des sozioökonomischen Status angeordnet.

Quelle: OECD, PISA-2012-Datenbank, Tabelle II.2.6.

StatLink inis ht htp://dx.doi.org/10.1787/888932964813 


\section{Resiliente Schülerinnen und Schüler}

Viele sozioökonomisch benachteiligte Schülerinnen und Schüler, Schulen und Schulsysteme haben in PISA 2012 im internationalen Vergleich trotz Benachteiligung hohe Leistungen erzielt. Wie in Band IV erläutert, stehen die Höhe der Bildungsausgaben eines Landes bzw. einer Volkswirtschaft sowie sein Pro-Kopf-BIP mit den PISA-Ergebnissen lediglich in einem schwachen Zusammenhang. Über einen gewissen Schwellenwert hinaus ist nur ein begrenzter Anteil der im Ländervergleich festzustellenden Varianz der Durchschnittsergebnisse in Mathematik auf diese beiden Wohlstandsmessgrößen zurückzuführen.

In den OECD-Ländern schnitten 6,4\% der gesamten Schülerpopulation - fast eine Million Schülerinnen und Schüler trotz eines ungünstigen sozioökonomischen Hintergrunds im Vergleich zu Schülerinnen und Schülern mit ähnlichem Hintergrund aus anderen Ländern über Erwarten gut ab. In Hongkong (China), Korea, Macau (China), Shanghai (China), Singapur und Vietnam gehörten über die Hälfte der sozioökonomisch benachteiligten Schülerinnen und Schüler (die im untersten Quartil der Skala des sozioökonomischen Hintergrunds des jeweiligen Landes/der jeweiligen Volkswirtschaft liegen) bzw. 12,5\% der gesamten Schülerpopulation zu den 25\% der Schülerinnen und Schüler aller teilnehmenden Länder und Volkswirtschaften, die nach Berücksichtigung ihres sozioökonomischen Status die höchsten Ergebnisse erzielten. Sie schnitten nicht nur trotz ihres verhältnismäßig ungünstigen sozioökonomischen Hintergrunds im Vergleich zu Schülerinnen und Schülern mit ähnlichem Hintergrund aus anderen Ländern über Erwarten gut ab, viele dieser Schülerinnen und Schüler erreichten sogar die höchsten Stufen der PISA-Skalen. Im Rahmen der PISA-Studie werden diese Schülerinnen und Schüler als „resilient" bezeichnet, da es ihnen gelungen ist, schwierige sozioökonomische Umstände zu überwinden und in der Schule Erfolg zu haben ${ }^{8}$. Abbildung II.2.4 zeigt den Prozentsatz der resilienten Schülerinnen und Schüler - gemessen an der gesamten Schülerpopulation - in den einzelnen Ländern und Volkswirtschaften sowie die äußerst unterschiedlichen Profile der einzelnen Schulsysteme (Tabelle II.2.7a).

Der Anteil resilienter Schülerinnen und Schüler ist in der Türkei, Mexiko, Polen, Italien, Tunesien und Deutschland deutlich gestiegen, was bedeutet, dass diese Länder sozioökonomisch benachteiligten Schülerinnen und Schülern 2012 bessere Chancen geboten haben, hohe Leistungen zu erbringen, als dies im Jahr 2003 der Fall war (eine Erörterung der trendmäßigen Entwicklung der Chancengerechtigkeit und insbesondere der Resilienz der Schülerinnen und Schüler findet sich weiter unten).

\section{Durchschnittsergebnisse nach Berücksichtigung des sozioökonomischen Status}

Der Vergleich der Ergebnisse der Länder nach Berücksichtigung des sozioökonomischen Status erlaubt den Vergleich unterschiedlicher Bildungssysteme auf der Grundlage der Leistungen von Schülerinnen und Schülern mit ähnlichem sozioökonomischem Status. Eine einfache Gedankenübung besteht darin, die Leistungen von Schülerinnen und Schülern mit durchschnittlichem sozioökonomischem Status in den einzelnen OECD-Ländern zu analysieren (d.h. der Schülerinnen und Schüler, deren sozioökonomischer Status dem Mittelwert des Index bzw. null entspricht) und davon ausgehend die Ergebnisse des Schulsystems zu untersuchen. Hiermit wird auf die Frage eingegangen, wie die Durchschnittsergebnisse des jeweiligen Schulsystems ausfallen würden, wenn der sozioökonomische Status aller Schülerinnen und Schüler dem OECD-Durchschnitt entsprechen würde.

Die meisten Bildungssysteme schneiden vor und nach Berücksichtigung des sozioökonomischen Status ähnlich ab, es gibt jedoch Ausnahmen ${ }^{9}$. Aus Abbildung II.2.5 wird ersichtlich, dass Portugals Leistungen über dem OECD-Durchschnitt liegen würden, wenn der sozioökonomische Status berücksichtigt würde. Auch in der Türkei würden sich die Leistungen verbessern, so dass sie nicht mehr unter, sondern am Durchschnitt liegen würden. Unter den Partnerländern würde Vietnam seinen Platz in der Rangfolge deutlich verbessern, wenn der sozioökonomische Status entsprechend dieser einfachen Gedankenübung berücksichtigt würde (Tabelle II.2.4a). Relativ gesehen handelt es sich hierbei um die einzigen drei Länder, die ihre Position in der Länderrangfolge bei Berücksichtigung des sozioökonomischen Status um über zehn Plätze verbessern würden.

Die Bildungssysteme lassen sich ferner anhand der von sozioökonomisch begünstigten bzw. benachteiligten Schülerinnen und Schülern erzielten Leistungen vergleichen (also der Schülerinnen und Schüler im obersten bzw. im untersten Quartil des sozioökonomischen Status innerhalb der einzelnen Länder). Wie in Abbildung II.2.6 dargestellt, schneiden sozioökonomisch benachteiligte Schülerinnen und Schüler in Bildungssystemen im OECD-Raum, die eine hohe Verteilungsgerechtigkeit der Bildungserträge mit hohen mittleren Punktzahlen kombinieren, beispielsweise in Kanada, Estland, Japan und Korea, international sehr gut ab, ebenso wie ihre sozioökonomisch begünstigten Mitschülerinnen und Mitschüler.

Unter den Ländern und Volkswirtschaften, deren Ergebnisse über dem OECD-Durchschnitt liegen, schneiden sozioökonomisch benachteiligte Schülerinnen und Schüler in Kanada, Dänemark, Estland, Finnland, Irland und Macau (China) in der Rangliste um über fünf Plätze besser ab als ihre sozioökonomisch begünstigten Mitschülerinnen und Mitschüler. 
- Abbildung II.2.4 -

Prozentsatz resilienter Schüler

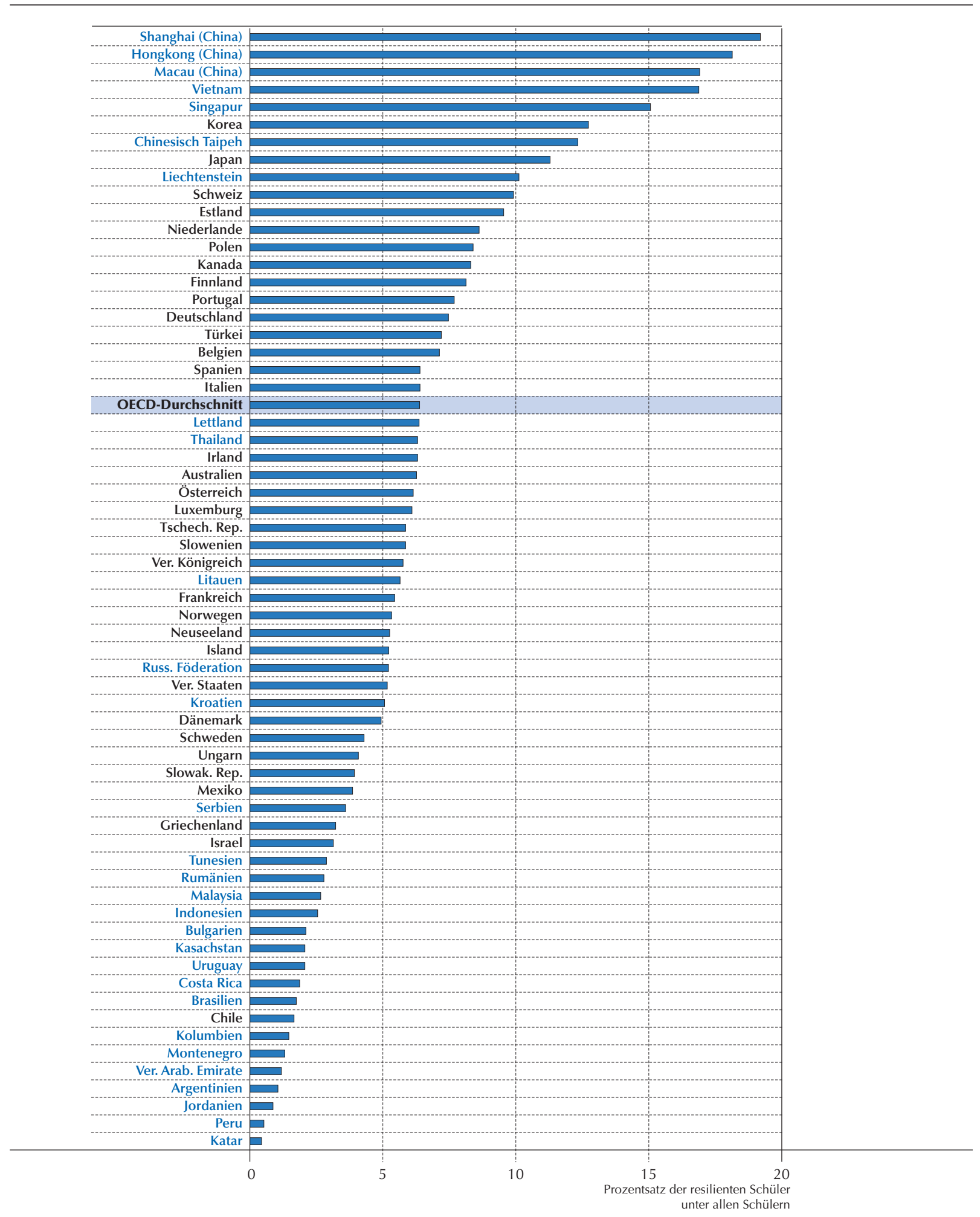

Anmerkung: Ein Schüler wird als resilient eingestuft, wenn er im Erhebungsland im untersten Quartil des PISA-Index des wirtschaftlichen, sozialen und kulturellen Status (ESCS) liegt und nach Berücksichtigung des sozioökonomischen Status unter den Schülern aus allen Ländern im obersten Quartil abschneidet.

Die Länder und Volkswirtschaften sind in absteigender Reihenfolge nach dem Prozentsatz der resilienten Schülerinnen und Schüler angeordnet.

Quelle: : OECD, PISA-2012-Datenbank, Tabelle II.2.7a.

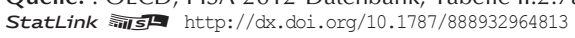


- Abbildung II.2.5 "

Durchschnittsergebnisse in Mathematik, vor und nach Berücksichtigung des sozioökonomischen Profils der Länder/Volkswirtschaften

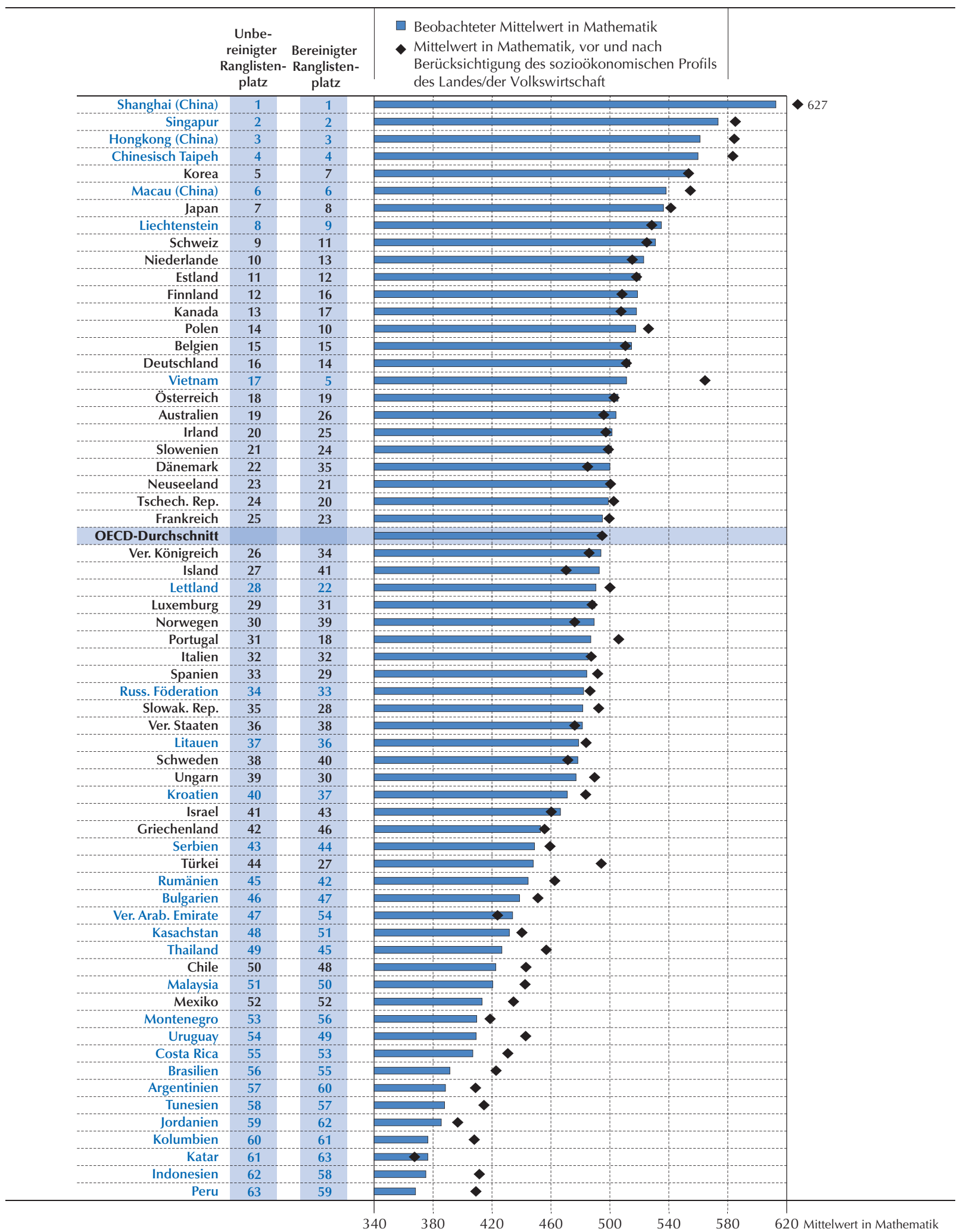

Die Länder/Volkswirtschaften sind in absteigender Reihenfolge nach dem beobachteten Mittelwert in Mathematik angeordnet.

Quelle: OECD, PISA-2012-Datenbank, Tabelle II.2.1.

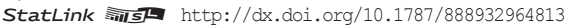


- Abbildung II.2.6 "

\section{Durchschnittliche Mathematikleistungen, nach nationalen Quartilen des sozioökonomischen \\ Status}

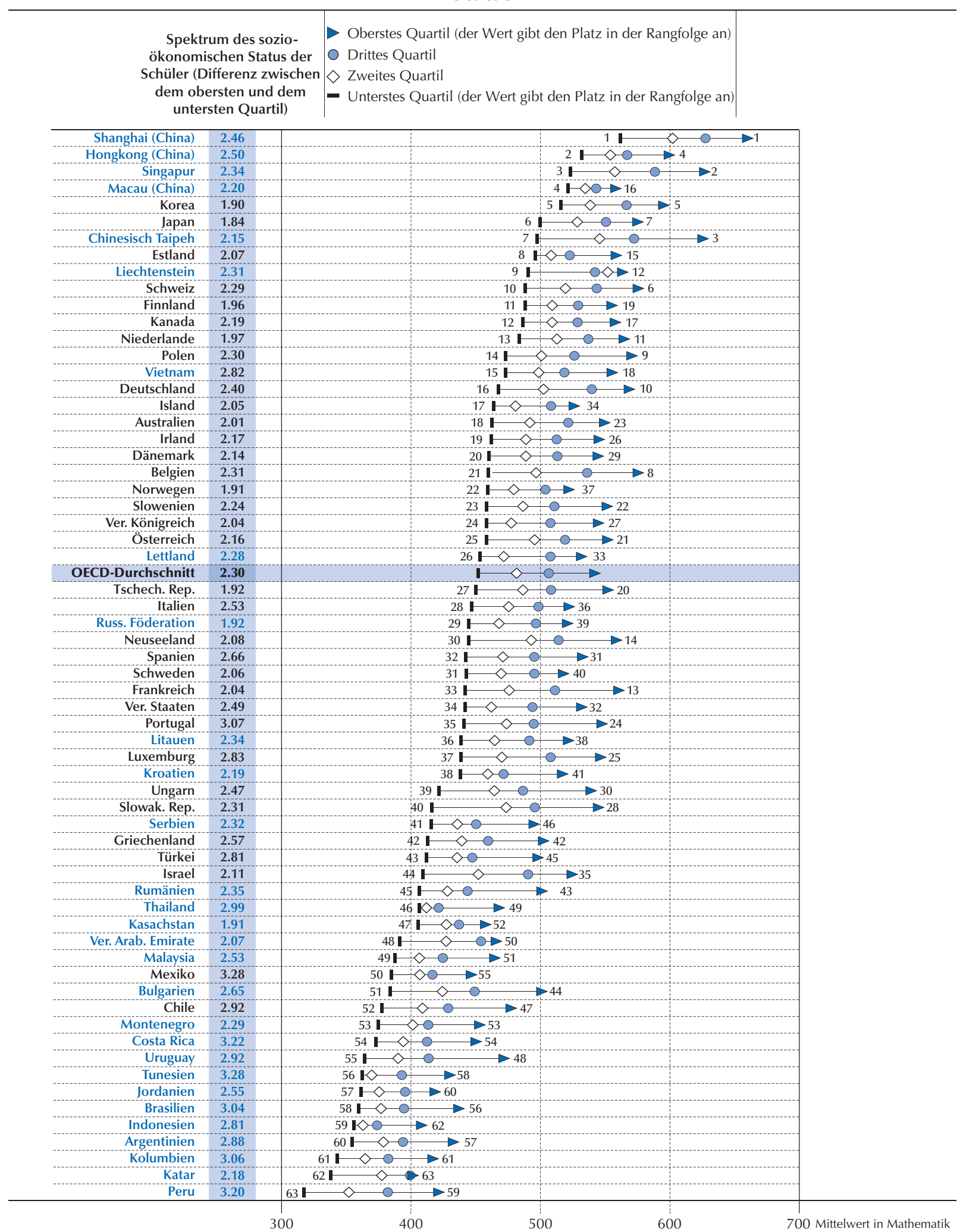

Die Länder und Volkswirtschaften sind in absteigender Reihenfolge nach den durchschnittlichen Leistungen der Schüler im untersten Quartil des PISA-Index des wirtschaftlichen, sozialen und kulturellen Status (ESCS) angeordnet.

Quelle: OECD, PISA-2012-Datenbank, Tabelle II.2.4a.

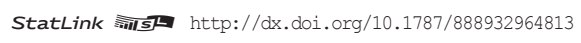


Unter den Bildungssystemen, deren mittlere Punktzahl in etwa dem OECD-Durchschnitt entspricht, ist der Abstand in Lettland, Norwegen und Island ebenso ausgeprägt. Unter den Ländern und Volkswirtschaften, deren Ergebnisse unter dem OECD-Durchschnitt liegen, schneiden sozioökonomisch benachteiligte Schülerinnen und Schüler in Italien, der Russischen Föderation und Schweden in der Rangliste um über fünf Plätze besser ab als ihre sozioökonomisch begünstigten Mitschülerinnen und Mitschüler.

Hingegen schneiden unter den Ländern, deren Ergebnisse über dem OECD-Durchschnitt liegen, in Belgien, Deutschland, Neuseeland und Polen sozioökonomisch begünstigte Schülerinnen und Schüler in der Rangliste um über fünf Plätze besser ab als ihre sozioökonomisch benachteiligten Mitschülerinnen und Mitschüler. Dieselbe Beobachtung lässt sich unter den Bildungssystemen, deren mittlere Punktzahl in etwa dem OECD-Durchschnitt entspricht, in der Tschechischen Republik, Frankreich und Portugal machen, ebenso wie in Bulgarien, Ungarn, Israel, Luxemburg, der Slowakischen Republik und Uruguay unter den Ländern, deren Ergebnisse unter dem OECD-Durchschnitt liegen (Abb. II.2.6).

Viele der mit einer sozioökonomischen Benachteiligung zusammenhängenden Faktoren lassen sich nicht direkt durch die Bildungspolitik beeinflussen, zumindest nicht auf kurze Sicht. Bis zu welchem Grad ist es den Schulen und der Bildungspolitik möglich, die Auswirkungen einer sozioökonomischen Benachteiligung auf die Schülerleistungen zu mildern? Der globale Zusammenhang zwischen sozioökonomischem Hintergrund und Schülerleistungen liefert einen Indikator für die Kapazität von Bildungssystemen, gleiche Bildungschancen anzubieten. Von noch größerer Relevanz ist dieser Zusammenhang indessen aus bildungspolitischer Sicht, da daran zu erkennen ist, inwieweit eine gerechte Chancenverteilung mit systemischen Bildungsaspekten verknüpft ist. Bessere Schulen für sozioökonomisch benachteiligte Schülerinnen und Schüler können einen Beitrag dazu leisten, sozioökonomisch bedingte Leistungsunterschiede zu verringern; aber die Länder müssen auch über andere Politikmaßnahmen nachdenken, die sich auf Familien auswirken, z.B. solche, die die Prävalenz von Armut, Mangelernährung und unangemessenen Wohnverhältnissen verringern oder das Bildungsniveau der Eltern anheben, sowie andere sozialpolitische Maßnahmen, mit denen sich die Leistungen der Schülerinnen und Schüler ebenfalls verbessern lassen.

\section{Leistungsvarianz zwischen Schulen}

Durchgehend hohe Standards in allen Schulen zu gewährleisten, stellt für alle Schulsysteme eine enorme Herausforderung dar. Ein Teil der Leistungsunterschiede zwischen den Schulen steht möglicherweise mit der sozioökonomischen Zusammensetzung der Schülerpopulation der Schulen oder mit anderen Merkmalen der Schülerschaft im Zusammenhang. Auch der Schulstandort kann Unterschiede zwischen den Schulen erklären. So bieten etwa Schulen in ländlichen bzw. städtischen Gebieten oftmals nicht dieselben Möglichkeiten oder haben quantitativ und qualitativ keinen Zugang zu denselben Ressourcen. In manchen Ländern und Volkswirtschaften gibt es darüber hinaus einen hohen Grad an räumlicher Segregation auf der Grundlage des Einkommens oder des kulturellen bzw. ethnischen Hintergrunds. In dezentralen Schulsystemen sind Leistungsunterschiede zwischen Schulen möglicherweise auch auf Unterschiede in Bezug auf die Art der zuständigen Bildungsbehörde zurückzuführen. In Kasten II.2.2 wird die Varianz der Schülerleistungen in den Ländern untersucht, die im Rahmen von PISA Daten auf regionaler/bundesstaatlicher Ebene erhoben haben.

\section{Kasten II.2.2 Standort, Regionen und Varianz der Schülerleistungen}

Australien, Belgien, Kanada, Italien, Mexiko, Spanien, das Vereinigte Königreich sowie die Partnerländer Brasilien, Kolumbien und die Vereinigten Arabischen Emirate haben genügend Daten auf subnationaler Ebene erhoben, um eine detaillierte Untersuchung darüber zu ermöglichen, inwiefern die Schülerleistungen in den verschiedenen Regionen und Standorten variieren. Anhang B2 jedes Bandes der Berichtsreihe PISA 2012 Ergebnisse enthält detaillierte Ergebnisse für die Länder, die Daten auf subnationaler Ebene bereitgestellt haben. In Abbildung II.2.a ist das Leistungsspektrum in den einzelnen Regionen verglichen mit der mittleren Punktzahl der Länder dargestellt.

Für jedes der vorstehend aufgeführten Länder wird in diesem Kasten beschrieben, welcher Anteil der Varianz der Schülerleistungen im Bereich Mathematik zwischen Schülerinnen und Schülern innerhalb von Schulen und Regionen, zwischen den Schulen innerhalb der Regionen bzw. zwischen den Regionen zu beobachten ist. Außerdem wird darin untersucht, inwiefern sich diese Varianz durch Unterschiede beim sozioökonomischen Status zwischen Schülern, Schulen und Regionen erklären lässt. Tabelle B2.II.25 gibt die Varianz auf jeder Ebene als Anteil an der insgesamt zwischen den OECD-Ländern beobachteten Leistungsvarianz wieder. 
- Abbildung II.2.a

\section{Durchschnittliche Mathematikleistungen in den Ländern und Regionen}

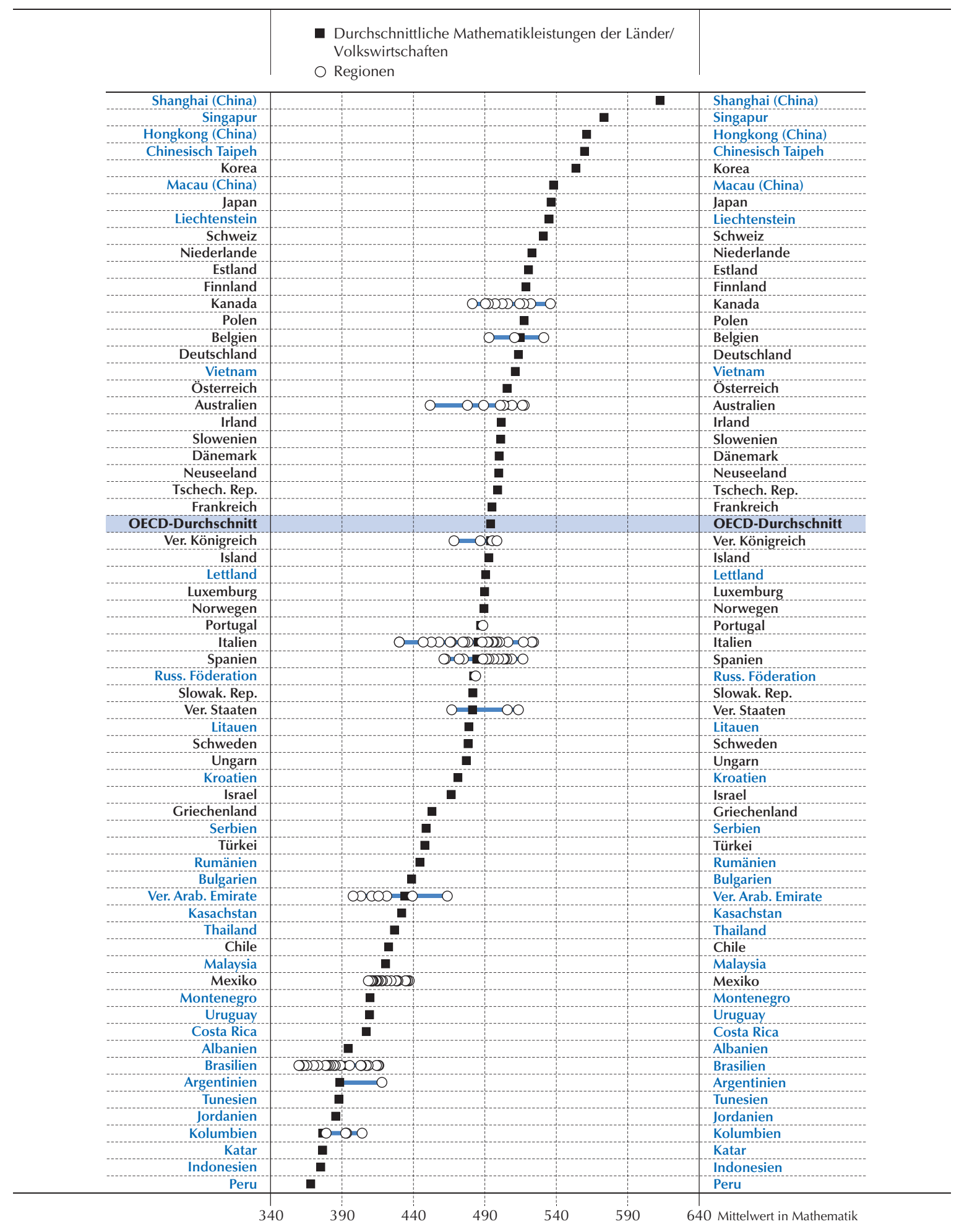

Anmerkung: Anhang B2 enthält eine Liste aller berücksichtigten Regionen sowie ihrer durchschnittlichen Mathematikleistungen.

Die Länder und Volkswirtschaften sind in absteigender Reihenfolge nach den Durchschnittsergebnissen im Bereich Mathematik angeordnet. Quelle: OECD, PISA-2012-Datenbank, Tabelle I.2.3a und B2.I.3.

StatLink 䨤鸟 http://dx.doi.org/10.1787/888932964813 
Auf Unterschiede bei den Durchschnittsergebnissen in Mathematik zwischen den einzelnen OECD-Ländern entfallen 10\% der Varianz der Schülerleistungen - was bedeutet, dass die Leistungsunterschiede zwischen den Ländern verglichen mit den Leistungsunterschieden zwischen den einzelnen Schülern relativ gering sind (vgl. Abb. IV.1.2 in Band IV). In den meisten Ländern macht die Leistungsvarianz zwischen den einzelnen Regionen unter 3\% der zwischen den OECD-Ländern beobachteten Varianz aus. In Italien jedoch entfallen nahezu 8\% der Gesamtvarianz der Schülerleistungen zwischen den OECD-Ländern auf die Leistungsvarianz zwischen den Regionen. Im Vereinigten Königreich gibt es praktisch keine Leistungsunterschiede zwischen England, Nordirland, Wales und Schottland; gleichwohl bestehen zwischen den Schulen innerhalb dieser Regionen Leistungsunterschiede.

Die Leistungsunterschiede zwischen den Schulen innerhalb einer Region sind in Belgien, wo sie 59\% der Gesamtvarianz der Schülerleistungen zwischen den OECD-Ländern ausmachen, und Italien (45\%) im Vergleich zum OECD-Durchschnitt von 36\% besonders groß. Die Varianz der Leistungen von Schülern, die ein und dieselbe Schule besuchen, sind in Australien, wo sie 68\% der Gesamtvarianz der Schülerleistungen zwischen den OECDLändern ausmachen, dem Vereinigten Königreich (65\%), Kanada (64\%) und Spanien (64\%) im Vergleich zum OECDDurchschnitt von 54\% relativ groß (Tabelle B2.II.25).

Unterschiede beim sozioökonomischen Status erklären einen relativ großen Anteil an der Leistungsdifferenz zwischen den Regionen, insbesondere in einigen Ländern, wie z.B. in Spanien und den Vereinigten Arabischen Emiraten, wo die Unterschiede beim sozioökonomischen Status zwischen Schülern, Schulen und Regionen über 85\% der zwischen den Regionen beobachteten Leistungsunterschiede erklären. Der auf diese Weise erklärte Anteil der Leistungsvarianz ist auch in Kolumbien (70\%), Belgien (70\%) und Mexiko (58\%) hoch. Dagegen erklären sozioökonomische Unterschiede in Kanada lediglich 16\% und in Australien lediglich 26\% der Leistungsunterschiede zwischen den Regionen. In Italien erklärt der sozioökonomische Status 47\% der Leistungsunterschiede zwischen den Regionen. In Belgien und im Vereinigten Königreich hängen die Leistungsunterschiede zwischen den Schulen innerhalb der Regionen eng mit dem sozioökonomischen Status zusammen. Im Vereinigten Königreich erklärt der sozioökonomische Status $62 \%$ der Leistungsunterschiede zwischen den Schulen innerhalb ein und derselben Region, während in Belgien 73\% der Leistungsunterschiede zwischen den Schulen innerhalb einer Region auf den sozioökonomischen Status zurückzuführen sind. Hingegen erklärt der sozioökonomische Status in Kanada, Mexiko und den Vereinigten Arabischen Emiraten weniger als 50\% der Varianz zwischen den Schulen innerhalb einer Region (im OECD-Vergleich sind $48 \%$ der Leistungsunterschiede zwischen den Schulen innerhalb einer Region auf den sozioökonomischen Status zurückzuführen). In den meisten Ländern machen sozioökonomische Disparitäten zwischen Schülern innerhalb einer Schule lediglich 3\% der innerhalb von Schulen beobachteten Leistungsunterschiede aus. In Spanien lassen sich indessen 9\% der innerhalb von Schulen beobachteten Leistungsunterschiede hierdurch erklären (Tabelle B2.II.25).

Leistungsunterschiede zwischen Schulen können auch mit der Qualität der Schule bzw. des Schulpersonals zusammenhängen oder dadurch bedingt sein, dass bildungspolitische Maßnahmen in einigen Schulen umgesetzt werden, nicht aber in anderen. Auch Politikmaßnahmen auf der Ebene der Schulsysteme könnten einen Teil der Erklärung liefern, weshalb die Schülerleistungen zwischen Schulen variieren. Schulsysteme, in denen die Leistungsvarianz zwischen Schulen gering ist, zeichnen sich im Allgemeinen dadurch aus, dass sie nicht nach Bildungsgängen, Schulart oder Fähigkeiten der Schülerinnen und Schüler differenzieren. In anderen Systemen wird versucht, dem Bedarf jeder Schülerin und jedes Schülers durch Schaffung verschiedener Bildungsgänge Rechnung zu tragen, wobei die Schüler gefordert sind, sich zu einem früheren oder späteren Zeitpunkt für einen dieser Bildungsgänge zu entscheiden. In Band IV der vorliegenden Publikation wird untersucht, wie sowohl schulpolitische Maßnahmen und Vorgehensweisen als auch Politikmaßnahmen auf Schulsystemebene variieren und mit Leistungsunterschieden zwischen Schülern und Schulen zusammenhängen.

Abbildung II.2.7 zeigt die Leistungsvarianz innerhalb der einzelnen Länder. Die Länge des Balkens stellt die Gesamtvarianz im jeweiligen Land als Anteil an der durchschnittlichen Leistungsvarianz im OECD-Raum dar. Der dunkle Teil des Balkens gibt den Anteil der zwischen Schulen beobachteten Varianz wieder, während der helle Teil den Anteil der innerhalb von Schulen beobachteten Varianz veranschaulicht. 
- Abbildung II.2.7

Gesamtvarianz der Mathematikleistungen sowie Varianz zwischen und innerhalb von Schulen

Ausgedrückt in Prozent der Varianz der Schülerleistungen in den OECD-Ländern

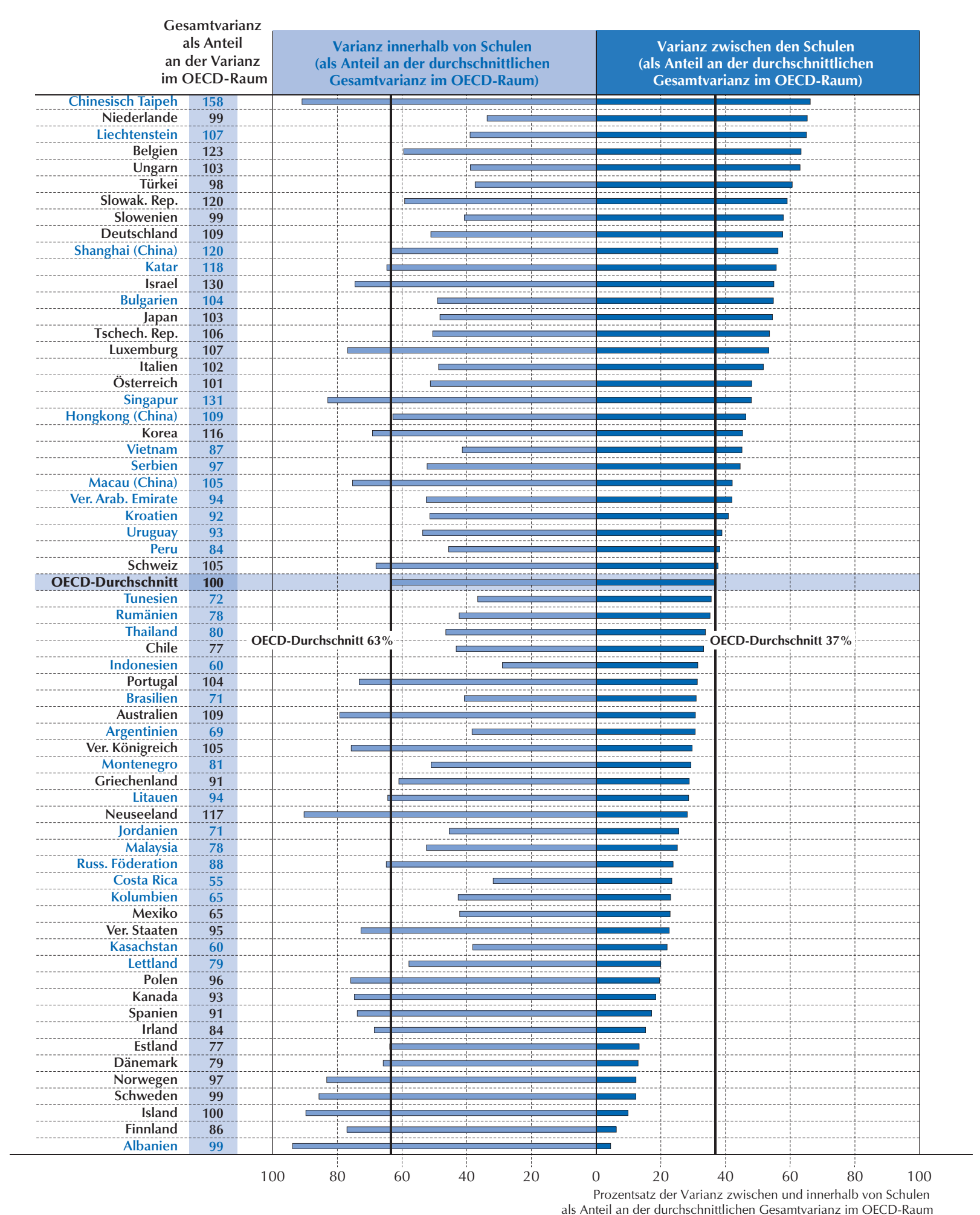

Die Länder/Volkswirtschaften sind in absteigender Reihenfolge der Varianz zwischen Schulen als Anteil an der Gesamtleistungsvarianz in den OECD Ländern angeordnet.

Quelle: OECD, PISA-2012-Datenbank, Tabelle II.2.8a.

StatLink 光IST http://dx.doi.org/10.1787/888932964813 
In den OECD-Ländern werden 37\% der Gesamtleistungsunterschiede zwischen Schulen und 63\% innerhalb von Schulen beobachtet. Die Leistungsunterschiede zwischen Schulen fallen in den einzelnen Schulsystemen sehr unterschiedlich aus ${ }^{10}$. So entfallen z.B. in Estland, Dänemark, Norwegen, Schweden, Island, Finnland und Albanien weniger als 15\% des OECDDurchschnitts bei der Gesamtvarianz der Schülerleistungen auf die Varianz zwischen Schulen. Da es Dänemark, Estland und Finnland zudem gelingt, eine überdurchschnittliche mittlere Punktzahl zu erreichen, können Eltern und Schüler in diesen Ländern davon ausgehen, dass letztere unabhängig von der besuchten Schule wahrscheinlich gute Leistungen erzielen werden. In Chinesisch Taipeh, den Niederlanden, Liechtenstein, Belgien, Ungarn und der Türkei hingegen macht die Varianz zwischen Schulen über 60\% der Durchschnittsvarianz im OECD-Raum aus. Die Gesamtvarianz in diesen Ländern fällt in der Tendenz überdurchschnittlich hoch aus, insbesondere in Chinesisch Taipeh und Belgien (Abb. II.2.7 und Tabelle II.2.8a).

\section{Leistungsunterschiede zwischen Schulen und sozioökonomische Disparitäten}

Im OECD-Durchschnitt lässt sich über die Hälfte der zwischen Schülern in verschiedenen Schulen beobachteten Leistungsunterschiede durch sozioökonomische Disparitäten zwischen Schülern und Schulen erklären (Abb. II.2.8). Der Bildungsgang der Schüler, der in PISA anhand des Schülerfragebogens und anderer Quellen ermittelt wird, spielt in vielen Ländern ebenfalls eine wichtige Rolle bei der Erklärung der Leistungsunterschiede zwischen Schulen und macht $40 \%$ der Varianz aus. Der Bildungsgang wird anhand der Stufe des Bildungsgangs (Sekundarstufe I oder II), der Ausrichtung des Lehrplans (allgemein- oder berufsbildend) und der Absicht (soll ein allgemeiner Abschluss erreicht werden, der den Zugang zu anderen Bildungsniveaus eröffnet, oder soll der unmittelbare Zugang zum Arbeitsmarkt ermöglicht werden) identifiziert. In den OECDLändern sind $71 \%$ der Leistungsunterschiede zwischen Schülern in unterschiedlichen Schulen auf eine Kombination aus dem sozioökonomischen Status und dem Bildungsgang der Schülerinnen und Schüler zurückzuführen (Tabelle II.2.9a).

Die Chancengerechtigkeit zwischen Schulen ist in Ländern größer, in denen die Bildungserträge, gemessen an der Stärke des Zusammenhangs zwischen Leistung und sozioökonomischem Status, allgemein gerechter verteilt sind - dies trifft auch auf Schulsysteme mit hohem durchschnittlichem Leistungsniveau in Mathematik zu, wie z.B. Kanada, Finnland und Hongkong (China). In den drei Ländern/Volkswirtschaften sind unter 45\% des Leistungsabstands zwischen Schulen auf sozioökonomische Disparitäten zurückzuführen. Sozioökonomische Disparitäten zwischen Schulen hängen in Luxemburg eng mit Leistungsunterschieden zusammen, wo 93\% der Leistungsvarianz zwischen Schulen durch den sozioökonomischen Status der Schüler und der Schulen erklärt werden. In Chile, Ungarn, Irland, Neuseeland, Peru und Slowenien sind über 75\% der Leistungsunterschiede zwischen Schulen auf den sozioökonomischen Status der Schüler und der Schulen zurückzuführen.

Die Bildungsgänge der Schülerinnen und Schüler stehen in Deutschland, Luxemburg und den Niederlanden in engem Zusammenhang mit Leistungsunterschieden zwischen den Schulen; in diesen drei Ländern sind über 80\% der Leistungsvarianz auf den jeweiligen Bildungsgang der Schülerinnen und Schüler zurückzuführen. Nach Berücksichtigung des sozioökonomischen Status der Schüler und der Schulen lassen sich in Belgien, Deutschland, Luxemburg, den Niederlanden, Portugal und Shanghai (China) mindestens 85\% der Leistungsunterschiede zwischen Schulen durch die Bildungsgänge und den sozioökonomischen Status der Schülerinnen und Schüler sowie durch das sozioökonomische Profil der Schulen erklären (Tabelle II.2.9a).

In den OECD-Ländern stehen der sozioökonomische Status der Schüler und der Schulen sowie die Bildungsgänge der Schüler mit den beobachteten Leistungsunterschieden (innerschulische Varianz) zwischen Schülern, die dieselbe Schule besuchen, in einem schwächeren Zusammenhang ${ }^{11}$. In Dänemark, Finnland, Neuseeland, Polen, Portugal, Spanien und Schweden lassen sich zwischen 9\% und 10\% der Leistungsvarianz innerhalb von Schulen durch Unterschiede beim sozioökonomischen Status der Schülerinnen und Schüler erklären. In Luxemburg, Macau (China), Malaysia, den Niederlanden und Portugal erklären sozioökonomischer Status und Bildungsgang zwischen 20\% und 38\% der Leistungsunterschiede innerhalb von Schulen (Tabelle II.2.9a).

Im Durchschnitt der OECD-Länder haben Schülerinnen und Schüler an sozioökonomisch begünstigten Schulen in Mathematik einen Leistungsvorsprung von 72 Punkten gegenüber Schülerinnen und Schülern, die sozioökonomisch weniger begünstigte Schulen besuchen. Innerhalb der Schulen erzielen sozioökonomisch bessergestellte Schülerinnen und Schüler in Mathematik 19 Punkte mehr als weniger begünstigte Schülerinnen und Schüler, die dieselbe Schule besuchen ${ }^{12}$. In Abbildung II.2.9 ist der durchschnittliche Leistungsabstand zwischen Schülerinnen und Schülern, die unterschiedliche Schulen besuchen, sowie zwischen Schülerinnen und Schülern, die dieselbe Schule besuchen, nach dem sozioökonomischen Status dargestellt (Tabelle II.2.9a). Die mit dem sozioökonomischen Status zusammenhängenden Unterschiede bei den Schülerleistungen zwischen den Schulen sind in Finnland, Mexiko und Spanien mit unter 30 Punkten relativ gering (41 Punkte entsprechen etwa einem Schuljahr). In Japan und den Niederlanden hingegen belaufen sich die entsprechenden Unterschiede auf über 140 Punkte. 
- Abbildung II.2.8 "

\section{Leistungsvarianz zwischen und innerhalb von Schulen, erklärt durch den} sozioökonomischen Status der Schüler und der Schulen

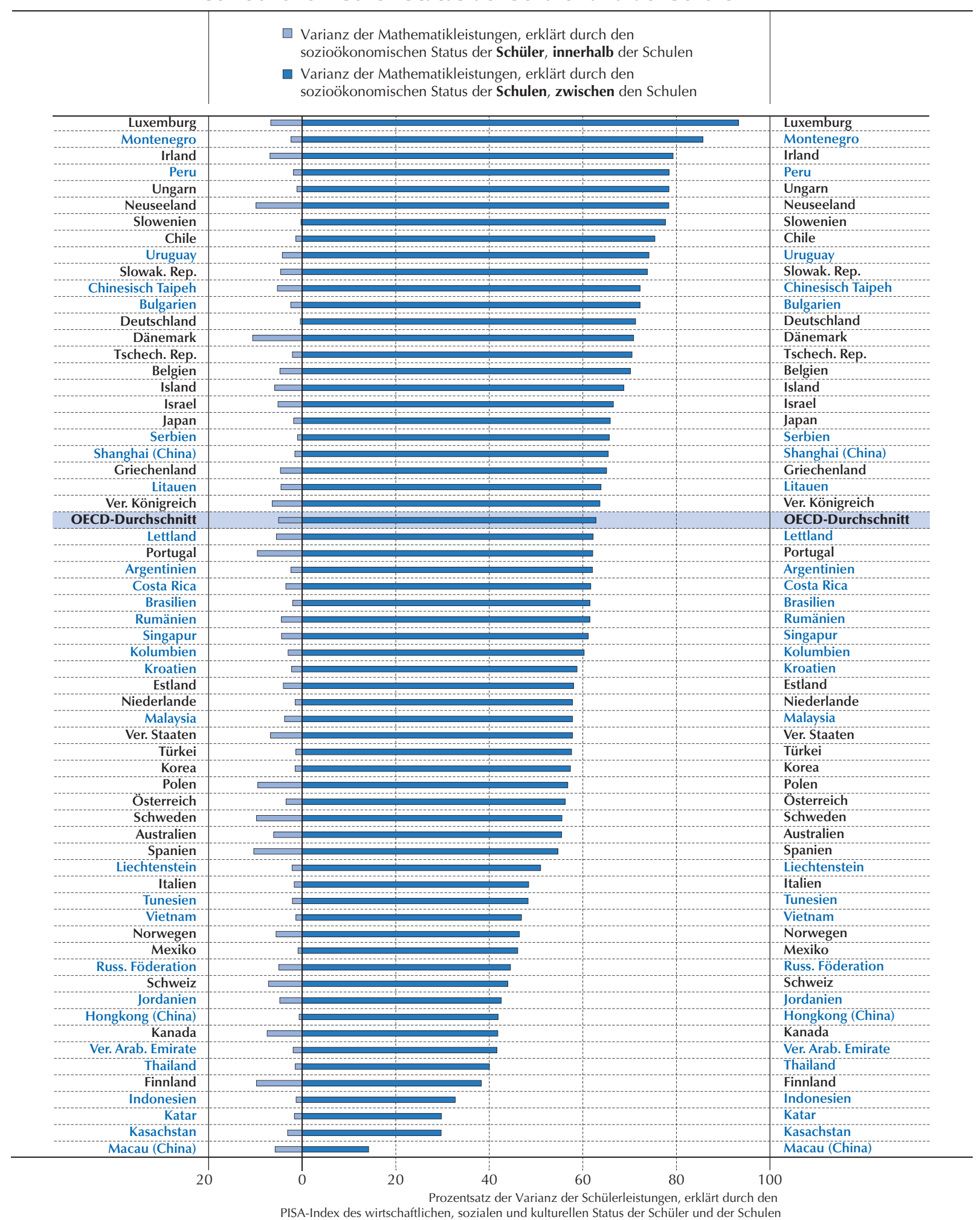

Die Länder/Volkswirtschaften sind in absteigender Reihenfolge nach dem prozentualen Anteil der Varianz der Mathematikleistungen zwischen den Schulen angeordnet, der durch den PISA-Index des wirtschaftlichen, sozialen und kulturellen Status der Schüler und der Schulen erklärt werden kann. Quelle: OECD, PISA-2012-Datenbank, Tabelle II.2.9a.

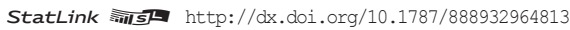


- Abbildung II.2.9

Leistungsunterschiede innerhalb und zwischen Schulen zwischen verschiedenen sozioökonomischen Gruppen

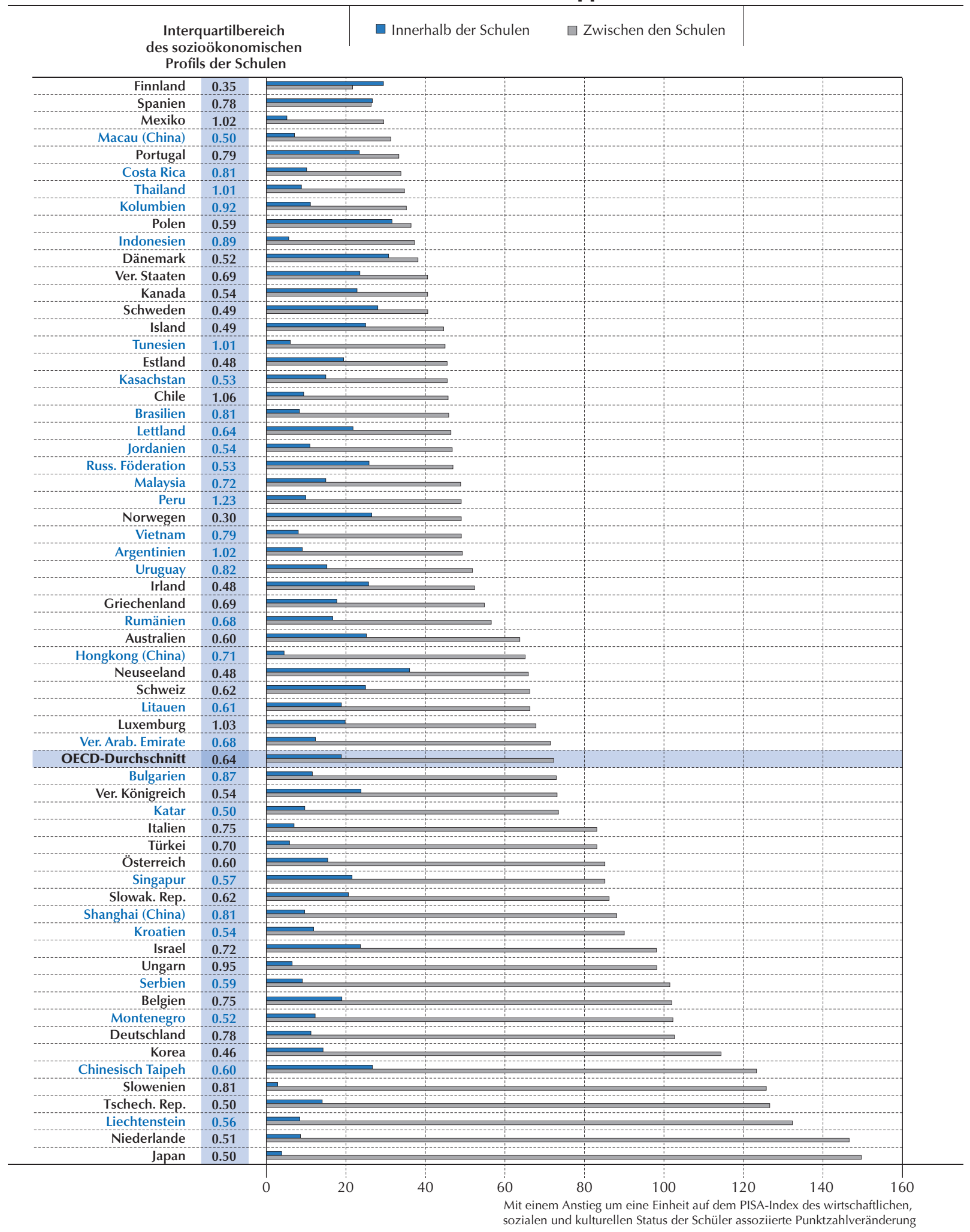

Die Länder und Volkswirtschaften sind in aufsteigender Reihenfolge nach der mit einem Anstieg um eine Einheit auf dem PISA-Index des wirtschaftlichen, sozialen und kulturellen Status der Schüler assoziierten Punktzahlveränderung zwischen Schulen angeordnet.

Quelle: OECD, PISA-2012-Datenbank, Tabelle II.2.9a und II.2.13a.

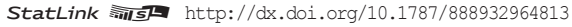


Schülerinnen und Schüler an sozioökonomisch begünstigten Schulen schneiden besser ab als Schülerinnen und Schüler an sozioökonomisch benachteiligten Schulen, allerdings erzielen sie im internationalen Vergleich nicht unbedingt besonders gute Leistungen. In Kasten II.2.3 wird erklärt, auf welche Art und Weise Schulen in PISA als sozioökonomisch begünstigt, durchschnittlich bzw. benachteiligt eingestuft werden, und werden die wichtigsten Merkmale der betreffenden Schulen beschrieben. Abbildung II.2.10 veranschaulicht die Leistungen der Schülerinnen und Schüler an sozioökonomisch begünstigten bzw. benachteiligten Schulen in den einzelnen Ländern. Im OECD-Durchschnitt beträgt der Leistungsabstand zwischen Schülerinnen und Schülern an sozioökonomisch begünstigten Schulen sowie Schülerinnen und Schülern an sozioökonomisch benachteiligten Schulen 104 Punkte. Während der Leistungsabstand in Finnland und Macau (China) relativ gering ist (er beträgt unter 40 Punkte, was zwar nach wie vor ein großer Abstand ist, aber dennoch unter dem OECD-Durchschnitt liegt), beträgt er in der Tschechischen Republik, den Niederlanden, der Slowakischen Republik und Chinesisch Taipeh über 150 Punkte.

\section{Kasten II.2.3 Welche Merkmale weisen Schülerinnen und Schüler auf, die sozioökonomisch begünstigte bzw. benachteiligte Schulen besuchen?}

Sozioökonomisch begünstigte (benachteiligte) Schulen sind Schulen, in denen der sozioökonomische Status eines Durchschnittsschülers bzw. das sozioökonomische Profil der Schule über (unter) dem sozioökonomischen Status eines Durchschnittsschülers im betreffenden Land bzw. dem durchschnittlichen sozioökonomischen Status des Landes/der Volkswirtschaft liegt. An jeder Schule nehmen 35 als Zufallsstichprobe ausgewählte Schülerinnen und Schüler an PISA teil (wegen näherer Einzelheiten vgl. PISA 2012 Technical Report [OECD, erscheint demnächst]). Da das sozioökonomische Profil der Schulen ausgehend von den Angaben dieser Schülerinnen und Schüler ermittelt wird, hängt die Genauigkeit der Schätzung von der Zahl der Schülerinnen und Schüler ab, die an den einzelnen Schulen jeweils an der Erhebung teilnehmen, sowie von der Vielfalt ihrer Antworten. Dies wurde bei der Einstufung der Schulen in sozioökonomisch begünstigte, sozioökonomisch benachteiligte oder sozioökonomisch durchschnittliche Schulen berücksichtigt. Wenn der Unterschied zwischen dem sozioökonomischen Profil einer Schule und dem sozioökonomischen Status eines Durchschnittsschülers des Landes (dem durchschnittlichen sozioökonomischen Status auf Ebene des Landes) nicht statistisch signifikant war, wurde die Schule als Schule mit durchschnittlichem sozioökonomischem Profil eingestuft. Wenn das Profil einer Schule statistisch signifikant über dem durchschnittlichen sozioökonomischen Status des Landes lag, wurde die Schule als sozioökonomisch begünstigte Schule eingestuft. Wenn das Profil der Schule unter dem durchschnittlichen sozioökonomischen Status des Landes lag, wurde die Schule als sozioökonomisch benachteiligte Schule eingestuft. Schulen, an denen nur wenige Schülerinnen und Schüler an PISA teilnahmen, wurden daher mit größerer Wahrscheinlichkeit als sozioökonomisch durchschnittliche Schulen eingestuft.

In den OECD-Ländern haben 73\% der Schülerinnen und Schüler, die sozioökonomisch begünstigte Schulen besuchen, mindestens einen Elternteil mit Tertiärabschluss (ISCED 5 bzw. 6), verglichen mit 33\% der Schülerinnen und Schüler an sozioökonomisch benachteiligten Schulen. Während $77 \%$ der Schülerinnen und Schüler an sozioökonomisch begünstigten Schulen Eltern haben, die als Fach- oder Führungskraft (ISCO-Berufshauptgruppe 1, 2 oder 3) tätig sind, trifft dies lediglich auf 32\% der Schülerinnen und Schüler an sozioökonomisch benachteiligten Schulen zu. Rund $61 \%$ der Schülerinnen und Schüler an sozioökonomisch begünstigten Schulen gegenüber $36 \%$ der Schülerinnen und Schüler an sozioökonomisch benachteiligten Schulen stehen im Elternhaus Gedichtbände zur Verfügung. Im Hinblick auf die Durchschnittswerte der einzelnen Länder bestehen indessen erhebliche Unterschiede. So haben unter den OECD-Ländern beispielsweise in Kanada, Finnland und Schweden über $80 \%$ der Schülerinnen und Schüler an sozioökonomisch begünstigten Schulen und über $50 \%$ der Schülerinnen und Schüler an sozioökonomisch benachteiligten Schulen Eltern mit einem Tertiärabschluss. Hingegen haben in Israel, Japan, Korea und den Vereinigten Staaten zwar über 80\% der Schülerinnen und Schüler an sozioökonomisch begünstigten Schulen Eltern, die über ein hohes Bildungsniveau verfügen, bei Schülerinnen und Schülern an sozioökonomisch benachteiligten Schulen trifft dies jedoch auf weniger als 40\% zu. Auch im Hinblick auf den Anteil der Schülerinnen und Schüler an sozioökonomisch begünstigten bzw. benachteiligten Schulen, deren Eltern einen angesehenen Beruf ausüben, bestehen erhebliche Unterschiede zwischen den einzelnen Schulsystemen (Tabelle II.2.10). 
- Abbildung II.2.10 "

\section{Mathematikleistungen der Schüler an sozioökonomisch begünstigten, durchschnittlichen und benachteiligten Schulen}

\begin{tabular}{|c|c|c|}
\hline \multicolumn{3}{|c|}{ Durchschnittlicher sozioökonomischer Status } \\
\hline $\begin{array}{c}\text { Sozio- } \\
\text { ökonomisch } \\
\text { benachteiligte } \\
\text { Schulen }{ }^{1}\end{array}$ & \begin{tabular}{|c|} 
Sozio- \\
ökonomisch \\
durchschnittliche \\
Schulen $^{1}$ \\
\end{tabular} & $\begin{array}{c}\text { Sozio- } \\
\text { ökonomisch } \\
\text { begünstigte } \\
\text { Schulen }^{1}\end{array}$ \\
\hline-1.06 & -0.35 & 0.25 \\
\hline-1.22 & -0.89 & -0.32 \\
\hline-0.71 & -0.33 & 0.45 \\
\hline-1.33 & -0.84 & 0.05 \\
\hline-0.04 & 0.36 & 0.77 \\
\hline-0.40 & 0.06 & 0.66 \\
\hline-0.28 & 0.11 & 0.73 \\
\hline-0.46 & 0.03 & 0.48 \\
\hline-0.12 & 0.38 & 0.90 \\
\hline-0.86 & -0.44 & 0.15 \\
\hline-0.74 & -0.22 & 0.47 \\
\hline-0.49 & -0.08 & 0.37 \\
\hline-2.46 & -1.85 & -0.78 \\
\hline 0.03 & 0.42 & 0.91 \\
\hline-0.08 & 0.39 & 0.97 \\
\hline 0.27 & 0.74 & 1.16 \\
\hline-0.30 & 0.24 & 0.77 \\
\hline-0.17 & 0.22 & 0.74 \\
\hline-0.95 & -0.32 & 0.32 \\
\hline-0.62 & -0.16 & 0.39 \\
\hline-0.79 & -0.23 & 0.52 \\
\hline-0.23 & 0.26 & 0.79 \\
\hline-0.44 & 0.19 & 0.82 \\
\hline-0.41 & 0.07 & 0.68 \\
\hline-0.56 & -0.02 & 0.60 \\
\hline-0.45 & 0.17 & 0.71 \\
\hline-0.51 & 0.02 & 0.58 \\
\hline-1.15 & -0.49 & 0.61 \\
\hline-0.26 & 0.23 & 0.68 \\
\hline-0.51 & 0.09 & 0.63 \\
\hline-0.46 & 0.12 & 0.68 \\
\hline-0.47 & 0.03 & 0.66 \\
\hline-0.48 & 0.17 & 0.78 \\
\hline-0.55 & -0.09 & 0.51 \\
\hline-0.76 & -0.13 & 0.45 \\
\hline-0.79 & -0.35 & 0.32 \\
\hline-0.65 & -0.08 & 0.55 \\
\hline-0.86 & -0.17 & 0.50 \\
\hline-0.81 & -0.37 & 0.12 \\
\hline-0.95 & -0.27 & 0.42 \\
\hline-2.04 & -1.37 & -0.40 \\
\hline-1.07 & -0.51 & 0.22 \\
\hline-2.05 & -1.49 & -0.63 \\
\hline-0.74 & -0.33 & 0.36 \\
\hline-0.39 & 0.18 & 0.67 \\
\hline-0.77 & -0.07 & 0.61 \\
\hline-0.26 & 0.29 & 0.80 \\
\hline-1.29 & -0.66 & 0.36 \\
\hline-1.36 & -0.76 & -0.04 \\
\hline-1.99 & -1.12 & -0.10 \\
\hline-0.97 & -0.29 & 0.46 \\
\hline-1.88 & -0.99 & 0.16 \\
\hline-1.51 & -0.89 & 0.26 \\
\hline-0.63 & -0.30 & 0.17 \\
\hline-1.87 & -1.23 & -0.11 \\
\hline-1.02 & -0.46 & 0.30 \\
\hline-2.38 & -1.88 & -0.85 \\
\hline-2.04 & -1.22 & -0.22 \\
\hline-0.03 & 0.42 & 0.81 \\
\hline-1.43 & -0.75 & 0.05 \\
\hline-2.12 & -1.28 & -0.40 \\
\hline-2.13 & -1.25 & -0.28 \\
\hline
\end{tabular}

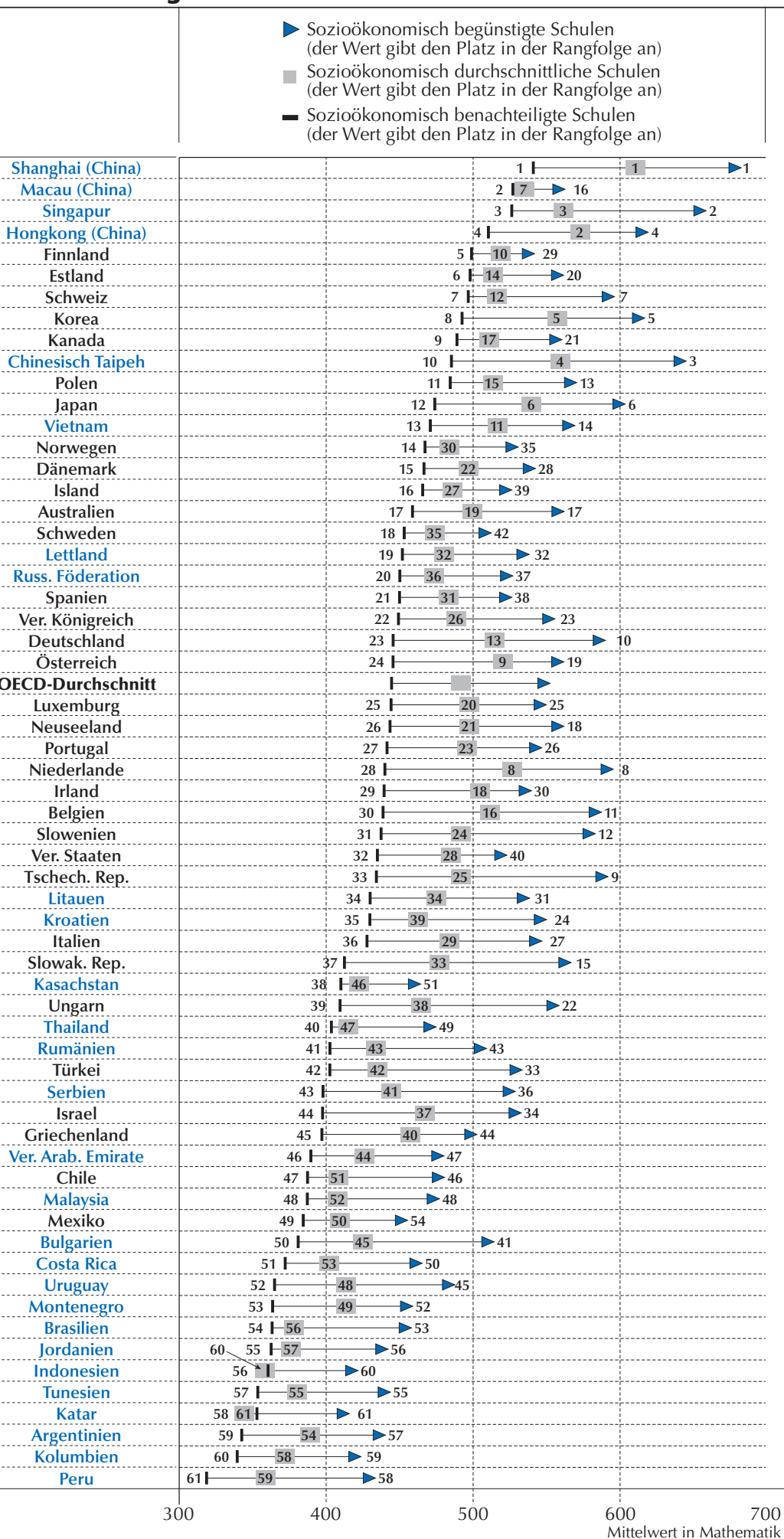

1. Eine sozioökonomisch benachteiligte Schule ist eine Schule mit Schülern, deren durchschnittlicher sozioökonomischer Status statistisch signifikant unter dem durchschnittlichen sozioökonomischen Status des Landes/derVolkswirtschaft liegt, eine sozioökonomisch durchschnittliche Schule ist eine Schule, an der es keinen Unterschied zwischen dem durchschnittlichen sozioökonomischen Status der Schulen und des Landes/der Volkswirtschaft gibt, und eine sozioökonomisch begünstigte Schule ist eine Schule mit Schülern, deren durchschnittlicher sozioökonomischer Status statistisch signifikant über dem durchschnittlichen sozioökonomischen Status des Landes/der Volkswirtschaft liegt. Die Länder/Volkswirtschaften sind in absteigender Reihenfolge nach den durchschnittlichen Leistungen der Schüler in sozioökonomisch benachteiligten Schulen angeordnet. Quelle: OECD, PISA-2012-Datenbank, Tabelle II.4.2.

StatLink त्राजम http://dx.doi.org/10.1787/888932964813 
In Abbildung II.2.10 ist darüber hinaus der durchschnittliche sozioökonomische Status der Schülerinnen und Schüler dargestellt, die sozioökonomisch begünstigte, durchschnittliche bzw. benachteiligte Schulen besuchen. Manchen Schülerinnen und Schülern, die Schulen mit einer sozioökonomisch relativ benachteiligten Schülerschaft besuchen, gelingt es, hohe Leistungen zu erbringen. So erzielen beispielsweise Schülerinnen und Schüler an sozioökonomisch benachteiligten Schulen in Hongkong (China), Macau (China), Shanghai (China) und Singapur in Mathematik im Durchschnitt über 500 Punkte. Der durchschnittliche sozioökonomische Status dieser Schülerinnen und Schüler liegt jedoch deutlich unter dem Durchschnitt, insbesondere in Hongkong (China), Macau (China) und Shanghai (China), wo er um über eine Einheit unter dem OECD-Durchschnitt liegt (Tabelle II.4.2).

\section{Kasten II.2.4 Verbesserung der PISA-Ergebnisse: Mexiko}

In Mexiko stiegen die Schulbesuchszahlen zwischen 1950 und 2007 um das Zehnfache von etwa 3 Millionen auf über 30 Millionen Schülerinnen und Schüler. Im Jahr 2003 befanden sich 58\% der 15-Jährigen in Klassenstufe 7 oder höher. Trotz der stetig wachsenden Bildungsbeteiligung besuchten 2012 dennoch weniger als 70\% der 15-Jährigen des Landes die Schule. Zusätzlich zu den niedrigen Schulbesuchsquoten sieht sich Mexiko auch bei der Gewährleistung einer qualitativ hochwertigen Bildung für alle Schülerinnen und Schüler erheblichen Herausforderungen gegenüber. Im Jahr 2012 erreichte über die Hälfte der mexikanischen Schülerinnen und Schüler in Mathematik nicht das Grundkompetenzniveau, und weniger als 1\% der Schülerinnen und Schüler gehörte zu den leistungsstärksten Schülern in PISA. Die Durchschnittsergebnisse liegen weiterhin deutlich unter dem OECD-Durchschnitt, und dies in einem Maß, dass der durchschnittliche 15-jährige mexikanische Schüler einen Leistungsrückstand von nahezu zwei Jahren gegenüber dem OECD-Durchschnitt hat.

Indessen hat Mexiko seine Ergebnisse in den vergangenen zehn Jahren verbessert. Zwischen 2003 und 2012 sind die Schülerleistungen in Lesekompetenz und Mathematik gestiegen. 2003 erzielten die 15-jährigen Schülerinnen und Schüler im PISA-Mathematiktest im Durchschnitt 385 Punkte, 2012 waren es 413 Punkte - d.h. sie haben sich durchschnittlich um 3,1 Punkte pro Jahr verbessert, was dem drittgrößten Anstieg in den OECD-Ländern entspricht; allerdings würde Mexiko selbst mit dieser Verbesserungsrate noch ein Vierteljahrhundert benötigen, um zum OECDDurchschnitt aufzuschließen. Am größten ist die Leistungszunahme unter den am schlechtesten abschneidenden Schülerinnen und Schülern: Sie verbesserten sich zwischen 2003 und 2012 um mehr als 40 Punkte - was einem Jahr formaler Schulbildung entspricht. Gleichzeitig ging der Anteil der 15-Jährigen mit Leistungen unter Kompetenzstufe 2 in Mathematik in diesem Zeitraum um 11 Prozentpunkte zurück. Obwohl unter den leistungsstärksten Schülerinnen und Schülern (die im 90. Perzentil der Kompetenzskala Mathematik angesiedelt sind) im Bereich Mathematik eine Leistungssteigerung um mehr als 20 Punkte beobachtet wurde, blieb der Prozentsatz der Schülerinnen und Schüler mit Leistungen auf Stufe 5 oder darüber in Mathematik zwischen 2003 und 2012 unverändert.

\section{Den Einfluss des sozioökonomischen Status auf die Leistungen verringern}

Mexiko ist es zwischen 2003 und 2012 ebenfalls gelungen, den Einfluss des sozialen Hintergrunds auf die Lernergebnisse zu mindern. Der Unterschied bei den Mathematikleistungen zwischen sozioökonomisch begünstigten und benachteiligten Schülerinnen und Schülern verringerte sich zwischen 2003 und 2012 von etwa 60 auf rund 40 Punkte. In diesem Kontext schwächte sich auch der Zusammenhang zwischen sozioökonomischem Status und Leistungsniveau ab: Während sich 2003 17\% der Varianz bei den Mathematikleistungen durch den sozioökonomischen Status erklären ließen, waren es 2012 nur noch 10\%. Das bedeutet mit anderen Worten, dass die Wahrscheinlichkeit, mit der ein 15-Jähriger zu den leistungsstarken oder leistungsschwachen Schülerinnen und Schülern zählt, 2012 weniger mit seinem sozioökonomischen Status in Zusammenhang stand als 2003. Die in Mexiko seit PISA 2003 in den Bereichen Mathematik und Chancengerechtigkeit beobachteten Verbesserungen sind effektiv weitgehend das Ergebnis von Leistungssteigerungen unter den sozioökonomisch benachteiligten und schlecht abschneidenden Schulen, so dass sich die in PISA festgestellten Leistungsunterschiede zwischen den Schulen in diesem Zeitraum verringert haben.

Auch die schulischen Erfahrungen der mexikanischen Schülerinnen und Schüler haben sich zwischen 2003 und 2012 verändert. Während 30\% der 15-jährigen Schülerinnen und Schüler 2003 angaben, in ihrer Schullaufbahn mindestens eine Klasse wiederholt zu haben, waren es 2012 nur 15\%. 2012 hatten die Schülerinnen und Schüler durchschnittlich etwa 15 Minuten mehr Mathematikunterricht pro Woche, verbrachten aber etwa 35 Minuten weniger 
mit Hausaufgaben pro Woche als die Schülerinnen und Schüler im Jahr 2003. Ferner stuften die Schülerinnen und Schüler die Disziplin im Klassenzimmer und die Lehrer-Schüler-Beziehungen 2012 besser ein als die Schüler im Jahr 2003. Außerdem ist der Anteil der auf Stundenbasis eingestellten Lehrkräfte von 33\% im Jahr 2000 auf 38\% im Jahr 2011 gestiegen (SEP, 2013). All diese Veränderungen bei der Klassenwiederholung, den Lernzeiten und dem Lernumfeld können nur dann als Verbesserungen gewertet werden, wenn sie zu einem besseren Unterricht und Lernprozess sowie zu besseren Schülerleistungen geführt haben. Das wäre beispielsweise nicht der Fall, wenn die Schülerinnen und Schüler am Ende des Schuljahres automatisch versetzt wurden, die erhöhte Lernzeit eine Verringerung des Kontakts der Schülerinnen und Schüler mit anderen ebenso wichtigen Fächern zur Folge hatte und die auf Stundenbasis eingestellten Lehrkräfte unter schlechteren Arbeitsbedingungen unterrichten mussten.

\section{Für eine gezielte Finanzierung und Unterstützung sowie unabhängige Beurteilung sorgen}

Mexiko hat in den vergangenen zehn Jahren einige Programme erstellt, um die Beteiligung an der Vorschulbildung zu fördern, Schulen in Not zu unterstützen und Anreize für Schüler zu schaffen, in der Schule zu verbleiben. Andere Reformen jüngeren Datums geben Aufschluss über den von Mexiko in der Bildungspolitik eingeschlagenen Kurs. Sie umfassen eine Lehrplanreform und die Einführung von Beurteilungs- und Evaluierungsmechanismen durch eine unabhängige Instanz. Da es sich hierbei um noch recht junge und komplexe Reformen handelt, bedarf es weiterer Untersuchungen, um beurteilen zu können, inwieweit diese Veränderungen bei den Evaluierungs- und Beurteilungsmechanismen sowie Lehrplänen das bessere Abschneiden Mexikos in der PISA-Erhebung erklären.

Im Rahmen dieser Reformen wurde die Vorschulerziehung 2002 obligatorisch (der Effekt dieser Maßnahme auf die Schülerleistungen im weiteren Schulverlauf wird erst in PISA 2015 sichtbar werden). Es wird weiter an der Verbesserung der Qualität der Kinderbetreuungseinrichtungen in den Großstädten für Kinder aus Niedrigeinkommensfamilien und Familien gearbeitet, in denen beide Elternteile berufstätig sind. Zu diesem Zweck werden ein nationales System von Kindertagesstätten eingerichtet und ein Rahmenlehrplan verbreitet, der Vorschuleinrichtungen bei der Aufstellung von Lehrplänen unterstützen soll, die am besten auf ihre Erfordernisse abgestimmt sind.

In Mexiko wird die Bildung einschließlich der Lehrererstausbildung und Lehrerfortbildung auf der Ebene der einzelnen Bundesstaaten verwaltet, es existieren aber Mechanismen, über die das Ministerium Maßnahmen im gesamten Land umsetzt. Es wurden mehrere gezielte Finanzierungs- und Unterstützungsprogramme umgesetzt, um insbesondere unter sozioökonomisch benachteiligten Schulen und Bundesstaaten eine gerechtere Verteilung der Unterstützungsund Finanzierungsleistungen zu fördern. Das Programa Escuelas de Calidad (PEC - Qualitätsschulen), das 2001 ins Leben gerufen wurde, erteilt Zuschüsse für die Finanzierung von Schulverbesserungsprojekten. Es gewährt den Schulen Autonomie und fördert mit Hilfe partizipativer Schulgremien die gemeinsame Entscheidungsfindung unter Schulleitern, Lehrkräften, Eltern, Gewerkschaftsvertretern, ehemaligen Schülerinnen und Schülern sowie lokalen Akteuren. Nahezu die Hälfte aller Schulen in Mexiko verfügt über ein derartiges Gremium. Der Plan Estratégico de Transformación Escolar (PETE) hilft den beteiligten Akteuren, einen jährlichen Arbeitsplan aufzustellen, für den ein Fünfjahreszuschuss gewährt wird. Etwa $70 \%$ der Zuschüsse für die ersten vier Jahre und $50 \%$ der Mittel für das fünfte Jahr sind für Anschaffungen, Infrastruktur und sonstige materielle Ressourcen bestimmt. Zwischen 2009 und 2012 wurde ferner die Estrategia para la Mejora del Logro Educativo (EIMLE - Strategie zur Verbesserung der Bildungsergebnisse) in mehr als 7000 der am schlechtesten abschneidenden Schulen umgesetzt, wo Mentoren Ausbildungsnetze für Lehrkräfte einrichteten und den Kapazitätsaufbau förderten. Das Programa Escuelas de Tiempo Completo (PETC - Ganztagsschule) zielt darauf ab, den Schultag um vier Stunden zu verlängern. Es konzentriert sich hauptsächlich auf die städtischen Randgruppen oder Bevölkerungsgruppen mit einem großen Anteil an indigenen, zugewanderten oder sonstigen leistungsschwachen Schülern. Drei Jahre nach seiner Gründung im Jahr 2007 umfasste das PETC-Programm mehr als 2000 Schulen in 30 Bundesstaaten. Unterdessen erreicht ConstruyeT, ein Programm, das sich auf die theoretische und praktische Ausbildung von Lehrkräften konzentriert, ein Drittel der mexikanischen Schulen und genießt die Unterstützung von UNICEF, UNDP, UNESCO sowie 29 NRO; im Rahmen des Programms für bessere Schulen wurden 19000 Schulen mit dem größten Reparaturbedarf vom Vorschul- bis zum Sekundarbereich I modernisiert. Wenngleich diese Programme meistens auf benachteiligte Schulen und Bundesstaaten zugeschnitten sind, kann den PISA-Daten nicht entnommen werden, inwieweit, wenn überhaupt, sie das Abschneiden Mexikos in der PISA-Erhebung verbessert haben (OECD, 2012a).

Andere Programme bieten Schülerinnen und Schülern Anreize zum Verbleib in der Schule. Oportunidades ist ein Programm mit Transferzahlungen, das darauf abzielt, die Gesundheit und Bildung von unter der Armutsgrenze 
lebenden Familien zu verbessern. Das Programm wurde 2002 ins Leben gerufen und erreichte 2012 über fünf Millionen Familien, denen es half, die Schulbesuchsquoten im Sekundarbereich zu erhöhen, insbesondere unter den Mädchen. Das Programa de Becas de Media Superior, ein weiteres Programm mit Transferzahlungen, zielt darauf ab, die Abbrecherquoten im Sekundarbereich II zu reduzieren. Auch wenn diese Programme zur Senkung der Abbrecherquoten beigetragen haben dürften, können andere Faktoren ebenfalls eine Rolle gespielt haben, zumal die Abbrecherquoten bereits vor der Umsetzung dieser Programme zu sinken begannen (SEP, 2013).

Im Jahr 2002 kündigte die Regierung die Gründung des Instituto Nacional para la Evaluación de la Educación (INEE) an, das mit der Messung und Evaluierung von Qualität und Leistung des Bildungssystems beauftragt ist. Dem Institut wurde vom Bildungsministerium 2013 vollständige Autonomie eingeräumt. Im Einklang mit der Idee, ein Informationssystem einzurichten, das es den beteiligten Akteuren ermöglichen würde, die Leistungen der Schulen weiter zu verfolgen, führte die Bundesregierung 2006 ein Programm zur Nationalen Beurteilung der akademischen Leistungen in Schulen (ENLACE) ein, um die Schülerleistungen im gesamten Land zu messen. Dieses fand in allen Klassenstufen ab Klasse 3 und in dieser bereits für Spanisch und Mathematik Anwendung. Mit der Weiterentwicklung von ENLACE wurden die Ergebnisse der Beurteilung dann aber auch für verschiedene, und in manchen Fällen, gegensätzliche Zwecke verwendet, und für die Lehrkräfte und Schulen stand in Bezug auf ihr Abschneiden bei den in ENLACE ermittelten Leistungen immer mehr auf dem Spiel. Dieser Druck, und die wachsenden Anreize, „auf den Test ausgerichtet zu unterrichten", haben Forderungen nach Änderungen der Testverfahren und/oder deren Nutzung laut werden lassen (Santiago et al., 2012; OECD, 2013).

2012 führte die Regierung eine tiefgreifende Reform der Lehrpläne ein, die Umfassende Reform der Grundbildung (RIEB), die die Schülerinnen und Schüler in den Mittelpunkt des Lerninteresses rückte. Das Hauptaugenmerk der Reform liegt auf diversen Konzepten, wie die Beurteilung der Lernbedürfnisse, erwartete Lernergebnisse, gemeinschaftliche Lernprozesse, Projektarbeit, Schüler-Selbstbeurteilung sowie Peer-Beurteilungen und kriteriumbasierte Benotung. Ferner fordert sie eine stärkere Koordination zwischen den verschiedenen Klassenstufen in der Grundschule und sorgt für eine Verbesserung der Kontinuität sowohl mit der Vorschule als auch der Sekundarschule I. Die Reform steht vor der Herausforderung, sicherzustellen, dass die vorgeschlagenen Änderungen letztlich im Klassenzimmer ankommen und tatsächlich bei den Lerninhalten und der Art und Weise, wie diese vermittelt werden, für Veränderungen sorgen. In Verbindung mit der Lehrplanreform setzt die RIEB auch Initiativen fort, die Mitte der 2000er Jahre ins Leben gerufen wurden und darin bestanden, das Schuljahr von 180 Tagen auf 200 Tage zu verlängern, und erzielte Fortschritte auf dem Weg zur Sicherung des Ganztagsunterrichts in allen Schulen. Ferner wurde der Schulbesuch im Sekundarbereich II 2012 obligatorisch, mit dem Ziel einer universellen Teilnahme bis 2022. Zu den weiteren laufenden Politikanstrengungen zählen die Professionalisierung des Unterrichts und Einführung eines Lehrer-Evaluierungssystems.

\section{Quelle:}

OECD (2013), Education Policy Outlook: Mexico, OECD Publishing.

OECD (2012a), Avances en las Reformas de la Educación Básica en México: Una Perspectiva de la OCDE (versión preliminar), OECD Publishing, www.sep.gob.mx/work/models/sep1/Resource/3048/2/images/Avances_en_las_reformas_de_la_educacion_basica.pdf.

Santiago, P., et al. (2012), OECD Reviews of Evaluation and Assessment in Education: Mexico 2012, OECD Reviews of Evaluation and Assessment in Education, OECD Publishing. http://dx.doi.org/10.1787/9789264172647-en

Subsecretaría de Educación Pública (SEP) (2013), Reporte de Indicadores Educativos, SEP, Distrito Federal, Mexico.

Zorrilla, M. and B. Barba (2008), “Reforma Educativa en México: Descentralización y Nuevos Actores”, Sinéctica, 30.

Im Allgemeinen hängen schwache Leistungen und sozioökonomische Benachteiligung auf Schulebene eng miteinander zusammen, doch einigen Schulsystemen gelingt es besser als anderen, diesen Zusammenhang abzuschwächen. Im Durchschnitt der OECD-Länder besuchen 18\% der Schülerinnen und Schüler sozioökonomisch benachteiligte Schulen, die schwache Ergebnisse erzielen, während $20 \%$ sozioökonomisch begünstigte, leistungsstarke Schulen besuchen ${ }^{13}$. Wie aus Abbildung II.2.11 hervorgeht, unterscheiden sich die entsprechenden Durchschnitte zwischen den einzelnen Ländern jedoch erheblich. In Finnland beispielsweise besuchen 4\% der Schülerinnen und Schüler sozioökonomisch benachteiligte, leistungsschwache Schulen, während 6\% sozioökonomisch begünstigte, leistungsstarke Schulen besuchen. In Norwegen, Estland, Kanada und Schweden besuchen weniger als $10 \%$ der Schülerinnen und Schüler sozioökonomisch benachteiligte, leistungsschwache Schulen, und weniger als 15\% besuchen sozioökonomisch begünstigte, leistungsstarke Schulen (Tabelle II.2.11). 
- Abbildung II.2.11

\section{Verteilung der Schüler in Bezug auf die Leistungen und sozioökonomischen Profile ${ }^{1}$ der Schulen}

$\square$ Hohe Leistungen, sozioökonomisch begünstigte Schule $\square$ Hohe Leistungen, sozioökonomisch durchschnittliche Schule $\square$ Hohe Leistungen, sozioökonomisch benachteiligte Schule

$\square$ Durchschnittl. Leistungen, sozioökonomisch begünstigte Schule

$\square$ Durchschnittl. Leistungen, sozioökonomisch durchschnittl. Schule $\square$ Durchschnittl. Leistungen, sozioökon. benachteiligte Schule $\square$ Schwache Leistungen, sozioökonomisch begünstigte Schule $\square$ Schwache Leistungen, sozioökonomisch durchschnittl. Schule

- Schwache Leistungen, sozioökonomisch benachteiligte Schule

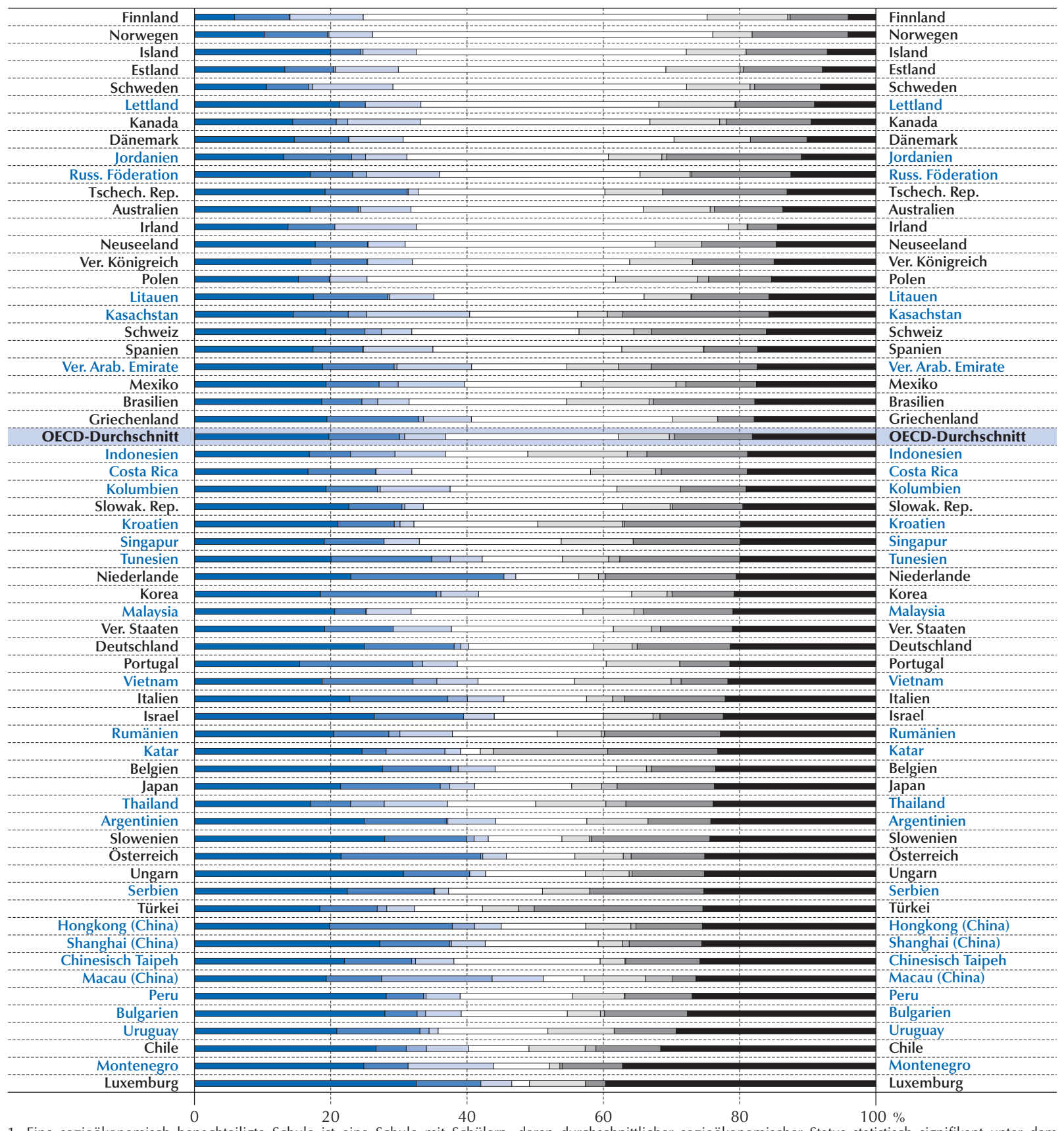

1. Eine sozioökonomisch benachteiligte Schule ist eine Schule mit Schülern, deren durchschnittlicher sozioökonomischer Status statistisch signifikant unter dem durchschnittlichen sozioökonomischen Status des Landes/der Volkswirtschaft liegt, eine sozioökonomisch durchschnittliche Schule ist eine Schule, an der es keinen Unterschied zwischen dem durchschnittlichen sozioökonomischen Status der Schulen und des Landes/der Volkswirtschaft gibt, und eine sozioökonomisch begünstigte Schule ist eine Schule mit Schülern, deren durchschnittlicher sozioökonomischer Status statistisch signifikant über dem durchschnittlichen sozioökonomischen Status des Landes/der Volkswirtschaft liegt. In einer leistungsschwachen Schule liegen die durchschnittlichen Leistungen der Schule statistisch signifikant unter den Durchschnittsergebnissen des Landes/der Volkswirtschaft, in einer durchschnittlich abschneidenden Schule besteht kein Unterschied zwischen den beiden, und in einer leistungsstarken Schule liegen die durchschnittlichen Leistungen der Schule statistisch siginifikant über den Durchschnittsergebnissen des Landes/der Volkswirtschaft. Kleine Schulen werden mit größerer Wahrscheinlichkeit als durchschnittliche Schulen eingestuft.

Die Länder/Volkswirtschaften sind in aufsteigender Reihenfolge nach dem Anteil der Schüler angeordnet, die leistungsschwächere Schulen besuchen, die zugleich sozioökonomisch benachteiligt sind.

Quelle: OECD, PISA-2012-Datenbank, Tabelle II.2.11.

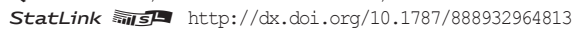




\section{ALLEN 15-JÄHRIGEN ZUGANG ZUR SCHULBILDUNG BIETEN}

Der Zugang zur Schulbildung ist eine unabdingbare Voraussetzung für Inklusion und Chancengerechtigkeit in der Bildung. In den meisten OECD-Ländern haben alle Schülerinnen und Schüler auf Grund der Schulpflicht Zugang zur Schulbildung. Unabhängig von den Leistungen oder selbst Gerechtigkeitserwägungen kann ein Bildungssystem, in dem ein Großteil der 15-Jährigen die Schule nicht besucht, nicht als qualitativ hochwertiges, gerechtes Bildungssystem betrachtet werden. Nahezu alle OECD-Länder haben den universellen Zugang zur Schulbildung vor mehr als einem Jahrzehnt verwirklicht, und die Schulbesuchsquoten im Primar- und Sekundarbereich I liegen sehr nahe bei 100\%. Einige der Länder, die der OECD in jüngster Zeit beigetreten sind, sowie einige Partnerländer haben dieses Ziel in jüngerer Zeit erreicht oder bewegen sich darauf zu.

Zwischen PISA 2003 und PISA 2012 erhöhte sich die Gesamtpopulation der 15-Jährigen, die mindestens Klasse 7 besuchen, in Indonesien und Brasilien um 485000 bzw. 425000 Schülerinnen und Schüler, was einem Anstieg um 16\% bzw. 18\% in diesem Zeitraum entspricht. Die Beschulung dieser neuen Schülerinnen und Schüler war eine große Leistung, da die höhere Bildungsbeteiligung in beiden Ländern keine Verschlechterung der Lernerträge zur Folge hatte. Unter den OECD-Ländern haben sich im selben Zeitraum in der Türkei und in Mexiko sowohl die absolute Zahl der neuen Schülerinnen und Schüler als auch der Prozentsatz der Schulbesucher insgesamt erhöht. All diese Länder ermöglichten mehr Schülerinnen und Schülern Zugang zur schulischen Bildung - und dies selbst zu einem Zeitpunkt, als die Zahl der 15-Jährigen schrumpfte, was bedeutet, dass die steigende Bildungsbeteiligung den besseren Kapazitäten zu verdanken ist, um die Schülerinnen und Schüler in den höheren Klassen in der Schule zu halten. In Brasilien sind diese Erfolge das Ergebnis von Politikmaßnahmen zur Minimierung von Klassenwiederholungen und Schulabbruch (Tabelle II.2.12).

In den meisten PISA-Teilnehmerländern und-volkswirtschaften ist der Anteil der 15-Jährigen, die die Schulenichtbesuchen, sehr gering; daher hätte die Einbeziehung von Proxy-Ergebnissen für diese Gruppe nur begrenzte Auswirkungen auf die mittlere Punktzahl eines Bildungssystems. So könnte beispielsweise unterstellt werden, dass die Schülerinnen und Schüler außerhalb des Bildungssystems lediglich die niedrigste der in PISA festgelegten Kompetenzstufen erreichen (d.h. das untere Ende der Stufe 1 auf der Mathematikgesamtskala bzw. 358 Punkte) und diese Schüler anhand ihres Anteils an der Population der 15-Jährigen gewichten. Wie aus Tabelle II.2.12 hervorgeht, würden sich die durchschnittlichen Leistungen nach Berücksichtigung der Ergebnisse der Personen außerhalb des Schulsystems in den meisten Ländern gar nicht oder nur sehr geringfügig verändern. Von den sechs Ländern und Volkswirtschaften, in denen die Leistungsveränderungen am größten sind (und über 15 Punkte betragen - Hongkong-China, Macau-China, Mexiko, Shanghai-China, die Türkei und Vietnam) würde nur Vietnam in der relativen Rangfolge eine signifikante Zahl an Plätzen verlieren (bis zu 24 Plätze im Länder-Ranking, gefolgt von Macau (China), das bis zu 10 Plätzen einbüßen würde).

\section{ENTWICKLUNG DER CHANCENGERECHTIGKEIT ZWISCHEN PISA 2003 UND PISA 2012}

Anhand einer Analyse der Daten aus verschiedenen PISA-Erhebungen lassen sich die Länder und Volkswirtschaften identifizieren, die auf dem Weg zu einem gerechteren Schulsystem vorangekommen sind. Im Jahr 2003 und im Durchschnitt der OECD-Länder mit vergleichbaren Daten für 2012 betrug die Steigung der sozioökonomischen Gradiente 39 Punkte, was bedeutet, dass ein sozioökonomisch benachteiligter Schüler (ein Schüler mit einem Punktwert von -1 auf dem PISA-Index des wirtschaftlichen, sozialen und kulturellen Status - ESCS) im PISA-Mathematiktest um 39 Punkte schlechter abschnitt als ein Schüler mit einem durchschnittlichen sozioökonomischen Status (d.h. einem ESCS-Wert von 0) (in Kasten II.2.5 wird beschrieben, wie sich ESCS-Indexwerte im Zeitverlauf vergleichen lassen). Bei sozioökonomisch begünstigten Schülern (mit einem Wert von 1 auf dem ESCS-Index) wurde davon ausgegangen, dass sie im Durchschnitt um 39 Punkte besser abschneiden als Schüler mit einem durchschnittlichen sozioökonomischen Status und um 78 Punkte besser als sozioökonomisch benachteiligte Schüler. In jenem Jahr ließen sich 17\% der Varianz bei den Schülerleistungen in Mathematik im Durchschnitt der OECD-Länder durch ihren sozioökonomischen Status erklären (OECD, 2004, und Tabelle II.2.5b).

Bis 2012 verharrte der Effekt des sozioökonomischen Status auf die Leistungen (die Steigung der sozioökonomischen Gradiente, für OECD-Länder mit vergleichbaren Daten aus PISA 2003 und PISA 2012) im OECD-Durchschnitt in Mathematik bei 39 Punkten, wohingegen der Grad, bis zu dem die Mathematikleistungen den Leistungen entsprachen, die der sozioökonomische Status erwarten ließ (die Stärke der sozioökonomischen Gradiente) von 17\% auf 15\% sank. Mit anderen Worten war es für die Schülerinnen und Schüler 2012 vergleichsweise einfacher als 2003, auf der Basis ihres sozioökonomischen Status formulierte Erwartungen hinsichtlich ihres Leistungsniveaus zu widerlegen. 


\section{Kasten II.2.5 Vergleich der Indizes zwischen PISA 2003 und PISA 2012}

PISA-Indizes, wie der PISA-Index des wirtschaftlichen, sozialen und kulturellen Status basieren auf Informationen aus dem Schülerfragebogen. In PISA 2012 ist jeder Index so skaliert, dass ein Indexwert von 0 dem OECD-Durchschnitt entspricht und ein Wert von 1 die durchschnittliche Standardabweichung in den OECD-Ländern zum Ausdruck bringt (vgl. Anhang A1 wegen näherer Einzelheiten zur Konstruktion der einzelnen Indizes). Entsprechend war in PISA 2003 jeder Index so skaliert, dass ein Indexwert von 0 dem OECD-Durchschnitt entsprach und ein Wert von 1 die durchschnittliche Standardabweichung in den OECD-Ländern zum Ausdruck brachte. Für den Vergleich der Entwicklung dieser Indizes im Zeitverlauf wurde die Indexskala von PISA 2012 verwendet, und alle Indexwerte für PISA 2003 wurden entsprechend neu skaliert. Folglich weichen die in diesem Bericht dargelegten Indexwerte für 2003 von den Werten ab, die in Lernen für die Welt von morgen: Erste Ergebnisse von PISA 2003 (OECD, 2004) enthalten sind.

Die Berechnung des prozentualen Anteils der resilienten Schülerinnen und Schüler muss ebenfalls angepasst werden, um im Zeitverlauf vergleichbar zu sein. Identifiziert wird ein resilienter Schüler anhand eines Vergleichs bestimmter Schüler mit den übrigen Schülern, die in einem beliebigen Jahr an der PISA-Erhebung teilnahmen. Da an der PISAErhebung 2012 mehr Länder und Volkswirtschaften teilnahmen als an der PISA-Erhebung 2003 muss die gepoolte Stichprobe für die Berechnung der Resilienz im Jahr 2012 zum Zwecke des Resilienzvergleichs im Zeitverlauf auf demselben Pool an Ländern und Volkswirtschaften basieren, die in PISA 2003 für die Schätzung der Resilienz zu Grunde gelegt wurden. Folglich weichen die im Zeitverlauf vergleichbaren Schätzungen der Resilienz in PISA 2012 von den Schätzungen ab, die für die Gesamtheit der Länder und Volkswirtschaften in PISA 2012 präsentiert wurden.

In der Türkei und Mexiko, wo sich sowohl die Steigung als auch die Stärke der sozioökonomischen Gradiente bei gleichzeitiger Leistungsverbesserung abflachten, war eine Entwicklung zu mehr Chancengerechtigkeit zu beobachten. In diesen beiden Ländern bedeutete die allgemeine Verbesserung der Mathematikleistungen zugleich auch, dass es für die Schülerinnen und Schüler 2012 einfacher war als 2003, die in Anbetracht ihres sozioökonomischen Status hinsichtlich ihrer Mathematikleistungen formulierten Erwartungen zu widerlegen, und dass der durchschnittliche Leistungsabstand zwischen sozioökonomisch begünstigten und sozioökonomisch benachteiligten Schülerinnen und Schülern schrumpft. Wenngleich der Leistungsabstand zwischen sozioökonomisch begünstigten und benachteiligten Schülerinnen und Schülern in Deutschland unverändert blieb, gelang es - gemessen an der abnehmenden Stärke der sozioökonomischen Gradiente mehr Schülerinnen und Schülern, diesen Zusammenhang zu überwinden. Am wichtigsten ist die Feststellung, dass in diesen drei Ländern die Verbesserung der Chancengerechtigkeit mit einer Steigerung der Mathematikleistungen einherging. In anderen Ländern und Volkswirtschaften, in denen sich die Mathematikleistungen verbesserten (Brasilien, Griechenland, Hongkong-China, Italien, Macau-China, Polen und Tunesien) blieb die Bildungsgerechtigkeit unverändert; nur in Portugal gingen Leistungsverbesserungen mit einem Rückgang der Chancengerechtigkeit einher, der sich hauptsächlich in der Stärke der sozioökonomischen Gradiente niederschlug. Diese Ergebnisse verdeutlichen, dass Leistungssteigerungen in den meisten Ländern und Volkswirtschaften nicht zu Lasten der Chancengerechtigkeit gehen müssen (Abb. II.2.12 und II.2.13).

Eine andere Möglichkeit festzustellen, ob sich Länder und Volkswirtschaften auf ein gerechteres Schulsystem zubewegen, besteht darin, zu untersuchen, wie sie die Resilienz der Schülerinnen und Schüler gefördert haben. Bei den resilienten Schülerinnen und Schülern handelt es sich um Schüler aus einem benachteiligen sozioökonomischen Umfeld (die im untersten Quartil der Verteilung des sozioökonomischen Status eines Landes oder einer Volkswirtschaft angesiedelt sind), deren Leistungen sich nach Bereinigung um den sozioökonomischen Status in allen Ländern im obersten Quartil bewegen. Länder und Volkswirtschaften, in denen der Anteil der resilienten Schülerinnen und Schüler wächst, sind jene, die dafür sorgen, dass sozioökonomisch benachteiligten Schülerinnen und Schülern mehr Chancen geboten werden, ein hohes Leistungsniveau zu erreichen.

In PISA 2003 galten 6,4\% der Schülerinnen und Schüler in den OECD-Ländern als resilient, bis 2012 war dieser Anteil geringfügig auf 6,1\% gesunken. Nur in Deutschland, Italien, Mexiko, Polen, Tunesien und der Türkei nahm der Anteil der resilienten Schülerinnen und Schüler um mehr als 1 Prozentpunkt zu. In elf Ländern und Volkswirtschaften schrumpfte der Anteil der resilienten Schülerinnen und Schüler - was mit anderen Worten bedeutet, dass in diesen Ländern/ Volkswirtschaften die Wahrscheinlichkeit geringer ist, dass sozioökonomisch benachteiligte Schülerinnen und Schüler hohe Leistungen erbringen (Abb. Il.2.14) (vgl. Kasten II.2.5 wegen einer Beschreibung der Resilienzberechnung für Vergleiche zwischen PISA-Erhebungen). 


\section{Veränderung der Stärke der sozioökonomischen Gradiente zwischen 2003 und 2012 und annualisierte Mathematikergebnisse}

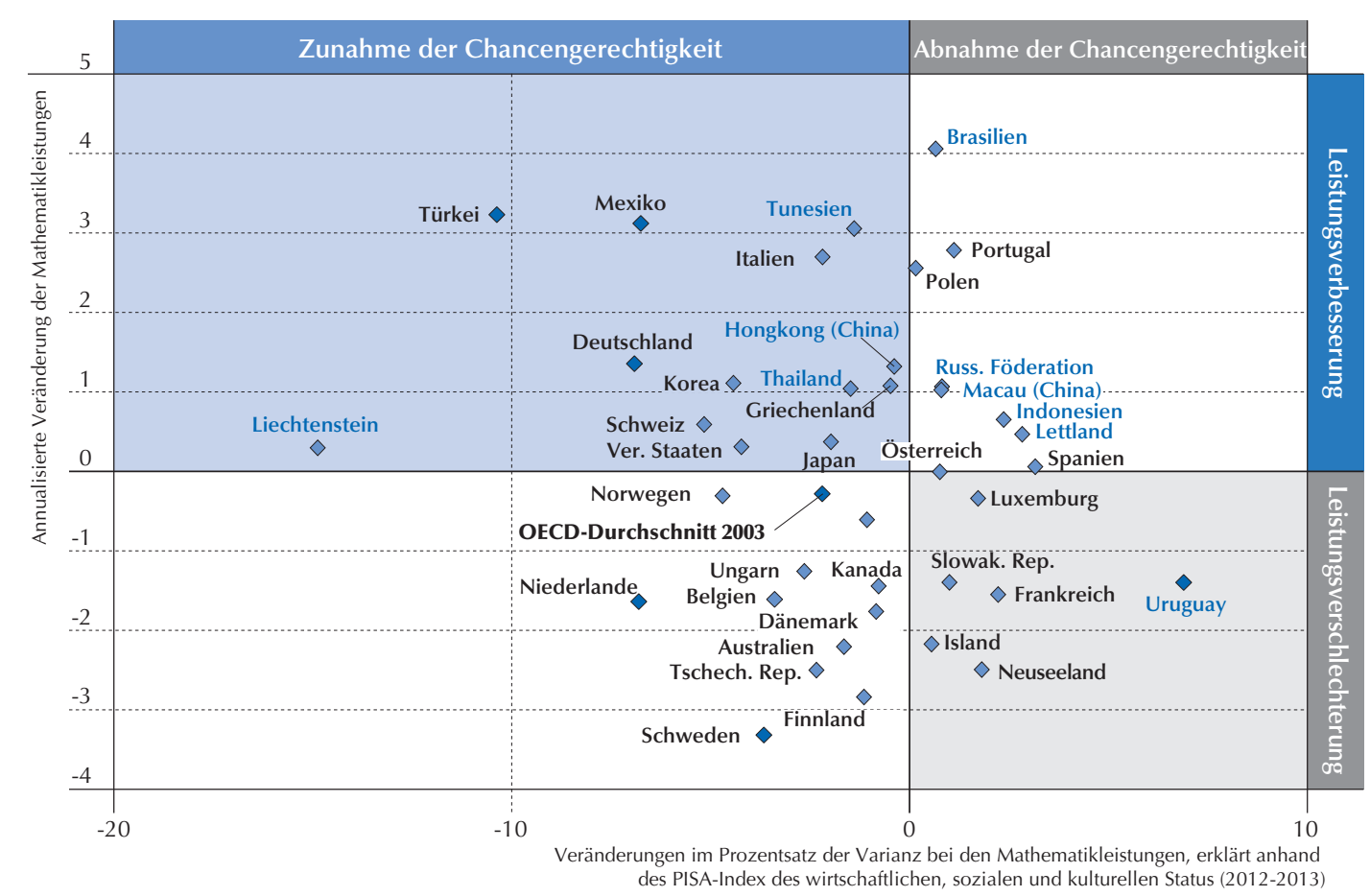

Anmerkung: Statistisch signifikante Veränderungen der Chancengerechtigkeit und der Schülerleistungen zwischen 2003 und 2012 sind durch einen dunkleren Farbton gekennzeichnet.

Die annualisierte Veränderung entspricht der jahresdurchschnittlichen Veränderung der PISA-Punktzahl von der ersten PISA-Teilnahme eines Landes/einer Volkswirtschaft bis zur Teilnahme an PISA 2012. Dabei wird jede PISA-Teilnahme eines Landes/einer Volkswirtschaft berücksichtigt. Wegen näherer Einzelheiten zur Berechnung der annualisierten Veränderung vgl. Anhang A5.

Angegeben sind nur Länder und Volkswirtschaften mit vergleichbaren Daten aus PISA 2003 und PISA 2012.

Um die Vergleichbarkeit im Zeitverlauf zu gewährleisten, wurden die Werte von PISA 2003 auf dem PISA-Index des wirtschaftlichen, sozialen und kulturellen Status entsprechend der Indexskala von PISA 2012 umskaliert. Die in dieser Abbildung dargestellten Ergebnisse aus PISA 2003 können daher von denen abweichen, die in Lernen für die Welt von morgen - erste Ergebnisse von PISA 2003 (OECD, 2004) wiedergegeben sind (vgl. Anhang A5 wegen näherer Einzelheiten).

Der OECD-Durchschnitt 2003 umfasst nur die Länder, deren Mathematikergebnisse und Werte auf dem PISA-Index des wirtschaftlichen, sozialen und kulturellen Status seit PISA 2003 vergleichbar sind.

Quelle: OECD, PISA-2012-Datenbank, Tabelle I.2.3b und II.2.9b.

StatLink : तils http://dx.doi.org/10.1787/888932964813

In Übereinstimmung mit den Verbesserungen der Schülerresilienz seit 2003 haben Schülerinnen und Schüler im untersten Quartil des sozioökonomischen Status in Brasilien, Italien, Mexiko, Polen, Tunesien und der Türkei ihre Mathematikleistungen zwischen PISA 2003 und PISA 2012 am stärksten verbessert. In diesen Ländern schnitt beispielsweise ein Schüler, der sich 2012 im untersten Quartil des PISA-Index des wirtschaftlichen, sozialen und kulturellen Status befand, im PISAMathematiktest um mehr als 25 Punkte besser ab als ein Schüler, der 2003 im untersten Quartil dieses Index lag; in Deutschland, Hongkong (China), Korea, Portugal und der Schweiz verbesserten Schülerinnen und Schüler im untersten Quartil des PISA-Index des wirtschaftlichen, sozialen und kulturellen Status ihre Leistungen um mindestens 15 Punkte. In Italien, Polen, Portugal und Tunesien war die unter den sozioökonomisch benachteiligten Schülerinnen und Schülern beobachtete Leistungssteigerung Teil einer generellen Verbesserung der Mathematikergebnisse unter allen Schülerinnen und Schülern, wohingegen die in Brasilien, Hongkong (China), Deutschland, Korea, Mexiko, der Schweiz und der Türkei unter den sozioökonomisch benachteiligten Schülerinnen und Schülern festgestellte Verbesserung deutlich größer ausfällt als die Verbesserung, die - wenn überhaupt - unter den Schülerinnen und Schülern aus sozioökonomisch begünstigten Milieus zu beobachten ist (Tabelle II.2.7b). 
- Abbildung II.2.13=

\section{Veränderung der Steigung der sozioökonomischen Gradiente zwischen 2003 und 2012 und annualisierte Mathematikergebnisse}

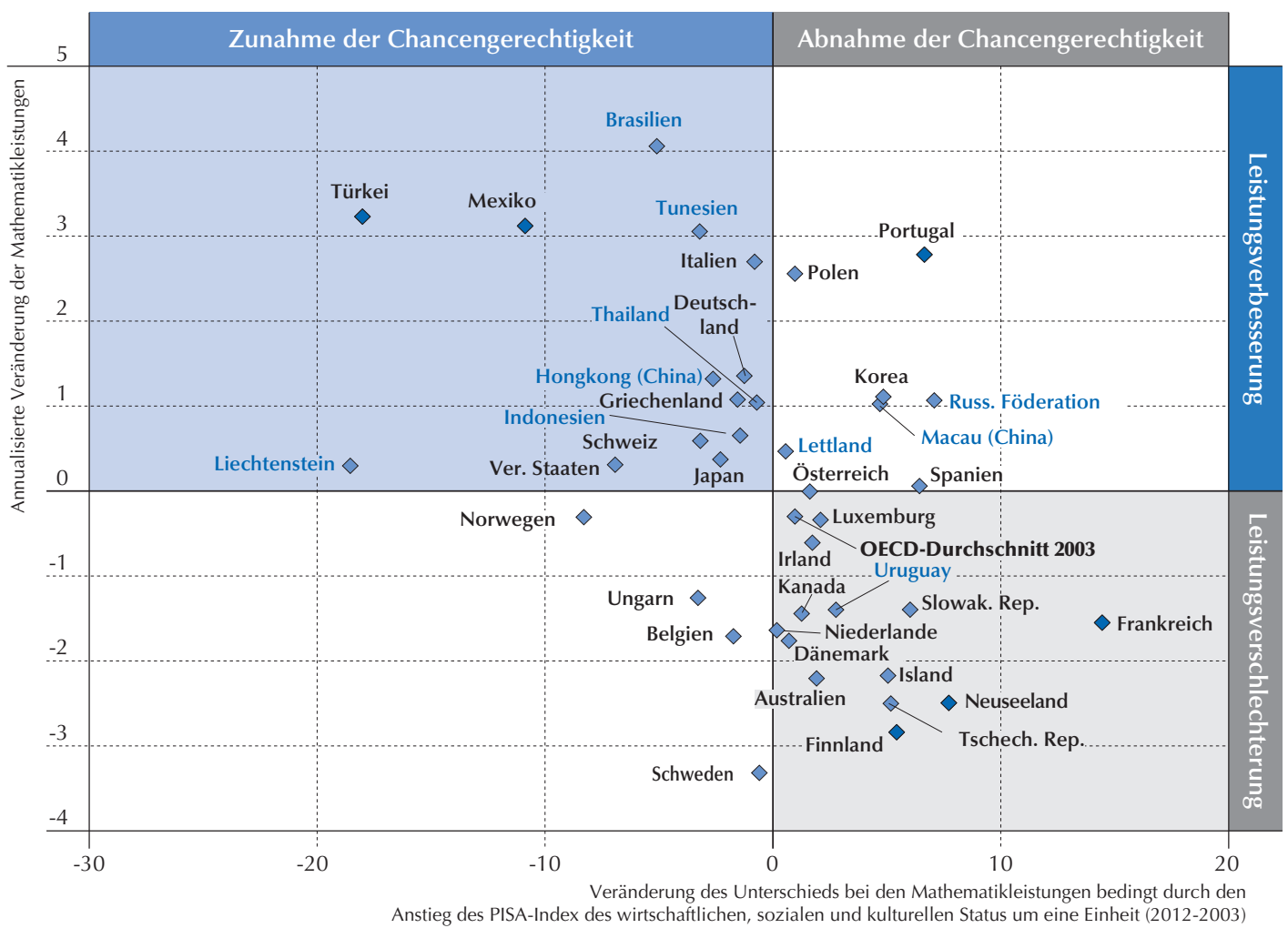

Anmerkung: Statistisch signifikante Veränderungen der Chancengerechtigkeit und der Schülerleistungen zwischen 2003 und 2012 sind durch einen dunkleren Farbton gekennzeichnet.

Die annualisierte Veränderung entspricht der jahresdurchschnittlichen Veränderung der PISA-Punktzahl von der ersten PISA-Teilnahme eines Landes/einer Volkswirtschaft bis zur Teilnahme an PISA 2012. Dabei wird jede PISA-Teilnahme eines Landes/einer Volkswirtschaft berücksichtigt. Wegen näherer Einzelheiten zur Berechnung der annualisierten Veränderung vgl. Anhang A5.

Angegeben sind nur Länder und Volkswirtschaften mit vergleichbaren Daten aus PISA 2003 und PISA 2012.

Um die Vergleichbarkeit im Zeitverlauf zu gewährleisten, wurden die Werte von PISA 2003 auf dem PISA-Index des wirtschaftlichen, sozialen und kulturellen Status entsprechend der Indexskala von PISA 2012 umskaliert. Die in dieser Abbildung dargestellten Ergebnisse aus PISA 2003 können daher von denen abweichen, die in Lernen für die Welt von morgen - erste Ergebnisse von PISA 2003 (OECD, 2004) wiedergegeben sind (vgl. Anhang A5 wegen näherer Einzelheiten).

Der OECD-Durchschnitt 2003 umfasst nur die Länder, deren Mathematikergebnisse und Werte auf dem PISA-Index des wirtschaftlichen, sozialen und kulturellen Status seit PISA 2003 vergleichbar sind.

Quelle: OECD, PISA-2012-Datenbank, Tabelle I.2.3b und II.2.9b.

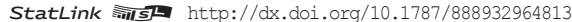

\section{Entwicklung des Profils und der Verteilung der Schülerinnen und Schüler in den Schulen}

In Anbetracht der Tatsache, dass die Mitschülerinnen und Mitschüler eine starke Inspirations- und Motivationsquelle für das Lernen darstellen können und angesichts des Stigmas, das sozioökonomisch benachteiligten und leistungsschwachen Schulen anhaftet, zielen Länder und Volkswirtschaften, die danach trachten, gerechtere Lernmöglichkeiten zu bieten, generell auch darauf ab, einen höheren Grad an sozialer Inklusion zu erreichen. Die Bildungspolitik kann bei der Förderung dieser beiden Elemente eine entscheidende Rolle spielen (OECD, 2012b).

Mit dem Konzept der sozialen Inklusion wird erfasst, inwieweit Schülerinnen und Schüler mit unterschiedlichem sozioökonomischem Status die gleiche Schule besuchen oder inwieweit verschiedene Schulen unterschiedliche sozioökonomische Profile aufweisen. Die Erhöhung der sozialen Inklusion in einem Schulsystem und mithin die Verringerung der sozioökonomischen Segregation gilt als die effizienteste Politikmaßnahme zur Steigerung der Chancengerechtigkeit auf kurze und lange Sicht und wird als sehr viel wirksamer angesehen, als die Einrichtung von Magnetschulen oder die Möglichkeit der Schulwahl (Eaton, 2001; Wells and Crain, 1997). Im Jahr 2003 und im Durchschnitt der OECD-Länder wurden 76\% der Varianz beim sozioökonomischen Status der Schüler innerhalb der 


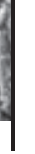

\section{- Abbildung II.2.14 " \\ Veränderung der Schülerresilienz gegenüber dem sozioökonomischen Status zwischen 2003 und 2012}

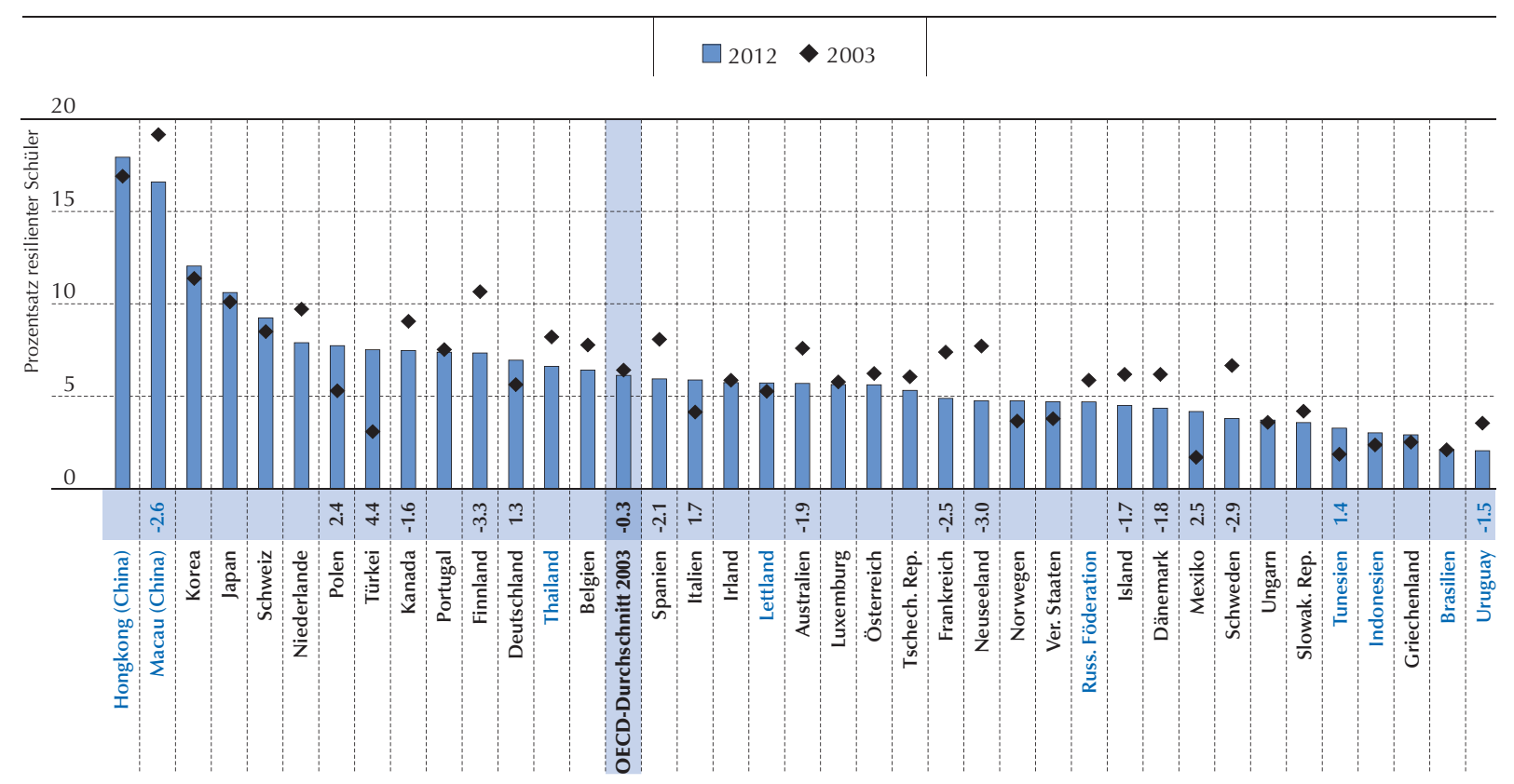

Anmerkung: Angegeben sind nur Länder und Volkswirtschaften mit vergleichbaren Daten aus PISA 2003 und PISA 2012.

Die Resilienzwerte für 2012 weichen u.U. von den in Abbildung II.2.4 dargestellten Werten ab, da sich die in dieser Abbildung verwendete gepoolte Referenzpopulation für resiliente Schüler auf die Länder und Volkswirtschaften beschränkt, für die vergleichbare Daten aus PISA 2003 und PISA 2012 vorliegen.

Der Prozentpunktunterschied beim Anteil der resilienten Schülerinnen und Schüler zwischen PISA 2003 und PISA 2012 (2012-2003) ist über dem Namen des Landes/der Volkswirtschaft angegeben. Aufgeführt sind nur statistisch signifikante Unterschiede.

Der OECD-Durchschnitt für 2003 umfasst nur die Länder, deren Ergebnisse im Bereich Mathematik seit dem Jahr 2003 vergleichbar sind.

Die Länder und Volkswirtschaften sind in absteigender Reihenfolge nach dem prozentualen Anteil der resilienten Schülerinnen und Schüler in PISA 2012 angeordnet.

Quelle: OECD, PISA-2012-Datenbank, Tabelle II.2.7b.

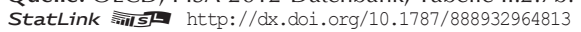

Schulen beobachtet. Am stärksten war die soziale Inklusion, d.h. die sozioökonomische Vielfalt, mit über $85 \%$ in den Schulen in Finnland, Island, Norwegen und Schweden. Am geringsten war sie (mit weniger als 65\%) in Brasilien, Ungarn, Mexiko, Thailand und der Türkei. In der erstgenannten Ländergruppe spiegelt die sozioökonomische Zusammensetzung jeder Schule die sozioökonomische Zusammensetzung des Landes/der Volkswirtschaft relativ nah wider; in der letztgenannten Gruppe der Länder/Volkswirtschaften ist die Wahrscheinlichkeit geringer, dass Schülerinnen und Schüler aus einem sozioökonomisch begünstigten Umfeld dieselbe Schule besuchen wie sozioökonomisch benachteiligte Schüler und umgekehrt.

Der Grad der sozialen Inklusion hat sich im Durchschnitt der OECD-Länder mit vergleichbaren Daten zwischen 2003 und 2012 nicht verändert. In Hongkong (China), Lettland und Neuseeland ließ er nach, er verbesserte sich hingegen in Italien, Japan, Korea, der Schweiz und der Türkei, was bedeutet, dass in den Schulen dieser Länder im Jahr 2012 Schülerinnen und Schüler aus unterschiedlichen sozioökonomischen Milieus stärker gemischt waren als 2003. In Italien und der Türkei verbesserten sich in diesem Zeitraum ebenfalls die Mathematikleistungen, was darauf hindeutet, dass eine stärkere schulische Inklusion mit Verbesserungen bei der sozioökonomischen Inklusion zusammenfallen kann (Abb. II.2.15). Der in Korea beobachtete Trend zu einer stärkeren sozialen Inklusion ist mit einem ähnlichen Grad an schulischer Inklusion kombiniert (Abb. II.2.15 und Tabelle II.2.1b) ${ }^{14}$. Das bedeutet, dass die Schulen in Korea bei der Zulassung aus schulischer Sicht leistungsstarker Schülerinnen und Schüler genauso selektiv geblieben sind, der sozioökonomische Faktor in diesen Ländern für die Zulassung aber eine geringere Rolle spielt. In Korea ist die Wahrscheinlichkeit größer, dass die Schülerinnen und Schüler die Schule mit Schülern aus anderen sozioökonomischen Milieus besuchen und ebenso groß, dass sie dieselbe Schule besuchen wie Schülerinnen und Schüler mit unterschiedlichen schulischen Fähigkeiten. 
- Abbildung II.2.15 "

\section{Veränderungen der sozialen Inklusion zwischen 2003 und 2012}

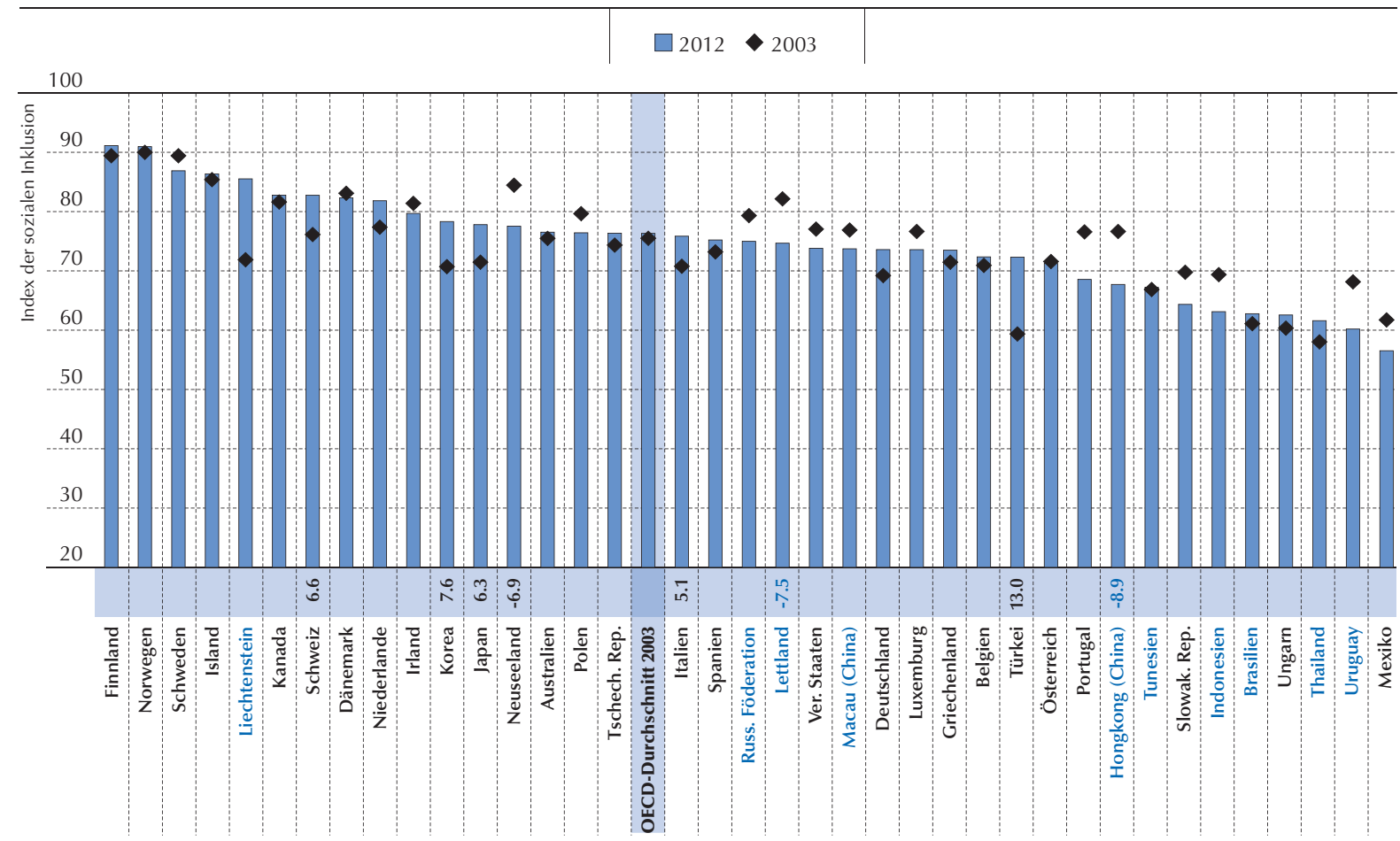

Anmerkung: Angegeben sind nur Länder und Volkswirtschaften mit vergleichbaren Daten aus PISA 2003 und PISA 2012.

Der Index der sozialen Inklusion entspricht dem Prozentsatz der Gesamtvarianz des in den Schulen beobachteten sozioökonomischen Status der Schülerinnen und Schüler.

Der Prozentpunktunterschied beim Index der sozialen Inklusion ist über dem Namen des Landes/der Volkswirtschaft angegeben. Aufgeführt sind nur statistisch signifikante Unterschiede.

Der OECD-Durchschnitt für 2003 umfasst nur die Länder, deren Ergebnisse im Bereich Mathematik seit dem Jahr 2003 vergleichbar sind.

Um die Vergleichbarkeit im Zeitverlauf zu gewährleisten, wurden die Werte von PISA 2003 auf dem PISA-Index des wirtschaftlichen, sozialen und kulturellen Status entsprechend der Indexskala von PISA 2012 umskaliert. Die in dieser Abbildung dargestellten Ergebnisse aus PISA 2003 können daher von denen abweichen, die in Lernen für die Welt von morgen: Erste Ergebnisse von PISA 2003 (OECD, 2004) wiedergegeben sind (vgl. Anhang A5 wegen näherer Einzelheiten).

Die Länder und Volkswirtschaften sind in absteigender Reihenfolge nach dem Index der sozialen Inklusion in PISA 2012 angeordnet.

Quelle: OECD, PISA-2012-Datenbank, Tabelle II.2.13b.

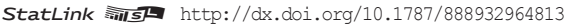

Die bisherige Diskussion setzt sich lediglich mit der Chancengerechtigkeit in der Bildung, d.h. der Bildungsgerechtigkeit in Zusammenhang mit dem sozioökonomischen Hintergrund auseinander, wobei das Konzept der Chancengerechtigkeit sich allgemeiner noch auf andere Quellen von Benachteiligungen für Schülerinnen und Schüler bezieht. In Kapitel 3 dieses Bandes werden andere potenzielle Quellen für Benachteiligungen von Schülerinnen und Schülern untersucht, darunter ihr Wohnort, die Art von Familie, aus der sie stammen, darunter die Frage, ob sie einen Migrationshintergrund haben, sowie das Ausmaß, in dem verschiedene Länder und Volkswirtschaften in der Lage sind, den Einfluss dieser Benachteiligungen auf die Leistungen in Mathematik in Grenzen zu halten. In Kapitel 3 werden ferner die Länder und Volkswirtschaften identifiziert, denen es gelungen ist, die Auswirkungen dieser Merkmale auf die Schülerleistungen zu reduzieren. 


\section{Anmerkungen}

1. Im vorliegenden Band stehen im Folgenden die Mathematikleistungen im Mittelpunkt. Es mag zwar signifikante Unterschiede zwischen den verschiedenen Erhebungsbereichen von PISA geben, diese liegen jedoch außerhalb des Untersuchungsrahmens dieses Bandes. Daher beziehen sich alle Hinweise auf Schülerleistungen im Folgenden auf Mathematikleistungen. Der prozentuale Anteil der Varianz der Schülerleistungen, der sich durch Unterschiede beim sozioökonomischen Status der Schülerinnen und Schüler erklären lässt, liegt bei 14,8\%. Diese Zahl entspricht dem Korrelationskoeffizienten einer einfachen Regression der Schülerleistungen auf den sozioökonomischen Status.

2. Es handelt sich also um Schülerinnen und Schüler, die eine Einheit auf dem PISA-Index des sozialen, wirtschaftlichen und kulturellen Status auseinanderliegen. Dieser Index ist derart standardisiert, dass der Mittelwert für die Schülerinnen und Schüler in den OECDLändern 0 entspricht und die Standardabweichung 1 beträgt.

3. Hierbei wird die Varianz der Schülerleistungen anhand der statistischen Varianz der Messgrößen der Schülerleistungen, z.B. der Punktzahl auf der PISA-Mathematikskala, gemessen.

4. Im Mittelpunkt steht hier die Stärke des Zusammenhangs zwischen Schülerleistungen und sozioökonomischem Status, gemessen anhand der erklärten Varianz bzw. anhand des Korrelationskoeffizienten einer einfachen Regression der Leistung auf den sozioökonomischen Status.

5. In anderen Bänden dieses Berichts wird auf die Leistungssteigerungen anderer Länder in PISA eingegangen und werden die von diesen Ländern in jüngster Zeit eingeführten bildungspolitischen Reformen beschrieben (z.B. Deutschland in Kapitel 3 des vorliegenden Bandes, Brasilien, die Türkei, Korea und Estland in Band I, Japan und Portugal in Band III sowie Kolumbien, Israel, Polen und Tunesien in Band IV).

6. ISCO-Berufshauptgruppe 0 (Angehörige der regulären Streitkräfte) wurde in zwei Kategorien aufgeteilt und entweder den Führungskräften (ISCO 1) oder den Berufsgruppen der angelernten Bürokräfte bzw. der Dienstleistungsberufe (ISCO 3) zugerechnet. Studierende, Hausfrauen und unklar definierte Berufe wurden als Fehlwert kodiert.

7. Hier bedeutet ein hohes Bildungsniveau, dass ein oder beide Elternteile über einen Tertiärabschluss (ISCED 5 oder 6) verfügen; ein niedriges Bildungsniveau bedeutet, dass ein oder beide Elternteile höchstens einen Sekundarstufe-II-Abschluss (ISCED 2) erworben haben.

8. Diese PISA-Messgröße der Resilienz ist nützlich, um länderübergreifende Vergleiche vorzunehmen. Sie ist jedoch nicht in der Lage, differenziertere Informationen zu berücksichtigen. So können die Schülerinnen und Schüler beispielsweise zum Zeitpunkt des PISA-Tests in besserer oder schlechterer Tagesform sein, im betreffenden Jahr besser oder schlechter lernen oder sich zwar in manchen Fächern als resilient erweisen, nicht aber in anderen. Darüber hinaus ist der sozioökonomische Status eine allgemeine Messgröße, die keine nicht durch die Messung erfassten Unterschiede zwischen und innerhalb von Ländern und Kulturen widerspiegelt, die dazu führen würden, dass die Resilienz auf eine andere Art und Weise identifiziert würde. Da die Identifizierung resilienter Schülerinnen und Schüler auf Daten beruht, die die Ränder der Verteilung betreffen, ist diese Messgröße weniger präzise.

9. Dieser Vergleich beruht auf einer internationalen Messgröße des sozioökonomischen Status, lässt jedoch die Tatsache außer Acht, dass die Familien der betreffenden Schülerinnen und Schüler u.U. ganz unterschiedliche Positionen in ihrer jeweiligen Gesellschaft einnehmen. So wären Schülerinnen und Schüler mit einem im OECD-Durchschnitt liegenden sozioökonomischen Status in einem Land, dessen durchschnittlicher sozioökonomischer Status deutlich unter dem OECD-Durchschnitt liegt, im Vergleich zu ihren Mitschülerinnen und Mitschülern möglicherweise sozioökonomisch begünstigt. In manchen Ländern stellt ein im OECD-Durchschnitt liegender sozioökonomischer Status einen begünstigten Status dar, in anderen Ländern jedoch nicht. Trotz dieser Annahmen und der Einschränkungen, die damit verbunden sind, lassen sich einige nützliche Erkenntnisse aus der hier vorgestellten hypothetischen Analyse gewinnen.

10. Dabei ist zu beachten, dass die Ergebnisse auch davon abhängen, wie die Schulen in den einzelnen Ländern definiert und organisiert sind und welche Einheiten für die Stichprobenziehung gewählt wurden. In einigen Ländern z.B. wurden einige der Schulen in der PISAStichprobe als Verwaltungseinheiten definiert (selbst wenn sie, wie in Italien, mehrere geografisch getrennte Einrichtungen umfassen); in anderen Ländern wurden sie als jene Teile größerer Bildungseinrichtungen definiert, die von 15-Jährigen besucht werden; in manchen Ländern wurden Schulen wiederum als Schulgebäude definiert, in wieder anderen dagegen aus Sicht der Schulorganisation (z.B. als Einheiten, die eine eigene Schulleitung haben). Der PISA 2012 Technical Report (OECD, erscheint demnächst) liefert einen Überblick über die verschiedenen Methoden der Definition der Schulen. Zu beachten ist auch, dass die Varianz innerhalb der Schulen auf Grund der Art und Weise, wie die Schülerinnen und Schüler in der Stichprobe erfasst wurden, sowohl die Leistungsunterschiede zwischen den Klassen als auch zwischen den einzelnen Schülern umfasst. In Slowenien ist die primäre Stichprobeneinheit definiert als eine Gruppe von Schülerinnen und Schülern, die demselben Bildungsgang innerhalb einer Schule folgen. In diesem besonderen Fall ist die Varianz zwischen Schulen vielmehr die Varianz innerhalb von Schulen, aber zwischen unterschiedlichen Bildungsgängen.

11. In Anbetracht der Gestaltung von PISA mit Stichproben von 35 Schülerinnen und Schülern je Schule sowie der Schwierigkeiten, die damit einhergehen, den sozioökonomischen Status jedes einzelnen Schülers mit hoher Präzision zu messen, ist dies zu erwarten. Wegen weiterer Einzelheiten vgl. Hauser (2009), „On Quality and Equity in the Performance of Students and Schools", Mimeo verfügbar unter https://edsurveys.rti.org/PISA/documents/HauserQuality-Equity_in_PISA_rmh_053009a.pdf. 
12. Der Leistungsabstand zwischen den Schulen ist größer, da er mit zahlreichen Schulmerkmalen zusammenhängt, wie z.B. schulpolitischen Maßnahmen und Vorgehensweisen oder dem Schulstandort bzw. anderen Merkmalen, die in PISA nicht gemessen werden können und in der vorliegenden Untersuchung daher nicht berücksichtigt wurden. Außerdem stützt sich die Untersuchung des Leistungsabstands innerhalb von Schulen auf weniger Informationen sowie auf Daten, die notwendigerweise weniger präzise sind, da sie lediglich auf den von Einzelnen - den Schülern - gemachten Angaben beruhen, wohingegen in den Schuldaten Informationen aller in der betreffenden Schule an der Erhebung teilnehmenden Schüler zusammengefasst sind.

13. Die Definition leistungsstarker, durchschnittlich abschneidender und leistungsschwacher Schulen ähnelt der für die Identifizierung sozioökonomisch benachteiligter, durchschnittlicher und begünstigter Schulen verwendeten Definition. Die durchschnittlichen Leistungen der Schulen werden mit den durchschnittlichen Leistungen auf der Ebene des Schulsystems verglichen, und die Schulen werden als leistungsstark bzw. leistungsschwach eingestuft, wenn ihre durchschnittlichen Leistungen statistisch signifikant über/unter den durchschnittlichen Leistungen des Schulsystems liegen. Sowohl die Varianz der Schülerleistungen als auch die Zahl der Beobachtungen in den einzelnen Schulen sind im Standardfehler der Schätzung der durchschnittlichen Leistungen auf Schulebene und daher bei der Einstufung der Schulen als leistungsstark, durchschnittlich abschneidend bzw. leistungsschwach berücksichtigt.

14. Die schulische Inklusion ist das Ausmaß, in dem die Schülerinnen und Schüler mit unterschiedlichen schulischen Fähigkeiten und Bedürfnissen dieselbe Schule besuchen oder der Grad, bis zu dem Schulen unterschiedliche Durchschnittsleistungen aufweisen. Ferner misst die schulische Inklusion die Wahrscheinlichkeit, mit der die leistungsstarken und leistungsschwachen Schülerinnen und Schüler eines Landes oder einer Volkswirtschaft dieselbe Schule besuchen. In PISA wird die schulische Inklusion gemessen als der Prozentsatz der Varianz bei den Mathematikleistungen, die innerhalb der Schulen anzutreffen ist; sie kann auch als das Ausmaß interpretiert werden, in dem die Schülerinnen und Schüler in einer Schule die schulische Verteilung im Land/in der Volkswirtschaft widerspiegeln. Der Index der schulischen Inklusion reicht von 0 bis 100, wobei näher bei 100 liegende Werte auf eine stärkere Inklusion hindeuten.

\section{Literaturverzeichnis}

Eaton, S.E. (2001), The Other Boston Busing Story: What's Won and Lost across the Boundary Line, Yale University Press, New Haven.

OECD (erscheint demnächst), PISA 2012 Technical Report, PISA, OECD Publishing.

OECD (2013), Education Policy Outlook: Mexico, OECD Publishing, http://www.oecd.org/edu/EDUCATION\%2OPOLICY\%20 OUTLOOK\%2OMEXICO_EN.pdf.

OECD (2012a), Avances en las Reformas de la Educación Básica en México: Una Perspectiva de la OCDE (vorläufige Ausgabe), OECD Publishing, www.sep.gob.mx/work/models/sep1/Resource/3048/2/images/Avances_en_las_reformas_de_la_educacion_basica.pdf.

OECD (2012b), Equity and Quality in Education: Supporting Disadvantaged Students and Schools, OECD Publishing, http://dx.doi.org/ 10.1787/9789264130852-en.

OECD (2004), Lernen für die Welt von morgen: Erste Ergebnisse von PISA 2003, PISA, W. Bertelsmann Verlag, http://dx.doi. org/10.1787/ 9783827416377-de.

Santiago, P., et al. (2012), OECD Reviews of Evaluation and Assessment in Education: Mexico 2012, OECD Reviews of Evaluation and Assessment in Education, OECD Publishing, http://dx.doi.org/10.1787/9789264172647-en.

Subsecretaría de Educación Pública (SEP) (2013), Reporte de Indicadores Educativos, SEP, Distrito Federal, Mexiko.

Wells, A.S. und R.L. Crain (1997), Stepping over the Color Line: African-American Students in White Suburban Schools, Yale University Press, New Haven.

Zorrilla, M. und B. Barba (2008), "Reforma Educativa en México: Descentralización y Nuevos Actores", Sinéctica, 30. 



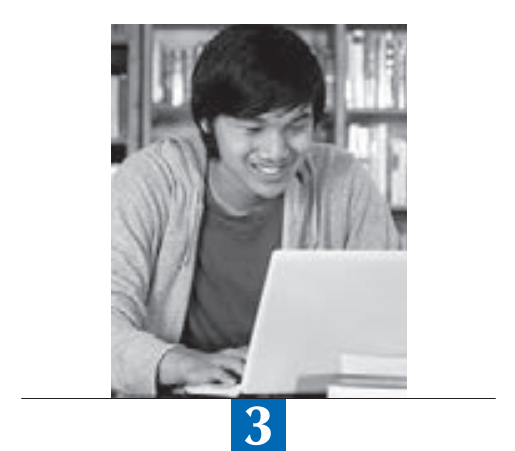

\section{Die Herausforderung der Vielfalt}

Dieses Kapitel untersucht verschiedene Aspekte der Merkmale von Schülerinnen und Schülern sowie Schulen, die die Bildungsergebnisse beeinflussen, darunter die Familienstruktur, der Erwerbsstatus der Eltern, der Schulstandort, ein etwaiger Migrationshintergrund und die zu Hause gesprochene Sprache. Darüber hinaus werden die Trends der Mathematikleistungen von Schülern mit Migrationshintergrund bis 2012 erörtert. 
Der sozioökonomische Status ist nur ein Aspekt des Hintergrunds von Schülerinnen und Schülern, der die Mathematikleistungen beeinflusst. Zu den weiteren Faktoren gehören die Familienstruktur, der Schulstandort, ein etwaiger Migrationshintergrund und die zu Hause gesprochene Sprache (im Vergleich zur Testsprache der PISA-Erhebung). All diese Faktoren haben Auswirkungen auf die Arbeit von Schulen und Lehrkräften, und die Art und Weise, in der die Schulen und Lehrkräfte sich mit ihnen auseinandersetzen, hat Auswirkungen auf die Bildungsergebnisse. In vielen Fällen hängen diese Faktoren auch eng mit dem sozioökonomischen Status der Schülerinnen und Schüler und der Schulen zusammen. Im vorliegenden Kapitel wird der Zusammenhang zwischen den Schülerleistungen und diesen Faktoren untersucht.

Im OECD-Raum leben rd. 14\% der 15-jährigen Schülerinnen und Schüler in Alleinerziehendenhaushalten (Tabelle II.3.1), mehr als 10\% leben in Familien, in denen der Vater oder die Mutter nicht erwerbstätig ist (Tabelle II.3.2), 11\% haben einen Migrationshintergrund (Tabelle II.3.4a), 6\% sind Schülerinnen und Schüler mit Migrationshintergrund, die zu Hause normalerweise eine andere Sprache als die Unterrichtssprache sprechen (Tabelle II.3.5), und 9\% besuchen eine Schule in einer kleinen ländlichen Gemeinde (Tabelle II.3.3a). Dieses Kapitel untersucht die Bildungsgerechtigkeit bei Schülergruppen, die einige dieser individuellen und schulbezogenen Merkmale teilen. Die Analyse der Chancengerechtigkeit bei verschiedenen Schülergruppen kann den politischen Entscheidungsträgern helfen, die Bildungs- und Sozialpolitik auf die Bedürfnisse einer immer vielfältiger werdenden Schülerpopulation auszurichten oder sie daran anzupassen.

\section{Ergebnisse der Datenanalyse}

- In den OECD-Ländern ist der Anteil der Schülerinnen und Schüler mit Migrationshintergrund zwischen 2003 und 2012 von 9\% auf 11\% gestiegen, während der Leistungsrückstand der Schüler mit Migrationshintergrund gegenüber den Schülern ohne Migrationshintergrund, die einen ähnlichen sozioökonomischen Status aufweisen, im gleichen Zeitraum um 11 Punkte gesunken ist.

- Im OECD-Raum erzielen Schülerinnen und Schüler, die eine Schule mit einem Migrationsanteil von über 25\% besuchen, tendenziell schlechtere Leistungen als Schüler in Schulen ohne Migrationsanteil, nach Berücksichtigung des sozioökonomischen Hintergrunds der Schüler geht der Unterschied in den Mathematikergebnissen jedoch um mehr als die Hälfte zurück, von 19 Punkten auf 7 Punkte.

\section{FAMILIENSTRUKTUR UND SCHÜLERLEISTUNGEN}

Die Familie ist normalerweise der erste Ort, an dem Kinder zum Lernen motiviert werden können. Manche Eltern lesen ihren Kindern Geschichten vor, helfen ihnen bei den Hausaufgaben und/oder beteiligen sich aktiv an schulischen Aktivitäten (OECD, 2012a). Die Unterstützung der Eltern kann auf alle Schüler ermutigend wirken, was zusätzlich gefördert werden kann, wenn die Eltern sich mit Lehr- oder Verwaltungskräften der Schulen treffen, um sich über die Lernfortschritte ihrer Kinder zu informieren ${ }^{1}$.

In den OECD-Ländern stammen rd. 14\% der 15-jährigen Schülerinnen und Schüler, die an PISA 2012 teilnahmen, aus Alleinerziehendenhaushalten. Viele von ihnen stammen außerdem aus sozioökonomisch benachteiligten Milieus. Im Durchschnitt der OECD-Länder sind Schüler aus Alleinerziehendenhaushalten generell gegenüber Schülern aus anderen Familientypen benachteiligt, weil ihre Eltern ein niedrigeres Bildungsniveau haben oder einen Beruf mit einem geringeren Status ausüben, oder weil die Ausstattung des Elternhauses mit verschiedenen Gütern nach Eigenangabe der Schüler schlechter ist (Tabelle II.3.1).

In Abbildung II.3.1 sind die durchschnittlichen Mathematikleistungen von Schülerinnen und Schülern aus Alleinerziehendenhaushalten im Vergleich zu Schülern aus anderen Familientypen ${ }^{2}$ dargestellt, und zwar vor und nach Berücksichtigung des sozioökonomischen Hintergrunds. Im OECD-Vergleich beträgt der Leistungsabstand zwischen Schülern aus Alleinerziehendenhaushalten und Schülern aus anderen Familientypen vor Berücksichtigung des sozioökonomischen Status 15 Punkte - was dem Lernfortschritt von fast einem halben Schuljahr entspricht.

Bei Schülerinnen und Schülern aus Alleinerziehendenhaushalten ist die Wahrscheinlichkeit, in ihrem Land im untersten Quartil der Mathematikleistungsverteilung zu liegen, 1,23-mal höher als bei Schülern aus anderen Familientypen; dies wird als das "relative Risiko" bezeichnet, dem Schüler aus Alleinerziehendenhaushalten im Vergleich zu Schülern aus anderen Familientypen ausgesetzt sind (Tabelle II.3.1). In Kasten II.3.1 wird eine andere Möglichkeit beschrieben, die Relevanz von Risikofaktoren wie Familienstruktur oder Migrationshintergrund in der gesamten Schülerpopulation zu bewerten. 
- Abbildung II.3.1

Leistungsunterschied im Bereich Mathematik, nach Familientyp

Leistungsunterschiede vor und nach Berücksichtigung des sozioökomischen Status

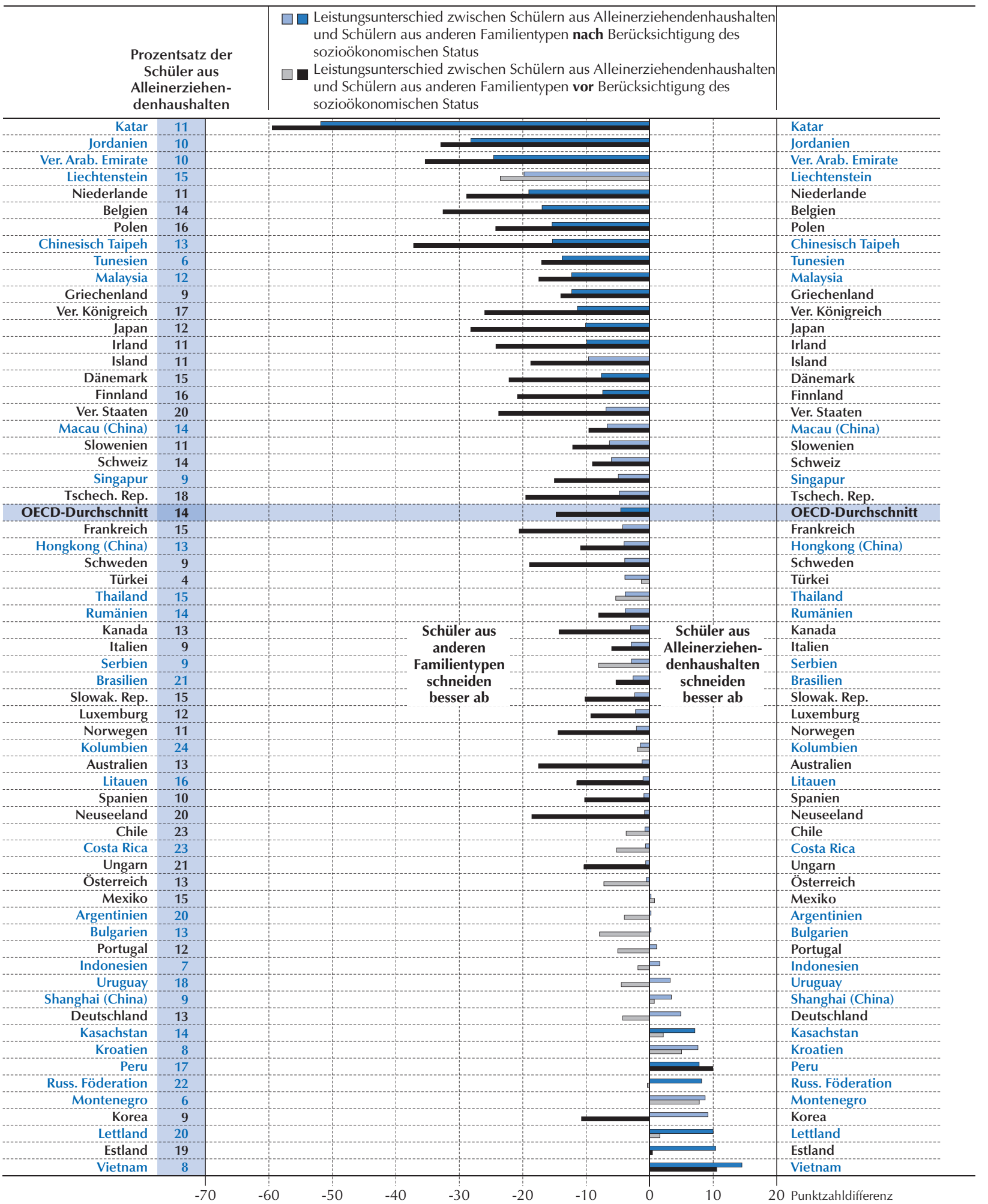

Anmerkung: Statistisch signifikante Punktzahldifferenzen sind in dunkleren Farbtönen gekennzeichnet.

Die Länder und Volkswirtschaften sind in aufsteigender Reihenfolge nach der Punktzahldifferenz zwischen Schülern aus Alleinerziehendenhaushalten und Schülern aus anderen Familientypen nach Berücksichtigung des sozioökonomischen Status angeordnet.

Quelle: OECD, PISA-2012-Datenbank, Tabelle II.3.1.

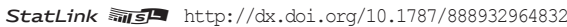




\section{Kasten II.3.1 Populationsrelevanz oder zuschreibbares Risiko}

Eine Möglichkeit, die Bedeutung von Risikofaktoren zu messen, ist die „Populationsrelevanz". Die Populationsrelevanz drückt aus, inwieweit ein Gesamtergebnis, wie z.B. schwache Mathematikergebnisse, mit der Zugehörigkeit zu einer potenziell benachteiligten Bevölkerungsgruppe zusammenhängt. Im Kontext von Alleinerziehendenhaushalten misst die Populationsrelevanz, in welchem Ausmaß die Inzidenz schwacher Mathematikleistungen in der Schülerpopulation insgesamt reduziert werden könnte, wenn das Risiko schwacher schulischer Leistungen bei Schülern aus Alleinerziehendenhaushalten genauso hoch wäre wie bei Schülern aus anderen Familientypen. Aus der Analyse geht hervor, dass der Anteil der leistungsschwachen Schülerinnen und Schüler um 3\% gesenkt werden könnte, wenn das Risiko schwacher schulischer Leistungen bei Schülern aus Alleinerziehendenhaushalten durch staatliche Maßnahmen auf das gleiche Niveau wie bei Schülern aus anderen Familientypen gesenkt werden könnte (Tabelle II.3.1). Während das relative Risiko schwacher schulischer Leistungen lediglich angibt, in welchem Ausmaß Schülerinnen und Schüler, die einer bestimmten Bevölkerungsgruppe angehören, anfällig gegenüber Leistungsschwäche sind, bietet die Populationsrelevanz einen absoluten Messwert für die Prävalenz dieses Risikos in der gesamten Schülerpopulation. Die Populationsrelevanz hängt ab von dem relativen Risiko, das sich aus der Zugehörigkeit zu einer gefährdeten Gruppe ergibt, und von der relativen Größe der gefährdeten Gruppe.

Der Leistungsabstand zwischen den Schülern aus Alleinerziehendenhaushalten und den Schülern aus anderen Familientypen wird durch die Berücksichtigung des sozioökonomischen Status im Allgemeinen verringert und in einigen Fällen beseitigt. Es besteht zwar ein Zusammenhang zwischen der Familienstruktur und dem sozioökonomischen Status, die separaten Effekte der einzelnen Variablen auf die Schülerleistungen können durch die Analyse der PISA-Daten jedoch nicht isoliert werden. Die Tatsache, dass selbst nach Berücksichtigung des sozioökonomischen Status der Schülerinnen und Schüler erhebliche Leistungsunterschiede bestehen bleiben, deutet darauf hin, dass es einen unabhängigen Zusammenhang zwischen der Familienstruktur und den Bildungschancen gibt.

Schülerinnen und Schüler aus Alleinerziehendenhaushalten erzielen nach Berücksichtigung des sozioökonomischen Status im Bereich Mathematik durchschnittlich fünf Punkte weniger als Schüler aus anderen Familientypen. In Brasilien, Chile, Kolumbien, Costa Rica, Ungarn und den Vereinigten Staaten leben mehr als 20\% der Schüler in Alleinerziehendenhaushalten, und sie schneiden nach Berücksichtigung des sozioökonomischen Status genauso gut ab wie ihre Mitschüler aus anderen Familientypen (vor Berücksichtigung dieses Faktors ist ihre Leistung in den Vereinigten Staaten, Ungarn und Brasilien jedoch schwächer). In Lettland und der Russischen Föderation machen diese Schüler ebenfalls mehr als 20\% der Schülerpopulation aus, und sie erzielen bessere Ergebnisse als ihre Mitschüler. Dagegen zeigt Abbildung II.3.1, dass der Abstand zwischen diesen beiden Schülergruppen in Katar, Jordanien, den Vereinigten Arabischen Emiraten und den Niederlanden besonders groß ist und nach Berücksichtigung des sozioökonomischen Status mindestens 19 Punkte beträgt. In Polen, Chinesisch Taipeh, Tunesien, Malaysia, Griechenland, dem Vereinigten Königreich und Japan beträgt der Unterschied mehr als 10 Punkte (Tabelle II.3.1). In Katar, Polen, den Vereinigten Staaten, Jordanien, Dänemark, den Vereinigten Arabischen Emiraten und Finnland ist die Populationsrelevanz höher als 6\%, was bedeutet, dass der Anteil der leistungsschwachen Schülerinnen und Schüler um mehr als 6\% zurückgehen würde, wenn das Risiko schwacher schulischer Leistungen bei den Schülern aus Alleinerziehendenhaushalten so gering wäre wie bei Schülern aus anderen Familientypen (Populationsrelevanz) (Tabelle II.3.1).

Die Evidenz, dass Schülerinnen und Schüler aus Alleinerziehendenhaushalten verhältnismäßig schwache Leistungen erbringen, ist zwar entmutigend, die im Ländervergleich festzustellende Varianz der Leistungsunterschiede deutet jedoch darauf hin, dass dieser Zusammenhang nicht unvermeidbar ist. Die Leistungsunterschiede können durch die staatliche Politik, insbesondere die Bildungspolitik, verringert werden, indem es Alleinerziehenden erleichtert wird, den Bildungserfolg ihrer Kinder zu unterstützen und zu fördern (Pong, Dronkers und Hampden-Thompson, 2004). Auf Ebene der Schulsysteme sowie der einzelnen Schulen könnte z.B. überlegt werden, wie und in welcher Form das Engagement von Alleinerziehenden, die wenig Zeit für Schulaktivitäten haben, gefördert werden sollte. Die bildungspolitischen Maßnahmen müssen im Zusammenspiel mit anderen Politikmaßnahmen gesehen werden, z.B. sozialpolitischen Maßnahmen und Regelungen für die Kinderbetreuung. 


\section{ERWERBSSTATUS DER ELTERN: ZIELAUSRICHTUNG DER BILDUNGSPOLITIK DURCH SOZIALPOLITISCHE MASSNAHMEN FÜR ARBEITSLOSE}

Bildungspolitische und andere sozialpolitische Maßnahmen sind für die Verbesserung der schulischen Leistungen von entscheidender Bedeutung. Da sich die Arbeitslosigkeit der Eltern in den einzelnen Ländern und Volkswirtschaften unterschiedlich auf die Schülerleistungen auswirkt, liegt der Schluss nahe, dass es einigen Ländern/Volkswirtschaften gelingt, die potenziell negativen Auswirkungen der Arbeitslosigkeit auf die Bildungserträge abzumildern.

In den OECD-Ländern gaben durchschnittlich 11\% der 15-jährigen Schülerinnen und Schüler den aktuellen Erwerbsstatus ihres Vaters als "nicht erwerbstätig“ (Voll- oder Teilzeit) an. Sie gaben an, dass ihr Vater derzeit entweder arbeitslos ist (nicht erwerbstätig, aber auf Arbeitsuche), oder einen anderen Erwerbsstatus hat (Haushaltspflichten, Ruhestand usw.). Rund 28\% der 15-Jährigen gaben für ihre Mutter einen ähnlichen Erwerbsstatus an. Der Erwerbsstatus der Eltern hängt eng mit dem sozioökonomischen Status zusammen, wobei große Leistungsunterschiede zwischen den Schülern, deren Eltern erwerbstätig sind, und den Schülern, deren Eltern nicht erwerbstätig sind, festzustellen sind. Aber selbst nach Berücksichtigung des sozioökonomischen Status schneiden die Schülerinnen und Schüler, die angaben, dass ihr Vater nicht erwerbstätig ist, im OECD-Raum um sechs Punkte schlechter ab als die Schüler, die angaben, dass ihr Vater erwerbstätig ist. Die Schüler, die angaben, dass ihre Mutter nicht erwerbstätig ist, erzielen acht Punkte weniger als die Schüler, die angaben, dass ihre Mutter erwerbstätig ist. Das relative Risiko schwacher schulischer Leistungen ist bei Schülern mit einem erwerbslosen Elternteil mehr als 1,4-mal höher als bei anderen Schülern, unabhängig davon, welcher Elternteil erwerbslos ist. Die Populationsrelevanz beträgt fast $5 \%$, wenn der Vater eines Schülers erwerbslos ist und mehr als 9\%, wenn die Mutter eines Schülers erwerbslos ist (Tabelle II.3.2).

In der Slowakischen Republik, Schweden, Belgien, der Tschechischen Republik, Island, Ungarn und Dänemark ist der Erwerbsstatus der Eltern (sowohl des Vaters als auch der Mutter) mit Leistungsunterschieden verbunden, die nach Berücksichtigung des sozioökonomischen Status von 12 Punkten in Dänemark bei Erwerbslosigkeit des Vaters bis zu 24 bzw. 22 Punkten in Belgien und der Slowakischen Republik bei Erwerbslosigkeit der Mutter reichen (Abb. II.3.2). In Norwegen, Deutschland und Frankreich sowie in Kroatien, Montenegro, Serbien und Shanghai (China) sind große Unterschiede nur in Bezug auf den Erwerbsstatus der Mutter zu beobachten. Israel verzeichnet den größten Leistungsunterschied in Bezug auf den Erwerbsstatus der Mutter (41 Punkte), in Bezug auf den Erwerbsstatus des Vaters ist jedoch kein Unterschied festzustellen. Die Populationsrelevanz ist am höchsten in der Slowakischen Republik, Rumänien, den Vereinigten Arabischen Emiraten und Ungarn, wo der Anteil der leistungsschwachen Schülerinnen und Schüler um mehr als $10 \%$ zurückgehen würde, wenn das Risiko schwacher schulischer Leistungen bei Schülern mit erwerbslosem Vater so niedrig wäre wie bei Schülern, deren Vater erwerbstätig ist, sowie in Israel, Montenegro, der Slowakischen Republik und den Vereinigten Arabischen Emiraten, wo der Anteil der leistungsschwachen Schüler um mehr als 20\% zurückgehen würde, wenn das Risiko schwacher schulischer Leistungen bei Schülern mit erwerbsloser Mutter so niedrig wäre wie bei Schülern, deren Mutter erwerbstätig ist (Tabelle II.3.2).

\section{STANDORT DER SCHULEN UND LEISTUNGSUNTERSCHIEDE ZWISCHEN VERSCHIEDENEN GEOGRAFISCHEN GEBIETEN}

In einigen Ländern bestehen je nach Schulstandort erhebliche Unterschiede zwischen den Schülerleistungen und dem sozioökonomischen bzw. organisatorischen Profil der Schulsysteme. Um die Leistungsunterschiede zwischen den Schulsystemen und Regionen innerhalb der einzelnen Länder zu erfassen, haben einige Länder im Rahmen der PISA-Erhebung Daten auf regionaler Ebene gesammelt. Die auf regionaler Ebene erfassten Ergebnisse sind in Anhang B2 dieses Bandes wiedergegeben. Die Leistungsunterschiede zwischen den Regionen der Länder, die diese Daten im Rahmen der PISA-Studie erhoben haben, werden in Kasten II.2.2 beschrieben.

Die Leistungsvarianz in Bezug auf geografische Merkmale kann auch unter Betrachtung des Standorts der einzelnen Schulen analysiert werden. Die Länder unterscheiden sich stark in Bezug auf die Einwohnerdichte sowie die Merkmale und Verteilung der Bevölkerung auf die verschiedenen Arten von Gemeinden (Tabelle II.3.3a), und diese Unterschiede dürfen bei der Interpretation der Leistungen der Schülerinnen und Schüler in diesen verschiedenen Gemeindetypen nicht außer Acht gelassen werden. Großstädte und dicht besiedelte Gebiete bieten tendenziell entscheidende Vorteile für Schulen, wie etwa ein reichhaltigeres kulturelles Umfeld, einen attraktiveren Arbeitsplatz für Lehrkräfte, eine größere Schulauswahl und bessere Beschäftigungsaussichten, die zur Motivation der Schüler beitragen können. Sie bringen jedoch oft auch größere sozioökonomische Herausforderungen mit sich. Darüber hinaus sind nicht alle Schüler dazu in der Lage, die Vorteile, die städtische Ballungszentren bieten, zu nutzen. Es gibt z.B. Schüler, die aus sozioökonomisch benachteiligten Milieus stammen, zu Hause eine andere Sprache als die Unterrichtssprache sprechen oder nur ein Elternteil haben, das sich um sie kümmert. 


\section{Leistungsunterschied im Bereich Mathematik, nach Erwerbsstatus der Eltern}

Leistungsunterschiede vor und nach Berücksichtigung des sozioökomischen Status

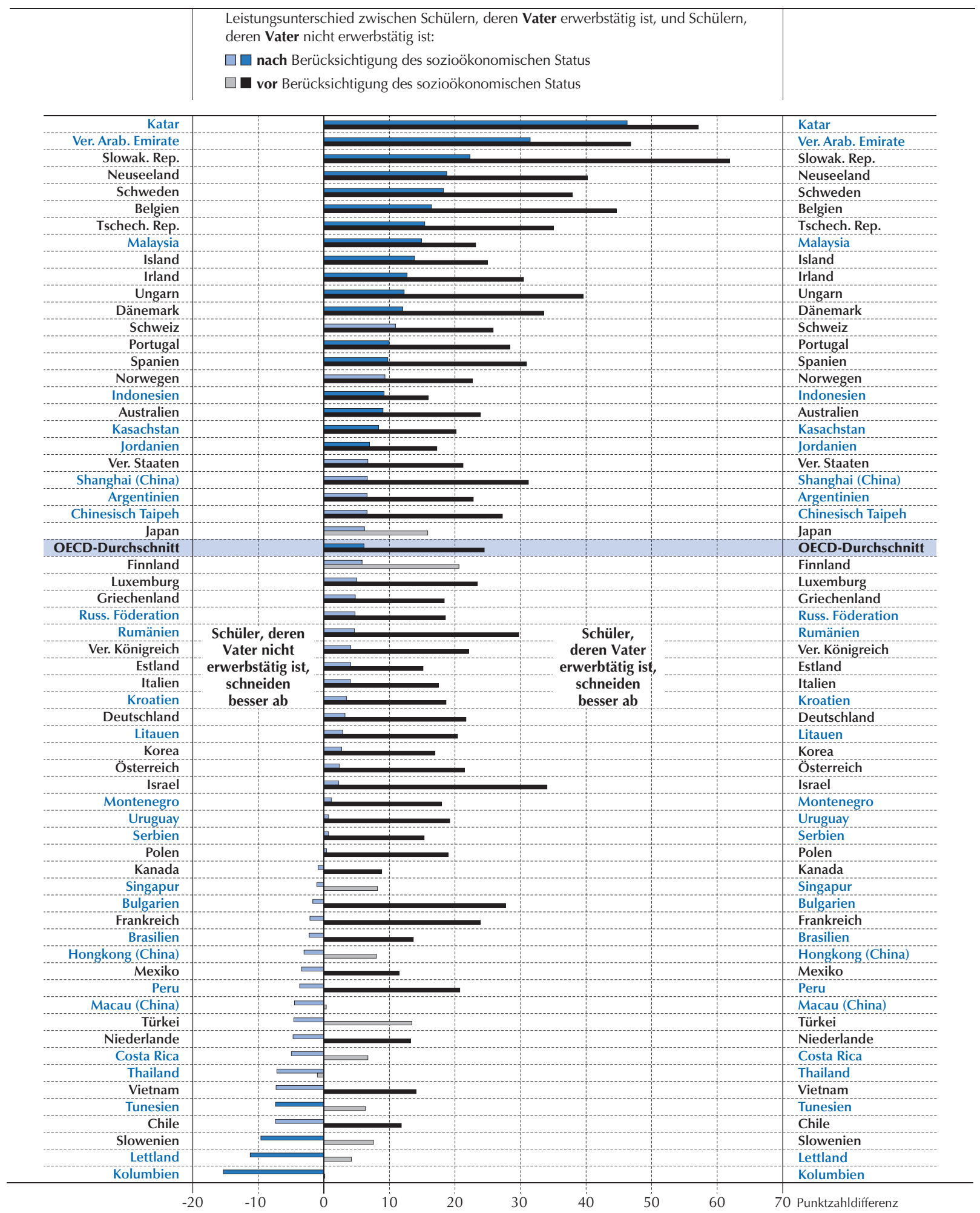

Anmerkung: Statistisch signifikante Punktzahldifferenzen sind in dunkleren Farbtönen gekennzeichnet.

Die Länder und Volkswirtschaften sind in absteigender Reihenfolge nach der Punktzahldifferenz zwischen Schülern, deren Vater/Mutter erwerbstätig ist, und Schülern, deren Vater/Mutter nicht erwerbstätig ist, nach Berücksichtigung des sozioökonomischen Status angeordnet.

Quelle: OECD, PISA-2012-Datenbank, Tabelle II.3.2.

StatLink त्तiाst http://dx.doi.org/10.1787/888932964832 
- Abbildung II.3.2 [Teil 2/2]

Leistungsunterschied im Bereich Mathematik, nach Erwerbsstatus der Eltern

Leistungsunterschiede vor und nach Berücksichtigung des sozioökomischen Status

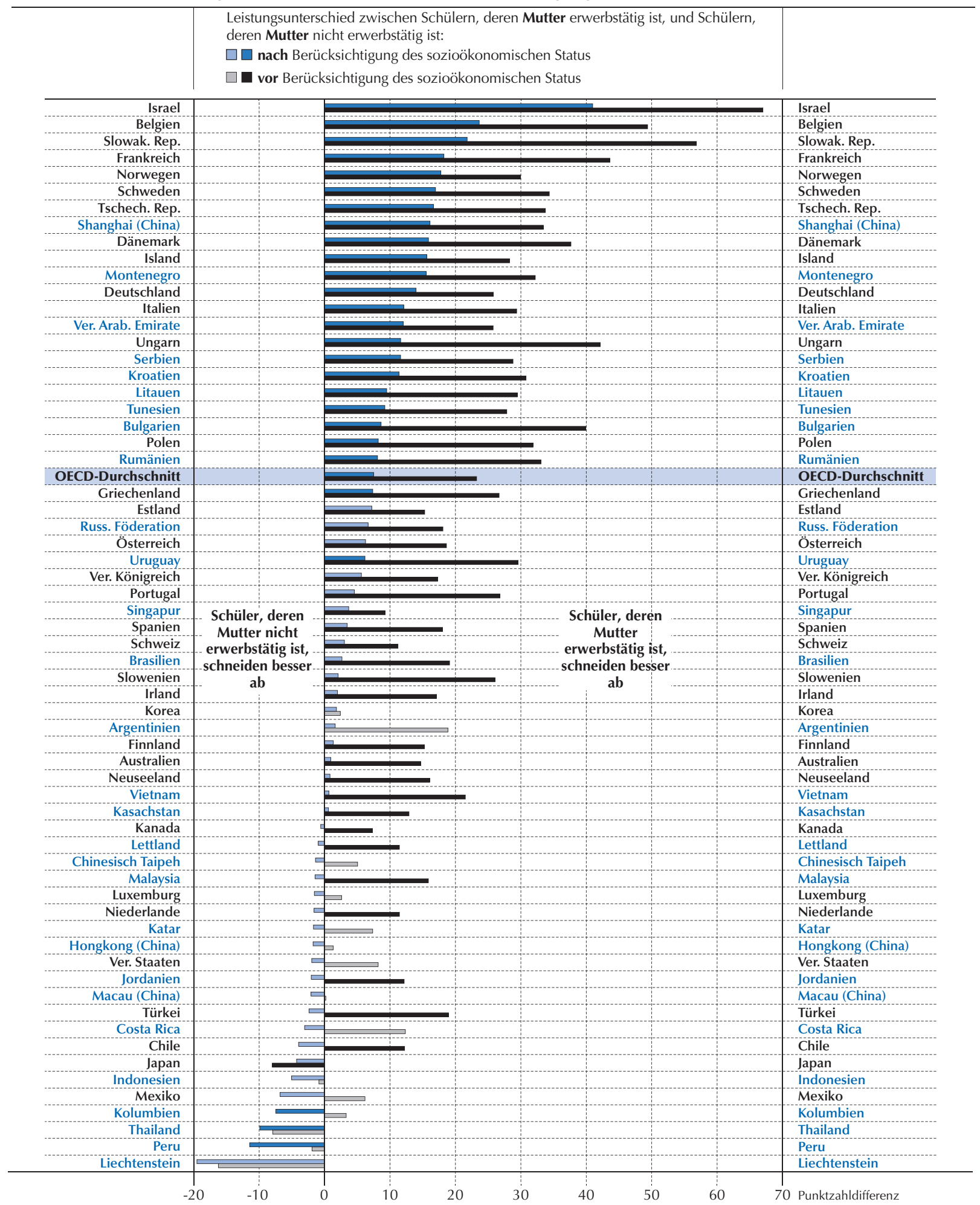

Anmerkung: Statistisch signifikante Punktzahldifferenzen sind in dunkleren Farbtönen gekennzeichnet.

Die Länder und Volkswirtschaften sind in absteigender Reihenfolge nach der Punktzahldifferenz zwischen Schülern, deren Vater/Mutter erwerbstätig ist, und Schülern, deren Vater/Mutter nicht erwerbstätig ist, nach Berücksichtigung des sozioökonomischen Status angeordnet.

Quelle: OECD, PISA-2012-Datenbank, Tabelle II.3.2.

StatLink 尚IIS http://dx.doi.org/10.1787/888932964832 
Im Durchschnitt der OECD-Länder erzielen Schüler in Städten (3 000 bis rd. 100000 Einwohner) nach Berücksichtigung des sozioökonomischen Status 11 Punkte mehr als Schüler in ländlichen Ortschaften (weniger als 3000 Einwohner). Schüler in Großstädten (mehr als 100000 Einwohner) schneiden nach Berücksichtigung des sozioökonomischen Status um 4 Punkte besser ab als Schüler in Städten (Tabelle II.3.3a).

Wie Abbildung II.3.3 zeigt, weisen die Schülerinnen und Schüler in Shanghai (China), Singapur, Hongkong (China), Chinesisch Taipeh, Vietnam, Macau (China), Korea, Japan, Liechtenstein, Polen, der Schweiz, Estland, Deutschland, den Niederlanden, Finnland, Kanada, Österreich und der Tschechischen Republik in allen Schulen unabhängig von ihrem Standort nach Berücksichtigung des sozioökonomischen Status Ergebnisse über dem OECD-Durchschnitt auf. In all diesen Ländern, mit Ausnahme von Vietnam, Japan und Polen, beträgt der Leistungsunterschied zwischen Schülern in ländlichen Gebieten und Schülern in Großstädten weniger als 10 Punkte (Tabelle II.3.3a).

Schüler in ländlichen Gebieten schneiden im Allgemeinen tendenziell schlechter ab als Schüler, die Schulen an anderen Standorten besuchen. Der Unterschied ist besonders groß, wenn die Leistungen mit den Leistungen von Schülern in Großstädten verglichen werden, obwohl auch Unterschiede zwischen den Schülern in ländlichen Gebieten und den Schülern in Städten festzustellen sind. So schneiden Schüler in Slowenien beispielsweise in Großstädten nach Berücksichtigung des sozioökonomischen Status um 74 Punkte besser ab als Schüler in ländlichen Gebieten, dieser Leistungsabstand ist zum großen Teil (65 Punkte) aber bereits zwischen Städten und ländlichen Gebieten festzustellen. Beim Vergleich von Schülerinnen und Schülern mit ähnlichem sozioökonomischem Status sind die größten Leistungsunterschiede zwischen Schülern in ländlichen Gebieten und Schülern in Großstädten in Bulgarien, Ungarn, Peru, Katar und Slowenien festzustellen (mehr als 41 Punkte, was einem Vorsprung von einem Schuljahr entspricht). Schüler in Großstädten sind im Durchschnitt sozioökonomisch bessergestellt als Schüler in Städten. In Belgien, Dänemark, Irland und der Türkei schneiden Schüler in Städten vor Berücksichtigung des sozioökonomischen Status besser ab als Schüler in Großstädten, die Unterschiede sind jedoch nicht markant. In den Vereinigten Staaten schneiden die Schüler in Großstädten im Durchschnitt schlechter ab als die Schüler in ländlichen Gebieten, selbst wenn sie sozioökonomisch bessergestellt sind (Tabelle II.3.3a).

Abbildung II.3.3 bietet außerdem einen länderübergreifenden Vergleich der Leistung von Schülern in Großstädten nach Berücksichtigung des sozioökonomischen Status. Shanghai (China), Chinesisch Taipeh, Singapur, Hongkong (China), Vietnam, Macau (China), Korea und Japan verzeichnen bei Schülern in Großstädten die besten Mathematikleistungen durchschnittlich mindestens 548 Punkte, wenigstens ein Schuljahr mehr als der OECD-Durchschnitt.

Der zwischen den Schulen im ländlichen Raum und den Schulen in Großstädten festzustellende Unterschied im sozioökonomischen Status variiert in den einzelnen Ländern erheblich. Am größten sind die Unterschiede in Mexiko, Bulgarien, Peru, Chile, Kolumbien, Vietnam, Thailand, Brasilien, Portugal, Ungarn, Tunesien und Costa Rica, wo der Abstand beim PISA-Index des wirtschaftlichen, sozialen und kulturellen Status mehr als eine Einheit beträgt (Tabelle II.3.3a).

Die Leistungsunterschiede resultieren teilweise aus dem unterschiedlichen sozioökonomischen Status von Schülern in Großstädten und Schülern im ländlichen Raum und/oder aus anderen Faktoren, die mit sozioökonomischen Disparitäten zusammenhängen können, die Auswirkungen auf die Schülerleistungen haben. Ein Vergleich der Ergebnisse vor und nach Berücksichtigung des sozioökonomischen Status zeigt, inwieweit die mit dem Schulstandort zusammenhängenden Unterschiede bei den Schülerleistungen mit Unterschieden beim sozioökonomischen Status der verschiedenen Schulstandorte innerhalb der einzelnen Länder assoziiert sind. Ein großer Leistungsunterschied sowohl vor als auch nach Berücksichtigung des sozioökonomischen Status deutet darauf hin, dass sich die sozioökonomischen Profile von städtischen und ländlichen Gebieten erheblich unterscheiden. So beträgt der durchschnittliche Leistungsabstand zwischen Schülern in ländlichen Räumen und Schülern in Städten im OECD-Vergleich beispielsweise 20 Punkte, er sinkt jedoch auf 11 Punkte, wenn Schüler mit ähnlichem sozioökonomischem Status verglichen werden. Der Unterschied zwischen Schulen in ländlichen Gebieten und Schulen in Großstädten ist größer und sinkt nach Berücksichtigung des sozioökonomischen Status von 31 Punkten auf 13 Punkte (Tabelle II.3.3a).

In PISA 2003 und in den OECD-Ländern, die an der PISA-Erhebung von 2012 teilgenommen haben, erreichten die Schülerinnen und Schüler im ländlichen Raum im Bereich Mathematik durchschnittlich 472 Punkte, während die Schüler in Städten 497 Punkte erreichten und die Schüler in Großstädten 513 Punkte erzielten. Bis 2012 hatten sich die Mathematikleistungen der Schüler in ländlichen Räumen und in Städten nicht geändert, in Großstädten dagegen waren sie um sieben Punkte gesunken. In den Ländern und Volkswirtschaften, die vergleichbare Daten für 2003 und 2012 aufweisen und in diesem Zeitraum Verbesserungen bei den Mathematikleistungen erzielt haben, verteilt sich die 


\section{Durchschnittsergebnisse im Bereich Mathematik, nach Schulstandort, nach Berücksichtigung des sozioökomischen Status}

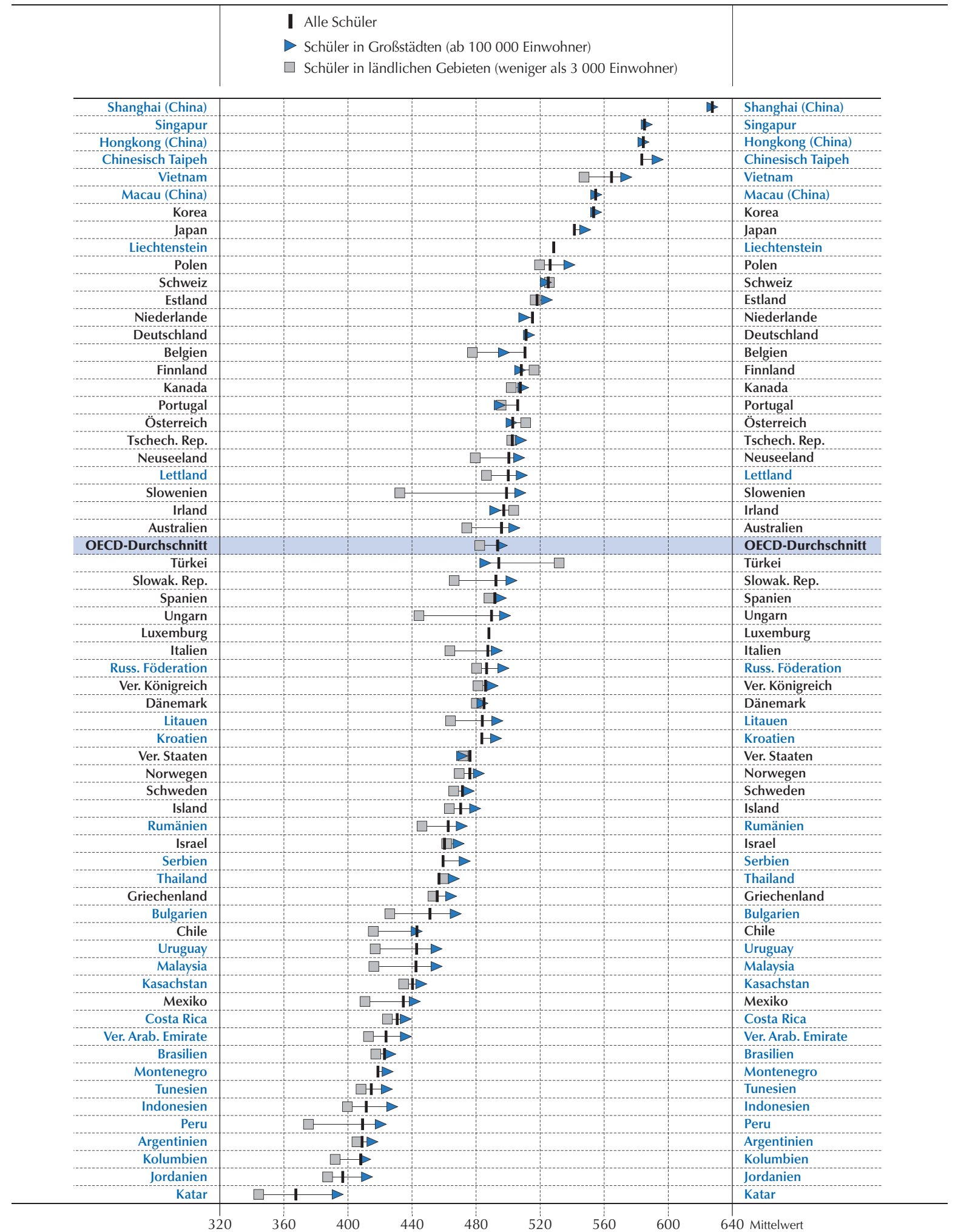

Die Länder und Volkswirtschaften sind in absteigender Reihenfolge nach dem Durchschnittsergebnis aller Schüler nach Berücksichtigung des sozioökonomischen Status angeordnet.

Quelle: OECD, PISA-2012-Datenbank, Tabelle II.2.1 und II.3.3a.

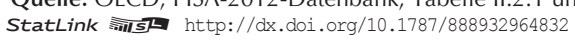


beobachtete Verbesserung auf alle Arten von Gemeinden. Die einzige Ausnahme ist die Türkei, wo sich ein großer Teil der im Bereich Mathematik beobachteten Verbesserung auf Schüler in Städten konzentriert, die ihre Mathematikergebnisse zwischen PISA 2003 und PISA 2012 nach Berücksichtigung der sozioökonomischen Unterschiede um 59 Punkte verbessern konnten (Tabelle II.3.3b).

\section{BILDUNGSGERECHTIGKEIT FÜR SCHÜLERINNEN UND SCHÜLER MIT MIGRATIONSHINTERGRUND}

Migration ist kein neues Phänomen, auf Grund der Bevölkerungsalterung und des drohenden Fachkräftemangels steht dieses Thema jedoch in vielen OECD-Ländern auf der politischen Tagesordnung ganz oben. Schülerinnen und Schüler mit Migrationshintergrund bilden sowohl innerhalb der Länder als auch im Ländervergleich eine heterogene Gruppe. Sie unterscheiden sich im Hinblick auf ihr Herkunftsland, ihre Sprache und ihre Kultur, und sie bringen ein breites Spektrum unterschiedlicher Kompetenzen, Kenntnisse und Motivationen in ihre Schulen ein. Obwohl eine große Untergruppe der Migranten hochqualifiziert ist, ist dies bei vielen anderen, die sozial benachteiligt sind, nicht der Fall. Ein solcher Nachteil kann zusammen mit kulturellen und ethnischen Unterschieden zu Spaltungen und Ungleichheiten zwischen der im Inland geborenen und der zugewanderten Bevölkerung führen. Diese Problematik geht weit über die Frage hinaus, wie die Migrationsströme kanalisiert und gesteuert werden können; es geht darum, wie die Zuwanderer auf eine Art und Weise in die Gesellschaft der Aufnahmeländer integriert werden können, die sowohl für die Zuwanderer als auch für die Bevölkerung der Aufnahmeländer annehmbar ist.

Die Integration zugewanderter Schülerinnen und Schüler in die Schule stellt für die meisten Länder eine Herausforderung dar, der Erfolg eines Landes bei der Integration der Migrantenkinder in die Gesellschaft ist jedoch ein Schlüsselindikator für die Wirksamkeit der Sozialpolitik im Allgemeinen und der Bildungspolitik im Besonderen. Der Unterschied beim selbst nach Berücksichtigung des sozioökonomischen Status vorhandenen - Leistungsabstand zwischen Schülerinnen und Schülern mit und ohne Migrationshintergrund zwischen den Ländern lässt darauf schließen, dass der Politik bei der Beseitigung solcher Leistungsdifferenzen eine wichtige Rolle zukommt. Angesichts der Vielfalt der zugewanderten Schülerpopulation in den verschiedenen Ländern ist die Entwicklung einer auf die konkreten Bedürfnisse dieser Schüler ausgerichteten Bildungspolitik - insbesondere im Bereich des Sprachunterrichts - jedoch keine leichte Aufgabe.

Die Bildungspolitik allein ist wahrscheinlich nicht ausreichend, um alle Probleme in Bezug auf die Leistungsunterschiede zwischen Schülern mit und ohne Migrationshintergrund zu lösen. So korrelieren die PISA-Ergebnisse der Schülerinnen und Schüler mit Migrationshintergrund stärker (und zwar negativ) mit der Konzentration sozioökonomischer Benachteiligung in den Schulen als mit der Konzentration der Migranten an sich oder mit der Konzentration der Schüler, die zu Hause eine andere Sprache als die Unterrichtssprache sprechen. Die Reduzierung der Konzentration der Benachteiligung in den Schulen erfordert möglicherweise Änderungen in anderen sozialpolitischen Bereichen, wie z.B. die Wohnungspolitik und Sozialleistungen, um eine ausgewogenere soziale Zusammensetzung in den Schulen zu fördern.

\section{Auswirkungen anderer sozialpolitischer Maßnahmen auf das Profil der Schülerinnen und Schüler mit Migrationshintergrund}

Bei der Interpretation von Leistungsdifferenzen zwischen Schülern mit und ohne Migrationshintergrund gilt es, die Unterschiede in Bezug auf den sozioökonomischen, bildungsbezogenen und sprachlichen Hintergrund der Zuwandererpopulation zu berücksichtigen. Die Zusammensetzung der Zuwandererpopulation wird durch die Einwanderungspolitik und -praxis beeinflusst, und die Kriterien für die Aufnahme von Migranten sind je nach Land sehr unterschiedlich. Während einige Länder jedes Jahr eine relativ große Zahl an Zuwanderern aufnehmen und dabei oft wenig selektiv vorgehen, sind die Zuwandererströme in anderen Ländern wesentlich geringer bzw. Gegenstand einer stärkeren Selektion. Zudem bestehen zwischen den Ländern Unterschiede in Bezug darauf, wie stark die soziale, bildungsbezogene oder berufliche Stellung der Zuwanderungskandidaten bei der Entscheidung über die Erteilung der Zuwanderungserlaubnis oder der Staatsbürgerschaft berücksichtigt wird. Die Zusammensetzung vergangener Migrationsströme spiegelt sich in der Regel in der künftiger Zuwanderungsströme wider, da die bestehenden Netzwerke die Migration aus denselben Herkunftsländern begünstigen. Außerdem lassen sich manche Migrationsströme auf Grund internationaler Verträge (Freizügigkeitsabkommen, Flüchtlingskonvention usw.) oder allgemein anerkannter Menschenrechte (wie dem Recht der Zuwanderer ebenso wie der Staatsangehörigen, mit ihren Familien zusammenzuleben) u.U. nicht ohne weiteres beschränken. Folglich sind die Migranten in einigen Ländern besser qualifiziert oder sozial bessergestellt als in anderen. Für die OECD-Länder ist festzustellen: 
- Australien, Kanada und Neuseeland verfolgen eine Einwanderungspolitik, die besser qualifizierte Zuwanderer begünstigt.

- Die Vereinigten Staaten haben ein Migrationssystem, das tendenziell den Familiennachzug begünstigt, und zwar sowohl von unmittelbaren Familienangehörigen - wie in anderen Ländern - als auch von Eltern, Geschwistern und erwachsenen Kindern.

- In den 1960er und 1970er Jahren wurde in Dänemark, Deutschland, Luxemburg, Norwegen, Österreich, Schweden und der Schweiz eine befristete Arbeitsmigration ausländischer Arbeitskräfte gefördert, von denen sich dann viele auf Dauer in diesen Ländern niederließen. In den letzten zehn Jahren hat die Zuwanderung in diesen Ländern mit Ausnahme von Deutschland wieder zugenommen. In Deutschland, Österreich und der Schweiz sowie in geringerem Umfang in Schweden verfügen Zuwanderer mit geringerer Wahrscheinlichkeit über einen Abschluss des Sekundarbereichs II und mit größerer über einen Tertiärabschluss. Infolgedessen gibt es in der Regel zwei unterschiedliche Migrantenkategorien: einerseits geringqualifizierte und andererseits hochqualifizierte Kräfte.

- Frankreich und das Vereinigte Königreich haben hohe Zahlen von Zuwanderern aus ehemaligen Kolonien, die die Sprache des Aufnahmelands bei der Einreise häufig bereits beherrschen.

- Finnland, Griechenland, Irland, Italien, Portugal und Spanien verzeichneten Anfang der 2000er Jahre eine drastische Zunahme der Zuwandererzahlen.

\section{Ein hohes Leistungsniveau in einer vielfältigen Schülerpopulation}

In PISA wird im Hinblick auf den Migrationsstatus zwischen drei Schülerkategorien unterschieden: Schülerinnen und Schüler ohne Migrationshintergrund (sie werden auch als einheimische Schüler bezeichnet und sind in dem Land geboren, in dem sie im Rahmen von PISA geprüft wurden, oder haben mindestens einen im Inland geborenen Elternteil) ${ }^{3}$, Schülerinnen und Schüler der zweiten Generation (sie sind im Inland geboren, ihre Eltern sind aber im Ausland geboren), und Schülerinnen und Schüler der ersten Generation (sie sind im Ausland geboren und haben im Ausland geborene Eltern) ${ }^{4}$. Dieses Kapitel konzentriert sich zunächst auf die Gesamtgruppe der Schülerinnen und Schüler mit Migrationshintergrund (erste und zweite Generation) und analysiert anschließend getrennt für beide Untergruppen die Bildungsgerechtigkeit.

Im OECD-Durchschnitt haben 11\% der im Rahmen von PISA 2012 beurteilten Schülerinnen und Schüler einen Migrationshintergrund. Diese zugewanderten Schüler sind tendenziell sozioökonomisch benachteiligt gegenüber den Schülern ohne Migrationshintergrund. Sie erzielen außerdem im PISA-Mathematiktest durchschnittlich 34 Punkte weniger als Schüler ohne Migrationshintergrund, wobei der Abstand nach Berücksichtigung der sozioökonomischen Unterschiede durchschnittlich 21 Punkte beträgt. Bei Schülerinnen und Schülern mit Migrationshintergrund ist die Wahrscheinlichkeit, im untersten Quartil der Leistungsverteilung zu liegen, effektiv 1,7-mal höher als bei Schülern ohne Migrationshintergrund. Wenn das Risiko schwacher schulischer Leistungen durch bildungspolitische Maßnahmen auf das Niveau gesenkt werden könnte, das bei Schülern ohne Migrationshintergrund zu verzeichnen ist, würde der Anteil der leistungsschwachen Schüler in der Gesamtpopulation um 7\% zurückgehen (Tabelle II.3.4a).

In Kanada, Neuseeland und Australien ist die Schülerpopulation mit Migrationshintergrund weit größer als im OECDDurchschnitt (29\%, 26\% und 23\%), und sowohl die Schüler mit als auch die Schüler ohne Migrationshintergrund erzielen im Durchschnitt Ergebnisse, die weit über dem OECD-Mittelwert liegen (mehr als 500 Punkte). In Australien erzielen die Schüler mit Migrationshintergrund selbst nach Berücksichtigung der sozioökonomischen Unterschiede 29 Punkte mehr als die Schüler ohne Migrationshintergrund. In Kanada und Neuseeland schneiden beide Gruppen gleich gut ab. Das Gleiche gilt für Irland, der Anteil der Schüler mit Migrationshintergrund (10\%) liegt aber näher am OECD-Durchschnitt (11\%). In der Gruppe der Partnerländer und -volkswirtschaften haben Macau (China), Hongkong (China), Liechtenstein und Singapur ebenfalls einen hohen Anteil an zugewanderten Schülern, wobei sowohl die Schüler mit als auch die Schüler ohne Migrationshintergrund im Durchschnitt eine hohe Leistung erzielen. In Macau (China) und Hongkong (China) schneiden die Schüler mit Migrationshintergrund nach Berücksichtigung des sozioökonomischen Status besser ab als die Schüler ohne Migrationshintergrund, in Singapur schneiden die beiden Gruppen gleich gut ab, und in Liechtenstein erzielen die Schüler mit Migrationshintergrund im Bereich Mathematik durchschnittlich 40 Punkte weniger als die Schüler ohne Migrationshintergrund (Tabelle II.3.4a).

In der Gruppe der OECD-Länder schneiden die Schüler mit und ohne Migrationshintergrund in Kanada, Neuseeland, Israel, dem Vereinigten Königreich und Irland gleich gut ab (das Gleiche gilt für die Slowakische Republik, die Türkei und Chile, der Anteil der Schülerpopulation mit Migrationshintergrund liegt in diesen Ländern aber unter 1\%). In Belgien, Frankreich, der Schweiz, Österreich, Schweden, Spanien, Luxemburg, Norwegen, den Niederlanden und 
- Abbildung II.3.4"

Leistungsunterschied in Mathematik zwischen Schülern mit und ohne Migrationshintergrund

Vor und nach Berücksichtigung des sozioökomischen Status

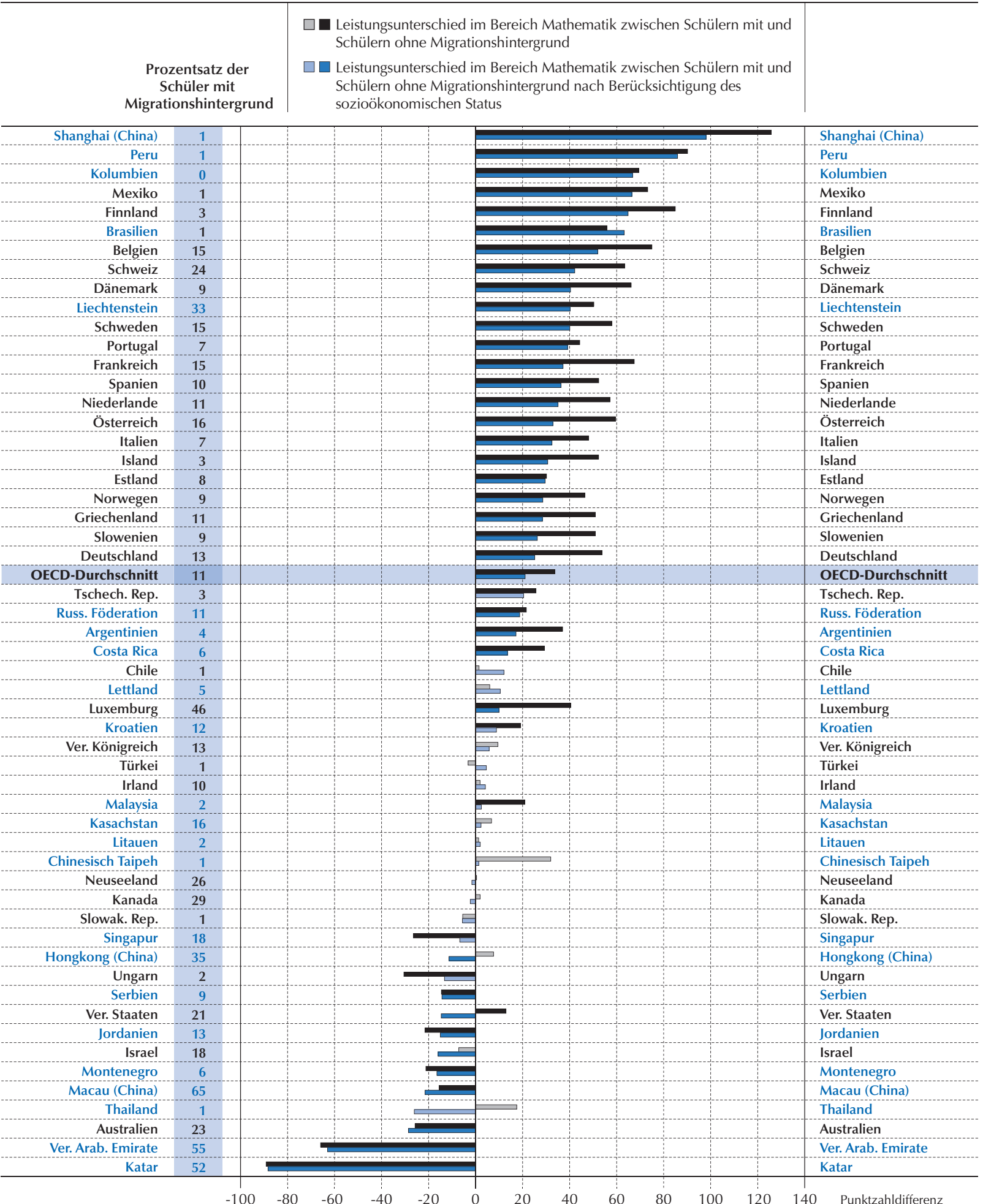

Anmerkung: Statistisch signifikante Punktzahldifferenzen sind in dunkleren Farbtönen gekennzeichnet.

Die Länder und Volkswirtschaften sind in absteigender Reihenfolge nach der Punktzahldifferenz zwischen den Schülern mit und den Schülern ohne Migrationshintergrund nach Berücksichtigung des sozioökonomischen Status angeordnet.

Quelle: OECD, PISA-2012-Datenbank, Tabelle II.3.4a.

StatLink न्ना1s http://dx.doi.org/10.1787/888932964832 
Dänemark machen Schüler mit Migrationshintergrund mindestens 9\% der Schülerpopulation aus, und bei ihnen ist die Wahrscheinlichkeit, im untersten Quartil der Leistungsverteilung zu liegen, 2,0- bis 2,4-mal höher als bei Schülern ohne Migrationshintergrund. Das gleiche Muster ist in Kolumbien, Peru, Finnland und Mexiko zu beobachten, in allen Fällen ist der Anteil der zugewanderten Schülerinnen und Schüler jedoch relativ gering - 1\% bzw. 3\%. In der Gruppe der Partnervolkswirtschaften ist dieses Muster in Shanghai (China) und Brasilien festzustellen, der Anteil der zugewanderten Schüler ist jedoch unerheblich. Wenn das Risiko schwacher schulischer Leistungen in Luxemburg bei den zugewanderten Schülern genauso hoch wäre wie bei den Schülern ohne Migrationshintergrund, würde der Anteil der leistungsschwachen Schüler in dem Land um 31\% zurückgehen, während er in der Schweiz um 24\% sinken würde. Der Anteil der leistungsschwachen Schüler würde in diesem Szenario in Belgien, Frankreich, Österreich, Schweden, Dänemark und Deutschland ebenfalls erheblich zurückgehen. In der Gruppe der Partnerländer und -volkswirtschaften ist die Populationsrelevanz der Schülerinnen und Schüler mit Migrationshintergrund nur in Liechtenstein ähnlich hoch (Tabelle II.3.4a).

\section{Ein höheres Leistungsniveau in einer zunehmend vielfältigen Schülerpopulation}

In PISA 2003 hatten 9\% der Schülerinnen und Schüler im OECD-Raum einen Migrationshintergrund. Sie erzielten im Bereich Mathematik 47 Punkte weniger als ihre Mitschüler ohne Migrationshintergrund. Wenn Schüler mit ähnlichem sozioökonomischem Status verglichen wurden, war der Leistungsunterschied geringer - 33 Punkte - aber immer noch vorhanden. Bis 2012 stieg der Anteil der Schülerinnen und Schüler mit Migrationshintergrund in den OECDLändern, für die vergleichbare Daten für 2003 und 2012 vorliegen, auf 11\%, und der Vorsprung der Schülerinnen und Schüler ohne Migrationshintergrund ging bei den Mathematikleistungen um etwa 10 Punkte zurück. Die Verringerung des Leistungsabstands der Schülerinnen und Schüler mit Migrationshintergrund im Bereich Mathematik (um rd. 10 Punkte) ist selbst bei einem Vergleich zwischen Schülern mit und ohne Migrationshintergrund zu beobachten, die einen ähnlichen sozioökonomischen Hintergrund aufweisen. Darüber hinaus war der sozioökonomische Status der Schülerinnen und Schüler mit Migrationshintergrund 2012 etwas besser als 2003 (der sozioökonomische Status der Schüler ohne Migrationshintergrund hat sich in diesem Zeitraum ebenfalls verbessert). Diese Ergebnisse lassen darauf schließen, dass die Schülerinnen und Schüler mit Migrationshintergrund 2012 im OECD-Durchschnitt sozioökonomisch weniger benachteiligt waren und einen geringeren Leistungsrückstand aufwiesen als 2003. Trotz dieser Veränderungen und Verbesserungen wiesen die zugewanderten Schülerinnen und Schüler 2012 bei den Mathematikleistungen jedoch noch einen erheblichen Rückstand gegenüber ihren Mitschülern ohne Migrationshintergrund auf, wenn auch in geringerem Maße als 2003 (Abb. II.3.5).

In der Gruppe der Länder und Volkswirtschaften, in denen sowohl 2003 als auch 2012 wenigstens 5\% der Schülerpopulation einen Migrationshintergrund aufwiesen, hat sich der Leistungsunterschied im Bereich Mathematik zwischen Schülern mit und ohne Migrationshintergrund von 2003 bis 2012 in Belgien, Deutschland, Neuseeland, der Schweiz und den Vereinigten Staaten verringert (Abb. II.3.5). In Belgien, Deutschland und der Schweiz ist der Rückgang darauf zurückzuführen, dass sich die Leistungen der Schülerinnen und Schüler mit Migrationshintergrund stärker verbessert haben als die Leistungen der Schülerinnen und Schüler ohne Migrationshintergrund. In Deutschland ging der Leistungsrückstand der Schüler mit Migrationshintergrund zurück: 2003 schnitten die Schüler ohne Migrationshintergrund im Bereich Mathematik um 81 Punkte besser ab als die Schüler mit Migrationshintergrund, 2012 war dieser Unterschied auf 54 Punkte gesunken (Kasten II.3.2 beschreibt Deutschlands Verbesserung bei PISA sowie den jüngsten bildungspolitischen Kurs des Landes). In Belgien und der Schweiz ist der Rückgang sogar festzustellen, wenn Schüler mit ähnlichem sozioökonomischem Status verglichen werden. In den Vereinigten Staaten ging der Leistungsunterschied zwischen Schülern mit und ohne Migrationshintergrund, die einen ähnlichen sozioökonomischen Status aufweisen, im Betrachtungszeitraum um 23 Punkte zurück. In Australien war 2003 kein Unterschied in den Mathematikleistungen zwischen den Schülern mit und ohne Migrationshintergrund festzustellen, 2012 schnitten die zugewanderten Schüler besser ab als die Schüler ohne Migrationshintergrund. Nur in Frankreich und Italien vergrößerte sich der Leistungsrückstand der Schüler mit Migrationshintergrund von 2003 bis 2012. Während dieser Anstieg in Italien weitgehend auf den Rückgang des sozioökonomischen Status von zugewanderten Schülern zurückzuführen ist, ist in Frankreich auch bei einem Vergleich von Schülern mit ähnlichem sozioökonomischem Status ein Anstieg des Leistungsrückstands festzustellen (Abb. II.3.5).

Ein rascher Anstieg des Anteils der Schülerinnen und Schüler mit Migrationshintergrund - insbesondere in Ländern und Volkswirtschaften, in denen der Anteil der Zuwanderer vorher gering war - stellt die Bildungssysteme vor Herausforderungen. Schülerinnen und Schüler mit Migrationshintergrund haben oft unterschiedliche Bildungsbedürfnisse, insbesondere wenn sich ihre Muttersprache von der Sprache des Aufnahmelands unterscheidet, sie können jedoch 


\section{Veränderung der Mathematikleistungen der Schüler mit Migrationshintergrund zwischen 2003 und 2012}

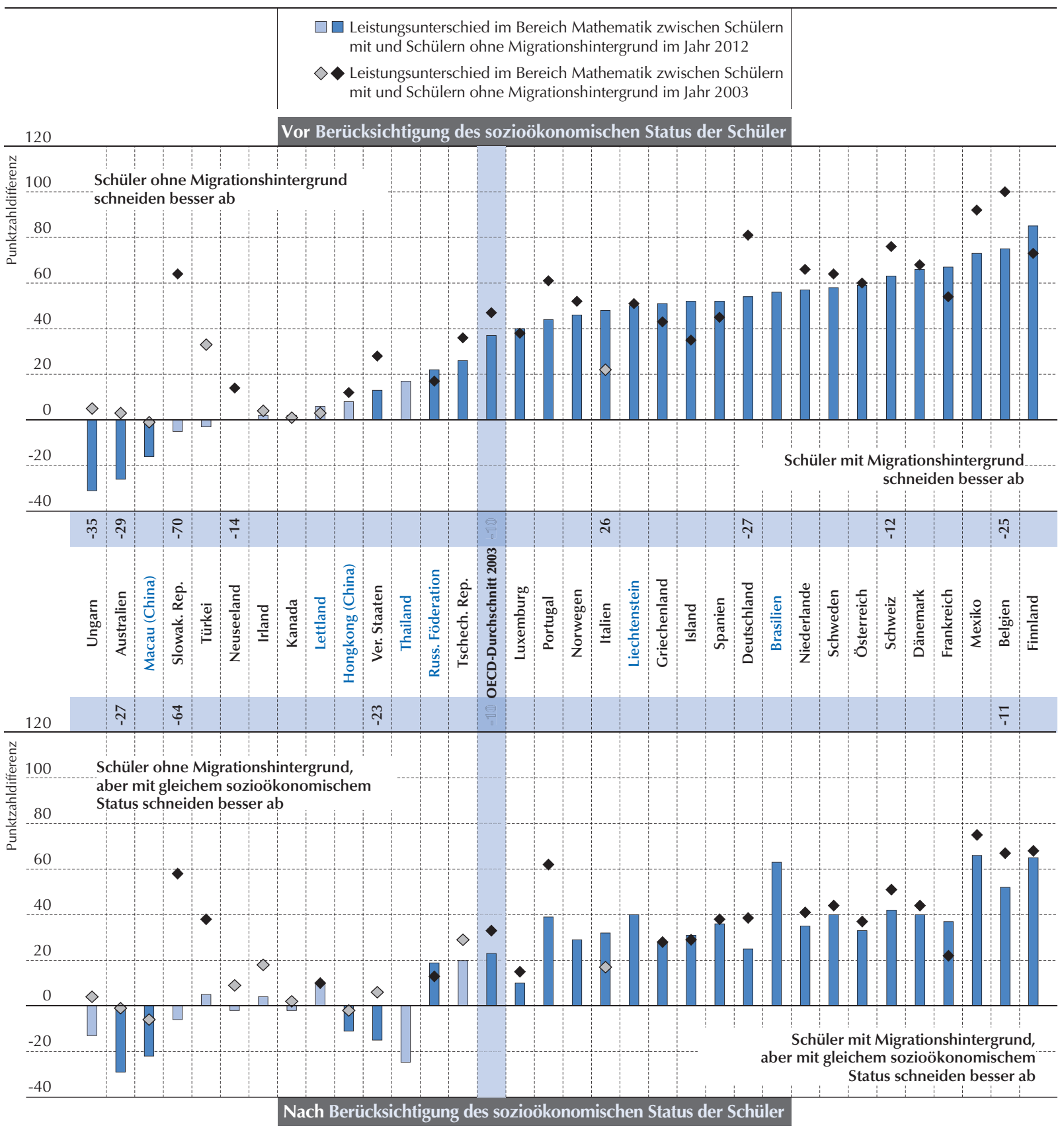

Anmerkung: Statistisch signifikante Leistungsunterschiede im Bereich Mathematik zwischen Schülern mit und ohne Migrationshintergrund in den Jahren 2003 und 2012 sind in dunkleren Farbtönen gekennzeichnet.

Angegeben sind nur Länder und Volkswirtschaften mit vergleichbaren Daten aus PISA 2003 und PISA 2012.

Die Veränderung der Punktzahldifferenz im Bereich Mathematik zwischen Schülern mit und ohne Migrationshintergrund vor Berücksichtigung des sozioökonomischen Status zwischen 2003 und 2012 wird über dem Namen des Landes/der Volkswirtschaft angezeigt, und der Unterschied nach Berücksichtigung des sozioökonomischen Status wird unter dem Namen des Landes/der Volkswirtschaft angezeigt. Nur statistisch signifikante Unterschiede werden aufgeführt.

Der OECD-Durchschnitt für 2003 umfasst nur die Länder, deren Ergebnisse im Bereich Mathematik seit dem Jahr 2003 vergleichbar sind.

Um die Vergleichbarkeit im Zeitverlauf zu gewährleisten, wurden die Werte von PISA 2003 auf dem PISA-Index des wirtschaftlichen, sozialen und kulturellen Status entsprechend der Indexskala von PISA 2012 umskaliert. Die in dieser Abbildung dargestellten Ergebnisse aus PISA 2003 können daher von denen abweichen, die in Lernen für die Welt von morgen: Erste Ergebnisse von PISA 2003 (OECD, 2004) wiedergegeben sind (vgl. Anhang A5 wegen näherer Einzelheiten).

Die Länder und Volkswirtschaften sind in aufsteigender Reihenfolge nach der Punktzahldifferenz zwischen den Schülern mit und ohne Migrationshintergrund vor Berücksichtigung des sozioökonomischen Status im Jahr 2012 angeordnet.

Quelle: OECD, PISA-2012-Datenbank, Tabelle II.3.4b

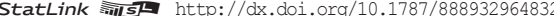


auch unterschiedliche Stärken und Talente besitzen, und die Schulsysteme müssen sich sowohl dieser Bedürfnisse als auch dieser Stärken bewusst sein, um den Erfolg der zugewanderten Schüler zu ermöglichen. Der Anteil der Schülerinnen und Schüler mit Migrationshintergrund ist zwischen 2003 und 2012 in Kanada, Irland, Italien, Spanien und den Vereinigten Staaten um mindestens 5 Prozentpunkte gestiegen, während in Luxemburg und Liechtenstein ein Anstieg von mindestens 10 Prozentpunkten zu verzeichnen war. 2003 hatten in den Schulsystemen von Irland, Italien und Spanien nur sehr wenige Schülerinnen und Schüler einen Migrationshintergrund, bis 2012 hatte sich der Anteil der Schüler mit Migrationshintergrund jedoch fast verdreifacht (Abb. II.3.6). In Irland besteht nur ein sehr geringer Zusammenhang zwischen dem Anstieg des Anteils der zugewanderten Schüler und Veränderungen bei den schulischen Problemen dieser Gruppe (Abb. II.3.5).

- Abbildung II.3.6 "

Veränderung des Anteils der Schüler mit Migrationshintergrund zwischen 2003 und 2012 Prozentsatz der Schüler mit Migrationshintergrund

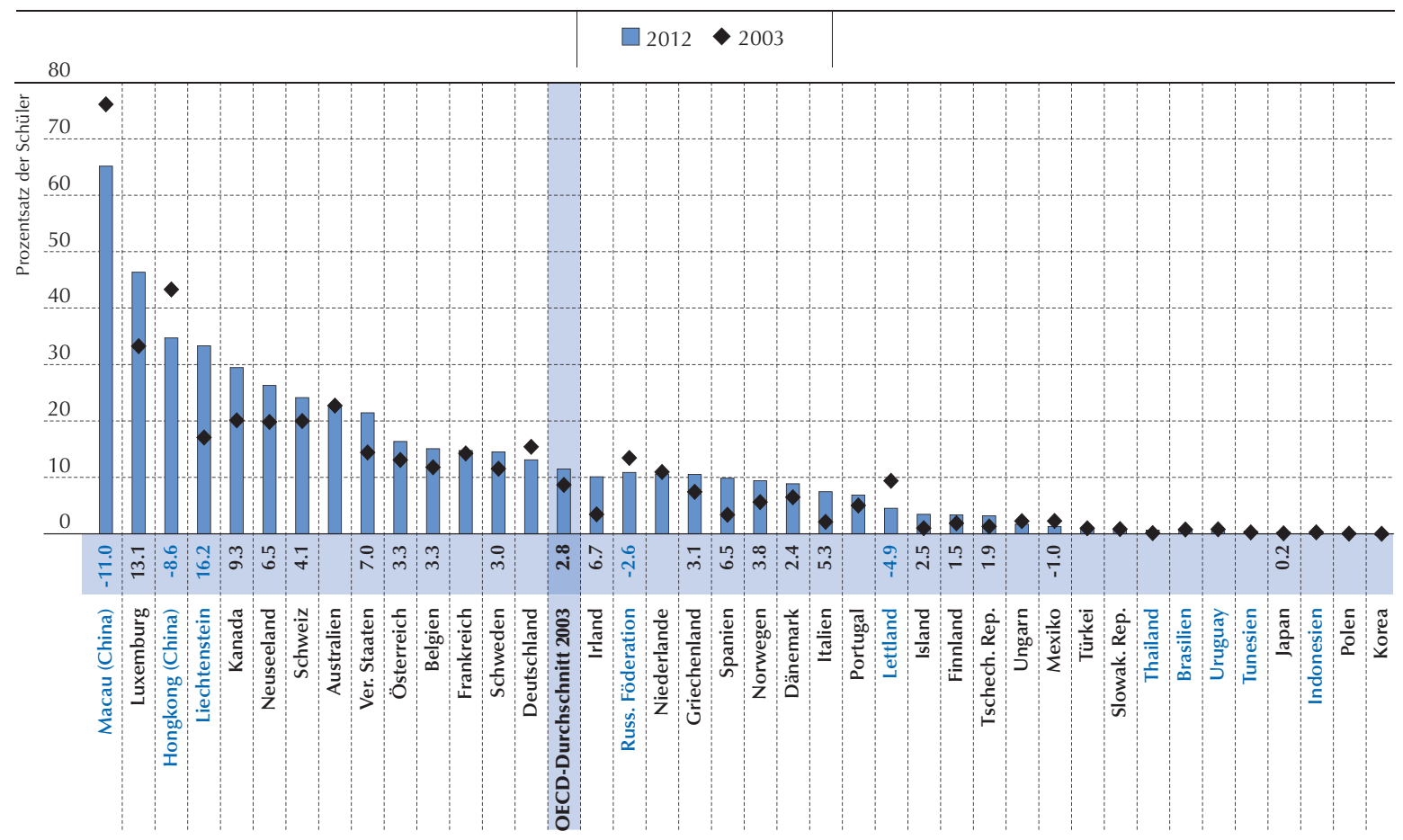

Anmerkung: Angegeben sind nur Länder und Volkswirtschaften mit vergleichbaren Daten aus PISA 2003 und PISA 2012

Der Prozentpunktunterschied beim Anteil der Schüler mit Migrationshintergrund zwischen 2003 und 2012 wird über dem Namen des Landes/der Volkswirtschaft angezeigt. Nur statistisch signifikante Unterschiede werden aufgeführt.

Der OECD-Durchschnitt für 2003 umfasst nur die Länder, deren Ergebnisse seit PISA 2003 vergleichbar sind.

Die Länder und Volkswirtschaften sind in absteigender Reihenfolge nach dem Prozentsatz der Schüler mit Migrationshintergrund im Jahr 2012 angeordnet. Quelle: OECD, PISA-2012-Datenbank, Tabelle II.3.4b.

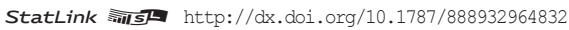

\section{Kasten II.3.2 Verbesserung der PISA-Ergebnisse: Deutschland}

In PISA 2000 lagen die Ergebnisse der deutschen Schülerinnen und Schüler nahe am OECD-Durchschnitt, sie zeigten jedoch, dass mehr als jeder fünfte Schüler im Bereich Lesekompetenz unter Kompetenzstufe 2 lag und dass Deutschland im Hinblick auf die soziale Gerechtigkeit in der Bildung zu den OECD-Ländern mit den schlechtesten Ergebnissen gehörte (OECD, 2010a). Da Deutschland ein Bundesstaat ist, in dem die einzelnen Länder Bildungshoheit genießen, konnte eine breit gefasste Bildungsreform nur durch konzertierte Anstrengungen der Bundesländer durchgeführt werden. Der "PISA-Schock", der auf die Veröffentlichung der Ergebnisse von PISA 2000 folgte, führte zu einem Ruck, der Reformmaßnahmen zur Förderung einer besseren Qualität und einer größeren Chancengerechtigkeit im Schulsystem einleitete.

EXZELLENZ DURCH CHANCENGERECHTIGKEIT: ALLEN SCHÜLERINNEN UND SCHÜLERN DIE VORAUSSETZUNGEN ZUM ERFOLG SICHERN - BAND II 
Deutschland hat seine Leistungen in den Bereichen Lesekompetenz und Mathematik in den letzten zehn Jahren kontinuierlich verbessert und gleichzeitig die Bildungsgerechtigkeit insgesamt erhöht. Die Durchschnittsergebnisse haben sich im Bereich Mathematik pro Jahr um durchschnittlich 1,4 Punkte verbessert, von 503 Punkten im Jahr 2003 auf 514 Punkte im Jahr 2012. Damit hat Deutschland die Mathematikleistungen von 2003 bis 2012 von einem durchschnittlichen Niveau zu einem erheblich über dem OECD-Durchschnitt liegenden Niveau gesteigert. Die Schülerleistungen im Bereich Lesekompetenz verbesserten sich um 1,8 Punkte pro Jahr, von 484 Punkten im Jahr 2000 auf 508 Punkte im Jahr 2012. Die Verbesserung war in beiden Fächern hauptsächlich eine Folge von Leistungssteigerungen unter leistungsschwachen und benachteiligten Schülerinnen und Schülern. In PISA 2012 erzielten die besonders leistungsschwachen Schüler (die leistungsschwächsten 10\%) im Bereich Mathematik über 20 Punkte mehr als die entsprechende Gruppe in PISA 2003. Ähnliche Verbesserungen waren 2012 im Bereich Lesekompetenz zu verzeichnen, wo die besonders leistungsschwachen Schüler fast 50 Punkte besser abschnitten als die entsprechende Gruppe in PISA 2000.

Da die leistungsschwachen Schülerinnen und Schüler mit größerer Wahrscheinlichkeit aus benachteiligten Verhältnissen kommen, spiegelt die im Bereich Mathematik verzeichnete und auf leistungsschwache Schüler zurückzuführende Verbesserung auch eine größere Chancengerechtigkeit im Bildungssystem wider. Während die benachteiligten Schülerinnen und Schüler (Schüler im untersten Quartil des PISA-Index des wirtschaftlichen, sozialen und kulturellen Status) ihre Mathematikleistungen um 20 Punkte verbesserten, war bei den Schülern im zweiten, dritten und obersten Quartil der Skala des sozioökonomischen Hintergrunds keine solcheVerbesserung festzustellen. Der sozioökonomische Status ist deshalb in PISA 2012 ein schwächerer Prädiktor für die Mathematikleistungen als in PISA 2003. Im Jahr 2003 waren 24\% der Varianz der Mathematikleistungen auf den sozioökonomischen Status der Schüler zurückzuführen, im Jahr 2012 waren es nur noch 17\%. In diesem Zeitraum entwickelte sich Deutschland von einem Land mit einer unter dem OECD-Durchschnitt liegenden Bildungsgerechtigkeit zu einem Land mit einem durchschnittlichen Niveau an Chancengerechtigkeit. Verbesserungen der Chancengerechtigkeit sind auch bei Schülerinnen und Schülern mit Migrationshintergrund zu beobachten: Während zugewanderte Schüler 2003 noch durchschnittlich 81 Punkte weniger erzielten als Schüler ohne Migrationshintergrund, war dieser Rückstand 2012 auf 53 Punkte gesunken.

\section{Von den PISA-Ergebnissen herbeigeführte Reformen}

Im Anschluss an die Ergebnisse von PISA 2000 definierte die Ständige Konferenz der Kultusminister der Länder (KMK) sieben Aktionsbereiche zur Verbesserung der Qualität des Bildungssystems. Obwohl diese und andere noch folgende bildungsbezogene Empfehlungen der KMK nicht verbindlich waren, wurden die meisten Vorschläge in den meisten Bundesländern umgesetzt. Um insbesondere die Leistung der sozial benachteiligten Kinder und der Kinder mit Migrationshintergrund zu fördern und die Bildungschancen aller Schüler durch innerschulische und außerschulische Aktivitäten zu verbessern, begann die Bundesregierung, die Länder beim Ausbau der Ganztagsschulen zu unterstützen. Während 2002 nur jede zehnte Schule eine Ganztagsschule war, war ihr Anteil 2012 auf über 50\% gestiegen. Die Teilnahme am Ganztagsangebot ist bisher zwar nur in einigen Schulen verbindlich, die Schulen bleiben jedoch den ganzen Tag geöffnet, stellen ein Mittagessen bereit und bieten Freizeitangebote sowie den Unterricht ergänzende Angebote und Nachmittagsunterricht für die Schüler, die dies benötigen oder wünschen. Jeder dritte Schüler besucht eine Ganztagsschule (KMK, 2013).

Eine weitere wichtige Empfehlung der KMK war die Entwicklung verbindlicher Standards und ergebnisorientierter Evaluierungen. Im Anschluss an die Empfehlung der KMK wurden 2003 und 2004 in den Jahrgangsstufen 4, 9 und 10 Bildungsstandards für die Fächer Deutsch und Mathematik eingeführt. In den Jahrgangsstufen 9 und 10 wurden darüber hinaus Standards für den Fremdsprachenunterricht (Englisch, Französisch) sowie für die Fächer Biologie, Chemie und Physik eingeführt. Seit 2004 basieren die Lehrpläne aller Bundesländer auf diesen Standards. Ferner wurden Prüfungen eingeführt, um sowohl auf Länder-als auch auf Bundesebene zu messen, inwieweit diese Standards erfüllt werden. Sie werden in allen 16 Bundesländern durchgeführt und überprüfen die Leistung einzelner Schulen und des Schulsystems insgesamt. Die Evaluierung der Jahrgangsstufen 3 und 8 erfolgt in jeder Schule. Auf Grund dieser Änderungen war die Wahrscheinlichkeit, eine Schule zu besuchen, die an einem Schulleistungsvergleich auf der Basis nationaler oder regionaler Benchmarks teilnimmt, bei den Schülern, die an PISA 2012 teilnahmen, um 22 Prozentpunkte höher, und die Wahrscheinlichkeit, eine Schule zu besuchen, in der Beurteilungen verwendet 
werden, um die Fortschritte der Schule im Jahresvergleich zu überprüfen, war 13 Prozentpunkte höher. Außerdem ist die Wahrscheinlichkeit höher, eine Schule zu besuchen, in der Schülerbeurteilungen verwendet werden, um zu evaluieren, ob die Schüler/Klassen das erwartete Leistungsniveau erreicht haben und Aspekte im Lehrplan zu identifizieren, die verbessert werden könnten. Die nationalen Ergebnisse dieser Überprüfungen werden in Bildung in Deutschland, einer alle zwei Jahre erscheinenden Bestandsaufnahme des Bildungswesens auf allen Ebenen, erfasst.

2001 signalisierte die KMK die Notwendigkeit, die Lehrkräfte weiter zu qualifizieren, insbesondere im Hinblick auf die Kompetenzen in den Bereichen schulische Diagnostik und Unterrichtsmethoden. Die Veränderungen in diesen Bereichen finden ihren Niederschlag in den Angaben der Schülerinnen und Schüler und der Schulleitungen im Rahmen von PISA. Die Wahrscheinlichkeit, eine Schule zu besuchen, in der der Unterricht der Lehrkräfte durch Kollegenbeurteilungen überprüft wird, war 2012 um 20 Prozentpunkte höher als 2003, und die Wahrscheinlichkeit, eine Schule zu besuchen, in der der Unterricht der Lehrkräfte durch Prüfungsleistungen der Schüler überprüft wird, war um 10 Prozentpunkte höher. Die Reformen der letzten zehn Jahre zielten darauf, die pädagogische Ausbildung von neuen Lehrkräften zu verbessern und den Schwerpunkt verstärkt auf die pädagogischen und psychologischen Kompetenzen zu legen. Die Lehrerfortbildung wird ausgeweitet, um sicherzustellen, dass in naher Zukunft alle Lehrkräfte daran teilnehmen.

In den meisten Bundesländern ist das Schulsystem gegliedert in eine vierjährige Grundschule und ein darauf folgendes dreigliedriges Schulsystem (Hauptschule, Realschule und Gymnasium), wobei es für jeden Bildungsgang bestimmte Übergänge gibt. Während die Hauptschule zu einem Sekundarschulabschluss führt, der zur Aufnahme einer Berufsausbildung berechtigt, bietet die Realschule den Übergang zu einem berufsbildenden und einem allgemeinbildenden Zweig für Schüler, die einen berufsorientierten bzw. technischen Bildungsgang oder ein Hochschulstudium anstreben. Da sich die Anforderungen auf dem Arbeitsmarkt geändert haben und die Nachfrage nach Fachkräften gestiegen ist und da der demografische Wandel in den Einzugsgebieten von ländlichen Schulen zu einem Bevölkerungsrückgang geführt hat, haben viele Bundesländer begonnen, die beiden beruflichen Bildungsgänge zu verschmelzen.

Parallel zu diesen Reformanstrengungen hat sich das Profil der deutschen Schülerpopulation durch den sozialen und demografischen Wandel verändert. In PISA 2012 erzielten die sozioökonomisch benachteiligten Schülerinnen und Schüler mit Migrationshintergrund auf dem PISA-Index des wirtschaftlichen, sozialen und kulturellen Status einen höheren Wert als 2003, und der Anteil der zugewanderten Schüler der ersten oder zweiten Generation ging insgesamt zurück. Darüber hinaus hatte ein deutscher Schüler im untersten Quartil der sozioökonomischen Verteilung 2003 auf dem ESCS-Index einen Wert von -1,34, während ein Schüler im untersten Quartil dieses Index 2012 einen Wert von -0,99 hatte (unter den Schülern im oberen Quartil der sozioökonomischen Verteilung war keine solche Veränderung festzustellen). In ähnlicher Weise verbesserte sich auch der sozioökonomische Status der Schüler mit Migrationshintergrund zwischen PISA 2003 und PISA 2012 (Tabelle II.2.3b und II.3.4b).

Diese Veränderungen bedeuten im Wesentlichen, dass sich die Unterschiede des sozioökonomischen Status zwischen benachteiligten und begünstigten Schülerinnen und Schülern und zwischen Schülern mit und ohne Migrationshintergrund zwischen PISA 2003 und PISA 2012 verringert haben. Diese Ergebnisse deuten darauf hin, dass die in Deutschland im Zeitverlauf bei PISA festzustellende Verbesserung nicht nur auf bestimmte Politikmaßnahmen oder Programme sondern auch auf Veränderungen im sozialen und demografischen Profil der Schülerinnen und Schüler zurückzuführen sein könnte (Tabelle I.2.4, I.4.4 und I.5.4).

\section{Quelle:}

KMK (Sekretariat der Ständigen Konferenz der Kultusminister der Länder in der Bundesrepublik Deutschland) (2013), Allgemein bildende Schulen in Ganztagsform in den Ländern in der Bundesrepublik Deutschland, Statistik 2007 bis 2011, Bonn.

OECD (2011), Lessons from PISA for the United States, Strong Performers and Successful Reformers in Education, OECD Publishing. http://dx.doi.org/10.1787/9789264096660-en

OECD (2010a), PISA 2009 Ergebnisse: Lernfortschritte im globalen Wettbewerb: Veränderungen bei den Schülerleistungen seit 2000 (Band V), PISA, OECD Publishing. http://dx.doi.org/10.1787/9789264091580-de 


\section{Sprachminderheiten unter den Schülerinnen und Schülern mit Migrationshintergrund}

Die größte Herausforderung besteht für viele Schüler mit zugewanderten Eltern darin, sich an eine neue Sprache und ein neues Lernumfeld zu gewöhnen. Die gefährdetsten Schüler mit Migrationshintergrund sind Schüler, die bei der Einreise bereits älter sind, die Sprache des Aufnahmelands nicht sprechen und aus einem Land kommen, in dem die Bildungsstandards nicht so hoch sind wie im Aufnahmeland. Diese Schülerinnen und Schüler würden von Politikmaßnahmen und Programmen profitieren, die diese vielfältigen Nachteile berücksichtigen. Nicht alle Schüler mit Migrationshintergrund stehen vor den gleichen Herausforderungen, einige sind möglicherweise in einem Aufnahmeland, das eine ähnliche Sprache und Kultur hat wie ihr Herkunftsland. Wenn diese spezifischen Probleme ignoriert werden, kann dies dazu führen, dass die zugewanderten Schülerinnen und Schüler in einem kritischen Alter mit schlechten Integrationsaussichten marginalisiert werden (OECD, 2012b).

Im OECD-Durchschnitt sind 6\% der 15-Jährigen zugewanderte Schüler, die zu Hause eine andere Sprache als dieTestsprache sprechen. In Luxemburg fällt etwa ein Drittel der Schüler in diese Kategorie, während dies in Kanada, Neuseeland, der Schweiz und den Vereinigten Staaten bei rd. 12\% der Fall ist. In Australien, Österreich, Belgien, Dänemark, Frankreich, Deutschland, Griechenland, Irland, Israel, Italien, den Niederlanden, Norwegen, Slowenien, Spanien, Schweden und dem Vereinigten Königreich sind mehr als 3\% der Schüler zugewanderte Schüler, die zu Hause eine andere Sprache als die Testsprache sprechen. In der Gruppe der Partnerländer und -volkswirtschaften fallen in Katar und den Vereinigten Arabischen Emiraten etwa 25\% der Schülerinnen und Schüler in diese Kategorie, während dies in Singapur bei 14\%, in

\section{Kasten II.3.3 Sprachminderheiten unter den Schülerinnen und Schülern ohne Migrationshintergrund}

Nur 4\% der 15-jährigen Schüler im OECD-Raum sind Schüler ohne Migrationshintergrund, die die Testsprache zu Hause nicht sprechen. Dieser Anteil variiert in den einzelnen Ländern jedoch erheblich: In Luxemburg beträgt ihr Anteil 53\%, in Belgien und Spanien 14\%, in Italien 10\%, in der Slowakischen Republik 7\%, in der Türkei $6 \%$ und in Estland, der Schweiz und Kanada zwischen 3\% und 4\%. Diese Schüler sind in allen Ländern, mit Ausnahme von Kanada, gegenüber anderen Schülern ohne Migrationshintergrund sozioökonomisch benachteiligt. Der Unterschied im sozioökonomischen Status ist in der Slowakischen Republik und der Türkei am größten, und er ist in der Schweiz sowie in Estland und Luxemburg relativ gering. Der Leistungsunterschied im Bereich Mathematik ist zwischen den Schülern ohne Migrationshintergrund, die die Testsprache zu Hause nicht sprechen, und denjenigen, die die Testsprache zu Hause sprechen, in der Slowakischen Republik besonders ausgeprägt, da die beiden Gruppen dort selbst nach Berücksichtigung des sozioökonomischen Status 50 Punkte auseinander liegen. In Italien und der Schweiz liegen die beiden Gruppen rd. 23 Punkte auseinander, und in Estland beträgt der Unterschied 14 Punkte. In Kanada und Luxemburg schneiden alle Schüler ohne Migrationshintergrund unabhängig von der Sprache, die sie zu Hause sprechen, gleich gut ab, während dies in Belgien und Spanien nach Berücksichtigung des sozioökonomischen Status der Fall ist (Tabelle II.3.8).

In 15 Partnerländern und -volkswirtschaften stellen die Schüler ohne Migrationshintergrund, die die Testsprache zu Hause nicht sprechen, einen erheblichen Anteil der Schülerpopulation, und die Unterschiede zwischen den einzelnen Ländern und Volkswirtschaften sind noch größer. Der Anteil dieser Schüler an der Schülerpopulation beträgt in Indonesien, Thailand, Malaysia und Singapur mehr als 40\%, in Chinesisch Taipeh, Katar und Bulgarien zwischen 10\% und 17\% und in Kasachstan, Litauen, Lettland, den Vereinigten Arabischen Emiraten, der Russischen Föderation, Macau (China), Peru, Jordanien und Serbien zwischen 3\% und 10\%. In Bulgarien, Peru, Singapur, Thailand, Indonesien, Chinesisch Taipeh, Lettland, der Russischen Föderation, Serbien, Litauen und Hongkong (China) sind diese Schüler sozioökonomisch benachteiligt gegenüber anderen Schülern ohne Migrationshintergrund. In den Vereinigten Arabischen Emiraten, Katar, Hongkong (China), Macau (China), Jordanien, Malaysia und Kasachstan sind sie gegenüber anderen Schülern ohne Migrationshintergrund begünstigt. Besonders ausgeprägt (15 oder mehr Punkte) sind die Leistungsunterschiede zu Gunsten dieser Schüler ohne Migrationshintergrund, die die Testsprache zu Hause nicht sprechen, in Katar, Malaysia und den Vereinigten Arabischen Emiraten. In Singapur, Chinesisch Taipeh, Litauen, Bulgarien, Peru und Liechtenstein schneiden die Schüler ohne Migrationshintergrund, die die Testsprache zu Hause sprechen, um mehr als 10 Punkte besser ab als die Schüler ohne Migrationshintergrund, die die Testsprache zu Hause nicht sprechen (Tabelle II.3.5). 
Liechtenstein bei 11\%, in Macau (China) bei 7\% und in Hongkong (China) bei 4\% der Fall ist. In einigen Ländern stellen Schüler ohne Migrationshintergrund, die die Testsprache zu Hause nicht sprechen, ebenfalls einen erheblichen Anteil der Gesamtbevölkerung. Kasten II.3.3 beschreibt die PISA-Ergebnisse für diese Schülergruppe (Tabelle II.3.5).

In Österreich, Belgien, Frankreich, Neuseeland, Schweden und der Schweiz betrug das zuschreibbare Risiko oder die Populationsrelevanz (eine Messgröße für den Anteil der Population, der von einem bestimmten Merkmal betroffen ist, das Schüler dem Risiko schwacher schulischer Leistungen aussetzt) der Schüler mit Migrationshintergrund, die die Testsprache zu Hause nicht sprechen, mindestens 6\%, während es in Luxemburg einen Wert von 29\% erreichte. In diesen Ländern ist das Risiko, im untersten Quartil der Leistungsverteilung zu liegen, bei Schülern mit Migrationshintergrund mehr als doppelt so hoch wie bei anderen Schülern. In der Gruppe dieser Länder liegt der Leistungsunterschied zwischen Schülern ohne Migrationshintergrund, die die Testsprache zu Hause sprechen, und Schülern ohne Migrationshintergrund, die die Testsprache nicht zu Hause sprechen, in Österreich, Belgien, Luxemburg, Neuseeland und Schweden nach Berücksichtigung des sozioökonomischen Status bei 30-53 Punkten, während der Unterschied zwischen diesen beiden Gruppen in der Schweiz 45 Punkte und in Belgien 53 Punkte beträgt (Tabelle II.3.5 und Abb. II.3.7).

Wer die Sprache des Aufenthaltslands bei der Ankunft nicht versteht, hat einen Nachteil; ebenso gravierend ist aber auch der unzureichende Kontakt mit dieser Sprache außerhalb der Schule. Politikmaßnahmen zur Unterstützung von Schülern mit Migrationshintergrund, die die Testsprache zu Hause nicht sprechen, sollten sowohl die Schule als auch das Elternhaus erfassen. Eine häufig diskutierte Möglichkeit besteht darin, den Eltern Sprachunterricht zu erteilen und sie zu ermutigen, sich für die Bildung ihres Kindes einzusetzen, falls dies nicht bereits der Fall ist. Dies kann den Schülern helfen, ihre Sprachkenntnisse zu verbessern, was ihre schulischen Leistungen fördert und die Integration in die Gesellschaft des Aufnahmelands erleichtert (wegen einer Liste der in den einzelnen OECD-Ländern in diesem Bereich umgesetzten Politikmaßnahmen vgl. den OECD-Bericht über die Bildung von Migranten [OECD, 2010b]).

\section{Schülerinnen und Schüler der ersten und zweiten Generation}

Der hier verwendete Begriff „Schülerinnen und Schüler mit Migrationshintergrund“ erfasst Schüler, deren Eltern im Ausland geboren sind, die aber selbst im Erhebungsland (zweite Generation) oder in einem anderen Land (erste Generation) geboren sind. Ein Leistungsvergleich zwischen den Schülern der ersten Generation und den Schülern der zweiten Generation kann Informationen über die Merkmale der verschiedenen Migrantenkohorten bieten, während ein Vergleich dieser beiden Gruppen mit Schülern ohne Migrationshintergrund Erkenntnisse zu der Frage liefern kann, inwieweit es den Schulsystemen gelingt, die zugewanderten Schülerinnen und Schüler in die Schulen zu integrieren und welche Rolle die Zuwanderungspolitik dabei spielt.

Im OECD-Durchschnitt beläuft sich der Anteil der Schüler der ersten Generation auf rd. 5\%, während der Anteil der Schüler der zweiten Generation 6\% beträgt. Beim sozioökonomischen Status gibt es im Allgemeinen keine erheblichen Unterschiede zwischen den beiden Gruppen. Die Schüler der zweiten Generation schneiden im PISA-Mathematiktest allerdings durchschnittlich um 10 Punkte besser ab als die Schüler der ersten Generation. Der Unterschied zwischen den Schülern ohne Migrationshintergrund und den Schülern der ersten Generation beträgt im OECD-Raum mehr als 45 Punkte, während der Leistungsunterschied zwischen den Schülern ohne Migrationshintergrund und den Schülern der zweiten Generation 31 Punkte beträgt. Bei Berücksichtigung des sozioökonomischen Status verringert sich der Abstand jedoch auf 29 bzw. 18 Punkte, weil die Schüler mit Migrationshintergrund generell gegenüber den Schülern ohne Migrationshintergrund benachteiligt sind. Wenn das Risiko schwacher schulischer Leistungen im OECD-Durchschnitt bei den Schülern der ersten und der zweiten Generation so niedrig wäre wie bei den Schülern ohne Migrationshintergrund, würde der Anteil der leistungsschwachen Schüler um etwa 4\% zurückgehen (Tabelle II.3.6a).

In Australien, Kanada und Irland liegen die Mathematikleistungen der Schülerinnen und Schüler der ersten Generation und der zweiten Generation und der Schülerinnen und Schüler ohne Migrationshintergrund im Durchschnitt nahe bei oder über dem OECD-Durchschnitt. Mit Ausnahme der Schüler der zweiten Generation in Irland entfällt in diesen Ländern auf alle drei Gruppen ein Anteil von wenigstens 8\% der gesamten Schülerpopulation. In Neuseeland erzielen die Schülerinnen und Schüler der ersten Generation Ergebnisse, die nahe bei oder über dem Durchschnitt liegen, bei den Schülern der zweiten Generation ist dies jedoch nicht der Fall. In Ungarn erzielen die Schülerinnen und Schüler der ersten Generation Ergebnisse, die über dem OECD-Durchschnitt liegen, sie machen jedoch nur 1\% der gesamten Schülerpopulation aus. Wie Abbildung II.3.8 zeigt, führen diese Unterschiede dazu, dass der Anteil der Schüler der ersten Generation und der zweiten Generation, die im Bereich Mathematik Ergebnisse über Kompetenzstufe 3 erzielen, im Vergleich zu den Schülern ohne Migrationshintergrund höher oder niedriger ausfällt. 


\title{
- Abbildung II.3.7
}

\section{Leistungsunterschied im Bereich Mathematik, nach Migrationsstatus und sprachlichem Hintergrund}

\author{
Vor und nach Berücksichtigung des sozioökomischen Status
}

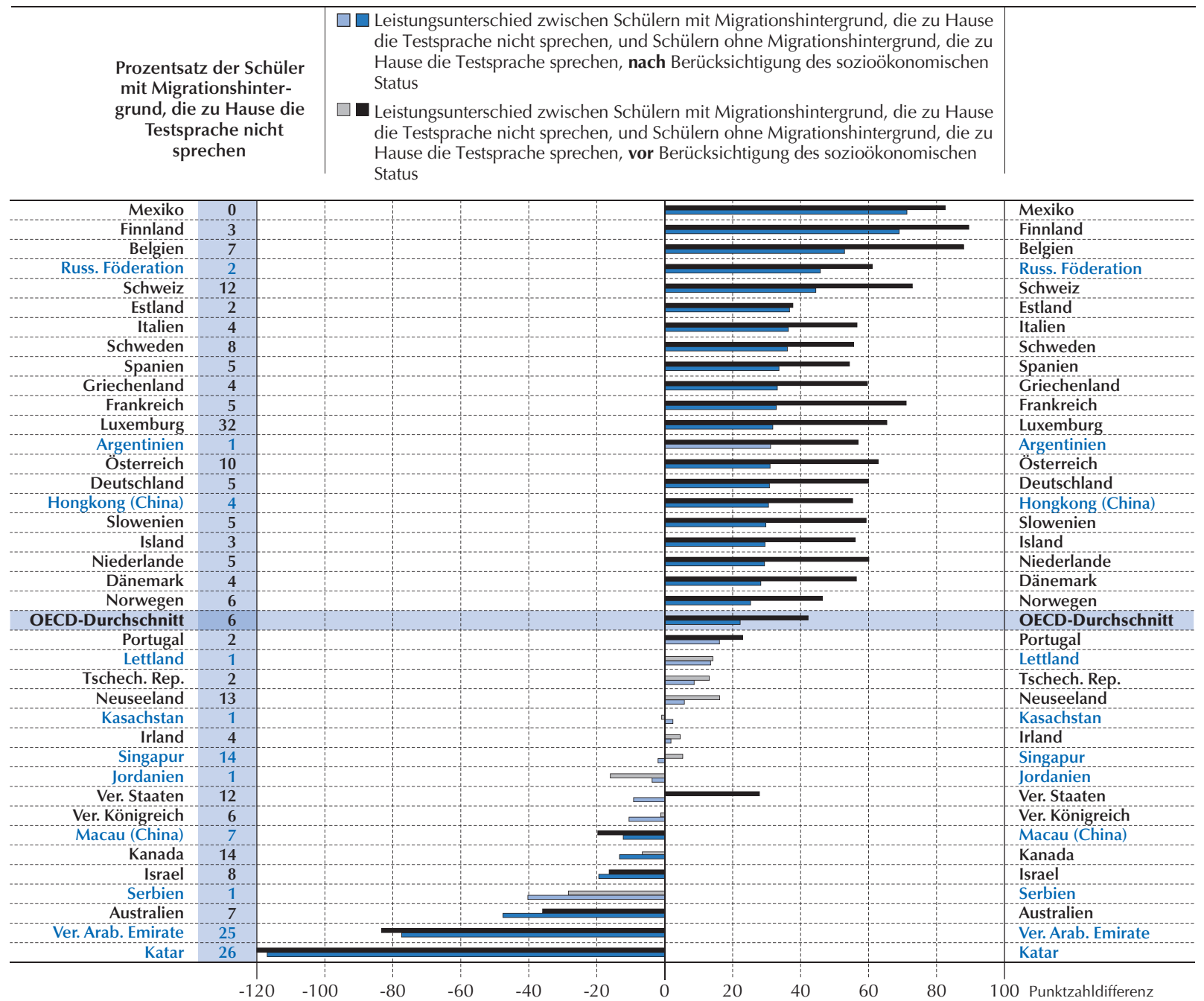

Anmerkung: Statistisch signifikante Punktzahldifferenzen sind in dunkleren Farbtönen gekennzeichnet

Die Länder und Volkswirtschaften sind in absteigender Reihenfolge nach der Punktzahldifferenz zwischen Schülern mit Migrationshintergrund, die die Testsprache zu Hause nicht sprechen, und Schülern ohne Migrationshintergrund, die die Testsprache zu Hause sprechen, angeordnet.

Quelle: OECD, PISA-2012-Datenbank, Tabelle II.3.5.

StatLink *iताs http://dx.doi.org/10.1787/888932964832

\section{„Nachteil des späten Zuwanderungsalters"}

Je älter ein zugewanderter Schüler bei der Einreise in das Aufnahmeland ist, desto schlechter ist generell das Ergebnis im PISA-Mathematiktest (Tabelle II.3.8). Dieser „Nachteil des späten Zuwanderungsalters“ scheint mit einer mangelhaften Beherrschung der Testsprache zusammenzuhängen (OECD, 2012b). Schwierigkeiten, sich an eine andere Kultur oder ein anderes Schulsystem oder an unterschiedliche nationale Bildungsstandards anzupassen, können bei Schülerinnen und Schülern mit Migrationshintergrund ebenfalls zu schwächeren Leistungen beitragen. Schüler der ersten Generation, die im Schulalter der Sekundarstufe I aus einem weniger entwickelten Land eingereist sind und zu Hause die Testsprache des Aufnahmelands nicht sprachen, stellen eine besonders gefährdete Gruppe dar. Diese Schülerinnen und Schüler müssen sowohl rasch die Testsprache lernen, als auch versuchen, das Bildungsniveau ihrer Mitschüler im Aufnahmeland zu erreichen, während sie zugleich mit der Schwierigkeit zurechtkommen müssen, sich in einer neuen Schule und in einem neuen sozialen Umfeld einzuleben. 
- Abbildung II.3.8 "

Prozentsatz der Schüler mit Mathematikleistungen unter bzw. über dem Grundkompetenzniveau (Stufe 2), nach Migrationshintergrund

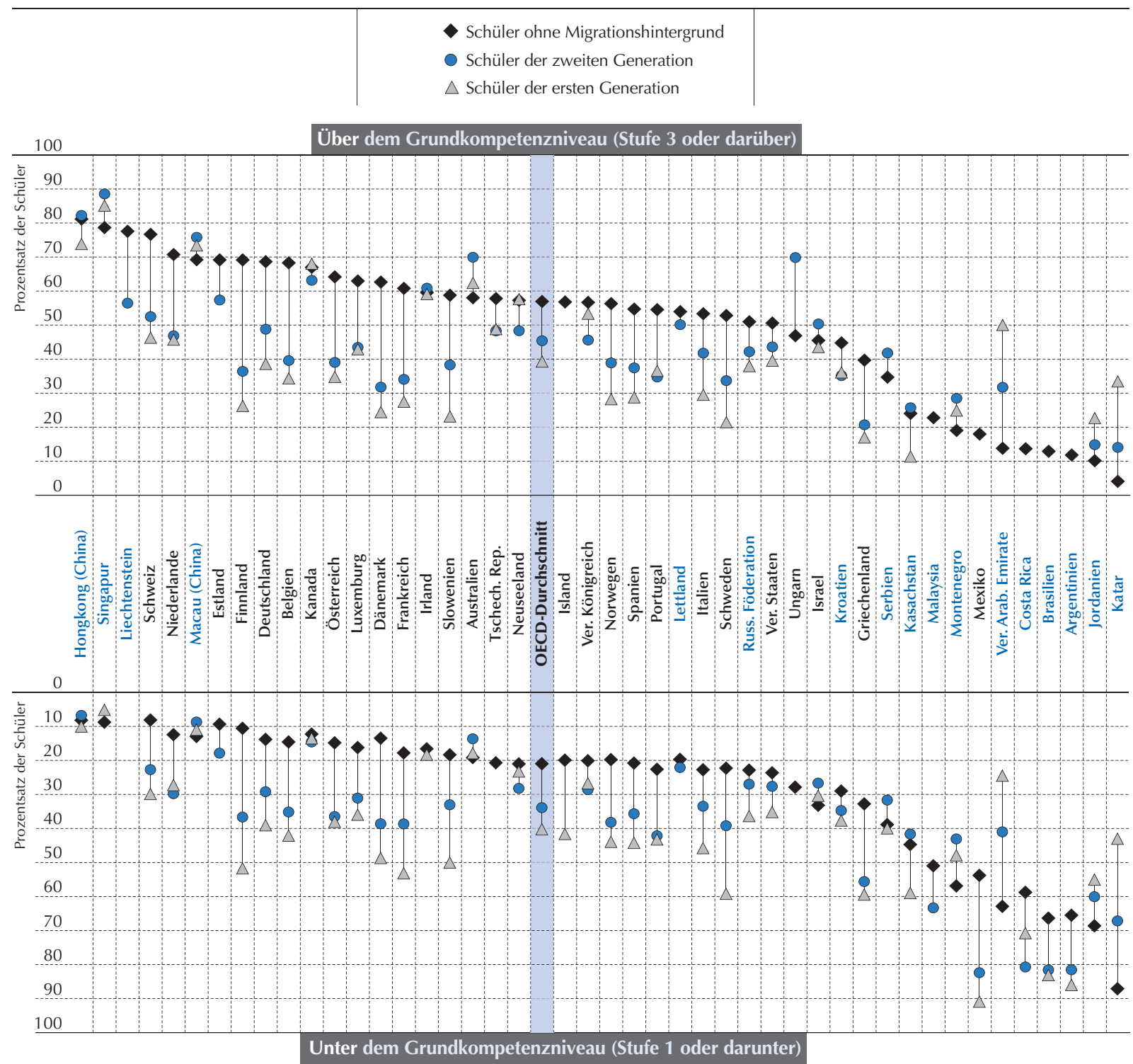

Anmerkung: Diese Abbildung zeigt nur Länder/Volkswirtschaften, bei denen Daten für wenigstens eine Kategorie von Schülern mit Migrationshintergrund vorliegen (erste oder zweite Generation).

Die Länder und Volkswirtschaften sind in absteigender Reihenfolge nach dem Prozentsatz der Schüler ohne Migrationshintergrund, die mindestens Kompetenzstufe 3 erreicht haben, angeordnet.

Quelle: OECD, PISA-2012-Datenbank, Tabelle II.3.7.

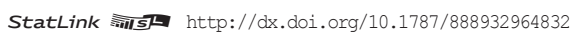

In einigen Fällen ist die späte Zuwanderung auf die Zuwanderungspolitik zurückzuführen. In den meisten Ländern wird die Familienzusammenführung nur erlaubt, wenn die Migranten über eine angemessene Wohnung und ein ausreichendes Einkommen verfügen. Diese Anforderungen werden zwar in guter Absicht gestellt, sie können jedoch dazu führen, dass die Kinder Jahre bis zur Einreise warten müssen, was ihnen die Integration in die Schule und die Gesellschaft des Aufnahmelands erschwert (Heath und Kilpi-Jakonen, 2012). Sprachunterricht für ältere Schüler mit Migrationshintergrund ist von entscheidender Bedeutung. 


\section{DIE KONZENTRATION DER BENACHTEILIGUNG}

Leistungsschwäche bei Schülerinnen und Schülern mit Migrationshintergrund kann teilweise mit der Tatsache zusammenhängen, dass diese Schüler tendenziell in benachteiligten Schulen konzentriert sind (OECD, 2012b). Migranten ziehen nach der Einreise in ein anderes Land tendenziell in ein Stadtviertel, wo bereits andere Migranten wohnen, die oft aus dem gleichen Land kommen und den gleichen sozioökonomischen Status haben. Dadurch bauen sie ein Netzwerk von Verwandten und Freunden auf, die ihre Kultur teilen und den neu zugezogenen Migranten bei Behördengängen und Arbeitsuche helfen können. Darüber hinaus kann die frühzeitige Selektion und Aufteilung der Schüler auf verschiedene Bildungsgänge dazu führen, dass Schülerinnen und Schüler mit ähnlicher Herkunft unabhängig von dem Stadtteil, in dem sie leben, in die gleiche Schule eingestuft werden. Studien über dieses Phänomen zeigen, dass die Konzentration von Schülern mit Migrationshintergrund an sich nicht zwangsläufig negative Auswirkungen hat, falls soziale und öffentliche Dienstleistungen angeboten werden, die eine vergleichbare Qualität aufweisen wie andernorts und unter der Voraussetzung, dass die räumliche Konzentration ethnischer Gruppen nicht zu dauerhaften ethnischen Enklaven führt, die wenig Möglichkeiten für Mobilität nach außen - und nach oben - bieten (Damm und Rosholm, 2010; Edin, Fredriksson und Aslund, 2004).

Dieser Abschnitt untersucht, inwieweit die Konzentration von Schülern mit Migrationshintergrund oder aus sprachlichen Minderheiten mit schlechteren Bildungserträgen zusammenhängt. Die Konzentration von Schülern mit Migrationshintergrund oder von Schülern, die die Testsprache zu Hause nicht sprechen, wird gemessen als der Anteil der Schüler, die in den einzelnen Schulen diese Merkmale aufweisen ${ }^{5}$.

Die Ergebnisse von PISA 2012 deuten darauf hin, dass die Schülerinnen und Schüler, die eine Schule mit einem hohen Anteil von zugewanderten Schülern besuchen, nach Berücksichtigung der sozioökonomischen Profile der Schüler und der Schule genauso gut abschneiden wie die Schüler, die eine Schule besuchen, in der der Anteil von Schülern mit Migrationshintergrund gering ist. Im OECD-Raum erzielen Schüler, die eine Schule mit einer hohen Konzentration von Migranten besuchen (d.h. wo mehr als ein Viertel der Schüler Migranten sind), tendenziell schlechtere Ergebnisse als Schüler in Schulen, in denen es keine Schüler mit Migrationshintergrund gibt. Der beobachtete Unterschied zwischen diesen beiden Gruppen beträgt 18 Punkte, nach Berücksichtigung des sozioökonomischen Status der Schüler und Schulen wird der Unterschied aber mehr als halbiert und beträgt nur noch fünf Punkte. Griechenland und Belgien sind in der Tat die einzigen Länder mit einem großen Anteil zugewanderter Schüler (mehr als 10\%), die nach Berücksichtigung des sozioökonomischen Status einen großen Leistungsunterschied aufweisen (40 Punkte bzw. 30 Punkte) (Tabelle II.3.9).

In Estland, Portugal und Ungarn gibt es nach Berücksichtigung des sozioökonomischen Status ebenfalls große Unterschiede, die Migrantenbevölkerung ist jedoch kleiner. In den Niederlanden, Deutschland und Irland sind vor Berücksichtigung des sozioökonomischen Status große Leistungsunterschiede zwischen diesen beiden Schultypen festzustellen, die meisten Unterschiede hängen jedoch eng mit sozioökonomischen Disparitäten zusammen, da sie nach Berücksichtigung des sozioökonomischen Status nicht mehr zu beobachten sind. Ein ähnliches Muster ist in Slowenien, Italien, Argentinien und Finnland festzustellen, in diesen Ländern/Volkswirtschaften ist die Migrantenbevölkerung jedoch kleiner (weniger als 10\%). In 14 von 35 Ländern, für die vergleichbare Daten vorliegen, erzielen Schüler in Schulen mit einer hohen Konzentration von Schülern mit Migrationshintergrund vor Berücksichtigung der sozioökonomischen Ungleichheiten schlechtere Ergebnisse. Nach Berücksichtigung des sozioökonomischen Status sinkt die Zahl dieser Länder/Volkswirtschaften auf sieben, und in den meisten Fällen wird der Leistungsunterschied so stark reduziert oder sogar halbiert, dass er praktisch nicht mehr signifikant ist (Tabelle II.3.9).

Ein ähnliches Muster ist zu beobachten, wenn die Konzentration von Schülern untersucht wird, die die Testsprache zu Hause nicht sprechen, wenngleich die Leistungsunterschiede größer sind (Tabelle II.3.10). Im OECD-Raum beträgt der Leistungsunterschied der Schülerinnen und Schüler im Bereich Mathematik zwischen den Schülern in Schulen mit einer hohen Konzentration an Schülern, die die Testsprache zu Hause nicht sprechen, und den Schülern in Schulen, in denen alle Schüler die Testsprache zu Hause sprechen, vor Berücksichtigung des sozioökonomischen Profils der Schüler und Schulen fast 30 Punkte, dieser Unterschied verschwindet jedoch nach Berücksichtigung des sozioökonomischen Status. In 16 der 42 Länder, für die Daten vorliegen, sind in diesen Schulen zwar große Unterschiede in den Mathematikleistungen festzustellen, nach Berücksichtigung des sozioökonomischen Status sind diese Unterschiede mit Ausnahme von sechs Ländern aber nicht mehr zu beobachten. Vor Berücksichtigung des sozioökonomischen Status sind die größten Unterschiede in den Niederlanden, Bulgarien, Italien, der Slowakischen Republik, Slowenien, Thailand, der Türkei, Mexiko und Litauen festzustellen. Nach Berücksichtigung des sozioökonomischen Status sind die größten Unterschiede in den Mathematikleistungen in Hongkong (China), der Schweiz, Indonesien, Griechenland und Peru zu beobachten. 


\section{- Abbildung II.3.9 "}

\section{Anteil der Schüler mit Migrationshintergrund in sozioökonomisch benachteiligten, durchschnittlichen und begünstigten Schulen ${ }^{1}$}

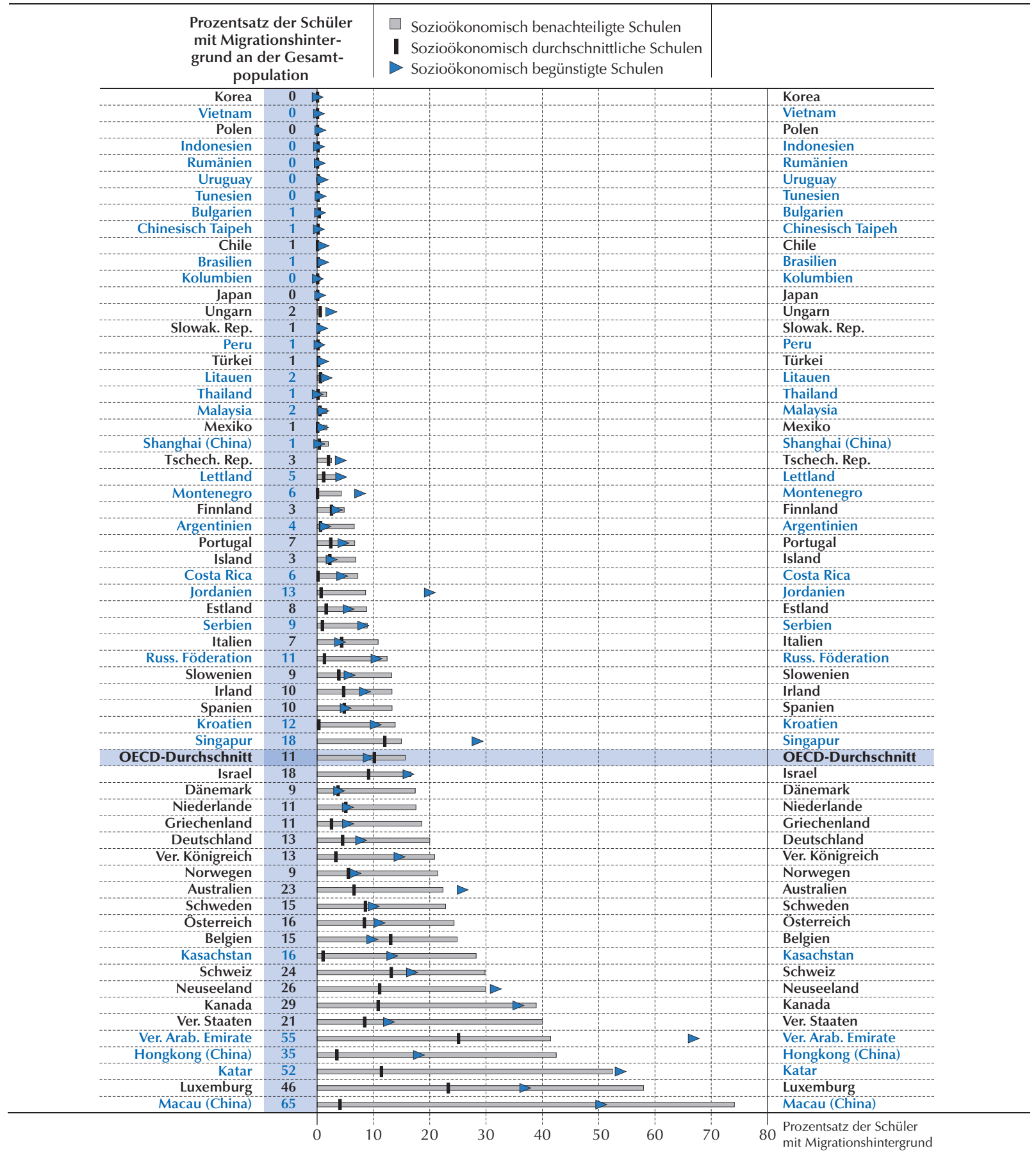

Zur Interpretation dieser Abbildung: In den Ländern und Volkswirtschaften machen Schüler mit Migrationshintergrund durchschnittlich $11 \%$ der gesamten Schülerpopulation aus, unter den Schülern, die eine sozioökonomisch benachteiligte Schule besuchen, beträgt ihr Anteil jedoch $16 \%$, während er sich bei den Schülern, die eine durchschnittliche Schule besuchen, auf 5\% beläuft und bei den Schülern, die eine sozioökonomisch begünstigte Schule besuchen, $9 \%$ beträgt. 1. Eine sozioökonomisch benachteiligte Schule ist eine Schule mit Schülern, deren durchschnittlicher sozioökonomischer Status statistisch signifikant unter dem durchschnittlichen sozioökonomischen Status des Landes/der Volkswirtschaft liegt, eine sozioökonomisch durchschnittliche Schule ist eine Schule, an der es keinen Unterschied zwischen dem durchschnittlichen sozioökonomischen Status der Schüler und des Landes/der Volkswirtschaft gibt, und eine sozioökonomisch begünstigte Schule ist eine Schule mit Schülern, deren durchschnittlicher sozioökonomischer Status statistisch signifikant über dem durchschnittlichen sozioökonomischen Status des Landes/der Volkswirtschaft liegt.

Die Länder und Volkswirtschaften sind in aufsteigender Reihenfolge nach dem Anteil der Schüler mit Migrationshintergrund in sozioökonomisch benachteiligten Schulen angeordnet.

Quelle: OECD, PISA-2012-Datenbank, Tabelle II.3.4a und II.4.2.

StatLink त्ताज http://dx.doi.org/10.1787/888932964832 
Schülerinnen und Schüler mit Migrationshintergrund und Schüler, die die Testsprache zu Hause nicht sprechen, sind tendenziell in benachteiligten Schulen konzentriert (Abb.II.3.9). So beträgt beispielsweise in den Vereinigten Staaten der Anteil der Schüler mit Migrationshintergrund in benachteiligten Schulen 40\%, während er sich in begünstigten Schulen auf 13\% der Schülerpopulation beläuft (Tabelle II.4.2). In Hongkong (China), Macau (China) und Luxemburg beträgt der Unterschied im Hinblick auf den Anteil der Schüler mit Migrationshintergrund, die eine begünstigte Schule besuchen, und den Anteil dieser Schüler, die eine benachteiligte Schule besuchen, mehr als 20 Prozentpunkte. Ein ähnliches Muster ist bei Schülern mit Migrationshintergrund zu beobachten, die zu Hause die Testsprache nicht sprechen.

\section{LEISTUNG, MIGRATIONSSTATUS UND HERKUNFTSLAND}

Mit der steigenden Zahl der Migranten erhöht sich auch die Vielfalt der sozialen Verhältnisse - und der Bildungserträge. Einige PISA-Teilnehmerländer sammeln Informationen über das Geburtsland der zugewanderten Schüler und ihrer Eltern, die Aufschluss darüber geben, wie der Leistungsstand dieser Schüler ist und inwieweit die Aufnahmeländer die Bedürfnisse der Schüler mit Migrationshintergrund erfüllen. Die Ergebnisse aus diesen Ländern zeigen, dass Schüler mit Migrationshintergrund aus dem gleichen Land und mit einem ähnlichen sozioökonomischen Status in den verschiedenen Schulsystemen sehr unterschiedliche Leistungen erzielen.

Die in den Partnerländern geborenen Schüler mit Migrationshintergrund weisen große Leistungsunterschiede auf. So erzielen die Schülerinnen und Schüler mit Migrationshintergrund aus der Russischen Föderation, die in Deutschland leben, nach Berücksichtigung des sozioökonomischen Status beispielsweise 75 Punkte mehr als diejenigen, die in Griechenland leben. Schüler türkischer Herkunft, die in Belgien leben, erzielen fast 55 Punkte mehr als türkische Schüler mit Migrationshintergrund und ähnlichem sozioökonomischem Status, die in Finnland leben. In den Aufnahmeländern mit vergleichbaren PISA-Daten erzielen Schüler mit Migrationshintergrund aus Vietnam im Bereich Mathematik durchschnittlich unabhängig von ihrem Zielland gute Ergebnisse. Diejenigen, die in Australien leben, erzielen ein Durchschnittsergebnis von 548 Punkten, während diejenigen, die in der Tschechischen Republik leben, 524 Punkte erzielen.

In der Gruppe der Schülerinnen und Schüler mit Migrationshintergrund aus den OECD-Ländern gibt es in den einzelnen Aufnahmeländern genauso große Leistungsunterschiede. So erzielen die Schüler mit Migrationshintergrund aus Frankreich, die in der Schweiz leben, nach Berücksichtigung des sozioökonomischen Status beispielsweise 60 Punkte mehr als die französischen Schüler mit Migrationshintergrund, die in Israel leben. Schüler portugiesischer Herkunft, die in der Schweiz leben, schneiden um 58 Punkte besser ab als Schüler aus dem gleichen Herkunftsland mit ähnlichem sozioökonomischem Status, die in Luxemburg leben. Die Schüler mit Migrationshintergrund aus Deutschland, die in Österreich leben, erzielen 37 Punkte mehr als diejenigen, die in der Schweiz leben (Tabelle II.3.11).

Die erheblichen Leistungsunterschiede zwischen Schülern mit ähnlichem sozioökonomischem Status aus dem gleichen Herkunftsland lassen darauf schließen, dass die Leistungen dieser Schüler durch die Schulen und die Bildungspolitik in den Aufnahmeländern beeinflusst werden. Die Zuwanderungspolitik, kulturelle Ähnlichkeiten zwischen den Migranten und dem Aufnahmeland und sozialpolitische Maßnahmen erklären zwar auch einige dieser Leistungsunterschiede, einige Bildungssysteme scheinen die Integration der zugewanderten Schülerinnen und Schüler jedoch besser zu fördern als andere.

Einige Schülergruppen mit Migrationshintergrund erzielen unabhängig von dem Land, in das sie eingewandert sind, hohe Leistungen. So gehören beispielsweise alle Schüler mit Migrationshintergrund aus China, die in Australien oder Neuseeland leben, zu den zehn leistungsstärksten zugewanderten Schülergruppen aller Aufnahmeländer, und das Gleiche gilt für zugewanderte Schüler aus Korea, die in Neuseeland leben, sowie für zugewanderte Schüler aus Indien und Vietnam, die in Australien leben. Schüler deutscher Herkunft, die in Österreich oder Luxemburg leben, sind die einzigen Schüler mit Migrationshintergrund, die zu diesen leistungsstärksten Gruppen gehören und nicht aus einem asiatischen Land kommen.

Die durchschnittliche Mathematikleistung all dieser Gruppen beträgt mindestens 548 Punkte (Tabelle II.3.11), das Äquivalent von über einem vollen Schuljahr mehr als der OECD-Durchschnitt. Diese Schülerinnen und Schüler zeigen, dass es möglich ist, in der Schule Erfolg zu haben, selbst wenn sie mit der Herausforderung konfrontiert sind, sich an ein neues Land, ein neues Schulsystem und, in vielen Fällen, eine neue Kultur und Sprache anzupassen.

Länder, in denen die Zuwanderung von Schülern aus unterschiedlichen sozialen Verhältnissen gerade erst anzusteigen beginnt, können aus den Erfahrungen der Systeme lernen, die bereits seit längerer Zeit mit dieser Herausforderung konfrontiert sind und diese Schüler erfolgreich in ihr Schulsystem integriert haben. Die Tatsache, dass Schülerinnen und Schüler mit Migrationshintergrund aus dem gleichen Herkunftsland und mit dem gleichen kulturellen Hintergrund und sozioökonomischen Status in ihren Aufnahmeländern so unterschiedliche Ergebnisse erzielen, zeigt, dass die Bildungsund Sozialpolitik nicht nur einen Einfluss auf die Leistungen dieser Schüler haben kann, sondern ihnen auch helfen kann, das Beste aus den in ihrem Aufnahmeland zur Verfügung stehenden Möglichkeiten zu machen. 


\section{Anmerkungen}

1. Es ist viel über den Zusammenhang zwischen der Familienstruktur und den Schülerleistungen geschrieben worden, und das Engagement der Eltern ist nur einer der Aspekte, der in diesen Studien behandelt wurde. Besonderes Augenmerk galt der wirtschaftlichen Situation der Familien und insbesondere der Belastung, der sie im Fall von Änderungen der Familienzusammensetzung sowie einer unsicheren Einkommenssituation ausgesetzt sind. Vgl. beispielsweise Buchmann und Hannum (2001) wegen einer Untersuchung dieses Zusammenhangs im Ländervergleich, McLanahan und Sandefur (1994) wegen einer Analyse der Auswirkungen auf die Schüler, Raley, Frisco und Wildsmith (2005) wegen einer Studie des Status und der Belastung von Alleinerziehendenhaushalten im Vergleich zu Paarhaushalten sowie Jeynes (2005) wegen einer Erörterung der Frage des Engagements der Eltern in Alleinerziehendenhaushalten. Eine klassische Studie der Unterschiede in der Sprachverwendung zwischen verschiedenen sozialen Schichten unter Berücksichtigung der Eltern-Kind-Interaktion sowie des Zusammenhangs zwischen Sprachniveau und Wohlstand findet sich in Brice Heath (1983). Vgl. auch Band IV dieses Berichts wegen einer Untersuchung der Unterschiede, die in Bezug auf die Art und den Grad des Engagements der Eltern in den Schulen in ausgewählten PISA-Ländern festzustellen sind.

2. Die Schüler gaben an, wer normalerweise zu Hause mit ihnen zusammenlebt: a) Mutter (einschließlich Stief- oder Pflegemutter), b) Vater (einschließlich Stief- oder Pflegevater), c) Bruder/Brüder (einschließlich Stiefbruder/-brüder), d) Schwester(n) (einschließlich Stiefschwestern), e) Großeltern, sonstige (z.B. Cousin oder Cousine). Schüler aus Alleinerziehendenhaushalten werden definiert als Schüler, die "Nein" und "Ja" auf a) und b) oder "Ja" und "Nein" auf a) und b) antworteten. Sie gaben also an, dass sie mit einem Elternteil, aber nicht mit dem anderen zusammenleben. Alle anderen Antworten werden als "sonstige“ eingeordnet, es sei denn, die Schüler beantworteten diese Frage überhaupt nicht.

3. Dies bedeutet, dass auch im Ausland geborene Schülerinnen und Schüler, die mindestens einen im Erhebungsland geborenen Elternteil haben, als Schüler ohne Migrationshintergrund eingestuft werden.

4. Fehlen Informationen über einen Elternteil, so wird unterstellt, dass dieser Elternteil denselben Migrationshintergrund hat wie der Elternteil, über den Informationen vorliegen. Fehlen Angaben zum Geburtsland des Schülers, wird die Variable als Fehlwert kodiert.

5. Es wurden Robustheitstests durchgeführt, indem Schulen mit wenigen Beobachtungen ausgeklammert wurden, die Ergebnisse haben sich aber nicht wesentlich geändert.

\section{Literaturverzeichnis}

Brice Heath, S. (1983), Ways with Words: Language, Life, and Work in Communities and Classrooms, Cambridge University Press, New York und Cambridge.

Buchmann, C. und E. Hannum (2001), "Stratification in Developing Countries: A Review of Theories and Research", Annual Review of Sociology, Vol. 27, S. 77-102.

Damm, A. und M. Rosholm (2010), "Employment Effects of Spatial Dispersal of Refugees," Review of Economics of the Household, Springer, Vol. 8(1), S. 105-146.

Edin, P., P. Fredriksson und O. Åslund (2004), "Settlement Policies and the Economic Success of Immigrants," Journal of Population Economics, Springer, Vol. 17(1), S. 133-155.

Heath, A. und E. Kilpi-Jakonen (2012), "Immigrant Children's Age at Arrival and Assessment Results", OECD Education Working Papers, No. 75, OECD Publishing, http://dx.doi.org/10.1787/5k993zsz6g7h-en

Jeynes, W.H. (2005), "A Meta-Analysis of the Relation of Parental Involvement to Urban Elementary School Student Academic Achievement", Urban Education, Vol. 40, No. 3, S. 237-269.

KMK (Sekretariat der Ständigen Konferenz der Kultusminister der Länder in der Bundesrepublik Deutschland) (2013), Allgemein bildende Schulen in Ganztagsform in den Ländern in der Bundesrepublik Deutschland, Statistik 2007 bis 2011, Bonn.

McLanahan, S. und G.D. Sandefur (1994), Growing Up with a Single Parent: What Hurts, What Helps, Harvard University Press, Cambridge, Massachusetts.

OECD (2012a), Let's Read Them a Story! The Parent Factor in Education, PISA, OECD Publishing, http://dx.doi. org/10.1787/9789264176232-en.

OECD (2012b), Untapped Skills: Realising the Potential of Immigrant Students, PISA, OECD Publishing, http://dx.doi. org/10.1787/9789264172470-en.

OECD (2011), Lessons from PISA for the United States, Strong Performers and Successful Reformers in Education, OECD Publishing. http://dx.doi.org/10.1787/9789264096660-en. 
OECD (2010a), PISA 2009 Ergebnisse: Lernfortschritte im globalen Wettbewerb: Veränderungen bei den Schülerleistungen seit 2000 (Band 5), PISA, OECD Publishing. http://dx.doi.org/10.1787/9789264091580-en.

OECD (2010b), Closing the Gap for Immigrant Students: Policies, Practice and Performance, OECD Reviews of Migrant Education, OECD Publishing, http://dx.doi.org/10.1787/9789264075788-en.

Pong, S., J. Dronkers und G. Hampden-Thompson (2004), "Family Policies and Children's School Achievement in Single- Versus Two-Parent Families", Journal of Marriage and Family, Vol. 65, Issue 3, S. 681-699.

Raley, R.K., M.L. Frisco und E. Wildsmith (2005), "Maternal Cohabitation and Educational Success", Sociology of Education, April 2005, Vol. 78, No. 2, S. 144-164. 

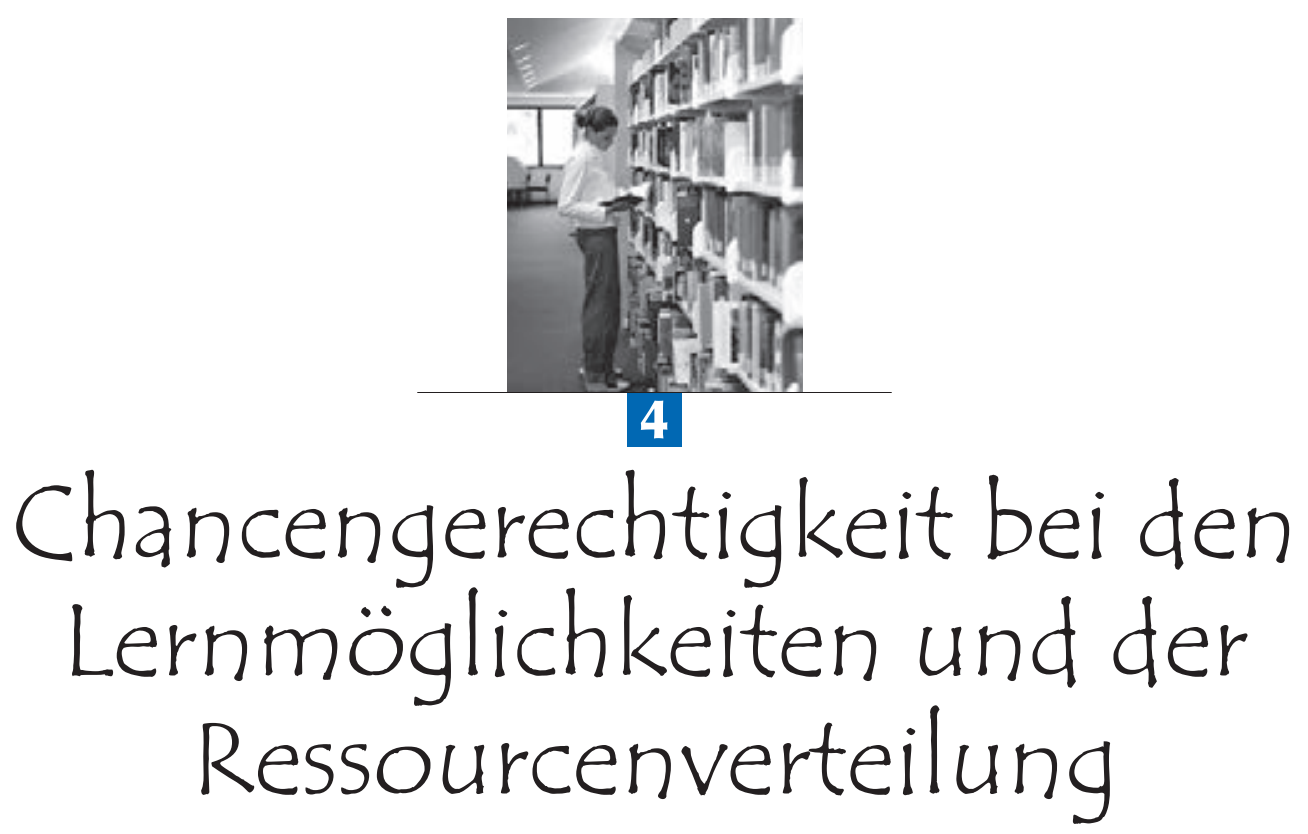

In diesem Kapitel wird das Konzept der Bildungsgerechtigkeit im Hinblick darauf untersucht, wie es mit der Häufigkeit, mit der Schülerinnen und Schüler im Unterricht mit bestimmten Mathematikaufgaben in Kontakt kommen, der Qualität und der Anzahl der Lehrkräfte, der Schuldisziplin und der Teilnahme an vorschulischer Bildung zusammenhängt. Es wird der enge Zusammenhang analysiert, der zwischen diesen Ressourcen, dem sozioökonomischen Hintergrund und den Mathematikleistungen besteht. 
In früheren Forschungsarbeiten wurde ein Zusammenhang zwischen dem Grad des Kontakts mit fachlichen Inhalten in der Schule, also den "Lernmöglichkeiten", und den Schülerleistungen aufgezeigt (Schmidt et al., 2001). Aufbauend auf existierenden Messgrößen der Lernmöglichkeiten (Carroll, 1963; Wiley und Harnischfeger, 1974; Sykes, Schneider und Planck, 2009; Schmidt et al., 2001) wurden die Schülerinnen und Schüler im Rahmen von PISA 2012 u.a. zu den mathematischen Theorien, Begriffen und Inhalten befragt, mit denen sie sich in der Schule u.U. beschäftigt hatten, bzw. dazu, wie häufig diese verschiedenen Themen in ihrem Unterricht behandelt wurden. Wie bereits Band I zu entnehmen war, variierten die Antworten ganz erheblich zwischen den einzelnen Systemen, Schulen und Schülern. Wenn diese Unterschiede auf Schüler- bzw. Schulmerkmale zurückzuführen sind, wie den sozioökonomischen Status der Schüler bzw. der Schulen, den Anteil der Schülerinnen und Schüler mit Migrations- bzw. minoritätssprachlichem Hintergrund oder die Größe der Kommune, in der sich eine Schule befindet, kann Chancenungerechtigkeit in der Bildung bestehen

\section{Ergebnisse der Datenanalyse}

- Der Kontakt mit Aufgaben der reinen Mathematik in der Schule (Lernmöglichkeiten) und die Vertrautheit mit grundlegenden Konzepten der Algebra und der Geometrie haben einen stärkeren Effekt auf die Leistungen, wenn die gesamte Schülerpopulation davon profitiert.

- Stärker ausgeprägt sind die Disparitäten bei der Intensität des Kontakts mit reiner Mathematik in Schulsystemen, in denen die Schüler entsprechend ihrer Leistungen auf verschiedene Schulen verteilt werden, sowie - angesichts des starken Zusammenhangs zwischen Leistungen und sozioökonomischem Hintergrund - in Systemen, in denen eine unbeabsichtigte Konsequenz der Aufteilung entsprechend der Leistungen eine Aufteilung nach sozioökonomischem Hintergrund ist.

- Im OECD-Durchschnitt erzielen Schülerinnen und Schüler, die eigenen Angaben zufolge über ein Jahr an Vorschulbildung teilgenommen hatten, im Bereich Mathematik 53 Punkte mehr - was einem Leistungsvorsprung von über einem Schuljahr entspricht - als Schülerinnen und Schüler, die nicht an Vorschulbildung teilgenommen hatten.

- Im OECD-Raum wird sozioökonomisch benachteiligten Schulen mindestens eine gleich hohe, wenn nicht höhere Zahl an Lehrkräften im Verhältnis zur Schülerzahl zugeteilt wie sozioökonomisch begünstigten Schulen; sozioökonomisch benachteiligte Schulen haben aber tendenziell große Schwierigkeiten, qualifizierte Lehrkräfte anzuwerben.

In Band I dieser Veröffentlichung wird eine Reihe von Indizes des Kontakts und der Vertrautheit mit reiner Mathematik definiert und beschrieben, die auf den Schülerangaben basieren. Die Schülerinnen und Schüler wurden gefragt, wie vertraut ihnen verschiedene mathematische Begriffe sind. Sie machten auch Angaben darüber, wie häufig sie verschiedenen Arten von Mathematikaufgaben im Unterricht begegnet waren, darunter Aufgaben der reinen Mathematik und der angewandten Mathematik. Diese Indizes liefern eine Messgröße der Arten der Mathematik-Lernmöglichkeiten, die sich Schülerinnen und Schülern in der Pflichtschulzeit bieten. Sie spiegeln die aktuellen Erfahrungen der 15-jährigen Schülerinnen und Schüler in der Schule wider, aber auch ihre Erfahrungen aus der Zeit bevor sie an PISA teilgenommen haben. Die Selbstangaben der Schüler entsprechen zwar per definitionem einer subjektiven Einschätzung der Häufigkeit, mit der sie mit verschiedenen Arten von Mathematikaufgaben konfrontiert wurden, sie können aber auch durch andere Eindrücke der Schüler beeinflusst werden, wie z.B. ihrem Grad der Vertrautheit oder ihrer Versiertheit im Umgang mit diesen Arten von Aufgaben. In Band I wird untersucht, welcher Zusammenhang zwischen den Antworten und den Leistungen der Schüler in den einzelnen Ländern besteht. In diesem Abschnitt geht es um die Vertrautheit mit grundlegenden Konzepten der Algebra und der Geometrie (z.B. "Quadratfunktion", "Lineare Gleichung", "Polygon“ oder „Kosinus") und den Kontakt mit Aufgaben der reinen Mathematik im Unterricht (z.B. „Löse: $2 x+3=7$ “ oder „Berechne das Volumen einer Kiste mit den Seitenlängen 3m, $4 \mathrm{~m}$ und $5 \mathrm{~m}$ ”).

Abbildung II.4.1 zeigt die wichtigsten Messgrößen der Chancengerechtigkeit beim Kontakt mit reiner Mathematik und ihre Zusammenhänge mit den durchschnittlichen Leistungen sowie die wichtigsten Messgrößen der Verteilungsgerechtigkeit der Bildungserträge. Sie enthält wichtige Daten und Ergebnisse, die in diesem Kapitel erörtert werden.

Die zwischen den Schulen zu beobachtenden Unterschiede beim Grad des Kontakts der Schüler mit grundlegenden Konzepten der reinen Mathematik in Algebra und Geometrie hängen eng mit den Leistungsunterschieden zwischen Schülern zusammen, die sozioökonomisch begünstigte und sozioökonomisch benachteiligte Schulen besuchen². Zwar sind nicht alle Leistungsunterschiede zwischen diesen beiden Schülergruppen auf Unterschiede beim Grad des Kontakts mit reiner Mathematik zurückzuführen, ein großer Teil davon ist es jedoch. Abbildung II.4.2 zeigt den Zusammenhang zwischen diesen Disparitäten. 
- Abbildung II.4.1

\section{Überblick über die PISA-Messgrößen der Chancengerechtigkeit beim Kontakt mit reiner Mathematik}

\begin{tabular}{|c|c|c|c|c|c|c|c|c|}
\hline & \multicolumn{4}{|c|}{$\begin{array}{l}\text { Höhere Bildungsqualität oder -gerechtigkeit als im OECD-Durchschnitt } \\
\text { Kein statistischer Unterschied zum OECD-Durchschnitt } \\
\text { Geringere Bildungsqualität oder -gerechtigkeit als im OECD-Durchschnitt }\end{array}$} & \multirow[b]{3}{*}{$\begin{array}{l}\text { Varianz beim } \\
\text { Index des } \\
\text { Kontakts } \\
\text { mit reiner } \\
\text { Mathematik }\end{array}$} & \multirow{3}{*}{\begin{tabular}{|} 
Innerschulische \\
Varianz beim \\
Index des \\
Kontakts \\
mit reiner \\
Mathematik als \\
Anteil an der \\
Summe der \\
Varianz zwischen \\
und innerhalb \\
der Schulen
\end{tabular}} & \multirow{3}{*}{$\begin{array}{l}\text { Innerschulische } \\
\text { Varianz des } \\
\text { sozioökono- } \\
\text { mischen Status } \\
\text { als Anteil an } \\
\text { der Summe der } \\
\text { Varianz } \\
\text { zwischen und } \\
\text { innerhalb der } \\
\text { Schulen }\end{array}$} & \multirow{3}{*}{$\begin{array}{c}\text { Innerschulische } \\
\text { Varianz der } \\
\text { Schülerleistungen } \\
\text { als Anteil an } \\
\text { der Summe der } \\
\text { Varianz } \\
\text { zwischen und } \\
\text { innerhalb der } \\
\text { Schulen }\end{array}$} \\
\hline & \multirow{2}{*}{$\begin{array}{l}\text { Durchschnitt- } \\
\text { liche Schüler- } \\
\text { leistungen in } \\
\text { Mathematik }\end{array}$} & \multirow{2}{*}{\begin{tabular}{|c|} 
Stärke des \\
Zusammenhangs \\
zwischen \\
Schülerleistungen und \\
sozioökonomischem \\
Status ${ }^{1}$ \\
\\
\\
Prozentsatz der \\
erklärten Varianz der \\
Schülerleistungen
\end{tabular}} & \multirow{2}{*}{\begin{tabular}{|c|}
$\begin{array}{c}\text { Leistungsunterschiede } \\
\text { nach sozio- } \\
\text { ökonomischem } \\
\text { Status: Steigung der } \\
\text { sozioökonomischen } \\
\text { Gradiente }\end{array}$ \\
Mit einem Anstieg \\
um eine Einheit beim \\
sozioökonomischen \\
Status assoziierte \\
Punktzahlveränderung
\end{tabular}} & \multirow[b]{2}{*}{$\begin{array}{l}\text { Indexmittel } \\
\text { des Kontakts } \\
\text { mit reiner } \\
\text { Mathematik }\end{array}$} & & & & \\
\hline & & & & & & & & \\
\hline OECD-Durchschnitt & 494 & 14.8 & 39 & 1.70 & 0.37 & 80.4 & 75.6 & 64.1 \\
\hline Shanghai (China) & 613 & 15.1 & 41 & 2.30 & 0.21 & 82.8 & 66.8 & 53.1 \\
\hline Singapur & 573 & 14.4 & 44 & 2.23 & 0.41 & 83.5 & 76.4 & 63.3 \\
\hline Hongkong (China) & 561 & 7.5 & 27 & 1.83 & 0.40 & 92.8 & 67.7 & 57.6 \\
\hline Chinesisch Taipeh & 560 & 17.9 & 58 & 1.98 & 0.33 & 81.9 & 76.7 & 57.9 \\
\hline Korea & 554 & 10.1 & 42 & 2.07 & 0.27 & 73.6 & 78.3 & 60.4 \\
\hline Macau (China) & 538 & 2.6 & 17 & 2.20 & 0.32 & 86.1 & 73.7 & 58.2 \\
\hline Japan & 536 & 9.8 & 41 & 2.05 & 0.22 & 71.6 & 77.8 & 47.0 \\
\hline Liechtenstein & 535 & 7.6 & 28 & 1.55 & 0.57 & 53.7 & 85.5 & 37.5 \\
\hline Schweiz & 531 & 12.8 & 38 & 1.41 & 0.50 & 59.6 & 82.7 & 64.4 \\
\hline Niederlande & 523 & 11.5 & 40 & 1.50 & 0.45 & 68.2 & 81.8 & 34.1 \\
\hline Estland & 521 & 8.6 & 29 & 2.00 & 0.21 & 92.0 & 81.5 & 82.7 \\
\hline Finnland & 519 & 9.4 & 33 & 1.72 & 0.35 & 87.9 & 91.1 & 92.5 \\
\hline Kanada & 518 & 9.4 & 31 & 1.98 & 0.37 & 89.0 & 82.8 & 80.2 \\
\hline Polen & 518 & 16.6 & 41 & 1.83 & 0.30 & 92.5 & 76.4 & 79.5 \\
\hline Belgien & 515 & 19.6 & 49 & 1.83 & 0.52 & 72.1 & 72.4 & 48.6 \\
\hline Deutschland & 514 & 16.9 & 43 & 1.66 & 0.43 & 66.7 & 73.6 & 47.0 \\
\hline Vietnam & 511 & 14.6 & 29 & 1.96 & 0.22 & 83.3 & 58.3 & 47.9 \\
\hline Österreich & 506 & 15.8 & 43 & 1.54 & 0.47 & 57.3 & 71.2 & 51.6 \\
\hline Australien & 504 & 12.3 & 42 & 1.69 & 0.49 & 80.1 & 76.5 & 72.1 \\
\hline Irland & 501 & 14.6 & 38 & 1.47 & 0.37 & 90.9 & 79.7 & 81.8 \\
\hline Slowenien & 501 & 15.6 & 42 & 1.93 & 0.32 & 78.7 & 74.6 & 41.3 \\
\hline Dänemark & 500 & 16.5 & 39 & 1.62 & 0.36 & 87.7 & 82.3 & 83.5 \\
\hline Neuseeland & 500 & 18.4 & 52 & 1.51 & 0.51 & 82.7 & 77.5 & 76.2 \\
\hline Tschech. Rep. & 499 & 16.2 & 51 & 1.80 & 0.29 & 71.2 & 76.4 & 48.5 \\
\hline Frankreich & 495 & 22.5 & 57 & 1.87 & 0.32 & w & w & w \\
\hline Ver. Königreich & 494 & 12.5 & 41 & 1.63 & 0.43 & 82.2 & 79.4 & 71.8 \\
\hline Island & 493 & 7.7 & 31 & 1.14 & 0.39 & 95.8 & 86.4 & 90.1 \\
\hline Lettland & 491 & 14.7 & 35 & 2.03 & 0.22 & 88.9 & 74.7 & 74.4 \\
\hline Luxemburg & 490 & 18.3 & 37 & 1.45 & 0.51 & 85.8 & 73.6 & 59.0 \\
\hline Norwegen & 489 & 7.4 & 32 & $\mathrm{~m}$ & $\mathrm{~m}$ & $\mathrm{~m}$ & 91.0 & 87.1 \\
\hline Portugal & 487 & 19.6 & 35 & 1.73 & 0.37 & 89.5 & 68.6 & 70.1 \\
\hline Italien & 485 & 10.1 & 30 & 1.83 & 0.39 & 68.0 & 75.9 & 48.5 \\
\hline Spanien & 484 & 15.8 & 34 & 1.87 & 0.44 & 88.0 & 75.2 & 81.2 \\
\hline Russ. Föderation & 482 & 11.4 & 38 & 2.10 & 0.16 & 94.7 & 75.0 & 73.2 \\
\hline Slowak. Rep. & 482 & 24.6 & 54 & 1.70 & 0.32 & 67.2 & 64.4 & 50.1 \\
\hline Ver. Staaten & 481 & 14.8 & 35 & 2.00 & 0.41 & 89.5 & 73.8 & 76.3 \\
\hline Litauen & 479 & 13.8 & 36 & 1.65 & 0.27 & 91.8 & 78.7 & 69.3 \\
\hline Schweden & 478 & 10.6 & 36 & 0.77 & 0.31 & 92.5 & 86.9 & 87.5 \\
\hline Ungarn & 477 & 23.1 & 47 & 1.96 & 0.29 & 72.4 & 62.6 & 38.1 \\
\hline Kroatien & 471 & 12.0 & 36 & 2.07 & 0.32 & 87.7 & 75.9 & 55.7 \\
\hline Israel & 466 & 17.2 & 51 & 1.81 & 0.41 & 80.0 & 74.6 & 57.6 \\
\hline Griechenland & 453 & 15.5 & 34 & 1.91 & 0.34 & 93.1 & 73.5 & 67.9 \\
\hline Serbien & 449 & 11.7 & 34 & 2.04 & 0.29 & 89.1 & 78.0 & 54.0 \\
\hline Türkei & 448 & 14.5 & 32 & 1.92 & 0.30 & 85.1 & 72.3 & 38.2 \\
\hline Rumänien & 445 & 19.3 & 38 & 2.02 & 0.40 & 78.0 & 64.4 & 54.6 \\
\hline Bulgarien & 439 & 22.3 & 42 & 1.96 & 0.45 & 82.0 & 59.6 & 47.2 \\
\hline Ver. Arab. Emirate & 434 & 9.8 & 33 & 2.13 & 0.50 & 80.3 & 73.9 & 55.6 \\
\hline Kasachstan & 432 & 8.0 & 27 & 1.97 & 0.32 & 90.5 & 76.8 & 63.5 \\
\hline Thailand & 427 & 9.9 & 22 & 1.70 & 0.29 & 85.2 & 61.6 & 57.9 \\
\hline Chile & 423 & 23.1 & 34 & 1.70 & 0.34 & 75.2 & 47.2 & 56.6 \\
\hline Malaysia & 421 & 13.4 & 30 & 1.59 & 0.36 & 88.1 & 71.5 & 67.6 \\
\hline Mexiko & 413 & 10.4 & 19 & 1.78 & 0.43 & 82.3 & 56.5 & 64.8 \\
\hline Montenegro & 410 & 12.7 & 33 & 1.90 & 0.40 & 92.8 & 80.6 & 63.5 \\
\hline Uruguay & 409 & 22.8 & 37 & 1.64 & 0.47 & 76.3 & 60.2 & 58.0 \\
\hline Costa Rica & 407 & 18.9 & 24 & 1.53 & 0.52 & 79.4 & 61.8 & 57.6 \\
\hline Albanien & 394 & $\mathrm{~m}$ & $m$ & 2.09 & 0.42 & 93.1 & 0.0 & 95.4 \\
\hline Brasilien & 391 & 15.7 & 26 & 1.43 & 0.51 & 72.4 & 62.8 & 56.9 \\
\hline Argentinien & 388 & 15.1 & 26 & 1.35 & 0.48 & 74.7 & 66.5 & 55.6 \\
\hline Tunesien & 388 & 12.4 & 22 & 1.23 & 0.36 & 94.4 & 67.2 & 50.7 \\
\hline Jordanien & 386 & 8.4 & 22 & 2.15 & 0.57 & 84.8 & 79.6 & 64.0 \\
\hline Kolumbien & 376 & 15.4 & 25 & 1.76 & 0.51 & 83.6 & 63.2 & 64.9 \\
\hline Katar & 376 & 5.6 & 27 & 1.72 & 0.67 & 76.1 & 75.5 & 53.8 \\
\hline Indonesien & 375 & 9.6 & 20 & 1.60 & 0.33 & 81.9 & 63.1 & 48.0 \\
\hline Peru & 368 & 23.4 & 33 & 1.79 & 0.51 & 79.8 & 54.2 & 54.4 \\
\hline
\end{tabular}

1. Einstufige bivariate Regression der Mathematikleistungen auf den PISA-Index des wirtschaftlichen, sozialen und kulturellen Status (ESCS), wobei die Steigung dem ESCS-Regressionskoeffizienten und die Stärke dem Korrelationskoeffizienten $\left(R^{2}\right)^{*} 100$ entspricht.

Die Länder und Volkswirtschaften sind in absteigender Reihenfolge nach den Durchschnittsergebnissen im Bereich Mathematik angeordnet.

Quelle: OECD, PISA-2012-Datenbank, Tabelle II.2.1, II.2.8a, II.2.13a und II.4.1. 


\section{Umfang der Leistungsunterschiede, die mit dem Kontakt der Schüler mit reiner Mathematik zusammenhängen, nach sozioökonomischem Profil der Schulen}

Zwischen Schülern in sozioökonomisch begünstigten und sozioökonomisch benachteiligten Schulen

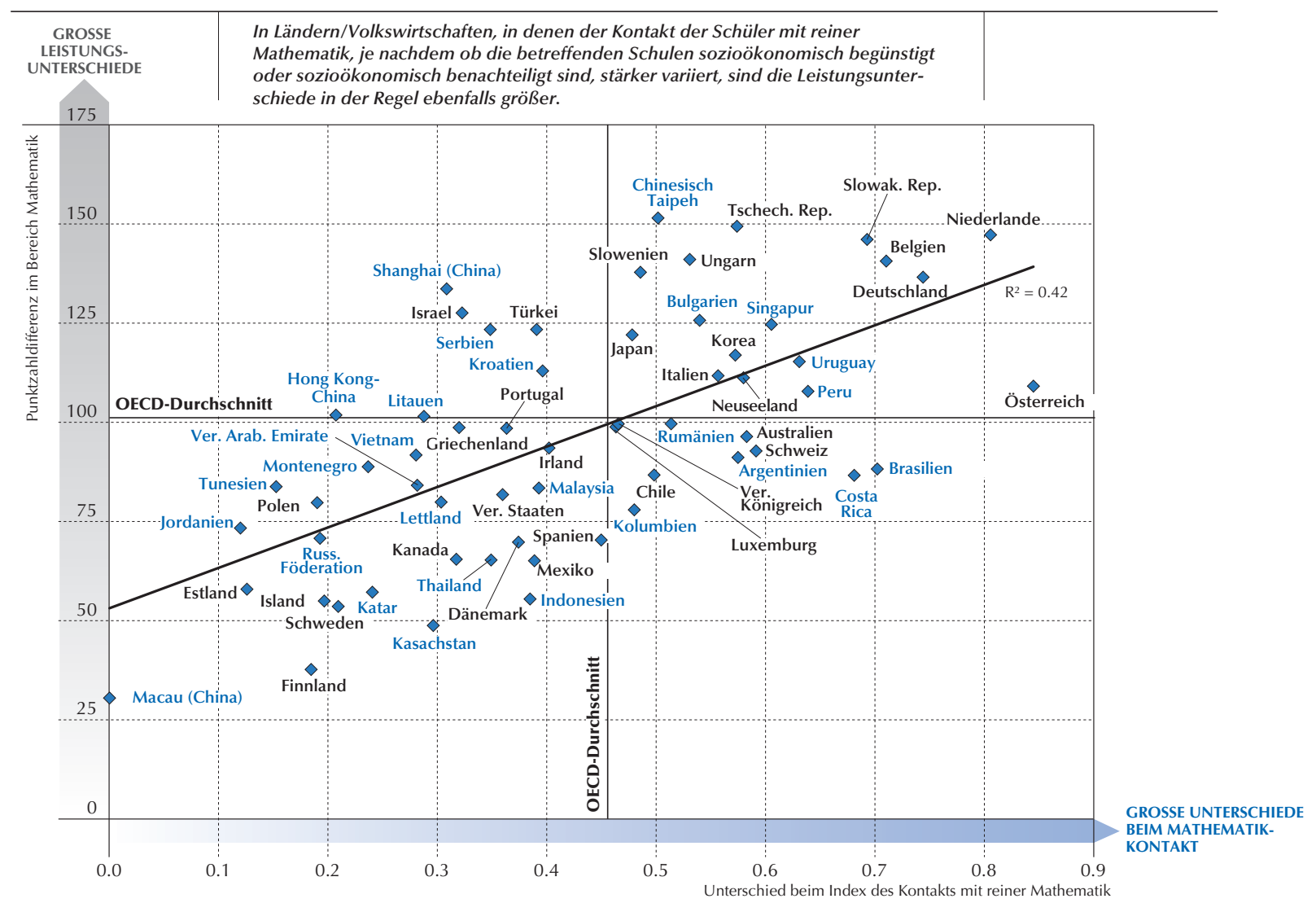

Anmerkung: Je nach der schulischen Organisation (integratives System vs. institutionelle Differenzierung entsprechend der Leistungen) ist im Vergleich zwischen den Schulen mit Unterschieden bei bestimmten Aspekten der Lernmöglichkeiten zu rechnen, mit denen auf die unterschiedlichen Bedürfnisse der Schüler geantwortet werden soll.

Quelle: OECD, PISA-2012-Datenbank, Tabelle II.4.2 und II.4.3.

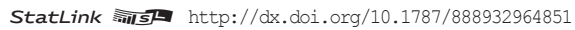

In Ländern mit hohen Durchschnittsergebnissen in Mathematik und einem hohen Niveau an Verteilungsgerechtigkeit der Bildungserträge sind die Unterschiede zwischen den Schülern sozioökonomisch begünstigter und sozioökonomisch benachteiligter Schulen geringer, und zwar sowohl im Hinblick auf die Mathematikleistungen als auch auf den Kontakt mit reiner Mathematik (Abb. II.4.1). So weisen beispielsweise Estland, Finnland und Kanada, die im unteren linken Quadranten von Abbildung II.4.2 liegen, einen geringen Leistungsabstand zwischen Schülern auf, die sozioökonomisch begünstigte und sozioökonomisch benachteiligte Schulen besuchen. Unter den Schulsystemen mit hohen durchschnittlichen Mathematikleistungen und einem hohen Grad an Verteilungsgerechtigkeit bei den Bildungserträgen (gemessen an der Stärke des Zusammenhangs zwischen den Leistungen und dem sozioökonomischen Hintergrund der Schüler) (Abb. II.4.1) sind nur in Japan und Korea große Unterschiede bei den Schülerleistungen und durchschnittliche Disparitäten bei den Lernmöglichkeiten zwischen sozioökonomisch begünstigten und sozioökonomisch benachteiligten Schulen festzustellen (oberer rechter Quadrant von Abb. II.4.2).

Abbildung II.4.2 zeigt zudem, dass die zwischen sozioökonomisch begünstigten und sozioökonomisch benachteiligten Schulen beobachteten Unterschiede bei den Mathematikleistungen einerseits und der Intensität des Kontakts mit reiner Mathematik andererseits im Durchschnitt der OECD-Länder sehr stark untereinander korrelieren (die Korrelation zwischen diesen beiden Messgrößen beträgt 0,65). Im OECD-Durchschnitt beläuft sich der Leistungsunterschied im Bereich Mathematik auf 104 Punkte. Die Unterschiede beim Kontakt mit Mathematik je nach dem sozioökonomischen Profil der Schulen sind ebenfalls groß (sie machen auf dem Index des Kontakts mit reiner Mathematik mehr als 0,45 bzw. drei Viertel einer Standardabweichung aus, d.h. 0,60). Im Durchschnitt gaben die Schülerinnen und Schüler in 
sozioökonomisch begünstigten Schulen an, dass sie „häufig“ von komplexeren Konzepten der Algebra und Geometrie gehört und im Unterricht im Allgemeinen auch „häufig“ Aufgaben der reinen Mathematik begegnet sind. Die Schülerinnen und Schüler sozioökonomisch benachteiligter Schulen hatten eigenen Angaben zufolge zwischen "ein- oder zweimal" und „einige Male“ im Unterricht von diesen Themen gehört.

\section{DISPARITÄTEN BEIM KONTAKT MIT REINER MATHEMATIK, BEIM SOZIOÖKONOMISCHEN HINTERGRUND UND BEI DEN LEISTUNGEN}

Von Schülerinnen und Schülern, die in der Schule nicht mit mathematischen Konzepten und Verfahren konfrontiert werden, kann nicht erwartet werden, dass sie sich den Stoff selbst beibringen, und noch weniger, dass sie darin überragende Leistungen erzielen. Durch die Zuteilung von mehr und besseren Ressourcen können nur Grundvoraussetzungen geschaffen werden; was im Unterricht vermittelt wird - und wie es vermittelt wird - ist, was letztlich bestimmt, ob diese Ressourcen dem Hauptziel des Schulsystems dienen können: nämlich allen Schülerinnen und Schülern auf gerechte Weise eine qualitativ hochwertige Bildung zu vermitteln. Die Breite und Tiefe der Unterrichtsinhalte und die Lehrmethoden sind für den Lernprozess der Schülerinnen und Schüler, insbesondere im Fach Mathematik, von entscheidender Bedeutung.

Die Lernmöglichkeiten können sich aus vielerlei Gründen zwischen verschiedenen Schülern und Schulen unterscheiden. Wenn die Schüler im Schulsystem auf verschiedeneSchulen aufgeteiltwerden, z.B. aufallgemeinbildende und berufsbildende Bildungsgänge, kann der Grad ihres Kontakts mit Mathematik variieren, je nachdem welche Schule sie besuchen. Der Kontakt mit verschiedenen mathematischen Konzepten und die Erfahrung im Umgang mit Mathematikaufgaben können sich auch zwischen Schülern innerhalb einer bestimmten Schule unterscheiden, vor allem wenn die Schüler in Leistungsgruppen eingeteilt und ihnen unterschiedliche Inhalte vermittelt werden. Die Unterrichtsinhalte und die Lehrmethoden können sich innerhalb einer Schule auch unterscheiden, wenn 15-jährige Schülerinnen und Schüler unterschiedliche Klassenstufen besuchen oder wenn sie verschiedene inhaltliche Schwerpunkte wählen.

\section{Disparitäten innerhalb der Länder}

Im Durchschnitt der OECD-Länder gaben die 15-jährigen Schülerinnen und Schüler an, dass sie „einige Male“ von mathematischen Konzepten der Algebra und Geometrie gehört, jedoch "häufig" Aufgaben der reinen Mathematik begegnet sind. Es sind zwar signifikante Unterschiede zwischen den einzelnen Schulsystemen festzustellen, die Unterschiede zwischen den Schulen innerhalb eines Landes und zwischen den Schülern innerhalb einer Schule sind aber noch größer ${ }^{3}$. Der Großteil der Unterschiede ist unter Schülern zu beobachten, die dieselbe Schule besuchen (65\%); die Unterschiede zwischen den Schulen innerhalb eines Landes machen 17\% der Gesamtunterschiede aus, und auf die Unterschiede zwischen den einzelnen Ländern und Volkswirtschaften entfallen die übrigen 18\% der Unterschiede 4 .

Schulsysteme, die ein hohes durchschnittliches Leistungsniveau und eine große Chancengerechtigkeit in der Bildung verbinden, bieten in der Regel allen ihren Schülern häufigen Kontakt mit Konzepten der reinen Mathematik (gemessen an überdurchschnittlichen Mittelwerten auf dem Index des Kontakts mit reiner Mathematik und einer unterdurchschnittlichen Gesamt- und zwischenschulischen Varianz auf diesem Index). Von den neun Ländern, die ein hohes Leistungsniveau und ein großes Maß an Bildungsgerechtigkeit vorweisen können, ist Liechtenstein das einzige Land, in dem der Mittelwert auf dem Index des Kontakts mit reiner Mathematik unter dem Durchschnitt liegt. In dieser Gruppe verzeichnen lediglich Australien, Hongkong (China) und Liechtenstein eine überdurchschnittliche Varianz des Kontakts mit reiner Mathematik. Nur in Liechtenstein ist die Varianz groß und sind die zwischenschulischen Unterschiede überdurchschnittlich stark ausgeprägt. Japan und Korea sind die einzigen Länder in dieser Gruppe, wo die zwischenschulischen Unterschiede beim Kontakt mit reiner Mathematik oberhalb des Durchschnitts angesiedelt sind (Abb. II.4.1).

\section{Unterschiede beim Kontakt mit Mathematik und durchschnittliche Mathematikleistungen in verschiedenen Schulsystemen}

Geringere Disparitäten beim Kontakt mit mathematischen Konzepten sind mit höheren Durchschnittsergebnissen assoziiert, insbesondere in den Schulsystemen, in denen die Häufigkeit des Kontakts und die Vertrautheit mit Konzepten der reinen Mathematik größer sind als im OECD-Durchschnitt. Die Begegnung mit Aufgaben der reinen Mathematik im Schulunterricht und die Vertrautheit mit grundlegenden Konzepten der Algebra und der Geometrie haben einen stärkeren Effekt auf die Durchschnittsleistungen, wenn die gesamte Schülerpopulation davon profitiert. Wie Abbildung II.4.3 zeigt, weisen die Länder, die höhere Leistungsniveaus erreichen, in der Tendenz geringere Disparitäten beim Kontakt mit reiner Mathematik auf. 
Aus Abbildung II.4.3 ist zudem ersichtlich, dass ein starker Zusammenhang zwischen den Unterschieden beim Kontakt mit reiner Mathematik und den Durchschnittsleistungen festzustellen ist, wenn die Schulsysteme einen häufigen Kontakt mit Konzepten und Verfahren der reinen Mathematik gewährleisten. Estland, Japan und Korea erzielten z.B. Ergebnisse weit über dem OECD-Durchschnitt, außerdem ist der Grad des Kontakts mit reiner Mathematik in diesen Ländern ebenfalls weit über dem OECD-Durchschnitt angesiedelt, und die Unterschiede bei den Lernmöglichkeiten sind schwächer ausgeprägt als im Durchschnitt. Diese Strukturen sind auch in einigen Partnerländern und -volkswirtschaften zu beobachten, wobei Shanghai (China) ein besonders hohes durchschnittliches Leistungsniveau, das mit einem häufigen Kontakt mit Aufgaben der reinen Mathematik und großer Vertrautheit mit grundlegenden mathematischen Konzepten der Algebra und Geometrie assoziiert ist, sowie eine ausgesprochen geringe Varianz dieser Lernmöglichkeiten vorweisen kann.

- Abbildung II.4.3 "

\section{Zusammenhang zwischen den Mathematikleistungen und der Varianz des Kontakts der Schüler mit reiner Mathematik}

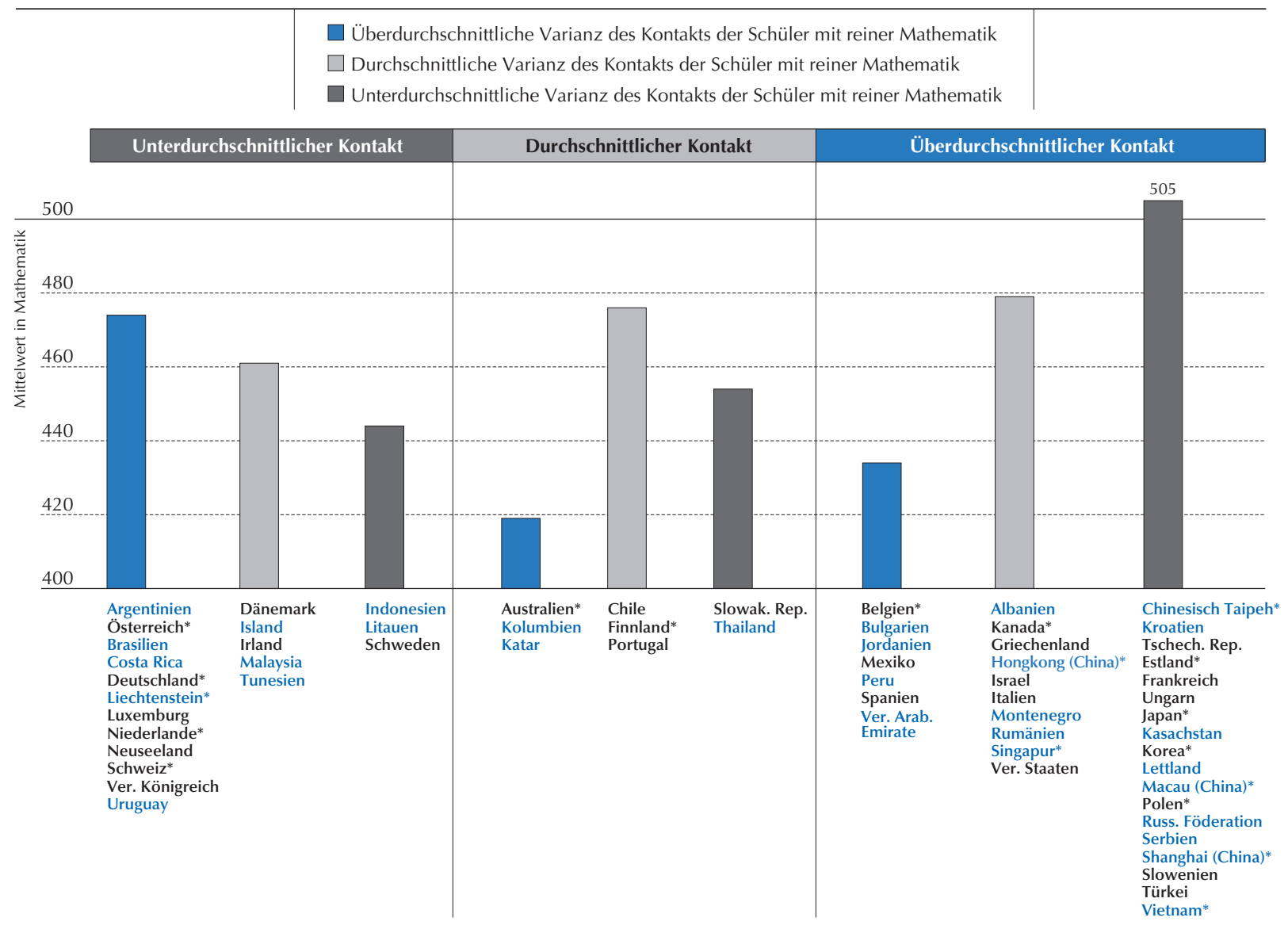

Anmerkung: Je nach der schulischen Organisation (integratives System vs. institutionelle Differenzierung entsprechend der Leistungen) ist im Vergleich zwischen den Schulen mit Unterschieden bei bestimmten Lernmöglichkeiten zu rechnen, mit denen auf die unterschiedlichen Bedürfnisse der Schüler geantwortet werden soll.

Länder mit über dem OECD-Durchschnitt liegenden Durchschnittsergebnissen in Mathematik sind mit einem Sternchen gekennzeichnet. Quelle: OECD, PISA-2012-Datenbank, Tabelle I.2.3a und II.4.1.

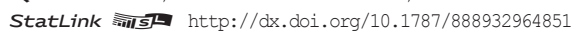

Wenn der Grad des Kontakts mit reiner Mathematik unter dem OECD-Durchschnitt liegt, besteht kein enger Zusammenhang zwischen den diesbezüglichen Disparitäten und den Durchschnittsergebnissen (wie auf der linken Seite von Abb. II.4.3 dargestellt). In einigen Ländern, wie in Costa Rica, Luxemburg und Uruguay, haben die Schüler weniger häufig Kontakt mit reiner Mathematik, ist die Varianz dieser Lernmöglichkeiten geringer und sind die Durchschnittsergebnisse schlechter, in anderen Ländern wiederum, wie in Österreich, Deutschland, den Niederlanden, Neuseeland und der Schweiz, wo die Schüler ebenfalls weniger häufig Kontakt mit Mathematik haben, sind die diesbezüglichen Disparitäten jedoch groß und werden überdurchschnittliche Leistungen erzielt (Abb. II.4.3). 


\section{Zwischenschulische Unterschiede bei den Lernmöglichkeiten, dem sozioökonomischen Hintergrund und den Leistungen}

In den meisten Schulsystemen, in denen auf die eine oder andere Weise eine Selektion der Schülerinnen und Schüler vorgenommen wird, werden die Schüler in der Regel entsprechend ihrer Leistungen auf verschiedene Schulen verteilt. Wie in Kapitel 2 untersucht wurde, sind die Leistungen tendenziell eng mit dem sozioökonomischen Hintergrund verknüpft, so dass eine unbeabsichtigte Folge der Aufteilung der Schüler entsprechend ihrer Leistungen häufig eine Aufteilung der Schüler nach ihrem sozioökonomischen Hintergrund ist. Die Ergebnisse der PISA-Erhebung 2012 zeigen, dass die Disparitäten bei der Intensität des Kontakts mit reiner Mathematik in Systemen stärker ausgeprägt sind, in denen die Schüler entsprechend ihrer Leistungen auf verschiedene Schulen verteilt werden, sowie - angesichts des Zusammenhangs zwischen Leistungen und sozioökonomischem Hintergrund - in Systemen, in denen eine unbeabsichtigte Konsequenz der Aufteilung entsprechend der Leistungen eine Aufteilung nach sozioökonomischem Hintergrund ist. Es ist eine Assoziation festzustellen zwischen großen zwischenschulischen Unterschieden bei den Lernmöglichkeiten, dem sozioökonomischen Hintergrund sowie den Leistungen und Systemen, die einen niedrigeren Grad an Verteilungsgerechtigkeit bei den Bildungserträgen und in manchen Fällen niedrigere Durchschnittsergebnisse aufweisen.

- Abbildung II.4.4 "

\section{Zwischenschulische Unterschiede beim Kontakt mit reiner Mathematik, beim sozioökonomischen} Status und bei den Leistungen

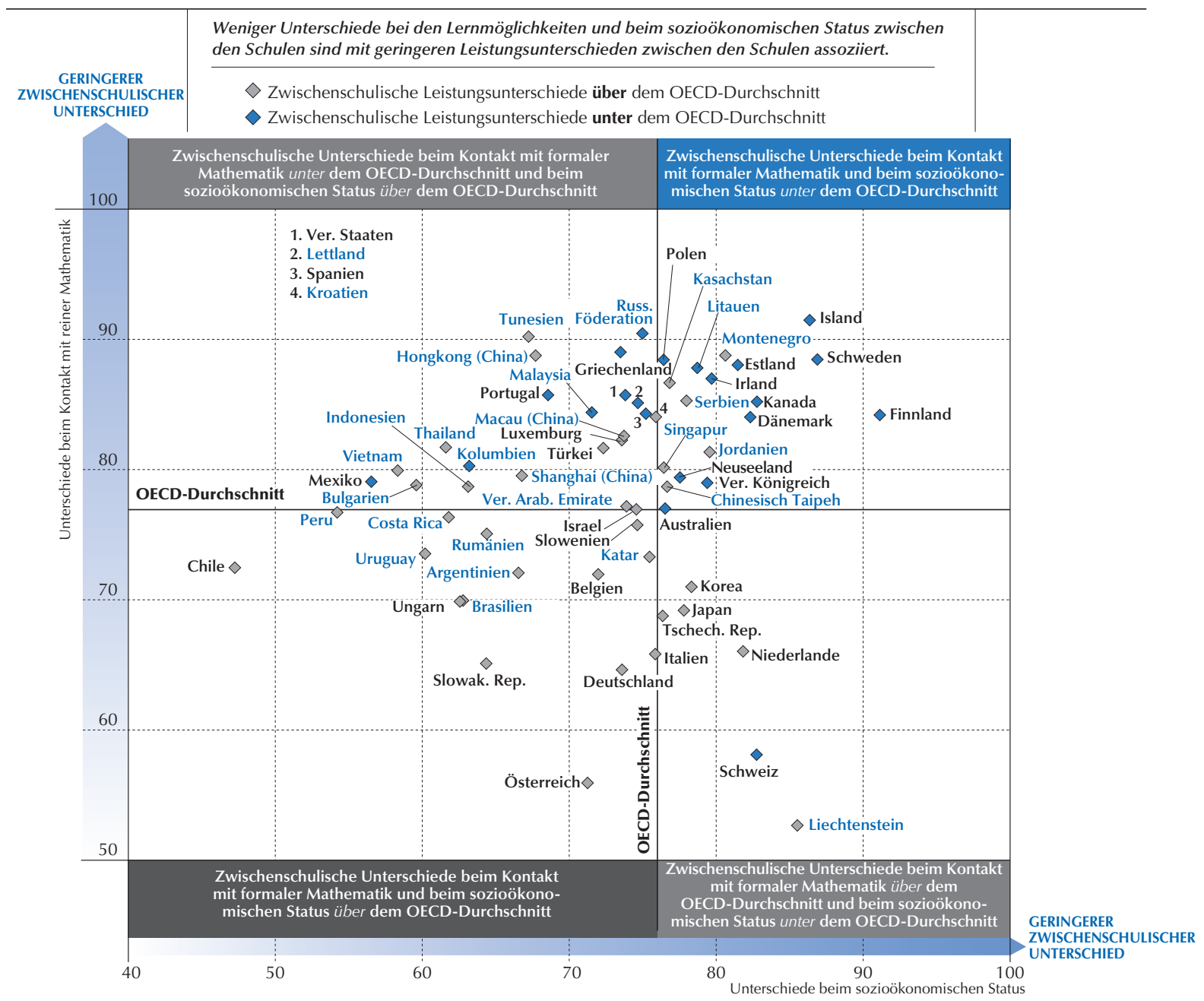

Anmerkung: Je nach der schulischen Organisation (integratives System vs. institutionelle Differenzierung entsprechend der Leistungen) ist im Vergleich zwischen den Schulen mit Unterschieden bei bestimmten Lernmöglichkeiten zu rechnen, mit denen auf die unterschiedlichen Bedürfnisse der Schüler geantwortet werden soll.

Quelle: OECD, PISA-2012-Datenbank, Tabelle II.2.8a, II.2.13a und II.4.1. 
Wie aus Abbildung II.4.1 ersichtlich, weisen Kanada, Dänemark, Estland, Finnland, Island und Schweden unterdurchschnittliche zwischenschulische Unterschiede bei den Leistungen, dem sozioökonomischen Hintergrund und dem Kontakt mit reiner Mathematik auf. Diesen Systemen gelingt es nicht nur, die zwischenschulischen Unterschiede im Hinblick auf diese drei Indikatoren auf ein Mindestmaß zu reduzieren, sondern auch ein überdurchschnittliches Maß an Verteilungsgerechtigkeit bei den Bildungserträgen (gemessen an der Stärke des Zusammenhangs zwischen dem sozioökonomischen Hintergrund und den Leistungen der Schüler), zu gewährleisten. Die einzige Ausnahme ist Dänemark, wo die Bildungsgerechtigkeit dem Durchschnitt entspricht. Kanada, Dänemark, Estland und Finnland erreichen überdurchschnittliche Leistungen, Island weist durchschnittliche Leistungen auf, und nur Schweden erzielt unterdurchschnittliche Leistungen.

Im Gegensatz dazu sind in Argentinien, Brasilien, Chile, Ungarn und der Slowakischen Republik große zwischenschulische Unterschiede bei den Leistungen, dem sozioökonomischen Hintergrund und dem Kontakt mit reiner Mathematik zu beobachten. In diesen Schulsystemen liegen die Durchschnittsleistungen unter dem OECD-Durchschnitt. Chile, Ungarn und die Slowakische Republik weisen zudem ein unterdurchschnittliches Maß an Verteilungsgerechtigkeit der Bildungserträge auf, während die Bildungsgerechtigkeit in Argentinien und Brasilien dem OECD-Durchschnitt entspricht (gemessen an der Stärke des Zusammenhangs zwischen dem sozioökonomischen Hintergrund und den Leistungen der Schüler).

Die zwischenschulischen Unterschiede und die Gesamtvarianz beim Kontakt mit reiner Mathematik sind in Österreich, Belgien, Deutschland, Liechtenstein, den Niederlanden und der Schweiz (Ländern mit überdurchschnittlichen Leistungen) sowie in Argentinien, Brasilien und Italien (Ländern mit unterdurchschnittlichen Leistungen) oberhalb des Durchschnitts angesiedelt. In all diesen Ländern, außer der Schweiz, sind die zwischenschulischen Leistungsunterschiede schwächer ausgeprägt als im Durchschnitt. Die sozioökonomischen Unterschiede zwischen den Schulen sind in Argentinien und Brasilien größer als im Durchschnitt, liegen in Österreich, Belgien, Deutschland und Italien beim Durchschnitt und sind in Liechtenstein, den Niederlanden und der Schweiz weniger groß als im Durchschnitt. Außer Italien und Liechtenstein erreicht keines dieser Länder ein überdurchschnittliches Maß an Verteilungsgerechtigkeit der Bildungserträge (gemessen an der Stärke des Zusammenhangs zwischen dem sozioökonomischen Hintergrund und den Leistungen der Schüler).

\section{CHANCENGERECHTIGKEIT BEI DER VERTEILUNG DER BILDUNGSRESSOURCEN}

Eine potenzielle Ursache für Chancenungerechtigkeit bei Lernerträgen und Lernmöglichkeiten ist in der Verteilung der Ressourcen auf Schüler- und Schulebene zu sehen. Ein positiver Zusammenhang zwischen dem sozioökonomischen Profil der Schulen und der Quantität bzw. Qualität der Ressourcen bedeutet, dass sozioökonomisch begünstigte Schulen mehr oder bessere Ressourcen erhalten; ein negativer Zusammenhang impliziert, dass mehr oder bessere Ressourcen für sozioökonomisch benachteiligte Schulen bereitgestellt werden. Kein Zusammenhang zwischen den beiden Messgrößen heißt, dass von sozioökonomisch benachteiligten Schülerinnen und Schülern besuchte Schulen mit genauso großer Wahrscheinlichkeit Zugang zu besseren oder mehr Ressourcen haben wie von sozioökonomisch begünstigten Schülerinnen und Schülern besuchte Schulen.

In Abbildung II.4.5 ist der Zusammenhang zwischen dem sozioökonomischen Profil der Schulen - d.h. der durchschnittlichen Position ihrer Schüler auf dem PISA-Index des wirtschaftlichen, sozialen und kulturellen Status - sowie einer Reihe von Schulmerkmalen, wie der Schüler/Lehrer-Quote, dem Anteil der Vollzeitlehrkräfte, dem Index des Lehrermangels und dem Index der Qualität der Ausstattung mit Lehr- und Sachmitteln, dargestellt (vgl. Band IV wegen weiterer Analysen und näherer Einzelheiten über diese Indizes). Zusammenhänge, die sozioökonomisch benachteiligte Schulen betreffen, in denen die Ressourcenausstattung den Angaben der Schulleitungen zufolge in quantitativer und/oder qualitativer Hinsicht besser ist, sind in einem helleren Blauton unterlegt; Zusammenhänge, die sozioökonomisch benachteiligte Schulen betreffen, in denen die Ressourcenausstattung den Angaben der Schulleitungen zufolge in quantitativer und/oder qualitativer Hinsicht geringer ist, sind durch eine graue Schattierung markiert. Wenn der Zusammenhang in einem Schulsystem insgesamt stärker ist als im OECD-Durchschnitt, ist die Korrelation durch Fettdruck gekennzeichnet.

\section{Mehr ist nicht immer besser}

Im Fall von Schülerinnen und Schülern sozioökonomisch benachteiligter Schulen ist die Quantität der Ressourcen nicht unbedingt mit der Qualität der Ressourcen gleichbedeutend. Im Allgemeinen besuchen sozioökonomisch benachteiligtere Schüler Schulen mit niedrigeren Schüler/Lehrer-Quoten; sozioökonomisch begünstigtere Schüler besuchen jedoch Schulen, die über einen höheren Anteil an Lehrkräften mit Hochschulabschluss verfügen.

Die PISA-Ergebnisse legen den Schluss nahe, dass viele Schülerinnen und Schüler doppelt benachteiligt sind, da sie selbst einen sozioökonomisch ungünstigen Hintergrund haben und zudem eine Schule besuchen, in der die Qualität der Ausstattung mit Lehrkräften geringer ist. Im Verhältnis zur Größe der Schülerpopulation der Schulen teilen die OECD-Länder 
- Abbildung II.4.5 "

\section{Überblick über die PISA-Messgrößen der Chancengerechtigkeit bei der Verteilung der Bildungsressourcen}

\begin{tabular}{|c|c|c|c|c|c|c|c|}
\hline \multirow[b]{4}{*}{ OECD-Durchschnitt } & \multicolumn{7}{|c|}{$\begin{array}{l}\text { Sozioökonomisch benachteiligte Schulen verfügen mit größerer Wahrscheinlichkeit über mehr oder bessere Ressourcen; } \\
\text { die Korrelation ist stärker als } 0,25 \\
\text { Sozioökonomisch begünstigte Schulen verfügen mit größerer Wahrscheinlichkeit über mehr oder bessere Ressourcen; } \\
\text { die Korrelation ist stärker als } 0,25\end{array}$} \\
\hline & \multicolumn{7}{|c|}{ Einfache Korrelation zwischen dem mittleren sozioökonomischen Profil der Schulen und: } \\
\hline & $\begin{array}{c}\text { Schüler/Lehrer- } \\
\text { Quote }^{1}\end{array}$ & \begin{tabular}{|c|} 
Zusammensetzung \\
und Qualifikationen \\
des Mathematik- \\
Lehrkörpers \\
(Anteil der Lehr- \\
kräfte mit Hoch- \\
schulabschluss) \\
\end{tabular} & $\begin{array}{l}\text { Das Schulklima } \\
\text { beeinträchtigende } \\
\text { schülerbezogene } \\
\text { Faktoren }\end{array}$ & $\begin{array}{c}\text { Anteil der Schüler, } \\
\text { die die Schule } \\
\text { ohne Abschluss } \\
\text { verlassen }\end{array}$ & $\begin{array}{c}\text { Elterlicher } \\
\text { Leistungsdruck }\end{array}$ & $\begin{array}{l}\text { Teilnahme an } \\
\text { Unterricht } \\
\text { außerhalb der } \\
\text { Schulzeit }\end{array}$ & $\begin{array}{l}\text { Mit Hausaufgaben } \\
\text { oder } \\
\text { selbstständigem } \\
\text { Lernen verbrachte } \\
\text { Zeit }\end{array}$ \\
\hline & 0.16 & 0.14 & 0.30 & -0.28 & 0.31 & 0.10 & 0.18 \\
\hline ¿ Australien & -0.05 & 0.02 & 0.52 & -0.31 & 0.36 & 0.14 & 0.25 \\
\hline Österreich & -0.11 & 0.60 & 0.23 & -0.22 & 0.25 & 0.12 & 0.23 \\
\hline : & 0.59 & 0.61 & 0.56 & -0.36 & 0.30 & 0.17 & 0.31 \\
\hline Kanada & 0.20 & 0.02 & 0.36 & -0.31 & 0.41 & 0.10 & 0.18 \\
\hline Chile & -0.03 & 0.19 & 0.45 & -0.34 & 0.44 & 0.08 & 0.16 \\
\hline Tschech. Rep. & 0.05 & 0.28 & 0.31 & -0.18 & 0.28 & 0.02 & 0.14 \\
\hline Dänemark & 0.20 & 0.09 & 0.35 & -0.30 & 0.35 & 0.00 & 0.05 \\
\hline Estland & 0.45 & 0.00 & 0.09 & -0.12 & 0.13 & 0.02 & 0.04 \\
\hline Finnland & 0.36 & 0.01 & 0.01 & 0.02 & 0.14 & 0.05 & 0.05 \\
\hline $\begin{array}{l}\text { Frankreich } \\
\end{array}$ & w & $\mathrm{w}$ & w & w & $w$ & $\mathrm{w}$ & $\mathrm{w}$ \\
\hline Deutschland & 0.19 & 0.00 & 0.29 & -0.18 & 0.13 & 0.08 & 0.14 \\
\hline Griechenland & 0.18 & 0.19 & 0.14 & -0.37 & 0.35 & 0.21 & 0.20 \\
\hline Ungarn & -0.04 & 0.16 & 0.47 & -0.43 & 0.49 & 0.20 & 0.32 \\
\hline Island & 0.42 & 0.18 & -0.01 & -0.07 & 0.24 & 0.05 & 0.11 \\
\hline Irland & 0.32 & -0.08 & 0.42 & -0.33 & 0.56 & 0.10 & 0.15 \\
\hline Israel & -0.03 & 0.21 & 0.14 & -0.20 & 0.37 & -0.06 & 0.07 \\
\hline Italien & 0.40 & 0.30 & 0.41 & -0.35 & 0.30 & 0.24 & 0.38 \\
\hline Japan & 0.30 & 0.18 & 0.34 & -0.39 & 0.44 & 0.31 & 0.33 \\
\hline Korea & 0.27 & 0.02 & 0.25 & -0.24 & 0.42 & 0.36 & 0.28 \\
\hline Luxemburg & 0.17 & 0.46 & 0.47 & -0.38 & -0.06 & 0.06 & 0.16 \\
\hline Mexiko & 0.02 & 0.01 & 0.12 & -0.02 & 0.10 & 0.09 & 0.16 \\
\hline Niederlande & 0.43 & 0.51 & 0.21 & -0.34 & 0.39 & 0.12 & 0.22 \\
\hline Neuseeland & 0.15 & 0.21 & 0.53 & -0.80 & 0.44 & 0.14 & 0.24 \\
\hline Norwegen & 0.27 & 0.00 & 0.28 & C & 0.47 & 0.09 & 0.12 \\
\hline Polen & 0.07 & -0.07 & 0.04 & -0.05 & 0.07 & 0.01 & 0.03 \\
\hline Portugal & 0.41 & -0.15 & 0.17 & 0.08 & 0.38 & 0.12 & 0.17 \\
\hline Slowak. Rep. & 0.04 & -0.15 & 0.25 & -0.28 & 0.30 & -0.01 & 0.16 \\
\hline Slowenien & 0.25 & 0.43 & 0.27 & -0.23 & 0.27 & 0.04 & 0.16 \\
\hline Spanien & 0.17 & -0.04 & 0.45 & -0.31 & 0.27 & 0.04 & 0.08 \\
\hline Schweden & 0.26 & 0.12 & 0.43 & -0.49 & 0.40 & 0.11 & 0.17 \\
\hline Schweiz & -0.07 & 0.18 & 0.08 & $\mathrm{C}$ & -0.10 & 0.06 & 0.12 \\
\hline Türkei & -0.37 & 0.04 & 0.31 & -0.19 & 0.21 & 0.05 & 0.04 \\
\hline Ver. Königreich & -0.18 & 0.00 & 0.35 & -0.29 & 0.48 & 0.16 & 0.31 \\
\hline Ver. Staaten & 0.02 & -0.02 & 0.42 & -0.31 & 0.47 & 0.14 & 0.25 \\
\hline § Albanien & $\mathrm{m}$ & $\mathrm{m}$ & $\mathrm{m}$ & $\mathrm{m}$ & $\mathrm{m}$ & $\mathrm{m}$ & $\mathrm{m}$ \\
\hline Argentinien & 0.05 & 0.17 & 0.33 & -0.24 & 0.15 & 0.04 & 0.10 \\
\hline Brasilien & -0.21 & -0.01 & 0.38 & -0.21 & 0.31 & 0.05 & 0.13 \\
\hline Bulgarien & -0.02 & 0.00 & 0.23 & -0.39 & 0.40 & 0.17 & 0.33 \\
\hline Chinesisch Taipeh & -0.01 & 0.02 & 0.36 & -0.20 & 0.29 & 0.29 & 0.36 \\
\hline Kolumbien & -0.07 & -0.04 & 0.25 & -0.06 & 0.07 & 0.12 & 0.18 \\
\hline Costa Rica & 0.18 & 0.15 & 0.43 & -0.41 & 0.22 & 0.13 & 0.22 \\
\hline گroatien & 0.22 & 0.42 & 0.20 & -0.22 & 0.19 & 0.10 & 0.24 \\
\hline Hongkong (China) & 0.04 & 0.04 & 0.21 & 0.02 & -0.07 & 0.20 & 0.14 \\
\hline Indonesien & -0.11 & 0.20 & 0.17 & -0.19 & -0.06 & 0.14 & 0.16 \\
\hline Jordanien & -0.07 & -0.01 & 0.06 & -0.18 & 0.19 & -0.03 & 0.04 \\
\hline Kasachstan & 0.22 & 0.21 & -0.04 & -0.04 & 0.20 & 0.08 & 0.13 \\
\hline Lettland & 0.37 & 0.16 & 0.01 & -0.14 & 0.13 & 0.11 & 0.17 \\
\hline Liechtenstein & 0.50 & 0.46 & 0.45 & $\mathrm{C}$ & -0.56 & 0.01 & 0.12 \\
\hline Litauen & 0.05 & 0.05 & 0.24 & -0.17 & 0.15 & 0.04 & 0.16 \\
\hline Macau (China) & -0.05 & -0.09 & 0.26 & -0.23 & 0.16 & 0.15 & 0.16 \\
\hline Malaysia & 0.08 & -0.10 & 0.41 & -0.23 & 0.30 & 0.11 & 0.18 \\
\hline Montenegro & 0.40 & 0.27 & 0.20 & -0.25 & -0.07 & 0.05 & 0.16 \\
\hline Peru & 0.20 & -0.05 & 0.29 & -0.14 & 0.18 & 0.08 & 0.13 \\
\hline Katar & 0.07 & -0.09 & -0.02 & -0.06 & 0.19 & \begin{tabular}{c|c|}
-0.03 \\
\end{tabular} & 0.13 \\
\hline Rumänien & -0.19 & 0.24 & 0.27 & -0.24 & 0.06 & 0.16 & 0.25 \\
\hline Russ. Föderation & 0.35 & 0.27 & 0.21 & -0.07 & 0.26 & 0.06 & 0.09 \\
\hline Serbien & 0.29 & 0.07 & 0.24 & -0.21 & 0.31 & 0.03 & 0.10 \\
\hline Shanghai (China) & -0.26 & 0.26 & 0.17 & -0.35 & 0.19 & 0.24 & 0.35 \\
\hline Singapur & 0.11 & 0.36 & 0.47 & -0.17 & 0.38 & 0.13 & 0.18 \\
\hline Thailand & 0.11 & 0.03 & 0.12 & -0.28 & 0.30 & 0.22 & 0.24 \\
\hline Tunesien & 0.05 & 0.03 & -0.08 & -0.19 & 0.23 & 0.03 & 0.07 \\
\hline Ver. Arab. Emirate & -0.05 & -0.05 & 0.11 & -0.22 & 0.26 & -0.03 & 0.11 \\
\hline Uruguay & -0.08 & 0.23 & 0.54 & -0.35 & 0.25 & 0.09 & 0.10 \\
\hline Vietnam & 0.12 & 0.10 & 0.20 & -0.26 & 0.24 & 0.21 & 0.20 \\
\hline
\end{tabular}

Anmerkung: Die Daten sind durch Fettdruck gekennzeichnet, wenn die Korrelation innerhalb eines Landes/einer Volkswirtschaft signifikant vom OECD-Durchschnitt abweicht. 1. Eine negative Korrelation steht für günstigere Werte für sozioökonomisch begünstigte Schülerinnen und Schüler

Quelle: OECD, PISA-2012-Datenbank, Tabelle II.4.6. 
sozioökonomisch benachteiligten Schulen mindestens eine gleich hohe, wenn nicht höhere Zahl an Mathematiklehrkräften zu wie sozioökonomisch begünstigten Schulen. Wie aus Abbildung II.4.6 hervorgeht, haben sozioökonomisch benachteiligte Schulen in der Regel jedoch große Schwierigkeiten, qualifizierte Lehrkräfte anzuwerben. So ist beispielsweise in den Niederlanden der Anteil der qualifizierten Lehrkräfte in sozioökonomisch begünstigten Schulen dreimal höher als der Anteil der qualifizierten Lehrkräfte in sozioökonomisch benachteiligten Schulen (52\% gegenüber 14\%), während die Schüler/LehrerQuote in sozioökonomisch begünstigten Schulen um 28\% höher ist als in sozioökonomisch benachteiligten Schulen (18 gegenüber 14 Schüler je Lehrkraft). Eine ähnliche Situation ist in Belgien, Kroatien, Griechenland, Island, Italien, Kasachstan, Luxemburg, Montenegro, der Russischen Föderation und Slowenien zu beobachten. In Österreich ist die Schüler/LehrerQuote in sozioökonomisch begünstigten Schulen niedriger und der Anteil der Lehrkräfte mit Hochschulabschluss höher als in sozioökonomisch benachteiligten Schulen. Das heißt, dass in sozioökonomisch benachteiligten Schulen mehr Schüler auf eine Lehrkraft kommen und diese Lehrkräfte in der Regel einen niedrigeren Bildungsabschluss besitzen. Eine ähnliche Situation ist in Rumänien, Shanghai (China) und Uruguay festzustellen.

Für viele Länder stellt es immer noch eine große Herausforderung dar, eine gerechte Verteilung der Ressourcen zu gewährleisten, wenn auch nicht, was deren Quantität anbelangt, so doch in Bezug auf ihre Qualität. Wie Abbildung II.4.7 zeigt, erklären der sozioökonomische Hintergrund der Schüler und das sozioökonomische Profil der Schule einen großen Anteil der Varianz der Lehrerqualität zwischen den Schulen. In Kroatien, Liechtenstein, Luxemburg, den Niederlanden und Slowenien stehen zwischen $17 \%$ und $27 \%$ der Varianz der Lehrerqualität zwischen den Schulen mit diesen Faktoren in Zusammenhang, in Österreich und Belgien über 35\%. In Band IV wird diese Analyse weitergeführt, indem die Wechselbeziehungen zwischen sozioökonomischem Hintergrund und Ressourcen sowie Schulpolitik und -praxis eingehender untersucht werden.

\section{Schwieriges Schulumfeld}

In sozioökonomisch benachteiligten Schulen ist die Disziplin häufig schlecht. Wie aus Abbildung II.4.8 ersichtlich, sind die Unterschiede bei der Schuldisziplin zwischen sozioökonomisch begünstigten und sozioökonomisch benachteiligten Schulen in Kroatien, Ungarn, Shanghai (China) und Slowenien mit einer Differenz von mehr als der Hälfte einer Einheit auf dem Index der Schuldisziplin besonders ausgeprägt, wohingegen in Estland, Jordanien, Lettland, Norwegen, Peru und Thailand zwischen den Schulen keine erkennbaren, mit dem sozioökonomischen Profil der Schulen in Zusammenhang stehenden Unterschiede beim Grad der Disziplin festzustellen sind. Wie Abbildung II.4.9 zeigt, hängt die Disziplin in manchen Schulsystemen stark mit dem sozioökonomischen Status zusammen, während der Zusammenhang in anderen Systemen viel schwächer ist. Die Varianz zwischen den Schulsystemen bei der Stärke dieses Zusammenhangs deutet darauf hin, dass die von Schulsystemen und Schulen verfolgte Politik bei der Verstärkung oder Verringerung dieser Unterschiede eine Rolle spielt.

Wenngleich all diese Faktoren mehr oder weniger mit den Schülerleistungen zusammenhängen können, ist klar, dass sie nicht die Art von förderlichem Lernumfeld schaffen, das sozioökonomisch benachteiligte Schülerinnen und Schüler brauchen. Es ist schwer vorstellbar, wie es Schulen mit einem unzureichenden Grad an Disziplin gelingen soll, einen Ausgleich für den Mangel an Ressourcen und Unterstützung zu schaffen, unter dem sozioökonomisch benachteiligte Schülerinnen und Schüler zuhause u.U. leiden, und sie zu befähigen, ihr Potenzial auszuschöpfen.

\section{Lernmöglichkeiten außerhalb der Schule und Erwartungen der Eltern an die Schulen}

Die Eltern spielen in verschiedener Hinsicht eine wichtige Rolle bei der Bildung ihrer Kinder, indem sie u.a. zusätzliche Lernmöglichkeiten durch Programme außerhalb der Schule oder privaten Nachhilfeunterricht zur Verbesserung oder Unterstützung der schulischen Leistungen organisieren, hohe Erwartungen in ihre Kinder und die von ihnen besuchte Schule setzen, fordern, dass diese Erwartungen erfüllt werden, und Druck auf die Schulen ausüben, an die Schüler höhere Leistungsanforderungen zu stellen. In all diesen Bereichen steht der sozioökonomische Hintergrund in einem engen Zusammenhang mit den Bildungsressourcen im Elternhaus.

In allen Ländern und Volkswirtschaften, die an PISA 2012 teilgenommen haben, verbringen sozioökonomisch begünstigte Schülerinnen und Schüler in der Regel nach der Schule mehr Zeit mit Hausaufgaben oder selbstständigem Lernen. Der Zusammenhang zwischen dem sozioökonomischen Hintergrund eines Schülers und seinem Zeitaufwand für Hausaufgaben (für alle Fächer) ist in Belgien, Bulgarien, Ungarn, Italien, Japan, Shanghai (China), Chinesisch Taipeh und dem Vereinigten Königreich relativ stark (die Korrelation liegt bei über 0,3). Sozioökonomisch begünstigte Schülerinnen und Schüler verbringen in der Regel mehr Zeit mit Unterricht außerhalb der Schule, der von Privatunternehmen organisiert und von ihren Eltern bezahlt wird, als sozioökonomisch benachteiligte Schülerinnen und Schüler; besonders stark ist der Zusammenhang in Japan und Korea (mit einer Korrelation von über 0,3) (Abb. II.4.5). 
- Abbildung II.4.6 "

\section{Anzahl und Qualität der Lehrkräfte, nach sozioökonomischem Profil der Schulen}

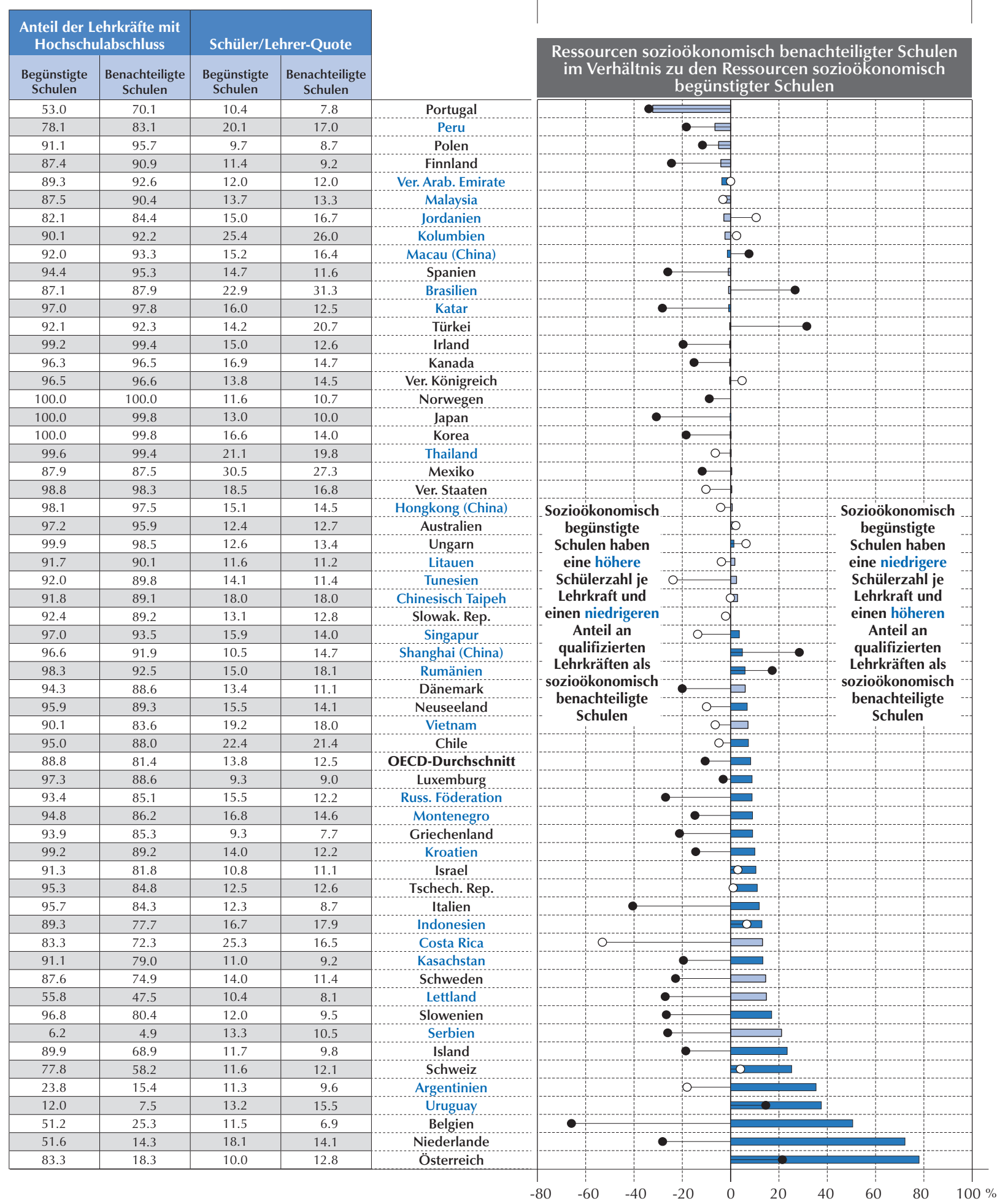

Anmerkung: Statistisch signifikante Unterschiede bei den Ressourcen zwischen Schülern sozioökonomisch begünstigter und sozioökonomisch benachteiligter Schulen sind durch einen dunkleren Farbton gekennzeichnet.

Die Länder und Volkswirtschaften sind in aufsteigender Reihenfolge nach dem Anteil an qualifizierten Lehrkräften in sozioökonomisch begünstigten Schulen im Verhältnis zum Anteil an qualifizierten Lehrkräften in sozioökonomisch benachteiligten Schulen angeordnet.

Quelle: OECD, PISA-2012-Datenbank, Tabelle II.4.8 und II.4.9.

StatLink त्नाls http://dx.doi.org/10.1787/888932964851 
- Abbildung II.4.7

Unterschiede bei der Lehrerqualität, erklärt durch das sozioökonomische Profil der Schüler und der Schulen

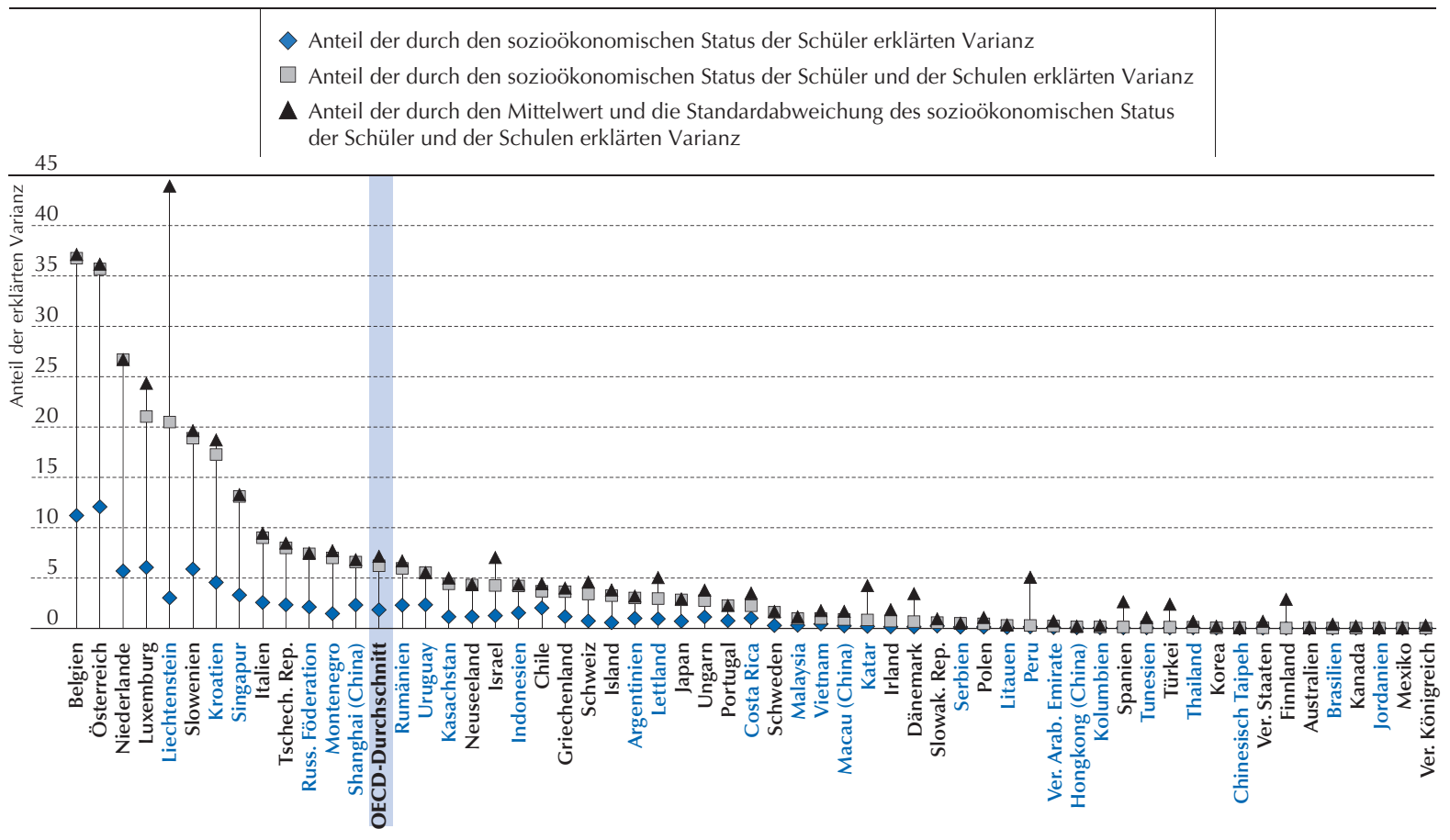

Die Länder und Volkswirtschaften sind in absteigender Reihenfolge nach der Varianz des Prozentsatzes der Lehrkräfte mit Hochschulabschluss angeordnet, die durch den sozioökonomischen Status der Schüler und der Schulen erklärt ist.

Quelle: OECD, PISA-2012-Datenbank, Tabelle II.4.9.

StatLink a्गारु http://dx.doi.org/10.1787/888932964851

\section{- Abbildung II.4.8 "}

\section{Unterschiede bei der Schuldisziplin, nach sozioökonomischem Profil der Schulen}

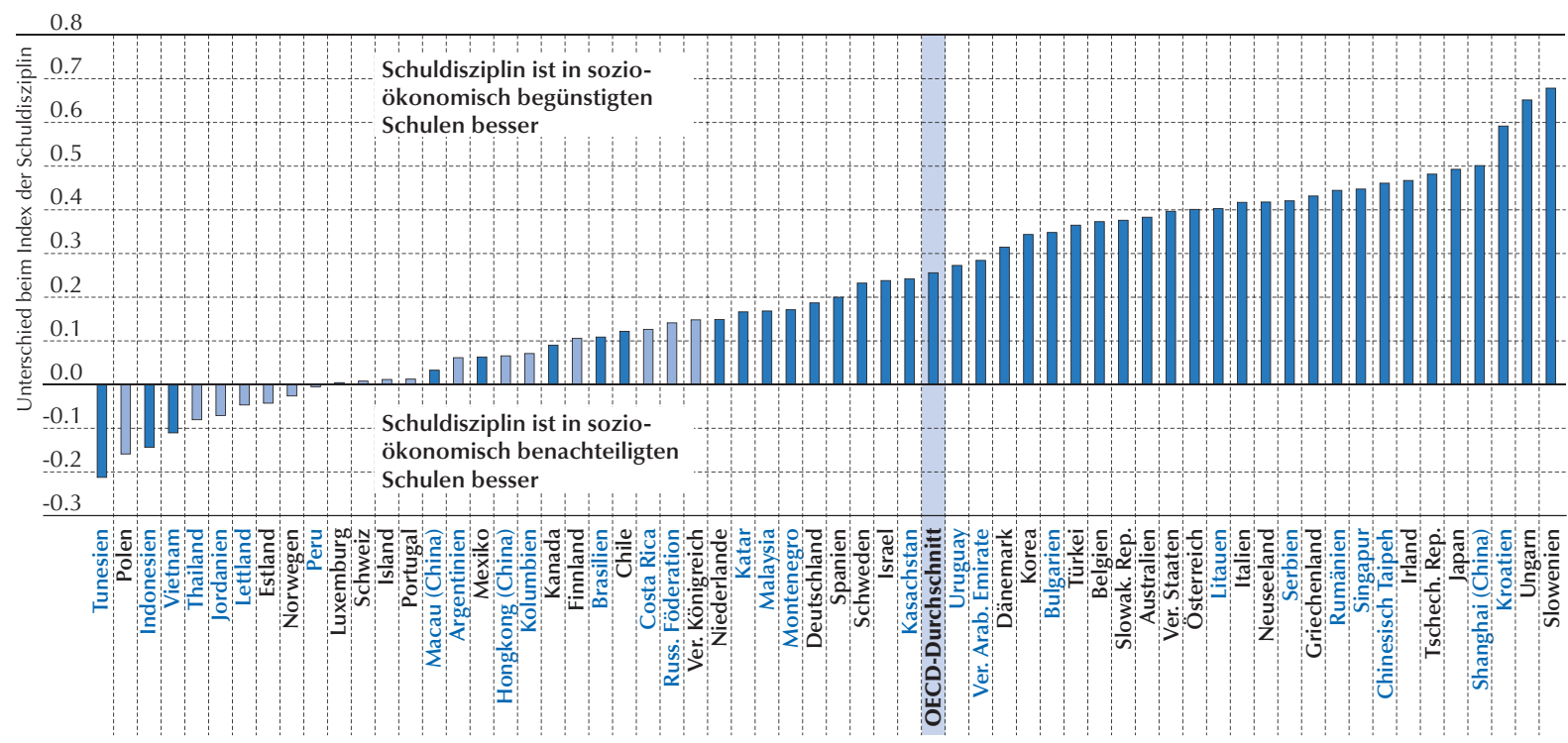

Anmerkung: Statistisch signifikante Unterschiede beim Index der Schuldisziplin zwischen Schülern sozioökonomisch benachteiligter und sozioökonomisch begünstigter Schulen sind durch einen dunkleren Farbton gekennzeichnet.

Die Länder und Volkswirtschaften sind in absteigender Reihenfolge nach dem Unterschied zwischen sozioökonomisch benachteiligten und sozioökonomisch begünstigten Schulen angeordnet.

Quelle: OECD, PISA-2012-Datenbank, Tabelle II.4.10.

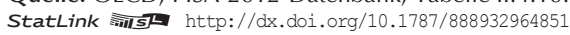


- Abbildung II.4.9 "

Unterschiede bei der Schuldisziplin, erklärt durch das sozioökonomische Profil der Schüler und der Schulen

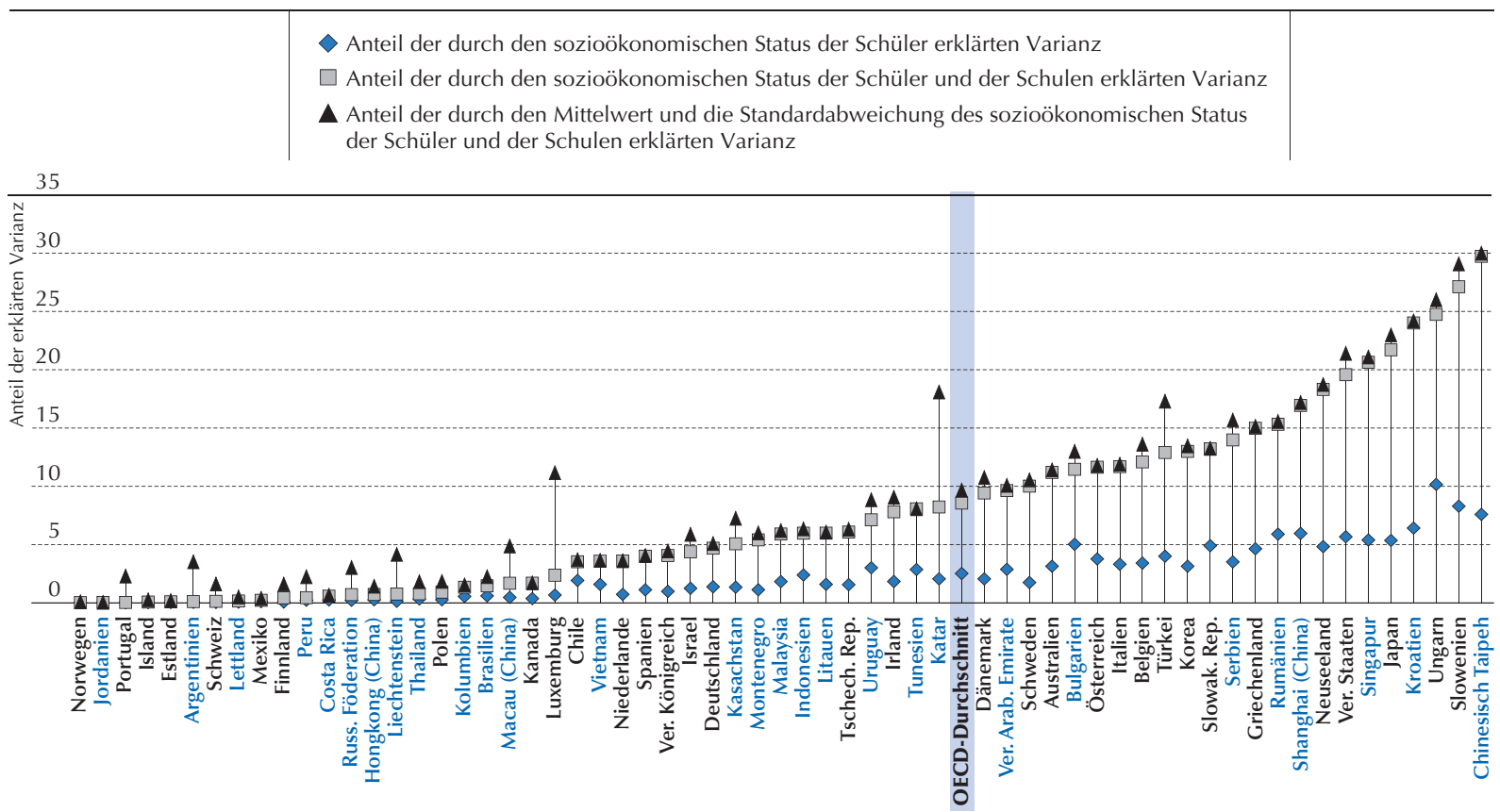

Die Länder und Volkswirtschaften sind in absteigender Reihenfolge nach der Varianz des Index der Schuldisziplin angeordnet, die durch den sozioökonomischen Status der Schüler und der Schulen, erklärt ist.

Quelle: OECD, PISA-2012-Datenbank, Tabelle II.4.10.

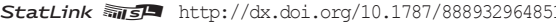

Die Wünsche der Eltern für die Bildung ihrer Kinder hängen ebenfalls stark mit dem sozioökonomischen Status zusammen. Die Eltern sozioökonomisch begünstigter Schülerinnen und Schüler haben höhere Erwartungen im Hinblick auf die Bildung ihrer Kinder als die Eltern sozioökonomisch benachteiligter Schülerinnen und Schüler. Sozioökomisch bessergestellte Eltern üben auch mehr Druck auf die Schulen aus, höhere Leistungsanforderungen an die Schüler zu stellen. In allen Ländern und Volkswirtschaften (außer Hongkong-China, Indonesien, Liechtenstein, Luxemburg, Montenegro und der Schweiz) besuchen sozioökonomisch begünstigtere Schülerinnen und Schüler zumeist Schulen, in denen die Schulleitungen im Fragebogen folgende Aussage ankreuzten:, Es gibt einen ständigen Druck von Seiten vieler Eltern, die von unserer Schule erwarten, dass wir sehr hohe Leistungsanforderungen stellen und sicherstellen, dass diese von unseren Schülerinnen/ Schülern erreicht werden“ (Abb. II.4.5).

\section{LERNMÖGLICHKEITEN, RESSOURCEN, LEISTUNGEN UND SOZIOÖKONOMISCHER STATUS}

Die Schülerleistungen hängen mit dem sozioökonomischen Status zusammen, sowohl auf Schul- als auch auf Schülerebene, sowie mit den Ressourcen und den Lernmöglichkeiten, die den Schülern und den Schulen zur Verfügung stehen. Im OECD-Raum sind 49\% der Leistungsunterschiede zwischen Schülern, die verschiedene Schulen besuchen, durch Unterschiede beim Zugang zu Lernmöglichkeiten und Ressourcen bedingt. Der durchschnittliche Leistungsunterschied in Mathematik zwischen sozioökonomisch begünstigteren und sozioökonomisch weniger begünstigten Schulen sinkt nach Berücksichtigung dieser Unterschiede von 69 auf 35 Punkte. Unterschiede bei Lernmöglichkeiten und Ressourcen sind auch für 39\% der zwischen Schülern derselben Schule zu beobachtenden Leistungsunterschiede verantwortlich. Unterschiede bei der Schuldisziplin erklären 17\% der Leistungsunterschiede, und auf die Lehrerqualität sind $8 \%$ der Leistungsunterschiede zurückzuführen (Tabelle II.4.9). Abbildung II.4.10 zeigt die zwischenschulischen Leistungsunterschiede vor und nach Berücksichtigung der zwischen Schülern und zwischen Schulen zu beobachtenden Unterschiede bei Lernmöglichkeiten und Bildungsressourcen für die Länder mit verfügbaren Daten 5 . 
- Abbildung II.4.10 "

\section{Leistungsunterschiede, die mit Unterschieden beim Kontakt mit reiner Mathematik und bei den Ressourcen zusammenhängen ${ }^{1}$}

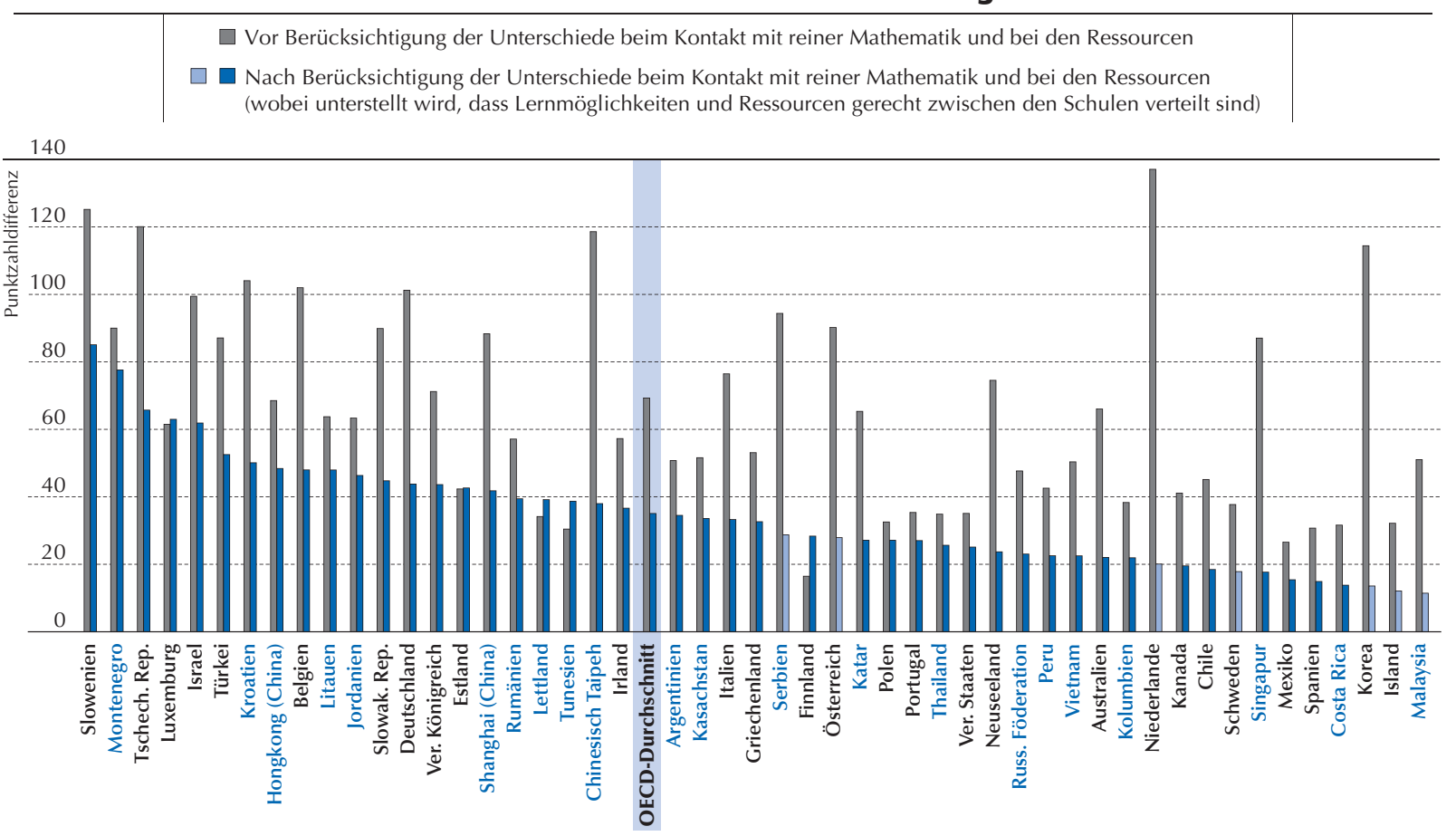

Anmerkung: Statistisch signifikante Punktzahldifferenzen sind durch einen dunkleren Farbton gekennzeichnet.

1. Punktzahldifferenzen zwischen Schulen, die sich auf dem PISA-Index des wirtschaftlichen, sozialen und kulturellen Status um eine Einheit unterscheiden.

Die Länder und Volkswirtschaften sind in absteigender Reihenfolge nach der Punktzahldifferenz zwischen zwei Schülern in Schulen, die sich auf dem PISA-Index des wirtschaftlichen, sozialen und kulturellen Status um eine Einheit unterscheiden, nach Berücksichtigung der Unterschiede bei Lernmöglichkeiten und Ressourcen, angeordnet.

Quelle: OECD, PISA-2012-Datenbank, Tabelle II.4.11.

StatLink त्ञात्रा http://dx.doi.org/10.1787/888932964851

\section{TEILNAHME AN VORSCHULBILDUNG}

Viele der Ungleichheiten, die innerhalb der Schulsysteme existieren, sind bereits bei der Einschulung der Schüler vorhanden und bestehen während der Schullaufbahn fort (Alexander, Entwisle und Olson, 1997; Downey, von Hippel und Broh, 2004). Da diese Ungleichheiten in der Regel bei Nichtteilnahme an Bildung zunehmen, trägt ein frühzeitiger Eintritt ins Schulsystem möglicherweise dazu bei, sie zu verringern. Bei einem frühzeitigen Eintritt in den vorschulischen Bereich sind Kinder besser auf die formale Bildung vorbereitet und schließen diese eher erfolgreich ab.

In Abbildung II.4.11 ist der Leistungsvorsprung in Mathematik von Schülerinnen und Schülern, die angaben, dass sie über ein Jahr an Vorschulbildung teilgenommen hatten, gegenüber solchen, bei denen das nicht der Fall war, dargestellt, sowohl vor als auch nach Berücksichtigung des sozioökonomischen Hintergrunds der Schülerinnen und Schüler. In allen Ländern mit verfügbaren Daten, außer in Estland und Lettland, lagen die Leistungen der Schülerinnen und Schüler, die über ein Jahr an Vorschulbildung teilgenommen hatten, über denen der Schülerinnen und Schüler, bei denen dies nicht der Fall war. An dieser Feststellung ändert sich in allen Ländern mit verfügbaren Daten (außer in Estland und Lettland) auch nach Berücksichtigung des sozioökonomischen Hintergrunds nichts. Im Durchschnitt der OECD-Länder beläuft sich der Vorsprung vor Berücksichtigung des sozioökonomischen Hintergrunds auf mehr als 53 Punkte und nach Berücksichtigung des sozioökonomischen Hintergrunds auf 31 Punkte. Der Unterschied zwischen diesen beiden Werten legt den Schluss nahe, dass eine mehr als einjährige Teilnahme an Vorschulbildung in gewissem Maße mit dem sozioökonomischen Hintergrund assoziiert ist; unabhängig davon besteht jedoch schon ein enger Zusammenhang zwischen der vorschulischen Bildung und den Leistungen im Alter von 15 Jahren. Schülerinnen und Schüler, die nicht an Vorschulbildung teilgenommen haben, erzielen mit 1,84-mal größerer Wahrscheinlichkeit Ergebnisse am unteren Ende der Leistungsverteilung. 
- Abbildung II.4.11

Leistungsunterschiede im Bereich Mathematik, nach Teilnahme an Vorschulbildung

Zwischen Schülern, die über ein Jahr an Vorschulbildung teilgenommen haben, und solchen, bei denen dies nicht der Fall war

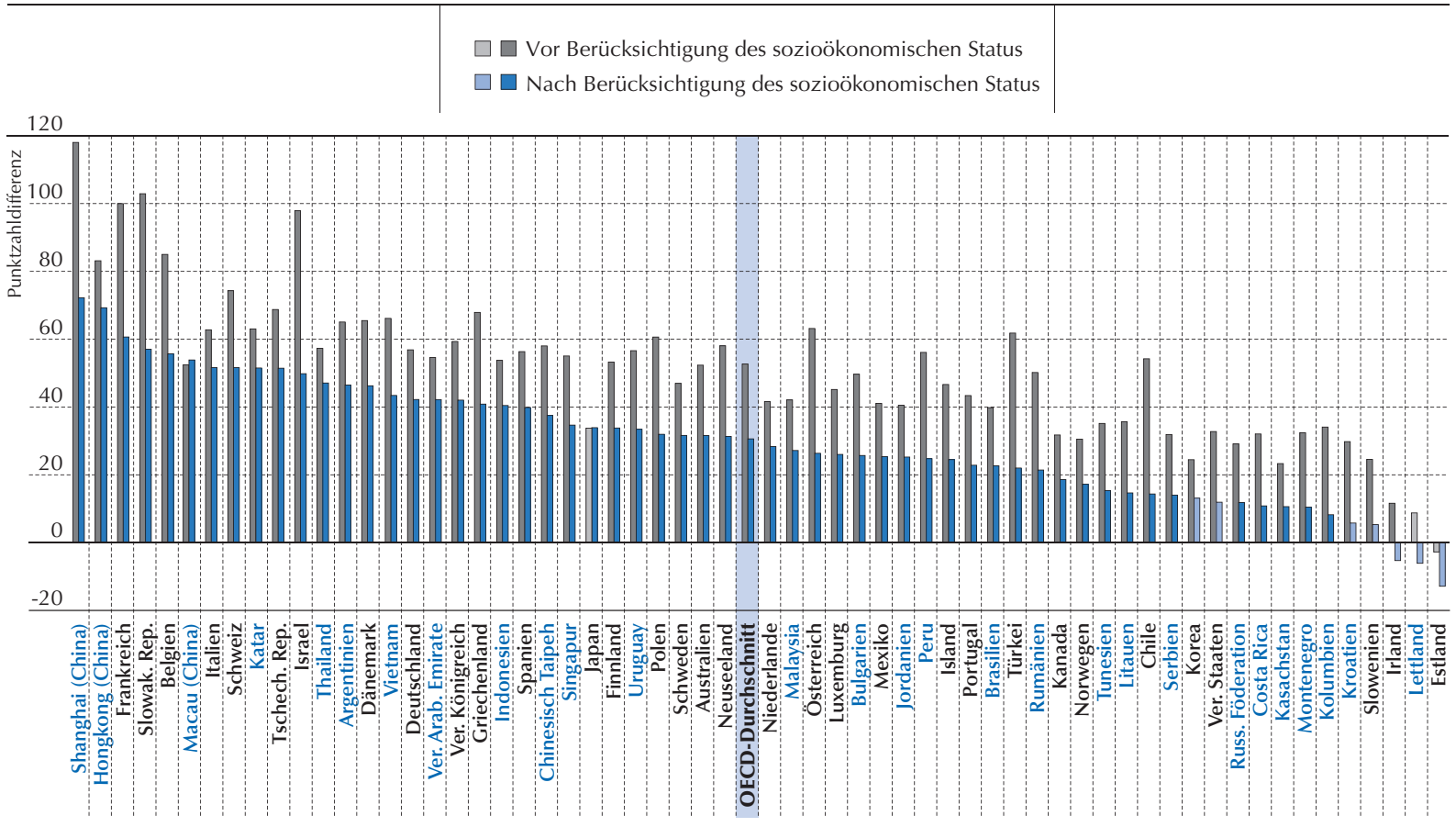

Anmerkung: Statistisch signifikante Punktzahldifferenzen sind durch einen dunkleren Farbton gekennzeichnet.

Die Länder und Volkswirtschaften sind in absteigender Reihenfolge nach der Punktzahldifferenz im Bereich Mathematik zwischen Schülern, die angaben, dass sie über ein Jahr an Vorschulbildung teilgenommen haben (ISCED 0), und solchen, bei denen dies nicht der Fall war, nach Berücksichtigung des sozioökonomischen Hintergrunds angeordnet.

Quelle: OECD, PISA-2012-Datenbank, Tabelle II.4.12.

StatLink त्ञाI

In Frankreich und der Slowakischen Republik erzielen Schülerinnen und Schüler, die angaben, dass sie über ein Jahr an Vorschulbildung teilgenommen haben, im Mathematiktest mindestens 100 Punkte mehr als Schülerinnen und Schüler, bei denen dies nicht der Fall war. In Frankreich haben lediglich 2\% der Schülerinnen und Schüler an keiner Form vorschulischer Bildung teilgenommen, wohingegen 92\% über ein Jahr an Vorschulbildung teilgenommen haben. Die Schülerinnen und Schüler, die nicht an Vorschulbildung teilgenommen haben, stammen großenteils aus sozioökonomisch benachteiligten Familien. Selbst nach Berücksichtigung des sozioökonomischen Hintergrunds der Schüler besteht der starke Zusammenhang zwischen der Teilnahme an Vorschulbildung und den Leistungen fort, die Leistungsunterschiede gehen jedoch um die Hälfte zurück. Die Situation ist in Shanghai (China) ähnlich, wo sich der Leistungsabstand vor Berücksichtigung des sozioökonomischen Hintergrunds auf 118 Punkte beläuft, sich aber nach Berücksichtigung des sozioökonomischen Hintergrunds auf 72 Punkte verringert. Nach Berücksichtigung des sozioökonomischen Hintergrunds sind die Punktzahldifferenzen zwischen Schülern, die nicht an vorschulischer Bildung teilgenommen haben, und denjenigen, die über ein Jahr lang an Vorschulbildung teilgenommen haben, in Belgien, der Tschechischen Republik, Frankreich, Hongkong (China), Italien, Macau (China), Katar, Shanghai (China), der Slowakischen Republik und der Schweiz am größten (mindestens 50 Punkte). In dieser Ländergruppe beträgt die Populationsrelevanz in allen Ländern allerdings weniger als 5\% (OECD-Durchschnitt der Populationsrelevanz), außer in Shanghai (China) (7\%), der Slowakischen Republik (11\%) und Katar (17\%) (Tabelle II.4.12).

Abbildung II.4.12 zeigt die Länder, in denen die Beteiligungsquoten verhältnismäßig niedrig sind (d.h. wo der Anteil der Schüler, die nicht an Vorschulbildung teilgenommen haben, hoch ist) und das relative Risiko schwacher schulischer Leistungen bei den Schülern, die nicht an vorschulischer Bildung teilgenommen haben, besonders hoch ist. Indonesien und die Türkei weisen ein hohes relatives Risiko und sehr niedrige Beteiligungsquoten auf. In Kroatien, Litauen, Montenegro, Katar und Tunesien sind ebenfalls niedrige Beteiligungsquoten und ein relatives Risiko, das nicht unerheblich ist, selbst wenn es unter dem OECD-Durchschnitt liegt, zu beobachten. Dagegen ist das relative Risiko in Frankreich, Israel, Shanghai (China) und der Slowakischen Republik zwar sehr hoch, betrifft aber nur wenige Schülerinnen und Schüler. 
- Abbildung II.4.12 "

Vorschulbildung, Mathematikleistungen und sozioökonomischer Status der Schüler

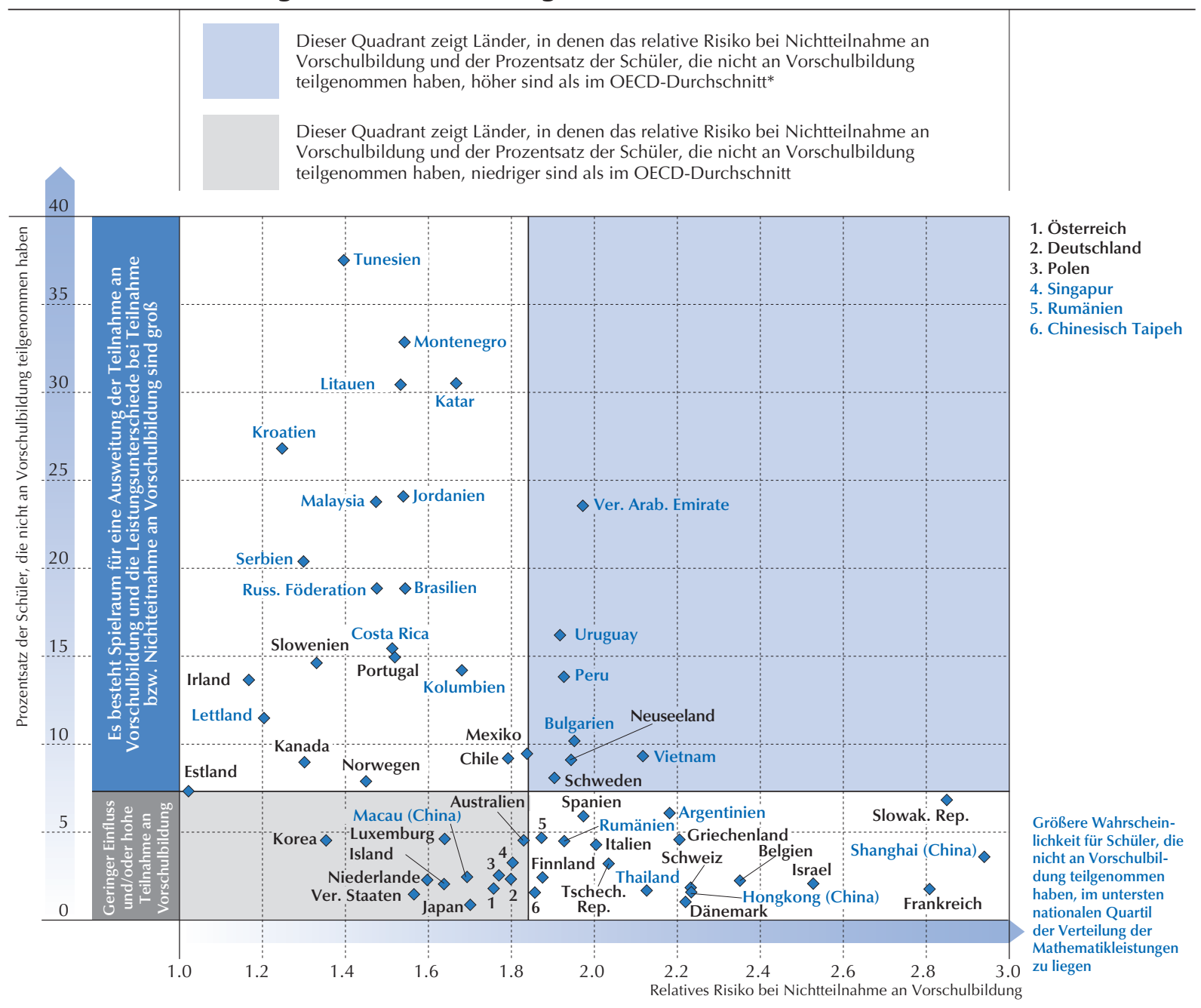

*Die Türkei und Indonesien weisen einen hohen Prozentsatz an Schülern auf, die nicht an Vorschulbildung teilgenommen haben (70\% bzw. 46\%), sowie ein hohes relatives Risiko. In Kasachstan ist ebenfalls eine hohe Quote der Nichtteilnahme (65\%), aber ein unter dem Durchschnitt liegendes relatives Risiko festzustellen. Diese Länder sind in der Abbildung nicht aufgeführt, um die Unterschiede zwischen den anderen Ländern deutlicher sichtbar zu machen.

Quelle: OECD, PISA-2012-Datenbank, Tabelle II.4.12

StatLink त्ञाI

In praktisch allen Ländern ist kein signifikanter Unterschied bei den Leistungen zwischen sozioökonomisch begünstigten und sozioökonomisch benachteiligten Schülerinnen und Schülern zu beobachten, wenn der Zusammenhang zwischen der Teilnahme an Vorschulbildung und den Mathematikleistungen im Alter von 15 Jahren untersucht wird (Tabelle II.4.13). In 32 OECD-Ländern und 22 Partnerländern und -volkswirtschaften ziehen sozioökonomisch benachteiligte und sozioökonomisch begünstigte Schülerinnen und Schüler in gleichem Maße Nutzen aus vorschulischer Bildung. In den OECD-Ländern, außer in Kanada und Estland, schneiden Schülerinnen und Schüler mit Migrationshintergrund, die an vorschulischer Bildung teilgenommen haben, genauso gut ab wie Schülerinnen und Schüler mit Migrationshintergrund, die nicht an Vorschulbildung teilgenommen haben (Tabelle II.4.14).

Neben der Teilnahme an vorschulischer Bildung haben viele andere Faktoren Einfluss auf die schulischen Leistungen der 15-Jährigen, und die hier angegebenen Schätzwerte sind nur begrenzt aussagefähig, weil viele dieser Faktoren nicht in ihnen berücksichtigt sind. Band IV dieses Berichts befasst sich eingehender mit diesen Aspekten und untersucht, wie sie sich seit PISA 2003 entwickelt haben. Die Trenddaten zeigen, dass Fragen der Chancengerechtigkeit im Zusammenhang mit vorschulischer Bildung in vielen Ländern an Brisanz gewinnen und dass sozioökonomisch benachteiligte Schülerinnen und Schüler, diejenigen also, die den größten Nutzen aus vorschulischer Bildung ziehen würden, in der Vorschulbildung noch immer unterrepräsentiert sind. 


\section{Anmerkungen}

1. Wegen einer detaillierten Analyse der Lernmöglichkeiten im Rahmen von PISA 2012, vgl. Schmidt et al. (2013).

2. Sozioökonomisch begünstigte (sozioökonomisch benachteiligte) Schulen sind Schulen, in denen der typische Schüler der Schule bzw. das sozioökonomische Profil der Schule über (unter) dem sozioökonomischen Status des typischen Schülers des Landes bzw. dem durchschnittlichen sozioökonomischen Status des Landes liegt. An jeder Schule nehmen 35 als Zufallsstichprobe ausgewählte Schülerinnen und Schüler an PISA teil (wegen näherer Einzelheiten vgI. PISA 2012 Technical Report (OECD, [erscheint demnächst]). Das sozioökonomische Profil der Schulen wird ausgehend von den Angaben dieser Schüler ermittelt. Deshalb hängt die Genauigkeit der Schätzung von der Zahl der Schüler ab, die an den einzelnen Schulen tatsächlich an der Erhebung teilnehmen, sowie von der Variationsbreite ihrer Antworten. Bei der Einstufung der Schulen in sozioökonomisch begünstigte, sozioökonomisch benachteiligte oder sozioökonomisch durchschnittliche Schulen wurde diese Frage der Genauigkeit berücksichtigt. Wenn der Unterschied zwischen dem sozioökonomischen Profil einer Schule und dem sozioökonomischen Status des typischen Schülers des Landes (dem durchschnittlichen sozioökonomischen Status auf Ebene des Landes) nicht statistisch signifikant war, wurde die Schule als Schule mit durchschnittlichem sozioökonomischem Profil eingestuft. Wenn das Profil einer Schule statistisch signifikant über dem durchschnittlichen sozioökonomischen Status des Landes lag, wurde die Schule als sozioökonomisch begünstigte Schule eingestuft. Wenn das Profil der Schule unter dem durchschnittlichen sozioökonomischen Status des Landes lag, wurde die Schule als sozioökonomisch benachteiligte Schule eingestuft.

3. Diese Ergebnisse hängen auch davon ab, wie die Schulen in den einzelnen Ländern definiert und organisiert sind und welche Einheiten für die Stichprobenziehung gewählt wurden. In einigen Ländern z.B. wurden einige der Schulen in der PISA-Stichprobe als Verwaltungseinheiten definiert (selbst wenn sie, wie in Italien, mehrere geografisch getrennte Einrichtungen umfassen); in anderen Ländern wurden sie als jene Teile größerer Bildungseinrichtungen definiert, die von 15-Jährigen besucht werden; in manchen Ländern wurden Schulen wiederum als Schulgebäude definiert, in wieder anderen dagegen aus Sicht der Schulorganisation (z.B. als Einheiten, die eine eigene Schulleitung haben). Der PISA 2012 Technical Report (OECD, erscheint demnächst) liefert einen Überblick über die verschiedenen Methoden der Definition der Schulen. Wegen der Art und Weise, in der die Schülerstichprobe erhoben wurde, ist in der Varianz innerhalb der Schulen sowohl die Varianz zwischen verschiedenen Klassen als auch zwischen verschiedenen Schülerinnen und Schülern enthalten. In Slowenien ist die primäre Stichprobeneinheit definiert als eine Gruppe von Schülerinnen und Schülern, die demselben Bildungsgang innerhalb einer Schule folgen. In diesem besonderen Fall entspricht die Varianz zwischen den Schulen tatsächlich der Varianz innerhalb einer Schule, aber zwischen unterschiedlichen Bildungsgängen.

4. Dies sind die Ergebnisse einer einfachen Varianzzerlegung eines Drei-Ebenen-Modells, bei dem Schüler in Schulen und Schulen in Länder geschachtelt sind. Die Ergebnisse basieren auf der gepoolten Stichprobe aller Länder und Volkswirtschaften, die an PISA 2012 teilgenommen haben.

5. Alle in Tabelle II.4.11 gezeigten Modelle berücksichtigen den sozioökonomischen Status auf Schüler- und Schulebene. Dann werden individuelle Schülermerkmale (Geschlecht, Migrationsstatus und zu Hause gesprochene Sprache) sowie der Schulstandort einbezogen. Diese Variablen werden in allen Einzelmodellen verwendet. Anschließend kommen nacheinander Variablen auf Schul- und Schülerebene hinzu, die dieVerfügbarkeit und die Qualität der Ressourcen messen. Am Ende werden alleVariablen in einem einzigen Modell kombiniert. In einigen Fällen sind auf Ebene der Schüler, der Schulen und der Länder keine Daten für manche dieser Variablen verfügbar, deshalb sind sie im kombinierten Modell nicht enthalten. Im Anschluss daran wird ein Basismodell präsentiert, in dem nur der sozioökonomische Status auf Schüler- und Schulebene berücksichtigt wird und in dem alle Schüler, Schulen und Länder, für die keine Daten vorliegen, ausgeklammert wurden, was einen direkteren Vergleich mit den Schätzwerten des kombinierten Modells ermöglicht. In Abbildung II.4.10 werden die Ergebnisse dieser beiden zuletzt genannten Modelle, des kombinierten Modells und des Basismodells, dargestellt; alle Beobachtungen mit fehlenden Werten wurden gelöscht.

\section{Literaturverzeichnis}

Alexander, K.L., D.R. Entwisle und L.S. Olson (2007), "Lasting Consequences of the Summer Learning Gap", American Sociological Review, Vol. 72, S. 167-180.

Carroll, J.B. (1963), "A model of School Learning", Teachers College Record, Vol. 64, No. 8, S. 723-733.

Downey D. B., P.T. von Hippel und B.A. Broh (2004), "Are Schools the Great Equalizer? Cognitive Inequality during the Summer Months and the School Year", American Sociological Review, Oktober 2004, Vol. 69, No. 5, S. 613-635.

OECD (erscheint demnächst), PISA 2012 Technical Report, PISA, OECD Publishing.

Schmidt, W.H., L.S. Cogan und P. Zoido (2013), "Schooling Matters: Opportunity to Learn in PISA 2012", OECD Education Working Papers, No. 95, OECD Publishing, http://dx.doi.org/10.1787/5k3vOhldmchl-en.

Schmidt, W.H., et al. (2001), Why Schools Matter: A Cross-National Comparison of Curriculum and Learning, Jossey-Bass, San Francisco.

Sykes, G., B. Schneider und D.N. Plank (2009), Handbook of Education Policy Research, Routledge, New York.

Wiley, D.E. und A. Harnischfeger (1974), “Explosion of a Myth: Quantity of Schooling and Exposure to Instruction, Major Educational Vehicles", Educational Researcher, Vol. 3, No. 4, S. 7-12. 



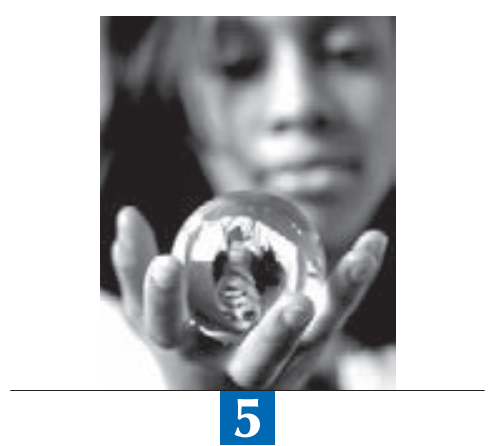

\section{Politikimplikationen der Frage der Bildungsgerechtigkeit}

In diesem Kapitel werden die verschiedenen Messgrößen der Bildungsgerechtigkeit analysiert, die im Rahmen von PISA erstellt werden, und verschiedene Politikoptionen zur Steigerung der Schülerleistungen und der Chancengerechtigkeit im Hinblick auf Bildungserträge und Bildungsmöglichkeiten untersucht. 
Der familiäre Hintergrund hat Einfluss auf den Bildungserfolg, und durch die Schule wird dieser Einfluss häufig verstärkt. Obwohl schlechte schulische Leistungen nicht automatisch von einer sozioökonomischen Benachteiligung herrühren, scheint der sozioökonomische Hintergrund der Schüler und der Schulen starke Auswirkungen auf die Lernerträge zu haben. In vielen Ländern reproduzieren die Schulen die bereits existierenden Muster sozioökonomischer Vorteile, anstatt eine ausgewogenere Verteilung der Bildungschancen und Bildungserträge zu gewährleisten, sei es, weil Familien aus begünstigten Milieus eher in der Lage sind, den Bildungseffekt der Schulen zu verstärken und zu verbessern, weil Schülerinnen und Schüler aus bessergestellten Familien bessere Schulen besuchen oder weil es den Schulen einfach leichter fällt, junge Menschen zu bilden und zu fördern, die aus begünstigten Verhältnissen stammen.

Abgesehen vom sozioökonomischen Hintergrund stehen noch andere Merkmale der Schülerinnen und Schüler in engem Zusammenhang mit ihren Leistungen in PISA. Merkmale wie die Familienstruktur, die berufliche Stellung der Eltern, ein etwaiger Migrationshintergrund sowie die im Elternhaus gesprochene Sprache sind nicht nur mit Leistungsunterschieden assoziiert, sondern auch miteinander verknüpft. Kinder von Eltern mit niedrigem Bildungsniveau, die in der Regel ebenfalls sozioökonomisch benachteiligt sind, gehören z.B. auch mit größerer Wahrscheinlichkeit zur Gruppe der Schüler, deren Eltern nicht berufstätig sind. In einigen Ländern fällt es Migranten schwer, eine Stelle zu finden, die ihrem Bildungs- und Kompetenzniveau entspricht; sie sind daher u.U. gezwungen, wenig angesehenen Berufen nachzugehen, was wiederum zur Folge hat, dass ihre Kinder einen ungünstigeren sozioökonomischen Hintergrund haben.

Kurz gesagt ist sozioökonomische Benachteiligung eng mit vielen der Schüler- und Schulmerkmale verknüpft, die mit dem Leistungsniveau assoziiert sind. Häufig ist es schwierig, den Effekt des sozioökonomischen Hintergrunds auf die Schülerleistungen vom Effekt anderer Faktoren zu trennen. Um aus den hier vorgestellten Ergebnissen Schlussfolgerungen für die Bildungspolitik und -praxis zu ziehen, ist es daher wichtig, die Wechselwirkungen zwischen all diesen Faktoren zu verstehen.

Die Ergebnisse der Analysen dieses Bandes zeigen im Allgemeinen, dass zwischen den Ländern große Unterschiede in Bezug darauf bestehen, inwieweit sich der sozioökonomische Hintergrund der Schüler auf deren Lernerfolg auswirkt, woraus sich schließen lässt, dass Länder und Volkswirtschaften es in der Tat schaffen können, ein hohes Leistungsniveau mit einem hohen Maß an Bildungsgerechtigkeit zu verbinden. Unterschiede zwischen den Ländern im Hinblick auf die Stärke des Zusammenhangs zwischen Faktoren auf Schüler- sowie Schulebene und den Schülerleistungen sowie dem sozioökonomischen Hintergrund machen deutlich, dass die von Schulsystemen und Schulen verfolgte Politik und Praxis Auswirkungen sowohl auf die Bildungsgerechtigkeit als auch auf die Schülerleistungen hat.

Dieser Band zeigt, wie wichtig es ist, bei der Analyse von Leistungsunterschieden den sozioökonomischen Hintergrund zu betrachten. Schüler aus Alleinerziehendenhaushalten erzielen beispielsweise in der Regel schlechtere Ergebnisse als Schüler aus anderen Familientypen; in vielen Ländern und Volkswirtschaften ist dieser Leistungsunterschied jedoch nicht mehr zu erkennen, wenn der sozioökonomische Hintergrund der Schüler berücksichtigt wurde. Auch Schulen mit einem hohen Anteil an Schülern mit Migrationshintergrund erzielen im Allgemeinen weniger gute Leistungen. Wenn jedoch der sozioökonomische Hintergrund der Schüler und vor allem das sozioökonomische Profil der Schulen selbst - d.h. der Grad der Konzentration sozioökonomischer Nachteile innerhalb der Schulen - berücksichtigt wird, kann ein großer Teil, wenn nicht sogar der gesamte Leistungsrückstand dieser Schulen durch den ungünstigen sozioökonomischen Hintergrund ihrer Schüler, sowohl derer mit Migrationshintergrund als auch derer ohne, erklärt werden.

Die Verteilung der Ressourcen auf die verschiedenen Schulen steht mit der Chancengerechtigkeit im Hinblick auf Bildungserträge und Bildungsmöglichkeiten in Zusammenhang. Von einigen Ausnahmen abgesehen, versuchen die OECD-Länder sozioökonomisch benachteiligten Schulen mindestens eine gleiche, wenn nicht höhere Zahl von Lehrkräften je Schüler zuzuteilen wie sozioökonomisch begünstigten Schulen. Dennoch berichten sozioökonomisch benachteiligte Schulen noch immer von großen Schwierigkeiten bei der Anwerbung qualifizierter Lehrkräfte. Mit anderen Worten führen mehr Ressourcen in sozioökonomisch benachteiligten Schulen nicht zwangsläufig zu einer besseren Qualität der Ressourcenausstattung. Diese Erkenntnis lässt vermuten, dass viele Schülerinnen und Schüler doppelt benachteiligt sind, weil sie selbst einen sozioökonomisch ungünstigen Hintergrund haben und zudem eine Schule mit einer schlechteren Ressourcenausstattung besuchen. In vielen Ländern besteht außerdem ein starker Zusammenhang zwischen dem sozioökonomischen Hintergrund der Schüler und ihrem Erfolg in der Schule. In einigen dieser Länder werden diese Disparitäten durch große Unterschiede beim sozioökonomischen Profil der Schülerpopulation der Schulen noch verstärkt.

Dieser Band liefert auch Evidenz für den engen Zusammenhang zwischen den Bildungsmöglichkeiten, die an den Angaben der Schüler zur Intensität ihres Kontakts und ihrer Vertrautheit mit reiner Mathematik gemessen wird, und dem 
sozioökonomischen Hintergrund der Schüler und ihren Leistungen. Einige Schulsysteme tendieren dazu, die Schüler entsprechend ihrer Leistungen auf verschiedene Klassen oder Schulen aufzuteilen. Daten aus PISA zeigen, dass die Schulsysteme, in denen die Schüler entsprechend ihrer Leistung auf verschiedene Schulen verteilt werden, in der Regel auch diejenigen sind, in denen eine Segregation der Schüler in Bezug auf ihren sozioökonomischen Hintergrund und die Intensität ihres Kontakts mit reiner Mathematik festzustellen ist. Dass sozioökonomisch benachteiligte Schüler in manchen Schulsystemen weniger mit grundlegenden mathematischen Konzepten in Kontakt kommen und weniger mit ihnen vertraut sind, deutet darauf hin, dass diese Schulsysteme größere Anstrengungen unternehmen müssen, um den Bildungsbedarf dieser Schülerinnen und Schüler vor Ende der Pflichtschulzeit zu decken.

In Finnland ist es Pädagogen dank Früherkennungsmechanismen, z.B. regelmäßigen individuellen Beurteilungen der Schülerinnen und Schüler durch mehrere Lehrergruppen, und besonderen Bildungsangeboten möglich, Bereiche zu identifizieren, in denen die Schüler vor besonderen Schwierigkeiten stehen. Leistungsschwache Schüler erhalten dann häufig bereits zu einem frühen Zeitpunkt ihrer schulischen Laufbahn die notwendige Unterstützung, d.h. bevor die Probleme akut werden und sie ihren Bildungsweg nicht mehr im selben Tempo fortsetzen können wie ihre Mitschüler. In anderen Schulsystemen müssen solche Schüler hingegen Klassen wiederholen, so dass sie immer wieder dieselben für sie nicht ausreichenden Bildungsangebote erhalten, bis es dann nicht mehr möglich ist, sie weiter Klassen wiederholen zu lassen. In Israel (vgl. Kasten IV.1.4) und Deutschland (Kasten II.3.2) wurden Programme konzipiert, die Schülern mit Migrationshintergrund oder aus Minderheitengruppen mehr Lernchancen bieten, indem für sie zusätzliche Unterrichtsstunden eingerichtet werden (Deutschland) oder sie die Möglichkeit erhalten, an kleineren Unterrichtsgruppen teilzunehmen (Israel).

Die in diesem Bericht durchgeführte Analyse der Leistungsfähigkeit der Schulen stützt sich auf Daten, die das Schulangebot gegen Ende der Primar- oder in der Sekundarstufe beschreiben. Eine Erhebung wie PISA bewertet jedoch die gesamte Lernentwicklung der Schüler, so dass die Ergebnisse eines Landes in PISA - oder jeder anderen Erhebung dieser Art - auch von der Qualität der Betreuung und den Anregungen abhängen, die die Schülerinnen und Schüler in ihrer frühen Kindheit und den Vorschuljahren erhalten haben, sowie von den Lernmöglichkeiten, die ihnen sowohl in der Schule als auch zu Hause von der Elementar- bis in die Sekundarschulzeit geboten wurden.

Die Verbesserung von Bildungsqualität und Bildungsgerechtigkeit erfordert daher eine langfristig orientierte und breit angelegte Perspektive. Für einige Länder kann das bedeuten, dass eine gesunde Entwicklung der Kleinkinder gesichert oder die frühkindliche Bildung, Betreuung und Erziehung verbessert werden muss. In anderen Ländern könnten sozioökonomische Reformen mit dem Ziel angezeigt sein, den Familien eine bessere Betreuung ihrer Kinder zu ermöglichen. Und in vielen Ländern werden hierfür wohl intensivere Anstrengungen zur Verstärkung der sozioökonomischen Integration und zur Verbesserung des Bildungsangebots der Schulen unternommen werden müssen.

\section{ZUSAMMENHANGSMUSTER ZWISCHEN SCHÜLERLEISTUNGEN UND SOZIOÖKONOMISCHEM HINTERGRUND}

Australien, Kanada, Estland, Finnland, Hongkong (China), Japan, Korea, Liechtenstein und Macau (China) zeichnen sich durch über dem OECD-Durchschnitt liegende Durchschnittsergebnisse und einen schwachen Zusammenhang zwischen dem sozioökonomischen Hintergrund und den Schülerleistungen aus (Stärke der sozioökonomischen Gradiente). In Vietnam entspricht die Stärke dieses Zusammenhangs in etwa dem Durchschnitt, während die Leistungsunterschiede, die mit sozioökonomischen Disparitäten assoziiert sind (Steigung der sozioökonomischen Gradiente), weniger groß sind als im Durchschnitt (Abb. II.5.1a). Diese Länder verbinden ein hohes Leistungsniveau mit einer großen Chancengerechtigkeit in der Bildung, was zeigt, dass diese beiden Aspekte nicht unvereinbar sind.

Das Hauptaugenmerk liegt in dieser Untersuchung zwar auf dem sozioökonomischen Kontext, viele dieser Länder erzielen jedoch auch ein großes Maß an Chancengerechtigkeit in anderen Bereichen. Sozioökonomische Nachteile sind vielfach eine Proxy-Variable bzw. ein Anhaltspunkt für andere Formen der Benachteiligung. Dies ist allerdings nicht immer der Fall. In Finnland z.B., wo die Leistungsunterschiede zwischen sozioökomisch begünstigten und benachteiligten Schülern gering sind, erzielen Schüler mit Migrationshintergrund - deutlich - schlechtere Ergebnisse als Schüler ohne Migrationshintergrund, und dies selbst nach Berücksichtigung ihres sozioökonomischen Status. In Polen erzielen Schulen in ländlichen Gebieten wesentlich schlechtere Ergebnisse als Schulen in städtischen Räumen, und zwar unabhängig von ihrem sozioökonomischen Profil. 
Für leistungsschwache Schüler - Schüler also, die das Basisniveau, d.h. Stufe 2 auf der PISA-Mathematikskala, nicht erreichen - besteht die Gefahr, dass es ihnen später nicht gelingt, sich erfolgreich in den Arbeitsmarkt und die Gesellschaft insgesamt zu integrieren. Eine gerechte Gesellschaft ist nicht möglich, wenn ein erheblicher Prozentsatz der Schülerinnen und Schüler nicht mit den Anforderungen mithalten kann, die an sie gestellt werden. Das Gleiche gilt, wenn ein großer Prozentsatz der 15-Jährigen überhaupt nicht zur Schule geht.

Wie kann der Effekt des sozialen Hintergrunds abgeschwächt werden, damit alle Schülerinnen und Schüler ihr Potenzial ausschöpfen können? Die in diesem Band beschriebenen Zusammenhänge zwischen dem familiären Hintergrund und den Leistungen folgen in den einzelnen Ländern sehr unterschiedlichen Mustern; deshalb müssen die Strategien zur Verbesserung der Situation auf die jeweiligen Bedingungen abgestimmt werden. Die Abbildungen II.5.1a und b zeigen die wichtigsten Merkmale des Zusammenhangs zwischen dem sozioökonomischen Profil der Schüler und der Schulen und den Leistungen im Vergleich der verschiedenen Bildungssysteme.

In den Abbildungen II.5.2 bis II.5.13 sind für die verschiedenen Länder und Volkswirtschaften, die an PISA 2012 teilgenommen haben, die Durchschnittsergebnisse und die sozioökonomische Zusammensetzung der Schülerpopulation in den einzelnen Schulen dargestellt. Wie überall in diesem Band, wird die sozioökonomische Zusammensetzung der Schulen an der durchschnittlichen Position ihrer Schülerinnen und Schüler auf dem PISA-Index des wirtschaftlichen, sozialen und kulturellen Status (ESCS) gemessen. Jeder Kreis in diesen Abbildungen entspricht einer Schule, wobei sich die Größe des Kreises nach der Anzahl der 15-Jährigen richtet, die die Schule besuchen. Die Muster verdeutlichen, wie sehr die Schülerinnen und Schüler entsprechend ihrer Leistung oder ihres sozioökonomischen Status auf verschiedene Schulen aufgeteilt sind. Die Abbildungen zeigen die Gesamtleistungsunterschiede zwischen verschiedenen sozioökonomischen Gruppen bzw. die Gradiente des Zusammenhangs zwischen Leistungen und sozioökonomischem Hintergrund (dünne schwarze Linie). In den Abbildungen sind zudem die durchschnittlichen Leistungsunterschiede zwischen Schulen mit unterschiedlichem sozioökonomischem Profil dargestellt, und zwar durch die zwischenschulische Gradiente, die der grauen Linie entspricht, und die durchschnittliche innerschulische Gradiente, die der blauen Linie entspricht. Schulen, die oberhalb der zwischenschulischen Gradiente (graue Linie) angeordnet sind, erzielen bessere Leistungen, als der sozioökonomische Hintergrund ihrer Schüler erwarten ließe; Schulen, die unterhalb der zwischenschulischen Gradiente liegen, erzielen schlechtere Leistungen, als ausgehend vom sozioökonomischen Hintergrund ihrer Schüler zu erwarten wäre.

Die Abbildungen geben einen Überblick über die drei Ebenen, auf denen der Zusammenhang zwischen dem Hintergrund der Schüler und den Schülerleistungen zum Tragen kommt. Die erste bezieht sich auf die Stärke des Zusammenhangs zwischen Schülerleistungen und sozioökonomischem Status in einem bestimmten Land, was an dem Teil der Varianz der Leistungen der Schüler gemessen wird, der Unterschieden bei ihrem sozioökonomischen Hintergrund zuzuschreiben ist. Auf der zweiten Ebene zeigt sich, inwieweit die Varianz der Durchschnittsleistungen im Vergleich zwischen den Schulen auf die Varianz des durchschnittlichen sozioökonomischen Hintergrunds der Schülerpopulation der Schulen zurückzuführen ist. Auf der dritten Ebene drückt sich der Zusammenhang zwischen den Schülerleistungen und dem sozioökonomischen Status innerhalb bestimmter Schulen aus, d.h. inwieweit die Varianz der Schülerleistungen innerhalb einer bestimmten Schule sich aus Unterschieden beim sozioökonomischen Hintergrund der Schüler dieser Schulen erklärt. Ebenfalls von Bedeutung sind die sozioökonomische Varianz und die Gesamtleistungsunterschiede innerhalb eines Landes. Mit diesen Abbildungen ist es zwar nicht möglich, alle Ungleichheiten zu erfassen, die innerhalb einzelner Länder u.U. zu beobachten sind, sie können aber einen verlässlichen Anhaltspunkt für die Chancengerechtigkeit in der Bildung geben, vor allem aus internationaler Sicht.

Die Analyse dieser Verteilungsmuster kann politisch Verantwortlichen bei der Gestaltung von Maßnahmen zur Verbesserung der Chancengerechtigkeit in der Bildung helfen (Willms, 2006). Als mögliche Optionen, die auch miteinander kombiniert werden können, sind zu nennen:

- Behebung von Leistungsschwächen, ungeachtet des Hintergrunds der Schülerinnen und Schüler, mit Hilfe von Maßnahmen, die entweder auf leistungsschwache Schulen oder auf leistungsschwache Schüler innerhalb bestimmter Schulen zugeschnitten sind, je nachdem inwieweit das Problem der Leistungsschwäche auf bestimmte Schulen konzentriert ist. Wo die Leistungsunterschiede zwischen den Schulen relativ groß sind, können die Maßnahmen auf leistungsschwache Schulen ausgerichtet werden; wo sie relativ gering sind, können die Maßnahmen direkt auf leistungsschwache Schüler innerhalb einzelner Schulen abzielen. Entsprechende Maßnahmen umfassen häufig spezielle Lehrpläne oder zusätzliche Unterrichtsmittel für bestimmte Schüler auf der Basis des Niveaus ihrer schulischen Leistungen. In manchen Schulsystemen sind z.B. früh greifende Präventionsprogramme vorgesehen, die auf Kinder ausgerichtet sind, bei denen schon im vorschulischen Bereich die Gefahr eines späteren schulischen Versagens 
- Abbildung II.5.1a -

\section{Überblick über die PISA-Messgrößen der Bildungsgerechtigkeit}

\begin{tabular}{|c|c|c|c|c|c|}
\hline & \multicolumn{5}{|c|}{$\begin{array}{l}\text { Höhere Bildungsqualität oder -gerechtigkeit als im OECD-Durchschnitt } \\
\text { Kein statistischer Unterschied gegenüber dem OECD-Durchschnitt } \\
\text { Geringere Bildungsqualität oder -gerechtigkeit als im OECD-Durchschnitt }\end{array}$} \\
\hline & \multirow[b]{2}{*}{$\begin{array}{l}\text { Durchschnittliche } \\
\text { Schülerleistungen }\end{array}$} & \multirow[b]{2}{*}{$\begin{array}{r}\text { Prozentsatz } \\
\text { der Schüler } \\
\text { unter Stufe } 2 \\
\end{array}$} & $\begin{array}{c}\begin{array}{c}\text { Stärke des Zusammenhangs } \\
\text { zwischen Schülerleistungen } \\
\text { und sozioökonomischem } \\
\text { Status }\end{array} \\
\end{array}$ & $\begin{array}{l}\text { Leistungsunterschiede } \\
\text { nach sozioökonomischem } \\
\text { Status: Steigung der } \\
\text { sozioökonomischen } \\
\text { Gradiente }\end{array}$ & $\begin{array}{c}\text { Länge der Projektion der } \\
\text { Gradiente }\end{array}$ \\
\hline & & & $\begin{array}{l}\text { Prozentsatz der } \\
\text { erklärten Varianz der } \\
\text { Schülerleistungen }\end{array}$ & $\begin{array}{l}\text { Mit einem Anstieg } \\
\text { um eine Einheit beim } \\
\text { sozioökonomischen } \\
\text { Status assoziierte } \\
\text { Punktzahlveränderung }\end{array}$ & $\begin{array}{c}\text { Differenz zwischen dem } \\
\text { 95. und dem 5. Perzentil } \\
\text { des ESCS }\end{array}$ \\
\hline OECD-Durchschnitt & 494 & 23.1 & 14.8 & 39 & 2.83 \\
\hline Shanghai (China) & 613 & 3.8 & 15.1 & 41 & 3.00 \\
\hline Singapur & 573 & 8.3 & 14.4 & 44 & 2.98 \\
\hline Hongkong (China) & 561 & 8.6 & 7.5 & 27 & 3.10 \\
\hline Chinesisch Taipeh & 560 & 12.9 & 17.9 & 58 & 2.69 \\
\hline Korea & 554 & 9.2 & 10.1 & 42 & 2.38 \\
\hline Macau (China) & 538 & 10.9 & 2.6 & 17 & 2.94 \\
\hline Japan & 536 & 11.2 & 9.8 & 41 & 2.22 \\
\hline Liechtenstein & 535 & 14.1 & 7.6 & 28 & 3.02 \\
\hline Schweiz & 531 & 12.5 & 12.8 & 38 & 2.85 \\
\hline Niederlande & 523 & 14.9 & 11.5 & 40 & 2.41 \\
\hline Estland & 521 & 10.6 & 8.6 & 29 & 2.48 \\
\hline Finnland & 519 & 12.3 & 9.4 & 33 & 2.39 \\
\hline Kanada & 518 & 13.9 & 9.4 & 31 & 2.71 \\
\hline Polen & 518 & 14.5 & 16.6 & 41 & 2.74 \\
\hline Belgien & 515 & 19.1 & 19.6 & 49 & 2.75 \\
\hline Deutschland & 514 & 17.8 & 16.9 & 43 & 2.91 \\
\hline Vietnam & 511 & 14.3 & 14.6 & 29 & 3.84 \\
\hline Österreich & 506 & 18.8 & 15.8 & 43 & 2.72 \\
\hline Australien & 504 & 19.8 & 12.3 & 42 & 2.48 \\
\hline Irland & 501 & 17.0 & 14.6 & 38 & 2.65 \\
\hline Slowenien & 501 & 20.3 & 15.6 & 42 & 2.69 \\
\hline Dänemark & 500 & 17.0 & 16.5 & 39 & 2.57 \\
\hline Neuseeland & 500 & 22.8 & 18.4 & 52 & 2.58 \\
\hline Tschech. Rep. & 499 & 21.2 & 16.2 & 51 & 2.37 \\
\hline Frankreich & 495 & 22.5 & 22.5 & 57 & 2.54 \\
\hline Ver. Königreich & 494 & 21.9 & 12.5 & 41 & 2.53 \\
\hline Island & 493 & 21.6 & 7.7 & 31 & 2.55 \\
\hline Lettland & 491 & 20.1 & 14.7 & 35 & 2.77 \\
\hline Luxemburg & 490 & 24.5 & 18.3 & 37 & 3.48 \\
\hline Norwegen & 489 & 22.5 & 7.4 & 32 & 2.36 \\
\hline Portugal & 487 & 25.0 & 19.6 & 35 & 3.74 \\
\hline Italien & 485 & 24.8 & 10.1 & 30 & 3.13 \\
\hline Spanien & 484 & 23.7 & 15.8 & 34 & 3.26 \\
\hline Russ. Föderation & 482 & 24.1 & 11.4 & 38 & 2.34 \\
\hline Slowak. Rep. & 482 & 27.6 & 24.6 & 54 & 2.89 \\
\hline Ver. Staaten & 481 & 26.0 & 14.8 & 35 & 3.12 \\
\hline Litauen & 479 & 26.2 & 13.8 & 36 & 2.75 \\
\hline Schweden & 478 & 27.2 & 10.6 & 36 & 2.47 \\
\hline Ungarn & 477 & 28.2 & 23.1 & 47 & 3.02 \\
\hline Kroatien & 471 & 30.0 & 12.0 & 36 & 2.76 \\
\hline Israel & 466 & 33.7 & 17.2 & 51 & 2.56 \\
\hline Griechenland & 453 & 35.8 & 15.5 & 34 & 3.12 \\
\hline Serbien & 449 & 39.1 & 11.7 & 34 & 2.87 \\
\hline Türkei & 448 & 42.2 & 14.5 & 32 & 3.64 \\
\hline Rumänien & 445 & 41.0 & 19.3 & 38 & 3.01 \\
\hline Bulgarien & 439 & 43.9 & 22.3 & 42 & 3.23 \\
\hline Ver. Arab. Emirate & 434 & 46.5 & 9.8 & 33 & 2.79 \\
\hline Kasachstan & 432 & 45.5 & 8.0 & 27 & 2.34 \\
\hline Thailand & 427 & 50.0 & 9.9 & 22 & 3.79 \\
\hline Chile & 423 & 51.7 & 23.1 & 34 & 3.66 \\
\hline Malaysia & 421 & 52.0 & 13.4 & 30 & 3.24 \\
\hline Mexiko & 413 & 54.9 & 10.4 & 19 & 4.10 \\
\hline Montenegro & 410 & 56.9 & 12.7 & 33 & 2.82 \\
\hline Uruguay & 409 & 56.0 & 22.8 & 37 & 3.67 \\
\hline Costa Rica & 407 & 60.1 & 18.9 & 24 & 3.93 \\
\hline Albanien & 394 & 60.8 & $\mathrm{~m}$ & $\mathrm{~m}$ & $\mathrm{~m}$ \\
\hline Brasilien & 391 & 67.3 & 15.7 & 26 & 3.80 \\
\hline Argentinien & 388 & 66.7 & 15.1 & 26 & 3.60 \\
\hline Tunesien & 388 & 67.9 & 12.4 & 22 & 4.11 \\
\hline Jordanien & 386 & 68.7 & 8.4 & 22 & 3.16 \\
\hline Kolumbien & 376 & 74.0 & 15.4 & 25 & 3.83 \\
\hline Katar & 376 & 69.7 & 5.6 & 27 & 2.93 \\
\hline Indonesien & 375 & 75.9 & 9.6 & 20 & 3.60 \\
\hline Peru & 368 & 74.7 & 23.4 & 33 & 4.14 \\
\hline
\end{tabular}

Die Länder und Volkswirtschaften sind in absteigender Reihenfolge nach den Durchschnittsergebnissen im Bereich Mathematik angeordnet.

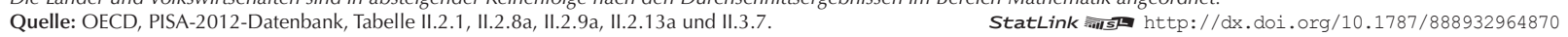


Abbildung II.5.1b "

\section{Überblick über die PISA-Messgrößen der Bildungsgerechtigkeit (Forts.)}

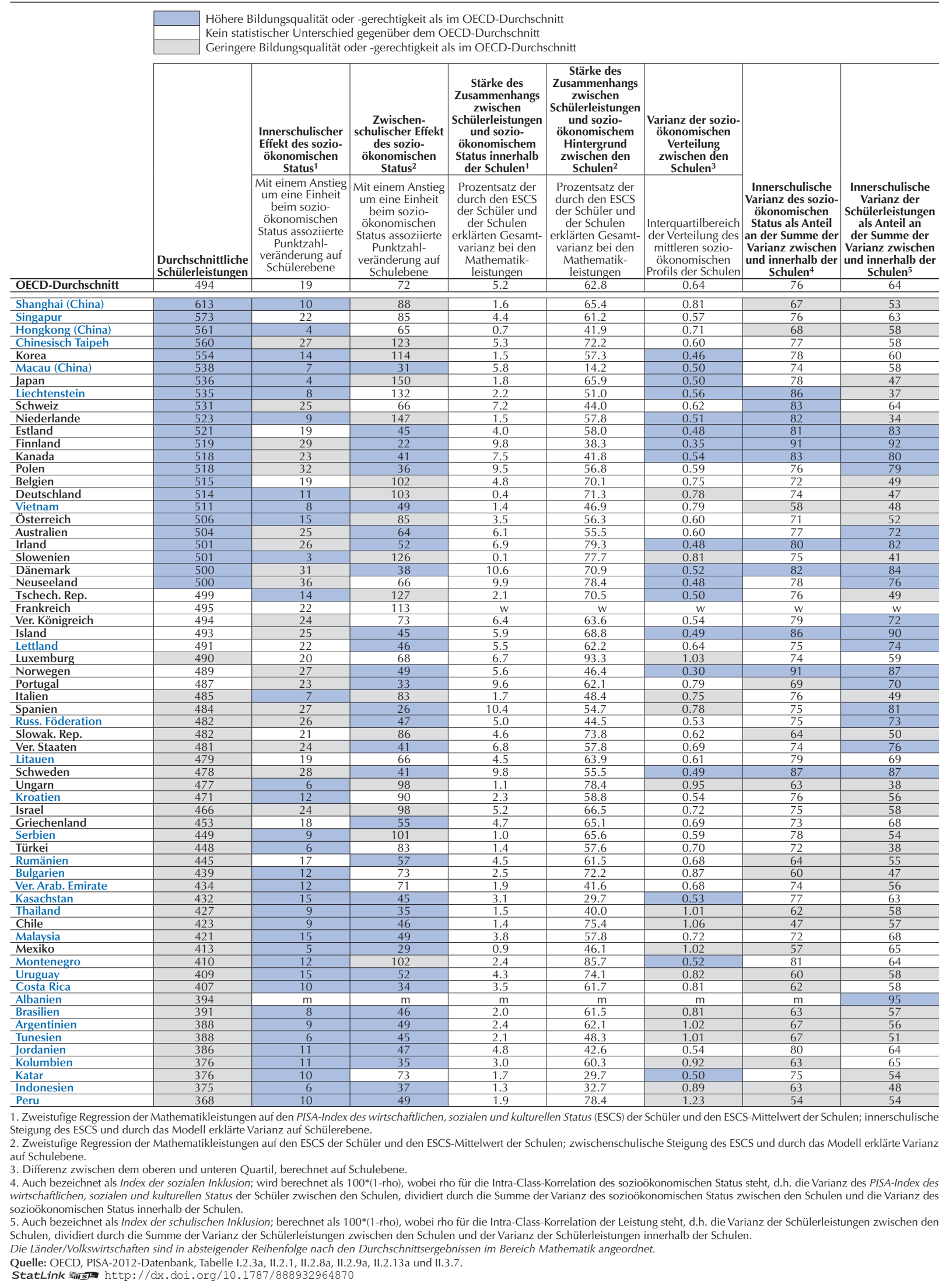


gesehen wird, während es in anderen Schulsystemen später einsetzende Präventions- oder Nachhilfeprogramme für Kinder gibt, denen es nicht gelingt, in den ersten Jahren der Grundschule mit dem normalen Unterrichtstempo Schritt zu halten. Einige leistungsorientierte Programme zielen darauf ab, Schülerinnen und Schülern mit besonders guten schulischen Leistungen auf ihre Bedürfnisse zugeschnittene Curricula anzubieten, z.B. Hochbegabtenprogramme. Klassenwiederholungen werden zuweilen ebenfalls als leistungsorientierte Maßnahmen betrachtet, da die Entscheidung, einen Schüler das Schuljahr wiederholen zu lassen, gewöhnlich auf dessen schulischer Leistung basiert. Vielfach werden den betreffenden Schülern bei einer Klassenwiederholung jedoch keine anderen Unterrichtsinhalte oder zusätzliche Unterrichtsressourcen geboten; deshalb fallen Klassenwiederholungen nicht unter die hier verwendete Definition einer leistungsorientierten Maßnahme. Wie in Band IV dieses Berichts aufgezeigt wird, sind Klassenwiederholungen eine kostspielige Option, die sich bei der Gestaltung von Maßnahmen zur Erhöhung der Leistung und der Bildungsgerechtigkeit nur selten empfiehlt. Leistungsorientierte Maßnahmen sind in der Regel auf den unteren Bereich des Leistungsspektrums ausgerichtet, unabhängig vom sozioökonomischen Hintergrund der Schüler; ihr Ziel besteht darin, leistungsschwachen Schülern dabei zu helfen, das Niveau ihrer Mitschüler zu erreichen. In diesem Band und in Band IV dieses Berichts wird beschrieben, wie beispielsweise Kolumbien (Kasten IV.4.3), Mexiko (Kasten II.2.4) und Polen (Kasten IV.2.1) die Informationsinfrastruktur in ihrem Bildungssystem verbessert haben, um Schüler bzw. Schulen, die vor besonderen Schwierigkeiten stehen, besser identifizieren zu können.

- Gezielte Ausrichtung auf Kinder aus benachteiligtem Milieu durch spezielle Lehrpläne, zusätzliche Unterrichtsressourcen oder wirtschaftliche Unterstützung. Solche Maßnahmen sind im Fall einer relativ starken sozioökonomischen Gradiente angezeigt, die die Leistungsvarianz zu einem wesentlichen Teil erklärt. Auch hier können die Maßnahmen wieder entweder auf der Schul- oder der Schülerebene ansetzen, je nach der Stärke der sozialen Gradiente zwischen den Schulen sowie dem Grad der Segregation der Schulen nach ihrem sozioökonomischen Profil. Manchmal werden die Schüler dabei auf der Basis anderer Risikofaktoren als ihrem sozioökonomischen Hintergrund ausgewählt, z.B. der Tatsache, dass sie erst seit kurzem im jeweiligen Land leben, dass sie einer ethnischen Minderheit angehören oder dass sie in einer ländlichen oder einkommensschwachen Gemeinde leben. Das distinktive Merkmal solcher Programme ist, dass die Schüler auf der Basis des sozioökonomischen Status ihrer Familie und nicht nach ihren kognitiven Fähigkeiten ausgewählt werden. Wie in den Kästen II.3.2 und IV.1.4 beschrieben, setzen Länder wie Deutschland und Israel gezielt bei Schülern mit Migrationshintergrund oder Schulen in kleinen, ländlichen Gemeinden an. Damit können sozioökonomische Nachteile zwar nicht direkt beseitigt werden, wegen der engen Zusammenhänge zwischen verschiedenen Ursachen sozioökonomischer Benachteiligung kann mit solchen Maßnahmen aber Ungleichheiten im Allgemeinen begegnet werden. Auf sozioökonomisch benachteiligte Kinder ausgerichtete Maßnahmen können auf die Erhöhung ihrer Schulleistungen abgestellt sein, sie können aber auch auf eine Verbesserung ihrer wirtschaftlichen Lage abzielen. Der Schwerpunkt liegt hier auf der Verbesserung der wirtschaftlichen Bedingungen von Schülern aus armen Familien, anstatt auf dem Angebot spezieller Lehrpläne oder zusätzlicher Unterrichtsressourcen. Gute Beispiele für derartige Maßnahmen sind an Auflagen geknüpfte Geldleistungen, wie es sie in Brasilien, Kolumbien und Mexiko gibt; dort werden Eltern finanziell unterstützt, wenn ihre Kinder zur Schule gehen. Kostenloser Schultransport oder kostenlose Mittagessensangebote für Schüler aus armen Familien sind weitere Beispiele solcher Maßnahmen. Die Zahlung von Transferleistungen an finanziell schlechtgestellte Familien ist generell eines der wichtigsten Politikinstrumente auf nationaler Ebene. Zwischen diesen kompensatorischen Maßnahmen und sozioökonomisch ausgerichteten Maßnahmen lässt sich nicht immer eine klare Trennlinie ziehen. Beispielsweise gibt es in einigen Ländern und Volkswirtschaften ausgleichende Finanzierungsmodalitäten, bei denen sich die Ressourcenallokation für die Schulen am sozioökonomischen Profil ihrer Schüler orientiert. In gewissem Sinne handelt es sich hier um kompensatorische Maßnahmen, die aber insofern auch als sozioökonomisch ausgerichtet betrachtet werden könnten, als sie darauf abzielen, Schülern aus benachteiligten Verhältnissen zusätzliche Bildungsmittel zukommen zu lassen. Wie in den verschiedenen Bänden dieses Berichts beschrieben wird, haben Länder wie Brasilien (vgl. Kasten I.2.4), Deutschland (Kasten II.3.2), Israel (vgl. Kasten IV.1.4), Mexiko (Kasten II.2.4) und die Türkei (vgl. Kasten I.2.5) bereits gezielte Politikmaßnahmen zur Verbesserung der Bildungsergebnisse leistungsschwacher Schulen oder Schüler ergriffen bzw. jenen Regionen und Schulen, in denen der Bedarf am größten ist, mehr Ressourcen zugeteilt.

- Einsatz allgemeinerer Maßnahmen, die auf eine Anhebung des Leistungsniveaus aller Schülerinnen und Schüler ausgerichtet sind. Solche Maßnahmen dürften vor allem in Ländern mit schwächer ausgeprägten Gradienten und einer geringeren Varianz der Schülerleistungen zweckmäßig sein. Sie können darauf abzielen, die Lehrinhalte und den Lehrplanrhythmus zu verändern, die Unterrichtsmethoden zu verbessern, Ganztagsschulen einzuführen, das Einschulungsalter zu ändern oder die für den Unterricht vorgesehene Zeit zu erhöhen. Einige Länder, wie Dänemark und Deutschland, haben auf die Ergebnisse von PISA 2000 mit umfangreichen Schul- und Lehrplanreformen reagiert, die einige solcher Änderungen beinhalteten. Es wurden auch Anstrengungen unternommen, um zu erreichen, dass sich die Eltern 
stärker für Schulangelegenheiten engagieren, indem sie ihren Kindern z.B. zu Hause größere Unterstützung zukommen lassen oder sich mehr an der Schulverwaltung beteiligen. Viele allgemeine Maßnahmen sind darauf ausgerichtet, die Vorgehensweisen der Lehrkräfte zu ändern oder die Rechenschaftspflicht der Schulen und der Schulsysteme durch Beurteilungen der Schülerleistungen zu erhöhen. Wie in diesem und anderen Bänden dieses Berichts beschrieben, haben manche Länder systemweite Reformen eingeführt, die auf eine Entwicklung hin zu integrativeren Schulen (Polen) bzw. einer weniger starken Aufteilung der Schüler auf verschiedene Bildungsgänge (Deutschland) abzielen. Mit diesen Reformen soll den Effekten verschiedener Formen von Chancenungleichheit mit unterschiedlichen Ursachen, wie sozioökonomische Benachteiligung, Migrationshintergrund oder schwierige familiäre Verhältnisse, gleichzeitig begegnet werden. Einige Länder richten das Augenmerk in erster Linie darauf, die Gesamtqualität der Bildungsressourcen zu verbessern - u.a. durch die Anwerbung und Anstellung hochqualifizierter Lehrkräfte - und diese besseren Ressourcen allen Schulen und vor allem sozioökonomisch benachteiligten Schulen zur Verfügung zu stellen. Länder, die ihre PISAErgebnisse verbessert haben, so z.B. Brasilien (vgl. Kasten I.2.4), Kolumbien (vgl. Kasten IV.4.3), Estland (vgl. Kasten I.5.1), Israel (vgl. IV.1.4), Japan (vgl. Kasten III.3.1) und Polen (vgl. Kasten IV.2.1), haben Maßnahmen eingeleitet, um die Qualität ihrer Lehrkräfte zu steigern. Sie haben die Anforderungen für die Erlangung der Lehrbefähigung erhöht, Anreize für besonders fähige Hochschulabsolventen zum Einstieg in den Lehrberuf geschaffen, die Vergütung von Lehrkräften erhöht, um den Beruf attraktiver zu machen und mehr Lehrkräfte im Lehrberuf zu halten, oder Anreize für die Teilnahme an Lehrerfortbildungen eingerichtet.

- Einbindung ausgegrenzter Schülerinnen und Schüler in die allgemeinen Schul- und Klassenstrukturen. Inklusionsmaßnahmen sind häufig darauf ausgerichtet, Schüler mit Behinderungen in normale Schulklassen zu integrieren, anstatt sie in gesonderten Klassen oder Schulen zu unterrichten. In diesem Band gelten als Inklusionsmaßnahmen Maßnahmen, die darauf abzielen, Schüler stärker zu integrieren, die möglicherweise unter Segregation leiden, z.B. auf Grund einer sozioökonomischen Benachteiligung. Einige Inklusionsmaßnahmen sind darauf angelegt, die Segregation zwischen den Schulen nach sozioökonomischen Kriterien durch eine Neuaufteilung der Einzugsgebiete der Schulen, durch die Zusammenlegung von Schulen oder durch die Einrichtung von Magnetschulen in Niedrigeinkommensgebieten zu reduzieren. Wie in Band IV dieses Berichts erörtert, sind viele Schulsysteme stark gegliedert. In stark gegliederten Systemen können für die Schulen stärkere Anreize bestehen, sich die besten Schüler herauszusuchen; die Anreize, schwierige Schüler zu unterstützen, sind demgegenüber geringer, wenn es möglich ist, sie an andere Schulen zu verweisen. Einige der Schulsysteme, denen es in den letzten Jahren gelungen ist, Leistung und Bildungsgerechtigkeit zu verbessern, sind inklusiver geworden. In Polen (vgl. Kasten IV.2.1) beispielsweise wurde das Bildungssystem reformiert, indem das Alter für die Aufteilung auf verschiedene Bildungsgänge angehoben wurde, und auch in Deutschland (Kasten II.3.2) ist eine Tendenz hin zu einer geringeren Stratifizierung des Schulsystems zu beobachten.

Im übrigen Teil dieses Kapitels werden die verschiedenen Politikoptionen beschrieben, die sich den Ländern bieten, je nachdem wie die Schülerleistungen mit dem sozialen Umfeld der Schüler und der Schulen zusammenhängen. Wenn der Anteil der leistungsschwachen Schülerinnen und Schüler groß ist, sollten die Politikmaßnahmen auf diese Schüler abzielen. Kasten II.5.1 liefert Orientierungshilfen zur Gestaltung von Maßnahmen, mit denen Schülerleistungen und Chancengerechtigkeit in anderen Fällen gesteigert werden können. Allgemeiner ausgerichtete Maßnahmen bieten sich z.B. vor allem für die Länder im oberen linken Quadranten der Länderübersicht an. Maßnahmen, die sozioökonomischen Nachteilen entgegenwirken sollen, dürften in den Ländern in den rechten Quadranten wirkungsvoller sein, und Maßnahmen zur Behebung von Leistungsschwächen dürften in den Ländern in den unteren Quadranten am sinnvollsten sein.

\section{Kasten II.5.1 Ein Rahmen für Maßnahmen zur Verbesserung der Schülerleistungen und der Bildungsgerechtigkeit}

In diesem Band werden zwei wichtige Messgrößen der Bildungsgerechtigkeit identifiziert: zum einen die Stärke des Zusammenhangs zwischen Leistung und sozioökonomischem Hintergrund (Stärke der sozioökonomischen Gradiente), zum anderen der Umfang der Leistungsunterschiede zwischen verschiedenen sozioökonomischen Gruppen (Steigung der sozioökonomischen Gradiente). In der folgenden typologischen Darstellung werden Länder ausgehend von ihren Durchschnittsergebnissen danach eingeordnet, ob sie nach diesen beiden Messgrößen über oder unter dem OECD-Durchschnitt liegen. 


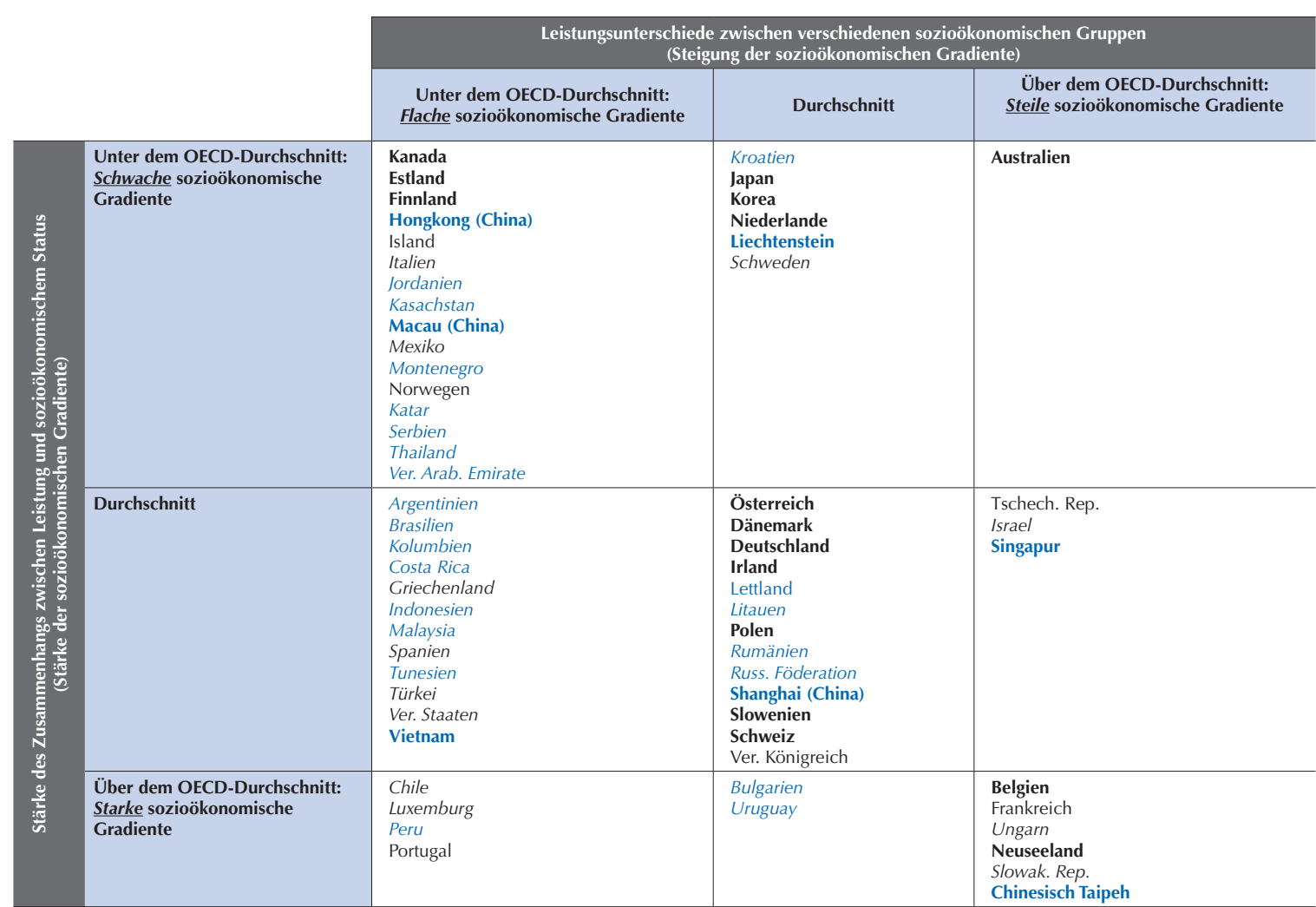

Anmerkung: Länder und Volkswirtschaften mit über dem OECD-Durchschnitt liegenden Durchschnittsergebnissen in Mathematik sind durch Fettdruck gekennzeichnet. Länder und Volkswirtschaften mit unter dem OECD-Durchschnitt liegenden Durchschnittsergebnissen in Mathematik sind durch Kursivdruck gekennzeichnet.

Die Betrachtung dieser beiden Dimensionen der Chancengerechtigkeit in der Bildung zusammen mit den oben beschriebenen Politikoptionen kann den politischen Entscheidungsträgern dabei helfen, einen Weg aufzuzeichnen, um die Bildungsqualität anzuheben und die Bildungsgerechtigkeit zu erhöhen.

\begin{tabular}{|c|c|c|}
\hline & \multicolumn{2}{|c|}{ Die Leistungsunterschiede innerhalb der sozioökonomischen Verteilung sind: } \\
\hline & Gering: Flache Gradiente & Groß: Steile Gradiente \\
\hline $\begin{array}{l}\text { Der Effekt des } \\
\text { sozioökonomischen } \\
\text { Status auf die Leistung } \\
\text { ist schwach }\end{array}$ & $\begin{array}{l}\text { Wenn die Leistungsunterschiede innerhalb der } \\
\text { sozioökonomischen Verteilung gering sind und die Schüler } \\
\text { häufig bessere (bzw. schlechtere) Leistungen erzielen als } \\
\text { in Anbetracht ihres sozioökonomischen Hintergrunds zu } \\
\text { erwarten wäre, besteht eines der wichtigsten Ziele für die } \\
\text { Politik darin, die Schülerleistungen insgesamt anzuheben. } \\
\text { In diesem Fall ist der Einsatz allgemeiner Maßnahmen in } \\
\text { der Regel am wirksamsten. Zu dieser Art von Maßnahmen } \\
\text { gehören die Veränderung der Lehrpläne sowie der } \\
\text { Unterrichtsmethoden und/oder die Verbesserung der Qualität } \\
\text { der Lehrkräfte, indem z.B. die Anforderungen für die } \\
\text { Erlangung der Lehrbefähigung erhöht werden, Anreize für } \\
\text { besonders fähige Hochschulabsolventen zum Einstieg in den } \\
\text { Lehrberuf geschaffen werden, die Vergütung von Lehrkräften } \\
\text { erhöht wird, um den Beruf attraktiver zu machen und mehr } \\
\text { Lehrkräfte im Lehrberuf zu halten, und/oder Anreize für die } \\
\text { Teilnahme an Lehrerfortbildungen eingerichtet werden. }\end{array}$ & $\begin{array}{l}\text { Wenn die Leistungsunterschiede innerhalb der sozioökonomischen Verteilung } \\
\text { groß sind und die Schüler häufig bessere (bzw. schlechtere) Leistungen } \\
\text { erzielen als in Anbetracht ihres sozioökonomischen Hintergrunds zu erwarten } \\
\text { wäre, besteht eines der wichtigsten Ziele für die Politik darin, die Ergebnisse } \\
\text { der besonders leistungsschwachen Schüler zu erhöhen, unabhängig von } \\
\text { ihrem sozioökonomischen Hintergrund. In diesem Fall würde eine alleinige } \\
\text { Ausrichtung der Maßnahmen auf sozioökonomisch benachteiligte Schüler } \\
\text { einigen Schülern, die bereits verhältnismäßig gute Leistungen erzielen, } \\
\text { zusätzliche Unterstützung bieten, während manche Schüler, die nicht } \\
\text { zwangsläufig sozioökonomisch benachteiligt sind, aber schlechte Leistungen } \\
\text { erbringen, keine Unterstützung erhielten. Die Maßnahmen können auf } \\
\text { leistungsschwache Schüler ausgerichtet werden, wenn diese Schüler } \\
\text { einfach identifiziert werden können, oder auf leistungsschwache Schulen, } \\
\text { insbesondere wenn sich die Leistungsschwächen auf bestimmte Schulen } \\
\text { konzentrieren. Zu den Beispielen solcher Maßnahmen zählen Evaluationen, } \\
\text { Feedback und Beurteilungen für Schüler, Lehrkräfte und Schulen, die } \\
\text { Einrichtung früh greifender Präventionsprogramme, die Einführung } \\
\text { angepasster Lehrpläne oder das Angebot zusätzlicher Unterrichtsstunden für } \\
\text { Schüler mit Lernschwierigkeiten. }\end{array}$ \\
\hline $\begin{array}{l}\text { Der Effekt des } \\
\text { sozioökonomischen } \\
\text { Status auf die Leistung } \\
\text { ist stark }\end{array}$ & $\begin{array}{l}\text { Wenn die Leistungsunterschiede innerhalb der sozioöko- } \\
\text { nomischen Verteilung gering sind, die Schüler aber die } \\
\text { in Anbetracht ihres sozioökonomischen Hintergrunds zu } \\
\text { erwartenden Leistungen erzielen, besteht eines der wichtigsten } \\
\text { Ziele für die Politik darin, die Hindernisse für hohe Leistungen } \\
\text { abzubauen, die auf sozioökonomische Benachteiligungen } \\
\text { zurückzuführen sind. In diesem Fall sind wirksame } \\
\text { kompensatorische Maßnahmen gezielt auf sozioökonomisch } \\
\text { benachteiligte Schüler und Schulen auszurichten, indem } \\
\text { ihnen zusätzliche Förderung, Ressourcen und Unterstützung } \\
\text { angeboten werden. Programme zur Übernahme der Kosten } \\
\text { des Mittagessens oder kostenfrei zur Verfügung gestellte } \\
\text { Lehrbücher für Schüler aus benachteiligten Familien sind } \\
\text { weitere Beispiele. }\end{array}$ & $\begin{array}{l}\text { Wenn die Leistungsunterschiede innerhalb der sozioökonomischen } \\
\text { Verteilung groß sind und die Schüler die in Anbetracht ihres sozioöko- } \\
\text { nomischen Hintergrunds zu erwartenden Leistungen erzielen, besteht eines } \\
\text { der wichtigsten Ziele für die Politik darin, die Leistungsunterschiede zu } \\
\text { reduzieren und die Leistungen zu steigern, vor allem unter sozioökonomisch } \\
\text { benachteiligten Schülern. Eine Kombination aus Maßnahmen zur } \\
\text { Behebung von Leistungsschwächen und zur Verringerung der Effekte } \\
\text { sozioökonomischer Benachteiligungen ist in diesem Fall in der Regel am } \\
\text { wirksamsten, da allgemeine Maßnahmen im Hinblick auf eine gleichzeitige } \\
\text { Verbesserung der Chancengerechtigkeit und der Schülerleistungen u.U. } \\
\text { weniger wirksam sind. }\end{array}$ \\
\hline
\end{tabular}


Ein weiterer wichtiger Aspekt, über den nachgedacht werden muss, ist die Frage, ob diese Maßnahmen auf Schüler oder Schulen ausgerichtet werden sollten. Vielfach ist es u.U. nicht sehr kosteneffektiv, auf einzelne Schüler abzuzielen, die in bestimmten Fächern Schwierigkeiten haben oder die in oder außerhalb der Schule vor besonderen Problemen stehen. Die im Rahmen von PISA gewonnene Evidenz kann Hinweise darauf liefern, wann Eingriffe auf Schul- oder auf Schülerebene die besseren Ergebnisse versprechen. Zwei Indikatoren liefern diesbezüglich Orientierungshilfen: Der Umfang der zwischen den Schulen bestehenden Unterschiede beim sozioökonomischen Profil und der Zusammenhang zwischen dem sozioökonomischen Profil einer Schule und ihren Durchschnittsergebnissen.

Wo im Vergleich zwischen den Schulen wenig Unterschiede zu beobachten sind, bietet es sich an, die Maßnahmen auf bestimmte Schüler innerhalb der Schulen auszurichten. Wenn demgegenüber im Vergleich der Schulen große Leistungsunterschiede oder sozioökonomische Diskrepanzen bestehen, kann es sich empfehlen, auf bestimmte Schulen - z.B. Schulen mit niedrigen Ergebnissen oder ungünstigem sozioökonomischem Profil - abzuzielen. Die Ausrichtung auf sozioökonomisch benachteiligte Schulen kann sich als wirkungsvoll erweisen, wenn zwischen den Schulen große, mit ihrem sozioökonomischen Profil in Zusammenhang stehende Leistungsunterschiede festzustellen sind.

\section{ÜBERPROPORTIONAL HOHER ANTEIL LEISTUNGSSCHWACHER SCHÜLER}

Wenn eine große Zahl von Schülerinnen und Schülern das Grundkompetenzniveau nicht erreicht, dürften Maßnahmen am wirkungsvollsten sein, die auf leistungsschwache Schüler ausgerichtet sind, unabhängig vom Grad an Bildungsgerechtigkeit, der in den fraglichen Ländern/Volkswirtschaften herrscht. Besonders hoch ist der Anteil der Schülerinnen und Schüler, die in Mathematik Kompetenzstufe 2 nicht erreichen, mit über 40\% in Albanien, Argentinien, Brasilien, Bulgarien, Chile, Kolumbien, Costa Rica, Indonesien, Jordanien, Kasachstan, Malaysia, Mexiko, Montenegro, Peru, Katar, Rumänien, Thailand, Tunesien, der Türkei, den Vereinigten Arabischen Emiraten und Uruguay (Abb. II.5.1). In diesen Ländern ist kein starker Zusammenhang zwischen den Leistungen und dem sozioökonomischen Hintergrund der Schüler festzustellen (außer in Bulgarien, Chile, Costa Rica, Peru und Uruguay), und die Leistungsunterschiede zwischen verschiedenen sozioökonomischen Gruppen sind geringer als im OECD-Durchschnitt (außer in Bulgarien, Rumänien und Uruguay, wo sie dem OECD-Durchschnitt entsprechen). Die Leistungen sind generell gering, und es bestehen keine großen Leistungsunterschiede zwischen den Schülern, auch wenn beim sozioökonomischen Hintergrund u.U. erhebliche Unterschiede zu beobachten sind. In diesen Ländern ist der Abstand zwischen den obersten und den untersten 5\% der Schüler in der sozioökonomischen Verteilung größer als im OECDDurchschnitt (außer in Kasachstan, wo er geringer ist als im Durchschnitt, sowie in Montenegro und in den Vereinigten Arabischen Emiraten, wo er dem Durchschnitt entspricht). Zudem gibt es in vielen dieser Länder zahlreiche 15-Jährige, die nicht zur Schule gehen und folglich auch nicht an PISA teilnahmen. Weil diese Schüler höchstwahrscheinlich sozioökonomisch benachteiligten Gruppen angehören, wirkt die Schülerpopulation in diesen Ländern und Volkswirtschaften homogener als die Gesamtpopulation der 15-Jährigen. Außerdem kann es sein, dass die PISA-Messgrößen des sozioökonomischen Status in einigen dieser Länder keine hinreichende Unterscheidung der verschiedenen Stufen der Benachteiligung gestatten. In Abbildung II.5.2 werden die Profile einiger dieser Länder gegenübergestellt.

Einige Länder in dieser Gruppe, z.B. Brasilien, Kolumbien und Mexiko, haben Maßnahmen zur Unterstützung sozioökonomisch benachteiligter Schülerinnen und Schüler eingeführt. Der eher geringe Anteil der Varianz der Schülerleistungen, der sich aus dem sozioökonomischen Hintergrund erklärt, legt den Schluss nahe, dass auf Leistungsschwächen an sich ebenso viel Aufmerksamkeit gerichtet werden muss wie auf sozioökonomische Benachteiligungen.

\section{UNTERSCHIEDLICHE STEIGUNG UND STÄRKE DER SOZIOÖKONOMISCHEN GRADIENTE}

Für Schulverwaltungen stellt sich häufig die Frage, ob Bemühungen um eine Steigerung der Schülerleistungen hauptsächlich auf leistungsschwache Schülerinnen und Schüler oder auf Schülerinnen und Schüler aus sozioökonomisch benachteiligten Verhältnissen abzielen sollten. Die Leistungsunterschiede innerhalb der sozioökonomischen Verteilung sowie der Anteil der Leistungsvarianz, der sich aus dem sozioökonomischen Status erklärt, stellen nützliche Indikatoren für die Beantwortung dieser Frage dar. Es gilt zu unterscheiden zwischen der Steigung der sozialen Gradiente, die sich auf den durchschnittlichen Umfang des Leistungsabstands bezieht, der mit einem gegebenen Unterschied beim sozioökonomischen Status verbunden ist, und ihrer Stärke, die sich darauf bezieht, wie stark die Leistungen der Schülerinnen und Schüler mit den auf ihrem sozioökonomischen Status basierenden Vorhersagen übereinstimmen. 
- Abbildung II.5.2 -

Zusammenhang zwischen den Leistungen der Schulen und ihrem sozioökonomischen Profil in Ländern mit einem hohen Anteil von Schülern, die unter Stufe 2 abschneiden: Indonesien, Kasachstan, Peru und Tunesien
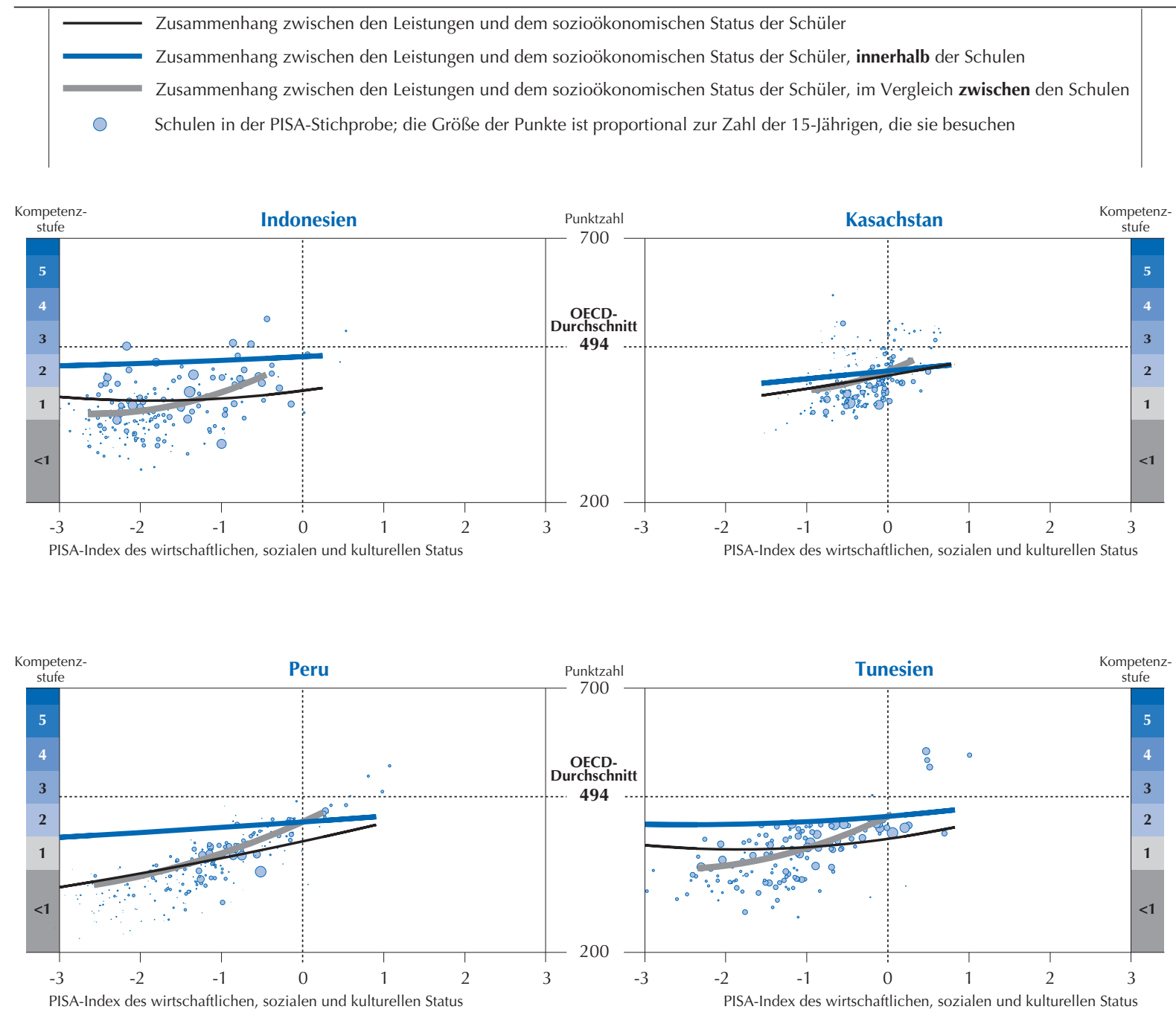

Quelle: OECD, PISA-2012-Datenbank.

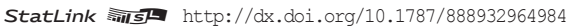

In Ländern mit verhältnismäßig flachen Gradienten - d.h. Länder, in denen die durch den sozioökonomischen Status bedingten Leistungsunterschiede gering sind - würden Maßnahmen, die speziell auf Schüler aus sozial benachteiligten Verhältnissen ausgerichtet sind, für sich allein genommen den Bedürfnissen vieler leistungsschwacher Schüler des Landes nicht gerecht werden.

Allgemein ausgerichtete Maßnahmen für Länder, in denen die Leistungsunterschiede gering sind und ein schwacher Zusammenhang zwischen Leistung und sozioökonomischem Status besteht

Unter den Ländern und Volkswirtschaften, die an PISA 2012 teilgenommen haben, sind Estland, Finnland, Hongkong (China), Kanada und Macau (China) die einzigen, deren Schulsysteme überdurchschnittliche Leistungen und überdurchschnittliche Bildungsgerechtigkeit vereinen, sowohl im Hinblick auf die Stärke des Zusammenhangs zwischen sozioökonomischem Status und Leistungen als auch hinsichtlich des Umfangs der Leistungsunterschiede zwischen verschiedenen sozioökonomischen Gruppen (Abb. II.5.1a). Innerhalb dieser Gruppe sind selbst große Unterschiede beim sozioökonomischen Status der Schüler (wie in Hongkong-China und Macau-China, wo die Länge der Gradiente über dem OECD-Durchschnitt liegt) im 


\section{Zusammenhang zwischen den Leistungen der Schulen und ihrem sozioökonomischen Profil in Ländern mit hohen Leistungen sowie flachen und schwachen Gradienten: Kanada und Vietnam}
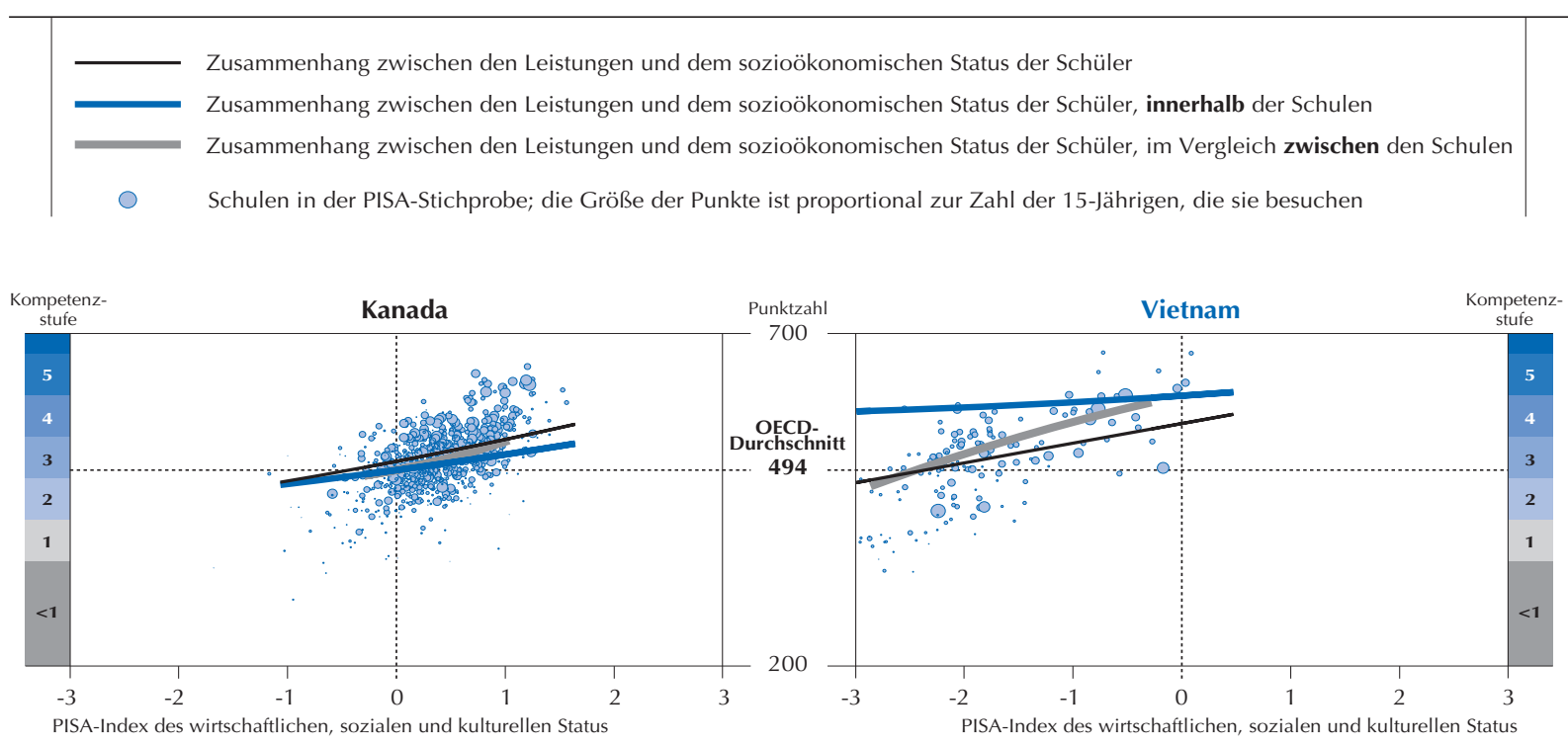

Quelle: OECD, PISA-2012-Datenbank.

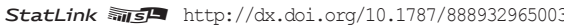

Durchschnitt nicht mit großen Leistungsunterschieden zwischen den Schülern verbunden. Generell dürften allgemeine Maßnahmen, die alle Schülerinnen und Schüler erreichen, diesen Ländern am ehesten helfen, die Schülerleistungen zu verbessern und weiterhin eine überdurchschnittliche Verteilungsgerechtigkeit bei den Bildungserträgen sicherzustellen. Japan, Korea und Liechtenstein zeichnen sich ebenfalls durch überdurchschnittliche Leistungen und einen schwachen Zusammenhang zwischen sozioökonomischem Status und Leistungen aus, allerdings liegen die mit dem sozioökonomischen Status verbundenen Leistungsunterschiede in diesen Ländern um den Durchschnitt. Neben universellen Maßnahmen könnten diese Länder gezielte Maßnahmen für leistungsschwache Schüler erwägen, die sich u.U. nicht zwangsläufig über ihren sozioökonomischen Status definieren lassen (z.B. Schüler mit Migrationshintergrund in Finnland), oder für leistungsschwache Schulen, wenn die zwischenschulischen Leistungsunterschiede sehr groß sind. In keinem der leistungsstarken Länder ist die sozioökonomische Gradiente flach und stark zugleich.

Bei Ländern, deren Durchschnittsergebnisse unter oder um den OECD-Durchschnitt liegen, impliziert eine gerechtere Verteilung der Bildungserträge, dass alle Schülerinnen und Schüler unabhängig von ihrem Hintergrund gleich gut - oder schlecht - abschneiden oder dass die Leistungsunterschiede zwischen begünstigten und benachteiligten Schülern gering sind. Island und Norwegen verzeichnen ein durchschnittliches Leistungsniveau und hohe Bildungsgerechtigkeit. Unter den OECD-Ländern weisen Italien und Mexiko ein Leistungsniveau unter dem OECD-Durchschnitt und eine hohe Bildungsgerechtigkeit auf; allerdings beträgt der Anteil der leistungsschwachen Schüler in Italien rd. 25\% und in Mexiko $55 \%$. Unter den Partnerländern verfügen Jordanien, Kasachstan, Katar, Montenegro, Thailand und die Vereinigten Arabischen Emirate über ein hohes Niveau an Bildungsgerechtigkeit, aber auch einen großen Anteil an Schülerinnen und Schülern, die unter dem Grundkompetenzniveau abschneiden. In Griechenland, Spanien, den Vereinigten Staaten und Vietnam liegen die durch den sozioökonomischen Status bedingten Leistungsunterschiede (Steigung der Gradiente) ebenfalls unter dem Durchschnitt, doch im Hinblick auf den Zusammenhang zwischen dem sozioökonomischen Status und den Leistungen (Stärke der Gradiente) befinden sich diese Länder in der Nähe des OECD-Durchschnitts. Diese Merkmale treffen zwar auch auf Argentinien, Brasilien, Indonesien, Kolumbien, Malaysia, Tunesien und die Türkei zu, doch in all diesen Ländern beträgt der Anteil der leistungsschwachen Schülerinnen und Schüler über $40 \%$.

Diese Daten deuten darauf hin, dass in vielen dieser Länder ein vergleichsweise geringerer Anteil der leistungsschwachen Schüler aus benachteiligten Verhältnissen stammt und der Zusammenhang zwischen Leistungen und sozioökonomischem Status schwach ist. Daher würden Maßnahmen, die speziell auf Schüler aus benachteiligten Verhältnissen zugeschnitten sind, für sich allein genommen den Bedürfnissen vieler der leistungsschwächeren Schüler in diesen Ländern nicht 
gerecht werden. Ebenso wie in leistungsstarken Ländern dürften auch in diesen Ländern allgemeine Maßnahmen, die auf alle Schulen sowie alle Schülerinnen und Schüler ausgerichtet sind, wirkungsvoller sein, um die Leistung zu steigern und zugleich eine weiterhin hohe Bildungsgerechtigkeit sicherzustellen.

Maßnahmen, die auf leistungsschwache Schüler zugeschnitten sind, könnten hingegen eine größere Wirkung zeigen als Maßnahmen für sozioökonomisch benachteiligte Schüler. Wenn beispielsweise das Ziel darin besteht, dass die meisten Schülerinnen und Schüler ein bestimmtes Mindestleistungsniveau erreichen, würden Maßnahmen, die auf sozioökonomisch benachteiligte Schüler ausgerichtet sind, auch einen großen Anteil von Schülern miteinbeziehen, die bereits gute Leistungen erzielen. In Ländern, in denen ein großer Anteil der Schülerinnen und Schüler unter dem Grundkompetenzniveau abschneidet, sind u.U. speziell auf diese Schüler zugeschnittene Maßnahmen erforderlich, damit sie nicht den Anschluss verlieren. Zwischen den Ländern, deren Ergebnisse unter dem OECD-Durchschnitt liegen, bestehen erhebliche Unterschiede beim Anteil der Schülerinnen und Schüler, deren Leistungen unter dem Grundkompetenzniveau liegen. In einigen Ländern, wie z.B. Italien, entspricht ihr Anteil ungefähr dem OECD-Durchschnitt, während in anderen Ländern, wie Argentinien, Brasilien, Indonesien, Kolumbien, Malaysia, Tunesien und der Türkei, der Anteil der Schüler mit Leistungen unter dem Grundkompetenzniveau von $42 \%$ in der Türkei bis $76 \%$ in Indonesien reicht. Bei einem hohen Anteil von Schülerinnen und Schülern mit Leistungen unter dem Grundkompetenzniveau sind gezielte Maßnahmen erforderlich, die auf diese Schüler und die Schulen, die sie besuchen, ausgerichtet sind.

- Abbildung II.5.4 "

\section{Zusammenhang zwischen den Leistungen der Schulen und ihrem sozioökonomischen Profil in Ländern mit durchschnittlichen oder niedrigen Leistungen sowie flachen und schwachen Gradienten: Brasilien, Italien, Mexiko und die Vereinigten Staaten}
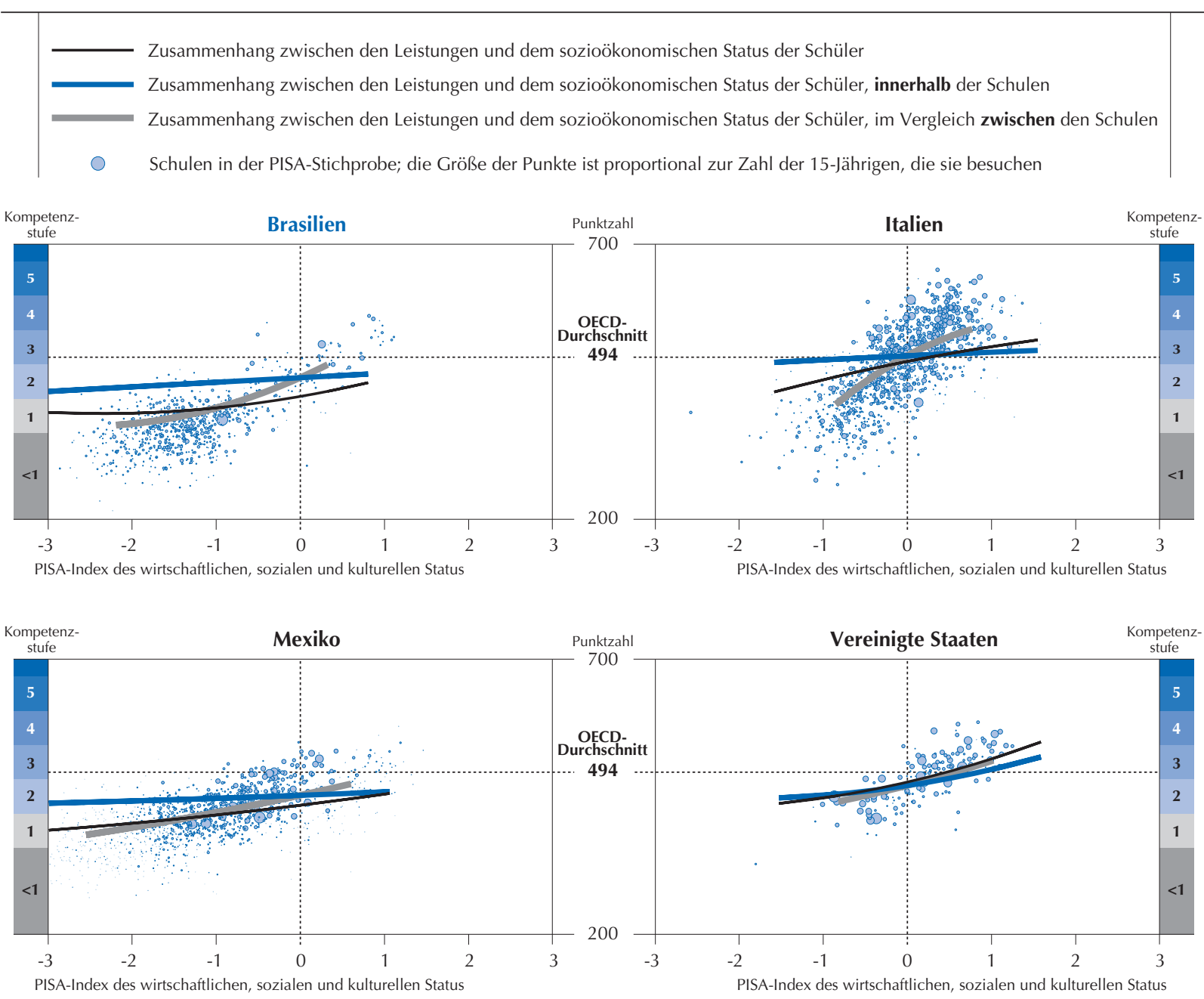

Quelle: : OECD, PISA-2012-Datenbank.

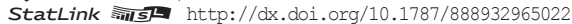




\section{Maßnahmen, die bei der sozioökonomischen Benachteiligung ansetzen, für Länder mit geringen Leistungsunterschieden und einem starken Zusammenhang zwischen Leistung und sozioökonomischem Status}

Ist die sozioökonomische Gradiente flach, aber stark, was bedeutet, dass die Leistungsunterschiede auf Grund des sozioökonomischen Status gering sind, aber ein enger Zusammenhang zwischen sozioökonomischem Status und Leistung besteht, lässt sich u.U. mit einer Kombination aus allgemeinen Maßnahmen sowie Maßnahmen, die speziell auf benachteiligte Schüler und Schulen ausgerichtet sind, die größte Wirkung erzielen. Ein möglicher Ansatz, um den starken Zusammenhang zwischen sozioökonomischem Status und Leistung aufzubrechen, besteht in einer flexibleren Gestaltung der Bildungssysteme, z.B. durch Übergangsmöglichkeiten zwischen verschiedenen Bildungsgängen in gegliederten Schulsystemen; ein weiterer besteht darin, mehr und bessere Ressourcen und Lernmöglichkeiten für benachteiligte Schüler verfügbar zu machen. Bestimmte allgemeine Maßnahmen, wie z.B. eine Verlängerung oder qualitative Verbesserung der in der Schule verbrachten Zeit, können ebenfalls die Bildungsgerechtigkeit erhöhen, da sie einen größeren Effekt auf benachteiligte Schülerinnen und Schüler haben dürften. Chile, Costa Rica, Peru und Portugal sind die einzigen Länder, in denen die sozioökonomische Gradiente flach und stark zugleich ist. In allen diesen Ländern außer Portugal schneiden mehr als $40 \%$ der Schülerinnen und Schüler unter dem Grundkompetenzniveau in Mathematik ab. Maßnahmen, die auf leistungsschwache Schüler und Schulen zugeschnitten sind, sowie allgemeine Maßnahmen, die eine Leistungssteigerung auf breiter Basis anstreben, sind in diesen Ländern möglicherweise am wirksamsten.

\section{- Abbildung II.5.5}

\section{Zusammenhang zwischen den Leistungen der Schulen und ihrem sozioökonomischen Profil in Ländern mit durchschnittlichen oder niedrigen Leistungen sowie flachen und starken Gradienten: Chile, Griechenland, Malaysia und die Türkei}
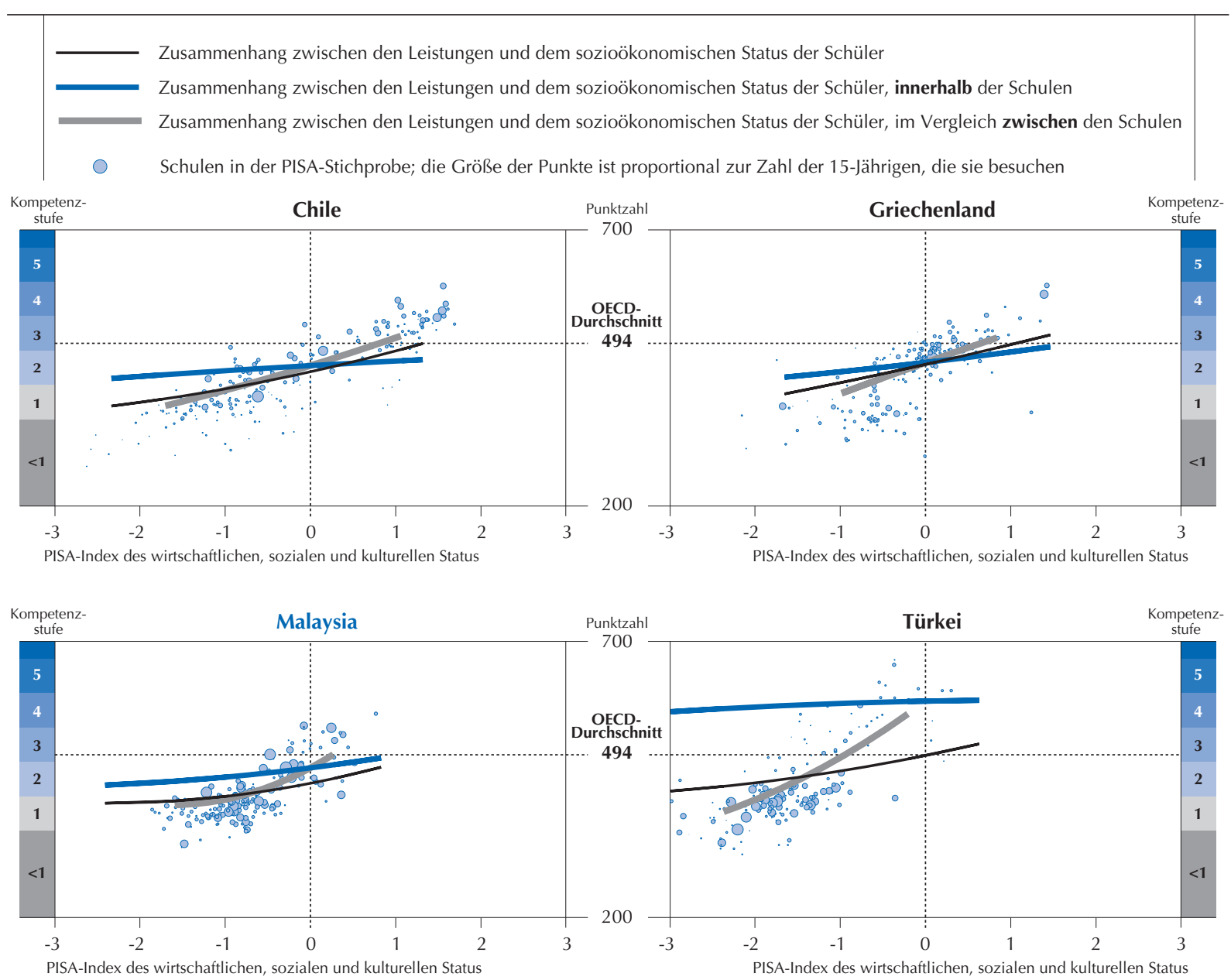

Quelle: OECD, PISA-2012-Datenbank.

StatLink ints http://dx.doi.org/10.1787/888932965041 


\section{Maßnahmen, die sowohl bei der Leistung als auch bei der sozioökonomischen Benachteiligung ansetzen, für Länder mit großen Leistungsunterschieden und einem starken Zusammenhang zwischen Leistung und sozioökonomischem Status}

In Ländern, in denen die Leistungsunterschiede zwischen verschiedenen sozioökonomischen Gruppen sehr groß sind (steile Gradiente), ist von Maßnahmen, die gezielt auf sozioökonomisch benachteiligte Schülerinnen und Schüler ausgerichtet sind, eine größere Wirksamkeit zu erwarten, insbesondere wenn die Stärke des Zusammenhangs zwischen Leistung und sozioökonomischem Status ebenfalls über dem Durchschnitt liegt.

Neuseeland und Chinesisch Taipeh sind die einzigen beiden leistungsstarken Länder bzw. Volkswirtschaften, in denen die Verteilungsgerechtigkeit der Bildungserträge unter dem Durchschnitt liegt. In beiden Ländern stellen Maßnahmen, die auf leistungsschwache und/oder benachteiligte Schülerinnen und Schüler ausgerichtet sind, eine Handlungsoption dar, da die steile Steigung der Gradiente darauf hindeutet, dass leistungsschwache Schüler ihre Leistungen rasch steigern könnten, wenn sich ihr sozioökonomischer Status verbessern würde. Der überdurchschnittlich starke Zusammenhang zwischen sozioökonomischem Status und Leistung signalisiert jedoch, dass in diesen Ländern sehr wenige Schülerinnen und Schüler die mit dem sozioökonomischen Status verbundenen Hindernisse für hohe Leistungen überwinden. Daher müssen diese Länder sozioökonomisch benachteiligten Schülerinnen und Schülern auch bessere Chancen bieten, höhere Leistungen zu erzielen. Eine Kombination von Maßnahmen, mit denen die Bildungsgerechtigkeit verbessert, zugleich aber das hohe Leistungsniveau auf Systemebene aufrechterhalten wird, scheint für diese Länder die empfehlenswerteste Vorgehensweise.

Belgien, Österreich und Singapur verzeichnen ebenfalls hohe Leistungen und große Leistungsunterschiede zwischen verschiedenen sozioökonomischen Gruppen, doch die Stärke des Zusammenhangs zwischen Leistung und sozioökonomischem Status ist nur durchschnittlich ausgeprägt. Um diese großen Differenzen zu verringern, können sich diese Länder auf kompensatorische Maßnahmen zur Unterstützung benachteiligter Schüler und Schulen konzentrieren, damit diese ebenso viele Bildungsmöglichkeiten und Ressourcen erhalten wie begünstigtere Schüler bzw. Schulen.

In Ländern mit niedrigen Leistungen und geringer Bildungsgerechtigkeit, wie z.B. Ungarn und die Slowakische Republik, könnten Maßnahmen, die sowohl Leistungsschwächen als auch sozioökonomischer Benachteiligung entgegenwirken, zweckmäßig sein, um diejenigen Schüler zu erreichen, die am dringendsten Unterstützung benötigen - wobei es sich

\section{- Abbildung II.5.6}

\section{Zusammenhang zwischen den Leistungen der Schulen und ihrem sozioökonomischen Profil in Ländern mit hohen Leistungen sowie steilen und starken Gradienten: Deutschland und Neuseeland}
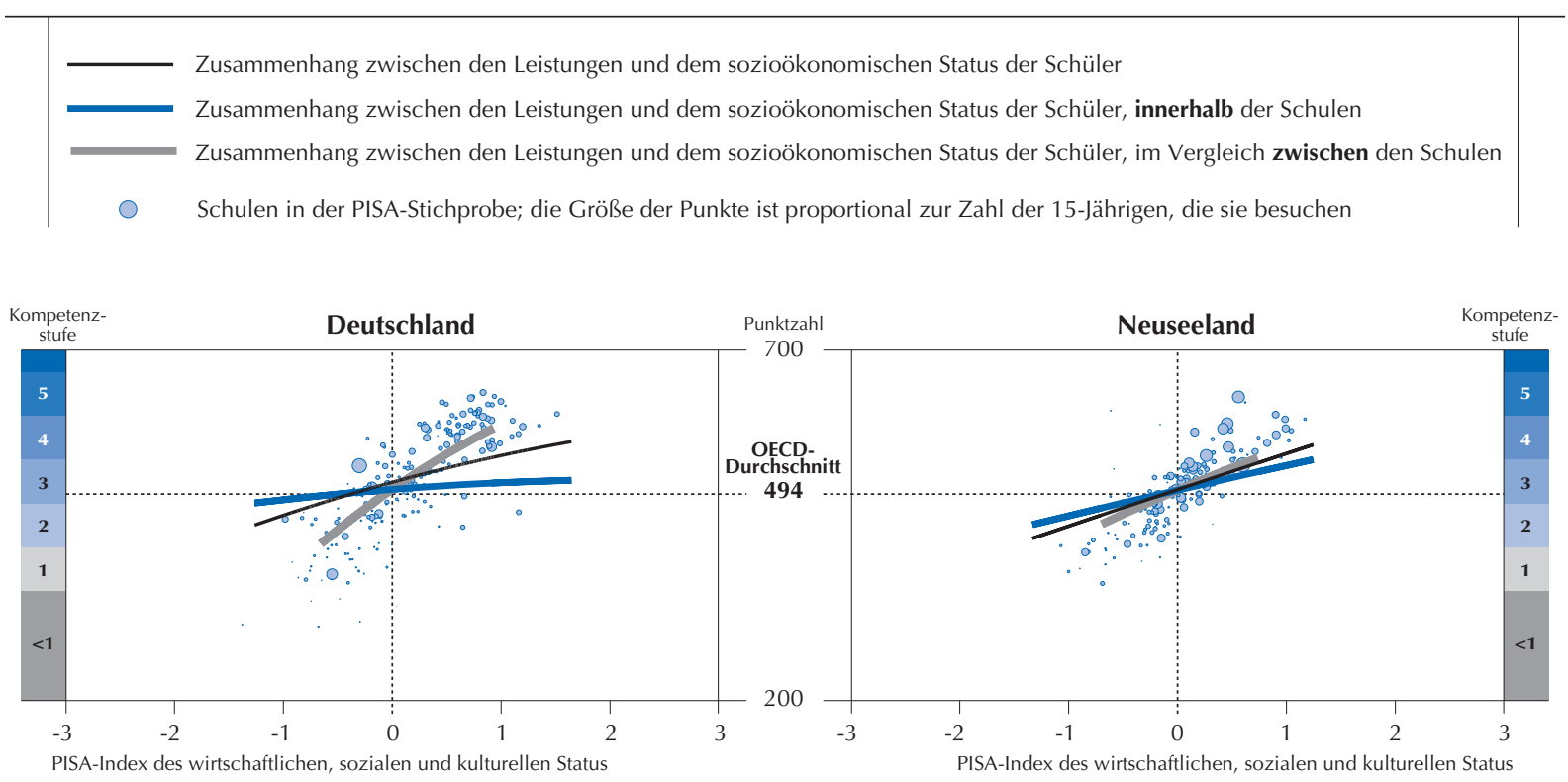

Quelle: OECD, PISA-2012-Datenbank.

StatLink 需可 http://dx.doi.org/10.1787/888932965060 
in diesen Fällen oft um ein und dieselben Schülerinnen und Schüler handelt. Die steile Steigung der Gradiente lässt den Schluss zu, dass bei einer Verbesserung des sozioökonomischen Status eine rasche Leistungssteigerung erfolgt. Allerdings deutet der starke Zusammenhang zwischen dem sozioökonomischen Status und den Leistungen darauf hin, dass wenige Schülerinnen und Schüler besser abschneiden als auf der Grundlage ihres sozioökonomischen Status zu erwarten ist. Reformen, die das Schulsystem flexibler machen, so dass benachteiligte und leistungsschwache Schülerinnen und Schüler Zugang zu besseren Ressourcen und/oder mehr und besseren Lernmöglichkeiten erhalten, können sich ebenfalls positiv auswirken. Bulgarien, Luxemburg und Uruguay weisen das gleiche Profil auf; allerdings sind in diesen Ländern die durch den sozioökonomischen Status bedingten Leistungsunterschiede nur durchschnittlich stark ausgeprägt. Dies lässt den Schluss zu, dass sich allgemeine Maßnahmen, die auf eine Steigerung der Leistungen aller Schülerinnen und Schüler ausgerichtet sind, ebenfalls als wirkungsvoll erweisen könnten.

In Ländern, in denen die Auswirkungen des sozioökonomischen Status auf die Leistungen hoch sind (steile Steigung), aber nur ein Teil der Leistungsvarianz durch den sozioökonomischen Status erklärt wird (schwache sozioökonomische Gradiente), sind Maßnahmen, die auf benachteiligte Schüler zugeschnitten sind, möglicherweise weniger wirkungsvoll. In diesen Ländern gibt es in der Regel eine große Gruppe leistungsschwacher Schülerinnen und Schüler, die nicht aus benachteiligten Verhältnissen stammen. Unter den leistungsstarken Ländern, die an PISA 2012 teilgenommen haben, ist Australien das einzige

- Abbildung II.5.7 -

\section{Zusammenhang zwischen den Leistungen der Schulen und ihrem sozioökonomischen Profil in Ländern mit niedrigen Leistungen sowie steilen und starken Gradienten: Ungarn, Israel, Singapur und die Slowakische Republik}

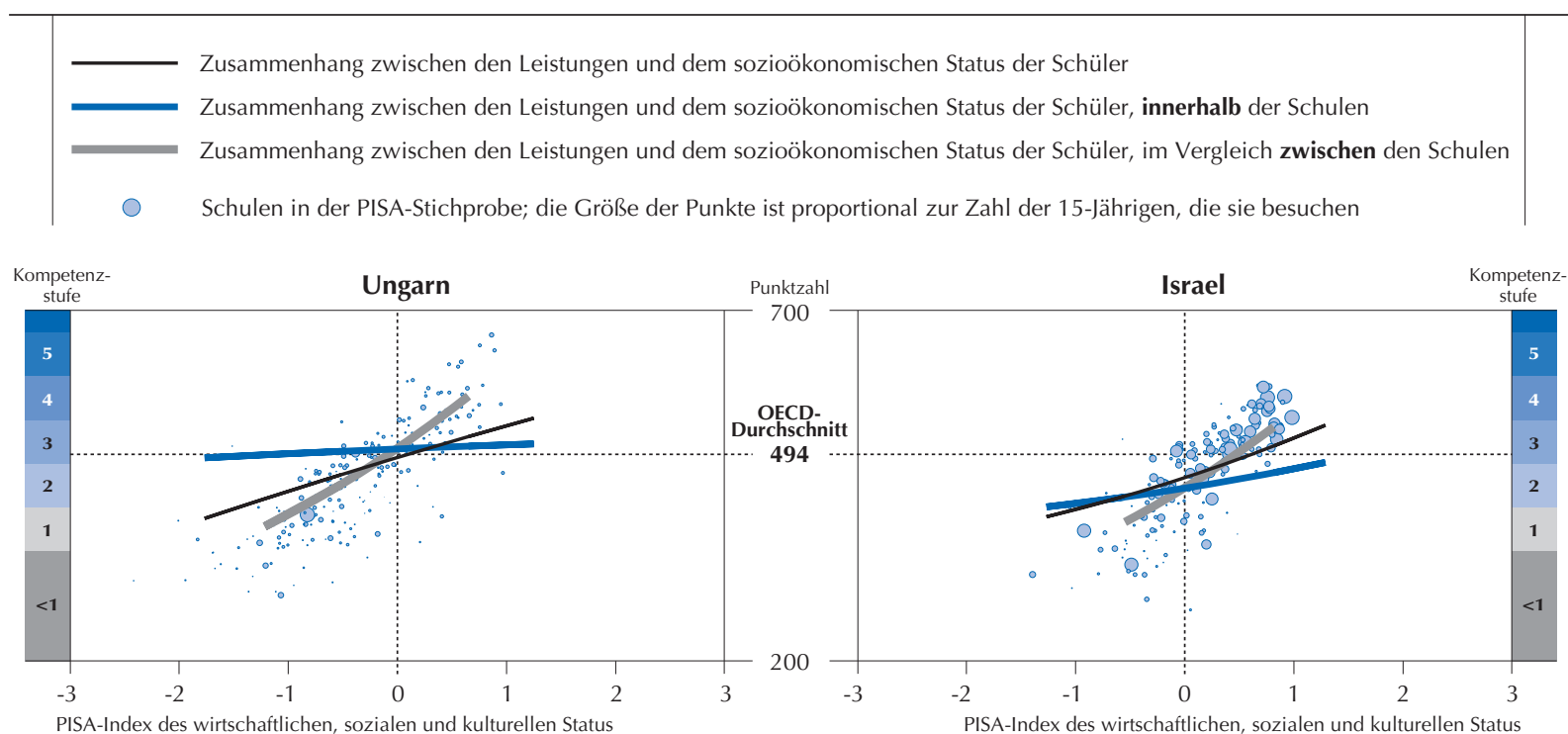

PISA-Index des wirtschaftlichen, sozialen und kulturellen Status

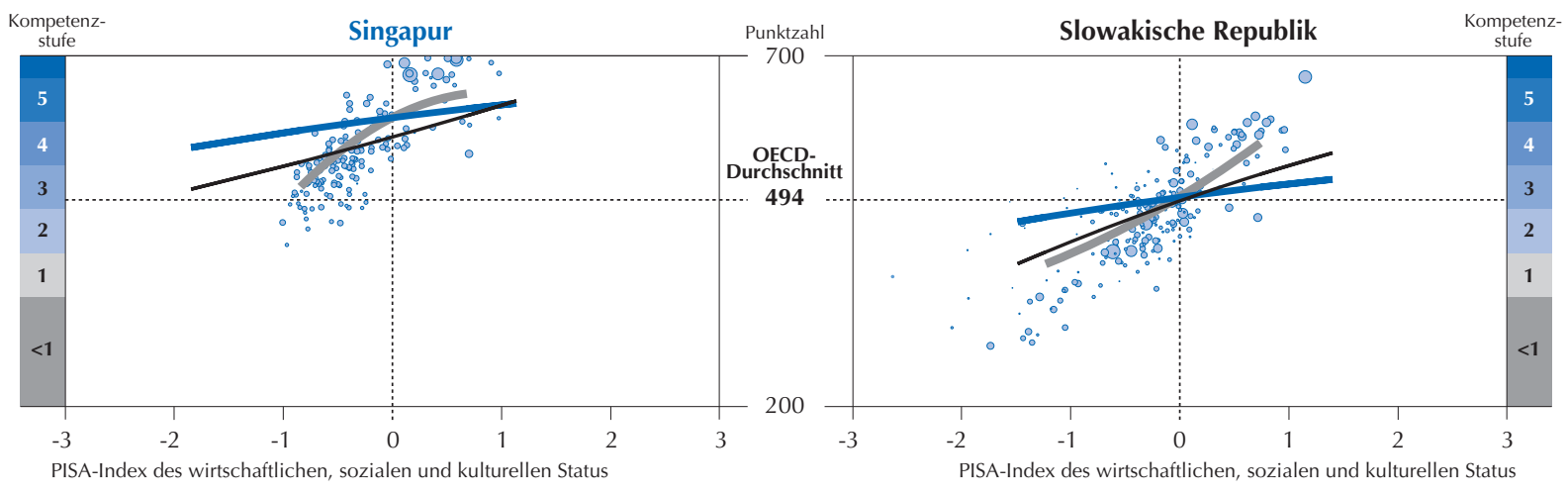

Quelle: OECD, PISA-2012-Datenbank.

StatLink 年IIS http://dx.doi.org/10.1787/888932965079 
mit einem schwachen Zusammenhang zwischen Leistung und sozioökonomischem Status und überdurchschnittlichen Leistungsunterschieden zwischen verschiedenen sozioökonomischen Gruppen. Diese großen Unterschiede deuten darauf hin, dass Australien durch Maßnahmen, die auf benachteiligte Schüler und Schulen ausgerichtet sind, eine Verbesserung der Bildungsgerechtigkeit erzielen und zugleich das hohe Leistungsniveau aufrechterhalten könnte. Vietnam ist das einzige leistungsstarke Land, in dem die mit dem sozioökonomischen Status verbundenen Leistungsunterschiede gering sind und die Stärke des Zusammenhangs zwischen den Leistungen und dem sozioökonomischen Status ungefähr dem Durchschnitt entspricht. In diesem Fall könnten Maßnahmen, die auf leistungsschwache Schüler und Schulen zugeschnitten sind, dazu beitragen, die Bildungsgerechtigkeit zu verbessern und zugleich das hohe Leistungsniveau aufrechtzuerhalten.

\section{GROSSE SOZIOÖKONOMISCHE DISPARITÄTEN}

Wichtig für die Interpretation des Zusammenhangs zwischen Leistung und sozioökonomischem Status ist auch die Kenntnis des Umfangs der sozioökonomischen Disparitäten innerhalb der betreffenden Länder. Beispielsweise haben Bulgarien, Japan, Korea, Luxemburg, Schweden und Uruguay sozioökonomische Gradienten mit ähnlichen Steigungen (mit dem sozioökonomischen Status verbundene Leistungsunterschiede zwischen 36 und 42 Punkten); doch die Bandbreite der Werte auf dem PISA-Index des wirtschaftlichen, sozialen und kulturellen Status (Länge der Gradiente) zwischen dem 5. und

- Abbildung II.5.8 "

\section{Zusammenhang zwischen den Leistungen der Schulen und ihrem sozioökonomischen Profil in Ländern mit starken sozioökonomischen Disparitäten und durchschnittlicher Bildungsgerechtigkeit: Bulgarien, Kolumbien, Costa Rica und Irland}
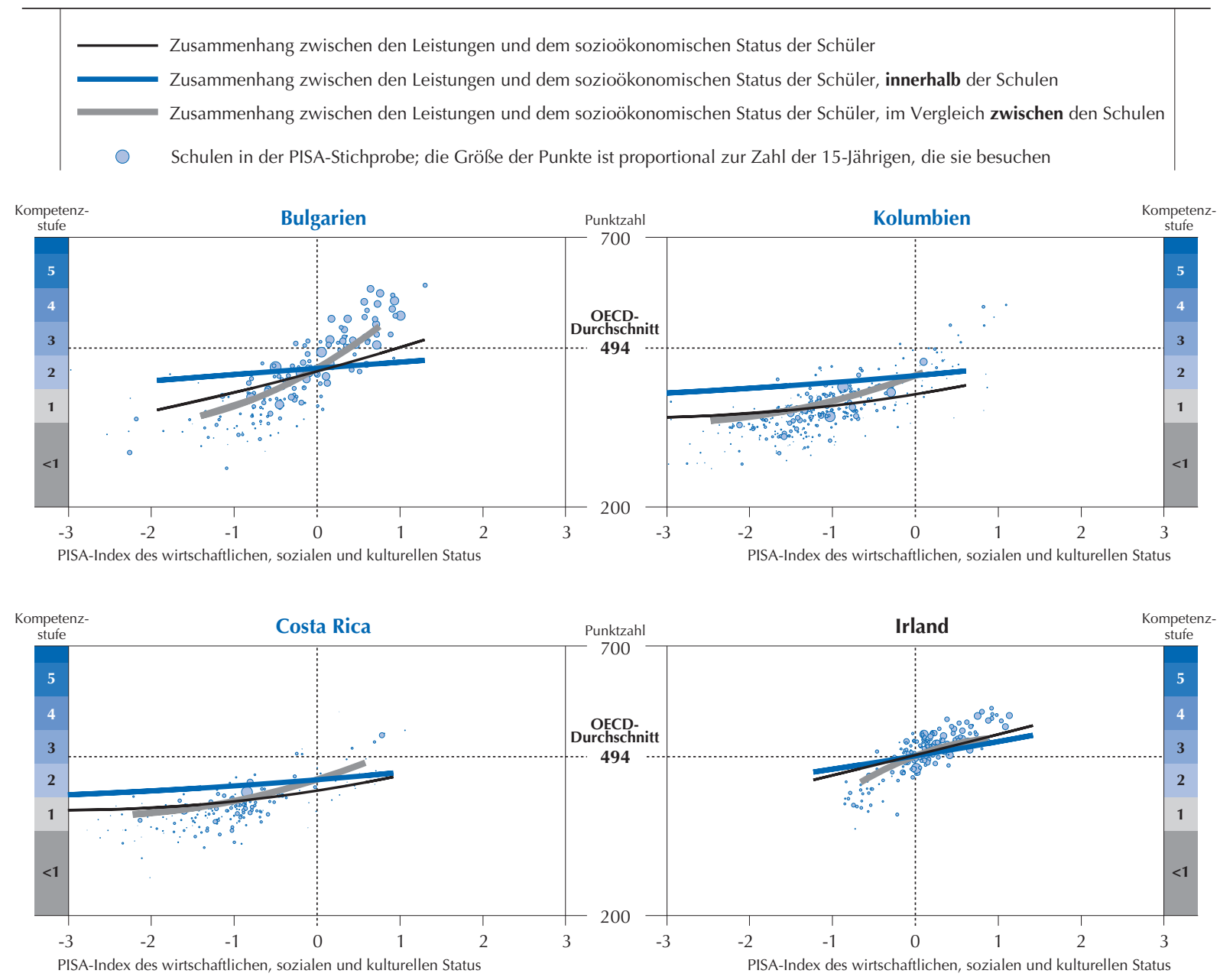

Quelle: OECD, PISA-2012-Datenbank.

StatLink 完政 http://dx.doi.org/10.1787/888932965098 
95. Perzentil der Schülerinnen und Schüler umfasst in Bulgarien, Luxemburg und Uruguay mindestens 3,2 Einheiten auf dem Index (mehr als das Dreifache des durchschnittlichen Unterschieds im sozioökonomischen Status von zwei zufällig ausgewählten Schülern im OECD-Raum), aber weniger als 2,5 Einheiten in Japan, Korea und Schweden. Anders ausgedrückt: Die Schülerpopulation der 15-Jährigen in Bulgarien, Luxemburg und Uruguay ist in sozioökonomischer Hinsicht wesentlich heterogener als die Schülerpopulation in Japan, Korea und Schweden. Dieser Unterschied erklärt zum Teil, warum der sozioökonomische Status in Schweden beispielsweise nur einen unterdurchschnittlichen Anteil der Leistungsvarianz ausmacht, während er in Uruguay überdurchschnittlich starke Auswirkungen auf die Leistung hat. Die auf diese Weise gemessene sozioökonomische Heterogenität ist im OECD-Raum in Chile, Mexiko, Portugal und der Türkei am größten; viele Partnerländer und -volkswirtschaften weisen jedoch ebenfalls eine über dem OECD-Durchschnitt liegende sozioökonomische Heterogenität auf, insbesondere Brasilien, Costa Rica, Kolumbien, Peru, Thailand, Tunesien, Uruguay und Vietnam. In allen diesen Ländern und Volkswirtschaften können kompensatorische Maßnahmen, die gezielt auf benachteiligte Schüler und Schulen ausgerichtet sind, zur Verbesserung der Leistungen und der Bildungsgerechtigkeit beitragen.

- Abbildung II.5.9 -

\section{Zusammenhang zwischen den Leistungen der Schulen und ihrem sozioökonomischen Profil in Ländern mit hoher Bildungsgerechtigkeit, aber starken sozioökonomischen Disparitäten: Hongkong (China), Jordanien, Katar und Thailand}
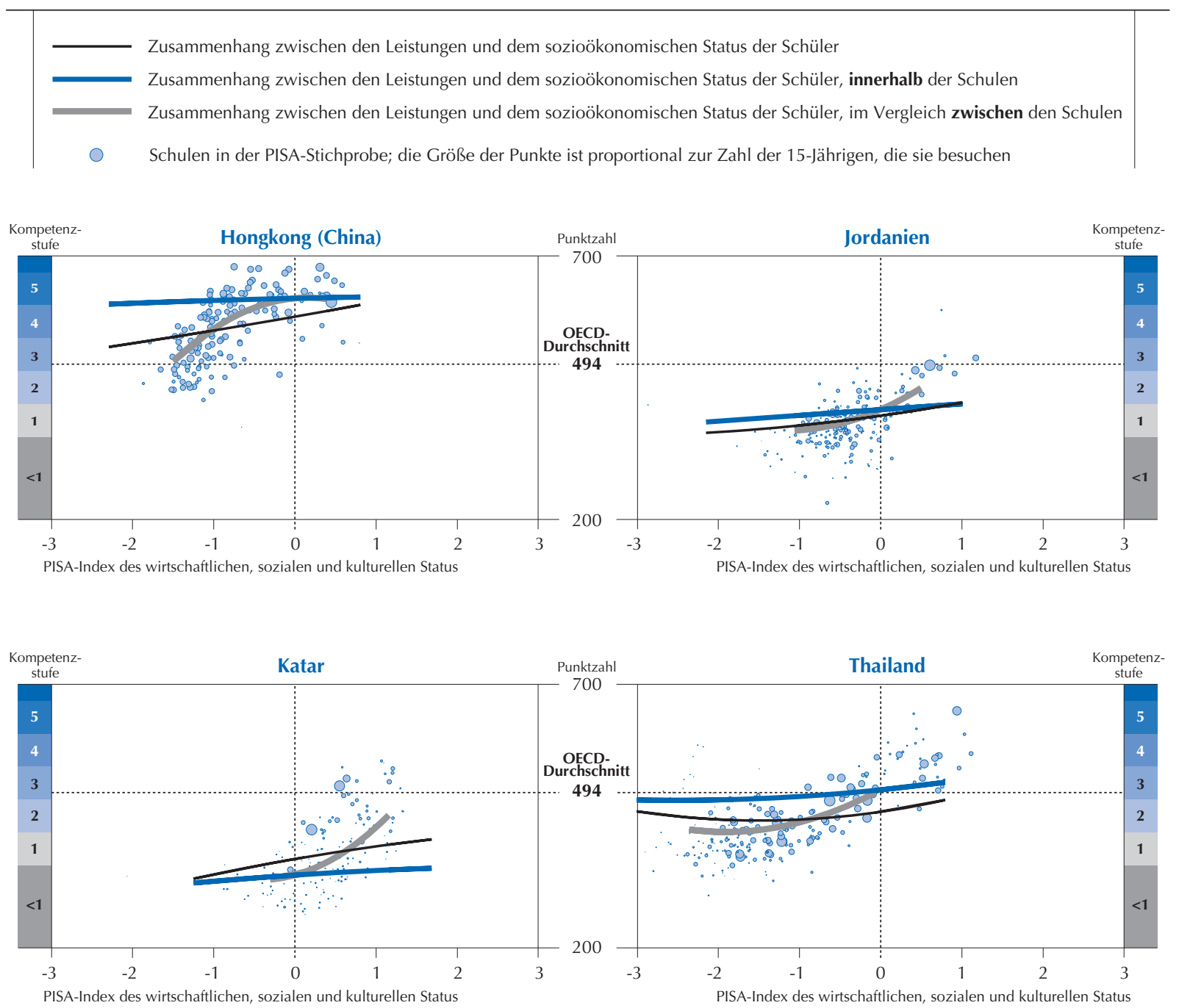

Quelle: OECD, PISA-2012-Datenbank.

StatLink त्ञाज http://dx.doi.org/10.1787/888932965117 
In Ländern mit großen Disparitäten und einem schwachen Zusammenhang zwischen sozioökonomischem Status und Leistung, wie z.B. Mexiko und Thailand, wären kompensatorische Maßnahmen zur Unterstützung der besonders benachteiligten Schülerinnen und Schüler wirkungsvoll. Wenn dagegen die sozioökonomischen Disparitäten geringer sind und einen schwächeren Effekt auf die Leistungen haben, wie es beispielsweise in Finnland, Japan, Korea, Norwegen und der Tschechischen Republik der Fall ist, dürften Maßnahmen, die auf Sozialreformen ausgerichtet sind, nicht der effektivste Weg zur Verbesserung der Schülerleistungen sein, da sie aller Wahrscheinlichkeit nach nicht viele Schüler erreichen.

\section{GEZIELTE AUSRICHTUNG VON MASSNAHMEN AUF LEISTUNGSSCHWACHE UND SOZIOÖKONOMISCH BENACHTEILIGTE SCHULEN}

Bei großen Leistungsunterschieden bzw. sozioökonomischen Disparitäten zwischen einzelnen Schulen sind u.U. Maßnahmen effektiver, die gezielt auf Schulen anstatt Schüler ausgerichtet sind, insbesondere wenn ein starker und deutlich erkennbarer Zusammenhang zwischen dem sozioökonomischen Profil und den Durchschnittsleistungen einer Schule besteht.

Der Zusammenhang zwischen dem sozioökonomischen Profil einer Schule und den Schülerleistungen kann aus verschiedenen Perspektiven betrachtet werden. Zum einen kann man sich darauf konzentrieren, wie groß der erwartete Leistungsvorsprung in Mathematik bei Schülern ist, die Schulen mit einer begünstigteren Schülerpopulation besuchen. Ein zweiter Aspekt ist, wie sehr die Leistungen der einzelnen Schüler diesen Vorhersagen tatsächlich entsprechen, d.h. die Stärke des Zusammenhangs ${ }^{1}$. All diese Faktoren spielen in Ländern, in denen Unterschiede im sozioökonomischen Profil der Schulen starke Auswirkungen auf die Lernmöglichkeiten der Schülerinnen und Schüler haben, eine wichtige Rolle. In Ländern, in denen große Unterschiede festgestellt werden, dürfte es mit Maßnahmen, die auf benachteiligte Schulen ausgerichtet sind, eher gelingen, die Leistungen und die Bildungsgerechtigkeit zu verbessern.

Wie Abbildung II.5.1b zeigt, sind in Uruguay 74\% der Leistungsunterschiede zwischen den einzelnen Schulen auf sozioökonomische Faktoren zurückzuführen, d.h. die Schulen fallen in der Regel in zwei Kategorien: Schulen mit höherem Leistungsniveau und begünstigterem sozioökonomischem Profil und Schulen mit niedrigerem Leistungsniveau und einer benachteiligteren Schülerpopulation. Vor allem aber ist die Varianz des sozioökonomischen Profils der Schulen groß, d.h. zwischen begünstigten und benachteiligten Schulen bestehen große Unterschiede beim sozioökonomischen Status. Demgegenüber sind in Island die mit dem sozioökonomischen Profil der Schulen verbundenen Leistungsdisparitäten zwar ebenfalls vergleichsweise groß (69\%), doch insgesamt sind die Unterschiede zwischen den sozioökonomischen Profilen

- Abbildung II.5.10

\section{Zusammenhang zwischen den Leistungen der Schulen und ihrem sozioökonomischen Profil in Ländern mit starken zwischenschulischen Gradienten: Island und Uruguay}
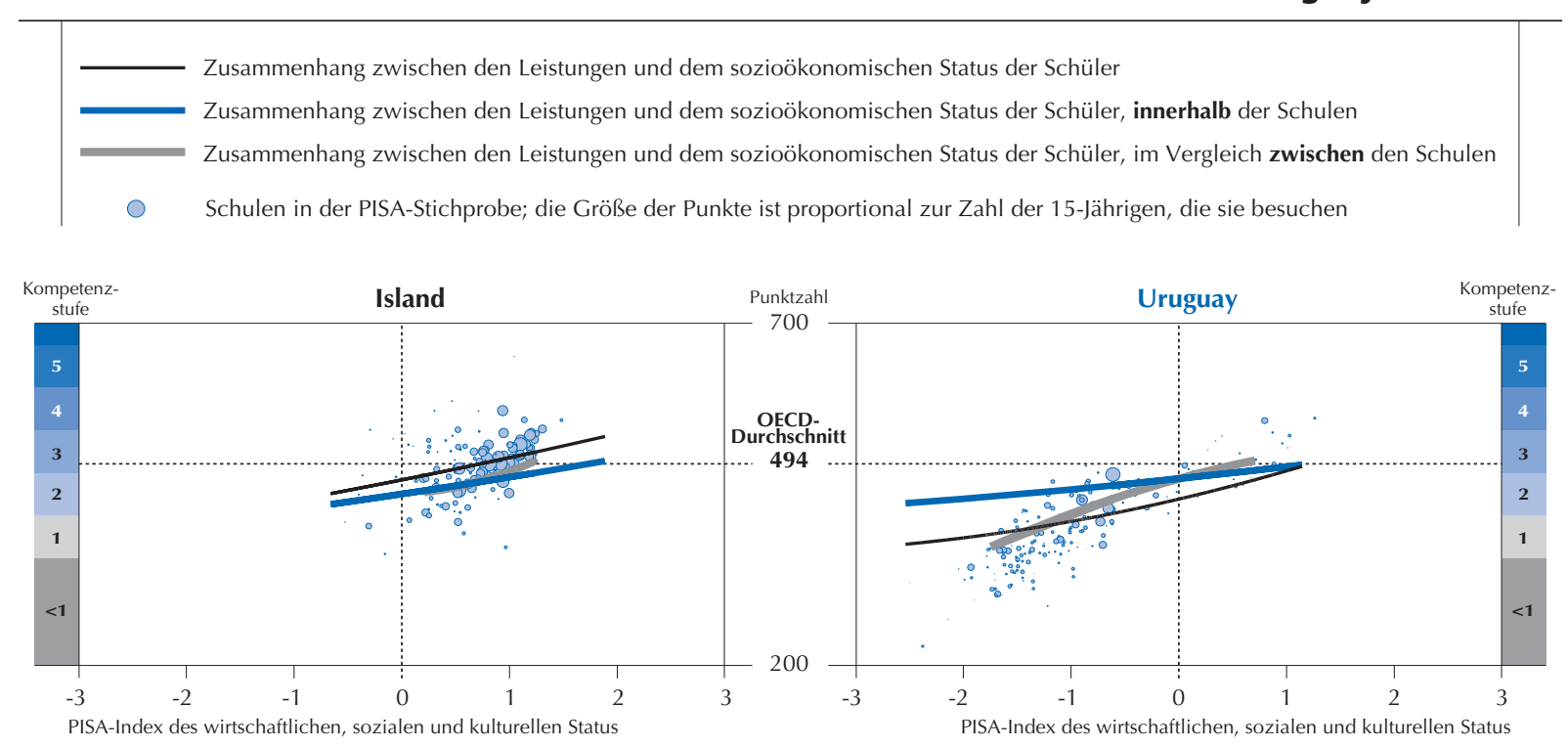

Quelle: OECD, PISA-2012-Datenbank.

StatLink त्ञाI http://dx.doi.org/10.1787/888932965136 
der Schulen wesentlich geringer. Dies erklärt, warum Island alles in allem über eines der gerechteren Bildungssysteme verfügt, während Uruguay eines der ungerechtesten Systeme hat. In Ländern, in denen die Leistungsvarianz überwiegend durch zwischen den Schulen bestehende sozioökonomische Unterschiede bedingt ist, sollten prioritär Maßnahmen ergriffen werden, die auf eine Verringerung der sozialen Segregation abzielen, da solche Disparitäten zwischen den Schulen in der Regel die Ungerechtigkeiten des Systems verstärken.

Ähnliche Gegensätze sind auch zwischen Ländern zu beobachten, in denen die mit einem unterschiedlichen sozioökonomischen Status verbundenen Leistungsdifferenzen relativ gering sind. In Norwegen und Portugal beispielsweise sind die zwischenschulischen Leistungsdifferenzen in Mathematik relativ gering (ungefähr 49 bzw. 33 Punkte, verglichen mit einem OECD-Durchschnitt von über 70 Punkten). Beim sozioökonomischen Profil bestehen jedoch erhebliche Unterschiede zwischen den Schulen. In Portugal, wo sich 62\% der Leistungsvarianz durch das sozioökonomische Profil der Schulen erklären lassen, ist das sozioökonomische Profil einer Schule ein wesentlich besserer Prädiktor der Schülerleistungen als in Norwegen, wo 46\% der Leistungsvarianz auf das sozioökonomische Profil zurückgeführt werden können. Das bedeutet, dass in Norwegen viele Schulen ein anderes Leistungsniveau aufweisen, als auf Grund ihres sozioökonomischen Profils zu erwarten wäre, wohingegen in Portugal ein enger Zusammenhang zwischen dem sozioökonomischen Status und dem Leistungsniveau der Schulen besteht. Auf benachteiligte Schulen ausgerichtete Maßnahmen dürften daher in Portugal mit höherer Wahrscheinlichkeit Wirkung zeigen. In Norwegen ist leistungsschwächeren Schulen, die nicht unbedingt benachteiligt sind, mit solchen Maßnahmen nicht geholfen.

Gezielte Maßnahmen für benachteiligte Schulen können in Ländern zweckmäßig sein, in denen große Unterschiede beim sozioökonomischen Profil bestehen (d.h. die zwischenschulischen sozioökonomischen Unterschiede machen einen großen Anteil der Varianz beim sozioökonomischen Status aus). Derartige Maßnahmen können sich dort als besonders effektiv erweisen, wo der Zusammenhang zwischen den Leistungen und dem sozioökonomischen Status auf Schulebene deutlich erkennbar ist, entweder weil große Leistungsunterschiede zwischen Schulen mit unterschiedlichem sozioökonomischem Profil bestehen oder weil ein starker Zusammenhang zwischen dem sozioökonomischen Profil einer Schule und den durchschnittlichen Schülerleistungen an dieser Schule besteht.

Wichtig wäre eine gezielte Ausrichtung von Maßnahmen auf benachteiligte Schulen u.U. in Ungarn, Shanghai (China) und der Slowakischen Republik, wo die sozioökonomischen Unterschiede zwischen den Schulen groß sind - sie machen einen überdurchschnittlich hohen Anteil der Varianz beim sozioökonomischen Status aus (37\%, 33\% bzw.

- Abbildung II.5.11

\section{Zusammenhang zwischen den Leistungen der Schulen und ihrem sozioökonomischen Profil in Ländern mit schwachen zwischenschulischen Gradienten: Norwegen und Portugal}

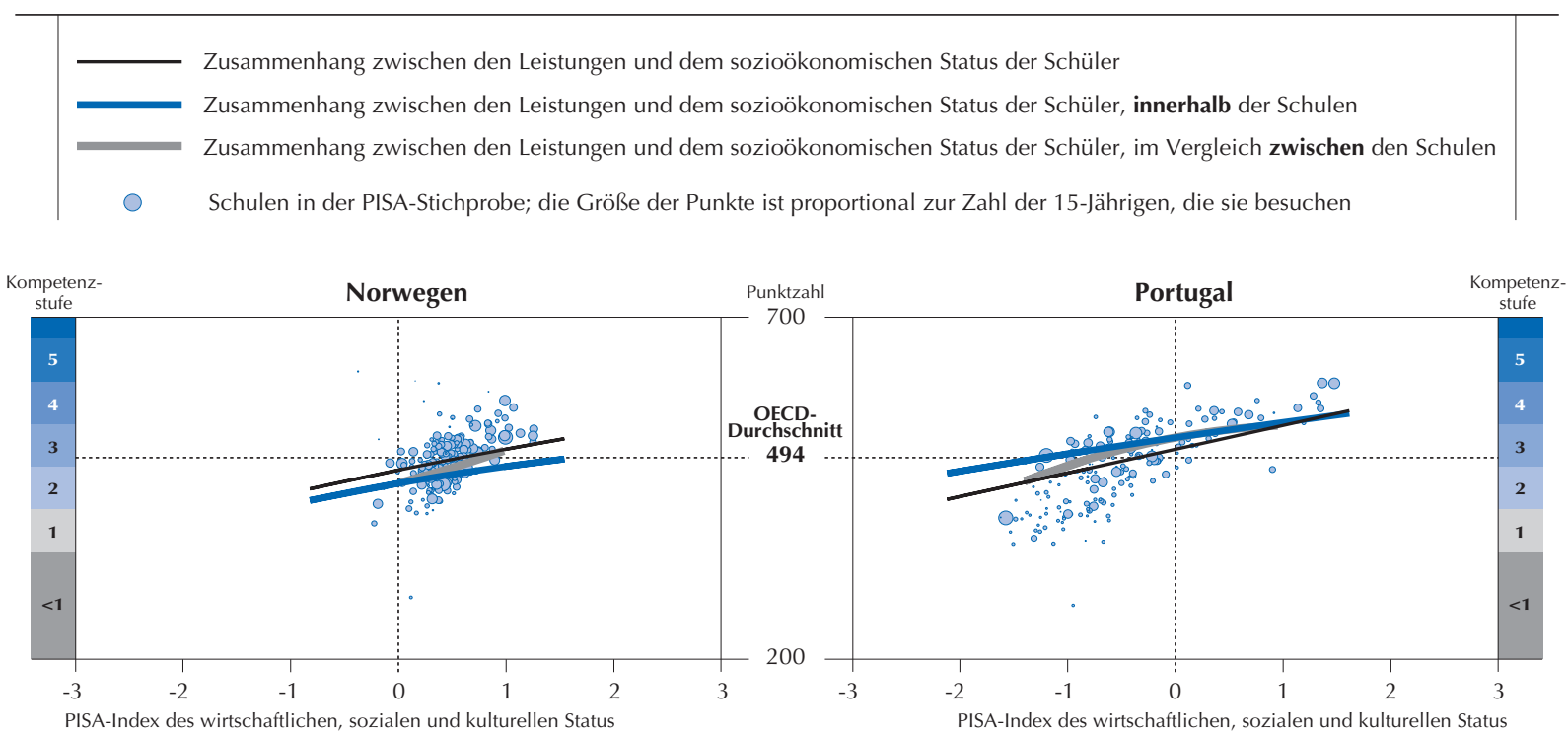

Quelle: OECD, PISA-2012-Datenbank.

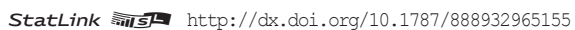


36\%, verglichen mit einem OECD-Durchschnittswert von 24\%, [Abb. II.5.1b]) - und wo die zwischenschulischen Leistungsunterschiede zwischen verschiedenen sozioökonomischen Gruppen (98 Punkte, 88 Punkte bzw. 86 Punkte) über dem OECD-Durchschnitt von 72 Punkten liegen. In allen drei Ländern besteht ein starker Zusammenhang zwischen den durchschnittlichen Leistungen der Schulen und ihrem sozioökonomischen Profil, das zwischen 65\% der Varianz der durchschnittlichen Ergebnisse auf Schulebene in Shanghai (China) und 78\% in Ungarn erklärt (verglichen mit einem OECD-Durchschnittswert von 63\%). Argentinien, Brasilien, Bulgarien, Chile, Costa Rica, Kolumbien, Peru, Portugal, Rumänien und Uruguay weisen ebenfalls große zwischenschulische Unterschiede beim sozioökonomischen Profil sowie einen starken Zusammenhang zwischen Leistungen und sozioökonomischem Status auf. In all diesen Ländern sind die mit dem sozioökonomischen Profil der Schulen verbundenen Leistungsunterschiede zwischen den Schulen unterdurchschnittlich ausgeprägt (außer in Bulgarien, wo sie dem Durchschnitt entsprechen); doch die Stärke des Zusammenhangs zwischen den durchschnittlichen Leistungen der Schulen und ihrem sozioökonomischen Profil liegt am oder über dem Durchschnitt. Der Anteil der Leistungsunterschiede, der sich durch die Varianz beim sozioökonomischen Profil erklären lässt, reicht von $60 \%$ in Kolumbien bis $78 \%$ in Peru. Hongkong (China) ist die einzige Volkswirtschaft, in der die zwischenschulischen Unterschiede im sozioökonomischen Profil dem OECD-Durchschnitt entsprechen, die Stärke des Zusammenhangs zwischen dem sozioökonomischen Profil und den Leistungen der Schulen aber unter dem Durchschnitt liegt.

Die gezielte Ausrichtung von Maßnahmen auf leistungsschwache Schulen könnte ebenfalls eine Option sein, insbesondere wenn der sozioökonomische Status der Schule einen starken Zusammenhang mit den Leistungsunterschieden aufweist. Die im Rahmen von PISA gewonnene Evidenz deutet darauf hin, dass Maßnahmen, die auf leistungsschwache Schulen ausgerichtet sind, in Belgien, Deutschland, der Slowakischen Republik, Slowenien, derTschechischen Republik und Ungarn besonders wirkungsvoll sein könnten. In all diesen Ländern machen zwischenschulische Unterschiede mindestens die Hälfte der Leistungsvarianz aus. Die Schulsysteme dieser Länder verteilen die Schülerinnen und Schüler in der Regel ihren Leistungen entsprechend auf verschiedene Schulen. Folglich ist es nicht überraschend, dass es in diesen Ländern erhebliche zwischenschulische Leistungsunterschiede gibt. Der durchschnittliche Effekt des sozioökonomischen Profils der Schulen auf die Leistungen (zwischenschulische sozioökonomische Gradiente) reicht von 86 Punkten in der Slowakischen Republik bis hin zu 127 Punkten in der Tschechischen Republik, verglichen mit einem OECD-Durchschnittswert von 72 Punkten. In all diesen Ländern lassen sich mehr als $70 \%$ der Leistungsunterschiede durch den sozioökonomischen Status der Schüler und der Schulen erklären, verglichen mit einem OECD-Durchschnittswert von 63\% (Abb. II.5.1b). Sozioökonomische Disparitäten zwischen verschiedenen Schulen machen in Ungarn und in der Slowakischen Republik mehr als 35\% der Varianz beim sozioökonomischen Status aus. Die innerschulischen Leistungsunterschiede zwischen verschiedenen sozioökonomischen Gruppen liegen nur in der Slowakischen Republik über dem Durchschnitt.

\section{GEZIELTE AUSRICHTUNG VON MASSNAHMEN AUF BESTIMMTE SCHÜLERINNEN UND SCHÜLER INNERHALB VON SCHULEN}

Bis zu einem gewissen Grad ist damit zu rechnen, dass in Schulsystemen, die Schülerinnen und Schüler ihren Fähigkeiten entsprechend auf verschiedene Schulen aufteilen, die Leistungsunterschiede innerhalb der einzelnen Schulen sowohl insgesamt als auch bezogen auf den sozioökonomischen Status geringer ausfallen. Die Kehrseite der Medaille besteht darin, dass in diesen Ländern ein größerer Teil der Leistungsunterschiede auf soziale Disparitäten zwischen den Schulen zurückzuführen ist als auf soziale Disparitäten innerhalb der Schulen. Dadurch ergeben sich bei der Analyse anhand der innerschulischen Gradienten selbst für Korea und Vietnam ähnliche Ergebnisse, obwohl gemessen an der zwischenschulischen Gradiente Korea zu den Ländern mit der geringsten Bildungsgerechtigkeit zählt, Vietnam dagegen zu den Ländern mit der höchsten Bildungsgerechtigkeit. In keinem Land entfallen auf sozioökonomische Unterschiede innerhalb der Schulen mehr als 11\% der Varianz der Schülerleistungen.

In Dänemark, Finnland, Neuseeland, Polen, Portugal, Schweden und Spanien lassen sich mindestens 9\% der innerschulischen Leistungsunterschiede durch Unterschiede beim sozioökonomischen Status erklären - ein überdurchschnittlich starker Zusammenhang zwischen Leistung und sozioökonomischem Status innerhalb von Schulen. In all diesen Ländern würden sich Maßnahmen auf Schulebene, zusätzlich zu Maßnahmen auf Systemebene, am ehesten als wirksam erweisen.

Abbildung II.5.13 zeigt für alle in den vorangegangenen Kapiteln nicht als Beispiele aufgeführten OECD-Länder sowie Partnerländer und -volkswirtschaften den Zusammenhang zwischen den Leistungen und dem sozioökonomischen Profil der Schulen. 
- Abbildung II.5.12 "

Zusammenhang zwischen den Leistungen der Schulen und ihrem sozioökonomischen Profil in Ländern mit starken innerschulischen Leistungsunterschieden:

Dänemark, Finnland, Spanien und die Schweiz
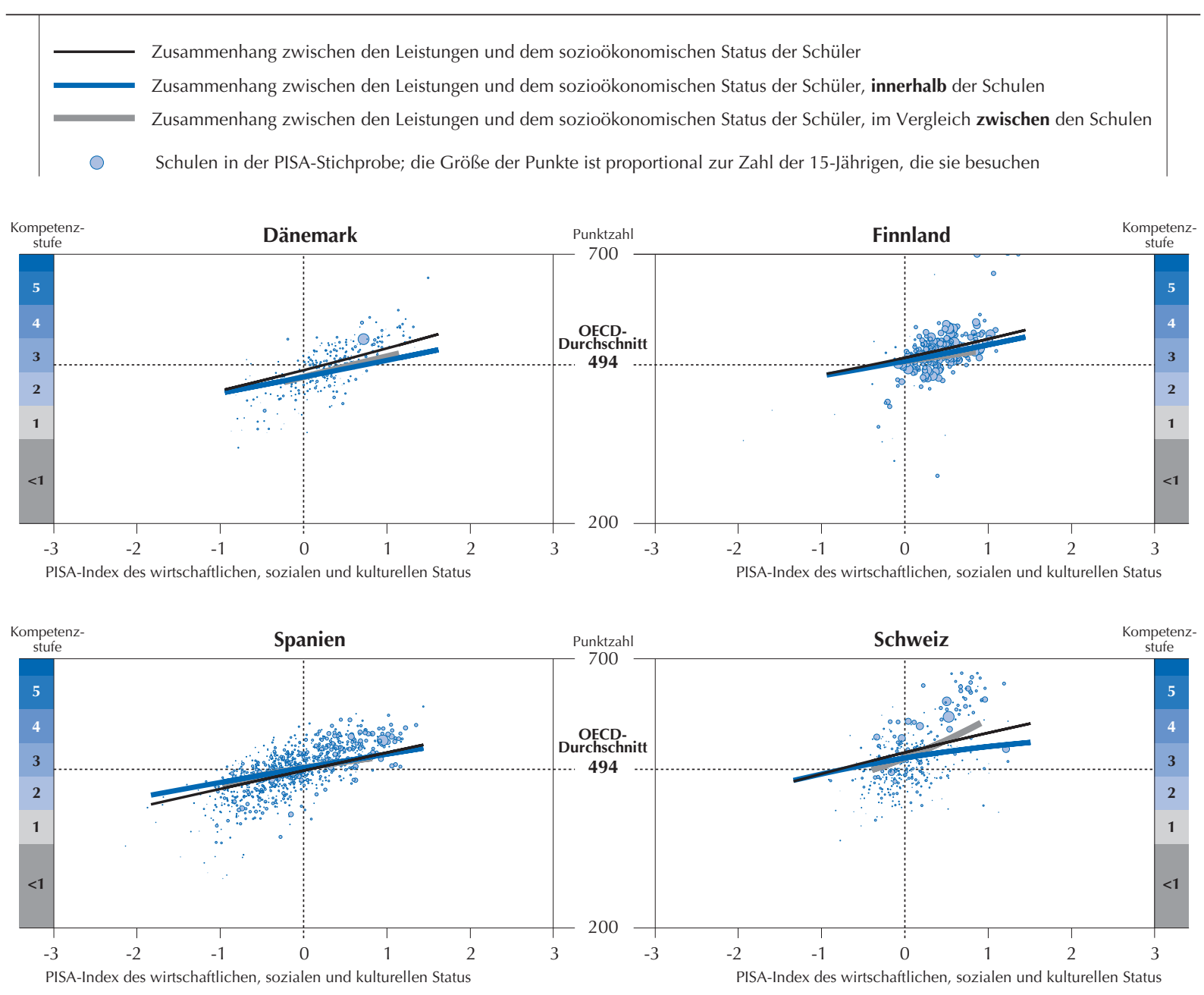

Quelle: OECD, PISA-2012-Datenbank.

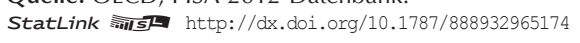


- Abbildung II.5.13 [Teil 1/5] "

\section{Zusammenhang zwischen den Leistungen der Schulen und ihrem sozioökonomischen Profil in allen anderen Ländern und Volkswirtschaften}
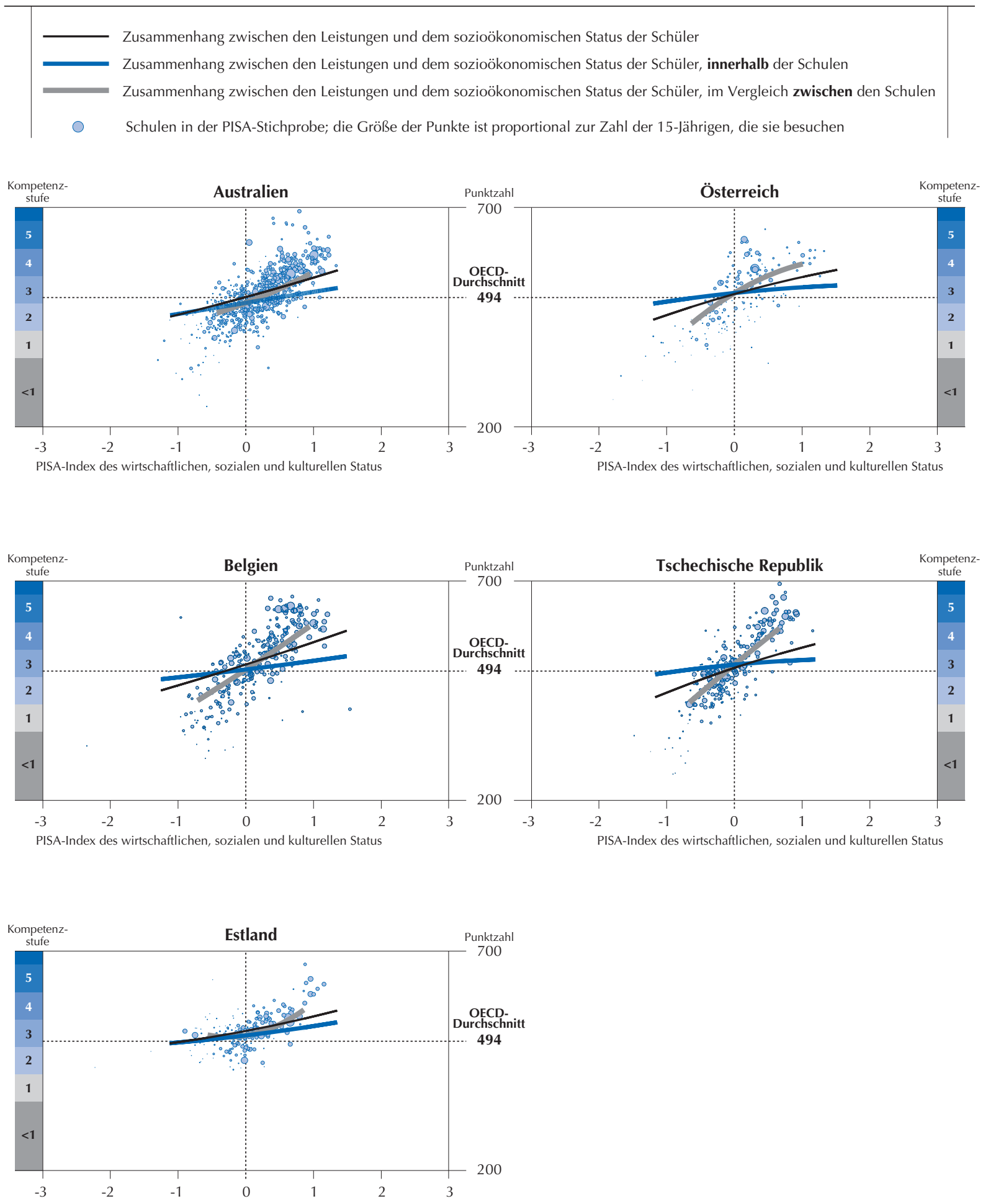

PISA-Index des wirtschaftlichen, sozialen und kulturellen Status

Quelle: OECD, PISA-2012-Datenbank.

StatLink *ills http://dx.doi.org/10.1787/888932965193

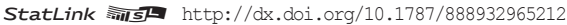


- Abbildung II.5.13 [Teil 2/5] "

\section{Zusammenhang zwischen den Leistungen der Schulen und ihrem sozioökonomischen Profil in allen anderen Ländern und Volkswirtschaften}
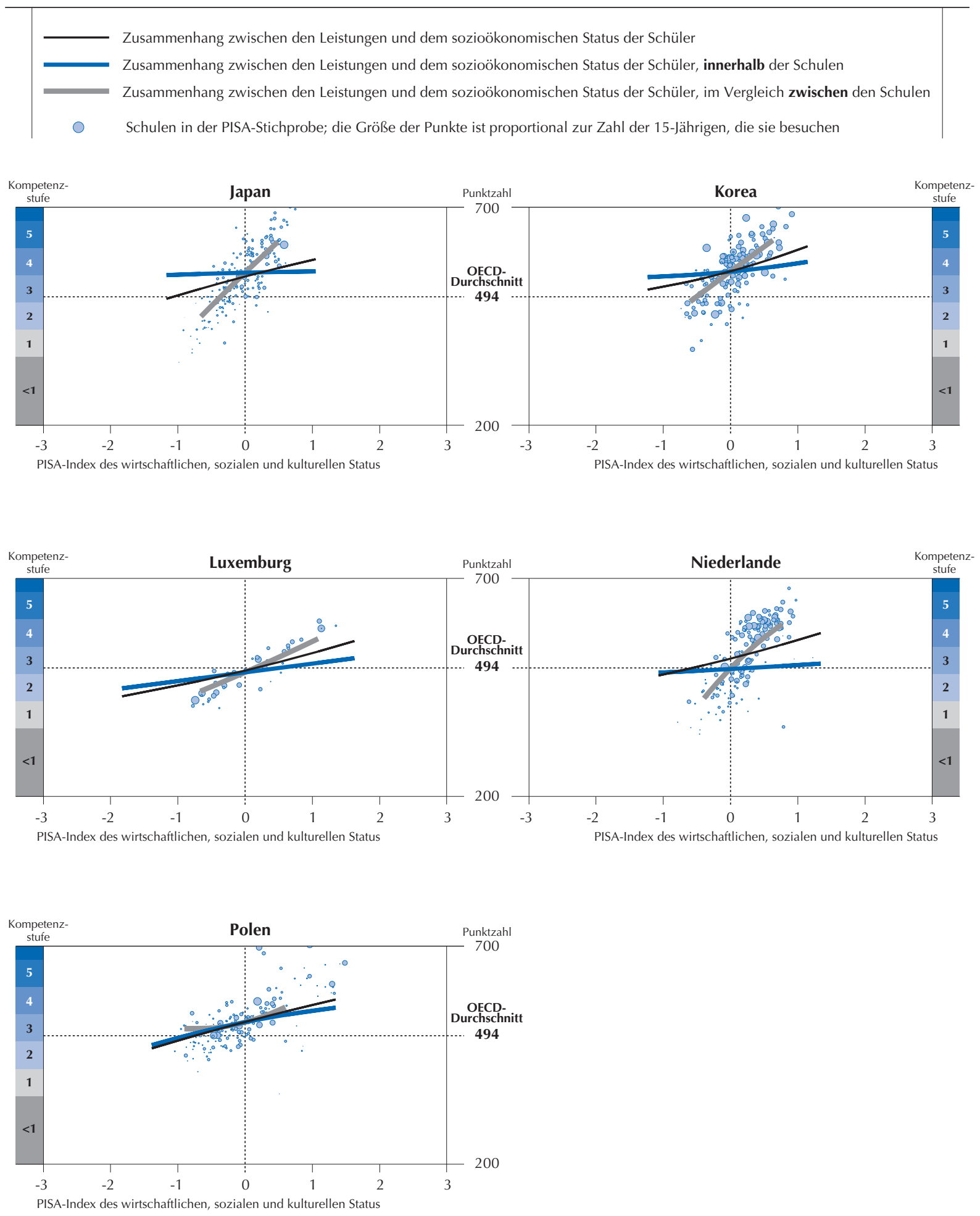

Quelle: OECD, PISA-2012-Datenbank.

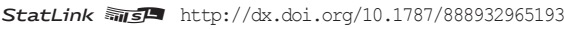

StatLink त्नाlा ht htp://dx.doi.org/10.1787/888932965212 
- Abbildung II.5.13 [Teil 3/5] "

\section{Zusammenhang zwischen den Leistungen der Schulen und ihrem sozioökonomischen Profil in allen anderen Ländern und Volkswirtschaften}
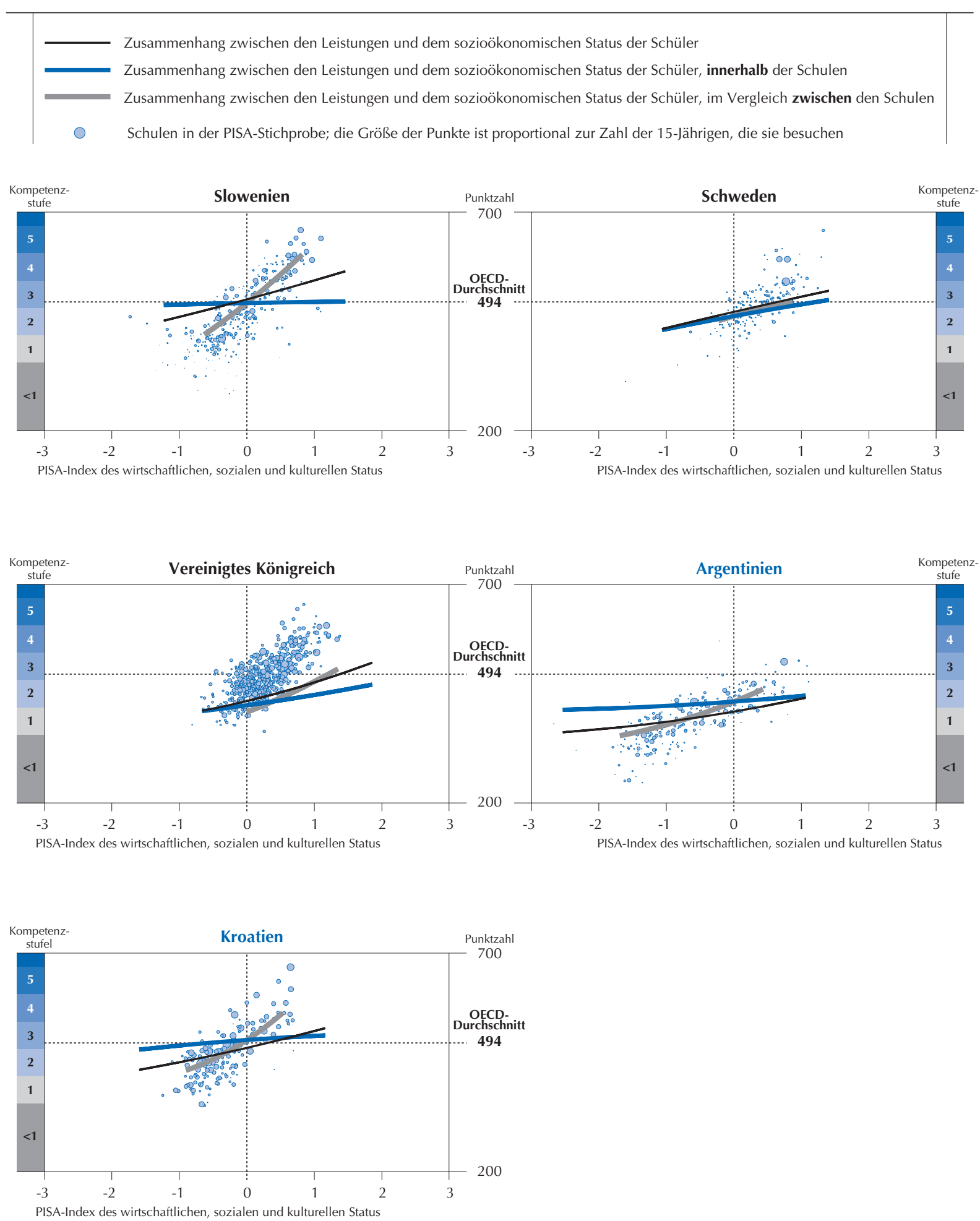

Quelle: : OECD, PISA-2012-Datenbank.

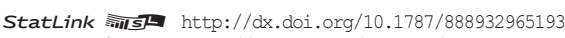
StatLink ints http://dx.doi.org/10.1787/888932965212 
- Abbildung II.5.13 [Teil 4/5] "

\section{Zusammenhang zwischen den Leistungen der Schulen und ihrem sozioökonomischen Profil in allen anderen Ländern und Volkswirtschaften}
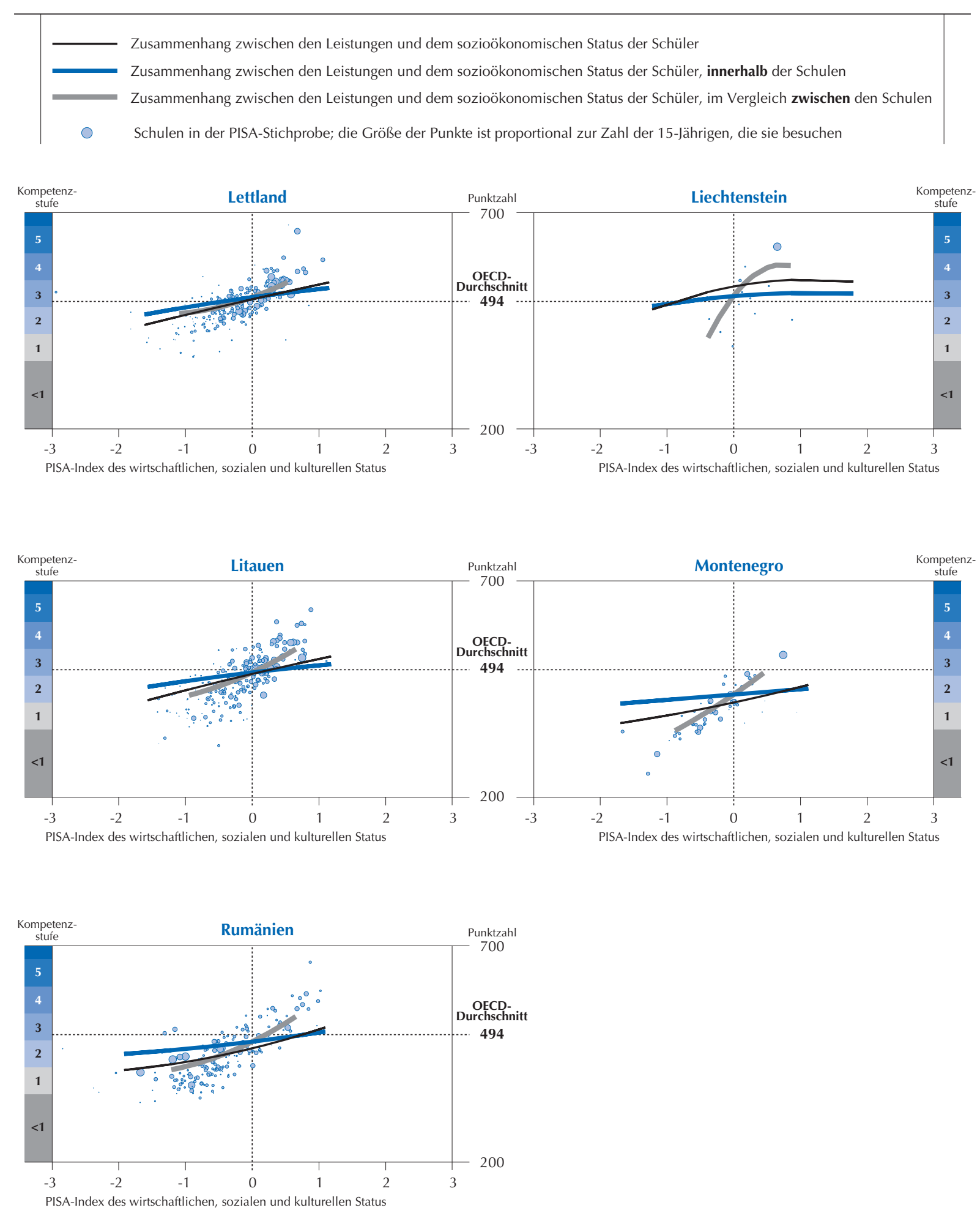

Quelle: OECD, PISA-2012-Datenbank.

StatLink तins http://dx.doi.org/10.1787/888932965193

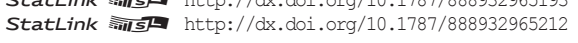


- Abbildung II.5.13 [Teil 5/5] "

Zusammenhang zwischen den Leistungen der Schulen und ihrem sozioökonomischen Profil in allen anderen Ländern und Volkswirtschaften
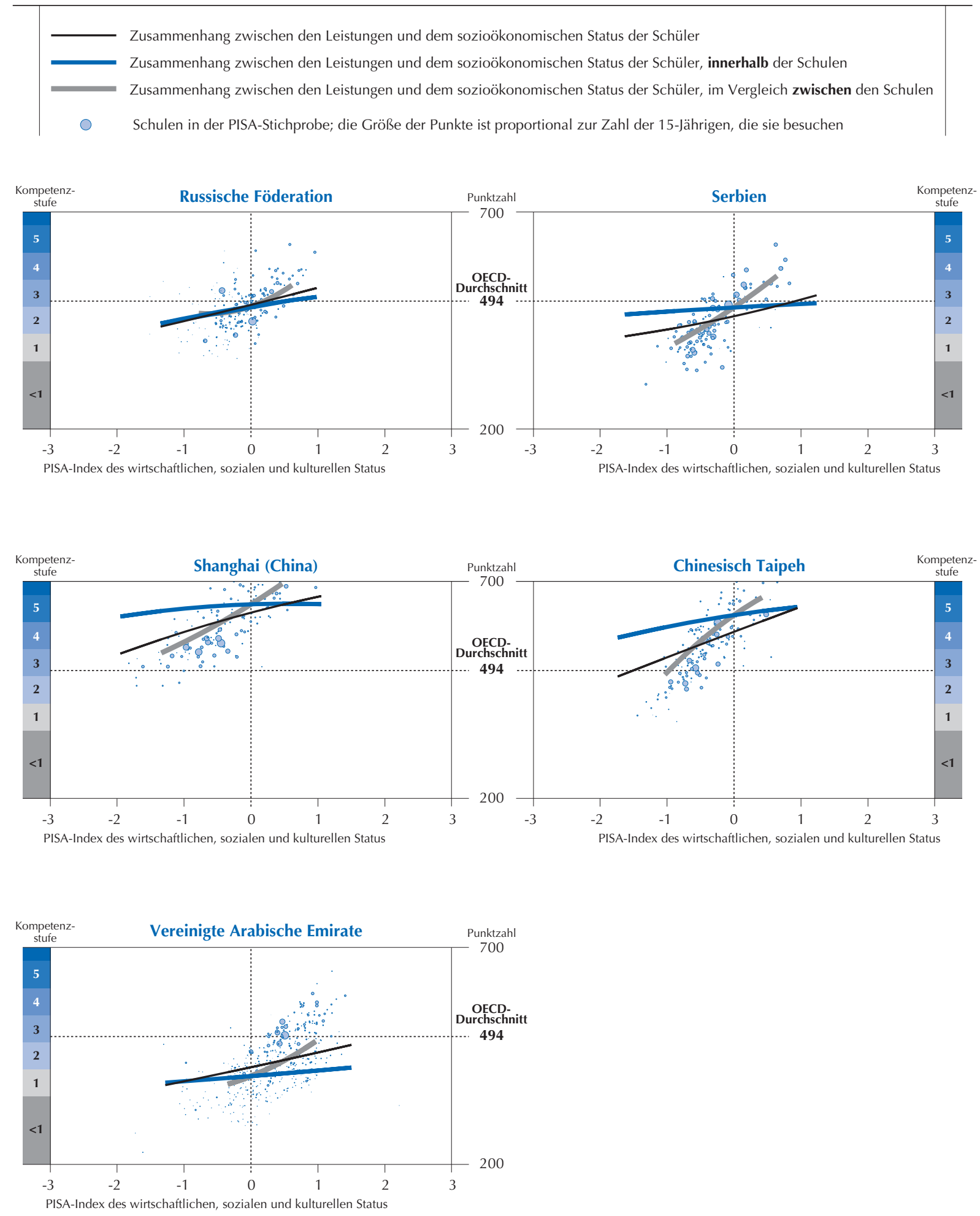

Quelle: OECD, PISA-2012-Datenbank.

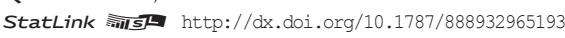
StatLink 需细 http://dx.doi.org/10.1787/888932965212 


\section{Anmerkung}

1. Dabei ist zu beachten, dass die Ergebnisse auch davon abhängen, wie die Schulen in den einzelnen Ländern/Volkswirtschaften definiert und organisiert sind und welche Einheiten für die Stichprobenziehung gewählt wurden. In einigen Ländern z.B. wurden einige der Schulen in der PISA-Stichprobe als Verwaltungseinheiten definiert (selbst wenn sie, wie in Italien, mehrere geografisch getrennte Einrichtungen umfassen); in anderen Ländern wurden sie als jene Teile größerer Bildungseinrichtungen definiert, die von 15-Jährigen besucht werden; in manchen Ländern wurden Schulen wiederum als Schulgebäude definiert, in wieder anderen dagegen aus Sicht der Schulorganisation (z.B. als Einheiten, die eine eigene Schulleitung haben). Der PISA 2012 Technical Report (OECD, erscheint demnächst) liefert einen Überblick über die verschiedenen Methoden der Definition der Schulen. Wegen der Art und Weise, in der die Schülerstichprobe erhoben wurde, ist in der Varianz innerhalb der Schulen sowohl die Varianz zwischen verschiedenen Klassen als auch zwischen verschiedenen Schülerinnen und Schülern enthalten. In Slowenien ist die primäre Stichprobeneinheit definiert als eine Gruppe von Schülerinnen und Schülern, die demselben Bildungsgang innerhalb einer Schule folgen. In diesem besonderen Fall entspricht die Varianz zwischen den Schulen tatsächlich der Varianz innerhalb einer Schule, aber zwischen unterschiedlichen Bildungsgängen.

\section{Literaturverzeichnis}

OECD (erscheint demnächst), PISA 2012 Technical Report, PISA, OECD Publishing.

Willms, J.D. (2006), Learning Divides: Ten Policy Questions About the Performance and Equity of Schools and Schooling Systems, UNESCO Institute for Statistics, Montreal. 


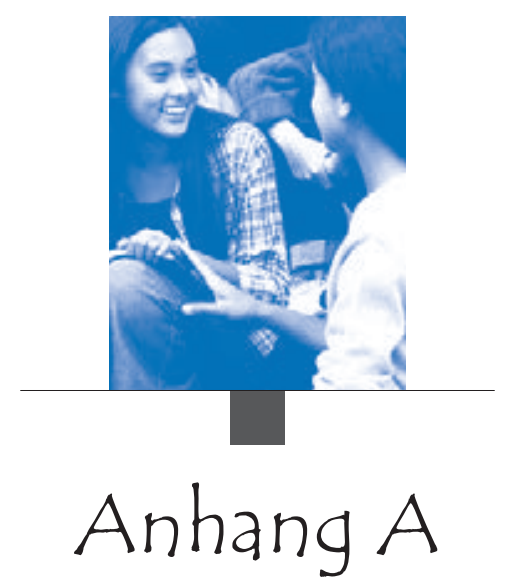

PISA 2012 - TECHNISCHE HINWEISE

Alle Abbildungen und Tabellen in Anhang A sind online verfügbar

Anhang A1: Konstruktion der auf den Kontextfragebogen für Schüler, Schulen und Eltern beruhenden Mathematikskalen und -indizes http://dx.doi.org/10.1787/888932937073

Anhang A2: $\quad$ PISA-Zielpopulation, PISA-Stichproben und Definition der Schulen http://dx.doi.org/10.1787/888932937092

Anhang A3: Technische Hinweise zu den in diesem Band enthaltenen Analysen

Anhang A4: Qualitätssicherung

Anhang A5: $\quad$ Technische Einzelheiten der Trendanalysen http://dx.doi.org/10.1787/888932937054

\section{Anmerkungen zu Zypern:}

Anmerkung der Türkei: Die Informationen in diesem Bericht zu „Zypern“ beziehen sich auf den südlichen Teil der Insel. Es existiert keine den türkischen und den griechischen Bevölkerungsteil der Insel gemeinsam vertretende Instanz. Die Türkei erkennt die Türkische Republik Nordzypern (TRNZ) an. Bis im Rahmen der Vereinten Nationen eine dauerhafte und gerechte Lösung gefunden ist, wird sich die Türkei ihre Stellungnahme zur "Zypernfrage" vorbehalten.

Anmerkung aller in der OECD vertretenen EU-Mitgliedstaaten und der Europäischen Union: Die Republik Zypern wird von allen Mitgliedern der Vereinten Nationen mit Ausnahme der Türkei anerkannt. Die Informationen in diesem Bericht beziehen sich auf das Gebiet, das sich de facto unter der Kontrolle der Regierung der Republik Zypern befindet.

\section{Anmerkung zu Israel:}

Die statistischen Daten für Israel wurden von den zuständigen israelischen Stellen bereitgestellt, die für sie verantwortlich zeichnen. Die Verwendung dieser Daten durch die OECD erfolgt unbeschadet des völkerrechtlichen Status der Golanhöhen, von Ost-Jerusalem und der israelischen Siedlungen im Westjordanland. 


\section{ANHANG A1 \\ KONSTRUKTION DER AUF DEN KONTEXTFRAGEBOGEN FÜR SCHÜLER, SCHULEN UND ELTERN BERUHENDEN MATHEMATIKSKALEN UND -INDIZES}

\section{Gestaltung, Analyse und Skalierung der Mathematiktests von PISA 2012}

Die in PISA 2012 verwendeten Mathematikaufgaben wurden von einem von der OECD beauftragten internationalen Konsortium von Bildungsforschungseinrichtungen unter der Leitung einer Gruppe von Mathematikexperten aus den Teilnehmerländern entwickelt. Die Teilnehmerländer schlugen Stimulusmaterial und Aufgaben vor, die in den drei Jahren bis zur Testdurchführung im Jahr 2012 in einem iterativen Prozess geprüft, getestet und optimiert wurden. Der Entwicklungsprozess umfasste mehrere Runden, in denen die Teilnehmerländer und -volkswirtschaften Kommentare abgeben konnten, sowie einen kleineren Pilotversuch und einen formellen Feldtest mit Stichproben der Population der 15-Jährigen (ungefähr 1000 Schülerinnen und Schüler) aus den teilnehmenden Ländern und Volkswirtschaften. Die Expertengruppe Mathematik machte eine Empfehlung für die endgültige Auswahl der Aufgaben, in der von den Teilnehmerländern und -volkswirtschaften eingereichtes Material berücksichtigt wurde. Die Auswahl wurde sowohl im Hinblick auf die technische Qualität der Aufgaben - die anhand der Ergebnisse des Feldtests bewertet wurde - als auch auf ihre kulturelle Angemessenheit und den Grad des Interesses getroffen, den sie bei 15-Jährigen hervorrufen dürften, was von den Teilnehmerländern beurteilt wurde. Ein weiteres wesentliches Kriterium für die Auswahl des Materials insgesamt war, wie gut es in den in Band I beschriebenen Rahmen passte, was die Wahrung eines ausgewogenen Verhältnisses zwischen den verschiedenen Kontext-, Inhalts- und Prozesskategorien anbelangte. Darüber hinaus wurde darauf geachtet, dass der Aufgabenkatalog ein breites Spektrum von Schwierigkeitsgraden abdeckte, um eine genaue Messung und Beschreibung der Mathematikkompetenz aller 15-jährigen Schülerinnen und Schüler, von den leistungsschwächsten bis zu den leistungsstärksten, zu ermöglichen.

Über 110 gedruckte Mathematikaufgaben kamen in PISA 2012 zum Einsatz, die Schülerinnen und Schüler in der Stichprobe sahen jedoch jeweils nur einen kleinen Teil der Aufgaben, da ihnen unterschiedliche Testhefte ausgehändigt wurden. Die für PISA 2012 ausgewählten Mathematikaufgaben waren zu halbstündigen Aufgabenblöcken zusammengelegt. Diese wurden mit Aufgabenblöcken aus den Bereichen Lesekompetenz und Naturwissenschaften zu Testheften zusammengefasst, die jeweils vier Aufgabenblöcke enthielten. Die teilnehmenden Schülerinnen und Schüler wurden daraufhin zwei Stunden lang geprüft. Da bei der PISA-Erhebung 2012 die Mathematikkompetenz im Mittelpunkt stand, enthielt jedes Testheft mindestens einen Aufgabenblock mit Mathematikaufgaben. Die Blöcke wurden unterschiedlich angeordnet, so dass jeder Aufgabenblock in den Testheften in jeder der vier möglichen Positionen erschien und jedes Paar von Aufgabenblöcken in mindestens einem der 13 verwendeten Testhefte enthalten war.

Dank dieses Testaufbaus, der sich mit der Gestaltung der vergangenen PISA-Erhebungen deckte, war es möglich, eine einzige Leistungsskala für Mathematik zu konstruieren, auf der jede Aufgabe einem bestimmten, ihrem Schwierigkeitsgrad entsprechenden Punkt und die Leistung jedes Schülers einem bestimmten, seiner geschätzten Mathematikkompetenz entsprechenden Punkt zugeordnet werden konnte. Eine Beschreibung des Modellierungsverfahrens, das zur Konstruktion dieser Skala verwendet wurde, findet sich im PISA 2012 Technical Report (OECD, erscheint demnächst).

Der relative Schwierigkeitsgrad einer Testaufgabe kann am Prozentsatz der Testteilnehmer gemessen werden, die die einzelnen Aufgaben richtig beantworten. Die relative Leistungsfähigkeit der Schülerinnen und Schüler, die an einem bestimmten Test teilnehmen, lässt sich anhand des Anteils der Testaufgaben ermessen, die sie richtig beantworten. Der Zusammenhang zwischen der Schwierigkeit der Testaufgaben und der Leistung der Schülerinnen und Schüler kann auf einer einzigen kontinuierlichen Skala aufgezeigt werden. Durch die Konstruktion einer Skala, die den Schwierigkeitsgrad jeder Aufgabe anzeigt, ist es möglich, die Mathematikkompetenzstufe zu ermitteln, die einer bestimmten Aufgabe entspricht. Durch die Übertragung der Leistung der Schülerinnen und Schüler auf diese Skala ist es dann möglich, den Grad der Mathematikkompetenz zu beschreiben, über den sie verfügen.

Die Position des Leistungsniveaus der Schülerinnen und Schüler auf dieser Skala wird mit der im Test konkret verwendeten Aufgabengruppe in Beziehung gesetzt. Die einzelnen in der Erhebung verwendeten Aufgaben wurden allerdings so gestaltet, dass sie repräsentativ für die PISA-Definition der Mathematikkompetenz sind, ebenso wie die Stichprobe der 2012 an PISA teilnehmenden Schülerinnen und Schüler so ausgewählt wurde, dass sie repräsentativ für alle 15-Jährigen in den Teilnehmerländern und -volkswirtschaften ist. Im geschätzten Leistungsniveau der Schülerinnen und Schüler spiegelt sich die Art der Aufgaben wider, von denen anzunehmen ist, dass sie sie erfolgreich lösen können. Dies bedeutet, dass die Schülerinnen und Schüler in der Lage sein dürften, Aufgaben bis zu dem Schwierigkeitsgrad zu lösen, der ihrer Position auf der Skala entspricht (auch wenn dies vielleicht nicht immer der Fall ist). Umgekehrt werden sie Aufgaben über dem Schwierigkeitsgrad, der ihrer Position auf der Skala entspricht, wahrscheinlich nicht lösen können (auch wenn dies bei manchen Aufgaben doch der Fall sein kann).

Je weiter das Kompetenzniveau eines Schülers über dem Schwierigkeitsgrad einer gegebenen Aufgabe liegt, desto höher ist die Wahrscheinlichkeit, dass er die Aufgabe (und andere Aufgaben mit ähnlichem Schwierigkeitsgrad) erfolgreich lösen kann; je weiter das Kompetenzniveau eines Schülers unter dem Schwierigkeitsgrad einer gegebenen Aufgabe liegt, desto geringer ist die Wahrscheinlichkeit, dass er die Aufgabe - und andere ähnlich schwierige Aufgaben - lösen kann. 


\section{Definition der Kompetenzstufen im Bereich Mathematik in PISA 2012}

Im Rahmen von PISA 2012 wurde eine Gesamtskala Mathematik erstellt, die sich auf die Gesamtheit der im Rahmen der Erhebung gestellten Mathematikaufgaben stützt; diese Gesamtskala wird durch Skalen für drei Prozesskategorien und vier Inhaltskategorien ergänzt. Das Maßsystem für die Gesamtskala Mathematik basiert auf einem Mittelwert für die OECD-Länder, der in PISA 2003 auf 500 gesetzt wurde, mit einer Standardabweichung von 100. Um leichter interpretieren zu können, was die Punktzahlen der Schülerinnen und Schüler konkret bedeuten, wurde die Skala nach einer Reihe statistischer Prinzipien in Stufen unterteilt. Danach wurde, ausgehend von den Aufgaben, die den verschiedenen Stufen zugeordnet sind, beschrieben, welche Art von Kenntnissen und Fertigkeiten zur Lösung dieser Aufgaben jeweils nötig sind.

In PISA 2012 lässt das Schwierigkeitsspektrum der Aufgaben die Beschreibung von sechs Mathematikkompetenzstufen zu: Stufe 1 ist die niedrigste Stufe, dann folgen Stufe 2, Stufe 3 usw. bis hin zu Stufe 6.

Schülerinnen und Schüler, deren Leistung im Bereich von Stufe 1 liegt, können Aufgaben der Kompetenzstufe 1 (und andere ähnliche Aufgaben) wahrscheinlich erfolgreich lösen, dürften jedoch nicht imstande sein, Aufgaben auf höheren Stufen zu lösen. Stufe 6 entspricht den Aufgaben, die die größten Anforderungen an die Mathematikkompetenz und -kenntnisse der Schülerinnen und Schüler stellen. Schülerinnen und Schüler mit Punktzahlen in diesem Bereich dürften in der Lage sein, Mathematikaufgaben dieser Stufe ebenso wie alle anderen PISA-Mathematikaufgaben erfolgreich zu lösen.

Die Konstruktion der Kompetenzskalen erfolgt bei PISA nach einer Standardmethode. Auf der Grundlage der Ergebnisse, die die Schülerinnen und Schüler bei den Testaufgaben erzielen, wird ihre Punktzahl ermittelt und in einem bestimmten Teil der Skala positioniert, wodurch diese Punktzahl einem festgelegten Kompetenzniveau zugeordnet werden kann. Die Stufe, auf der die Punktzahl eines Schülers liegt, ist die höchste Stufe, auf der zu erwarten ist, dass er den Großteil einer nach dem Zufallsprinzip erfolgten Auswahl von Testaufgaben dieser Stufe richtig beantwortet. So wäre z.B. bei einem Test, der aus Aufgaben besteht, die sich gleichmäßig auf Stufe 3 verteilen, von allen Schülerinnen und Schülern, deren Punktzahl Stufe 3 zuzuordnen ist, anzunehmen, dass sie mindestens 50\% der Aufgaben richtig lösen. Da eine Stufe einer ganzen Bandbreite verschiedener Schwierigkeitsgrade und Leistungsniveaus entspricht, variiert die Erfolgsquote innerhalb dieses Spektrums. Schülerinnen und Schüler am unteren Ende der Stufe werden wahrscheinlich knapp über 50\% der gleichmäßig über die Stufe verteilten Aufgaben erfolgreich lösen können, wohingegen Schülerinnen und Schüler am oberen Ende der Stufe deutlich über 70\% dieser Aufgaben richtig beantworten dürften.

Abbildung I.2.21 in Band I enthält nähere Angaben zur Art der mathematischen Kompetenzen, Kenntnisse und Verständniskapazitäten, die auf den einzelnen Stufen der Mathematikskala erforderlich sind.

\section{Indizes zu den Kontextfragebogen}

Dieser Abschnitt erklärt die auf den Kontextfragebogen für Schülerinnen und Schüler sowie Schulen beruhenden Indizes, die in PISA 2012 verwendet werden.

Bei mehreren PISA-Messgrößen handelt es sich um Indizes, die Antworten von Schülern, ihren Eltern oder Schulvertretern (in der Regel Schulleiterinnen und Schulleiter) auf eine Reihe miteinander verknüpfter Fragen zusammenfassen. Die Fragen wurden auf der Grundlage theoretischer Überlegungen und früherer Forschungsarbeiten aus einem größeren Fragenkatalog ausgewählt. Dieser konzeptionelle Rahmen wird in PISA 2012 Assessment and Analytical Framework (OECD, 2013) eingehend beschrieben. Zur Bestätigung des theoretisch erwarteten Verhaltens der Indizes und zur Validierung ihrer Vergleichbarkeit zwischen den verschiedenen Ländern und Volkswirtschaften wurden Strukturgleichungsmodelle verwendet. Zu diesem Zweck wurde eine Modellrechnung für jedes Land separat und für alle OECD-Länder zusammen durchgeführt. Wegen einer ausführlichen Beschreibung anderer PISA-Indizes und Einzelheiten zu den gewählten Methoden vgI. PISA 2012 Technical Report (OECD, erscheint demnächst).

Es gibt zwei Arten von Indizes: einfache Indizes und Skalenindizes.

Einfache Indizes sind Variablen, die durch arithmetische Transformation oder Umkodierung eines oder mehrerer Items in den einzelnen Erhebungen jeweils auf genau dieselbe Art und Weise konstruiert werden. Hier werden die Antworten je Item (item response) zur Berechnung aussagekräftiger Variablen verwendet, etwa bei der Umkodierung der vierstelligen ISCO-08-Codes zur Erstellung des Index der höchsten beruflichen Stellung der Eltern (HISEI) oder bei der Berechnung der Schüler/Lehrer-Quote auf der Grundlage der Daten aus dem Schulleiterfragebogen.

Skalenindizes sind Variablen, die durch Skalierung mehrerer Items konstruiert werden. Sofern nicht anders vermerkt, wurden die fraglichen Indizes mit Hilfe einer gewichteten Likelihood-Schätzung (WLE - weighted likelihood estimate) (Warm, 1989) auf der Grundlage eines einparametrigen Item-Response-Modells skaliert (im Fall von Items mit mehr als zwei Antwortkategorien wurde ein Modell abgestufter Punktwerte - Partial Credit Model - eingesetzt). Wegen Einzelheiten zur Konstruktion der einzelnen Skalenindizes vgl. PISA 2012 Technical Report (OECD, erscheint demnächst). Die Skalierung erfolgte generell in drei Stufen:

- Die Itemparameter wurden auf der Basis gleich großer Teilstichproben von Schülerinnen und Schülern aus allen teilnehmenden Ländern und Volkswirtschaften geschätzt.

- Die Schätzungen wurden für alle Schüler und alle Schulen durch die im vorangegangenen Schritt ermittelten Itemparameter geankert.

- Die Indizes wurden dann standardisiert, so dass der mittlere Indexwert für die OECD-Schülerpopulation gleich 0 und die Standardabweichung gleich 1 gesetzt wurde (wobei den Ländern bei dem Standardisierungsprozess dieselbe Gewichtung gegeben wurde). 
Den verschiedenen Antwortkategorien der Fragen wurden in der Reihenfolge, in der letztere in den Schüler-, Schulleiter- und Elternfragebogen erschienen, sequenzielle Codes zugewiesen. Diese Codes wurden zum Zweck der Konstruktion der Indizes bzw. Skalen z.T. umgepolt; wenn dies der Fall war, wird in diesem Abschnitt jeweils darauf hingewiesen. Negative Werte bei einem Index lassen nicht zwangsläufig auf negative Antworten der Schülerinnen und Schüler auf die gestellten Fragen schließen. Ein negativer Wert weist lediglich darauf hin, dass die betreffenden Befragten weniger positiv antworteten als der Durchschnitt der Befragten in den OECD-Ländern. Analog dazu bedeutet ein positiver Wert bei einem Index, dass die jeweiligen Befragten positivere Antworten gaben als der Durchschnitt der Befragten in den OECD-Ländern. Die bei den folgenden Beschreibungen in Klammern $<>$ gesetzten Begriffe wurden in den nationalen Fassungen der Schüler-, Schulleiter- und Elternfragebogen durch den entsprechenden nationalen Ausdruck ersetzt. So wurde z.B. der Begriff <Abschluss entsprechend ISCED-Stufe 5A> in den Vereinigten Staaten übersetzt in "Bachelor's degree, post-graduate certificate program, Master's degree program or first professional degree program“. Desgleichen wurde der Ausdruck < Testsprachenunterricht> in Luxemburg übersetzt in "Deutschunterricht" oder "Französischunterricht", je nachdem ob die Schülerinnen und Schüler die deutsche oder die französische Fassung der Erhebungsinstrumente erhielten.

Neben den in diesem Anhang beschriebenen einfachen Indizes und Skalenindizes gibt es eine Reihe von Variablen aus den Fragebogen, die Einzelitems entsprechen, die nicht zur Konstruktion der Indizes verwendet wurden. Diese nicht umkodierten Variablen weisen das Präfix „ST" für die Items im Schülerfragebogen, „SC" für die Items im Schulleiterfragebogen sowie „PA" für die Items im Elternfragebogen auf. Alle Kontextfragebogen sowie die internationale PISA-Datenbank mit sämtlichen Variablen sind auf www.pisa.oecd.org verfügbar.

\section{Skalierung der Fragebogenindizes für Trendanalysen}

Um Informationen über die Merkmale der Schülerinnen und Schüler und der Schulen zu sammeln, füllen sowohl die Schülerinnen und Schüler als auch die Schulen in PISA einen Hintergrundfragebogen aus. Da einige Fragen in PISA 2003 und PISA 2012 unverändert blieben, können die Antworten auf diese Fragen im Zeitverlauf verglichen werden. In diesem Bericht wurden nur Fragen, deren Wortlaut genau gleich geblieben ist, für Trendanalysen verwendet. Fragen mit geringfügigen oder großen Änderungen im Wortlaut wurden im Zeitverlauf nicht verglichen, weil unmöglich festzustellen ist, ob beobachtete Änderungen in einer Antwort auf Änderungen im gemessenen Konstrukt oder auf Änderungen in der Art, wie das Konstrukt gemessen wird, zurückzuführen sind.

Darüber hinaus werden Fragebogen-Items, wie in diesem Anhang beschrieben, in PISA für die Konstruktion von Indizes verwendet. Wenn die für die Konstruktion von Indizes verwendeten Fragen in PISA 2003 und PISA 2012 genau gleich geblieben sind, werden die entsprechenden Indizes verglichen. In PISA werden zwei Arten von Indizes verwendet: einfache Indizes und Skalenindizes.

Durch einfache Indizes wird eine Reihe von Antworten auf Fragebogen-Items umkodiert. Für Trendanalysen werden die in PISA 2003 beobachteten Werte ebenso wie einfache Antworten auf Fragebogen-Items direkt mit PISA 2012 verglichen. Dies gilt für Indizes wie die Schüler/Lehrer-Quote und die Einteilung in Leistungsgruppen in Mathematik.

Skalenindizeshingegen basieren aufWLE-Schätzungen, dieumskaliertwerden müssen, umüber mehrerePISA-Erhebungsrunden vergleichbar zu sein. Skalenindizes wie der PISA-Index des wirtschaftlichen, sozialen und kulturellen Status, der Index des Zugehörigkeitsgefühls, der Index der Einstellung zur Schule, der Index der intrinsischen Lernmotivation in Mathematik, der Index der instrumentellen Lernmotivation in Mathematik, der Index der Selbstwirksamkeit in Mathematik, der Index des Selbstkonzepts in Mathematik, der Index der Mathematikangst, der Index des Lehrermangels, der Index der Qualität der räumlichen Bedingungen, der Index der Ausstattung mit Lehr- und Sachmitteln, der Index der Schuldisziplin, der Index der Lehrer-Schüler-Beziehungen, der Index der Arbeitshaltung der Lehrkräfte, der Index der schülerbezogenen Faktoren für das Schulklima und der Index der lehrkräftebezogenen Faktoren für das Schulklima wurden in PISA 2012 so skaliert, dass der OECD-Durchschnittswert gleich 0 und die durchschnittliche Standardabweichung in den OECD-Ländern gleich 1 gesetzt wurde. Dieselben Skalen wurden in PISA 2003 so skaliert, dass der OECD-Durchschnitt gleich 0 und die Standardabweichung gleich 1 gesetzt wurde. Da die in Lernen für die Welt von morgen: Erste Ergebnisse von PISA 2003 (OECD, 2004) aufgeführten Werte auf unterschiedlichen Skalen basieren, können sie nicht mit den in diesem Band aufgeführten Werten verglichen werden. Um diese Skalenindizes vergleichbar zu machen, wurden die Werte von 2003 unter Verwendung der Parameterschätzungen von PISA 2012 auf den Wertebereich der Skala von 2012 umskaliert.

Diese umskalierten Indizes sind verfügbar unter www.pisa.oecd.org. Sie können unter Verwendung der Ländernamen, sowie der Schulund Schüleridentifikationsnummern in den entsprechenden Datensatz von PISA 2003 integriert werden. Der umskalierte PISA-Index des wirtschaftlichen, sozialen und kulturellen Status kann außerdem in den Datensatz von PISA 2000, PISA 2006 und PISA 2009 integriert werden.

\section{Einfache Indizes auf Schülerebene}

\section{Alter}

Die Variable ALTER ist berechnet als Differenz zwischen dem mittleren Monat und dem Jahr, in dem die Tests durchgeführt wurden, und dem Geburtsmonat und -jahr der Schülerinnen und Schüler, ausgedrückt in Jahren und Monaten.

\section{Bildungsgang}

In PISA 2012 wurden die für 15-jährige Schülerinnen und Schüler in den einzelnen Ländern angebotenen Bildungsgänge mit Hilfe der Unterlagen über den bisherigen Bildungsweg der Schülerinnen und Schüler sowie des Schülerfragebogens (ST02) erfasst. Alle 
Bildungsgänge wurden nach ISCED klassifiziert (OECD, 1999). In der internationalen PISA-Datenbank werden alle nationalen Bildungsgänge in Form einer Variablen (PROGN) angegeben, bei der die ersten sechs Ziffern dem Code des nationalen Zentrums und die letzten beiden Ziffern dem nationalen Code für den jeweiligen Bildungsgang entsprechen.

Die folgenden international vergleichbaren Indizes wurden aus den Daten über die Bildungsgänge abgeleitet:

- Der Index der Stufe des Bildungsgangs (ISCEDL) gibt an, ob sich die Schülerinnen und Schüler (1) in der Primarstufe (ISCED 1), (2) in der Sekundarstufe I oder (3) in der Sekundarstufe II befinden.

- Der Index der Bezeichnung des Bildungsgangs (ISCEDD) gibt die Bezeichnung des Bildungsgangs an: (1) „A“ (allgemeinbildender Zweig, der den Zugang zur nächsthöheren Bildungsstufe ermöglicht), (2) „B“ (Bildungsgang, der den Zugang zur nächsthöheren berufsbildenden Stufe ermöglicht), (3) „C" (Bildungsgang, der auf den direkten Arbeitsmarktzugang ausgerichtet ist), (4) „M" (modularer Bildungsgang, der mehrere dieser Merkmale kombiniert).

- Der Index der Lehrplanausrichtung (ISCEDO) gibt an, ob die Lehrplaninhalte des betreffenden Bildungsgangs (1) allgemeinbildend, (2) berufsvorbereitend, (3) berufsbildend oder (4) modular sind, d.h. mehrere oder alle dieser Elemente kombinieren.

\section{Berufliche Stellung der Eltern}

Im Schülerfragebogen (ST12, ST16) wurden mit Hilfe von offenen Fragen berufsspezifische Daten über die Väter und Mütter der Schülerinnen und Schüler erhoben. Die Antworten wurden in vierstellige ISCO-Codes kodiert (ILO, 1990) und dann im sozioökonomischen Index (SEI) von Ganzeboom et al. (1992) abgebildet. Je höher die SEI-Werte sind desto höher ist die berufliche Stellung. Auf diese Weise wurden die folgenden drei Indizes erstellt:

- Der Index der beruflichen Stellung der Mutter (OCOD1).

- Der Index der beruflichen Stellung des Vaters (OCOD2).

- Der Index der höchsten beruflichen Stellung der Eltern (HISEI) entspricht dem SEI-Wert des Elternteils mit der jeweils höheren beruflichen Stellung bzw. dem einzigen verfügbaren SEI-Wert eines Elternteils.

Einige der Analysen unterscheiden zwischen vier verschiedenen Kategorien von Berufen auf Basis der Berufshauptgruppen gemäß ISCOKodierung der höchsten beruflichen Stellung der Eltern: Hilfsarbeitskräfte (ISCO 9), angelernte Fachkräfte (ISCO 6, 7 und 8), angelernte Bürokräfte oder in einem Dienstleistungsberuf Tätige (ISCO 4 und 5), Fach- oder Führungskräfte (ISCO 1, 2 und 3). Diese Klassifizierung folgt derselben Methodik, die auch in anderen OECD-Publikationen wie Bildung auf einen Blick (OECD, 2013b) und dem OECD Skills Outlook (OECD, 2013c) verwendet wird ${ }^{1}$.

\section{Bildungsabschluss der Eltern}

Das Bildungsniveau der Eltern wird auf der Grundlage der Angaben der Schülerinnen und Schüler im Schülerfragebogen (ST13, ST14, ST17 und ST18) nach ISCED eingestuft (OECD, 1999).

Wie in PISA 2000, 2003, 2006 und 2009 wurden die Indizes konstruiert, indem für jeden Elternteil der höchste Abschluss ausgewählt und einer der folgenden Kategorien zugeordnet wurde: (0) Kein Abschluss, (1) ISCED 1 (Primarbereich), (2) ISCED 2 (Sekundarbereich I), (3) ISCED 3B oder 3C (berufsbildender/vorberuflicher Sekundarbereich II), (4) ISCED 3A (Sekundarbereich II) und/oder ISCED 4 (nichttertiärer postsekundärer Bereich), (5) ISCED 5B (berufsbildender Tertiärbereich) und (6) ISCED 5A, 6 (theoretisch orientierter Tertiär- und Postgraduiertenbereich). Anhand dieser Kategorien wurden die folgenden drei Indizes konzipiert:

- Index des Bildungsabschlusses der Mutter (MISCED).

- Index des Bildungsabschlusses des Vaters (FISCED).

- Index des höchsten Bildungsabschlusses der Eltern (HISCED), entspricht der ISCED-Stufe des Elternteils mit dem jeweils höheren Bildungsabschluss.

Der höchste Bildungsabschluss der Eltern wurde ferner in die Anzahl der Ausbildungsjahre (PARED) umgerechnet. Wegen der Umrechnung des Bildungsstands in Ausbildungsjahre vgl. Tabelle A1.1.

\section{Migrationsstatus und sprachlicher Hintergrund}

Die Daten über das Geburtsland der Schülerinnen und Schüler sowie ihrer Eltern wurden anhand von länderspezifischen ISO-Codes auf ähnliche Weise erhoben wie in PISA 2000, PISA 2003, PISA 2006 und PISA 2009. Die ISO-Codes der Geburtsländer der Schülerinnen und Schüler sowie ihrer Eltern sind in der internationalen PISA-Datenbank verfügbar (COBN_S, COBN_M und COBN_F).

Der Index des Migrationshintergrunds (IMMIG) umfasst folgende Kategorien: (1) Schülerinnen und Schüler ohne Migrationshintergrund (im Erhebungsland geborene Schüler mit mindestens einem im Erhebungsland geborenen Elternteil; in einem anderen Land geborene Schüler, die aber wenigstens einen Elternteil haben, der im Erhebungsland geboren ist, gelten ebenfalls als Schüler ohne Migrationshintergrund), (2) Schülerinnen und Schüler mit Migrationshintergrund der zweiten Generation (im Erhebungsland geborene Schüler mit in einem anderen Land geborenen Eltern) und (3) Schülerinnen und Schüler mit Migrationshintergrund der ersten Generation (nicht im Erhebungsland geborene Schülerinnen und Schüler mit ebenfalls in einem anderen Land geborenen Eltern). Schülerinnen und Schüler, die zu ihrem eigenen Geburtsland, dem ihrer Mutter oder dem ihres Vaters oder zu allen dreien keine Angaben machten, wurden bei dieser Variablen mit Fehlwerten erfasst. 
[Teil 1/1]

Tabelle A1.1 Bildungsabschluss der Eltern, umgerechnet in Bildungsjahre

\begin{tabular}{|c|c|c|c|c|c|c|c|}
\hline & & $\begin{array}{c}\text { Abschluss ISCED } 1 \\
\text { (Primarbereich) }\end{array}$ & $\begin{array}{l}\text { Abschluss ISCED } 2 \\
\text { (Sekundarbereich I) }\end{array}$ & \begin{tabular}{|c|} 
Abschluss ISCED \\
3B oder 3C \\
(Sekundarbereich II, \\
zur Berufsreife oder zu \\
ISCED-5B-Programmen \\
führend)
\end{tabular} & $\begin{array}{c}\text { Abschluss ISCED 3A } \\
\text { (Sekundarbereich II, } \\
\text { Zugang zu ISCED-5A- } \\
\text { und 5B-Programmen) } \\
\text { oder ISCED 4 } \\
\text { (postsekundärer } \\
\text { nichttertiärer Bereich) }\end{array}$ & $\begin{array}{c}\text { Abschluss ISCED 5A } \\
\text { (universitärer Tertiär- } \\
\text { bereich) oder ISCED } 6 \\
\text { (weiterführende } \\
\text { Forschungsprogramme) }\end{array}$ & $\begin{array}{c}\text { Abschluss } \\
\text { ISCED 5B } \\
\text { (nichtuniversitärer } \\
\text { Tertiärbereich) }\end{array}$ \\
\hline \multirow{35}{*}{ 这 } & Australien & 6.0 & 10.0 & 11.0 & 12.0 & 15.0 & 14.0 \\
\hline & Österreich & 4.0 & 9.0 & 12.0 & 12.5 & 17.0 & 15.0 \\
\hline & Belgien 1 & 6.0 & 9.0 & 12.0 & 12.0 & 17.0 & 15.0 \\
\hline & Kanada & 6.0 & 9.0 & 12.0 & 12.0 & 17.0 & 15.0 \\
\hline & Chile & 6.0 & 8.0 & 12.0 & 12.0 & 17.0 & 16.0 \\
\hline & Tschech. Rep. & 5.0 & 9.0 & 11.0 & 13.0 & 16.0 & 16.0 \\
\hline & Dänemark & 7.0 & 10.0 & 13.0 & 13.0 & 18.0 & 16.0 \\
\hline & Estland & 6.0 & 9.0 & 12.0 & 12.0 & 16.0 & 15.0 \\
\hline & Finnland & 6.0 & 9.0 & 12.0 & 12.0 & 16.5 & 14.5 \\
\hline & Frankreich & 5.0 & 9.0 & 12.0 & 12.0 & 15.0 & 14.0 \\
\hline & Deutschland & 4.0 & 10.0 & 13.0 & 13.0 & 18.0 & 15.0 \\
\hline & Griechenland & 6.0 & 9.0 & 11.5 & 12.0 & 17.0 & 15.0 \\
\hline & Ungarn & 4.0 & 8.0 & 10.5 & 12.0 & 16.5 & 13.5 \\
\hline & Island & 7.0 & 10.0 & 13.0 & 14.0 & 18.0 & 16.0 \\
\hline & Irland & 6.0 & 9.0 & 12.0 & 12.0 & 16.0 & 14.0 \\
\hline & Israel & 6.0 & 9.0 & 12.0 & 12.0 & 15.0 & 15.0 \\
\hline & Italien & 5.0 & 8.0 & 12.0 & 13.0 & 17.0 & 16.0 \\
\hline & Japan & 6.0 & 9.0 & 12.0 & 12.0 & 16.0 & 14.0 \\
\hline & Korea & 6.0 & 9.0 & 12.0 & 12.0 & 16.0 & 14.0 \\
\hline & Luxemburg & 6.0 & 9.0 & 12.0 & 13.0 & 17.0 & 16.0 \\
\hline & Mexiko & 6.0 & 9.0 & 12.0 & 12.0 & 16.0 & 14.0 \\
\hline & Niederlande & 6.0 & 10.0 & 13.0 & 12.0 & 16.0 & 15.0 \\
\hline & Neuseeland & 5.5 & 10.0 & 11.0 & 12.0 & 15.0 & 14.0 \\
\hline & Norwegen & 6.0 & 9.0 & 12.0 & 12.0 & 16.0 & 14.0 \\
\hline & Polen & $\mathrm{a}$ & 8.0 & 11.0 & 12.0 & 16.0 & 15.0 \\
\hline & Portugal & 6.0 & 9.0 & 12.0 & 12.0 & 17.0 & 15.0 \\
\hline & Slowak. Republik ${ }^{2}$ & 4.0 & 9.0 & 12.0 & 13.0 & 18.0 & 16.0 \\
\hline & Slowenien & 4.0 & 8.0 & 11.0 & 12.0 & 16.0 & 15.0 \\
\hline & Spanien & 5.0 & 8.0 & 10.0 & 12.0 & 16.5 & 13.0 \\
\hline & Schweden & 6.0 & 9.0 & 11.5 & 12.0 & 16.0 & 14.0 \\
\hline & Schweiz & 6.0 & 9.0 & 12.5 & 12.5 & 17.5 & 14.5 \\
\hline & Türkei & 5.0 & 8.0 & 11.0 & 11.0 & 15.0 & 13.0 \\
\hline & Ver. Königreich (ohne Schottland) & 6.0 & 9.0 & 12.0 & 13.0 & 16.0 & 15.0 \\
\hline & Ver. Königreich (Schottland) & 7.0 & 9.0 & 11.0 & 13.0 & 17.0 & 15.0 \\
\hline & Ver. Staaten & 6.0 & 9.0 & a & 12.0 & 16.0 & 14.0 \\
\hline \multirow{31}{*}{ 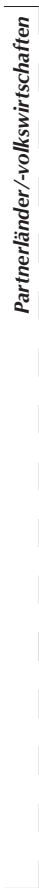 } & Albanien & 6.0 & 9.0 & 12.0 & 12.0 & 16.0 & 16.0 \\
\hline & Argentinien & 6.0 & 10.0 & 12.0 & 12.0 & 17.0 & 14.5 \\
\hline & Aserbaidschan & 4.0 & 9.0 & 11.0 & 11.0 & 17.0 & 14.0 \\
\hline & Brasilien & 4.0 & 8.0 & 11.0 & 11.0 & 16.0 & 14.5 \\
\hline & Bulgarien & 4.0 & 8.0 & 10.0 & 12.0 & 17.5 & 15.0 \\
\hline & Kolumbien & 5.0 & 9.0 & 11.0 & 11.0 & 15.5 & 14.0 \\
\hline & Costa Rica & 6.0 & 9.0 & 11.0 & 12.0 & 14.0 & 16.0 \\
\hline & Kroatien & 4.0 & 8.0 & 11.0 & 12.0 & 17.0 & 15.0 \\
\hline & Hongkong (China) & 6.0 & 9.0 & 11.0 & 13.0 & 16.0 & 14.0 \\
\hline & Indonesien & 6.0 & 9.0 & 12.0 & 12.0 & 15.0 & 14.0 \\
\hline & Jordanien & 6.0 & 10.0 & 12.0 & 12.0 & 16.0 & 14.5 \\
\hline & Kasachstan & 4.0 & 9.0 & 11.5 & 12.5 & 15.0 & 14.0 \\
\hline & Lettland & 4.0 & 8.0 & 11.0 & 11.0 & 16.0 & 14.0 \\
\hline & Liechtenstein & 5.0 & 9.0 & 11.0 & 13.0 & 17.0 & 14.0 \\
\hline & Litauen & 3.0 & 8.0 & 11.0 & 11.0 & 16.0 & 15.0 \\
\hline & Macau (China) & 6.0 & 9.0 & 11.0 & 12.0 & 16.0 & 15.0 \\
\hline & Malaysia & 6.0 & 9.0 & 11.0 & 13.0 & 15.0 & 16.0 \\
\hline & Montenegro & 4.0 & 8.0 & 11.0 & 12.0 & 16.0 & 15.0 \\
\hline & Peru & 6.0 & 9.0 & 11.0 & 11.0 & 17.0 & 14.0 \\
\hline & Katar & 6.0 & 9.0 & 12.0 & 12.0 & 16.0 & 15.0 \\
\hline & Rumänien & 4.0 & 8.0 & 11.5 & 12.5 & 16.0 & 14.0 \\
\hline & Russ. Föderation & 4.0 & 9.0 & 11.5 & 12.0 & 15.0 & a \\
\hline & Serbien & 4.0 & 8.0 & 11.0 & 12.0 & 17.0 & 14.5 \\
\hline & Shanghai (China) & 6.0 & 9.0 & 12.0 & 12.0 & 16.0 & 15.0 \\
\hline & Singapur & 6.0 & 8.0 & 10.0 & 11.0 & 16.0 & 13.0 \\
\hline & Chinesisch Taipeh & 6.0 & 9.0 & 12.0 & 12.0 & 16.0 & 14.0 \\
\hline & Thailand & 6.0 & 9.0 & 12.0 & 12.0 & 16.0 & 14.0 \\
\hline & Tunesien & 6.0 & 9.0 & 12.0 & 13.0 & 17.0 & 16.0 \\
\hline & Ver. Arab. Emirate & 5.0 & 9.0 & 12.0 & 12.0 & 16.0 & 15.0 \\
\hline & Uruguay & 6.0 & 9.0 & 12.0 & 12.0 & 17.0 & 15.0 \\
\hline & Vietnam & 5.0 & 9.0 & 12.0 & 12.0 & 17.0 & $\mathrm{a}$ \\
\hline
\end{tabular}

1. In Belgien deckt sich die Unterscheidung zwischen Universitäten und sonstigen tertiären Bildungseinrichtungen nicht mit der Unterscheidung zwischen ISCED 5A und ISCED 5B. 2. In der Slowakischen Republik dauern universitäre Studiengänge (ISECD 5A) in der Regel fünf Jahre, für eine Promotion (ISCED 6) sind weitere drei Jahre erforderlich. Hochschulabsolventen haben somit 18 Bildungsjahre und Promovierte 21 Bildungsjahre absolviert.

Quelle: OECD, PISA-2012-Datenbank.

StatLink inils http://dx.doi.org/10.1787/888932937073 
Die Schülerinnen und Schüler gaben die Sprache an, die sie in der Regel zu Hause sprechen. Die Daten werden in länderspezifischen Sprachencodes erfasst, die mit folgenden beiden Werten in die Variable LANGN umkodiert wurden: (1) Die zu Hause gesprochene Sprache ist mit der Testsprache identisch und (2) die zu Hause gesprochene Sprache ist nicht mit der Testsprache identisch.

\section{Relative Klassenstufe}

Die Daten zur Klassenstufe der Schülerinnen und Schüler können sowohl dem Schülerfragebogen (ST01) als auch den Unterlagen über den bisherigen Bildungsweg der Schülerinnen und Schüler entnommen werden. Wie bei allen Variablen, die sowohl in den Unterlagen über den bisherigen Bildungsweg als auch in dem Fragebogen zu finden sind, werden Unstimmigkeiten zwischen den beiden Quellen während der Datenbereinigung geprüft und geklärt. Zur Berücksichtigung der Varianz zwischen den Ländern gibt der relative Klassenstufenindex (GRADE) an, ob sich die Schülerinnen und Schüler in Bezug auf ein gegebenes Land in der Regelklassenstufe (Wert $=0$ ) oder über bzw. unter der Regelklassenstufe befinden (+ x Klassenstufen, - x Klassenstufen).

Der Zusammenhang zwischen Klassenstufe und Schülerleistungen wurde mit Hilfe eines Mehrebenenmodells geschätzt, in dem folgende Hintergrundvariablen berücksichtigt wurden: a) der PISA-Index des wirtschaftlichen, sozialen und kulturellen Status, b) der PISA-Index des wirtschaftlichen, sozialen und kulturellen Status quadriert, c) der Mittelwert der Schule auf dem PISA-Index des wirtschaftlichen, sozialen und kulturellen Status, d) ein Indikator für im Ausland geborene Schülerinnen und Schüler der ersten Generation, e) der Prozentsatz der Schülerinnen und Schüler der ersten Generation in der Schule sowie $f$ ) das Geschlecht.

In Tabelle A1.2 sind die Ergebnisse des Mehrebenenmodells dargestellt. Spalte 1 in Tabelle A1.2 enthält eine Schätzung der mit einer Klassenstufe (oder einem Schuljahr) assoziierten Punktzahldifferenz. Schätzen lässt sich diese Differenz für die 32 OECD-Länder, in denen eine beträchtliche Zahl 15-Jähriger in den PISA-Stichproben mindestens zwei unterschiedliche Klassenstufen besuchte. Da nicht davon ausgegangen werden kann, dass die 15-Jährigen nach dem Zufallsprinzip auf die Klassenstufen verteilt sind, mussten für die oben genannten Umfeldfaktoren, die einen Einfluss auf die Zuordnung der Schülerinnen und Schüler zu bestimmten Klassenstufen haben können, Anpassungen vorgenommen werden. Diese Anpassungen sind in den Spalten 2-7 der Tabelle dargestellt. Es ist zwar möglich, den typischen Leistungsunterschied zwischen Schülerinnen und Schülern aus zwei aufeinanderfolgenden Klassenstufen, korrigiert um Auswahl- und Kontexteffekte, zu schätzen, dieser Unterschied kann jedoch nicht automatisch mit den Lernfortschritten gleichgesetzt werden, die die Schülerinnen und Schüler im vorausgegangenen Schuljahr gemacht haben, sondern sollte vielmehr als Untergrenze der erzielten Fortschritte interpretiert werden. Dies ist nicht nur darauf zurückzuführen, dass unterschiedliche Schülerinnen und Schüler getestet wurden, sondern auch auf die Tatsache, dass der Inhalt der PISA-Tests nicht ausdrücklich auf den Lehrstoff abstellte, den die Schülerinnen und Schüler im vorausgegangenen Schuljahr durchgenommen hatten, sondern ganz allgemein auf die Bewertung der kumulativen Lernstände von Schülerinnen und Schülern bis zum Alter von 15 Jahren. Wenn z.B. der Lehrplan für die Klassenstufen, die 15-Jährige besuchen, hauptsächlich einen anderen Stoff abdeckt als das in PISA verwendete Material (das wiederum in den Lehrplänen früherer Schuljahre enthalten gewesen sein könnte), dann wird der beobachtete Leistungsunterschied den Schülerfortschritt unterschätzen.

\section{Zeitaufwand für das Lernen}

Der Index der Lernzeit im Testsprachenunterricht (LMINS) wurde durch Multiplikation der Angaben der Schülerinnen und Schüler zur durchschnittlichen Dauer des Testsprachenunterrichts in Minuten mit der Zahl der Unterrichtsstunden in der Testsprache pro Woche berechnet (ST69 und ST70). Vergleichbare Indizes wurden für Mathematik (MMINS) und Naturwissenschaften (SMINS) berechnet.

\section{Skalenindizes auf Schülerebene Instrumentelle Lernmotivation in Mathematik}

Der Index der instrumentellen Lernmotivation in Mathematik (INSTMOT) beruht auf den Angaben der Schülerinnen und Schüler darüber, inwiefern sie den Aussagen in ST29 über Mathematik völlig zustimmen, eher zustimmen, eher nicht zustimmen oder überhaupt nicht zustimmen: Ich gebe mir in Mathematik Mühe, weil es mir in meinem späteren Job weiterhelfen wird; Mathematik zu lernen lohnt sich, weil es meine Berufs- und Karriereaussichten verbessert; Mathematik ist für mich ein wichtiges Fach, weil ich es für mein späteres Studium brauche; ich werde viele Dinge in Mathematik lernen, die mir dabei helfen werden, einen Job zu bekommen.

Für Trendanalysen wurden die Werte des Index der instrumentellen Lernmotivation in Mathematik von PISA 2003 umskaliert, um die Vergleichbarkeit mit den Werten von PISA 2012 zu gewährleisten. Folglich können die in diesem Bericht dargelegten Werte für den Index der instrumentellen Lernmotivation in Mathematik für PISA 2003 von den Werten abweichen, die in Lernen für die Welt von morgen: Erste Ergebnisse von PISA 2003 (OECD, 2004) enthalten sind.

\section{Schuldisziplin}

Der Index der Schuldisziplin (DISCLIMA) beruht auf den Angaben der Schülerinnen und Schüler darüber, wie oft Folgendes im Mathematikunterricht vorkommt (ST81): a) die Schülerinnen und Schüler hören der Lehrerin/dem Lehrer nicht zu, b) im Klassenzimmer ist es oft laut, und es geht drunter und drüber, c) die Lehrerin/der Lehrer muss lange warten, bis die Schülerinnen und Schüler ruhig werden, $d$ ) die Schülerinnen und Schüler können nicht ungestört arbeiten und e) die Schülerinnen und Schüler fangen erst lange nach Beginn der Stunde an zu arbeiten. Höhere Werte auf diesem Index weisen auf eine bessere Schuldisziplin hin.

Für Trendanalysen wurden die Werte des Index der Schuldisziplin von PISA 2003 umskaliert, um die Vergleichbarkeit mit den Werten von PISA 2012 zu gewährleisten. Folglich können die in diesem Bericht dargelegten Werte für den Index der Schuldisziplin für PISA 2003 von den Werten abweichen, die in Lernen für die Welt von morgen: Erste Ergebnisse von PISA 2003 (OECD, 2004) enthalten sind. 
[Teil 1/1]

Mehrebenenmodell zur Schätzung von Klasseneffekten im Bereich Mathematik nach Berücksichtigung Tabelle A1.2 mehrerer Hintergrundvariablen

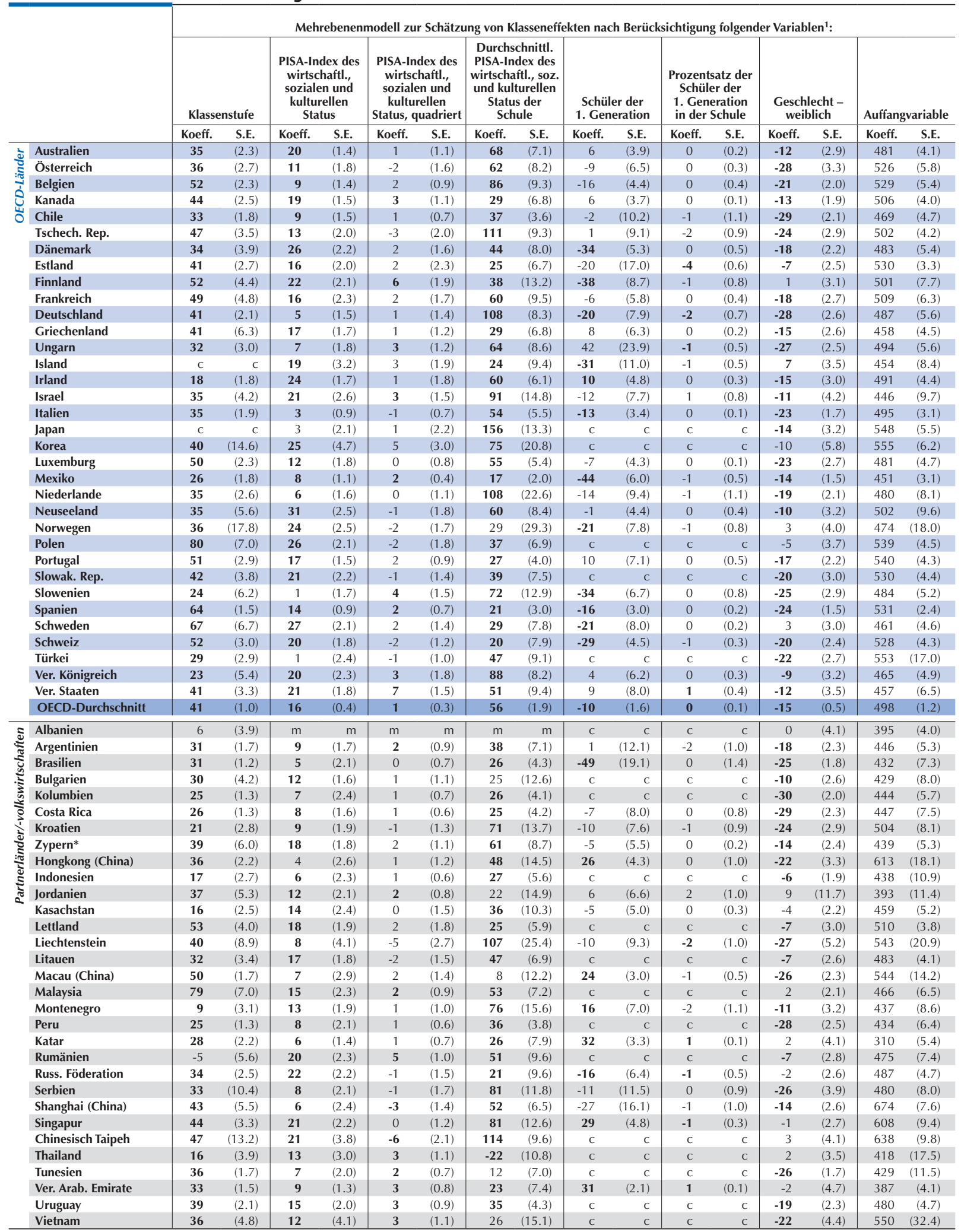

Anmerkung: Statistisch signifikante Werte sind durch Fettdruck gekennzeichnet (vgl. Anhang A3).

1. Mehrebenen-Regressionsmodell (auf Schüler- und Schulebene): Die Leistungen im Bereich Mathematik sind auf die verschiedenen Variablen in dieser Tabelle regressiert.

* Vgl. Anmerkungen am Anfang dieses Anhangs.

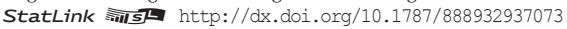




\section{Lehrer-Schüler-Beziehungen}

Der Index der Lehrer-Schüler-Beziehungen (STUDREL) beruht auf den Angaben der Schülerinnen und Schüler dazu, inwiefern sie den folgenden Aussagen über die Lehrerinnen und Lehrer an ihrer Schule zustimmten (ST86): a) die Schülerinnen und Schüler kommen mit den meisten Lehrerinnen und Lehrern gut aus, b) den meisten Lehrerinnen und Lehrern ist es wichtig, dass sich die Schülerinnen und Schüler wohl fühlen, c) die meisten meiner Lehrerinnen und Lehrer interessieren sich für das, was ich zu sagen habe, $d$ ) wenn ich zusätzliche Hilfe brauche, bekomme ich sie von meinen Lehrerinnen und Lehrern, und e) die meisten Lehrerinnen und Lehrer behandeln mich fair. Höhere Werte auf diesem Index weisen auf positive Lehrer-Schüler-Beziehungen hin.

Für Trendanalysen wurden die Werte des Index der Lehrer-Schüler-Beziehungen von PISA 2003 umskaliert, um die Vergleichbarkeit mit den Werten von PISA 2012 zu gewährleisten. Folglich können die in diesem Bericht dargelegten Werte für den Index der LehrerSchüler-Beziehungen für PISA 2003 von den Werten abweichen, die in Lernen für die Welt von morgen: Erste Ergebnisse von PISA 2003 (OECD, 2004) enthalten sind.

\section{Wirtschaftlicher, sozialer und kultureller Status}

Der PISA-Index des wirtschaftlichen, sozialen und kulturellen Status (ESCS) wurde aus den folgenden drei Indizes abgeleitet: dem Index der höchsten beruflichen Stellung der Eltern (HISEI), dem Index des höchsten Bildungsabschlusses der Eltern in Ausbildungsjahren gemäß ISCED (PARED) und dem Index der Ausstattung des Elternhauses (HOMEPOS). Der Index der Ausstattung des Elternhauses (HOMEPOS) umfasst alle Items der Indizes WEALTH, CULTPOSS und HEDRES ebenso wie die Zahl der Bücher im Elternhaus, die in eine vierstufige Kategorienvariable umkodiert wurde (0-10 Bücher, 11-25 bzw. 26-100 Bücher, 101-200 bzw. 201-500 Bücher, über 500 Bücher).

Der PISA-Index des wirtschaftlichen, sozialen und kulturellen Status (ESCS) wurde aus einer Hauptkomponentenanalyse standardisierter Variablen (in der jede Variable einen OECD-Mittelwert von 0 und eine Standardabweichung von 1 hat) abgeleitet, wobei die Faktorwerte für die erste Hauptkomponente als Messgrößen des ESCS-Index dienten.

Die Hauptkomponentenanalyse wurde ebenfalls für jedes Teilnehmerland bzw. jede Teilnehmervolkswirtschaft durchgeführt, um festzustellen, inwieweit die Indexkomponenten in den verschiedenen Ländern bzw. Volkswirtschaften auf ähnliche Art und Weise operieren. Aus der Analyse ging hervor, dass die Strukturen für die Gewichtung der einzelnen Faktoren in den Ländern sehr ähnlich waren und dass alle drei Komponenten in gleichem Ausmaß im Index berücksichtigt wurden (wegen Einzelheiten zur Reliabilität und zur Faktorgewichtung vgl. PISA 2012 Technical Report, OECD, erscheint demnächst).

Für Schülerinnen und Schüler, für die Daten zu einer der Komponenten fehlten, erfolgte die Berechnung der Komponenten auf der Grundlage einer Regression der anderen beiden Variablen unter Hinzufügung einer Zufallsfehlerkomponente. Die Endwerte auf dem PISA-Index des wirtschaftlichen, sozialen und kulturellen Status (ESCS) für 2012 haben einen OECD-Mittelwert von 0 und eine Standardabweichung von 1.

Der ESCS wurde für alle Schülerinnen und Schüler, die an den fünf Erhebungsrunden teilgenommen haben, berechnet, und die ESCSIndizes für Trendanalysen wurden erstellt, indem die für die Ableitung standardisierter Werte in 2012 verwendeten Parameter auf die ESCS-Komponenten früherer Zyklen angewendet wurden. Diese Werte sind folglich mit den ESCS-Werten, die in den Datenbanken für die früheren Zyklen aufgeführt werden, nicht direkt vergleichbar, wenngleich die Unterschiede bei den Erhebungsrunden 2006 und 2009 nicht groß sind. In den früheren Erhebungsrunden wurden die ESCS-Werte unter Verwendung anderer Algorithmen berechnet, so dass die Unterschiede bei den Erhebungsrunden 2000 und 2003 größer sind.

\section{Änderungen bei der Berechnung des sozioökonomischen Status in PISA 2012}

Die Berechnung des sozioökonomischen Status erfolgte zwar nach dem Schema der früheren Testzyklen, in PISA 2012 wurde jedoch eine wichtige Aktualisierung in Bezug auf die Kodierung des Berufs der Eltern vorgenommen. Vor PISA 2012 wurde die Internationale Standardklassifikation der Berufe von 1988 (ISCO-88) für die Kodierung des Berufs der Eltern herangezogen. 2012 war ISCO-88 jedoch fast 25 Jahre alt, und die Verwendung dieser Klassifikation als Kodierungssystem für Berufe war nicht länger tragbar $^{2}$. Es wurde deshalb beschlossen, dieses Kodierungssystem für Berufe in PISA 2012 durch ISCO-08 zu ersetzen.

Der Übergang von ISCO-88 zu ISCO-08 erforderte eine Aktualisierung der Kodierung des internationalen sozioökonomischen Index der beruflichen Stellung der Eltern (ISEI). In PISA 2012 wurde deshalb ein modifiziertes Quantifizierungssystem für ISCO-08 verwendet (ISEI-08), das von Harry Ganzeboom (2010) entwickelt wurde. Die Konstruktion des ISEI-08 basiert auf einer aus den kombinierten Datensätzen des „International Social Survey Programme“ (ISSP) von 2002-2007 abgeleiteten Datenbank mit validen Daten zum Bildungsabschluss, Beruf und (persönlichen) Einkommen von 198500 Männern und Frauen (Ganzeboom, 2010). Die für diesen Zweck verwendete Methodik entspricht der bei der Konstruktion des ISEI für ISCO-68 und ISCO-88 verwendeten und in verschiedenen Publikationen beschriebenen Methodik (Ganzeboom, de Graff und Treiman, 1992; Ganzeboom und Treiman, 1996; Ganzeboom und Treiman, 2003) $)^{3}$.

Die Hauptunterschiede im Vergleich zur früheren ISEI-Konstruktion sind die Folgenden:

- Es wurde eine neue Datenbank verwendet, die aktueller und größer ist und einen umfassenderen Ländervergleich ermöglicht als die frühere Datenbank. 
- Die Konstruktion des neuen ISEl basiert auf Daten, die sowohl Frauen als auch Männer erfassen, während früher nur Daten zu Männern für die Skalenschätzung herangezogen wurden. Die Daten zu Einkommen wurden um die geleisteten Arbeitsstunden bereinigt, um zu berücksichtigen, dass die Teilzeitbeschäftigung in vielen Ländern bei Männern und Frauen unterschiedlich ausgeprägt ist.

Der Übergang von ISCO-88/ISEI-88 zu ISCO-08/ISEI-08 ging mit mehreren Validierungsschritten einher, darunter a) ein Vergleich der Verteilungen von ISEI-88 und ISEI-08 in Bezug auf Bandbreite, Mittelwert und Standardabweichungen sowohl für die Berufe der Mütter als auch für die Berufe der Väter und $b$ ) ein Vergleich der Korrelationen zwischen den beiden ISEI-Indikatoren und der Leistung, wobei die Berufe der Mütter und Väter wiederum getrennt betrachtet wurden.

Um Trends für alle Zyklen von 2000 bis 2012 zu erhalten, basierte die Berechnung der Indizes WEALTH, HEDRES, CULTPOSS und HOMEPOS für diesen Turnus auf Daten aller Erhebungsrunden von 2000 bis 2012. Der HOMEPOS ist von besonderer Bedeutung, da er für die Berechnung des ESCS verwendet wird. Diese Indizes wurden für 2012 dann so standardisiert, dass der OECD-Mittelwert gleich 0 und die Standardabweichung gleich 1 gesetzt wurden. Das bedeutet, dass die auf der Grundlage der früheren Erhebungsrunden berechneten Indizes der Skala von 2012 entsprechen und folglich nicht direkt mit den in der Datenbank gespeicherten Indizes der früheren Erhebungsrunden vergleichbar sind. Um die Itemparameter für die Skalierung zu schätzen, wurde eine Kalibrierungsstichprobe aus allen Erhebungsrunden verwendet, wobei für die früheren Zyklen jeweils 500 Schülerinnen und Schüler aus allen Ländern erfasst wurden, während für 2012750 Schülerinnen und Schüler berücksichtigt wurden, da jedes einzelne Schülerfragebogen-Item im Jahr 2012 nur in zwei Drittel der Fragebogen enthalten war.

Die zur Berechnung der Indizes verwendeten Items haben sich zwischen den einzelnen Erhebungsrunden bis zu einem gewissen Grad verändert, wenngleich sie von 2006 bis 2012 weitgehend gleich geblieben sind. In den früheren Erhebungsrunden fehlten im Allgemeinen einige Items, die in den späteren Erhebungen enthalten sind, es wurde jedoch davon ausgegangen, dass eine Ausklammerung der Items, die nur in den späteren Runden vorhanden waren, den früheren Zyklen zu viel Gewicht beimessen würde. Deshalb wurde eine Obermenge aller in den 5 Erhebungsrunden aufgeführten Items (mit Ausnahme länderspezifischer Items) verwendet, und aus dieser Menge wurden internationale Itemparameter abgeleitet.

Der zweite Schritt bestand in der WLE-Schätzung für die Indizes, wobei die Parameter bei den internationalen Items verankert wurden, während die länderspezifischen Itemparameter geschätzt wurden. Das gleiche Verfahren wurde in den früheren Zyklen angewendet.

\section{Wohlstand der Familie}

Der Index des Wohlstands der Familie (WEALTH) beruht auf den Angaben der Schülerinnen und Schüler darüber, ob sie zu Hause über Folgendes verfügen: ein eigenes Zimmer, einen Internetanschluss, eine Geschirrspülmaschine (als länderspezifischer Gegenstand), einen DVD-Player und drei weitere länderspezifische Gegenstände (einige der in ST26 aufgelisteten Items); ebenfalls berücksichtigt wurden ihre Angaben zur Zahl der Handys, Fernseher, Computer, Autos sowie Zimmer mit Badewanne oder Dusche in ihrem Zuhause (ST27).

\section{Bildungsressourcen im Elternhaus}

Der Index der Bildungsressourcen im Elternhaus (HEDRES) beruht auf Items, die zur Messung der im Elternhaus vorhandenen Bildungsressourcen dienen, darunter ein Schreibtisch und ein ruhiger Platz zum Lernen, ein Computer, den die Schülerinnen und Schüler für Schularbeiten verwenden können, Lernsoftware, Bücher, die den Schülerinnen und Schülern bei den Schularbeiten helfen, technische Nachschlagewerke sowie ein Wörterbuch (einige der in ST26 aufgelisteten Items).

\section{Besitz von Kulturgütern}

Der Kulturgüterindex (CULTPOSS) beruht auf den Angaben der Schülerinnen und Schüler über das Vorhandensein der folgenden Dinge in ihrem Zuhause: klassische Literatur, Gedichtbände und Kunstwerke (einige der in ST26 aufgelisteten Items).

\section{Das Rotationsprinzip des Schülerfragebogens}

Eine wesentliche Innovation in PISA 2012 ist die Anwendung des Rotationsverfahrens für den Schülerfragebogen. Einer der Hauptgründe für die Rotationsmethode, die bei der kognitiven Beurteilung bereits seit längerer Zeit angewandt wird, bestand darin, den Erfassungsgrad des Schülerfragebogens zu erweitern. Tabelle A1.3 bietet einen Überblick über das Rotationsverfahren und den Inhalt der in der Haupterhebung verwendeten Fragebogenformulare.

Der PISA 2012 Technical Report (OECD, erscheint demnächst) enthält alle Einzelheiten zum Rotationsprinzip des in PISA 2012 verwendeten Schülerfragebogens, darunter die Auswirkungen in Bezug auf a) die Leistungsschätzungen, $b$ ) internationale Berichte und Trends, c) weitere Analysen, d) die Struktur und Dokumentation der internationalen Datenbank und e) die Logistik. Das Rotationsverfahren hat unwesentliche Auswirkungen auf die Leistungsschätzungen und die Korrelationen zwischen den Leistungsschätzungen und den Kontext-Konstrukten. Die internationale Datenbank (verfügbar unter www.pisa.oecd.org) enthält alle Hintergrundvariablen für alle Schülerinnen und Schüler, wobei Variablen, bei denen die der Variablen zu Grunde liegende Frage von den Schülerinnen und Schülern beantwortet wurde, deren Antworten widerspiegeln, während ein eindeutiger Fehlwert verzeichnet ist, wenn die der Variablen zu Grunde liegende Frage nicht gestellt wurde. Die Rotation ermöglicht die Schätzung einer vollständigen Kovarianzmatrix, was zur Folge hat, dass alle Variablen mit allen anderen Variablen korreliert werden können. Schlussfolgerungen in Bezug auf die Frage, ob ein Effekt in einem Mehrebenenmodell als signifikant gelten würde, werden davon nicht beeinflusst. 
Tabelle A1.3 Rotationsverfahren beim Schülerfragebogen

\begin{tabular}{|l|l|l|l}
\hline Formular A & $\begin{array}{l}\text { Gemeinsamer Fragenkatalog (alle } \\
\text { Formulare) }\end{array}$ & $\begin{array}{l}\text { Fragenkatalog } 1 \text { - Einstellungen zu Mathematik/ } \\
\text { Problemlösen }\end{array}$ & $\begin{array}{l}\text { Fragenkatalog } 3 \text { - Lernmöglichkeiten/ } \\
\text { Lernstrategien }\end{array}$ \\
\hline Formular B & $\begin{array}{l}\text { Gemeinsamer Fragenkatalog (alle } \\
\text { Formulare) }\end{array}$ & $\begin{array}{l}\text { Fragenkatalog 2 - Schulklima/Einstellungen zur } \\
\text { Schule/Angst }\end{array}$ & $\begin{array}{l}\text { Fragenkatalog } 1 \text { - Einstellungen zu Mathematik/ } \\
\text { Problemlösen }\end{array}$ \\
\hline Formular C & $\begin{array}{l}\text { Gemeinsamer Fragenkatalog (alle } \\
\text { Formulare) }\end{array}$ & $\begin{array}{l}\text { Fragenkatalog } 3 \text { - Lernmöglichkeiten/ } \\
\text { Lernstrategien }\end{array}$ & $\begin{array}{l}\text { Fragenkatalog } 2 \text { - Schulklima/Einstellungen zur } \\
\text { Schule/Angst }\end{array}$ \\
\hline
\end{tabular}

Anmerkung: Wegen näherer Angaben zu den Fragen in den einzelnen Fragebogen vgl. PISA 2012 Technical Report (OECD, erscheint demnächst).

\section{Einfache Indizes auf Schulebene Schul- und Klassengröße}

Der Index der Schulgröße (SCHSIZE) wurde aus der Addition der Zahl der Jungen und Mädchen an der jeweiligen Schule abgeleitet (SC07).

\section{Schüler/Lehrer-Quote}

Die Schüler/Lehrer-Quote (STRATIO) wurde durch Division der Schulgröße durch die Gesamtzahl der Lehrkräfte (SC09) berechnet. Die Zahl der Teilzeitlehrkräfte wurde bei der Berechnung dieses Index mit 0,5 und die Zahl der Vollzeitlehrkräfte mit 1,0 gewichtet.

Die Schüler/Mathematiklehrer-Quote (SMRATIO) wurde durch Division der Schulgröße durch die Gesamtzahl der Mathematiklehrkräfte berechnet (SC10Q11 und SC10Q12). Die Zahl der Teilzeit-Mathematiklehrkräfte wurde bei der Berechnung dieses Index mit 0,5 und die Zahl der Vollzeit-Mathematiklehrkräfte mit 1,0 gewichtet.

\section{Schultyp}

Schulen werden entweder als öffentliche oder private Einrichtungen eingestuft, je nachdem ob die letzte Entscheidungsgewalt über ihre Angelegenheiten einem privaten Träger oder einer staatlichen Behörde obliegt (SC01). Diese Information wird mit den Daten aus SC02 über den aus öffentlichen Mitteln finanzierten Anteil am Gesamtbudget kombiniert, um den Index des Schultyps (SCHLTYPE) zu erstellen. Dieser Index umfasst drei Kategorien: (1) vom Staat unabhängige Privatschulen, die einer nichtstaatlichen Organisation oder einem Verwaltungsrat unterstehen, dessen Mitglieder nicht von einer staatlichen Stelle ausgewählt wurden, und die weniger als $50 \%$ ihrer Kernfinanzierung von staatlichen Stellen beziehen, (2) vom Staat abhängige Privatschulen, die einer nichtstaatlichen Organisation oder einem Verwaltungsrat unterstehen, dessen Mitglieder nicht von einer staatlichen Stelle ausgewählt wurden, und die mehr als 50\% ihrer Kernfinanzierung von staatlichen Quellen beziehen, und (3) öffentliche Schulen, die der Kontrolle und Verwaltung durch eine staatliche Stelle bzw. Bildungsbehörde unterstehen.

\section{Verfügbarkeit von Computern}

Der Index der Verfügbarkeit von Computern (RATCMP15) beruht auf der Division der Zahl der Computer, die den Schülerinnen und Schülern der von 15-Jährigen in der Regel besuchten Klassenstufe (Regelklassenstufe) für Bildungszwecke zur Verfügung stehen (SC11Q02), durch die Zahl der Schülerinnen und Schüler in der Regelklassenstufe für 15-Jährige (SC11Q01). Die Formulierung der Fragen zur Computerverfügbarkeit wurde zwischen 2006 und 2009 geändert. Die Daten zur Verfügbarkeit von Computern für 2012 können folglich mit den entsprechenden Daten für 2009, nicht aber mit den Daten für 2006 oder frühere Erhebungsrunden verglichen werden.

Der Index der Verfügbarkeit von Computern mit Internetverbindung (COMPWEB) beruht auf der Division der Zahl der Computer, die den Schülerinnen und Schülern der Regelklassenstufe für 15-Jährige für Bildungszwecke zur Verfügung stehen und an das Internet angeschlossen sind (SC11Q03), durch die Zahl der Computer, die den Schülerinnen und Schülern der Regelklassenstufe für 15-Jährige für Bildungszwecke zur Verfügung stehen (SC11Q02).

\section{Anzahl der Lehrkräfte an den Schulen}

Der Anteil der Lehrkräfte, die eine Lehrbefähigung haben (PROPCERT), wurde durch Division der Zahl der Lehrkräfte mit Lehrbefähigung (SC09Q21 plus 0,5*SC09Q22) durch die Gesamtzahl der Lehrkräfte (SC09Q11 plus 0,5*SC09Q12) berechnet. Der Anteil der Lehrkräfte, die über eine ISCED-5A-Qualifikation verfügen (PROPQUAL), wurde durch Division der Zahl der entsprechend qualifizierten Lehrkräfte (SC09Q31 plus 0,5*SC09Q32) durch die Gesamtzahl der Lehrkräfte (SC09Q11 plus 0,5*SC09Q12) berechnet. Der Anteil der Mathematiklehrkräfte (PROPMATH) wurde durch Division der Zahl der Mathematiklehrkräfte (SC10Q11 plus 0,5*SC10Q12) durch die Gesamtzahl der Lehrkräfte (SC09Q11 plus 0,5*SC09Q12) berechnet. Der Anteil der Mathematiklehrkräfte, die über eine ISCED-5AQualifikation verfügen (PROPMA5A), wurde durch Division der Zahl der Mathematiklehrkräfte, die über eine ISCED-5A-Qualifikation verfügen (SC10Q21 plus 0,5*SC10Q22) durch die Zahl der Mathematiklehrkräfte (SC10Q11 plus 0,5*SC10Q12) ermittelt.

Zwar wurden die Schulleitungen sowohl in PISA 2003 als auch in PISA 2012 zu den Lehrkräften an ihrer Schule befragt, doch die Formulierung der Fragen zum Anteil der Lehrer mit ISCED-5A-Qualifikation änderte sich, so dass keine Vergleiche möglich waren. 


\section{Selektivität der Schulen}

Der Index der Selektivität der Schulen (SCHSEL) beruht auf den Angaben der Schulleitungen darüber, wie oft die folgenden beiden Faktoren bei der Aufnahme von Schülerinnen und Schülern in die Schulen berücksichtigt wurden, wobei eine Skala mit den Antwortkategorien "nie“, "manchmal“ und „immer" verwendet wurde (SC32Q02 und SC32Q03): bisherige Schulleistungen/Zeugnisnoten (einschließlich Aufnahmeprüfungen) und Empfehlungen der letzten Schule. Dieser Index umfasst die folgenden drei Kategorien: (1) Schulen, an denen diese beiden Faktoren bei der Aufnahme von Schülerinnen und Schülern „nie“ berücksichtigt werden, (2) Schulen, an denen mindestens einer der beiden Faktoren "manchmal“, aber kein Faktor „immer" berücksichtigt wird, und (3) Schulen, an denen mindestens einer dieser beiden Faktoren bei der Aufnahme von Schülerinnen und Schülern „immer“ berücksichtigt wird.

Zwar wurden die Schulleitungen sowohl in PISA 2003 als auch in PISA 2012 zu den Kriterien der Schulen für die Aufnahme von Schülerinnen und Schülern befragt, doch die Formulierung der Fragen änderte sich, so dass keine Vergleiche möglich waren.

\section{Einteilung in Leistungsgruppen}

Der Index der Einteilung in Leistungsgruppen im Mathematikunterricht (ABGMATH) beruht auf den beiden Items, bei denen die Schulleitungen angeben, ob ihre Schule für unterschiedliche Begabungen in Mathematik „in allen Klassen“, „in einigen Klassen“ oder „in keiner Klasse“ differenzierten Unterricht anbietet (SC15Q01: in den Klassen werden in Mathematik die gleichen Inhalte, jedoch mit unterschiedlichen Schwierigkeitsgraden unterrichtet; SC15Q02: in verschiedenen Klassen werden in Mathematik unterschiedliche Inhalte mit unterschiedlichen Schwierigkeitsgraden unterrichtet). Dieser Index umfasst die folgenden drei Kategorien: (1) in keiner Klasse werden in Mathematik unterschiedliche Inhalte bzw. Inhalte mit unterschiedlichem Schwierigkeitsgrad unterrichtet (d.h. Antwort „in keiner Klasse“ sowohl bei SC15Q01 als auch bei SC15Q02), (2) in einigen Klassen werden in Mathematik unterschiedliche Inhalte bzw. Inhalte mit unterschiedlichem Schwierigkeitsgrad unterrichtet (d.h. Antwort „in einigen Klassen“ bei entweder SC15Q01 oder SC15Q02), (3) in allen Klassen werden in Mathematik unterschiedliche Inhalte bzw. Inhalte mit unterschiedlichem Schwierigkeitsgrad unterrichtet (d.h. Antwort „in allen Klassen“ bei entweder SC15Q01 oder SC15Q02).

\section{Von der Schule angebotene außercurriculare Aktivitäten}

Der Index der außercurricularen Mathematikaktivitäten an Schulen (MACTIV) beruht auf den Angaben der Schulleitungen dazu, ob ihre Schulen für Schülerinnen und Schüler in der Regelklassenstufe für 15-Jährige im Schuljahr der PISA-Erhebung die folgenden Aktivitäten angeboten haben (SC16 bzw. SC21 für das letztgenannte Item): a) Mathematik-AG, b) Mathematikwettbewerbe, c) AG zu Inhalten aus den Informations- und Kommunikationstechnologien und d) zusätzliche Mathematikkurse. Dieser Index wurde durch Addition der Zahl der von einer Schule angebotenen Aktivitäten erstellt. Bei der Frage nach „zusätzlichen Mathematikkursen“ (SC21) zählt es als eine Aktivität, wenn die Schulleitung ",ausschließlich Begabungsförderung in Mathematik", "ausschließlich Stütz-/Förderunterricht in Mathematik“ oder „,keine Differenzierung bezüglich des bisherigen Leistungsniveaus der Schülerinnen und Schüler" angegeben hat; wenn die Schulleitung „,beides: Begabungsförderung und Stütz-/Förderunterricht in Mathematik“ angegeben hat, zählt dies als zwei Aktivitäten.

Der Index der außercurricularen kreativen Aktivitäten an Schulen (CREACTIV) beruht auf den Angaben der Schulleitungen dazu, ob ihre Schulen für Schülerinnen und Schüler in der Regelklassenstufe für 15-Jährige im Schuljahr der PISA-Erhebung die folgenden Aktivitäten angeboten haben (SC16): a) Band, Orchester oder Chor, b) Schultheater oder Musical und c) künstlerische Aktivitäten. Dieser Index wurde durch Addition der Zahl der von einer Schule angebotenen Aktivitäten erstellt.

\section{Nutzung von Leistungsbeurteilungen}

Die Schulleitungen wurden dazu befragt, ob Informationen über die Leistungen der Schülerinnen und Schüler für folgende Zwecke genutzt werden (SC18): a) um die Eltern über die Fortschritte ihres Kindes zu informieren, b) um Entscheidungen über Klassenwiederholungen, Rück- oder Höherstufungen von Schülerinnen und Schülern zu treffen, c) um Schülerinnen und Schüler in leistungsdifferenzierte Gruppen einzuteilen, d) um die Leistungen der Schule mit landesspezifischen oder nationalen Leistungsstandards zu vergleichen, e) um die Entwicklung des Leistungsniveaus der Schule von Jahr zu Jahr zu beobachten, f) um die Effektivität der Lehrkräfte zu beurteilen, g) um herauszufinden, was am Unterricht oder Lehrplan verbessert werden kann und $h$ ) um die Schule mit anderen Schulen zu vergleichen. Der Index der Nutzung von Leistungsbeurteilungen (ASSESS) wurde aus diesen acht Items durch Addition der Zahl der "Ja"-Antworten der Schulleitungen auf diese Fragen ermittelt.

\section{Schulautonomie: Ressourcenallokation}

Die Schulleitungen wurden dazu befragt, ob die "Schulleitung", die "Lehrerinnen und Lehrer", der "Schulverwaltungsrat", die "regionale oder lokale Bildungsbehörde“ oder die "nationale Bildungsbehörde“ maßgebliche Verantwortung für die folgenden Aufgaben/Bereiche tragen (SC33): a) Einstellung von Lehrkräften, b) Entlassung von Lehrkräften, c) Festlegung des Anfangsgehalts der Lehrerinnen/Lehrer, $d$ ) Entscheidungen über Gehaltserhöhungen der Lehrerinnen/Lehrer, e) Festlegung des Schulbudgets und $f$ ) Entscheidungen über die Verwendung des Budgets innerhalb der Schule. Der Index der Schulautonomie bei der Ressourcenallokation (RESPRES) beruht auf diesen sechs Items. Dazu wurde das Verhältnis zwischen der Zahl der Items, für die die Verantwortung bei den „Schulleitungen“ und/oder „Lehrerinnen und Lehrern“ liegt, und der Zahl der Items, für die sie bei der "regionalen oder lokalen Bildungsbehörde“ und/oder der "nationalen Bildungsbehörde“ liegt, berechnet. PositiveWerte auf diesem Index weisen auf eine vergleichsweise größere Autonomie der Schulen gegenüber der lokalen, regionalen oder nationalen Bildungsbehörde hin. Bei diesem Index beträgt der Mittelwert für die OECD-Länder 0 und die Standardabweichung 1.

Zwar wurden die Schulleitungen sowohl in PISA 2003 als auch in PISA 2012 zu der Schulautonomie bei der Ressourcenallokation befragt, doch die Formulierung der Fragen änderte sich, so dass keine Vergleiche möglich waren. 


\section{Schulautonomie: Unterrichtsinhalte und Leistungsbeurteilung}

Die Schulleitungen wurden dazu befragt, ob die "Schulleitung", die „Lehrerinnen und Lehrer", der "Schulverwaltungsrat", die "regionale oder lokale Bildungsbehörde“ oder die "nationale Bildungsbehörde“ maßgebliche Verantwortung für die folgenden Aufgaben/Bereiche tragen (SC33): a) Festlegung von Kriterien für die Schülerbeurteilung, b) Wahl der verwendeten Schulbücher, c) Bestimmung des Lehrstoffs und d) Entscheidungen über das Kursangebot. Der Index der Schulautonomie bei Unterrichtsinhalten und Leistungsbeurteilung (RESPCUR) beruht auf diesen vier Items. Dazu wurde das Verhältnis zwischen der Zahl der Items, für die die Verantwortung bei den "Schulleitungen" und/oder „Lehrerinnen und Lehrern" liegt, und der Zahl der Items, für die sie bei der "regionalen oder lokalen Bildungsbehörde" und/oder der "nationalen Bildungsbehörde" liegt, berechnet. Positive Werte auf diesem Index weisen auf eine vergleichsweise größere Autonomie der Schulen gegenüber der lokalen, regionalen oder nationalen Bildungsbehörde hin. Bei diesem Index beträgt der Mittelwert für die OECD-Länder 0 und die Standardabweichung 1.

Zwar wurden die Schulleitungen sowohl in PISA 2003 als auch in PISA 2012 zu der Schulautonomie bei Aufnahmeregelungen und Unterrichtsgestaltung befragt, doch die Formulierung der Fragen änderte sich, so dass keine Vergleiche möglich waren.

\section{Skalenindizes auf Schulebene}

\section{Führungsrolle der Schulleitung}

Der Index der Führungsrolle der Schulleitung bei der Formulierung und Vermittlung der Ziele und Lehrplanentwicklung der Schule (LEADCOM) beruht auf den Angaben der Schulleitungen darüber, wie oft sie im vergangenen Schuljahr an ihren Schulen folgende Aufgaben wahrgenommen haben (SC34): a) auf Basis von erzielten Schülerleistungen die pädagogischen Ziele der Schule weiterentwickeln, b) sicherstellen, dass die Fortbildungsaktivitäten der Lehrerinnen und Lehrer auf die Lehrziele abgestimmt werden, c) sicherstellen, dass die Arbeit der Lehrerinnen und Lehrer mit den Lehrzielen der Schule übereinstimmt und d) mit den Lehrerinnen und Lehrern die pädagogischen Zielsetzungen der Schule an den Fachbereichskonferenzen diskutieren. Der Index der Führungsrolle der Schulleitung bei der Unterrichtsgestaltung (LEADINST) beruht auf den Angaben der Schulleitungen darüber, wie oft sie im vergangenen Schuljahr an ihren Schulen folgende Aufgaben wahrgenommen haben (SC34): a) Lehrmethoden fördern, die auf neuester Bildungsforschung basieren, b) Lehrerinnen und Lehrer loben, deren Schülerinnen und Schüler aktiv am Lernen teilnehmen, und c) die Aufmerksamkeit der Lehrerinnen und Lehrer auf die Bedeutung der Entwicklung von Kritikfähigkeit und Sozialkompetenz der Schülerinnen und Schüler lenken. Der Index der Führungsrolle der Schulleitung bei der Förderung von Unterrichtsverbesserungen und beruflicher Weiterbildung (LEADPD) beruht auf den Angaben der Schulleitungen darüber, wie oft sie im vergangenen Schuljahr an ihren Schulen folgende Aufgaben wahrgenommen haben (SC34): a) wenn eine Lehrkraft Probleme in ihrer Klasse hat, die Initiative ergreifen, um darüber zu sprechen, b) sich um Probleme in Bezug auf störendes Verhalten in den Klassen kümmern, und c) wenn eine Lehrkraft ein Problem in einer Klasse anspricht, das Problem gemeinsam lösen. Der Index der Führungsrolle der Schulleitung bei der Einbeziehung der Lehrkräfte (LEADTCH) beruht auf den Angaben der Schulleitungen darüber, wie oft sie im vergangenen Schuljahr an ihren Schulen folgende Aufgaben wahrgenommen haben (SC34): a) den Lehrerinnen und Lehrern Möglichkeiten anbieten, bei Entscheidungen, die die Schule betreffen, teilzuhaben, b) die Lehrerinnen und Lehrer beim Aufbau einer Schulkultur der kontinuierlichen Verbesserung mit einbinden und c) die Lehrerinnen und Lehrer bitten, an der Bewertung der Führungspraxis teilzunehmen. Höhere Werte auf diesen Indizes weisen auf eine stärkere Führungsrolle der Schulleitung hin.

\section{Lehrermangel}

Der Index des Lehrermange/s (TCSHORT) beruht auf vier Items, die messen, inwieweit aus Sicht der Schulleitungen der Unterricht an ihren Schulen durch folgende Faktoren beeinträchtigt wird (SC14): a) einen Mangel an qualifizierten Lehrkräften für naturwissenschaftliche Fächer, b) einen Mangel an qualifizierten Mathematiklehrkräften, c) einen Mangel an qualifizierten Lehrkräften für den Testsprachenunterricht und d) einen Mangel an qualifizierten Lehrkräften in anderen Fächern. Höhere Werte auf diesem Index weisen darauf hin, dass an der betreffenden Schule laut den Angaben der Schulleitung ein größerer Lehrkräftemangel besteht.

Für Trendanalysen wurden die Werte des Index des Lehrermangels von PISA 2003 umskaliert, um die Vergleichbarkeit mit den Werten von PISA 2012 zu gewährleisten. Folglich können die in diesem Bericht dargelegten Werte für den Index des Lehrermange/s für PISA 2003 von den Werten abweichen, die in Lernen für die Welt von morgen: Erste Ergebnisse von PISA 2003 (OECD, 2004) enthalten sind.

\section{Ausstattung der Schulen mit Lehr- und Sachmitteln}

Der Index der Ausstattung der Schulen mit Lehr- und Sachmitteln (SCMATEDU) beruht auf sechs Items, die messen, inwieweit aus Sicht der Schulleitungen der Unterricht an ihren Schulen durch folgende Faktoren beeinträchtigt wird (SC14): a) fehlende oder unzulängliche Ausstattung für naturwissenschaftliche Labors, b) fehlendes oder unzulängliches Unterrichtsmaterial, c) fehlende oder unzulängliche Computerausstattung für den Unterricht, $d$ ) fehlende oder unzulängliche Internetanschlussmöglichkeit, e) fehlende oder unzulängliche Computersoftware für den Unterricht und f) fehlendes oder unzulängliches Büchereimaterial. Da alle Items für die Skalierung umgepolt wurden, weisen höhere Werte auf diesem Index auf eine höhere Qualität der Ausstattung der Schule mit Lehr- und Sachmitteln hin.

Für Trendanalysen wurden die Werte des Index der Ausstattung mit Lehr- und Sachmitteln von PISA 2003 umskaliert, um die Vergleichbarkeit mit den Werten von PISA 2012 zu gewährleisten. Folglich können die in diesem Bericht dargelegten Werte für den Index der Ausstattung mit Lehr- und Sachmitteln für PISA 2003 von den Werten abweichen, die in Lernen für die Welt von morgen: Erste 
Ergebnisse von PISA 2003 (OECD, 2004) enthalten sind. Eine der in PISA 2012 enthaltenen Fragen, auf denen der Index der Ausstattung mit Lehr- und Sachmitteln beruht („fehlende oder unzulängliche Internetanschlussmöglichkeit“) war im Fragebogen zu PISA 2003 nicht enthalten. Bei der Schätzung des Index für PISA 2003 wird diese Frage als fehlend behandelt, und unter der Annahme, dass der Zusammenhang zwischen den Items bei Hinzunahme neuer Fragen unverändert bleibt, sind die Werte für PISA 2003 und PISA 2012 auf dem Index der Ausstattung mit Lehr- und Sachmitteln nach der Umskalierung vergleichbar.

\section{Qualität der räumlichen Bedingungen der Schulen}

Der Index der Qualität der räumlichen Bedingungen (SCMATBUI) beruht auf drei Items, die messen, inwieweit aus Sicht der Schulleitungen der Unterricht an ihren Schulen durch folgende Faktoren beeinträchtigt wird (SC14): a) fehlendes oder unzulängliches Schulgebäude oder Schulgelände, $b$ ) fehlendes oder unzulängliches Heizungs-, Belüftungs- und Beleuchtungssystem und c) fehlende oder unzulängliche Unterrichtsräume (z.B. Klassenzimmer). Da alle Items für die Skalierung umgepolt wurden, weisen höhere Werte auf diesem Index auf eine höhere Qualität der räumlichen Infrastruktur hin.

Für Trendanalysen wurden die Werte des Index der Qualität der räumlichen Bedingungen von PISA 2003 umskaliert, um die Vergleichbarkeit mit den Werten von PISA 2012 zu gewährleisten. Folglich können die in diesem Bericht dargelegten Werte für den Index der Qualität der räumlichen Bedingungen für PISA 2003 von den Werten abweichen, die in Lernen für die Welt von morgen: Erste Ergebnisse von PISA 2003 (OECD, 2004) enthalten sind.

\section{Lehrerverhalten}

Der Index der lehrkräftebezogenen Faktoren für das Schulklima (TEACCLIM) beruht auf den Angaben der Schulleitungen darüber, wie sehr das Lernen der Schülerinnen und Schüler an ihren Schulen durch die folgenden Faktoren beeinträchtigt wird (SC22): a) Schülerinnen und Schüler werden nicht dazu ermutigt, ihr Potenzial voll auszuschöpfen, b) schlechte Lehrer-Schüler-Beziehung, c) Lehrerinnen und Lehrer müssen leistungsheterogene Klassen unterrichten, $d$ ) Lehrerinnen und Lehrer müssen multikulturelle Klassen unterrichten (d.h. Schülerinnen und Schüler unterschiedlicher Sprache und Kultur), e) niedrige Erwartungen der Lehrpersonen gegenüber den Schülerinnen und Schülern, f) Lehrerinnen und Lehrer gehen nicht auf individuelle Bedürfnisse der Schülerinnen und Schüler ein, $g$ ) Abwesenheit von Lehrerinnen und Lehrern, $h$ ) Widerstand des Lehrkörpers gegenüber Veränderungen, i) Lehrerinnen und Lehrer sind mit den Schülerinnen und Schülern zu streng, j) Lehrerinnen und Lehrer erscheinen verspätet zum Unterricht und $k$ ) Lehrerinnen und Lehrer erscheinen nicht gut vorbereitet zum Unterricht. Da alle Items für die Skalierung umgepolt wurden, weisen höhere Werte auf diesem Index auf ein positiv wirkendes Verhalten der Lehrkräfte hin.

Für Trendanalysen wurden die Werte des Index der lehrkräftebezogenen Faktoren für das Schulklima von PISA 2003 umskaliert, um die Vergleichbarkeit mit den Werten von PISA 2012 zu gewährleisten. Folglich können die in diesem Bericht dargelegten Werte für den Index der lehrkräftebezogenen Faktoren für das Schulklima für PISA 2003 von den Werten abweichen, die in Lernen für die Welt von morgen: Erste Ergebnisse von PISA 2003 (OECD, 2004) enthalten sind. Vier der in PISA 2012 enthaltenen Fragen, auf denen der Index der lehrkräftebezogenen Faktoren für das Schulklima beruht ["Lehrerinnen und Lehrer müssen leistungsheterogene Klassen unterrichten“, „Lehrerinnen und Lehrer müssen multikulturelle Klassen unterrichten (d.h. Schülerinnen und Schüler unterschiedlicher Sprache und Kultur)", "Lehrerinnen und Lehrer erscheinen verspätet zum Unterricht" und "Lehrerinnen und Lehrer erscheinen nicht gut vorbereitet zum Unterricht"], waren im Fragebogen zu PISA 2003 nicht enthalten. Bei der Schätzung des Index für PISA 2003 werden diese Indizes als fehlend behandelt, und unter der Annahme, dass der Zusammenhang zwischen den Items bei Hinzunahme neuer Fragen unverändert bleibt, sind die Werte für PISA 2003 und für PISA 2012 auf dem Index der lehrkräftebezogenen Faktoren für das Schulklima nach der Umskalierung vergleichbar.

\section{Schülerverhalten}

Der Index der schülerbezogenen Faktoren für das Schulklima (STUDCLIM) beruht auf den Angaben der Schulleitungen darüber, wie sehr das Lernen der Schülerinnen und Schüler an ihren Schulen durch die folgenden Faktoren beeinträchtigt wird (SC22): a) Schülerinnen und Schüler fehlen unentschuldigt in der Schule, b) Schülerinnen und Schüler schwänzen einzelne Unterrichtsstunden, c) Schülerinnen und Schüler erscheinen verspätet zum Unterricht, $d$ ) Schülerinnen und Schüler nehmen an obligatorischen Schulveranstaltungen (z.B. Sporttag) oder Ausflügen nicht teil, e) fehlender Respekt der Schülerinnen und Schüler vor den Lehrpersonen, $f$ ) Störung des Unterrichts durch Schülerinnen und Schüler, g) Konsum von Alkohol oder illegalen Drogen durch Schülerinnen und Schüler und $h$ ) Einschüchtern oder Schikanieren von Schülerinnen und Schülern durch Mitschülerinnen und Mitschüler. Da alle Items für die Skalierung umgepolt wurden, weisen höhere Werte auf diesem Index auf ein positiv wirkendes Schülerverhalten hin.

Für Trendanalysen wurden die Werte des Index der schülerbezogenen Faktoren für das Schulklima von PISA 2003 umskaliert, um die Vergleichbarkeit mit den Werten von PISA 2012 zu gewährleisten. Folglich können die in diesem Bericht dargelegten Werte für den Index der schülerbezogenen Faktoren für das Schulklima für PISA 2003 von den Werten abweichen, die in Lernen für die Welt von morgen: Erste Ergebnisse von PISA 2003 (OECD, 2004) enthalten sind. Zwei der in PISA 2012 enthaltenen Fragen, auf denen der Index der schülerbezogenen Faktoren für das Schulklima beruht [„Schülerinnen und Schüler erscheinen verspätet zum Unterricht" und "Schülerinnen und Schüler nehmen an obligatorischen Schulveranstaltungen (z.B. Sporttag) oder Ausflügen nicht teil“], waren im Fragebogen zu PISA 2003 nicht enthalten. Bei der Schätzung des Index für PISA 2003 werden diese Fragen als fehlend behandelt, und unter der Annahme, dass der Zusammenhang zwischen den Items bei Hinzunahme neuer Fragen unverändert bleibt, sind die Werte für PISA 2003 und für PISA 2012 auf dem Index der schülerbezogenen Faktoren für das Schulklima nach der Umskalierung vergleichbar. 


\section{Arbeitshaltung der Lehrkräfte}

Der Index der Arbeitshaltung der Lehrkräfte (TCMORALE) beruht auf den Angaben der Schulleitungen darüber, wie sehr sie den folgenden Aussagen zu den Lehrkräften an ihren Schulen zustimmen (SC26): a) Stimmung und Arbeitshaltung der Lehrkräfte an dieser Schule sind sehr gut, $b$ ) die Lehrkräfte arbeiten mit großem Engagement, c) die Lehrkräfte sind stolz auf diese Schule und d) die Lehrkräfte legen großen Wert auf schulische Leistung. Da alle Items für die Skalierung umgepolt wurden, weisen höhere Werte auf diesem Index auf eine positivere Arbeitshaltung der Lehrkräfte hin.

Für Trendanalysen wurden die Werte des Index der Arbeitshaltung der Lehrkräfte von PISA 2003 umskaliert, um die Vergleichbarkeit mit den Werten von PISA 2012 zu gewährleisten. Folglich können die in diesem Bericht dargelegten Werte für den Index der Arbeitshaltung der Lehrkräfte für PISA 2003 von den Werten abweichen, die in Lernen für die Welt von morgen: Erste Ergebnisse von PISA 2003 (OECD, 2004) enthalten sind.

\section{Fragen, auf denen die drei Indizes der Lernmöglichkeiten beruhen}

Sechs Fragen aus dem Schülerfragebogen dienten dazu, sowohl die inhaltlichen als auch die zeitlichen Aspekte der Lernmöglichkeiten abzudecken. Diese Fragen sind nachstehend abgebildet. 
Frage 1

Wie häufig bist du dieser Art von Aufgabe im Mathematikunterricht begegnet?

(Bitte in jeder Zeile nur ein Kästchen ankreuzen.)

a) Mit Hilfe eines Zugfahrplanes ausrechnen, wie lange man von einem Ort zum anderen brauchen würde.

b) Ausrechnen, um wie viel teurer ein Computer wird, wenn man die Mehrwertsteuer darauf schlägt.

c) Ausrechnen, wie viele Quadratmeter Fliesen du brauchtest, um einen Fußboden damit auszulegen.

d) Wissenschaftliche Tabellen in einem Artikel verstehen.

e) Eine Gleichung wie $6 x^{2}+5=29$ lösen.

f) Auf einer Karte mit einem Maßstab von 1:10.000 die tatsächliche Entfernung zwischen zwei Orten bestimmen.

g) Eine Gleichung wie $2(x+3)=(x+3)(x-3)$ lösen.

h) Den wöchentlichen Verbrauch eines Elektrogerätes berechnen.

i) Eine Gleichung wie $3 x+5=17$ lösen.

\begin{tabular}{|c|c|c|c|}
\hline Häufig & Manchmal & Selten & Nie \\
\hline$\sqcup 1$ & $\sqcup 2$ & $\sqcup 3$ & $\sqcup 4$ \\
\hline$\sqcup 1$ & $\sqcup 2$ & $\sqcup 3$ & $\sqcup 4$ \\
\hline$\square 1$ & $\square 2$ & $\square 3$ & $\square 4$ \\
\hline$\square 1$ & $\square 2$ & $\square 3$ & $\square 4$ \\
\hline$\square 1$ & $\square 2$ & $\square 3$ & $\square 4$ \\
\hline$\sqcup 1$ & $\sqcup 2$ & $\sqcup 3$ & $\sqcup 4$ \\
\hline$\sqcup 1$ & $\sqcup 2$ & $\sqcup 3$ & $\sqcup 4$ \\
\hline$\sqcup 1$ & $\sqcup 2$ & $\sqcup 3$ & $\sqcup 4$ \\
\hline$\sqcup 1$ & $\sqcup 2$ & $\sqcup 3$ & $\sqcup 4$ \\
\hline
\end{tabular}

Frage 2

Wenn du über mathematische Begriffe nachdenkst: Wie vertraut sind dir die folgenden Begriffe? (Bitte in jeder Zeile nur ein Kästchen ankreuzen.)

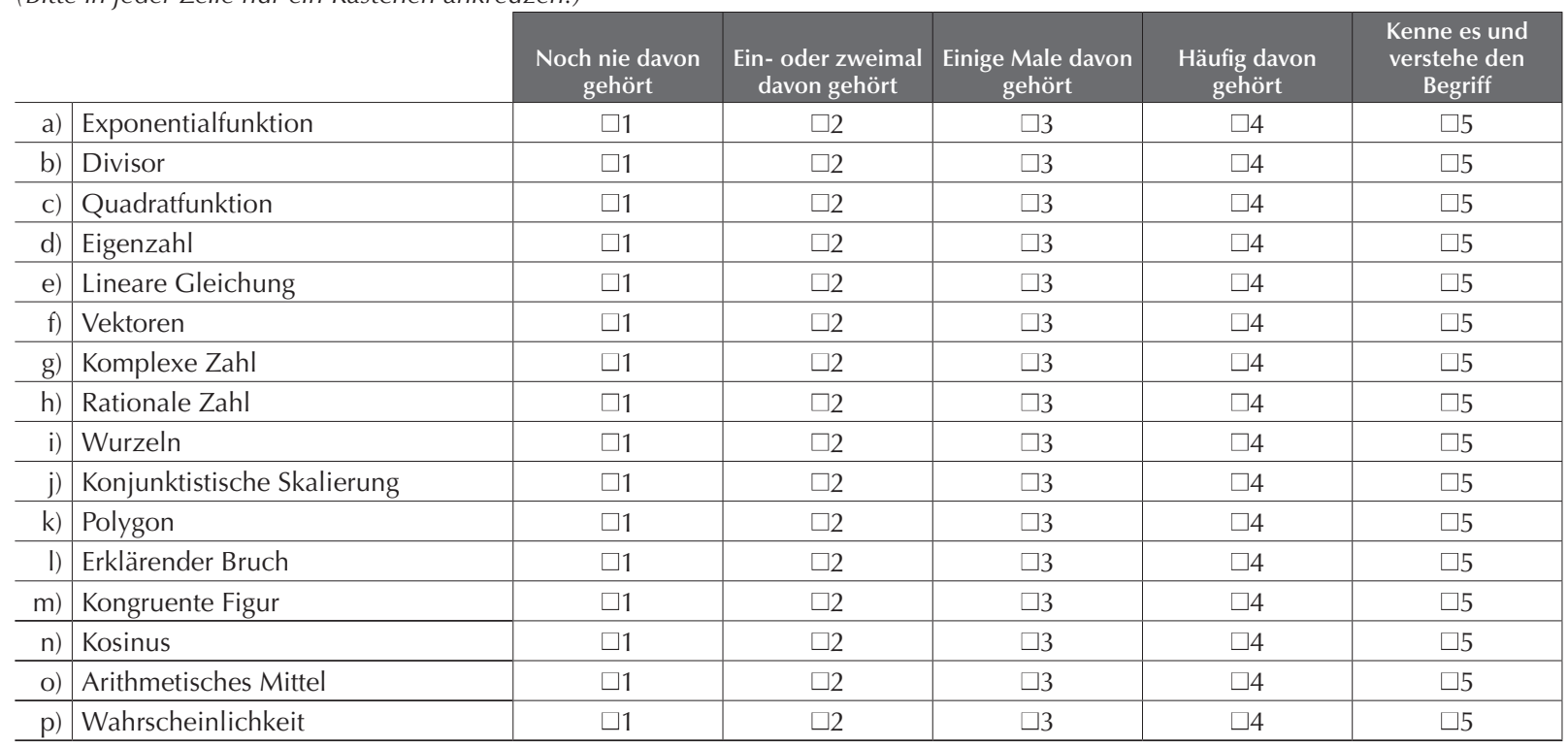

Die folgenden vier Fragen beziehen sich auf die Erfahrungen, die die Schülerinnen und Schüler in der Schule mit verschiedenen Arten von Mathematikaufgaben gemacht haben. Sie enthalten einige Aufgabenbeschreibungen und dunkelblaue Kästen, in denen jeweils eine Mathematikaufgabe angegeben ist. Die Schülerinnen und Schüler mussten jede dieser Aufgaben durchlesen, ohne sie zu lösen.

Frage 3

Im Kasten stehen zwei Textaufgaben. Normalerweise handelt die Aufgabe von einer realen Situation, aber die Zahlen, die Personen und die Orte, die angegeben sind, wurden frei erfunden. Alle Informationen, die du brauchst, sind im Text enthalten. Hier sind zwei Beispiele:

1) Anna ist zwei Jahre älter als Bettina, und Bettina ist viermal so alt wie Samuel. Wenn Bettina 30 wird, wie alt ist dann Samuel?

2) Herr Schmidt hat ein Fernsehgerät und ein Bett gekauft. Der Fernseher kostet 499 EUR, aber er hat einen Rabatt von $10 \%$ erhalten. Das Bett kostet 159 EUR. Außerdem hat er noch 15 EUR für die Lieferung bezahlt. Wie viel Geld hat Herr Schmidt ausgegeben?

Wir möchten wissen, wie viel Erfahrung du mit dieser Art von Aufgabe in der Schule gemacht hast. Du musst sie nicht lösen! (Bitte in jeder Zeile nur ein Kästchen ankreuzen.)

a) Wie häufig bist du dieser Art von Aufgabe im Mathematikunterricht begegnet?

b) Wie häufig bist du dieser Art von Aufgabe in Tests begegnet, die du in der Schule gemacht hast?

\begin{tabular}{|c|c|c|c|}
\hline Häufig & Manchmal & Selten & Nie \\
$\square 1$ & $\square 2$ & $\square 3$ & $\square 4$ \\
\hline$\sqcup 1$ & $\sqcup 2$ & $\sqcup 3$ & $\sqcup 4$ \\
\hline
\end{tabular}


Frage 4

Unten siehst du Beispiele von Aufgaben, für die man andere mathematische Fähigkeiten braucht

1) Löse: $2 x+3=7$.

2) Berechne das Volumen einer Kiste mit den Seitenlängen $3 \mathrm{~m}, 4 \mathrm{~m}$ und $5 \mathrm{~m}$.

Wir möchten wissen, wie viel Erfahrung du mit dieser Art von Aufgabe in der Schule gemacht hast. Du musst sie nicht lösen! (Bitte in jeder Zeile nur ein Kästchen ankreuzen.)

a) Wie häufig bist du dieser Art von Aufgabe im Mathematikunterricht begegnet?

b) Wie häufig bist du dieser Art von Aufgabe in Tests begegnet, die du in der Schule gemacht hast?

\begin{tabular}{|c|c|c|c|c|}
\cline { 2 - 5 } & Häufig & Manchmal & Selten & Nie \\
\hline & $\sqcup 1$ & $\sqcup 2$ & $\sqcup 3$ & $\sqcup 4$ \\
\hline Tests & $\sqcup 1$ & $\sqcup 2$ & $\sqcup 3$ & $\sqcup 4$ \\
\hline
\end{tabular}

\section{Frage 5}

Beim nächsten Aufgabentyp musst du dein mathematisches Wissen einsetzen und Schlussfolgerungen daraus ziehen. Hier sind zwei Beispiele.

1) Bei dieser Aufgabe musst du geometrische Sätze anwenden:

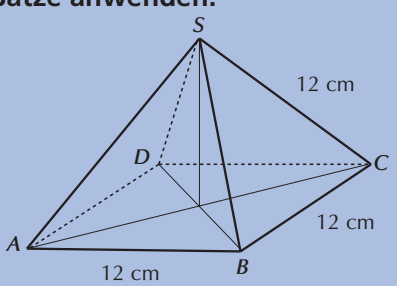

Bestimme die Höhe der Pyramide!

2) Hier musst du wissen, was eine Primzahl ist:

Wenn $n$ irgendeine Zahl ist: kann dann $(n+1)^{2}$ eine Primzahl sein?

Wir möchten wissen, wie viel Erfahrung du mit dieser Art von Aufgabe in der Schule gemacht hast. Du musst sie nicht lösen! (Bitte in jeder Zeile nur ein Kästchen ankreuzen.)

a) Wie häufig bist du dieser Art von Aufgabe im

Mathematikunterricht begegnet?

b) Wie häufig bist du dieser Art von Aufgabe in Tests begegnet, die du in der Schule gemacht hast?

\begin{tabular}{l|c|c|c|c|} 
& Häufig & Manchmal & Selten & Nie \\
\hline Tests & $\square 1$ & $\square 2$ & $\square 3$ & $\square 4$ \\
\hline t? & $\sqcup 1$ & $\sqcup 2$ & $\sqcup 3$ & $\sqcup 4$ \\
\hline
\end{tabular}

\section{Frage 6}

Bei diesem Aufgabentyp musst du das passende mathematische Wissen anwenden, um eine brauchbare Antwort auf ein Alltagsproblem zu finden. Hier sind zwei Beispiele.

\section{Beispiel 1}

Ein Fernsehreporter sagt: "Diese Grafik zeigt, dass es zwischen 1998 und 1999 eine enorme Zunahme von Raubüberfällen gegeben hat."

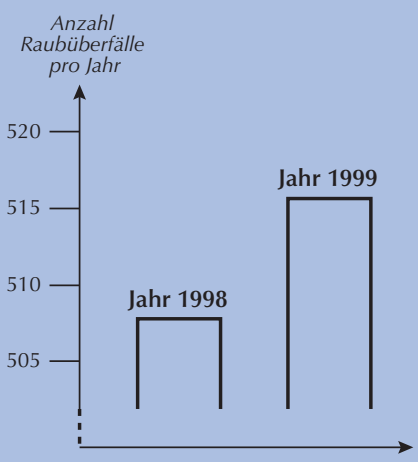




\section{Beispiel 2}

Das Verhältnis zwischen empfohlener maximaler Herzfrequenz und dem Alter einer Person wurde lange mit der folgenden Formel beschrieben:

Maximale empfohlene Herzfrequenz = 220 - Alter

Neuere Forschungsergebnisse haben nun gezeigt, dass diese Formel leicht angepasst werden sollte. Die neue Formel lautet:

Maximale empfohlene Herzfrequenz = $208-(0,7$ x Alter $)$

Ab welchem Alter beginnt die maximale empfohlene Herzfrequenz gemäß der neuen Formel zu steigen? Gib deinen Lösungsweg an.

Wir möchten wissen, wie viel Erfahrung du mit dieser Art von Aufgabe in der Schule gemacht hast. Du musst sie nicht lösen! (Bitte in jeder Zeile nur ein Kästchen ankreuzen.)

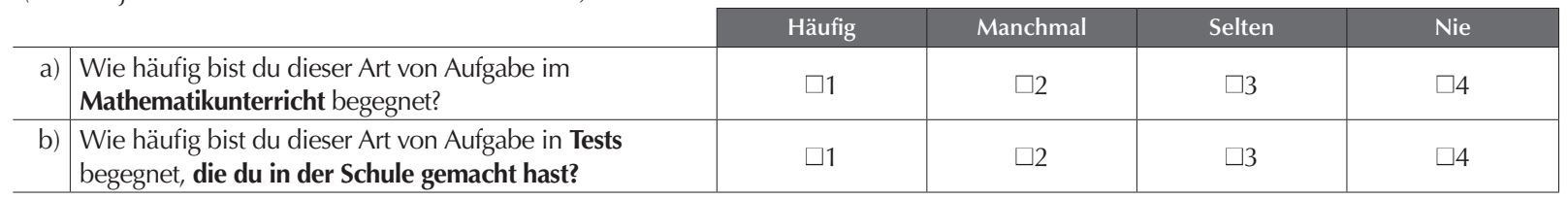

\section{Die drei Indizes der Lernmöglichkeiten}

Aus diesen Fragen wurden drei Indizes konstruiert:

\section{- Index des Kontakts mit Textaufgaben}

Dieser Index wurde anhand der Angaben zur Häufigkeit für den Aufgabentyp der Textaufgabe (Frage 3) wie folgt kodiert: häufig $=3$, manchmal $=2$, selten $=1$ und nie $=0$

- Index des Kontakts mit angewandter Mathematik

Dieser Index wurde als der Mittelwert für die Aufgaben der angewandten Mathematik konstruiert, die sich auf einen mathematischen (Frage 5) bzw. einen realen Kontext (Frage 6) beziehen. Jede der beiden Fragen wurde separat nach folgendem Schema skaliert: häufig $=3$, manchmal $=2$, selten $=1$ und nie $=0$.

- Index des Kontakts mit reiner Mathematik

Dieser Index wurde als Durchschnitt dreier Skalen gebildet.

- Zwei separate Skalen wurden auf Basis der Vertrautheit der Schülerinnen und Schüler mit 7 der 13 mathematischen Inhaltsbereiche in Frage 2 konstruiert. Die fünf Antwortkategorien für ihre Vertrautheit mit den in der Frage genannten mathematischen Begriffen wurden von 0 bis 4 skaliert, wobei 0 für "noch nie davon gehört" und 4 für "kenne es und verstehe den Begriff" steht. Aus den Häufigkeitscodes für drei Themen - Exponentialfunktion, Quadratfunktion und lineare Gleichung - wurde der Durchschnittswert ermittelt, um die Vertrautheit mit Algebra zu definieren. Analog dazu diente der Durchschnitt aus vier Themen - Vektoren, Polygon, kongruente Figur und Kosinus - zur Definition einer Geometrie-Skala.

- Die dritte Skala beruht auf dem Item, bei dem die Schülerinnen und Schüler angaben, wie oft sie Aufgaben begegnet waren, die als reine Mathematik definiert sind (Frage 4). Die Häufigkeitskategorien wurden so kodiert, dass "häufig", "manchmal" und "selten" mit 1 gleichgesetzt wurden und "nie" mit 0, so dass sich eine dichotome Variable ergab. Aus dem Durchschnitt der Algebraaufgaben, Geometrieaufgaben und Aufgaben der reinen Mathematik wurde der Index "reine Mathematik" gebildet, dessen Werte ähnlich wie bei den anderen drei Indizes von 0 bis 3 reichten. 


\section{Anmerkungen}

1. Dabei ist zu beachten, dass die ISCO-Kodierung 0 „Angehörige der regulären Streitkräfte" wie folgt umkodiert wurde: "Offiziere" wurden als „Führungskräfte“ (ISCO 1) kodiert, „Angehörige der regulären Streitkräfte in sonstigen Rängen“ (Fahrer, Schützen, Matrosen, Angehörige der Streitkräfte allgemein) als „Bediener von Anlagen und Maschinen“ (ISCO 8). Ferner wurden alle mit "97" beginnenden Antworten (Hausfrauen, Studierende und unklar definierte Berufe) als Fehlwert kodiert.

2. Die Aktualisierung von ISCO-88 auf ISCO-08 betraf hauptsächlich a) eine zutreffendere Einstufung der IT-Berufe, b) eine Unterscheidung nach militärischen Rängen und c) eine Revision der Kategorien zur Einstufung von Führungskräften.

3. Die Informationen zu ISCO-08 und ISEI-08 stammen aus http://www.ilo.org/public/eng/ish/bureau/stat/isco/index.htm und http://home.fsw.vu.nl/hbg.ganzeboom/isco08.

\section{Literaturverzeichnis}

Ganzeboom, H.B.G. (2010), "A new international socio-economic index [ISEI] of occupational status for the International Standard Classification of Occupation 2008 [ISCO-08] constructed with data from the ISSP 2002-2007; with an analysis of quality of occupational measurement in ISSP", Beitrag zur Annual Conference of International Social Survey Programme, Lissabon, 1. Mai 2010.

Ganzeboom, H.B.G. und D.J. Treiman (2003), "Three Internationally Standardised Measures for Comparative Research on Occupational Status ", in Jürgen H.P. Hoffmeyer-Zlotnik und Christof Wolf (Hrsg.), Advances in Cross-National Comparison: A European Working Book for Demographic and Socio-Economic Variables, Kluwer Academic Press, New York.

Ganzeboom, H.B.G. und D.J. Treiman (1996), "Internationally Comparable Measures of Occupational Status for the 1988 International Standard Classification of Occupations", Social Science Research, Vol. 25, S. 201-239.

Ganzeboom, H.B.G., P. de Graaf und D.J. Treiman (1992), "A Standard International Socio-Economic Index of Occupational Status", Social Science Research, Vol. 21, Issue 1, S. 1-56.

Ganzeboom, H.B.G., R. Luijkx und D.J. Treiman (1989), "InterGenerational Class Mobility in Comparative Perspective", Research in Social Stratification and Mobility, Vol. 8, S. 3-79.

ILO (1990), ISCO-88: Internationale Standardklassifikation der Berufe, Internationales Arbeitsamt, Genf.

OECD (erscheint demnächst), PISA 2012 Technical Report, OECD Publishing.

OECD (2013a), PISA 2012 Assessment and Analytical Framework: Mathematics, Reading, Science, Problem Solving and Financial Literacy, PISA, OECD Publishing, http://dx.doi.org/10.1787/9789264190511-en.

OECD (2013b), Bildung auf einen Blick 2013: OECD Indikatoren, W. Bertelsmann Verlag, Bielefeld, http://dx.doi.org/10.1787/eag-2013.de.

OECD (2013c), OECD Skills Outlook 2013: First Results from the Survey of Adult Skills, OECD Publishing, http://dx.doi. org/10.1787/9789264204256-en.

OECD (2004), Lernen für die Welt von morgen: Erste Ergebnisse von PISA 2003, Elsevier, http://dx.doi.org/10.1787/9789264063556-de.

OECD (1999), Classifying Educational Programmes: Manual for ISCED-97 Implemention in OECD Countries, OECD Publishing, www. oecd.org/education/skills-beyond-school/1962350.pdf.

Warm, T.A. (1989), "Weighted likelihood estimation of ability in item response theory", Psychometrika, Volume 54, Issue 3, S. 427-450, http://dx.doi.org/10.1007/BF02294627. 


\section{ANHANG A2}

\section{PISA-ZIELPOPULATION, PISA-STICHPROBEN UND DEFINITION DER SCHULEN}

\section{Definition der PISA-Zielpopulation}

PISA 2012 untersucht die kumulativen Bildungserträge und Lernergebnisse zu einem Zeitpunkt, an dem sich die meisten Jugendlichen noch in der Phase der Erstausbildung befinden.

Eine große Herausforderung bei einer internationalen Erhebung besteht darin sicherzustellen, dass die internationale Vergleichbarkeit der Zielpopulationen in den verschiedenen Ländern gewährleistet ist.

Auf Grund länderspezifischer Unterschiede im Hinblick auf die Art und die Verbreitung von Einrichtungen des Elementarbereichs, das reguläre Einschulungsalter sowie die institutionelle Struktur des Bildungssystems sind Definitionen der Zielpopulation, die sich auf bestimmte Klassenstufen beziehen, für internationale Vergleiche ungeeignet. Daher werden bei internationalen Vergleichen von Schulleistungen die Populationen in der Regel in Bezug auf ein Zielalter definiert. In einigen früheren internationalen Erhebungen wurden die Zielpopulationen auch auf der Basis der Klassenstufe definiert, die jeweils die breiteste Erfassung einer bestimmten Alterskohorte gewährleistet. Dieser Ansatz hat den Nachteil, dass leichte Abweichungen bei der altersmäßigen Verteilung der Schülerinnen und Schüler auf die einzelnen Klassenstufen häufig dazu führen, dass in verschiedenen Ländern oder in verschiedenen Bildungssystemen innerhalb einzelner Länder unterschiedliche Zielklassenstufen ausgewählt werden, was die Vergleichbarkeit der Ergebnisse zwischen den einzelnen Ländern und zuweilen auch innerhalb der Länder ernsthaft in Frage stellt. Da gewöhnlich nicht alle Schülerinnen und Schüler der gewünschten Altersgruppe in klassenstufenbasierten Stichproben repräsentiert sind, kann es darüber hinaus zu potenziell gravierenderen Verzerrungen der Ergebnisse kommen, wenn die unterrepräsentierten Schülerinnen und Schüler in einigen Ländern üblicherweise die nächsthöhere Klassenstufe, in anderen Ländern wiederum die nächstuntere Klassenstufe besuchen. Dadurch blieben in der einen Ländergruppe Schülerinnen und Schüler mit einem potenziell höheren Leistungsniveau unberücksichtigt, während in der anderen Ländergruppe Schüler mit einem potenziell niedrigeren Leistungsniveau nicht erfasst würden.

Um dieses Problem zu vermeiden, wird in PISA eine altersbezogene Definition der Zielpopulation verwendet, d.h. eine Definition, die von der institutionellen Struktur der Bildungssysteme der jeweiligen Länder unabhängig ist. PISA erfasst Schülerinnen und Schüler, die zu Beginn der Testperiode zwischen 15 Jahren und 3 (vollen) Monaten und 16 Jahren und 2 (vollen) Monaten alt waren - wobei eine Abweichung von plus/minus einem Monat akzeptiert wird - und die eine Bildungseinrichtung mit Klassenstufe 7 oder darüber besuchen, unabhängig davon, welche Klassenstufe oder Art von Bildungseinrichtung sie besuchen und ob es sich um eine Ganztagsoder Halbtagsschule handelt. Bildungseinrichtungen werden in dieser Veröffentlichung generell als "Schulen“ bezeichnet, obwohl einige (insbesondere manche Formen berufsbildender Einrichtungen) im landesüblichen Sprachgebrauch u.U. nicht Schulen genannt werden. Wie auf Grund dieser Definition zu erwarten, betrug das Durchschnittsalter der teilnehmenden Schülerinnen und Schüler im OECD-Raum 15 Jahre und 9 Monate. Die Varianz des Durchschnittsalters in den verschiedenen Ländern belief sich auf 2 Monate und 5 Tage (0,18 Jahre), wobei das niedrigste Durchschnittsalter 15 Jahre und 8 Monate und das höchste 15 Jahre und 10 Monate war.

Auf Grund dieser Populationsdefinition trifft PISA Aussagen über die Kenntnisse und Fähigkeiten einer Gruppe von Schülerinnen und Schülern, die in einer vergleichbaren Referenzperiode geboren sind, aber über unterschiedliche inner- wie außerschulische Bildungserfahrungen verfügen können. In PISA gelten diese Kenntnisse und Fähigkeiten als die Erträge der Bildung in einem für alle Länder gleich angesetzten Alter. Je nach der von den einzelnen Ländern verfolgten Politik in Bezug auf Einschulung, Auswahl und Versetzung können sich die betreffenden Schülerinnen und Schüler auf ein engeres oder breiteres Spektrum von Klassenstufen in verschiedenen Bildungssystemen, Bildungsgängen oder -zweigen verteilen. Diese Abweichungen sollten beim Vergleich der PISAErgebnisse in den verschiedenen Ländern berücksichtigt werden, da die beobachteten Unterschiede zwischen Schülern im Alter von 15 Jahren auf Grund konvergierender Bildungserfahrungen später möglicherweise nicht mehr zu erkennen sind.

Wenn ein Land auf den Leistungsskalen für Lesekompetenz, Naturwissenschaften und Mathematik eine wesentlich höhere Punktzahl erreicht als ein anderes, ist daraus nicht automatisch zu folgern, dass die Schulen oder bestimmte Teile des Bildungssystems in diesem Land effektiver sind als in dem anderen. Man kann daraus aber durchaus schließen, dass der kumulative Effekt der Lernerfahrungen in dem Land mit der höheren Punktzahl von der frühen Kindheit bis zum Alter von 15 Jahren, sowohl im schulischen als auch im außerschulischen Umfeld, zu positiveren Ergebnissen in den von PISA getesteten Grundbildungsbereichen geführt hat.

In der PISA-Zielpopulation nicht enthalten sind Schülerinnen und Schüler des jeweiligen Landes, die eine Schule im Ausland besuchen. Erfasst sind hingegen ausländische Schülerinnen und Schüler, die eine Schule im Erhebungsland besuchen.

Um den Ländern entgegenzukommen, die zum Zweck nationaler Analysen nach Klassenstufen aufgeschlüsselte Ergebnisse wünschten, wurde in PISA 2012 eine Stichprobenoption zur Ergänzung der altersbezogenen Stichprobe durch eine klassenstufenbezogene Stichprobe angeboten. 


\section{Erfassung der PISA-Schülerpopulation}

Alle Länder waren um eine möglichst breite Erfassung der 15-jährigen Schülerinnen und Schüler in ihren nationalen Stichproben bemüht, auch solcher in Sondereinrichtungen. Daher erreichte PISA 2012 einen für internationale Erhebungen dieser Art beispiellosen Grad der Populationserfassung.

Die PISA-Stichprobenstandards erlaubten es den Ländern, bis zu insgesamt 5\% der relevanten Population auszuschließen, sei es durch Ausschluss von Schulen oder durch Ausschluss von Schülern innerhalb der Schulen. Mit Ausnahme von acht Ländern, nämlich Luxemburg (8,40\%), Kanada (6,38\%), Dänemark (6,18\%), Norwegen (6,11\%), Estland (5,80\%), Schweden (5,44\%), dem Vereinigten Königreich $(5,43 \%)$ und den Vereinigten Staaten (5,35\%), konnten alle Länder diese Regel einhalten, und in 30 Ländern und Volkswirtschaften lag die Gesamtausschlussrate bei weniger als $2 \%$. Werden sprachlich bedingte Ausschlüsse berücksichtigt, d.h. aus der Gesamtausschlussrate herausgerechnet, liegen die Ausschlussraten in Norwegen, Schweden, dem Vereinigten Königreich und den Vereinigten Staaten nicht mehr über 5\%. Wegen Einzelheiten vgl. www.pisa.oecd.org.

Zu den Ausschlüssen innerhalb der oben genannten Grenzen zählen:

- Auf Schulebene: a) Schulen, die in schwer erreichbaren Gegenden liegen oder in denen die Durchführung der PISA-Erhebung als nicht praktikabel angesehen wurde; b) Schulen, deren Unterrichtsangebot sich auf die Kategorien beschränkt, die unter der Rubrik "Ausschlüsse innerhalb der Schulen“ definiert sind, wie z.B. Blindenschulen. Der Prozentsatz der 15-Jährigen, die solche Schulen besuchten, musste weniger als 2,5\% der angestrebten Grundgesamtheit auf Länderebene ausmachen (maximal 0,5\% für a) und maximal 2\% für b)). Der PISA 2012 Technical Report (OECD, erscheint demnächst) enthält Angaben über die Größenordnung, Art und Begründung der Ausschlüsse auf Schulebene.

- Auf Schülerebene: a) Schülerinnen und Schüler mit kognitiver Behinderung; b) Schülerinnen und Schüler mit funktionaler Behinderung; c) Schülerinnen und Schüler mit unzureichender Kenntnis der Testsprache; d) sonstige Schülerinnen und Schüler, eine Kategorie, die von den nationalen Zentren definiert und vom Internationalen Konsortium genehmigt werden muss; e) Schülerinnen und Schüler, die im Haupttestbereich in einer Unterrichtssprache unterrichtet werden, für die keine Unterlagen verfügbar waren. Es war nicht möglich, Schülerinnen und Schüler allein auf Grund eines niedrigen Leistungsniveaus oder normaler Disziplinprobleme auszuschließen. Der Prozentsatz der innerhalb der Schulen ausgeschlossenen 15-Jährigen musste niedriger sein als 2,5\% der auf Länderebene angestrebten Grundgesamtheit.

Tabelle A2.1 beschreibt die Zielpopulation der an PISA 2012 teilnehmenden Länder. Nähere Einzelheiten über die Zielpopulation und die Anwendung der PISA-Stichprobenstandards enthält der PISA 2012 Technical Report (OECD, erscheint demnächst).

- Spalte 1 zeigt die Gesamtzahl der 15-Jährigen gemäß den aktuellsten vorliegenden Informationen, d.h. für die meisten Länder aus dem Vorjahr der Erhebung, d.h. 2011.

- Spalte 2 zeigt die Zahl der 15-Jährigen in Klassenstufe 7 oder darüber (gemäß obiger Definition), also die in Betracht kommende Population.

- Spalte 3 zeigt die angestrebte Grundgesamtheit auf Länderebene. Die Länder konnten von vornherein - hauptsächlich aus praktischen Gründen - bis zu 0,5\% der Schülerinnen und Schüler aus der in Betracht kommenden Population ausschließen. In folgenden Fällen wurde diese Höchstgrenze überschritten, was aber im Einvernehmen mit dem PISA-Konsortium geschah: Belgien hat 0,23\% seiner Schülerpopulation ausgeschlossen, weil es sich um Schüler handelte, die gleichzeitig einer Erwerbstätigkeit nachgingen; Kanada hat Schüler in Indigenengebieten (Territories and Aboriginal Reserves) ausgeschlossen, insgesamt 1,14\% seiner Zielpopulation; Chile hat Schüler ausgeschlossen, die auf den Osterinseln, auf den Juan-Fernández-Inseln und in der Antarktis leben und 0,04\% der Zielpopulation ausmachen; Indonesien hat wegen Durchführungsproblemen Schüler aus zwei Provinzen, insgesamt 1,55\% seiner Population, ausgeschlossen; Irland hat Schüler aus drei Inselschulen vor seiner Westküste ausgeschlossen, die 0,05\% seiner Zielpopulation ausmachen; Lettland hat 0,08\% seiner Zielpopulation ausgeschlossen, weil es sich um Schüler von Fernschulen handelte; in Serbien wurden 2,11\% der Schüler ausgeschlossen, die im Kosovo in serbischer Sprache unterrichtet werden.

- Spalte 4 zeigt die Zahl der Schülerinnen und Schüler in Schulen, die von der angestrebten Grundgesamtheit auf Länderebene bei der Stichprobenziehung oder zu einem späteren Zeitpunkt während der Datensammlung ausgeschlossen wurden.

- Spalte 5 zeigt die Größe der angestrebten Grundgesamtheit auf Länderebene nach Abzug der Schülerinnen und Schüler in den ausgeschlossenen Schulen. Dies wird durch Subtraktion von Spalte 4 von Spalte 3 ermittelt.

- Spalte 6 zeigt den Prozentsatz der Schülerinnen und Schüler in den ausgeschlossenen Schulen. Dieser wird durch Division von Spalte 4 durch Spalte 3, multipliziert mit 100, ermittelt.

- Spalte 7 zeigt die Zahl der an PISA 2012 teilnehmenden Schülerinnen und Schüler. Dabei ist zu beachten, dass die im Rahmen nationaler Optionen zusätzlich einbezogenen Schülerinnen und Schüler in diesem Wert in manchen Fällen nicht berücksichtigt sind.

- Spalte 8 zeigt die gewichtete Zahl der teilnehmenden Schülerinnen und Schüler, d.h. die Zahl der Schülerinnen und Schüler in der definierten Grundgesamtheit auf Länderebene, die durch die PISA-Stichprobe repräsentiert wird.

- Jedes Land war bestrebt, den Grad der Erfassung der PISA-Zielpopulation innerhalb der in die Stichprobe einbezogenen Schulen zu maximieren. Für jede einbezogene Schule wurden zunächst alle in Betracht kommenden Schülerinnen und Schüler, d.h. alle 15-Jährigen, unabhängig von ihrer Klasse, aufgelistet. Auch die von der Stichprobe auszuschließenden Schülerinnen und Schüler 
[Teil 1/2]

Tabelle A2.1 PISA-Zielpopulationen und -Stichproben

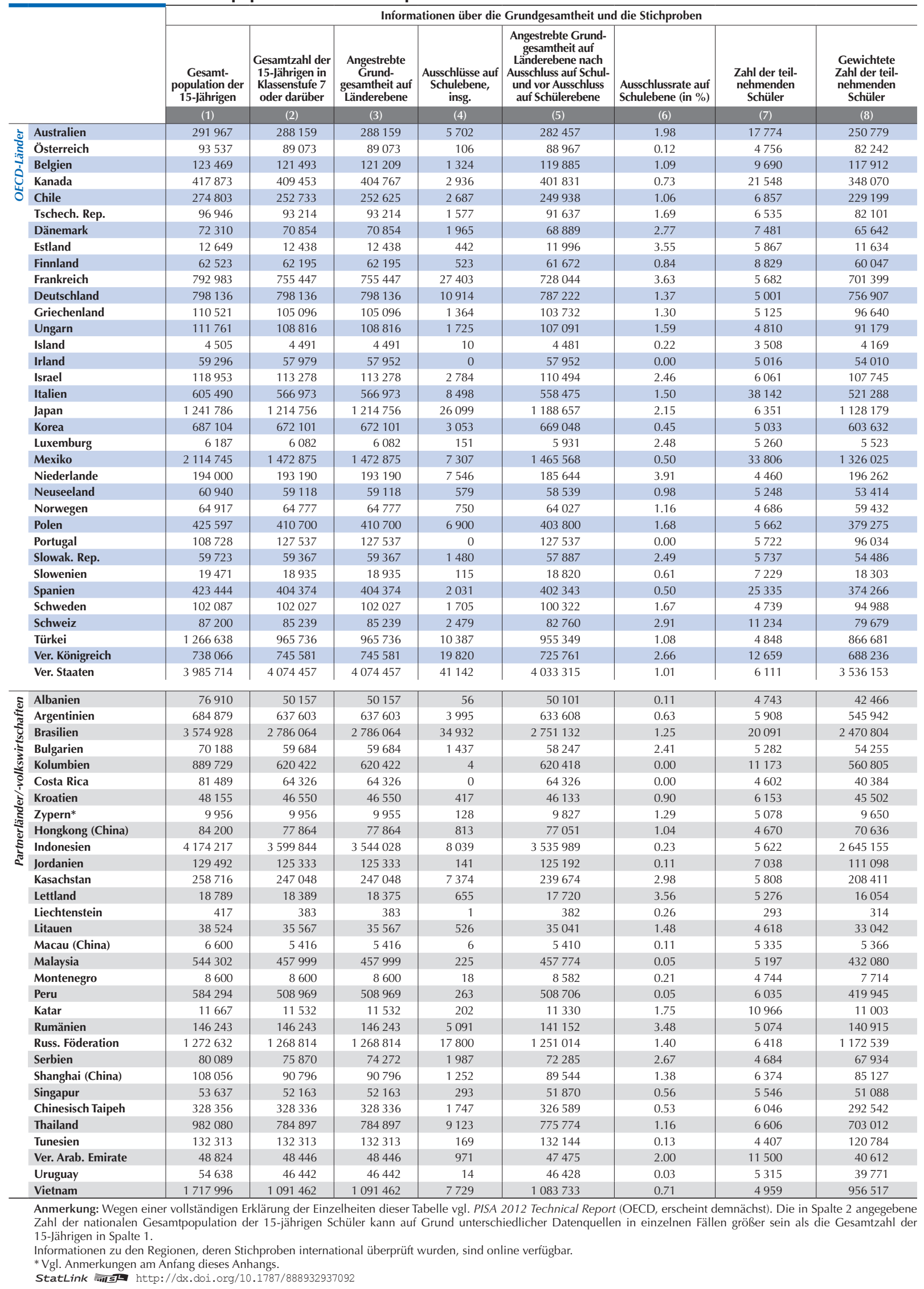


[Teil 2/2]

Tabelle A2.1 PISA-Zielpopulationen und -Stichproben

\begin{tabular}{|c|c|c|c|c|c|c|c|c|}
\hline & & \multicolumn{4}{|c|}{ Informationen über die Grundgesamtheit und die Stichproben } & \multicolumn{3}{|c|}{ Erfassungsindizes } \\
\hline & & $\begin{array}{l}\text { Zahl der } \\
\text { ausgeschloss. } \\
\text { Schüler }\end{array}$ & $\begin{array}{l}\text { Gewichtete Zahl } \\
\text { der ausgeschloss. } \\
\text { Schüler }\end{array}$ & $\begin{array}{l}\text { Ausschlussrate } \\
\text { innerhalb der } \\
\text { Schulen (in \%) }\end{array}$ & $\begin{array}{c}\text { Ausschlussrate insg. } \\
\text { (in \%) }\end{array}$ & $\begin{array}{l}\text { Erfassungsindex 1: } \\
\text { Erfassung der } \\
\text { angestrebten } \\
\text { Grundgesamtheit } \\
\text { auf Länderebene }\end{array}$ & $\begin{array}{l}\text { Erfassungsindex 2: } \\
\text { Erfassung der } \\
\text { Schülerpopulation } \\
\text { auf Länderebene }\end{array}$ & $\begin{array}{l}\text { Erfassungsindex 3: } \\
\text { Erfassung der } \\
\text { Population }\end{array}$ \\
\hline & & $(9)$ & (10) & $(11)$ & (12) & (13) & $(14)$ & $(15)$ \\
\hline \multirow{34}{*}{ 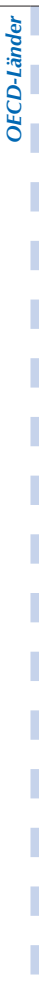 } & Australien & 505 & 5282 & 2.06 & 4.00 & 0.960 & 0.960 & 0.859 \\
\hline & Österreich & 46 & 1011 & 1.21 & 1.33 & 0.987 & 0.987 & 0.879 \\
\hline & Belgien & 39 & 367 & 0.31 & 1.40 & 0.986 & 0.984 & 0.955 \\
\hline & Kanada & 1796 & 21013 & 5.69 & 6.38 & 0.936 & 0.926 & 0.833 \\
\hline & Chile & 18 & 548 & 0.24 & 1.30 & 0.987 & 0.987 & 0.834 \\
\hline & Tschech. Rep. & 15 & 118 & 0.14 & 1.83 & 0.982 & 0.982 & 0.847 \\
\hline & Dänemark & 368 & 2381 & 3.50 & 6.18 & 0.938 & 0.938 & 0.908 \\
\hline & Estland & 143 & 277 & 2.33 & 5.80 & 0.942 & 0.942 & 0.920 \\
\hline & Finnland & 225 & 653 & 1.08 & 1.91 & 0.981 & 0.981 & 0.960 \\
\hline & Frankreich & 52 & 5828 & 0.82 & 4.42 & 0.956 & 0.956 & 0.885 \\
\hline & Deutschland & 8 & 1302 & 0.17 & 1.54 & 0.985 & 0.985 & 0.948 \\
\hline & Griechenland & 136 & 2304 & 2.33 & 3.60 & 0.964 & 0.964 & 0.874 \\
\hline & Ungarn & 27 & 928 & 1.01 & 2.58 & 0.974 & 0.974 & 0.816 \\
\hline & Island & 155 & 156 & 3.60 & 3.81 & 0.962 & 0.962 & 0.925 \\
\hline & Irland & 271 & 2524 & 4.47 & 4.47 & 0.955 & 0.955 & 0.911 \\
\hline & Israel & 114 & 1884 & 1.72 & 4.13 & 0.959 & 0.959 & 0.906 \\
\hline & Italien & 741 & 9855 & 1.86 & 3.33 & 0.967 & 0.967 & 0.861 \\
\hline & Japan & 0 & 0 & 0.00 & 2.15 & 0.979 & 0.979 & 0.909 \\
\hline & Korea & 17 & 2238 & 0.37 & 0.82 & 0.992 & 0.992 & 0.879 \\
\hline & Luxemburg & 357 & 357 & 6.07 & 8.40 & 0.872 & 0.916 & 0.893 \\
\hline & Mexiko & 58 & 3247 & 0.24 & 0.74 & 0.993 & 0.993 & 0.627 \\
\hline & Niederlande & 27 & 1056 & 0.54 & 4.42 & 0.956 & 0.956 & 1.012 \\
\hline & Neuseeland & 255 & 2030 & 3.66 & 4.61 & 0.954 & 0.954 & 0.876 \\
\hline & Norwegen & 278 & 3133 & 5.01 & 6.11 & 0.939 & 0.939 & 0.916 \\
\hline & Polen & 212 & 11566 & 2.96 & 4.59 & 0.954 & 0.954 & 0.891 \\
\hline & Portugal & 124 & 1560 & 1.60 & 1.60 & 0.984 & 0.984 & 0.883 \\
\hline & Slowak. Rep. & 29 & 246 & 0.45 & 2.93 & 0.971 & 0.971 & 0.912 \\
\hline & Slowenien & 84 & 181 & 0.98 & 1.58 & 0.984 & 0.984 & 0.940 \\
\hline & Spanien & 959 & 14931 & 3.84 & 4.32 & 0.957 & 0.957 & 0.884 \\
\hline & Schweden & 201 & 3789 & 3.84 & 5.44 & 0.946 & 0.946 & 0.930 \\
\hline & Schweiz & 256 & 1093 & 1.35 & 4.22 & 0.958 & 0.958 & 0.914 \\
\hline & Türkei & 21 & 3684 & 0.42 & 1.49 & 0.985 & 0.985 & 0.684 \\
\hline & Ver. Königreich & 486 & 20173 & 2.85 & 5.43 & 0.946 & 0.946 & 0.932 \\
\hline & Ver. Staaten & 319 & 162194 & 4.39 & 5.35 & 0.946 & 0.946 & 0.887 \\
\hline \multirow{31}{*}{ 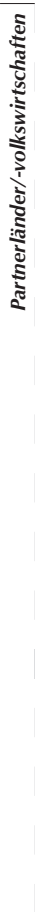 } & Albanien & 1 & 10 & 0.02 & 0.14 & 0.999 & 0.999 & 0.552 \\
\hline & Argentinien & 12 & 641 & 0.12 & 0.74 & 0.993 & 0.993 & 0.797 \\
\hline & Brasilien & 44 & 4900 & 0.20 & 1.45 & 0.986 & 0.986 & 0.691 \\
\hline & Bulgarien & 6 & 80 & 0.15 & 2.55 & 0.974 & 0.974 & 0.773 \\
\hline & Kolumbien & 23 & 789 & 0.14 & 0.14 & 0.999 & 0.999 & 0.630 \\
\hline & Costa Rica & 2 & 12 & 0.03 & 0.03 & 1.000 & 1.000 & 0.496 \\
\hline & Kroatien & 91 & 627 & 1.36 & 2.24 & 0.978 & 0.978 & 0.945 \\
\hline & Zypern* & 157 & 200 & 2.03 & 3.29 & 0.967 & 0.967 & 0.969 \\
\hline & Hongkong (China) & 38 & 518 & 0.73 & 1.76 & 0.982 & 0.982 & 0.839 \\
\hline & Indonesien & 2 & 860 & 0.03 & 0.26 & 0.997 & 0.982 & 0.634 \\
\hline & Jordanien & 19 & 304 & 0.27 & 0.39 & 0.996 & 0.996 & 0.858 \\
\hline & Kasachstan & 25 & 951 & 0.45 & 3.43 & 0.966 & 0.966 & 0.806 \\
\hline & Lettland & 14 & 76 & 0.47 & 4.02 & 0.960 & 0.959 & 0.854 \\
\hline & Liechtenstein & 13 & 13 & 3.97 & 4.22 & 0.958 & 0.958 & 0.753 \\
\hline & Litauen & 130 & 867 & 2.56 & 4.00 & 0.960 & 0.960 & 0.858 \\
\hline & Macau (China) & 3 & 3 & 0.06 & 0.17 & 0.998 & 0.998 & 0.813 \\
\hline & Malaysia & 7 & 554 & 0.13 & 0.18 & 0.998 & 0.998 & 0.794 \\
\hline & Montenegro & 4 & 8 & 0.10 & 0.31 & 0.997 & 0.997 & 0.897 \\
\hline & Peru & 8 & 549 & 0.13 & 0.18 & 0.998 & 0.998 & 0.719 \\
\hline & Katar & 85 & 85 & 0.77 & 2.51 & 0.975 & 0.975 & 0.943 \\
\hline & Rumänien & 0 & 0 & 0.00 & 3.48 & 0.965 & 0.965 & 0.964 \\
\hline & Russ. Föderation & 69 & 11940 & 1.01 & 2.40 & 0.976 & 0.976 & 0.921 \\
\hline & Serbien & 10 & 136 & 0.20 & 2.87 & 0.971 & 0.951 & 0.848 \\
\hline & Shanghai (China) & 8 & 107 & 0.13 & 1.50 & 0.985 & 0.985 & 0.788 \\
\hline & Singapur & 33 & 315 & 0.61 & 1.17 & 0.988 & 0.988 & 0.952 \\
\hline & Chinesisch Taipeh & 44 & 2029 & 0.69 & 1.22 & 0.988 & 0.988 & 0.891 \\
\hline & Thailand & 12 & 1144 & 0.16 & 1.32 & 0.987 & 0.987 & 0.716 \\
\hline & Tunesien & 5 & 130 & 0.11 & 0.24 & 0.998 & 0.998 & 0.913 \\
\hline & Ver. Arab. Emirate & 11 & 37 & 0.09 & 2.09 & 0.979 & 0.979 & 0.832 \\
\hline & Uruguay & 15 & 99 & 0.25 & 0.28 & 0.997 & 0.997 & 0.728 \\
\hline & Vietnam & 1 & 198 & 0.02 & 0.73 & 0.993 & 0.993 & 0.557 \\
\hline
\end{tabular}

Anmerkung: Wegen einer vollständigen Erklärung der Einzelheiten dieser Tabelle vgl. PISA 2012 Technical Report (OECD, erscheint demnächst). Die in Spalte 2 angegebene Zahl der nationalen Gesamtpopulation der 15-jährigen Schüler kann auf Grund unterschiedlicher Datenquellen in einzelnen Fällen größer sein als die Gesamtzahl der 15-lährigen in Spalte 1.

Informationen zu den Regionen, deren Stichproben international überprüft wurden, sind online verfügbar.

$*$ Vgl. Anmerkungen am Anfang dieses Anhangs.

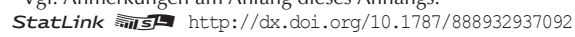

\begin{tabular}{ll|l|l} 
EXZELLENZ DURCH CHANCENGERECHTIGKEIT: ALLEN SCHÜLERINNEN UND SCHÜLERN DIEVORAUSSETZUNGEN ZUM ERFOLG SICHERN - BAND II @ OECD 2014 & 161
\end{tabular} 
[Teil 1/1]

Tabelle A2.2 Ausschlüsse

\begin{tabular}{|c|c|c|c|c|c|c|c|c|c|c|c|c|c|}
\hline & & \multicolumn{6}{|c|}{ Zahl der ausgeschlossenen Schüler (ungewichtet) } & \multicolumn{6}{|c|}{ Zahl der ausgeschlossenen Schüler (gewichtet) } \\
\hline & & $\begin{array}{l}\text { Schüler } \\
\text { mit } \\
\text { funktion. } \\
\text { Behind. } \\
\text { (Code 1) }\end{array}$ & $\begin{array}{c}\text { Schüler } \\
\text { mit kognit. } \\
\text { Behind. } \\
\text { (Code 2) }\end{array}$ & $\begin{array}{c}\text { Schüler } \\
\text { mit } \\
\text { Sprach- } \\
\text { problemen } \\
\text { (Code 3) }\end{array}$ & $\begin{array}{c}\text { Aus } \\
\text { anderen } \\
\text { Gründen } \\
\text { ausge- } \\
\text { schloss. } \\
\text { Schüler } \\
\text { (Code 4) }\end{array}$ & $\begin{array}{c}\text { Mangels } \\
\text { verfügbarer } \\
\text { Unterlagen } \\
\text { in der } \\
\text { Unterrichts- } \\
\text { sprache } \\
\text { ausgeschloss. } \\
\text { Schüler } \\
\text { (Code 5) }\end{array}$ & $\begin{array}{l}\text { Ausge- } \\
\text { schloss. } \\
\text { Schüler } \\
\text { insg. }\end{array}$ & $\begin{array}{c}\text { Schüler mit } \\
\text { funktion. } \\
\text { Behind. } \\
\text { (Code 1) }\end{array}$ & $\begin{array}{l}\text { Schüler } \\
\text { mit kognit. } \\
\text { Behind. } \\
\text { (Code 2) }\end{array}$ & $\begin{array}{c}\text { Schüler mit } \\
\text { Sprach- } \\
\text { problemen } \\
\text { (Code 3) }\end{array}$ & $\begin{array}{c}\text { Aus } \\
\text { anderen } \\
\text { Gründen } \\
\text { ausge- } \\
\text { schloss. } \\
\text { Schüler } \\
\text { (Code 4) }\end{array}$ & \begin{tabular}{|c|} 
Mangels \\
verfügbarer \\
Unterlagen \\
in der \\
Unterrichts- \\
sprache \\
ausgeschloss. \\
Schüler \\
(Code 5)
\end{tabular} & $\begin{array}{c}\text { Ausge- } \\
\text { schloss. } \\
\text { Schüler } \\
\text { insg. }\end{array}$ \\
\hline & & (1) & $(2)$ & (3) & (4) & (5) & (6) & (7) & (8) & (9) & (10) & (11) & (12) \\
\hline \multirow{34}{*}{ 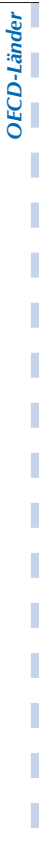 } & Australien & 39 & 395 & 71 & 0 & 0 & 505 & 471 & 3925 & 886 & 0 & 0 & 5282 \\
\hline & Österreich & 11 & 24 & 11 & 0 & 0 & 46 & 332 & 438 & 241 & 0 & 0 & 1011 \\
\hline & Belgien & 5 & 22 & 12 & 0 & 0 & 39 & 24 & 154 & 189 & 0 & 0 & 367 \\
\hline & Kanada & 82 & 1593 & 121 & 0 & 0 & 1796 & 981 & 18682 & 1350 & 0 & 0 & 21013 \\
\hline & Chile & 3 & 15 & 0 & 0 & 0 & 18 & 74 & 474 & 0 & 0 & 0 & 548 \\
\hline & Tschech. Rep. & 1 & 8 & 6 & 0 & 0 & 15 & 1 & 84 & 34 & 0 & 0 & 118 \\
\hline & Dänemark & 10 & 204 & 112 & 42 & 0 & 368 & 44 & 1469 & 559 & 310 & 0 & 2381 \\
\hline & Estland & 7 & 134 & 2 & 0 & 0 & 143 & 14 & 260 & 3 & 0 & 0 & 277 \\
\hline & Finnland & 5 & 80 & 101 & 15 & 24 & 225 & 43 & 363 & 166 & 47 & 35 & 653 \\
\hline & Frankreich & 52 & 0 & 0 & 0 & 0 & 52 & 5828 & 0 & 0 & 0 & 0 & 5828 \\
\hline & Deutschland & 0 & 4 & 4 & 0 & 0 & 8 & 0 & 705 & 597 & 0 & 0 & 1302 \\
\hline & Griechenland & 3 & 18 & 4 & 111 & 0 & 136 & 49 & 348 & 91 & 1816 & 0 & 2304 \\
\hline & Ungarn & 1 & 15 & 2 & 9 & 0 & 27 & 36 & 568 & 27 & 296 & 0 & 928 \\
\hline & Island & 5 & 105 & 27 & 18 & 0 & 155 & 5 & 105 & 27 & 18 & 0 & 156 \\
\hline & Irland & 13 & 159 & 33 & 66 & 0 & 271 & 121 & 1521 & 283 & 599 & 0 & 2524 \\
\hline & Israel & 9 & 91 & 14 & 0 & 0 & 114 & 133 & 1492 & 260 & 0 & 0 & 1884 \\
\hline & Italien & 64 & 566 & 111 & 0 & 0 & 741 & 596 & 7899 & 1361 & 0 & 0 & 9855 \\
\hline & Japan & 0 & 0 & 0 & 0 & 0 & 0 & 0 & 0 & 0 & 0 & 0 & 0 \\
\hline & Luxemburg & 6 & 261 & 90 & 0 & 0 & 357 & 6 & 261 & 90 & 0 & 0 & 357 \\
\hline & Mexiko & 21 & 36 & 1 & 0 & 0 & 58 & 812 & 2390 & 45 & 0 & 0 & 3247 \\
\hline & Niederlande & 5 & 21 & 1 & 0 & 0 & 27 & 188 & 819 & 50 & 0 & 0 & 1056 \\
\hline & Neuseeland & 27 & 118 & 99 & 0 & 11 & 255 & 235 & 926 & 813 & 0 & 57 & 2030 \\
\hline & Norwegen & 11 & 192 & 75 & 0 & 0 & 278 & 120 & 2180 & 832 & 0 & 0 & 3133 \\
\hline & Polen & 23 & 89 & 6 & 88 & 6 & 212 & 1470 & 5187 & 177 & 4644 & 89 & 11566 \\
\hline & Portugal & 69 & 48 & 7 & 0 & 0 & 124 & 860 & 605 & 94 & 0 & 0 & 1560 \\
\hline & Korea & 2 & 15 & 0 & 0 & 0 & 17 & 223 & 2015 & 0 & 0 & 0 & 2238 \\
\hline & Slowak. Rep. & 2 & 14 & 0 & 13 & 0 & 29 & 22 & 135 & 0 & 89 & 0 & 246 \\
\hline & Slowenien & 13 & 27 & 44 & 0 & 0 & 84 & 23 & 76 & 81 & 0 & 0 & 181 \\
\hline & Spanien & 56 & 679 & 224 & 0 & 0 & 959 & 618 & 11330 & 2984 & 0 & 0 & 14931 \\
\hline & Schweden & 120 & 0 & 81 & 0 & 0 & 201 & 2218 & 0 & 1571 & 0 & 0 & 3789 \\
\hline & Schweiz & 7 & 99 & 150 & 0 & 0 & 256 & 41 & 346 & 706 & 0 & 0 & 1093 \\
\hline & Türkei & 5 & 14 & 2 & 0 & 0 & 21 & 757 & 2556 & 371 & 0 & 0 & 3684 \\
\hline & Ver. Königreich & 40 & 405 & 41 & 0 & 0 & 486 & 1468 & 15514 & 3191 & 0 & 0 & 20173 \\
\hline & Ver. Staaten & 37 & 219 & 63 & 0 & 0 & 319 & 18399 & 113965 & 29830 & 0 & 0 & 162194 \\
\hline \multirow{31}{*}{ 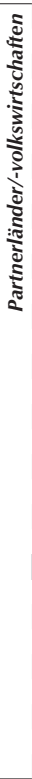 } & Albanien & 0 & 0 & 1 & 0 & 0 & 1 & 0 & 0 & 10 & 0 & 0 & 10 \\
\hline & Argentinien & 1 & 11 & 0 & 0 & 0 & 12 & 84 & 557 & 0 & 0 & 0 & 641 \\
\hline & Brasilien & 17 & 27 & 0 & 0 & 0 & 44 & 1792 & 3108 & 0 & 0 & 0 & 4900 \\
\hline & Bulgarien & 6 & 0 & 0 & 0 & 0 & 6 & 80 & 0 & 0 & 0 & 0 & 80 \\
\hline & Kolumbien & 12 & 10 & 1 & 0 & 0 & 23 & 397 & 378 & 14 & 0 & 0 & 789 \\
\hline & Costa Rica & 0 & 2 & 0 & 0 & 0 & 2 & 0 & 12 & 0 & 0 & 0 & 12 \\
\hline & Kroatien & 10 & 78 & 3 & 0 & 0 & 91 & 69 & 539 & 19 & 0 & 0 & 627 \\
\hline & Zypern* & 8 & 54 & 60 & 35 & 0 & 157 & 9 & 64 & 72 & 55 & 0 & 200 \\
\hline & Hongkong (China) & 4 & 33 & 1 & 0 & 0 & 38 & 57 & 446 & 15 & 0 & 0 & 518 \\
\hline & Indonesien & 1 & 0 & 1 & 0 & 0 & 2 & 426 & 0 & 434 & 0 & 0 & 860 \\
\hline & Jordanien & 8 & 6 & 5 & 0 & 0 & 19 & 109 & 72 & 122 & 0 & 0 & 304 \\
\hline & Kasachstan & 9 & 16 & 0 & 0 & 0 & 25 & 317 & 634 & 0 & 0 & 0 & 951 \\
\hline & Lettland & 3 & 7 & 4 & 0 & 0 & 14 & 8 & 45 & 24 & 0 & 0 & 76 \\
\hline & Liechtenstein & 1 & 7 & 5 & 0 & 0 & 13 & 1 & 7 & 5 & 0 & 0 & 13 \\
\hline & Litauen & 10 & 120 & 0 & 0 & 0 & 130 & 66 & 801 & 0 & 0 & 0 & 867 \\
\hline & Macau (China) & 0 & 1 & 2 & 0 & 0 & 3 & 0 & 1 & 2 & 0 & 0 & 3 \\
\hline & Malaysia & 3 & 4 & 0 & 0 & 0 & 7 & 274 & 279 & 0 & 0 & 0 & 554 \\
\hline & Montenegro & 3 & 1 & 0 & 0 & 0 & 4 & 7 & 1 & 0 & 0 & 0 & 8 \\
\hline & Peru & 3 & 5 & 0 & 0 & 0 & 8 & 269 & 280 & 0 & 0 & 0 & 549 \\
\hline & Katar & 23 & 43 & 19 & 0 & 0 & 85 & 23 & 43 & 19 & 0 & 0 & 85 \\
\hline & Rumänien & 0 & 0 & 0 & 0 & 0 & 0 & 0 & 0 & 0 & 0 & 0 & 0 \\
\hline & Russ. Föderation & 25 & 40 & 4 & 0 & 0 & 69 & 4345 & 6934 & 660 & 0 & 0 & 11940 \\
\hline & Serbien & 4 & 4 & 2 & 0 & 0 & 10 & 53 & 55 & 28 & 0 & 0 & 136 \\
\hline & Shanghai (China) & 1 & 6 & 1 & 0 & 0 & 8 & 14 & 80 & 14 & 0 & 0 & 107 \\
\hline & Singapur & 5 & 17 & 11 & 0 & 0 & 33 & 50 & 157 & 109 & 0 & 0 & 315 \\
\hline & Chinesisch Taipeh & 6 & 36 & 2 & 0 & 0 & 44 & 296 & 1664 & 70 & 0 & 0 & 2029 \\
\hline & Thailand & 2 & 10 & 0 & 0 & 0 & 12 & 13 & 1131 & 0 & 0 & 0 & 1144 \\
\hline & Tunesien & 4 & 1 & 0 & 0 & 0 & 5 & 104 & 26 & 0 & 0 & 0 & 130 \\
\hline & Ver. Arab. Emirate & 3 & 7 & 1 & 0 & 0 & 11 & 26 & 9 & 2 & 0 & 0 & 37 \\
\hline & Uruguay & 9 & 6 & 0 & 0 & 0 & 15 & 66 & 33 & 0 & 0 & 0 & 99 \\
\hline & Vietnam & 0 & 1 & 0 & 0 & 0 & 1 & 0 & 198 & 0 & 0 & 0 & 198 \\
\hline
\end{tabular}

Ausschlusscodes:

Code 1 Funktionale Behinderung - die Schülerin/der Schüler hat eine mittelschwere bis schwere dauerhafte körperliche Behinderung.

Code 2 Kognitive Behinderung - die Schülerin/der Schüler hat eine mentale oder emotionale Behinderung und wurde nach entsprechenden Tests bzw. nach der professionellen Meinung qualifizierter Kräfte als kognitiv retardiert eingestuft.

Code 3 Unzureichende Kenntnis der Testsprache - keine der Testsprachen des jeweiligen Landes ist die Muttersprache der Schülerin/des Schülers und die Schülerin/der Schüler lebt seit weniger als einem Jahr im betreffenden Land.

Code 4 Sonstige - von den nationalen Zentren definierte und dem internationalen Konsortium genehmigte Ausschlussgründe.

Code 5 Keine Unterlagen in der Unterrichtssprache verfügbar.

Anmerkung: Wegen einer ausführlicheren Erklärung der in dieser Tabelle enthaltenen Einzelheiten vgl. PISA 2012 Technical Report (OECD, erscheint demnächst).

Informationen zu den Regionen, deren Stichproben international überprüft wurden, sind online verfügbar.

*Vgl. Anmerkungen am Anfang dieses Anhangs.

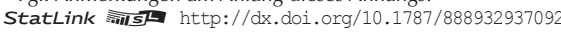


mussten in der Stichprobendokumentation aufgeführt sein; dazu musste eine Liste erstellt werden, in der die Gründe für ihren Ausschluss anzugeben waren. Spalte 9 informiert über die Gesamtzahl der ausgeschlossenen Schülerinnen und Schüler, die in Tabelle A2.2 näher beschrieben und in spezifische Kategorien unterteilt sind.

- Der in Spalte 10 angegebene Wert entspricht der gewichteten Zahl der ausgeschlossenen Schülerinnen und Schüler, d.h. der Gesamtzahl der Schüler in der definierten Grundgesamtheit auf Länderebene, die durch die Zahl der aus der Stichprobe ausgeschlossenen Schülerinnen und Schüler repräsentiert wird, die ebenfalls in Tabelle A2.2 näher beschrieben und in verschiedene Kategorien unterteilt sind. Der Ausschluss der Schülerinnen und Schüler erfolgte auf der Basis von fünf Kategorien: a) Schülerinnen und Schüler mit kognitiver Behinderung, d.h. die eine mentale oder emotionale Behinderung aufweisen und kognitiv retardiert sind, so dass sie nicht in der Lage sind, an einer Erhebung unter den PISA-Testbedingungen teilzunehmen; $b$ ) Schülerinnen und Schüler mit funktionaler Behinderung, d.h. die an einer mittelschweren bis schweren dauerhaften körperlichen Behinderung leiden, so dass sie nicht in der Lage sind, an einer Erhebung unter den PISA-Testbedingungen teilzunehmen; c) Schülerinnen und Schüler mit unzureichender Kenntnis der Testsprache, die keine der Testsprachen des Landes hinreichend lesen oder sprechen können und daher die Sprachbarriere in der Testsituation nicht hätten überwinden können (in der Regel können Schülerinnen und Schüler, die weniger als ein Jahr Unterricht in der Testsprache hatten, von der Erhebung ausgeschlossen werden); d) sonstige Schülerinnen und Schüler, eine Kategorie, die von den nationalen Zentren definiert und vom Internationalen Konsortium genehmigt werden muss; e) Schülerinnen und Schüler, die in dem Haupttestbereich in einer Unterrichtssprache unterrichtet werden, für die keine Unterlagen verfügbar waren.

- Spalte 11 zeigt den Prozentsatz der Schülerinnen und Schüler, die innerhalb der Schulen ausgeschlossen wurden. Er ist berechnet als die gewichtete Zahl der ausgeschlossenen Schülerinnen und Schüler (Spalte 10), dividiert durch die gewichtete Zahl der ausgeschlossenen und der teilnehmenden Schülerinnen und Schüler (Spalte 8 plus Spalte 10), multipliziert mit 100.

- Spalte 12 zeigt die Ausschlussrate insgesamt, die dem gewichteten Prozentsatz der angestrebten Grundgesamtheit auf Länderebene entspricht, der von PISA auf Schulebene oder auf innerschulischer Ebene von der Erhebung ausgeschlossen wurde. Sie wird wie folgt berechnet: Ausschlussrate auf Schulebene (Spalte 6 dividiert durch 100), zuzüglich Ausschlussrate innerhalb der Schulen (Spalte 11 dividiert durch 100), multipliziert mit 1, abzüglich der Ausschlussrate auf Schulebene (Spalte 6 dividiert durch 100). Dieses Ergebnis wird dann mit 100 multipliziert.

- Spalte 13 zeigt einen Index für den Erfassungsgrad der angestrebten Grundgesamtheit auf Länderebene durch die PISA-Stichprobe. Kanada, Dänemark, Estland, Luxemburg, Norwegen, Schweden, das Vereinigte Königreich und die Vereinigten Staaten waren die einzigen Länder, in denen der Erfassungsgrad unter 95\% lag.

- Spalte 14 zeigt einen Index für den Erfassungsgrad der 15-jährigen Schülerinnen und Schüler durch die PISA-Stichprobe. Dieser Index misst den Gesamtanteil der nationalen Schülerpopulation, der durch die nach den Ausschlüssen verbleibende Schülerstichprobe erfasst wird. Dabei werden Ausschlüsse sowohl auf Schulebene als auch auf Schülerebene berücksichtigt. Nahe bei 100 liegende Werte deuten darauf hin, dass die PISA-Stichprobe das gesamte Schulsystem nach Definition für PISA 2012 repräsentiert. Der Index ergibt sich aus der gewichteten Zahl der teilnehmenden Schülerinnen und Schüler (Spalte 8), dividiert durch die gewichtete Zahl der teilnehmenden und der ausgeschlossenen Schüler (Spalte 8 plus Spalte 10), multipliziert mit der definierten Grundgesamtheit auf Länderebene (Spalte 5), dividiert durch die in Betracht kommende Population (Spalte 2).

- Spalte 15 zeigt einen Index des prozentualen Anteils der erfassten Schülerpopulation. Dieser Index ergibt sich aus der gewichteten Gesamtzahl der teilnehmenden Schülerinnen und Schüler (Spalte 8), dividiert durch die Gesamtpopulation der 15-jährigen Schülerinnen und Schüler (Spalte 1).

Dieser hohe Erfassungsgrad trägt zur Vergleichbarkeit der Erhebungsergebnisse bei. Selbst wenn man z.B. annimmt, dass die ausgeschlossenen Schülerinnen und Schüler durchgehend schlechtere Ergebnisse erzielt hätten als die teilnehmenden und dass diese Korrelation mittelstark ausgeprägt ist, würde eine Ausschlussrate in einer Größenordnung von 5\% wahrscheinlich nur zu einer Überzeichnung der nationalen Durchschnittsergebnisse um weniger als 5 Punkte führen (auf einer Skala mit einem internationalen Mittelwert von 500 Punkten und einer Standardabweichung von 100 Punkten). Diese Feststellung basiert auf folgenden Berechnungen: Beträgt die Korrelation zwischen der Ausschlussneigung und den Schülerleistungen 0,3, sind die resultierenden Durchschnittsergebnisse bei einer Ausschlussrate von 1\% um 1 Punkt, von 5\% um 3 Punkte und von 10\% um 6 Punkte überzeichnet. Beträgt die Korrelation zwischen der Ausschlussneigung und den Schülerleistungen 0,5, sind die resultierenden Durchschnittsergebnisse bei einer Ausschlussrate von 1\% um 1 Punkt, von 5\% um 5 Punkte und von 10\% um 10 Punkte überzeichnet. Für diese Berechnungen wurde ein Modell verwendet, in dem von einer bivariaten Normalverteilung der Teilnahmeneigung und der Leistungen ausgegangen wurde. Wegen näherer Einzelheiten vgl. PISA 2012 Technical Report (OECD, erscheint demnächst).

\section{Stichprobenziehung und Beteiligungsquoten}

Die Genauigkeit jeglicher Untersuchungsergebnisse hängt von der Qualität der Informationen ab, auf denen die nationalen Stichproben basieren, sowie von den Stichprobenziehungsverfahren. Für PISA wurden Qualitätsstandards, Verfahren, Instrumente und Verifikationsmechanismen entwickelt, die gewährleisteten, dass die nationalen Stichproben vergleichbare Daten ergaben und die Ergebnisse ohne Vorbehalte miteinander verglichen werden können.

Die meisten PISA-Stichproben wurden nach dem Konzept der zweistufigen geschichteten Stichproben konzipiert (soweit die Länder unterschiedliche Stichprobenkonzepte anwandten, sind diese dokumentiert im PISA 2012 Technical Report, OECD, erscheint 
[Teil 1/2]

Tabelle A2.3 Beteiligungsquoten

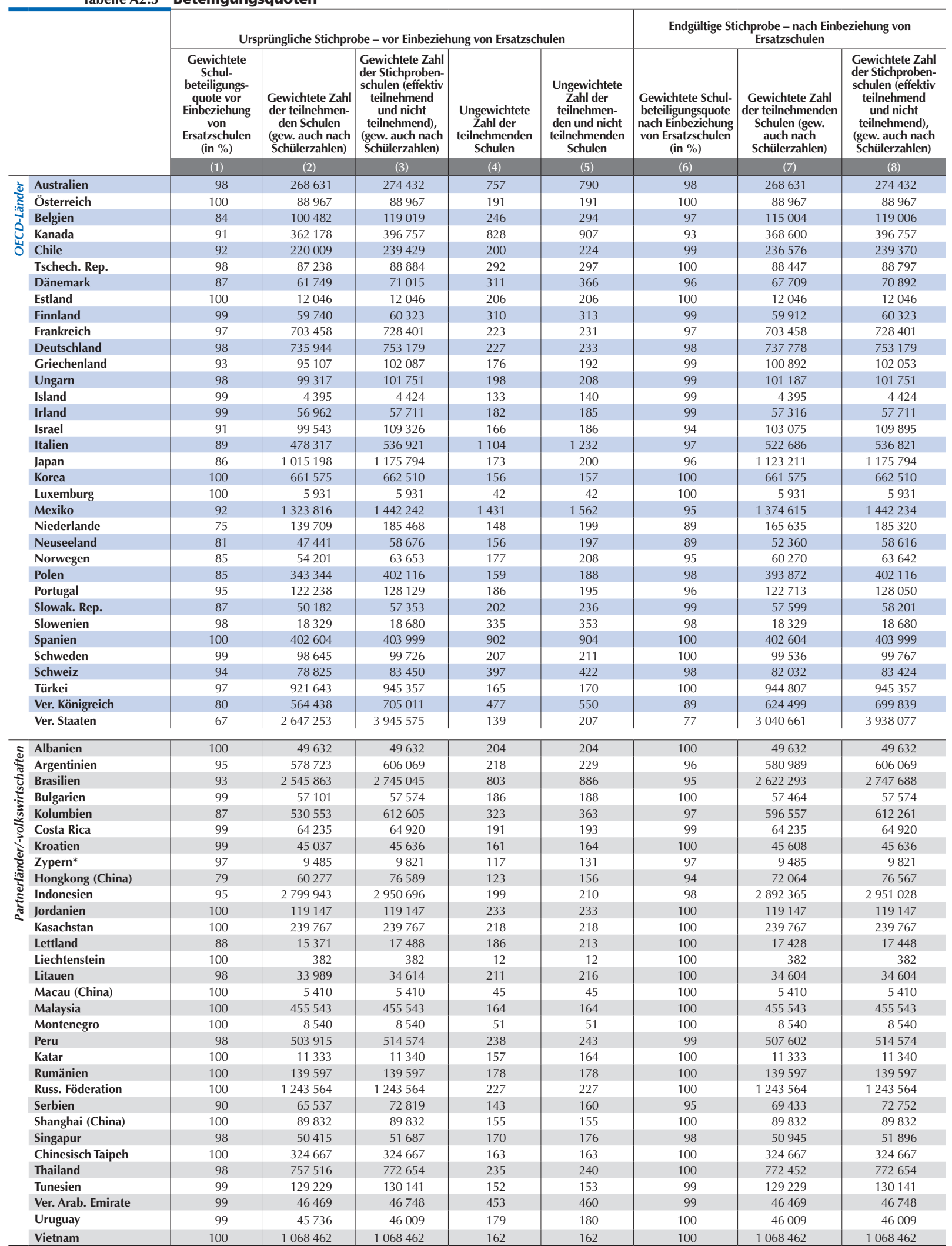

Informationen zu den Regionen, deren Stichproben international überprüft wurden, sind online verfügbar.

* Vgl. Anmerkungen am Anfang dieses Anhangs.

StatLink तinst http://dx.doi.org/10.1787/888932937092 
[Teil 2/2]

Tabelle A2.3 Beteiligungsquoten

\begin{tabular}{|c|c|c|c|c|c|c|c|c|}
\hline & & \multicolumn{2}{|c|}{$\begin{array}{c}\text { Endgültige Stichprobe - nach Einbeziehung } \\
\text { von Ersatzschulen }\end{array}$} & \multicolumn{5}{|c|}{ Endgültige Stichprobe - Schüler innerhalb der Schulen nach Einbeziehung von Ersatzschulen } \\
\hline & & $\begin{array}{l}\text { Ungewichtete Zahl } \\
\text { der teilnehmenden } \\
\text { Schulen }\end{array}$ & $\begin{array}{c}\text { Ungewichtete Zahl } \\
\text { der teilnehmenden } \\
\text { u. nicht teilnehmen- } \\
\text { den Schulen }\end{array}$ & $\begin{array}{c}\text { Gewichtete Schüler- } \\
\text { beteiligungsquote } \\
\text { nach Einbeziehung } \\
\text { von Ersatzschulen } \\
\text { (in \%) }\end{array}$ & $\begin{array}{c}\text { Gewichtete Zahl } \\
\text { der teilnehmenden } \\
\text { Schüler }\end{array}$ & $\begin{array}{c}\text { Gewichtete Zahl } \\
\text { der Stichproben- } \\
\text { schüler (effektiv } \\
\text { teilnehmend u. nicht } \\
\text { teilnehmend) }\end{array}$ & $\begin{array}{l}\text { Ungewichtete Zahl } \\
\text { der teilnehmenden } \\
\text { Schüler }\end{array}$ & $\begin{array}{c}\text { Ungewichtete } \\
\text { Zahl der Stich- } \\
\text { probenschüler } \\
\text { (effektiv teil- } \\
\text { nehmend u. nich } \\
\text { teilnehmend) }\end{array}$ \\
\hline & & (9) & $(10)$ & (11) & (12) & (13) & (14) & (15) \\
\hline \multirow{34}{*}{ 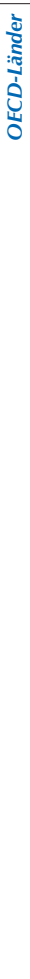 } & Australien & 757 & 790 & 87 & 213495 & 246012 & 17491 & 20799 \\
\hline & Österreich & 191 & 191 & 92 & 75393 & 82242 & 4756 & 5318 \\
\hline & Belgien & 282 & 294 & 91 & 103914 & 114360 & 9649 & 10595 \\
\hline & Kanada & 840 & 907 & 81 & 261928 & 324328 & 20994 & 25835 \\
\hline & Chile & 221 & 224 & 95 & 214558 & 226689 & 6857 & 7246 \\
\hline & Tschech. Rep. & 295 & 297 & 90 & 73536 & 81642 & 6528 & 7222 \\
\hline & Dänemark & 339 & 366 & 89 & 56096 & 62988 & 7463 & 8496 \\
\hline & Estland & 206 & 206 & 93 & 10807 & 11634 & 5867 & 6316 \\
\hline & Finnland & 311 & 313 & 91 & 54126 & 59653 & 8829 & 9789 \\
\hline & Frankreich & 223 & 231 & 89 & 605371 & 676730 & 5641 & 6308 \\
\hline & Deutschland & 228 & 233 & 93 & 692226 & 742416 & 4990 & 5355 \\
\hline & Griechenland & 188 & 192 & 97 & 92444 & 95580 & 5125 & 5301 \\
\hline & Ungarn & 204 & 208 & 93 & 84032 & 90652 & 4810 & 5184 \\
\hline & Island & 133 & 140 & 85 & 3503 & 4135 & 3503 & 4135 \\
\hline & Irland & 183 & 185 & 84 & 45115 & 53644 & 5016 & 5977 \\
\hline & Israel & 172 & 186 & 90 & 91181 & 101288 & 6061 & 6727 \\
\hline & Italien & 1186 & 1232 & 93 & 473104 & 510005 & 38084 & 41003 \\
\hline & Japan & 191 & 200 & 96 & 1034803 & 1076786 & 6351 & 6609 \\
\hline & Korea & 156 & 157 & 99 & 595461 & 603004 & 5033 & 5101 \\
\hline & Luxemburg & 42 & 42 & 95 & 5260 & 5523 & 5260 & 5523 \\
\hline & Mexiko & 1468 & 1562 & 94 & 1193866 & 1271639 & 33786 & 35972 \\
\hline & Niederlande & 177 & 199 & 85 & 148432 & 174697 & 4434 & 5215 \\
\hline & Neuseeland & 177 & 197 & 85 & 40397 & 47703 & 5248 & 6206 \\
\hline & Norwegen & 197 & 208 & 91 & 51155 & 56286 & 4686 & 5156 \\
\hline & Polen & 182 & 188 & 88 & 325389 & 371434 & 5629 & 6452 \\
\hline & Portugal & 187 & 195 & 87 & 80719 & 92395 & 5608 & 6426 \\
\hline & Slowak. Rep. & 231 & 236 & 94 & 50544 & 53912 & 5737 & 6106 \\
\hline & Slowenien & 335 & 353 & 90 & 16146 & 17849 & 7211 & 7921 \\
\hline & Spanien & 902 & 904 & 90 & 334382 & 372042 & 26443 & 29027 \\
\hline & Schweden & 209 & 211 & 92 & 87359 & 94784 & 4739 & 5141 \\
\hline & Schweiz & 410 & 422 & 92 & 72116 & 78424 & 11218 & 12138 \\
\hline & Türkei & 169 & 170 & 98 & 850830 & 866269 & 4847 & 4939 \\
\hline & Ver. Königreich & 505 & 550 & 86 & 528231 & 613736 & 12638 & 14649 \\
\hline & Ver. Staaten & 161 & 207 & 89 & 2429718 & 2734268 & 6094 & 6848 \\
\hline \multirow{31}{*}{ 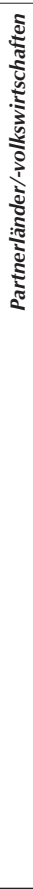 } & Albanien & 204 & 204 & 92 & 39275 & 42466 & 4743 & 5102 \\
\hline & Argentinien & 219 & 229 & 88 & 457294 & 519733 & 5804 & 6680 \\
\hline & Brasilien & 837 & 886 & 90 & 2133035 & 2368438 & 19877 & 22326 \\
\hline & Bulgarien & 187 & 188 & 96 & 51819 & 54145 & 5280 & 5508 \\
\hline & Kolumbien & 352 & 363 & 93 & 507178 & 544862 & 11164 & 12045 \\
\hline & Costa Rica & 191 & 193 & 89 & 35525 & 39930 & 4582 & 5187 \\
\hline & Kroatien & 163 & 164 & 92 & 41912 & 45473 & 6153 & 6675 \\
\hline & Zypern* & 117 & 131 & 93 & 8719 & 9344 & 5078 & 5458 \\
\hline & Hongkong (China) & 147 & 156 & 93 & 62059 & 66665 & 4659 & 5004 \\
\hline & Indonesien & 206 & 210 & 95 & 2478961 & 2605254 & 5579 & 5885 \\
\hline & Jordanien & 233 & 233 & 95 & 105493 & 111098 & 7038 & 7402 \\
\hline & Kasachstan & 218 & 218 & 99 & 206053 & 208411 & 5808 & 5874 \\
\hline & Lettland & 211 & 213 & 91 & 14579 & 16039 & 5276 & 5785 \\
\hline & Liechtenstein & 12 & 12 & 93 & 293 & 314 & 293 & 314 \\
\hline & Litauen & 216 & 216 & 92 & 30429 & 33042 & 4618 & 5018 \\
\hline & Macau (China) & 45 & 45 & 99 & 5335 & 5366 & 5335 & 5366 \\
\hline & Malaysia & 164 & 164 & 94 & 405983 & 432080 & 5197 & 5529 \\
\hline & Montenegro & 51 & 51 & 94 & 7233 & 7714 & 4799 & 5117 \\
\hline & Peru & 240 & 243 & 96 & 398193 & 414728 & 6035 & 6291 \\
\hline & Katar & 157 & 164 & 100 & 10966 & 10996 & 10966 & 10996 \\
\hline & Rumänien & 178 & 178 & 98 & 137860 & 140915 & 5074 & 5188 \\
\hline & Russ. Föderation & 227 & 227 & 97 & 1141317 & 1172539 & 6418 & 6602 \\
\hline & Serbien & 152 & 160 & 93 & 60366 & 64658 & 4681 & 5017 \\
\hline & Shanghai (China) & 155 & 155 & 98 & 83821 & 85127 & 6374 & 6467 \\
\hline & Singapur & 172 & 176 & 94 & 47465 & 50330 & 5546 & 5887 \\
\hline & Chinesisch Taipeh & 163 & 163 & 96 & 281799 & 292542 & 6046 & 6279 \\
\hline & Thailand & 239 & 240 & 99 & 695088 & 702818 & 6606 & 6681 \\
\hline & Tunesien & 152 & 153 & 90 & 108342 & 119917 & 4391 & 4857 \\
\hline & Ver. Arab. Emirate & 453 & 460 & 95 & 38228 & 40384 & 11460 & 12148 \\
\hline & Uruguay & 180 & 180 & 90 & 35800 & 39771 & 5315 & 5904 \\
\hline & Vietnam & 162 & 162 & 100 & 955222 & 956517 & 4959 & 4966 \\
\hline
\end{tabular}

Informationen zu den Regionen, deren Stichproben international überprüft wurden, sind online verfügbar.

*Vgl. Anmerkungen am Anfang dieses Anhangs.
StatLink *ilsh http://dx.doi.org/10.1787/888932937092 
demnächst). Im ersten Schritt wurde eine Stichprobe einzelner Schulen gezogen, die von 15-jährigen Schülerinnen und Schülern besucht wurden. Die Schulstichprobe wurde systematisch gezogen, mit Wahrscheinlichkeiten proportional zur Größe, die wiederum in Abhängigkeit zur geschätzten Zahl der in Betracht kommenden (15-jährigen) Schülerinnen und Schüler stand. In jedem Land wurden mindestens 150 Schulen (sofern vorhanden) ausgewählt, wobei die Anforderungen für die nationalen Analysen allerdings häufig eine etwas größere Stichprobe bedingten. Gleichzeitig mit der Stichprobenziehung wurden Ersatzschulen ermittelt für den Fall, dass eine der ausgewählten Schulen nicht an der PISA-2012-Erhebung teilnehmen wollte.

In Island, Katar, Liechtenstein, Luxemburg und Macau (China) wurden alle Schulen und alle innerhalb der Schulen in Betracht kommenden Schülerinnen und Schüler in die Stichprobe aufgenommen.

Experten des PISA-Konsortiums führten in den meisten Teilnehmerländern die Auswahl der Stichproben durch; in den Ländern, die ihre Stichproben selbst auswählten, überwachten sie diesen Prozess. In der zweiten Phase des Auswahlprozesses wurden Schülerstichproben innerhalb der für die Stichprobe ermittelten Schulen gezogen. Nach der Auswahl der Schulen wurde eine Liste aller 15-jährigen Schülerinnen und Schüler an den für die Stichprobe gezogenen Schulen erstellt. Aus dieser Liste wurden 35 Schülerinnen und Schüler mit gleicher Wahrscheinlichkeit ausgewählt (wenn es weniger als 35 gab, wurden alle 15-jährigen Schülerinnen und Schüler ausgewählt). Die Zahl der für die Stichproben ausgewählten Schülerinnen und Schüler je Schule konnte von 35 abweichen, durfte jedoch 20 nicht unterschreiten.

Die Qualitätsstandards der PISA-Daten setzten eine Mindestteilnahmequote sowohl für die Schulen als auch für die Schülerinnen und Schüler voraus. Diese Standards wurden aufgestellt, um mögliche beteiligungsbedingte Verzerrungen zu minimieren. Bei den Ländern, die diese Standards erfüllten, dürften etwaige Verzerrungen infolge von Nichtbeteiligung unerheblich, d.h. in der Regel geringer als der Stichprobenfehler sein.

Für die ursprünglich ausgewählten Schulen war eine Mindestbeteiligungsquote von $85 \%$ erforderlich. Wenn die ursprüngliche Beteiligungsquote auf Schulebene zwischen 65\% und 85\% lag, konnte durch die Einbeziehung von Ersatzschulen immer noch eine akzeptable Quote erzielt werden. Dieses Verfahren war mit dem Risiko eines erhöhten Beteiligungsbias verbunden. Daher wurden die Teilnehmerländer ersucht, so viele der ursprünglich für die Stichprobe gezogenen Schulen wie möglich zur Teilnahme zu veranlassen. Wenn die Beteiligungsquote der Schülerinnen und Schüler zwischen 25\% und 50\% lag, galt die betreffende Schule nicht als teilnehmende Schule, die diesbezüglichen Daten wurden aber in die Datenbank aufgenommen und bei den verschiedenen Schätzungen berücksichtigt. Daten von Schulen mit einer Schülerbeteiligungsquote von weniger als $25 \%$ wurden nicht in die Datenbank aufgenommen.

Für PISA 2012 wurde auch eine Mindestschülerbeteiligungsquote von 80\% innerhalb der teilnehmenden Schulen festgesetzt. Diese Mindestquote musste auf Landesebene und nicht zwangsläufig von jeder teilnehmenden Schule erreicht werden. Folgesitzungen waren in Schulen erforderlich, in denen zu wenig Schülerinnen und Schüler an der ersten Testsitzung teilgenommen hatten. Die Schülerbeteiligungsquoten wurden für alle ursprünglich ausgewählten Schulen sowie für alle Schulen, d.h. die ursprünglich ausgewählten und die Ersatzschulen, auf der Basis der Teilnahme der Schülerinnen und Schüler an der ersten Testsitzung wie auch an etwaigen Folgesitzungen errechnet. Als teilnehmende Schüler galten Schüler, die bei der ersten Testsitzung oder einer Folgesitzung anwesend waren. Schüler, die sich nur an der Beantwortung der Fragebogen beteiligt hatten, wurden in die internationale Datenbank aufgenommen und bei den Statistiken in dieser Veröffentlichung berücksichtigt, wenn sie zumindest Angaben zur beruflichen Tätigkeit des Vaters oder der Mutter gemacht hatten.

Tabelle A2.3 gibt Auskunft über die Beteiligungsquoten auf Schüler- und auf Schulebene, vor und nach Einbeziehung von Ersatzschulen.

- Spalte 1 zeigt die gewichtete Beteiligungsquote der Schulen vor Einbeziehung von Ersatzschulen. Diese wird durch Division von Spalte 2 durch Spalte 3 ermittelt.

- Spalte 2 zeigt die gewichtete Zahl der teilnehmenden Schulen vor Einbeziehung von Ersatzschulen (gewichtet nach der Schülerzahl).

- Spalte 3 zeigt die gewichtete Zahl der für die Stichprobe gezogenen Schulen vor Einbeziehung von Ersatzschulen (sowohl effektiv teilnehmende als auch nicht teilnehmende Schulen, gewichtet nach der Schülerzahl).

- Spalte 4 zeigt die ungewichtete Zahl der teilnehmenden Schulen vor Einbeziehung von Ersatzschulen.

- Spalte 5 zeigt die ungewichtete Zahl der teilnehmenden und nicht teilnehmenden Schulen vor Einbeziehung von Ersatzschulen.

- Spalte 6 zeigt die gewichtete Schulbeteiligungsquote nach Einbeziehung von Ersatzschulen. Diese wird durch Division von Spalte 7 durch Spalte 8 ermittelt.

- Spalte 7 zeigt die gewichtete Zahl der teilnehmenden Schulen nach Einbeziehung von Ersatzschulen (gewichtet nach der Schülerzahl).

- Spalte 8 zeigt die gewichtete Zahl der für die Stichprobe gezogenen Schulen nach Einbeziehung von Ersatzschulen (sowohl effektiv teilnehmende als auch nicht teilnehmende Schulen, gewichtet nach der Schülerzahl).

- Spalte 9 zeigt die ungewichtete Zahl der teilnehmenden Schulen nach Einbeziehung von Ersatzschulen.

- Spalte 10 zeigt die ungewichtete Zahl der teilnehmenden und nicht teilnehmenden Schulen nach Einbeziehung von Ersatzschulen. 
- Spalte 11 zeigt die gewichtete Schülerbeteiligungsquote nach Einbeziehung von Ersatzschulen. Diese wird durch Division von Spalte 12 durch Spalte 13 ermittelt.

- Spalte 12 zeigt die gewichtete Zahl der teilnehmenden Schülerinnen und Schüler.

- Spalte 13 zeigt die gewichtete Zahl der Stichprobenschüler (am Test teilnehmende und nicht teilnehmende Schülerinnen und Schüler).

- Spalte 14 zeigt die ungewichtete Zahl der teilnehmenden Schülerinnen und Schüler. Zu beachten ist, dass Schüler von Schulen mit einer Schülerbeteiligungsquote von weniger als 50\% nicht in die (gewichteten und ungewichteten) Quoten einbezogen wurden.

- Spalte 15 zeigt die ungewichtete Zahl der Stichprobenschüler (am Test teilnehmende und nicht teilnehmende Schülerinnen und Schüler). Zu beachten ist, dass Schülerinnen und Schüler von Schulen, in denen weniger als die Hälfte der in Betracht kommenden Schüler am Test teilgenommen hat, nicht in den (gewichteten und ungewichteten) Quoten berücksichtigt wurden.

\section{Definition der Schulen}

In einigen Ländern wurden anstelle von Schulen Untereinheiten von Schulen für die Stichprobe herangezogen, was die Schätzung der Anteile der zwischenschulischen Varianz beeinflussen kann. In Deutschland, Japan, Österreich, Rumänien, Slowenien, der Tschechischen Republik und Ungarn wurden Schulen mit mehr als einem Bildungsgang in Einheiten unterteilt, die den jeweiligen Bildungsgängen entsprechen. In den Niederlanden wurden Schulen, die Sekundarstufe I und II umfassen, in die jeweiligen Stufen unterteilt. Was Belgien betrifft, so wurden in der Flämischen Gemeinschaft bei auf mehrere Standorte verteilten Schulen die verschiedenen Einzeleinrichtungen in die Stichprobe einbezogen, während in der französischen Gemeinschaft im Fall solcher Schulen die jeweils größere Verwaltungseinheit berücksichtigt wurde. In Australien wurden im Fall von Schulen mit mehr als einem Standort die verschiedenen Einzeleinrichtungen erfasst. In Argentinien, Dubai (Vereinigte Arabische Emirate) und Kroatien wurden im Fall solcher Schulen die einzelnen Standorte berücksichtigt. In Spanien wurden die Schulen im Baskenland, die Mehrsprachenmodelle anbieten, für die Stichprobenziehung in die einzelnen Programme unterteilt.

\section{Klassenstufen}

Die Schülerinnen und Schüler, die an den Tests von PISA 2012 teilgenommen haben, besuchen unterschiedliche Klassenstufen. Der Anteil der Schülerinnen und Schüler in den einzelnen Klassenstufen ist in Tabelle A2.4a nach Ländern geordnet aufgeführt und in Tabelle A2.4b für die einzelnen Länder nach Geschlecht aufgeschlüsselt. 
[Teil 1/1]

Tabelle A2.4a Prozentsatz der Schüler in den einzelnen Klassenstufen

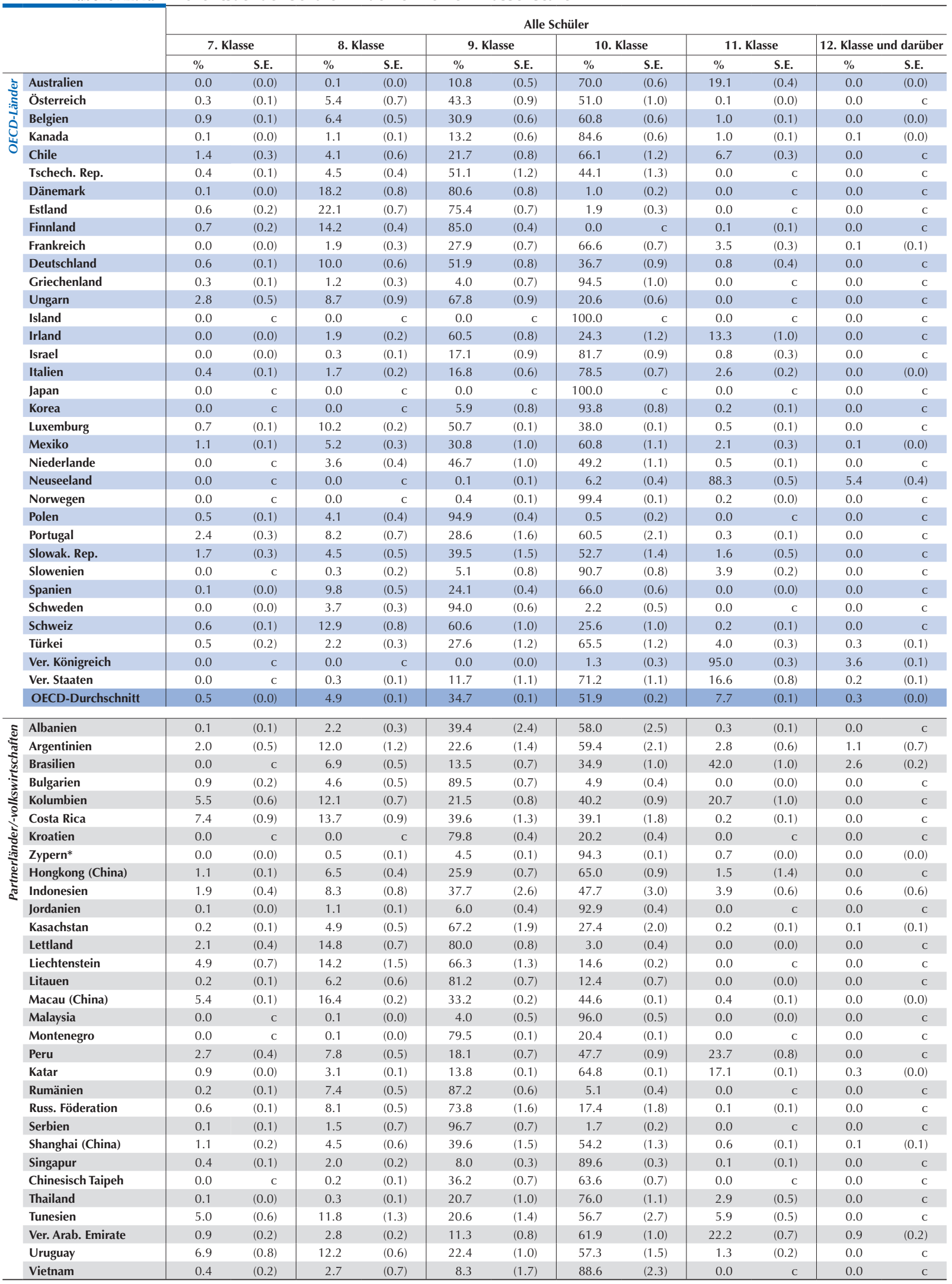

Informationen zu den Regionen, deren Stichproben international überprüft wurden, sind online verfügbar.

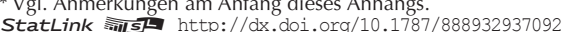


[Teil 1/2]

Tabelle A2.4b Prozentsatz der Schüler in den einzelnen Klassenstufen, nach Geschlecht

\begin{tabular}{|c|c|c|c|c|c|c|c|c|c|c|c|c|c|}
\hline & & \multicolumn{12}{|c|}{ Jungen } \\
\hline & & \multicolumn{2}{|c|}{ 7. Klasse } & \multicolumn{2}{|c|}{ 8. Klasse } & \multicolumn{2}{|c|}{ 9. Klasse } & \multicolumn{2}{|c|}{ 10. Klasse } & \multicolumn{2}{|c|}{ 11. Klasse } & \multicolumn{2}{|c|}{ 12. Klasse und darüber } \\
\hline & & $\%$ & S.E. & $\%$ & S.E. & $\%$ & S.E. & $\%$ & S.E. & $\%$ & S.E. & $\%$ & S.E. \\
\hline \multirow{35}{*}{ 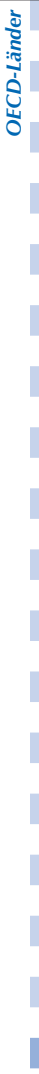 } & Australien & 0.0 & $\mathrm{c}$ & 0.1 & $(0.0)$ & 13.1 & $(0.9)$ & 69.2 & $(0.9)$ & 17.5 & $(0.6)$ & 0.0 & $(0.0)$ \\
\hline & Österreich & 0.3 & $(0.1)$ & 6.0 & $(0.9)$ & 44.8 & (1.4) & 48.9 & (1.5) & 0.0 & c & 0.0 & c \\
\hline & Belgien & 1.0 & $(0.1)$ & 7.1 & $(0.6)$ & 33.8 & $(0.9)$ & 57.1 & $(1.0)$ & 1.0 & $(0.2)$ & 0.0 & $(0.0)$ \\
\hline & Kanada & 0.1 & $(0.1)$ & 1.3 & $(0.2)$ & 14.8 & $(0.8)$ & 82.7 & $(0.8)$ & 0.9 & $(0.1)$ & 0.1 & $(0.1)$ \\
\hline & Chile & 1.4 & $(0.4)$ & 5.0 & $(0.9)$ & 24.2 & $(1.0)$ & 63.1 & $(1.6)$ & 6.4 & $(0.4)$ & 0.0 & c \\
\hline & Tschech. Rep. & 0.7 & $(0.2)$ & 5.5 & $(0.6)$ & 54.9 & $(2.0)$ & 39.0 & $(2.1)$ & 0.0 & c & 0.0 & c \\
\hline & Dänemark & 0.1 & $(0.0)$ & 23.4 & $(1.0)$ & 75.7 & $(1.0)$ & 0.8 & $(0.3)$ & 0.0 & c & 0.0 & c \\
\hline & Estland & 0.8 & $(0.3)$ & 25.7 & $(1.0)$ & 71.7 & $(1.1)$ & 1.7 & $(0.4)$ & 0.0 & c & 0.0 & c \\
\hline & Finnland & 0.9 & $(0.4)$ & 16.2 & $(0.6)$ & 82.8 & $(0.7)$ & 0.0 & c & 0.1 & $(0.1)$ & 0.0 & c \\
\hline & Frankreich & 0.1 & $(0.1)$ & 2.3 & $(0.4)$ & 30.8 & $(0.9)$ & 63.5 & $(1.0)$ & 3.2 & $(0.5)$ & 0.1 & $(0.1)$ \\
\hline & Deutschland & 0.9 & $(0.2)$ & 11.6 & $(0.7)$ & 53.6 & (1.1) & 33.2 & (1.2) & 0.7 & $(0.3)$ & 0.0 & c \\
\hline & Griechenland & 0.4 & $(0.2)$ & 1.8 & $(0.6)$ & 4.8 & $(1.0)$ & 93.0 & (1.4) & 0.0 & c & 0.0 & c \\
\hline & Ungarn & 3.9 & $(0.6)$ & 12.1 & (1.5) & 67.1 & (1.3) & 17.0 & $(0.8)$ & 0.0 & c & 0.0 & c \\
\hline & Island & 0.0 & c & 0.0 & c & 0.0 & c & 100.0 & C & 0.0 & c & 0.0 & c \\
\hline & Irland & 0.0 & c & 2.4 & $(0.3)$ & 63.6 & $(1.0)$ & 21.1 & (1.4) & 13.0 & (1.3) & 0.0 & c \\
\hline & Israel & 0.1 & $(0.1)$ & 0.3 & $(0.1)$ & 18.9 & $(1.3)$ & 79.6 & (1.3) & 1.2 & $(0.5)$ & 0.0 & c \\
\hline & Italien & 0.5 & $(0.2)$ & 2.1 & $(0.3)$ & 19.3 & $(0.7)$ & 75.8 & $(0.7)$ & 2.3 & $(0.2)$ & 0.0 & c \\
\hline & Japan & 0.0 & c & 0.0 & c & 0.0 & c & 100.0 & c & 0.0 & c & 0.0 & c \\
\hline & Korea & 0.0 & c & 0.0 & c & 6.4 & $(1.2)$ & 93.4 & $(1.2)$ & 0.2 & $(0.1)$ & 0.0 & c \\
\hline & Luxemburg & 0.7 & $(0.1)$ & 10.7 & $(0.2)$ & 51.1 & $(0.2)$ & 37.0 & $(0.2)$ & 0.6 & $(0.1)$ & 0.0 & c \\
\hline & Mexiko & 1.3 & $(0.2)$ & 6.3 & $(0.3)$ & 33.0 & (1.1) & 57.2 & (1.2) & 2.1 & $(0.5)$ & 0.0 & $(0.0)$ \\
\hline & Niederlande & 0.0 & c & 4.4 & $(0.6)$ & 49.5 & $(1.1)$ & 45.7 & $(1.2)$ & 0.4 & $(0.1)$ & 0.0 & c \\
\hline & Neuseeland & 0.0 & c & 0.0 & c & 0.2 & $(0.1)$ & 7.0 & $(0.5)$ & 88.0 & $(0.7)$ & 4.8 & $(0.5)$ \\
\hline & Norwegen & 0.0 & c & 0.0 & c & 0.6 & $(0.1)$ & 99.1 & $(0.1)$ & 0.3 & $(0.0)$ & 0.0 & c \\
\hline & Polen & 0.9 & $(0.2)$ & 5.7 & $(0.6)$ & 93.0 & $(0.6)$ & 0.4 & $(0.2)$ & 0.0 & c & 0.0 & c \\
\hline & Portugal & 2.6 & $(0.5)$ & 9.9 & $(0.9)$ & 30.1 & $(1.7)$ & 57.0 & $(2.2)$ & 0.4 & $(0.2)$ & 0.0 & c \\
\hline & Slowak. Rep. & 1.5 & $(0.3)$ & 5.4 & $(0.8)$ & 40.1 & $(2.0)$ & 51.5 & $(2.1)$ & 1.5 & $(0.5)$ & 0.0 & c \\
\hline & Slowenien & 0.0 & c & 0.4 & $(0.3)$ & 6.3 & $(1.0)$ & 90.2 & (1.0) & 3.1 & (0.4) & 0.0 & c \\
\hline & Spanien & 0.1 & $(0.1)$ & 11.8 & (0.6) & 25.8 & (0.6) & 62.2 & $(0.7)$ & 0.1 & $(0.1)$ & 0.0 & c \\
\hline & Schweden & 0.1 & $(0.1)$ & 4.6 & $(0.5)$ & 93.7 & $(0.8)$ & 1.7 & $(0.6)$ & 0.0 & c & 0.0 & c \\
\hline & Schweiz & 0.5 & $(0.1)$ & 13.9 & $(0.9)$ & 60.6 & $(1.7)$ & 24.7 & $(2.0)$ & 0.2 & $(0.1)$ & 0.0 & c \\
\hline & Türkei & 0.3 & $(0.1)$ & 2.6 & $(0.5)$ & 33.2 & $(1.5)$ & 60.3 & (1.5) & 3.2 & $(0.4)$ & 0.3 & $(0.1)$ \\
\hline & Ver. Königreich & 0.0 & c & 0.0 & c & 0.0 & $(0.0)$ & 1.7 & $(0.4)$ & 94.7 & $(0.4)$ & 3.7 & $(0.2)$ \\
\hline & Ver. Staaten & 0.0 & c & 0.4 & $(0.2)$ & 14.6 & (1.1) & 69.8 & (1.1) & 14.9 & $(0.9)$ & 0.3 & $(0.2)$ \\
\hline & OECD-Durchschnitt & 0.6 & $(0.1)$ & 5.9 & $(0.1)$ & 35.6 & $(0.2)$ & 50.1 & $(0.2)$ & 7.5 & $(0.1)$ & 0.3 & $(0.1)$ \\
\hline ฐ & Albanien & 0.1 & $(0.1)$ & 2.9 & $(0.4)$ & 42.9 & $(2.7)$ & 53.8 & (2.8) & 0.2 & $(0.1)$ & 0.0 & c \\
\hline 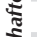 & Argentinien & 2.8 & $(0.8)$ & 15.0 & $(1.7)$ & 25.8 & (1.9) & 52.6 & (2.6) & 3.0 & (0.9) & 0.8 & $(0.5)$ \\
\hline క్ర్య & Brasilien & 0.0 & c & 9.0 & $(0.7)$ & 15.8 & $(0.8)$ & 36.1 & $(1.1)$ & 37.2 & (1.0) & 1.9 & $(0.2)$ \\
\hline 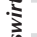 & Bulgarien & 1.3 & $(0.3)$ & 5.8 & $(0.7)$ & 88.2 & $(1.0)$ & 4.6 & $(0.4)$ & 0.0 & c & 0.0 & c \\
\hline$\stackrel{\bar{n}}{=}$ & Kolumbien & 7.4 & $(0.8)$ & 13.5 & (1.0) & 22.1 & $(1.0)$ & 38.8 & (1.4) & 18.2 & $(1.2)$ & 0.0 & c \\
\hline$\frac{1}{1}$ & Costa Rica & 9.3 & $(1.3)$ & 16.4 & (1.2) & 38.5 & (1.5) & 35.7 & $(2.0)$ & 0.0 & $(0.0)$ & 0.0 & c \\
\hline 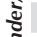 & Kroatien & 0.0 & c & 0.0 & c & 82.0 & $(0.6)$ & 18.0 & $(0.6)$ & 0.0 & c & 0.0 & c \\
\hline : & Zypern* & 0.0 & $(0.0)$ & 0.5 & $(0.1)$ & 4.7 & $(0.1)$ & 94.0 & $(0.2)$ & 0.7 & $(0.1)$ & 0.0 & c \\
\hline ఏ & Hongkong (China) & 1.2 & $(0.2)$ & 6.9 & $(0.5)$ & 27.5 & $(0.7)$ & 63.0 & (1.0) & 1.4 & (1.3) & 0.0 & c \\
\hline ฮั & Indonesien & 2.3 & $(0.4)$ & 10.0 & (1.1) & 38.5 & (3.0) & 45.5 & (3.7) & 3.1 & (0.6) & 0.6 & $(0.6)$ \\
\hline & Jordanien & 0.1 & $(0.1)$ & 0.8 & $(0.2)$ & 5.7 & $(0.6)$ & 93.4 & $(0.6)$ & 0.0 & c & 0.0 & c \\
\hline & Kasachstan & 0.3 & $(0.1)$ & 5.5 & $(0.6)$ & 68.4 & $(2.4)$ & 25.4 & (2.6) & 0.2 & $(0.1)$ & 0.2 & $(0.2)$ \\
\hline & Lettland & 3.6 & $(0.8)$ & 18.0 & $(0.9)$ & 76.4 & (1.3) & 2.0 & $(0.3)$ & 0.0 & $(0.0)$ & 0.0 & c \\
\hline & Liechtenstein & 4.5 & $(1.2)$ & 16.5 & (2.1) & 69.4 & $(2.2)$ & 9.6 & (0.6) & 0.0 & C & 0.0 & c \\
\hline & Litauen & 0.2 & $(0.1)$ & 7.3 & (0.6) & 82.2 & $(0.9)$ & 10.4 & $(0.8)$ & 0.0 & $(0.0)$ & 0.0 & c \\
\hline & Macau (China) & 7.1 & $(0.2)$ & 19.3 & $(0.2)$ & 33.3 & $(0.2)$ & 40.0 & $(0.2)$ & 0.2 & $(0.1)$ & 0.0 & $(0.0)$ \\
\hline & Malaysia & 0.0 & c & 0.1 & $(0.1)$ & 5.1 & $(0.7)$ & 94.7 & $(0.7)$ & 0.0 & c & 0.0 & c \\
\hline & Montenegro & 0.0 & c & 0.1 & $(0.1)$ & 82.0 & $(0.3)$ & 17.9 & $(0.3)$ & 0.0 & c & 0.0 & c \\
\hline & Peru & 3.1 & $(0.5)$ & 9.1 & $(0.8)$ & 19.5 & $(0.7)$ & 46.2 & (1.0) & 22.1 & (0.9) & 0.0 & c \\
\hline & Katar & 1.2 & $(0.1)$ & 3.6 & $(0.1)$ & 14.0 & $(0.1)$ & 64.6 & $(0.2)$ & 16.1 & $(0.2)$ & 0.4 & $(0.0)$ \\
\hline & Rumänien & 0.3 & $(0.2)$ & 6.5 & $(0.6)$ & 88.7 & $(0.7)$ & 4.5 & $(0.4)$ & 0.0 & c & 0.0 & c \\
\hline & Russ. Föderation & 0.7 & $(0.2)$ & 8.9 & $(0.7)$ & 73.7 & $(1.5)$ & 16.7 & (1.8) & 0.1 & $(0.1)$ & 0.0 & c \\
\hline & Serbien & 0.1 & $(0.1)$ & 1.9 & $(0.9)$ & 96.7 & $(1.0)$ & 1.4 & $(0.2)$ & 0.0 & c & 0.0 & c \\
\hline & Shanghai (China) & 1.3 & $(0.3)$ & 5.3 & $(0.8)$ & 41.6 & (1.6) & 51.2 & (1.4) & 0.6 & $(0.1)$ & 0.0 & $(0.0)$ \\
\hline & Singapur & 0.4 & $(0.1)$ & 2.0 & $(0.3)$ & 8.3 & $(0.4)$ & 89.3 & $(0.5)$ & 0.0 & $(0.0)$ & 0.0 & c \\
\hline & Chinesisch Taipeh & 0.0 & c & 0.2 & $(0.2)$ & 37.4 & (1.5) & 62.4 & (1.5) & 0.0 & c & 0.0 & c \\
\hline & Thailand & 0.1 & $(0.1)$ & 0.4 & $(0.2)$ & 22.9 & (1.3) & 74.1 & (1.5) & 2.5 & $(0.5)$ & 0.0 & c \\
\hline & Tunesien & 6.3 & $(0.8)$ & 14.6 & (1.6) & 21.9 & (1.6) & 52.3 & (3.0) & 4.9 & $(0.5)$ & 0.0 & c \\
\hline & Ver. Arab. Emirate & 1.3 & $(0.3)$ & 3.1 & $(0.3)$ & 12.9 & $(0.9)$ & 60.3 & $(1.2)$ & 21.8 & $(1.0)$ & 0.6 & $(0.1)$ \\
\hline & Uruguay & 9.4 & $(1.3)$ & 13.1 & $(0.8)$ & 24.0 & $(1.1)$ & 52.4 & (1.9) & 1.2 & $(0.2)$ & 0.0 & c \\
\hline & Vietnam & 0.7 & $(0.3)$ & 3.5 & $(0.8)$ & 10.5 & $(2.2)$ & 85.3 & $(2.8)$ & 0.0 & c & 0.0 & $\mathrm{c}$ \\
\hline
\end{tabular}

Informationen zu den Regionen, deren Stichproben international überprüft wurden, sind online verfügbar.

*Vgl. Anmerkungen am Anfang dieses Anhangs.
StatLink त्राIsh http://dx.doi.org/10.1787/888932937092 
[Teil 2/2]

Tabelle A2.4b Prozentsatz der Schüler in den einzelnen Klassenstufen, nach Geschlecht

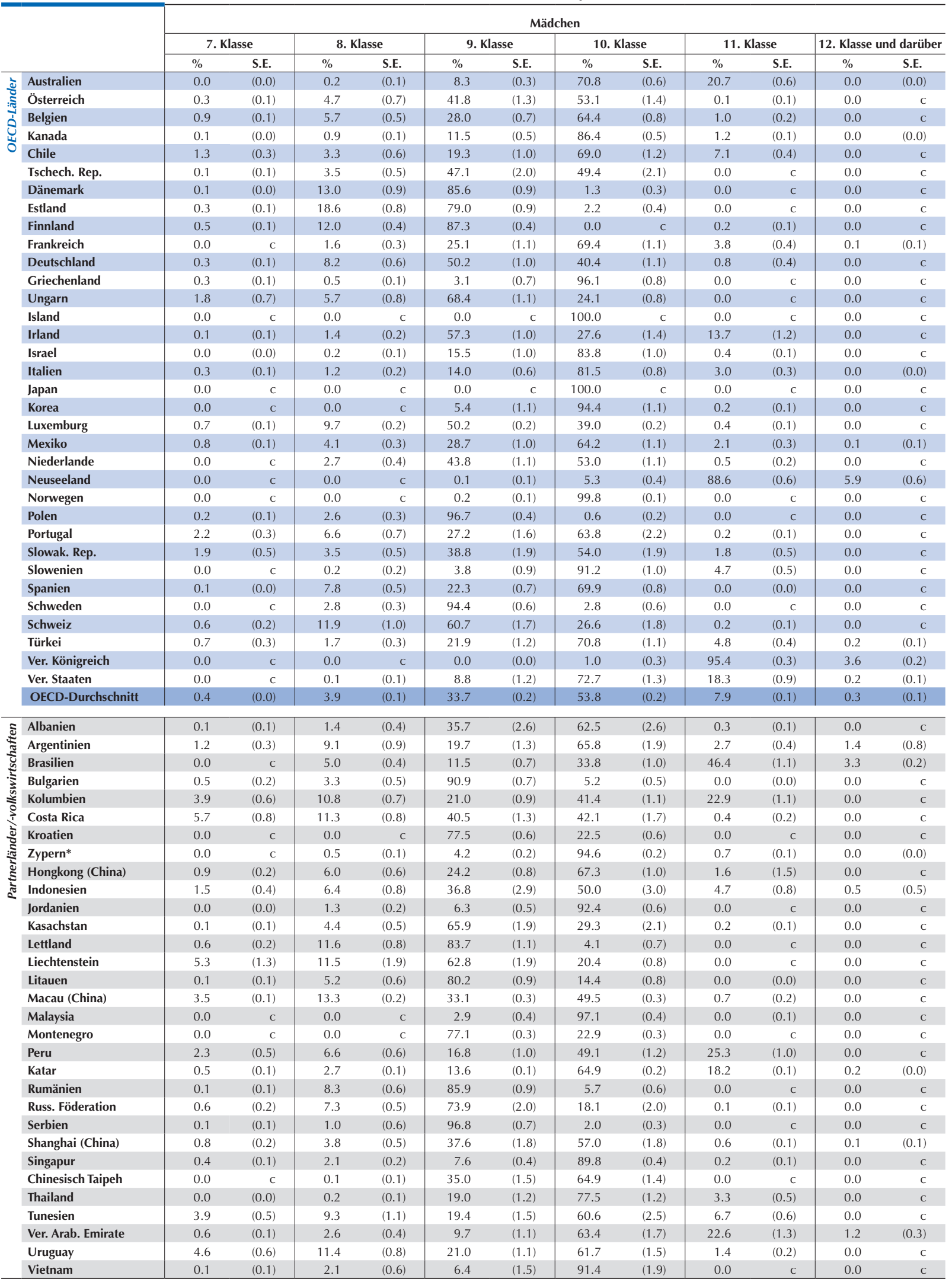

Informationen zu den Regionen, deren Stichproben international überprüft wurden, sind online verfügbar.

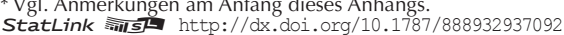




\section{ANHANG A3}

\section{TECHNISCHE HINWEISE ZU DEN IN DIESEM BAND ENTHALTENEN ANALYSEN}

\section{Methoden und Definitionen}

\section{Relatives Risiko oder erhöhte Wahrscheinlichkeit}

Das relative Risiko ist eine Messgröße für die kausale Abhängigkeit zwischen einem Antezedens- und einem Konsequenzfaktor. Das relative Risiko ist nichts anderes als das Verhältnis zwischen zwei Risiken, d.h. dem Risiko der Beobachtung eines Ergebnisses bei Anwesenheit des Antezedens und dem Risiko der Beobachtung eines Ergebnisses bei Abwesenheit des Antezedens. Abbildung A3.1 stellt die im Folgenden verwendete Einteilung dar.

- Abbildung A3.1

Bezeichnungen in einer zweidimensionalen Tabelle

\begin{tabular}{c|c|c}
$p_{11}$ & $p_{12}$ & $p_{1 .}$ \\
\hline$p_{21}$ & $p_{22}$ & $p_{2 .}$ \\
\hline$p_{.1}$ & $p_{.2}$ & $p_{. .}$ \\
\hline
\end{tabular}

$p_{\text {.. }}$ ist gleich $\frac{n_{. .}}{n_{. .}}$, wobei $n_{\text {.. }}$ die Gesamtzahl der Schülerinnen und Schüler ist und $p_{\text {.. }}$ daher gleich 1 ist, $p_{i, .} p_{j}$ stehen jeweils für die bedingte Wahrscheinlichkeit in jeder Zeile bzw. jeder Spalte. Die bedingte Wahrscheinlichkeit entspricht der bedingten Häufigkeit, dividiert durch die Gesamtzahl der Schüler. $p_{i j}$ schließlich bezeichnet die Wahrscheinlichkeit für jede Zelle und entspricht der Zahl der Beobachtungen in einer bestimmten Zelle, dividiert durch die Gesamtzahl der Beobachtungen.

In PISA entsprechen die Zeilen dem Antezedensfaktor - mit der ersten Zeile für das Vorhandensein und der zweiten Zeile für das Nichtvorhandensein des Antezedensfaktors. Die Spalten entsprechen dem Konsequenzfaktor: In der ersten Spalte ist das Vorhandensein und in der zweiten Spalte das Nichtvorhandensein des Konsequenzfaktors vermerkt. Das relative Risiko entspricht dann:

$R R=\frac{\left(p_{11} / p_{1 .}\right)}{\left(p_{21} / p_{2 .}\right)}$

\section{Zuschreibbares Risiko oder Populationsrelevanz}

Das zuschreibbare Risiko, das im Text und in den Tabellen dieses Bandes auch als Populationsrelevanz bezeichnet wird, wird folgendermaßen interpretiert: Wenn der Risikofaktor ausgeschlossen werden könnte, würde die Häufigkeit des Auftretens des Konsequenzmerkmals in der Population um den betreffenden Koeffizienten verringert werden. Das zuschreibbare Risiko ist gleich (vgl. Abb. A3.1 wegen der in der folgenden Formel verwendeten Einteilung):

$A R=\frac{\left(p_{11} p_{22}\right)-\left(p_{12} p_{21}\right)}{\left(p_{.1} p_{2 .}\right)}$

Die Koeffizienten werden mit 100 multipliziert, um das Ergebnis als Prozentsatz auszudrücken.

\section{Statistiken auf der Basis von Mehrebenenmodellen}

Statistiken auf der Basis von Mehrebenenmodellen umfassen Varianzkomponenten (zwischen- und innerschulische Varianz), den aus diesen Komponenten abgeleiteten Index der Inklusion sowie die Regressionskoeffizienten, sofern angegeben. Mehrebenenmodelle werden im Allgemeinen als zweistufige Regressionsmodelle (Schüler- und Schulebene) mit normalverteilten Residuen spezifiziert und nach der Maximum-Likelihood-Methode geschätzt. Wenn es sich bei der abhängigen Variablen um die Mathematikleistungen handelt, wurden für die Schätzung fünf plausible Werte für die Ergebnisse jedes Schülers auf der Mathematikskala verwendet. Die Modelle wurden unter Verwendung der Software Mplus ${ }^{\circledR}$ geschätzt.

Bei Mehrebenenmodellen werden sowohl auf der Schüler- als auch auf der Schulebene Gewichtungen vorgenommen. Das Ziel dieser Gewichtungen besteht darin, den Unterschieden in derWahrscheinlichkeit Rechnung zu tragen, dass die Schüler in der Stichprobe gezogen werden. Da bei PISA ein zweistufiges Stichprobenziehungsverfahren angewendet wird, sind diese Unterschiede auf Faktoren sowohl auf der Schul- als auch auf der Schülerebene zurückzuführen. Für die Mehrebenenmodelle wurden endgültige Schülergewichtungen (W_FSTUWT) herangezogen. Die innerschulischen Gewichtungen entsprechen der endgültigen Schülergewichtung, wurden jedoch umskaliert, um dem Stichprobenumfang innerhalb jeder Schule zu entsprechen. Die zwischenschulischen Gewichtungen entsprechen der Summe der endgültigen Schülergewichtungen (W_FSTUWT) innerhalb jeder Schule. Die Definition der zwischenschulischen Gewichtungen hat sich gegenüber PISA 2009 geändert. 
Der Index der Inklusion wird folgendermaßen definiert und geschätzt:

$100 * \frac{\sigma_{w}^{2}}{\sigma_{w}^{2}+\sigma_{b}^{2}}$

wo $\sigma_{w}^{2}$ und $\sigma_{b}^{2}$, den Schätzwerten für die inner- bzw. zwischenschulische Varianz entsprechen.

Die Ergebnisse in Mehrebenenmodellen und insbesondere die Schätzung der Anteile der zwischenschulischen Varianz hängen davon ab, wie die Schulen in den einzelnen Ländern definiert und organisiert sind und welche Einheiten für die Stichprobenziehung gewählt wurden. In einigen Ländern z.B. wurden einige der Schulen in der PISA-Stichprobe als Verwaltungseinheiten definiert (selbst wenn sie, wie in Italien, mehrere geografisch getrennte Einrichtungen umfassen); in anderen Ländern wurden sie als die Teile größerer Bildungseinrichtungen definiert, die von 15-Jährigen besucht werden; in manchen Ländern wurden Schulen wiederum als Schulgebäude definiert, in wieder anderen dagegen aus Sicht der Schulorganisation (z.B. als Einheiten, die eine eigene Schulleitung haben). Der PISA 2012 Technical Report (OECD, erscheint demnächst) und Anhang A2 liefern einen Überblick über die verschiedenen Methoden der Definition der Schulen. In Slowenien ist die primäre Stichprobeneinheit definiert als eine Gruppe von Schülerinnen und Schülern, die demselben Bildungsgang innerhalb einer Schule folgen. In diesem besonderen Fall entspricht die Varianz zwischen den Schulen tatsächlich der Varianz innerhalb einer Schule, aber zwischen unterschiedlichen Bildungsgängen. Die Verwendung von Schichtungsvariablen bei der Auswahl der Schulen kann auch die Schätzung der Anteile der zwischenschulischen Varianz beeinflussen, insbesondere wenn die Schichtungsvariablen mit zwischenschulischen Unterschieden assoziiert sind.

Wegen der Art und Weise, in der die Schülerstichprobe erhoben wurde, ist in der Varianz innerhalb der Schulen sowohl die Varianz zwischen verschiedenen Klassen als auch zwischen verschiedenen Schülerinnen und Schülern enthalten.

Bei der multiplen Imputation wird jeder fehlende Wert durch einen Satz plausibler Werte ersetzt, die die Unsicherheit in Bezug auf den richtigen zu imputierenden Wert darstellen. Die multiplen imputierten Datensätze werden anschließend mit Hilfe von Standardverfahren untersucht, um vollständige Daten zu erhalten, und die Ergebnisse aus diesen Analysen werden kombiniert. Fünf imputierte Werte werden für jeden fehlenden Wert berechnet. Je nach Struktur der fehlenden Werte können verschiedene Methoden verwendet werden. Für willkürlich fehlende Datenstrukturen kann auf das MCMC (Markov-Chain-Monte-Carlo)-Verfahren zurückgegriffen werden.

Dieses Verfahren wird zusammen mit dem SAS-Verfahren MI für die Mehrebenenanalysen in diesem Band angewandt. Die multiple Imputation wird für jedes Modell und jedes Land separat durchgeführt, außer für das Modell mit allen Variablen (Tabelle IV.1.12a, IV.1.12b und IV.1.12c), in dem die Daten für die einzelnen Modelle auf der Grundlage imputierter Daten konstruiert wurden, wie das Modell für das Lernumfeld, das Modell für die Selektion und Gruppierung von Schülern usw. Wenn kontinuierliche Werte für fehlende diskrete Variablen generiert werden, werden diese auf den nächsten diskreten Wert der jeweiligen Variablen gerundet. Jeder der fünf plausiblen Messwerte wird durch die Mplus ${ }$ Software unter Verwendung einer der fünf imputierten Datensätze analysiert, wobei es sich um kombinierte Datensätze handelt, da die zwischen den Imputationen liegende Varianz berücksichtigt wurde.

\section{Standardfehler und Signifikanztests}

Die in diesem Bericht enthaltenen Statistiken stellen Schätzwerte der in den einzelnen Ländern erreichten Leistung auf der Basis der Schülerstichproben dar und nicht etwa Werte, die ausgehend von den Antworten sämtlicher Schülerinnen und Schüler eines Landes auf sämtliche Fragen errechnet worden wären. Daher ist es wichtig, die mögliche Höhe des Messfehlers in diesen Schätzungen zu ermitteln. In PISA wird bei jeder Schätzung ein Messfehler angegeben, der durch den Standardfehler (S.E.) ausgedrückt ist. Mit Hilfe von Konfidenzintervallen können Schlüsse über die Populationsmittelwerte und -anteile gezogen werden, die die mit den Stichprobenschätzungen verbundene Unsicherheit widerspiegeln. Unter Annahme einer Normalverteilung kann davon ausgegangen werden, dass das tatsächlich beobachtete statistische Ergebnis einer gegebenen Population in 95 von 100 Wiederholungsmessungen mit unterschiedlichen Stichproben derselben Population innerhalb des Konfidenzintervalls liegen würde.

Die Leser sind häufig in erster Linie daran interessiert, ob sich ein bestimmter Wert für ein gegebenes Land von einem zweiten Wert für dasselbe Land oder für ein anderes Land unterscheidet, z.B. ob in einem bestimmten Land Mädchen bessere Leistungen erzielen als Jungen. In den Tabellen und Abbildungen dieses Berichts werden Unterschiede als statistisch signifikant bezeichnet, wenn die betreffenden kleineren oder größeren Differenzen in weniger als 5\% der Fälle beobachtet würden, obwohl die entsprechenden Populationswerte in Wirklichkeit nicht voneinander abweichen. Entsprechend wird das Risiko, dass eine Korrelation als signifikant eingestuft wird, wenn effektiv keine Korrelation zwischen zwei Messgrößen besteht, auf 5\% begrenzt.

Für sämtliche Teile des Berichts wurden Signifikanztests durchgeführt, um die statistische Signifikanz der vorgenommenen Vergleiche zu prüfen.

\section{Geschlechtsspezifische Unterschiede und Unterschiede zwischen den Mittelwerten der Untergruppen}

Die geschlechtsspezifischen Unterschiede bei den Schülerleistungen oder anderen Indizes wurden auf ihre statistische Signifikanz hin getestet. Positive Unterschiede weisen auf höhere Punktzahlen für Jungen hin, negative Unterschiede auf höhere Punktzahlen für Mädchen. Die in den Tabellen dieses Bands fettgedruckten Unterschiede sind im Allgemeinen bei einem Konfidenzniveau von 95\% statistisch signifikant. 
Desgleichen wurden die Unterschiede zwischen anderen Schülergruppen (z.B. Schülerinnen und Schüler mit und ohne Migrationshintergrund) auf ihre statistische Signifikanz hin getestet. Die Definitionen der Untergruppen finden sich im Allgemeinen in den Tabellen und im Begleittext zu den Analysen. Alle in den Tabellen von Anhang B dieses Berichts fettgedruckten Unterschiede sind bei einem Konfidenzniveau von $95 \%$ statistisch signifikant.

\section{Unterschiede zwischen den Mittelwerten der Untergruppen, nach Berücksichtigung der anderen Variablen}

Für viele Tabellen wurden Vergleiche des beobachteten Unterschieds („vor Berücksichtigung der anderen Variablen“) und nach Berücksichtigung der anderen Variablen wie des PISA-Index des wirtschaftlichen, sozialen und kulturellen Status der Schüler (ESCS) zwischen den Untergruppen angestellt. Die bereinigten Unterschiede wurden mittels linearer Regression geschätzt und bei einem Konfidenzniveau von 95\% auf ihre Signifikanz hin getestet. Signifikante Unterschiede sind durch Fettdruck gekennzeichnet.

\section{Leistungsunterschiede zwischen dem obersten und dem untersten Quartil der PISA-Indizes und -Skalen}

Unterschiede bei den Durchschnittsleistungen zwischen dem obersten und dem untersten Quartil auf den PISA-Indizes und -Skalen wurden auf ihre statistische Signifikanz hin getestet. Angaben in Fettdruck weisen darauf hin, dass bei einem Konfidenzniveau von 95\% ein statistisch signifikanter Unterschied zwischen der Leistung des obersten und des untersten Quartils der Schüler auf dem jeweiligen Index besteht.

\section{Unterschiede zwischen Untergruppen von Schulen}

In diesem Band werden die Schulen anhand verschiedener Aspekte wie Ressourcenallokation oder Leistungen verglichen. Zu diesem Zweck werden die Schulen nach dem sozioökonomischen Status der Schüler bzw. der Schulen, dem Status öffentliche bzw. private Schule, dem Sekundarbereich I bzw. II sowie dem Schulstandort in Kategorien eingeteilt. Die Unterschiede zwischen den Untergruppen von Schulen werden folgendermaßen auf ihre statistische Signifikanz hin getestet:

- Sozioökonomischer Status der Schüler: Die Schüler im obersten ESCS-Quartil werden mit den Schülern im untersten ESCS-Quartil verglichen. Wenn der Unterschied bei einem Konfidenzniveau von 95\% statistisch signifikant ist, sind beide Werte durch Fettdruck gekennzeichnet. Das zweite und das dritte Quartil gehen nicht in den Vergleich ein.

- Sozioökonomischer Status der Schulen: Sozioökonomisch begünstigte Schulen werden mit sozioökonomisch benachteiligten Schulen verglichen. Wenn der Unterschied bei einem Konfidenzniveau von 95\% statistisch signifikant ist, sind beide Werte durch Fettdruck gekennzeichnet. Sozioökonomisch durchschnittliche Schulen gehen nicht in den Vergleich ein.

- Öffentliche und private Schulen: Vom Staat abhängige und unabhängige Privatschulen werden zusammen als Privatschulen betrachtet. Die fettgedruckten Werte in den Tabellen von Anhang B dieses Bandes weisen bei einem Konfidenzniveau von 95\% auf statistisch signifikante Unterschiede zwischen öffentlichen und privaten Schulen hin.

- Bildungsniveau: Die Schüler im Sekundarbereich II werden mit den Schülern im Sekundarbereich I verglichen. Wenn der Unterschied bei einem Konfidenzniveau von 95\% statistisch signifikant ist, sind beide Werte durch Fettdruck gekennzeichnet.

- Schulstandort: Zum Zweck der Signifikanztests werden „Schulen in Kleinstädten“ und "Schulen in Städten“ zusammen als Gruppe betrachtet. Die Werte für "Schulen in mittleren Großstädten oder Großstädten“ sind in den Tabellen von Anhang B dieses Bandes durch Fettdruck gekennzeichnet, wenn der Unterschied zu dieser mittleren Kategorie („Schulen in Kleinstädten“ und „Schulen in Städten") bei einem Konfidenzniveau von 95\% signifikant ist. Die Werte für „Schulen in Dörfern, Ortschaften oder ländlichen Gemeinden" sind hingegen durch Fettdruck gekennzeichnet, wenn der Unterschied zu dieser mittleren Kategorie signifikant ist. Die Unterschiede zwischen den beiden äußeren Kategorien wurden nicht auf ihre Signifikanz hin getestet.

\section{Veränderung der Leistung je Indexeinheit}

Für viele Tabellen wurde die Veränderung der Schülerleistungen je Indexeinheit berechnet. Angaben in Fettdruck weisen auf Veränderungen hin, die bei einem Konfidenzniveau von 95\% statistisch signifikant von null abweichen.

\section{Relatives Risiko oder erhöhte Wahrscheinlichkeit}

Die fettgedruckten Werte in den Tabellen von Anhang B dieses Berichts weisen darauf hin, dass das relative Risiko bei einem Konfidenzniveau von 95\% statistisch signifikant von 1 abweicht. Zur Berechnung der statistischen Signifikanz um den Wert von 1 (Nullhypothese) wird unterstellt, dass die statistische Maßzahl für das relative Risiko bei einer Nullhypothese eher einer Log-Normalverteilung als einer Normalverteilung folgt.

\section{Zuschreibbares Risiko oder Populationsrelevanz}

Die fettgedruckten Werte in den Tabellen von Anhang B dieses Berichts weisen darauf hin, dass das zuschreibbare Risiko bei einem Konfidenzniveau von $95 \%$ statistisch signifikant von 0 abweicht.

\section{Standardfehler in Statistiken, die auf Mehrebenenmodellen basieren}

Für Statistiken auf der Basis von Mehrebenenmodellen (wie z.B. die Schätzung derVarianzkomponenten und der Regressionskoeffizienten aus zweistufigen Regressionsmodellen) erfolgt die Schätzung der Standardfehler nicht mit der üblichen Replikationsmethode, die die 
Schichtung und den Auswahlsatz endlicher Populationen berücksichtigt. Stattdessen basieren die Standardfehler auf Modellen: Bei ihrer Berechnung wird unterstellt, dass die Schulen sowie die Schülerinnen und Schüler in den Schulen durch Zufallsstichproben aus einer theoretischen unendlichen Population von Schulen sowie Schülerinnen und Schülern ausgewählt werden, die den unterstellten Parametern des Modells entspricht (wobei die Stichprobenwahrscheinlichkeit in der Gewichtung der Schulen und Schülerinnen und Schüler widergespiegelt wird).

Der Standardfehler für den geschätzten Index der Inklusion wird berechnet, indem dafür mittels der Delta-Methode aus den (modellbasierten) Standardfehlern für die Varianzkomponenten eine approximative Verteilung abgeleitet wird.

\section{Standardfehler bei der Trendanalyse der Ergebnisse: Linking-Fehler}

Standardfehler in den Schätzungen der Leistungstrends mussten bereinigt werden, da das Equating-Verfahren, das den Vergleich der Ergebnisse aus verschiedenen PISA-Erhebungen ermöglicht, eine Zufallsfehlerkomponente einführt, die mit Leistungsveränderungen bei den Link-Items verknüpft ist. Diese eher konservativen Standardfehler (die größer sind als die Standardfehler, die vor Einführung des Linking-Fehlers geschätzt wurden) spiegeln nicht nur die Messpräzision und die Stichprobenvarianz wie für die herkömmlichen PISA-Ergebnisse, sondern auch den Linking-Fehler wider (vgl. Anhang A5 wegen einer technischen Beschreibung des Linking-Fehlers).

Link-Items stellen nur einen Unterkatalog aller zur Ableitung von PISA-Ergebnissen verwendeten Items dar. Wenn für die Angleichung von PISA-Punktzahlen im Zeitverlauf unterschiedliche Items verwendet würden, könnte der Vergleich der Ergebnisse für eine Gruppe von Schülerinnen und Schülern im Zeitverlauf unterschiedlich ausfallen. Infolgedessen ist in den Standardfehlern bei den Schätzungen der Leistungsveränderungen einer bestimmten Gruppe (z.B. ein Land oder eine Volkswirtschaft, eine Region, Jungen, Mädchen, Schülerinnen und Schüler mit Migrationshintergrund, Schülerinnen und Schüler ohne Migrationshintergrund, sozioökonomisch begünstigte Schülerinnen und Schüler, Schülerinnen und Schüler in öffentlichen Schulen usw.) im Zeitverlauf in den Bereichen Mathematik, Lesekompetenz oder Naturwissenschaften zusätzlich zum Stichproben- und Imputationsfehler, der bei den Schätzungen der Ergebnisse für ein bestimmtes Jahr üblicherweise gegeben ist, ein Linking-Fehler einbezogen. Da die Position in der Verteilung (eine Veränderung der Auffangvariable) durch das Equating-Verfahren ungewisser wird, dieses Verfahren aber keine Veränderung in der Verteilungsvarianz nach sich zieht, bleibt der Linking-Fehler in den Standardfehlern bei ortsinvarianten Schätzungen unberücksichtigt. Ortsinvariante Schätzungen umfassen beispielsweise Schätzungen für Varianzen, Regressionskoeffizienten für Kovariaten auf Schüleroder Schulebene sowie Korrelationskoeffizienten.

Die fettgedruckten Werte in den Tabellen zu Leistungstrends, die in Anhang B dieses Berichts aufgeführt sind, weisen darauf hin, dass die Leistungsveränderung dieser bestimmten Gruppe bei einem Konfidenzniveau von 95\% statistisch signifikant von 0 abweicht. Die für die Berechnung der statistischen Signifikanz des angegebenen Trends verwendeten Standardfehler enthalten einen Linking-Fehler. 


\section{ANHANG A4 QUALITÄTSSICHERUNG}

Wie bereits in allen früheren PISA-Erhebungen wurden in sämtlichen Teilen von PISA 2012 Qualitätssicherungsverfahren durchgeführt.

Die einheitliche Qualität und sprachliche Äquivalenz der in PISA 2012 verwendeten Erhebungsinstrumente wurden dadurch gesichert, dass den Ländern gleichwertige Originalfassungen der Erhebungsinstrumente in Englisch und Französisch zur Verfügung gestellt wurden; Länder, in denen die Schülerleistungen in keiner dieser zwei Sprachen erhoben wurden, mussten ausgehend von den beiden Originalfassungen zwei unabhängige Übersetzungen erstellen lassen und diese dann zusammenführen. Dazu wurden genaue Übersetzungs- und Bearbeitungsrichtlinien aufgestellt und Anweisungen hinsichtlich der Auswahl und Ausbildung der Übersetzer gegeben. Für jedes Land wurden die Übersetzung und das Format der Erhebungsinstrumente (einschließlich des Testmaterials, der Kodieranweisungen, der Fragebogen und der Handbücher) durch vom PISA-Konsortium eingesetzte erfahrene Übersetzer überprüft, bevor sie im Feldtest und in der Haupterhebung von PISA 2012 eingesetzt wurden. Die Muttersprache dieser Übersetzer war die Unterrichtssprache des betreffenden Landes, und sie verfügten über ausreichende Kenntnisse bezüglich der Bildungssysteme. Nähere Informationen über die PISA-Übersetzungsverfahren enthält der PISA 2012 Technical Report (OECD, erscheint demnächst).

Die Erhebung wurde mit Hilfe standardisierter Verfahren durchgeführt. Das PISA-Konsortium stellte umfassende Handbücher zur Verfügung, die den Ablauf der Erhebung erklärten und u.a. präzise Anweisungen für die Arbeit der Schulkoordinatoren sowie Vorlagen für die Testleiter zum Gebrauch bei den Testsitzungen enthielten. Vorgeschlagene Anpassungen der Erhebungsmethoden oder vorgeschlagene Änderungen der Testsitzungen wurden vor der Überprüfung dem PISA-Konsortium zur Genehmigung vorgelegt. Das PISA-Konsortium überprüfte dann die Übersetzungen und die Anpassung der Handbücher für die verschiedenen Länder.

Um die Glaubwürdigkeit von PISA im Hinblick auf Validität und Unvoreingenommenheit zu gewährleisten und einen einheitlichen Ablauf der Testsitzungen zu fördern, wurden die Testleiterinnen und Testleiter in den Teilnehmerländern nach folgenden Kriterien ausgewählt: Die Leiterinnen und Leiter der vorgesehenen PISA-Testsitzungen durften nicht zugleich die Fachlehrer der teilnehmenden Schülerinnen und Schüler in den Bereichen Lesen, Mathematik und Naturwissenschaften sein; die Testleiterinnen und Testleiter sollten nicht Mitglieder des Kollegiums einer Schule sein, an der sie die Leitung der PISA-Tests übernahmen; nach Möglichkeit sollten die Testleiterinnen und Testleiter auch nicht zum Kollegium einer für die PISA-Stichprobe gezogenen Schule gehören. Die Teilnehmerländer organisierten für die Testleiterinnen und Testleiter eine individuelle Schulung.

Es war Aufgabe der Teilnehmerländer sicherzustellen, dass die Testleiterinnen und Testleiter mit den Schulkoordinatoren bei der Vorbereitung der Testsitzung zusammenarbeiteten, u.a. bei der Aktualisierung der Unterlagen über den bisherigen Bildungsweg der Schülerinnen und Schüler sowie der Identifizierung ausgeschlossener Schülerinnen und Schüler, dass für die kognitiven Items keine zusätzliche Zeit eingeräumt wurde (während es erlaubt war, für die Beantwortung des Schülerfragebogens mehr Zeit zuzugestehen), dass kein Instrument vor Beginn der zwei einstündigen Teile der kognitiven Sitzung bekannt gegeben wurde, dass die Testleiterinnen und Testleiter den Stand der Schülerbeteiligung auf den Unterlagen über die Bildungswege der Schülerinnen und Schüler festhielten und ein Formular für den Sitzungsbericht ausfüllten, dass kein kognitives Instrument fotokopiert werden konnte, dass kein kognitives Instrument von dem Schulpersonal vor der Testsitzung eingesehen werden konnte und dass die Testleiterinnen und Testleiter das Material unmittelbar nach Abschluss der Testsitzungen an das nationale Zentrum zurückgaben.

Die nationalen Projektmanager wurden dazu angehalten, eine Folgesitzung zu organisieren, wenn mehr als $15 \%$ der PISAStichprobenschüler nicht an der ursprünglichen Testsitzung teilnehmen konnten.

Für das Qualitätsmonitoring zuständige nationale Vertreter des PISA-Konsortiums besuchten alle nationalen Zentren, um die Verfahren der Datenerhebung zu überprüfen. Darüber hinaus besuchten „School Quality Monitors" aus dem PISA-Konsortium eine Stichprobe von sieben Schulen während der Erhebung. Nähere Informationen über die Feldoperationen enthält der PISA 2012 Technical Report (OECD, erscheint demnächst).

Um eine konsistente und genaue Anwendung der in den PISA-Handbüchern dargelegten Kodieranweisungen zu gewährleisten, wurden entsprechende Verfahren entwickelt. Die nationalen Projektleiter wurden gebeten, dem Konsortium Änderungsvorschläge zur Genehmigung vorzulegen. Es wurden Reliabilitätsuntersuchungen durchgeführt, um die Konsistenz des Kodierungsprozesses zu analysieren.

Eine speziell für PISA konzipierte Software erleichterte die Dateneingabe, deckte weitverbreitete Fehler während der Dateneingabe auf und vereinfachte den Prozess der Datenbereinigung. Die nationalen Projektleiter wurden durch Schulungen mit diesen Verfahren vertraut gemacht.

Eine Beschreibung der für PISA angewandten Verfahren zur Qualitätssicherung und deren Ergebnisse enthält der PISA 2012 Technical Report (OECD, erscheint demnächst). 
Die Ergebnisse der Datenbeurteilung zeigen, dass die technischen Standards von PISA in allen PISA-2012-Teilnehmerländern und -volkswirtschaften in vollem Umfang eingehalten wurden; die einzige Ausnahme war Albanien. Albanien unterbreitete unvollständige Daten zur beruflichen Stellung der Eltern, die offenbar auch nicht zutreffend waren, weil ein enges Spektrum von Berufen zu häufig genannt wurde. Da es nicht gelang, diese Probleme während der Phase der Datenbereinigung auszuräumen, wurden für Albanien keine Daten zum Beruf der Eltern oder auf diesen Daten beruhende Indizes in die internationale Datenbank aufgenommen. In Analysen, die sich auf diese Indizes stützen, wurden die Daten aus Albanien nicht berücksichtigt. 


\section{ANHANG A5 \\ TECHNISCHE EINZELHEITEN DER TRENDANALYSEN}

\section{Vergleich der Schülerleistungen in den Bereichen Mathematik, Lesekompetenz und Naturwissenschaften in den verschiedenen PISA-Erhebungsrunden}

In den PISA-Erhebungen 2003, 2006, 2009 und 2012 wurde jeweils dieselbe Gesamtskala Mathematik verwendet, so dass die Punkte auf dieser Skala im Zeitverlauf unmittelbar miteinander vergleichbar sind. Dasselbe gilt für die seit PISA 2000 verwendete Gesamtskala Lesekompetenz und die seit PISA 2006 verwendete Gesamtskala Naturwissenschaften. Die Vergleichbarkeit der Punktwerte im Zeitverlauf ist dank der Verwendung von Link-Items möglich, die in allen Erhebungen zum Einsatz kamen und sich für das EquatingVerfahren zur Anpassung der Leistungsskalen nutzen lassen. Die Items, die in allen Erhebungen zum Einsatz kommen, sind ein Unterkatalog aller Items, aus denen sich die Erhebung zusammensetzt, da PISA seinen Item-Pool stufenweise erneuert. Infolgedessen knüpfen von den insgesamt 110 Items im PISA-Mathematiktest 201284 an Items aus PISA 2003, 48 an Items aus PISA 2006 und 35 an Items aus PISA 2009 an. Die Zahl der an die PISA-Erhebung 2003 gekoppelten Items in PISA 2012 ist größer als die Zahl der an die PISA-Erhebungen 2006 oder 2009 geknüpften Items, da Mathematik in den PISA-Erhebungen 2003 und 2012 den Schwerpunktbereich darstellte. In PISA 2006 und PISA 2009 war Mathematik ein untergeordneter Bereich, und alle in diesen Erhebungen enthaltenen Mathematik-Items waren Link-Items. Der PISA 2012 Technical Report (OECD, erscheint demnächst) liefert die technischen Einzelheiten zum Equating der Gesamtskalen Mathematik, Lesekompetenz und Naturwissenschaften für die Ermittlung von Leistungstrends.

\section{Linking-Fehler}

Die Standardfehler der Schätzwerte für die Leistungstrends mussten angepasst werden, da durch das Equating-Verfahren, das den Vergleich von Punktzahlen aus verschiedenen PISA-Erhebungen ermöglicht, ein Zufallsfehler im Zusammenhang mit den Leistungsveränderungen bei den Link-Items entsteht. Diese konservativeren Standardfehler (die größer sind als die Standardfehler, die vor der Einführung des Linking-Fehlers geschätzt wurden) spiegeln nicht nur die Messpräzision und Stichprobenvarianz wie für die herkömmlichen PISA-Ergebnisse, sondern auch den in Tabelle A5.1 dargestellten Linking-Fehler wider.

Link-Items stellen nur einen Unterkatalog aller zur Ableitung von PISA-Ergebnissen verwendeten Items dar. Wenn für die Angleichung von PISA-Punktzahlen im Zeitverlauf unterschiedliche Items verwendet würden, könnte der Vergleich der Ergebnisse für eine Gruppe von Schülerinnen und Schülern im Zeitverlauf unterschiedlich ausfallen. Infolgedessen ist in den Standardfehlern bei den Schätzungen der Leistungsveränderungen einer bestimmten Gruppe (z.B. ein Land oder eine Volkswirtschaft, eine Region, Jungen, Mädchen, Schülerinnen und Schüler mit Migrationshintergrund, Schülerinnen und Schüler ohne Migrationshintergrund, sozioökonomisch begünstigte Schülerinnen und Schüler, Schülerinnen und Schüler in öffentlichen Schulen usw.) im Zeitverlauf in den Bereichen Mathematik, Lesekompetenz oder Naturwissenschaften zusätzlich zum Stichproben- und Imputationsfehler, der bei den Schätzungen der Ergebnisse für ein bestimmtes Jahr üblicherweise gegeben ist, ein Linking-Fehler vorhanden. Da die Position in der Verteilung (eine Veränderung der Auffangvariable) durch das Equating-Verfahren ungewisser wird, dieses Verfahren aber keine Veränderung in der Verteilungsvarianz nach sich zieht, bleibt der Linking-Fehler in den Standardfehlern bei ortsinvarianten Schätzungen unberücksichtigt. Ortsinvariante Schätzungen umfassen beispielsweise Schätzungen für Varianzen, Regressionskoeffizienten für Kovariaten auf Schüleroder Schulebene sowie Korrelationskoeffizienten.

\section{Linking-Fehler bei Leistungsvergleichen zwischen zwei PISA-Erhebungen}

In den nachstehenden Gleichungen wird beschrieben, wie Linking-Fehler zwischen zwei PISA-Erhebungen berechnet werden. Angenommen wir haben $L$ Punkte in $K$ Units. Wird $i$ für die Indexierung von Items in einer Unit und $j$ für die Indexierung von Units verwendet, steht für die geschätzte Schwierigkeit von Item $i$ in Unit $j$ im Jahr y, ergibt beispielsweise ein Vergleich zwischen PISA 2006 und PISA 2003:

$$
c_{i j}=\hat{\mu}_{i j}^{2006}-\hat{\mu}_{i j}^{2003}
$$

Der Wert (Gesamtpunkzahl) von Unit $j$ ist $m_{j}$, so dass gilt:

$$
\sum_{j=1}^{K} m_{j}=L
$$

und

Des Weiteren gilt:

$$
\begin{gathered}
\bar{m}=\frac{1}{K} \sum_{j=1}^{K} m_{j} \\
c_{. j}=\frac{1}{m_{j}} \sum_{j=1}^{m_{j}} c_{i j}
\end{gathered}
$$


und

$$
\bar{c}=\frac{1}{N} \sum_{j=1}^{K} \sum_{i=1}^{m_{j}} c_{i j}
$$

dann lautet der Linking-Fehler unter Berücksichtigung des Clustering wie folgt:

$$
\text { error }_{2006,2003}=\sqrt{\frac{\sum_{j=1}^{K} m_{j}^{2}(c . j-\bar{c})^{2}}{K(K-1) \bar{m}^{2}}}
$$

Dieser Ansatz für die Schätzung der Linking-Fehler wurde in den PISA-Erhebungen 2006, 2009 und 2012 verwendet. Die LinkingFehler bei Vergleichen von Ergebnissen aus PISA 2012 mit denen früherer Erhebungen sind in Tabelle A5.1 aufgeführt.

[Teil 1/1]

Tabelle A5.1 Linking-Fehler bei Leistungsvergleichen zwischen PISA 2012 und früheren Erhebungen

\begin{tabular}{l|c|c|c}
\hline \multicolumn{1}{c|}{ Vergleich } & Mathematik & Lesekompetenz & Naturwissenschaften \\
\hline Zwischen PISA 2000 und PISA 2012 & & 5.923 & 5.604 \\
\hline Zwischen PISA 2003 und PISA 2012 & 1.931 & 5.580 & 3.512 \\
\hline Zwischen PISA 2006 und PISA 2012 & 2.084 & 2.602 & 2.006 \\
\hline Zwischen PISA 2009 und PISA 2012 & 2.294 & \\
\hline
\end{tabular}

Anmerkung: Vergleiche zwischen den Ergebnissen in PISA 2012 und früheren Erhebungen können nur vorgenommen werden, wenn das Fach vor 2012 bereits Schwerpunktbereich war. Folglich sind weder Vergleiche der Mathematikleistungen zwischen PISA 2012 und PISA 2000 noch Vergleiche der Leistungen in Naturwissenschaften zwischen PISA 2012 und PISA 2000 oder PISA 2003 möglich.

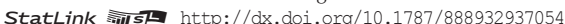

\section{Linking-Fehler bei anderen Vergleichsformen von Schülerleistungen}

Für den Linking-Fehler bei anderen Leistungsvergleichen gibt es keine einfache theoretische Lösung, wie für den Linking-Fehler bei Vergleichen zwischen zwei PISA-Erhebungen. Der oben beschriebene Linking-Fehler zwischen zwei PISA-Erhebungen kann aber für eine empirische Schätzung der Größenordnung des Linking-Fehlers beim Vergleich des prozentualen Anteils der Schülerinnen und Schüler auf einer bestimmten Kompetenzstufe oder der Größenordnung des mit der Schätzung der annualisierten und kurvilinearen Veränderung einhergehenden Linking-Fehlers verwendet werden.

In der empirischen Schätzung dieser Linking-Fehler wird davon ausgegangen, dass die Größenordnung des Linking-Fehlers einer Normalverteilung folgt, mit einem Mittelwert von 0 und einer Standardabweichung, die dem in Tabelle A5.1 dargelegten LinkingFehler entspricht. Dieser Verteilung werden 500 Messfehler entnommen und zum ersten plausiblen Wert für jede Erhebung vor 2012 hinzugefügt. Der gesuchte Schätzwert (Veränderung beim prozentualen Anteil der Schülerinnen und Schüler auf einer bestimmten Kompetenzstufe oder die annualisierte Veränderung) wird für jede der 500 Wiederholungsmessungen berechnet. Danach wird die Standardabweichung dieser 500 als der Linking-Fehler für die annualisierte Veränderung, die quadratische Veränderung sowie die Veränderung beim Prozentsatz der Schülerinnen und Schüler mit Leistungen auf einer bestimmten Kompetenzstufe verwendet. Die zur Bereinigung von Standardfehlern bei der Berechnung der Veränderung im prozentualen Anteil der Schülerinnen und Schüler auf jeder Kompetenzstufe verwendeten Werte sind in Tabelle A5.2 und die Werte, die für die Anpassung der linearen und quadratischen Terme in den für die Schätzung der annualisierten und kurvilinearen Veränderung genutzten Regressionsmodelle verwendet wurden, in Tabelle A5.3 aufgeführt.

\section{Leistungsvergleiche: Unterschiede zwischen zwei Erhebungen und annualisierte Veränderung}

Um die Leistungsentwicklung zu evaluieren, werden in den Analysen die Leistungsveränderungen zwischen zwei Erhebungszyklen und die annualisierte Leistungsveränderung wiedergegeben. Vergleiche zwischen zwei Erhebungen (z.B. die Leistungsveränderungen eines Landes/einer Volkswirtschaft zwischen PISA 2003 und PISA 2012 oder die Leistungsveränderungen einer Untergruppe) werden wie folgt berechnet:

$$
\Delta_{2012-t}=P I S A_{2012}-P I S A_{t}
$$

wobei $\Delta_{2012-t}$ für den Leistungsunterschied zwischen PISA 2012 und einer früheren PISA-Erhebung steht, in der $t$ jeden der folgenden Werte annehmen kann: 2000, 2003, 2006 oder 2009. PISA 2012 steht für die in PISA 2012 im Bereich Mathematik, Lesekompetenz oder Naturwissenschaften erzielte Punktzahl und PISA für die in einer früheren Erhebung (2000, 2003, 2006 oder 2009) im Bereich Mathematik, Lesekompetenz oder Naturwissenschaften verzeichnete Punktzahl. Der Standardfehler der Leistungsveränderung $\sigma\left(\Delta_{2012-t}\right)$ entspricht:

$$
\sigma\left(\Delta_{2012-t}\right)=\sqrt{\sigma_{2012}^{2}+\sigma_{t}^{2}+\text { error }_{2012, t}^{2}}
$$

wobei $\sigma_{2012}$ für den in $P_{I S A_{2012}}$ beobachteten Standardfehler, $\sigma_{t}$ für den in PISA $_{t}$ beobachteten Standardfehler und error $2012, t$ für den Linking-Fehler bei Vergleichen der Ergebnisse in den Bereichen Mathematik, Lesekompetenz oder Naturwissenschaften zwischen der PISA-Erhebung 2012 und einer früheren Erhebung $(t)$ steht. Der Wert für error ${ }_{2012, t}$ ist Tabelle A5.1 zu entnehmen. 
[Teil 1/3]

Tabelle A5.2 Linking-Fehler bei Vergleichen der Kompetenzstufen zwischen PISA 2012 und früheren Erhebungen

\begin{tabular}{|c|c|c|c|c|c|c|c|c|c|c|c|}
\hline & & \multicolumn{10}{|c|}{ Vergleich der Mathematikleistungen zwischen PISA 2012 und ... } \\
\hline & & \multicolumn{6}{|c|}{ PISA 2003} & \multicolumn{2}{|c|}{ PISA 2006} & \multicolumn{2}{|c|}{ PISA 2009} \\
\hline & & \multicolumn{3}{|c|}{ Unter Stufe 2} & \multicolumn{3}{|c|}{ Auf Stufe 5 oder darüber } & \multirow{2}{*}{\begin{tabular}{|c|} 
Unter Stufe 2 \\
Alle Schüler \\
\end{tabular}} & \multirow{2}{*}{\begin{tabular}{|c|}
$\begin{array}{c}\text { Auf Stufe } 5 \\
\text { oder darüber }\end{array}$ \\
Alle Schüler \\
\end{tabular}} & \multirow{2}{*}{\begin{tabular}{|l|} 
Unter Stufe 2 \\
Alle Schüler \\
\end{tabular}} & $\begin{array}{c}\text { Auf Stufe } 5 \\
\text { oder darüber }\end{array}$ \\
\hline & & Alle Schüler & Jungen & Mädchen & Alle Schüler & Jungen & Mädchen & & & & \\
\hline ¿ & Australien & 0.534 & 0.462 & 0.612 & 0.435 & 0.477 & 0.393 & 0.588 & 0.464 & 0.634 & 0.498 \\
\hline : & Österreich & 0.566 & 0.567 & 0.579 & 0.501 & 0.537 & 0.470 & 0.610 & 0.530 & $\mathrm{~m}$ & $\mathrm{~m}$ \\
\hline บำ & Belgien & 0.484 & 0.476 & 0.495 & 0.556 & 0.572 & 0.543 & 0.521 & 0.596 & 0.556 & 0.637 \\
\hline 氙 & Kanada & 0.457 & 0.385 & 0.530 & 0.539 & 0.583 & 0.498 & 0.484 & 0.577 & 0.518 & 0.615 \\
\hline 0 & Chile & m & $\mathrm{m}$ & m & m & m & m & 0.934 & 0.094 & 0.995 & 0.099 \\
\hline & Tschech. Rep. & 0.532 & 0.410 & 0.670 & 0.437 & 0.429 & 0.456 & 0.582 & 0.455 & 0.630 & 0.486 \\
\hline & Dänemark & 0.601 & 0.554 & 0.657 & 0.379 & 0.400 & 0.359 & 0.653 & 0.402 & 0.703 & 0.430 \\
\hline & Estland & $\mathrm{m}$ & $\mathrm{m}$ & $\mathrm{m}$ & $\mathrm{m}$ & $\mathrm{m}$ & $\mathrm{m}$ & 0.457 & 0.538 & 0.490 & 0.577 \\
\hline & Finnland & 0.400 & 0.452 & 0.348 & 0.445 & 0.435 & 0.465 & 0.429 & 0.485 & 0.462 & 0.520 \\
\hline & Frankreich & 0.541 & 0.568 & 0.519 & 0.471 & 0.487 & 0.462 & 0.587 & 0.497 & 0.631 & 0.528 \\
\hline & Deutschland & 0.445 & 0.404 & 0.494 & 0.518 & 0.554 & 0.482 & 0.482 & 0.543 & 0.517 & 0.586 \\
\hline & Griechenland & 1.029 & 0.927 & 1.133 & 0.192 & 0.240 & 0.149 & 1.099 & 0.206 & 1.163 & 0.221 \\
\hline & Ungarn & 0.640 & 0.586 & 0.699 & 0.374 & 0.387 & 0.370 & 0.680 & 0.397 & 0.723 & 0.428 \\
\hline & Island & 0.560 & 0.567 & 0.555 & 0.419 & 0.370 & 0.477 & 0.594 & 0.447 & 0.640 & 0.481 \\
\hline & Irland & 0.542 & 0.440 & 0.655 & 0.426 & 0.509 & 0.353 & 0.584 & 0.459 & 0.627 & 0.491 \\
\hline & Israel & $\mathrm{m}$ & $\mathrm{m}$ & $\mathrm{m}$ & $\mathrm{m}$ & $\mathrm{m}$ & $\mathrm{m}$ & 0.785 & 0.376 & 0.836 & 0.399 \\
\hline & Italien & 0.635 & 0.562 & 0.714 & 0.350 & 0.427 & 0.270 & 0.683 & 0.375 & 0.735 & 0.402 \\
\hline & Japan & 0.421 & 0.365 & 0.487 & 0.740 & 0.787 & 0.694 & 0.448 & 0.788 & 0.479 & 0.843 \\
\hline & Korea & 0.326 & 0.300 & 0.365 & 0.660 & 0.618 & 0.714 & 0.355 & 0.727 & 0.383 & 0.774 \\
\hline & Luxemburg & 0.555 & 0.607 & 0.509 & 0.377 & 0.445 & 0.312 & 0.603 & 0.397 & 0.652 & 0.426 \\
\hline & Mexiko & 0.998 & 0.998 & 0.999 & 0.062 & 0.088 & 0.038 & 1.079 & 0.064 & 1.154 & 0.067 \\
\hline & Niederlande & 0.473 & 0.446 & 0.504 & 0.622 & 0.720 & 0.522 & 0.507 & 0.659 & 0.541 & 0.698 \\
\hline & Neuseeland & 0.657 & 0.691 & 0.632 & 0.420 & 0.497 & 0.344 & 0.706 & 0.451 & 0.759 & 0.478 \\
\hline & Norwegen & 0.600 & 0.524 & 0.683 & 0.329 & 0.283 & 0.385 & 0.642 & 0.347 & 0.683 & 0.374 \\
\hline & Polen & 0.537 & 0.602 & 0.486 & 0.574 & 0.639 & 0.515 & 0.572 & 0.624 & 0.615 & 0.669 \\
\hline & Portugal & 0.516 & 0.483 & 0.556 & 0.458 & 0.531 & 0.387 & 0.566 & 0.482 & 0.608 & 0.508 \\
\hline & Slowak. Rep. & 0.691 & 0.698 & 0.694 & 0.286 & 0.331 & 0.243 & 0.721 & 0.319 & 0.771 & 0.343 \\
\hline & Slowenien & $\mathrm{m}$ & $\mathrm{m}$ & $\mathrm{m}$ & $\mathrm{m}$ & $\mathrm{m}$ & $\mathrm{m}$ & 0.711 & 0.491 & 0.767 & 0.520 \\
\hline & Spanien & 0.619 & 0.543 & 0.699 & 0.377 & 0.464 & 0.290 & 0.671 & 0.402 & 0.714 & 0.431 \\
\hline & Schweden & 0.696 & 0.661 & 0.735 & 0.296 & 0.297 & 0.302 & 0.757 & 0.324 & 0.814 & 0.346 \\
\hline & Schweiz & 0.414 & 0.278 & 0.555 & 0.636 & 0.672 & 0.606 & 0.446 & 0.682 & 0.478 & 0.730 \\
\hline & Türkei & 1.008 & 0.911 & 1.111 & 0.220 & 0.289 & 0.154 & 1.085 & 0.235 & 1.158 & 0.253 \\
\hline & Ver. Königreich & $\mathrm{m}$ & $\mathrm{m}$ & $\mathrm{m}$ & $\mathrm{m}$ & $\mathrm{m}$ & $\mathrm{m}$ & 0.575 & 0.317 & 0.628 & 0.348 \\
\hline & Ver. Staaten & 0.735 & 0.697 & 0.777 & 0.382 & 0.409 & 0.358 & 0.787 & 0.404 & 0.836 & 0.430 \\
\hline$\Xi$ & Albanien & $\mathrm{m}$ & $\mathrm{m}$ & $\mathrm{m}$ & $\mathrm{m}$ & $\mathrm{m}$ & $\mathrm{m}$ & $\mathrm{m}$ & $\mathrm{m}$ & 0.810 & 0.033 \\
\hline Е્ฮ & Argentinien & $\mathrm{m}$ & $\mathrm{m}$ & $\mathrm{m}$ & $\mathrm{m}$ & $\mathrm{m}$ & $\mathrm{m}$ & 0.906 & 0.019 & 0.970 & 0.021 \\
\hline క్ర్య & Brasilien & 0.900 & 1.042 & 0.773 & 0.068 & 0.081 & 0.059 & 0.968 & 0.072 & 1.031 & 0.075 \\
\hline . & Bulgarien & $\mathrm{m}$ & $\mathrm{m}$ & $\mathrm{m}$ & $\mathrm{m}$ & $\mathrm{m}$ & $\mathrm{m}$ & 0.777 & 0.230 & 0.830 & 0.245 \\
\hline$=$ & Kolumbien & $\mathrm{m}$ & $\mathrm{m}$ & $\mathrm{m}$ & $\mathrm{m}$ & $\mathrm{m}$ & $\mathrm{m}$ & 0.778 & 0.022 & 0.829 & 0.024 \\
\hline$\frac{1}{1}$ & Costa Rica & $\mathrm{m}$ & $\mathrm{m}$ & $\mathrm{m}$ & $\mathrm{m}$ & $\mathrm{m}$ & $\mathrm{m}$ & $\mathrm{m}$ & $\mathrm{m}$ & 1.179 & 0.043 \\
\hline वे & Kroatien & $\mathrm{m}$ & $\mathrm{m}$ & $\mathrm{m}$ & $\mathrm{m}$ & $\mathrm{m}$ & $\mathrm{m}$ & 0.804 & 0.248 & 0.859 & 0.263 \\
\hline : & Dubai (VAE) & $\mathrm{m}$ & $\mathrm{m}$ & $\mathrm{m}$ & $\mathrm{m}$ & $\mathrm{m}$ & $\mathrm{m}$ & $\mathrm{m}$ & $\mathrm{m}$ & 0.731 & 0.390 \\
\hline ఏँ & Hongkong (China) & 0.250 & 0.224 & 0.287 & 0.805 & 0.695 & 0.940 & 0.277 & 0.864 & 0.295 & 0.917 \\
\hline ฏ & Indonesien & 0.715 & 0.662 & 0.776 & 0.025 & 0.021 & 0.036 & 0.758 & 0.025 & 0.812 & 0.026 \\
\hline & Jordanien & $\mathrm{m}$ & $\mathrm{m}$ & $\mathrm{m}$ & $\mathrm{m}$ & $\mathrm{m}$ & $\mathrm{m}$ & 1.017 & 0.052 & 1.081 & 0.053 \\
\hline & Kasachstan & $\mathrm{m}$ & $\mathrm{m}$ & $\mathrm{m}$ & $\mathrm{m}$ & $\mathrm{m}$ & $\mathrm{m}$ & m & m & 1.216 & 0.060 \\
\hline & Lettland & 0.638 & 0.725 & 0.557 & 0.439 & 0.412 & 0.469 & 0.677 & 0.455 & 0.725 & 0.484 \\
\hline & Liechtenstein & 0.552 & 0.680 & 0.479 & 1.055 & 1.440 & 0.697 & 0.579 & 1.065 & 0.610 & 1.147 \\
\hline & Litauen & $\mathrm{m}$ & $\mathrm{m}$ & $\mathrm{m}$ & $\mathrm{m}$ & $\mathrm{m}$ & $\mathrm{m}$ & 0.863 & 0.337 & 0.927 & 0.364 \\
\hline & Macau (China) & 0.343 & 0.309 & 0.383 & 0.697 & 0.754 & 0.643 & 0.369 & 0.755 & 0.395 & 0.806 \\
\hline & Malaysia & $\mathrm{m}$ & $\mathrm{m}$ & $\mathrm{m}$ & $\mathrm{m}$ & $\mathrm{m}$ & $\mathrm{m}$ & $\mathrm{m}$ & $\mathrm{m}$ & 0.984 & 0.091 \\
\hline & Montenegro & $\mathrm{m}$ & $\mathrm{m}$ & $\mathrm{m}$ & $\mathrm{m}$ & $\mathrm{m}$ & $\mathrm{m}$ & 0.840 & 0.064 & 0.891 & 0.069 \\
\hline & Peru & $\mathrm{m}$ & $\mathrm{m}$ & $\mathrm{m}$ & $\mathrm{m}$ & $\mathrm{m}$ & $\mathrm{m}$ & $\mathrm{m}$ & $\mathrm{m}$ & 0.760 & 0.055 \\
\hline & Katar & $\mathrm{m}$ & $\mathrm{m}$ & $\mathrm{m}$ & $\mathrm{m}$ & $\mathrm{m}$ & $\mathrm{m}$ & 0.577 & 0.082 & 0.616 & 0.089 \\
\hline & Rumänien & $\mathrm{m}$ & $\mathrm{m}$ & $\mathrm{m}$ & $\mathrm{m}$ & $\mathrm{m}$ & $\mathrm{m}$ & 1.101 & 0.164 & 1.169 & 0.176 \\
\hline & Russ. Föderation & 0.804 & 0.890 & 0.723 & 0.344 & 0.321 & 0.375 & 0.871 & 0.363 & 0.933 & 0.392 \\
\hline & Serbien & m & $\mathrm{m}$ & $\mathrm{m}$ & $\mathrm{m}$ & $\mathrm{m}$ & $\mathrm{m}$ & 0.939 & 0.157 & 1.011 & 0.168 \\
\hline & Shanghai (China) & $\mathrm{m}$ & $\mathrm{m}$ & $\mathrm{m}$ & $\mathrm{m}$ & $\mathrm{m}$ & $\mathrm{m}$ & $\mathrm{m}$ & $\mathrm{m}$ & 0.194 & 0.776 \\
\hline & Singapur & $\mathrm{m}$ & $\mathrm{m}$ & $\mathrm{m}$ & $\mathrm{m}$ & $\mathrm{m}$ & $\mathrm{m}$ & $\mathrm{m}$ & $\mathrm{m}$ & 0.293 & 0.894 \\
\hline & Chinesisch Taipeh & $\mathrm{m}$ & $\mathrm{m}$ & $\mathrm{m}$ & $\mathrm{m}$ & $\mathrm{m}$ & $\mathrm{m}$ & 0.327 & 0.625 & 0.354 & 0.673 \\
\hline & Thailand & 0.911 & 1.048 & 0.810 & 0.085 & 0.063 & 0.108 & 0.974 & 0.093 & 1.039 & 0.104 \\
\hline & Tunesien & 0.804 & 0.643 & 0.955 & 0.056 & 0.040 & 0.074 & 0.857 & 0.059 & 0.911 & 0.062 \\
\hline & Ver. Arab. Emirate* & $\mathrm{m}$ & $\mathrm{m}$ & $\mathrm{m}$ & $\mathrm{m}$ & $\mathrm{m}$ & $\mathrm{m}$ & $\mathrm{m}$ & $\mathrm{m}$ & 0.942 & 0.112 \\
\hline & Uruguay & 0.817 & 0.793 & 0.846 & 0.065 & 0.105 & 0.035 & 0.881 & 0.069 & 0.944 & 0.075 \\
\hline
\end{tabular}

Anmerkung: Der Linking-Fehler wird empirisch berechnet, indem zu den Ergebnissen aller Schülerinnen und Schüler in PISA 2000, PISA 2003, PISA 2006 oder PISA 2009 eine Zufallsfehlerkomponente aus einer Normalverteilung hinzugefügt wird, mit einem Mittelwert von 0 und einer Standardabweichung, die den in Tabelle A5.1 dargelegten Linking-Fehlern entspricht. Der Prozentsatz der Schülerinnen und Schüler auf jeder Kompetenzstufe wird dann für jede der 500 Wiederholungsmessungen berechnet. Die Standardabweichung bei den beobachteten Koeffizienten ist das Ergebnis des hinzugefügten Messfehlers und entspricht dem aufgeführten Linking-Fehler.

* Vereinigte Arabische Emirate ohne Dubai.

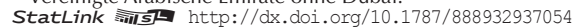


[Teil 2/3]

Tabelle A5.2 Linking-Fehler bei Vergleichen der Kompetenzstufen zwischen PISA 2012 und früheren Erhebungen

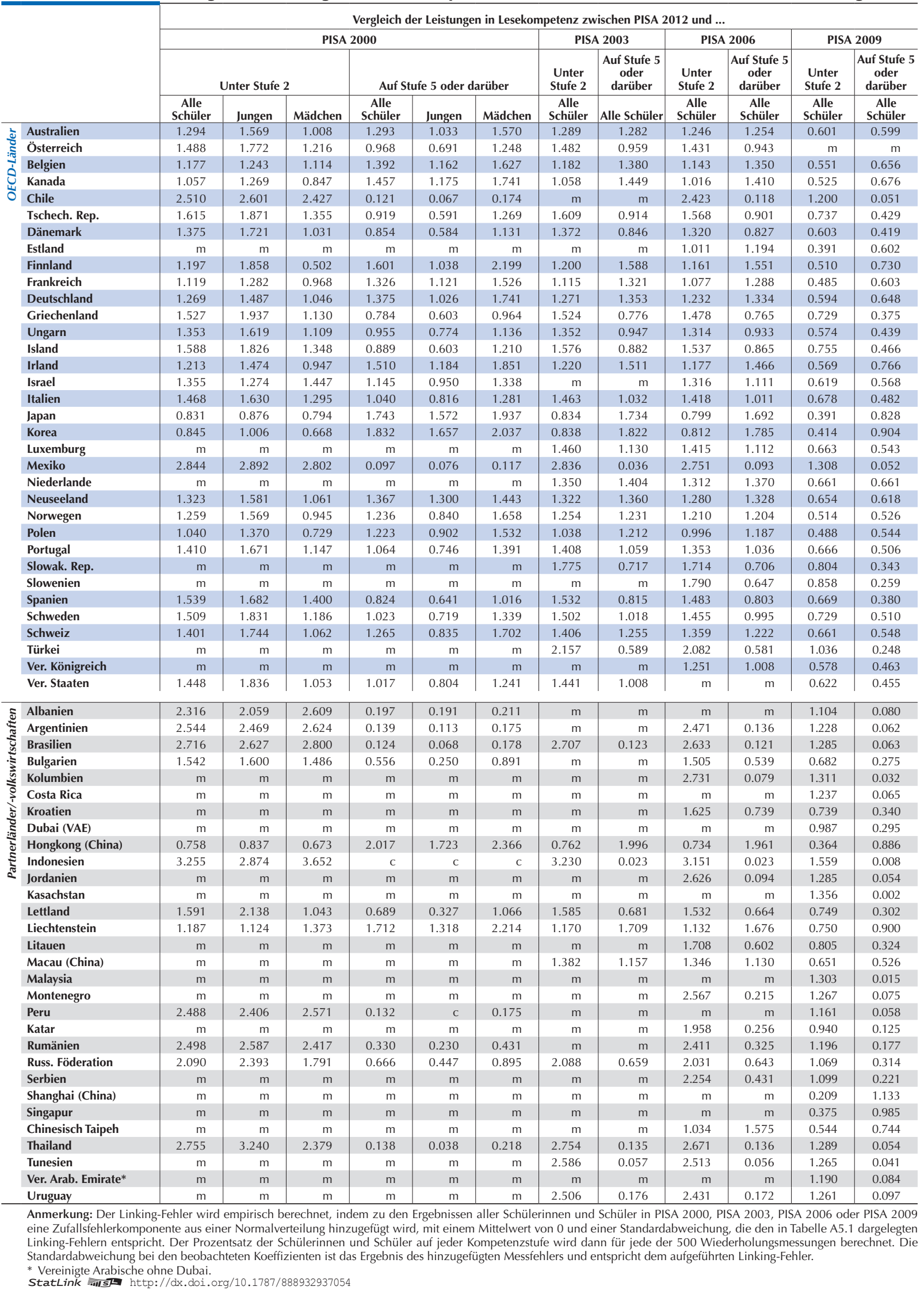


[Teil 3/3]

Tabelle A5.2 Linking-Fehler bei Vergleichen der Kompetenzstufen zwischen PISA 2012 und früheren Erhebungen

\begin{tabular}{|c|c|c|c|c|c|c|c|c|c|}
\hline & & \multicolumn{8}{|c|}{ Vergleich der Leistungen in Naturwissenschaften zwischen PISA 2012 und... } \\
\hline & & \multicolumn{6}{|c|}{ PISA 2006} & \multicolumn{2}{|c|}{ PISA 2009} \\
\hline & & \multicolumn{3}{|c|}{ Unter Stufe 2} & \multicolumn{3}{|c|}{ Auf Stufe 5 oder darüber } & \multirow{2}{*}{$\begin{array}{c}\text { Unter Stufe } 2 \\
\text { Alle Schüler }\end{array}$} & \multirow{2}{*}{\begin{tabular}{|c|}
$\begin{array}{c}\text { Auf Stufe } 5 \text { ode } \\
\text { darüber }\end{array}$ \\
Alle Schüler \\
\end{tabular}} \\
\hline & & Alle Schüler & Jungen & Mädchen & Alle Schüler & Jungen & Mädchen & & \\
\hline \multirow{34}{*}{ 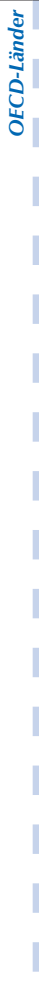 } & Australien & 0.702 & 0.699 & 0.708 & 0.816 & 0.779 & 0.855 & 0.419 & 0.486 \\
\hline & Österreich & 0.935 & 0.912 & 0.963 & 0.704 & 0.742 & 0.669 & $\mathrm{~m}$ & $\mathrm{~m}$ \\
\hline & Belgien & 0.805 & 0.748 & 0.867 & 0.767 & 0.764 & 0.772 & 0.451 & 0.433 \\
\hline & Kanada & 0.584 & 0.585 & 0.584 & 0.856 & 0.933 & 0.783 & 0.338 & 0.478 \\
\hline & Chile & 1.563 & 1.488 & 1.639 & 0.143 & 0.207 & 0.087 & 0.888 & 0.079 \\
\hline & Tschech. Rep. & 0.836 & 0.719 & 0.970 & 0.605 & 0.444 & 0.786 & 0.456 & 0.361 \\
\hline & Dänemark & 0.922 & 0.872 & 0.975 & 0.519 & 0.573 & 0.478 & 0.540 & 0.277 \\
\hline & Estland & 0.506 & 0.560 & 0.456 & 0.933 & 0.929 & 0.941 & 0.310 & 0.518 \\
\hline & Finnland & 0.457 & 0.518 & 0.398 & 1.040 & 0.864 & 1.236 & 0.259 & 0.585 \\
\hline & Frankreich & 0.830 & 0.761 & 0.899 & 0.634 & 0.718 & 0.562 & 0.489 & 0.326 \\
\hline & Deutschland & 0.717 & 0.676 & 0.768 & 0.892 & 0.970 & 0.814 & 0.430 & 0.501 \\
\hline & Griechenland & 1.222 & 1.308 & 1.146 & 0.279 & 0.342 & 0.224 & 0.722 & 0.165 \\
\hline & Ungarn & 1.073 & 1.186 & 0.971 & 0.606 & 0.677 & 0.542 & 0.639 & 0.365 \\
\hline & Island & 0.940 & 0.930 & 0.957 & 0.484 & 0.496 & 0.476 & 0.486 & 0.288 \\
\hline & Irland & 0.748 & 0.826 & 0.680 & 0.677 & 0.691 & 0.668 & 0.425 & 0.401 \\
\hline & Israel & 0.957 & 0.877 & 1.038 & 0.557 & 0.736 & 0.388 & 0.537 & 0.337 \\
\hline & Italien & 1.014 & 0.959 & 1.075 & 0.516 & 0.566 & 0.465 & 0.607 & 0.303 \\
\hline & Japan & 0.499 & 0.521 & 0.478 & 1.093 & 1.285 & 0.888 & 0.313 & 0.612 \\
\hline & Korea & 0.499 & 0.586 & 0.404 & 0.976 & 1.129 & 0.809 & 0.293 & 0.584 \\
\hline & Luxemburg & 0.947 & 0.751 & 1.156 & 0.650 & 0.603 & 0.705 & 0.548 & 0.386 \\
\hline & Mexiko & 2.072 & 1.952 & 2.190 & 0.022 & 0.028 & 0.017 & 1.195 & 0.014 \\
\hline & Niederlande & 0.879 & 0.668 & 1.106 & 0.911 & 0.968 & 0.856 & 0.541 & 0.548 \\
\hline & Neuseeland & 0.796 & 0.677 & 0.923 & 0.803 & 0.900 & 0.707 & 0.433 & 0.451 \\
\hline & Norwegen & 0.864 & 0.812 & 0.921 & 0.551 & 0.521 & 0.585 & 0.486 & 0.298 \\
\hline & Polen & 0.620 & 0.708 & 0.545 & 0.813 & 0.795 & 0.835 & 0.334 & 0.484 \\
\hline & Portugal & 0.953 & 0.928 & 0.982 & 0.422 & 0.442 & 0.407 & 0.522 & 0.221 \\
\hline & Slowak. Rep. & 1.013 & 1.100 & 0.924 & 0.424 & 0.463 & 0.386 & 0.566 & 0.253 \\
\hline & Slowenien & 0.918 & 1.222 & 0.600 & 0.758 & 0.832 & 0.685 & 0.542 & 0.414 \\
\hline & Spanien & 0.884 & 0.840 & 0.932 & 0.501 & 0.591 & 0.411 & 0.517 & 0.286 \\
\hline & Schweden & 0.973 & 0.918 & 1.033 & 0.454 & 0.447 & 0.466 & 0.560 & 0.254 \\
\hline & Schweiz & 0.740 & 0.725 & 0.760 & 0.712 & 0.665 & 0.765 & 0.443 & 0.389 \\
\hline & Türkei & 1.492 & 1.514 & 1.480 & 0.246 & 0.296 & 0.203 & 0.870 & 0.130 \\
\hline & Ver. Königreich & 0.718 & 0.648 & 0.790 & 0.808 & 0.862 & 0.768 & 0.411 & 0.452 \\
\hline & Ver. Staaten & 0.938 & 0.946 & 0.938 & 0.507 & 0.546 & 0.476 & 0.527 & 0.288 \\
\hline \multirow{30}{*}{ 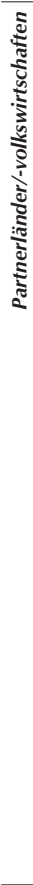 } & Albanien & $\mathrm{m}$ & $\mathrm{m}$ & $\mathrm{m}$ & $\mathrm{m}$ & $\mathrm{m}$ & $\mathrm{m}$ & 0.808 & 0.051 \\
\hline & Argentinien & 1.800 & 1.660 & 1.941 & 0.053 & 0.066 & 0.047 & 1.025 & 0.027 \\
\hline & Brasilien & 1.755 & 1.616 & 1.882 & 0.038 & 0.049 & 0.034 & 1.019 & 0.017 \\
\hline & Bulgarien & 1.207 & 1.248 & 1.169 & 0.264 & 0.249 & 0.286 & 0.723 & 0.149 \\
\hline & Kolumbien & 1.891 & 2.043 & 1.768 & 0.012 & 0.022 & 0.004 & 1.111 & 0.005 \\
\hline & Costa Rica & $\mathrm{m}$ & $\mathrm{m}$ & $\mathrm{m}$ & $\mathrm{m}$ & m & $\mathrm{m}$ & 1.026 & 0.036 \\
\hline & Kroatien & 0.965 & 1.036 & 0.895 & 0.456 & 0.465 & 0.452 & 0.572 & 0.284 \\
\hline & Dubai (VAE) & $\mathrm{m}$ & $\mathrm{m}$ & $\mathrm{m}$ & $\mathrm{m}$ & $\mathrm{m}$ & $\mathrm{m}$ & 0.720 & 0.182 \\
\hline & Hongkong (China) & 0.299 & 0.304 & 0.296 & 1.454 & 1.556 & 1.341 & 0.167 & 0.873 \\
\hline & Indonesien & 1.740 & 1.763 & 1.728 & c & $\mathrm{c}$ & c & 0.932 & c \\
\hline & Jordanien & 1.669 & 1.530 & 1.808 & 0.051 & 0.057 & 0.053 & 0.936 & 0.028 \\
\hline & Kasachstan & $\mathrm{m}$ & $\mathrm{m}$ & $\mathrm{m}$ & $\mathrm{m}$ & m & $\mathrm{m}$ & 1.048 & 0.025 \\
\hline & Lettland & 0.953 & 1.016 & 0.898 & 0.460 & 0.470 & 0.457 & 0.566 & 0.288 \\
\hline & Liechtenstein & 0.597 & 0.867 & 0.380 & 0.728 & 0.928 & 0.584 & 0.269 & 0.423 \\
\hline & Litauen & 0.869 & 0.924 & 0.819 & 0.501 & 0.382 & 0.628 & 0.489 & 0.320 \\
\hline & Macau (China) & 0.685 & 0.640 & 0.742 & 0.656 & 0.820 & 0.494 & 0.434 & 0.383 \\
\hline & Malaysia & $\mathrm{m}$ & $\mathrm{m}$ & m & $\mathrm{m}$ & m & $\mathrm{m}$ & 1.058 & 0.026 \\
\hline & Montenegro & 1.689 & 1.595 & 1.793 & 0.067 & 0.071 & 0.070 & 1.035 & 0.042 \\
\hline & Peru & $\mathrm{m}$ & $\mathrm{m}$ & $\mathrm{m}$ & $\mathrm{m}$ & $\mathrm{m}$ & $\mathrm{m}$ & 0.822 & 0.000 \\
\hline & Katar & 1.126 & 0.940 & 1.328 & 0.132 & 0.124 & 0.143 & 0.657 & 0.071 \\
\hline & Rumänien & 1.861 & 1.923 & 1.810 & 0.129 & 0.129 & 0.130 & 1.122 & 0.094 \\
\hline & Russ. Föderation & 1.298 & 1.333 & 1.267 & 0.398 & 0.390 & 0.407 & 0.801 & 0.230 \\
\hline & Serbien & 1.482 & 1.599 & 1.369 & 0.117 & 0.115 & 0.125 & 0.844 & 0.061 \\
\hline & Shanghai (China) & m & m & $\mathrm{m}$ & m & m & m & 0.150 & 1.006 \\
\hline & Singapur & $\mathrm{m}$ & $\mathrm{m}$ & $\mathrm{m}$ & $\mathrm{m}$ & $\mathrm{m}$ & $\mathrm{m}$ & 0.307 & 0.650 \\
\hline & Chinesisch Taipeh & 0.751 & 0.742 & 0.763 & 0.764 & 0.788 & 0.747 & 0.480 & 0.426 \\
\hline & Thailand & 1.781 & 1.899 & 1.696 & 0.135 & 0.092 & 0.172 & 1.060 & 0.078 \\
\hline & Tunesien & 1.794 & 1.703 & 1.877 & 0.022 & 0.033 & 0.021 & 1.049 & 0.014 \\
\hline & Ver. Arab. Emirate* & $\mathrm{m}$ & $\mathrm{m}$ & m & $\mathrm{m}$ & m & $\mathrm{m}$ & 0.758 & 0.075 \\
\hline & Uruguay & 1.352 & 1.225 & 1.468 & 0.096 & 0.157 & 0.049 & 0.760 & 0.052 \\
\hline
\end{tabular}

Anmerkung: Der Linking-Fehler wird empirisch berechnet, indem zu den Ergebnissen aller Schülerinnen und Schüler in PISA 2000, PISA 2003, PISA 2006 oder PISA 2009 eine Zufallsfehlerkomponente aus einer Normalverteilung hinzugefügt wird, mit einem Mittelwert von 0 und einer Standardabweichung, die den in Tabelle A5.1 dargelegten Linking-Fehlern entspricht. Der Prozentsatz der Schülerinnen und Schüler auf jeder Kompetenzstufe wird dann für jede der 500 Wiederholungsmessungen berechnet. Die Standardabweichung bei den beobachteten Koeffizienten ist das Ergebnis des hinzugefügten Messfehlers und entspricht dem aufgeführten Linking-Fehler.

* Vereinigte Arabische Emirate ohne Dubai.

StatLink ailst http://dx.doi.org/10.1787/888932937054 
[Teil 1/1]

Linking-Fehler bei Vergleichen der annualisierten und kurvilinearen Veränderung zwischen PISA 2012 Tabelle A5.3 und früheren Erhebungen

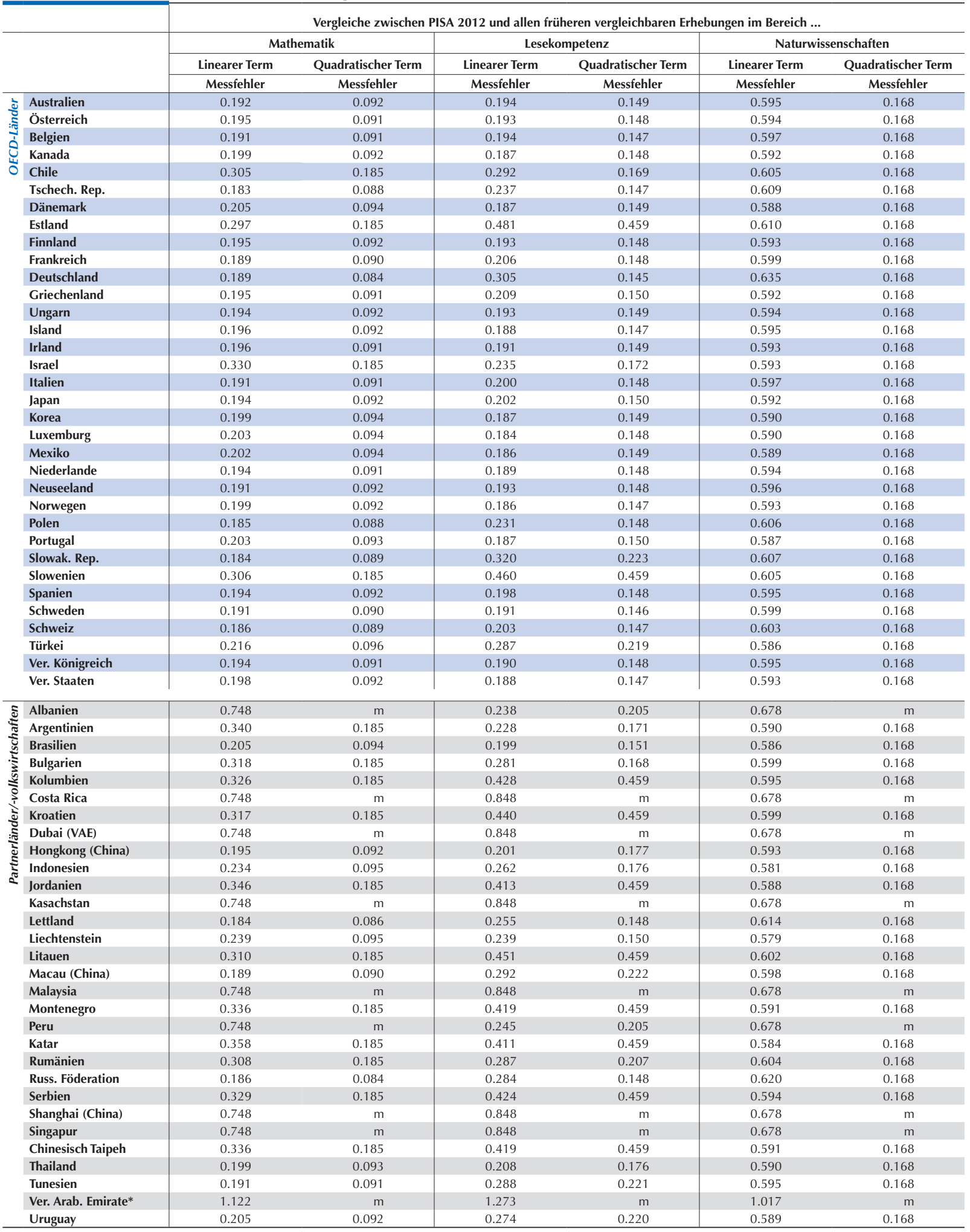

Anmerkung: Der Linking-Fehler wird empirisch berechnet, indem zu den Ergebnissen aller Schülerinnen und Schüler in PISA 2000, PISA 2003, PISA 2006 oder PISA 2009 eine Zufallsfehlerkomponente aus einer Normalverteilung hinzugefügt wird, mit einem Mittelwert von 0 und einer Standardabweichung, die den in Tabelle A5.1 dargelegten Linking-Fehlern entspricht. Der lineare und quadratische Term eines Regressionsmodells wird dann für jede der 500 Wiederholungsmessungen berechnet. Die Standardabweichung bei den beobachteten Koeffizienten ist das Ergebnis des hinzugefügten Messfehlers und entspricht dem aufgeführten Linking-Fehler.

* Vereinigte Arabische Emirate ohne Dubai.

StatLink 체이 http://dx.doi.org/10.1787/888932937054 
Ein zweiter, in PISA wiedergegebener Analysekatalog bezieht sich auf annualisierte Leistungsveränderungen. Bei der annualisierten Veränderung handelt es sich um die jahresdurchschnittliche Veränderungsrate, die im Lauf der PISA-Teilnahme eines Landes/ einer Volkswirtschaft beobachtet wurde. Die annualisierte Veränderung entspricht der durchschnittlichen Rate, mit der sich die Durchschnittsergebnisse eines Landes/einer Volkswirtschaft in den Bereichen Mathematik, Lesekompetenz und Naturwissenschaften im Verlauf der Teilnahme an den PISA-Erhebungen verändert haben. So deutet eine positive annualisierte Veränderung von $x$ Punkten darauf hin, dass das Land/die Volkswirtschaft seine/ihre Leistungen seit der ersten PISA-Erhebung, aus der vergleichbare Ergebnisse vorliegen, um x Punkte pro Jahr verbessert hat. Bei Ländern und Volkswirtschaften, die nur an zwei PISA-Erhebungen teilgenommen haben, entspricht die annualisierte Veränderung der Differenz zwischen den beiden Erhebungen, dividiert durch die Zahl der Jahre, die zwischen den Erhebungen verstrichen sind.

Die annualisierte Leistungsveränderung wird anhand einer OLS-Regression auf individueller Ebene der Form

$$
\operatorname{PISA}_{i}=\beta_{0}+\beta_{1} \text { year }_{i}+\varepsilon_{i}
$$

berechnet, in der PISA für die Punktzahl eines Schülers $i$ in Mathematik, Lesekompetenz oder Naturwissenschaften steht, year ${ }_{i}$ für das Jahr, in dem der Schüler $i$ an der PISA-Erhebung teilnahm und $\varepsilon_{i}$ für einen Fehlerterm steht, der die Abweichung der Leistungen von Schüler $i$ vom Mittelwert der Gruppe wiedergibt. Bei dieser Spezifikation gibt die Schätzung für $\beta_{1}$ die annualisierte Veränderungsrate an. Ebenso wie bei Vergleichen zwischen zwei PISA-Erhebungen ein Linking-Fehler hinzugerechnet wird, enthalten auch die Standardfehler für $\beta_{1}$ einen Linking-Fehler:

$$
\sigma_{\text {link }}\left(\beta_{1}\right)=\sqrt{\sigma^{2}\left(\beta_{1}\right)+\text { error }_{\text {annual }}^{2}}
$$

wobei error $_{\text {annual }}$ dem Linking-Fehler entspricht, der in einem Regressionsmodell mit dem linearen Term assoziiert wird. Dieser ist in Tabelle A5.3 dargestellt.

Die annualisierte Veränderung ist eine robustere Messgröße der Fortschritte, die ein Land/eine Volkswirtschaft bei der Verbesserung der Bildungsergebnisse erzielt hat, da sie auf Informationen aus allen Erhebungen basiert. Sie reagiert dementsprechend weniger auf anormale Messungen, die die PISA-Trends eines Landes/einer Volkswirtschaft verändern könnten, wenn die Ergebnisse nur zwischen zwei Erhebungen verglichen werden. Berechnet wird die annualisierte Veränderung als die Linie, die die Leistungsveränderungen eines Landes/einerVolkswirtschaft im Lauf der PISA-Teilnahme am besten darstellt. Die annualisierteVeränderung ergibt sich aus der Regression des Jahres der Teilnahme der einzelnen Schülerinnen und Schüler an PISA auf ihre PISA-Ergebnisse. Die annualisierte Veränderung trägt auch der Tatsache Rechnung, dass der Zeitraum zwischen zwei PISA-Erhebungen in einigen Ländern und Volkswirtschaften weniger als 3 Jahre beträgt. Das ist für jene Länder und Volkswirtschaften der Fall, die im Rahmen von PISA+ an den PISA-Erhebungen 2000 oder 2009 teilnahmen: Sie führten die jeweilige Erhebung statt in den Jahren 2000 bzw. 2009 im Jahr 2001 oder 2002 bzw. im Jahr 2010 durch. In Abbildung A5.1 wird der Wert der annualisierten Veränderung der Mathematikleistungen mit der zwischen PISA 2012 und PISA 2003 bei den Mathematikergebnissen beobachteten Differenz verglichen. Die Abbildungen A5.2 und A5.3 liefern dieselben Informationen für die Bereiche Lesekompetenz und Naturwissenschaften: Dort wird die annualisierte Leistungsveränderung mit der Differenz zwischen PISA 2012 und PISA 2000 bzw. PISA 2006 verglichen. Im Allgemeinen und insbesondere beim Vergleich der Leistungen im Bereich Naturwissenschaften zwischen PISA 2006 und PISA 2012 liefert die annualisierte Veränderung ein Ergebnis, das dem Leistungsunterschied zwischen zwei Erhebungen ähnelt. Sobald mehr Erhebungen berücksichtigt werden, beginnt die annualisierte Veränderung vom beobachteten Trend abzuweichen, wodurch sich ein vollständigeres Bild der von einem Land/einer Volkswirtschaft in PISA erzielten Fortschritte ergibt.

\section{Vergleich der Items und nicht leistungsbezogenen Skalen in den verschiedenen PISA-Erhebungsrunden}

Um Informationen über die Merkmale der Schülerinnen und Schüler und der Schulen zu sammeln, werden sowohl die Schülerinnen und Schüler als auch die Schulen in PISA gebeten, einen Hintergrundfragebogen auszufüllen. Da einige Fragen in PISA 2003 und PISA 2012 unverändert blieben, ist ein Vergleich der Antworten auf diese Fragen im Zeitverlauf möglich. In diesem Bericht wurden für Trendanalysen nur Fragen verwendet, deren Wortlaut gleich geblieben ist, Fragen mit geringfügigen oder großen Änderungen im Wortlaut wurden im Zeitverlauf nicht verglichen, weil unmöglich festzustellen ist, ob beobachtete Änderungen in einer Antwort auf Änderungen im gemessenen Konstrukt oder auf Änderungen in der Art, wie das Konstrukt gemessen wird, zurückzuführen sind.

Darüber hinaus werden, wie in Anhang A1 beschrieben, Fragebogen-Items in PISA für die Konstruktion von Indizes verwendet. Wenn die für die Konstruktion von Indizes verwendeten Fragen in PISA 2003 und PISA 2012 genau gleich geblieben sind, werden die entsprechenden Indizes verglichen. In PISA werden zwei Arten von Indizes verwendet: einfache Indizes und Skalenindizes.

Durch einfache Indizes wird eine Reihe von Antworten auf Fragebogen-Items umkodiert. Für Trendanalysen werden die in PISA 2003 beobachteten Werte ebenso wie einfache Antworten auf Fragebogen-Items direkt mit PISA 2012 verglichen. Dies gilt für Indizes wie die Schüler/Lehrer-Quote und die Einteilung in Leistungsgruppen in Mathematik.

Skalenindizes hingegen basieren auf WLE-Schätzungen, die umskaliert werden müssen, um über mehrere PISA-Erhebungsrunden vergleichbar zu sein. Skalenindizes wie der PISA-Index des wirtschaftlichen, sozialen und kulturellen Status, der Index des Zugehörig- 
- Abbildung A5.1 -

Annualisierte Veränderung der Mathematikleistungen seit PISA 2003 und beobachtete Leistungsdifferenz zwischen PISA 2012 und PISA 2003

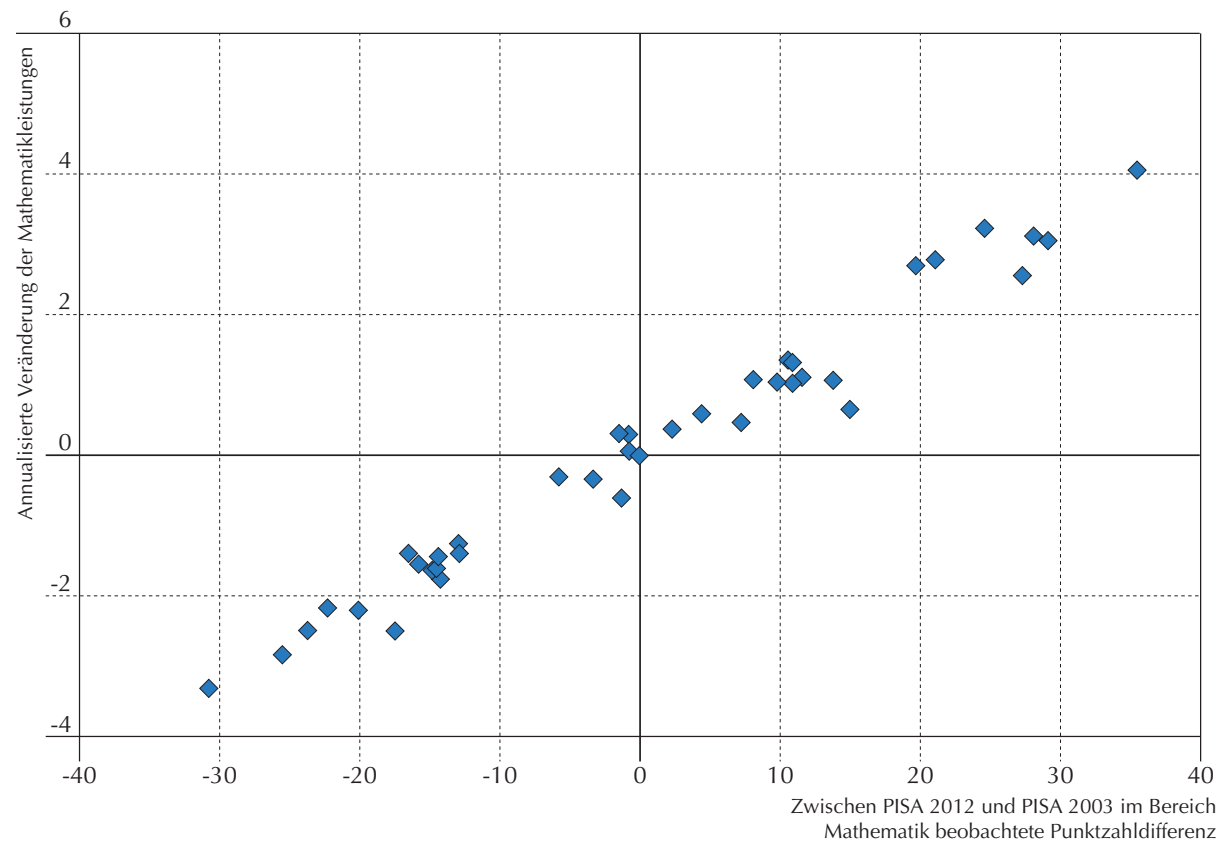

Anmerkung: Die annualisierte Veränderung entspricht der jahresdurchschnittlichen Veränderung der PISA-Punktzahl von der ersten PISA-Teilnahme eines Landes/einer Volkswirtschaft bis zur Teilnahme an PISA 2012. Dabei wird jede PISA-Teilnahme eines Landes/einer Volkswirtschaft berücksichtigt. Quelle: OECD, PISA-2012-Datenbank, Tabelle 1.2.3b.

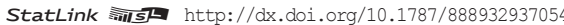

- Abbildung A5.2 "

Annualisierte Veränderung der Leistungen in Lesekompetenz seit PISA 2000 und beobachtete Leistungsdifferenz zwischen PISA 2012 und PISA 2000

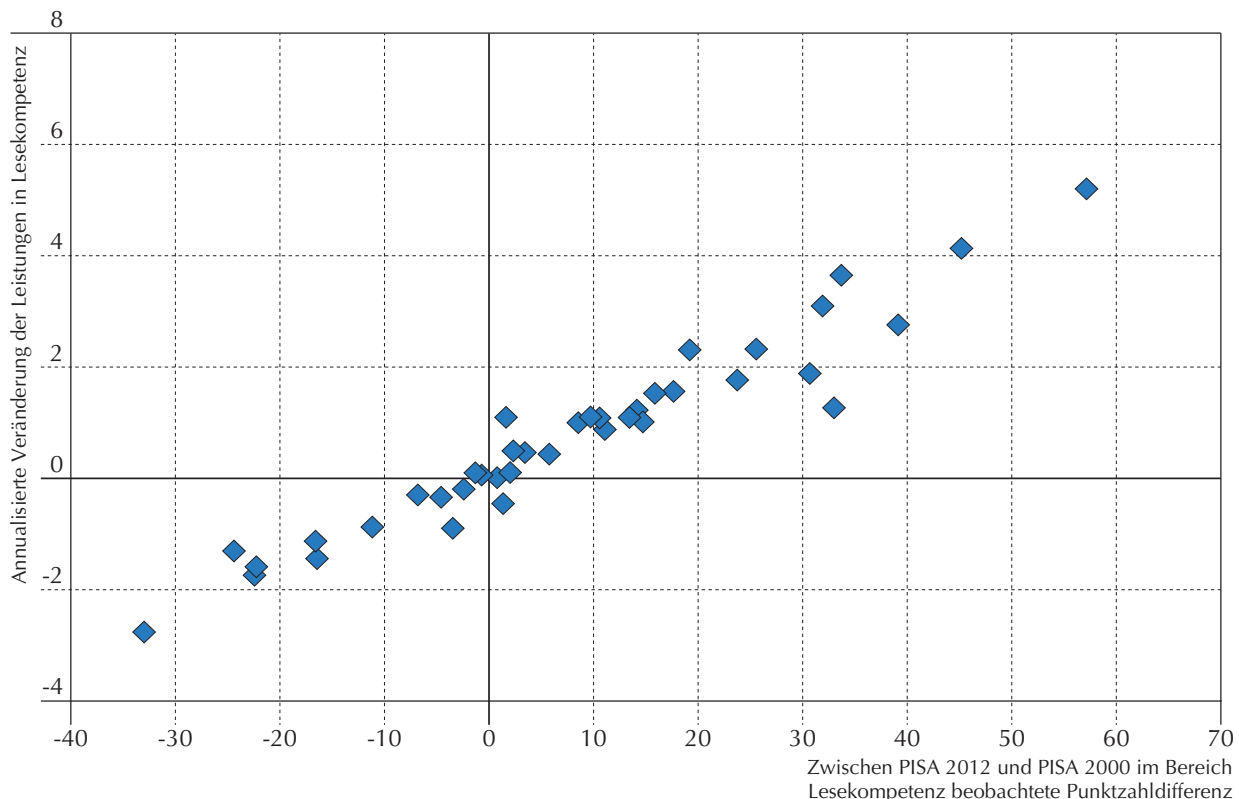

Anmerkung: Die annualisierte Veränderung entspricht der jahresdurchschnittlichen Veränderung der PISA-Punktzahl von der ersten PISA-Teilnahme eines Landes/einer Volkswirtschaft bis zur Teilnahme an PISA 2012. Dabei wird jede PISA-Teilnahme eines Landes/einer Volkswirtschaft berücksichtigt. Quelle: OECD, PISA-2012-Datenbank, Tabelle I.4.3b.

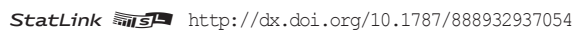


- Abbildung A5.3 "

Annualisierte Veränderung der Leistungen in Naturwissenschaften seit PISA 2006 und beobachtete Leistungsdifferenz zwischen PISA 2012 und PISA 2006

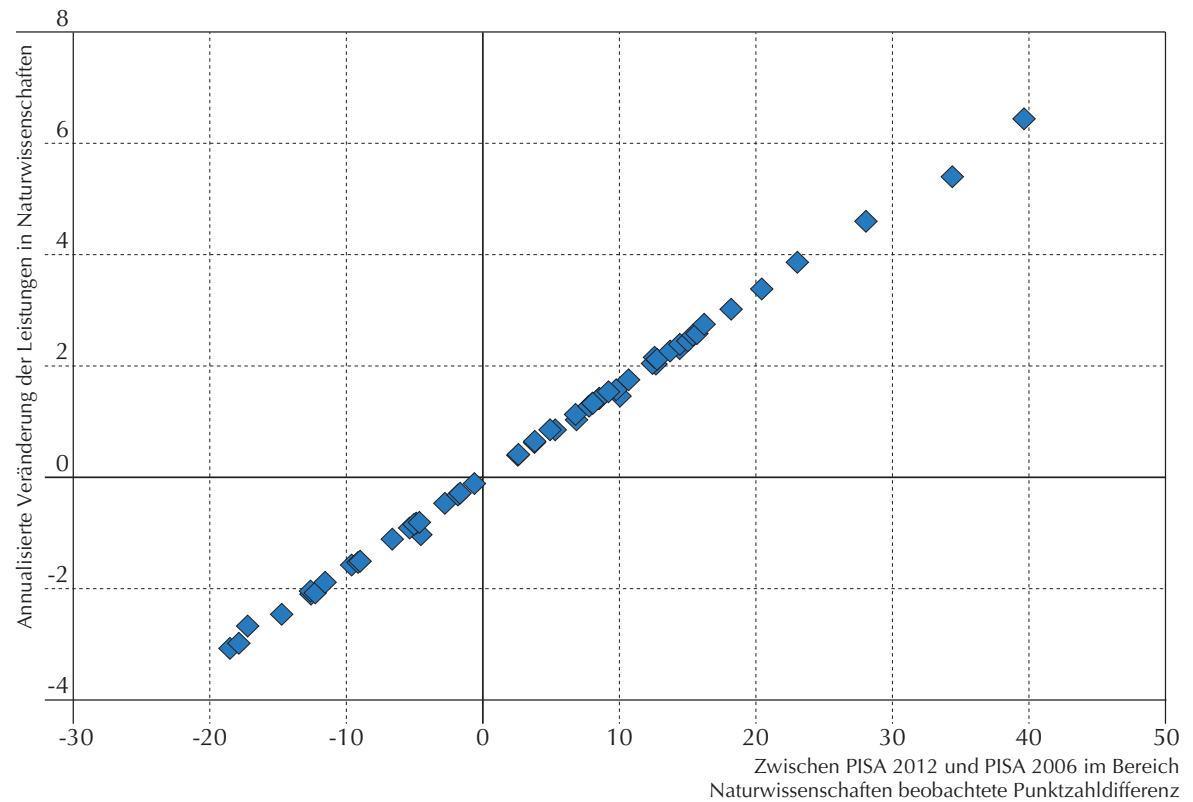

Anmerkung: Die annualisierte Veränderung entspricht der jahresdurchschnittlichen Veränderung der PISA-Punktzahl von der ersten PISA-Teilnahme eines Landes/einer Volkswirtschaft bis zur Teilnahme an PISA 2012. Dabei wird jede PISA-Teilnahme eines Landes/einer Volkswirtschaft berücksichtigt Quelle: OECD, PISA-2012-Datenbank, Tabelle I.5.3b.

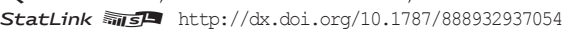

keitsgefühls, der Index der Einstellung zur Schule, der Index der intrinsischen Motivation für Lernen im Bereich Mathematik, der Index der instrumentellen Motivation für Lernen im Bereich Mathematik, der Index der Selbstwirksamkeit in Mathematik, der Index des Selbstkonzepts in Mathematik, der Index der Mathematikangst, der Index des Lehrermangels, der Index der räumlichen Bedingungen, der Index der Qualität der Ausstattung mit Lehr- und Sachmitteln, der Index der Schuldisziplin, der Index der Lehrer-SchülerBeziehungen, der Index der Arbeitshaltung der Lehrkräfte, der Index der schülerbezogenen Faktoren für das Schulklima und der Index der lehrkräftebezogenen Faktoren für das Schulklima wurden in PISA 2012 so skaliert, dass der OECD-Durchschnittswert gleich 0 und die durchschnittliche Standardabweichung in den OECD-Ländern gleich 1 gesetzt wurde. In PISA 2003 wurden dieselben Skalen so skaliert, dass der OECD-Durchschnitt gleich 0 und die Standardabweichung gleich 1 gesetzt wurde. Da die in Lernen für die Welt von morgen: Erste Ergebnisse von PISA 2003 (OECD, 2004) aufgeführten Werte auf unterschiedlichen Skalen basieren, können sie nicht mit den in diesem Band aufgeführten Werten verglichen werden. Um diese Skalenindizes vergleichbar zu machen, wurden die Werte von 2003 unter Verwendung der Parameterschätzungen von PISA 2012 auf den Wertebereich der Skala von 2012 umskaliert.

Um Veränderungen bei diesen Items und Skalen zu evaluieren, werden Schätzwertänderungen zwischen zwei Erhebungen, in der Regel PISA 2003 und PISA 2012, in den Analysen aufgeführt. Vergleiche zwischen zwei Erhebungen (z.B. die Veränderungen eines Landes/einer Volkswirtschaft beim Index der Mathematikangst zwischen PISA 2003 und PISA 2012 oder die Veränderungen bei diesem Index für eine Untergruppe) werden berechnet als:

$$
\Delta_{2012, t}=P I S A_{2012}-P I S A_{t}
$$

wobei $\Delta_{2012, t}$ für die Differenz beim Index zwischen PISA 2012 und einer früheren Erhebung, PISA 2012 für den in PISA 2012 beobachteten Indexwert und $P I S A_{t}$ für den in einer früheren Erhebung (2000, 2003, 2006 oder 2009) verzeichneten Indexwert steht. Der Standardfehler der Leistungsveränderung $\sigma\left(\Delta_{2012-t}\right)$ entspricht:

$$
\sigma\left(\Delta_{2012-t}\right)=\sqrt{\sigma_{2012}^{2}+\sigma_{t}^{2}}
$$

wobei $\sigma_{2012}$ für den in PISA 2012 und $\sigma_{t}$ für den in PISA $A_{t}$ beobachteten Standardfehler steht. Diese Vergleiche fußen auf einem identischen Aufgabenkatalog; es besteht keine Ungewissheit in Bezug auf die Auswahl der Aufgaben für Equating-Zwecke, so dass kein LinkingFehler hinzugerechnet werden muss. 
Wenngleich für Trendvergleiche nur Skalenindizes valide sind, die in PISA 2003 und PISA 2012 dieselben Items zu Grunde legten, bedeutet das nicht, dass Indizes aus PISA 2012, die sich auf genau dieselben Items erstrecken wie in PISA 2003 sowie neue FragebogenItems, nicht mit Indizes aus PISA 2003 verglichen werden können, denen ein kleinerer Item-Pool zu Grunde lag. In diesen Fällen wurden beispielsweise die Trendanalysen zum Index des Zugehörigkeitsgefüh/s durchgeführt, indem in PISA 2003 Items, die im Kontext von PISA 2012 gestellt wurden, aber nicht im Schülerfragebogen zu PISA 2003 enthalten waren, als Fehlwerte behandelt wurden. Während also der Skalierung des Index des Zugehörigkeitsgefühls in PISA 2012 die Gesamtheit der Informationen zu Grunde lag, wurde der Index des Zugehörigkeitsgefüh/s aus PISA 2003 unter der Annahme skaliert, dass, wenn die Items von 2012, die 2003 fehlten, 2003 effektiv gestellt worden wären, der Gesamtindex und die Indexvarianz genauso ausgefallen wären, wie sie bei den gemeinsamen Items von 2003 beobachtet wurden. Das ist insofern eine vertretbare Annahme, als sowohl in PISA 2003 als auch in PISA 2012 die für die Konstruktion der Skala verwendeten Fragebogen-Items Faktoren sind, die einen besonderen Einfluss auf die Konstruktion der Skala haben.

\section{OECD-Durchschnitt}

Im gesamten Bericht fungiert der OECD-Durchschnitt als Referenzgröße. Berechnet wird er als der Durchschnitt der OECD-Länder, in dem alle Länder gleich gewichtet sind. Einige OECD-Länder haben an bestimmten Erhebungen nicht teilgenommen, andere OECDLänder verfügen für einige Erhebungen nicht über vergleichbare Ergebnisse, wiederum andere haben bestimmte Fragen nicht in ihre Fragebogen aufgenommen oder diese von Erhebung zu Erhebung erheblich geändert. Aus diesem Grund ist der OECD-Durchschnitt in Tabellen und Abbildungen, die Trendentwicklungen darstellen, als erhebungsspezifisch angegeben, d.h. er erstreckt sich jeweils nur auf die Länder, für die in der jeweiligen Erhebung vergleichbare Informationen zur Verfügung stehen. Somit umfasst der „OECDDurchschnitt für 2003“ nur jene OECD-Länder, die über vergleichbare Informationen seit der Erhebung 2003 verfügen, selbst wenn er sich auf Ergebnisse bezieht, die auch Gegenstand der PISA-Erhebung 2012 sind und zu denen mehr Länder vergleichbare Informationen haben. Diese Einschränkung schafft valide Vergleichsmöglichkeiten des OECD-Durchschnitts im Zeitverlauf.

\section{Literaturverzeichnis}

OECD (erscheint demnächst), PISA 2012 Technical Report, PISA, OECD Publishing.

OECD (2004), Lernen für die Welt von morgen: Erste Ergebnisse von PISA 2003, PISA, W. Bertelsmann Verlag, Bielefeld. 


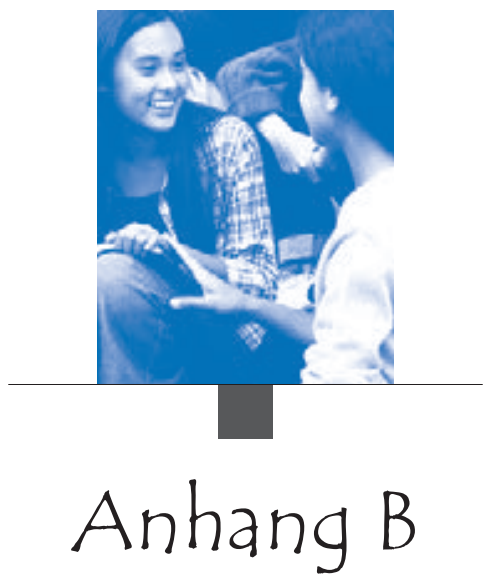

PISA-2012-ERGEBNISSE

Alle Tabellen in Anhang B sind online verfügbar

Anhang B1: Ergebnisse für Länder und Volkswirtschaften http://dx.doi.org/10.1787/888932964908 http://dx.doi.org/10.1787/888932964927 http://dx.doi.org/10.1787/888932964946

Anhang B2: Ergebnisse für einzelne Regionen innerhalb der Länder (nur auf Englisch verfügbar) http://dx.doi.org/10.1787/888932964965

Anhang B3: Liste der online verfügbaren Tabellen (nur auf Englisch verfügbar)

Die Nummerierung der Tabellen ist nicht durchlaufend, da einige Tabellen nur online erscheinen und in dieser Publikation nicht enthalten sind.

\section{Anmerkungen zu Zypern:}

Anmerkung der Türkei: Die Informationen in diesem Bericht zu „Zypern“ beziehen sich auf den südlichen Teil der Insel. Es existiert keine den türkischen und den griechischen Bevölkerungsteil der Insel gemeinsam vertretende Instanz. Die Türkei erkennt die Türkische Republik Nordzypern (TRNZ) an. Bis im Rahmen der Vereinten Nationen eine dauerhafte und gerechte Lösung gefunden ist, wird sich die Türkei ihre Stellungnahme zur "Zypernfrage" vorbehalten.

Anmerkung aller in der OECD vertretenen EU-Mitgliedstaaten und der Europäischen Union: Die Republik Zypern wird von allen Mitgliedern der Vereinten Nationen mit Ausnahme der Türkei anerkannt. Die Informationen in diesem Bericht beziehen sich auf das Gebiet, das sich de facto unter der Kontrolle der Regierung der Republik Zypern befindet.

Die statistischen Daten für Israel wurden von den zuständigen israelischen Stellen bereitgestellt, die für sie verantwortlich zeichnen. Die Verwendung dieser Daten durch die OECD erfolgt unbeschadet des völkerrechtlichen Status der Golanhöhen, von Ost-Jerusalem und der israelischen Siedlungen im Westjordanland. 


\section{ANHANG B1}

\section{ERGEBNISSE FÜR LÄNDER UND VOLKSWIRTSCHAFTEN}

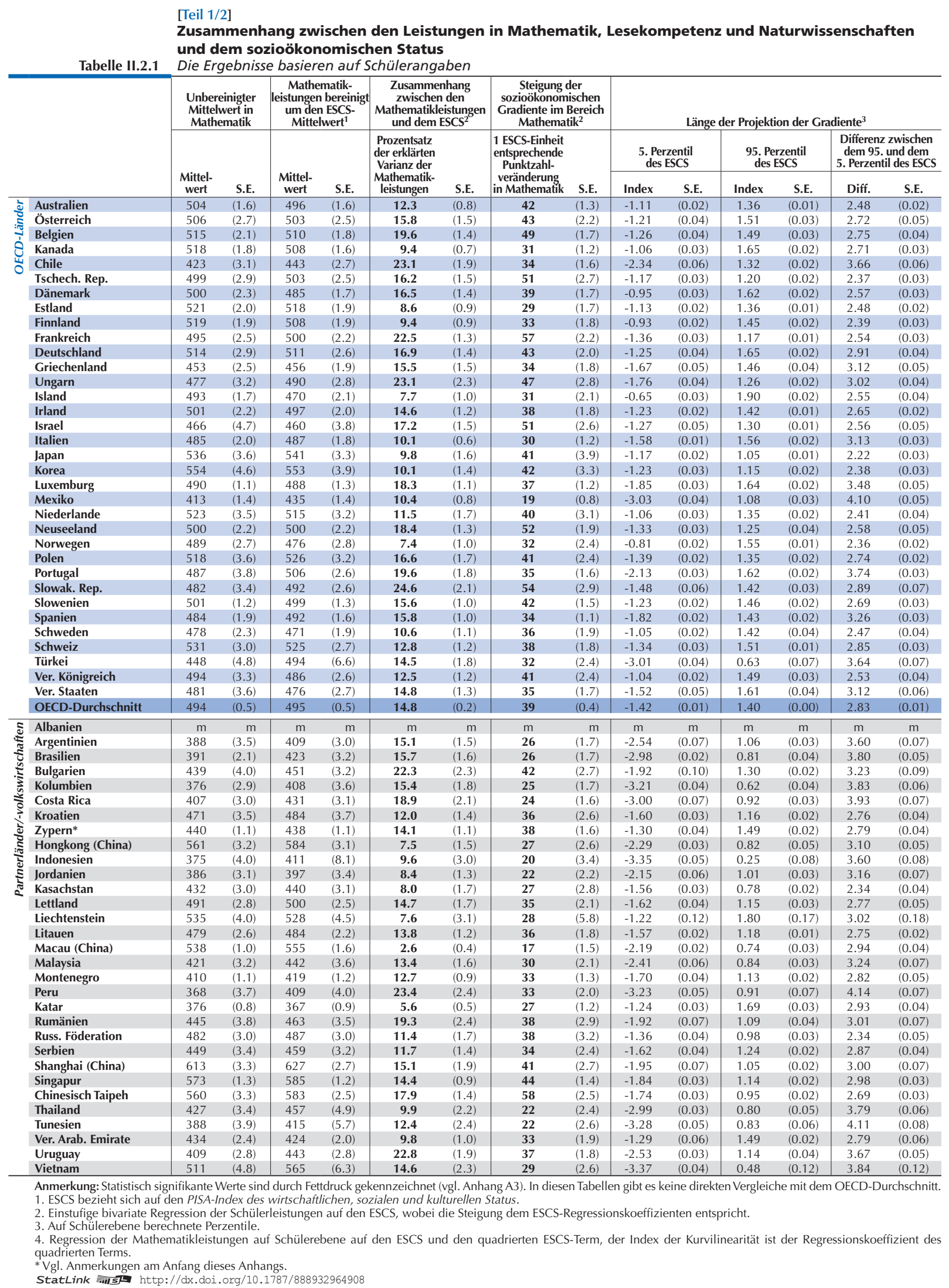


[Teil 2/2]

Zusammenhang zwischen den Leistungen in Mathematik, Lesekompetenz und Naturwissenschaften

und dem sozioökonomischen Status

Tabelle II.2.1 Die Ergebnisse basieren auf Schülerangaben

\begin{tabular}{|c|c|c|c|c|c|c|c|c|c|c|c|}
\hline & & Index der Kurvi & arität ${ }^{4}$ & $\begin{array}{r}\text { Zusammenhang } \mathrm{z} \\
\text { den Leseleistung } \\
\text { dem ESCS }\end{array}$ & $\begin{array}{l}\text { vischen } \\
\mathrm{n} \text { und }\end{array}$ & $\begin{array}{l}\text { Steigung d } \\
\text { sozioökonom } \\
\text { Gradiente im } \\
\text { Lesekompet }\end{array}$ & $\begin{array}{l}\text { rhen } \\
\text { ceich } \\
\text { az }\end{array}$ & $\begin{array}{r}\text { Zusammenhang } \\
\text { den Leistung } \\
\text { Naturwissenscha } \\
\text { dem ESCS }\end{array}$ & $\begin{array}{l}\text { ischen } \\
\text { in } \\
\text { en und }\end{array}$ & $\begin{array}{l}\text { Steigung } \\
\text { sozioökonom } \\
\text { Gradiente im } \\
\text { Naturwissensc }\end{array}$ & $\begin{array}{l}\text { hen } \\
\text { reich } \\
\text { ften }\end{array}$ \\
\hline & & $\begin{array}{l}1 \text { quadrierten } \\
\text { ESCS-Einheit } \\
\text { entsprechende } \\
\text { Punktzahl- } \\
\text { veränderung in } \\
\text { Mathematik }\end{array}$ & S.E. & $\begin{array}{l}\text { Prozentsatz der } \\
\text { erklärten Varianz } \\
\text { der Leseleistungen }\end{array}$ & S.E. & $\begin{array}{l}1 \text { ESCS-Einheit } \\
\text { entsprechende } \\
\text { Punktzahl- } \\
\text { veränderung in } \\
\text { Mathematik }\end{array}$ & S.E. & $\begin{array}{c}\text { Prozentsatz der } \\
\text { erklärten Varianz } \\
\text { der Leistungen } \\
\text { in Naturwissen- } \\
\text { schaften }\end{array}$ & S.E. & $\begin{array}{l}1 \text { ESCS-Einheit } \\
\text { entsprechende } \\
\text { Punktzahl- } \\
\text { veränderung in } \\
\text { Naturwissen- } \\
\text { schaften }\end{array}$ & S.E. \\
\hline $\bar{y}$ & Australien & 2.0 & (1.1) & 12.0 & $(0.8)$ & 42 & (1.3) & 11.9 & $(0.7)$ & 43 & (1.3) \\
\hline 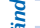 & Österreich & -4.6 & $(2.0)$ & 15.3 & (1.6) & 42 & $(2.3)$ & 18.3 & $(1.7)$ & 46 & $(2.2)$ \\
\hline 莲 & Belgien & 1.7 & $(1.3)$ & 18.2 & $(1.4)$ & 47 & $(1.8)$ & 19.2 & $(1.4)$ & 48 & $(1.7)$ \\
\hline ક & Kanada & 1.7 & $(1.0)$ & 8.1 & $(0.7)$ & 30 & (1.3) & 7.8 & $(0.7)$ & 29 & (1.4) \\
\hline ప & Chile & 3.3 & $(0.8)$ & 20.4 & (1.8) & 31 & (1.5) & 20.2 & (1.9) & 32 & (1.7) \\
\hline & Tschech. Rep. & -5.0 & (2.9) & 14.8 & (1.5) & 46 & $(2.7)$ & 14.3 & $(1.7)$ & 46 & (3.1) \\
\hline & Dänemark & 1.3 & (1.5) & 15.3 & $(1.3)$ & 39 & (1.9) & 15.7 & (1.5) & 43 & $(2.2)$ \\
\hline & Estland & 3.7 & $(2.2)$ & 6.8 & $(1.0)$ & 26 & (1.9) & 7.4 & $(0.9)$ & 27 & (1.9) \\
\hline & Finnland & 1.6 & $(1.7)$ & 7.5 & $(0.9)$ & 33 & $(2.2)$ & 7.9 & $(0.9)$ & 33 & $(2.1)$ \\
\hline & Frankreich & 4.3 & (1.5) & 18.7 & (1.5) & 58 & $(2.9)$ & 21.5 & $(1.3)$ & 58 & $(2.4)$ \\
\hline & Deutschland & -4.5 & (1.6) & 15.0 & (1.4) & 37 & $(2.0)$ & 17.1 & (1.4) & 42 & $(2.2)$ \\
\hline & Griechenland & 1.3 & $(1.2)$ & 12.0 & $(1.3)$ & 34 & (2.1) & 13.7 & (1.4) & 33 & (1.9) \\
\hline & Ungarn & -1.4 & $(1.8)$ & 20.0 & $(2.1)$ & 42 & $(2.3)$ & 22.4 & $(2.2)$ & 44 & $(2.3)$ \\
\hline & Island & 1.3 & (1.9) & 6.3 & (1.0) & 30 & (2.3) & 7.5 & $(0.9)$ & 33 & $(2.1)$ \\
\hline & Irland & 0.5 & (1.5) & 15.1 & $(1.2)$ & 39 & $(1.9)$ & 14.5 & $(1.2)$ & 41 & $(2.0)$ \\
\hline & Israel & 5.5 & $(1.2)$ & 11.2 & (1.4) & 44 & (2.9) & 14.7 & (1.4) & 48 & (2.9) \\
\hline & Italien & -3.2 & $(0.7)$ & 9.7 & $(0.7)$ & 31 & (1.1) & 9.6 & $(0.7)$ & 30 & (1.1) \\
\hline & Japan & -2.6 & (2.4) & 7.9 & (1.5) & 38 & (3.9) & 7.3 & (1.4) & 36 & (3.9) \\
\hline & Korea & 6.6 & $(2.2)$ & 7.9 & $(1.2)$ & 33 & $(2.8)$ & 6.7 & $(1.1)$ & 29 & (2.6) \\
\hline & Luxemburg & 2.6 & (1.1) & 15.6 & $(1.0)$ & 37 & $(1.3)$ & 20.0 & $(1.0)$ & 42 & $(1.1)$ \\
\hline & Mexiko & 1.3 & $(0.4)$ & 10.9 & $(0.9)$ & 21 & $(0.9)$ & 10.5 & $(0.8)$ & 18 & $(0.8)$ \\
\hline & Niederlande & 3.1 & (1.8) & 10.8 & (1.7) & 39 & (3.2) & 12.5 & $(1.8)$ & 43 & (3.1) \\
\hline & Neuseeland & -0.3 & $(2.0)$ & 16.5 & (1.4) & 52 & $(2.4)$ & 18.2 & $(1.5)$ & 54 & $(2.3)$ \\
\hline & Norwegen & -1.6 & (1.6) & 6.3 & $(1.0)$ & 33 & (2.7) & 6.9 & (1.0) & 34 & $(2.8)$ \\
\hline & Polen & -2.1 & $(1.7)$ & 13.4 & (1.6) & 36 & $(2.2)$ & 14.4 & $(1.7)$ & 36 & (2.4) \\
\hline & Portugal & 0.1 & $(1.1)$ & 16.5 & $(1.7)$ & 31 & $(1.8)$ & 18.7 & $(1.7)$ & 32 & (1.6) \\
\hline & Slowak. Rep. & -3.9 & $(2.0)$ & 24.1 & $(2.1)$ & 56 & (3.3) & 26.4 & $(2.0)$ & 56 & $(2.9)$ \\
\hline & Slowenien & 1.7 & (1.5) & 14.2 & (1.1) & 40 & (1.6) & 14.1 & $(1.0)$ & 39 & (1.5) \\
\hline & Spanien & -0.3 & $(0.7)$ & 12.5 & (1.0) & 31 & $(1.3)$ & 13.6 & $(0.9)$ & 31 & (1.1) \\
\hline & Schweden & -1.1 & (1.4) & 9.1 & $(1.1)$ & 38 & (2.5) & 10.4 & $(1.2)$ & 38 & $(2.4)$ \\
\hline & Schweiz & -1.5 & $(1.7)$ & 13.9 & (1.0) & 38 & (1.4) & 15.4 & (1.0) & 40 & (1.4) \\
\hline & Türkei & 3.3 & $(1.2)$ & 14.5 & $(1.8)$ & 30 & $(2.1)$ & 11.0 & (1.6) & 24 & (1.8) \\
\hline & Ver. Königreich & 5.5 & (1.5) & 11.8 & (1.1) & 40 & $(2.3)$ & 13.5 & $(1.2)$ & 45 & (2.3) \\
\hline & Ver. Staaten & 6.5 & $(1.2)$ & 12.6 & (1.3) & 33 & $(1.8)$ & 14.2 & (1.4) & 36 & (1.8) \\
\hline & OECD-Durchschnitt & 0.8 & $(0.3)$ & 13.1 & $(0.2)$ & 38 & $(0.4)$ & 14.0 & $(0.2)$ & 38 & $(0.4)$ \\
\hline ฐ & Albanien & $\mathrm{m}$ & $\mathrm{m}$ & $\mathrm{m}$ & $\mathrm{m}$ & $\mathrm{m}$ & $\mathrm{m}$ & $\mathrm{m}$ & $\mathrm{m}$ & $\mathrm{m}$ & $\mathrm{m}$ \\
\hline$\underset{\mathbb{t}}{\mathbb{t}}$ & Argentinien & 3.1 & (1.1) & 11.9 & (1.5) & 29 & $(2.1)$ & 15.1 & $(1.8)$ & 29 & $(2.2)$ \\
\hline$=$ & Brasilien & 5.6 & (1.1) & 11.0 & $(1.1)$ & 24 & $(1.3)$ & 13.5 & (1.4) & 25 & (1.5) \\
\hline$\stackrel{n}{=}$ & Bulgarien & 2.5 & (1.6) & 21.9 & $(2.2)$ & 53 & (2.9) & 23.8 & $(2.3)$ & 47 & $(2.8)$ \\
\hline $\mathrm{s}_{0}$ & Kolumbien & 3.5 & $(0.9)$ & 15.6 & (1.9) & 28 & (1.9) & 12.7 & $(1.8)$ & 23 & (1.8) \\
\hline 亏 & Costa Rica & 4.0 & $(0.9)$ & 14.7 & $(2.1)$ & 23 & $(1.7)$ & 16.3 & $(2.2)$ & 23 & $(1.7)$ \\
\hline$\frac{1}{2}$ & Kroatien & 2.6 & (1.6) & 11.2 & (1.4) & 34 & $(2.5)$ & 9.8 & $(1.2)$ & 31 & $(2.3)$ \\
\hline ‡े & Zypern* & 2.3 & $(1.2)$ & 8.2 & (0.8) & 35 & (1.9) & 13.7 & $(1.0)$ & 39 & (1.7) \\
\hline$\tau$ & Hongkong (China) & 0.9 & $(1.4)$ & 5.2 & $(1.2)$ & 20 & (2.5) & 6.0 & $(1.3)$ & 21 & (2.3) \\
\hline$\frac{2}{2 \pi}$ & Indonesien & 5.4 & (1.9) & 6.2 & $(2.2)$ & 17 & (3.1) & 8.4 & (2.5) & 18 & $(2.7)$ \\
\hline కั้ & Jordanien & 3.0 & $(1.3)$ & 4.8 & (1.0) & 19 & $(2.1)$ & 7.0 & $(1.2)$ & 21 & $(2.2)$ \\
\hline ‡ & Kasachstan & 1.6 & $(1.8)$ & 12.2 & (1.4) & 34 & $(2.1)$ & 9.5 & (1.4) & 30 & (2.3) \\
\hline & Lettland & -1.0 & $(1.7)$ & 14.0 & $(2.0)$ & 36 & $(2.7)$ & 11.9 & $(1.7)$ & 30 & $(2.2)$ \\
\hline & Liechtenstein & -12.3 & (5.1) & 7.0 & (3.1) & 25 & (5.6) & 9.7 & (3.4) & 29 & $(5.2)$ \\
\hline & Litauen & -1.7 & (1.4) & 11.3 & $(1.1)$ & 32 & $(1.7)$ & 11.8 & $(1.2)$ & 32 & (1.9) \\
\hline & Macau (China) & 0.8 & (1.4) & 1.5 & $(0.4)$ & 11 & (1.4) & 2.1 & $(0.6)$ & 13 & (1.8) \\
\hline & Malaysia & 6.2 & $(1.1)$ & 7.7 & (1.4) & 23 & $(2.2)$ & 10.3 & (1.4) & 25 & (1.9) \\
\hline & Montenegro & 2.9 & (1.4) & 10.9 & (1.0) & 34 & (1.5) & 11.6 & $(0.9)$ & 32 & (1.4) \\
\hline & Peru & 1.4 & $(1.2)$ & 23.6 & (2.6) & 37 & (2.3) & 21.7 & (2.5) & 30 & (1.8) \\
\hline & Katar & -2.7 & $(0.7)$ & 5.2 & $(0.5)$ & 29 & (1.4) & 5.4 & $(0.5)$ & 28 & $(1.2)$ \\
\hline & Rumänien & 6.0 & $(1.0)$ & 16.5 & $(2.0)$ & 38 & (2.6) & 16.9 & $(2.1)$ & 34 & $(2.5)$ \\
\hline & Russ. Föderation & 1.0 & $(2.3)$ & 13.1 & (1.6) & 43 & (3.2) & 14.6 & (1.9) & 43 & (3.1) \\
\hline & Serbien & 3.7 & (1.6) & 8.7 & $(1.2)$ & 30 & $(2.3)$ & 8.8 & $(1.2)$ & 29 & $(2.2)$ \\
\hline & Shanghai (China) & -3.9 & $(1.8)$ & 15.6 & (1.8) & 33 & $(2.0)$ & 15.3 & $(2.0)$ & 33 & $(2.1)$ \\
\hline & Singapur & 1.7 & (1.4) & 15.2 & $(0.9)$ & 43 & (1.4) & 16.5 & (1.0) & 46 & (1.6) \\
\hline & Chinesisch Taipeh & -0.5 & (1.8) & 15.1 & (1.4) & 42 & $(2.2)$ & 16.7 & (1.4) & 40 & (1.8) \\
\hline & Thailand & 7.5 & (1.4) & 10.0 & (1.9) & 21 & (2.1) & 8.2 & (1.8) & 19 & (2.1) \\
\hline & Tunesien & 5.9 & (1.4) & 9.2 & (1.8) & 21 & $(2.2)$ & 8.2 & $(1.7)$ & 18 & $(2.0)$ \\
\hline & Ver. Arab. Emirate & 0.8 & $(1.1)$ & 7.1 & $(0.9)$ & 30 & (1.9) & 8.9 & $(1.0)$ & 33 & $(2.1)$ \\
\hline & Uruguay & 4.4 & $(1.2)$ & 17.5 & (1.8) & 35 & (2.0) & 19.8 & $(1.8)$ & 37 & (1.9) \\
\hline & Vietnam & -0.1 & (1.4) & 11.7 & $(2.1)$ & 23 & $(2.3)$ & 10.1 & $(1.8)$ & 22 & $(2.2)$ \\
\hline
\end{tabular}

Anmerkung: Statistisch signifikante Werte sind durch Fettdruck gekennzeichnet (vgl. Anhang A3). In diesen Tabellen gibt es keine direkten Vergleiche mit dem OECD-Durchschnitt.

1. ESCS bezieht sich auf den PISA-Index des wirtschaftlichen, sozialen und kulturellen Status.

2. Einstufige bivariate Regression der Schülerleistungen auf den ESCS, wobei die Steigung dem ESCS-Regressionskoeffizienten entspricht.

3. Auf Schülerebene berechnete Perzentile.

4. Regression der Mathematikleistungen auf Schülerebene auf den ESCS und den quadrierten ESCS-Term, der Index der Kurvilinearität ist der Regressionskoeffizient des quadrierten Terms.

Vgl. Anmerkungen am Anfang dieses Anhangs.

StatLink त्नाls http://dx.doi.org/10.1787/888932964908 
[Teil 1/7]

Merkmale des sozioökonomischen Status, nach Quartilen des sozioökonomischen Status innerhalb der einzelnen Länder

Tabelle II.2.2 Die Ergebnisse basieren auf Schülerangaben

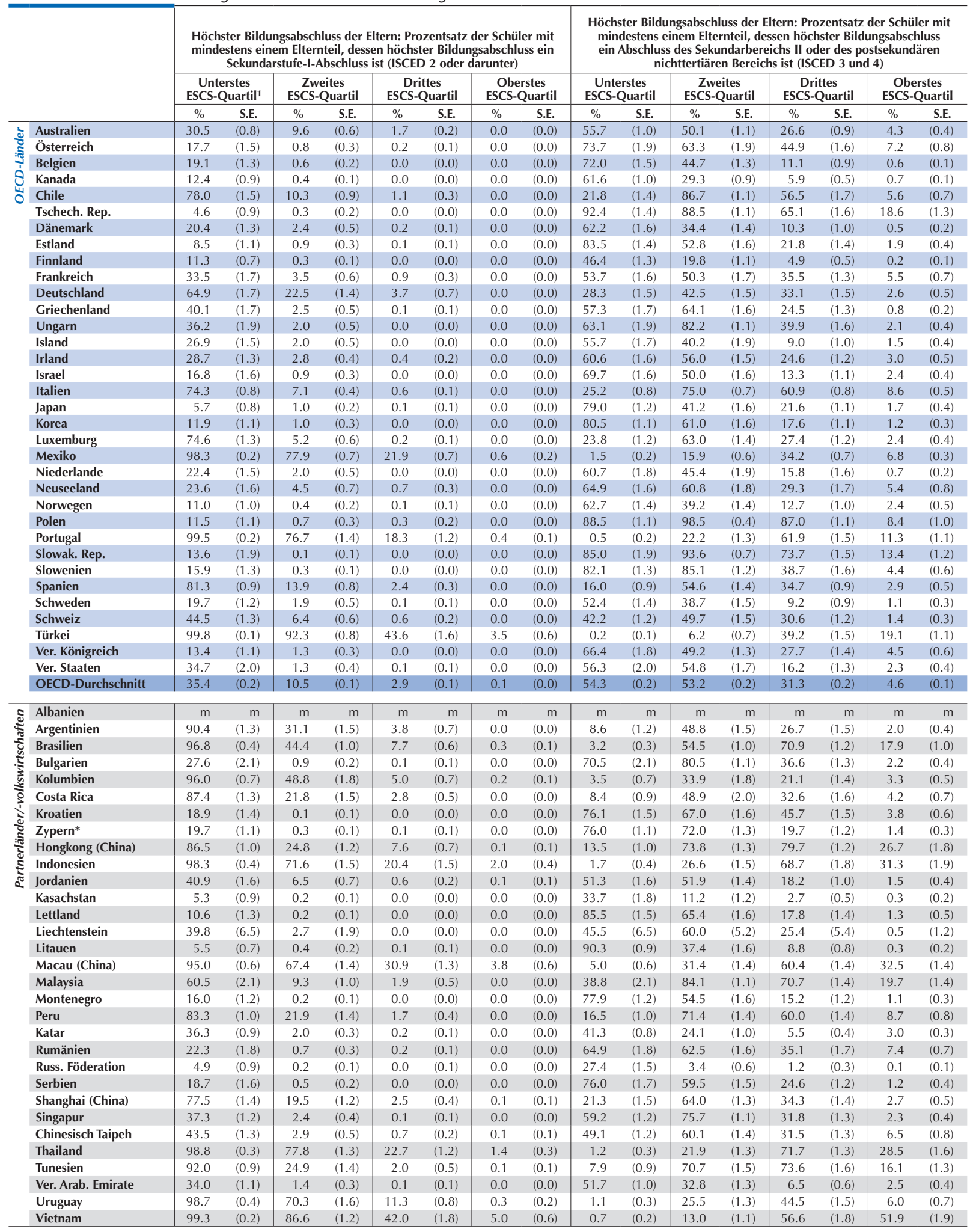

1. ESCS bezieht sich auf den PISA-Index des wirtschaftlichen, sozialen und kulturellen Status.

2. Die Variable, die die Anzahl der im Elternhaus vorhandenen Bücher angibt, wurde folgendermaßen kodiert: 0-10 Bücher wurden als 5 Bücher kodiert, $11-25$ Bücher als

18 Bücher, 26-100 Bücher als 63 Bücher, 101-200 Bücher als 150,5 Bücher, 201-500 Bücher als 350,5 Bücher und über 500 Bücher als 750,5 Bücher.

* Vgl. Anmerkungen am Anfang dieses Anhangs.

StatLink तinst http://dx.doi.org/10.1787/888932964908 
[Teil 2/7]

Merkmale des sozioökonomischen Status, nach Quartilen des sozioökonomischen Status innerhalb der einzelnen Länder

Tabelle II.2.2 Die Ergebnisse basieren auf Schülerangaben

\begin{tabular}{|c|c|c|c|c|c|c|c|c|c|c|c|c|c|c|c|c|}
\hline & \multicolumn{8}{|c|}{$\begin{array}{c}\text { Höchster Bildungsabschluss der Eltern: Prozentsatz der Schüler mit } \\
\text { mindestens einem Elternteil, dessen höchster Bildungsabschluss } \\
\text { ein Abschluss des Tertiärbereichs oder eines weiterführenden } \\
\text { Forschungsprogramms ist (ISCED } 5 \text { oder 6) }\end{array}$} & \multicolumn{8}{|c|}{$\begin{array}{l}\text { Höchste berufliche Stellung der Eltern: Prozentsatz der Schüler mit } \\
\text { mindestens einem Elternteil, der als Hilfsarbeitskraft tätig ist (ISCO 9) }\end{array}$} \\
\hline & \multicolumn{2}{|c|}{$\begin{array}{c}\text { Unterstes } \\
\text { ESCS-Quartil }\end{array}$} & \multicolumn{2}{|c|}{$\begin{array}{c}\text { Zweites } \\
\text { ESCS-Quartil }\end{array}$} & \multicolumn{2}{|c|}{$\begin{array}{c}\text { Drittes } \\
\text { ESCS-Quartil }\end{array}$} & \multicolumn{2}{|c|}{$\begin{array}{c}\text { Oberstes } \\
\text { ESCS-Quartil }\end{array}$} & \multicolumn{2}{|c|}{$\begin{array}{c}\text { Unterstes } \\
\text { ESCS-Quartil }\end{array}$} & \multicolumn{2}{|c|}{$\begin{array}{c}\text { Zweites } \\
\text { ESCS-Quartil }\end{array}$} & \multicolumn{2}{|c|}{$\begin{array}{c}\text { Drittes } \\
\text { ESCS-Quartil }\end{array}$} & \multicolumn{2}{|c|}{$\begin{array}{c}\text { Oberstes } \\
\text { ESCS-Quartil }\end{array}$} \\
\hline & $\%$ & S.E. & $\%$ & S.E. & $\%$ & S.E. & $\%$ & S.E. & $\%$ & S.E. & $\%$ & S.E. & $\%$ & S.E. & $\%$ & S.E. \\
\hline Australien & 13.8 & $(0.7)$ & 40.3 & $(1.0)$ & 71.7 & $(0.9)$ & 95.7 & $(0.4)$ & 33.7 & $(1.0)$ & 7.2 & $(0.5)$ & 0.5 & $(0.1)$ & 0.1 & $(0.1)$ \\
\hline Österreich & 8.6 & (1.0) & 35.9 & (1.9) & 54.9 & (1.5) & 92.8 & $(0.8)$ & 42.2 & $(1.8)$ & 21.7 & (1.5) & 3.1 & $(0.5)$ & 0.2 & $(0.1)$ \\
\hline Belgien & 8.9 & $(0.8)$ & 54.7 & (1.3) & 88.9 & $(0.9)$ & 99.4 & $(0.1)$ & 40.3 & (1.4) & 18.8 & $(1.2)$ & 1.2 & $(0.3)$ & 0.0 & $(0.0)$ \\
\hline Kanada & 26.0 & $(1.0)$ & 70.2 & $(0.9)$ & 94.1 & $(0.5)$ & 99.3 & $(0.1)$ & 29.9 & (1.1) & 9.4 & $(0.6)$ & 1.0 & $(0.2)$ & 0.2 & $(0.1)$ \\
\hline Chile & 0.3 & $(0.2)$ & 3.0 & $(0.5)$ & 42.4 & (1.7) & 94.4 & $(0.7)$ & 41.9 & (1.7) & 39.2 & (1.6) & 17.7 & $(1.2)$ & 0.8 & $(0.3)$ \\
\hline Tschech. Rep. & 3.0 & $(0.7)$ & 11.3 & (1.1) & 34.8 & (1.6) & 81.4 & (1.3) & 49.2 & (1.9) & 21.3 & (1.5) & 4.9 & $(0.7)$ & 0.3 & $(0.3)$ \\
\hline Dänemark & 17.4 & (1.4) & 63.3 & (1.4) & 89.5 & $(1.0)$ & 99.5 & $(0.2)$ & 26.2 & $(1.2)$ & 6.7 & $(0.7)$ & 0.5 & $(0.3)$ & 0.0 & $(0.0)$ \\
\hline Estland & 8.0 & $(0.9)$ & 46.2 & (1.6) & 78.1 & (1.4) & 98.1 & $(0.4)$ & 48.4 & $(1.8)$ & 24.3 & (1.5) & 2.8 & $(0.5)$ & 0.0 & $(0.0)$ \\
\hline Finnland & 42.4 & (1.2) & 79.8 & (1.1) & 95.0 & $(0.5)$ & 99.8 & $(0.1)$ & 31.2 & $(1.2)$ & 7.6 & $(0.6)$ & 0.5 & $(0.2)$ & 0.1 & $(0.2)$ \\
\hline Frankreich & 12.8 & $(1.0)$ & 46.1 & (1.6) & 63.7 & (1.3) & 94.5 & $(0.7)$ & 37.6 & (1.8) & 12.8 & $(1.0)$ & 1.0 & $(0.3)$ & 0.1 & $(0.1)$ \\
\hline Deutschland & 6.7 & $(0.8)$ & 35.1 & (1.6) & 63.0 & (1.6) & 97.4 & $(0.5)$ & 36.6 & (1.5) & 16.4 & (1.4) & 2.9 & $(0.6)$ & 0.1 & $(0.1)$ \\
\hline Griechenland & 2.5 & (0.5) & 33.4 & (1.5) & 75.4 & (1.3) & 99.2 & $(0.2)$ & 48.0 & (1.5) & 23.4 & (1.5) & 3.8 & $(0.6)$ & 0.3 & $(0.1)$ \\
\hline Ungarn & 0.8 & $(0.3)$ & 15.7 & (1.0) & 60.0 & (1.6) & 97.9 & $(0.4)$ & 55.2 & $(2.0)$ & 30.1 & (1.5) & 8.8 & $(1.0)$ & 0.2 & $(0.1)$ \\
\hline Island & 17.4 & (1.2) & 57.8 & (1.9) & 91.0 & (1.0) & 98.5 & (0.4) & 26.9 & (1.6) & 8.1 & $(1.0)$ & 1.0 & $(0.3)$ & 0.3 & $(0.2)$ \\
\hline Irland & 10.7 & (1.1) & 41.1 & (1.5) & 74.9 & (1.2) & 97.0 & $(0.5)$ & 30.5 & (1.5) & 12.8 & $(1.0)$ & 2.5 & $(0.5)$ & 0.0 & $(0.0)$ \\
\hline Israel & 13.5 & (0.9) & 49.1 & (1.7) & 86.8 & (1.1) & 97.6 & $(0.4)$ & 37.4 & $(2.1)$ & 7.8 & $(0.8)$ & 0.3 & $(0.2)$ & 0.0 & $(0.0)$ \\
\hline Italien & 0.5 & $(0.1)$ & 17.9 & $(0.6)$ & 38.6 & $(0.8)$ & 91.4 & $(0.5)$ & 53.9 & $(0.9)$ & 32.2 & $(0.8)$ & 7.1 & $(0.5)$ & 0.3 & $(0.1)$ \\
\hline Japan & 15.3 & $(1.0)$ & 57.9 & (1.6) & 78.3 & $(1.1)$ & 98.3 & $(0.4)$ & 30.8 & $(1.3)$ & 11.8 & $(1.0)$ & 1.7 & $(0.3)$ & 0.1 & $(0.1)$ \\
\hline Korea & 7.6 & $(0.9)$ & 38.0 & (1.5) & 82.3 & $(1.0)$ & 98.8 & $(0.3)$ & 29.1 & (1.4) & 5.4 & $(0.7)$ & 0.7 & $(0.3)$ & 0.0 & $(0.0)$ \\
\hline Luxemburg & 1.5 & $(0.3)$ & 31.9 & (1.3) & 72.3 & $(1.2)$ & 97.6 & $(0.4)$ & 53.0 & (1.5) & 23.8 & $(1.2)$ & 2.4 & $(0.4)$ & 0.1 & $(0.1)$ \\
\hline Mexiko & 0.2 & $(0.1)$ & 6.2 & $(0.4)$ & 44.0 & $(0.8)$ & 92.5 & $(0.4)$ & 32.9 & $(1.0)$ & 38.5 & $(0.8)$ & 20.3 & $(0.6)$ & 1.2 & $(0.2)$ \\
\hline Niederlande & 16.8 & (1.5) & 52.6 & $(2.0)$ & 84.2 & (1.6) & 99.3 & $(0.2)$ & 28.7 & $(1.8)$ & 4.4 & $(0.7)$ & 0.2 & $(0.2)$ & 0.1 & $(0.1)$ \\
\hline Neuseeland & 11.4 & (0.9) & 34.8 & (1.6) & 70.1 & (1.7) & 94.6 & $(0.8)$ & 40.7 & (1.6) & 11.1 & (1.1) & 0.3 & $(0.2)$ & 0.1 & $(0.1)$ \\
\hline Norwegen & 26.3 & (1.5) & 60.3 & (1.4) & 87.2 & $(1.0)$ & 97.6 & $(0.5)$ & 20.8 & $(1.2)$ & 3.4 & $(0.6)$ & 0.3 & $(0.2)$ & 0.0 & $(0.0)$ \\
\hline Polen & 0.0 & $(0.0)$ & 0.9 & $(0.3)$ & 12.7 & $(1.2)$ & 91.6 & (1.0) & 67.8 & $(1.7)$ & 47.4 & (1.6) & 7.7 & $(0.9)$ & 0.0 & $(0.0)$ \\
\hline Portugal & 0.0 & $(0.0)$ & 1.0 & $(0.3)$ & 19.8 & (1.1) & 88.4 & (1.1) & 52.9 & (1.7) & 38.7 & (1.5) & 12.3 & $(1.2)$ & 0.3 & $(0.2)$ \\
\hline Slowak. Rep. & 1.4 & (0.4) & 6.3 & $(0.7)$ & 26.3 & (1.5) & 86.7 & $(1.2)$ & 45.5 & $(2.1)$ & 36.4 & $(1.7)$ & 11.8 & (1.4) & 0.1 & $(0.1)$ \\
\hline Slowenien & 2.0 & $(0.7)$ & 14.6 & $(1.2)$ & 61.2 & (1.6) & 95.5 & $(0.6)$ & 47.8 & (1.6) & 16.5 & $(1.2)$ & 2.3 & $(0.5)$ & 0.1 & $(0.0)$ \\
\hline Spanien & 2.7 & $(0.4)$ & 31.5 & $(1.4)$ & 62.9 & $(0.9)$ & 97.1 & $(0.5)$ & 48.5 & $(1.2)$ & 29.6 & (0.9) & 7.7 & $(0.6)$ & 0.1 & $(0.1)$ \\
\hline Schweden & 27.9 & (1.3) & 59.4 & (1.6) & 90.7 & $(0.9)$ & 98.9 & $(0.3)$ & 25.6 & (1.4) & 8.5 & (0.9) & 0.4 & $(0.2)$ & 0.0 & $(0.0)$ \\
\hline Schweiz & 13.3 & (0.8) & 44.0 & (1.5) & 68.8 & $(1.2)$ & 98.6 & $(0.3)$ & 38.2 & (1.3) & 8.2 & $(0.7)$ & 0.7 & $(0.3)$ & 0.0 & $(0.0)$ \\
\hline Türkei & 0.1 & $(0.1)$ & 1.5 & $(0.3)$ & 17.2 & (1.1) & 77.4 & (1.3) & 54.6 & (2.4) & 43.9 & $(1.8)$ & 29.4 & (1.5) & 3.2 & $(0.5)$ \\
\hline Ver. Königreich & 20.2 & (1.4) & 49.6 & (1.4) & 72.2 & (1.4) & 95.5 & $(0.6)$ & 20.3 & $(1.2)$ & 6.9 & (1.3) & 0.5 & $(0.2)$ & 0.1 & $(0.1)$ \\
\hline Ver. Staaten & 9.0 & $(0.9)$ & 43.9 & (1.6) & 83.6 & (1.3) & 97.7 & $(0.4)$ & 23.7 & (1.3) & 7.5 & $(0.8)$ & 0.9 & $(0.3)$ & 0.2 & $(0.1)$ \\
\hline OECD-Durchschnitt & 10.3 & $(0.2)$ & 36.3 & $(0.2)$ & 65.8 & $(0.2)$ & 95.3 & $(0.1)$ & 39.1 & $(0.3)$ & 18.9 & $(0.2)$ & 4.7 & $(0.1)$ & 0.3 & $(0.0)$ \\
\hline
\end{tabular}

\begin{tabular}{|c|c|c|c|c|c|c|c|c|c|c|c|c|c|c|c|c|}
\hline Albanien & $\mathrm{m}$ & $\mathrm{m}$ & $\mathrm{m}$ & $\mathrm{m}$ & $\mathrm{m}$ & $\mathrm{m}$ & $\mathrm{m}$ & $\mathrm{m}$ & $\mathrm{m}$ & $\mathrm{m}$ & $\mathrm{m}$ & $\mathrm{m}$ & $\mathrm{m}$ & $\mathrm{m}$ & $\mathrm{m}$ & $\mathrm{m}$ \\
\hline Argentinien & 1.1 & $(0.4)$ & 20.1 & $(1.2)$ & 69.5 & $(1.7)$ & 97.9 & $(0.4)$ & 51.3 & (1.4) & 39.8 & (1.5) & 18.1 & (1.5) & 0.7 & $(0.3)$ \\
\hline Brasilien & 0.0 & $(0.0)$ & 1.2 & $(0.2)$ & 21.3 & (1.1) & 81.8 & (1.0) & 32.9 & $(1.2)$ & 29.3 & (1.1) & 12.4 & $(0.8)$ & 1.5 & $(0.3)$ \\
\hline Bulgarien & 1.9 & $(0.4)$ & 18.6 & $(1.2)$ & 63.3 & (1.3) & 97.8 & (0.4) & 53.7 & (1.7) & 30.1 & (1.4) & 10.4 & (0.9) & 0.5 & $(0.2)$ \\
\hline Kolumbien & 0.5 & $(0.2)$ & 17.3 & (1.4) & 73.9 & (1.7) & 96.5 & $(0.5)$ & 58.1 & (1.6) & 46.2 & (1.7) & 27.1 & (1.4) & 3.1 & $(0.5)$ \\
\hline Costa Rica & 4.2 & (0.9) & 29.4 & $(2.2)$ & 64.5 & (1.6) & 95.8 & $(0.7)$ & 31.7 & (3.1) & 32.6 & (1.9) & 18.1 & (1.3) & 0.4 & $(0.2)$ \\
\hline Kroatien & 5.1 & $(0.6)$ & 32.8 & (1.6) & 54.3 & (1.6) & 96.2 & $(0.6)$ & 47.7 & (1.6) & 27.4 & (1.4) & 9.2 & $(0.7)$ & 0.4 & $(0.2)$ \\
\hline Zypern* & 4.2 & $(0.5)$ & 27.8 & $(1.3)$ & 80.2 & $(1.3)$ & 98.6 & $(0.3)$ & 44.8 & (1.7) & 17.6 & (1.1) & 3.6 & $(0.5)$ & 0.4 & $(0.2)$ \\
\hline Hongkong (China) & 0.1 & $(0.1)$ & 1.3 & $(0.4)$ & 12.7 & $(1.0)$ & 73.2 & (1.9) & 30.1 & (1.2) & 28.6 & (1.4) & 5.4 & $(0.8)$ & 0.0 & $(0.0)$ \\
\hline Indonesien & 0.0 & $(0.0)$ & 1.8 & $(0.5)$ & 10.9 & (1.1) & 66.6 & (2.1) & 53.4 & (3.7) & 39.4 & (2.9) & 30.9 & (2.4) & 4.4 & $(0.8)$ \\
\hline Jordanien & 7.9 & $(0.7)$ & 41.6 & (1.3) & 81.2 & (1.1) & 98.5 & $(0.4)$ & 55.2 & (1.7) & 21.0 & (1.3) & 2.1 & $(0.4)$ & 0.3 & $(0.2)$ \\
\hline Kasachstan & 61.0 & (1.8) & 88.6 & (1.1) & 97.3 & $(0.5)$ & 99.7 & $(0.2)$ & 45.6 & $(2.2)$ & 23.9 & (1.4) & 2.0 & $(0.4)$ & 0.0 & $(0.0)$ \\
\hline Lettland & 3.9 & $(0.8)$ & 34.3 & (1.6) & 82.3 & (1.4) & 98.7 & $(0.5)$ & 38.6 & $(2.2)$ & 18.7 & (1.3) & 6.1 & (1.0) & 0.2 & $(0.1)$ \\
\hline Liechtenstein & 14.4 & $(4.0)$ & 37.6 & $(5.0)$ & 74.3 & (5.4) & 99.7 & $(0.9)$ & 35.5 & (5.7) & 0.0 & $(0.0)$ & 2.8 & (2.0) & 0.0 & $(0.0)$ \\
\hline Litauen & 4.2 & $(0.7)$ & 62.2 & (1.6) & 91.2 & $(0.8)$ & 99.7 & $(0.2)$ & 55.6 & (1.4) & 30.9 & (1.6) & 4.2 & (0.6) & 0.3 & $(0.2)$ \\
\hline Macau (China) & 0.0 & $(0.0)$ & 1.2 & $(0.3)$ & 8.8 & $(0.7)$ & 63.7 & (1.4) & 19.6 & $(1.0)$ & 13.2 & $(1.0)$ & 3.0 & $(0.5)$ & 0.8 & $(0.2)$ \\
\hline Malaysia & 0.7 & $(0.3)$ & 6.7 & $(0.8)$ & 27.4 & (1.5) & 80.2 & (1.4) & 48.6 & (1.8) & 39.5 & (1.5) & 12.8 & (1.0) & 0.7 & $(0.3)$ \\
\hline Montenegro & 6.2 & $(0.6)$ & 45.2 & (1.6) & 84.7 & $(1.2)$ & 99.0 & $(0.3)$ & 43.6 & (1.7) & 22.4 & (1.3) & 7.3 & $(0.8)$ & 0.4 & $(0.2)$ \\
\hline Peru & 0.2 & $(0.1)$ & 6.8 & $(0.6)$ & 38.2 & (1.3) & 91.3 & $(0.8)$ & 23.8 & (1.5) & 44.4 & (1.3) & 29.3 & (1.0) & 4.4 & $(0.7)$ \\
\hline Katar & 22.4 & $(0.8)$ & 73.9 & $(1.0)$ & 94.3 & $(0.4)$ & 97.0 & $(0.3)$ & 23.1 & $(0.9)$ & 4.1 & $(0.4)$ & 0.7 & $(0.2)$ & 0.8 & $(0.2)$ \\
\hline Rumänien & 12.8 & (1.1) & 36.6 & (1.6) & 64.8 & (1.7) & 92.6 & $(0.7)$ & 54.5 & $(2.0)$ & 45.6 & (1.6) & 22.9 & (1.4) & 0.9 & $(0.3)$ \\
\hline Russ. Föderation & 67.7 & (1.5) & 96.4 & $(0.6)$ & 98.8 & $(0.3)$ & 99.9 & $(0.1)$ & 42.8 & (1.9) & 16.5 & (1.1) & 1.7 & $(0.4)$ & 0.0 & $(0.0)$ \\
\hline Serbien & 5.3 & $(0.7)$ & 40.0 & (1.4) & 75.4 & $(1.2)$ & 98.8 & (0.4) & 26.8 & (1.5) & 18.1 & $(1.2)$ & 5.3 & $(0.7)$ & 0.0 & $(0.0)$ \\
\hline Shanghai (China) & 1.2 & $(0.3)$ & 16.6 & $(1.0)$ & 63.2 & (1.3) & 97.2 & $(0.5)$ & 41.8 & (1.9) & 14.9 & (1.1) & 2.8 & $(0.5)$ & 0.0 & $(0.0)$ \\
\hline Singapur & 3.5 & $(0.5)$ & 21.8 & (1.1) & 68.2 & (1.4) & 97.7 & $(0.4)$ & 25.7 & (1.4) & 5.1 & $(0.8)$ & 0.5 & $(0.2)$ & 0.0 & $(0.0)$ \\
\hline Chinesisch Taipeh & 7.4 & $(0.6)$ & 36.9 & (1.5) & 67.9 & $(1.2)$ & 93.4 & $(0.8)$ & 50.8 & (1.4) & 28.3 & (1.3) & 6.3 & $(0.7)$ & 0.5 & $(0.2)$ \\
\hline Thailand & 0.0 & $(0.0)$ & 0.3 & $(0.2)$ & 5.6 & $(0.7)$ & 70.1 & (1.7) & 66.3 & (1.8) & 52.5 & (1.6) & 31.8 & (1.5) & 4.4 & $(0.6)$ \\
\hline Tunesien & 0.2 & $(0.1)$ & 4.3 & $(0.7)$ & 24.3 & (1.6) & 83.8 & (1.3) & 20.5 & (1.7) & 32.4 & (1.6) & 23.1 & (1.4) & 1.3 & $(0.3)$ \\
\hline Ver. Arab. Emirate & 14.3 & $(0.9)$ & 65.8 & (1.3) & 93.3 & $(0.6)$ & 97.5 & $(0.4)$ & 13.1 & (1.0) & 2.9 & $(0.4)$ & 0.7 & $(0.2)$ & 0.4 & $(0.1)$ \\
\hline Uruguay & 0.2 & $(0.2)$ & 4.3 & $(0.7)$ & 44.2 & (1.4) & 93.7 & $(0.7)$ & 29.5 & (1.4) & 33.2 & (1.5) & 17.5 & (1.1) & 1.6 & (0.4) \\
\hline Vietnam & 0.0 & $(0.0)$ & 0.3 & $(0.2)$ & 1.5 & $(0.4)$ & 43.1 & (2.0) & 90.6 & (1.3) & 88.0 & (1.2) & 69.1 & (2.0) & 16.1 & (1.7) \\
\hline
\end{tabular}

1. ESCS bezieht sich auf den PISA-Index des wirtschaftlichen, sozialen und kulturellen Status.

2. Die Variable, die die Anzahl der im Elternhaus vorhandenen Bücher angibt, wurde folgendermaßen kodiert: 0-10 Bücher wurden als 5 Bücher kodiert, 11-25 Bücher als

18 Bücher, 26-100 Bücher als 63 Bücher, 101-200 Bücher als 150,5 Bücher, 201-500 Bücher als 350,5 Bücher und über 500 Bücher als 750,5 Bücher.

$*$ Vgl. Anmerkungen am Anfang dieses Anhangs.

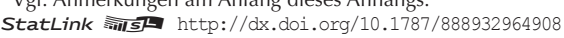


Merkmale des sozioökonomischen Status, nach Quartilen des sozioökonomischen Status innerhalb der einzelnen Länder

Tabelle II.2.2 Die Ergebnisse basieren auf Schülerangaben

\begin{tabular}{|c|c|c|c|c|c|c|c|c|c|c|c|c|c|c|c|c|c|}
\hline & & \multicolumn{8}{|c|}{$\begin{array}{l}\text { Höchste berufliche Stellung der Eltern: Prozentsatz der Schüler mit } \\
\text { mindestens einem Elternteil, der als angelernte Fachkraft tätig ist } \\
\text { (ISCO 6, } 7 \text { und 8) }\end{array}$} & \multicolumn{8}{|c|}{$\begin{array}{c}\text { Höchste berufliche Stellung der Eltern: Prozentsatz der Schüler mit } \\
\text { mindestens einem Elternteil, der als angelernte Bürokraft oder in einem } \\
\text { Dienstleistungsberuf tätig ist (ISCO } 4 \text { und 5) }\end{array}$} \\
\hline & & \multicolumn{2}{|c|}{$\begin{array}{c}\text { Unterstes } \\
\text { ESCS-Quartil }\end{array}$} & \multicolumn{2}{|c|}{$\begin{array}{c}\text { Zweites } \\
\text { ESCS-Quartil }\end{array}$} & \multicolumn{2}{|c|}{$\begin{array}{c}\text { Drittes } \\
\text { ESCS-Quartil }\end{array}$} & \multicolumn{2}{|c|}{$\begin{array}{c}\text { Oberstes } \\
\text { ESCS-Quartil }\end{array}$} & $\begin{array}{r}\text { Un } \\
\text { ESCS }\end{array}$ & $\begin{array}{l}\text { stes } \\
\text { uartil }\end{array}$ & $\begin{array}{r}\mathrm{ZV} \\
\mathrm{ESCS}\end{array}$ & $\begin{array}{l}\text { tes } \\
\text { uartil }\end{array}$ & $\begin{array}{r}\text { Dr } \\
\text { ESCS } \\
\end{array}$ & tes & $\begin{array}{r}\mathrm{Ob} \\
\mathrm{ESCS}\end{array}$ & $\begin{array}{l}\text { stes } \\
\text { uartil }\end{array}$ \\
\hline & & $\%$ & S.E. & $\%$ & S.E. & $\%$ & S.E. & $\%$ & S.E. & $\%$ & S.E. & $\%$ & S.E. & $\%$ & S.E. & $\%$ & S.E. \\
\hline ذ & Australien & 16.0 & $(0.6)$ & 0.6 & $(0.2)$ & 0.0 & $(0.0)$ & 0.0 & $(0.0)$ & 40.7 & (1.0) & 25.7 & $(1.0)$ & 3.7 & $(0.5)$ & 0.5 & $(0.1)$ \\
\hline & Österreich & 9.0 & $(0.9)$ & 0.8 & $(0.3)$ & 0.1 & $(0.1)$ & 0.0 & $(0.0)$ & 46.1 & (1.9) & 48.6 & (1.6) & 22.1 & (1.3) & 3.2 & (0.6) \\
\hline & Belgien & 16.3 & $(0.8)$ & 2.0 & $(0.3)$ & 0.1 & $(0.1)$ & 0.0 & $(0.0)$ & 38.6 & $(1.2)$ & 44.6 & $(1.1)$ & 12.7 & $(0.8)$ & 0.3 & $(0.1)$ \\
\hline 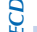 & Kanada & 9.9 & $(0.7)$ & 1.0 & $(0.2)$ & 0.0 & $(0.0)$ & 0.0 & $(0.0)$ & 46.9 & $(1.2)$ & 32.6 & $(1.0)$ & 7.7 & $(0.5)$ & 0.7 & $(0.2)$ \\
\hline ప & Chile & 37.4 & (1.7) & 10.0 & $(0.8)$ & 1.9 & $(0.4)$ & 0.0 & $(0.0)$ & 19.7 & (1.5) & 44.6 & (1.4) & 40.2 & (1.6) & 5.8 & $(0.5)$ \\
\hline & Tschech. Rep. & 4.7 & (1.0) & 0.7 & $(0.3)$ & 0.1 & $(0.1)$ & 0.0 & $(0.0)$ & 44.0 & (1.8) & 54.5 & (1.9) & 32.3 & (1.7) & 6.1 & $(0.9)$ \\
\hline & Dänemark & 10.4 & (1.0) & 0.7 & $(0.2)$ & 0.0 & $(0.0)$ & 0.0 & $(0.0)$ & 58.6 & (1.6) & 47.4 & (1.6) & 5.2 & $(0.6)$ & 0.2 & $(0.1)$ \\
\hline & Estland & 6.9 & $(0.8)$ & 0.6 & $(0.3)$ & 0.1 & $(0.1)$ & 0.0 & $(0.0)$ & 41.7 & (1.9) & 41.5 & $(1.4)$ & 10.3 & (1.0) & 0.3 & $(0.2)$ \\
\hline & Finnland & 3.4 & $(0.4)$ & 0.2 & $(0.1)$ & 0.0 & $(0.0)$ & 0.0 & $(0.0)$ & 54.3 & (1.5) & 34.3 & (1.3) & 5.5 & $(0.6)$ & 0.1 & $(0.1)$ \\
\hline & Frankreich & 15.9 & (1.1) & 0.9 & $(0.3)$ & 0.0 & $(0.0)$ & 0.0 & $(0.0)$ & 42.1 & (1.8) & 46.1 & $(1.5)$ & 8.8 & (1.0) & 0.1 & $(0.1)$ \\
\hline & Deutschland & 7.3 & $(0.9)$ & 1.0 & $(0.4)$ & 0.0 & $(0.0)$ & 0.0 & $(0.0)$ & 51.1 & (1.6) & 46.2 & (1.9) & 22.6 & (1.3) & 2.1 & $(0.5)$ \\
\hline & Griechenland & 17.2 & $(1.2)$ & 3.0 & $(0.4)$ & 0.2 & $(0.1)$ & 0.0 & $(0.0)$ & 32.8 & (1.5) & 52.4 & $(1.3)$ & 24.6 & $(1.0)$ & 0.6 & $(0.2)$ \\
\hline & Ungarn & 12.6 & (1.4) & 1.7 & $(0.4)$ & 0.3 & $(0.2)$ & 0.0 & $(0.0)$ & 30.3 & (1.6) & 50.7 & (1.6) & 30.8 & (1.5) & 2.8 & $(0.6)$ \\
\hline & Island & 9.2 & (1.0) & 0.6 & $(0.3)$ & 0.0 & $(0.0)$ & 0.1 & $(0.1)$ & 47.7 & (1.6) & 31.7 & $(1.5)$ & 4.2 & $(0.7)$ & 0.7 & $(0.3)$ \\
\hline & Irland & 11.1 & (1.0) & 1.0 & $(0.3)$ & 0.0 & $(0.0)$ & 0.0 & $(0.0)$ & 52.0 & (1.6) & 45.5 & (1.4) & 12.9 & (1.1) & 0.8 & $(0.2)$ \\
\hline & Israel & 7.1 & $(0.8)$ & 0.1 & $(0.1)$ & 0.0 & $(0.0)$ & 0.0 & $(0.0)$ & 42.7 & (2.1) & 19.7 & $(1.3)$ & 2.5 & $(0.5)$ & 0.5 & $(0.2)$ \\
\hline & Italien & 11.2 & $(0.6)$ & 2.3 & $(0.2)$ & 0.2 & $(0.0)$ & 0.0 & $(0.0)$ & 31.9 & $(0.8)$ & 51.3 & $(0.9)$ & 35.6 & $(0.8)$ & 4.4 & $(0.4)$ \\
\hline & Japan & 12.1 & $(0.9)$ & 1.9 & $(0.4)$ & 0.1 & $(0.1)$ & 0.0 & $(0.0)$ & 54.0 & (1.4) & 57.1 & (1.5) & 27.0 & (1.1) & 1.3 & $(0.3)$ \\
\hline & Korea & 10.8 & (1.0) & 0.6 & $(0.2)$ & 0.0 & $(0.0)$ & 0.0 & $(0.0)$ & 50.6 & (1.5) & 49.3 & (1.7) & 24.7 & (1.3) & 1.7 & $(0.4)$ \\
\hline & Luxemburg & 14.6 & (1.1) & 2.7 & $(0.5)$ & 0.0 & $(0.0)$ & 0.0 & $(0.0)$ & 28.7 & (1.4) & 53.1 & (1.5) & 28.0 & (1.3) & 2.0 & $(0.4)$ \\
\hline & Mexiko & 51.3 & (1.3) & 15.1 & $(0.6)$ & 4.0 & $(0.4)$ & 0.1 & $(0.0)$ & 15.1 & $(0.7)$ & 40.4 & $(0.7)$ & 50.2 & $(0.8)$ & 10.2 & $(0.4)$ \\
\hline & Niederlande & 6.8 & $(0.9)$ & 0.2 & $(0.1)$ & 0.0 & $(0.0)$ & 0.0 & $(0.0)$ & 51.2 & (2.1) & 26.6 & $(1.2)$ & 3.2 & $(0.6)$ & 0.5 & $(0.3)$ \\
\hline & Neuseeland & 12.0 & (1.0) & 0.1 & $(0.1)$ & 0.1 & $(0.1)$ & 0.0 & $(0.0)$ & 36.7 & (1.7) & 27.2 & (1.3) & 5.2 & $(0.7)$ & 0.6 & $(0.2)$ \\
\hline & Norwegen & 5.3 & $(0.6)$ & 0.0 & $(0.0)$ & 0.1 & $(0.1)$ & 0.0 & $(0.0)$ & 59.9 & (1.5) & 22.0 & $(1.2)$ & 2.0 & $(0.4)$ & 0.3 & $(0.2)$ \\
\hline & Polen & 5.8 & $(0.7)$ & 0.4 & $(0.2)$ & 0.0 & $(0.0)$ & 0.0 & $(0.0)$ & 25.8 & (1.6) & 45.1 & (1.6) & 22.6 & (1.3) & 0.8 & $(0.3)$ \\
\hline & Portugal & 21.1 & $(1.5)$ & 6.0 & $(0.7)$ & 1.3 & $(0.3)$ & 0.0 & $(0.0)$ & 23.1 & $(1.3)$ & 44.8 & (1.6) & 48.8 & (1.6) & 6.6 & $(0.9)$ \\
\hline & Slowak. Rep. & 18.1 & (1.9) & 2.5 & $(0.6)$ & 0.5 & $(0.3)$ & 0.0 & $(0.0)$ & 35.8 & (1.8) & 56.4 & (1.6) & 40.8 & (1.6) & 9.7 & $(0.8)$ \\
\hline & Slowenien & 11.0 & (1.1) & 0.7 & $(0.3)$ & 0.0 & $(0.0)$ & 0.0 & $(0.0)$ & 36.5 & (1.3) & 40.8 & (1.7) & 19.5 & (1.3) & 1.0 & $(0.3)$ \\
\hline & Spanien & 16.3 & $(0.9)$ & 6.3 & $(0.5)$ & 1.7 & $(0.3)$ & 0.0 & $(0.0)$ & 32.7 & $(1.2)$ & 49.1 & (1.1) & 33.2 & (1.1) & 2.8 & $(0.3)$ \\
\hline & Schweden & 6.3 & $(0.8)$ & 0.0 & $(0.0)$ & 0.0 & $(0.0)$ & 0.0 & $(0.0)$ & 60.5 & (1.4) & 38.0 & (1.7) & 4.8 & $(0.6)$ & 0.3 & $(0.2)$ \\
\hline & Schweiz & 4.8 & $(0.6)$ & 0.5 & $(0.2)$ & 0.1 & $(0.1)$ & 0.0 & $(0.0)$ & 47.8 & (1.1) & 36.4 & (1.6) & 8.3 & $(0.9)$ & 0.5 & $(0.1)$ \\
\hline & Türkei & 20.4 & (1.6) & 11.4 & (1.1) & 4.4 & $(0.7)$ & 0.4 & $(0.2)$ & 24.3 & $(2.0)$ & 40.4 & (1.7) & 51.1 & (1.9) & 31.8 & (1.9) \\
\hline & Ver. Königreich & 8.7 & $(0.8)$ & 0.5 & $(0.2)$ & 0.0 & $(0.0)$ & 0.0 & $(0.0)$ & 58.6 & (1.7) & 37.4 & (1.5) & 6.4 & $(0.6)$ & 0.5 & $(0.2)$ \\
\hline & Ver. Staaten & 21.0 & $(1.5)$ & 1.6 & $(0.5)$ & 0.1 & $(0.1)$ & 0.0 & $(0.0)$ & 45.2 & (1.9) & 32.2 & (1.5) & 8.7 & $(0.9)$ & 0.4 & $(0.3)$ \\
\hline & OECD-Durchschnitt & 13.3 & $(0.2)$ & 2.3 & $(0.1)$ & 0.4 & $(0.0)$ & 0.0 & $(0.0)$ & 41.4 & $(0.3)$ & 41.6 & $(0.2)$ & 19.6 & $(0.2)$ & 2.9 & $(0.1)$ \\
\hline ฐ & Albanien & $\mathrm{m}$ & $\mathrm{m}$ & $\mathrm{m}$ & $\mathrm{m}$ & $\mathrm{m}$ & $\mathrm{m}$ & $\mathrm{m}$ & $\mathrm{m}$ & $\mathrm{m}$ & $\mathrm{m}$ & $\mathrm{m}$ & $\mathrm{m}$ & $\mathrm{m}$ & $\mathrm{m}$ & $\mathrm{m}$ & $\mathrm{m}$ \\
\hline 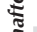 & Argentinien & 21.9 & $(1.5)$ & 9.0 & $(1.0)$ & 3.4 & $(0.6)$ & 0.0 & $(0.0)$ & 23.9 & (1.5) & 42.1 & $(1.7)$ & 44.2 & (1.8) & 7.2 & $(0.7)$ \\
\hline క్ర & Brasilien & 28.8 & (1.1) & 11.3 & $(0.7)$ & 3.4 & $(0.4)$ & 0.1 & $(0.0)$ & 36.5 & (1.1) & 50.1 & $(0.9)$ & 44.8 & $(1.2)$ & 11.0 & $(0.7)$ \\
\hline. & Bulgarien & 12.4 & (1.4) & 2.7 & $(0.6)$ & 0.8 & $(0.3)$ & 0.1 & $(0.1)$ & 30.9 & (1.6) & 45.0 & (1.4) & 28.5 & (1.4) & 2.8 & $(0.5)$ \\
\hline 产 & Kolumbien & 16.2 & (1.3) & 9.9 & $(0.9)$ & 3.8 & $(0.6)$ & 0.4 & $(0.2)$ & 24.1 & (1.5) & 39.4 & $(1.7)$ & 54.2 & (1.6) & 19.4 & (1.4) \\
\hline 20 & Costa Rica & 31.2 & $(3.0)$ & 13.3 & $(1.5)$ & 2.9 & $(0.6)$ & 0.1 & $(0.1)$ & 34.1 & $(2.0)$ & 45.6 & $(1.9)$ & 43.9 & (1.9) & 4.4 & $(0.9)$ \\
\hline$\stackrel{1}{2}$ & Kroatien & 5.2 & $(0.6)$ & 0.9 & $(0.3)$ & 0.3 & $(0.2)$ & 0.0 & $(0.0)$ & 45.4 & (1.8) & 55.8 & (1.4) & 37.5 & (1.8) & 5.3 & $(0.7)$ \\
\hline ֻัญ & Zypern* & 8.8 & $(0.9)$ & 1.0 & $(0.3)$ & 0.1 & $(0.1)$ & 0.0 & $(0.0)$ & 43.1 & (1.5) & 60.0 & $(1.4)$ & 43.2 & (1.6) & 6.2 & $(0.8)$ \\
\hline : & Hongkong (China) & 21.9 & $(1.2)$ & 5.6 & $(0.8)$ & 0.4 & $(0.2)$ & 0.0 & $(0.0)$ & 44.9 & (1.5) & 54.0 & (1.7) & 33.7 & (1.5) & 3.6 & $(0.6)$ \\
\hline$\Xi$ & Indonesien & 36.5 & (3.5) & 32.2 & (3.0) & 16.1 & (1.8) & 1.5 & $(0.4)$ & 10.0 & (1.3) & 26.0 & $(2.1)$ & 42.5 & $(2.2)$ & 21.8 & $(2.3)$ \\
\hline ปั้ & Jordanien & 6.9 & (1.0) & 0.7 & $(0.2)$ & 0.0 & $(0.0)$ & 0.0 & $(0.0)$ & 26.0 & (1.2) & 29.3 & $(1.5)$ & 10.0 & (1.0) & 0.7 & $(0.3)$ \\
\hline & Kasachstan & 11.1 & (1.0) & 1.5 & $(0.4)$ & 0.0 & $(0.0)$ & 0.0 & $(0.0)$ & 36.0 & (2.3) & 32.0 & (1.6) & 4.9 & $(0.7)$ & 0.7 & $(0.3)$ \\
\hline & Lettland & 17.6 & (1.8) & 2.6 & $(0.6)$ & 0.2 & $(0.1)$ & 0.0 & $(0.0)$ & 42.5 & (2.1) & 49.5 & (1.9) & 19.5 & (1.4) & 1.3 & $(0.3)$ \\
\hline & Liechtenstein & 5.7 & (3.2) & 0.0 & $(0.0)$ & 0.0 & $(0.0)$ & 0.0 & $(0.0)$ & 30.4 & (5.3) & 13.3 & (3.6) & 10.0 & (3.2) & 0.0 & $(0.0)$ \\
\hline & Litauen & 13.5 & (1.2) & 3.7 & $(0.5)$ & 0.4 & $(0.2)$ & 0.0 & $(0.0)$ & 29.3 & (1.3) & 41.5 & (1.5) & 12.7 & (1.1) & 0.6 & $(0.2)$ \\
\hline & Macau (China) & 15.5 & (1.1) & 3.9 & $(0.5)$ & 0.8 & $(0.2)$ & 0.1 & $(0.1)$ & 62.3 & (1.3) & 74.0 & $(1.2)$ & 65.8 & (1.5) & 29.8 & $(1.3)$ \\
\hline & Malaysia & 27.2 & $(2.0)$ & 13.5 & (1.1) & 2.1 & $(0.5)$ & 0.0 & $(0.0)$ & 21.5 & (1.4) & 35.8 & (1.6) & 33.4 & (1.5) & 5.1 & $(0.7)$ \\
\hline & Montenegro & 12.7 & (1.1) & 2.7 & $(0.6)$ & 0.2 & $(0.1)$ & 0.0 & $(0.0)$ & 40.1 & (1.7) & 49.6 & $(1.5)$ & 36.7 & $(2.0)$ & 3.4 & $(0.7)$ \\
\hline & Peru & 62.0 & (1.7) & 19.5 & (1.1) & 4.6 & $(0.5)$ & 0.6 & $(0.3)$ & 13.9 & (1.0) & 34.7 & (1.4) & 54.8 & (1.2) & 21.4 & $(1.2)$ \\
\hline & Katar & 2.2 & $(0.3)$ & 0.1 & $(0.1)$ & 0.0 & $(0.0)$ & 0.0 & $(0.0)$ & 34.4 & (1.1) & 17.0 & $(0.9)$ & 5.0 & $(0.4)$ & 3.2 & $(0.3)$ \\
\hline & Rumänien & 11.4 & (1.3) & 3.9 & $(0.8)$ & 1.4 & $(0.4)$ & 0.0 & $(0.0)$ & 31.0 & (1.7) & 41.9 & $(1.8)$ & 34.9 & (1.6) & 3.2 & $(0.6)$ \\
\hline & Russ. Föderation & 5.8 & $(0.7)$ & 0.3 & $(0.2)$ & 0.1 & $(0.1)$ & 0.0 & $(0.0)$ & 46.4 & $(2.0)$ & 47.7 & $(1.5)$ & 13.4 & $(0.9)$ & 1.2 & $(0.4)$ \\
\hline & Serbien & 10.5 & (1.0) & 3.2 & $(0.6)$ & 0.9 & $(0.3)$ & 0.0 & $(0.0)$ & 60.0 & (1.6) & 60.8 & (1.5) & 40.4 & (1.5) & 5.9 & $(0.8)$ \\
\hline & Shanghai (China) & 5.5 & $(0.8)$ & 0.6 & $(0.2)$ & 0.0 & $(0.0)$ & 0.0 & $(0.0)$ & 43.1 & (1.7) & 44.6 & $(1.5)$ & 15.9 & (1.1) & 1.3 & $(0.3)$ \\
\hline & Singapur & 10.0 & $(0.8)$ & 0.4 & $(0.2)$ & 0.0 & $(0.0)$ & 0.0 & $(0.0)$ & 49.9 & (1.4) & 27.1 & (1.3) & 3.7 & $(0.5)$ & 0.1 & $(0.1)$ \\
\hline & Chinesisch Taipeh & 11.5 & $(0.9)$ & 1.5 & $(0.4)$ & 0.0 & $(0.0)$ & 0.1 & $(0.1)$ & 35.7 & (1.5) & 52.9 & $(1.4)$ & 31.8 & (1.3) & 5.6 & $(0.9)$ \\
\hline & Thailand & 20.4 & (1.4) & 16.3 & $(1.2)$ & 8.5 & $(0.9)$ & 1.2 & $(0.3)$ & 12.7 & $(1.0)$ & 26.5 & (1.5) & 41.9 & (1.4) & 20.9 & (1.1) \\
\hline & Tunesien & 64.4 & (1.9) & 38.1 & (1.6) & 9.0 & (1.0) & 0.5 & $(0.2)$ & 14.2 & $(1.2)$ & 23.7 & (1.6) & 40.3 & (1.7) & 10.9 & $(1.1)$ \\
\hline & Ver. Arab. Emirate & 2.6 & $(0.4)$ & 0.0 & $(0.0)$ & 0.1 & $(0.1)$ & 0.0 & $(0.0)$ & 33.6 & (1.5) & 11.1 & $(0.9)$ & 3.7 & $(0.5)$ & 2.1 & $(0.4)$ \\
\hline & Uruguay & 42.6 & (1.6) & 16.0 & (1.1) & 6.9 & $(0.8)$ & 0.6 & $(0.2)$ & 26.6 & (1.4) & 45.4 & (1.4) & 57.0 & (1.3) & 12.8 & $(1.2)$ \\
\hline & Vietnam & 4.1 & (1.1) & 3.2 & $(0.7)$ & 3.3 & $(0.6)$ & 1.2 & $(0.3)$ & 5.3 & $(0.8)$ & 8.5 & $(0.9)$ & 25.0 & (1.7) & 32.7 & $(1.7)$ \\
\hline
\end{tabular}

1. ESCS bezieht sich auf den PISA-Index des wirtschaftlichen, sozialen und kulturellen Status.

2. Die Variable, die die Anzahl der im Elternhaus vorhandenen Bücher angibt, wurde folgendermaßen kodiert: 0-10 Bücher wurden als 5 Bücher kodiert, $11-25$ Bücher als 18 Bücher, 26-100 Bücher als 63 Bücher, 101-200 Bücher als 150,5 Bücher, 201-500 Bücher als 350,5 Bücher und über 500 Bücher als 750,5 Bücher

$*$ Vgl. Anmerkungen am Anfang dieses Anhangs.

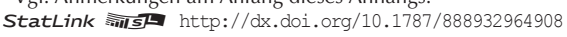


[Teil 4/7]

Merkmale des sozioökonomischen Status, nach Quartilen des sozioökonomischen Status innerhalb der einzelnen Länder

Tabelle II.2.2 Die Ergebnisse basieren auf Schülerangaben

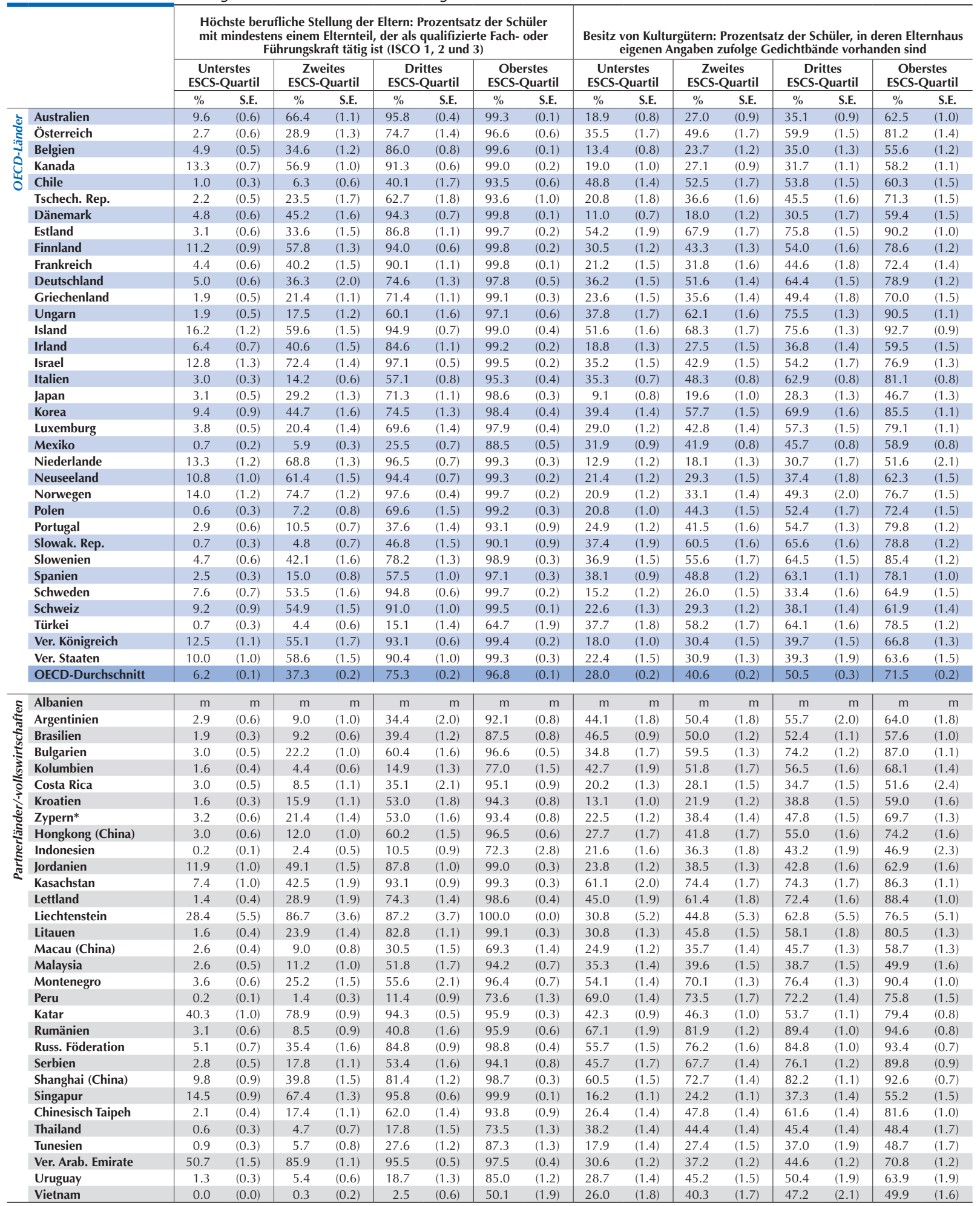

1. ESCS bezieht sich auf den PISA-Index des wirtschaftlichen, sozialen und kulturellen Status.

2. Die Variable, die die Anzahl der im Elternhaus vorhandenen Bücher angibt, wurde folgendermaßen kodiert: 0-10 Bücher wurden als 5 Bücher kodiert, $11-25$ Bücher als 18

Bücher, 26-100 Bücher als 63 Bücher, 101-200 Bücher als 150,5 Bücher, 201-500 Bücher als 350,5 Bücher und über 500 Bücher als 750,5 Bücher.

$* \mathrm{Vgl}$. Anmerkungen am Anfang dieses Anhangs.

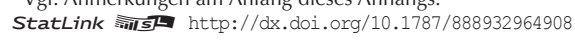


[Teil 5/7]

Merkmale des sozioökonomischen Status, nach Quartilen des sozioökonomischen Status innerhalb der einzelnen Länder

Tabelle II.2.2 Die Ergebnisse basieren auf Schülerangaben

\begin{tabular}{|c|c|c|c|c|c|c|c|c|c|c|c|c|c|c|c|c|c|}
\hline & & \multicolumn{8}{|c|}{$\begin{array}{l}\text { Besitz von Kulturgütern: Prozentsatz der Schüler, in deren Elternhaus } \\
\text { eigenen Angaben zufolge Kunstwerke vorhanden sind }\end{array}$} & \multicolumn{8}{|c|}{$\begin{array}{l}\text { Besitz von Kulturgütern: Prozentsatz der Schüler, in deren Elternhaus } \\
\text { eigenen Angaben zufolge ein Schreibtisch zum Lernen vorhanden ist }\end{array}$} \\
\hline & & \multicolumn{2}{|c|}{$\begin{array}{c}\text { Unterstes } \\
\text { ESCS-Quartil }\end{array}$} & \multicolumn{2}{|c|}{$\begin{array}{c}\text { Zweites } \\
\text { ESCS-Quartil }\end{array}$} & \multicolumn{2}{|c|}{$\begin{array}{c}\text { Drittes } \\
\text { ESCS-Quartil }\end{array}$} & \multicolumn{2}{|c|}{$\begin{array}{c}\text { Oberstes } \\
\text { ESCS-Quartil }\end{array}$} & $\begin{array}{l}\text { Un } \\
\text { ESCS }\end{array}$ & $\begin{array}{l}\text { stes } \\
\text { uartil }\end{array}$ & $\begin{aligned} \mathrm{Z} \\
\mathrm{ESCS}\end{aligned}$ & tuartil & $\begin{array}{r}\text { D } \\
\text { ESCS }\end{array}$ & uartil & $\begin{aligned} \mathrm{Ob} \\
\mathrm{ESCS}\end{aligned}$ & $\begin{array}{l}\text { tes } \\
\text { uartil }\end{array}$ \\
\hline & & $\%$ & S.E. & $\%$ & S.E. & $\%$ & S.E. & $\%$ & S.E. & $\%$ & S.E. & $\%$ & S.E. & $\%$ & S.E. & $\%$ & S.E. \\
\hline ذ & Australien & 58.7 & (1.0) & 68.7 & $(0.9)$ & 79.1 & $(0.8)$ & 90.1 & $(0.6)$ & 82.7 & $(0.8)$ & 90.4 & $(0.5)$ & 94.7 & $(0.4)$ & 98.9 & $(0.2)$ \\
\hline ב & Österreich & 55.8 & (1.5) & 70.7 & (1.4) & 76.7 & $(1.3)$ & 87.5 & (1.1) & 92.4 & $(0.9)$ & 96.5 & $(0.6)$ & 98.1 & $(0.4)$ & 99.4 & $(0.2)$ \\
\hline & Belgien & 35.4 & (1.1) & 52.8 & (1.3) & 60.4 & (1.5) & 80.7 & $(0.8)$ & 90.8 & $(0.7)$ & 96.8 & $(0.6)$ & 98.5 & $(0.4)$ & 99.5 & $(0.1)$ \\
\hline 0 & Kanada & 58.4 & (1.1) & 70.9 & (1.0) & 76.7 & $(0.9)$ & 90.9 & $(0.5)$ & 77.2 & $(1.0)$ & 83.6 & $(0.7)$ & 90.7 & $(0.6)$ & 96.3 & $(0.4)$ \\
\hline o & Chile & 22.1 & (1.2) & 38.0 & (1.4) & 56.0 & (1.5) & 70.9 & (1.3) & 50.0 & (1.7) & 69.0 & (1.5) & 76.9 & (1.5) & 89.7 & $(0.6)$ \\
\hline & Tschech. Rep. & 33.8 & (1.8) & 40.8 & (1.8) & 53.1 & (1.5) & 68.7 & (1.4) & 97.5 & (0.6) & 98.9 & $(0.3)$ & 99.8 & $(0.1)$ & 99.7 & $(0.2)$ \\
\hline & Dänemark & 56.4 & (1.7) & 71.3 & (1.3) & 79.2 & (1.5) & 94.2 & $(0.6)$ & 80.4 & $(1.2)$ & 89.7 & $(0.9)$ & 92.7 & $(0.8)$ & 98.5 & $(0.4)$ \\
\hline & Estland & 45.9 & (1.8) & 60.7 & (1.5) & 66.1 & (1.5) & 81.6 & (1.3) & 96.4 & (0.6) & 97.6 & $(0.5)$ & 97.4 & (0.5) & 99.3 & $(0.2)$ \\
\hline & Finnland & 61.2 & (1.5) & 73.3 & $(1.2)$ & 79.0 & $(1.1)$ & 90.7 & $(0.8)$ & 89.7 & $(0.9)$ & 93.6 & $(0.6)$ & 95.2 & $(0.4)$ & 98.6 & $(0.3)$ \\
\hline & Frankreich & 17.6 & $(1.2)$ & 31.0 & (1.6) & 41.8 & (1.3) & 67.0 & (1.4) & 96.1 & (0.6) & 98.0 & $(0.5)$ & 98.9 & $(0.3)$ & 99.8 & $(0.1)$ \\
\hline & Deutschland & 53.6 & (1.6) & 65.5 & (1.7) & 76.0 & (1.5) & 86.9 & $(0.9)$ & 93.5 & (0.8) & 96.5 & $(0.7)$ & 98.3 & $(0.4)$ & 99.2 & (0.4) \\
\hline & Griechenland & 42.4 & (1.6) & 61.9 & (1.6) & 73.0 & $(1.2)$ & 83.8 & (1.1) & 95.4 & $(0.8)$ & 98.4 & $(0.4)$ & 98.1 & $(0.5)$ & 99.5 & $(0.2)$ \\
\hline & Ungarn & 29.8 & (1.6) & 45.9 & (1.6) & 56.9 & (1.6) & 74.3 & (1.3) & 94.9 & $(0.7)$ & 98.2 & $(0.4)$ & 98.9 & $(0.4)$ & 99.4 & (0.3) \\
\hline & Island & 77.7 & (1.5) & 90.0 & (1.0) & 94.1 & $(0.8)$ & 98.7 & (0.4) & 90.3 & (1.0) & 94.4 & (0.9) & 95.7 & $(0.7)$ & 99.6 & $(0.2)$ \\
\hline & Irland & 55.3 & (1.5) & 69.2 & (1.2) & 77.7 & $(1.2)$ & 88.3 & $(1.0)$ & 77.2 & (1.3) & 89.1 & $(0.9)$ & 93.3 & (0.9) & 97.9 & $(0.4)$ \\
\hline & Israel & 46.8 & (1.7) & 63.8 & (1.4) & 75.1 & (1.4) & 88.7 & $(0.8)$ & 89.9 & (1.1) & 95.0 & $(0.9)$ & 97.6 & $(0.5)$ & 99.0 & (0.4) \\
\hline & Italien & 52.8 & (0.9) & 64.0 & $(0.7)$ & 75.5 & $(0.6)$ & 85.0 & $(0.5)$ & 94.1 & $(0.4)$ & 97.1 & $(0.3)$ & 98.3 & $(0.2)$ & 99.0 & (0.1) \\
\hline & Japan & 17.9 & $(1.0)$ & 27.3 & (1.4) & 36.2 & $(1.2)$ & 53.8 & (1.5) & 90.2 & $(1.2)$ & 95.2 & $(0.8)$ & 96.8 & $(0.5)$ & 98.7 & $(0.3)$ \\
\hline & Korea & 24.0 & $(1.2)$ & 40.1 & (1.4) & 55.2 & (1.7) & 76.4 & $(1.2)$ & 91.3 & (0.8) & 96.6 & $(0.5)$ & 98.0 & $(0.4)$ & 99.4 & $(0.2)$ \\
\hline & Luxemburg & 56.2 & (1.3) & 71.1 & (1.4) & 80.9 & $(1.1)$ & 92.0 & $(0.8)$ & 94.5 & $(0.6)$ & 96.5 & $(0.5)$ & 97.8 & $(0.5)$ & 99.4 & $(0.2)$ \\
\hline & Mexiko & 13.8 & (0.6) & 25.4 & (1.0) & 35.5 & $(0.7)$ & 59.6 & (1.0) & 61.4 & (0.9) & 69.6 & $(0.7)$ & 80.1 & $(0.7)$ & 90.4 & (0.4) \\
\hline & Niederlande & 52.6 & (1.7) & 65.5 & $(1.2)$ & 73.5 & (1.4) & 88.6 & (1.5) & 90.7 & $(0.9)$ & 95.2 & $(0.6)$ & 97.6 & $(0.5)$ & 99.4 & $(0.2)$ \\
\hline & Neuseeland & 51.7 & (1.6) & 68.6 & (1.6) & 74.9 & (1.3) & 90.5 & $(0.9)$ & 67.6 & (1.5) & 84.3 & (1.1) & 90.4 & $(0.9)$ & 96.2 & $(0.6)$ \\
\hline & Norwegen & 70.1 & (1.3) & 81.0 & $(1.2)$ & 89.3 & $(1.1)$ & 96.6 & $(0.5)$ & 92.2 & $(0.7)$ & 96.1 & $(0.6)$ & 98.1 & $(0.4)$ & 99.8 & $(0.1)$ \\
\hline & Polen & 31.6 & (1.5) & 50.3 & (1.6) & 56.5 & (1.6) & 69.0 & (1.8) & 93.8 & (0.8) & 98.9 & $(0.4)$ & 98.5 & $(0.4)$ & 99.0 & $(0.3)$ \\
\hline & Portugal & 43.5 & (1.5) & 58.4 & (1.4) & 69.9 & (1.5) & 81.3 & (1.2) & 89.2 & $(0.9)$ & 95.7 & $(0.6)$ & 97.4 & $(0.5)$ & 99.1 & $(0.3)$ \\
\hline & Slowak. Rep. & 39.8 & (1.8) & 59.5 & (1.5) & 69.1 & (1.3) & 81.2 & (1.3) & 74.2 & (2.1) & 94.7 & $(0.8)$ & 96.3 & (0.6) & 98.2 & (0.6) \\
\hline & Slowenien & 54.6 & (1.6) & 69.3 & (1.5) & 75.8 & (1.4) & 90.8 & (1.0) & 98.6 & $(0.4)$ & 99.7 & $(0.1)$ & 99.7 & $(0.1)$ & 99.7 & $(0.2)$ \\
\hline & Spanien & 39.3 & $(0.9)$ & 51.2 & $(1.2)$ & 67.9 & $(1.1)$ & 81.7 & (1.0) & 95.8 & $(0.5)$ & 97.8 & $(0.3)$ & 98.8 & $(0.2)$ & 99.4 & $(0.1)$ \\
\hline & Schweden & 63.7 & (1.8) & 75.6 & (1.3) & 80.9 & $(1.2)$ & 94.4 & $(0.7)$ & 81.5 & (1.4) & 90.2 & $(0.8)$ & 92.1 & $(0.8)$ & 97.3 & (0.5) \\
\hline & Schweiz & 48.8 & (1.4) & 59.5 & (1.1) & 70.0 & (1.3) & 82.3 & $(1.1)$ & 96.5 & $(0.4)$ & 97.4 & $(0.4)$ & 98.2 & $(0.4)$ & 99.2 & $(0.2)$ \\
\hline & Türkei & 10.6 & (0.9) & 22.4 & (1.3) & 36.6 & (1.5) & 57.1 & (1.8) & 61.7 & (1.6) & 88.3 & $(1.0)$ & 93.3 & $(0.8)$ & 98.4 & $(0.4)$ \\
\hline & Ver. Königreich & 43.7 & (1.6) & 56.5 & (1.4) & 65.9 & $(1.2)$ & 84.0 & $(1.0)$ & 72.0 & (1.1) & 82.6 & $(1.1)$ & 88.8 & (0.9) & 97.2 & (0.5) \\
\hline & Ver. Staaten & 49.3 & (2.1) & 64.8 & (1.5) & 75.2 & (1.3) & 87.6 & $(0.9)$ & 61.4 & (1.4) & 74.8 & $(1.3)$ & 81.0 & (1.1) & 94.0 & $(0.7)$ \\
\hline & OECD-Durchschnitt & 44.6 & $(0.2)$ & 58.4 & $(0.2)$ & 67.9 & $(0.2)$ & 82.2 & $(0.2)$ & 85.3 & $(0.2)$ & 92.2 & $(0.1)$ & 94.9 & $(0.1)$ & 98.2 & $(0.1)$ \\
\hline$=$ & Albanien & $\mathrm{m}$ & $\mathrm{m}$ & $m$ & $\mathrm{~m}$ & $\mathrm{~m}$ & $\mathrm{~m}$ & $\mathrm{~m}$ & $\mathrm{~m}$ & $\mathrm{~m}$ & $\mathrm{~m}$ & $\mathrm{~m}$ & $\mathrm{~m}$ & $\mathrm{~m}$ & $\mathrm{~m}$ & $\mathrm{~m}$ & $\mathrm{~m}$ \\
\hline 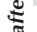 & Argentinien & 17.5 & (1.4) & 26.3 & (1.4) & 37.2 & (1.6) & 57.6 & (1.6) & 69.1 & (1.8) & 80.9 & (1.6) & 87.9 & (1.0) & 94.2 & $(0.8)$ \\
\hline కัป & Brasilien & 24.6 & (1.0) & 30.5 & $(1.2)$ & 41.1 & $(1.2)$ & 57.6 & (1.5) & 45.5 & (1.1) & 57.8 & (1.1) & 71.3 & $(0.7)$ & 85.5 & $(0.8)$ \\
\hline$=$ & Bulgarien & 36.5 & (1.5) & 59.3 & (1.5) & 67.9 & (1.4) & 81.4 & $(1.2)$ & 76.6 & (1.9) & 94.1 & $(0.7)$ & 96.2 & $(0.7)$ & 98.2 & $(0.4)$ \\
\hline$\sum_{5}^{2}$ & Kolumbien & 28.7 & (1.6) & 43.8 & (1.6) & 55.8 & $(1.4)$ & 73.4 & (1.5) & 43.1 & (2.1) & 63.0 & (1.8) & 75.2 & (1.6) & 90.9 & (1.1) \\
\hline$=$ & Costa Rica & 22.5 & (1.4) & 33.4 & (1.8) & 54.9 & (1.8) & 73.2 & (1.5) & 60.0 & $(2.0)$ & 79.1 & $(1.2)$ & 90.2 & (1.0) & 95.4 & $(0.7)$ \\
\hline 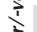 & Kroatien & 35.3 & (1.5) & 52.1 & (1.5) & 65.9 & $(1.4)$ & 80.4 & $(1.1)$ & 96.4 & $(0.5)$ & 98.7 & $(0.3)$ & 98.9 & $(0.4)$ & 99.3 & $(0.2)$ \\
\hline ¿ & Zypern* & 47.9 & (1.8) & 69.4 & (1.3) & 78.5 & $(1.3)$ & 88.8 & $(1.0)$ & 94.8 & $(0.5)$ & 98.3 & $(0.4)$ & 98.3 & $(0.4)$ & 99.6 & $(0.2)$ \\
\hline : & Hongkong (China) & 15.4 & $(1.2)$ & 25.3 & (1.1) & 40.3 & (1.5) & 60.7 & (1.7) & 83.7 & $(1.0)$ & 93.3 & $(0.7)$ & 94.8 & $(0.7)$ & 98.3 & $(0.3)$ \\
\hline ఏ & Indonesien & 32.5 & (1.6) & 48.6 & $(2.0)$ & 62.1 & $(1.7)$ & 70.3 & (1.6) & 47.8 & (1.9) & 68.9 & $(1.8)$ & 77.2 & (1.3) & 91.0 & $(0.9)$ \\
\hline ऐ) & Jordanien & 20.4 & (1.1) & 34.5 & (1.6) & 43.0 & (1.6) & 60.8 & (1.5) & 34.8 & (1.6) & 63.9 & (1.6) & 77.2 & (1.1) & 92.3 & $(0.7)$ \\
\hline & Kasachstan & 45.8 & (1.4) & 61.8 & $(2.2)$ & 62.7 & (1.8) & 77.3 & $(1.7)$ & 81.1 & (1.7) & 93.3 & $(0.9)$ & 96.0 & $(0.7)$ & 99.4 & $(0.3)$ \\
\hline & Lettland & 43.7 & (2.1) & 58.1 & (1.5) & 62.1 & $(1.4)$ & 79.3 & $(1.5)$ & 94.8 & $(0.9)$ & 98.6 & $(0.4)$ & 98.9 & $(0.2)$ & 99.6 & $(0.2)$ \\
\hline & Liechtenstein & 63.8 & (5.8) & 69.7 & (5.8) & 84.4 & (4.3) & 90.3 & $(4.0)$ & 98.5 & (1.6) & 98.7 & $(1.3)$ & 98.5 & (1.5) & 100.0 & $(0.0)$ \\
\hline & Litauen & 51.7 & (1.4) & 65.7 & (1.4) & 72.0 & $(1.3)$ & 87.5 & $(1.0)$ & 97.1 & $(0.5)$ & 99.1 & $(0.3)$ & 99.1 & $(0.3)$ & 99.6 & $(0.2)$ \\
\hline & Macau (China) & 14.5 & $(1.0)$ & 25.1 & $(1.2)$ & 37.9 & (1.3) & 57.2 & (1.4) & 72.8 & (1.3) & 83.0 & $(1.0)$ & 90.9 & $(0.8)$ & 96.2 & $(0.5)$ \\
\hline & Malaysia & 36.7 & (1.3) & 45.5 & (1.5) & 48.4 & (1.5) & 58.1 & (1.4) & 74.1 & (1.5) & 87.0 & $(1.1)$ & 89.1 & $(0.8)$ & 94.5 & $(0.7)$ \\
\hline & Montenegro & 45.6 & (1.3) & 63.6 & (1.7) & 74.8 & (1.4) & 91.9 & (0.9) & 94.7 & $(0.5)$ & 97.7 & $(0.5)$ & 98.4 & $(0.4)$ & 99.4 & $(0.2)$ \\
\hline & Peru & 35.3 & (1.6) & 42.0 & (1.5) & 48.5 & (1.6) & 57.7 & $(2.1)$ & 49.8 & (1.5) & 74.1 & (1.3) & 87.1 & (1.0) & 93.9 & $(0.6)$ \\
\hline & Katar & 43.7 & (0.9) & 57.7 & (1.0) & 66.0 & $(0.8)$ & 85.4 & $(0.8)$ & 58.8 & (1.0) & 84.0 & $(0.7)$ & 90.2 & (0.6) & 97.4 & (0.3) \\
\hline & Rumänien & 28.8 & $(1.2)$ & 49.7 & (1.5) & 64.0 & $(1.6)$ & 76.3 & (1.6) & 86.9 & $(1.2)$ & 96.7 & $(0.6)$ & 98.1 & $(0.4)$ & 99.2 & $(0.3)$ \\
\hline & Russ. Föderation & 38.1 & (1.7) & 57.1 & (1.5) & 61.6 & $(1.7)$ & 78.5 & (1.8) & 97.0 & (0.4) & 97.9 & $(0.4)$ & 99.3 & $(0.2)$ & 99.3 & $(0.2)$ \\
\hline & Serbien & 37.4 & (1.6) & 58.8 & (1.6) & 73.8 & (1.4) & 85.1 & (1.3) & 95.7 & (0.6) & 99.0 & $(0.3)$ & 99.5 & $(0.2)$ & 99.6 & $(0.2)$ \\
\hline & Shanghai (China) & 29.3 & (1.4) & 50.7 & (1.6) & 64.0 & (1.4) & 82.0 & $(1.1)$ & 94.1 & $(0.7)$ & 98.1 & $(0.4)$ & 98.6 & $(0.4)$ & 99.6 & $(0.2)$ \\
\hline & Singapur & 24.9 & (1.2) & 38.4 & (1.2) & 51.3 & (1.5) & 70.1 & (1.4) & 80.9 & (1.0) & 91.9 & $(0.8)$ & 96.8 & $(0.5)$ & 98.9 & $(0.3)$ \\
\hline & Chinesisch Taipeh & 16.8 & (1.0) & 37.9 & (1.3) & 51.1 & (1.3) & 72.0 & (1.4) & 85.2 & (0.9) & 95.6 & $(0.5)$ & 97.1 & $(0.4)$ & 99.3 & $(0.2)$ \\
\hline & Thailand & 52.4 & (1.6) & 66.0 & (1.4) & 71.7 & $(1.1)$ & 75.1 & $(1.2)$ & 68.2 & (1.6) & 83.7 & $(1.2)$ & 92.2 & $(0.9)$ & 95.5 & $(0.7)$ \\
\hline & Tunesien & 17.1 & $(1.2)$ & 27.9 & (1.3) & 39.5 & (1.6) & 56.5 & $(2.1)$ & 72.4 & (1.8) & 88.3 & $(1.0)$ & 96.3 & $(0.6)$ & 98.9 & $(0.3)$ \\
\hline & Ver. Arab. Emirate & 33.1 & (1.1) & 48.9 & (1.2) & 57.0 & $(1.3)$ & 79.0 & (1.3) & 63.8 & (1.2) & 84.5 & $(0.9)$ & 92.6 & $(0.6)$ & 96.5 & $(0.4)$ \\
\hline & Uruguay & 19.8 & $(1.2)$ & 34.2 & (1.3) & 49.9 & $(1.7)$ & 75.1 & (1.4) & 62.4 & (1.5) & 85.1 & $(1.0)$ & 90.8 & $(0.9)$ & 97.2 & $(0.6)$ \\
\hline & Vietnam & 27.3 & (1.6) & 43.6 & (1.8) & 49.4 & (1.5) & 61.3 & (1.9) & 87.4 & (1.3) & 96.9 & $(0.6)$ & 98.5 & $(0.4)$ & 99.8 & $(0.1)$ \\
\hline
\end{tabular}

1. ESCS bezieht sich auf den PISA-Index des wirtschaftlichen, sozialen und kulturellen Status.

2. Die Variable, die die Anzahl der im Elternhaus vorhandenen Bücher angibt, wurde folgendermaßen kodiert: 0-10 Bücher wurden als 5 Bücher kodiert, 11-25 Bücher als 18 Bücher, 26-100 Bücher als 63 Bücher, 101-200 Bücher als 150,5 Bücher, 201-500 Bücher als 350,5 Bücher und über 500 Bücher als 750,5 Bücher

$*$ Vgl. Anmerkungen am Anfang dieses Anhangs.

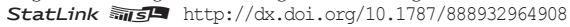


[Teil 6/7]

Merkmale des sozioökonomischen Status, nach Quartilen des sozioökonomischen Status innerhalb der einzelnen Länder

Tabelle II.2.2 Die Ergebnisse basieren auf Schülerangaben

\begin{tabular}{|c|c|c|c|c|c|c|c|c|c|c|c|c|c|c|c|c|c|}
\hline & & \multicolumn{8}{|c|}{$\begin{array}{c}\text { Bildungsressourcen im Elternhaus: Prozentsatz der Schüler, in deren } \\
\text { Elternhaus eigenen Angaben zufolge ein ruhiger Platz zum Lernen } \\
\text { vorhanden ist }\end{array}$} & \multicolumn{8}{|c|}{$\begin{array}{l}\text { Bildungsressourcen im Elternhaus: Prozentsatz der Schüler, in deren } \\
\text { Elternhaus eigenen Angaben zufolge ein Wörterbuch vorhanden ist }\end{array}$} \\
\hline & & \multicolumn{2}{|c|}{$\begin{array}{c}\text { Unterstes } \\
\text { ESCS-Quartil }\end{array}$} & \multicolumn{2}{|c|}{$\begin{array}{c}\text { Zweites } \\
\text { ESCS-Quartil }\end{array}$} & \multicolumn{2}{|c|}{$\begin{array}{c}\text { Drittes } \\
\text { ESCS-Quartil }\end{array}$} & \multicolumn{2}{|c|}{$\begin{array}{c}\text { Oberstes } \\
\text { ESCS-Quartil }\end{array}$} & $\begin{aligned} \text { Un } \\
\text { ESCS }\end{aligned}$ & stes & $\begin{array}{r}\mathrm{Zn} \\
\mathrm{ESCS}\end{array}$ & ites & $\begin{aligned} \text { Dr } \\
\text { ESCS }\end{aligned}$ & $\begin{array}{l}\text { tes } \\
\text { uartil }\end{array}$ & $\begin{aligned} \mathrm{Ob} \\
\mathrm{ESCS}\end{aligned}$ & $\begin{array}{l}\text { estes } \\
\text { Quartil }\end{array}$ \\
\hline & & $\%$ & S.E. & $\%$ & S.E. & $\%$ & S.E. & $\%$ & S.E. & $\%$ & S.E. & $\%$ & S.E. & $\%$ & S.E. & $\%$ & S.E. \\
\hline \pm & Australien & 79.2 & $(0.9)$ & 87.7 & $(0.7)$ & 91.5 & (0.6) & 96.5 & $(0.4)$ & 91.7 & $(0.6)$ & 95.2 & $(0.4)$ & 98.2 & $(0.3)$ & 99.7 & $(0.1)$ \\
\hline $\mathbb{T}$ & Österreich & 92.6 & $(0.8)$ & 97.3 & $(0.4)$ & 97.9 & $(0.5)$ & 98.7 & $(0.3)$ & 96.7 & $(0.6)$ & 97.8 & $(0.5)$ & 99.1 & $(0.4)$ & 99.6 & $(0.3)$ \\
\hline & Belgien & 87.5 & $(0.9)$ & 93.5 & $(0.7)$ & 95.5 & $(0.5)$ & 98.3 & $(0.3)$ & 93.9 & $(0.8)$ & 98.1 & $(0.3)$ & 99.1 & $(0.3)$ & 99.4 & $(0.2)$ \\
\hline 0 & Kanada & 87.0 & $(0.8)$ & 90.2 & $(0.6)$ & 93.1 & $(0.6)$ & 97.9 & $(0.3)$ & 91.1 & (0.6) & 96.2 & $(0.4)$ & 97.6 & $(0.4)$ & 99.5 & $(0.2)$ \\
\hline ప & Chile & 70.1 & (1.3) & 79.9 & (1.0) & 85.6 & $(0.9)$ & 91.1 & $(0.8)$ & 95.6 & $(0.9)$ & 98.7 & $(0.3)$ & 99.1 & $(0.3)$ & 99.3 & $(0.2)$ \\
\hline & Tschech. Rep. & 87.4 & $(1.2)$ & 93.5 & (1.0) & 94.9 & $(0.7)$ & 97.7 & $(0.5)$ & 85.5 & (1.6) & 94.9 & $(0.8)$ & 98.0 & $(0.5)$ & 99.1 & $(0.3)$ \\
\hline & Dänemark & 81.5 & (1.3) & 91.4 & $(0.8)$ & 92.9 & $(0.7)$ & 97.0 & $(0.6)$ & 90.6 & $(0.9)$ & 96.6 & $(0.5)$ & 98.4 & $(0.4)$ & 99.5 & $(0.2)$ \\
\hline & Estland & 88.0 & $(1.2)$ & 93.0 & $(0.8)$ & 93.9 & $(0.7)$ & 97.3 & $(0.4)$ & 85.5 & (1.1) & 93.7 & $(0.8)$ & 96.2 & $(0.6)$ & 98.7 & $(0.3)$ \\
\hline & Finnland & 91.6 & $(0.8)$ & 95.1 & $(0.5)$ & 95.9 & $(0.5)$ & 98.4 & $(0.3)$ & 78.9 & $(1.2)$ & 89.6 & $(0.9)$ & 94.2 & $(0.7)$ & 98.9 & $(0.3)$ \\
\hline & Frankreich & 90.7 & $(0.9)$ & 94.8 & $(0.6)$ & 96.3 & $(0.7)$ & 98.8 & $(0.3)$ & 96.5 & $(0.6)$ & 97.9 & $(0.5)$ & 99.0 & $(0.3)$ & 99.9 & $(0.1)$ \\
\hline & Deutschland & 93.7 & $(0.9)$ & 97.0 & $(0.5)$ & 97.1 & $(0.5)$ & 98.8 & $(0.3)$ & 93.4 & (1.0) & 97.3 & $(0.5)$ & 98.1 & $(0.4)$ & 99.5 & $(0.2)$ \\
\hline & Griechenland & 77.0 & (1.1) & 84.8 & (1.1) & 87.4 & (1.1) & 92.6 & $(0.7)$ & 93.6 & $(0.8)$ & 97.6 & $(0.5)$ & 97.9 & $(0.5)$ & 99.5 & $(0.2)$ \\
\hline & Ungarn & 85.6 & (1.4) & 92.6 & (1.0) & 93.8 & $(0.8)$ & 96.4 & $(0.6)$ & 81.1 & (1.6) & 94.3 & $(0.8)$ & 97.9 & $(0.5)$ & 99.4 & $(0.2)$ \\
\hline & Island & 93.3 & $(0.8)$ & 95.5 & $(0.6)$ & 96.5 & $(0.7)$ & 98.7 & $(0.3)$ & 90.9 & (1.1) & 96.0 & $(0.6)$ & 98.4 & $(0.4)$ & 99.6 & $(0.2)$ \\
\hline & Irland & 81.9 & $(1.2)$ & 89.5 & $(0.9)$ & 90.2 & $(0.8)$ & 96.2 & $(0.6)$ & 94.6 & $(0.9)$ & 97.7 & $(0.5)$ & 99.1 & $(0.3)$ & 99.6 & $(0.2)$ \\
\hline & Israel & 87.2 & $(1.2)$ & 92.4 & $(0.8)$ & 95.2 & $(0.7)$ & 98.2 & $(0.4)$ & 93.4 & $(0.8)$ & 97.7 & $(0.6)$ & 98.4 & $(0.4)$ & 99.7 & $(0.2)$ \\
\hline & Italien & 88.0 & $(0.5)$ & 91.2 & $(0.5)$ & 94.3 & $(0.4)$ & 95.5 & $(0.4)$ & 97.8 & $(0.3)$ & 98.7 & $(0.2)$ & 99.5 & $(0.1)$ & 99.5 & $(0.1)$ \\
\hline & Japan & 80.0 & (1.1) & 88.0 & $(0.8)$ & 88.4 & $(0.9)$ & 93.3 & $(0.6)$ & 95.3 & $(0.7)$ & 97.9 & $(0.3)$ & 99.2 & $(0.2)$ & 99.4 & $(0.2)$ \\
\hline & Korea & 68.2 & (1.4) & 80.3 & (1.3) & 85.6 & $(0.9)$ & 93.3 & $(0.8)$ & 92.4 & $(0.8)$ & 96.9 & $(0.5)$ & 98.8 & $(0.3)$ & 99.9 & $(0.1)$ \\
\hline & Luxemburg & 89.5 & $(0.8)$ & 93.3 & $(0.7)$ & 96.1 & $(0.5)$ & 97.9 & $(0.4)$ & 95.8 & $(0.5)$ & 97.8 & $(0.4)$ & 98.4 & $(0.4)$ & 99.8 & $(0.1)$ \\
\hline & Mexiko & 61.0 & $(0.9)$ & 69.0 & $(0.8)$ & 73.7 & $(0.8)$ & 83.7 & $(0.5)$ & 95.6 & $(0.4)$ & 97.7 & $(0.2)$ & 98.0 & $(0.2)$ & 98.5 & $(0.2)$ \\
\hline & Niederlande & 94.5 & $(0.7)$ & 96.4 & $(0.6)$ & 98.5 & $(0.4)$ & 98.6 & $(0.4)$ & 92.4 & $(1.0)$ & 96.2 & $(0.6)$ & 98.8 & $(0.3)$ & 100.0 & $(0.0)$ \\
\hline & Neuseeland & 74.5 & (1.7) & 87.8 & $(0.9)$ & 88.2 & $(1.0)$ & 95.7 & $(0.6)$ & 87.6 & (1.1) & 95.3 & $(0.7)$ & 96.5 & $(0.7)$ & 99.0 & $(0.3)$ \\
\hline & Norwegen & 90.8 & $(0.9)$ & 93.3 & $(0.7)$ & 96.1 & $(0.6)$ & 97.9 & $(0.5)$ & 84.2 & $(1.2)$ & 90.7 & $(0.9)$ & 97.0 & $(0.5)$ & 98.2 & $(0.4)$ \\
\hline & Polen & 91.0 & $(1.0)$ & 97.2 & $(0.4)$ & 96.5 & $(0.6)$ & 97.9 & $(0.5)$ & 96.6 & $(0.6)$ & 98.7 & $(0.4)$ & 98.5 & $(0.4)$ & 99.5 & $(0.2)$ \\
\hline & Portugal & 91.1 & $(0.8)$ & 95.4 & $(0.7)$ & 96.9 & $(0.5)$ & 97.9 & $(0.3)$ & 92.1 & $(0.6)$ & 97.1 & $(0.5)$ & 98.3 & $(0.4)$ & 99.4 & $(0.2)$ \\
\hline & Slowak. Rep. & 74.9 & (1.7) & 87.9 & (1.0) & 90.5 & (0.9) & 95.5 & $(0.8)$ & 79.2 & (1.6) & 95.8 & $(0.7)$ & 96.8 & $(0.5)$ & 99.0 & $(0.3)$ \\
\hline & Slowenien & 88.8 & (1.0) & 94.5 & $(0.6)$ & 93.8 & $(0.8)$ & 98.0 & $(0.4)$ & 84.9 & (1.1) & 92.9 & $(0.8)$ & 95.8 & $(0.7)$ & 98.7 & $(0.3)$ \\
\hline & Spanien & 88.9 & $(0.6)$ & 92.6 & $(0.5)$ & 94.0 & $(0.5)$ & 96.5 & $(0.4)$ & 98.1 & $(0.3)$ & 99.0 & $(0.3)$ & 99.1 & $(0.3)$ & 99.7 & $(0.1)$ \\
\hline & Schweden & 86.7 & (1.1) & 93.0 & $(0.7)$ & 93.8 & $(0.8)$ & 96.9 & $(0.5)$ & 83.3 & (1.2) & 89.7 & $(0$ & 93.8 & $(0.7)$ & 98.6 & $(0.3)$ \\
\hline & Schweiz & 93.4 & $(0.7)$ & 96.6 & $(0.4)$ & 97.5 & $(0.4)$ & 98.0 & $(0.4)$ & 90.3 & $(0.8)$ & 95.4 & $(0$ & 97.5 & $(0.4)$ & 99.3 & $(0.2)$ \\
\hline & Türkei & 63.7 & (1.6) & 85.1 & (1.2) & 88.2 & $(1.0)$ & 94.9 & $(0.8)$ & 93.0 & $(0.7)$ & 97.5 & $(0.5)$ & 98.2 & $(0.4)$ & 99.3 & $(0.2)$ \\
\hline & Ver. Königreich & 78.4 & (1.5) & 87.1 & $(0.9)$ & 90.3 & $(0.8)$ & 96.3 & $(0.5)$ & 85.9 & (1.0) & 93.3 & $(0.7)$ & 96.3 & $(0.5)$ & 99.6 & $(0.1)$ \\
\hline & Ver. Staaten & 80.1 & (1.3) & 85.4 & (1.0) & 91.4 & $(0.8)$ & 97.2 & $(0.4)$ & 80.9 & (1.2) & 88.7 & $(0.9)$ & 95.2 & $(0.7)$ & 98.5 & $(0.4)$ \\
\hline & OECD-Durchschnitt & 84.1 & $(0.2)$ & 90.7 & $(0.1)$ & 92.7 & $(0.1)$ & 96.4 & $(0.1)$ & 90.5 & $(0.2)$ & 95.8 & $(0.1)$ & 97.8 & $(0.1)$ & 99.3 & $(0.0)$ \\
\hline 5 & Albanien & $\mathrm{m}$ & $\mathrm{m}$ & $\mathrm{m}$ & $\mathrm{m}$ & $\mathrm{m}$ & $m$ & $\mathrm{~m}$ & $\mathrm{~m}$ & $\mathrm{~m}$ & $\mathrm{~m}$ & $\mathrm{~m}$ & $\mathrm{~m}$ & $\mathrm{~m}$ & $\mathrm{~m}$ & $\mathrm{~m}$ & $\mathrm{~m}$ \\
\hline 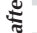 & Argentinien & 68.4 & (1.6) & 72.2 & $(2.0)$ & 79.2 & (1.1) & 89.0 & $(0.9)$ & 94.5 & $(0.8)$ & 97.6 & $(0.5)$ & 97.9 & $(0.8)$ & 99.2 & $(0.4)$ \\
\hline క్ర & Brasilien & 70.0 & $(0.9)$ & 74.1 & $(0.7)$ & 79.6 & $(0.7)$ & 88.1 & $(0.8)$ & 88.3 & $(0.6)$ & 93.2 & $(0.5)$ & 95.3 & $(0.5)$ & 98.1 & $(0.2)$ \\
\hline$\stackrel{n}{\Sigma}$ & Bulgarien & 61.7 & (1.7) & 72.2 & (1.1) & 71.8 & (1.3) & 78.7 & $(1.2)$ & 63.4 & $(2.1)$ & 87.2 & (1.1) & 94.7 & $(0.7)$ & 98.1 & $(0.4)$ \\
\hline$\widehat{\widehat{t}}$ & Kolumbien & 64.1 & (1.6) & 67.9 & (1.3) & 75.0 & (1.4) & 82.9 & (1.1) & 96.6 & $(0.5)$ & 98.6 & $(0.3)$ & 98.5 & $(0.4)$ & 99.4 & $(0.3)$ \\
\hline ङ & Costa Rica & 71.7 & (1.6) & 79.7 & (1.4) & 87.5 & (1.0) & 92.4 & $(0.8)$ & 92.8 & $(0.9)$ & 96.5 & $(0.6)$ & 97.6 & $(0.6)$ & 98.1 & $(0.5)$ \\
\hline 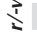 & Kroatien & 80.8 & $(0.9)$ & 84.5 & (1.2) & 89.7 & $(0.9)$ & 90.1 & $(0.9)$ & 93.5 & $(0.7)$ & 96.8 & $(0.5)$ & 97.8 & $(0.4)$ & 99.6 & $(0.2)$ \\
\hline 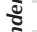 & Zypern* & 81.3 & (1.1) & 88.3 & (1.0) & 92.5 & $(0.9)$ & 96.2 & (0.6) & 91.6 & $(0.7)$ & 97.2 & $(0.4)$ & 97.7 & $(0.5)$ & 99.2 & $(0.3)$ \\
\hline : & Hongkong (China) & 68.2 & (1.6) & 80.0 & (1.3) & 86.4 & (1.1) & 93.2 & $(0.8)$ & 96.9 & $(0.5)$ & 98.8 & $(0.3)$ & 99.1 & $(0.3)$ & 99.5 & $(0.2)$ \\
\hline$\Xi$ & Indonesien & 36.1 & (1.5) & 53.3 & (1.9) & 64.5 & $(1.8)$ & 71.7 & (1.6) & 87.3 & (1.1) & 93.7 & $(0.7)$ & 96.3 & $(0.6)$ & 97.6 & $(0.7)$ \\
\hline ปั & Jordanien & 68.7 & (1.5) & 85.4 & (1.2) & 87.2 & $(1.0)$ & 94.6 & $(0.6)$ & 68.9 & (1.5) & 86.9 & (1.1) & 90.0 & (0.9) & 95.6 & $(0.6)$ \\
\hline & Kasachstan & 79.1 & $(1.2)$ & 89.3 & $(0.9)$ & 88.4 & (1.1) & 94.5 & $(0.7)$ & 75.2 & (1.6) & 89.6 & $(1.1)$ & 93.8 & $(0.8)$ & 97.7 & $(0.5)$ \\
\hline & Lettland & 86.2 & (1.5) & 91.8 & (1.2) & 93.9 & $(0.8)$ & 96.3 & $(0.6)$ & 83.0 & (1.6) & 91.5 & (1.3) & 97.6 & $(0.4)$ & 98.6 & $(0.4)$ \\
\hline & Liechtenstein & 97.2 & $(2.0)$ & 98.5 & (1.5) & 98.5 & (1.5) & 98.7 & (1.3) & 90.7 & (3.4) & 97.0 & $(2.1)$ & 98.7 & (1.3) & 100.0 & $(0.0)$ \\
\hline & Litauen & 87.4 & $(0.9)$ & 92.9 & $(0.7)$ & 92.3 & $(0.8)$ & 96.8 & $(0.5)$ & 81.7 & (1.2) & 88.2 & $(0.9)$ & 93.5 & $(0.7)$ & 97.6 & $(0.4)$ \\
\hline & Macau (China) & 63.9 & (1.4) & 75.5 & (1.2) & 84.3 & $(0.9)$ & 89.9 & (0.9) & 96.0 & $(0.5)$ & 96.7 & $(0.5)$ & 97.9 & $(0.4)$ & 99.0 & $(0.3)$ \\
\hline & Malaysia & 50.3 & (1.5) & 62.9 & (1.4) & 71.3 & (1.4) & 80.0 & $(1.2)$ & 92.8 & $(0.8)$ & 96.6 & $(0.6)$ & 95.6 & $(0.6)$ & 98.5 & $(0.3)$ \\
\hline & Montenegro & 86.2 & (1.0) & 90.2 & (1.0) & 92.2 & $(0.8)$ & 95.9 & $(0.8)$ & 87.1 & (1.1) & 94.5 & $(0.7)$ & 96.4 & $(0.6)$ & 99.4 & $(0.3)$ \\
\hline & Peru & 69.2 & (1.8) & 76.6 & (1.2) & 82.8 & (1.0) & 87.6 & (0.9) & 96.5 & $(0.5)$ & 98.3 & $(0.4)$ & 99.4 & $(0.2)$ & 99.8 & $(0.1)$ \\
\hline & Katar & 78.6 & $(0.8)$ & 87.2 & $(0.6)$ & 92.8 & $(0.5)$ & 97.9 & $(0.3)$ & 70.9 & $(0.8)$ & 87.6 & $(0.6)$ & 93.7 & $(0.5)$ & 97.5 & $(0.3)$ \\
\hline & Rumänien & 83.6 & $(1.2)$ & 91.9 & $(0.9)$ & 96.1 & $(0.6)$ & 97.2 & $(0.6)$ & 73.2 & (1.6) & 85.0 & (1.3) & 90.6 & (1.0) & 94.8 & $(0.7)$ \\
\hline & Russ. Föderation & 82.5 & (1.0) & 87.9 & $(0.9)$ & 87.9 & $(0.8)$ & 94.9 & (0.6) & 80.6 & (1.6) & 92.2 & (1.0) & 95.8 & $(0.7)$ & 98.6 & (0.3) \\
\hline & Serbien & 90.7 & $(0.8)$ & 96.0 & $(0.5)$ & 96.4 & $(0.5)$ & 96.9 & $(0.6)$ & 81.3 & (1.3) & 92.7 & $(0.8)$ & 95.6 & $(0.7)$ & 99.0 & $(0.3)$ \\
\hline & Shanghai (China) & 80.4 & (1.5) & 91.9 & $(0.9)$ & 94.9 & $(0.6)$ & 97.7 & $(0.5)$ & 97.5 & $(0.5)$ & 99.2 & $(0.2)$ & 99.1 & $(0.3)$ & 99.6 & $(0.2)$ \\
\hline & Singapur & 62.4 & $(1.2)$ & 77.3 & (1.1) & 85.6 & $(0.9)$ & 91.2 & $(0.7)$ & 95.9 & $(0.6)$ & 98.1 & $(0.3)$ & 99.0 & $(0.3)$ & 99.5 & $(0.3)$ \\
\hline & Chinesisch Taipeh & 64.7 & $(1.2)$ & 80.4 & (1.0) & 85.1 & $(0.9)$ & 90.8 & $(0.7)$ & 91.7 & $(0.7)$ & 97.8 & $(0.3)$ & 99.4 & $(0.2)$ & 99.3 & $(0.2)$ \\
\hline & Thailand & 60.5 & (1.5) & 70.7 & (1.4) & 78.8 & (1.1) & 85.8 & $(1.2)$ & 70.5 & (1.6) & 80.6 & (1.4) & 88.9 & $(1.1)$ & 95.4 & $(0.8)$ \\
\hline & Tunesien & 60.2 & (1.5) & 76.1 & (1.3) & 89.4 & $(1.0)$ & 92.2 & $(0.9)$ & 65.1 & (1.6) & 83.1 & (1.3) & 92.1 & $(0.9)$ & 94.9 & $(0.7)$ \\
\hline & Ver. Arab. Emirate & 78.7 & (1.1) & 86.1 & $(0.8)$ & 90.3 & $(0.7)$ & 95.5 & $(0.5)$ & 81.0 & $(0.8)$ & 92.5 & $(0.6)$ & 95.2 & $(0.6)$ & 99.1 & $(0.2)$ \\
\hline & Uruguay & 77.4 & (1.3) & 86.3 & (1.1) & 89.8 & $(0.9)$ & 96.2 & $(0.5)$ & 95.8 & $(0.6)$ & 98.0 & $(0.5)$ & 99.3 & $(0.3)$ & 99.2 & $(0.3)$ \\
\hline & Vietnam & 70.4 & $(2.2)$ & 85.5 & (1.4) & 86.9 & $(1.2)$ & 92.0 & $(0.9)$ & 44.5 & $(2.0)$ & 66.1 & $(2.2)$ & 81.6 & $(1.5)$ & 91.9 & (1.3) \\
\hline
\end{tabular}

1. ESCS bezieht sich auf den PISA-Index des wirtschaftlichen, sozialen und kulturellen Status.

2. Die Variable, die die Anzahl der im Elternhaus vorhandenen Bücher angibt, wurde folgendermaßen kodiert: 0-10 Bücher wurden als 5 Bücher kodiert, $11-25$ Bücher als 18 Bücher, 26-100 Bücher als 63 Bücher, 101-200 Bücher als 150,5 Bücher, 201-500 Bücher als 350,5 Bücher und über 500 Bücher als 750,5 Bücher.

$*$ Vgl. Anmerkungen am Anfang dieses Anhangs.

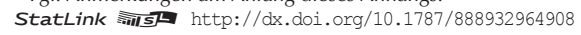


[Teil 7/7]

Merkmale des sozioökonomischen Status, nach Quartilen des sozioökonomischen Status innerhalb der einzelnen Länder

Tabelle II.2.2 Die Ergebnisse basieren auf Schülerangaben

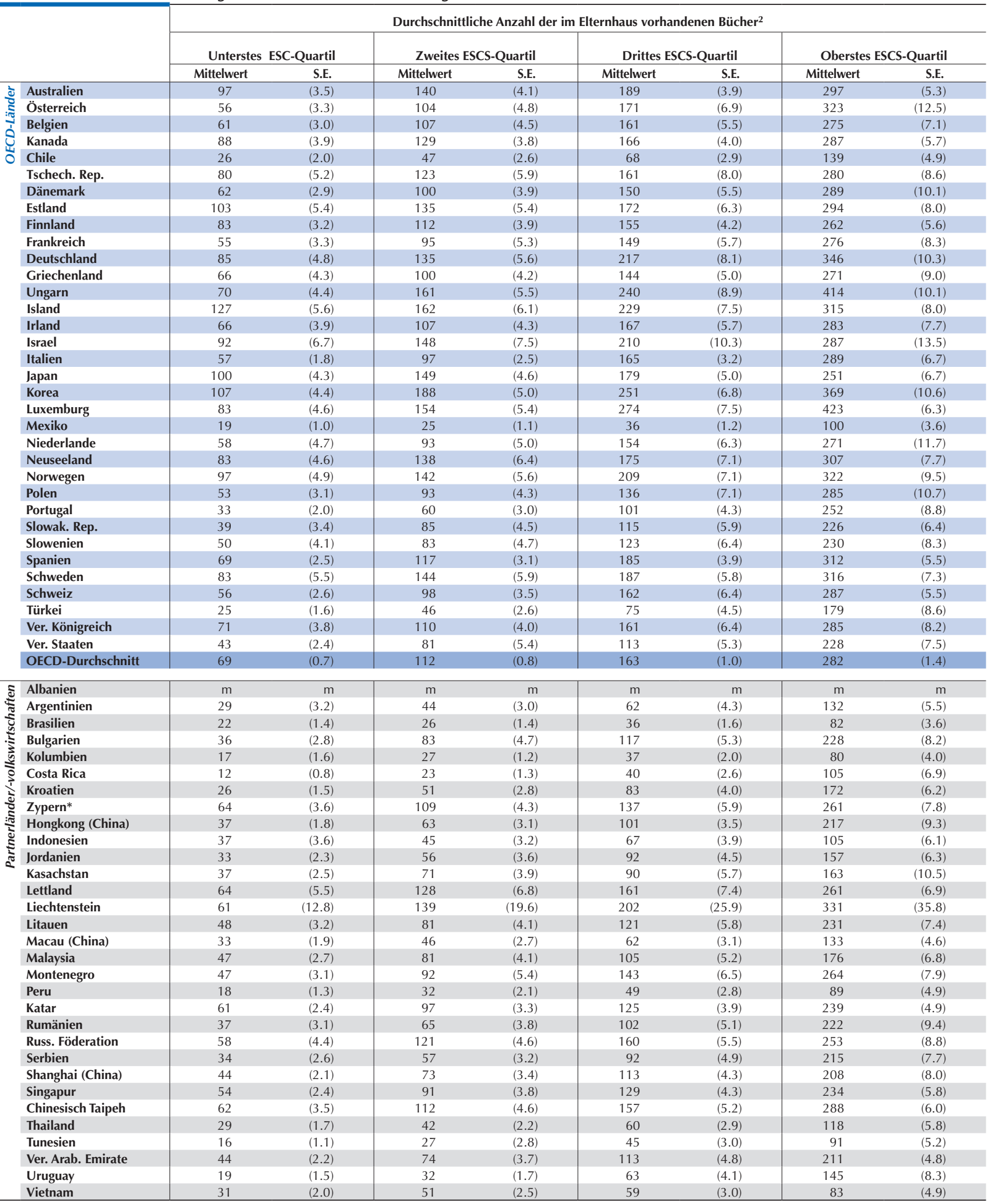

1. ESCS bezieht sich auf den PISA-Index des wirtschaftlichen, sozialen und kulturellen Status.

2. Die Variable, die die Anzahl der im Elternhaus vorhandenen Bücher angibt, wurde folgendermaßen kodiert: 0-10 Bücher wurden als 5 Bücher kodiert, $11-25$ Bücher als 18 Bücher, 26-100 Bücher als 63 Bücher, 101-200 Bücher als 150,5 Bücher, 201-500 Bücher als 350,5 Bücher und über 500 Bücher als 750,5 Bücher.

* Vgl. Anmerkungen am Anfang dieses Anhangs.

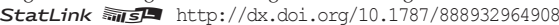


[Teil 1/2]

Merkmale des sozioökonomischen Status im Ländervergleich

Tabelle II.2.3 Die Ergebnisse basieren auf Schülerangaben

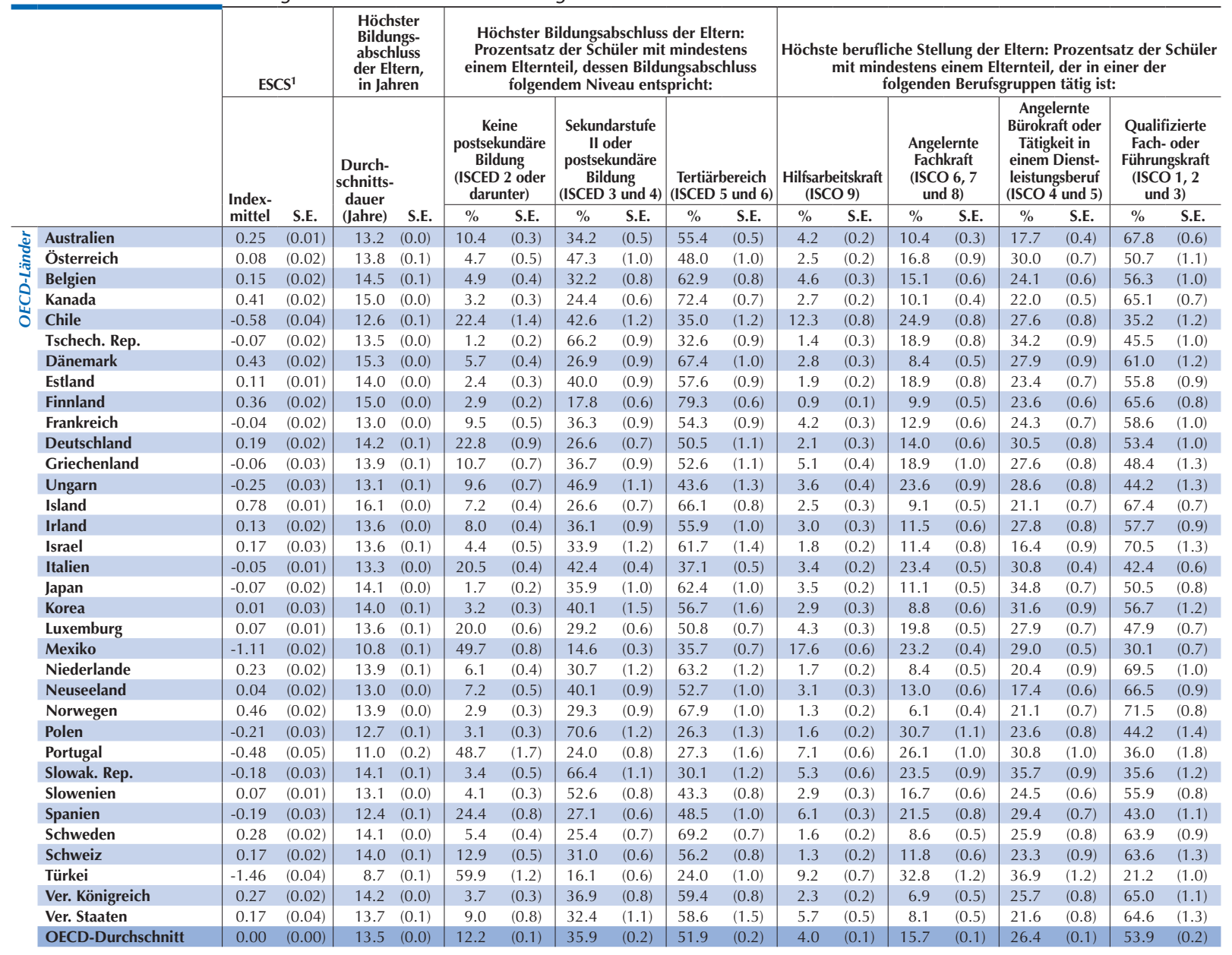

\begin{tabular}{|c|c|c|c|c|c|c|c|c|c|c|c|c|c|c|c|c|c|c|}
\hline Albanien & $\mathrm{m}$ & $\mathrm{m}$ & 12.1 & $(0.1)$ & 24.4 & $(0.9)$ & 46.3 & $(0.8)$ & 29.3 & $(0.8)$ & 6.0 & $(0.4)$ & 37.4 & $(1.1)$ & 19.0 & $(0.8)$ & 37.7 & $(1.0)$ \\
\hline Argentinien & -0.72 & $(0.04)$ & 12.5 & $(0.1)$ & 31.3 & (1.3) & 21.5 & $(0.7)$ & 47.1 & (1.3) & 8.6 & (0.6) & 27.5 & (1.1) & 29.4 & (1.0) & 34.6 & (1.6) \\
\hline Brasilien & -1.17 & $(0.02)$ & 10.2 & $(0.1)$ & 37.3 & $(0.8)$ & 36.6 & $(0.7)$ & 26.0 & $(0.8)$ & 10.9 & $(0.4)$ & 19.0 & $(0.6)$ & 35.6 & $(0.6)$ & 34.5 & $(0.7)$ \\
\hline Bulgarien & -0.28 & $(0.04)$ & 13.7 & $(0.1)$ & 7.2 & $(0.8)$ & 47.5 & (1.0) & 45.4 & $(1.2)$ & 4.0 & (0.4) & 23.7 & (1.1) & 26.8 & $(0.8)$ & 45.5 & (1.4) \\
\hline Kolumbien & -1.26 & $(0.04)$ & 10.9 & $(0.1)$ & 37.5 & $(1.3)$ & 15.4 & $(0.8)$ & 47.0 & (1.5) & 7.6 & $(0.5)$ & 33.7 & $(1.2)$ & 34.3 & $(0.9)$ & 24.5 & (1.0) \\
\hline Costa Rica & -0.98 & $(0.04)$ & 12.1 & $(0.1)$ & 28.0 & (1.1) & 23.5 & (1.0) & 48.5 & (1.3) & 11.9 & (1.0) & 20.7 & $(1.2)$ & 32.0 & (1.1) & 35.4 & (1.4) \\
\hline Kroatien & -0.34 & $(0.02)$ & 13.7 & $(0.0)$ & 4.8 & $(0.4)$ & 48.2 & $(0.8)$ & 47.1 & $(0.8)$ & 1.6 & $(0.2)$ & 21.2 & $(0.7)$ & 36.0 & $(0.9)$ & 41.2 & (1.1) \\
\hline Zypern* & 0.09 & $(0.01)$ & 14.1 & $(0.0)$ & 5.1 & $(0.3)$ & 42.2 & $(0.7)$ & 52.7 & $(0.7)$ & 2.5 & $(0.3)$ & 16.6 & $(0.6)$ & 38.2 & $(0.8)$ & 42.8 & $(0.8)$ \\
\hline Hongkong (China) & -0.79 & $(0.05)$ & 11.4 & $(0.1)$ & 29.7 & (1.4) & 48.4 & (1.1) & 21.8 & (2.0) & 7.0 & $(0.5)$ & 16.0 & $(0.8)$ & 34.0 & $(1.2)$ & 42.9 & (2.0) \\
\hline Indonesien & -1.80 & $(0.05)$ & 10.1 & $(0.1)$ & 48.1 & (1.7) & 32.1 & (1.1) & 19.8 & (1.5) & 21.6 & (1.9) & 32.0 & $(2.1)$ & 25.1 & (1.4) & 21.3 & (1.7) \\
\hline Jordanien & -0.42 & $(0.02)$ & 13.6 & $(0.1)$ & 12.0 & $(0.6)$ & 30.7 & $(0.8)$ & 57.3 & (1.1) & 1.9 & $(0.2)$ & 19.6 & $(0.8)$ & 16.5 & $(0.6)$ & 61.9 & $(0.9)$ \\
\hline Kasachstan & -0.32 & $(0.02)$ & 14.2 & $(0.0)$ & 1.4 & $(0.2)$ & 12.0 & $(0.7)$ & 86.6 & $(0.8)$ & 3.1 & $(0.3)$ & 17.9 & $(0.9)$ & 18.4 & $(0.9)$ & 60.5 & (1.3) \\
\hline Lettland & -0.26 & $(0.03)$ & 13.3 & $(0.1)$ & 2.7 & $(0.4)$ & 42.6 & $(1.2)$ & 54.7 & (1.3) & 5.1 & $(0.5)$ & 15.9 & $(0.8)$ & 28.1 & (1.0) & 50.8 & (1.3) \\
\hline Liechtenstein & 0.30 & $(0.05)$ & 13.6 & $(0.2)$ & 10.9 & $(2.0)$ & 33.0 & (2.4) & 56.1 & (2.5) & 2.1 & $(0.9)$ & 9.8 & (1.6) & 13.2 & (1.8) & 75.0 & (2.3) \\
\hline Litauen & -0.13 & $(0.02)$ & 14.0 & $(0.0)$ & 1.5 & $(0.2)$ & 34.2 & $(0.9)$ & 64.3 & $(0.9)$ & 4.4 & $(0.4)$ & 22.8 & $(0.7)$ & 21.0 & $(0.6)$ & 51.8 & (0.9) \\
\hline Macau (China) & -0.89 & $(0.01)$ & 10.5 & $(0.0)$ & 49.3 & $(0.6)$ & 32.3 & $(0.6)$ & 18.4 & $(0.5)$ & 5.0 & $(0.3)$ & 9.2 & $(0.4)$ & 58.0 & $(0.7)$ & 27.8 & $(0.6)$ \\
\hline Malaysia & -0.72 & $(0.03)$ & 12.7 & $(0.1)$ & 17.9 & $(0.9)$ & 53.3 & $(0.9)$ & 28.8 & $(1.2)$ & 10.7 & $(0.7)$ & 25.4 & $(1.0)$ & 24.0 & $(0.8)$ & 40.0 & (1.4) \\
\hline Montenegro & -0.25 & $(0.01)$ & 13.7 & $(0.0)$ & 4.0 & $(0.3)$ & 37.2 & $(0.7)$ & 58.8 & $(0.8)$ & 3.9 & $(0.3)$ & 18.4 & $(0.6)$ & 32.5 & $(0.8)$ & 45.2 & $(0.8)$ \\
\hline Peru & -1.23 & $(0.05)$ & 11.5 & $(0.1)$ & 26.7 & (1.3) & 39.2 & $(1.0)$ & 34.1 & (1.5) & 21.7 & $(1.2)$ & 25.4 & $(0.8)$ & 31.2 & $(0.7)$ & 21.7 & (1.3) \\
\hline Katar & 0.44 & $(0.01)$ & 14.3 & $(0.0)$ & 9.8 & $(0.3)$ & 18.6 & $(0.4)$ & 71.7 & $(0.4)$ & 0.6 & $(0.1)$ & 7.1 & $(0.3)$ & 15.0 & $(0.4)$ & 77.3 & $(0.4)$ \\
\hline Rumänien & -0.47 & $(0.04)$ & 13.6 & $(0.1)$ & 5.8 & $(0.6)$ & 42.5 & (1.1) & 51.7 & $(1.2)$ & 4.2 & $(0.5)$ & 31.0 & $(1.2)$ & 27.8 & $(1.0)$ & 37.1 & (1.6) \\
\hline Russ. Föderation & -0.11 & $(0.02)$ & 13.9 & $(0.0)$ & 1.3 & $(0.3)$ & 8.0 & $(0.5)$ & 90.7 & $(0.6)$ & 1.6 & $(0.2)$ & 15.3 & $(0.8)$ & 27.2 & $(0.8)$ & 56.0 & (1.1) \\
\hline Serbien & -0.30 & $(0.02)$ & 13.5 & $(0.1)$ & 4.8 & $(0.5)$ & 40.3 & (1.0) & 54.9 & (1.1) & 3.6 & $(0.3)$ & 12.5 & $(0.6)$ & 41.8 & (1.0) & 42.0 & (1.1) \\
\hline Shanghai (China) & -0.36 & $(0.04)$ & 12.8 & $(0.1)$ & 24.9 & (1.3) & 30.6 & $(0.8)$ & 44.5 & (1.3) & 1.5 & $(0.2)$ & 14.9 & $(0.8)$ & 26.2 & $(0.9)$ & 57.4 & (1.3) \\
\hline Singapur & -0.26 & $(0.01)$ & 12.4 & $(0.0)$ & 10.0 & $(0.4)$ & 42.2 & $(0.7)$ & 47.8 & $(0.7)$ & 2.6 & $(0.2)$ & 7.8 & $(0.5)$ & 20.2 & $(0.6)$ & 69.4 & $(0.6)$ \\
\hline Chinesisch Taipeh & -0.40 & $(0.02)$ & 13.0 & $(0.1)$ & 11.8 & $(0.6)$ & 36.8 & $(0.8)$ & 51.4 & (1.1) & 3.3 & $(0.3)$ & 21.5 & $(0.9)$ & 31.5 & $(0.6)$ & 43.8 & (1.2) \\
\hline Thailand & -1.35 & $(0.04)$ & 10.1 & $(0.1)$ & 50.2 & (1.4) & 30.8 & $(0.7)$ & 19.0 & $(1.2)$ & 11.6 & $(0.7)$ & 38.8 & $(1.2)$ & 25.5 & $(0.8)$ & 24.2 & (1.2) \\
\hline Tunesien & -1.19 & $(0.05)$ & 11.9 & $(0.1)$ & 29.8 & (1.3) & 42.1 & (1.0) & 28.1 & $(1.2)$ & 28.0 & (1.3) & 19.4 & $(0.9)$ & 22.3 & $(0.9)$ & 30.4 & (1.4) \\
\hline Ver. Arab. Emirate & 0.32 & $(0.02)$ & 14.1 & $(0.1)$ & 8.9 & $(0.4)$ & 23.4 & $(0.6)$ & 67.7 & $(0.9)$ & 0.7 & $(0.1)$ & 4.3 & $(0.3)$ & 12.6 & $(0.6)$ & 82.4 & $(0.6)$ \\
\hline Uruguay & -0.88 & $(0.03)$ & 11.4 & $(0.1)$ & 45.1 & (1.1) & 19.3 & $(0.6)$ & 35.6 & (1.0) & 16.5 & $(0.7)$ & 20.5 & $(0.6)$ & 35.5 & $(0.8)$ & 27.6 & (1.0) \\
\hline Vietnam & -1.81 & $(0.05)$ & 9.8 & $(0.1)$ & 58.2 & (1.5) & 30.5 & (1.0) & 11.2 & (1.0) & 2.9 & $(0.5)$ & 66.0 & (1.7) & 17.9 & $(0.9)$ & 13.2 & (1.1) \\
\hline
\end{tabular}

1. ESCS bezieht sich auf den PISA-Index des wirtschaftlichen, sozialen und kulturellen Status.

2. Die Variable, die die Anzahl der im Elternhaus vorhandenen Bücher angibt, wurde folgendermaßen kodiert: 0-10 Bücher wurden als 5 Bücher kodiert, $11-25$ Bücher als

18 Bücher, 26-100 Bücher als 63 Bücher, 101-200 Bücher als 150,5 Bücher, 201-500 Bücher als 350,5 Bücher und über 500 Bücher als 750,5 Bücher.

$*$ Vgl. Anmerkungen am Anfang dieses Anhangs.

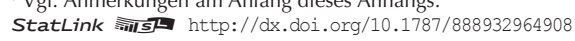


[Teil 2/2]

Merkmale des sozioökonomischen Status im Ländervergleich

Tabelle II.2.3 Die Ergebnisse basieren auf Schülerangaben

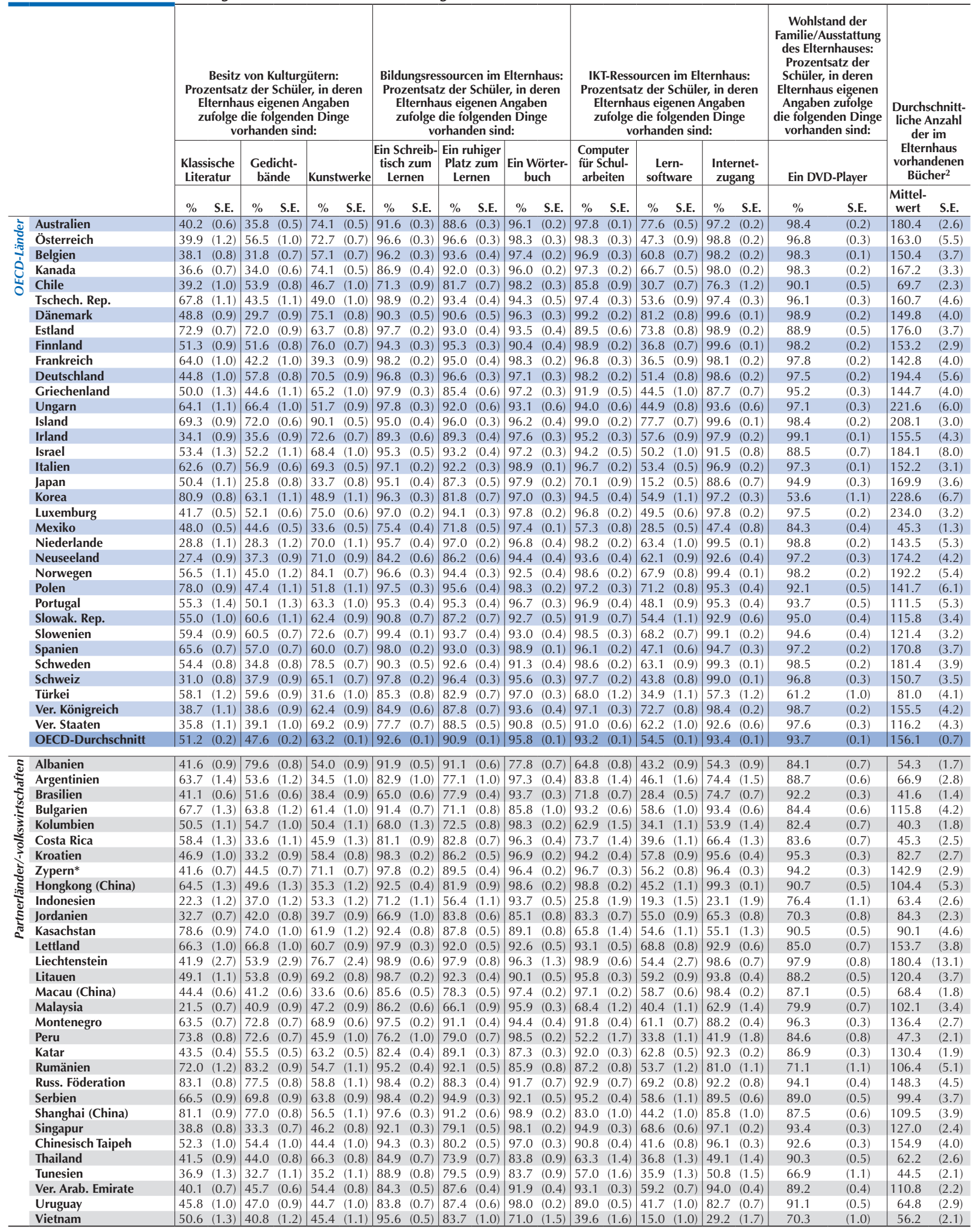

1. ESCS bezieht sich auf den PISA-Index des wirtschaftlichen, sozialen und kulturellen Status.

2. Die Variable, die die Anzahl der im Elternhaus vorhandenen Bücher angibt, wurde folgendermaßen kodiert: 0-10 Bücher wurden als 5 Bücher kodiert, 11-25 Bücher als 18 Bücher, 26-100 Bücher als 63 Bücher, 101-200 Bücher als 150,5 Bücher, 201-500 Bücher als 350,5 Bücher und über 500 Bücher als 750,5 Bücher.

* Vgl. Anmerkungen am Anfang dieses Anhangs.

StatLink त्तils http://dx.doi.org/10.1787/888932964908 
[Teil 1/1]

Sozioökonomischer Status der Schüler und Mathematikleistungen

Nach nationalen Quartilen des PISA-Index des wirtschaftlichen, sozialen und kulturellen Status; die Ergebnisse Tabelle II.2.4a basieren auf Schülerangaben

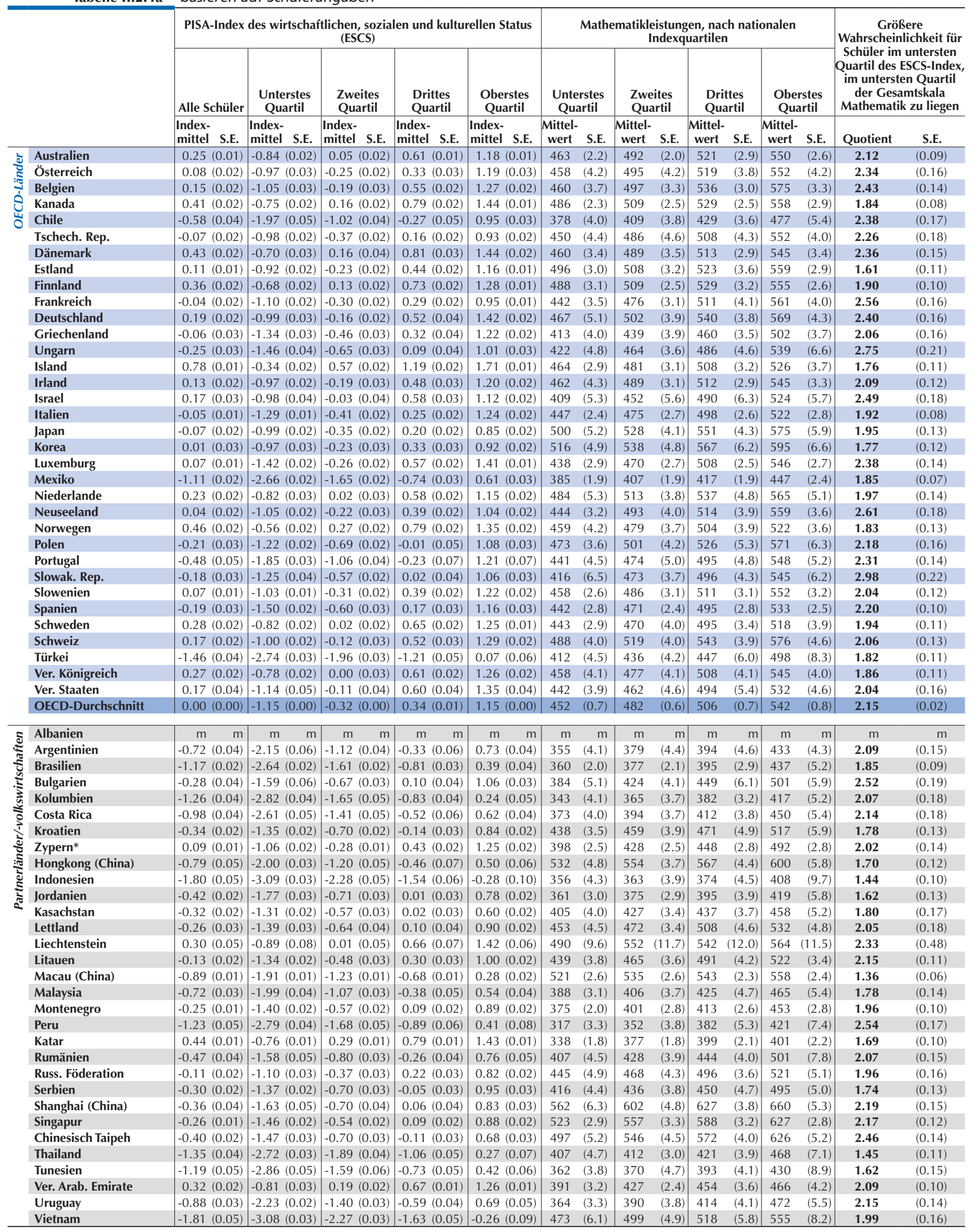

Anmerkung: Statistisch signifikante Werte sind durch Fettdruck gekennzeichnet (vgl. Anhang A3).

$*$ Vgl. Anmerkungen am Anfang dieses Anhangs.

StatLink 部SL http://dx.doi.org/10.1787/888932964908 
[Teil 1/3]

Veränderung beim sozioökonomischen Status und bei den Mathematikleistungen der Schüler zwischen 2003 und 2012

Nach nationalen Quartilen des PISA-Index des wirtschaftlichen, sozialen und kulturellen Status; die Ergebnisse Tabelle II.2.4b basieren auf Schülerangaben

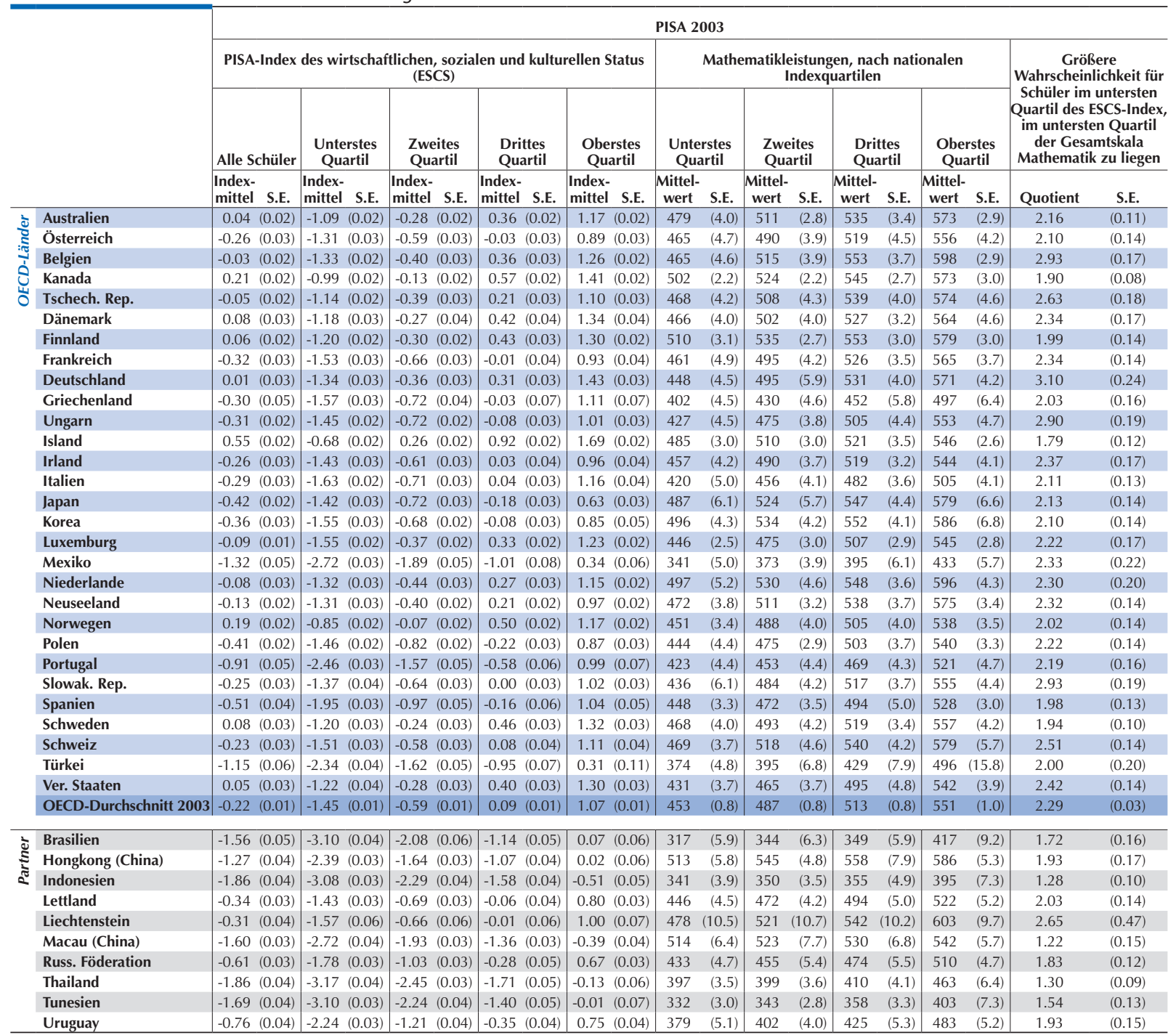

Anmerkung: Statistisch signifikante Werte sind durch Fettdruck gekennzeichnet (vgl. Anhang A3).

Angegeben sind nur Länder und Volkswirtschaften mit vergleichbaren Daten aus PISA 2003 und PISA 2012.

Um die Vergleichbarkeit im Zeitverlauf zu gewährleisten, wurden die Werte von PISA 2003 auf dem PISA-Index des wirtschaftlichen, sozialen und kulturellen Status entsprechend der Indexskala von PISA 2012 umskaliert. Die in dieser Tabelle dargestellten Ergebnisse aus PISA 2003 können daher von denen abweichen, die in Lernen für die Welt von morgen: Erste Ergebnisse von PISA 2003 (OECD, 2004) wiedergegeben sind (vgl. Anhang A5 wegen näherer Einzelheiten)

StatLink ints http://dx.doi.org/10.1787/888932964908 
[Teil 2/3]

Veränderung beim sozioökonomischen Status und bei den Mathematikleistungen der Schüler zwischen 2003 und 2012

Nach nationalen Quartilen des PISA-Index des wirtschaftlichen, sozialen und kulturellen Status; die Ergebnisse Tabelle II.2.4b basieren auf Schülerangaben

\begin{tabular}{|c|c|c|c|c|c|c|c|c|c|c|c|c|c|c|c|c|c|c|c|c|c|}
\hline & \multicolumn{20}{|c|}{ PISA 2012} \\
\hline & & \multicolumn{10}{|c|}{$\begin{array}{l}\text { PISA-Index des wirtschaftlichen, sozialen und kulturellen Status } \\
\text { (ESCS) }\end{array}$} & \multicolumn{8}{|c|}{$\begin{array}{c}\text { Mathematikleistungen, } \\
\text { nach nationalen Indexquartilen }\end{array}$} & \multirow{2}{*}{\multicolumn{2}{|c|}{\begin{tabular}{|c} 
Größere \\
Wahrscheinlichkeit für \\
Schüler im untersten \\
Quartil des ESCS-Index \\
im untersten Quartil \\
der Gesamtskala \\
Mathematik zu liegen
\end{tabular}}} \\
\hline & & \multirow{2}{*}{\multicolumn{2}{|c|}{\begin{tabular}{|l} 
Alle Schüler \\
$\begin{array}{l}\text { Index- } \\
\text { mittel S.E. }\end{array}$
\end{tabular}}} & \multicolumn{2}{|c|}{$\begin{array}{c}\text { Unterstes } \\
\text { Quartil }\end{array}$} & \multicolumn{2}{|c|}{$\begin{array}{l}\text { Zweites } \\
\text { Quartil }\end{array}$} & \multicolumn{2}{|c|}{$\begin{array}{l}\text { Drittes } \\
\text { Quartil }\end{array}$} & \multicolumn{2}{|c|}{$\begin{array}{c}\text { Oberstes } \\
\text { Quartil }\end{array}$} & \multicolumn{2}{|c|}{$\begin{array}{l}\text { Unterstes } \\
\text { Quartil }\end{array}$} & \multicolumn{2}{|c|}{$\begin{array}{l}\text { Zweites } \\
\text { Quartil }\end{array}$} & \multicolumn{2}{|c|}{$\begin{array}{l}\text { Drittes } \\
\text { Quartil }\end{array}$} & \multicolumn{2}{|c|}{$\begin{array}{c}\text { Oberstes } \\
\text { Quartil }\end{array}$} & & \\
\hline & & & & $\begin{array}{l}\text { Index } \\
\text { mittel }\end{array}$ & S.E. & $\begin{array}{l}\text { Index } \\
\text { mittel }\end{array}$ & S.E. & $\begin{array}{l}\text { Index- } \\
\text { mittel }\end{array}$ & S.E. & $\begin{array}{l}\text { Index- } \\
\text { mittel }\end{array}$ & S.E. & $\begin{array}{c}\text { Mittel- } \\
\text { wert }\end{array}$ & S.E. & $\begin{array}{c}\text { Mittel- } \\
\text { wert }\end{array}$ & S.E. & $\begin{array}{c}\text { Mittel- } \\
\text { wert }\end{array}$ & S.E. & $\begin{array}{c}\text { Mittel- } \\
\text { wert }\end{array}$ & S.E. & Quotient & S.E. \\
\hline \multirow{30}{*}{ 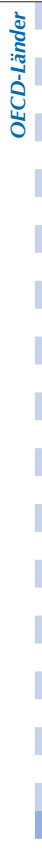 } & Australien & 0.25 & $(0.01)$ & -0.84 & $(0.02)$ & 0.05 & $(0.02)$ & \begin{tabular}{|l|}
0.61 \\
\end{tabular} & $(0.01)$ & 1.18 & $(0.01)$ & 463 & $(2.2)$ & 492 & $(2.0)$ & 521 & (2.9) & 550 & $(2.5)$ & 2.14 & $(0.09)$ \\
\hline & Österreich & 0.08 & $(0.02)$ & -0.97 & $(0.03)$ & -0.25 & $(0.02)$ & 0.33 & $(0.03)$ & 1.19 & $(0.03)$ & 458 & $(4.2)$ & 495 & $(4.2)$ & 519 & (3.8) & 552 & (4.1) & 2.36 & $(0.16)$ \\
\hline & Belgien & 0.15 & $(0.02)$ & -1.05 & $(0.03)$ & -0.19 & $(0.03)$ & 0.55 & $(0.02)$ & 1.27 & $(0.02)$ & 460 & (3.6) & 497 & (3.3) & 536 & (3.0) & 575 & (3.3) & 2.43 & $(0.15)$ \\
\hline & Kanada & 0.41 & $(0.02)$ & -0.75 & $(0.02)$ & 0.16 & $(0.02)$ & 0.79 & $(0.02)$ & 1.44 & $(0.01)$ & 486 & (2.4) & 509 & (2.4) & 529 & (2.5) & 558 & (2.9) & 1.86 & $(0.08)$ \\
\hline & Tschech. Rep. & -0.07 & $(0.02)$ & -0.98 & $(0.02)$ & -0.37 & $(0.02)$ & 0.16 & $(0.02)$ & 0.93 & $(0.02)$ & 450 & (4.3) & 486 & (4.4) & 508 & (4.3) & 552 & $(4.0)$ & 2.27 & $(0.18)$ \\
\hline & Dänemark & 0.43 & $(0.02)$ & -0.70 & $(0.03)$ & 0.16 & $(0.04)$ & 0.81 & $(0.03)$ & 1.44 & $(0.02)$ & 460 & (3.5) & 489 & (3.5) & 513 & (3.0) & 545 & (3.4) & 2.39 & $(0.16)$ \\
\hline & Finnland & 0.36 & $(0.02)$ & -0.68 & $(0.02)$ & 0.13 & $(0.02)$ & 0.73 & $(0.02)$ & 1.28 & $(0.01)$ & 488 & (3.1) & 509 & (2.5) & 529 & (3.2) & 555 & (2.6) & 1.89 & $(0.10)$ \\
\hline & Frankreich & -0.04 & $(0.02)$ & -1.10 & $(0.02)$ & -0.30 & $(0.02)$ & 0.29 & $(0.02)$ & 0.95 & $(0.01)$ & 442 & (3.5) & 476 & $(3.2)$ & 511 & (4.1) & 561 & (3.9) & 2.57 & $(0.16)$ \\
\hline & Deutschland & 0.19 & $(0.02)$ & -0.99 & $(0.03)$ & -0.16 & $(0.03)$ & 0.52 & $(0.04)$ & 1.42 & $(0.02)$ & 467 & (5.1) & 503 & (3.9) & 540 & (3.8) & 569 & (4.3) & 2.41 & $(0.17)$ \\
\hline & Griechenland & -0.06 & $(0.03)$ & -1.34 & $(0.03)$ & -0.46 & $(0.03)$ & 0.32 & $(0.04)$ & 1.22 & $(0.02)$ & 413 & (3.9) & 439 & $(4.0)$ & 459 & (3.4) & 502 & (3.7) & 2.05 & $(0.17)$ \\
\hline & Ungarn & -0.25 & $(0.03)$ & -1.46 & $(0.04)$ & -0.65 & $(0.03)$ & 0.09 & $(0.04)$ & 1.01 & $(0.03)$ & 422 & (4.8) & 464 & (3.7) & 487 & (4.6) & 539 & (6.6) & 2.74 & $(0.21)$ \\
\hline & Island & 0.78 & $(0.01)$ & -0.34 & $(0.02)$ & 0.57 & $(0.02)$ & 1.19 & $(0.02)$ & 1.71 & $(0.01)$ & 464 & (3.0) & 481 & (3.2) & 508 & (3.3) & 526 & (3.7) & 1.75 & $(0.11)$ \\
\hline & Irland & 0.13 & $(0.02)$ & -0.97 & $(0.02)$ & -0.19 & $(0.03)$ & 0.48 & $(0.03)$ & 1.20 & $(0.02)$ & 462 & (4.4) & 489 & (3.2) & 513 & (2.9) & 545 & (3.3) & 2.11 & $(0.12)$ \\
\hline & Italien & -0.05 & $(0.01)$ & -1.29 & $(0.01)$ & -0.41 & $(0.02)$ & 0.25 & $(0.02)$ & 1.24 & $(0.02)$ & 447 & (2.4) & 475 & (2.7) & 498 & (2.6) & 522 & (2.8) & 1.91 & $(0.08)$ \\
\hline & Japan & -0.07 & $(0.02)$ & -0.99 & $(0.02)$ & -0.35 & $(0.02)$ & 0.20 & $(0.02)$ & 0.85 & $(0.02)$ & 500 & $(5.2)$ & 528 & (4.1) & 551 & (4.3) & 575 & (5.9) & 1.97 & $(0.13)$ \\
\hline & Korea & 0.01 & $(0.03)$ & -0.97 & $(0.03)$ & -0.23 & $(0.03)$ & 0.33 & $(0.03)$ & 0.92 & $(0.02)$ & 516 & (4.9) & 538 & (4.8) & 567 & $(6.2)$ & 595 & (6.6) & 1.76 & $(0.11)$ \\
\hline & Luxemburg & 0.07 & $(0.01)$ & -1.42 & $(0.02)$ & -0.26 & $(0.02)$ & 0.57 & $(0.02)$ & 1.41 & $(0.01)$ & 439 & (2.9) & 470 & (2.7) & 508 & (2.6) & 547 & (2.7) & 2.38 & $(0.13)$ \\
\hline & Mexiko & -1.11 & $(0.02)$ & -2.66 & $(0.02)$ & -1.65 & $(0.03)$ & -0.74 & $(0.03)$ & 0.61 & $(0.03)$ & 385 & (1.9) & 407 & (1.9) & 417 & (1.9) & 447 & (2.4) & 1.84 & $(0.07)$ \\
\hline & Niederlande & 0.23 & $(0.02)$ & -0.82 & $(0.03)$ & 0.02 & $(0.03)$ & 0.58 & $(0.02)$ & 1.15 & $(0.02)$ & 484 & (5.2) & 513 & (3.7) & 537 & $(4.9)$ & 565 & (5.1) & 2.02 & $(0.15)$ \\
\hline & Neuseeland & 0.04 & $(0.02)$ & -1.05 & $(0.02)$ & -0.22 & $(0.03)$ & 0.39 & $(0.02)$ & 1.04 & $(0.02)$ & 445 & $(3.2)$ & 493 & (3.9) & 514 & $(4.0)$ & 559 & (3.6) & 2.64 & $(0.19)$ \\
\hline & Norwegen & 0.46 & $(0.02)$ & -0.56 & $(0.02)$ & 0.27 & $(0.02)$ & 0.79 & $(0.02)$ & 1.35 & $(0.02)$ & 459 & $(4.2)$ & 479 & (3.7) & 504 & (3.9) & 522 & (3.7) & 1.83 & (0.13) \\
\hline & Polen & -0.21 & $(0.03)$ & -1.22 & $(0.02)$ & -0.69 & $(0.02)$ & -0.01 & $(0.05)$ & 1.08 & $(0.03)$ & 473 & (3.6) & 501 & $(4.1)$ & 526 & (5.2) & 571 & (6.3) & 2.17 & $(0.16)$ \\
\hline & Portugal & -0.48 & $(0.05)$ & -1.85 & $(0.03)$ & -1.06 & $(0.04)$ & -0.23 & $(0.07)$ & 1.21 & $(0.07)$ & 441 & (4.5) & 474 & (4.9) & 495 & (4.8) & 548 & (5.2) & 2.34 & $(0.15)$ \\
\hline & Slowak. Rep. & -0.18 & $(0.03)$ & -1.25 & $(0.04)$ & -0.57 & $(0.02)$ & 0.02 & $(0.04)$ & 1.06 & $(0.03)$ & 416 & (6.5) & 473 & (3.7) & 496 & (4.4) & 545 & (6.2) & 2.99 & $(0.23)$ \\
\hline & Spanien & -0.19 & $(0.03)$ & -1.50 & $(0.02)$ & -0.60 & $(0.03)$ & 0.17 & $(0.03)$ & 1.16 & $(0.03)$ & 442 & (2.8) & 471 & (2.4) & 495 & $(2.8)$ & 533 & (2.5) & 2.19 & $(0.10)$ \\
\hline & Schweden & 0.28 & $(0.02)$ & -0.82 & $(0.02)$ & 0.02 & $(0.02)$ & 0.65 & $(0.02)$ & 1.25 & $(0.01)$ & 443 & $(2.9)$ & 470 & (3.9) & 495 & (3.5) & 518 & (3.9) & 1.95 & $(0.12)$ \\
\hline & Schweiz & 0.17 & $(0.02)$ & -1.00 & $(0.02)$ & -0.12 & $(0.03)$ & 0.52 & $(0.03)$ & 1.29 & $(0.02)$ & 488 & $(4.0)$ & 519 & $(4.1)$ & 543 & (3.9) & 576 & (4.6) & 2.06 & $(0.13)$ \\
\hline & Türkei & -1.46 & $(0.04)$ & -2.74 & $(0.03)$ & -1.96 & $(0.03)$ & -1.21 & $(0.05)$ & 0.07 & $(0.06)$ & 412 & $(4.5)$ & 435 & $(4.2)$ & 447 & (5.9) & 498 & $(8.3)$ & 1.83 & $(0.12)$ \\
\hline & Ver. Staaten & 0.17 & $(0.04)$ & -1.14 & $(0.05)$ & -0.11 & $(0.04)$ & 0.60 & $(0.04)$ & 1.35 & $(0.03)$ & 442 & (3.9) & 462 & (4.6) & 493 & (5.4) & 532 & $(4.8)$ & 2.06 & $(0.17)$ \\
\hline & OECD-Durchschnitt 2003 & 0.00 & $(0.00)$ & -1.15 & $(0.00)$ & -0.33 & $(0.01)$ & 0.34 & $(0.01)$ & 1.15 & $(0.01)$ & 454 & $(0.7)$ & 484 & $(0.7)$ & 509 & $(0.7)$ & 544 & $(0.8)$ & 2.17 & $(0.03)$ \\
\hline \multirow{10}{*}{ ఏ } & Brasilien & -1.17 & $(0.02)$ & -2.64 & $(0.02)$ & -1.61 & $(0.02)$ & -0.81 & $(0.03)$ & 0.39 & $(0.04)$ & 360 & $(2.0)$ & 377 & (2.1) & 395 & (2.9) & 437 & (5.3) & 1.84 & $(0.09)$ \\
\hline & Hongkong (China) & -0.79 & $(0.05)$ & -2.00 & $(0.03)$ & -1.20 & $(0.05)$ & -0.46 & $(0.07)$ & 0.50 & $(0.06)$ & 532 & $(4.8)$ & 554 & (3.8) & 567 & (4.4) & 599 & (5.8) & 1.71 & $(0.12)$ \\
\hline & Indonesien & -1.80 & $(0.05)$ & -3.09 & $(0.03)$ & -2.28 & $(0.05)$ & -1.54 & $(0.06)$ & -0.28 & $(0.10)$ & 356 & (4.3) & 363 & (3.9) & 374 & (4.5) & 408 & (9.8) & 1.43 & $(0.09)$ \\
\hline & Lettland & $\mid-0.26$ & $(0.03)$ & -1.39 & $(0.03)$ & -0.64 & $(0.04)$ & 0.11 & $(0.04)$ & 0.90 & $(0.02)$ & 453 & (4.5) & 472 & (3.5) & 507 & (4.6) & 532 & (4.8) & 2.06 & $(0.17)$ \\
\hline & Liechtenstein & 0.30 & $(0.05)$ & -0.89 & $(0.08)$ & 0.01 & $(0.06)$ & 0.66 & $(0.07)$ & 1.42 & $(0.06)$ & 490 & (9.6) & 552 & (11.2) & 544 & (12.3) & 561 & (11.7) & 2.32 & $(0.45)$ \\
\hline & Macau (China) & -0.89 & $(0.01)$ & -1.91 & $(0.01)$ & -1.23 & $(0.01)$ & -0.68 & $(0.01)$ & 0.28 & $(0.02)$ & 521 & (2.6) & 535 & (2.6) & 543 & (2.3) & 558 & (2.4) & 1.36 & $(0.06)$ \\
\hline & Russ. Föderation & -0.11 & $(0.02)$ & -1.10 & $(0.03)$ & -0.37 & $(0.03)$ & 0.22 & $(0.03)$ & 0.82 & $(0.02)$ & 445 & $(4.8)$ & 468 & (4.3) & 496 & (3.6) & 521 & (5.1) & 1.95 & $(0.16)$ \\
\hline & Thailand & -1.35 & $(0.04)$ & -2.72 & $(0.03)$ & -1.89 & $(0.04)$ & -1.06 & $(0.05)$ & 0.27 & $(0.07)$ & 407 & $(4.7)$ & 412 & (3.0) & 421 & (3.9) & 468 & (7.1) & 1.43 & $(0.12)$ \\
\hline & Tunesien & -1.19 & $(0.05)$ & -2.86 & $(0.05)$ & -1.59 & $(0.06)$ & -0.73 & $(0.05)$ & 0.42 & $(0.06)$ & 362 & (3.8) & 370 & (4.8) & 393 & (4.1) & 430 & (8.8) & 1.61 & $(0.15)$ \\
\hline & Uruguay & -0.88 & $(0.03)$ & -2.23 & $(0.02)$ & -1.40 & $(0.03)$ & -0.59 & $(0.04)$ & 0.69 & $(0.05)$ & 364 & (3.3) & 390 & (3.7) & 414 & (4.1) & 472 & (5.5) & 2.15 & $(0.14)$ \\
\hline
\end{tabular}

Anmerkung: Statistisch signifikante Werte sind durch Fettdruck gekennzeichnet (vgl. Anhang A3).

Angegeben sind nur Länder und Volkswirtschaften mit vergleichbaren Daten aus PISA 2003 und PISA 2012.

Um die Vergleichbarkeit im Zeitverlauf zu gewährleisten, wurden die Werte von PISA 2003 auf dem PISA-Index des wirtschaftlichen, sozialen und kulturellen Status entsprechend der Indexskala von PISA 2012 umskaliert. Die in dieser Tabelle dargestellten Ergebnisse aus PISA 2003 können daher von denen abweichen, die in Lernen für die Welt von morgen: Erste Ergebnisse von PISA 2003 (OECD, 2004) wiedergegeben sind (vgl. Anhang A5 wegen näherer Einzelheiten).

StatLink ints http://dx.doi.org/10.1787/888932964908 
[Teil 3/3]

Veränderung beim sozioökonomischen Status und bei den Mathematikleistungen der Schüler zwischen 2003 und 2012

Nach nationalen Quartilen des PISA-Index des wirtschaftlichen, sozialen und kulturellen Status; die Ergebnisse Tabelle II.2.4b basieren auf Schülerangaben

\begin{tabular}{|c|c|c|c|c|c|c|c|c|c|c|c|c|c|c|c|c|}
\hline & \multicolumn{15}{|c|}{ Veränderung zwischen 2003 und 2012 (PISA 2012 - PISA 2003) } \\
\hline & & \multicolumn{5}{|c|}{$\begin{array}{l}\text { PISA-Index des wirtschaftlichen, sozialen und kulturellen Status } \\
\text { (ESCS) }\end{array}$} & \multicolumn{8}{|c|}{$\begin{array}{l}\text { Mathematikleistungen, nach nationalen } \\
\text { Indexquartilen }\end{array}$} & \multirow{2}{*}{\multicolumn{2}{|c|}{\begin{tabular}{|c} 
Größere \\
Wahrscheinlichkeit für \\
Schüler im untersten \\
Quartil des ESCS-Index \\
im untersten Quartil \\
der Gesamtskala \\
Mathematik zu liegen
\end{tabular}}} \\
\hline & & Alle Schüler & $\begin{array}{l}\text { Unterstes } \\
\text { Quartil }\end{array}$ & $\begin{array}{l}\text { Zweites } \\
\text { Quartil }\end{array}$ & $\begin{array}{l}\text { Drittes } \\
\text { Quartil }\end{array}$ & $\begin{array}{c}\text { Oberstes } \\
\text { Quartil }\end{array}$ & \multicolumn{2}{|c|}{$\begin{array}{l}\text { Unterstes } \\
\text { Quartil }\end{array}$} & \multicolumn{2}{|c|}{$\begin{array}{l}\text { Zweites } \\
\text { Quartil }\end{array}$} & \multicolumn{2}{|c|}{$\begin{array}{l}\text { Drittes } \\
\text { Quartil }\end{array}$} & \multicolumn{2}{|c|}{$\begin{array}{l}\text { Oberstes } \\
\text { Quartil }\end{array}$} & & \\
\hline & & Dif. S.E. & Dif. S.E. & Dif. S.E. & Dif. S.E. & Dif. S.E. & $\begin{array}{l}\text { Punkt- } \\
\text { diff. }\end{array}$ & S.E. & $\begin{array}{l}\text { Punkt- } \\
\text { diff. }\end{array}$ & S.E. & $\begin{array}{l}\text { Punkt- } \\
\text { diff. }\end{array}$ & S.E. & $\begin{array}{l}\text { Punkt- } \\
\text { diff. }\end{array}$ & S.E. & $\begin{array}{l}\text { Quotient- } \\
\text { diff. }\end{array}$ & S.E. \\
\hline \multirow{30}{*}{ 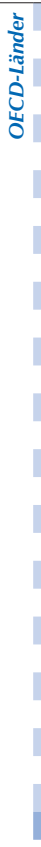 } & Australien & $0.21(0.02)$ & $\mathbf{0 . 2 5}(0.03)$ & $\mathbf{0 . 3 2}(0.03)$ & $\mathbf{0 . 2 5}(0.03)$ & $0.00(0.02)$ & -17 & $(4.6)$ & -19 & (3.5) & -13 & $(4.5)$ & -24 & (3.8) & -0.02 & $(0.14)$ \\
\hline & Österreich & $0.34(0.04)$ & $\mathbf{0 . 3 4}(0.04)$ & $\mathbf{0 . 3 4}(0.04)$ & $0.36(0.04)$ & $\mathbf{0 . 3 0}(0.04)$ & -7 & $(6.3)$ & 6 & (5.7) & 1 & (5.9) & -4 & (5.9) & 0.26 & $(0.21)$ \\
\hline & Belgien & $\mathbf{0 . 1 7}(0.03)$ & $0.28(0.04)$ & $\mathbf{0 . 2 1}(0.04)$ & $\mathbf{0 . 1 9}(0.04)$ & $0.01(0.03)$ & -5 & (5.9) & -18 & (5.1) & -18 & $(4.8)$ & -23 & (4.4) & -0.50 & $(0.22)$ \\
\hline & Kanada & $\mathbf{0 . 2 0}(0.02)$ & $\mathbf{0 . 2 4}(0.03)$ & $\mathbf{0 . 2 9}(0.03)$ & $\mathbf{0 . 2 3}(0.03)$ & $0.03(0.02)$ & -15 & (3.2) & -15 & (3.3) & -16 & (3.7) & -15 & (4.1) & -0.04 & $(0.11)$ \\
\hline & Tschech. Rep. & $-0.01(0.03)$ & $\mathbf{0 . 1 6}(0.03)$ & $0.02(0.03)$ & $-0.05(0.04)$ & $-\mathbf{0 . 1 7}(0.03)$ & -18 & (6.0) & -22 & (6.1) & -31 & (5.9) & -22 & (6.1) & -0.37 & $(0.26)$ \\
\hline & Dänemark & $\mathbf{0 . 3 5}(0.04)$ & $\mathbf{0 . 4 7}(0.04)$ & $\mathbf{0 . 4 3}(0.05)$ & $\mathbf{0 . 3 9}(0.05)$ & $\mathbf{0 . 0 9}(0.04)$ & -6 & (5.3) & -13 & (5.3) & -13 & (4.4) & -20 & (5.7) & 0.05 & $(0.23)$ \\
\hline & Finnland & $0.31(0.03)$ & $\mathbf{0 . 5 2}(0.03)$ & $\mathbf{0 . 4 3}(0.03)$ & $\mathbf{0 . 2 9}(0.03)$ & $-0.01(0.02)$ & -22 & (4.4) & -26 & (3.7) & -24 & (4.4) & -24 & (4.0) & -0.10 & $(0.17)$ \\
\hline & Frankreich & $\mathbf{0 . 2 8}(0.03)$ & $\mathbf{0 . 4 3}(0.04)$ & $\mathbf{0 . 3 6}(0.04)$ & $\mathbf{0 . 3 1}(0.04)$ & $0.02(0.04)$ & -19 & (6.0) & -19 & (5.3) & -15 & (5.4) & -5 & (5.4) & 0.22 & 1) \\
\hline & Deutschland & $0.19(0.04)$ & $0.35(0.04)$ & $\mathbf{0 . 2 0}(0.04)$ & $\mathbf{0 . 2 1}(0.05)$ & $-0.01(0.04)$ & 19 & (6.8) & 8 & (7.1) & 9 & (5.5) & -3 & (6.0) & -0.69 & $(0.29)$ \\
\hline & Griechenland & $\mathbf{0 . 2 4}(0.06)$ & $\mathbf{0 . 2 3}(0.05)$ & $\mathbf{0 . 2 5}(0.05)$ & $\mathbf{0 . 3 5}(0.08)$ & $0.12(0.08)$ & 11 & (6.0) & 10 & (6.0) & 8 & (6.7) & 5 & (7.4) & 0.02 & $(0.23)$ \\
\hline & Ungarn & $0.06(0.04)$ & $-0.01(0.04)$ & $0.07(0.04)$ & $\mathbf{0 . 1 7}(0.05)$ & $0.01(0.04)$ & -5 & (6.5) & -11 & (5.4) & -19 & (6.4) & -14 & (8.1) & -0.16 & $(0.28)$ \\
\hline & Island & $\mathbf{0 . 2 3}(0.02)$ & $\mathbf{0 . 3 4}(0.03)$ & $\mathbf{0 . 3 0}(0.03)$ & $\mathbf{0 . 2 6}(0.03)$ & $0.02(0.02)$ & -21 & $(4.2)$ & -29 & (4.4) & -13 & $(4.8)$ & -20 & (4.5) & -0.03 & $(0.17)$ \\
\hline & Irland & $0.39(0.04)$ & $\mathbf{0 . 4 5}(0.04)$ & $\mathbf{0 . 4 1}(0.04)$ & $\mathbf{0 . 4 5}(0.05)$ & $\mathbf{0 . 2 3}(0.05)$ & 5 & (6.1) & -1 & (4.9) & -6 & (4.3) & 1 & (5.3) & -0.26 & $(0.21)$ \\
\hline & Italien & $\mathbf{0 . 2 3}(0.03)$ & $\mathbf{0 . 3 4}(0.02)$ & $\mathbf{0 . 3 0}(0.03)$ & $\mathbf{0 . 2 1}(0.03)$ & $\mathbf{0 . 0 8}(0.04)$ & 27 & (5.5) & 20 & (4.9) & 16 & (4.4) & 17 & (5.0) & -0.20 & $(0.15)$ \\
\hline & Japan & $\mathbf{0 . 3 5}(0.03)$ & $\mathbf{0 . 4 3}(0.03)$ & $\mathbf{0 . 3 7}(0.03)$ & $\mathbf{0 . 3 8}(0.03)$ & $0.22(0.03)$ & 12 & (8.0) & 4 & (7.0) & 4 & (6.1) & -4 & (8.9) & -0.16 & $(0.19)$ \\
\hline & Korea & $\mathbf{0 . 3 8}(0.04)$ & $\mathbf{0 . 5 8}(0.04)$ & $\mathbf{0 . 4 5}(0.04)$ & $\mathbf{0 . 4 0}(0.05)$ & 0.07 (0.05) & 19 & (6.5) & 4 & (6.4) & 14 & (7.4) & 9 & (9.5) & -0.34 & $(0.18)$ \\
\hline & Luxemburg & $\mathbf{0 . 1 6}(0.02)$ & $\mathbf{0 . 1 3}(0.03)$ & $\mathbf{0 . 1 1}(0.03)$ & $\mathbf{0 . 2 4}(0.02)$ & $\mathbf{0 . 1 8}(0.02)$ & -7 & (3.8) & -5 & $(4.1)$ & 1 & (3.9) & 1 & (3.8) & 0.16 & $(0.21)$ \\
\hline & Mexiko & $\mathbf{0 . 2 1}(0.06)$ & $0.06(0.04)$ & $\mathbf{0 . 2 4}(0.05)$ & $0.27(0.08)$ & $\mathbf{0 . 2 8}(0.07)$ & 44 & (5.4) & 34 & (4.3) & 21 & (6.4) & 13 & (6.2) & -0.50 & (0 \\
\hline & Niederlande & $\mathbf{0 . 3 2}(0.03)$ & $\mathbf{0 . 5 1}(0.04)$ & $\mathbf{0 . 4 6}(0.04)$ & $\mathbf{0 . 3 1}(0.04)$ & $0.00(0.03)$ & -14 & (7.4) & -17 & (5.9) & -11 & (6.1) & -32 & (6.6) & -0.28 & $(0$ \\
\hline & Neuseeland & $\mathbf{0 . 1 7}(0.03)$ & $\mathbf{0 . 2 7}(0.04)$ & $\mathbf{0 . 1 7}(0.03)$ & $\mathbf{0 . 1 8}(0.03)$ & $\mathbf{0 . 0 7}(0.03)$ & -28 & (5.0) & -19 & (5.1) & -24 & (5.5) & -16 & (5.0) & 0.32 & $(0.24)$ \\
\hline & Norwegen & $\mathbf{0 . 2 8}(0.03)$ & $\mathbf{0 . 2 9}(0.03)$ & $\mathbf{0 . 3 4}(0.03)$ & $\mathbf{0 . 2 9}(0.03)$ & $\mathbf{0 . 1 8}(0.03)$ & 8 & (5.4) & -9 & (5.4) & -1 & (5.6) & -16 & (5.1) & -0.19 & $(0.19)$ \\
\hline & Polen & $\mathbf{0 . 2 0}(0.04)$ & $\mathbf{0 . 2 4}(0.03)$ & $\mathbf{0 . 1 3}(0.03)$ & $\mathbf{0 . 2 1}(0.06)$ & $\mathbf{0 . 2 1}(0.05)$ & 29 & (5.7) & 26 & (5.0) & 23 & (6.4) & 31 & (7.1) & -0.05 & $(0.21)$ \\
\hline & Portugal & $\mathbf{0 . 4 2}(0.07)$ & $\mathbf{0 . 6 0}(0.04)$ & $\mathbf{0 . 5 1}(0.06)$ & $0.35(0.10)$ & $\mathbf{0 . 2 3}(0.10)$ & 18 & (6.3) & 21 & (6.6) & 26 & (6.5) & 27 & (7.1) & 0.15 & $(0.22)$ \\
\hline & Slowak. Rep. & $0.06(0.04)$ & $\mathbf{0 . 1 2}(0.06)$ & \begin{tabular}{|ll}
$\mathbf{0 . 0 7}$ & $(0.04)$
\end{tabular} & $0.02(0.05)$ & $0.04(0.05)$ & -21 & (8.9) & -10 & (5.6) & -22 & (5.7) & -11 & (7.6) & 0.06 & $(0.29)$ \\
\hline & Spanien & $\mathbf{0 . 3 2}(0.05)$ & $\mathbf{0 . 4 6}(0.03)$ & $\mathbf{0 . 3 7}(0.06)$ & $\mathbf{0 . 3 4}(0.07)$ & $\mathbf{0 . 1 3}(0.06)$ & -5 & (4.3) & -1 & $(4.2)$ & 2 & (5.8) & 5 & (3.8) & 0.21 & $(0.17)$ \\
\hline & Schweden & $\mathbf{0 . 1 9}(0.03)$ & $\mathbf{0 . 3 8}(0.04)$ & $\mathbf{0 . 2 6}(0.04)$ & $\mathbf{0 . 1 9}(0.04)$ & $\mathbf{- 0 . 0 7}(0.03)$ & -25 & (5.0) & -24 & $(5.7)$ & -24 & (4.9) & -40 & (5.7) & 0.01 & $(0.15)$ \\
\hline & Schweiz & $\mathbf{0 . 4 0}(0.04)$ & $0.51(0.04)$ & $\mathbf{0 . 4 6}(0.04)$ & $\mathbf{0 . 4 5}(0.05)$ & $\mathbf{0 . 1 8}(0.05)$ & 19 & (5.5) & 1 & (6.1) & 4 & (5.7) & -3 & (7.3) & -0.44 & $(0.19)$ \\
\hline & Türkei & $-\mathbf{0 . 3 1}(0.07)$ & $\mathbf{- 0 . 4 0}(0.04)$ & $-\mathbf{0 . 3 3}(0.06)$ & $\mathbf{- 0 . 2 6}(0.09)$ & $-0.23(0.12)$ & 38 & (6.6) & 41 & (7.9) & 18 & (9.9) & 2 & $(17.8)$ & -0.18 & $(0.24)$ \\
\hline & Ver. Staaten & $\mathbf{0 . 1 2}(0.05)$ & $0.08(0.06)$ & $\mathbf{0 . 1 7}(0.05)$ & $0.20(0.06)$ & $0.04(0.05)$ & 11 & (5.4) & -3 & (5.9) & -2 & (7.2) & -9 & (6.1) & -0.36 & $(0.22)$ \\
\hline & OECD-Durchschnitt 2003 & $\left|\begin{array}{ll}0.22 & (0.01)\end{array}\right|$ & $0.30(0.01)$ & $0.27(0.01)$ & $0.25(0.01)$ & $0.08(0.01)$ & 1 & (1.1) & -3 & $(1.0)$ & -4 & (1.1) & -7 & (1.3) & -0.12 & $(0.04)$ \\
\hline \multirow{10}{*}{ ఏ } & Brasilien & \begin{tabular}{|l|l|}
$\mathbf{0 . 3 9}$ & $(0.05)$ \\
\end{tabular} & $0.46(0.04)$ & $\mathbf{0 . 4 7}(0.06)$ & $\mathbf{0 . 3 3}(0.06)$ & $\mathbf{0 . 3 2}(0.07)$ & 43 & (6.2) & 33 & (6.6) & 45 & (6.6) & 20 & (10.6) & 0.11 & $(0.18)$ \\
\hline & Hongkong (China) & $\mathbf{0 . 4 8}(0.06)$ & $\mathbf{0 . 3 9}(0.04)$ & $\mathbf{0 . 4 4}(0.05)$ & $\mathbf{0 . 6 1}(0.09)$ & $\mathbf{0 . 4 9}(0.09)$ & 19 & (7.5) & 9 & (6.1) & 9 & (9.1) & 13 & (7.9) & -0.22 & $(0.21)$ \\
\hline & Indonesien & $0.06(0.06)$ & $-0.02(0.04)$ & $0.00(0.06)$ & $0.04(0.08)$ & $0.23(0.11)$ & 15 & (5.8) & 13 & $(5.2)$ & 19 & $(6.7)$ & 14 & $(12.2)$ & 0.14 & $(0.14)$ \\
\hline & Lettland & \begin{tabular}{|l|l|}
$\mathbf{0 . 0 9}$ & $(0.04)$ \\
\end{tabular} & $0.05(0.04)$ & $0.04(0.05)$ & $\mathbf{0 . 1 6}(0.05)$ & $\mathbf{0 . 1 0}(0.04)$ & 7 & (6.3) & 0 & (5.5) & 14 & (6.8) & 9 & $(7.0)$ & 0.03 & $(0.22)$ \\
\hline & Liechtenstein & $\mathbf{0 . 6 1}(0.07)$ & $0.68(0.10)$ & $\mathbf{0 . 6 8}(0.08)$ & $\mathbf{0 . 6 7}(0.09)$ & $\mathbf{0 . 4 2}(0.09)$ & 12 & (14.2) & 31 & (15.5) & 3 & (16.0) & -42 & $(15.2)$ & -0.34 & $(0.65)$ \\
\hline & Macau (China) & $\mathbf{0 . 7 1}(0.03)$ & $\mathbf{0 . 8 1}(0.04)$ & $\mathbf{0 . 7 0}(0.03)$ & $\mathbf{0 . 6 8}(0.03)$ & $\mathbf{0 . 6 7}(0.05)$ & 7 & (6.9) & 12 & $(8.1)$ & 13 & (7.2) & 16 & (6.2) & 0.15 & $(0.16)$ \\
\hline & Russ. Föderation & $\mathbf{0 . 5 0}(0.04)$ & $0.69(0.04)$ & $\mathbf{0 . 6 6}(0.04)$ & $\mathbf{0 . 5 0}(0.06)$ & $0.15(0.04)$ & 11 & (6.7) & 13 & (6.9) & 22 & (6.5) & 11 & (6.9) & 0.13 & $(0.20)$ \\
\hline & Thailand & 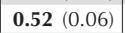 & $\mathbf{0 . 4 6}(0.05)$ & $\mathbf{0 . 5 6}(0.05)$ & $\mathbf{0 . 6 5}(0.07)$ & $\mathbf{0 . 4 0}(0.09)$ & 10 & (5.9) & 13 & $(4.7)$ & 12 & (5.6) & 5 & (9.6) & 0.13 & $(0.15)$ \\
\hline & Tunesien & $\mathbf{0 . 5 0}(0.06)$ & $0.24(0.06)$ & $\mathbf{0 . 6 5}(0.07)$ & $\mathbf{0 . 6 7}(0.08)$ & $0.43(0.09)$ & 31 & (4.8) & 27 & (5.5) & 35 & (5.2) & 27 & (11.5) & 0.07 & $(0.20)$ \\
\hline & Uruguay & $\mathbf{- 0 . 1 2}(0.05)$ & $0.00(0.04)$ & $\mathbf{- 0 . 1 9}(0.05)$ & $\mathbf{- 0 . 2 4}(0.06)$ & $-0.07(0.06)$ & -15 & (6.1) & -12 & (5.4) & -11 & (6.7) & -11 & (7.6) & 0.21 & $(0.20)$ \\
\hline
\end{tabular}

Anmerkung: Statistisch signifikante Werte sind durch Fettdruck gekennzeichnet (vgl. Anhang A3).

Angegeben sind nur Länder und Volkswirtschaften mit vergleichbaren Daten aus PISA 2003 und PISA 2012.

Um die Vergleichbarkeit im Zeitverlauf zu gewährleisten, wurden die Werte von PISA 2003 auf dem PISA-Index des wirtschaftlichen, sozialen und kulturellen Status entsprechend der Indexskala von PISA 2012 umskaliert. Die in dieser Tabelle dargestellten Ergebnisse aus PISA 2003 können daher von denen abweichen, die in Lernen für die Welt von morgen: Erste Ergebnisse von PISA 2003 (OECD, 2004) wiedergegeben sind (vgl. Anhang A5 wegen näherer Einzelheiten).

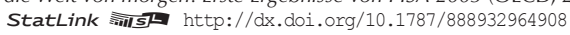


[Teil 1/4]

Schülerleistungen und ausgewählte Merkmale des sozioökonomischen Status im Ländervergleich Tabelle II.2.5 Die Ergebnisse basieren auf Schülerangaben

\begin{tabular}{|c|c|c|c|c|c|c|c|c|c|c|c|c|c|c|c|c|c|c|c|c|c|}
\hline & & \multicolumn{20}{|c|}{ Höchster Bildungsabschluss der Eltern } \\
\hline & & \multicolumn{4}{|c|}{$\begin{array}{c}\text { Prozentsatz der Schüler, } \\
\text { deren Eltern einen } \\
\text { niedrigen (höchstens } \\
\text { Sekundarstufe I: ISCED } 2 \\
\text { oder darunter) bzw. } \\
\text { hohen (Tertiärbereich } \\
\text { oder weiterführende } \\
\text { Forschungsprogramme: } \\
\text { ISCED } 5 \text { oder 6) } \\
\text { Bildungsabschluss } \\
\text { aufweisen }\end{array}$} & \multicolumn{3}{|c|}{\begin{tabular}{|} 
Durchschnittliche \\
Leistungen der Schüler \\
mit Eltern, deren höchster \\
Bildungsabschluss niedrig \\
(höchstens Sekundarstufe I: \\
ISCED 2 oder darunter) \\
bzw. hoch (Tertiärbereich \\
oder weiterführende \\
Forschungsprogramme: \\
ISCED 5 oder 6) ist \\
\end{tabular}} & 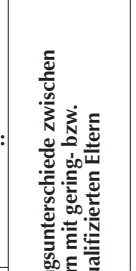 & 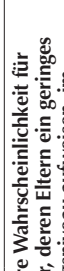 & 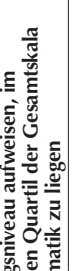 & 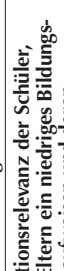 & 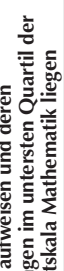 & 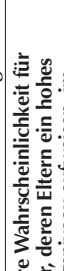 & 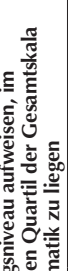 & 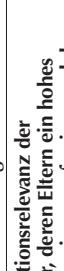 & 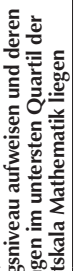 & $\mid$ & 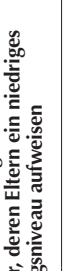 & 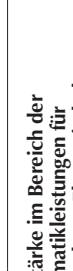 & 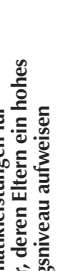 \\
\hline & & $\begin{array}{r}\text { Nied } \\
\text { Bild } \\
\text { niv }\end{array}$ & $\begin{array}{l}\text { riges } \\
\text { ungs- } \\
\text { eau }\end{array}$ & $\begin{array}{r}\text { Ho } \\
\text { Bildu } \\
\text { nive }\end{array}$ & $\begin{array}{l}\text { hes } \\
\text { Ings- } \\
\text { eau }\end{array}$ & $\begin{array}{l}\text { Niedriges } \\
\text { Bildungs- } \\
\text { niveau }\end{array}$ & $\begin{array}{r}\text { Hoh } \\
\text { Bildu } \\
\text { nive }\end{array}$ & $\begin{array}{l}\text { hes } \\
\text { ungs- } \\
\text { eau }\end{array}$ & 窇总总 & 总 & 年 & 震 & 言焉 & 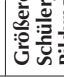 & 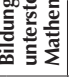 & 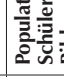 & . & 离 & 害焉 & 党吾 & 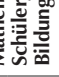 \\
\hline & & $\%$ & S.E. & $\%$ & S.E. & $\begin{array}{c}\text { Mittel- } \\
\text { wert }\end{array}$ & $\begin{array}{c}\text { Mittel- } \\
\text { wert }\end{array}$ & S.E. & $\begin{array}{l}\text { Punkt- } \\
\text { diff. } \quad \text { S.E. }\end{array}$ & $\begin{array}{l}\text { Quo- } \\
\text { tient }\end{array}$ & S.E. & $\%$ & S.E. & $\begin{array}{l}\text { Quo- } \\
\text { tient }\end{array}$ & S.E. & $\%$ & S.E. & $\begin{array}{l}\text { Effekt- } \\
\text { stärke }\end{array}$ & S.E. & $\begin{array}{l}\text { Effekt- } \\
\text { stärke }\end{array}$ & S.E. \\
\hline & Australien & 10.4 & $(0.3)$ & 55.4 & $(0.5)$ & $461 \quad(2.9)$ & 528 & $(2.1)$ & $66 \quad(3.3)$ & 1.83 & $(0.08)$ & 8.0 & $(0.7)$ & 0.55 & $(0.03)$ & -32.9 & $(2.7)$ & -0.6 & $(0.0)$ & 0.5 & $(0.0)$ \\
\hline & Österreich & 4.7 & $(0.5)$ & 48.0 & $(1.0)$ & $429 \quad(9.0)$ & 524 & (3.3) & 95 (9.5) & 2.43 & $(0.25)$ & 6.3 & (1.1) & 0.62 & $(0.04)$ & -22.5 & (3.2) & -0.9 & $(0.1)$ & 0.4 & $(0.0)$ \\
\hline & Belgien & 4.9 & $(0.4)$ & 62.9 & $(0.8)$ & $453 \quad(8.0)$ & 539 & (2.6) & $86 \quad(8.4)$ & 2.00 & $(0.17)$ & 4.7 & $(0.9)$ & 0.52 & $(0.04)$ & -43.5 & (4.5) & -0.7 & $(0.1)$ & 0.6 & $(0.0)$ \\
\hline & Kanada & 3.2 & $(0.3)$ & 72.4 & $(0.7)$ & $458 \quad(5.6)$ & 530 & (2.1) & 72 (5.6) & 2.20 & $(0.16)$ & 3.7 & $(0.5)$ & 0.63 & $(0.03)$ & -37.1 & (4.3) & -0.7 & $(0.1)$ & 0.4 & $(0.0)$ \\
\hline 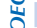 & Chile & 22.4 & (1.4) & 35.0 & $(1.2)$ & $\begin{array}{ll}377 & (3.5)\end{array}$ & 458 & $(4.0)$ & $82 \quad(4.9)$ & 2.37 & $(0.16)$ & 23.5 & $(2.4)$ & 0.46 & $(0.04)$ & -23.5 & $(2.7)$ & -0.8 & $(0.1)$ & 0.7 & $(0.1)$ \\
\hline & Tschech. Rep. & 1.2 & $(0.2)$ & 32.6 & $(0.9)$ & $406(17.8)$ & 529 & $(4.2)$ & $123(18.1)$ & 2.70 & $(0.43)$ & 2.0 & $(0.7)$ & 0.67 & $(0.06)$ & -12.0 & (2.6) & -1.1 & $(0.2)$ & 0.5 & $(0.0)$ \\
\hline & Dänemark & 5.7 & $(0.4)$ & 67.4 & (1.0) & $443 \quad(5.3)$ & 516 & $(2.2)$ & $\begin{array}{|ll|}73 & (5.7) \\
\end{array}$ & 2.28 & $(0.18)$ & 6.9 & (1.0) & 0.55 & $(0.04)$ & -44.1 & (5.3) & -0.8 & $(0.1)$ & 0.5 & $(0.0)$ \\
\hline & Estland & 2.4 & $(0.3)$ & 57.6 & $(0.9)$ & $478 \quad(7.1)$ & 531 & (2.6) & 53 (7.4) & 1.89 & $(0.25)$ & 2.1 & $(0.6)$ & 0.77 & $(0.04)$ & -15.2 & (3.5) & -0.6 & $(0.1)$ & 0.3 & $(0.0)$ \\
\hline & Finnland & 2.9 & $(0.2)$ & 79.3 & $(0.6)$ & $466 \quad(7.6)$ & 528 & (1.9) & 62 (7.6) & 2.04 & $(0.17)$ & 2.9 & $(0.5)$ & 0.60 & $(0.03)$ & -46.3 & (5.5) & -0.7 & $(0.1)$ & 0.4 & $(0.0)$ \\
\hline & Frankreich & 9.5 & $(0.5)$ & 54.3 & $(0.9)$ & $431 \quad(4.7)$ & 524 & (3.1) & $93 \quad(5.3)$ & 2.37 & $(0.16)$ & 11.5 & (1.3) & 0.53 & $(0.04)$ & -33.8 & (3.6) & -0.9 & $(0.1)$ & 0.6 & $(0.0)$ \\
\hline & Deutschland & 22.8 & (0.9) & 50.5 & (1.1) & $481 \quad(4.4)$ & 543 & (3.3) & 62 & 1.95 & $(0.13)$ & 17.8 & $(2.1)$ & 0.60 & $(0.04)$ & -25.5 & (3.3) & -0.6 & $(0.0)$ & 0.4 & $(0.0)$ \\
\hline & Griechenland & 10.7 & $(0.7)$ & 52.6 & (1.1) & $403 \quad(4.5)$ & 473 & (3.1) & 70 (5.7) & 2.11 & $(0.18)$ & 10.6 & (1.6) & 0.60 & $(0.05)$ & -26.6 & $(4.2)$ & -0.7 & $(0.1)$ & 0.5 & $(0.0)$ \\
\hline & Ungarn & 9.6 & $(0.7)$ & 43.6 & (1.3) & $395 \quad(6.6)$ & 508 & $(5.0)$ & \begin{tabular}{|ll}
113 & $(8.6)$ \\
\end{tabular} & 3.01 & $(0.26)$ & 16.1 & $(2.0)$ & 0.51 & $(0.05)$ & -26.9 & (3.7) & -1.1 & $(0.1)$ & 0.6 & $(0.1)$ \\
\hline & Island & 7.2 & $(0.4)$ & 66.1 & $(0.8)$ & $456 \quad(5.4)$ & 504 & (2.3) & $49 \quad(6.0)$ & 1.67 & $(0.14)$ & 4.6 & $(1.0)$ & 0.73 & $(0.04)$ & -21.9 & (4.6) & -0.5 & $(0.1)$ & 0.3 & $(0.0)$ \\
\hline & Irland & 8.0 & $(0.4)$ & 55.9 & (1.0) & $458 \quad(5.1)$ & 521 & $(2.2)$ & $63 \quad(4.9)$ & 1.77 & $(0.14)$ & 5.8 & $(1.0)$ & 0.56 & $(0.04)$ & -32.7 & (3.7) & -0.6 & $(0.1)$ & 0.5 & $(0.0)$ \\
\hline & Israel & 4.4 & $(0.5)$ & 61.7 & (1.4) & 377 (6.4) & 499 & (4.7) & $122(7.5)$ & 2.64 & $(0.21)$ & 6.7 & $(1.0)$ & 0.41 & $(0.03)$ & -57.5 & (6.1) & -1.0 & $(0.1)$ & 0.8 & $(0.0)$ \\
\hline & Italien & 20.5 & $(0.4)$ & 37.1 & $(0.5)$ & $450 \quad(2.4)$ & 497 & (2.5) & $\begin{array}{|ll|}48 & (3.0) \\
\end{array}$ & 1.76 & $(0.06)$ & 13.5 & $(0.9)$ & 0.80 & $(0.03)$ & -7.9 & (1.3) & -0.5 & $(0.0)$ & 0.2 & $(0.0)$ \\
\hline & Japan & 1.7 & $(0.2)$ & 62.4 & (1.0) & $484(12.2)$ & 557 & (4.2) & 74 (12.6) & 2.01 & $(0.32)$ & 1.7 & $(0.6)$ & 0.52 & $(0.04)$ & -42.8 & (5.3) & -0.6 & $(0.1)$ & 0.5 & $(0.1)$ \\
\hline & Korea & 3.2 & $(0.3)$ & 56.7 & (1.6) & $509 \quad(9.7)$ & 574 & (5.1) & $65 \quad(9.7)$ & 1.66 & $(0.19)$ & 2.1 & $(0.6)$ & 0.59 & $(0.04)$ & -30.7 & (4.4) & -0.5 & $(0.1)$ & 0.5 & $(0.0)$ \\
\hline & Luxemburg & 20.0 & $(0.6)$ & 50.8 & $(0.7)$ & $440 \quad(3.1)$ & 518 & (1.8) & 78 (3.6) & 2.23 & $(0.15)$ & 19.7 & $(2.0)$ & 0.52 & $(0.03)$ & -32.3 & $(2.7)$ & -0.7 & $(0.0)$ & 0.6 & $(0.0)$ \\
\hline & Mexiko & 49.7 & $(0.8)$ & 35.7 & $(0.7)$ & $397 \quad(1.5)$ & 433 & (1.8) & $37 \quad(2.1)$ & 1.79 & $(0.06)$ & 28.2 & (1.6) & 0.60 & $(0.02)$ & -16.6 & (1.2) & -0.5 & $(0.0)$ & 0.4 & $(0.0)$ \\
\hline & Niederlande & 6.1 & $(0.4)$ & 63.2 & $(1.2)$ & $478 \quad(7.9)$ & 537 & (3.9) & 59 (7.5) & 1.76 & $(0.19)$ & 4.4 & (1.1) & 0.71 & $(0.06)$ & -22.1 & (6.4) & -0.6 & $(0.1)$ & 0.3 & $(0.0)$ \\
\hline & Neuseeland & 7.2 & $(0.5)$ & 52.7 & (1.0) & $449 \quad(5.5)$ & 528 & (3.1) & 79 (5.4) & 1.90 & $(0.17)$ & 6.1 & $(1.1)$ & 0.61 & $(0.04)$ & -25.7 & (3.5) & -0.7 & $(0.1)$ & 0.5 & $(0.0)$ \\
\hline & Norwegen & 2.9 & $(0.3)$ & 67.9 & (1.0) & $424 \quad(8.5)$ & 502 & (2.6) & 78 (8.7) & 2.30 & $(0.25)$ & 3.6 & $(0.7)$ & 0.65 & $(0.04)$ & -31.6 & (5.3) & -0.8 & $(0.1)$ & 0.3 & $(0.0)$ \\
\hline & Polen & 3.1 & $(0.3)$ & 26.3 & (1.3) & $439 \quad(8.1)$ & 568 & (5.5) & $\begin{array}{|ll|}128 & (9.8)\end{array}$ & 2.50 & $(0.24)$ & 4.4 & $(0.8)$ & 0.33 & $(0.04)$ & -21.3 & (2.1) & -1.0 & $(0.1)$ & 0.8 & $(0.0)$ \\
\hline & Portugal & 48.7 & (1.7) & 27.3 & (1.6) & $461 \quad(4.0)$ & 531 & (4.1) & $71 \quad(5.1)$ & 2.23 & $(0.18)$ & 37.4 & (3.1) & 0.47 & $(0.05)$ & -16.7 & (2.5) & -0.6 & $(0.0)$ & 0.6 & $(0.1)$ \\
\hline & Slowak. Rep. & 3.4 & $(0.5)$ & 30.1 & $(1.2)$ & $345(11.0)$ & 519 & (5.5) & $\mid 173(13.0)$ & 3.44 & $(0.28)$ & 7.7 & (1.3) & 0.63 & $(0.06)$ & -12.6 & (2.5) & -1.6 & $(0.1)$ & 0.5 & $(0.1)$ \\
\hline & Slowenien & 4.1 & $(0.3)$ & 43.3 & $(0.8)$ & $443 \quad(6.8)$ & 530 & (2.0) & $87 \quad(7.7)$ & 1.94 & $(0.23)$ & 3.7 & $(0.9)$ & 0.54 & $(0.04)$ & -25.1 & (2.6) & -0.7 & $(0.1)$ & 0.5 & $(0.0)$ \\
\hline & Spanien & 24.4 & $(0.8)$ & 48.5 & $(1.0)$ & $450 \quad(2.9)$ & 507 & $(2.2)$ & $57 \quad(3.2)$ & 1.89 & $(0.09)$ & 17.8 & (1.6) & 0.56 & $(0.03)$ & -27.3 & (2.7) & -0.6 & $(0.0)$ & 0.5 & $(0.0)$ \\
\hline & Schweden & 5.4 & $(0.4)$ & 69.2 & $(0.7)$ & $429 \quad(6.7)$ & 490 & $(2.6)$ & $61 \quad(7.1)$ & 1.96 & $(0.18)$ & 5.0 & $(0.9)$ & 0.76 & $(0.06)$ & -19.4 & (5.4) & -0.7 & $(0.1)$ & 0.3 & $(0.0)$ \\
\hline & Schweiz & 12.9 & $(0.5)$ & 56.2 & $(0.8)$ & $475 \quad(4.0)$ & 549 & (3.3) & 74 (4.1) & 2.18 & $(0.12)$ & 13.2 & $(1.2)$ & 0.65 & $(0.04)$ & -24.9 & (3.3) & -0.7 & $(0.0)$ & 0.4 & $(0.0)$ \\
\hline & Türkei & 59.9 & $(1.2)$ & 24.0 & (1.0) & $428 \quad(3.7)$ & 488 & (7.5) & $59 \quad(6.2)$ & 1.89 & $(0.17)$ & 34.6 & (3.9) & 0.57 & $(0.06)$ & -11.5 & (1.9) & -0.6 & $(0.0)$ & 0.6 & $(0.1)$ \\
\hline & Ver. Königreich & 3.7 & $(0.3)$ & 59.4 & $(0.8)$ & $450 \quad(7.2)$ & 511 & (3.4) & $61 \quad(7.3)$ & 1.82 & (0.19) & 2.9 & $(0.7)$ & 0.77 & $(0.06)$ & -15.8 & (4.4) & -0.6 & (0.1) & 0.3 & $(0.0)$ \\
\hline & Ver. Staaten & 9.0 & $(0.8)$ & 58.6 & (1.5) & $441 \quad(5.2)$ & 501 & (4.0) & $61 \quad(5.9)$ & 1.75 & $(0.15)$ & 6.4 & $(1.2)$ & 0.55 & $(0.04)$ & -35.4 & (4.8) & -0.6 & (0.1) & 0.5 & $(0.0)$ \\
\hline & OECD-Durchschnitt & 12.2 & $(0.1)$ & 51.9 & $(0.2)$ & $440 \quad(1.2)$ & 517 & (0.6) & $\begin{array}{ll}77 & (1.3)\end{array}$ & 2.13 & $(0.03)$ & 10.2 & $(0.3)$ & 0.59 & $(0.01)$ & -27.1 & $(0.7)$ & -0.7 & $(0.0)$ & 0.5 & $(0.0)$ \\
\hline ฮ & Albanien & 24.4 & (0.9) & 29.3 & (0.8) & $394 \quad(3.4)$ & 395 & (3.2) & $1 \quad(4.2)$ & 1.01 & $(0.11)$ & 0.2 & $(2.7)$ & 0.99 & $(0.08)$ & -0.2 & (2.5) & 0.0 & (0.0) & 0.0 & $(0.0)$ \\
\hline$=$ & Argentinien & 31.3 & (1.3) & 47.1 & (1.3) & 362 & 410 & (4.1) & $48 \quad(4.2)$ & 1.93 & $(0.17)$ & 22.6 & (3.1) & 0.53 & $(0.04)$ & -28.4 & (3.5) & -0.6 & $(0.0)$ & 0.5 & $(0.0)$ \\
\hline 5 & Brasilien & 37.3 & $(0.8)$ & 26.0 & $(0.8)$ & $366 \quad(1.9)$ & 424 & (4.4) & 58 (4.5) & 1.78 & $(0.09)$ & 22.5 & $(2.0)$ & 0.61 & $(0.04)$ & -11.3 & (1.6) & -0.6 & $(0.0)$ & 0.5 & $(0.0)$ \\
\hline & Bulgarien & 7.2 & $(0.8)$ & 45.4 & $(1.2)$ & $\begin{array}{ll}357 & (9.6)\end{array}$ & 468 & (4.8) & $111(10.4)$ & 2.68 & $(0.25)$ & 10.7 & (1.6) & 0.51 & $(0.04)$ & -28.5 & (3.6) & -1.1 & (0.1) & 0.6 & $(0.0)$ \\
\hline & Kolumbien & 37.5 & (1.3) & 47.0 & (1.5) & $352 \quad(3.1)$ & 396 & (3.6) & $43 \quad(3.8)$ & 1.90 & $(0.16)$ & 25.2 & (3.5) & 0.54 & $(0.05)$ & -27.8 & (4.2) & -0.6 & $(0.0)$ & 0.5 & $(0.0)$ \\
\hline & Costa Rica & 28.0 & (1.1) & 48.5 & (1.3) & $379 \quad(3.3)$ & 423 & (3.7) & $43 \quad(4.1)$ & 1.89 & $(0.14)$ & 20.0 & (2.6) & 0.63 & $(0.05)$ & -21.9 & (3.8) & -0.6 & (0.1) & 0.4 & $(0.1)$ \\
\hline 1 & Kroatien & 4.8 & $(0.4)$ & 47.1 & $(0.8)$ & $416 \quad(6.1)$ & 482 & (4.6) & $65 \quad(7.3)$ & 2.04 & $(0.23)$ & 4.7 & $(1.0)$ & 0.82 & $(0.05)$ & -9.0 & (2.6) & -0.7 & (0.1) & 0.2 & $(0.0)$ \\
\hline ఏ & Zypern* & 5.1 & $(0.3)$ & 52.7 & $(0.7)$ & $389 \quad(6.3)$ & 461 & (2.0) & 72 (6.6) & 1.91 & $(0.18)$ & 4.4 & $(0.9)$ & 0.63 & $(0.04)$ & -24.5 & (3.7) & -0.6 & (0.1) & 0.5 & $(0.0)$ \\
\hline 8 & Hongkong (China) & 29.7 & (1.4) & 21.8 & $(2.0)$ & $537 \quad(4.1)$ & 601 & (5.3) & 64 (6.6) & 1.59 & $(0.11)$ & 14.9 & $(2.2)$ & 0.48 & $(0.07)$ & -12.9 & (2.8) & -0.4 & $(0.0)$ & 0.5 & $(0.1)$ \\
\hline & Indonesien & 48.1 & (1.7) & 19.8 & (1.5) & $361 \quad(3.5)$ & 403 & (10.3) & $42(10.4)$ & 1.37 & $(0.09)$ & 15.2 & (3.1) & 0.75 & $(0.09)$ & -5.2 & (2.2) & -0.4 & $(0.1)$ & 0.5 & $(0.1)$ \\
\hline & Jordanien & 12.0 & $(0.6)$ & 57.3 & (1.1) & 355 (3.9) & 401 & (4.1) & $46 \quad(5.0)$ & 1.69 & $(0.15)$ & 7.7 & (1.6) & 0.67 & $(0.04)$ & -23.7 & (4.0) & -0.5 & $(0.1)$ & 0.4 & $(0.0)$ \\
\hline ‡ & Kasachstan & 1.4 & $(0.2)$ & 86.6 & $(0.8)$ & $391(12.6)$ & 435 & (3.2) & 44 (12.6) & 1.99 & $(0.34)$ & 1.3 & $(0.4)$ & 0.66 & $(0.07)$ & -41.6 & (12.9) & -0.6 & $(0.2)$ & 0.4 & $(0.1)$ \\
\hline & Lettland & 2.7 & $(0.4)$ & 54.7 & (1.3) & $442(11.3)$ & 508 & (3.7) & 66 (10.7) & 2.08 & $(0.30)$ & 2.8 & $(0.9)$ & 0.61 & $(0.06)$ & -27.1 & (5.6) & -0.6 & $(0.1)$ & 0.5 & $(0.1)$ \\
\hline & Liechtenstein & 10.9 & $(2.0)$ & 56.1 & (2.5) & $476(14.6)$ & 544 & (5.9) & $68(16.4)$ & 2.14 & $(0.53)$ & 11.0 & $(5.2)$ & 0.82 & $(0.19)$ & -11.5 & (12.8) & -0.8 & $(0.2)$ & 0.2 & $(0.1)$ \\
\hline & Litauen & 1.5 & $(0.2)$ & 64.3 & $(0.9)$ & $413(13.2)$ & 496 & $(2.7)$ & 83 (13.1) & 2.30 & $(0.29)$ & 1.9 & $(0.5)$ & 0.50 & $(0.03)$ & -46.7 & (4.4) & -0.8 & $(0.2)$ & 0.5 & $(0.0)$ \\
\hline & Macau (China) & 49.3 & $(0.6)$ & 18.4 & $(0.5)$ & $531 \quad(1.6)$ & 554 & (3.0) & $23 \quad(3.6)$ & 1.27 & $(0.06)$ & 11.8 & $(2.2)$ & 0.80 & $(0.06)$ & -3.8 & (1.1) & -0.2 & $(0.0)$ & 0.2 & $(0.0)$ \\
\hline & Malaysia & 17.9 & $(0.9)$ & 28.8 & $(1.2)$ & $396 \quad(3.7)$ & 442 & (5.1) & $46 \quad(5.8)$ & 1.47 & $(0.11)$ & 7.8 & (1.7) & 0.77 & $(0.07)$ & -6.9 & (2.3) & -0.4 & $(0.1)$ & 0.4 & $(0.1)$ \\
\hline & Montenegro & 4.0 & $(0.3)$ & 58.8 & $(0.8)$ & $349 \quad(5.0)$ & 425 & (1.5) & $76 \quad(5.1)$ & 2.28 & $(0.23)$ & 4.9 & $(0.9)$ & 0.60 & $(0.04)$ & -30.6 & (4.3) & -0.9 & $(0.1)$ & 0.4 & $(0.0)$ \\
\hline & Peru & 26.7 & (1.3) & 34.1 & (1.5) & $324 \quad(3.1)$ & 403 & (5.5) & 79 (6.2) & 2.24 & $(0.17)$ & 24.9 & $(2.3)$ & 0.48 & $(0.04)$ & -21.7 & (3.0) & -0.8 & $(0.1)$ & 0.6 & $(0.1)$ \\
\hline & Katar & 9.8 & $(0.3)$ & 71.7 & $(0.4)$ & $328 \quad(2.5)$ & 391 & (1.1) & $64 \quad(2.7)$ & 1.63 & $(0.10)$ & 5.8 & $(0.9)$ & 0.66 & $(0.03)$ & -32.8 & (4.1) & -0.7 & $(0.0)$ & 0.5 & $(0.0)$ \\
\hline & Rumänien & 5.8 & $(0.6)$ & 51.7 & $(1.2)$ & $402 \quad(6.5)$ & 458 & $(4.7)$ & $56 \quad(7.5)$ & 1.77 & $(0.19)$ & 4.3 & $(1.1)$ & 0.73 & $(0.06)$ & -16.3 & (4.3) & -0.6 & $(0.1)$ & 0.3 & $(0.1)$ \\
\hline & Russ. Föderation & 1.3 & $(0.3)$ & 90.7 & (0.6) & $416(13.5)$ & 487 & (3.1) & 71 (13.6) & 1.99 & $(0.42)$ & 1.2 & $(0.5)$ & 0.60 & $(0.07)$ & -56.8 & (14.5) & -0.8 & $(0.1)$ & 0.5 & $(0.1)$ \\
\hline & Serbien & 4.8 & $(0.5)$ & 54.9 & (1.1) & $\begin{array}{ll}397 & (8.3)\end{array}$ & 460 & (3.9) & $63 \quad(8.4)$ & 1.94 & $(0.21)$ & 4.3 & (1.1) & 0.78 & $(0.05)$ & -13.5 & (3.5) & -0.6 & $(0.1)$ & 0.3 & $(0.0)$ \\
\hline & Shanghai (China) & 24.9 & (1.3) & 44.5 & (1.3) & $564 \quad(5.5)$ & 643 & (3.6) & 79 (6.0) & 2.10 & $(0.15)$ & 21.5 & (2.5) & 0.50 & $(0.04)$ & -28.5 & (2.9) & -0.7 & $(0.1)$ & 0.6 & $(0.0)$ \\
\hline & Singapur & 0.0 & $(0.4)$ & 47.8 & $(0.7)$ & $525 \quad(3.9)$ & 602 & (2.0) & 76 (4.6) & 1.71 & $(0.11)$ & 6.6 & $(0.9)$ & 0.50 & $(0.03)$ & -31.7 & (2.8) & -0.5 & $(0.0)$ & 0.5 & $(0.0)$ \\
\hline & Chinesisch Taipeh & 11.8 & $(0.6)$ & 51.4 & (1.1) & $493 \quad(6.0)$ & 591 & (3.4) & 99 (6.6) & 2.06 & $(0.14)$ & 11.2 & (1.3) & 0.48 & $(0.03)$ & -36.5 & (3.4) & -0.7 & $(0.1)$ & 0.6 & $(0.0)$ \\
\hline & Thailand & 50.2 & (1.4) & 19.0 & $(1.2)$ & $412 \quad(3.2)$ & 474 & (6.8) & $61 \quad(6.8)$ & 1.40 & $(0.11)$ & 16.6 & (3.8) & 0.45 & $(0.06)$ & -11.6 & (1.8) & -0.4 & $(0.1)$ & 0.7 & $(0.1)$ \\
\hline & Tunesien & 29.8 & (1.3) & 28.1 & $(1.2)$ & $366 \quad(3.4)$ & 417 & (7.6) & $52 \quad(7.7)$ & 1.56 & $(0.14)$ & 14.3 & $(2.9)$ & 0.65 & $(0.07)$ & -10.8 & (2.6) & -0.5 & $(0.1)$ & 0.5 & $(0.1)$ \\
\hline & Ver. Arab. Emirate & 8.9 & $(0.4)$ & 67.7 & $(0.9)$ & $384 \quad(3.1)$ & 453 & (2.8) & 69 (3.6) & 1.86 & $(0.11)$ & 7.1 & $(0.9)$ & 0.48 & $(0.02)$ & -54.5 & (4.0) & -0.7 & $(0.0)$ & 0.7 & $(0.0)$ \\
\hline & Uruguay & 45.1 & (1.1) & 35.6 & (1.0) & $378 \quad(2.9)$ & 444 & (4.2) & $65 \quad(4.9)$ & 2.25 & $(0.18)$ & $\mid 36.1$ & (3.1) & 0.50 & $(0.04)$ & -21.6 & (2.5) & -0.7 & $(0.0)$ & 0.6 & $(0.0)$ \\
\hline & Vietnam & 58.2 & (1.5) & 11.2 & (1.0) & $\begin{array}{ll}492 & (4.4)\end{array}$ & 559 & (9.7) & $67 \quad(9.3)$ & 1.93 & $(0.19)$ & 35.0 & (4.3) & 0.47 & $(0.10)$ & -6.3 & (1.6) & -0.6 & (0.1) & 0.6 & $(0.1)$ \\
\hline
\end{tabular}

EXZELLENZ DURCH CHANCENGERECHTIGKEIT: ALLEN SCHÜLERINNEN UND SCHÜLERN DIEVORAUSSETZUNGEN ZUM ERFOLG SICHERN - BAND II O OEECD 2014 203 
[Teil 2/4]

Schülerleistungen und ausgewählte Merkmale des sozioökonomischen Status im Ländervergleich Tabelle II.2.5 Die Ergebnisse basieren auf Schülerangaben

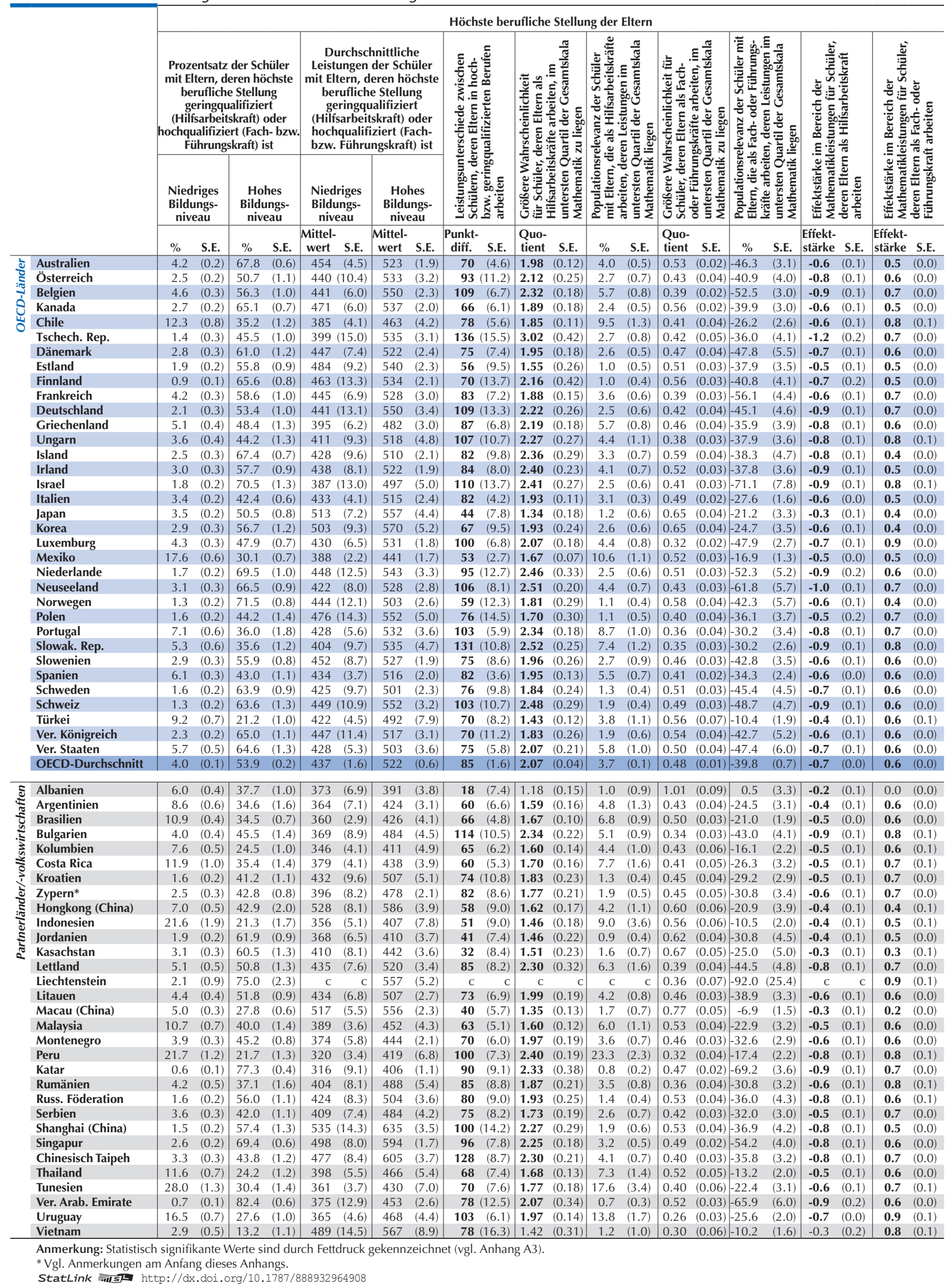


[Teil 3/4]

Schülerleistungen und ausgewählte Merkmale des sozioökonomischen Status im Ländervergleich Tabelle II.2.5 Die Ergebnisse basieren auf Schülerangaben

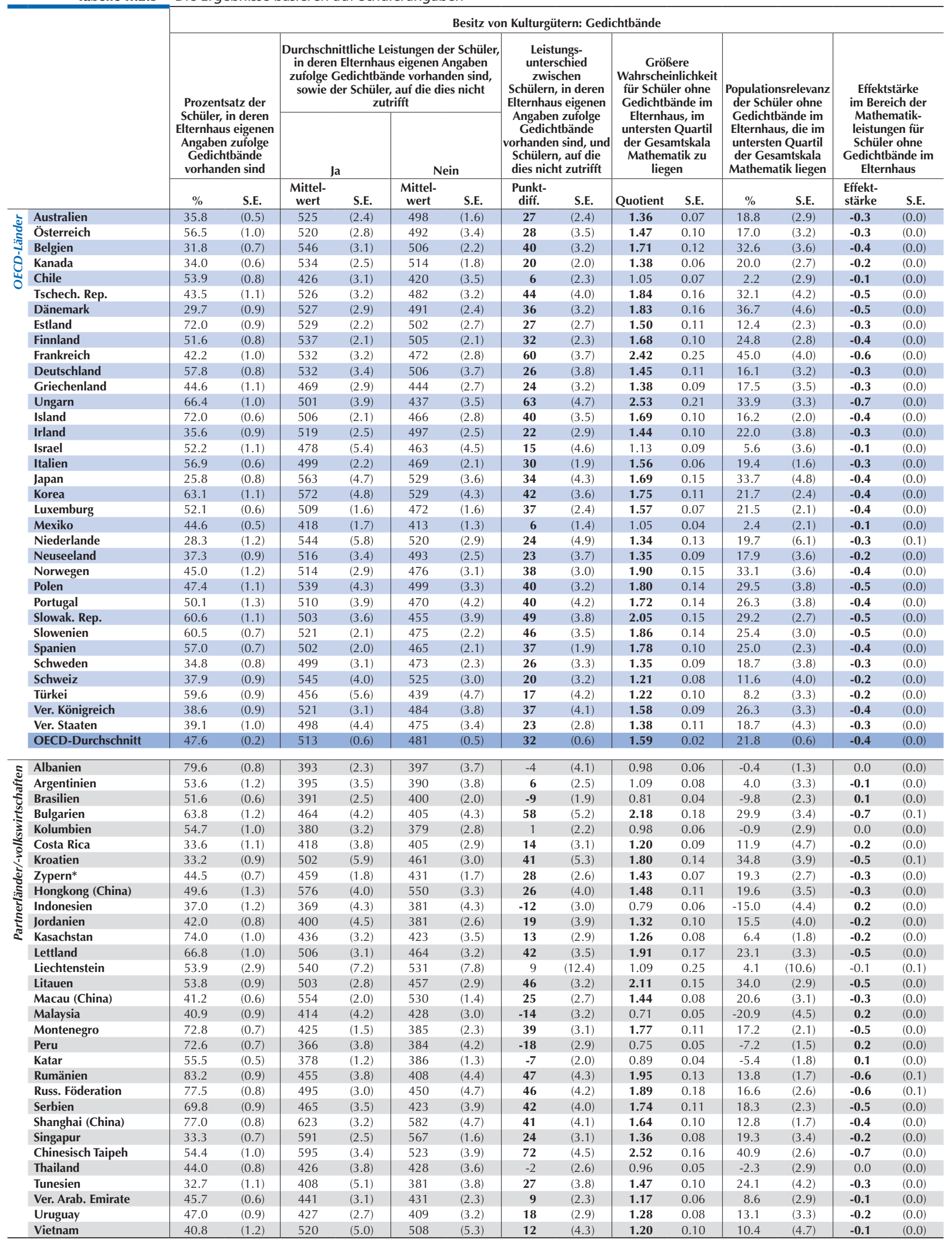

Anmerkung: Statistisch signifikante Werte sind durch Fettdruck gekennzeichnet (vgl. Anhang A3).

* Vgl. Anmerkungen am Anfang dieses Anhangs.

StatLink त्तilst http://dx.doi.org/10.1787/888932964908 
[Teil 4/4]

Schülerleistungen und ausgewählte Merkmale des sozioökonomischen Status im Ländervergleich Tabelle II.2.5 Die Ergebnisse basieren auf Schülerangaben

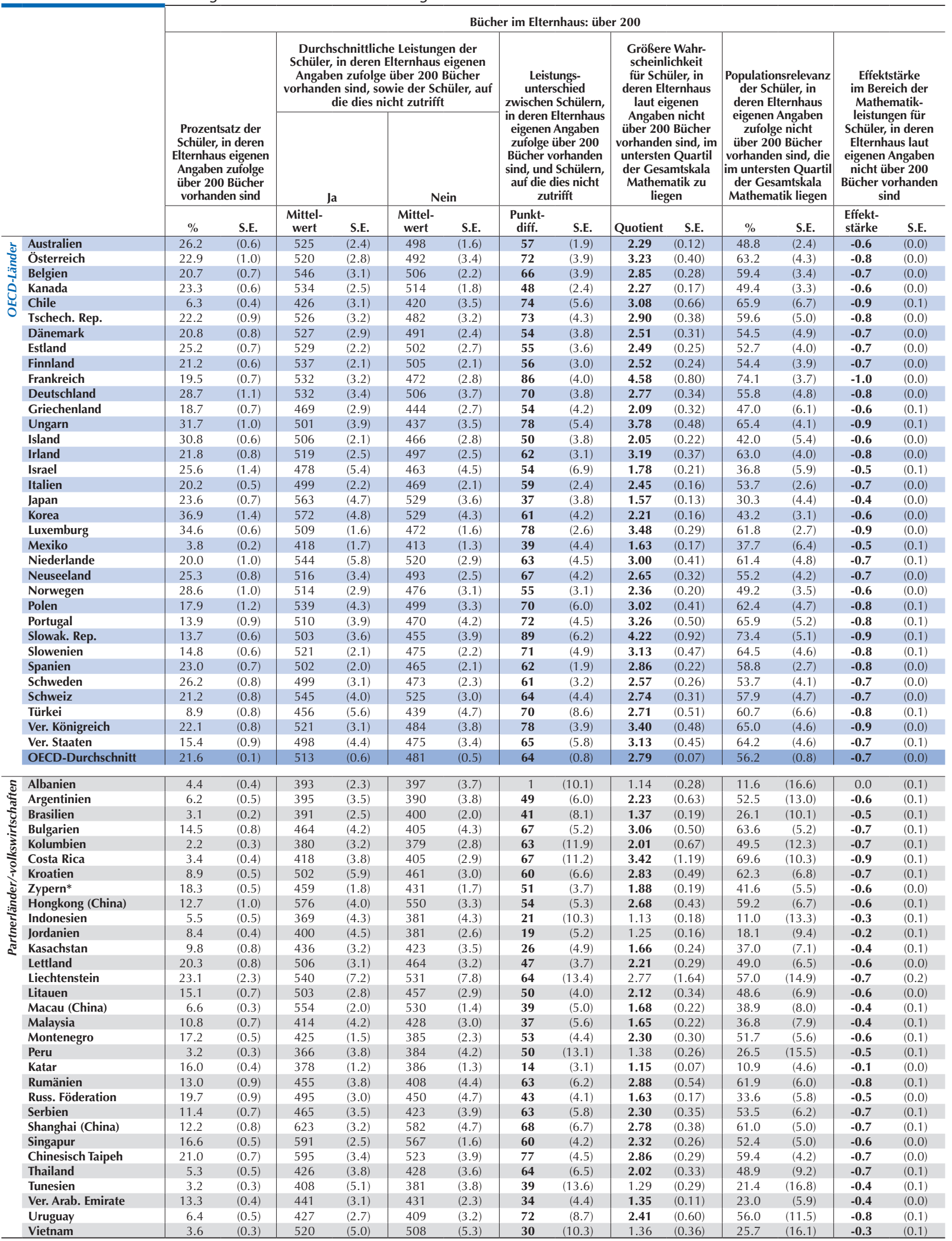

Anmerkung: Statistisch signifikante Werte sind durch Fettdruck gekennzeichnet (vgl. Anhang A3).

* Vgl. Anmerkungen am Anfang dieses Anhangs.

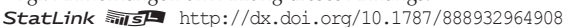


[Teil 1/1]

Zusammenhang zwischen Mathematikleistungen und Merkmalen des sozioökonomischen Status Tabelle II.2.6 Die Ergebnisse basieren auf Schülerangaben

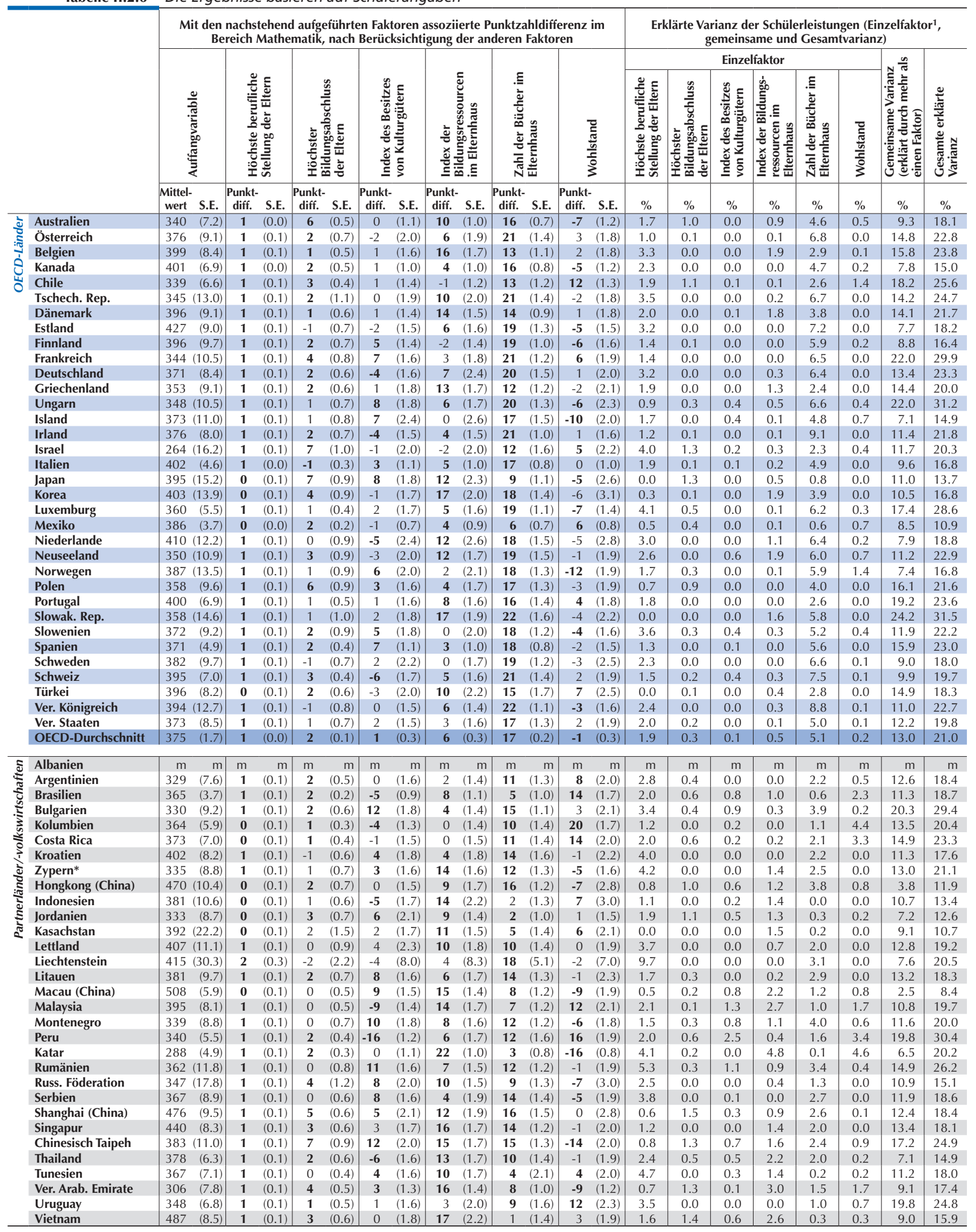

Anmerkung: Statistisch signifikante Werte sind durch Fettdruck gekennzeichnet (vgl. Anhang A3).

1. Die auf einen Einzelfaktor zurückgehende Varianz ist die Varianz, die dem jeweiligen Faktor nach Abzug der durch die anderen Faktoren des Modells erklärten Varianz zuzuschreiben ist.

$*$ Vgl. Anmerkungen am Anfang dieses Anhangs.

StatLink त्ताls http://dx.doi.org/10.1787/888932964908 
Teil 1/1]

Prozentsatz der resilienten Schüler und der Leistungsschwachen unter den sozioökonomisch benachteiligten Schülern, nach Geschlecht

Tabelle II.2.7a Die Ergebnisse basieren auf Schülerangaben

Resiliente und sozioökonomisch benachteiligte Leistungsschwache

\begin{tabular}{|c|c|c|c|c|c|c|c|c|c|c|c|c|c|c|c|c|c|}
\hline & \multicolumn{8}{|c|}{ Resiliente Schüler ${ }^{1}$} & \multicolumn{8}{|c|}{ Sozioökonomisch benachteiligte leistungsschwache Schüler ${ }^{2}$} \\
\hline & & \multicolumn{2}{|c|}{ Alle Schüler } & \multicolumn{2}{|c|}{ Jungen } & \multicolumn{2}{|c|}{ Mädchen } & $\begin{array}{l}\text { Unte } \\
\text { (Jur } \\
\text { Mä }\end{array}$ & $\begin{array}{l}\text { chied } \\
\text { en - } \\
\text { hen) }\end{array}$ & Alle & chüler & & & Mäc & chen & $\begin{array}{l}\text { Unte } \\
\text { (Jur } \\
\text { Mä }\end{array}$ & $\begin{array}{l}\text { chied } \\
\text { en - } \\
\text { hen) }\end{array}$ \\
\hline & & $\%$ & S.E. & $\%$ & S.E. & $\%$ & S.E. & $\begin{array}{l}\text { Diff. } \\
\text { in } \%\end{array}$ & S.E. & $\%$ & S.E. & $\%$ & S.E. & $\%$ & S.E. & $\begin{array}{l}\text { Diff. } \\
\text { in } \%\end{array}$ & S.E. \\
\hline & Australien & 6.3 & $(0.3)$ & 7.1 & $(0.5)$ & 5.4 & $(0.4)$ & 1.6 & $(0.5)$ & 4.9 & $(0.2)$ & 4.4 & $(0.4)$ & 5.3 & $(0.3)$ & -0.9 & $(0.5)$ \\
\hline & Österreich & 6.1 & $(0.5)$ & 6.9 & $(0.8)$ & 5.3 & $(0.6)$ & 1.6 & $(0.9)$ & 4.8 & $(0.5)$ & 3.9 & $(0.8)$ & 5.8 & $(0.8)$ & -1.8 & (1.2) \\
\hline & Belgien & 7.1 & $(0.4)$ & 7.3 & $(0.5)$ & 6.9 & $(0.5)$ & 0.4 & $(0.6)$ & 5.0 & $(0.4)$ & 4.6 & $(0.5)$ & 5.3 & $(0.6)$ & -0.7 & $(0.7)$ \\
\hline & Kanada & 8.3 & $(0.4)$ & 9.2 & $(0.5)$ & 7.5 & $(0.5)$ & 1.7 & $(0.6)$ & 3.3 & $(0.2)$ & 3.0 & $(0.3)$ & 3.5 & $(0.4)$ & -0.5 & $(0.5)$ \\
\hline 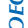 & Chile & 1.7 & $(0.3)$ & 2.2 & $(0.4)$ & 1.1 & $(0.3)$ & 1.1 & $(0.5)$ & 8.1 & $(0.8)$ & 6.3 & $(1.0)$ & 9.8 & $(1.0)$ & -3.5 & $(1.1)$ \\
\hline & Tschech. Rep. & 5.9 & $(0.5)$ & 6.6 & $(0.7)$ & 5.1 & $(0.5)$ & 1.5 & $(0.8)$ & 5.2 & $(0.5)$ & 4.7 & $(0.7)$ & 5.9 & $(0.8)$ & -1.2 & (1.1) \\
\hline & Dänemark & 4.9 & $(0.4)$ & 5.6 & $(0.5)$ & 4.2 & $(0.5)$ & 1.4 & $(0.7)$ & 4.7 & (0.5) & 4.1 & $(0.6)$ & 5.2 & $(0.8)$ & -1.1 & $(0.9)$ \\
\hline & Estland & 9.5 & $(0.6)$ & 9.3 & $(0.9)$ & 9.7 & $(0.8)$ & -0.4 & $(1.3)$ & 1.8 & $(0.3)$ & 1.5 & $(0.3)$ & 2.1 & $(0.3)$ & -0.6 & $(0.5)$ \\
\hline & Finnland & 8.1 & $(0.5)$ & 8.6 & $(0.6)$ & 7.6 & $(0.7)$ & 1.0 & $(0.9)$ & 3.2 & $(0.3)$ & 3.8 & $(0.5)$ & 2.6 & $(0.4)$ & 1.2 & $(0.6)$ \\
\hline & Frankreich & 5.4 & $(0.4)$ & 6.1 & $(0.6)$ & 4.8 & $(0.6)$ & 1.3 & $(0.8)$ & 5.8 & $(0.5)$ & 5.3 & $(0.6)$ & 6.3 & $(0.7)$ & -1.1 & $(0.9)$ \\
\hline & Deutschland & 7.5 & $(0.5)$ & 8.7 & $(0.8)$ & 6.2 & $(0.7)$ & 2.5 & (1.1) & 4.3 & $(0.5)$ & 4.1 & $(0.6)$ & 4.5 & $(0.6)$ & -0.4 & $(0.7)$ \\
\hline & Griechenland & 3.2 & $(0.4)$ & 3.5 & $(0.5)$ & 2.9 & $(0.6)$ & 0.6 & $(0.7)$ & 7.3 & $(0.6)$ & 7.3 & $(0.8)$ & 7.4 & $(0.8)$ & -0.1 & $(1.1)$ \\
\hline & Ungarn & 4.1 & $(0.4)$ & 4.4 & (0.6) & 3.8 & $(0.5)$ & 0.7 & $(0.7)$ & 5.9 & $(0.5)$ & 5.2 & $(0.8)$ & 6.5 & $(0.8)$ & -1.3 & (1.1) \\
\hline & Island & 5.2 & $(0.4)$ & 5.3 & $(0.6)$ & 5.2 & $(0.6)$ & 0.1 & $(0.9)$ & 6.5 & $(0.5)$ & 6.6 & $(0.7)$ & 6.4 & $(0.6)$ & 0.1 & $(1.0)$ \\
\hline & Irland & 6.3 & $(0.4)$ & 7.3 & $(0.6)$ & 5.3 & $(0.5)$ & 2.0 & $(0.8)$ & 4.1 & $(0.5)$ & 3.8 & $(0.7)$ & 4.5 & $(0.5)$ & -0.7 & $(0.7)$ \\
\hline & Israel & 3.1 & $(0.3)$ & 3.8 & $(0.5)$ & 2.5 & $(0.4)$ & 1.4 & $(0.6)$ & 9.9 & $(0.8)$ & 8.8 & $(1.0)$ & 11.0 & $(0.9)$ & -2.2 & (1.2) \\
\hline & Italien & 6.4 & $(0.3)$ & 6.9 & $(0.3)$ & 5.9 & $(0.4)$ & 1.0 & $(0.4)$ & 4.7 & $(0.3)$ & 4.3 & $(0.3)$ & 5.3 & $(0.4)$ & -1.0 & $(0.5)$ \\
\hline & Japan & 11.3 & $(0.5)$ & 12.6 & $(0.8)$ & 9.9 & $(0.7)$ & 2.7 & $(1.0)$ & 2.4 & $(0.5)$ & 2.6 & $(0.6)$ & 2.3 & $(0.5)$ & 0.2 & $(0.5)$ \\
\hline & Korea & 12.7 & $(0.9)$ & 12.4 & $(1.2)$ & 13.1 & $(1.0)$ & -0.7 & (1.3) & 1.9 & $(0.3)$ & 2.2 & $(0.5)$ & 1.6 & $(0.3)$ & 0.6 & $(0.6)$ \\
\hline & Luxemburg & 6.1 & $(0.4)$ & 7.4 & $(0.6)$ & 4.8 & $(0.5)$ & 2.7 & $(0.7)$ & 5.1 & $(0.4)$ & 3.7 & $(0.5)$ & 6.5 & $(0.6)$ & -2.8 & $(0.8)$ \\
\hline & Mexiko & 3.9 & $(0.2)$ & 4.3 & $(0.3)$ & 3.5 & $(0.3)$ & 0.8 & $(0.3)$ & 4.3 & $(0.2)$ & 3.7 & $(0.3)$ & 5.0 & $(0.3)$ & -1.3 & $(0.3)$ \\
\hline & Niederlande & 8.6 & $(0.8)$ & 8.6 & $(0.9)$ & 8.7 & $(1.1)$ & -0.1 & $(1.2)$ & 3.7 & $(0.5)$ & 3.4 & $(0.6)$ & 4.0 & $(0.6)$ & -0.6 & $(0.7)$ \\
\hline & Neuseeland & 5.3 & $(0.4)$ & 5.9 & $(0.6)$ & 4.6 & (0.6) & 1.3 & $(0.8)$ & 5.8 & $(0.5)$ & 5.7 & $(0.7)$ & 5.9 & $(0.7)$ & -0.2 & (1.0) \\
\hline & Norwegen & 5.3 & $(0.5)$ & 5.6 & $(0.5)$ & 5.0 & $(0.6)$ & 0.6 & $(0.6)$ & 6.1 & $(0.6)$ & 6.1 & $(0.7)$ & 6.1 & $(0.7)$ & 0.1 & $(0.8)$ \\
\hline & Polen & 8.4 & $(0.6)$ & 7.5 & $(0.8)$ & 9.2 & $(0.7)$ & -1.7 & $(0.9)$ & 2.7 & $(0.3)$ & 2.8 & $(0.5)$ & 2.7 & $(0.4)$ & 0.2 & $(0.7)$ \\
\hline & Portugal & 7.7 & $(0.6)$ & 8.3 & $(0.9)$ & 7.0 & $(0.6)$ & 1.3 & $(0.9)$ & 3.5 & $(0.4)$ & 3.8 & $(0.6)$ & 3.3 & $(0.5)$ & 0.5 & $(0.7)$ \\
\hline & Slowak. Rep. & 3.9 & $(0.4)$ & 4.1 & $(0.5)$ & 3.8 & $(0.6)$ & 0.3 & $(0.8)$ & 8.1 & $(0.8)$ & 7.0 & $(0.8)$ & 9.3 & $(1.1)$ & -2.3 & $(0.9)$ \\
\hline & Slowenien & 5.9 & $(0.4)$ & 5.9 & $(0.7)$ & 5.8 & $(0.6)$ & 0.1 & (1.1) & 4.1 & $(0.5)$ & 3.7 & $(0.7)$ & 4.5 & $(0.5)$ & -0.8 & $(0.7)$ \\
\hline & Spanien & 6.4 & $(0.4)$ & 7.1 & $(0.6)$ & 5.7 & $(0.4)$ & 1.4 & $(0.6)$ & 4.1 & $(0.3)$ & 3.8 & $(0.4)$ & 4.5 & $(0.4)$ & -0.7 & $(0.5)$ \\
\hline & Schweden & 4.3 & $(0.3)$ & 4.5 & $(0.5)$ & 4.1 & $(0.4)$ & 0.4 & $(0.7)$ & 6.4 & $(0.5)$ & 6.8 & $(0.8)$ & 6.0 & $(0.6)$ & 0.9 & $(0.9)$ \\
\hline & Schweiz & 9.9 & $(0.6)$ & 11.7 & $(0.8)$ & 8.2 & $(0.6)$ & 3.5 & $(0.9)$ & 2.9 & $(0.3)$ & 2.5 & $(0.4)$ & 3.3 & $(0.4)$ & -0.8 & $(0.5)$ \\
\hline & Türkei & 7.2 & $(0.5)$ & 7.1 & $(0.6)$ & 7.3 & $(0.7)$ & -0.3 & $(0.8)$ & 2.4 & $(0.3)$ & 2.4 & $(0.5)$ & 2.4 & $(0.4)$ & 0.0 & $(0.6)$ \\
\hline & Ver. Königreich & 5.8 & $(0.4)$ & 6.0 & $(0.5)$ & 5.5 & $(0.6)$ & 0.5 & $(0.8)$ & 5.2 & $(0.6)$ & 4.4 & $(0.7)$ & 5.9 & $(0.8)$ & -1.5 & $(0.9)$ \\
\hline & Ver. Staaten & 5.2 & $(0.5)$ & 5.8 & $(0.6)$ & 4.5 & $(0.6)$ & 1.3 & $(0.7)$ & 5.6 & $(0.6)$ & 5.8 & $(0.8)$ & 5.4 & $(0.7)$ & 0.3 & $(0.8)$ \\
\hline & OECD-Durchschnitt & 6.4 & $(0.1)$ & 6.9 & $(0.1)$ & 5.9 & $(0.1)$ & 1.0 & $(0.1)$ & 4.8 & $(0.1)$ & 4.5 & $(0.1)$ & 5.2 & $(0.1)$ & -0.7 & $(0.1)$ \\
\hline$\Phi$ & Albanien & $\mathrm{m}$ & $\mathrm{m}$ & $\mathrm{m}$ & $\mathrm{m}$ & $\mathrm{m}$ & $\mathrm{m}$ & $\mathrm{m}$ & $\mathrm{m}$ & $\mathrm{m}$ & $\mathrm{m}$ & $\mathrm{m}$ & $\mathrm{m}$ & $\mathrm{m}$ & $\mathrm{m}$ & $\mathrm{m}$ & $\mathrm{m}$ \\
\hline 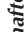 & Argentinien & 1.1 & $(0.3)$ & 1.2 & $(0.3)$ & 0.9 & $(0.3)$ & 0.3 & $(0.4)$ & 10.8 & $(0.8)$ & 8.7 & $(0.9)$ & 12.8 & $(1.0)$ & -4.1 & (1.1) \\
\hline & Brasilien & 1.7 & $(0.2)$ & 2.0 & $(0.3)$ & 1.5 & $(0.2)$ & 0.6 & $(0.4)$ & 7.5 & $(0.4)$ & 5.8 & $(0.5)$ & 9.0 & $(0.5)$ & -3.2 & $(0.6)$ \\
\hline & Bulgarien & 2.1 & $(0.3)$ & 2.2 & $(0.4)$ & 2.0 & $(0.4)$ & 0.1 & $(0.5)$ & 10.0 & $(0.8)$ & 10.4 & $(1.1)$ & 9.6 & $(0.9)$ & 0.8 & (1.0) \\
\hline & Kolumbien & 1.5 & $(0.3)$ & 2.2 & $(0.5)$ & 0.8 & $(0.2)$ & 1.4 & $(0.6)$ & 8.6 & $(0.8)$ & 6.7 & $(0.9)$ & 10.2 & $(1.0)$ & -3.5 & $(1.0)$ \\
\hline 亏 & Costa Rica & 1.9 & $(0.3)$ & 2.4 & $(0.4)$ & 1.4 & $(0.3)$ & 0.9 & $(0.5)$ & 4.8 & $(0.7)$ & 3.2 & $(0.6)$ & 6.3 & $(1.0)$ & -3.1 & $(1.0)$ \\
\hline & Kroatien & 5.1 & $(0.4)$ & 5.4 & $(0.6)$ & 4.7 & $(0.6)$ & 0.7 & $(0.9)$ & 4.7 & $(0.4)$ & 4.3 & $(0.6)$ & 5.1 & $(0.6)$ & -0.8 & $(0.8)$ \\
\hline$=$ & Zypern* & 1.9 & $(0.2)$ & 2.3 & $(0.3)$ & 1.4 & $(0.3)$ & 0.8 & $(0.5)$ & 10.3 & $(0.5)$ & 11.0 & $(0.5)$ & 9.5 & $(0.8)$ & 1.5 & $(0.9)$ \\
\hline : & Hongkong (China) & 18.1 & (1.1) & 17.6 & $(1.2)$ & 18.7 & $(1.2)$ & -1.1 & $(1.2)$ & 0.8 & $(0.2)$ & 0.8 & $(0.3)$ & 0.8 & $(0.2)$ & 0.0 & $(0.3)$ \\
\hline పँ & Indonesien & 2.5 & $(0.5)$ & 3.2 & $(0.8)$ & 1.9 & $(0.4)$ & 1.4 & $(0.9)$ & 5.5 & $(0.6)$ & 5.0 & $(0.7)$ & 6.0 & $(0.8)$ & -1.0 & $(0.8)$ \\
\hline & Jordanien & 0.9 & $(0.2)$ & 0.8 & $(0.4)$ & 0.9 & $(0.3)$ & -0.1 & $(0.5)$ & 11.5 & $(0.6)$ & 11.6 & $(1.0)$ & 11.4 & $(0.9)$ & 0.2 & (1.4) \\
\hline$\approx$ & Kasachstan & 2.1 & $(0.4)$ & 2.4 & $(0.6)$ & 1.7 & $(0.4)$ & 0.7 & $(0.5)$ & 7.7 & $(0.6)$ & 7.8 & $(0.7)$ & 7.5 & $(0.8)$ & 0.3 & $(0.9)$ \\
\hline & Lettland & 6.4 & $(0.5)$ & 6.2 & $(0.7)$ & 6.5 & $(0.7)$ & -0.2 & (1.0) & 2.8 & $(0.6)$ & 2.9 & $(0.8)$ & 2.7 & $(0.6)$ & 0.2 & (0.8) \\
\hline & Liechtenstein & 10.1 & $(1.7)$ & 11.8 & (2.6) & 8.2 & $(1.8)$ & 3.6 & (3.1) & 3.2 & (1.3) & 2.2 & $(1.7)$ & 4.4 & (1.9) & -2.2 & (2.6) \\
\hline & Litauen & 5.6 & $(0.4)$ & 5.8 & $(0.6)$ & 5.5 & $(0.7)$ & 0.3 & (1.0) & 5.0 & $(0.4)$ & 4.8 & $(0.6)$ & 5.1 & $(0.6)$ & -0.3 & $(0.8)$ \\
\hline & Macau (China) & 16.9 & $(0.5)$ & 18.2 & $(0.7)$ & 15.6 & $(0.7)$ & 2.6 & $(1.0)$ & 0.7 & $(0.1)$ & 0.7 & $(0.2)$ & 0.7 & $(0.2)$ & 0.0 & $(0.2)$ \\
\hline & Malaysia & 2.7 & $(0.3)$ & 2.7 & $(0.4)$ & 2.6 & $(0.5)$ & 0.0 & $(0.6)$ & 7.1 & $(0.5)$ & 7.6 & $(0.8)$ & 6.6 & $(0.6)$ & 1.1 & $(1.0)$ \\
\hline & Montenegro & 1.3 & $(0.2)$ & 1.5 & $(0.3)$ & 1.2 & $(0.3)$ & 0.3 & $(0.4)$ & 12.1 & $(0.4)$ & 11.5 & $(0.6)$ & 12.7 & $(0.7)$ & -1.2 & $(1.0)$ \\
\hline & Peru & 0.5 & $(0.1)$ & 0.7 & $(0.2)$ & 0.4 & $(0.2)$ & 0.3 & $(0.3)$ & 12.6 & $(0.9)$ & 10.7 & $(0.9)$ & 14.5 & $(1.3)$ & -3.8 & (1.3) \\
\hline & Katar & 0.4 & $(0.1)$ & 0.5 & $(0.1)$ & 0.4 & $(0.1)$ & 0.2 & $(0.1)$ & 19.2 & $(0.4)$ & 18.7 & $(0.5)$ & 19.7 & $(0.6)$ & -1.0 & $(0.8)$ \\
\hline & Rumänien & 2.8 & $(0.5)$ & 3.1 & $(0.6)$ & 2.5 & $(0.5)$ & 0.6 & $(0.5)$ & 6.5 & $(0.6)$ & 6.4 & $(0.7)$ & 6.5 & $(0.8)$ & -0.1 & $(0.8)$ \\
\hline & Russ. Föderation & 5.2 & $(0.6)$ & 5.6 & $(0.8)$ & 4.8 & $(0.7)$ & 0.8 & $(0.8)$ & 5.2 & $(0.5)$ & 4.7 & $(0.6)$ & 5.7 & $(0.7)$ & -1.0 & $(0.7)$ \\
\hline & Serbien & 3.6 & $(0.4)$ & 4.0 & $(0.5)$ & 3.2 & $(0.6)$ & 0.8 & $(0.7)$ & 7.1 & $(0.7)$ & 6.1 & $(0.8)$ & 8.0 & $(0.9)$ & -1.9 & $(0.9)$ \\
\hline & Shanghai (China) & 19.2 & $(1.0)$ & 20.3 & $(1.0)$ & 18.1 & $(1.2)$ & 2.2 & (1.1) & 0.4 & $(0.1)$ & 0.4 & $(0.2)$ & 0.3 & $(0.1)$ & 0.1 & $(0.2)$ \\
\hline & Singapur & 15.1 & $(0.5)$ & 14.5 & $(0.7)$ & 15.6 & $(0.7)$ & -1.1 & (1.0) & 1.3 & $(0.2)$ & 1.6 & $(0.3)$ & 1.0 & $(0.2)$ & 0.6 & $(0.4)$ \\
\hline & Chinesisch Taipeh & 12.3 & $(0.6)$ & 12.6 & $(0.7)$ & 12.1 & $(0.8)$ & 0.5 & $(0.9)$ & 2.9 & $(0.3)$ & 3.2 & $(0.5)$ & 2.5 & $(0.4)$ & 0.7 & $(0.5)$ \\
\hline & Thailand & 6.3 & $(0.6)$ & 5.4 & $(0.6)$ & 7.0 & $(0.7)$ & -1.6 & $(0.8)$ & 2.9 & $(0.4)$ & 2.9 & $(0.5)$ & 2.9 & $(0.5)$ & 0.0 & $(0.7)$ \\
\hline & Tunesien & 2.9 & $(0.4)$ & 4.1 & $(0.6)$ & 1.9 & $(0.4)$ & 2.2 & $(0.6)$ & 6.2 & $(0.7)$ & 4.6 & $(0.7)$ & 7.6 & $(0.9)$ & -2.9 & $(0.8)$ \\
\hline & Ver. Arab. Emirate & 1.2 & $(0.2)$ & 1.2 & $(0.2)$ & 1.2 & $(0.2)$ & 0.0 & $(0.3)$ & 12.8 & $(0.6)$ & 13.1 & $(0.9)$ & 12.5 & $(1.0)$ & 0.6 & (1.3) \\
\hline & Uruguay & 2.1 & $(0.3)$ & 2.3 & $(0.4)$ & 1.9 & $(0.3)$ & 0.4 & $(0.5)$ & 9.1 & $(0.6)$ & 8.0 & $(0.8)$ & 10.1 & $(0.8)$ & -2.1 & $(1.0)$ \\
\hline & Vietnam & 16.9 & $(1.0)$ & 15.9 & $(1.2)$ & 17.8 & $(1.2)$ & -1.9 & $(1.2)$ & 0.4 & $(0.1)$ & 0.4 & $(0.2)$ & 0.4 & $(0.2)$ & 0.0 & $(0.3)$ \\
\hline
\end{tabular}

Anmerkung: Statistisch signifikante Werte sind durch Fettdruck gekennzeichnet (vgl. Anhang A3).

1. Ein benachteiligter Schüler wird als resilient eingestuft, wenn er im Erhebungsland/in der Erhebungsvolkswirtschaft im untersten Quartil des PISA-Index des wirtschaftlichen, sozialen und kulturellen Status (ESCS) liegt und nach Berücksichtigung des sozioökonomischen Status unter den Schülern aus allen Ländern/Volkswirtschaften im obersten Quartil abschneidet.

2. Ein Schüler wird als benachteiligter leistungsschwacher Schüler eingestuft, wenn er im Erhebungsland/in der Erhebungsvolkswirtschaft im untersten Quartil des PISA-Index des wirtschaftlichen, sozialen und kulturellen Status liegt und nach Berücksichtigung des sozioökonomischen Status unter den Schülern aus allen Ländern/Volkswirtschaften im untersten Quartil abschneidet.

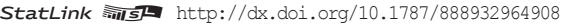


[Teil 1/1]

Veränderung beim Prozentsatz der resilienten Schüler unter den sozioökonomisch benachteiligten Schülern zwischen 2003 und 2012, nach Geschlecht

Tabelle II.2.7b Die Ergebnisse basieren auf Schülerangaben

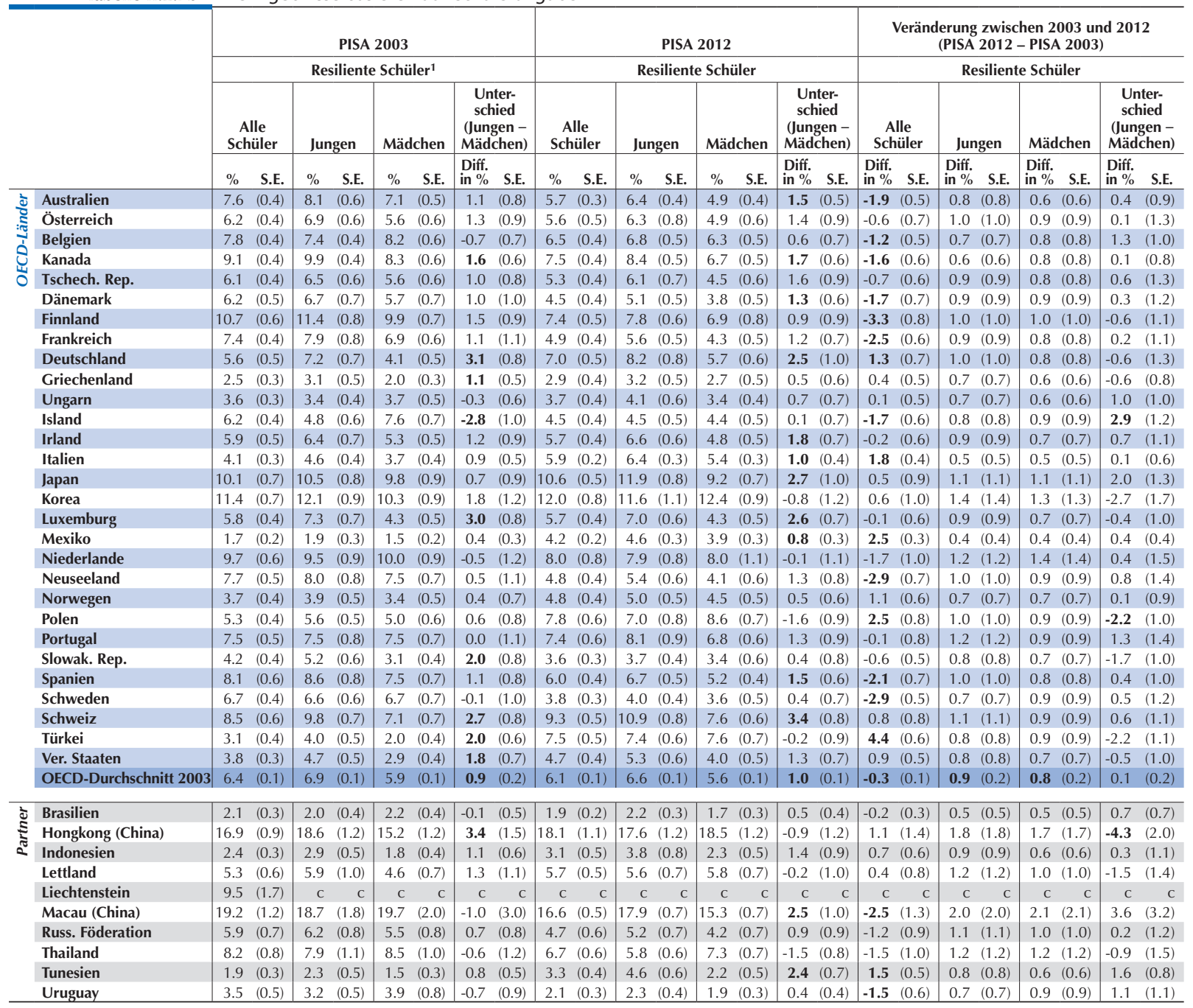

Anmerkung: Statistisch signifikante Werte sind durch Fettdruck gekennzeichnet (vgl. Anhang A3)

Die Resilienzwerte für 2012 weichen u.U. von den in Tabelle II.2.7a dargestellten Werten ab, da sich die in dieser Tabelle verwendete gepoolte Referenzpopulation für resiliente Schüler auf die Länder und Volkswirtschaften beschränkt, für die vergleichbare Daten aus PISA 2003 und PISA 2012 vorliegen.

Angegeben sind nur Länder und Volkswirtschaften mit vergleichbaren Daten aus PISA 2003 und PISA 2012.

1. Ein benachteiligter Schüler wird als resilient eingestuft, wenn er im Erhebungsland/in der Erhebungsvolkswirtschaft im untersten Quartil des PISA-Index des wirtschaftlichen, sozialen und kulturellen Status (ESCS) liegt und nach Berücksichtigung des sozioökonomischen Status unter den Schülern aus allen Ländern/Volkswirtschaften im obersten Quartil abschneidet.

StatLink 完开起 http://dx.doi.org/10.1787/888932964908 
[Teil 1/1]

Varianz der Mathematikleistungen

Tabelle II.2.8a Die Ergebnisse basieren auf Schülerangaben

\begin{tabular}{|c|c|c|c|c|c|c|c|c|c|c|c|c|c|c|}
\hline & \multicolumn{2}{|c|}{ Mittelwerte ${ }^{1}$} & \multicolumn{2}{|c|}{$\begin{array}{l}\text { Gesamtvarianz } \\
\text { der Mathematik- } \\
\text { leistungen }^{2}\end{array}$} & \multicolumn{2}{|c|}{$\begin{array}{c}\text { Varianz der } \\
\text { Mathematik- } \\
\text { leistungen zwischen } \\
\text { den Schulen }\end{array}$} & \multicolumn{2}{|c|}{$\begin{array}{c}\text { Varianz der } \\
\text { Mathematik- } \\
\text { leistungen innerhalb } \\
\text { der Schulen }\end{array}$} & \multicolumn{3}{|c|}{$\begin{array}{c}\text { In Prozent der } \\
\text { durchschnittl. Gesamtvarianz } \\
\text { der Mathematikleistungen in } \\
\text { den OECD-Ländern }\end{array}$} & \multicolumn{2}{|c|}{$\begin{array}{c}\text { Index der schulischer } \\
\text { Inklusion }^{5}\end{array}$} \\
\hline & & $\begin{array}{c}\text { Punkt- } \\
\text { zahl }\end{array}$ & S.E. & Varianz & S.E. & Varianz & S.E. & Varianz & S.E. & $\begin{array}{l}\text { Gesamt- } \\
\text { varianz }\end{array}$ & $\begin{array}{c}\text { Varianz } \\
\text { zwischen } \\
\text { den } \\
\text { Schulen }\end{array}$ & \begin{tabular}{|c} 
Varianz \\
innerhalb \\
der \\
Schulen
\end{tabular} & Index & S.E. \\
\hline \multirow[t]{35}{*}{ छे } & Australien & 504 & (1.6) & 9273 & (228) & 2602 & (228) & 6720 & (106) & 109.4 & 30.7 & 79.3 & 72.1 & (1.8) \\
\hline & Österreich & 506 & (2.7) & 8554 & (315) & 4080 & (373) & 4346 & (113) & 100.9 & 48.1 & 51.3 & 51.6 & (2.4) \\
\hline & Belgien & 515 & $(2.1)$ & 10459 & (283) & 5366 & (423) & 5075 & $(130)$ & 123.3 & 63.3 & 59.8 & 48.6 & (2.3) \\
\hline & Kanada & 518 & (1.8) & 7896 & (143) & 1563 & (134) & 6342 & (116) & 93.1 & 18.4 & 74.7 & 80.2 & $(1.4)$ \\
\hline & Chile & 423 & (3.1) & 6522 & (237) & 2817 & (243) & 3669 & (95) & 76.9 & 33.2 & 43.3 & 56.6 & $(2.2)$ \\
\hline & Tschech. Rep. & 499 & (2.9) & 9016 & (308) & 4544 & (445) & 4285 & (148) & 106.3 & 53.6 & 50.5 & 48.5 & $(2.8)$ \\
\hline & Dänemark & 500 & $(2.3)$ & 6741 & (213) & 1100 & (153) & 5582 & $(171)$ & 79.5 & 13.0 & 65.8 & 83.5 & $(2.0)$ \\
\hline & Estland & 521 & (2.0) & 6546 & (189) & 1129 & (187) & 5412 & (156) & 77.2 & 13.3 & 63.8 & 82.7 & (2.4) \\
\hline & Finnland & 519 & (1.9) & 7276 & (198) & 530 & $(93)$ & 6533 & (153) & 85.8 & 6.3 & 77.0 & 92.5 & $(1.2)$ \\
\hline & Frankreich & 495 & (2.5) & 9500 & (326) & w & w & w & w & 112.0 & w & w & w & w \\
\hline & Deutschland & 514 & (2.9) & 9275 & (317) & 4890 & (389) & 4333 & $(100)$ & 109.4 & 57.7 & 51.1 & 47.0 & $(2.1)$ \\
\hline & Griechenland & 453 & (2.5) & 7709 & (235) & 2441 & (305) & 5173 & (127) & 90.9 & 28.8 & 61.0 & 67.9 & (2.9) \\
\hline & Ungarn & 477 & (3.2) & 8767 & (450) & 5346 & (532) & 3296 & (84) & 103.4 & 63.0 & 38.9 & 38.1 & (2.5) \\
\hline & Island & 493 & (1.7) & 8456 & (241) & 834 & (144) & 7610 & (320) & 99.7 & 9.8 & 89.7 & 90.1 & (1.7) \\
\hline & Irland & 501 & (2.2) & 7155 & (213) & 1297 & (191) & 5815 & (139) & 84.4 & 15.3 & 68.6 & 81.8 & (2.3) \\
\hline & Israel & 466 & (4.7) & 11008 & (382) & 4659 & (499) & 6320 & $(176)$ & 129.8 & 54.9 & 74.5 & 57.6 & $(2.8)$ \\
\hline & Italien & 485 & (2.0) & 8609 & (213) & 4381 & (215) & 4130 & (56) & 101.5 & 51.7 & 48.7 & 48.5 & (1.3) \\
\hline & Japan & 536 & (3.6) & 8748 & (409) & 4620 & (441) & 4094 & (106) & 103.2 & 54.5 & 48.3 & 47.0 & (2.5) \\
\hline & Korea & 554 & (4.6) & 9818 & (426) & 3840 & (482) & 5864 & (180) & 115.8 & 45.3 & 69.2 & 60.4 & $(3.2)$ \\
\hline & Luxemburg & 490 & (1.1) & 9102 & (182) & 4525 & (2193) & 6516 & (348) & 107.3 & 53.4 & 76.8 & 59.0 & $(11.0)$ \\
\hline & Mexiko & 413 & (1.4) & 5516 & (107) & 1940 & (108) & 3578 & (54) & 65.1 & 22.9 & 42.2 & 64.8 & (1.3) \\
\hline & Niederlande & 523 & (3.5) & 8394 & (385) & 5534 & (474) & 2858 & (94) & 99.0 & 65.3 & 33.7 & 34.1 & $(2.2)$ \\
\hline & Neuseeland & 500 & $(2.2)$ & 9923 & (243) & 2387 & (338) & 7658 & (198) & 117.0 & 28.2 & 90.3 & 76.2 & $(2.7)$ \\
\hline & Norwegen & 489 & (2.7) & 8188 & (240) & 1045 & (168) & 7063 & (176) & 96.5 & 12.3 & 83.3 & 87.1 & $(1.8)$ \\
\hline & Polen & 518 & (3.6) & 8168 & (341) & 1659 & (329) & 6433 & (152) & 96.3 & 19.6 & 75.9 & 79.5 & (3.4) \\
\hline & Portugal & 487 & (3.8) & 8828 & (257) & 2653 & (282) & 6212 & (159) & 104.1 & 31.3 & 73.3 & 70.1 & (2.5) \\
\hline & Slowak. Rep. & 482 & (3.4) & 10171 & (496) & 5008 & (536) & 5020 & (163) & 119.9 & 59.1 & 59.2 & 50.1 & (2.9) \\
\hline & Slowenien & 501 & $(1.2)$ & 8404 & (187) & 4904 & (462) & 3453 & (98) & 99.1 & 57.8 & 40.7 & 41.3 & (2.5) \\
\hline & Spanien & 484 & (1.9) & 7698 & (129) & 1454 & (117) & 6263 & (112) & 90.8 & 17.2 & 73.9 & 81.2 & (1.3) \\
\hline & Schweden & 478 & (2.3) & 8420 & (235) & 1042 & (168) & 7266 & $(177)$ & 99.3 & 12.3 & 85.7 & 87.5 & $(1.8)$ \\
\hline & Schweiz & 531 & (3.0) & 8892 & $(274)$ & 3196 & (296) & 5771 & $(151)$ & 104.9 & 37.7 & 68.1 & 64.4 & $(2.3)$ \\
\hline & Türkei & 448 & (4.8) & 8296 & (555) & 5140 & (657) & 3173 & (95) & 97.8 & 60.6 & 37.4 & 38.2 & (3.3) \\
\hline & Ver. Königreich & 494 & (3.3) & 8935 & (330) & 2517 & (289) & 6421 & $(160)$ & 105.4 & 29.7 & 75.7 & 71.8 & (2.5) \\
\hline & Ver. Staaten & 481 & (3.6) & 8077 & (233) & 1916 & (215) & 6164 & $(162)$ & 95.2 & 22.6 & 72.7 & 76.3 & $(2.2)$ \\
\hline & OECD-Durchschnitt & 494 & $(0.5)$ & 8481 & (51) & 3126 & $(87)$ & 5372 & $(27)$ & 100.0 & 36.9 & 63.3 & 64.1 & $(0.5)$ \\
\hline & Albanien & 394 & $(2.0)$ & 8372 & (256) & 380 & (87) & 7958 & (219) & 98.7 & 4.5 & 93.9 & 95.4 & $(1.0)$ \\
\hline$\underset{\mathbb{N}}{\mathbb{E}}$ & Argentinien & 388 & (3.5) & 5891 & (266) & 2597 & (241) & 3253 & (87) & 69.5 & 30.6 & 38.4 & 55.6 & $(2.3)$ \\
\hline క్ & Brasilien & 391 & $(2.1)$ & 6041 & (253) & 2623 & (277) & 3457 & (80) & 71.2 & 30.9 & 40.8 & 56.9 & $(2.7)$ \\
\hline.$\stackrel{5}{\Sigma}$ & Bulgarien & 439 & (4.0) & 8821 & (411) & 4647 & $(470)$ & 4160 & (108) & 104.0 & 54.8 & 49.1 & 47.2 & (2.7) \\
\hline$\sum_{0}^{2}$ & Kolumbien & 376 & (2.9) & 5527 & (254) & 1953 & (246) & 3618 & (111) & 65.2 & 23.0 & 42.7 & 64.9 & (2.9) \\
\hline 吾 & Costa Rica & 407 & (3.0) & 4674 & (247) & 1984 & (302) & 2700 & $(82)$ & 55.1 & 23.4 & 31.8 & 57.6 & (3.8) \\
\hline בิ & Kroatien & 471 & (3.5) & 7829 & (451) & 3466 & (509) & 4360 & (133) & 92.3 & 40.9 & 51.4 & 55.7 & (3.9) \\
\hline छे & Zypern* & 440 & (1.1) & 8675 & (158) & 2791 & (581) & 5814 & (156) & 102.3 & 32.9 & 68.6 & 67.6 & $(4.8)$ \\
\hline : & Hongkong (China) & 561 & (3.2) & 9277 & $(370)$ & 3924 & (348) & 5330 & (159) & 109.4 & 46.3 & 62.9 & 57.6 & $(2.2)$ \\
\hline పัँ & Indonesien & 375 & $(4.0)$ & 5093 & $(463)$ & 2665 & (438) & 2457 & (75) & 60.1 & 31.4 & 29.0 & 48.0 & $(4.1)$ \\
\hline 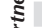 & Jordanien & 386 & (3.1) & 6019 & $(413)$ & 2166 & (378) & 3852 & (98) & 71.0 & 25.5 & 45.4 & 64.0 & $(4.1)$ \\
\hline ๘ & Kasachstan & 432 & (3.0) & 5067 & (250) & 1861 & (241) & 3234 & (87) & 59.7 & 21.9 & 38.1 & 63.5 & (3.1) \\
\hline & Lettland & 491 & $(2.8)$ & 6705 & $(248)$ & 1691 & (298) & 4908 & (163) & 79.1 & 19.9 & 57.9 & 74.4 & (3.6) \\
\hline & Liechtenstein & 535 & $(4.0)$ & 9111 & (710) & 5513 & (1419) & 3305 & (336) & 107.1 & 65.0 & 39.0 & 37.5 & (6.8) \\
\hline & Litauen & 479 & (2.6) & 7942 & (243) & 2424 & (286) & 5463 & (138) & 93.6 & 28.6 & 64.4 & 69.3 & $(2.7)$ \\
\hline & Macau (China) & 538 & $(1.0)$ & 8929 & (205) & 3568 & (749) & 6385 & (240) & 105.3 & 42.1 & 75.3 & 58.2 & $(4.4)$ \\
\hline & Malaysia & 421 & (3.2) & 6581 & (263) & 2129 & (284) & 4449 & (143) & 77.6 & 25.1 & 52.5 & 67.6 & (3.2) \\
\hline & Montenegro & 410 & (1.1) & 6835 & (177) & 2485 & (737) & 4324 & (174) & 80.6 & 29.3 & 51.0 & 63.5 & (7.3) \\
\hline & Peru & 368 & (3.7) & 7118 & (371) & 3244 & (364) & 3865 & (103) & 83.9 & 38.3 & 45.6 & 54.4 & (2.8) \\
\hline & Katar & 376 & $(0.8)$ & 9973 & $(148)$ & 4722 & (661) & 5487 & (307) & 117.6 & 55.7 & 64.7 & 53.8 & (3.7) \\
\hline & Rumänien & 445 & (3.8) & 6618 & (361) & 2986 & (330) & 3591 & (98) & 78.0 & 35.2 & 42.3 & 54.6 & $(2.8)$ \\
\hline & Russ. Föderation & 482 & (3.0) & 7461 & (270) & 2018 & (250) & 5502 & (151) & 88.0 & 23.8 & 64.9 & 73.2 & (2.6) \\
\hline & Serbien & 449 & (3.4) & 8225 & (402) & 3776 & (479) & 4431 & (138) & 97.0 & 44.5 & 52.3 & 54.0 & (3.3) \\
\hline & Shanghai (China) & 613 & (3.3) & 10199 & $(460)$ & 4767 & (473) & 5401 & (205) & 120.3 & 56.2 & 63.7 & 53.1 & $(2.7)$ \\
\hline & Singapur & 573 & (1.3) & 11102 & (194) & 4070 & (503) & 7033 & (213) & 130.9 & 48.0 & 82.9 & 63.3 & $(3.2)$ \\
\hline & Chinesisch Taipeh & 560 & (3.3) & 13368 & $(444)$ & 5613 & (632) & 7710 & (259) & 157.6 & 66.2 & 90.9 & 57.9 & $(3.2)$ \\
\hline & Thailand & 427 & (3.4) & 6759 & (353) & 2866 & (354) & 3941 & (107) & 79.7 & 33.8 & 46.5 & 57.9 & (3.0) \\
\hline & Tunesien & 388 & (3.9) & 6113 & $(480)$ & 3017 & (486) & 3104 & (82) & 72.1 & 35.6 & 36.6 & 50.7 & $(4.1)$ \\
\hline & Ver. Arab. Emirate & 434 & (2.4) & 8014 & (213) & 3559 & (312) & 4453 & (106) & 94.5 & 42.0 & 52.5 & 55.6 & $(2.2)$ \\
\hline & Uruguay & 409 & (2.8) & 7869 & (307) & 3297 & (376) & 4546 & (121) & 92.8 & 38.9 & 53.6 & 58.0 & (3.0) \\
\hline & Vietnam & 511 & (4.8) & 7357 & (455) & 3823 & $(476)$ & 3509 & (110) & 86.7 & 45.1 & 41.4 & 47.9 & $(3.2)$ \\
\hline
\end{tabular}

1. Die für diese Tabelle berechneten Statistiken wurden für alle Schüler geschätzt, unabhängig davon, ob Daten über ihren sozioökonomischen Status vorlagen oder nicht.

2. Die Gesamtvarianz der Schülerleistungen wird aus dem Quadrat der Standardabweichung für alle Schüler berechnet.

3. In einigen Ländern/Volkswirtschaften wurden anstelle von Schulen Untereinheiten von Schulen für die Stichprobe herangezogen, was die Schätzung der Anteile der Varianz zwischen Schulen beeinflussen kann (vgl. Anhang A3).

4. Auf Grund der unausgewogenen Konzentration der Daten entspricht die Summe der Varianzkomponenten zwischen und innerhalb von Schulen nicht unbedingt der Gesamtvarianz, da es sich um Schätzungen auf der Basis einer Stichprobe handelt.

5. Der Index der schulischen Inklusion wird berechnet als 100*(1-rho), wobei rho für die Intra-Class-Korrelation der Leistung steht, d.h. die Varianz der Schülerleistungen zwischen den Schulen, dividiert durch die Summe der Varianz der Schülerleistungen zwischen den Schulen und der Varianz der Schülerleistungen innerhalb der Schulen.

* Vgl. Anmerkungen am Anfang dieses Anhangs.

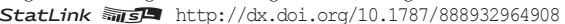


[Teil 1/3]

Veränderung bei der Varianz der Mathematikleistungen zwischen 2003 und 2012 Tabelle II.2.8b Die Ergebnisse basieren auf Schülerangaben

\begin{tabular}{|c|c|c|c|c|c|c|c|c|c|c|c|}
\hline & \multicolumn{10}{|c|}{ PISA 2003} \\
\hline & & \multicolumn{2}{|c|}{ Mittelwerte ${ }^{1}$} & \multicolumn{2}{|c|}{$\begin{array}{c}\text { Gesamtvarianz der } \\
\text { Mathematikleistungen }{ }^{2}\end{array}$} & \multicolumn{2}{|c|}{$\begin{array}{c}\text { Varianz der } \\
\text { Mathematikleistungen } \\
\text { zwischen den Schulen }\end{array}$} & \multicolumn{2}{|c|}{$\begin{array}{l}\text { Varianz der } \\
\text { Mathematikleistungen } \\
\text { innerhalb der Schulen }\end{array}$} & \multicolumn{2}{|c|}{$\begin{array}{l}\text { Index der schulischen } \\
\text { Inklusion }^{5}\end{array}$} \\
\hline & & Punktzahl & S.E. & Varianz & S.E. & Varianz & S.E. & Varianz & S.E. & Index & S.E. \\
\hline \multirow{30}{*}{ 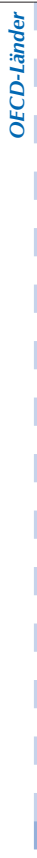 } & Australien & 524 & (2.1) & 9106 & (286) & 1957 & (233) & 7138 & (117) & 78.5 & $(2.1)$ \\
\hline & Österreich & 506 & (3.3) & 8668 & (311) & 4237 & (348) & 4290 & (127) & 50.3 & $(2.2)$ \\
\hline & Belgien & 529 & $(2.3)$ & 12076 & (391) & 6251 & (606) & 5718 & (134) & 47.8 & (2.6) \\
\hline & Kanada & 532 & $(1.8)$ & 7588 & (170) & 1301 & (121) & 6290 & (128) & 82.9 & (1.4) \\
\hline & Tschech. Rep. & 516 & (3.5) & 9207 & (358) & 4460 & (452) & 4690 & (139) & 51.3 & $(2.8)$ \\
\hline & Dänemark & 514 & $(2.7)$ & 8341 & (264) & 1015 & (175) & 7308 & (211) & 87.8 & (1.9) \\
\hline & Finnland & 544 & (1.9) & 7004 & (180) & 318 & $(61)$ & 6664 & (164) & 95.4 & $(0.8)$ \\
\hline & Frankreich & 511 & (2.5) & 8410 & (329) & w & w & w & w & w & $w$ \\
\hline & Deutschland & 503 & (3.3) & 10528 & (363) & 5991 & (582) & 4484 & (140) & 42.8 & (2.6) \\
\hline & Griechenland & 445 & (3.9) & 8806 & (329) & 2824 & (397) & 5872 & (186) & 67.5 & (3.3) \\
\hline & Ungarn & 490 & $(2.8)$ & 8746 & (366) & 4488 & (438) & 4065 & (89) & 47.5 & $(2.5)$ \\
\hline & Island & 515 & (1.4) & 8168 & (218) & 307 & (108) & 8152 & (363) & 96.4 & $(1.2)$ \\
\hline & Irland & 503 & (2.4) & 7270 & (214) & 1081 & (168) & 6124 & (145) & 85.0 & $(2.0)$ \\
\hline & Italien & 466 & (3.1) & 9158 & (359) & 4522 & (389) & 4528 & (90) & 50.0 & $(2.2)$ \\
\hline & Japan & 534 & $(4.0)$ & 10110 & (553) & 5350 & (595) & 4738 & (163) & 47.0 & (2.9) \\
\hline & Korea & 542 & (3.2) & 8536 & (394) & 3523 & $(422)$ & 4972 & (120) & 58.5 & (3.1) \\
\hline & Luxemburg & 493 & $(1.0)$ & 8440 & (175) & 4196 & (2622) & 6143 & (493) & 59.4 & $(16.0)$ \\
\hline & Mexiko & 385 & (3.6) & 7301 & (316) & 3253 & (303) & 4020 & (115) & 55.3 & (2.4) \\
\hline & Niederlande & 538 & (3.1) & 8562 & (432) & 5198 & (502) & 3343 & (112) & 39.1 & (2.6) \\
\hline & Neuseeland & 523 & (2.3) & 9664 & (229) & 1677 & (234) & 7988 & (217) & 82.6 & $(2.1)$ \\
\hline & Norwegen & 495 & (2.4) & 8473 & (212) & 557 & (101) & 7925 & (210) & 93.4 & (1.1) \\
\hline & Polen & 490 & (2.5) & 8145 & (242) & 980 & (148) & 7149 & (171) & 87.9 & (1.6) \\
\hline & Portugal & 466 & (3.4) & 7681 & (292) & 2481 & (308) & 5216 & (151) & 67.8 & (2.9) \\
\hline & Slowak. Rep. & 498 & (3.3) & 8708 & (432) & 3634 & (350) & 4866 & (157) & 57.2 & (2.5) \\
\hline & Spanien & 485 & (2.4) & 7828 & (223) & 1746 & (211) & 6066 & (154) & 77.6 & $(2.2)$ \\
\hline & Schweden & 509 & (2.6) & 8979 & (340) & 831 & (211) & 8133 & (250) & 90.7 & $(2.2)$ \\
\hline & Schweiz & 527 & (3.4) & 9680 & (403) & 3532 & (424) & 6098 & (212) & 63.3 & (3.1) \\
\hline & Türkei & 423 & (6.7) & 10973 & (1116) & 6006 & (1243) & 4890 & (167) & 44.9 & (5.4) \\
\hline & Ver. Staaten & 483 & $(2.9)$ & 9074 & (245) & 2198 & $(261)$ & 6807 & (154) & 75.6 & (2.3) \\
\hline & OECD-Durchschnitt 2003 & 500 & $(0.6)$ & 8801 & (70) & 3027 & (118) & 5800 & (35) & 66.8 & $(0.7)$ \\
\hline \multirow{10}{*}{ 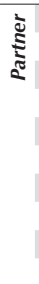 } & Brasilien & 356 & $(4.8)$ & 9946 & (588) & 4754 & (650) & 5261 & (144) & 52.5 & (3.5) \\
\hline & Hongkong (China) & 550 & $(4.5)$ & 10040 & (602) & 4806 & (499) & 5184 & (197) & 51.9 & (2.3) \\
\hline & Indonesien & 360 & (3.9) & 6483 & (332) & 3066 & (397) & 3409 & (94) & 52.7 & (3.4) \\
\hline & Lettland & 483 & $(3.7)$ & 7729 & (292) & 1554 & (210) & 5994 & (180) & 79.4 & (2.3) \\
\hline & Liechtenstein & 536 & $(4.1)$ & 9846 & (879) & 5235 & $(1442)$ & 4644 & (609) & 47.0 & $(6.7)$ \\
\hline & Macau (China) & 527 & (2.9) & 7566 & (420) & 1163 & (289) & 6410 & (468) & 84.6 & (3.8) \\
\hline & Russ. Föderation & 468 & $(4.2)$ & 8513 & (356) & 2534 & (336) & 6017 & (176) & 70.4 & $(2.9)$ \\
\hline & Thailand & 417 & (3.0) & 6718 & (295) & 2325 & (288) & 4480 & (126) & 65.8 & (2.8) \\
\hline & Tunesien & 359 & (2.5) & 6721 & (320) & 2844 & (345) & 3881 & (110) & 57.7 & (3.1) \\
\hline & Uruguay & 422 & (3.3) & 9938 & (320) & 3959 & (356) & 5920 & (155) & 59.9 & (2.3) \\
\hline
\end{tabular}

Anmerkung: Statistisch signifikante Werte sind durch Fettdruck gekennzeichnet (vgl. Anhang A3).

Angegeben sind nur Länder und Volkswirtschaften mit vergleichbaren Daten aus PISA 2003 und PISA 2012.

1. Die für diese Tabelle berechneten Statistiken wurden für alle Schüler geschätzt, unabhängig davon, ob Daten über ihren sozioökonomischen Status vorlagen oder nicht. 2. Die Gesamtvarianz der Schülerleistungen wird aus dem Quadrat der Standardabweichung für alle Schüler berechnet.

3. In einigen Ländern/Volkswirtschaften wurden anstelle von Schulen Untereinheiten von Schulen für die Stichprobe herangezogen, was die Schätzung der Anteile der Varianz zwischen Schulen beeinflussen kann (vgl. Anhang A3).

4. Auf Grund der unausgewogenen Konzentration der Daten entspricht die Summe der Varianzkomponenten zwischen und innerhalb von Schulen nicht unbedingt der Gesamtvarianz, da es sich um Schätzungen auf der Basis einer Stichprobe handelt.

5. Der Index der schulischen Inklusion wird berechnet als 100*(1-rho), wobei rho für die Intra-Class-Korrelation der Leistung steht, d.h. die Varianz der Schülerleistungen zwischen den Schulen, dividiert durch die Summe der Varianz der Schülerleistungen zwischen den Schulen und der Varianz der Schülerleistungen innerhalb der Schulen.

StatLink 完正四 http://dx.doi.org/10.1787/888932964908 
[Teil 2/3]

Veränderung bei der Varianz der Mathematikleistungen zwischen 2003 und 2012

Tabelle II.2.8b Die Ergebnisse basieren auf Schülerangaben

\begin{tabular}{|c|c|c|c|c|c|c|c|c|c|c|c|}
\hline & \multicolumn{10}{|c|}{ PISA 2012} \\
\hline & & \multicolumn{2}{|c|}{ Mittelwerte $^{1}$} & \multicolumn{2}{|c|}{$\begin{array}{c}\text { Gesamtvarianz der } \\
\text { Mathematikleistungen }{ }^{2}\end{array}$} & \multicolumn{2}{|c|}{$\begin{array}{c}\text { Varianz der } \\
\text { Mathematikleistungen } \\
\text { zwischen den Schulen }\end{array}$} & \multicolumn{2}{|c|}{$\begin{array}{c}\text { Varianz der } \\
\text { Mathematikleistungen } \\
\text { innerhalb der Schulen }{ }^{4}\end{array}$} & \multicolumn{2}{|c|}{$\begin{array}{l}\text { Index der schulischen } \\
\text { Inklusion }^{5}\end{array}$} \\
\hline & & Punktzahl & S.E. & Varianz & S.E. & Varianz & S.E. & Varianz & S.E. & Index & S.E. \\
\hline \multirow{30}{*}{ 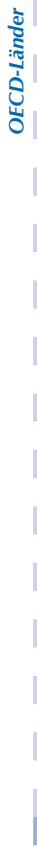 } & Australien & 504 & (1.6) & 9273 & (228) & 2602 & $(228)$ & 6720 & (106) & 72.1 & $\begin{array}{l}(1.8) \\
\end{array}$ \\
\hline & Österreich & 506 & $(2.7)$ & 8554 & (315) & 4080 & $(373)$ & 4346 & (113) & 51.6 & (2.4) \\
\hline & Belgien & 515 & $(2.1)$ & 10459 & (283) & 5366 & $(423)$ & 5075 & (130) & 48.6 & (2.3) \\
\hline & Kanada & 518 & (1.8) & 7896 & (143) & 1563 & (134) & 6342 & (116) & 80.2 & (1.4) \\
\hline & Tschech. Rep. & 499 & $(2.9)$ & 9016 & (308) & 4544 & $(445)$ & 4285 & (148) & 48.5 & (2.8) \\
\hline & Dänemark & 500 & $(2.3)$ & 6741 & (213) & 1100 & (153) & 5582 & (171) & 83.5 & (2.0) \\
\hline & Finnland & 519 & (1.9) & 7276 & (198) & 530 & (93) & 6533 & (153) & 92.5 & $(1.2)$ \\
\hline & Frankreich & 495 & (2.5) & 9500 & (326) & w & w & w & w & w & w \\
\hline & Deutschland & 514 & $(2.9)$ & 9275 & (317) & 4890 & (389) & 4333 & (100) & 47.0 & (2.1) \\
\hline & Griechenland & 453 & (2.5) & 7709 & (235) & 2441 & (305) & 5173 & (127) & 67.9 & (2.9) \\
\hline & Ungarn & 477 & (3.2) & 8767 & (450) & 5346 & (532) & 3296 & (84) & 38.1 & (2.5) \\
\hline & Island & 493 & $(1.7)$ & 8456 & (241) & 834 & (144) & 7610 & (320) & 90.1 & (1.7) \\
\hline & Irland & 501 & $(2.2)$ & 7155 & (213) & 1297 & (191) & 5815 & (139) & 81.8 & (2.3) \\
\hline & Italien & 485 & $(2.0)$ & 8609 & (213) & 4381 & (215) & 4130 & (56) & 48.5 & (1.3) \\
\hline & Japan & 536 & (3.6) & 8748 & (409) & 4620 & $(441)$ & 4094 & (106) & 47.0 & (2.5) \\
\hline & Korea & 554 & (4.6) & 9818 & (426) & 3840 & $(482)$ & 5864 & (180) & 60.4 & (3.2) \\
\hline & Luxemburg & 490 & (1.1) & 9102 & (182) & 4525 & (2193) & 6516 & (348) & 59.0 & (11.0) \\
\hline & Mexiko & 413 & (1.4) & 5516 & (107) & 1940 & (108) & 3578 & (54) & 64.8 & $(1.3)$ \\
\hline & Niederlande & 523 & (3.5) & 8394 & (385) & 5534 & $(474)$ & 2858 & (94) & 34.1 & $(2.2)$ \\
\hline & Neuseeland & 500 & $(2.2)$ & 9923 & (243) & 2387 & (338) & 7658 & (198) & 76.2 & $(2.7)$ \\
\hline & Norwegen & 489 & $(2.7)$ & 8188 & (240) & 1045 & (168) & 7063 & (176) & 87.1 & (1.8) \\
\hline & Polen & 518 & (3.6) & 8168 & (341) & 1659 & (329) & 6433 & (152) & 79.5 & (3.4) \\
\hline & Portugal & 487 & (3.8) & 8828 & (257) & 2653 & $(282)$ & 6212 & (159) & 70.1 & (2.5) \\
\hline & Slowak. Rep. & 482 & (3.4) & 10171 & (496) & 5008 & $(536)$ & 5020 & (163) & 50.1 & (2.9) \\
\hline & Spanien & 484 & (1.9) & 7698 & (129) & 1454 & (117) & 6263 & (112) & 81.2 & (1.3) \\
\hline & Schweden & 478 & $(2.3)$ & 8420 & (235) & 1042 & $(168)$ & 7266 & (177) & 87.5 & (1.8) \\
\hline & Schweiz & 531 & $(3.0)$ & 8892 & (274) & 3196 & (296) & 5771 & (151) & 64.4 & (2.3) \\
\hline & Türkei & 448 & $(4.8)$ & 8296 & (555) & 5140 & $(657)$ & 3173 & (95) & 38.2 & (3.3) \\
\hline & Ver. Staaten & 481 & (3.6) & 8077 & (233) & 1916 & $(215)$ & 6164 & (162) & 76.3 & $(2.2)$ \\
\hline & OECD-Durchschnitt 2003 & 494 & $(0.5)$ & 8481 & (51) & 3117 & (86) & 5372 & (27) & 64.2 & $(0.5)$ \\
\hline \multirow{10}{*}{ ఏ } & Brasilien & 391 & $(2.1)$ & 6041 & (253) & 2623 & $(277)$ & 3457 & (80) & 56.9 & $(2.7)$ \\
\hline & Hongkong (China) & 561 & $(3.2)$ & 9277 & (370) & 3924 & (348) & 5330 & (159) & 57.6 & $(2.2)$ \\
\hline & Indonesien & 375 & $(4.0)$ & 5093 & (463) & 2665 & $(438)$ & 2457 & (75) & 48.0 & $(4.1)$ \\
\hline & Lettland & 491 & $(2.8)$ & 6705 & (248) & 1691 & (298) & 4908 & (163) & 74.4 & (3.6) \\
\hline & Liechtenstein & 535 & $(4.0)$ & 9111 & (710) & 5513 & (1419) & 3305 & (336) & 37.5 & (6.8) \\
\hline & Macau (China) & 538 & $(1.0)$ & 8931 & (177) & 4442 & $(4943)$ & 6181 & (254) & 58.2 & $(27.3)$ \\
\hline & Russ. Föderation & 482 & (3.0) & 7461 & (270) & 2018 & $(250)$ & 5502 & (151) & 73.2 & (2.6) \\
\hline & Thailand & 427 & (3.4) & 6759 & (353) & 2866 & (354) & 3941 & (107) & 57.9 & (3.0) \\
\hline & Tunesien & 388 & (3.9) & 6113 & $(480)$ & 3017 & $(486)$ & 3104 & $(82)$ & 50.7 & $(4.1)$ \\
\hline & Uruguay & 409 & $(2.8)$ & 7869 & $(307)$ & 3297 & $(376)$ & 4546 & (121) & 58.0 & (3.0) \\
\hline
\end{tabular}

Anmerkung: Statistisch signifikante Werte sind durch Fettdruck gekennzeichnet (vgl. Anhang A3).

Angegeben sind nur Länder und Volkswirtschaften mit vergleichbaren Daten aus PISA 2003 und PISA 2012

1. Die für diese Tabelle berechneten Statistiken wurden für alle Schüler geschätzt, unabhängig davon, ob Daten über ihren sozioökonomischen Status vorlagen oder nicht.

2. Die Gesamtvarianz der Schülerleistungen wird aus dem Quadrat der Standardabweichung für alle Schüler berechnet.

3. In einigen Ländern/Volkswirtschaften wurden anstelle von Schulen Untereinheiten von Schulen für die Stichprobe herangezogen, was die Schätzung der Anteile der Varianz zwischen Schulen beeinflussen kann (vgl. Anhang A3).

4. Auf Grund der unausgewogenen Konzentration der Daten entspricht die Summe der Varianzkomponenten zwischen und innerhalb von Schulen nicht unbedingt der Gesamtvarianz da es sich um Schätzungen auf der Basis einer Stichprobe handelt.

5. Der Index der schulischen Inklusion wird berechnet als 100*(1-rho), wobei rho für die Intra-Class-Korrelation der Leistung steht, d.h. die Varianz der Schülerleistungen zwischen den Schulen, dividiert durch die Summe der Varianz der Schülerleistungen zwischen den Schulen und der Varianz der Schülerleistungen innerhalb der Schulen. StatLink 체대 http://dx.doi.org/10.1787/888932964908 
[Teil 3/3]

Veränderung bei der Varianz der Mathematikleistungen zwischen 2003 und 2012 Tabelle II.2.8b Die Ergebnisse basieren auf Schülerangaben

\begin{tabular}{|c|c|c|c|c|c|c|c|c|c|c|c|c|c|c|}
\hline & \multicolumn{10}{|c|}{$\begin{array}{l}\text { Veränderung zwischen } 2003 \text { und } 2012 \\
\text { (PISA } 2012 \text { - PISA 2003) }\end{array}$} & \multicolumn{3}{|c|}{$\begin{array}{c}\text { Veränderung zwischen } 2003 \text { und } 2012 \\
\text { in \% der Varianz von } 2003 \\
\text { (PISA } 2012 \text { - PISA 2003)/PISA } 2003\end{array}$} \\
\hline & & \multicolumn{2}{|c|}{ Mittelwerte $^{1}$} & \multicolumn{2}{|c|}{$\begin{array}{l}\text { Gesamtvarianz } \\
\text { der Mathematik- } \\
\text { leistungen }^{2}\end{array}$} & \multicolumn{2}{|c|}{\begin{tabular}{|c|} 
Varianz der \\
Mathematik- \\
leistungen zwischen \\
den Schulen ${ }^{3}$
\end{tabular}} & \multicolumn{2}{|c|}{\begin{tabular}{|c|} 
Varianz der \\
$\begin{array}{c}\text { Mathematik- } \\
\text { leistungen innerhalb } \\
\text { der Schulen }\end{array}$ \\
\end{tabular}} & \multicolumn{2}{|c|}{$\begin{array}{l}\text { Index der } \\
\text { schulischen } \\
\text { Inklusion }\end{array}$} & \multirow{2}{*}{\begin{tabular}{|c|}
$\begin{array}{c}\text { Gesamt- } \\
\text { varianz }\end{array}$ \\
$\begin{array}{c}\text { Veränderung } \\
\text { in } \%\end{array}$ \\
\end{tabular}} & \multirow{2}{*}{\begin{tabular}{|c|}
$\begin{array}{c}\text { Varianz } \\
\text { zwischen } \\
\text { Schulen }\end{array}$ \\
$\begin{array}{c}\text { Veränderung } \\
\text { in \% }\end{array}$ \\
\end{tabular}} & \multirow{2}{*}{\begin{tabular}{|c|c|}
$\begin{array}{c}\text { Varianz } \\
\text { innerhalb } \\
\text { von Schulen }\end{array}$ \\
$\begin{array}{c}\text { Veränderung } \\
\text { in \% }\end{array}$
\end{tabular}} \\
\hline & & $\begin{array}{c}\text { Punkt- } \\
\text { diff. }\end{array}$ & S.E. & Var. Diff. & S.E. & Var. Diff. & S.E. & Var. Diff. & S.E. & Diff. & S.E. & & & \\
\hline \multirow{30}{*}{ 空 } & Australien & -20 & $(2.7)$ & 167 & (366) & 644 & (326) & -418 & (158) & -6.4 & (2.8) & 1.8 & 32.9 & -5.9 \\
\hline & Österreich & 0 & $(4.2)$ & -114 & $(443)$ & -157 & (510) & 55 & (171) & 1.3 & (3.3) & -1.3 & -3.7 & 1.3 \\
\hline & Belgien & -15 & $(3.1)$ & -1617 & $(483)$ & -885 & (739) & -644 & (187) & 0.8 & (3.4) & -13.4 & -14.2 & -11.3 \\
\hline & Kanada & -14 & (2.6) & 308 & $(222)$ & 262 & (181) & 52 & (172) & -2.6 & (2.0) & 4.1 & 20.1 & 0.8 \\
\hline & Tschech. Rep. & -17 & $(4.6)$ & -191 & $(472)$ & 84 & (634) & -405 & (203) & -2.7 & $(4.0)$ & -2.1 & 1.9 & -8.6 \\
\hline & Dänemark & -14 & (3.6) & -1600 & (339) & 85 & (233) & -1725 & $(272)$ & -4.3 & $(2.8)$ & -19.2 & 8.4 & -23.6 \\
\hline & Finnland & -26 & $(2.7)$ & 272 & (268) & 212 & (111) & -131 & (225) & -3.0 & (1.5) & 3.9 & 66.8 & -2.0 \\
\hline & Frankreich & -16 & (3.5) & 1089 & (463) & w & w & w & w & w & w & 13.0 & w & w \\
\hline & Deutschland & 11 & $(4.4)$ & -1253 & $(482)$ & -1101 & $(700)$ & -151 & $(172)$ & 4.2 & (3.4) & -11.9 & -18.4 & -3.4 \\
\hline & Griechenland & 8 & (4.6) & -1097 & (404) & -383 & (501) & -699 & (226) & 0.4 & (4.4) & -12.5 & -13.6 & -11.9 \\
\hline & Ungarn & -13 & $(4.3)$ & 20 & $(580)$ & 858 & (689) & -769 & (122) & -9.4 & (3.5) & 0.2 & 19.1 & -18.9 \\
\hline & Island & -22 & $(2.2)$ & 288 & (325) & 527 & $(180)$ & -543 & (483) & -6.2 & $(2.1)$ & 3.5 & 171.5 & -6.7 \\
\hline & Irland & -1 & (3.3) & -116 & (302) & 215 & (254) & -309 & (201) & -3.2 & (3.0) & -1.6 & 19.9 & -5.0 \\
\hline & Italien & 20 & (3.7) & -549 & (417) & -140 & (444) & -398 & (106) & -1.5 & (2.6) & -6.0 & -3.1 & -8.8 \\
\hline & Japan & 2 & (5.4) & -1362 & $(688)$ & -730 & (740) & -644 & (194) & 0.0 & (3.8) & -13.5 & -13.6 & -13.6 \\
\hline & Korea & 12 & (5.6) & 1282 & (581) & 317 & (640) & 892 & (216) & 1.9 & (4.5) & 15.0 & 9.0 & 17.9 \\
\hline & Luxemburg & -3 & (1.5) & 662 & (252) & 329 & (3 418) & 373 & (603) & -0.4 & (19.4) & 7.8 & 7.9 & 6.1 \\
\hline & Mexiko & 28 & (3.9) & -1785 & (334) & -1314 & (322) & -442 & (128) & 9.6 & (2.8) & -24.4 & -40.4 & -11.0 \\
\hline & Niederlande & -15 & $(4.7)$ & -167 & (579) & 336 & (690) & -484 & (147) & -5.1 & (3.4) & -2.0 & 6.5 & -14.5 \\
\hline & Neuseeland & -24 & $(3.2)$ & 260 & (334) & 710 & (411) & -330 & (294) & -6.4 & (3.4) & 2.7 & 42.4 & -4.1 \\
\hline & Norwegen & -6 & (3.6) & -285 & (321) & 488 & (196) & -863 & (275) & -6.3 & $(2.2)$ & -3.4 & 87.7 & -10.9 \\
\hline & Polen & 27 & $(4.4)$ & 23 & (418) & 679 & (361) & -716 & (229) & -8.5 & (3.8) & 0.3 & 69.4 & -10.0 \\
\hline & Portugal & 21 & $(5.1)$ & 1147 & (389) & 172 & (417) & 996 & (219) & 2.3 & (3.8) & 14.9 & 6.9 & 19.1 \\
\hline & Slowak. Rep. & -17 & $(4.8)$ & 1463 & (658) & 1373 & (640) & 154 & (227) & -7.2 & (3.8) & 16.8 & 37.8 & 3.2 \\
\hline & Spanien & -1 & (3.1) & -130 & (258) & -292 & (241) & 197 & (190) & 3.5 & (2.5) & -1.7 & -16.7 & 3.2 \\
\hline & Schweden & -31 & (3.4) & -559 & (413) & 211 & (270) & -866 & (307) & -3.3 & $(2.8)$ & -6.2 & 25.4 & -10.7 \\
\hline & Schweiz & 4 & $(4.5)$ & -789 & (487) & -337 & (517) & -327 & (260) & 1.0 & (3.9) & -8.1 & -9.5 & -5.4 \\
\hline & Türkei & 25 & $(8.3)$ & -2677 & (1 246) & -865 & (1 406) & -1717 & (192) & -6.7 & (6.3) & -24.4 & -14.4 & -35.1 \\
\hline & Ver. Staaten & -2 & $(4.7)$ & -997 & (338) & -282 & (338) & -642 & (223) & 0.7 & (3.2) & -11.0 & -12.8 & -9.4 \\
\hline & OECD-Durchschnitt 2003 & -4 & $(0.8)$ & -263 & (88) & 84 & (148) & -359 & (45) & -2.3 & $(0.9)$ & -2.5 & 17.3 & -6.2 \\
\hline \multirow{10}{*}{ ఏ } & Brasilien & 35 & $(5.3)$ & -3905 & (640) & -2131 & (706) & -1805 & (165) & 4.3 & (4.4) & -39.3 & -44.8 & -34.3 \\
\hline & Hongkong (China) & 11 & (5.6) & -762 & (707) & -882 & (608) & 146 & (253) & 5.7 & (3.2) & -7.6 & -18.3 & 2.8 \\
\hline & Indonesien & 15 & (5.6) & -1390 & (570) & -401 & (591) & -952 & (120) & -4.7 & (5.3) & -21.4 & -13.1 & -27.9 \\
\hline & Lettland & 7 & (4.6) & -1024 & (383) & 137 & (364) & -1086 & (243) & -5.0 & (4.3) & -13.2 & 8.8 & -18.1 \\
\hline & Liechtenstein & -1 & $(5.7)$ & -735 & $(1130)$ & 278 & (2023) & -1339 & (695) & -9.5 & (9.5) & -7.5 & 5.3 & -28.8 \\
\hline & Macau (China) & 11 & (3.0) & 1365 & (455) & 3279 & (4952) & -230 & (532) & -26.5 & $(27.5)$ & 18.0 & 282.0 & -3.6 \\
\hline & Russ. Föderation & 14 & $(5.2)$ & -1051 & $(447)$ & -516 & (418) & -515 & (232) & 2.8 & (3.9) & -12.4 & -20.4 & -8.6 \\
\hline & Thailand & 10 & $(4.6)$ & 41 & (460) & 541 & (456) & -539 & (166) & -7.9 & (4.1) & 0.6 & 23.3 & -12.0 \\
\hline & Tunesien & 29 & $(4.7)$ & -608 & (577) & 173 & (596) & -777 & (137) & -7.0 & (5.1) & -9.1 & 6.1 & -20.0 \\
\hline & Uruguay & -13 & $(4.3)$ & -2069 & (443) & -662 & (518) & -1374 & (197) & -2.0 & (3.7) & -20.8 & -16.7 & -23.2 \\
\hline
\end{tabular}

Anmerkung: Statistisch signifikante Werte sind durch Fettdruck gekennzeichnet (vgl. Anhang A3).

Angegeben sind nur Länder und Volkswirtschaften mit vergleichbaren Daten aus PISA 2003 und PISA 2012

1. Die für diese Tabelle berechneten Statistiken wurden für alle Schüler geschätzt, unabhängig davon, ob Daten über ihren sozioökonomischen Status vorlagen oder nicht.

2. Die Gesamtvarianz der Schülerleistungen wird aus dem Quadrat der Standardabweichung für alle Schüler berechnet.

3. In einigen Ländern/Volkswirtschaften wurden anstelle von Schulen Untereinheiten von Schulen für die Stichprobe herangezogen, was die Schätzung der Anteile der Varianz zwischen Schulen beeinflussen kann (vgl. Anhang A3).

4. Auf Grund der unausgewogenen Konzentration der Daten entspricht die Summe der Varianzkomponenten zwischen und innerhalb von Schulen nicht unbedingt der Gesamtvarianz, da es sich um Schätzungen auf der Basis einer Stichprobe handelt.

5. Der Index der schulischen Inklusion wird berechnet als 100*(1-rho), wobei rho für die Intra-Class-Korrelation der Leistung steht, d.h. die Varianz der Schülerleistungen zwischen den Schulen, dividiert durch die Summe der Varianz der Schülerleistungen zwischen den Schulen und der Varianz der Schülerleistungen innerhalb der Schulen.

StatLink त्राI http://dx.doi.org/10.1787/888932964908 
[Teil 1/2]

Zusammenhang zwischen den Mathematikleistungen und dem sozioökonomischen Status, zwischen und innerhalb von Schulen ${ }^{1}$

Tabelle II.2.9a Die Ergebnisse basieren auf Schülerangaben

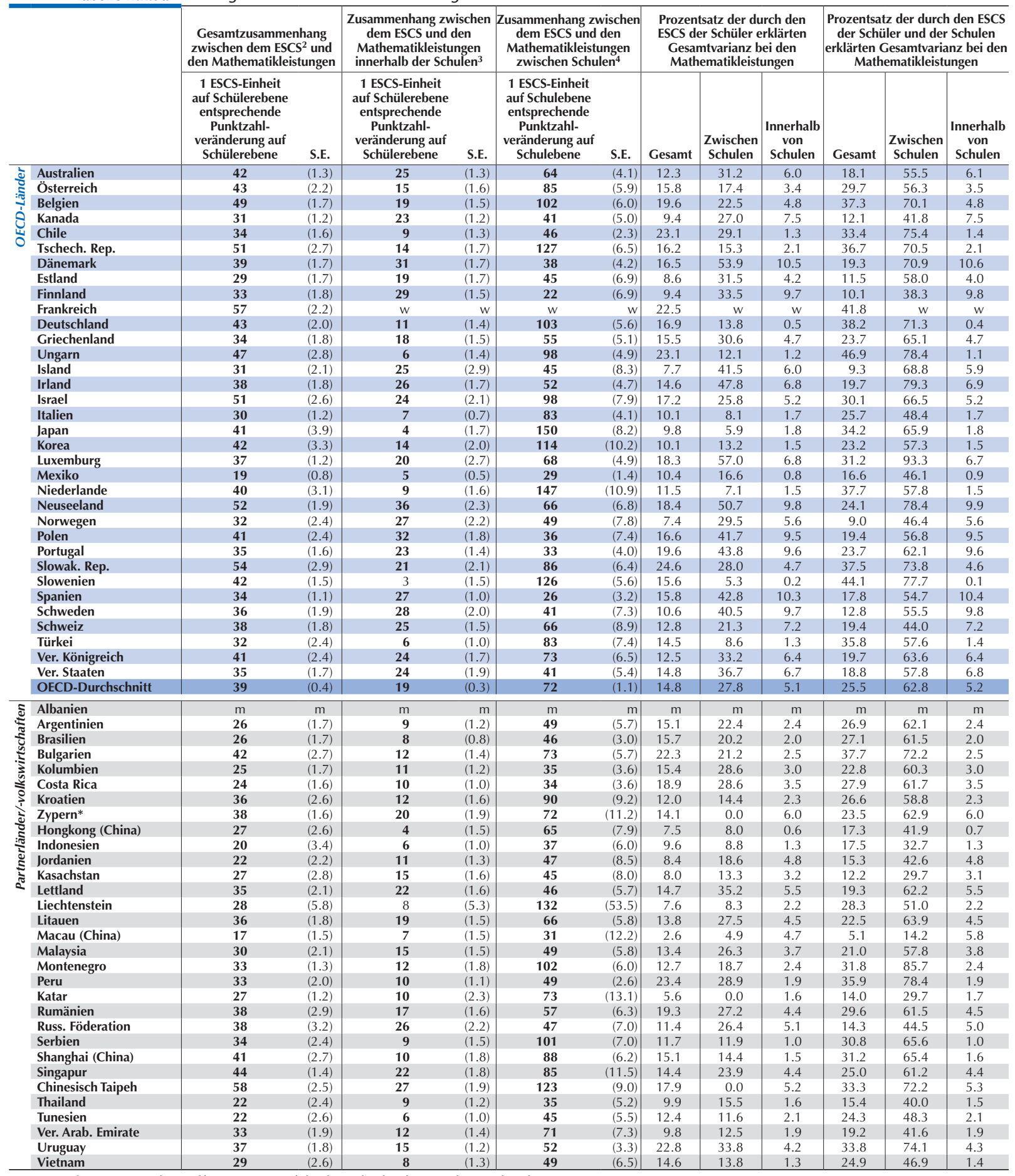

Anmerkung: Statistisch signifikante Werte sind durch Fettdruck gekennzeichnet (vgl. Anhang A3).

1. In einigen Ländern wurden Untereinheiten von Schulen anstelle von Schulen als Verwaltungseinheiten für die Stichprobe herangezogen, was die Schätzungen der Effekte auf Schulebene beeinflussen kann (vgl. Anhang A3).

2. ESCS bezieht sich auf den PISA-Index des wirtschaftlichen, sozialen und kulturellen Status.

3. Zweistufige Regression der Mathematikleistungen auf den ESCS der Schüler und den ESCS-Mittelwert der Schulen: innerschulische Steigung des ESCS und durch das Modell erklärte Varianz auf Schülerebene.

4. Zweistufige Regression der Mathematikleistungen auf den ESCS der Schüler und den ESCS-Mittelwert der Schulen: zwischenschulische Steigung des ESCS und durch das Modell erklärte Varianz auf Schulebene.

5. Zweistufige Regression der Mathematikleistungen auf den ESCS der Schüler, den quadrierten ESCS auf Schülerebene, den ESCS-Mittelwert der Schulen und den quadrierten ESCS-Mittelwert der Schulen. Der innerschulische Index der Kurvilinearität entspricht dem Koeffizienten des quadrierten ESCS auf Schülerebene.

6. Zweistufige Regression der Mathematikleistungen auf den ESCS der Schüler, den quadrierten ESCS auf Schülerebene, den ESCS-Mittelwert der Schulen und den quadrierten ESCS-Mittelwert der Schulen: Der zwischenschulische Index der Kurvilinearität entspricht dem Koeffizienten des quadrierten ESCS-Mittelwerts der Schulen.

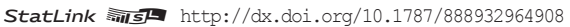


[Teil 2/2]

Zusammenhang zwischen den Mathematikleistungen und dem sozioökonomischen Status, zwischen und innerhalb von Schulen ${ }^{1}$

Tabelle II.2.9a Die Ergebnisse basieren auf Schülerangaben

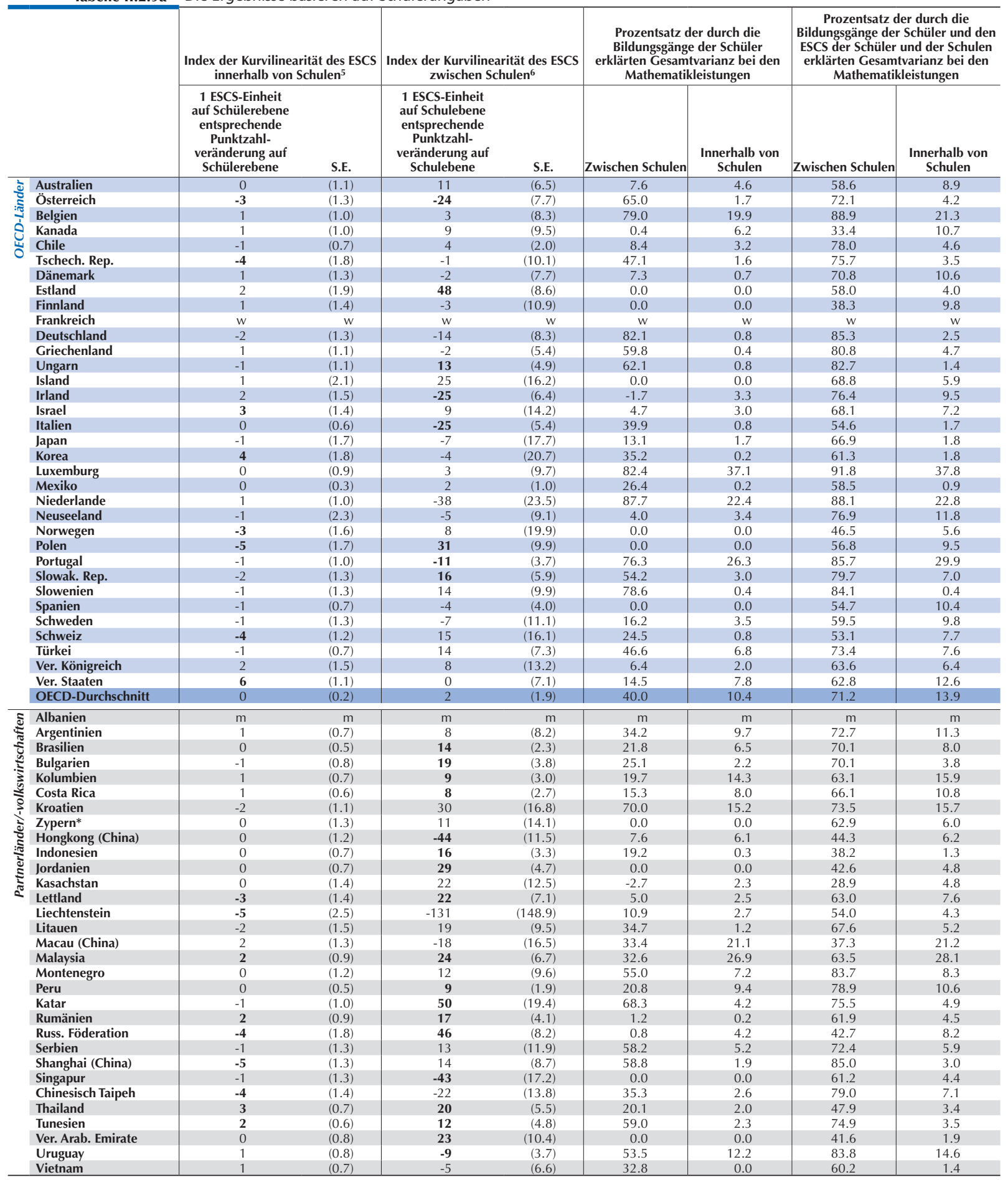

Anmerkung: Statistisch signifikante Werte sind durch Fettdruck gekennzeichnet (vgl. Anhang A3).

1. In einigen Ländern wurden Untereinheiten von Schulen anstelle von Schulen als Verwaltungseinheiten für die Stichprobe herangezogen, was die Schätzungen der Effekte auf Schulebene beeinflussen kann (vgl. Anhang A3).

2. ESCS bezieht sich auf den PISA-Index des wirtschaftlichen, sozialen und kulturellen Status.

3. Zweistufige Regression der Mathematikleistungen auf den ESCS der Schüler und den ESCS-Mittelwert der Schulen: innerschulische Steigung des ESCS und durch das Modell erklärte Varianz auf Schülerebene.

4. Zweistufige Regression der Mathematikleistungen auf den ESCS der Schüler und den ESCS-Mittelwert der Schulen: zwischenschulische Steigung des ESCS und durch das Modell erklärte Varianz auf Schulebene.

5. Zweistufige Regression der Mathematikleistungen auf den ESCS der Schüler, den quadrierten ESCS auf Schülerebene, den ESCS-Mittelwert der Schulen und den quadrierten ESCS-Mittelwert der Schulen. Der innerschulische Index der Kurvilinearität entspricht dem Koeffizienten des quadrierten ESCS auf Schülerebene.

6. Zweistufige Regression der Mathematikleistungen auf den ESCS der Schüler, den quadrierten ESCS auf Schülerebene, den ESCS-Mittelwert der Schulen und den quadrierten ESCS-Mittelwert der Schulen: Der zwischenschulische Index der Kurvilinearität entspricht dem Koeffizienten des quadrierten ESCS-Mittelwerts der Schulen.

StatLink 需占四 http://dx.doi.org/10.1787/888932964908 
Teil 1/3]

Von 2003 bis 2012 festzustellende Veränderung des Zusammenhangs zwischen den Mathematik-

leistungen und dem sozioökonomischen Status, zwischen und innerhalb von Schulen

Tabelle II.2.9b Die Ergebnisse basieren auf Schülerangaben

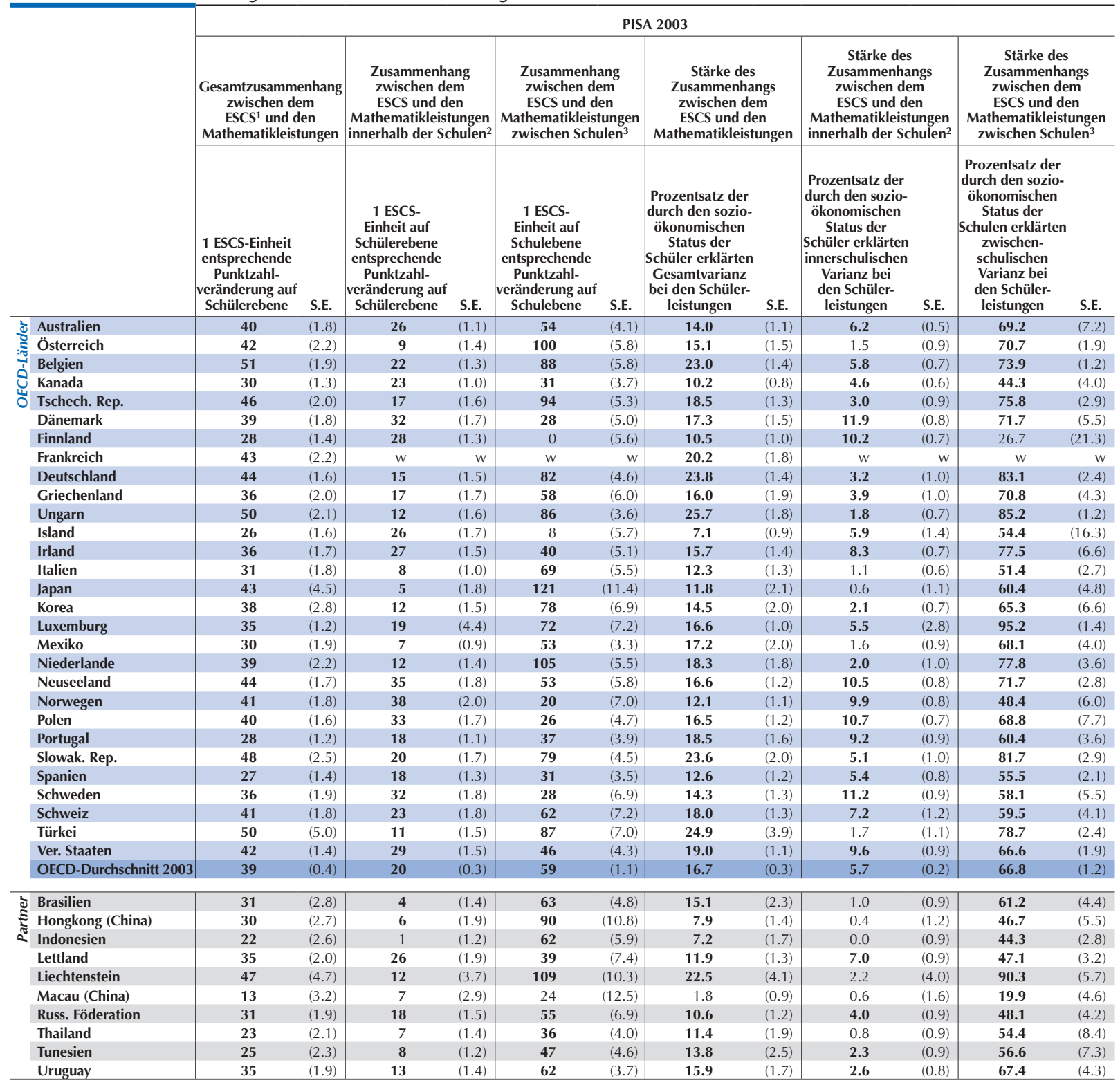

Anmerkung: Statistisch signifikante Werte sind durch Fettdruck gekennzeichnet (vgl. Anhang A3).

Angegeben sind nur Länder und Volkswirtschaften mit vergleichbaren Daten aus PISA 2003 und PISA 2012.

Um die Vergleichbarkeit im Zeitverlauf zu gewährleisten, wurden die Werte von PISA 2003 auf dem PISA-Index des wirtschaftlichen, sozialen und kulturellen Status entsprechend der Indexskala von PISA 2012 umskaliert. Die in dieser Tabelle dargestellten Ergebnisse aus PISA 2003 können daher von denen abweichen, die in Lernen für die Welt von morgen: Erste Ergebnisse von PISA 2003 (OECD, 2004) wiedergegeben sind (vgl. Anhang A5 wegen näherer Einzelheiten).

1. ESCS bezieht sich auf den PISA-Index des wirtschaftlichen, sozialen und kulturellen Status.

2. Zweistufige Regression der Mathematikleistungen auf den ESCS der Schüler und den ESCS-Mittelwert der Schulen: innerschulische Steigung des ESCS und durch das Modell erklärte Varianz auf Schülerebene.

3. Zweistufige Regression der Mathematikleistungen auf den ESCS der Schüler und den ESCS-Mittelwert der Schulen: zwischenschulische Steigung des ESCS und durch das Modell erklärte Varianz auf Schulebene.

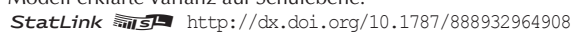


[Teil 2/3]

Von 2003 bis 2012 festzustellende Veränderung des Zusammenhangs zwischen den Mathematikleistungen und dem sozioökonomischen Status, zwischen und innerhalb von Schulen

Tabelle II.2.9b Die Ergebnisse basieren auf Schülerangaben

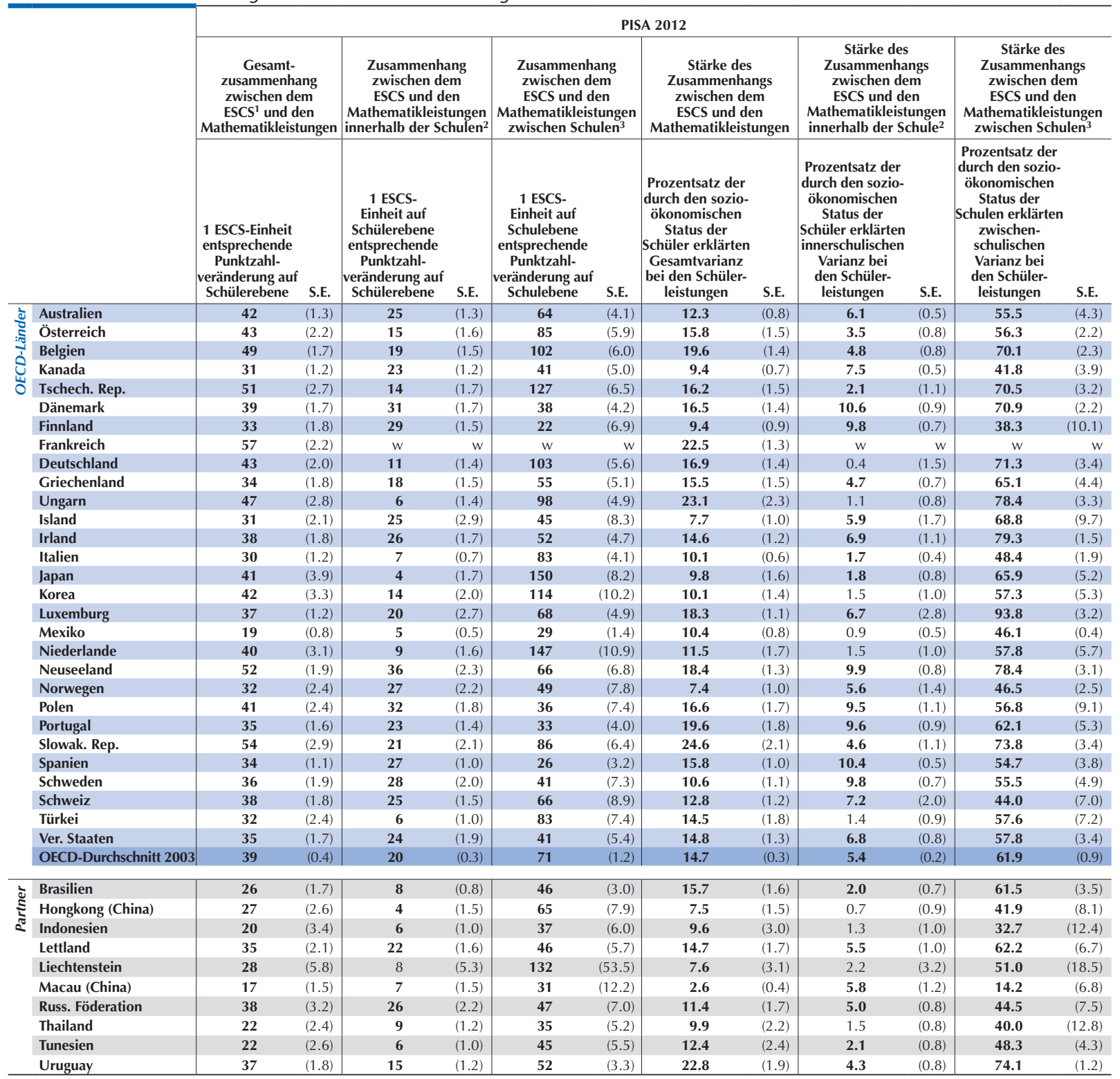

Anmerkung: Statistisch signifikante Werte sind durch Fettdruck gekennzeichnet (vgl. Anhang A3).

Angegeben sind nur Länder und Volkswirtschaften mit vergleichbaren Daten aus PISA 2003 und PISA 2012

Um die Vergleichbarkeit im Zeitverlauf zu gewährleisten, wurden die Werte von PISA 2003 auf dem PISA-Index des wirtschaftlichen, sozialen und kulturellen Status entsprechend der Indexskala von PISA 2012 umskaliert. Die in dieser Tabelle dargestellten Ergebnisse aus PISA 2003 können daher von denen abweichen, die in Lernen für die Welt von morgen: Erste Ergebnisse von PISA 2003 (OECD, 2004) wiedergegeben sind (vgl. Anhang A5 wegen näherer Einzelheiten).

1. ESCS bezieht sich auf den PISA-Index des wirtschaftlichen, sozialen und kulturellen Status.

2. Zweistufige Regression der Mathematikleistungen auf den ESCS der Schüler und den ESCS-Mittelwert der Schulen: innerschulische Steigung des ESCS und durch das Modell erklärte Varianz auf Schülerebene.

3. Zweistufige Regression der Mathematikleistungen auf den ESCS der Schüler und den ESCS-Mittelwert der Schulen: zwischenschulische Steigung des ESCS und durch das Modell erklärte Varianz auf Schulebene.

StatLink 쳎ㄴ http://dx.doi.org/10.1787/888932964908 
Von 2003 bis 2012 festzustellende Veränderung des Zusammenhangs zwischen den Mathematik-

leistungen und dem sozioökonomischen Status, zwischen und innerhalb von Schulen

Die Ergebnisse basieren auf Schülerangaben

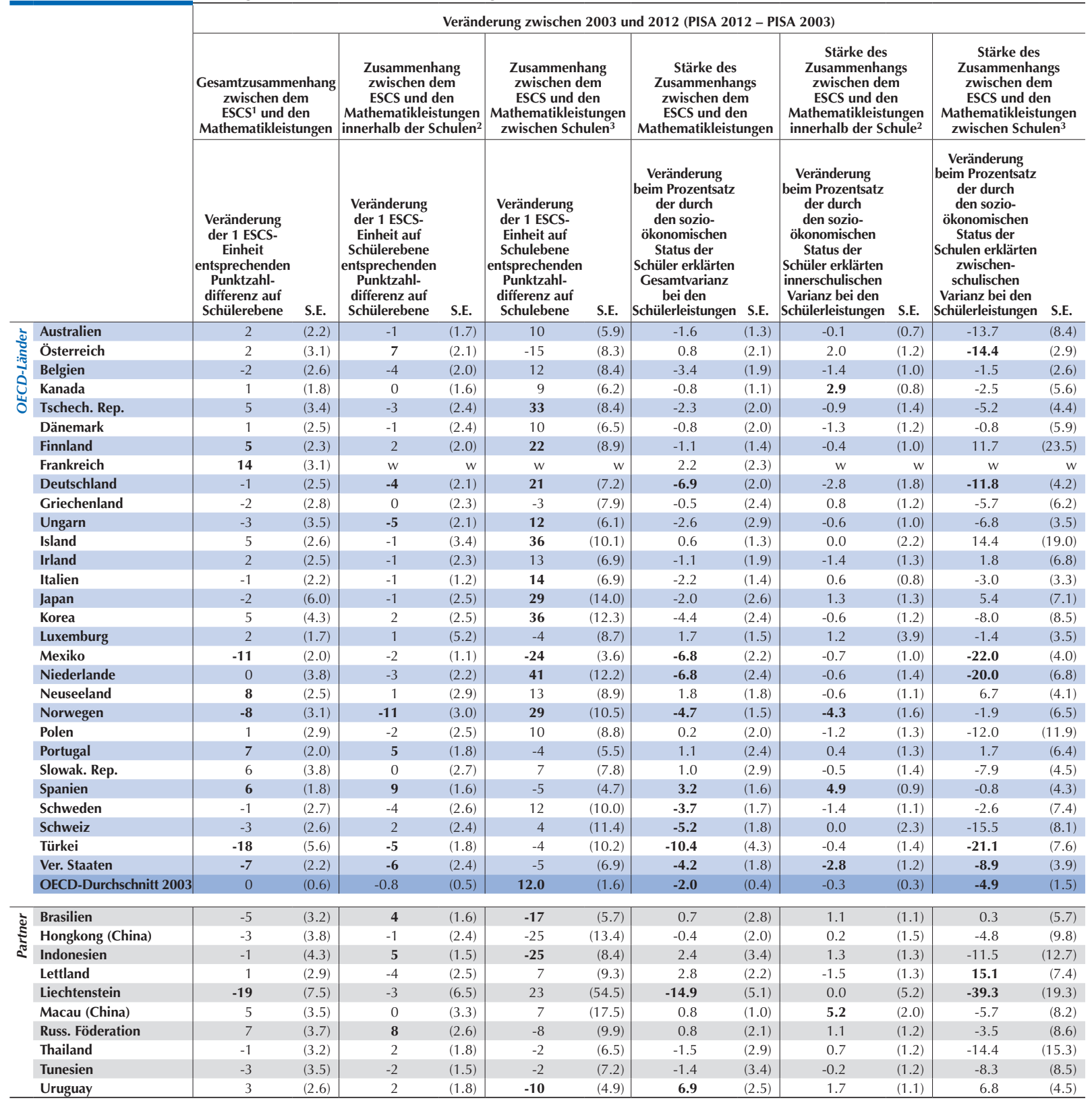

Anmerkung: Statistisch signifikante Werte sind durch Fettdruck gekennzeichnet (vgl. Anhang A3).

Angegeben sind nur Länder und Volkswirtschaften mit vergleichbaren Daten aus PISA 2003 und PISA 2012.

Um die Vergleichbarkeit im Zeitverlauf zu gewährleisten, wurden die Werte von PISA 2003 auf dem PISA-Index des wirtschaftlichen, sozialen und kulturellen Status entsprechend der Indexskala von PISA 2012 umskaliert. Die in dieser Tabelle dargestellten Ergebnisse aus PISA 2003 können daher von denen abweichen, die in Lernen für die Welt von morgen: Erste Ergebnisse von PISA 2003 (OECD, 2004) wiedergegeben sind (vgl. Anhang A5 wegen näherer Einzelheiten).

1. ESCS bezieht sich auf den PISA-Index des wirtschaftlichen, sozialen und kulturellen Status.

2. Zweistufige Regression der Mathematikleistungen auf den ESCS der Schüler und den ESCS-Mittelwert der Schulen: innerschulische Steigung des ESCS und durch das Modell erklärte Varianz auf Schülerebene.

3. Zweistufige Regression der Mathematikleistungen auf den ESCS der Schüler und den ESCS-Mittelwert der Schulen: zwischenschulische Steigung des ESCS und durch das Modell erklärte Varianz auf Schulebene.

StatLink inis http://dx.doi.org/10.1787/888932964908 
[Teil 1/3]

Bildungsabschluss und Beruf der Eltern sowie Ausstattung des Elternhauses der Schüler, nach dem sozioökonomischen Profil der Schulen

Tabelle II.2.10 Die Ergebnisse basieren auf Schülerangaben

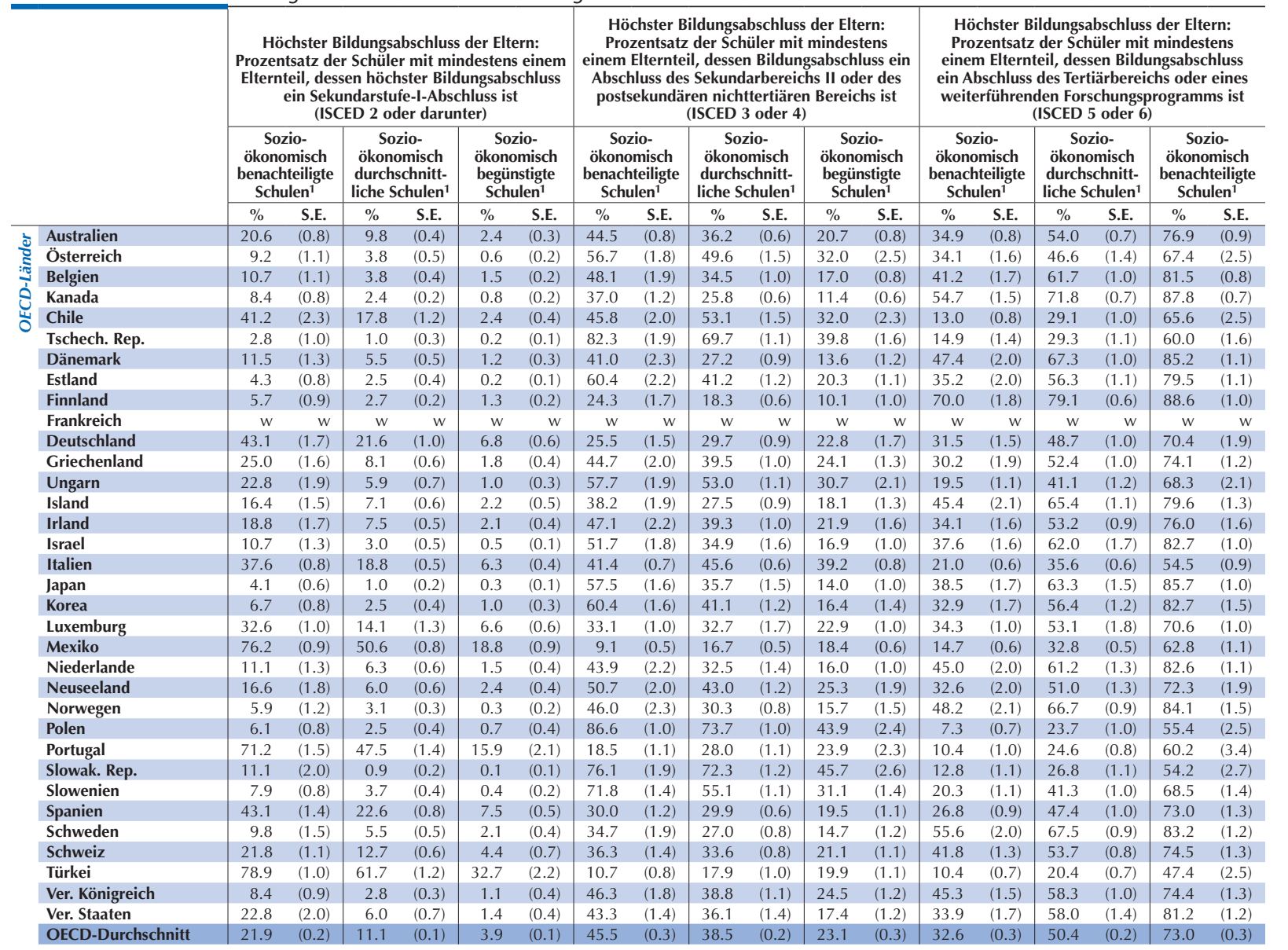

\begin{tabular}{|c|c|c|c|c|c|c|c|c|c|c|c|c|c|c|c|c|c|c|c|}
\hline \multirow{31}{*}{ 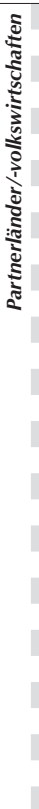 } & Albanien & $\mathrm{m}$ & $\mathrm{m}$ & $\mathrm{m}$ & $\mathrm{m}$ & $\mathrm{m}$ & $\mathrm{m}$ & $\mathrm{m}$ & $\mathrm{m}$ & $\mathrm{m}$ & $\mathrm{m}$ & $\mathrm{m}$ & $\mathrm{m}$ & $\mathrm{m}$ & $\mathrm{m}$ & $\mathrm{m}$ & $\mathrm{m}$ & $\mathrm{m}$ & $\mathrm{m}$ \\
\hline & Argentinien & 54.4 & (1.5) & 29.3 & (1.6) & 10.2 & (0.9) & 21.1 & (1.3) & 25.6 & (1.0) & 17.6 & (1.2) & 24.5 & (1.1) & 45.0 & (1.4) & 72.1 & (1.6) \\
\hline & Brasilien & 60.8 & (0.9) & 35.4 & (0.9) & 8.7 & (0.8) & 28.1 & $(0.9)$ & 43.2 & (0.9) & 36.9 & (2.0) & 11.0 & $(0.5)$ & 21.4 & $(0.7)$ & 54.4 & (2.3) \\
\hline & Bulgarien & 16.7 & (1.8) & 3.8 & $(0.7)$ & 0.4 & (0.1) & 60.3 & (1.4) & 53.2 & (1.2) & 29.4 & (1.3) & 23.0 & (1.2) & 43.1 & (1.1) & 70.2 & (1.4) \\
\hline & Kolumbien & 64.7 & (1.9) & 36.4 & (1.3) & 12.5 & (1.1) & 12.3 & (1.3) & 18.2 & (1.4) & 14.7 & (1.3) & 23.0 & (1.3) & 45.4 & (1.2) & 72.8 & (1.6) \\
\hline & Costa Rica & 50.2 & (2.3) & 26.2 & (1.0) & 4.6 & (1.0) & 18.4 & (1.4) & 28.3 & (1.5) & 19.6 & (1.4) & 31.4 & (2.3) & 45.5 & (1.2) & 75.8 & (1.8) \\
\hline & Kroatien & 10.7 & (0.9) & 2.7 & (0.4) & 0.3 & $(0.2)$ & 55.0 & (1.7) & 51.3 & (1.1) & 32.7 & (1.7) & 34.3 & (1.4) & 46.0 & (1.0) & 66.9 & (1.8) \\
\hline & Zypern* & 10.2 & $(0.8)$ & 3.8 & (0.5) & 0.6 & $(0.2)$ & 57.0 & (1.2) & 43.4 & (1.2) & 23.0 & (1.2) & 32.8 & (1.2) & 52.9 & (1.3) & 76.4 & (1.2) \\
\hline & Hongkong (China) & 47.6 & (1.6) & 28.3 & (1.1) & 6.6 & $(0.9)$ & 45.5 & (1.4) & 54.8 & (1.3) & 42.2 & (3.0) & 6.9 & $(0.8)$ & 16.9 & (1.0) & 51.2 & (3.6) \\
\hline & Indonesien & 69.4 & (1.3) & 48.2 & (1.6) & 16.9 & (1.5) & 23.3 & (1.5) & 36.9 & (1.7) & 39.0 & (2.4) & 7.3 & $(0.8)$ & 14.9 & $(0.9)$ & 44.1 & (3.3) \\
\hline & Jordanien & 25.9 & (1.5) & 10.5 & $(0.7)$ & 2.0 & $(0.4)$ & 38.4 & (1.8) & 32.4 & (1.1) & 17.7 & (1.6) & 35.7 & (1.7) & 57.1 & (1.2) & 80.3 & (1.7) \\
\hline & Kasachstan & 3.0 & $(0.7)$ & 1.2 & $(0.3)$ & 0.5 & $(0.2)$ & 22.4 & (2.1) & 12.2 & (1.0) & 4.3 & $(0.5)$ & 74.6 & (2.1) & 86.6 & $(0.9)$ & 95.2 & $(0.6)$ \\
\hline & Lettland & 7.0 & (1.5) & 2.3 & $(0.4)$ & 0.3 & $(0.1)$ & 66.4 & (2.4) & 45.1 & (1.3) & 22.2 & $(1.3)$ & 26.6 & $(2.2)$ & 52.6 & (1.4) & 77.5 & $(1.3)$ \\
\hline & Liechtenstein & c & c & 10.5 & (2.6) & c & c & c & c & 35.8 & (3.5) & c & c & c & c & 53.7 & (4.0) & c & c \\
\hline & Litauen & 3.2 & $(0.6)$ & 1.2 & $(0.2)$ & 0.5 & $(0.2)$ & 58.1 & (1.6) & 33.4 & (1.0) & 13.4 & (1.0) & 38.7 & (1.6) & 65.4 & (1.1) & 86.1 & (1.1) \\
\hline & Macau (China) & 61.0 & $(0.8)$ & 51.3 & (1.6) & 28.3 & (1.1) & 29.5 & $(0.8)$ & 33.4 & (1.4) & 36.3 & $(1.2)$ & 9.4 & (0.6) & 15.3 & (1.1) & 35.4 & (1.1) \\
\hline & Malaysia & 32.7 & (1.8) & 16.2 & (1.0) & 5.8 & $(0.7)$ & 56.5 & (1.6) & 57.6 & (1.5) & 43.8 & (2.0) & 10.8 & (1.0) & 26.2 & (1.1) & 50.3 & (2.4) \\
\hline & Montenegro & 7.1 & $(0.6)$ & 3.6 & $(0.7)$ & 1.2 & $(0.3)$ & 44.8 & (1.1) & 41.9 & (1.3) & 26.6 & (1.0) & 48.1 & (1.1) & 54.5 & (1.4) & 72.2 & (1.0) \\
\hline & Peru & 52.6 & (1.6) & 21.9 & (1.4) & 4.3 & $(0.5)$ & 33.4 & $(1.3)$ & 47.1 & (1.5) & 37.6 & (2.1) & 14.0 & (0.8) & 31.0 & $(1.2)$ & 58.1 & (2.3) \\
\hline & Katar & 20.4 & $(0.6)$ & 8.3 & (0.6) & 2.3 & $(0.2)$ & 23.9 & $(0.7)$ & 19.7 & $(0.9)$ & 13.9 & $(0.5)$ & 55.7 & $(0.7)$ & 71.9 & (1.0) & 83.9 & $(0.5)$ \\
\hline & Rumänien & 13.2 & (1.8) & 4.0 & $(0.5)$ & 0.6 & $(0.2)$ & 52.8 & (1.9) & 44.6 & (1.5) & 28.6 & (2.0) & 34.0 & (2.3) & 51.4 & (1.5) & 70.8 & (2.1) \\
\hline & Russ. Föderation & 2.7 & $(0.8)$ & 1.2 & $(0.3)$ & 0.2 & $(0.1)$ & 16.8 & (1.6) & 7.5 & $(0.5)$ & 2.3 & $(0.4)$ & 80.5 & (1.7) & 91.3 & $(0.7)$ & 97.5 & $(0.4)$ \\
\hline & Serbien & 9.3 & (1.2) & 3.9 & $(0.5)$ & 0.6 & $(0.3)$ & 50.0 & (1.6) & 42.8 & (1.3) & 23.3 & (1.7) & 40.7 & (1.3) & 53.3 & (1.2) & 76.1 & (1.8) \\
\hline & Shanghai (China) & 49.6 & (2.0) & 22.4 & (1.5) & 5.9 & $(0.7)$ & 30.3 & (1.5) & 35.5 & $(1.2)$ & 25.3 & (1.3) & 20.1 & (1.0) & 42.1 & $(1.2)$ & 68.8 & (1.5) \\
\hline & Singapur & 17.8 & $(0.9)$ & 9.3 & $(0.7)$ & 1.4 & $(0.3)$ & 51.1 & (1.1) & 45.7 & (1.0) & 24.8 & (1.3) & 31.1 & (1.1) & 45.0 & (1.0) & 73.8 & (1.3) \\
\hline & Chinesisch Taipeh & 21.3 & (1.5) & 10.9 & $(0.7)$ & 3.0 & $(0.6)$ & 45.3 & (1.1) & 39.4 & (1.0) & 23.8 & (1.4) & 33.3 & (1.4) & 49.8 & $(1.2)$ & 73.2 & (1.6) \\
\hline & Thailand & 72.4 & $(1.2)$ & 47.8 & (1.3) & 23.1 & (1.3) & 22.5 & (1.1) & 37.8 & $(1.2)$ & 34.4 & (1.5) & 5.2 & $(0.5)$ & 14.4 & (1.0) & 42.4 & $(2.2)$ \\
\hline & Tunesien & 52.7 & (1.8) & 27.8 & (1.0) & 8.2 & $(0.9)$ & 34.7 & (1.9) & 48.2 & (1.3) & 39.9 & (2.0) & 12.6 & (1.0) & 24.0 & (1.1) & 51.8 & (2.3) \\
\hline & Ver. Arab. Emirate & 21.5 & (1.0) & 7.6 & $(0.7)$ & 1.1 & $(0.2)$ & 38.7 & $(0.9)$ & 24.8 & (1.1) & 10.6 & $(0.6)$ & 39.8 & $(1.2)$ & 67.6 & (1.5) & 88.4 & (0.6) \\
\hline & Uruguay & 65.9 & (1.5) & 43.3 & (1.1) & 11.0 & (1.8) & 15.3 & (1.0) & 23.0 & (1.0) & 20.3 & $(1.2)$ & 18.8 & $(0.9)$ & 33.8 & $(0.9)$ & 68.7 & (2.4) \\
\hline & Vietnam & 77.8 & (1.4) & 60.4 & (1.6) & 26.2 & $(2.8)$ & 19.1 & (1.3) & 32.1 & (1.5) & 45.6 & (2.2) & 3.0 & $(0.5)$ & 7.5 & $(0.7)$ & 28.2 & (2.9) \\
\hline
\end{tabular}

1. Eine sozioökonomisch benachteiligte Schule ist eine Schule mit Schülern, deren durchschnittlicher sozioökonomischer Status statistisch signifikant unter dem durchschnittlichen sozioökonomischen Status des Landes/der Volkswirtschaft liegt; eine sozioökonomisch durchschnittliche Schule ist eine Schule, an der es keinen Unterschied zwischen dem durchschnittlichen sozioökonomischen Status der Schüler und des Landes/der Volkswirtschaft gibt; und eine sozioökonomisch begünstigte Schule ist eine Schule mit Schülern, deren durchschnittlicher sozioökonomischer Status statistisch signifikant über dem durchschnittlichen sozioökonomischen Status des Landes/der Volkswirtschaft liegt.

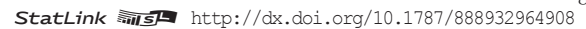


[Teil 2/3]

Bildungsabschluss und Beruf der Eltern sowie Ausstattung des Elternhauses der Schüler, nach dem sozioökonomischen Profil der Schulen

Tabelle II.2.10 Die Ergebnisse basieren auf Schülerangaben

\begin{tabular}{|c|c|c|c|c|c|c|c|c|c|c|c|c|c|c|c|c|c|c|c|}
\hline & \multicolumn{6}{|c|}{$\begin{array}{l}\text { Höchste berufliche Stellung der Eltern: } \\
\text { Prozentsatz der Schüler mit mindestens einem } \\
\text { Elternteil, der als Hilfsarbeitskraft tätig ist } \\
\text { (ISCO 9) }\end{array}$} & \multicolumn{6}{|c|}{$\begin{array}{l}\text { Höchste berufliche Stellung der Eltern: } \\
\text { Prozentsatz der Schüler mit mindestens einem } \\
\text { Elternteil, der als angelernte Fachkraft tätig ist } \\
\text { (ISCO 6,7 und 8) }\end{array}$} & \multicolumn{6}{|c|}{$\begin{array}{c}\text { Höchste berufliche Stellung der Eltern: } \\
\text { Prozentsatz der Schüler mit mindestens einem } \\
\text { Elternteil, der als angelernte Bürokraft oder in } \\
\text { einem Dienstleistungsberuf tätig ist } \\
\text { (ISCO } 4 \text { und 5) }\end{array}$} \\
\hline & & \multicolumn{2}{|c|}{$\begin{array}{c}\begin{array}{c}\text { Sozio- } \\
\text { ökonomisch } \\
\text { benachteiligte } \\
\text { Schulen }\end{array} \\
\end{array}$} & $\begin{array}{r}\text { So } \\
\text { ökon } \\
\text { durch } \\
\text { liche S }\end{array}$ & $\begin{array}{l}\text { io- } \\
\text { misch } \\
\text { chnitt- } \\
\text { hulen } 1\end{array}$ & $\begin{array}{r}\text { So } \\
\text { ökon } \\
\text { begü } \\
\text { Sch }\end{array}$ & $\begin{array}{l}\text { io- } \\
\text { nisch } \\
\text { tigte } \\
\text { en }\end{array}$ & $\begin{array}{r}\text { So } \\
\text { ökon } \\
\text { benac } \\
\text { Sch }\end{array}$ & $\begin{array}{l}\text { io- } \\
\text { misch } \\
\text { teiligte } \\
\text { len }\end{array}$ & $\begin{array}{r}\text { So } \\
\text { ökon } \\
\text { durch } \\
\text { iche S }\end{array}$ & $\begin{array}{l}\text { io- } \\
\text { nisch } \\
\text { hnittl- } \\
\text { hulen }\end{array}$ & $\begin{array}{r}\text { So } \\
\text { ökon: } \\
\text { begü } \\
\text { Sch }\end{array}$ & $\begin{array}{l}\text { io- } \\
\text { misch } \\
\text { stigte } \\
\text { len }\end{array}$ & $\begin{array}{r}\text { So } \\
\text { ökon } \\
\text { benac } \\
\text { Sch } \\
\end{array}$ & \begin{tabular}{|l|} 
io- \\
misch \\
teiligte \\
len
\end{tabular} & $\begin{array}{r}\mathrm{S} \\
\text { öko } \\
\text { durc } \\
\text { liche }\end{array}$ & $\begin{array}{l}\text { io- } \\
\text { misch } \\
\text { chnitt- } \\
\text { hulen }{ }^{1}\end{array}$ & $\begin{array}{r}\text { So } \\
\text { ökon } \\
\text { begi } \\
\text { Sch }\end{array}$ & $\begin{array}{l}\text { io- } \\
\text { misch } \\
\text { stigte } \\
\text { len } 1\end{array}$ \\
\hline & & $\%$ & S.E. & $\%$ & S.E. & $\%$ & S.E. & $\%$ & S.E. & $\%$ & S.E. & $\%$ & S.E. & $\%$ & S.E. & $\%$ & S.E. & $\%$ & S.E. \\
\hline & Australien & 8.7 & $(0.6)$ & 3.7 & $(0.3)$ & 1.1 & $(0.2)$ & 20.0 & $(0.9)$ & 10.0 & (0.4) & 2.6 & $(0.4)$ & 25.3 & $(0.9)$ & 19.2 & $(0.5)$ & 7.8 & $(0.6)$ \\
\hline כָ & Österreich & 4.8 & $(0.7)$ & 1.8 & $(0.3)$ & 0.7 & $(0.2)$ & 28.8 & (1.8) & 15.5 & $(1.2)$ & 3.9 & $(0.5)$ & 38.6 & (1.5) & 32.2 & $(1.2)$ & 15.8 & (1.4) \\
\hline & Belgien & 10.5 & $(0.8)$ & 3.8 & $(0.4)$ & 0.9 & $(0.2)$ & 29.2 & (1.5) & 14.2 & $(0.8)$ & 4.8 & $(0.5)$ & 31.8 & $(0.9)$ & 27.1 & $(0.8)$ & 14.7 & (0.8) \\
\hline 8 & Kanada & 5.7 & $(0.7)$ & 2.6 & $(0.2)$ & 0.6 & $(0.2)$ & 18.6 & (1.0) & 9.9 & (0.5) & 3.9 & $(0.5)$ & 30.6 & (1.4) & 23.1 & $(0.6)$ & 12.9 & $(0.8)$ \\
\hline u & Chile & 21.7 & (1.6) & 10.4 & (1.1) & 2.3 & $(0.4)$ & 36.6 & (1.3) & 25.3 & $(1.2)$ & 10.5 & $(0.9)$ & 28.9 & (1.2) & 35.3 & (1.7) & 21.0 & (1.3) \\
\hline 0 & Tschech. Rep. & 3.6 & $(0.9)$ & 1.0 & $(0.2)$ & 0.2 & $(0.2)$ & 32.0 & (2.0) & 19.7 & $(0.8)$ & 3.9 & $(0.6)$ & 44.0 & (2.1) & 35.2 & (1.3) & 21.6 & (1.5) \\
\hline & Dänemark & 5.7 & $(0.8)$ & 2.5 & $(0.3)$ & 1.0 & $(0.3)$ & 15.4 & $(1.2)$ & 8.7 & (0.6) & 1.6 & $(0.4)$ & 38.8 & (1.6) & 29.8 & $(0.9)$ & 13.7 & (1.3) \\
\hline & Estland & 3.5 & $(0.7)$ & 1.9 & $(0.2)$ & 0.4 & $(0.2)$ & 36.3 & (1.5) & 19.0 & (1.0) & 4.6 & $(0.7)$ & 31.0 & (1.7) & 25.8 & (1.0) & 11.5 & $(0.8)$ \\
\hline & Finnland & 1.7 & $(0.4)$ & 0.9 & $(0.1)$ & 0.2 & $(0.1)$ & 19.4 & $(1.7)$ & 9.4 & $(0.5)$ & 2.6 & $(0.6)$ & 31.5 & (1.2) & 24.1 & $(0.7)$ & 14.1 & (1.1) \\
\hline & Frankreich & w & w & w & w & w & w & w & w & w & w & w & w & w & w & w & w & w & w \\
\hline & Deutschland & 4.3 & $(0.7)$ & 1.8 & $(0.3)$ & 0.4 & $(0.2)$ & 25.4 & (1.5) & 13.9 & (0.9) & 3.2 & $(0.5)$ & 42.6 & (1.6) & 31.7 & $(1.0)$ & 17.0 & (1.4) \\
\hline & Griechenland & 11.0 & $(1.2)$ & 4.2 & $(0.4)$ & 1.2 & $(0.3)$ & 35.8 & (1.7) & 17.4 & (1.1) & 6.1 & $(0.7)$ & 32.4 & $(2.0)$ & 30.5 & (1.1) & 18.0 & (1.0) \\
\hline & Ungarn & 8.0 & (1.1) & 2.6 & $(0.5)$ & 1.0 & $(0.2)$ & 44.4 & (2.1) & 21.3 & $(1.2)$ & 7.5 & (0.9) & 29.0 & (1.8) & 36.4 & $(1.2)$ & 20.5 & (1.4) \\
\hline & Island & 6.1 & (1.1) & 2.4 & $(0.3)$ & 0.6 & $(0.3)$ & 19.0 & (1.6) & 9.0 & $(0.7)$ & 3.4 & $(0.6)$ & 29.0 & $(2.0)$ & 22.0 & $(0.9)$ & 14.6 & $(1.1)$ \\
\hline & Irland & 9.2 & (1.4) & 2.4 & $(0.3)$ & 0.7 & $(0.2)$ & 18.7 & $(2.1)$ & 12.4 & $(0.7)$ & 5.0 & $(0.8)$ & 37.5 & (2.1) & 30.4 & $(0.9)$ & 16.0 & (1.4) \\
\hline & Israel & 4.3 & $(0.7)$ & 1.3 & $(0.3)$ & 0.4 & $(0.2)$ & 25.9 & $(2.1)$ & 9.5 & $(1.2)$ & 2.1 & $(0.4)$ & 24.2 & (1.9) & 1 & (1.0) & 7.0 & $(0.7)$ \\
\hline & Italien & 6.9 & $(0.4)$ & 3.0 & $(0.2)$ & 0.8 & $(0.1)$ & 40.7 & (1.0) & 22.1 & $(0.6)$ & 9.0 & $(0.5)$ & 33.8 & $(0.8)$ & 33.3 & $(0.6)$ & 24.6 & $(0.7)$ \\
\hline & Japan & 5.9 & (0.6) & 3.3 & $(0.4)$ & 1.6 & $(0.3)$ & 19.7 & $(1.2)$ & 10.5 & (0.6) & 3.4 & $(0.4)$ & 41.6 & (1.3) & 35.9 & (1.0) & 26.3 & (1.3) \\
\hline & Korea & 6.9 & $(0.8)$ & 1.8 & $(0.3)$ & 0.8 & $(0.3)$ & 15.8 & (1.3) & 7.8 & $(0.5)$ & 3.5 & $(0.5)$ & 38.8 & (1.5) & 33.1 & (1.0) & 21.1 & (1.3) \\
\hline & Luxemburg & 6.9 & (0.6) & 3.0 & $(0.7)$ & 1.5 & $(0.3)$ & 31.2 & (0.9) & 17.5 & (1.2) & 6.3 & (0.5) & 34.2 & (1.1) & 29.0 & (1.7) & 19.6 & (0.9) \\
\hline & Mexiko & 36.7 & (1.4) & 12.0 & (0.6) & 3.4 & $(0.2)$ & 29.2 & $(0.8)$ & 27.0 & (0.6) & 12.4 & $(0.6)$ & 22.5 & (0.9) & 35.6 & $(0.8)$ & 28.5 & $(0.9)$ \\
\hline & Niederland & 4.0 & $(0.8)$ & 1.3 & $(0.3)$ & 0.8 & $(0.5)$ & 17.9 & $(1.2)$ & 7.3 & $(0.7)$ & 2.6 & $(0.5)$ & 32.3 & (1.6) & 1.4 & $(0.9)$ & 3.7 & $(0.9)$ \\
\hline & Neuseeland & 6.8 & (1.0) & 2.8 & $(0.4)$ & 0.6 & $(0.3)$ & 26.2 & (2.2) & 12.5 & $(0.6)$ & 3.5 & $(0.6)$ & 24.5 & (1.5) & 19.0 & $(0.8)$ & 7.8 & (0.9) \\
\hline & Norwegen & 2.6 & $(0.6)$ & 1.5 & $(0.2)$ & 0.0 & $(0.0)$ & 10.0 & (1.6) & 6.5 & (0.4) & 2.3 & (0.5) & 32.2 & (2.2) & 22.2 & $(0.7)$ & 9.4 & (1.0) \\
\hline & Polen & 2.5 & $(0.4)$ & 1.5 & $(0.3)$ & 0.6 & $(0.2)$ & 53.8 & (2.3) & 27.8 & (1.1) & 9.4 & (1.3) & 25.3 & (1.6) & .5 & (0.9) & 14.7 & (1.2) \\
\hline & Portuga & 12.1 & $(1.2)$ & 6.1 & $(0$. & 1.3 & (0. & 40.4 & $(1.8)$ & 24.7 & (1.3) & 6. & (1.4) & 31.0 & (1.5) & 5.0 & (1.3) & 21.3 & (2.9) \\
\hline & Slowak. Re & 12.0 & $(1.7)$ & 4.1 & $(0.7)$ & 1.3 & $(0.3)$ & 37.0 & (2.3) & 25.8 & (1.3) & 7.4 & $(1.2)$ & 40.8 & (2.0) & 8.1 & (1.5) & 26.9 & $(1.2)$ \\
\hline & Slowenien & 5.0 & (0.5) & 2.9 & (0.5) & 0.8 & $(0.3)$ & 32.9 & (1.3) & 13.9 & (0.8) & 4.4 & $(0.7)$ & 29.0 & (1.2) & 28.5 & (1.1) & 14.3 & (1.0) \\
\hline & Spanien & 10.2 & $(0.7)$ & 6.0 & $(0.5)$ & 1.7 & $(0.3)$ & 34.9 & $(1.2)$ & 21.4 & $(0.7)$ & 7.3 & $(0.7)$ & 32.5 & (1.4) & 32.7 & $(0.9)$ & 21.2 & (1.3) \\
\hline & Schweden & 3.2 & $(0.7)$ & 1.5 & (0.3) & 0.5 & $(0.2)$ & 15.5 & (1.4) & 9.1 & (0.6) & 1.7 & (0.4) & 39.0 & (2.0) & 27.7 & $(0.8)$ & 11.8 & (0.9) \\
\hline & Schweiz & 2.2 & $(0.4)$ & 1.4 & $(0.2)$ & 0.4 & $(0.2)$ & 21.2 & $(1.3)$ & 11.4 & $(0.7)$ & 3.1 & $(0.3)$ & 32.8 & (1.3) & 25.5 & $(0.9)$ & 9.8 & $(0.8)$ \\
\hline & Türkei & 12.4 & (1.3) & 9.8 & $(0.8)$ & 4.0 & $(0.7)$ & 43.4 & (2.5) & 33.0 & (1.5) & 19.2 & (1.4) & 34.3 & (2.1) & 38.4 & $(1.7)$ & 37.4 & $(2.1)$ \\
\hline & Ver. Königreich & 5.1 & $(0.6)$ & 1.9 & $(0.3)$ & 0.5 & $(0.1)$ & 14.2 & $(1.5)$ & 6.0 & $(0.4)$ & 2.3 & $(0.4)$ & 36.6 & (1.8) & 27.2 & $(0.8)$ & 12.6 & $(1.0)$ \\
\hline & Ver. Staaten & 12.2 & $(1.2)$ & 4.9 & $(0.7)$ & 1.4 & $(0.3)$ & 14.9 & (1.4) & 7.2 & $(0.6)$ & 3.5 & $(0.5)$ & 28.7 & (1.5) & 23.5 & $(0.8)$ & 12.9 & $(1.2)$ \\
\hline & OECD-Durchschnitt & 7.9 & $(0.2)$ & 3.4 & $(0.1)$ & 1.0 & (0.0) & 27.4 & $(0.3)$ & 15.3 & $(0.1)$ & 5.2 & $(0.1)$ & 33.0 & $(0.3)$ & 28.9 & $(0.2)$ & 16.7 & $(0.2)$ \\
\hline & & $\mathrm{m}$ & & $\mathrm{m}$ & 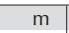 & 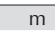 & & n & & $\mathrm{m}$ & & $\mathrm{m}$ & & $\mathrm{m}$ & & $\mathrm{m}$ & & $\mathrm{n}$ & $\mathrm{n}$ \\
\hline $\mathcal{E}$ & Argentinien & 14.1 & $(1.0)$ & 9.8 & (1.4) & 2.2 & $(0.4)$ & 43.3 & $(1.3)$ & 28.4 & (1.3) & 11.8 & (1.6) & 26.8 & (1.3) & 32.5 & $(1.7)$ & 28.5 & $(2.2)$ \\
\hline 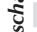 & Brasilien & 19.0 & $(0.9)$ & 10.0 & $(0.7)$ & 1.8 & $(0.3)$ & 26.7 & (1.0) & 20.4 & $(0.8)$ & 6.4 & $(0.6)$ & 35.1 & $(0.9)$ & 41.4 & $(0.8)$ & 26.4 & (1.5) \\
\hline$\stackrel{5}{\Sigma}$ & Bulgarien & 8.2 & (1.0) & 3.0 & $(0.6)$ & 0.7 & $(0.2)$ & 42.6 & (1.9) & 23.1 & $(1.2)$ & 6.7 & $(0.8)$ & 29.9 & (1.6) & 32.1 & (1.5) & 19.3 & $(1.2)$ \\
\hline$=$ & Kolumbien & 10.1 & $(0.8)$ & 9.0 & $(0.7)$ & 3.4 & $(0$. & 51.8 & (1.6) & 34.1 & (1.6) & 16.5 & (1.6) & 28.9 & (1.4) & 36.1 & (1.6) & 36.3 & (1.3) \\
\hline a & Costa Rica & 23.4 & $(2.7)$ & 10.1 & (1.0) & 1 & $(0$ & 30.9 & (3.3) & 20.6 & (1.2) & 8.8 & (1. & 30.2 & (1.8) & 38.6 & (1.6) & 20.5 & (1.9) \\
\hline 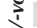 & Kroatien & 3.0 & $(0.5)$ & 1.3 & $(0.3)$ & 0.4 & $(0$. & 36.6 & (1.4) & 18.1 & (1.1) & 5.8 & $(0.8)$ & 39.6 & (1.5) & 41.2 & $(1.1)$ & 21.6 & $(2.1)$ \\
\hline ذँ & Zypern* & 4.3 & $(0.5)$ & 2.1 & $(0.4)$ & 0.8 & (0.3) & 29.6 & (1.3) & 13.7 & (0.8) & 5.3 & (0.6) & 42.6 & (1.2) & 43.1 & (1.3) & 26.6 & (1.3) \\
\hline & Hongkong (C & 11.9 & $(0.8)$ & 6.4 & $(0.7)$ & 1.1 & $(0.4)$ & 23.2 & $(1.0)$ & 16.4 & $(0.9)$ & 5.3 & $(0.9)$ & 41.7 & (1.5) & 36.8 & $(1.1)$ & 18.6 & (1.9) \\
\hline & Indonesien & 28.0 & (3.4) & 25.2 & (3.3) & 0 & - & 43.9 & (3.1) & 29.7 & (3.9) & 17.6 & (2.3) & 20.1 & (1.8) & 28.0 & (2.8) & 28.9 & $(2.7)$ \\
\hline ș & Jordanien & 3.8 & $(0.7)$ & 1.9 & $(0.3)$ & & $(0$. & 31.6 & (1.3) & 20.8 & (0. & 6. & (1. & 21.5 & (1.3) & 17.8 & $(0.9)$ & 9.1 & (1.0) \\
\hline ฝ & Kasachstan & 6.1 & $(0.8)$ & 3.3 & (0.5) & 1.1 & (U.2 & 30.9 & (2.2) & 19.4 & (1.1) & 7.3 & (0.8) & 18.3 & (1.9) & 21.3 & $(1.2)$ & 14.6 & (1.1) \\
\hline & Lettland & 12.5 & $(1.7)$ & 4.9 & $(0.6)$ & 0.9 & $(0.3)$ & 30.9 & (2.5) & 15.9 & (0.9) & 6.0 & $(0.7)$ & 35.4 & (2.5) & 32.6 & (1.1) & 16.5 & (1.3) \\
\hline & Liechtens & c & c & 2.8 & $(1.4)$ & c & C & c & c & 9.9 & (2.4) & c & 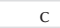 & c & C & 15.6 & $(2.7)$ & c & c \\
\hline & Litauen & 9.9 & 9) & 3.5 & $(0.4)$ & 1.4 & 4) & 39.6 & 1.4) & 22.8 & (1.0) & 7.5 & (0.9) & 3.6 & $.5)$ & 23.2 & $(0.7)$ & 14.1 & $(1.2)$ \\
\hline & Macau (C & 7.3 & (U.J) & & $(0.7)$ & 1.3 & & & $(0.6)$ & 7.7 & $(0$. & 4.2 & & 63.6 & & 61.9 & (1.5) & 46.3 & (1.2) \\
\hline & Malaysia & 21.6 & $(2.0)$ & 8.6 & $(0.8)$ & 3.1 & $(0.6$ & 38.4 & $(1.7)$ & 26.4 & (1.1) & 11.4 & $(1.3)$ & 20.2 & $(1.3)$ & 29.0 & $(1.2)$ & 20.4 & $(1.3)$ \\
\hline & Montenegro & 6.4 & $(0.7)$ & 4.0 & (0.6) & 1.6 & $(0.3)$ & 30.1 & (1.3) & 18.1 & (1.4) & 8.1 & $(0.7)$ & 35.8 & $(1.2)$ & 35.6 & (1.8) & 27.7 & $(1.2)$ \\
\hline & Peru & 44.2 & (1.9) & 15.2 & $(1.3)$ & 4.2 & $(0.6)$ & 29.3 & $(1.2)$ & 31.1 & (1.3) & 16.1 & (1.5) & 21.2 & (1.3) & 37.4 & (1.4) & 35.8 & (1.3) \\
\hline & Katar & & (1) & 0.9 & $(0.2)$ & & 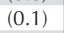 & 12.1 & $(0.6)$ & 8.6 & (0.6) & 3.2 & $(0.3)$ & 20.5 & $(0.8)$ & 17.2 & $(0.9)$ & 10.2 & $(0.4)$ \\
\hline & Rumänien & & $(1.2)$ & 3.7 & (0.5) & & & 49.3 & (2.0) & 31.7 & (1.5) & 13.3 & (1.3) & 27.5 & (1.8) & 33.3 & (1.4) & 20.7 & (1.7) \\
\hline & Russ & 3.8 & $(0.7)$ & 1.3 & $(0.2)$ & 0.4 & 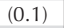 & 29.3 & (1.5) & 15.1 & $(1.0)$ & 4.9 & $(0.7)$ & 34.6 & (1.8) & 29.4 & $(1.1)$ & 17.6 & $(1.1)$ \\
\hline & Serbien & 5.7 & $(0.8)$ & 3.5 & $(0.4)$ & 1.2 & (0.4) & 18.5 & (1.4) & 12.1 & $(0.8)$ & 5.5 & $(0.8)$ & 53.9 & (1.6) & 43.6 & $(1.2)$ & 23.0 & (1.9) \\
\hline & Shanghai ( & 3.1 & $(0.7)$ & 1.3 & $(0.2)$ & 0.3 & $(0.1)$ & 29.1 & (1.9) & 13.3 & $(0.7)$ & 4.2 & $(0.5)$ & 33.0 & (1.4) & 29.1 & $(1.3)$ & 17.1 & (1.4) \\
\hline & Singapur & 4.9 & $(0.5)$ & 2.2 & $(0.3)$ & 0.5 & 02 & 11.3 & $(0.8)$ & 8.9 & $(0.7)$ & 1.5 & $(0.4)$ & 28.9 & (1.0) & 22.0 & (1.0) & 6.3 & $(0.7)$ \\
\hline & Chinesisch Ta & 5.5 & $(0.5)$ & 3.4 & (0.4) & 0.7 & (0. & 34.4 & (1.5) & 20.9 & (1.0) & 9.1 & (1.0) & 37.6 & (1.3) & 34.0 & $(0.9)$ & 21.6 & (1.5) \\
\hline & Thailand & 16.9 & (1.3) & 11.8 & (1.0) & 4.7 & $(0.4$ & 54.7 & (2.0) & 40.7 & (1.7) & 16.5 & (1.3) & 17.3 & (1.3) & 27.3 & (1.5) & 33.9 & (1.4) \\
\hline & Tunesien & 57.4 & $(2.1)$ & 23.9 & (1.5) & 4.6 & $(0.8)$ & 16.3 & $(1.3)$ & 25.5 & (1.6) & 12.8 & $(1.2)$ & 16.1 & (1.3) & 24.5 & (1.4) & 25.0 & (1.7) \\
\hline & Ver. Arab. Emirate & 1.7 & $(0.3)$ & 0.7 & $(0.2)$ & 0.1 & $(0.0)$ & 10.2 & $(0.9)$ & 4.1 & $(0.5)$ & 1.0 & $(0.3)$ & 23.9 & (1.7) & 14.0 & $(0.9)$ & 4.8 & $(0.5)$ \\
\hline & Uruguay & 26.2 & (1.2) & 15.4 & (1.1) & 2.0 & (0.6) & 27.9 & (1.2) & 20.9 & (1.0) & 7.2 & (1.1) & 36.7 & (1.0) & 39.6 & (1.4) & 26.5 & (2.0) \\
\hline & Vietnam & 3.4 & $(1.1)$ & 2.0 & $(0.5)$ & 3.4 & $(0.7)$ & 86.2 & (1.6) & 69.4 & (2.0) & 30.9 & (3.8) & 7.0 & $(0.9)$ & 17.7 & (1.6) & 34.5 & $(2.1)$ \\
\hline
\end{tabular}

1. Eine sozioökonomisch benachteiligte Schule ist eine Schule mit Schülern, deren durchschnittlicher sozioökonomischer Status statistisch signifikant unter dem durchschnittlichen sozioökonomischen Status des Landes/der Volkswirtschaft liegt; eine sozioökonomisch durchschnittliche Schule ist eine Schule, an der es keinen Unterschied zwischen dem durchschnittlichen sozioökonomischen Status der Schüler und des Landes/der Volkswirtschaft gibt; und eine sozioökonomisch begünstigte Schule ist eine Schule mit Schülern, deren durchschnittlicher sozioökonomischer Status statistisch signifikant über dem durchschnittlichen sozioökonomischen Status des Landes/der Volkswirtschaft liegt. StatLink 개내 http://dx.doi.org/10.1787/888932964908 
[Teil 3/3]

Bildungsabschluss und Beruf der Eltern sowie Ausstattung des Elternhauses der Schüler, nach dem sozioökonomischen Profil der Schulen

Tabelle II.2.10 Die Ergebnisse basieren auf Schülerangaben

\begin{tabular}{|c|c|c|c|c|c|c|c|c|c|c|c|c|c|c|c|c|c|c|c|}
\hline & & \multicolumn{6}{|c|}{$\begin{array}{l}\text { Höchste berufliche Stellung der Eltern: } \\
\text { Prozentsatz der Schüler mit mindestens einem } \\
\text { Elternteil, der als qualifizierte Fach- oder } \\
\text { Führungskraft tätig ist (ISCO 1, } 2 \text { und 3) }\end{array}$} & \multicolumn{6}{|c|}{$\begin{array}{l}\text { Besitz von Kulturgütern: Prozentsatz der } \\
\text { Schüler, in deren Elternhhaus eigenen Angaben } \\
\text { zufolge Gedichtbände vorhanden sind }\end{array}$} & \multicolumn{6}{|c|}{$\begin{array}{l}\text { Anzahl der Bücher im Elternhaus: Prozentsatz } \\
\text { der Schüler, in deren Elternhaus eigenen } \\
\text { Angaben zufolge mindestens } 200 \text { Bücher } \\
\text { vorhanden sind: }\end{array}$} \\
\hline & & \multicolumn{2}{|c|}{\begin{tabular}{|c|} 
Sozio- \\
ökonomisch \\
benachteiligte \\
Schulen ${ }^{1}$
\end{tabular}} & \multicolumn{2}{|c|}{$\begin{array}{l}\text { Sozio- } \\
\text { ökonomisch } \\
\text { durchschnitt- } \\
\text { liche Schulen } 1\end{array}$} & $\begin{array}{r}\text { So } \\
\text { ökon } \\
\text { begü } \\
\text { Sch }\end{array}$ & $\begin{array}{l}\text { io- } \\
\text { misch } \\
\text { stigte } \\
\text { len }\end{array}$ & $\begin{array}{r}\text { Sc } \\
\text { ökon } \\
\text { benac } \\
\text { Sch }\end{array}$ & $\begin{array}{l}\text { io- } \\
\text { misch } \\
\text { teiligte } \\
\text { len }\end{array}$ & $\begin{array}{r}\text { So } \\
\text { ökon } \\
\text { durch } \\
\text { liche S }\end{array}$ & $\begin{array}{l}\text { io- } \\
\text { misch } \\
\text { chnitt- } \\
\text { hulen }\end{array}$ & $\begin{array}{r}\text { So } \\
\text { ökon } \\
\text { begü } \\
\text { Sch }\end{array}$ & $\begin{array}{l}\text { io- } \\
\text { misch } \\
\text { stigte } \\
\text { len }\end{array}$ & $\begin{array}{r}\text { So } \\
\text { ökon } \\
\text { benac } \\
\text { Sch } \\
\end{array}$ & $\begin{array}{l}\text { io- } \\
\text { misch } \\
\text { teiligte } \\
\text { len }\end{array}$ & $\begin{array}{r}\text { So } \\
\text { ökon } \\
\text { durch } \\
\text { liche S }\end{array}$ & $\begin{array}{l}\text { io- } \\
\text { misch } \\
\text { chnitt- } \\
\text { hulen }\end{array}$ & $\begin{array}{r}\text { So } \\
\text { ökono } \\
\text { begü } \\
\text { Scht }\end{array}$ & $\begin{array}{l}\text { io- } \\
\text { misch } \\
\text { stigte } \\
\text { len }{ }^{1}\end{array}$ \\
\hline & & $\%$ & S.E. & $\%$ & S.E. & $\%$ & S.E. & $\%$ & S.E. & $\%$ & S.E. & $\%$ & S.E. & $\%$ & S.E. & $\%$ & S.E. & $\%$ & S.E. \\
\hline & Australien & 45.9 & $(1.0)$ & 67.1 & $(0.6)$ & 88.5 & $(0.7)$ & 25.3 & $(0.7)$ & 33.9 & (0.8) & 49.1 & $(1.0)$ & 15.9 & $(0.7)$ & 24.3 & $(0.8)$ & 39.9 & $(1.2)$ \\
\hline 8 & Österreich & 27.8 & (1.4) & 50.6 & (1.5) & 79.6 & $(1.7)$ & 44.1 & (1.5) & 56.1 & $(1.2)$ & 72.3 & $(2.1)$ & 7.3 & $(0.8)$ & 19.6 & (1.3) & 47.5 & $(2.8)$ \\
\hline & Belgien & 28.6 & $(1.1)$ & 54.9 & $(1.1)$ & 79.7 & $(0.9)$ & 21.0 & $(1.1)$ & 28.5 & (1.1) & 44.0 & $(1.2)$ & 8.4 & $(0.6)$ & 18.3 & $(0.9)$ & 33.6 & (1.3) \\
\hline & Kanada & 45.1 & (1.1) & 64.3 & $(0.7)$ & 82.6 & $(1.0)$ & 26.2 & (1.5) & 32.5 & $(0.8)$ & 43.0 & $(1.2)$ & 12.7 & $(1.0)$ & 22.6 & $(0.7)$ & 33.4 & (1.1) \\
\hline & Chile & 12.8 & (1.0) & 28.9 & (1.4) & 66.1 & (1.9) & 52.1 & (1.1) & 56.4 & (1.9) & 54.4 & (1.4) & 2.0 & $(0.3)$ & 4.3 & $(0.6)$ & 13.0 & $(0.9)$ \\
\hline 0 & Tschech. Rep. & 20.4 & (1.8) & 44.1 & $(1.1)$ & 74.3 & (1.5) & 28.1 & $(2.3)$ & 43.2 & (1.4) & 60.0 & $(1.7)$ & 10.6 & (1.4) & 18.7 & (1.0) & 43.9 & (2.1) \\
\hline & Dänemark & 40.1 & $(2.1)$ & 59.0 & (1.0) & 83.8 & $(1.3)$ & 18.4 & (1.5) & 27.1 & $(0.8)$ & 46.6 & $(2.1)$ & 10.8 & (1.1) & 19.0 & $(0.8)$ & 34.0 & (2.1) \\
\hline & Estland & 29.3 & (1.5) & 53.3 & $(1.2)$ & 83.5 & (1.1) & 64.4 & (2.0) & 70.8 & (1.1) & 81.7 & $(1.2)$ & 15.5 & $(1.2)$ & 23.0 & $(0.9)$ & 39.2 & (1.7) \\
\hline & Finnland & 47.4 & (1.9) & 65.5 & $(0.9)$ & 83.1 & $(1.1)$ & 40.8 & (1.9) & 52.3 & $(1.0)$ & 59.0 & (1.5) & 12.9 & (1.3) & 20.3 & $(0.8)$ & 32.5 & (2.0) \\
\hline & Frankreich & w & w & w & w & w & w & w & w & w & w & w & w & w & w & w & w & w & w \\
\hline & Deutschland & 27.7 & (1.5) & 52.6 & $(1.2)$ & 79.4 & (1.6) & 47.3 & $(1.7)$ & 56.3 & (1.1) & 70.2 & $(1.3)$ & 12.2 & $(1.0)$ & 25.9 & (1.7) & 49.5 & $(2.3)$ \\
\hline & Griechenland & 20.8 & (1.5) & 47.8 & $(1.2)$ & 74.6 & $(1.2)$ & 33.0 & $(1.7)$ & 42.8 & $(1.2)$ & 58.6 & $(1.7)$ & 8.8 & $(0.9)$ & 16.8 & $(0.9)$ & 31.8 & (1.5) \\
\hline & Ungarn & 18.7 & (1.3) & 39.7 & (1.4) & 70.9 & $(2.0)$ & 44.9 & (2.4) & 66.6 & (1.7) & 85.8 & (1.1) & 10.9 & (1.2) & 27.0 & (1.2) & 56.0 & $(2.0)$ \\
\hline & Island & 46.0 & $(1.7)$ & 66.6 & $(0.9)$ & 81.5 & $(1.2)$ & 62.5 & $(1.8)$ & 71.0 & $(0.9)$ & 79.4 & $(1.2)$ & 21.0 & (1.7) & 28.9 & $(0.8)$ & 40.2 & (1.5) \\
\hline & Irland & 34.6 & (2.3) & 54.8 & $(0.9)$ & 78.4 & (1.4) & 24.1 & $(2.3)$ & 34.7 & (1.0) & 45.0 & (1.5) & 7.4 & (1.2) & 19.2 & $(0.8)$ & 37.0 & (1.8) \\
\hline & Israel & 45.6 & $(2.3)$ & 70.0 & (1.5) & 90.5 & $(0.9)$ & 47.6 & $(2.1)$ & 49.1 & (1.8) & 59.9 & (1.6) & 17.2 & $(2.1)$ & 23.4 & $(2.3)$ & 35.7 & (3.2) \\
\hline & Italien & 18.7 & $(0.7)$ & 41.6 & $(0.6)$ & 65.6 & $(0.9)$ & 42.2 & (1.1) & 54.4 & $(0.8)$ & 74.3 & $(0.8)$ & 7.9 & $(0.5)$ & 16.6 & $(0.6)$ & 37.0 & (1.0) \\
\hline & Japan & 32.8 & (1.0) & 50.3 & $(1.0)$ & 68.7 & (1.4) & 13.8 & $(1.0)$ & 25.2 & (1.0) & 39.4 & $(1.7)$ & 18.2 & (1.0) & 21.0 & $(0.9)$ & 33.4 & (1.7) \\
\hline & Korea & 38.4 & (1.5) & 57.4 & (1.1) & 74.6 & (1.4) & 46.5 & (1.5) & 65.1 & (1.1) & 76.5 & (1.5) & 17.7 & (1.4) & 37.1 & (1.5) & 56.9 & (2.4) \\
\hline & Luxemburg & 27.7 & $(1.0)$ & 50.4 & (1.6) & 72.6 & (1.0) & 41.0 & $(1.0)$ & 54.7 & (1.9) & 65.3 & $(1.0)$ & 16.6 & $(0.7)$ & 37.3 & (1.7) & 56.8 & (1.1) \\
\hline & Mexiko & 11.5 & $(0.4)$ & 25.5 & $(0.7)$ & 55.6 & $(1.1)$ & 39.5 & $(1.0)$ & 44.7 & $(0.8)$ & 50.1 & $(0.9)$ & 1.8 & $(0.2)$ & 2.5 & $(0.2)$ & 7.4 & $(0.6)$ \\
\hline & Niederlande & 45.8 & (1.6) & 70.0 & (1.1) & 87.9 & $(0.9)$ & 20.2 & (1.6) & 24.3 & $(1.3)$ & 42.9 & (1.9) & 8.5 & $(1.1)$ & 16.6 & (1.5) & 36.8 & $(2.0)$ \\
\hline & Neuseeland & 42.6 & (2.4) & 65.7 & $(0.9)$ & 88.0 & $(1.3)$ & 29.1 & (1.4) & 36.3 & $(1.2)$ & 47.4 & $(2.0)$ & 12.9 & $(1.2)$ & 23.7 & $(1.2)$ & 40.3 & (1.9) \\
\hline & Norwegen & 55.2 & $(2.2)$ & 69.9 & $(0.8)$ & 88.3 & $(1.2)$ & 31.8 & $(2.5)$ & 42.9 & $(1.2)$ & 62.3 & (2.5) & 17.8 & (2.2) & 26.1 & $(0.9)$ & 46.1 & (3.0) \\
\hline & Polen & 18.4 & (1.0) & 44.3 & $(1.0)$ & 75.3 & (1.8) & 33.7 & (1.3) & 47.1 & (1.1) & 64.5 & (2.0) & 6.3 & $(0.8)$ & 14.2 & (1.0) & 40.2 & (2.7) \\
\hline & Portugal & 16.5 & $(1.0)$ & 34.2 & $(0.8)$ & 70.9 & $(4.1)$ & 35.6 & $(1.3)$ & 50.1 & (1.3) & 72.8 & $(2.1)$ & 4.7 & $(0.6)$ & 11.9 & $(0.7)$ & 32.9 & (1.9) \\
\hline & Slowak. Rep. & 10.2 & (1.2) & 32.0 & (1.3) & 64.5 & $(2.0)$ & 45.2 & (2.1) & 61.5 & (1.1) & 74.4 & (1.5) & 4.3 & $(0.8)$ & 10.8 & $(0.9)$ & 28.8 & (1.9) \\
\hline & Slowenien & 33.2 & $(1.2)$ & 54.7 & $(1.2)$ & 80.5 & $(1.3)$ & 44.6 & $(1.2)$ & 57.7 & $(1.2)$ & 78.8 & (1.3) & 4.8 & $(0.6)$ & 10.7 & $(0.7)$ & 30.3 & (1.6) \\
\hline & Spanien & 22.3 & $(0.8)$ & 39.9 & $(0.8)$ & 69.7 & (1.5) & 47.8 & $(1.1)$ & 55.6 & (0.9) & 68.7 & (1.3) & 10.4 & $(0.6)$ & 21.7 & $(0.6)$ & 38.3 & (1.2) \\
\hline & Schweden & 42.3 & $(1.8)$ & 61.6 & $(0.9)$ & 85.9 & (1.0) & 25.1 & $(1.5)$ & 32.4 & $(1.0)$ & 48.5 & $(2.1)$ & 14.2 & (1.5) & 25.2 & $(1.0)$ & 37.9 & (1.8) \\
\hline & Schweiz & 43.7 & (1.7) & 61.8 & $(0.9)$ & 86.7 & (1.0) & 29.7 & (1.5) & 35.2 & $(1.0)$ & 50.8 & (2.0) & 11.5 & $(0.9)$ & 16.6 & (1.1) & 39.2 & (1.9) \\
\hline & Türkei & 9.9 & $(0.9)$ & 18.8 & $(0.8)$ & 39.4 & (2.3) & 51.9 & $(1.7)$ & 59.6 & $(1.2)$ & 69.4 & $(2.1)$ & 2.9 & $(0.5)$ & 6.6 & $(0.7)$ & 20.3 & (2.2) \\
\hline & Ver. Königreich & 44.1 & (2.5) & 64.9 & $(0.9)$ & 84.6 & $(1.2)$ & 27.3 & (1.9) & 36.6 & (1.1) & 53.8 & (1.4) & 9.6 & $(0.7)$ & 20.0 & $(1.0)$ & 39.1 & (2.0) \\
\hline & Ver. Staaten & 44.2 & (1.9) & 64.5 & $(1.2)$ & 82.2 & (1.5) & 33.2 & $(1.7)$ & 38.0 & (1.4) & 46.0 & (1.5) & 5.7 & $(0.5)$ & 16.3 & $(1.2)$ & 22.7 & (1.5) \\
\hline & OECD-Durchschnitt & 31.7 & $(0.3)$ & 52.5 & $(0.2)$ & 77.0 & $(0.3)$ & 36.4 & $(0.3)$ & 46.5 & $(0.2)$ & 60.5 & $(0.3)$ & 10.5 & $(0.2)$ & 19.5 & $(0.2)$ & 36.7 & $(0.3)$ \\
\hline & Albanien & $\mathrm{m}$ & $m$ & $\mathrm{~m}$ & $\mathrm{~m}$ & $m$ & $\mathrm{~m}$ & $\mathrm{~m}$ & $\mathrm{~m}$ & $\mathrm{~m}$ & $\mathrm{~m}$ & $m$ & $\mathrm{~m}$ & $\mathrm{~m}$ & $\mathrm{~m}$ & $\mathrm{~m}$ & $\mathrm{~m}$ & $\mathrm{~m}$ & $\mathrm{~m}$ \\
\hline$\approx$ & Argentinien & 15.8 & $(1.2)$ & 29.2 & (1.4) & 57.5 & (3.4) & 50.5 & $(1.7)$ & 54.4 & (2.3) & 55.8 & $(1.8)$ & 2.1 & $(0.4)$ & 5.9 & $(0.7)$ & 10.8 & (1.1) \\
\hline J & Brasilien & 19.1 & $(0.7)$ & 28.1 & $(0.8)$ & 65.4 & (1.7) & 50.8 & (1.1) & 52.8 & (1.1) & 50.7 & (1.3) & 1.5 & $(0.2)$ & 2.4 & $(0.3)$ & 6.5 & $(0.8)$ \\
\hline$n$ & Bulgarien & 19.2 & $(1.0)$ & 41.7 & (1.6) & 73.3 & (1.7) & 44.6 & (1.9) & 63.0 & (1.7) & 83.4 & $(0.9)$ & 5.5 & $(0.7)$ & 11.5 & $(0.9)$ & 26.3 & $(1.5)$ \\
\hline 5 & Kolumbien & 9.3 & $(0.8)$ & 20.7 & $(1.2)$ & 43.8 & $(2.2)$ & 48.6 & $(2.1)$ & 55.7 & (1.3) & 59.1 & (1.7) & 0.7 & $(0.3)$ & 1.7 & $(0.2)$ & 4.4 & $(0.8)$ \\
\hline$\stackrel{n}{z}$ & Costa Rica & 15.5 & $(1.7)$ & 30.7 & $(1.1)$ & 68.9 & (2.5) & 23.7 & $(1.1)$ & 33.3 & $(1.4)$ & 46.2 & $(2.2)$ & 0.4 & $(0.2)$ & 1.8 & $(0.3)$ & 10.2 & (1.5) \\
\hline$\frac{1}{1}$ & Kroatien & 20.8 & $(1.1)$ & 39.5 & $(1.3)$ & 72.3 & $(2.2)$ & 18.9 & $(1.1)$ & 31.8 & (1.1) & 55.1 & $(2.3)$ & 3.1 & $(0.4)$ & 7.2 & $(0.7)$ & 20.1 & (1.6) \\
\hline อे & Zypern* & 23.4 & $(1.2)$ & 41.1 & $(1.3)$ & 67.3 & (1.3) & 35.6 & $(1.2)$ & 43.3 & (1.3) & 56.6 & $(1.2)$ & 11.0 & $(0.6)$ & 17.0 & $(0.9)$ & 28.5 & (1.1) \\
\hline$\tau$ & Hongkong (China) & 23.2 & $(1.0)$ & 40.4 & $(1.3)$ & 75.0 & (2.6) & 37.5 & $(1.8)$ & 50.4 & (1.5) & 66.0 & $(2.3)$ & 3.8 & $(0.5)$ & 11.0 & $(0.8)$ & 28.2 & $(2.1)$ \\
\hline$\frac{\pi}{2 \pi}$ & Indonesien & 7.9 & $(0.9)$ & 17.2 & $(1.3)$ & 45.3 & (3.8) & 33.2 & $(2.1)$ & 39.2 & (2.0) & 39.9 & $(2.3)$ & 3.0 & $(0.5)$ & 5.3 & $(0.7)$ & 9.5 & (1.3) \\
\hline$\cong$ & Jordanien & 43.0 & (1.4) & 59.4 & $(1.2)$ & 83.7 & (1.3) & 38.5 & (1.3) & 40.6 & $(1.0)$ & 49.6 & $(2.2)$ & 5.4 & $(0.6)$ & 7.9 & $(0.4)$ & 12.7 & (1.3) \\
\hline ป & Kasachstan & 44.6 & $(2.0)$ & 56.1 & $(1.1)$ & 77.0 & (1.4) & 69.8 & $(2.4)$ & 74.6 & (1.6) & 76.1 & (1.8) & 3.5 & $(0.7)$ & 7.9 & $(0.9)$ & 17.0 & (1.9) \\
\hline & Lettland & 21.3 & $(2.2)$ & 46.6 & $(1.2)$ & 76.6 & (1.4) & 49.9 & $(2.7)$ & 65.6 & (1.3) & 80.2 & (1.3) & 10.0 & (1.7) & 17.8 & $(1.0)$ & 31.4 & (1.8) \\
\hline & Liechtenstein & c & c & 71.6 & (3.5) & c & c & c & c & 54.7 & (4.1) & c & c & c & C & 19.3 & (3.6) & c & c \\
\hline & Litauen & 26.9 & (1.4) & 50.5 & $(1.0)$ & 77.0 & (1.5) & 34.7 & (1.4) & 53.2 & (1.4) & 72.5 & (1.5) & 5.6 & $(0.8)$ & 14.1 & $(0.8)$ & 26.4 & (1.8) \\
\hline & Macau (China) & 16.3 & $(0.8)$ & 26.9 & $(1.3)$ & 47.6 & (1.2) & 39.4 & $(0.9)$ & 36.3 & (1.5) & 47.0 & $(1.2)$ & 4.1 & $(0.3)$ & 4.9 & $(0.8)$ & 11.5 & $(0.8)$ \\
\hline & Malaysia & 19.9 & (1.3) & 36.0 & $(1.2)$ & 65.1 & $(2.2)$ & 43.2 & $(1.7)$ & 40.1 & $(1.3)$ & 39.6 & (1.6) & 6.9 & $(0.9)$ & 10.2 & $(1.0)$ & 15.6 & (1.5) \\
\hline & Montenegro & 27.7 & (1.1) & 42.2 & (1.7) & 62.6 & (1.4) & 62.4 & (1.1) & 74.0 & (1.4) & 82.1 & (1.0) & 10.1 & $(0.8)$ & 14.2 & $(1.2)$ & 26.3 & (1.0) \\
\hline & Peru & 5.2 & $(0.6)$ & 16.3 & (1.1) & 43.9 & (2.4) & 77.1 & $(1.3)$ & 73.9 & (1.4) & 66.9 & (1.3) & 1.9 & $(0.3)$ & 2.4 & $(0.3)$ & 5.3 & $(0.8)$ \\
\hline & Katar & 66.3 & $(0.9)$ & 73.3 & $(1.0)$ & 86.4 & $(0.5)$ & 53.8 & $(1.0)$ & 54.2 & (1.0) & 57.3 & $(0.7)$ & 10.9 & $(0.5)$ & 14.6 & $(0.8)$ & 20.5 & $(0.6)$ \\
\hline & Rumänien & 14.3 & (1.1) & 31.4 & (1.4) & 65.4 & (2.6) & 73.7 & (1.6) & 82.4 & (1.3) & 94.0 & $(0.7)$ & 4.1 & $(0.7)$ & 8.5 & $(0.7)$ & 28.9 & (2.0) \\
\hline & Russ. Föderation & 32.3 & $(1.3)$ & 54.2 & (1.0) & 77.2 & (1.5) & 62.5 & $(1.8)$ & 76.6 & (1.0) & 90.4 & $(0.8)$ & 8.3 & $(0.9)$ & 17.4 & (1.0) & 32.7 & (1.5) \\
\hline & Serbien & 21.9 & $(1.2)$ & 40.8 & $(1.1)$ & 70.3 & $(2.1)$ & 56.9 & $(1.8)$ & 69.8 & $(1.2)$ & 85.5 & $(1.3)$ & 3.5 & $(0.5)$ & 9.2 & $(0.7)$ & 25.6 & (1.8) \\
\hline & Shanghai (China) & 34.8 & $(1.6)$ & 56.3 & $(1.2)$ & 78.3 & (1.6) & 66.9 & $(1.7)$ & 76.4 & $(1.2)$ & 86.5 & $(1.0)$ & 3.9 & $(0.4)$ & 8.1 & $(0.7)$ & 24.3 & (1.7) \\
\hline & Singapur & 54.9 & (1.0) & 66.8 & (1.0) & 91.6 & $(0.8)$ & 24.5 & (1.0) & 29.7 & (1.0) & 50.6 & (1.7) & 7.4 & $(0.6)$ & 14.6 & $(0.8)$ & 31.7 & (1.5) \\
\hline & Chinesisch Taipeh & 22.5 & $(1.0)$ & 41.7 & (1.1) & 68.5 & (1.9) & 38.5 & $(1.2)$ & 53.4 & $(1.2)$ & 72.7 & (1.9) & 10.3 & $(0.8)$ & 19.0 & $(0.8)$ & 35.8 & (1.4) \\
\hline & Thailand & 11.1 & $(0.9)$ & 20.1 & (1.3) & 44.9 & (2.1) & 44.7 & (1.4) & 43.0 & (1.6) & 44.3 & (1.6) & 2.1 & $(0.4)$ & 3.1 & $(0.6)$ & 11.7 & (1.0) \\
\hline & Tunesien & 10.2 & (1.0) & 26.1 & (1.3) & 57.6 & $(2.2)$ & 24.5 & (1.6) & 32.8 & (1.3) & 41.7 & $(2.0)$ & 1.1 & $(0.3)$ & 2.5 & $(0.4)$ & 6.6 & $(0.9)$ \\
\hline & Ver. Arab. Emirate & 64.2 & $(1.7)$ & 81.2 & $(1.1)$ & 94.1 & $(0.6)$ & 41.2 & $(1.3)$ & 44.7 & (1.1) & 50.4 & $(0.9)$ & 6.4 & $(0.4)$ & 11.5 & $(0.6)$ & 20.3 & (1.1) \\
\hline & Uruguay & 9.2 & $(0.6)$ & 24.1 & $(0.9)$ & 64.4 & (2.8) & 40.0 & $(1.3)$ & 49.2 & (1.5) & 54.8 & $(2.3)$ & 2.6 & $(0.4)$ & 4.5 & $(0.6)$ & 15.8 & (1.8) \\
\hline & Vietnam & 3.4 & $(0.5)$ & 10.8 & $(1.0)$ & 31.2 & $(3.0)$ & 38.1 & $(2.3)$ & 41.1 & $(2.3)$ & 44.6 & $(1.4)$ & 1.9 & $(0.4)$ & 4.2 & $(0.5)$ & 5.3 & $(0.9)$ \\
\hline
\end{tabular}

1. Eine sozioökonomisch benachteiligte Schule ist eine Schule mit Schülern, deren durchschnittlicher sozioökonomischer Status statistisch signifikant unter dem durchschnittlichen sozioökonomischen Status des Landes/der Volkswirtschaft liegt; eine sozioökonomisch durchschnittliche Schule ist eine Schule, an der es keinen Unterschied zwischen dem durchschnittlichen sozioökonomischen Status der Schüler und des Landes/der Volkswirtschaft gibt; und eine sozioökonomisch begünstigte Schule ist eine Schule mit Schülern, deren durchschnittlicher sozioökonomischer Status statistisch signifikant über dem durchschnittlichen sozioökonomischen Status des Landes/der Volkswirtschaft liegt.

*Vgl. Anmerkungen am Anfang dieses Anhangs.

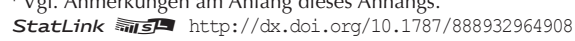


[Teil 1/2]

Leistungen und sozioökonomisches Profil der Schulen

Tabelle II.2.11 Die Ergebnisse basieren auf Schülerangaben

\begin{tabular}{|c|c|c|c|c|c|c|c|c|c|c|c|c|c|}
\hline & \multicolumn{6}{|c|}{$\begin{array}{l}\text { Prozentsatz der Schüler } \\
\text { (in Schulen aller sozioökonomischen Profile) }\end{array}$} & \multicolumn{6}{|c|}{$\begin{array}{l}\text { Prozentsatz der Schüler in Schulen, die sozioökonomisch benachteiligt } \\
\text { sind und ... }\end{array}$} \\
\hline & & \multicolumn{2}{|c|}{$\begin{array}{l}\text { Schulen mit schwachen } \\
\text { Mathematikleistungen }{ }^{1}\end{array}$} & \multicolumn{2}{|c|}{\begin{tabular}{|c|} 
Schulen mit \\
durchschnittlichen \\
Mathematikleistungen 1
\end{tabular}} & \multicolumn{2}{|c|}{\begin{tabular}{|l} 
Schulen mit guten \\
Mathematikleistungen
\end{tabular}} & \multicolumn{2}{|c|}{\begin{tabular}{|c|} 
schwache \\
$\begin{array}{c}\text { Mathematikleistungen } \\
\text { erbracht haben }\end{array}$ \\
\end{tabular}} & \multicolumn{2}{|c|}{$\begin{array}{c}\text { durchschnittliche } \\
\text { Mathematikleistungen } \\
\text { erbracht haben }\end{array}$} & $\begin{array}{r}\begin{array}{r}\text { Mathem } \\
\text { erbr }\end{array} \\
\end{array}$ & $\begin{array}{l}\text { istungen } \\
\text { aben } \\
\end{array}$ \\
\hline & & $\%$ & S.E. & $\%$ & S.E. & $\%$ & S.E. & $\%$ & S.E. & $\%$ & S.E. & $\%$ & S.E. \\
\hline 2 & Australien & 24.4 & (1.6) & 51.2 & $(2.1)$ & 24.4 & $(1.5)$ & 13.6 & $(1.1)$ & 9.8 & $(1.3)$ & 0.4 & $(0.3)$ \\
\hline$\checkmark$ & Österreich & 37.1 & (2.4) & 20.6 & (3.0) & 42.3 & (2.9) & 25.1 & $(2.7)$ & 7.1 & $(2.3)$ & 0.3 & (0.6) \\
\hline$\sqrt{1}$ & Belgien & 33.7 & (1.9) & 27.6 & (2.4) & 38.7 & $(2.0)$ & 23.5 & $(2.0)$ & 4.4 & $(1.3)$ & 1.1 & $(0.7)$ \\
\hline 8 & Kanada & 22.9 & (2.3) & 54.6 & (2.8) & 22.5 & $(2.1)$ & 9.5 & (1.6) & 10.2 & (1.7) & 1.7 & $(0.8)$ \\
\hline 밈 & Chile & 42.7 & (3.5) & 23.3 & (3.9) & 34.0 & (3.3) & 31.6 & (3.3) & 8.2 & $(2.0)$ & 3.0 & (1.5) \\
\hline & Tschech. Rep. & 31.3 & (3.1) & 37.3 & (3.8) & 31.4 & $(2.5)$ & 13.0 & $(2.2)$ & 8.5 & (2.4) & 0.2 & $(0.1)$ \\
\hline & Dänemark & 18.4 & (2.9) & 58.9 & (3.8) & 22.7 & (2.9) & 10.1 & (2.3) & 11.2 & (2.5) & 0.0 & $(0.1)$ \\
\hline & Estland & 19.9 & (2.7) & 59.4 & (3.5) & 20.7 & $(2.3)$ & 7.8 & (1.7) & 10.9 & $(1.8)$ & 0.3 & (0.6) \\
\hline & Finnland & 12.9 & (2.5) & 73.0 & (3.5) & 14.0 & (2.4) & 4.0 & (1.4) & 11.8 & $(2.6)$ & 0.1 & $(0.3)$ \\
\hline & Frankreich & w & w & w & w & w & w & w & w & w & w & w & w \\
\hline & Deutschland & 36.1 & (2.4) & 25.2 & $(2.8)$ & 38.7 & $(2.2)$ & 21.4 & $(2.3)$ & 5.6 & (1.5) & 1.0 & $(0.7)$ \\
\hline & Griechenland & 23.2 & (2.8) & 43.2 & $(4.0)$ & 33.6 & (3.3) & 17.8 & (2.6) & 6.7 & (1.9) & 0.7 & $(0.7)$ \\
\hline & Ungarn & 36.2 & (2.4) & 23.4 & $(2.7)$ & 40.4 & (2.6) & 25.2 & $(2.2)$ & 6.4 & $(2.3)$ & 0.0 & $(0.1)$ \\
\hline & Island & 19.0 & (2.6) & 56.2 & $(3.1)$ & 24.7 & $(1.1)$ & 7.1 & $(0.3)$ & 8.8 & $(0.3)$ & 0.4 & $(0.3)$ \\
\hline & Irland & 18.9 & (2.8) & 60.5 & (3.5) & 20.6 & (2.5) & 14.5 & (2.3) & 2.7 & (1.3) & 0.0 & $(0.0)$ \\
\hline & Israel & 32.4 & (2.7) & 28.0 & (3.3) & 39.6 & (3.7) & 22.4 & $(2.6)$ & 7.3 & $(2.1)$ & 0.0 & $(0.0)$ \\
\hline & Italien & 38.6 & $(1.7)$ & 21.3 & $(2.0)$ & 40.0 & (1.9) & 22.1 & $(1.4)$ & 3.8 & $(0.9)$ & 2.9 & $(0.7)$ \\
\hline & Japan & 40.5 & (2.9) & 22.2 & (3.1) & 37.3 & (3.0) & 23.7 & (2.4) & 4.4 & (1.6) & 1.4 & (1.0) \\
\hline & Korea & 30.6 & (2.6) & 33.2 & (3.8) & 36.2 & (3.5) & 20.8 & $(2.4)$ & 5.2 & (1.8) & 0.7 & $(0.7)$ \\
\hline & Luxemburg & 42.6 & (0.4) & 15.4 & $(0.4)$ & 42.0 & $(0.5)$ & 39.7 & $(0.1)$ & 8.2 & $(0.1)$ & 0.0 & $(0.0)$ \\
\hline & Mexiko & 29.5 & (1.5) & 40.7 & $(2.0)$ & 29.8 & $(1.7)$ & 17.5 & (1.4) & 13.9 & (1.3) & 2.8 & $(0.6)$ \\
\hline & Niederlande & 40.7 & (2.9) & 13.9 & (2.9) & 45.4 & (2.5) & 20.5 & (2.6) & 2.9 & $(0.9)$ & 0.0 & $(0.0)$ \\
\hline & Neuseeland & 25.6 & (2.6) & 48.9 & (3.4) & 25.5 & (2.3) & 14.6 & (2.3) & 6.8 & $(2.2)$ & 0.1 & $(0.1)$ \\
\hline & Norwegen & 18.2 & (3.1) & 62.1 & (3.7) & 19.7 & (3.1) & 4.1 & (1.6) & 5.8 & (1.8) & 0.2 & $(0.3)$ \\
\hline & Polen & 26.2 & (3.8) & 53.9 & $(4.2)$ & 19.9 & (3.1) & 15.3 & (3.0) & 12.1 & (2.5) & 0.1 & $(0.4)$ \\
\hline & Portugal & 28.8 & (3.3) & 37.7 & $(4.1)$ & 33.5 & $(3.7)$ & 21.4 & (3.0) & 10.8 & (3.0) & 1.4 & (1.5) \\
\hline & Slowak. Rep. & 30.2 & (3.3) & 38.9 & $(4.0)$ & 30.9 & (2.5) & 19.5 & (2.6) & 7.0 & (1.5) & 0.4 & $(0.4)$ \\
\hline & Slowenien & 42.0 & $(0.8)$ & 16.9 & (1.4) & 41.0 & $(1.1)$ & 24.3 & $(0.6)$ & 4.0 & $(0.7)$ & 1.1 & $(0.5)$ \\
\hline & Spanien & 25.3 & (2.3) & 49.9 & (3.1) & 24.8 & $(2.2)$ & 17.3 & (2.4) & 12.0 & $(2.2)$ & 0.1 & $(0.1)$ \\
\hline & Schweden & 18.5 & (3.0) & 64.2 & $(4.2)$ & 17.3 & (2.9) & 8.1 & $(2.2)$ & 9.3 & (2.4) & 0.6 & (0.6) \\
\hline & Schweiz & 35.5 & (3.1) & 37.1 & (3.1) & 27.4 & (2.8) & 16.1 & $(2.7)$ & 8.0 & (1.6) & 2.4 & (1.3) \\
\hline & Türkei & 52.5 & (3.6) & 19.3 & $(2.7)$ & 28.2 & $(2.8)$ & 25.4 & $(3.1)$ & 5.3 & (1.9) & 1.4 & $(1.0)$ \\
\hline & Ver. Königreich & 26.9 & (3.2) & 47.7 & (3.8) & 25.4 & (2.8) & 14.9 & (2.9) & 9.2 & $(2.2)$ & 0.1 & $(0.2)$ \\
\hline & Ver. Staaten & 32.9 & (3.9) & 37.9 & $(4.3)$ & 29.2 & (3.8) & 21.1 & (3.1) & 5.6 & $(2.0)$ & 0.0 & $(0.0)$ \\
\hline & OECD-Durchschnitt & 30.3 & (0.5) & 38.8 & $(0.6)$ & 30.8 & $(0.5)$ & 18.1 & $(0.4)$ & 7.5 & $(0.3)$ & 0.8 & $(0.1)$ \\
\hline ร & Albanien & 9.4 & (2.6) & 77.8 & (3.8) & 12.8 & (2.6) & $\mathrm{m}$ & $\mathrm{m}$ & $\mathrm{m}$ & $\mathrm{m}$ & $\mathrm{m}$ & $\mathrm{m}$ \\
\hline 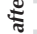 & Argentinien & 33.4 & (3.5) & 29.4 & (3.5) & 37.2 & (3.9) & 24.2 & (3.1) & 9.0 & $(2.2)$ & 0.2 & $(0.3)$ \\
\hline క్ & Brasilien & 33.3 & (2.3) & 39.8 & (3.0) & 26.9 & $(2.1)$ & 17.8 & (1.9) & 12.1 & $(2.1)$ & 2.3 & (1.0) \\
\hline 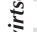 & Bulgarien & 40.5 & (3.4) & 25.7 & (3.5) & 33.9 & $(2.8)$ & 27.7 & (3.0) & 4.8 & (1.8) & 1.2 & (1.1) \\
\hline ज्ञ & Kolumbien & 28.7 & (3.1) & 44.1 & (3.8) & 27.3 & (3.4) & 19.0 & (2.8) & 9.3 & (2.3) & 0.4 & $(0.4)$ \\
\hline 亏 & Costa Rica & 32.3 & (3.4) & 41.0 & (3.4) & 26.7 & (3.4) & 18.9 & (3.0) & 9.5 & $(2.5)$ & 0.1 & $(0.3)$ \\
\hline T & Kroatien & 37.2 & (3.3) & 32.6 & (3.4) & 30.2 & $(2.5)$ & 19.8 & $(2.8)$ & 12.4 & $(2.3)$ & 0.8 & $(0.7)$ \\
\hline$\frac{2}{0}$ & Zypern* & 29.6 & $(0.8)$ & 34.7 & (1.8) & 35.7 & (1.4) & 20.1 & (1.1) & 13.4 & $(0.7)$ & 1.1 & (1.1) \\
\hline בิ & Hongkong (China) & 35.9 & (3.4) & 23.0 & $(3.7)$ & 41.1 & $(2.8)$ & 25.5 & (3.0) & 6.6 & $(2.4)$ & 3.3 & (1.5) \\
\hline 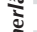 & Indonesien & 36.5 & (3.5) & 34.1 & $(4.4)$ & 29.4 & $(3.7)$ & 18.8 & (3.3) & 14.6 & (3.6) & 6.5 & $(2.1)$ \\
\hline ミ & Jordanien & 31.4 & (3.8) & 43.5 & $(4.2)$ & 25.1 & (3.1) & 10.9 & $(2.1)$ & 7.8 & $(2.2)$ & 2.0 & (1.1) \\
\hline$\tilde{2}$ & Kasachstan & 39.4 & (3.3) & 35.3 & (3.8) & 25.3 & (3.4) & 15.7 & $(3.2)$ & 4.4 & $(1.8)$ & 2.7 & $(1.2)$ \\
\hline & Lettland & 20.5 & (3.2) & 54.7 & (3.8) & 24.9 & (2.8) & 9.0 & (1.9) & 11.2 & (2.4) & 0.0 & $(0.0)$ \\
\hline & Liechtenstein & $\mathrm{C}$ & c & $\mathrm{C}$ & C & C & c & C & c & C & $\mathrm{C}$ & C & $\mathrm{c}$ \\
\hline & Litauen & 27.1 & (3.2) & 44.2 & $(4.1)$ & 28.6 & $(2.9)$ & 15.7 & $(2.2)$ & 6.9 & (1.4) & 0.3 & $(0.3)$ \\
\hline & Macau (China) & 33.8 & $(0.1)$ & 22.5 & (1.4) & 43.7 & (1.4) & 26.4 & $(0.0)$ & 9.0 & $(0.6)$ & 16.2 & (0.6) \\
\hline & Malaysia & 35.5 & (3.7) & 39.3 & (3.9) & 25.2 & $(3.2)$ & 21.0 & (2.6) & 7.5 & $(2.2)$ & 0.1 & $(0.3)$ \\
\hline & Montenegro & 46.4 & (2.1) & 22.3 & (2.4) & 31.4 & (3.4) & 37.2 & $(0.7)$ & 1.5 & $(0.7)$ & 0.0 & $(0.0)$ \\
\hline & Peru & 36.9 & (2.7) & 29.1 & (3.5) & 34.0 & (3.4) & 26.9 & (2.5) & 7.7 & (1.9) & 0.4 & $(0.4)$ \\
\hline & Katar & 56.1 & $(0.4)$ & 7.2 & $(0.5)$ & 36.7 & $(0.4)$ & 23.2 & $(0.3)$ & 1.9 & $(0.3)$ & 8.6 & $(0.1)$ \\
\hline & Rumänien & 40.3 & (3.5) & 29.5 & $(3.5)$ & 30.1 & (2.9) & 22.8 & (3.2) & 6.4 & (1.9) & 1.6 & (1.1) \\
\hline & Russ. Föderation & 27.3 & (2.9) & 47.5 & $(3.5)$ & 25.3 & (3.1) & 12.5 & $(2.1)$ & 7.3 & (2.0) & 2.1 & (1.0) \\
\hline & Serbien & 42.0 & (3.4) & 22.8 & (3.2) & 35.3 & (2.9) & 25.2 & (3.1) & 6.9 & $(2.2)$ & 0.1 & $(0.5)$ \\
\hline & Shanghai (China) & 37.2 & (2.6) & 25.1 & $(3.2)$ & 37.7 & $(2.4)$ & 25.6 & $(2.8)$ & 3.6 & (1.5) & 0.3 & $(0.6)$ \\
\hline & Singapur & 35.6 & (2.3) & 36.6 & $(2.6)$ & 27.8 & $(0.7)$ & 19.9 & $(1.8)$ & 10.6 & $(1.8)$ & 0.0 & $(0.0)$ \\
\hline & Chinesisch Taipeh & 36.8 & (3.2) & 30.8 & $(4.1)$ & 32.4 & (3.3) & 25.8 & (2.9) & 3.7 & (1.5) & 0.6 & $(0.7)$ \\
\hline & Thailand & 39.6 & (3.2) & 32.5 & $(3.7)$ & 27.9 & (3.4) & 23.9 & (3.0) & 10.3 & $(2.3)$ & 4.9 & (1.5) \\
\hline & Tunesien & 39.2 & (3.6) & 23.2 & $(4.0)$ & 37.5 & (3.5) & 20.0 & (3.0) & 6.8 & (2.0) & 2.7 & (1.4) \\
\hline & Ver. Arab. Emirate & 37.8 & (2.9) & 32.5 & $(2.9)$ & 29.7 & $(2.0)$ & 17.4 & (1.5) & 7.5 & (1.4) & 0.4 & $(0.5)$ \\
\hline & Uruguay & 38.4 & (2.6) & 27.2 & $(3.2)$ & 34.4 & $(2.3)$ & 29.3 & $(2.5)$ & 9.7 & $(2.1)$ & 1.3 & (1.1) \\
\hline & Vietnam & 30.0 & (3.7) & 34.4 & $(4.8)$ & 35.6 & $(4.2)$ & 21.7 & (3.3) & 14.2 & $(2.7)$ & 3.5 & $(1.3)$ \\
\hline
\end{tabular}

1. Fine sozioökonomisch benachteiligte Schule ist eine Schule mit Schülern, deren durchschnittlicher sozioökonomischer Status statistisch signifikant unter dem durchschnittlichen sozioökonomischen Status des Landes/der Volkswirtschaft liegt; eine sozioökonomisch durchschnittliche Schule ist eine Schule, an der es keinen Unterschied zwischen dem durchschnittlichen sozioökonomischen Status der Schüler und des Landes/der Volkswirtschaft gibt; und eine sozioökonomisch begünstigte Schule ist eine Schule mit Schülern, deren durchschnittlicher sozioökonomischer Status statistisch signifikant über dem durchschnittlichen sozioökonomischen Status des Landes/der Volkswirtschaft liegt.

*Vgl. Anmerkungen am Anfang dieses Anhangs.

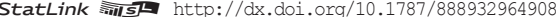


[Teil 2/2]

Leistungen und sozioökonomisches Profil der Schulen

Tabelle II.2.11 Die Ergebnisse basieren auf Schülerangaben

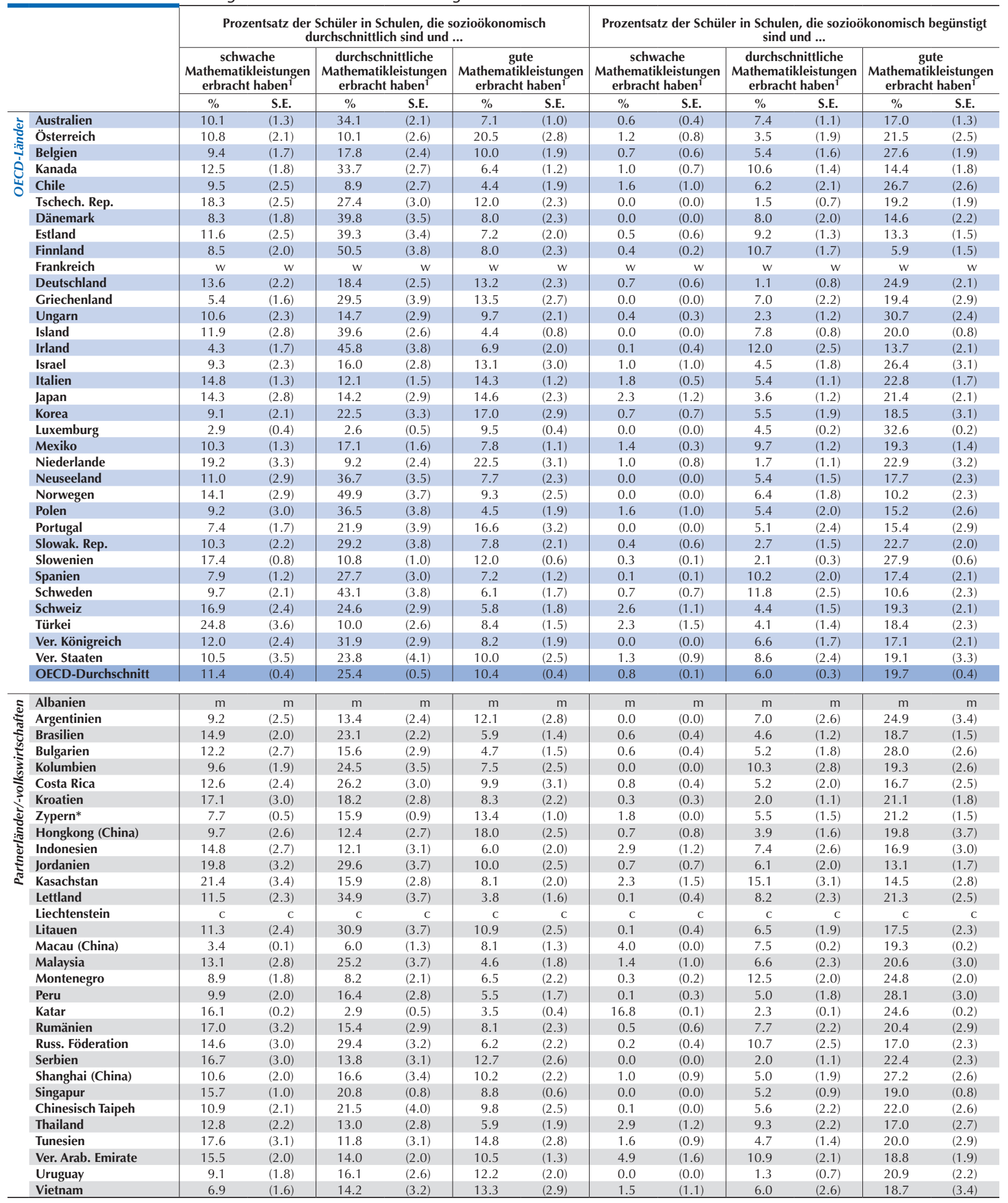

1. Eine sozioökonomisch benachteiligte Schule ist eine Schule mit Schülern, deren durchschnittlicher sozioökonomischer Status statistisch signifikant unter dem durchschnittlichen sozioökonomischen Status des Landes/der Volkswirtschaft liegt; eine sozioökonomisch durchschnittliche Schule ist eine Schule, an der es keinen Unterschied zwischen dem durchschnittlichen sozioökonomischen Status der Schüler und des Landes/der Volkswirtschaft gibt; und eine sozioökonomisch begünstigte Schule ist eine Schule mit Schülern, deren durchschnittlicher sozioökonomischer Status statistisch signifikant über dem durchschnittlichen sozioökonomischen Status des Landes/der Volkswirtschaft liegt.

* Vgl. Anmerkungen am Anfang dieses Anhangs.

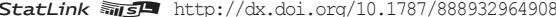


[Teil 1/2]

Tabelle II.2.12 Veränderung der Bildungsteilnahme 15-Jähriger zwischen 2003 und 2012

\begin{tabular}{|c|c|c|c|c|c|c|c|c|c|c|}
\hline & & \multicolumn{3}{|c|}{ PISA 2003} & \multicolumn{3}{|c|}{ PISA 2012} & \multicolumn{3}{|c|}{$\begin{array}{l}\text { Veränderung zwischen } 2003 \text { und } 2012 \\
\text { (PISA } 2012 \text { - PISA 2003) }\end{array}$} \\
\hline & & $\begin{array}{c}\text { Gesamt- } \\
\text { population der } \\
\text { 15-Jährigen }\end{array}$ & $\begin{array}{c}\text { Gesamtzahl } \\
\text { der 15-Jährigen } \\
\text { in Klassenstufe } \\
7 \text { oder darüber }\end{array}$ & \begin{tabular}{|} 
Erfassungs- \\
index 3: \\
Prozentsatz \\
der an Bildung \\
teilnehmenden \\
Bevölkerung
\end{tabular} & $\begin{array}{c}\text { Gesamt- } \\
\text { population der } \\
\text { 15-Jährigen }\end{array}$ & $\begin{array}{c}\text { Gesamtzahl } \\
\text { der 15-Jährigen } \\
\text { in Klassenstufe } \\
7 \text { oder darüber }\end{array}$ & \begin{tabular}{|} 
Erfassungs- \\
index 3: \\
Prozentsatz \\
der an Bildung \\
teilnehmenden \\
Bevölkerung
\end{tabular} & \begin{tabular}{|c} 
Gesamt- \\
population der \\
15-Jährigen
\end{tabular} & $\begin{array}{c}\text { Gesamtzahl } \\
\text { der 15-Jährigen } \\
\text { in Klassenstufe } \\
7 \text { oder darüber }\end{array}$ & $\begin{array}{c}\text { Erfassungs- } \\
\text { index 3: } \\
\text { Prozentsatz } \\
\text { der an Bildung } \\
\text { teilnehmenden } \\
\text { Bevölkerung }\end{array}$ \\
\hline \multirow{34}{*}{ 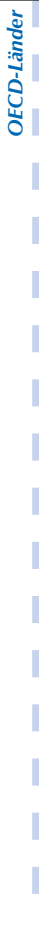 } & Australien & 268164 & 250635 & 93 & 291967 & 288159 & 99 & 23803 & 37524 & 5.2 \\
\hline & Österreich & 94515 & 89049 & 94 & 93537 & 89073 & 95 & -978 & 24 & 1.0 \\
\hline & Belgien & 120802 & 118185 & 98 & 123469 & 121493 & 98 & 2667 & 3308 & 0.6 \\
\hline & Kanada & 399265 & 399265 & 100 & 417873 & 409453 & 98 & 18608 & 10188 & -2.0 \\
\hline & Chile & $\mathrm{m}$ & $\mathrm{m}$ & $\mathrm{m}$ & 274803 & 252733 & 92 & $\mathrm{~m}$ & $\mathrm{~m}$ & $\mathrm{~m}$ \\
\hline & Tschech. Rep. & 130679 & 126348 & 97 & 96946 & 93214 & 96 & -33733 & -33134 & -0.5 \\
\hline & Dänemark & 59156 & 58188 & 98 & 72310 & 70854 & 98 & 13154 & 12666 & -0.4 \\
\hline & Estland & $\mathrm{m}$ & $\mathrm{m}$ & $\mathrm{m}$ & 12649 & 12438 & 98 & $\mathrm{~m}$ & $\mathrm{~m}$ & $\mathrm{~m}$ \\
\hline & Finnland & 61107 & 61107 & 100 & 62523 & 62195 & 99 & 1416 & 1088 & -0.5 \\
\hline & Frankreich & 809053 & 808276 & 100 & 792983 & 755447 & 95 & -16070 & -52829 & -4.6 \\
\hline & Deutschland & 951800 & 916869 & 96 & 798136 & 798136 & 100 & -153664 & -118733 & 3.7 \\
\hline & Griechenland & 111286 & 108314 & 97 & 110521 & 105096 & 95 & -765 & -3218 & -2.2 \\
\hline & Ungarn & 129138 & 123762 & 96 & 111761 & 108816 & 97 & -17377 & -14946 & 1.5 \\
\hline & Island & 4168 & 4112 & 99 & 4505 & 4491 & 100 & 337 & 379 & 1.0 \\
\hline & Irland & 61535 & 58997 & 96 & 59296 & 57979 & 98 & -2239 & -1018 & 1.9 \\
\hline & Israel & $\mathrm{m}$ & $\mathrm{m}$ & $\mathrm{m}$ & 118953 & 113278 & 95 & $\mathrm{~m}$ & $\mathrm{~m}$ & $\mathrm{~m}$ \\
\hline & Italien & 574611 & 574611 & 100 & 605490 & 566973 & 94 & 30879 & -7638 & -6.4 \\
\hline & Japan & 1365471 & 1328498 & 97 & 1241786 & 1214756 & 98 & -123685 & -113742 & 0.5 \\
\hline & Korea & 606722 & 606370 & 100 & 687104 & 672101 & 98 & 80382 & 65731 & -2.1 \\
\hline & Luxemburg & 4204 & 4204 & 100 & 6187 & 6082 & 98 & 1983 & 1878 & -1.7 \\
\hline & Mexiko & 2192452 & 1273163 & 58 & 2114745 & 1472875 & 70 & -77707 & 199712 & 11.6 \\
\hline & Niederlande & 194216 & 194216 & 100 & 194000 & 193190 & 100 & -216 & -1026 & -0.4 \\
\hline & Neuseeland & 55440 & 53293 & 96 & 60940 & 59118 & 97 & 5500 & 5825 & 0.9 \\
\hline & Norwegen & 56060 & 55648 & 99 & 64917 & 64777 & 100 & 8857 & 9129 & 0.5 \\
\hline & Polen & 589506 & 569294 & 97 & 425597 & 410700 & 96 & -163909 & -158594 & -0.1 \\
\hline & Portugal & 109149 & 99216 & 91 & 127537 & 127537 & 100 & 18388 & 28321 & 9.1 \\
\hline & Slowak. Rep. & 84242 & 81945 & 97 & 59723 & 59367 & 99 & -24519 & -22578 & 2.1 \\
\hline & Slowenien & $\mathrm{m}$ & $\mathrm{m}$ & $\mathrm{m}$ & 19471 & 18935 & 97 & $\mathrm{~m}$ & $\mathrm{~m}$ & $\mathrm{~m}$ \\
\hline & Spanien & 454064 & 418005 & 92 & 423444 & 404374 & 95 & -30620 & -13631 & 3.4 \\
\hline & Schweden & 112258 & 112258 & 100 & 102087 & 102027 & 100 & -10171 & -10231 & -0.1 \\
\hline & Schweiz & 83247 & 81020 & 97 & 87200 & 85239 & 98 & 3953 & 4219 & 0.4 \\
\hline & Türkei & 1351492 & 725030 & 54 & 1266638 & 965736 & 76 & -84854 & 240706 & 22.6 \\
\hline & Ver. Königreich & 768180 & 736785 & 96 & 745581 & 745581 & 100 & -22599 & 8796 & 4.1 \\
\hline & Ver. Staaten & 3979116 & 3979116 & 100 & 4074457 & 4074457 & 100 & 95341 & 95341 & 0.0 \\
\hline \multirow{31}{*}{ 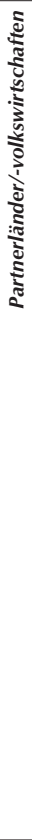 } & Albanien & $\mathrm{m}$ & $\mathrm{m}$ & $\mathrm{m}$ & 76910 & 50157 & 65 & $\mathrm{~m}$ & $\mathrm{~m}$ & $\mathrm{~m}$ \\
\hline & Argentinien & $\mathrm{m}$ & $\mathrm{m}$ & $\mathrm{m}$ & 684879 & 637603 & 93 & $\mathrm{~m}$ & $\mathrm{~m}$ & $\mathrm{~m}$ \\
\hline & Brasilien & 3618332 & 2359854 & 65 & 3574928 & 2786064 & 78 & -43404 & 426210 & 12.7 \\
\hline & Bulgarien & $\mathrm{m}$ & $\mathrm{m}$ & $\mathrm{m}$ & 70188 & 59684 & 85 & $\mathrm{~m}$ & $\mathrm{~m}$ & $\mathrm{~m}$ \\
\hline & Kolumbien & $\mathrm{m}$ & $\mathrm{m}$ & $\mathrm{m}$ & 889729 & 620422 & 70 & $\mathrm{~m}$ & $\mathrm{~m}$ & $\mathrm{~m}$ \\
\hline & Costa Rica & $\mathrm{m}$ & $\mathrm{m}$ & $\mathrm{m}$ & 81489 & 64326 & 79 & $\mathrm{~m}$ & $\mathrm{~m}$ & $\mathrm{~m}$ \\
\hline & Kroatien & $\mathrm{m}$ & $\mathrm{m}$ & $\mathrm{m}$ & 48155 & 46550 & 97 & $\mathrm{~m}$ & $\mathrm{~m}$ & $\mathrm{~m}$ \\
\hline & Zypern* & $\mathrm{m}$ & $\mathrm{m}$ & $\mathrm{m}$ & $\mathrm{m}$ & $\mathrm{m}$ & $\mathrm{m}$ & $\mathrm{m}$ & $\mathrm{m}$ & $\mathrm{m}$ \\
\hline & Hongkong (China) & 75000 & 72631 & 97 & 84200 & 77864 & 92 & 9200 & 5233 & -4.4 \\
\hline & Indonesien & 4281895 & 3113548 & 73 & 4174217 & 3599844 & 86 & -107678 & 486296 & 13.5 \\
\hline & Jordanien & $\mathrm{m}$ & $\mathrm{m}$ & $\mathrm{m}$ & 129492 & 125333 & 97 & $\mathrm{~m}$ & $\mathrm{~m}$ & $\mathrm{~m}$ \\
\hline & Kasachstan & $\mathrm{m}$ & $\mathrm{m}$ & $\mathrm{m}$ & 258716 & 247048 & 95 & $\mathrm{~m}$ & $\mathrm{~m}$ & $\mathrm{~m}$ \\
\hline & Lettland & 37544 & 37138 & 99 & 18789 & 18389 & 98 & -18755 & -18749 & -1.0 \\
\hline & Liechtenstein & 402 & 348 & 87 & 417 & 383 & 92 & 15 & 35 & 5.3 \\
\hline & Litauen & $\mathrm{m}$ & $\mathrm{m}$ & $\mathrm{m}$ & 38524 & 35567 & 92 & $\mathrm{~m}$ & $\mathrm{~m}$ & $\mathrm{~m}$ \\
\hline & Macau (China) & 8318 & 6939 & 83 & 6600 & 5416 & 82 & -1718 & -1523 & -1.4 \\
\hline & Malaysia & $\mathrm{m}$ & $\mathrm{m}$ & $\mathrm{m}$ & 544302 & 457999 & 84 & $\mathrm{~m}$ & $\mathrm{~m}$ & $\mathrm{~m}$ \\
\hline & Montenegro & $\mathrm{m}$ & $\mathrm{m}$ & $\mathrm{m}$ & 8600 & 8600 & 100 & $\mathrm{~m}$ & $\mathrm{~m}$ & $\mathrm{~m}$ \\
\hline & Peru & $\mathrm{m}$ & $\mathrm{m}$ & $\mathrm{m}$ & 584294 & 508969 & 87 & $\mathrm{~m}$ & $\mathrm{~m}$ & $\mathrm{~m}$ \\
\hline & Katar & $\mathrm{m}$ & $\mathrm{m}$ & $\mathrm{m}$ & 11667 & 11532 & 99 & $\mathrm{~m}$ & $\mathrm{~m}$ & $\mathrm{~m}$ \\
\hline & Rumänien & $\mathrm{m}$ & $\mathrm{m}$ & $\mathrm{m}$ & 146243 & 146243 & 100 & $\mathrm{~m}$ & $\mathrm{~m}$ & $\mathrm{~m}$ \\
\hline & Russ. Föderation & 2496216 & 2366285 & 95 & 1272632 & 1268814 & 100 & -1223584 & -1097471 & 4.9 \\
\hline & Serbien & 98729 & 92617 & 94 & 80089 & 75870 & 95 & -18640 & -16747 & 0.9 \\
\hline & Shanghai (China) & $\mathrm{m}$ & $\mathrm{m}$ & $\mathrm{m}$ & 108056 & 90796 & 84 & $\mathrm{~m}$ & $\mathrm{~m}$ & $\mathrm{~m}$ \\
\hline & Singapur & $\mathrm{m}$ & $\mathrm{m}$ & $\mathrm{m}$ & 53637 & 52163 & 97 & $\mathrm{~m}$ & $\mathrm{~m}$ & $\mathrm{~m}$ \\
\hline & Chinesisch Taipeh & $\mathrm{m}$ & $\mathrm{m}$ & $\mathrm{m}$ & 328356 & 328336 & 100 & $\mathrm{~m}$ & $\mathrm{~m}$ & $\mathrm{~m}$ \\
\hline & Thailand & 927070 & 778267 & 84 & 982080 & 784897 & 80 & 55010 & 6630 & -4.0 \\
\hline & Tunesien & 164758 & 164758 & 100 & 132313 & 132313 & 100 & -32445 & -32445 & 0.0 \\
\hline & Ver. Arab. Emirate & $\mathrm{m}$ & $\mathrm{m}$ & $\mathrm{m}$ & 48824 & 48446 & 99 & $\mathrm{~m}$ & $\mathrm{~m}$ & $\mathrm{~m}$ \\
\hline & Uruguay & 53948 & 40023 & 74 & 54638 & 46442 & 85 & 690 & 6419 & 10.8 \\
\hline & Vietnam & $\mathrm{m}$ & $\mathrm{m}$ & $\mathrm{m}$ & 1717996 & 1091462 & 64 & $\mathrm{~m}$ & $\mathrm{~m}$ & $\mathrm{~m}$ \\
\hline
\end{tabular}

Anmerkung: Diese Daten sind Anhang 2 von PISA 2003 sowie Anhang A2 der vorliegenden Publikation entnommen. Wenn der Anteil der Schüler ab Klassenstufe 7 bei über 100 lag, wurde der Wert für die Gesamtzahl der 15-ährigen entsprechend dem Anteil der an Bildung teilnehmenden 15-Jährigen festgelegt. Diese Daten sind verschiedenen nationalen Datenquellen entnommen, z.B. wird die Gesamtbevölkerung den nationalen Volkszählungen entnommen, die lediglich in bestimmten Jahren durchgeführt werden. Es ist daher möglich, dass die Bevölkerungsdaten auf einer älteren Quelle beruhen als die Schulbesuchsdaten. Diese Unterschiede können zu einer Schätzung der an Bildung teilnehmenden Bevölkerung führen, die größer ist als die Gesamtbevölkerung, was natürlich nicht möglich ist.

* Vgl. Anmerkungen am Anfang dieses Anhangs.

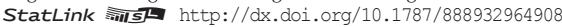


Tabelle II.2.12 Veränderung der Bildungsteilnahme 15-Jähriger zwischen 2003 und 2012

\begin{tabular}{|c|c|c|c|c|c|c|c|c|}
\hline & & \multirow{2}{*}{\begin{tabular}{|c|} 
Veränderung \\
zwischen 2003 und \\
2012 in Prozent \\
(PISA 2012 - PISA \\
2003)/(PISA 2003) \\
\\
\\
Gesamtzahl der \\
15-Jährigen in \\
Klassenstufe 7 \\
oder darüber
\end{tabular}} & \multirow[b]{2}{*}{$\begin{array}{l}\text { Durchschnitts- } \\
\text { leistungen in } \\
\text { Mathematik } \\
\text { in PISA } 2012\end{array}$} & \multirow{2}{*}{\begin{tabular}{|} 
Durchschnitts- \\
leistungen in \\
Mathematik in \\
PISA 2012, wenn \\
diejenigen, die \\
nicht an Bildung \\
teilnehmen, am \\
unteren Ende der \\
Kompetenzstufe 1 \\
(d.h. 357,77 \\
Punkte erzielen) \\
abschneiden würden
\end{tabular}} & \multirow{2}{*}{$\begin{array}{c}\text { Leistungs- } \\
\text { unterschiede in } \\
\text { Mathematik nach } \\
\text { Berücksichtigung } \\
\text { derjenigen, } \\
\text { die im Alter von } \\
15 \text { Jahren keine } \\
\text { Schule besuchen }\end{array}$} & \multirow[b]{2}{*}{$\begin{array}{l}\text { Rangplatz unter } \\
\text { allen Ländern vor } \\
\text { Berücksichtigung }\end{array}$} & \multirow[b]{2}{*}{$\begin{array}{l}\text { Rangplatz unter } \\
\text { allen Ländern nach } \\
\text { Berücksichtigung }\end{array}$} & \multirow[b]{2}{*}{$\begin{array}{l}\text { Veränderung des } \\
\text { Rangplatzes nach } \\
\text { Berücksichtigung } \\
\text { (vorher - nachher) }\end{array}$} \\
\hline & & & & & & & & \\
\hline \multirow{34}{*}{ 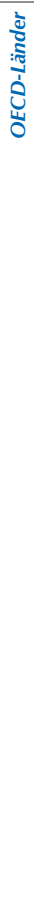 } & Australien & 15.0 & 504 & 502 & 2 & 19 & 17 & 2 \\
\hline & Österreich & 0.0 & 506 & 498 & 7 & 18 & 18 & 0 \\
\hline & Belgien & 2.8 & 515 & 512 & 3 & 15 & 14 & 1 \\
\hline & Kanada & 2.6 & 518 & 515 & 3 & 13 & 12 & 1 \\
\hline & Chile & $\mathrm{m}$ & 423 & 417 & 5 & 52 & 49 & 3 \\
\hline & Tschech. Rep. & -26.2 & 499 & 494 & 5 & 24 & 24 & 0 \\
\hline & Dänemark & 21.8 & 500 & 497 & 3 & 22 & 21 & 1 \\
\hline & Estland & $\mathrm{m}$ & 521 & 518 & 3 & 11 & 11 & 0 \\
\hline & Finnland & 1.8 & 519 & 518 & 1 & 12 & 10 & 2 \\
\hline & Frankreich & -6.5 & 495 & 488 & 6 & 25 & 27 & -2 \\
\hline & Deutschland & -12.9 & 514 & 514 & 0 & 16 & 13 & 3 \\
\hline & Griechenland & -3.0 & 453 & 448 & 5 & 43 & 42 & 1 \\
\hline & Ungarn & -12.1 & 477 & 474 & 3 & 40 & 37 & 3 \\
\hline & Island & 9.2 & 493 & 492 & 0 & 28 & 25 & 3 \\
\hline & Irland & -1.7 & 501 & 498 & 3 & 20 & 19 & 1 \\
\hline & Israel & $\mathrm{m}$ & 466 & 461 & 5 & 42 & 40 & 2 \\
\hline & Italien & -1.3 & 485 & 477 & 8 & 33 & 36 & -3 \\
\hline & Japan & -8.6 & 536 & 533 & 4 & 7 & 6 & 1 \\
\hline & Korea & 10.8 & 554 & 549 & 4 & 5 & 4 & 1 \\
\hline & Luxemburg & 44.7 & 490 & 488 & 2 & 30 & 29 & 1 \\
\hline & Mexiko & 15.7 & 413 & 396 & 17 & 54 & 55 & -1 \\
\hline & Niederlande & -0.5 & 523 & 522 & 1 & 10 & 8 & 2 \\
\hline & Neuseeland & 10.9 & 500 & 496 & 4 & 23 & 22 & 1 \\
\hline & Norwegen & 16.4 & 489 & 489 & 0 & 31 & 26 & 5 \\
\hline & Polen & -27.9 & 518 & 512 & 6 & 14 & 15 & -1 \\
\hline & Portugal & 28.5 & 487 & 487 & 0 & 32 & 30 & 2 \\
\hline & Slowak. Rep. & -27.6 & 482 & 481 & 1 & 36 & 33 & 3 \\
\hline & Slowenien & $\mathrm{m}$ & 501 & 497 & 4 & 21 & 20 & 1 \\
\hline & Spanien & -3.3 & 484 & 479 & 6 & 34 & 34 & 0 \\
\hline & Schweden & -9.1 & 478 & 478 & 0 & 39 & 35 & 4 \\
\hline & Schweiz & 5.2 & 531 & 527 & 4 & 9 & 7 & 2 \\
\hline & Türkei & 33.2 & 448 & 427 & 21 & 45 & 48 & -3 \\
\hline & Ver. Königreich & 1.2 & 494 & 494 & 0 & 27 & 23 & 4 \\
\hline & Ver. Staaten & 2.4 & 481 & 481 & 0 & 37 & 32 & 5 \\
\hline \multirow{31}{*}{ 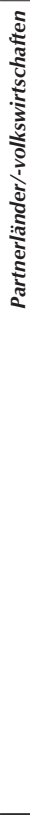 } & Albanien & $\mathrm{m}$ & 394 & 382 & 13 & 58 & 60 & -2 \\
\hline & Argentinien & $\mathrm{m}$ & 388 & 386 & 2 & 60 & 57 & 3 \\
\hline & Brasilien & 18.1 & 391 & 384 & 7 & 59 & 59 & 0 \\
\hline & Bulgarien & $\mathrm{m}$ & 439 & 427 & 12 & 48 & 47 & 1 \\
\hline & Kolumbien & $\mathrm{m}$ & 376 & 371 & 6 & 63 & 63 & 0 \\
\hline & Costa Rica & $\mathrm{m}$ & 407 & 397 & 10 & 57 & 54 & 3 \\
\hline & Kroatien & $\mathrm{m}$ & 471 & 467 & 4 & 41 & 39 & 2 \\
\hline & Zypern* & $\mathrm{m}$ & 440 & $\mathrm{~m}$ & $\mathrm{~m}$ & $\mathrm{~m}$ & $\mathrm{~m}$ & $\mathrm{~m}$ \\
\hline & Hongkong (China) & 7.2 & 561 & 546 & 15 & 3 & 5 & -2 \\
\hline & Indonesien & 15.6 & 375 & 373 & 2 & 65 & 62 & 3 \\
\hline & Jordanien & $\mathrm{m}$ & 386 & 385 & 1 & 62 & 58 & 4 \\
\hline & Kasachstan & $\mathrm{m}$ & 432 & 428 & 3 & 50 & 46 & 4 \\
\hline & Lettland & -50.5 & 491 & 488 & 3 & 29 & 28 & 1 \\
\hline & Liechtenstein & 10.1 & 535 & 521 & 14 & 8 & 9 & -1 \\
\hline & Litauen & $\mathrm{m}$ & 479 & 470 & 9 & 38 & 38 & 0 \\
\hline & Macau (China) & -21.9 & 538 & 506 & 32 & 6 & 16 & -10 \\
\hline & Malaysia & $\mathrm{m}$ & 421 & 411 & 10 & 53 & 51 & 2 \\
\hline & Montenegro & $\mathrm{m}$ & 410 & 410 & 0 & 55 & 52 & 3 \\
\hline & Peru & $\mathrm{m}$ & 368 & 367 & 1 & 66 & 64 & 2 \\
\hline & Katar & $\mathrm{m}$ & 376 & 376 & 0 & 64 & 61 & 3 \\
\hline & Rumänien & $\mathrm{m}$ & 445 & 445 & 0 & 46 & 43 & 3 \\
\hline & Russ. Föderation & -46.4 & 482 & 482 & 0 & 35 & 31 & 4 \\
\hline & Serbien & -18.1 & 449 & 444 & 5 & 44 & 44 & 0 \\
\hline & Shanghai (China) & $\mathrm{m}$ & 613 & 572 & 41 & 1 & 1 & 0 \\
\hline & Singapur & $\mathrm{m}$ & 573 & 568 & 6 & 2 & 2 & 0 \\
\hline & Chinesisch Taipeh & $\mathrm{m}$ & 560 & 560 & 0 & 4 & 3 & 1 \\
\hline & Thailand & 0.9 & 427 & 413 & 14 & 51 & 50 & 1 \\
\hline & Tunesien & -19.7 & 388 & 388 & 0 & 61 & 56 & 5 \\
\hline & Ver. Arab. Emirate & $\mathrm{m}$ & 434 & 433 & 1 & 49 & 45 & 4 \\
\hline & Uruguay & 16.0 & 409 & 402 & 8 & 56 & 53 & 3 \\
\hline & Vietnam & $\mathrm{m}$ & 511 & 455 & 56 & 17 & 41 & -24 \\
\hline
\end{tabular}

Note: Diese Daten sind Anhang 2 von PISA 2003 sowie Anhang A2 der vorliegenden Publikation entnommen. Wenn der Anteil der Schüler ab Klassenstufe 7 bei über 100 lag, wurde der Wert für die Gesamtzahl der 15-Jährigen entsprechend dem Anteil der an Bildung teilnehmenden 15-Jährigen festgelegt. Diese Daten sind verschiedenen nationalen Datenquellen entnommen, z.B. wird die Gesamtbevölkerung den nationalen Volkszählungen entnommen, die lediglich in bestimmten Jahren durchgeführt werden. Es ist daher möglich, dass die Bevölkerungsdaten auf einer älteren Quelle beruhen als die Schulbesuchsdaten. Diese Unterschiede können zu einer Schätzung der an Bildung teilnehmenden Bevölkerung führen, die größer ist als die Gesamtbevölkerung, was natürlich nicht möglich ist.

*Vgl. Anmerkungen am Anfang dieses Anhangs.

StatLink *inls http://dx.doi.org/10.1787/888932964908 
[Teil 1/2]

Sozioökonomischer Status der Schüler

Tabelle II.2.13a Die Ergebnisse basieren auf Schülerangaben

\begin{tabular}{|c|c|c|c|c|c|c|c|c|c|c|c|c|c|c|c|}
\hline & \multirow{2}{*}{\multicolumn{2}{|c|}{$\begin{array}{l}\text { PISA-Index des } \\
\text { wirtschaftlichen, } \\
\text { sozialen und } \\
\text { kulturellen Status } \\
\text { (ESCS) }\end{array}$}} & \multirow{2}{*}{\multicolumn{2}{|c|}{ Varianz des ESCS }} & \multirow{2}{*}{\multicolumn{2}{|c|}{$\begin{array}{l}\text { Verteilungs- } \\
\text { asymmetrie } \\
\text { des ESCS }\end{array}$}} & \multirow{2}{*}{\multicolumn{2}{|c|}{\begin{tabular}{|c} 
Prozentsatz \\
der Schüler mit \\
niedrigem ESCS ${ }^{1}$ \\
Erfasst durch \% der \\
Schüler mit einem \\
ESCS-Wert von \\
weniger als -1
\end{tabular}}} & \multicolumn{6}{|c|}{ Varianz der ESCS-Verteilung der Schüler } \\
\hline & & & & & & & & & & \multicolumn{2}{|c|}{$\begin{array}{l}\text { 25. Perzentil } \\
\text { des ESCS }\end{array}$} & $\begin{array}{r}75 . P \\
\text { des }\end{array}$ & $\begin{array}{l}\text { zentil } \\
\text { CSS }\end{array}$ & $\begin{array}{r}\text { Interquar } \\
\text { der ESCS } \\
\text { der S }\end{array}$ & $\begin{array}{l}\text { lbereich } \\
\text { erteilung } \\
\text { ü̈ler }\end{array}$ \\
\hline & & $\begin{array}{l}\text { Index- } \\
\text { mittel }\end{array}$ & S.E. & S.D. & S.E. & $\begin{array}{l}\text { Asym- } \\
\text { metrie }\end{array}$ & S.E. & $\%$ & S.E. & Index & S.E. & Index & S.E. & Bereich & S.E. \\
\hline & Australien & 0.25 & $(0.01)$ & 0.79 & $(0.01)$ & -0.46 & $(0.03)$ & 6.8 & $(0.3)$ & -0.31 & $(0.02)$ & 0.86 & $(0.01)$ & 1.17 & $(0.02)$ \\
\hline$\tau$ & Österreich & 0.08 & $(0.02)$ & 0.85 & $(0.01)$ & 0.02 & $(0.05)$ & 8.3 & $(0.6)$ & -0.53 & $(0.02)$ & 0.66 & $(0.03)$ & 1.19 & $(0.03)$ \\
\hline & Belgien & 0.15 & $(0.02)$ & 0.91 & $(0.02)$ & -0.33 & $(0.18)$ & 10.5 & $(0.6)$ & -0.57 & $(0.03)$ & 0.89 & $(0.03)$ & 1.46 & $(0.02)$ \\
\hline ن & Kanada & 0.41 & $(0.02)$ & 0.86 & $(0.01)$ & -0.39 & $(0.05)$ & 5.6 & $(0.4)$ & -0.20 & $(0.02)$ & 1.08 & $(0.01)$ & 1.28 & $(0.02)$ \\
\hline 空 & Chile & -0.58 & $(0.04)$ & 1.13 & $(0.02)$ & 0.13 & $(0.04)$ & 37.7 & $(1.5)$ & -1.38 & $(0.05)$ & 0.26 & $(0.06)$ & 1.64 & $(0.06)$ \\
\hline & Tschech. Rep. & -0.07 & $(0.02)$ & 0.75 & $(0.01)$ & 0.14 & $(0.06)$ & 9.1 & $(0.6)$ & -0.62 & $(0.02)$ & 0.47 & $(0.04)$ & 1.09 & $(0.03)$ \\
\hline & Dänemark & 0.43 & $(0.02)$ & 0.84 & $(0.01)$ & -0.35 & $(0.04)$ & 4.3 & $(0.4)$ & -0.23 & $(0.03)$ & 1.09 & $(0.03)$ & 1.32 & $(0.03)$ \\
\hline & Estland & 0.11 & $(0.01)$ & 0.81 & $(0.01)$ & 0.01 & $(0.04)$ & 7.8 & $(0.5)$ & -0.55 & $(0.02)$ & 0.78 & $(0.02)$ & 1.34 & $(0.02)$ \\
\hline & Finnland & 0.36 & $(0.02)$ & 0.77 & $(0.01)$ & -0.44 & $(0.04)$ & 4.0 & $(0.3)$ & -0.21 & $(0.02)$ & 0.99 & $(0.02)$ & 1.20 & $(0.02)$ \\
\hline & Frankreich & -0.04 & $(0.02)$ & 0.80 & $(0.01)$ & -0.33 & $(0.03)$ & 11.8 & $(0.6)$ & -0.62 & $(0.02)$ & 0.58 & $(0.02)$ & 1.21 & $(0.02)$ \\
\hline & Deutschland & 0.19 & $(0.02)$ & 0.93 & $(0.01)$ & -0.08 & $(0.04)$ & 9.8 & $(0.6)$ & -0.49 & $(0.03)$ & 0.95 & $(0.04)$ & 1.44 & $(0.03)$ \\
\hline & Griechenland & -0.06 & $(0.03)$ & 1.00 & $(0.01)$ & -0.09 & $(0.04)$ & 18.6 & $(1.0)$ & -0.81 & $(0.03)$ & 0.78 & $(0.04)$ & 1.59 & $(0.03)$ \\
\hline & Ungarn & -0.25 & $(0.03)$ & 0.96 & $(0.02)$ & -0.06 & $(0.07)$ & 23.7 & $(1.0)$ & -0.97 & $(0.03)$ & 0.52 & $(0.05)$ & 1.49 & $(0.03)$ \\
\hline & Island & 0.78 & $(0.01)$ & 0.81 & $(0.01)$ & -0.48 & $(0.04)$ & 1.9 & $(0.2)$ & 0.20 & $(0.02)$ & 1.42 & $(0.01)$ & 1.22 & $(0.02)$ \\
\hline & Irland & 0.13 & $(0.02)$ & 0.85 & $(0.01)$ & -0.15 & $(0.03)$ & 9.2 & $(0.6)$ & -0.53 & $(0.02)$ & 0.81 & $(0.03)$ & 1.34 & $(0.03)$ \\
\hline & Israel & 0.17 & $(0.03)$ & 0.85 & $(0.02)$ & -0.77 & $(0.08)$ & 8.8 & $(0.6)$ & -0.40 & $(0.03)$ & 0.82 & $(0.02)$ & 1.22 & $(0.02)$ \\
\hline & Italien & -0.05 & $(0.01)$ & 0.97 & $(0.01)$ & 0.08 & $(0.02)$ & 18.4 & $(0.4)$ & -0.76 & $(0.02)$ & 0.66 & $(0.02)$ & 1.42 & $(0.02)$ \\
\hline & Japan & -0.07 & $(0.02)$ & 0.71 & $(0.01)$ & -0.02 & $(0.03)$ & 10.0 & $(0.6)$ & -0.62 & $(0.02)$ & 0.49 & $(0.02)$ & 1.11 & $(0.02)$ \\
\hline & Korea & 0.01 & $(0.03)$ & 0.74 & $(0.01)$ & -0.28 & $(0.04)$ & 9.5 & $(0.6)$ & -0.54 & $(0.02)$ & 0.59 & $(0.03)$ & 1.13 & $(0.02)$ \\
\hline & Luxemburg & 0.07 & $(0.01)$ & 1.10 & $(0.01)$ & -0.29 & $(0.02)$ & 18.7 & $(0.6)$ & -0.71 & $(0.03)$ & 0.99 & $(0.02)$ & 1.70 & $(0.02)$ \\
\hline & Mexiko & -1.11 & $(0.02)$ & 1.27 & $(0.01)$ & 0.21 & $(0.02)$ & 55.9 & $(0.8)$ & -2.07 & $(0.02)$ & -0.19 & $(0.04)$ & 1.88 & $(0.03)$ \\
\hline & Niederlande & 0.23 & $(0.02)$ & 0.78 & $(0.01)$ & -0.50 & $(0.05)$ & 5.9 & $(0.5)$ & -0.31 & $(0.02)$ & 0.83 & $(0.03)$ & 1.14 & $(0.02)$ \\
\hline & Neuseeland & 0.04 & $(0.02)$ & 0.82 & $(0.01)$ & -0.26 & $(0.04)$ & 11.5 & $(0.6)$ & -0.56 & $(0.03)$ & 0.68 & $(0.02)$ & 1.24 & $(0.02)$ \\
\hline & Norwegen & 0.46 & $(0.02)$ & 0.76 & $(0.01)$ & -0.55 & $(0.09)$ & 2.6 & $(0.3)$ & -0.06 & $(0.03)$ & 1.01 & $(0.02)$ & 1.08 & $(0.03)$ \\
\hline & Polen & -0.21 & $(0.03)$ & 0.90 & $(0.01)$ & 0.41 & $(0.04)$ & 19.1 & $(1.0)$ & -0.92 & $(0.02)$ & 0.54 & $(0.07)$ & 1.46 & $(0.06)$ \\
\hline & Portugal & -0.48 & $(0.05)$ & 1.19 & $(0.02)$ & 0.43 & $(0.05)$ & 39.8 & $(1.6)$ & -1.42 & $(0.04)$ & 0.34 & $(0.11)$ & 1.76 & $(0.09)$ \\
\hline & Slowak. Rep. & -0.18 & $(0.03)$ & 0.92 & $(0.02)$ & 0.06 & $(0.07)$ & 15.0 & $(0.9)$ & -0.79 & $(0.02)$ & 0.42 & $(0.04)$ & 1.20 & $(0.04)$ \\
\hline & Slowenien & 0.07 & $(0.01)$ & 0.87 & $(0.01)$ & 0.08 & $(0.04)$ & 11.2 & $(0.4)$ & -0.63 & $(0.02)$ & 0.77 & $(0.02)$ & 1.40 & $(0.02)$ \\
\hline & Spanien & -0.19 & $(0.03)$ & 1.03 & $(0.01)$ & 0.01 & $(0.03)$ & 23.5 & $(0.8)$ & -0.96 & $(0.03)$ & 0.65 & $(0.04)$ & 1.61 & $(0.03)$ \\
\hline & Schweden & 0.28 & $(0.02)$ & 0.82 & $(0.01)$ & -0.41 & $(0.05)$ & 5.7 & $(0.3)$ & -0.33 & $(0.02)$ & 0.93 & $(0.02)$ & 1.26 & $(0.02)$ \\
\hline & Schweiz & 0.17 & $(0.02)$ & 0.89 & $(0.01)$ & -0.21 & $(0.03)$ & 10.4 & $(0.6)$ & -0.47 & $(0.03)$ & 0.90 & $(0.03)$ & 1.36 & $(0.02)$ \\
\hline & Türkei & -1.46 & $(0.04)$ & 1.10 & $(0.02)$ & 0.43 & $(0.04)$ & 68.7 & $(1.3)$ & -2.28 & $(0.03)$ & -0.71 & $(0.07)$ & 1.57 & $(0.05)$ \\
\hline & Ver. Königreich & 0.27 & $(0.02)$ & 0.80 & $(0.01)$ & -0.19 & $(0.03)$ & 5.6 & $(0.3)$ & -0.33 & $(0.02)$ & 0.90 & $(0.02)$ & 1.22 & $(0.02)$ \\
\hline & Ver. Staaten & 0.17 & $(0.04)$ & 0.97 & $(0.02)$ & -0.33 & $(0.04)$ & 13.4 & $(1.0)$ & -0.52 & $(0.05)$ & 0.91 & $(0.05)$ & 1.43 & $(0.04)$ \\
\hline & OECD-Durchschnitt & 0.00 & $(0.00)$ & 0.90 & $(0.00)$ & -0.16 & $(0.07)$ & 15.4 & $(0.1)$ & -0.66 & $(0.00)$ & 0.70 & $(0.01)$ & 1.36 & $(0.01)$ \\
\hline s & Albanien & $\mathrm{m}$ & $\mathrm{m}$ & $\mathrm{m}$ & $\mathrm{m}$ & $\mathrm{m}$ & $\mathrm{m}$ & $\mathrm{m}$ & $\mathrm{m}$ & $\mathrm{m}$ & $\mathrm{m}$ & $\mathrm{m}$ & $\mathrm{m}$ & $\mathrm{m}$ & $\mathrm{m}$ \\
\hline ప & Argentinien & -0.72 & $(0.04)$ & 1.11 & $(0.02)$ & -0.06 & $(0.05)$ & 41.2 & (1.5) & -1.51 & $(0.04)$ & 0.15 & $(0.06)$ & 1.66 & $(0.06)$ \\
\hline ड़ & Brasilien & -1.17 & $(0.02)$ & 1.17 & $(0.01)$ & 0.15 & $(0.02)$ & 57.5 & $(0.9)$ & -2.04 & $(0.03)$ & -0.33 & $(0.03)$ & 1.71 & $(0.03)$ \\
\hline. & Bulgarien & -0.28 & $(0.04)$ & 1.05 & $(0.03)$ & -0.38 & $(0.12)$ & 24.3 & $(1.2)$ & -0.99 & $(0.03)$ & 0.54 & $(0.05)$ & 1.53 & $(0.04)$ \\
\hline క్ & Kolumbien & -1.26 & $(0.04)$ & 1.18 & $(0.02)$ & -0.07 & $(0.04)$ & 56.4 & $(1.4)$ & -2.15 & $(0.06)$ & -0.40 & $(0.06)$ & 1.74 & $(0.05)$ \\
\hline$=$ & Costa Rica & -0.98 & $(0.04)$ & 1.24 & $(0.02)$ & -0.14 & $(0.04)$ & 49.2 & $(1.5)$ & -1.90 & $(0.05)$ & 0.02 & $(0.07)$ & 1.92 & $(0.06)$ \\
\hline$T$ & Kroatien & -0.34 & $(0.02)$ & 0.85 & $(0.01)$ & 0.23 & $(0.03)$ & 21.7 & $(0.7)$ & -0.94 & $(0.02)$ & 0.26 & $(0.04)$ & 1.20 & $(0.03)$ \\
\hline छे & Zypern* & 0.09 & $(0.01)$ & 0.91 & $(0.01)$ & -0.06 & $(0.03)$ & 10.7 & $(0.5)$ & -0.60 & $(0.02)$ & 0.82 & $(0.01)$ & 1.42 & $(0.02)$ \\
\hline$\therefore$ & Hongkong (China) & -0.79 & $(0.05)$ & 0.97 & $(0.02)$ & 0.09 & $(0.05)$ & 45.2 & $(2.0)$ & -1.53 & $(0.04)$ & -0.03 & $(0.08)$ & 1.49 & $(0.06)$ \\
\hline$\approx$ & Indonesien & -1.80 & $(0.05)$ & 1.10 & $(0.03)$ & 0.41 & $(0.06)$ & 76.7 & (1.9) & -2.64 & $(0.04)$ & -1.09 & $(0.09)$ & 1.55 & $(0.07)$ \\
\hline & Jordanien & -0.42 & $(0.02)$ & 1.02 & $(0.01)$ & -0.54 & $(0.05)$ & 27.8 & $(0.9)$ & -1.10 & $(0.03)$ & 0.36 & $(0.03)$ & 1.45 & $(0.03)$ \\
\hline$\approx$ & Kasachstan & -0.32 & $(0.02)$ & 0.75 & $(0.01)$ & -0.35 & $(0.05)$ & 20.1 & $(0.9)$ & -0.89 & $(0.03)$ & 0.28 & $(0.03)$ & 1.17 & $(0.03)$ \\
\hline & Lettland & -0.26 & $(0.03)$ & 0.89 & $(0.01)$ & 0.01 & $(0.04)$ & 24.3 & $(1.3)$ & -0.98 & $(0.03)$ & 0.49 & $(0.03)$ & 1.47 & $(0.03)$ \\
\hline & Liechtenstein & 0.30 & $(0.05)$ & 0.91 & $(0.03)$ & -0.25 & $(0.11)$ & 9.4 & $(1.6)$ & -0.28 & $(0.05)$ & 0.97 & $(0.06)$ & 1.25 & $(0.06)$ \\
\hline & Litauen & -0.13 & $(0.02)$ & 0.92 & $(0.01)$ & -0.18 & $(0.04)$ & 21.5 & $(0.8)$ & -0.89 & $(0.03)$ & 0.65 & $(0.02)$ & 1.54 & $(0.03)$ \\
\hline & Macau (China) & -0.89 & $(0.01)$ & 0.87 & $(0.01)$ & 0.36 & $(0.03)$ & 48.6 & $(0.6)$ & -1.49 & $(0.01)$ & -0.35 & $(0.01)$ & 1.14 & $(0.02)$ \\
\hline & Malaysia & -0.72 & $(0.03)$ & 0.99 & $(0.02)$ & -0.17 & $(0.04)$ & 40.5 & $(1.4)$ & -1.37 & $(0.03)$ & 0.04 & $(0.04)$ & 1.41 & $(0.03)$ \\
\hline & Montenegro & -0.25 & $(0.01)$ & 0.89 & $(0.01)$ & -0.13 & $(0.03)$ & 21.4 & $(0.5)$ & -0.89 & $(0.02)$ & 0.44 & $(0.02)$ & 1.33 & $(0.02)$ \\
\hline & Peru & -1.23 & $(0.05)$ & 1.23 & $(0.02)$ & 0.13 & $(0.04)$ & 59.9 & $(1.7)$ & -2.11 & $(0.06)$ & -0.38 & $(0.09)$ & 1.73 & $(0.07)$ \\
\hline & Katar & 0.44 & $(0.01)$ & 0.89 & $(0.01)$ & -0.75 & $(0.03)$ & 7.0 & $(0.2)$ & -0.03 & $(0.01)$ & 1.02 & $(0.01)$ & 1.05 & $(0.01)$ \\
\hline & Rumänien & -0.47 & $(0.04)$ & 0.94 & $(0.03)$ & -0.27 & $(0.15)$ & 25.9 & $(1.3)$ & -1.02 & $(0.02)$ & 0.15 & $(0.07)$ & 1.17 & $(0.06)$ \\
\hline & Russ. Föderation & -0.11 & $(0.02)$ & 0.76 & $(0.01)$ & -0.28 & $(0.04)$ & 12.3 & $(0.7)$ & -0.65 & $(0.03)$ & 0.51 & $(0.02)$ & 1.16 & $(0.02)$ \\
\hline & Serbien & -0.30 & $(0.02)$ & 0.90 & $(0.01)$ & 0.23 & $(0.03)$ & 24.0 & $(1.0)$ & -0.98 & $(0.03)$ & 0.39 & $(0.04)$ & 1.37 & $(0.03)$ \\
\hline & Shanghai (China) & -0.36 & $(0.04)$ & 0.96 & $(0.02)$ & -0.26 & $(0.04)$ & 27.2 & (1.4) & -1.08 & $(0.04)$ & 0.43 & $(0.04)$ & 1.51 & $(0.04)$ \\
\hline & Singapur & -0.26 & $(0.01)$ & 0.92 & $(0.01)$ & -0.27 & $(0.04)$ & 21.3 & $(0.5)$ & -0.88 & $(0.02)$ & 0.45 & $(0.02)$ & 1.33 & $(0.03)$ \\
\hline & Chinesisch Taipeh & -0.40 & $(0.02)$ & 0.84 & $(0.01)$ & -0.08 & $(0.04)$ & 24.7 & $(1.0)$ & -1.00 & $(0.03)$ & 0.22 & $(0.04)$ & 1.21 & $(0.03)$ \\
\hline & Thailand & -1.35 & $(0.04)$ & 1.17 & $(0.02)$ & 0.40 & $(0.04)$ & 64.4 & $(1.5)$ & -2.26 & $(0.03)$ & -0.56 & $(0.07)$ & 1.70 & $(0.05)$ \\
\hline & Tunesien & -1.19 & $(0.05)$ & 1.26 & $(0.02)$ & -0.10 & $(0.04)$ & 54.4 & $(1.6)$ & -2.15 & $(0.06)$ & -0.26 & $(0.08)$ & 1.89 & $(0.07)$ \\
\hline & Ver. Arab. Emirate & 0.32 & $(0.02)$ & 0.85 & $(0.01)$ & -0.81 & $(0.04)$ & 7.2 & $(0.4)$ & -0.14 & $(0.02)$ & 0.90 & $(0.01)$ & 1.03 & $(0.02)$ \\
\hline & Uruguay & -0.88 & $(0.03)$ & 1.13 & $(0.02)$ & 0.32 & $(0.03)$ & 50.4 & (1.1) & -1.77 & $(0.02)$ & -0.06 & $(0.05)$ & 1.71 & $(0.04)$ \\
\hline & Vietnam & -1.81 & $(0.05)$ & 1.12 & $(0.03)$ & 0.62 & $(0.04)$ & 78.9 & (1.6) & -2.57 & $(0.03)$ & -1.20 & $(0.08)$ & 1.36 & $(0.07)$ \\
\hline
\end{tabular}

1. Schüler mit einem niedrigen ESCS sind diejenigen mit einem Wert auf dem PISA-Index des wirtschaftlichen, sozialen und kulturellen Status von weniger als -1.

2. Verteilung des ESCS-Mittelwerts der Schulen, Perzentile berechnet auf Schülerebene.

3. Der Index der sozialen Inklusion wird berechnet als 100*(1-rho), wobei rho für die Intra-Class-Korrelation des sozioökonomischen Status steht, d.h. die Varianz des PISAIndex des wirtschaftlichen, sozialen und kulturellen Status der Schüler zwischen Schulen, dividiert durch die Summe der Varianz des sozioökonomischen Status der Schüler zwischen Schulen und der Varianz des sozioökonomischen Status der Schüler innerhalb von Schulen.

*Vgl. Anmerkungen am Anfang dieses Anhangs.

StatLink 完ISL http://dx.doi.org/10.1787/888932964908 
[Teil 2/2]

Sozioökonomischer Status der Schüler

Tabelle II.2.13a Die Ergebnisse basieren auf Schülerangaben

\begin{tabular}{|c|c|c|c|c|c|c|c|c|c|}
\hline & & \multicolumn{6}{|c|}{ Varianz der ESCS-Verteilung der Schulen ${ }^{2}$} & \multirow{2}{*}{\multicolumn{2}{|c|}{ Index der sozialen Inklusion }} \\
\hline & & \multicolumn{2}{|c|}{$\begin{array}{l}\text { 25. Perzentil des durchschnittlichen } \\
\text { ESCS der Schulen }\end{array}$} & \multicolumn{2}{|c|}{$\begin{array}{l}\text { 75. Perzentil des durchschnittlichen } \\
\text { ESCS der Schulen }\end{array}$} & \multicolumn{2}{|c|}{$\begin{array}{l}\text { Interquartilbereich der ESCS- } \\
\text { Verteilung der Schulen }\end{array}$} & & \\
\hline & & Index & S.E. & Index & S.E. & Bereich & S.E. & Index & S.E. \\
\hline \multirow{35}{*}{ 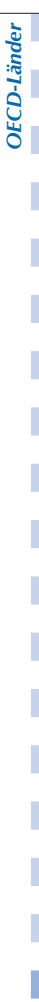 } & Australien & -0.05 & $(0.02)$ & 0.54 & $(0.01)$ & 0.60 & $(0.02)$ & 76.5 & $(1.1)$ \\
\hline & Österreich & -0.23 & $(0.04)$ & 0.36 & $(0.02)$ & 0.60 & $(0.04)$ & 71.2 & $(2.8)$ \\
\hline & Belgien & -0.24 & $(0.07)$ & 0.51 & $(0.05)$ & 0.75 & $(0.09)$ & 72.4 & $(2.1)$ \\
\hline & Kanada & 0.15 & $(0.02)$ & 0.70 & $(0.02)$ & 0.54 & $(0.03)$ & 82.8 & $(1.2)$ \\
\hline & Chile & -1.13 & $(0.07)$ & -0.08 & $(0.08)$ & 1.06 & $(0.11)$ & 47.2 & $(2.2)$ \\
\hline & Tschech. Rep. & -0.34 & $(0.02)$ & 0.17 & $(0.02)$ & 0.50 & $(0.03)$ & 76.4 & $(2.2)$ \\
\hline & Dänemark & 0.16 & $(0.04)$ & 0.68 & $(0.04)$ & 0.52 & $(0.05)$ & 82.3 & (1.6) \\
\hline & Estland & -0.14 & $(0.02)$ & 0.34 & $(0.04)$ & 0.48 & $(0.04)$ & 81.5 & $(2.0)$ \\
\hline & Finnland & 0.20 & $(0.03)$ & 0.55 & $(0.01)$ & 0.35 & $(0.03)$ & 91.1 & (1.0) \\
\hline & Frankreich & w & w & w & w & w & w & w & w \\
\hline & Deutschland & -0.18 & $(0.03)$ & 0.60 & $(0.03)$ & 0.78 & $(0.05)$ & 73.6 & (1.9) \\
\hline & Griechenland & -0.41 & $(0.11)$ & 0.27 & $(0.06)$ & 0.69 & $(0.11)$ & 73.5 & (2.6) \\
\hline & Ungarn & -0.70 & $(0.07)$ & 0.25 & $(0.08)$ & 0.95 & $(0.10)$ & 62.6 & (2.6) \\
\hline & Island & 0.54 & $(0.00)$ & 1.04 & $(0.00)$ & 0.49 & $(0.00)$ & 86.4 & (1.9) \\
\hline & Irland & -0.09 & $(0.03)$ & 0.39 & $(0.03)$ & 0.48 & $(0.04)$ & 79.7 & $(2.1)$ \\
\hline & Israel & -0.18 & $(0.04)$ & 0.54 & $(0.04)$ & 0.72 & $(0.05)$ & 74.6 & $(2.0)$ \\
\hline & Italien & -0.43 & $(0.02)$ & 0.32 & $(0.02)$ & 0.75 & $(0.03)$ & 75.9 & (1.1) \\
\hline & Japan & -0.32 & $(0.02)$ & 0.18 & $(0.03)$ & 0.50 & $(0.03)$ & 77.8 & (1.7) \\
\hline & Korea & -0.24 & $(0.06)$ & 0.23 & $(0.04)$ & 0.46 & $(0.07)$ & 78.3 & (1.9) \\
\hline & Luxemburg & -0.46 & $(0.00)$ & 0.57 & $(0.00)$ & 1.03 & $(0.00)$ & 73.6 & (3.4) \\
\hline & Mexiko & -1.64 & $(0.01)$ & -0.62 & $(0.04)$ & 1.02 & $(0.03)$ & 56.5 & (1.5) \\
\hline & Niederlande & 0.00 & $(0.03)$ & 0.51 & $(0.04)$ & 0.51 & $(0.05)$ & 81.8 & (1.8) \\
\hline & Neuseeland & -0.21 & $(0.01)$ & 0.27 & $(0.02)$ & 0.48 & $(0.02)$ & 77.5 & $(2.4)$ \\
\hline & Norwegen & 0.30 & $(0.02)$ & 0.60 & $(0.03)$ & 0.30 & $(0.03)$ & 91.0 & (1.4) \\
\hline & Polen & -0.54 & $(0.05)$ & 0.05 & $(0.06)$ & 0.59 & $(0.07)$ & 76.4 & $(2.2)$ \\
\hline & Portugal & -0.98 & $(0.05)$ & -0.19 & $(0.07)$ & 0.79 & $(0.07)$ & 68.6 & (3.3) \\
\hline & Slowak. Rep. & -0.48 & $(0.05)$ & 0.14 & $(0.04)$ & 0.62 & $(0.06)$ & 64.4 & (3.0) \\
\hline & Slowenien & -0.29 & $(0.01)$ & 0.52 & $(0.00)$ & 0.81 & $(0.01)$ & 74.6 & (1.9) \\
\hline & Spanien & -0.57 & $(0.04)$ & 0.21 & $(0.05)$ & 0.78 & $(0.06)$ & 75.2 & (1.4) \\
\hline & Schweden & 0.04 & $(0.02)$ & 0.53 & $(0.06)$ & 0.49 & $(0.06)$ & 86.9 & (1.4) \\
\hline & Schweiz & -0.14 & $(0.03)$ & 0.48 & $(0.04)$ & 0.62 & $(0.04)$ & 82.7 & (1.5) \\
\hline & Türkei & -1.83 & $(0.03)$ & -1.13 & $(0.05)$ & 0.70 & $(0.06)$ & 72.3 & (2.9) \\
\hline & Ver. Königreich & -0.02 & $(0.04)$ & 0.53 & $(0.02)$ & 0.54 & $(0.04)$ & 79.4 & $(2.0)$ \\
\hline & Ver. Staaten & -0.15 & $(0.05)$ & 0.54 & $(0.06)$ & 0.69 & $(0.08)$ & 73.8 & $(2.4)$ \\
\hline & OECD-Durchschnitt & -0.33 & $(0.01)$ & 0.32 & $(0.01)$ & 0.64 & $(0.01)$ & 75.6 & $(0.4)$ \\
\hline \multirow{31}{*}{ 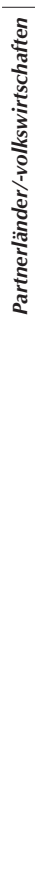 } & Albanien & $\mathrm{m}$ & $\mathrm{m}$ & $\mathrm{m}$ & $\mathrm{m}$ & $\mathrm{m}$ & $\mathrm{m}$ & $\mathrm{m}$ & $\mathrm{m}$ \\
\hline & Argentinien & -1.24 & $(0.04)$ & -0.23 & $(0.09)$ & 1.02 & $(0.10)$ & 66.5 & $(2.6)$ \\
\hline & Brasilien & -1.66 & $(0.02)$ & -0.85 & $(0.04)$ & 0.81 & $(0.04)$ & 62.8 & (2.3) \\
\hline & Bulgarien & -0.68 & $(0.05)$ & 0.18 & $(0.11)$ & 0.87 & $(0.12)$ & 59.6 & (3.0) \\
\hline & Kolumbien & -1.75 & $(0.08)$ & -0.83 & $(0.06)$ & 0.92 & $(0.09)$ & 63.2 & $(2.7)$ \\
\hline & Costa Rica & -1.47 & $(0.08)$ & -0.65 & $(0.07)$ & 0.81 & $(0.10)$ & 61.8 & $(3.1)$ \\
\hline & Kroatien & -0.66 & $(0.03)$ & -0.12 & $(0.03)$ & 0.54 & $(0.04)$ & 75.9 & $(2.2)$ \\
\hline & Zypern* & -0.25 & $(0.00)$ & 0.42 & $(0.00)$ & 0.67 & $(0.00)$ & 76.6 & (3.2) \\
\hline & Hongkong (China) & -1.20 & $(0.03)$ & -0.49 & $(0.15)$ & 0.71 & $(0.14)$ & 67.7 & (3.2) \\
\hline & Indonesien & -2.27 & $(0.03)$ & -1.38 & $(0.12)$ & 0.89 & $(0.11)$ & 63.1 & $(4.3)$ \\
\hline & Jordanien & -0.71 & $(0.03)$ & -0.17 & $(0.03)$ & 0.54 & $(0.04)$ & 79.6 & (2.8) \\
\hline & Kasachstan & -0.57 & $(0.02)$ & -0.04 & $(0.02)$ & 0.53 & $(0.03)$ & 76.8 & $(2.1)$ \\
\hline & Lettland & -0.58 & $(0.04)$ & 0.05 & $(0.08)$ & 0.64 & $(0.09)$ & 74.7 & (2.5) \\
\hline & Liechtenstein & 0.07 & $(0.00)$ & 0.64 & $(0.00)$ & 0.56 & $(0.00)$ & 85.5 & $(4.1)$ \\
\hline & Litauen & -0.45 & $(0.02)$ & 0.16 & $(0.03)$ & 0.61 & $(0.03)$ & 78.7 & (1.9) \\
\hline & Macau (China) & -1.21 & $(0.00)$ & -0.71 & $(0.00)$ & 0.50 & $(0.00)$ & 73.7 & $(4.7)$ \\
\hline & Malaysia & -1.08 & $(0.03)$ & -0.37 & $(0.07)$ & 0.72 & $(0.08)$ & 71.5 & (2.4) \\
\hline & Montenegro & -0.53 & $(0.00)$ & -0.01 & $(0.00)$ & 0.52 & $(0.00)$ & 80.6 & $(5.2)$ \\
\hline & Peru & -1.84 & $(0.05)$ & -0.62 & $(0.11)$ & 1.23 & $(0.10)$ & 54.2 & (2.6) \\
\hline & Katar & 0.21 & $(0.00)$ & 0.71 & $(0.00)$ & 0.50 & $(0.00)$ & 75.5 & $(3.2)$ \\
\hline & Rumänien & -0.80 & $(0.05)$ & -0.12 & $(0.06)$ & 0.68 & $(0.07)$ & 64.4 & (3.6) \\
\hline & Russ. Föderation & -0.39 & $(0.05)$ & 0.15 & $(0.03)$ & 0.53 & $(0.05)$ & 75.0 & (2.4) \\
\hline & Serbien & -0.62 & $(0.02)$ & -0.03 & $(0.06)$ & 0.59 & $(0.06)$ & 78.0 & (2.3) \\
\hline & Shanghai (China) & -0.73 & $(0.08)$ & 0.07 & $(0.06)$ & 0.81 & $(0.09)$ & 66.8 & (2.5) \\
\hline & Singapur & -0.59 & $(0.00)$ & -0.02 & $(0.03)$ & 0.57 & $(0.03)$ & 76.4 & (2.5) \\
\hline & Chinesisch Taipeh & -0.70 & $(0.02)$ & -0.10 & $(0.05)$ & 0.60 & $(0.06)$ & 76.7 & $(2.0)$ \\
\hline & Thailand & -1.87 & $(0.03)$ & -0.85 & $(0.04)$ & 1.01 & $(0.04)$ & 61.6 & (2.9) \\
\hline & Tunesien & -1.71 & $(0.08)$ & -0.70 & $(0.13)$ & 1.01 & $(0.14)$ & 67.2 & (3.0) \\
\hline & Ver. Arab. Emirate & 0.00 & $(0.02)$ & 0.68 & $(0.01)$ & 0.68 & $(0.02)$ & 73.9 & (1.8) \\
\hline & Uruguay & -1.44 & $(0.04)$ & -0.61 & $(0.03)$ & 0.82 & $(0.04)$ & 60.2 & (3.5) \\
\hline & Vietnam & -2.25 & $(0.02)$ & -1.45 & $(0.12)$ & 0.79 & $(0.12)$ & 58.3 & (3.4) \\
\hline
\end{tabular}

1. Schüler mit einem niedrigen ESCS sind diejenigen mit einem Wert auf dem PISA-Index des wirtschaftlichen, sozialen und kulturellen Status von weniger als -1.

2. Verteilung des ESCS-Mittelwerts der Schulen, Perzentile berechnet auf Schülerebene.

3. Der Index der sozialen Inklusion wird berechnet als 100*(1-rho), wobei rho für die Intra-Class-Korrelation des sozioökonomischen Status steht, d.h. die Varianz des PISAIndex des wirtschaftlichen, sozialen und kulturellen Status der Schüler zwischen Schulen, dividiert durch die Summe der Varianz des sozioökonomischen Status der Schüler zwischen Schulen und der Varianz des sozioökonomischen Status der Schüler innerhalb von Schulen.

$*$ Vgl. Anmerkungen am Anfang dieses Anhangs.

StatLink *its

EXZELLENZ DURCH CHANCENGERECHTIGKET: ALLEN SCHÜLERINNEN UND SCHÜLERN DIEVORAUSSETZUNGEN ZUM ERFOLG SICHERN - BAND II @ O OECD 2014227 
[Teil 1/3]

Veränderung beim sozioökonomischen Status der Schüler zwischen 2003 und 2012

Tabelle II.2.13b Die Ergebnisse basieren auf Schülerangaben

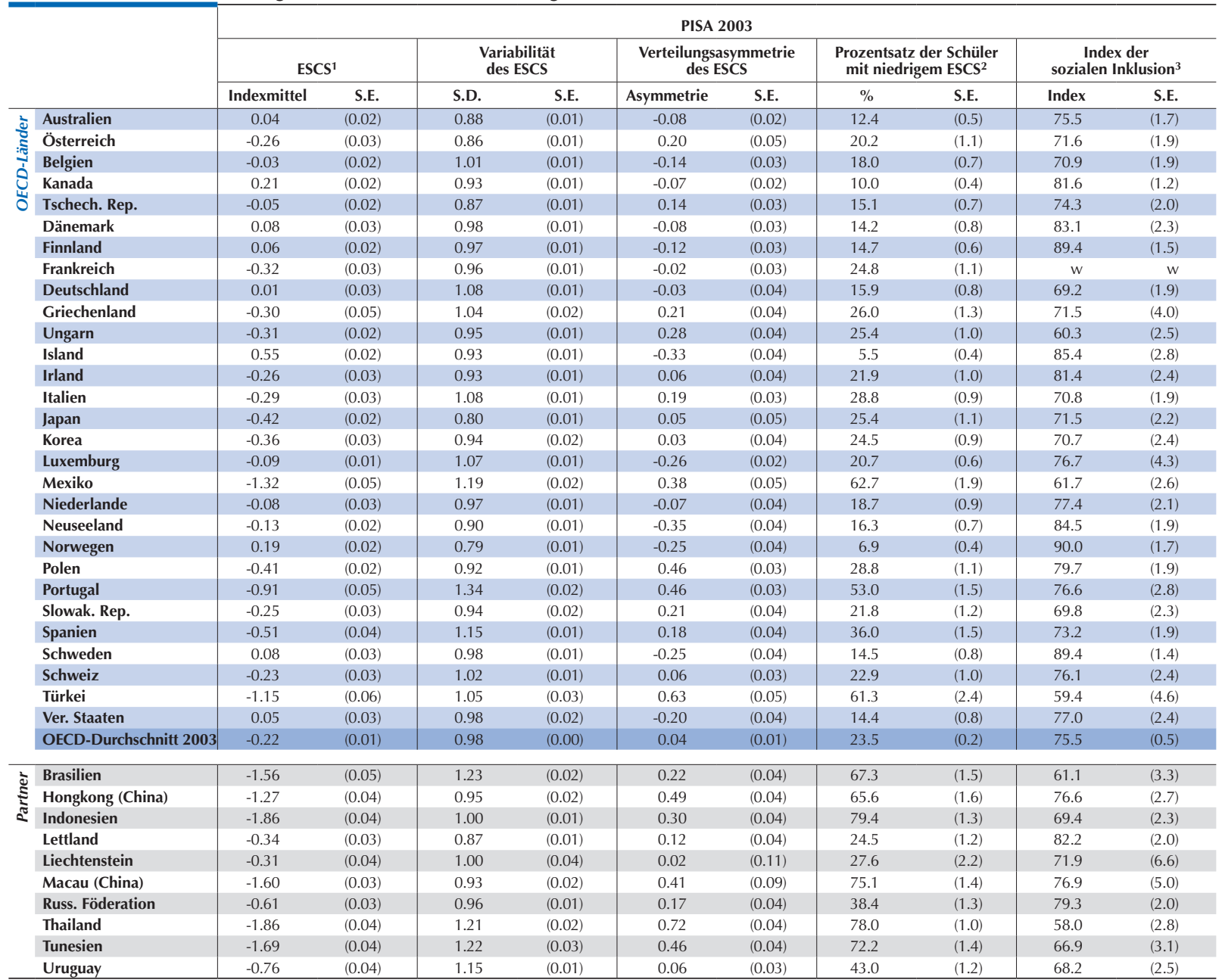

Anmerkung: Statistisch signifikante Werte sind durch Fettdruck gekennzeichnet (vgl. Anhang A3).

Angegeben sind nur Länder und Volkswirtschaften mit vergleichbaren Daten aus PISA 2003 und PISA 2012

Um die Vergleichbarkeit im Zeitverlauf zu gewährleisten, wurden die Werte von PISA 2003 auf dem PISA-Index des wirtschaftlichen, sozialen und kulturellen Status entsprechend der Indexskala von PISA 2012 umskaliert. Die in dieser Abbildung dargestellten Ergebnisse aus PISA 2003 können daher von denen abweichen, die in Lernen für die Welt von morgen: Erste Ergebnisse von PISA 2003 (OECD, 2004) wiedergegeben sind (vgl. Anhang A5 wegen näherer Einzelheiten).

1. ESCS bezieht sich auf den PISA-Index des wirtschaftlichen, sozialen und kulturellen Status.

2. Schüler mit einem niedrigen ESCS sind diejenigen mit einem Wert auf dem PISA-Index des wirtschaftlichen, sozialen und kulturellen Status von weniger als -1.

3. Der Index der sozialen Inklusion wird berechnet als $100^{*}(1-$ rho), wobei rho für die Intra-Class-Korrelation des sozioökonomischen Status steht, d.h. die Varianz des PISAIndex des wirtschaftlichen, sozialen und kulturellen Status der Schüler zwischen Schulen, dividiert durch die Summe der Varianz des sozioökonomischen Status der Schüler zwischen Schulen und der Varianz des sozioökonomischen Status der Schüler innerhalb von Schulen.

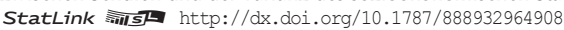


[Teil 2/3]

Veränderung beim sozioökonomischen Status der Schüler zwischen 2003 und 2012

Tabelle II.2.13b Die Ergebnisse basieren auf Schülerangaben

\begin{tabular}{|c|c|c|c|c|c|c|c|c|c|c|c|}
\hline & \multicolumn{10}{|c|}{ PISA 2012} \\
\hline & & \multicolumn{2}{|c|}{ ESCS $^{1}$} & \multicolumn{2}{|c|}{ Variabilität des ESCS } & \multicolumn{2}{|c|}{$\begin{array}{c}\text { Verteilungsasymmetrie } \\
\text { des ESCS }\end{array}$} & \multicolumn{2}{|c|}{$\begin{array}{c}\text { Prozentsatz der Schüler } \\
\text { mit niedrigem ESCS }\end{array}$} & \multicolumn{2}{|c|}{$\begin{array}{c}\text { Index der } \\
\text { sozialen Inklusion }\end{array}$} \\
\hline & & Indexmittel & S.E. & S.D. & S.E. & Asymmetrie & S.E. & $\%$ & S.E. & Index & S.E. \\
\hline \multirow{30}{*}{ 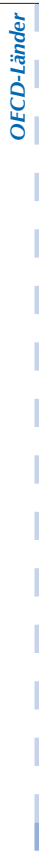 } & Australien & 0.25 & $(0.01)$ & 0.79 & $(0.01)$ & -0.46 & $(0.03)$ & 6.8 & $(0.3)$ & 76.5 & (1.1) \\
\hline & Österreich & 0.08 & $(0.02)$ & 0.85 & $(0.01)$ & 0.02 & $(0.05)$ & 8.3 & $(0.6)$ & 71.2 & (2.8) \\
\hline & Belgien & 0.15 & $(0.02)$ & 0.91 & $(0.02)$ & -0.33 & $(0.09)$ & 10.5 & $(0.6)$ & 72.4 & (2.1) \\
\hline & Kanada & 0.41 & $(0.02)$ & 0.86 & $(0.01)$ & -0.39 & $(0.05)$ & 5.6 & $(0.4)$ & 82.8 & (1.2) \\
\hline & Tschech. Rep. & -0.07 & $(0.02)$ & 0.75 & $(0.01)$ & 0.14 & $(0.06)$ & 9.1 & $(0.6)$ & 76.4 & (2.2) \\
\hline & Dänemark & 0.43 & $(0.02)$ & 0.84 & $(0.01)$ & -0.35 & $(0.04)$ & 4.3 & $(0.4)$ & 82.3 & (1.6) \\
\hline & Finnland & 0.36 & $(0.02)$ & 0.77 & $(0.01)$ & -0.44 & $(0.04)$ & 4.0 & $(0.3)$ & 91.1 & (1.0) \\
\hline & Frankreich & -0.04 & $(0.02)$ & 0.80 & $(0.01)$ & -0.33 & $(0.03)$ & 11.8 & $(0.6)$ & w & w \\
\hline & Deutschland & 0.19 & $(0.02)$ & 0.93 & $(0.01)$ & -0.08 & $(0.04)$ & 9.8 & $(0.6)$ & 73.6 & (1.9) \\
\hline & Griechenland & -0.06 & $(0.03)$ & 1.00 & $(0.01)$ & -0.09 & $(0.04)$ & 18.6 & $(1.0)$ & 73.5 & (2.6) \\
\hline & Ungarn & -0.25 & $(0.03)$ & 0.96 & $(0.02)$ & -0.06 & $(0.07)$ & 23.7 & (1.0) & 62.6 & (2.6) \\
\hline & Island & 0.78 & $(0.01)$ & 0.81 & $(0.01)$ & -0.48 & $(0.04)$ & 1.9 & $(0.2)$ & 86.4 & (1.9) \\
\hline & Irland & 0.13 & $(0.02)$ & 0.85 & $(0.01)$ & -0.15 & $(0.03)$ & 9.2 & $(0.6)$ & 79.7 & (2.1) \\
\hline & Italien & -0.05 & $(0.01)$ & 0.97 & $(0.01)$ & 0.08 & $(0.02)$ & 18.4 & (0.4) & 75.9 & (1.1) \\
\hline & Japan & -0.07 & $(0.02)$ & 0.71 & $(0.01)$ & -0.02 & $(0.03)$ & 10.0 & $(0.6)$ & 77.8 & (1.7) \\
\hline & Korea & 0.01 & $(0.03)$ & 0.74 & $(0.01)$ & -0.28 & $(0.04)$ & 9.5 & (0.6) & 78.3 & (1.9) \\
\hline & Luxemburg & 0.07 & $(0.01)$ & 1.10 & $(0.01)$ & -0.29 & $(0.02)$ & 18.7 & $(0.6)$ & 73.6 & (3.4) \\
\hline & Mexiko & -1.11 & $(0.02)$ & 1.27 & $(0.01)$ & 0.21 & $(0.02)$ & 55.9 & (0.8) & 56.5 & (1.5) \\
\hline & Niederlande & 0.23 & $(0.02)$ & 0.78 & $(0.01)$ & -0.50 & $(0.05)$ & 5.9 & $(0.5)$ & 81.8 & (1.8) \\
\hline & Neuseeland & 0.04 & $(0.02)$ & 0.82 & $(0.01)$ & -0.26 & $(0.04)$ & 11.5 & (0.6) & 77.5 & (2.4) \\
\hline & Norwegen & 0.46 & $(0.02)$ & 0.76 & $(0.01)$ & -0.55 & $(0.09)$ & 2.6 & $(0.3)$ & 91.0 & (1.4) \\
\hline & Polen & -0.21 & $(0.03)$ & 0.90 & $(0.01)$ & 0.41 & $(0.04)$ & 19.1 & (1.0) & 76.4 & (2.2) \\
\hline & Portugal & -0.48 & $(0.05)$ & 1.19 & $(0.02)$ & 0.43 & $(0.05)$ & 39.8 & (1.6) & 68.6 & (3.3) \\
\hline & Slowak. Rep. & -0.18 & $(0.03)$ & 0.92 & $(0.02)$ & 0.06 & $(0.07)$ & 15.0 & (0.9) & 64.4 & (3.0) \\
\hline & Spanien & -0.19 & $(0.03)$ & 1.03 & $(0.01)$ & 0.01 & $(0.03)$ & 23.5 & (0.8) & 75.2 & (1.4) \\
\hline & Schweden & 0.28 & $(0.02)$ & 0.82 & $(0.01)$ & -0.41 & $(0.05)$ & 5.7 & $(0.3)$ & 86.9 & (1.4) \\
\hline & Schweiz & 0.17 & $(0.02)$ & 0.89 & $(0.01)$ & -0.21 & $(0.03)$ & 10.4 & $(0.6)$ & 82.7 & (1.5) \\
\hline & Türkei & -1.46 & $(0.04)$ & 1.10 & $(0.02)$ & 0.43 & $(0.04)$ & 68.7 & (1.3) & 72.3 & (2.9) \\
\hline & Ver. Staaten & 0.17 & $(0.04)$ & 0.97 & $(0.02)$ & -0.33 & $(0.04)$ & 13.4 & $(1.0)$ & 73.8 & (2.4) \\
\hline & OECD-Durchschnitt 2003 & 0.00 & $(0.00)$ & 0.90 & $(0.00)$ & -0.15 & $(0.01)$ & 15.6 & $(0.1)$ & 76.3 & $(0.4)$ \\
\hline \multirow{10}{*}{ ఏ } & Brasilien & -1.17 & $(0.02)$ & 1.17 & $(0.01)$ & 0.15 & $(0.02)$ & 57.5 & (0.9) & 62.8 & (2.3) \\
\hline & Hongkong (China) & -0.79 & $(0.05)$ & 0.97 & $(0.02)$ & 0.09 & $(0.05)$ & 45.2 & $(2.0)$ & 67.7 & (3.2) \\
\hline & Indonesien & -1.80 & $(0.05)$ & 1.10 & $(0.03)$ & 0.41 & $(0.06)$ & 76.7 & (1.9) & 63.1 & (4.3) \\
\hline & Lettland & -0.26 & $(0.03)$ & 0.89 & $(0.01)$ & 0.01 & $(0.04)$ & 24.3 & (1.3) & 74.7 & (2.5) \\
\hline & Liechtenstein & 0.30 & $(0.05)$ & 0.91 & $(0.03)$ & -0.25 & $(0.11)$ & 9.4 & (1.6) & 85.5 & (4.1) \\
\hline & Macau (China) & -0.89 & $(0.01)$ & 0.87 & $(0.01)$ & 0.36 & $(0.03)$ & 48.6 & $(0.6)$ & 73.7 & (4.7) \\
\hline & Russ. Föderation & -0.11 & $(0.02)$ & 0.76 & $(0.01)$ & -0.28 & $(0.04)$ & 12.3 & $(0.7)$ & 75.0 & (2.4) \\
\hline & Thailand & -1.35 & $(0.04)$ & 1.17 & $(0.02)$ & 0.40 & $(0.04)$ & 64.4 & (1.5) & 61.6 & (2.9) \\
\hline & Tunesien & -1.19 & $(0.05)$ & 1.26 & $(0.02)$ & -0.10 & $(0.04)$ & 54.4 & (1.6) & 67.2 & (3.0) \\
\hline & Uruguay & -0.88 & $(0.03)$ & 1.13 & $(0.02)$ & 0.32 & $(0.03)$ & 50.4 & (1.1) & 60.2 & (3.5) \\
\hline
\end{tabular}

Anmerkung: Statistisch signifikante Werte sind durch Fettdruck gekennzeichnet (vgl. Anhang A3).

Angegeben sind nur Länder und Volkswirtschaften mit vergleichbaren Daten aus PISA 2003 und PISA 2012

Um die Vergleichbarkeit im Zeitverlauf zu gewährleisten, wurden die Werte von PISA 2003 auf dem PISA-Index des wirtschaftlichen, sozialen und kulturellen Status entsprechend der Indexskala von PISA 2012 umskaliert. Die in dieser Tabelle dargestellten Ergebnisse aus PISA 2003 können daher von denen abweichen, die in Lernen für die Welt von morgen: Erste Ergebnisse von PISA 2003 (OECD, 2004) wiedergegeben sind (vgl. Anhang A5 wegen näherer Einzelheiten).

1. ESCS bezieht sich auf den PISA-Index des wirtschaftlichen, sozialen und kulturellen Status.

2. Schüler mit einem niedrigen ESCS sind diejenigen mit einem Wert auf dem PISA-Index des wirtschaftlichen, sozialen und kulturellen Status von weniger als -1.

3. Der Index der sozialen Inklusion wird berechnet als 100*(1-rho), wobei rho für die Intra-Class-Korrelation des sozioökonomischen Status steht, d h die Varianz des PISAIndex des wirtschaftlichen, sozialen und kulturellen Status der Schüler zwischen Schulen, dividiert durch die Summe der Varianz des sozioökonomischen Status der Schüler zwischen Schulen und der Varianz des sozioökonomischen Status der Schüler innerhalb von Schulen.

StatLink 完正西 http://dx.doi.org/10.1787/888932964908 
[Teil 3/3]

Veränderung beim sozioökonomischen Status der Schüler zwischen 2003 und 2012

Tabelle II.2.13b Die Ergebnisse basieren auf Schülerangaben

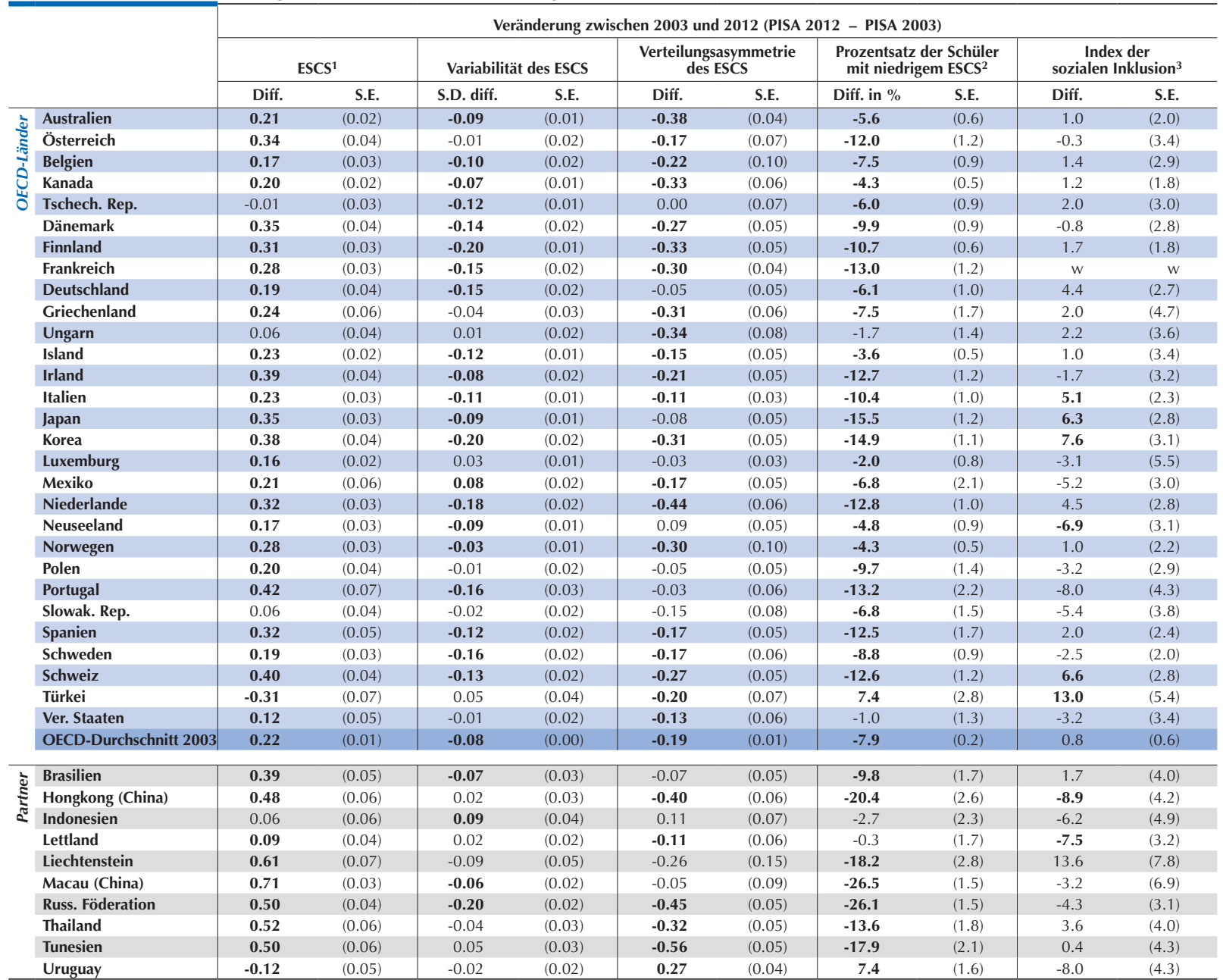

Anmerkung: Statistisch signifikante Werte sind durch Fettdruck gekennzeichnet (vgl. Anhang A3).

Angegeben sind nur Länder und Volkswirtschaften mit vergleichbaren Daten aus PISA 2003 und PISA 2012

Um die Vergleichbarkeit im Zeitverlauf zu gewährleisten, wurden die Werte von PISA 2003 auf dem PISA-Index des wirtschaftlichen, sozialen und kulturellen Status entsprechend der Indexskala von PISA 2012 umskaliert. Die in dieser Tabelle dargestellten Ergebnisse aus PISA 2003 können daher von denen abweichen, die in Lernen für die Welt von morgen: Erste Ergebnisse von PISA 2003 (OECD, 2004) wiedergegeben sind (vgl. Anhang A5 wegen näherer Einzelheiten).

1. ESCS bezieht sich auf den PISA-Index des wirtschaftlichen, sozialen und kulturellen Status.

2. Schüler mit einem niedrigen ESCS sind diejenigen mit einem Wert auf dem PISA-Index des wirtschaftlichen, sozialen und kulturellen Status von weniger als -1.

3. Der Index der sozialen Inklusion wird berechnet als $100^{*}(1$-rho), wo rho für die Intra-Class-Korrelation des sozioökonomischen Status steht, d.h. die Varianz des PISA-Index des wirtschaftlichen, sozialen und kulturellen Status der Schüler zwischen Schulen, dividiert durch die Summe der Varianz des sozioökonomischen Status der Schüler zwischen Schulen und der Varianz des sozioökonomischen Status der Schüler innerhalb von Schulen.

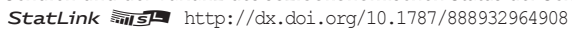


[Teil 1/2]

Schülerleistungen in Mathematik und Familientyp Tabelle II.3.1 Die Ergebnisse basieren auf Schülerangaben

\begin{tabular}{|c|c|c|c|c|c|c|c|c|c|c|c|c|c|}
\hline & & \multicolumn{4}{|c|}{ Prozentsatz der Schüler } & \multicolumn{4}{|c|}{$\begin{array}{c}\text { PISA-Index des wirtschaftlichen, sozialen und } \\
\text { kulturellen Status (ESCS) }\end{array}$} & \multicolumn{4}{|c|}{ Schülerleistungen in Mathematik } \\
\hline & & \multicolumn{2}{|c|}{$\begin{array}{c}\text { Schüler aus } \\
\text { Alleinerziehenden- } \\
\text { haushalten }\end{array}$} & \multicolumn{2}{|c|}{$\begin{array}{c}\text { Schüler aus anderen } \\
\text { Familientypen }\end{array}$} & \multicolumn{2}{|c|}{$\begin{array}{c}\text { Schüler aus } \\
\text { Alleinerziehenden- } \\
\text { haushalten }\end{array}$} & \multicolumn{2}{|c|}{$\begin{array}{c}\text { Schüler aus anderen } \\
\text { Familientypen }\end{array}$} & \multicolumn{2}{|c|}{$\begin{array}{c}\text { Schüler aus } \\
\text { Alleinerziehenden- } \\
\text { haushalten }\end{array}$} & $\begin{array}{r}\text { Schüler au } \\
\text { Familie }\end{array}$ & $\begin{array}{l}\text { anderen } \\
\text { ypen }\end{array}$ \\
\hline & & $\%$ & S.E. & $\%$ & S.E. & $\begin{array}{l}\text { Index- } \\
\text { mittel }\end{array}$ & S.E. & $\begin{array}{l}\text { Index- } \\
\text { mittel }\end{array}$ & S.E. & Mittelwert & S.E. & Mittelwert & S.E. \\
\hline ذँ & Australien & 13.5 & $(0.4)$ & 86.5 & $(0.4)$ & -0.07 & $(0.02)$ & 0.33 & $(0.01)$ & 495 & (2.6) & 513 & (1.8) \\
\hline & Österreich & 13.5 & $(0.7)$ & 86.5 & $(0.7)$ & -0.05 & $(0.04)$ & 0.11 & $(0.02)$ & 503 & $(4.9)$ & 510 & $(2.7)$ \\
\hline & Belgien & 13.6 & $(0.5)$ & 86.4 & $(0.5)$ & -0.12 & $(0.05)$ & 0.21 & $(0.02)$ & 493 & $(4.9)$ & 525 & $(2.2)$ \\
\hline తి & Kanada & 12.7 & $(0.3)$ & 87.3 & $(0.3)$ & 0.11 & $(0.03)$ & 0.48 & $(0.02)$ & 511 & $(3.2)$ & 525 & (1.9) \\
\hline & Chile & 22.6 & $(0.7)$ & 77.4 & $(0.7)$ & -0.60 & $(0.04)$ & -0.52 & $(0.04)$ & 426 & (3.6) & 430 & (3.1) \\
\hline & Tschech. Rep. & 17.6 & $(0.5)$ & 82.4 & $(0.5)$ & -0.30 & $(0.02)$ & 0.00 & $(0.02)$ & 486 & $(4.3)$ & 506 & (2.9) \\
\hline & Dänemark & 15.1 & $(0.6)$ & 84.9 & $(0.6)$ & 0.12 & $(0.04)$ & 0.50 & $(0.02)$ & 485 & $(4.0)$ & 508 & $(2.1)$ \\
\hline & Estland & 19.2 & $(0.7)$ & 80.8 & $(0.7)$ & -0.15 & $(0.03)$ & 0.19 & $(0.01)$ & 525 & (3.4) & 524 & (2.3) \\
\hline & Finnland & 15.9 & $(0.6)$ & 84.1 & $(0.6)$ & 0.02 & $(0.03)$ & 0.45 & $(0.01)$ & 507 & (3.3) & 528 & $(1.8)$ \\
\hline & Frankreich & 15.1 & $(0.6)$ & 84.9 & $(0.6)$ & -0.26 & $(0.03)$ & 0.03 & $(0.02)$ & 484 & $(4.4)$ & 505 & $(2.7)$ \\
\hline & Deutschland & 13.3 & $(0.6)$ & 86.7 & (0.6) & 0.03 & $(0.05)$ & 0.24 & $(0.02)$ & 520 & (4.9) & 524 & (3.1) \\
\hline & Griechenland & 8.7 & $(0.5)$ & 91.3 & $(0.5)$ & -0.09 & $(0.06)$ & -0.04 & $(0.03)$ & 444 & (6.8) & 458 & $(2.5)$ \\
\hline & Ungarn & 20.6 & $(0.8)$ & 79.4 & $(0.8)$ & -0.40 & $(0.04)$ & -0.18 & $(0.03)$ & 474 & (4.8) & 485 & (3.1) \\
\hline & Island & 10.7 & $(0.5)$ & 89.3 & $(0.5)$ & 0.52 & $(0.04)$ & 0.83 & $(0.01)$ & 481 & (5.9) & 500 & (1.9) \\
\hline & Irland & 10.9 & $(0.6)$ & 89.1 & (0.6) & -0.20 & $(0.04)$ & 0.21 & $(0.02)$ & 486 & (3.9) & 510 & (2.1) \\
\hline & Israel & $\mathrm{m}$ & $\mathrm{m}$ & $\mathrm{m}$ & $\mathrm{m}$ & $\mathrm{m}$ & $\mathrm{m}$ & $\mathrm{m}$ & $\mathrm{m}$ & $\mathrm{m}$ & $\mathrm{m}$ & $\mathrm{m}$ & $\mathrm{m}$ \\
\hline & Italien & 9.5 & $(0.3)$ & 90.5 & $(0.3)$ & -0.14 & $(0.03)$ & -0.03 & $(0.02)$ & 482 & (3.0) & 488 & (2.1) \\
\hline & Japan & 12.1 & $(0.6)$ & 87.9 & $(0.6)$ & -0.47 & $(0.03)$ & -0.01 & $(0.01)$ & 516 & (5.8) & 544 & (3.5) \\
\hline & Korea & 8.8 & $(0.5)$ & 91.2 & $(0.5)$ & -0.39 & $(0.05)$ & 0.08 & $(0.03)$ & 549 & (6.8) & 560 & (4.7) \\
\hline & Luxemburg & 12.2 & $(0.5)$ & 87.8 & $(0.5)$ & -0.08 & $(0.04)$ & 0.11 & $(0.02)$ & 485 & (3.8) & 494 & (1.4) \\
\hline & Mexiko & 15.2 & $(0.4)$ & 84.8 & $(0.4)$ & -1.02 & $(0.03)$ & -1.06 & $(0.03)$ & 423 & (2.2) & 422 & (1.3) \\
\hline & Niederlande & 11.2 & $(0.5)$ & 88.8 & $(0.5)$ & 0.02 & $(0.05)$ & 0.27 & $(0.02)$ & 501 & (6.3) & 530 & (3.4) \\
\hline & Neuseeland & 19.6 & $(0.8)$ & 80.4 & (0.8) & -0.23 & $(0.03)$ & 0.11 & $(0.02)$ & 489 & (3.8) & 507 & (2.5) \\
\hline & Norwegen & 10.7 & $(0.6)$ & 89.3 & $(0.6)$ & 0.13 & $(0.04)$ & 0.52 & $(0.02)$ & 481 & (5.5) & 495 & $(2.7)$ \\
\hline & Polen & 16.4 & $(0.7)$ & 83.6 & $(0.7)$ & -0.39 & $(0.04)$ & -0.16 & $(0.03)$ & 500 & (5.5) & 524 & (3.4) \\
\hline & Portugal & 12.3 & $(0.5)$ & 87.7 & $(0.5)$ & -0.62 & $(0.08)$ & -0.44 & $(0.05)$ & 489 & (5.7) & 494 & (3.6) \\
\hline & Slowak. Rep. & 14.9 & $(0.7)$ & 85.1 & $(0.7)$ & -0.28 & $(0.04)$ & -0.13 & $(0.03)$ & 481 & (5.3) & 492 & (3.5) \\
\hline & Slowenien & 10.8 & $(0.5)$ & 89.2 & $(0.5)$ & -0.05 & $(0.05)$ & 0.10 & $(0.01)$ & 495 & $(4.9)$ & 507 & $(1.3)$ \\
\hline & Spanien & 10.2 & $(0.3)$ & 89.8 & $(0.3)$ & -0.43 & $(0.04)$ & -0.15 & $(0.02)$ & 479 & (3.0) & 489 & $(1.8)$ \\
\hline & Schweden & 9.4 & $(0.5)$ & 90.6 & $(0.5)$ & -0.09 & $(0.04)$ & 0.33 & $(0.02)$ & 468 & (5.4) & 487 & (2.1) \\
\hline & Schweiz & 13.6 & $(0.4)$ & 86.4 & $(0.4)$ & 0.11 & $(0.03)$ & 0.19 & $(0.02)$ & 527 & (3.9) & 536 & (3.3) \\
\hline & Türkei & 4.2 & $(0.3)$ & 95.8 & $(0.3)$ & -1.33 & $(0.10)$ & -1.42 & $(0.04)$ & 456 & $(8.3)$ & 457 & (4.9) \\
\hline & Ver. Königreich & 16.6 & $(0.6)$ & 83.4 & $(0.6)$ & -0.01 & $(0.03)$ & 0.37 & $(0.02)$ & 481 & $(4.4)$ & 507 & (3.0) \\
\hline & Ver. Staaten & 20.3 & $(0.9)$ & 79.7 & $(0.9)$ & -0.19 & $(0.04)$ & 0.30 & $(0.04)$ & 468 & $(5.0)$ & 492 & (3.7) \\
\hline & OECD-Durchschnitt & 13.7 & $(0.1)$ & 86.3 & $(0.1)$ & -0.21 & $(0.01)$ & 0.06 & $(0.00)$ & 488 & $(0.8)$ & 502 & $(0.5)$ \\
\hline s & Albanien & $\mathrm{m}$ & $\mathrm{m}$ & $\mathrm{m}$ & $\mathrm{m}$ & $\mathrm{m}$ & $\mathrm{m}$ & $\mathrm{m}$ & $\mathrm{m}$ & $\mathrm{m}$ & $\mathrm{m}$ & $\mathrm{m}$ & $\mathrm{m}$ \\
\hline 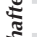 & Argentinien & 19.5 & $(0.7)$ & 80.5 & $(0.7)$ & -0.80 & $(0.07)$ & -0.64 & $(0.04)$ & 395 & (4.1) & 399 & (3.7) \\
\hline స్ స్ & Brasilien & 20.6 & $(0.5)$ & 79.4 & $(0.5)$ & -1.20 & $(0.03)$ & -1.10 & $(0.03)$ & 396 & (2.7) & 401 & (2.3) \\
\hline 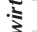 & Bulgarien & 12.7 & $(0.6)$ & 87.3 & (0.6) & -0.39 & $(0.05)$ & -0.20 & $(0.04)$ & 442 & (5.4) & 450 & (3.8) \\
\hline = & Kolumbien & 23.9 & $(0.8)$ & 76.1 & $(0.8)$ & -1.19 & $(0.05)$ & -1.17 & $(0.05)$ & 387 & (3.4) & 389 & (3.3) \\
\hline$\frac{1}{3}$ & Costa Rica & 22.6 & $(0.7)$ & 77.4 & $(0.7)$ & -1.08 & $(0.05)$ & -0.89 & $(0.05)$ & 408 & (3.5) & 414 & (3.1) \\
\hline के & Kroatien & 8.1 & $(0.5)$ & 91.9 & $(0.5)$ & -0.40 & $(0.04)$ & -0.33 & $(0.02)$ & 478 & (5.3) & 473 & (3.7) \\
\hline : & Zypern* & 8.9 & $(0.4)$ & 91.1 & $(0.4)$ & -0.26 & $(0.04)$ & 0.14 & $(0.01)$ & 425 & $(4.7)$ & 448 & $(1.3)$ \\
\hline ¿ั & Hongkong (China) & 13.3 & $(0.5)$ & 86.7 & $(0.5)$ & -1.01 & $(0.06)$ & -0.75 & $(0.05)$ & 555 & (4.3) & 566 & (3.4) \\
\hline 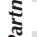 & Indonesien & 7.4 & $(0.5)$ & 92.6 & $(0.5)$ & -1.91 & $(0.09)$ & -1.75 & $(0.06)$ & 383 & (5.8) & 385 & $(4.3)$ \\
\hline & Jordanien & 9.7 & $(0.6)$ & 90.3 & $(0.6)$ & -0.58 & $(0.07)$ & -0.37 & $(0.02)$ & 367 & (5.6) & 400 & $(3.1)$ \\
\hline & Kasachstan & 14.1 & $(0.6)$ & 85.9 & $(0.6)$ & -0.47 & $(0.04)$ & -0.29 & $(0.02)$ & 435 & $(4.3)$ & 433 & (3.1) \\
\hline & Lettland & 20.1 & $(0.8)$ & 79.9 & $(0.8)$ & -0.41 & $(0.06)$ & -0.17 & $(0.03)$ & 498 & $(4.0)$ & 496 & (2.9) \\
\hline & Liechtenstein & 15.0 & $(2.2)$ & 85.0 & $(2.2)$ & 0.18 & $(0.14)$ & 0.31 & $(0.06)$ & 518 & (13.8) & 541 & $(5.0)$ \\
\hline & Litauen & 15.7 & $(0.6)$ & 84.3 & $(0.6)$ & -0.35 & $(0.04)$ & -0.05 & $(0.02)$ & 474 & $(4.1)$ & 485 & $(2.7)$ \\
\hline & Macau (China) & 13.6 & $(0.5)$ & 86.4 & $(0.5)$ & -1.03 & $(0.03)$ & -0.86 & $(0.01)$ & 533 & (3.6) & 543 & $(1.1)$ \\
\hline & Malaysia & 12.3 & $(0.6)$ & 87.7 & $(0.6)$ & -0.85 & $(0.06)$ & -0.67 & $(0.04)$ & 411 & (3.9) & 429 & (3.3) \\
\hline & Montenegro & 6.4 & $(0.4)$ & 93.6 & $(0.4)$ & -0.26 & $(0.05)$ & -0.23 & $(0.01)$ & 423 & (6.1) & 415 & (1.3) \\
\hline & Peru & 17.0 & $(0.5)$ & 83.0 & $(0.5)$ & -1.15 & $(0.07)$ & -1.21 & $(0.05)$ & 382 & (4.4) & 372 & (3.9) \\
\hline & Katar & 10.9 & $(0.3)$ & 89.1 & $(0.3)$ & 0.23 & $(0.03)$ & 0.51 & $(0.01)$ & 340 & (3.0) & 400 & (1.1) \\
\hline & Rumänien & 13.8 & $(0.7)$ & 86.2 & $(0.7)$ & -0.54 & $(0.05)$ & -0.42 & $(0.04)$ & 443 & $(4.5)$ & 451 & $(4.0)$ \\
\hline & Russ. Föderation & 22.3 & $(0.7)$ & 77.7 & $(0.7)$ & -0.26 & $(0.04)$ & -0.04 & $(0.03)$ & 488 & (3.8) & 488 & (3.2) \\
\hline & Serbien & 8.8 & $(0.4)$ & 91.2 & $(0.4)$ & -0.41 & $(0.05)$ & -0.25 & $(0.02)$ & 448 & (5.3) & 456 & (3.3) \\
\hline & Shanghai (China) & 9.4 & $(0.4)$ & 90.6 & $(0.4)$ & -0.42 & $(0.05)$ & -0.35 & $(0.04)$ & 615 & (4.8) & 615 & (3.1) \\
\hline & Singapur & 9.2 & $(0.4)$ & 90.8 & $(0.4)$ & -0.45 & $(0.04)$ & -0.22 & $(0.02)$ & 564 & (5.5) & 579 & (1.5) \\
\hline & Chinesisch Taipeh & 12.9 & $(0.4)$ & 87.1 & $(0.4)$ & -0.72 & $(0.04)$ & -0.34 & $(0.03)$ & 531 & (5.4) & 568 & (3.4) \\
\hline & Thailand & 14.7 & (0.6) & 85.3 & $(0.6)$ & -1.35 & $(0.06)$ & -1.28 & $(0.05)$ & 429 & $(4.7)$ & 435 & (3.6) \\
\hline & Tunesien & 6.2 & $(0.5)$ & 93.8 & $(0.5)$ & -1.27 & $(0.09)$ & -1.13 & $(0.05)$ & 379 & (6.9) & 396 & (4.3) \\
\hline & Ver. Arab. Emirate & 9.8 & $(0.4)$ & 90.2 & $(0.4)$ & 0.07 & $(0.04)$ & 0.40 & $(0.02)$ & 411 & $(4.4)$ & 446 & (2.5) \\
\hline & Uruguay & 18.4 & $(0.6)$ & 81.6 & (0.6) & -1.01 & $(0.05)$ & -0.79 & $(0.03)$ & 417 & (4.1) & 421 & (2.6) \\
\hline & Vietnam & 7.8 & $(0.4)$ & 92.2 & $(0.4)$ & -1.92 & $(0.06)$ & -1.78 & $(0.05)$ & 525 & $(6.2)$ & 514 & $(4.8)$ \\
\hline
\end{tabular}

Anmerkung: Diese Tabelle wurde nur für Schüler berechnet, für die Daten zum PISA-Index des wirtschaftlichen, sozialen und kulturellen Status vorliegen. Statistisch signifikante Werte sind durch Fettdruck gekennzeichnet (vgl. Anhang A3).

*Vgl. die Anmerkungen am Beginn dieses Anhangs.

StatLink तins http://dx.doi.org/10.1787/888932964927 
[Teil 2/2]

Schülerleistungen in Mathematik und Familientyp

Tabelle II.3.1 Die Ergebnisse basieren auf Schülerangaben

\begin{tabular}{|c|c|c|c|c|c|c|c|c|c|c|c|}
\hline & \multicolumn{2}{|c|}{\begin{tabular}{|c|} 
Leistungsunterschied \\
in Mathematik \\
zwischen Schülern aus \\
Alleinerziehenden- \\
haushalten und Schülern \\
aus anderen Familientypen, \\
vor Berücksichtigung \\
des ESCS
\end{tabular}} & \multicolumn{2}{|c|}{\begin{tabular}{|} 
Leistungsunterschied \\
in Mathematik \\
zwischen Schülern aus \\
Alleinerziehendenhaushalten \\
und Schülern aus anderen \\
Familientypen, nach \\
Berücksichtigung des ESCS
\end{tabular}} & \multicolumn{2}{|c|}{$\begin{array}{c}\text { Größere Wahrscheinlichkeit } \\
\text { für Schüler aus } \\
\text { Alleinerziehenden- } \\
\text { haushalten, im untersten } \\
\text { nationalen Quartil der } \\
\text { Gesamtskala Mathematik } \\
\text { zu liegen }\end{array}$} & \multicolumn{2}{|c|}{\begin{tabular}{|c|} 
Populationsrelevanz \\
der Schüler aus \\
Alleinerziehenden- \\
haushalten, die im untersten \\
nationalen Quartil der \\
Gesamtskala Mathematik \\
liegen
\end{tabular}} & \multicolumn{2}{|c|}{$\begin{array}{l}\text { Effektstärke bei den } \\
\text { Mathematikleistungen } \\
\text { für Schüler aus } \\
\text { Alleinerziehenden- } \\
\text { haushalten und anderen } \\
\text { Familientypen }\end{array}$} \\
\hline & & Punktdiff. & S.E. & Punktdiff. & S.E. & Quotient & S.E. & $\%$ & S.E. & Effektstärke & S.E. \\
\hline \multirow{35}{*}{ 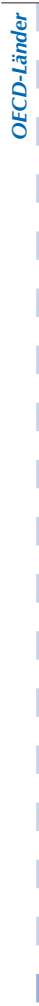 } & Australien & -18 & (2.5) & -1 & (2.5) & 1.27 & $(0.06)$ & 3.5 & $(0.7)$ & -0.2 & $(0.0)$ \\
\hline & Österreich & -7 & (4.9) & -1 & $(4.8)$ & 1.09 & $(0.10)$ & 1.2 & (1.3) & -0.1 & $(0.1)$ \\
\hline & Belgien & -33 & $(4.8)$ & -17 & $(4.2)$ & 1.43 & $(0.10)$ & 5.5 & $(1.2)$ & -0.3 & $(0.0)$ \\
\hline & Kanada & -14 & (3.1) & -3 & (3.0) & 1.24 & $(0.07)$ & 3.0 & $(0.9)$ & -0.2 & $(0.0)$ \\
\hline & Chile & -4 & (3.5) & -1 & (3.3) & 1.06 & $(0.09)$ & 1.3 & (2.0) & 0.0 & $(0.0)$ \\
\hline & Tschech. Rep. & -20 & $(4.2)$ & -5 & (4.5) & 1.24 & $(0.09)$ & 4.0 & (1.5) & -0.2 & $(0.0)$ \\
\hline & Dänemark & -22 & (3.8) & -8 & (3.5) & 1.49 & $(0.12)$ & 6.9 & (1.6) & -0.3 & $(0.0)$ \\
\hline & Estland & 0 & (3.8) & 10 & (3.7) & 0.95 & $(0.08)$ & -1.0 & (1.5) & 0.0 & $(0.0)$ \\
\hline & Finnland & -21 & $(3.2)$ & -7 & (3.0) & 1.41 & $(0.09)$ & 6.1 & (1.3) & -0.2 & $(0.0)$ \\
\hline & Frankreich & -21 & $(4.7)$ & -4 & $(4.2)$ & 1.25 & $(0.12)$ & 3.6 & (1.7) & -0.2 & $(0.0)$ \\
\hline & Deutschland & -4 & $(4.7)$ & 5 & (3.9) & 1.07 & $(0.09)$ & 1.0 & $(1.2)$ & 0.0 & $(0.0)$ \\
\hline & Griechenland & -14 & (6.5) & -12 & (6.0) & 1.33 & $(0.13)$ & 2.8 & (1.1) & -0.2 & $(0.1)$ \\
\hline & Ungarn & -10 & $(4.0)$ & -1 & (3.8) & 1.16 & $(0.09)$ & 3.1 & (1.7) & -0.1 & $(0.0)$ \\
\hline & Island & -19 & (6.3) & -10 & (6.1) & 1.41 & $(0.14)$ & 4.2 & (1.4) & -0.2 & $(0.1)$ \\
\hline & Irland & -24 & (3.7) & -10 & (3.4) & 1.42 & $(0.12)$ & 4.4 & $(1.2)$ & -0.3 & $(0.0)$ \\
\hline & Israel & $\mathrm{m}$ & $\mathrm{m}$ & $\mathrm{m}$ & $\mathrm{m}$ & $\mathrm{m}$ & $\mathrm{m}$ & $\mathrm{m}$ & $\mathrm{m}$ & $\mathrm{m}$ & $\mathrm{m}$ \\
\hline & Italien & -6 & $(2.7)$ & -3 & $(2.7)$ & 1.10 & $(0.06)$ & 0.9 & $(0.6)$ & -0.1 & $(0.0)$ \\
\hline & Japan & -28 & (5.1) & -10 & $(4.0)$ & 1.47 & $(0.12)$ & 5.3 & (1.3) & -0.3 & $(0.1)$ \\
\hline & Korea & -11 & (5.3) & 9 & (5.5) & 1.11 & $(0.09)$ & 1.0 & $(0.8)$ & -0.1 & $(0.1)$ \\
\hline & Luxemburg & -9 & (4.4) & -2 & (3.8) & 1.06 & $(0.09)$ & 0.7 & (1.1) & -0.1 & $(0.0)$ \\
\hline & Mexiko & 1 & $(2.1)$ & 0 & $(2.0)$ & 0.99 & $(0.06)$ & -0.2 & $(0.9)$ & 0.0 & $(0.0)$ \\
\hline & Niederlande & -29 & (5.4) & -19 & (5.3) & 1.45 & $(0.13)$ & 4.8 & (1.3) & -0.3 & $(0.1)$ \\
\hline & Neuseeland & -19 & (4.0) & -1 & (4.0) & 1.32 & $(0.10)$ & 5.9 & (1.7) & -0.2 & $(0.0)$ \\
\hline & Norwegen & -14 & (5.4) & -2 & (5.1) & 1.21 & $(0.11)$ & 2.2 & (1.2) & -0.2 & $(0.1)$ \\
\hline & Polen & -24 & $(4.0)$ & -15 & $(4.0)$ & 1.49 & $(0.10)$ & 7.4 & (1.4) & -0.3 & $(0.0)$ \\
\hline & Portugal & -5 & $(4.8)$ & 1 & (4.4) & 1.04 & $(0.09)$ & 0.4 & (1.1) & -0.1 & $(0.1)$ \\
\hline & Slowak. Rep. & -10 & (5.3) & -2 & (5.0) & 1.12 & $(0.09)$ & 1.7 & (1.3) & -0.1 & $(0.1)$ \\
\hline & Slowenien & -12 & (5.1) & -6 & $(4.7)$ & 1.18 & $(0.12)$ & 1.9 & $(1.2)$ & -0.1 & $(0.1)$ \\
\hline & Spanien & -10 & (2.6) & -1 & (2.3) & 1.15 & $(0.07)$ & 1.5 & $(0.7)$ & -0.1 & $(0.0)$ \\
\hline & Schweden & -19 & (5.3) & -4 & (5.5) & 1.34 & $(0.13)$ & 3.1 & (1.1) & -0.2 & $(0.1)$ \\
\hline & Schweiz & -9 & (3.7) & -6 & (3.6) & 1.10 & $(0.09)$ & 1.3 & $(1.2)$ & -0.1 & $(0.0)$ \\
\hline & Türkei & -1 & (6.9) & -4 & (6.5) & 1.05 & $(0.16)$ & 0.2 & $(0.7)$ & 0.0 & $(0.1)$ \\
\hline & Ver. Königreich & -26 & (4.3) & -11 & (3.8) & 1.33 & $(0.09)$ & 5.1 & (1.4) & -0.3 & $(0.0)$ \\
\hline & Ver. Staaten & -24 & (4.3) & -7 & (3.5) & 1.38 & $(0.10)$ & 7.2 & (1.7) & -0.3 & $(0.0)$ \\
\hline & OECD-Durchschnitt & -15 & $(0.8)$ & -4 & $(0.7)$ & 1.23 & $(0.02)$ & 3.0 & $(0.2)$ & -0.2 & $(0.0)$ \\
\hline \multirow{31}{*}{ 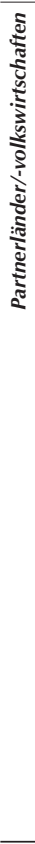 } & Albanien & $\mathrm{m}$ & $\mathrm{m}$ & $\mathrm{m}$ & $\mathrm{m}$ & $\mathrm{m}$ & $\mathrm{m}$ & $\mathrm{m}$ & $\mathrm{m}$ & $m$ & $m$ \\
\hline & Argentinien & -4 & (3.6) & 0 & (3.5) & 1.06 & $(0.10)$ & 1.1 & (1.8) & -0.1 & $(0.0)$ \\
\hline & Brasilien & -5 & (2.6) & -3 & (2.6) & 1.08 & $(0.07)$ & 1.6 & (1.3) & -0.1 & $(0.0)$ \\
\hline & Bulgarien & -8 & (4.3) & 0 & (4.0) & 1.09 & $(0.09)$ & 1.2 & (1.1) & -0.1 & $(0.0)$ \\
\hline & Kolumbien & -2 & (3.3) & -1 & (3.2) & 0.98 & $(0.09)$ & -0.5 & (2.1) & 0.0 & $(0.0)$ \\
\hline & Costa Rica & -5 & (3.1) & -1 & (2.8) & 1.09 & $(0.09)$ & 2.0 & (1.8) & -0.1 & $(0.0)$ \\
\hline & Kroatien & 5 & (5.5) & 8 & (5.1) & 0.94 & $(0.11)$ & -0.5 & $(0.9)$ & 0.1 & $(0.1)$ \\
\hline & Zypern* & -23 & (5.2) & -8 & (5.0) & 1.33 & $(0.11)$ & 2.9 & (1.0) & -0.2 & $(0.1)$ \\
\hline & Hongkong (China) & -11 & $(4.4)$ & -4 & (4.3) & 1.20 & $(0.09)$ & 2.6 & (1.2) & -0.1 & $(0.0)$ \\
\hline & Indonesien & -2 & $(4.0)$ & 2 & (4.1) & 1.06 & $(0.11)$ & 0.4 & $(0.8)$ & 0.0 & $(0.1)$ \\
\hline & Jordanien & -33 & (4.6) & -28 & (4.9) & 1.79 & $(0.13)$ & 7.1 & (1.1) & -0.4 & $(0.1)$ \\
\hline & Kasachstan & 2 & (3.6) & 7 & (3.5) & 0.92 & $(0.08)$ & -1.1 & (1.1) & 0.0 & $(0.1)$ \\
\hline & Lettland & 2 & (3.9) & 10 & (3.7) & 0.98 & $(0.10)$ & -0.5 & (2.0) & 0.0 & $(0.0)$ \\
\hline & Liechtenstein & -24 & $(15.5)$ & -20 & $(15.8)$ & 1.21 & $(0.34)$ & 3.0 & (5.1) & -0.3 & $(0.2)$ \\
\hline & Litauen & -11 & $(4.2)$ & -1 & (3.9) & 1.27 & $(0.10)$ & 4.0 & (1.5) & -0.1 & $(0.0)$ \\
\hline & Macau (China) & -10 & $(4.0)$ & -7 & $(4.0)$ & 1.19 & $(0.09)$ & 2.5 & $(1.2)$ & -0.1 & $(0.0)$ \\
\hline & Malaysia & -17 & (3.5) & -12 & (3.4) & 1.32 & $(0.10)$ & 3.8 & (1.1) & -0.2 & $(0.0)$ \\
\hline & Montenegro & 8 & $(6.2)$ & 9 & (6.0) & 0.90 & $(0.13)$ & -0.7 & $(0.8)$ & 0.1 & $(0.1)$ \\
\hline & Peru & 10 & (3.0) & 8 & $(2.7)$ & 0.83 & $(0.08)$ & -3.0 & (1.4) & 0.1 & $(0.0)$ \\
\hline & Katar & -59 & (3.2) & -52 & (3.1) & 2.09 & $(0.09)$ & 10.6 & $(0.8)$ & -0.6 & $(0.0)$ \\
\hline & Rumänien & -8 & $(3.7)$ & -4 & (3.4) & 1.10 & $(0.08)$ & 1.3 & (1.1) & -0.1 & $(0.0)$ \\
\hline & Russ. Föderation & 0 & (3.6) & 8 & (2.8) & 1.00 & $(0.06)$ & -0.1 & (1.4) & 0.0 & $(0.0)$ \\
\hline & Serbien & -8 & (4.5) & -3 & (4.3) & 1.09 & $(0.10)$ & 0.8 & (0.8) & -0.1 & $(0.1)$ \\
\hline & Shanghai (China) & 1 & (4.2) & 3 & (3.7) & 0.89 & $(0.09)$ & -1.1 & (0.9) & 0.0 & $(0.0)$ \\
\hline & Singapur & -15 & (5.9) & -5 & (5.5) & 1.24 & $(0.11)$ & 2.2 & (0.9) & -0.1 & $(0.1)$ \\
\hline & Chinesisch Taipeh & -37 & (5.4) & -15 & (4.5) & 1.47 & $(0.10)$ & 5.7 & (1.2) & -0.3 & $(0.0)$ \\
\hline & Thailand & -5 & (3.7) & -4 & (3.7) & 1.03 & $(0.10)$ & 0.4 & (1.4) & -0.1 & $(0.0)$ \\
\hline & Tunesien & -17 & (6.2) & -14 & (6.1) & 1.38 & $(0.16)$ & 2.3 & $(1.0)$ & -0.2 & $(0.1)$ \\
\hline & Ver. Arab. Emirate & -35 & (3.9) & -25 & (3.8) & 1.69 & $(0.11)$ & 6.4 & $(1.0)$ & -0.4 & $(0.0)$ \\
\hline & Uruguay & -4 & (3.8) & 3 & (3.2) & 1.03 & $(0.07)$ & 0.5 & (1.2) & -0.1 & $(0.0)$ \\
\hline & Vietnam & 11 & (4.6) & 15 & (4.4) & 0.85 & $(0.09)$ & -1.2 & $(0.7)$ & 0.1 & $(0.1)$ \\
\hline
\end{tabular}

Anmerkung: Diese Tabelle wurde nur für Schüler berechnet, für die Daten zum PISA-Index des wirtschaftlichen, sozialen und kulturellen Status vorliegen. Statistisch signifikante Werte sind durch Fettdruck gekennzeichnet (vgl. Anhang A3).

* Vgl. die Anmerkungen am Beginn dieses Anhangs.

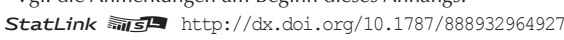


[Teil 1/3]

Schülerleistungen in Mathematik, Erwerbsstatus und sozioökonomischer Status der Eltern Tabelle II.3.2 Die Ergebnisse basieren auf Schülerangaben

\begin{tabular}{|c|c|c|c|c|c|c|c|c|c|c|c|c|c|c|c|c|c|}
\hline & & \multicolumn{8}{|c|}{ Prozentsatz der Schüler } & \multicolumn{8}{|c|}{ PISA-Index des wirtschaftlichen, sozialen und kulturellen Status (ESCS) } \\
\hline & & \multicolumn{4}{|c|}{ Aktueller Erwerbsstatus des Vaters } & \multicolumn{4}{|c|}{ Aktueller Erwerbsstatus der Mutter } & \multicolumn{4}{|c|}{ Aktueller Erwerbsstatus des Vaters } & \multicolumn{4}{|c|}{ Aktueller Erwerbsstatus der Mutter } \\
\hline & & \multicolumn{2}{|c|}{$\begin{array}{c}\text { Erwerbstätig in } \\
\text { Voll- oder Teilzeit }\end{array}$} & \multicolumn{2}{|c|}{$\begin{array}{c}\text { Nicht erwerbs- } \\
\text { tätig, aber auf } \\
\text { Arbeitsuche oder } \\
\text { anderer Status } \\
\text { (z.B. Haushalts- } \\
\text { pflichten oder } \\
\text { Ruhestand) }\end{array}$} & \multicolumn{2}{|c|}{$\begin{array}{c}\text { Erwerbstätig in } \\
\text { Voll- oder Teilzeit }\end{array}$} & \multicolumn{2}{|c|}{$\begin{array}{c}\text { Nicht erwerbs- } \\
\text { tätig, aber auf } \\
\text { Arbeitsuche oder } \\
\text { anderer Status } \\
\text { (z.B. Haushalts- } \\
\text { pflichten oder } \\
\text { Ruhestand) } \\
\end{array}$} & \multicolumn{2}{|c|}{$\begin{array}{c}\text { Erwerbstätig in } \\
\text { Voll- oder Teilzeit }\end{array}$} & \multicolumn{2}{|c|}{\begin{tabular}{|c|} 
Nicht erwerbs- \\
tätig, aber auf \\
Arbeitsuche oder \\
anderer Status \\
(z.B. Haushalts- \\
pflichten oder \\
Ruhestand)
\end{tabular}} & \multicolumn{2}{|c|}{$\begin{array}{c}\text { Erwerbstätig in } \\
\text { Voll- oder Teilzeit }\end{array}$} & \multicolumn{2}{|c|}{\begin{tabular}{|c} 
Nicht erwerbs- \\
tätig, aber auf \\
Arbeitsuche ode \\
anderer Status \\
(z.B. Haushalts- \\
pflichten oder \\
Ruhestand) \\
\end{tabular}} \\
\hline & & $\%$ & S.E. & $\%$ & S.E. & $\%$ & S.E. & $\%$ & S.E. & $\begin{array}{l}\text { Index- } \\
\text { mittel }\end{array}$ & S.E. & $\begin{array}{l}\text { Index- } \\
\text { mittel }\end{array}$ & S.E. & $\begin{array}{l}\text { Index- } \\
\text { mittel }\end{array}$ & S.E. & $\begin{array}{l}\text { Index- } \\
\text { mittel }\end{array}$ & S.E. \\
\hline \multirow{5}{*}{ 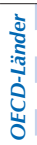 } & Australien & 90.9 & $(0.3)$ & 9.1 & $(0.3)$ & 74.8 & (0.6) & 25.2 & $(0.6)$ & 0.31 & $(0.01)$ & -0.05 & $(0.03)$ & 0.34 & $(0.01)$ & 0.01 & $(0.02)$ \\
\hline & Österreich & 92.6 & $(0.5)$ & 7.4 & $(0.5)$ & 80.0 & (0.6) & 20.0 & $(0.6)$ & 0.13 & $(0.02)$ & -0.32 & $(0.07)$ & 0.14 & $(0.02)$ & -0.15 & $(0.04)$ \\
\hline & Belgien & 88.8 & $(0.5)$ & 11.2 & $(0.5)$ & 76.6 & $(0.7)$ & 23.4 & $(0.7)$ & 0.25 & $(0.02)$ & -0.36 & $(0.06)$ & 0.29 & $(0.02)$ & -0.27 & $(0.04)$ \\
\hline & Kanada & 91.7 & $(0.3)$ & 8.3 & $(0.3)$ & 78.4 & $(0.5)$ & 21.6 & $(0.5)$ & 0.47 & $(0.02)$ & 0.15 & $(0.03)$ & 0.48 & $(0.01)$ & 0.22 & $(0.03)$ \\
\hline & Chile & 90.0 & $(0.5)$ & 10.0 & $(0.5)$ & 53.5 & $(0.9)$ & 46.5 & $(0.9)$ & -0.48 & $(0.03)$ & -1.03 & $(0.08)$ & -0.35 & $(0.03)$ & -0.82 & $(0.04)$ \\
\hline & Tschech. Rep. & 93.4 & $(0.5)$ & 6.6 & $(0.5)$ & 82.8 & $(0.8)$ & 17.2 & $(0.8)$ & -0.02 & $(0.02)$ & -0.41 & $(0.07)$ & 0.00 & $(0.02)$ & -0.35 & $(0.04)$ \\
\hline & Dänemark & 89.2 & $(0.5)$ & 10.8 & $(0.5)$ & 82.5 & $(0.8)$ & 17.5 & $(0.8)$ & 0.51 & $(0.02)$ & -0.05 & $(0.04)$ & 0.54 & $(0.02)$ & -0.05 & $(0.04)$ \\
\hline & Estland & 91.0 & $(0.5)$ & 9.0 & $(0.5)$ & 82.0 & $(0.6)$ & 18.0 & $(0.6)$ & 0.18 & $(0.01)$ & -0.19 & $(0.05)$ & 0.17 & $(0.02)$ & -0.12 & $(0.03)$ \\
\hline & Finnland & 87.5 & $(0.5)$ & 12.5 & $(0.5)$ & 85.2 & $(0.6)$ & 14.8 & $(0.6)$ & 0.44 & $(0.02)$ & -0.01 & $(0.04)$ & 0.44 & $(0.01)$ & 0.01 & $(0.04)$ \\
\hline & Frankreich & 90.2 & $(0.5)$ & 9.8 & $(0.5)$ & 78.4 & $(0.8)$ & 21.6 & $(0.8)$ & 0.04 & $(0.02)$ & -0.41 & $(0.05)$ & 0.08 & $(0.02)$ & -0.39 & $(0.03)$ \\
\hline & Deutschland & 93.3 & $(0.4)$ & 6.7 & $(0.4)$ & 77.2 & $(0.8)$ & 22.8 & $(0.8)$ & 0.26 & $(0.02)$ & -0.19 & $(0.08)$ & 0.26 & $(0.03)$ & -0.02 & $(0.04)$ \\
\hline & Griechenland & 81.4 & $(0.6)$ & 18.6 & $(0.6)$ & 56.8 & $(1.1)$ & 43.2 & (1.1) & 0.02 & $(0.03)$ & -0.38 & $(0.05)$ & 0.19 & $(0.03)$ & -0.39 & $(0.03)$ \\
\hline & Ungarn & 85.5 & $(0.7)$ & 14.5 & $(0.7)$ & 74.3 & $(0.8)$ & 25.7 & $(0.8)$ & -0.14 & $(0.03)$ & -0.72 & $(0.07)$ & -0.07 & $(0.03)$ & -0.75 & $(0.05)$ \\
\hline & Island & 93.9 & $(0.4)$ & 6.1 & $(0.4)$ & 84.2 & $(0.6)$ & 15.8 & $(0.6)$ & 0.82 & $(0.01)$ & 0.44 & $(0.06)$ & 0.86 & $(0.02)$ & 0.42 & $(0.04)$ \\
\hline & Irland & 81.7 & $(0.6)$ & 18.3 & $(0.6)$ & 62.8 & $(0.8)$ & 37.2 & $(0.8)$ & 0.24 & $(0.02)$ & -0.25 & $(0.03)$ & 0.28 & $(0.02)$ & -0.12 & $(0.03)$ \\
\hline & Israel & 88.4 & $(0.7)$ & 11.6 & $(0.7)$ & 71.9 & $(1.1)$ & 28.1 & (1.1) & 0.28 & $(0.03)$ & -0.35 & $(0.06)$ & 0.36 & $(0.03)$ & -0.25 & $(0.04)$ \\
\hline & Italien & 91.7 & $(0.3)$ & 8.3 & $(0.3)$ & 62.8 & $(0.5)$ & 37.2 & $(0.5)$ & 0.00 & $(0.01)$ & -0.45 & $(0.04)$ & 0.18 & $(0.02)$ & -0.43 & $(0.02)$ \\
\hline & Japan & 96.9 & $(0.2)$ & 3.1 & $(0.2)$ & 77.9 & $(0.8)$ & 22.1 & $(0.8)$ & -0.02 & $(0.02)$ & -0.27 & $(0.06)$ & -0.09 & $(0.01)$ & 0.01 & $(0.03)$ \\
\hline & Korea & 90.4 & $(0.6)$ & 9.6 & $(0.6)$ & 59.4 & $(0.9)$ & 40.6 & $(0.9)$ & 0.07 & $(0.03)$ & -0.26 & $(0.05)$ & 0.03 & $(0.03)$ & 0.02 & $(0.03)$ \\
\hline & Luxemburg & 90.3 & $(0.5)$ & 9.7 & $(0.5)$ & 72.1 & $(0.7)$ & 27.9 & $(0.7)$ & 0.16 & $(0.02)$ & -0.35 & $(0.05)$ & 0.11 & $(0.02)$ & 0.00 & $(0.03)$ \\
\hline & Mexiko & 84.8 & $(0.4)$ & 15.2 & $(0.4)$ & 40.7 & $(0.5)$ & 59.3 & $(0.5)$ & -0.95 & $(0.03)$ & -1.73 & $(0.04)$ & -0.69 & $(0.03)$ & -1.36 & $(0.02)$ \\
\hline & Niederlande & 91.8 & $(0.4)$ & 8.2 & $(0.4)$ & 77.6 & $(0.7)$ & 22.4 & $(0.7)$ & 0.29 & $(0.02)$ & -0.16 & $(0.07)$ & 0.32 & $(0.02)$ & -0.02 & $(0.03)$ \\
\hline & Neuseeland & 91.3 & $(0.5)$ & 8.7 & $(0.5)$ & 76.2 & $(0.7)$ & 23.8 & $(0.7)$ & 0.11 & $(0.02)$ & -0.31 & $(0.05)$ & 0.12 & $(0.02)$ & -0.18 & $(0.03)$ \\
\hline & Norwegen & 92.3 & $(0.5)$ & 7.7 & $(0.5)$ & 86.0 & $(0.7)$ & 14.0 & $(0.7)$ & 0.52 & $(0.02)$ & 0.08 & $(0.04)$ & 0.53 & $(0.02)$ & 0.12 & $(0.04)$ \\
\hline & Polen & 87.4 & $(0.7)$ & 12.6 & $(0.7)$ & 70.0 & $(0.8)$ & 30.0 & $(0.8)$ & -0.13 & $(0.03)$ & -0.58 & $(0.04)$ & -0.03 & $(0.03)$ & -0.62 & $(0.03)$ \\
\hline & Portugal & 85.8 & $(0.6)$ & 14.2 & $(0.6)$ & 74.2 & $(0.9)$ & 25.8 & $(0.9)$ & -0.36 & $(0.05)$ & -0.91 & $(0.06)$ & -0.30 & $(0.05)$ & -0.96 & $(0.05)$ \\
\hline & Slowak. Rep. & 85.8 & $(0.8)$ & 14.2 & $(0.8)$ & 75.8 & $(0.8)$ & 24.2 & $(0.8)$ & -0.06 & $(0.02)$ & -0.82 & $(0.05)$ & -0.01 & $(0.02)$ & -0.70 & $(0.05)$ \\
\hline & Slowenien & 88.6 & $(0.6)$ & 11.4 & (0.6) & 84.3 & $(0.6)$ & 15.7 & $(0.6)$ & 0.13 & $(0.02)$ & -0.28 & $(0.05)$ & 0.16 & $(0.01)$ & -0.42 & $(0.04)$ \\
\hline & Spanien & 84.5 & $(0.5)$ & 15.5 & $(0.5)$ & 66.7 & $(0.6)$ & 33.3 & $(0.6)$ & -0.06 & $(0.02)$ & -0.71 & $(0.03)$ & -0.04 & $(0.03)$ & -0.48 & $(0.03)$ \\
\hline & Schweden & 93.1 & $(0.5)$ & 6.9 & $(0.5)$ & 88.6 & $(0.6)$ & 11.4 & $(0.6)$ & 0.33 & $(0.02)$ & -0.23 & $(0.06)$ & 0.34 & $(0.02)$ & -0.17 & $(0.04)$ \\
\hline & Schweiz & 94.2 & $(0.3)$ & 5.8 & $(0.3)$ & 75.9 & $(0.6)$ & 24.1 & $(0.6)$ & 0.21 & $(0.02)$ & -0.19 & $(0.05)$ & 0.23 & $(0.02)$ & 0.01 & $(0.03)$ \\
\hline & Türkei & 71.1 & $(0.9)$ & 28.9 & $(0.9)$ & 14.5 & $(0.8)$ & 85.5 & $(0.8)$ & -1.27 & $(0.04)$ & -1.83 & $(0.04)$ & -0.83 & $(0.08)$ & -1.52 & $(0.03)$ \\
\hline & Ver. Königreich & 89.6 & $(0.4)$ & 10.4 & $(0.4)$ & 76.3 & (1.0) & 23.7 & $(1.0)$ & 0.35 & $(0.02)$ & -0.09 & $(0.05)$ & 0.35 & $(0.02)$ & 0.06 & $(0.04)$ \\
\hline & Ver. Staaten & 86.0 & $(0.8)$ & 14.0 & $(0.8)$ & 73.8 & $(0.9)$ & 26.2 & $(0.9)$ & 0.27 & $(0.04)$ & -0.14 & $(0.04)$ & 0.25 & $(0.03)$ & -0.03 & $(0.07)$ \\
\hline & OECD-Durchschnitt & 89.0 & $(0.1)$ & 11.0 & $(0.1)$ & 72.2 & $(0.1)$ & 27.8 & $(0.1)$ & 0.09 & $(0.00)$ & -0.39 & $(0.01)$ & 0.14 & $(0.00)$ & -0.28 & $(0.01)$ \\
\hline
\end{tabular}

\begin{tabular}{|c|c|c|c|c|c|c|c|c|c|c|c|c|c|c|c|c|c|}
\hline \multirow{31}{*}{ 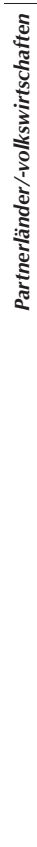 } & Albanien & $\mathrm{m}$ & $\mathrm{m}$ & $\mathrm{m}$ & $\mathrm{m}$ & $\mathrm{m}$ & $\mathrm{m}$ & $\mathrm{m}$ & $\mathrm{m}$ & $\mathrm{m}$ & $\mathrm{m}$ & $\mathrm{m}$ & $\mathrm{m}$ & $\mathrm{m}$ & $\mathrm{m}$ & $\mathrm{m}$ & $\mathrm{m}$ \\
\hline & Argentinien & 88.8 & $(0.6)$ & 11.2 & $(0.6)$ & 53.8 & $(1.2)$ & 46.2 & $(1.2)$ & -0.59 & $(0.04)$ & -1.19 & $(0.08)$ & -0.38 & $(0.05)$ & -1.04 & $(0.04)$ \\
\hline & Brasilien & 80.8 & $(0.4)$ & 19.2 & $(0.4)$ & 56.5 & $(0.5)$ & 43.5 & $(0.5)$ & -1.01 & $(0.02)$ & -1.60 & $(0.03)$ & -0.88 & $(0.03)$ & -1.51 & $(0.03)$ \\
\hline & Bulgarien & 86.4 & $(0.7)$ & 13.6 & $(0.7)$ & 79.3 & $(0.9)$ & 20.7 & (0.9) & -0.17 & $(0.03)$ & -0.86 & $(0.08)$ & -0.11 & $(0.03)$ & -0.87 & $(0.06)$ \\
\hline & Kolumbien & 84.3 & $(0.9)$ & 15.7 & $(0.9)$ & 53.4 & $(1.0)$ & 46.6 & (1.0) & -1.12 & $(0.04)$ & -1.70 & $(0.07)$ & -1.05 & $(0.04)$ & -1.47 & $(0.05)$ \\
\hline & Costa Rica & 87.9 & $(0.8)$ & 12.1 & $(0.8)$ & 44.7 & (1.1) & 55.3 & (1.1) & -0.85 & $(0.04)$ & -1.32 & $(0.08)$ & -0.61 & $(0.05)$ & -1.25 & $(0.04)$ \\
\hline & Kroatien & 71.6 & $(0.8)$ & 28.4 & $(0.8)$ & 62.2 & (1.0) & 37.8 & $(1.0)$ & -0.21 & $(0.02)$ & -0.63 & $(0.03)$ & -0.12 & $(0.02)$ & -0.70 & $(0.02)$ \\
\hline & Zypern* & 90.3 & $(0.5)$ & 9.7 & $(0.5)$ & 72.7 & $(0.6)$ & 27.3 & (0.6) & 0.18 & $(0.01)$ & -0.52 & $(0.04)$ & 0.25 & $(0.01)$ & -0.27 & $(0.03)$ \\
\hline & Hongkong (China) & 88.1 & $(0.5)$ & 11.9 & $(0.5)$ & 62.5 & $(1.0)$ & 37.5 & (1.0) & -0.73 & $(0.05)$ & -1.14 & $(0.05)$ & -0.75 & $(0.06)$ & -0.86 & $(0.05)$ \\
\hline & Indonesien & 80.1 & (1.0) & 19.9 & (1.0) & 38.9 & (1.1) & 61.1 & (1.1) & -1.71 & $(0.06)$ & -2.05 & $(0.05)$ & -1.65 & $(0.07)$ & -1.86 & $(0.05)$ \\
\hline & Jordanien & 75.1 & $(0.7)$ & 24.9 & $(0.7)$ & 17.4 & $(0.7)$ & 82.6 & $(0.7)$ & -0.29 & $(0.02)$ & -0.75 & $(0.05)$ & 0.09 & $(0.03)$ & -0.53 & $(0.02)$ \\
\hline & Kasachstan & 76.5 & (1.1) & 23.5 & (1.1) & 61.6 & $(1.3)$ & 38.4 & $(1.3)$ & -0.19 & $(0.02)$ & -0.65 & $(0.03)$ & -0.13 & $(0.02)$ & -0.60 & $(0.03)$ \\
\hline & Lettland & 86.3 & $(0.7)$ & 13.7 & $(0.7)$ & 78.7 & $(0.8)$ & 21.3 & $(0.8)$ & -0.16 & $(0.03)$ & -0.58 & $(0.06)$ & -0.17 & $(0.03)$ & -0.52 & $(0.05)$ \\
\hline & Liechtenstein & 91.4 & (1.9) & 8.6 & (1.9) & 66.3 & $(2.8)$ & 33.7 & (2.8) & 0.30 & $(0.06)$ & c & c & 0.32 & $(0.06)$ & 0.21 & $(0.09)$ \\
\hline & Litauen & 82.5 & $(0.6)$ & 17.5 & $(0.6)$ & 74.8 & $(0.8)$ & 25.2 & $(0.8)$ & -0.01 & $(0.02)$ & -0.51 & $(0.04)$ & 0.02 & $(0.02)$ & -0.56 & $(0.04)$ \\
\hline & Macau (China) & 88.8 & $(0.4)$ & 11.2 & $(0.4)$ & 76.3 & (0.6) & 23.7 & (0.6) & -0.84 & $(0.01)$ & -1.13 & $(0.04)$ & -0.86 & $(0.01)$ & -0.97 & $(0.02)$ \\
\hline & Malaysia & 86.0 & $(0.6)$ & 14.0 & $(0.6)$ & 39.6 & (1.0) & 60.4 & (1.0) & -0.67 & $(0.04)$ & -0.95 & $(0.05)$ & -0.37 & $(0.04)$ & -0.95 & $(0.03)$ \\
\hline & Montenegro & 73.8 & $(0.7)$ & 26.2 & $(0.7)$ & 50.5 & (0.8) & 49.5 & (0.8) & -0.10 & $(0.02)$ & -0.61 & $(0.02)$ & 0.03 & $(0.02)$ & -0.52 & $(0.02)$ \\
\hline & Peru & 84.0 & (0.8) & 16.0 & $(0.8)$ & 51.4 & $(0.8)$ & 48.6 & (0.8) & -1.08 & $(0.05)$ & -1.80 & $(0.07)$ & -1.08 & $(0.06)$ & -1.36 & (0.05) \\
\hline & Katar & 86.4 & (0.3) & 13.6 & $(0.3)$ & 38.0 & $(0.4)$ & 62.0 & $(0.4)$ & 0.51 & $(0.01)$ & 0.06 & $(0.03)$ & 0.65 & $(0.01)$ & 0.32 & $(0.01)$ \\
\hline & Rumänien & 74.1 & (1.0) & 25.9 & (1.0) & 60.5 & (1.2) & 39.5 & (1.2) & -0.28 & $(0.04)$ & -0.95 & $(0.05)$ & -0.19 & $(0.04)$ & -0.88 & $(0.04)$ \\
\hline & Russ. Föderation & 86.3 & (0.6) & 13.7 & (0.6) & 76.2 & (0.9) & 23.8 & (0.9) & -0.02 & $(0.03)$ & -0.37 & $(0.03)$ & -0.03 & $(0.02)$ & -0.33 & $(0.04)$ \\
\hline & Serbien & 77.2 & $(0.8)$ & 22.8 & $(0.8)$ & 59.4 & (1.1) & 40.6 & (1.1) & -0.18 & $(0.02)$ & -0.61 & $(0.03)$ & -0.08 & $(0.03)$ & -0.60 & $(0.03)$ \\
\hline & Shanghai (China) & 87.3 & $(0.5)$ & 12.7 & $(0.5)$ & 75.0 & (0.9) & 25.0 & (0.9) & -0.28 & $(0.03)$ & -0.89 & $(0.06)$ & -0.25 & $(0.03)$ & -0.69 & $(0.05)$ \\
\hline & Singapur & 92.6 & $(0.4)$ & 7.4 & $(0.4)$ & 63.3 & $(0.6)$ & 36.7 & $(0.6)$ & -0.23 & $(0.02)$ & -0.44 & $(0.05)$ & -0.21 & $(0.02)$ & -0.34 & $(0.03)$ \\
\hline & Chinesisch Taipeh & 87.9 & (0.5) & 12.1 & $(0.5)$ & 69.6 & $(0.6)$ & 30.4 & (0.6) & -0.34 & $(0.02)$ & -0.70 & $(0.04)$ & -0.35 & $(0.03)$ & -0.46 & $(0.03)$ \\
\hline & Thailand & 81.8 & $(0.6)$ & 18.2 & $(0.6)$ & 70.9 & $(0.6)$ & 29.1 & $(0.6)$ & -1.27 & $(0.05)$ & -1.55 & $(0.05)$ & -1.31 & $(0.05)$ & -1.40 & $(0.04)$ \\
\hline & Tunesien & 84.0 & $(0.7)$ & 16.0 & $(0.7)$ & 23.9 & (1.1) & 76.1 & (1.1) & -1.07 & $(0.05)$ & -1.68 & $(0.07)$ & -0.51 & $(0.09)$ & -1.39 & $(0.04)$ \\
\hline & Ver. Arab. Emirate & 80.9 & $(0.6)$ & 19.1 & $(0.6)$ & 27.0 & $(0.6)$ & 73.0 & $(0.6)$ & 0.44 & $(0.01)$ & -0.08 & $(0.04)$ & 0.65 & $(0.02)$ & 0.21 & $(0.02)$ \\
\hline & Uruguay & 89.5 & $(0.5)$ & 10.5 & $(0.5)$ & 65.5 & $(0.8)$ & 34.5 & $(0.8)$ & -0.76 & $(0.03)$ & -1.25 & $(0.05)$ & -0.65 & $(0.03)$ & -1.29 & $(0.03)$ \\
\hline & Vietnam & 53.2 & (1.6) & 46.8 & (1.6) & 36.3 & (1.7) & 63.7 & (1.7) & -1.46 & $(0.06)$ & -2.16 & $(0.04)$ & -1.35 & $(0.08)$ & -2.06 & $(0.03)$ \\
\hline
\end{tabular}

Anmerkung: Diese Tabelle wurde nur für Schüler berechnet, für die Daten zum PISA-Index des wirtschaftlichen, sozialen und kulturellen Status vorliegen. Statistisch signifikante Werte sind durch Fettdruck gekennzeichnet (vgl. Anhang A3).

$*$ Vgl. die Anmerkungen am Beginn dieses Anhangs.

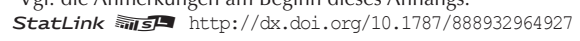


[Teil 2/3]

Schülerleistungen in Mathematik, Erwerbsstatus und sozioökonomischer Status der Eltern Tabelle II.3.2 Die Ergebnisse basieren auf Schülerangaben

\begin{tabular}{|c|c|c|c|c|c|c|c|c|c|c|c|c|c|c|c|}
\hline & \multicolumn{8}{|c|}{ Durchschnittsergebnisse in Mathematik } & \multirow{3}{*}{\multicolumn{2}{|c|}{$\begin{array}{l}\text { Leistungsunterschied } \\
\text { in Mathematik } \\
\text { zwischen Schülern, } \\
\text { deren Vater } \\
\text { erwerbstätig ist und } \\
\text { Schülern, deren Vater } \\
\text { nicht erwerbstätig ist }\end{array}$}} & \multirow{3}{*}{\multicolumn{2}{|c|}{$\begin{array}{l}\text { Leistungsunterschied } \\
\text { in Mathematik } \\
\text { zwischen Schülern, } \\
\text { deren Mutter } \\
\text { erwerbstätig ist } \\
\text { und Schülern, } \\
\text { deren Mutter nicht } \\
\text { erwerbstätig ist }\end{array}$}} & \multirow{3}{*}{\multicolumn{2}{|c|}{$\begin{array}{l}\text { Leistungsunterschied } \\
\text { in Mathematik } \\
\text { zwischen Schülern, } \\
\text { deren Vater } \\
\text { erwerbstätig ist und } \\
\text { Schülern, deren Vater } \\
\text { nicht erwerbstätig } \\
\text { ist, unter Berück- } \\
\text { sichtigung des ESCS }\end{array}$}} \\
\hline & & \multicolumn{4}{|c|}{ Aktueller Erwerbsstatus des Vaters } & \multicolumn{4}{|c|}{ Aktueller Erwerbsstatus der Mutter } & & & & & & \\
\hline & & $\begin{array}{c}\text { Erwerbst } \\
\text { oder }\end{array}$ & $\begin{array}{l}g \text { in Voll- } \\
\text { lzeit }\end{array}$ & $\begin{array}{r}\text { Nicht er } \\
\text { abe } \\
\text { Arbeitst } \\
\text { anderer } \\
\text { Haushal } \\
\text { oder Ru }\end{array}$ & $\begin{array}{l}\text { rbstätig, } \\
\text { auf } \\
\text { he oder } \\
\text { tus (z.B. } \\
\text { flichten } \\
\text { estand) }\end{array}$ & $\begin{array}{r}\text { Erwerbst } \\
\text { oder }\end{array}$ & $\begin{array}{l}\text { g in Voll- } \\
\text { ilzeit }\end{array}$ & $\begin{array}{r}\text { Nicht ery } \\
\text { aber au } \\
\text { suche od } \\
\text { Statu } \\
\text { Haushalt } \\
\text { oder Ru }\end{array}$ & $\begin{array}{l}\text { rbstätig, } \\
\text { Arbeit- } \\
\text { anderer } \\
\text { (z.B. } \\
\text { flichten } \\
\text { estand) }\end{array}$ & & & & & & \\
\hline & & $\begin{array}{c}\text { Mittel- } \\
\text { wert }\end{array}$ & S.E. & $\begin{array}{c}\text { Mittel- } \\
\text { wert }\end{array}$ & S.E. & $\begin{array}{c}\text { Mittel- } \\
\text { wert }\end{array}$ & S.E. & $\begin{array}{c}\text { Mittel- } \\
\text { wert }\end{array}$ & S.E. & $\begin{array}{l}\text { Punkt- } \\
\text { diff. }\end{array}$ & S.E. & $\begin{array}{c}\text { Punkt- } \\
\text { diff. }\end{array}$ & S.E. & $\begin{array}{l}\text { Punkt- } \\
\text { diff. }\end{array}$ & S.E. \\
\hline ¿ & Australien & 511 & $(1.7)$ & 487 & (3.5) & 511 & $(1.8)$ & 497 & $(2.5)$ & 24 & (3.3) & 15 & $(2.3)$ & 9 & $(3.2)$ \\
\hline 7 & Österreich & 509 & (2.6) & 488 & (7.8) & 511 & $(2.6)$ & 492 & $(4.4)$ & 21 & $(7.5)$ & 19 & $(4.1)$ & 2 & (5.9) \\
\hline & Belgien & 526 & $(2.1)$ & 482 & $(4.8)$ & 530 & $(2.0)$ & 481 & (3.7) & 45 & $(4.6)$ & 49 & $(3.4)$ & 16 & $(4.0)$ \\
\hline ف & Kanada & 524 & $(1.8)$ & 515 & $(4.2)$ & 523 & $(1.9)$ & 516 & $(2.6)$ & 9 & $(4.0)$ & 7 & $(2.4)$ & -1 & $(3.8)$ \\
\hline صِ & Chile & 426 & (3.1) & 415 & (5.1) & 430 & (3.3) & 418 & $(3.2)$ & 12 & $(4.6)$ & 12 & (2.5) & -7 & (3.9) \\
\hline & Tschech. Rep. & 504 & $(2.8)$ & 469 & $(9.1)$ & 506 & $(2.6)$ & 472 & $(5.9)$ & 35 & $(8.8)$ & 34 & $(5.6)$ & 15 & $(7.2)$ \\
\hline & Dänemark & 508 & $(2.2)$ & 474 & $(3.1)$ & 509 & $(2.1)$ & 472 & $(3.2)$ & 34 & (3.6) & 38 & $(3.2)$ & 12 & (3.5) \\
\hline & Estland & 524 & (2.1) & 508 & (5.3) & 525 & $(2.1)$ & 509 & (3.8) & 15 & (4.9) & 15 & (3.8) & 4 & (4.8) \\
\hline & Finnland & 525 & (1.8) & 504 & $(4.1)$ & 524 & (1.8) & 509 & $(4.3)$ & 21 & $(4.0)$ & 15 & $(4.1)$ & 6 & (3.7) \\
\hline & Frankreich & 503 & (2.5) & 479 & $(5.2)$ & 509 & $(2.7)$ & 465 & $(4.1)$ & 24 & (5.1) & 44 & (4.5) & -2 & $(5.1)$ \\
\hline & Deutschland & 523 & $(3.0)$ & 502 & (7.4) & 526 & (3.3) & 500 & $(4.7)$ & 22 & $(7.0)$ & 26 & $(4.9)$ & 3 & $(5.9)$ \\
\hline & Griechenland & 459 & $(2.5)$ & 440 & $(4.2)$ & 466 & $(2.6)$ & 440 & $(2.8)$ & 18 & $(4.1)$ & 27 & $(2.5)$ & 5 & (3.5) \\
\hline & Ungarn & 485 & (3.4) & 446 & $(6.2)$ & 490 & (3.4) & 448 & (4.5) & 40 & (6.8) & 42 & (4.6) & 12 & $(4.2)$ \\
\hline & Island & 498 & (1.8) & 473 & (6.7) & 500 & (1.9) & 472 & (3.4) & 25 & (6.7) & 28 & (3.8) & 14 & (6.2) \\
\hline & Irland & 510 & $(2.1)$ & 480 & $(4.1)$ & 509 & $(2.1)$ & 492 & (3.1) & 30 & (3.6) & 17 & $(2.7)$ & 13 & (3.0) \\
\hline & Israel & 477 & $(4.6)$ & 442 & (6.7) & 490 & $(4.7)$ & 423 & $(5.0)$ & 34 & (6.3) & 67 & $(5.4)$ & 2 & (5.9) \\
\hline & Italien & 489 & $(2.0)$ & 471 & (3.5) & 497 & $(2.2)$ & 468 & $(2.3)$ & 17 & (3.2) & 29 & $(2.2)$ & 4 & (2.9) \\
\hline & Japan & 542 & (3.6) & 526 & (9.3) & 538 & (3.5) & 546 & $(5.0)$ & 16 & $(9.4)$ & -8 & (3.6) & 6 & $(7.9)$ \\
\hline & Korea & 558 & $(4.7)$ & 541 & (6.5) & 557 & (4.3) & 554 & (5.7) & 17 & $(6.2)$ & 2 & (3.4) & 3 & (5.6) \\
\hline & Luxemburg & 496 & $(1.2)$ & 473 & (4.6) & 494 & (1.3) & 491 & $(2.9)$ & 23 & $(4.9)$ & 3 & $(3.2)$ & 5 & $(4.6)$ \\
\hline & Mexiko & 417 & $(1.3)$ & 405 & $(2.4)$ & 418 & (1.6) & 412 & $(1.4)$ & 11 & $(2.2)$ & 6 & $(1.3)$ & -3 & (1.9) \\
\hline & Niederlande & 528 & (3.5) & 515 & $(5.7)$ & 529 & (3.4) & 518 & $(4.7)$ & 13 & $(5.2)$ & 11 & (3.4) & -5 & $(5.2)$ \\
\hline & Neuseeland & 509 & $(2.2)$ & 469 & $(6.2)$ & 508 & $(2.2)$ & 492 & $(4.7)$ & 40 & (5.8) & 16 & $(4.6)$ & 19 & (5.1) \\
\hline & Norwegen & 495 & (2.6) & 472 & (6.7) & 496 & $(2.6)$ & 466 & $(4.2)$ & 23 & (6.5) & 30 & (3.9) & 9 & (6.3) \\
\hline & Polen & 522 & (3.7) & 503 & (4.8) & 528 & (3.8) & 496 & (3.7) & 19 & (4.3) & 32 & (3.3) & 0 & $(4.2)$ \\
\hline & Portugal & 496 & (3.5) & 468 & (5.2) & 498 & (3.8) & 471 & $(4.2)$ & 28 & (4.1) & 27 & (3.7) & 10 & (3.7) \\
\hline & Slowak. Rep. & 493 & (3.3) & 431 & (6.5) & 497 & $(3.2)$ & 440 & $(5.8)$ & 62 & (6.4) & 57 & $(5.1)$ & 22 & $(4.8)$ \\
\hline & Slowenien & 504 & (1.4) & 497 & (4.5) & 507 & (1.4) & 481 & $(4.0)$ & 8 & (4.9) & 26 & (4.3) & -10 & (4.5) \\
\hline & Spanien & 492 & $(2.0)$ & 461 & $(2.9)$ & 492 & $(2.0)$ & 474 & $(2.2)$ & 31 & (3.1) & 18 & $(2.2)$ & 10 & (3.1) \\
\hline & Schweden & 486 & $(2.2)$ & 448 & $(6.2)$ & 486 & $(2.2)$ & 452 & $(5.2)$ & 38 & (6.4) & 34 & (5.4) & 18 & $(6.2)$ \\
\hline & Schweiz & 535 & $(3.2)$ & 509 & $(6.1)$ & 535 & $(3.0)$ & 524 & $(4.1)$ & 26 & (6.1) & 11 & $(3.0)$ & 11 & $(5.8)$ \\
\hline & Türkei & 455 & $(5.0)$ & 441 & $(5.7)$ & 469 & (7.6) & 450 & (4.6) & 13 & $(4.1)$ & 19 & (5.5) & -5 & (3.7) \\
\hline & Ver. Königreich & 503 & $(3.0)$ & 481 & $(4.7)$ & 502 & $(2.9)$ & 485 & $(5.1)$ & 22 & (3.9) & 17 & (4.5) & 4 & (3.8) \\
\hline & Ver. Staaten & 488 & (3.7) & 467 & (5.3) & 485 & (3.6) & 477 & $(4.5)$ & 21 & $(4.7)$ & 8 & (3.6) & 7 & $(4.3)$ \\
\hline & OECD-Durchschnitt & 501 & $(0.5)$ & 476 & (1.0) & 503 & $(0.5)$ & 480 & $(0.7)$ & 25 & $(0.9)$ & 23 & $(0.7)$ & 6 & $(0.8)$ \\
\hline$=$ & Albanien & $\mathrm{m}$ & $\mathrm{m}$ & $\mathrm{m}$ & $\mathrm{m}$ & $\mathrm{m}$ & $\mathrm{m}$ & $\mathrm{m}$ & $\mathrm{m}$ & $\mathrm{m}$ & $\mathrm{m}$ & $\mathrm{m}$ & $\mathrm{m}$ & $\mathrm{m}$ & $\mathrm{m}$ \\
\hline$\stackrel{\pi}{\stackrel{\pi}{*}}$ & Argentinien & 395 & (3.5) & 372 & $(5.2)$ & 401 & (3.4) & 382 & (3.8) & 23 & $(4.2)$ & 19 & $(2.2)$ & 7 & $(4.5)$ \\
\hline$\frac{\pi}{5}$ & Brasilien & 397 & $(2.3)$ & 384 & $(2.4)$ & 402 & $(2.4)$ & 382 & $(2.1)$ & 14 & (2.5) & 19 & (1.9) & -2 & $(2.0)$ \\
\hline. & Bulgarien & 445 & (3.7) & 418 & $(6.1)$ & 450 & (3.9) & 410 & (5.5) & 28 & (5.0) & 40 & (5.3) & -2 & (4.4) \\
\hline 恿 & Kolumbien & 378 & (3.1) & 378 & $(4.4)$ & 379 & (3.0) & 376 & (3.3) & 0 & (3.4) & 3 & $(2.5)$ & -15 & $(3.1)$ \\
\hline ڤ్ & Costa Rica & 410 & (3.1) & 404 & $(5.2)$ & 415 & (3.8) & 403 & $(3.1)$ & 7 & (4.6) & 12 & (3.2) & -5 & (3.9) \\
\hline 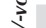 & Kroatien & 478 & (3.9) & 459 & (3.8) & 484 & $(4.1)$ & 453 & (3.4) & 19 & (3.2) & 31 & $(3.7)$ & 3 & (3.0) \\
\hline$\frac{8}{8}$ & Zypern* & 448 & (1.3) & 410 & $(4.1)$ & 451 & (1.6) & 426 & $(2.4)$ & 38 & (4.3) & 25 & (3.2) & 12 & $(4.3)$ \\
\hline : & Hongkong (China) & 565 & (3.3) & 557 & $(4.7)$ & 564 & (3.6) & 563 & (3.6) & 8 & (4.6) & 1 & (3.5) & -3 & $(4.1)$ \\
\hline 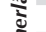 & Indonesien & 381 & $(4.2)$ & 365 & (4.5) & 377 & (4.9) & 378 & (3.8) & 16 & (3.5) & -1 & (3.0) & 9 & (3.3) \\
\hline 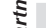 & Jordanien & 396 & $(2.9)$ & 378 & $(4.0)$ & 401 & $(5.8)$ & 389 & $(2.6)$ & 17 & $(3.2)$ & 12 & (4.5) & 7 & (3.3) \\
\hline & Kasachstan & 437 & (3.5) & 417 & $(4.2)$ & 437 & (3.4) & 424 & (3.3) & 20 & $(4.5)$ & 13 & (3.1) & 8 & (3.9) \\
\hline & Lettland & 493 & (3.0) & 489 & $(5.8)$ & 494 & (3.1) & 483 & (3.5) & 4 & $(6.0)$ & 11 & (3.9) & -11 & $(5.2)$ \\
\hline & Liechtenstein & 538 & (4.3) & C & $\mathrm{C}$ & 532 & (5.9) & 548 & $(8.0)$ & C & c & -16 & $(11.2)$ & C & c \\
\hline & Litauen & 486 & $(2.5)$ & 466 & $(4.3)$ & 488 & $(2.6)$ & 458 & (3.5) & 20 & (3.7) & 29 & (3.0) & 3 & (3.4) \\
\hline & Macau (China) & 540 & (1.1) & 540 & $(4.2)$ & 539 & $(1.2)$ & 540 & $(2.6)$ & 0 & (4.6) & 0 & (3.1) & -4 & (4.4) \\
\hline & Malaysia & 425 & (3.3) & 402 & $(4.2)$ & 431 & $(4.1)$ & 415 & (2.9) & 23 & (3.9) & 16 & $(2.9)$ & 15 & $(4.0)$ \\
\hline & Montenegro & 416 & (1.5) & 398 & $(2.4)$ & 428 & (1.7) & 396 & (1.6) & 18 & (3.1) & 32 & (2.5) & 1 & (3.0) \\
\hline & Peru & 373 & (3.9) & 352 & $(4.6)$ & 368 & $(4.1)$ & 370 & (3.7) & 21 & (3.7) & -2 & (2.6) & -4 & (2.7) \\
\hline & Katar & 389 & $(1.0)$ & 332 & $(2.1)$ & 386 & $(1.7)$ & 379 & $(1.1)$ & 57 & (2.4) & 7 & (2.2) & 46 & (2.4) \\
\hline & Rumänien & 454 & $(4.1)$ & 424 & $(4.0)$ & 459 & $(4.2)$ & 425 & (3.8) & 30 & (3.8) & 33 & (3.7) & 5 & $(2.9)$ \\
\hline & Russ. Föderation & 487 & $(3.0)$ & 468 & $(6.1)$ & 488 & $(2.9)$ & 470 & $(4.5)$ & 19 & (5.6) & 18 & (3.5) & 5 & (5.4) \\
\hline & Serbien & 455 & (3.6) & 439 & $(4.1)$ & 462 & $(3.8)$ & 433 & $(3.8)$ & 15 & (3.6) & 29 & (3.8) & 1 & (3.1) \\
\hline & Shanghai (China) & 617 & (3.1) & 586 & (6.7) & 622 & (3.0) & 588 & $(5.3)$ & 31 & (5.6) & 33 & (4.3) & 7 & $(4.8)$ \\
\hline & Singapur & 576 & $(1.5)$ & 568 & $(5.7)$ & 578 & $(1.7)$ & 569 & $(2.8)$ & 8 & $(6.2)$ & 9 & (3.6) & -1 & $(5.7)$ \\
\hline & Chinesisch Taipeh & 565 & (3.2) & 538 & $(6.1)$ & 563 & (3.5) & 558 & (4.5) & 27 & (5.7) & 5 & (4.2) & 7 & $(5.2)$ \\
\hline & Thailand & 428 & (3.6) & 429 & (4.5) & 426 & (3.6) & 433 & $(4.0)$ & -1 & (3.7) & -8 & (2.9) & -7 & (3.5) \\
\hline & Tunesien & 391 & (4.3) & 385 & (4.9) & 412 & (7.9) & 384 & (3.4) & 6 & (4.5) & 28 & (6.7) & -7 & (3.7) \\
\hline & Ver. Arab. Emirate & 446 & (2.5) & 399 & $(2.7)$ & 455 & (3.0) & 429 & $(2.6)$ & 47 & (3.0) & 26 & (2.9) & 31 & $(2.7)$ \\
\hline & Uruguay & 416 & (2.8) & 397 & (4.3) & 423 & (2.8) & 393 & (3.2) & 19 & (4.5) & 30 & (2.9) & 1 & $(4.2)$ \\
\hline & Vietnam & 519 & (5.9) & 505 & $(4.7)$ & 526 & (6.4) & 504 & (4.5) & 14 & (5.1) & 22 & (4.9) & -7 & (3.8) \\
\hline
\end{tabular}

Anmerkung: Diese Tabelle wurde nur für Schüler berechnet, für die Daten zum PISA-Index des wirtschaftlichen, sozialen und kulturellen Status vorliegen. Statistisch signifikante Werte sind durch Fettdruck gekennzeichnet (vgl. Anhang A3).

*Vgl. die Anmerkungen am Beginn dieses Anhangs.

StatLink त्ञाज http://dx.doi.org/10.1787/888932964927 
[Teil 3/3]

Schülerleistungen in Mathematik, Erwerbsstatus und sozioökonomischer Status der Eltern Tabelle II.3.2 Die Ergebnisse basieren auf Schülerangaben

\begin{tabular}{|c|c|c|c|c|c|c|c|c|c|c|c|c|c|c|c|}
\hline & & $\begin{array}{l}\text { Leistungs } \\
\text { in Ma } \\
\text { zwische } \\
\text { derer } \\
\text { erwer } \\
\text { und S } \\
\text { deren } M \\
\text { erwerk } \\
\text { unter } \\
\text { sichtigur }\end{array}$ & $\begin{array}{l}\text { terschied } \\
\text { ematik } \\
\text { schülern, } \\
\text { Mutter } \\
\text { ätig ist } \\
\text { ülern, } \\
\text { ter nicht } \\
\text { ätig ist, } \\
\text { erück- } \\
\text { des ESCS }\end{array}$ & $\begin{array}{r}\text { Gröf } \\
\text { Wahrsche } \\
\text { für Sc } \\
\text { deren Va } \\
\text { erwerbstä } \\
\text { untersten } \\
\text { der Gesa } \\
\text { Mathe } \\
\text { zu lie }\end{array}$ & $\begin{array}{l}\text { ere } \\
\text { nlichkeit } \\
\text { üler, } \\
\text { er nicht } \\
\text { ig ist, im } \\
\text { Quartil } \\
\text { ntskala } \\
\text { natik } \\
\text { gen }\end{array}$ & $\begin{array}{r}\text { Populat } \\
\text { der } \\
\text { deren } \\
\text { erw } \\
\text { ist u } \\
\text { unters } \\
\text { der G } \\
\text { Mather }\end{array}$ & $\begin{array}{l}\text { relevanz } \\
\text { üller, } \\
\text { er nicht } \\
\text { sätig } \\
\text { lie im } \\
\text { Quartil } \\
\text { ntskala } \\
\text { ik liegen }\end{array}$ & $\begin{array}{r}\text { Grö } \\
\text { Wahrsche } \\
\text { für Schül } \\
\text { Mutte } \\
\text { erwerbstä } \\
\text { unterster } \\
\text { der Gesa } \\
\text { Mathe } \\
\text { zu li }\end{array}$ & $\begin{array}{l}\text { ere } \\
\text { lichkeit } \\
\text { r, deren } \\
\text { nicht } \\
\text { ig ist, im } \\
\text { Quartil } \\
\text { ntskala } \\
\text { natik } \\
\text { sen }\end{array}$ & $\begin{array}{r}\text { Populat } \\
\text { der } \\
\text { dere } \\
\text { nicht e } \\
\text { ist ut } \\
\text { unters } \\
\text { der G } \\
\text { Mather }\end{array}$ & $\begin{array}{l}\text { Isrelevanz } \\
\text { hüler, } \\
\text { Mutter } \\
\text { erbstätig } \\
\text { die im } \\
\text { Quartil } \\
\text { imtskala } \\
\text { tik liegen }\end{array}$ & $\begin{array}{r}\text { Effekt } \\
\text { den } M \\
\text { leistu } \\
\text { Schüler, } \\
\text { nicht erv }\end{array}$ & $\begin{array}{l}\text { rke bei } \\
\text { ematik- } \\
\text { en für } \\
\text { ren Vater } \\
\text { bstätig ist }\end{array}$ & $\begin{array}{l}\text { Effekts } \\
\text { den } M \\
\text { leistu } \\
\text { Schül } \\
\text { Mutt } \\
\text { erwer }\end{array}$ & $\begin{array}{l}\text { rke bei } \\
\text { ematik- } \\
\text { en für } \\
\text { deren } \\
\text { nicht } \\
\text { ätig ist }\end{array}$ \\
\hline & & $\begin{array}{l}\text { Punkt- } \\
\text { diff. }\end{array}$ & S.E. & Quotient & S.E. & $\%$ & S.E. & Quotient & S.E. & $\%$ & S.E. & $\begin{array}{l}\text { Effekt- } \\
\text { stärke }\end{array}$ & S.E. & $\begin{array}{l}\text { Effekt- } \\
\text { stärke }\end{array}$ & S.E. \\
\hline む & Australien & 1 & $(2.2)$ & 1.40 & $(0.08)$ & 3.5 & $(0.7)$ & 1.29 & $(0.06)$ & 6.7 & $(1.2)$ & -0.25 & $(0.04)$ & -0.15 & $(0.02)$ \\
\hline $\bar{g}$ & Österreich & 6 & (3.6) & 1.47 & $(0.15)$ & 3.4 & (1.1) & 1.41 & $(0.09)$ & 7.6 & (1.7) & -0.22 & $(0.08)$ & -0.20 & $(0.04)$ \\
\hline & Belgien & 24 & (2.6) & 1.78 & $(0.10)$ & 8.0 & (1.0) & 1.86 & $(0.09)$ & 16.8 & (1.6) & -0.45 & $(0.04)$ & -0.50 & $(0.03)$ \\
\hline 5 & Kanada & -1 & (2.1) & 1.20 & $(0.08)$ & 1.7 & $(0.6)$ & 1.15 & $(0.05)$ & 3.1 & (1.1) & -0.10 & $(0.05)$ & -0.08 & $(0.03)$ \\
\hline 0 & Chile & -4 & (2.1) & 1.23 & $(0.14)$ & 2.3 & (1.3) & 1.21 & $(0.08)$ & 9.0 & (3.0) & -0.15 & $(0.06)$ & -0.15 & $(0.03)$ \\
\hline & Tschech. Rep. & 17 & (4.9) & 1.64 & $(0.21)$ & 4.0 & (1.4) & 1.53 & $(0.12)$ & 8.4 & (1.9) & -0.35 & $(0.09)$ & -0.35 & $(0.05)$ \\
\hline & Dänemark & 16 & (3.0) & 1.64 & $(0.10)$ & 6.4 & $(0.9)$ & 1.81 & $(0.10)$ & 12.4 & (1.4) & -0.42 & $(0.05)$ & -0.47 & $(0.04)$ \\
\hline & Estland & 7 & (3.7) & 1.27 & $(0.14)$ & 2.4 & $(1.2)$ & 1.29 & $(0.12)$ & 5.0 & (1.9) & -0.19 & $(0.06)$ & -0.19 & $(0.05)$ \\
\hline & Finnland & 1 & (3.5) & 1.43 & $(0.09)$ & 5.1 & (1.1) & 1.35 & $(0.09)$ & 5.0 & (1.3) & -0.24 & $(0.05)$ & -0.18 & $(0.05)$ \\
\hline & Frankreich & 18 & $(4.2)$ & 1.40 & $(0.13)$ & 3.8 & $(1.2)$ & 1.88 & $(0.15)$ & 16.0 & (2.5) & -0.25 & $(0.05)$ & -0.45 & $(0.05)$ \\
\hline & Deutschland & 14 & $(4.1)$ & 1.46 & $(0.16)$ & 3.0 & $(1.0)$ & 1.47 & $(0.12)$ & 9.6 & $(2.2)$ & -0.22 & $(0.07)$ & -0.26 & $(0.05)$ \\
\hline & Griechenland & 7 & (2.3) & 1.36 & $(0.09)$ & 6.2 & (1.5) & 1.45 & $(0.07)$ & 16.3 & (2.3) & -0.21 & $(0.05)$ & -0.31 & $(0.03)$ \\
\hline & Ungarn & 12 & (3.7) & 1.79 & $(0.15)$ & 10.3 & (1.9) & 1.91 & $(0.14)$ & 19.0 & (2.5) & -0.42 & $(0.07)$ & -0.46 & $(0.05)$ \\
\hline & Island & 16 & (3.8) & 1.33 & $(0.13)$ & 2.0 & $(0.8)$ & 1.46 & $(0.12)$ & 6.8 & (1.6) & -0.28 & $(0.07)$ & -0.32 & $(0.04)$ \\
\hline & Irland & 2 & (2.5) & 1.57 & $(0.10)$ & 9.4 & (1.5) & 1.35 & $(0.07)$ & 11.6 & (2.1) & -0.36 & $(0.04)$ & -0.20 & $(0.03)$ \\
\hline & Israel & 41 & (4.8) & 1.52 & $(0.13)$ & 5.7 & (1.4) & 2.37 & $(0.19)$ & 27.8 & (2.9) & -0.32 & $(0.06)$ & -0.68 & $(0.05)$ \\
\hline & Italien & 12 & (2.0) & 1.29 & (0.08) & 2.4 & $(0.6)$ & 1.49 & $(0.06)$ & 15.4 & (1.6) & -0.19 & $(0.03)$ & -0.32 & $(0.02)$ \\
\hline & Japan & -4 & (3.2) & 1.17 & $(0.18)$ & 0.5 & $(0.6)$ & 0.94 & $(0.05)$ & -1.2 & $(1.2)$ & -0.17 & $(0.10)$ & 0.09 & $(0.04)$ \\
\hline & Korea & 2 & (3.0) & 1.25 & $(0.11)$ & 2.3 & (1.0) & 1.13 & $(0.06)$ & 4.9 & (2.2) & -0.17 & $(0.06)$ & -0.02 & $(0.03)$ \\
\hline & Luxemburg & -2 & (2.7) & 1.36 & $(0.14)$ & 3.4 & (1.3) & 1.05 & $(0.06)$ & 1.5 & (1.7) & -0.25 & $(0.05)$ & -0.03 & $(0.03)$ \\
\hline & Mexiko & -7 & (1.2) & 1.23 & $(0.05)$ & 3.4 & $(0.7)$ & 1.09 & $(0.04)$ & 5.0 & (1.9) & -0.16 & $(0.03)$ & -0.08 & $(0.02)$ \\
\hline & Niederlande & -2 & (3.3) & 1.20 & $(0.10)$ & 1.6 & $(0.8)$ & 1.22 & $(0.10)$ & 4.6 & (2.0) & -0.15 & $(0.06)$ & -0.13 & $(0.04)$ \\
\hline & Neuseeland & 1 & $(4.2)$ & 1.74 & (0.15) & 6.0 & (1.2) & 1.35 & $(0.11)$ & 7.7 & $(2.2)$ & -0.40 & (0.06) & -0.16 & $(0.05)$ \\
\hline & Norwegen & 18 & (3.8) & 1.41 & $(0.14)$ & 3.1 & $(1.0)$ & 1.52 & (0.09) & 6.8 & $(1.2)$ & -0.25 & $(0.08)$ & -0.34 & $(0.04)$ \\
\hline & Polen & 8 & (3.0) & 1.26 & $(0.10)$ & 3.2 & (1.2) & 1.56 & (0.11) & 14.4 & (2.3) & -0.21 & $(0.05)$ & -0.36 & $(0.04)$ \\
\hline & Portugal & 5 & (3.1) & 1.45 & $(0.14)$ & 6.0 & (1.6) & 1.47 & (0.10) & 10.7 & (2.0) & -0.31 & $(0.04)$ & -0.29 & $(0.04)$ \\
\hline & Slowak. Rep. & 22 & (4.1) & 2.23 & $(0.16)$ & 14.9 & (1.9) & 2.18 & (0.13) & 22.2 & $(2.0)$ & -0.60 & $(0.06)$ & -0.57 & $(0.05)$ \\
\hline & Slowenien & 2 & (4.1) & 1.15 & $(0.14)$ & 1.7 & (1.5) & 1.35 & $(0.11)$ & 5.2 & (1.5) & -0.08 & $(0.05)$ & -0.29 & $(0.05)$ \\
\hline & Spanien & 3 & (1.9) & 1.52 & $(0.08)$ & 7.5 & (1.1) & 1.30 & $(0.06)$ & 9.0 & (1.6) & -0.36 & $(0.04)$ & -0.21 & $(0.03)$ \\
\hline & Schweden & 17 & (5.1) & 1.74 & $(0.15)$ & 4.9 & $(0.9)$ & 1.58 & (0.13) & 6.2 & (1.4) & -0.41 & $(0.07)$ & -0.38 & $(0.06)$ \\
\hline & Schweiz & 3 & (2.9) & 1.46 & $(0.13)$ & 2.6 & $(0.7)$ & 1.17 & $(0.07)$ & 4.0 & (1.5) & -0.27 & $(0.07)$ & -0.12 & $(0.03)$ \\
\hline & Türkei & -2 & (3.5) & 1.22 & $(0.08)$ & 5.9 & $(2.0)$ & 1.03 & $(0.10)$ & 2.8 & (7.6) & -0.15 & $(0.05)$ & -0.20 & $(0.05)$ \\
\hline & Ver. Königreich & 6 & (3.6) & 1.35 & $(0.09)$ & 3.5 & $(0.8)$ & 1.33 & $(0.12)$ & 7.2 & (2.6) & -0.24 & $(0.04)$ & -0.18 & $(0.05)$ \\
\hline & Ver. Staaten & -2 & (3.0) & 1.39 & $(0.10)$ & 5.2 & (1.4) & 1.16 & $(0.08)$ & 4.1 & (1.9) & -0.24 & $(0.05)$ & -0.09 & $(0.04)$ \\
\hline & OECD-Durchschnitt & 8 & $(0.6)$ & 1.44 & $(0.02)$ & 4.6 & $(0.2)$ & 1.43 & $(0.02)$ & 9.2 & $(0.4)$ & -0.27 & $(0.01)$ & -0.25 & $(0.01)$ \\
\hline & Albanien & $\mathrm{m}$ & $\mathrm{m}$ & $\mathrm{m}$ & $\mathrm{m}$ & $\mathrm{m}$ & $\mathrm{m}$ & $\mathrm{m}$ & $\mathrm{m}$ & $\mathrm{m}$ & $\mathrm{m}$ & $\mathrm{m}$ & $\mathrm{m}$ & $\mathrm{m}$ & $\mathrm{m}$ \\
\hline$=$ & Argentinien & 2 & (2.3) & 1.46 & $(0.12)$ & 4.9 & $(1.3)$ & 1.35 & $(0.08)$ & 13.9 & $(2.8)$ & -0.30 & $(0.05)$ & -0.25 & $(0.03)$ \\
\hline$=$ & Brasilien & 3 & (1.7) & 1.19 & $(0.05)$ & 3.5 & $(0.9)$ & 1.31 & $(0.05)$ & 11.8 & (1.8) & -0.18 & $(0.03)$ & -0.25 & $(0.02)$ \\
\hline .2 & Bulgarien & 9 & (3.7) & 1.49 & $(0.11)$ & 6.2 & (1.4) & 1.75 & $(0.14)$ & 13.4 & $(2.2)$ & -0.30 & $(0.05)$ & -0.44 & $(0.05)$ \\
\hline$\underline{\xi}$ & Kolumbien & -7 & (2.6) & 1.00 & $(0.09)$ & 0.0 & (1.4) & 1.07 & $(0.07)$ & 3.2 & (3.3) & 0.00 & $(0.05)$ & -0.04 & $(0.03)$ \\
\hline 亏ั & Costa Rica & -3 & (2.8) & 1.12 & $(0.11)$ & 1.5 & (1.3) & 1.14 & $(0.08)$ & 7.0 & $(4.0)$ & -0.10 & $(0.07)$ & -0.18 & $(0.05)$ \\
\hline$\frac{7}{1}$ & Kroatien & 11 & (3.2) & 1.28 & $(0.07)$ & 7.4 & (1.8) & 1.54 & $(0.12)$ & 17.0 & (3.0) & -0.21 & $(0.04)$ & -0.36 & $(0.04)$ \\
\hline 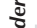 & Zypern* & 5 & (3.0) & 1.67 & $(0.13)$ & 6.1 & $(1.2)$ & 1.37 & $(0.08)$ & 9.1 & (1.8) & -0.42 & $(0.05)$ & -0.28 & $(0.04)$ \\
\hline$\approx$ & Hongkong (China) & -2 & (3.2) & 1.11 & $(0.08)$ & 1.3 & $(0.9)$ & 0.99 & $(0.06)$ & -0.3 & $(2.2)$ & -0.08 & $(0.05)$ & -0.01 & $(0.04)$ \\
\hline & Indonesien & -5 & $(2.7)$ & 1.32 & $(0.11)$ & 6.1 & (1.9) & 0.92 & $(0.07)$ & -5.5 & (5.0) & -0.23 & $(0.05)$ & 0.01 & $(0.04)$ \\
\hline 3 & Jordanien & -2 & (3.9) & 1.37 & $(0.11)$ & 8.3 & $(2.2)$ & 1.08 & $(0.10)$ & 6.4 & (6.8) & -0.23 & $(0.05)$ & -0.16 & $(0.05)$ \\
\hline$\approx$ & Kasachstan & 1 & (2.8) & 1.47 & $(0.14)$ & 9.9 & (2.6) & 1.28 & $(0.09)$ & 9.6 & (2.8) & -0.28 & $(0.06)$ & -0.18 & $(0.04)$ \\
\hline & Lettland & -1 & (3.4) & 1.14 & $(0.13)$ & 1.9 & (1.7) & 1.16 & $(0.09)$ & 3.3 & (1.8) & -0.05 & $(0.07)$ & -0.14 & $(0.05)$ \\
\hline & Liechtenstein & -20 & (10.9) & c & c & c & c & 0.77 & $(0.26)$ & -8.7 & (10.6) & c & c & 0.17 & $(0.12)$ \\
\hline & Litauen & 9 & (2.9) & 1.37 & $(0.09)$ & 6.2 & (1.5) & 1.52 & $(0.09)$ & 11.7 & (1.8) & -0.23 & $(0.04)$ & -0.34 & $(0.03)$ \\
\hline & Macau (China) & -2 & (3.1) & 0.97 & $(0.09)$ & -0.3 & $(1.0)$ & 1.05 & $(0.06)$ & 1.1 & (1.4) & 0.00 & $(0.05)$ & 0.00 & $(0.03)$ \\
\hline & Malaysia & -1 & (2.5) & 1.41 & $(0.11)$ & 5.4 & (1.4) & 1.18 & $(0.08)$ & 10.0 & $(4.1)$ & -0.29 & $(0.05)$ & -0.19 & $(0.03)$ \\
\hline & Montenegro & 16 & (2.6) & 1.39 & $(0.10)$ & 9.3 & $(2.1)$ & 1.70 & $(0.11)$ & 25.9 & (2.9) & -0.22 & $(0.04)$ & -0.40 & $(0.03)$ \\
\hline & Peru & -11 & (2.3) & 1.35 & $(0.10)$ & 5.3 & (1.4) & 0.91 & $(0.05)$ & -4.8 & $(2.6)$ & -0.25 & $(0.04)$ & 0.02 & $(0.03)$ \\
\hline & Katar & -2 & $(2.1)$ & 1.79 & $(0.08)$ & 9.7 & $(0.9)$ & 0.97 & $(0.05)$ & -2.0 & $(3.2)$ & -0.64 & $(0.03)$ & -0.07 & $(0.02)$ \\
\hline & Rumänien & 8 & $(2.7)$ & 1.55 & $(0.09)$ & 12.5 & (1.8) & 1.61 & $(0.11)$ & 19.4 & (2.9) & -0.38 & $(0.05)$ & -0.42 & $(0.04)$ \\
\hline & Russ. Föderation & 7 & (3.3) & 1.30 & $(0.14)$ & 3.9 & $(1.8)$ & 1.25 & $(0.08)$ & 5.6 & (1.7) & -0.21 & $(0.06)$ & -0.21 & $(0.04)$ \\
\hline & Serbien & 12 & $(3.2)$ & 1.18 & $(0.08)$ & 4.0 & (1.7) & 1.44 & $(0.10)$ & 15.2 & (2.9) & -0.17 & $(0.04)$ & -0.32 & $(0.04)$ \\
\hline & Shanghai (China) & 16 & (3.7) & 1.45 & $(0.13)$ & 5.4 & (1.6) & 1.60 & $(0.09)$ & 13.1 & (1.9) & -0.31 & $(0.05)$ & -0.33 & $(0.04)$ \\
\hline & Singapur & 4 & (3.2) & 1.09 & $(0.09)$ & 0.7 & $(0.7)$ & 1.12 & $(0.06)$ & 4.2 & (2.1) & -0.08 & $(0.06)$ & -0.09 & $(0.03)$ \\
\hline & Chinesisch Taipeh & -1 & (3.6) & 1.37 & $(0.10)$ & 4.3 & (1.1) & 1.09 & $(0.07)$ & 2.7 & $(2.0)$ & -0.23 & $(0.05)$ & -0.04 & $(0.04)$ \\
\hline & Thailand & -10 & $(2.7)$ & 0.96 & $(0.07)$ & -0.7 & $(1.3)$ & 0.85 & $(0.06)$ & -4.7 & (1.8) & 0.01 & $(0.04)$ & 0.10 & $(0.04)$ \\
\hline & Tunesien & 9 & (4.5) & 1.11 & $(0.11)$ & 1.7 & $(1.8)$ & 1.26 & $(0.14)$ & 16.5 & (6.9) & -0.08 & $(0.06)$ & -0.34 & $(0.07)$ \\
\hline & Ver. Arab. Emirate & 12 & (2.9) & 1.74 & $(0.08)$ & 12.4 & (1.1) & 1.38 & $(0.09)$ & 21.8 & $(4.1)$ & -0.56 & $(0.03)$ & -0.29 & $(0.03)$ \\
\hline & Uruguay & 6 & (2.6) & 1.27 & $(0.12)$ & 2.7 & $(1.2)$ & 1.47 & $(0.10)$ & 13.9 & (2.4) & -0.22 & $(0.05)$ & -0.34 & $(0.03)$ \\
\hline & Vietnam & 1 & (3.7) & 1.19 & $(0.10)$ & 8.0 & $(4.0)$ & 1.29 & $(0.12)$ & 15.4 & (5.6) & -0.16 & $(0.06)$ & -0.25 & $(0.06)$ \\
\hline
\end{tabular}

Anmerkung: Diese Tabelle wurde nur für Schüler berechnet, für die Daten zum PISA-Index des wirtschaftlichen, sozialen und kulturellen Status vorliegen. Statistisch signifikante Werte sind durch Fettdruck gekennzeichnet (vgl. Anhang A3).

*Vgl. die Anmerkungen am Beginn dieses Anhangs.

StatLink 규내 http://dx.doi.org/10.1787/888932964927 
[Teil 1/2]

Zusammenhang zwischen den Schülerleistungen in Mathematik und dem Schulstandort Tabelle II.3.3a Die Ergebnisse basieren auf Angaben der Schüler und der Schulleitungen

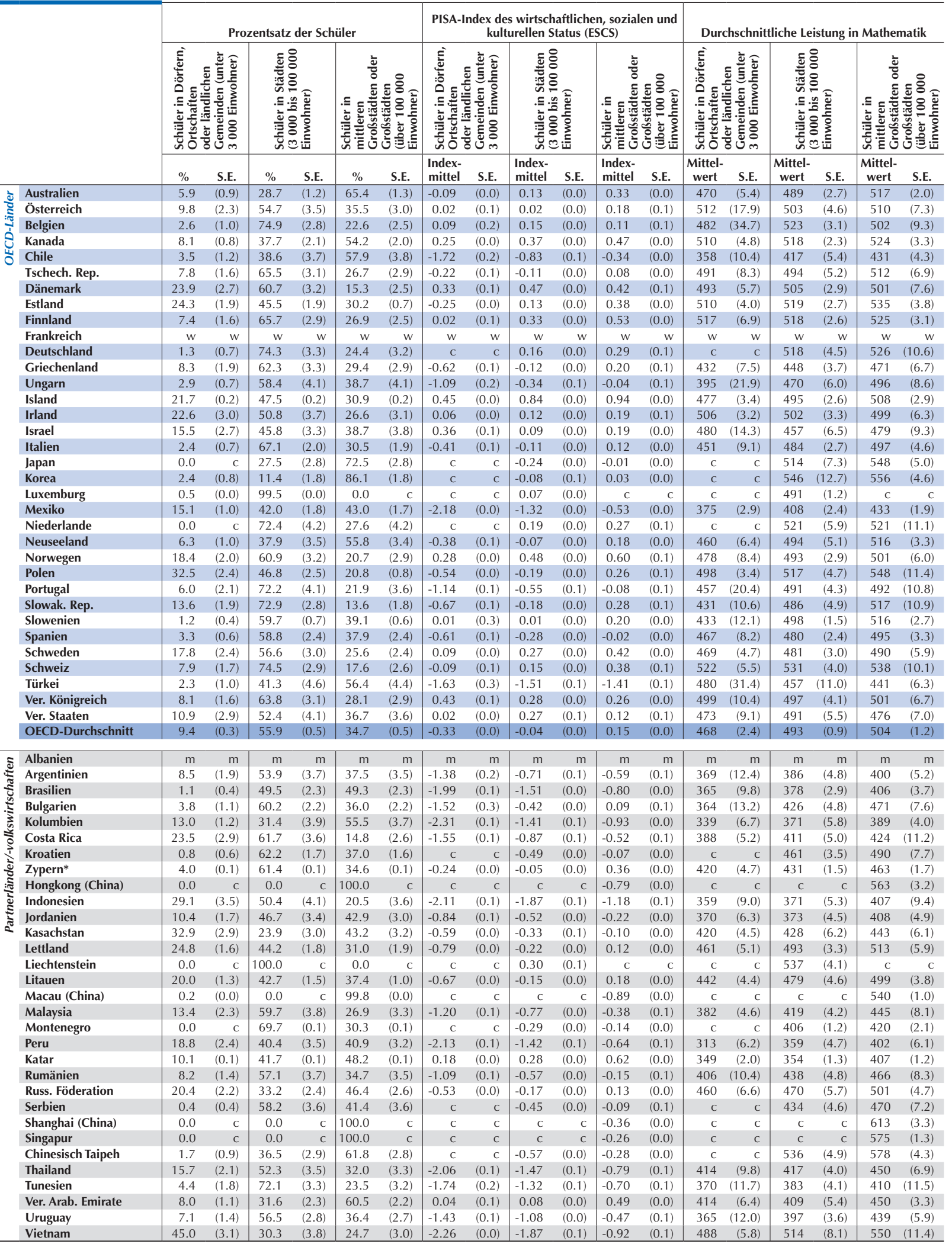

Anmerkung: Diese Tabelle wurde nur für Schüler berechnet, für die Daten zum PISA-Index des wirtschaftlichen, sozialen und kulturellen Status vorliegen. Statistisch signifikante Werte sind durch Fettdruck gekennzeichnet (vgl. Anhang A3).

*Vgl. die Anmerkungen am Beginn dieses Anhangs.

StatLink तinाs http://dx.doi.org/10.1787/888932964927 
[Teil 2/2]

Zusammenhang zwischen den Schülerleistungen in Mathematik und dem Schulstandort Tabelle II.3.3a Die Ergebnisse basieren auf Angaben der Schüler und der Schulleitungen

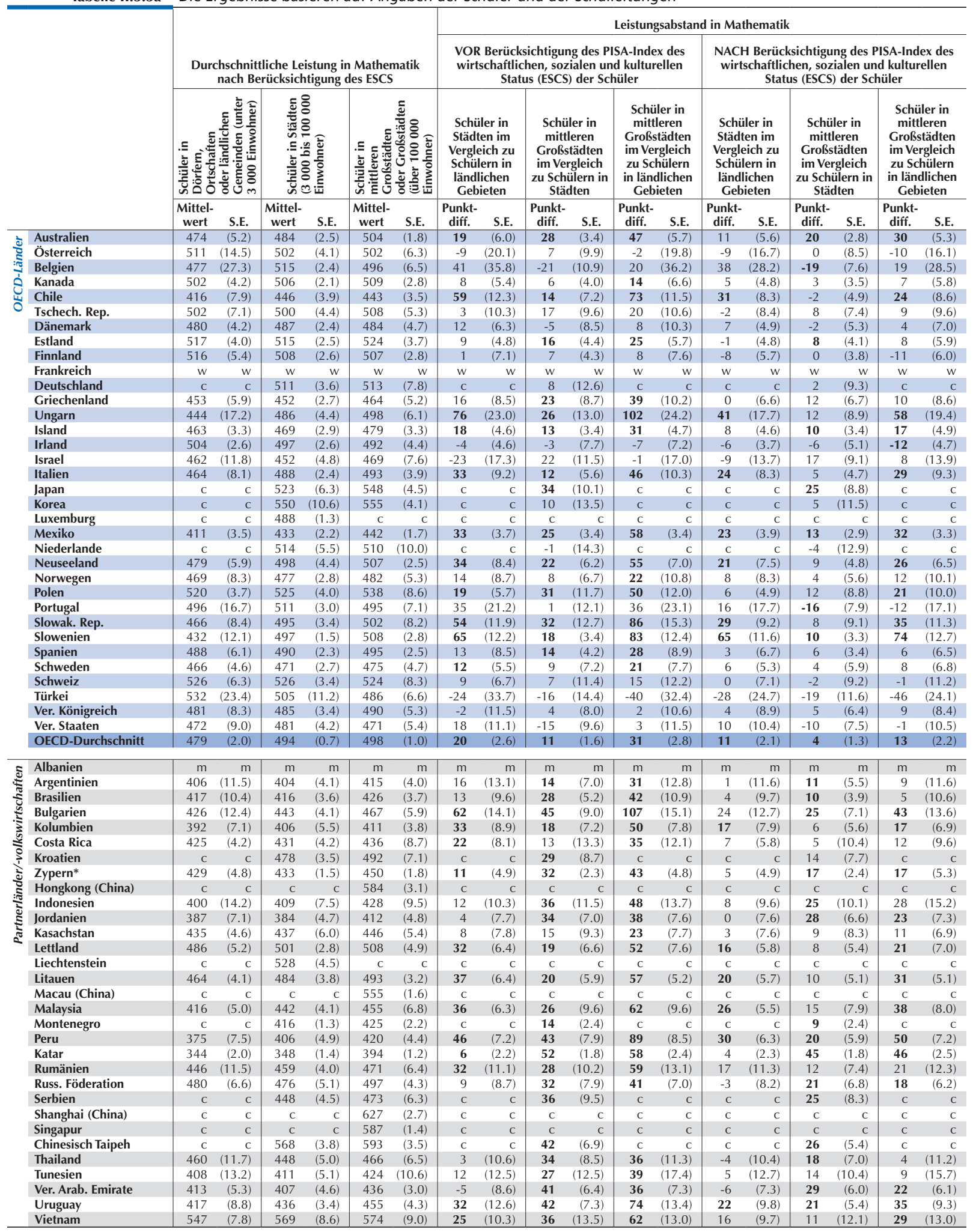

Anmerkung: Diese Tabelle wurde nur für Schüler berechnet, für die Daten zum PISA-Index des wirtschaftlichen, sozialen und kulturellen Status vorliegen. Statistisch signifikante Werte sind durch Fettdruck gekennzeichnet (vgl. Anhang A3).

*Vgl. die Anmerkungen am Beginn dieses Anhangs.

StatLink 完开且 http://dx.doi.org/10.1787/888932964927 
[Teil 1/3]

Von 2003 bis 2012 festzustellende Veränderung des Zusammenhangs zwischen Mathematikleistungen und Schulstandort

Tabelle II.3.3b Die Ergebnisse basieren auf Angaben der Schüler und der Schulleitungen

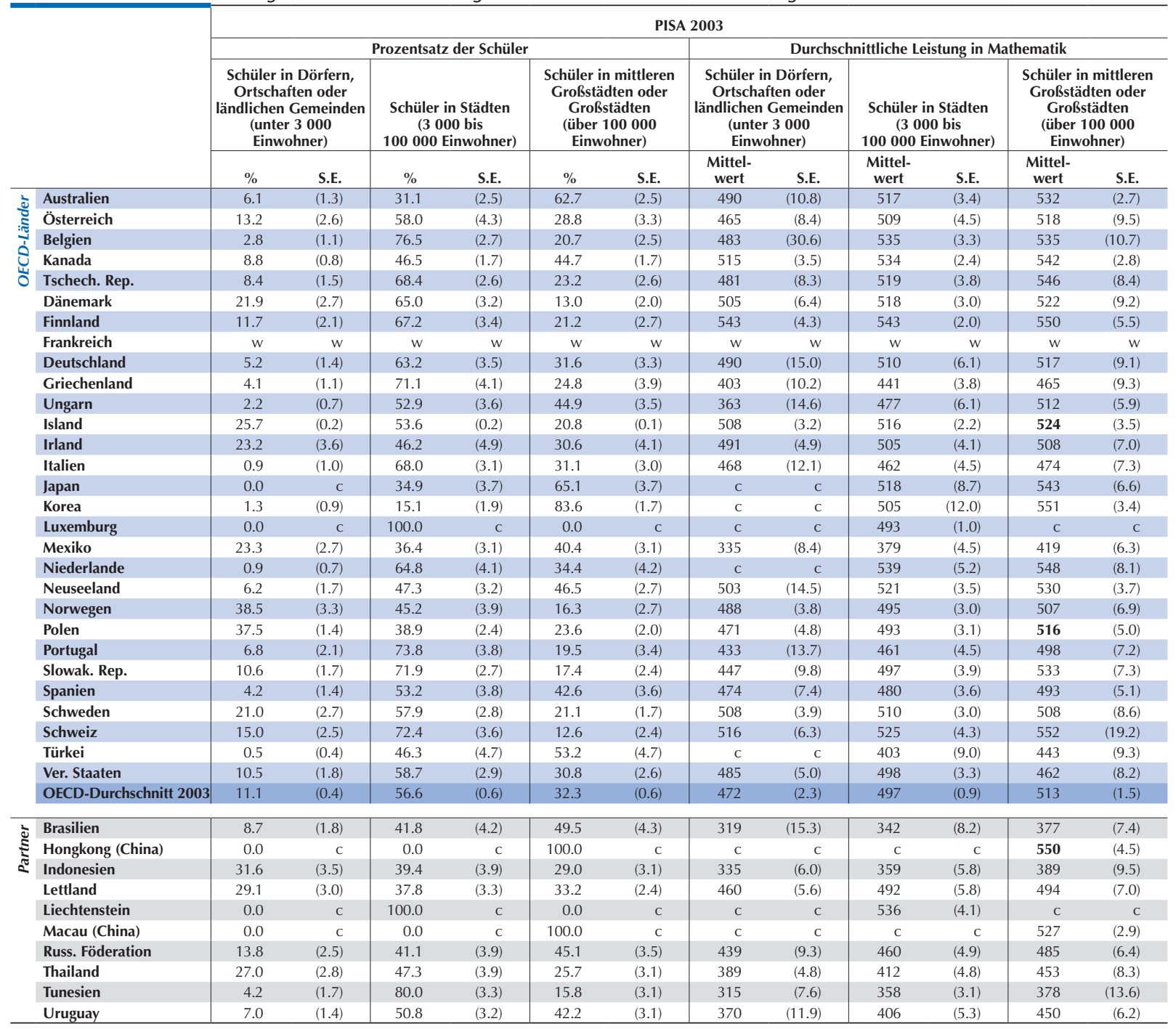

Anmerkung: Diese Tabelle wurde nur für Schüler berechnet, für die Daten zum PISA-Index des wirtschaftlichen, sozialen und kulturellen Status vorliegen. Statistisch signifikante Werte sind durch Fettdruck gekennzeichnet (vgl. Anhang A3).

Um die Vergleichbarkeit im Zeitverlauf zu gewährleisten, wurden die Werte von PISA 2003 auf dem PISA-Index des wirtschaftlichen, sozialen und kulturellen Status entsprechend der Indexskala von PISA 2012 umskaliert. Die in dieser Abbildung dargestellten Ergebnisse aus PISA 2003 können daher von denen abweichen, die in Lernen für die Welt von morgen: Erste Ergebnisse von PISA 2003 (OECD, 2004) wiedergegeben sind (vgl. Anhang A5 wegen näherer Einzelheiten).

Angegeben sind nur Länder und Volkswirtschaften mit vergleichbaren Daten aus PISA 2003 und PISA 2012

1. ESCS bezieht sich auf den PISA-Index des wirtschaftlichen, sozialen und kulturellen Status.

StatLink 完正四 http://dx.doi.org/10.1787/888932964927 
[Teil 2/3]

Von 2003 bis 2012 festzustellende Veränderung des Zusammenhangs zwischen Mathematikleistungen und Schulstandort

Tabelle II.3.3b Die Ergebnisse basieren auf Angaben der Schüler und der Schulleitungen

\begin{tabular}{|c|c|c|c|c|c|c|c|c|c|c|c|c|c|}
\hline & & \multicolumn{12}{|c|}{ PISA 2012} \\
\hline & & \multicolumn{6}{|c|}{ Prozentsatz der Schüler } & \multicolumn{6}{|c|}{ Durchschnittliche Leistung in Mathematik } \\
\hline & & \multicolumn{2}{|c|}{$\begin{array}{c}\text { Schüler in Dörfern, } \\
\text { Ortschaften oder } \\
\text { ländlichen Gemeinden } \\
\text { (unter } 3000 \text { Einwohner) }\end{array}$} & \multicolumn{2}{|c|}{$\begin{array}{l}\text { Schüler in Städten } \\
\text { (3 000 bis } 100000 \\
\text { Einwohner) }\end{array}$} & \multicolumn{2}{|c|}{$\begin{array}{l}\text { Schüler in mittleren } \\
\text { Großstädten oder } \\
\text { Großstädten } \\
\text { (über } 100000 \\
\text { Einwohner) }\end{array}$} & \multicolumn{2}{|c|}{$\begin{array}{l}\text { Schüler in Dörfern, } \\
\text { Ortschaften oder } \\
\text { ländlichen Gemeinden } \\
\text { (unter } 3000 \text { Einwohner) }\end{array}$} & \multicolumn{2}{|c|}{$\begin{array}{c}\text { Schüler in Städten } \\
\text { (3 } 000 \text { bis } 100000 \\
\text { Einwohner) }\end{array}$} & \multicolumn{2}{|c|}{$\begin{array}{c}\text { Schüler in mittleren } \\
\text { Großstädten oder } \\
\text { Großstädten } \\
\text { (über } 100000 \\
\text { Einwohner) }\end{array}$} \\
\hline & & $\%$ & S.E. & $\%$ & S.E. & $\%$ & S.E. & Mittelwert & S.E. & Mittelwert & S.E. & Mittelwert & S.E. \\
\hline \multirow{30}{*}{ 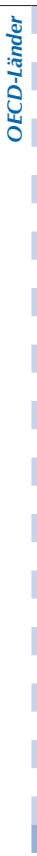 } & Australien & 5.9 & $(0.9)$ & 28.7 & $(1.2)$ & 65.4 & (1.3) & 470 & (5.4) & 489 & $(2.7)$ & 517 & (2.0) \\
\hline & Österreich & 9.8 & (2.3) & 54.7 & (3.5) & 35.5 & (3.0) & 512 & (17.9) & 503 & (4.6) & 510 & (7.3) \\
\hline & Belgien & 2.6 & (1.0) & 74.9 & (2.8) & 22.6 & (2.5) & 482 & $(34.7)$ & 523 & (3.1) & 502 & (9.3) \\
\hline & Kanada & 8.1 & $(0.8)$ & 37.7 & (2.1) & 54.2 & $(2.0)$ & 510 & (4.8) & 518 & (2.3) & 524 & (3.3) \\
\hline & Tschech. Rep. & 7.8 & (1.6) & 65.5 & (3.1) & 26.7 & (2.9) & 491 & (8.3) & 494 & $(5.2)$ & 512 & (6.9) \\
\hline & Dänemark & 23.9 & $(2.7)$ & 60.7 & $(3.2)$ & 15.3 & (2.5) & 493 & (5.7) & 505 & $(2.9)$ & 501 & (7.6) \\
\hline & Finnland & 7.4 & (1.6) & 65.7 & $(2.9)$ & 26.9 & (2.5) & 517 & (6.9) & 518 & (2.6) & 525 & (3.1) \\
\hline & Frankreich & w & w & w & w & w & w & w & w & w & w & w & w \\
\hline & Deutschland & 1.3 & $(0.7)$ & 74.3 & (3.3) & 24.4 & (3.2) & c & c & 518 & $(4.5)$ & 526 & $(10.6)$ \\
\hline & Griechenland & 8.3 & (1.9) & 62.3 & (3.3) & 29.4 & (2.9) & 432 & (7.5) & 448 & (3.7) & 471 & $(6.7)$ \\
\hline & Ungarn & 2.9 & $(0.7)$ & 58.4 & $(4.1)$ & 38.7 & $(4.1)$ & 395 & (21.9) & 470 & (6.0) & 496 & (8.6) \\
\hline & Island & 21.7 & $(0.2)$ & 47.5 & $(0.2)$ & 30.9 & $(0.2)$ & 477 & (3.4) & 495 & (2.6) & 508 & (2.9) \\
\hline & Irland & 22.6 & $(3.0)$ & 50.8 & (3.7) & 26.6 & (3.1) & 506 & (3.2) & 502 & (3.3) & 499 & (6.3) \\
\hline & Italien & 2.4 & $(0.7)$ & 67.1 & (2.0) & 30.5 & (1.9) & 451 & (9.1) & 484 & $(2.7)$ & 497 & (4.6) \\
\hline & Japan & 0.0 & c & 27.5 & (2.8) & 72.5 & (2.8) & c & c & 514 & (7.3) & 548 & (5.0) \\
\hline & Korea & 2.4 & $(0.8)$ & 11.4 & (1.8) & 86.1 & (1.8) & c & C & 546 & (12.7) & 556 & (4.6) \\
\hline & Luxemburg & 0.5 & $(0.0)$ & 99.5 & $(0.0)$ & 0.0 & C & C & c & 491 & (1.2) & c & $\mathrm{C}$ \\
\hline & Mexiko & 15.1 & (1.0) & 42.0 & (1.8) & 43.0 & (1.7) & 375 & (2.9) & 408 & (2.4) & 433 & (1.9) \\
\hline & Niederlande & 0.0 & c & 72.4 & $(4.2)$ & 27.6 & $(4.2)$ & c & c & 521 & (5.9) & 521 & (11.1) \\
\hline & Neuseeland & 6.3 & (1.0) & 37.9 & (3.5) & 55.8 & (3.4) & 460 & (6.4) & 494 & (5.1) & 516 & $(3.3)$ \\
\hline & Norwegen & 18.4 & $(2.0)$ & 60.9 & (3.2) & 20.7 & (2.9) & 478 & (8.4) & 493 & (2.9) & 501 & (6.0) \\
\hline & Polen & 32.5 & (2.4) & 46.8 & (2.5) & 20.8 & $(0.8)$ & 498 & (3.4) & 517 & $(4.7)$ & 548 & (11.4) \\
\hline & Portugal & 6.0 & $(2.1)$ & 72.2 & $(4.1)$ & 21.9 & (3.6) & 457 & (20.4) & 491 & (4.3) & 492 & $(10.8)$ \\
\hline & Slowak. Rep. & 13.6 & (1.9) & 72.9 & $(2.8)$ & 13.6 & (1.8) & 431 & (10.6) & 486 & (4.9) & 517 & $(10.9)$ \\
\hline & Spanien & 3.3 & $(0.6)$ & 58.8 & $(2.4)$ & 37.9 & (2.4) & 467 & $(8.2)$ & 480 & (2.4) & 495 & (3.3) \\
\hline & Schweden & 17.8 & (2.4) & 56.6 & (3.0) & 25.6 & (2.4) & 469 & $(4.7)$ & 481 & (3.0) & 490 & (5.9) \\
\hline & Schweiz & 7.9 & $(1.7)$ & 74.5 & (2.9) & 17.6 & (2.6) & 522 & (5.5) & 531 & (4.0) & 538 & $(10.1)$ \\
\hline & Türkei & 2.3 & $(1.0)$ & 41.3 & (4.6) & 56.4 & (4.4) & 480 & (31.4) & 457 & (11.0) & 441 & $(6.3)$ \\
\hline & Ver. Staaten & 10.9 & $(2.9)$ & 52.4 & $(4.1)$ & 36.7 & (3.6) & 473 & (9.1) & 491 & (5.5) & 476 & (7.0) \\
\hline & OECD-Durchschnitt 2003 & 9.3 & $(0.3)$ & 56.3 & $(0.6)$ & 34.4 & $(0.5)$ & 472 & $(2.8)$ & 495 & $(1.0)$ & 507 & (1.4) \\
\hline \multirow{10}{*}{ ఏ } & Brasilien & 1.1 & $(0.4)$ & 49.5 & $(2.3)$ & 49.3 & $(2.3)$ & 365 & (9.8) & 378 & (2.9) & 406 & $(3.7)$ \\
\hline & Hongkong (China) & 0.0 & c & 0.0 & c & 100.0 & c & c & c & c & c & 563 & $(3.2)$ \\
\hline & Indonesien & 29.1 & (3.5) & 50.4 & $(4.1)$ & 20.5 & (3.6) & 359 & $(9.0)$ & 371 & (5.3) & 407 & (9.4) \\
\hline & Lettland & 24.8 & (1.6) & 44.2 & $(1.8)$ & 31.0 & (1.9) & 461 & (5.1) & 493 & (3.3) & 513 & (5.9) \\
\hline & Liechtenstein & 0.0 & c & 100.0 & c & 0.0 & c & c & c & 537 & $(4.1)$ & c & $\mathrm{c}$ \\
\hline & Macau (China) & 0.2 & $(0.0)$ & 0.0 & c & 99.8 & $(0.0)$ & c & C & c & c & 540 & $(1.0)$ \\
\hline & Russ. Föderation & 20.4 & $(2.2)$ & 33.2 & $(2.4)$ & 46.4 & (2.6) & 460 & (6.6) & 470 & (5.7) & 501 & $(4.7)$ \\
\hline & Thailand & 15.7 & $(2.1)$ & 52.3 & (3.5) & 32.0 & (3.3) & 414 & (9.8) & 417 & $(4.0)$ & 450 & (6.9) \\
\hline & Tunesien & 4.4 & (1.8) & 72.1 & (3.3) & 23.5 & (3.2) & 370 & (11.7) & 383 & (4.1) & 410 & $(11.5)$ \\
\hline & Uruguay & 7.1 & (1.4) & 56.5 & $(2.8)$ & 36.4 & $(2.7)$ & 365 & (12.0) & 397 & (3.6) & 439 & (5.9) \\
\hline
\end{tabular}

Anmerkung: Diese Tabelle wurde nur für Schüler berechnet, für die Daten zum PISA-Index des wirtschaftlichen, sozialen und kulturellen Status vorliegen. Statistisch signifikante Werte sind durch Fettdruck gekennzeichnet (vgl. Anhang A3).

Um die Vergleichbarkeit im Zeitverlauf zu gewährleisten, wurden die Werte von PISA 2003 auf dem PISA-Index des wirtschaftlichen, sozialen und kulturellen Status entsprechend der Indexskala von PISA 2012 umskaliert. Die in dieser Abbildung dargestellten Ergebnisse aus PISA 2003 können daher von denen abweichen, die in Lernen für die Welt von morgen: Erste Ergebnisse von PISA 2003 (OECD 2004) wiedergegeben sind (vgl. Anhang A5 wegen näherer Einzelheiten).

Angegeben sind nur Länder und Volkswirtschaften mit vergleichbaren Daten aus PISA 2003 und PISA 2012

1. ESCS bezieht sich auf den PISA-Index des wirtschaftlichen, sozialen und kulturellen Status.

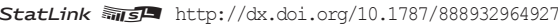


[Teil 3/3]

Von 2003 bis 2012 festzustellende Veränderung des Zusammenhangs zwischen Mathematikleistungen und Schulstandort

Tabelle II.3.3b Die Ergebnisse basieren auf Angaben der Schüler und der Schulleitungen

\begin{tabular}{|c|c|c|c|c|c|c|c|c|c|c|c|c|c|}
\hline & \multicolumn{12}{|c|}{ Veränderung zwischen 2003 und 2012 (PISA 2012 - PISA 2003) } \\
\hline & & \multicolumn{6}{|c|}{ Veränderung der durchschnittlichen Mathematikleistung } & \multicolumn{6}{|c|}{$\begin{array}{l}\text { Veränderung der durchschnittlichen Mathematikleistung } \\
\text { NACH Berücksichtigung des sozioökonomischen Status }\end{array}$} \\
\hline & & \multicolumn{2}{|c|}{\begin{tabular}{|} 
Schüler in Dörfern, \\
Ortschaften oder \\
ländlichen Gemeinden \\
(unter 3000 Einwohner)
\end{tabular}} & \multicolumn{2}{|c|}{$\begin{array}{l}\text { Schüler in Städten } \\
\text { (3 000 bis } 100000 \\
\text { Einwohner) }\end{array}$} & \multicolumn{2}{|c|}{$\begin{array}{l}\text { Schüler in mittleren } \\
\text { Großstädten oder } \\
\text { Großstädten } \\
\text { (über } 100000 \\
\text { Einwohner) }\end{array}$} & \multicolumn{2}{|c|}{$\begin{array}{l}\text { Schüler in Dörfern, } \\
\text { Ortschaften oder } \\
\text { ländlichen Gemeinden } \\
\text { (unter } 3000 \text { Einwohner) }\end{array}$} & \multicolumn{2}{|c|}{$\begin{array}{l}\text { Schüler in Städten } \\
(3000 \text { bis } \\
100000 \text { Einwohner })\end{array}$} & \multicolumn{2}{|c|}{$\begin{array}{l}\text { Schüler in mittleren } \\
\text { Großstädten oder } \\
\text { Großstädten } \\
\text { (über } 100000 \\
\text { Einwohner) }\end{array}$} \\
\hline & & $\begin{array}{l}\text { Punkt- } \\
\text { diff. }\end{array}$ & S.E. & $\begin{array}{l}\text { Punkt- } \\
\text { diff. }\end{array}$ & S.E. & $\begin{array}{l}\text { Punkt- } \\
\text { diff. }\end{array}$ & S.E. & $\begin{array}{l}\text { Punkt- } \\
\text { diff. }\end{array}$ & S.E. & $\begin{array}{l}\text { Punkt- } \\
\text { diff. }\end{array}$ & S.E. & $\begin{array}{l}\text { Punkt- } \\
\text { diff. }\end{array}$ & S.E. \\
\hline \multirow{30}{*}{ 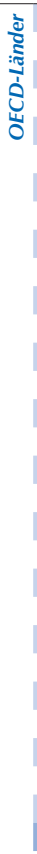 } & Australien & -20 & $(12.3)$ & -27 & $(4.8)$ & -15 & (3.9) & -29 & $(10.4)$ & -35 & $(4.2)$ & -23 & (3.6) \\
\hline & Österreich & 47 & $(19.8)$ & -6 & (6.7) & -8 & $(12.2)$ & 27 & $(17.5)$ & -16 & $(5.7)$ & -24 & (9.8) \\
\hline & Belgien & -1 & $(46.3)$ & -13 & (4.9) & -33 & $(14.3)$ & -23 & (29.5) & -24 & (3.8) & -32 & (9.9) \\
\hline & Kanada & -6 & (6.3) & -16 & (3.8) & -18 & $(4.7)$ & -13 & (6.0) & -23 & (3.7) & -21 & $(4.0)$ \\
\hline & Tschech. Rep. & 10 & (11.9) & -24 & (6.7) & -34 & (11.0) & 5 & (10.9) & -25 & (5.6) & -26 & (8.5) \\
\hline & Dänemark & -11 & (8.8) & -13 & (4.6) & -21 & $(12.1)$ & -28 & (6.8) & -26 & (3.8) & -28 & (6.9) \\
\hline & Finnland & -26 & (8.3) & -25 & (3.8) & -24 & (6.6) & -36 & (7.5) & -33 & (3.7) & -31 & (5.6) \\
\hline & Frankreich & w & w & w & w & w & w & w & w & w & w & w & w \\
\hline & Deutschland & c & c & 8 & $(7.8)$ & 9 & $(14.2)$ & c & c & 1 & (6.3) & 1 & (8.5) \\
\hline & Griechenland & 29 & $(12.7)$ & 7 & (5.7) & 6 & (11.6) & 20 & $(12.1)$ & -1 & (4.6) & -2 & (8.7) \\
\hline & Ungarn & 31 & (26.4) & -7 & (8.8) & -16 & (10.6) & 27 & $(24.6)$ & -15 & (6.3) & -16 & (7.3) \\
\hline & Island & -31 & (5.1) & -21 & (3.9) & -17 & (5.0) & -39 & $(5.2)$ & -27 & (3.9) & -20 & $(5.2)$ \\
\hline & Irland & 15 & (6.1) & -3 & (5.6) & -8 & (9.6) & 2 & (5.4) & -16 & $(4.4)$ & -25 & (5.9) \\
\hline & Italien & -17 & (15.3) & 22 & (5.6) & 23 & (8.8) & -16 & (12.8) & 14 & (4.9) & 17 & (8.1) \\
\hline & Japan & $\mathrm{c}$ & c & -4 & (11.5) & 5 & (8.5) & c & c & -16 & (9.8) & -10 & (7.5) \\
\hline & Korea & c & c & 41 & (17.6) & 5 & (6.1) & c & c & 19 & $(17.2)$ & -6 & (5.5) \\
\hline & Luxemburg & c & c & -3 & (2.5) & c & c & c & c & -8 & (2.5) & c & c \\
\hline & Mexiko & 39 & (9.1) & 29 & (5.5) & 15 & (6.9) & 41 & (8.0) & 28 & (5.2) & 7 & (5.3) \\
\hline & Niederlande & c & c & -18 & (8.1) & -27 & (13.9) & c & c & -32 & (8.1) & -36 & (11.7) \\
\hline & Neuseeland & -42 & (16.0) & -27 & (6.5) & -14 & (5.3) & -42 & $(14.0)$ & -33 & (5.6) & -25 & $(4.1)$ \\
\hline & Norwegen & -10 & (9.4) & -3 & (4.6) & -7 & (9.4) & -19 & (9.6) & -11 & (4.4) & -18 & (6.6) \\
\hline & Polen & 28 & (6.2) & 24 & (6.0) & 32 & (12.6) & 20 & (5.4) & 17 & (5.2) & 25 & (8.7) \\
\hline & Portugal & 23 & $(24.7)$ & 30 & (6.5) & -6 & (13.2) & 13 & (22.6) & 17 & (5.6) & -15 & (8.8) \\
\hline & Slowak. Rep. & -15 & $(14.6)$ & -12 & (6.6) & -16 & (13.2) & -21 & $(11.3)$ & -16 & (4.6) & -21 & $(10.6)$ \\
\hline & Spanien & -7 & $(11.2)$ & 0 & $(4.7)$ & 2 & (6.3) & -9 & $(9.7)$ & -9 & $(4.2)$ & -10 & $(4.8)$ \\
\hline & Schweden & -39 & (6.4) & -29 & (4.6) & -18 & (10.6) & -43 & (6.1) & -35 & (4.1) & -28 & (6.9) \\
\hline & Schweiz & 6 & (8.6) & 7 & (6.2) & -15 & $(21.7)$ & -4 & (8.4) & -9 & (5.5) & -26 & $(15.3)$ \\
\hline & Türkei & c & c & 54 & $(14.4)$ & -2 & (11.4) & c & c & 59 & $(10.6)$ & 15 & (8.8) \\
\hline & Ver. Staaten & -13 & (10.6) & -7 & (6.7) & 14 & $(11.0)$ & -18 & $(10.1)$ & -11 & (5.5) & 9 & $(9.1)$ \\
\hline & OECD-Durchschnitt 2003 & 0 & (3.5) & -1 & (1.4) & -8 & $(2.1)$ & -8 & (2.8) & -10 & (1.2) & -14 & (1.5) \\
\hline \multirow{10}{*}{ ฐ } & Brasilien & 46 & $(18.2)$ & 36 & (8.9) & 29 & (8.5) & 48 & $(14.3)$ & 30 & (7.5) & 14 & (7.5) \\
\hline & Hongkong (China) & c & c & c & c & 13 & (5.8) & c & c & c & c & -1 & $(5.2)$ \\
\hline & Indonesien & 24 & $(11.0)$ & 12 & (8.1) & 18 & (13.5) & 24 & (9.8) & 11 & (7.5) & 12 & $(10.7)$ \\
\hline & Lettland & 0 & $(7.8)$ & 1 & (7.0) & 19 & (9.3) & 4 & (7.5) & -3 & (6.1) & 13 & (9.0) \\
\hline & Liechtenstein & c & c & 1 & (6.1) & c & c & c & c & -23 & (6.8) & c & c \\
\hline & Macau (China) & c & c & c & c & 12 & (3.6) & c & c & c & c & 2 & (3.6) \\
\hline & Russ. Föderation & 21 & (11.6) & 9 & (7.8) & 17 & $(8.2)$ & 7 & $(13.0)$ & -7 & (7.0) & 1 & $(7.3)$ \\
\hline & Thailand & 25 & $(11.0)$ & 4 & (6.5) & -3 & (11.0) & 27 & (11.6) & -4 & (6.3) & -13 & (9.0) \\
\hline & Tunesien & 55 & $(14.1)$ & 25 & (5.5) & 31 & $(17.9)$ & 53 & (15.1) & 15 & (5.5) & 23 & $(14.2)$ \\
\hline & Uruguay & -6 & $(17.0)$ & -9 & $(6.7)$ & -11 & $(8.7)$ & -8 & $(14.3)$ & -5 & $(5.5)$ & -10 & $(6.1)$ \\
\hline
\end{tabular}

Anmerkung: Diese Tabelle wurde nur für Schüler berechnet, für die Daten zum PISA-Index des wirtschaftlichen, sozialen und kulturellen Status vorliegen. Statistisch signifikante Werte sind durch Fettdruck gekennzeichnet (vgl. Anhang A3).

Um die Vergleichbarkeit im Zeitverlauf zu gewährleisten, wurden die Werte von PISA 2003 auf dem PISA-Index des wirtschaftlichen, sozialen und kulturellen Status entsprechend der Indexskala von PISA 2012 umskaliert. Die in dieser Abbildung dargestellten Ergebnisse aus PISA 2003 können daher von denen abweichen, die in Lernen für die Welt von morgen: Erste Ergebnisse von PISA 2003 (OECD 2004) wiedergegeben sind (vol. Anhang A5 wegen näherer Einzelheiten).

Angegeben sind nur Länder und Volkswirtschaften mit vergleichbaren Daten aus PISA 2003 und PISA 2012.

1. ESCS bezieht sich auf den PISA-Index des wirtschaftlichen, sozialen und kulturellen Status.

StatLink त्ञाIs http://dx.doi.org/10.1787/888932964927 
[Teil 1/2]

Schülerleistungen in Mathematik und Migrationshintergrund

Tabelle II.3.4a Die Ergebnisse basieren auf Schülerangaben

\begin{tabular}{|c|c|c|c|c|c|c|c|c|c|c|c|c|c|c|c|}
\hline & \multicolumn{4}{|c|}{ Prozentsatz der Schüler } & \multicolumn{4}{|c|}{$\begin{array}{l}\text { PISA-Index des wirtschaftlichen, } \\
\text { sozialen und kulturellen Status (ESCS) }\end{array}$} & \multicolumn{4}{|c|}{ Schülerleistungen in Mathematik } & \multirow{2}{*}{\multicolumn{2}{|c|}{$\begin{array}{l}\text { Leistungs- } \\
\text { unterschied in } \\
\text { Mathematik } \\
\text { zwischen Schülern } \\
\text { mit und ohne } \\
\text { Migrations- } \\
\text { hintergrund }\end{array}$}} \\
\hline & & \multicolumn{2}{|c|}{$\begin{array}{l}\text { Ohne Migrations- } \\
\text { hintergrund }\end{array}$} & \multicolumn{2}{|c|}{$\begin{array}{l}\text { Mit Migrations- } \\
\text { hintergrund }\end{array}$} & $\begin{array}{r}\text { Ohne M } \\
\text { hinte }\end{array}$ & $\begin{array}{l}\text { rations- } \\
\text { rund }\end{array}$ & $\begin{array}{r}\text { Mit Mi } \\
\text { hinte }\end{array}$ & $\begin{array}{l}\text { ations- } \\
\text { rund }\end{array}$ & $\begin{array}{r}\text { Ohne M } \\
\text { hinte }\end{array}$ & $\begin{array}{l}\text { ations- } \\
\text { und }\end{array}$ & $\begin{array}{l}\text { Mit Mi } \\
\text { hinte }\end{array}$ & $\begin{array}{l}\text { rations- } \\
\text { grund }\end{array}$ & & \\
\hline & & $\%$ & S.E. & $\%$ & S.E. & $\begin{array}{l}\text { Index- } \\
\text { mittel }\end{array}$ & S.E. & $\begin{array}{l}\text { Index- } \\
\text { mittel }\end{array}$ & S.E. & $\begin{array}{c}\text { Mittel- } \\
\text { wert }\end{array}$ & S.E. & $\begin{array}{c}\text { Mittel- } \\
\text { wert }\end{array}$ & S.E. & $\begin{array}{c}\text { Punkt- } \\
\text { diff. }\end{array}$ & S.E. \\
\hline ¿ & Australien & 77.3 & $(0.7)$ & 22.7 & $(0.7)$ & 0.28 & $(0.01)$ & 0.21 & $(0.02)$ & 503 & $(1.5)$ & 528 & (3.8) & -26 & $(3.6)$ \\
\hline 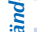 & Österreich & 83.6 & $(1.1)$ & 16.4 & $(1.1)$ & 0.19 & $(0.02)$ & -0.49 & $(0.05)$ & 517 & $(2.7)$ & 457 & $(4.9)$ & 59 & $(5.2)$ \\
\hline în & Belgien & 84.9 & $(0.9)$ & 15.1 & $(0.9)$ & 0.23 & $(0.02)$ & -0.29 & $(0.06)$ & 530 & (2.1) & 455 & $(5.2)$ & 75 & $(5.0)$ \\
\hline 仓ิ & Kanada & 70.5 & $(1.3)$ & 29.5 & (1.3) & 0.45 & $(0.02)$ & 0.32 & $(0.04)$ & 522 & $(1.8)$ & 520 & $(4.2)$ & 2 & $(4.4)$ \\
\hline$\ddot{0}$ & Chile & 99.1 & $(0.2)$ & 0.9 & $(0.2)$ & -0.58 & $(0.04)$ & -0.27 & $(0.17)$ & 424 & (3.0) & 422 & $(14.1)$ & 1 & $(13.3)$ \\
\hline & Tschech. Rep. & 96.8 & $(0.4)$ & 3.2 & $(0.4)$ & -0.06 & $(0.02)$ & -0.16 & $(0.07)$ & 501 & (2.8) & 475 & $(12.2)$ & 26 & $(11.8)$ \\
\hline & Dänemark & 91.1 & $(0.6)$ & 8.9 & $(0.6)$ & 0.49 & $(0.02)$ & -0.23 & $(0.04)$ & 508 & $(2.2)$ & 442 & $(3.2)$ & 66 & $(3.6)$ \\
\hline & Estland & 91.8 & $(0.5)$ & 8.2 & $(0.5)$ & 0.11 & $(0.01)$ & 0.09 & $(0.05)$ & 524 & (2.0) & 494 & (5.9) & 30 & $(5.8)$ \\
\hline & Finnland & 96.7 & $(0.2)$ & 3.3 & $(0.2)$ & 0.39 & $(0.02)$ & -0.26 & $(0.04)$ & 523 & (1.9) & 439 & (5.0) & 85 & $(5.0)$ \\
\hline & Frankreich & 85.2 & (1.1) & 14.8 & (1.1) & 0.05 & $(0.02)$ & -0.53 & $(0.04)$ & 508 & (2.7) & 441 & (6.0) & 67 & (6.9) \\
\hline & Deutschland & 86.9 & $(0.8)$ & 13.1 & $(0.8)$ & 0.30 & $(0.03)$ & -0.41 & $(0.04)$ & 528 & (3.2) & 475 & (5.5) & 54 & $(6.0)$ \\
\hline & Griechenland & 89.5 & $(0.8)$ & 10.5 & $(0.8)$ & 0.00 & $(0.03)$ & -0.68 & $(0.04)$ & 459 & (2.6) & 408 & (5.9) & 51 & (6.4) \\
\hline & Ungarn & 98.3 & $(0.2)$ & 1.7 & $(0.2)$ & -0.26 & $(0.03)$ & 0.12 & $(0.10)$ & 478 & (3.1) & 508 & (14.1) & -31 & (13.3) \\
\hline & Island & 96.5 & $(0.3)$ & 3.5 & $(0.3)$ & 0.81 & $(0.01)$ & 0.09 & $(0.08)$ & 498 & (1.8) & 445 & (8.5) & 52 & (8.6) \\
\hline & Irland & 89.9 & $(0.7)$ & 10.1 & $(0.7)$ & 0.12 & $(0.02)$ & 0.18 & $(0.06)$ & 503 & (2.3) & 501 & (4.6) & 2 & $(4.8)$ \\
\hline & Israel & 81.7 & $(1.2)$ & 18.3 & $(1.2)$ & 0.21 & $(0.03)$ & 0.04 & $(0.06)$ & 470 & (4.6) & 477 & (6.9) & -7 & $(5.7)$ \\
\hline & Italien & 92.5 & $(0.3)$ & 7.5 & $(0.3)$ & -0.01 & $(0.01)$ & -0.55 & $(0.03)$ & 490 & (2.0) & 442 & (3.3) & 48 & $(3.5)$ \\
\hline & Japan & 99.7 & $(0.1)$ & 0.3 & $(0.1)$ & -0.07 & $(0.02)$ & C & $\mathrm{C}$ & 539 & (3.5) & c & c & C & c \\
\hline & Korea & 100.0 & $(0.0)$ & 0.0 & $(0.0)$ & 0.01 & $(0.03)$ & $\mathrm{c}$ & c & 555 & (4.6) & c & c & c & c \\
\hline & Luxemburg & 53.6 & $(0.7)$ & 46.4 & $(0.7)$ & 0.47 & $(0.01)$ & -0.39 & $(0.02)$ & 511 & (1.7) & 470 & $(2.3)$ & 40 & $(3.3)$ \\
\hline & Mexiko & 98.7 & $(0.1)$ & 1.3 & $(0.1)$ & -1.10 & $(0.02)$ & -1.46 & $(0.14)$ & 416 & (1.3) & 343 & $(5.7)$ & 73 & $(5.5)$ \\
\hline & Niederlande & 89.4 & $(1.0)$ & 10.6 & $(1.0)$ & 0.31 & $(0.02)$ & -0.31 & $(0.05)$ & 531 & (3.4) & 474 & (7.5) & 57 & $(7.1)$ \\
\hline & Neuseeland & 73.7 & (1.5) & 26.3 & (1.5) & 0.05 & $(0.02)$ & 0.02 & $(0.04)$ & 503 & $(2.7)$ & 503 & $(4.7)$ & 0 & $(5.4)$ \\
\hline & Norwegen & 90.6 & $(0.9)$ & 9.4 & $(0.9)$ & 0.52 & $(0.02)$ & -0.08 & $(0.05)$ & 496 & (2.8) & 450 & (6.1) & 46 & $(6.6)$ \\
\hline & Polen & 99.8 & $(0.1)$ & 0.2 & $(0.1)$ & -0.21 & $(0.03)$ & $\mathrm{C}$ & $\mathrm{C}$ & 518 & (3.6) & c & c & c & c \\
\hline & Portugal & 93.1 & (0.6) & 6.9 & $(0.6)$ & -0.47 & $(0.05)$ & -0.62 & $(0.10)$ & 493 & (3.7) & 449 & $(7.2)$ & 44 & $(7.2)$ \\
\hline & Slowak. Rep. & 99.3 & $(0.2)$ & 0.7 & $(0.2)$ & -0.18 & $(0.03)$ & -0.18 & $(0.17)$ & 484 & (3.4) & 489 & $(21.2)$ & -5 & $(21.1)$ \\
\hline & Slowenien & 91.4 & $(0.4)$ & 8.6 & $(0.4)$ & 0.12 & $(0.01)$ & -0.50 & $(0.05)$ & 506 & $(1.2)$ & 456 & $(4.8)$ & 51 & $(5.0)$ \\
\hline & Spanien & 90.1 & (0.6) & 9.9 & $(0.6)$ & -0.14 & $(0.03)$ & -0.64 & $(0.04)$ & 491 & (1.7) & 439 & (4.6) & 52 & $(4.3)$ \\
\hline & Schweden & 85.5 & $(0.9)$ & 14.5 & $(0.9)$ & 0.36 & $(0.02)$ & -0.21 & $(0.05)$ & 490 & (2.3) & 432 & (4.9) & 58 & $(5.1)$ \\
\hline & Schweiz & 75.9 & $(0.9)$ & 24.1 & $(0.9)$ & 0.34 & $(0.02)$ & -0.34 & $(0.04)$ & 548 & (3.0) & 484 & (3.9) & 63 & $(3.2)$ \\
\hline & Türkei & 99.1 & $(0.2)$ & 0.9 & $(0.2)$ & -1.46 & $(0.04)$ & -1.21 & $(0.20)$ & 449 & (4.8) & 452 & (30.9) & -3 & $(31.1)$ \\
\hline & Ver. Königreich & 87.3 & (1.1) & 12.7 & (1.1) & 0.28 & $(0.02)$ & 0.19 & $(0.06)$ & 499 & (2.8) & 489 & $(8.2)$ & 9 & $(7.9)$ \\
\hline & Ver. Staaten & 78.5 & (2.0) & 21.5 & (2.0) & 0.34 & $(0.03)$ & -0.40 & $(0.08)$ & 487 & (3.6) & 474 & (6.2) & 13 & $(5.9)$ \\
\hline & OECD-Durchschnitt & 88.8 & $(0.1)$ & 11.2 & $(0.1)$ & 0.06 & $(0.00)$ & -0.29 & $(0.01)$ & 500 & $(0.5)$ & 462 & (1.7) & 34 & $(1.7)$ \\
\hline s & Albanien & 0.0 & c & 0.0 & c & $\mathrm{m}$ & $\mathrm{m}$ & $\mathrm{m}$ & $\mathrm{m}$ & $\mathrm{m}$ & $\mathrm{m}$ & $\mathrm{m}$ & $\mathrm{m}$ & c & c \\
\hline$\underset{\Xi}{\overparen{E}}$ & Argentinien & 96.1 & $(0.4)$ & 3.9 & $(0.4)$ & -0.68 & $(0.04)$ & -1.45 & $(0.09)$ & 392 & (3.4) & 355 & $(7.1)$ & 37 & $(6.7)$ \\
\hline క్ర్ల & Brasilien & 99.3 & $(0.1)$ & 0.7 & $(0.1)$ & -1.16 & $(0.02)$ & -0.88 & $(0.21)$ & 394 & (2.1) & 338 & (11.4) & 56 & $(11.0)$ \\
\hline$\stackrel{2}{\Xi}$ & Bulgarien & 99.5 & $(0.2)$ & 0.5 & $(0.2)$ & -0.27 & $(0.04)$ & c & c & 442 & (3.9) & c & c & c & c \\
\hline 商 & Kolumbien & 99.7 & $(0.1)$ & 0.3 & $(0.1)$ & -1.26 & $(0.04)$ & -1.36 & $(0.30)$ & 378 & (2.9) & 309 & $(13.1)$ & 69 & $(13.0)$ \\
\hline ङ & Costa Rica & 94.5 & $(0.7)$ & 5.5 & $(0.7)$ & -0.94 & $(0.04)$ & -1.60 & $(0.15)$ & 409 & (2.9) & 380 & (9.9) & 29 & $(9.6)$ \\
\hline \̦ & Kroatien & 87.9 & $(0.8)$ & 12.1 & $(0.8)$ & -0.30 & $(0.02)$ & -0.59 & $(0.04)$ & 474 & (3.6) & 455 & (5.4) & 19 & $(5.2)$ \\
\hline 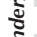 & Zypern* & 91.5 & $(0.4)$ & 8.5 & $(0.4)$ & 0.10 & $(0.01)$ & -0.07 & $(0.04)$ & 444 & (1.2) & 424 & $(4.7)$ & 21 & $(5.0)$ \\
\hline : & Hongkong (China) & 65.3 & (1.5) & 34.7 & (1.5) & -0.56 & $(0.06)$ & -1.22 & $(0.03)$ & 566 & (3.7) & 559 & (3.8) & 8 & $(4.4)$ \\
\hline ఏ & Indonesien & 99.8 & $(0.1)$ & 0.2 & $(0.1)$ & -1.80 & $(0.05)$ & c & c & 376 & $(4.0)$ & c & c & c & c \\
\hline ปั้ & Jordanien & 86.6 & $(0.7)$ & 13.4 & $(0.7)$ & -0.47 & $(0.02)$ & -0.19 & $(0.05)$ & 388 & (2.8) & 410 & (5.1) & -22 & $(4.4)$ \\
\hline & Kasachstan & 83.9 & $(1.7)$ & 16.1 & (1.7) & -0.29 & $(0.02)$ & -0.46 & $(0.05)$ & 433 & (3.1) & 427 & (5.8) & 7 & $(5.8)$ \\
\hline & Lettland & 95.5 & $(0.5)$ & 4.5 & $(0.5)$ & -0.26 & $(0.03)$ & -0.13 & $(0.09)$ & 492 & (2.8) & 486 & $(8.1)$ & 6 & $(7.8)$ \\
\hline & Liechtenstein & 66.7 & (2.9) & 33.3 & (2.9) & 0.44 & $(0.05)$ & 0.02 & $(0.11)$ & 554 & (5.6) & 504 & (8.6) & 50 & $(11.5)$ \\
\hline & Litauen & 98.3 & $(0.3)$ & 1.7 & $(0.3)$ & -0.13 & $(0.02)$ & -0.11 & $(0.10)$ & 480 & $(2.7)$ & 479 & (9.3) & 1 & $(9.8)$ \\
\hline & Macau (China) & 34.9 & $(0.6)$ & 65.1 & (0.6) & -0.69 & $(0.02)$ & -0.99 & $(0.01)$ & 530 & (2.1) & 545 & (1.3) & -16 & $(2.8)$ \\
\hline & Malaysia & 98.3 & $(0.3)$ & 1.7 & $(0.3)$ & -0.71 & $(0.03)$ & -1.33 & $(0.13)$ & 423 & (3.2) & 402 & (8.5) & 21 & (8.9) \\
\hline & Montenegro & 94.2 & $(0.4)$ & 5.8 & $(0.4)$ & -0.26 & $(0.01)$ & -0.12 & $(0.05)$ & 410 & (1.2) & 431 & (6.2) & -21 & $(6.5)$ \\
\hline & Peru & 99.5 & $(0.1)$ & 0.5 & $(0.1)$ & -1.22 & $(0.05)$ & -1.35 & $(0.21)$ & 370 & (3.6) & 280 & $(23.0)$ & 90 & $(22.5)$ \\
\hline & Katar & 48.0 & $(0.4)$ & 52.0 & $(0.4)$ & 0.42 & $(0.01)$ & 0.45 & $(0.01)$ & 335 & (1.1) & 424 & (1.3) & -89 & $(1.7)$ \\
\hline & Rumänien & 99.8 & $(0.1)$ & 0.2 & $(0.1)$ & -0.47 & $(0.04)$ & c & c & 445 & (3.8) & c & c & c & c \\
\hline & Russ. Föderation & 89.1 & $(0.8)$ & 10.9 & $(0.8)$ & -0.10 & $(0.02)$ & -0.17 & $(0.05)$ & 486 & (3.2) & 464 & (4.3) & 22 & $(4.5)$ \\
\hline & Serbien & 91.5 & $(0.8)$ & 8.5 & $(0.8)$ & -0.30 & $(0.02)$ & -0.29 & $(0.06)$ & 449 & (3.4) & 464 & $(7.0)$ & -15 & (6.2) \\
\hline & Shanghai (China) & 99.1 & $(0.2)$ & 0.9 & $(0.2)$ & -0.35 & $(0.04)$ & -1.05 & $(0.19)$ & 615 & (3.2) & 489 & $(15.2)$ & 126 & $(14.6)$ \\
\hline & Singapur & 81.7 & $(0.8)$ & 18.3 & $(0.8)$ & -0.34 & $(0.01)$ & 0.12 & $(0.04)$ & 570 & (1.6) & 596 & (3.6) & -26 & $(4.3)$ \\
\hline & Chinesisch Taipeh & 99.5 & $(0.1)$ & 0.5 & $(0.1)$ & -0.39 & $(0.02)$ & -0.92 & $(0.17)$ & 562 & (3.3) & 530 & (23.6) & 32 & $(23.1)$ \\
\hline & Thailand & 99.3 & $(0.4)$ & 0.7 & $(0.4)$ & -1.34 & $(0.04)$ & -3.24 & $(0.13)$ & 428 & (3.3) & 411 & $(57.1)$ & 17 & $(56.4)$ \\
\hline & Tunesien & 99.6 & $(0.1)$ & 0.4 & $(0.1)$ & -1.19 & $(0.05)$ & C & $\mathrm{c}$ & 389 & $(4.0)$ & c & $\mathrm{c}$ & $\mathrm{C}$ & C \\
\hline & Ver. Arab. Emirate & 45.1 & (1.4) & 54.9 & (1.4) & 0.27 & $(0.03)$ & 0.37 & $(0.02)$ & 400 & (2.4) & 466 & (2.6) & -66 & (3.1) \\
\hline & Uruguay & 99.5 & $(0.1)$ & 0.5 & (0.1) & -0.88 & $(0.03)$ & C & c & 412 & (2.6) & c & c & c & c \\
\hline & Vietnam & 99.9 & $(0.1)$ & 0.1 & $(0.1)$ & -1.81 & $(0.05)$ & c & c & 512 & (4.8) & c & c & c & c \\
\hline
\end{tabular}

Anmerkung: Diese Tabelle wurde nur für Schüler berechnet, für die Daten zum PISA-Index des wirtschaftlichen, sozialen und kulturellen Status vorliegen. Statistisch signifikante Werte sind durch Fettdruck gekennzeichnet (vgl. Anhang A3).

Schüler mit Migrationshintergrund sind Schüler, deren Eltern in einem anderen Land/einer anderen Volkswirtschaft als dem getesteten Land/der getesteten Volkswirtschaft geboren sind

*Vgl. die Anmerkungen am Beginn dieses Anhangs.

StatLink तinा St http://dx.doi.org/10.1787/888932964927 
[Teil 2/2]

Schülerleistungen in Mathematik und Migrationshintergrund Tabelle II.3.4a Die Ergebnisse basieren auf Schülerangaben

\begin{tabular}{|c|c|c|c|c|c|c|c|c|c|c|c|c|c|}
\hline & \multicolumn{2}{|c|}{$\begin{array}{c}\text { Leistungsunterschied in } \\
\text { Mathematik zwischen } \\
\text { Schülern mit und } \\
\text { ohne Migrations- } \\
\text { hintergrund NACH } \\
\text { Berücksichtigung des } \\
\text { sozioökonomischen } \\
\text { Status }\end{array}$} & \multicolumn{2}{|c|}{$\begin{array}{c}\text { Erhöhte } \\
\text { Wahrscheinlichkeit } \\
\text { für Schüler mit } \\
\text { Migrationshintergrund, } \\
\text { im untersten Quartil } \\
\text { der Gesamtskala } \\
\text { Mathematik zu liegen }\end{array}$} & \multicolumn{2}{|c|}{\begin{tabular}{|c|} 
Populationsrelevanz \\
der Schüler mit \\
Migrationshintergrund, \\
die im untersten Quartil \\
der Gesamtskala \\
Mathematik liegen
\end{tabular}} & \multicolumn{2}{|c|}{$\begin{array}{c}\text { Effektstärke bei den } \\
\text { Mathematikleistungen } \\
\text { für Schüler mit } \\
\text { Migrationshintergrund } \\
\text { (positive Werte } \\
\text { weisen auf Vorsprung } \\
\text { für Schüler ohne } \\
\text { Migrations- } \\
\text { hintergrund hin) }\end{array}$} & \multicolumn{2}{|c|}{\begin{tabular}{|c|} 
Erhöhte \\
Wahrscheinlichkeit \\
für Schüler ohne \\
Migrationshintergrund, \\
im untersten Quartil \\
der Gesamtskala \\
Mathematik zu liegen
\end{tabular}} & \multicolumn{2}{|c|}{$\begin{array}{l}\text { Populationsrelevanz } \\
\text { der Schüler ohne } \\
\text { Migrationshintergrund, } \\
\text { die im untersten Quarti } \\
\text { der Gesamtskala } \\
\text { Mathematik liegen }\end{array}$} \\
\hline & & $\begin{array}{l}\text { Punkt- } \\
\text { diff. }\end{array}$ & S.E. & Quotient & S.E. & $\%$ & S.E. & Effektstärke & S.E. & Quotient & S.E. & $\%$ & S.E. \\
\hline \multirow{35}{*}{ 芯 } & Australien & -29 & (3.4) & 0.80 & $(0.04)$ & -4.8 & (1.0) & 0.27 & $(0.03)$ & 1.25 & $(0.06)$ & 16.3 & (3.4) \\
\hline & Österreich & 33 & (4.9) & 2.16 & $(0.16)$ & 16.0 & (1.8) & -0.66 & $(0.06)$ & 0.46 & $(0.03)$ & -81.6 & (9.8) \\
\hline & Belgien & 52 & (3.9) & 2.37 & $(0.15)$ & 17.2 & (1.8) & -0.78 & $(0.05)$ & 0.42 & $(0.03)$ & -96.6 & (8.5) \\
\hline & Kanada & -2 & (3.9) & 1.07 & $(0.07)$ & 2.1 & (2.0) & -0.02 & $(0.05)$ & 0.93 & $(0.06)$ & -5.1 & (4.8) \\
\hline & Chile & 12 & (11.1) & 1.04 & $(0.33)$ & 0.0 & $(0.3)$ & -0.02 & $(0.16)$ & 0.98 & $(0.30)$ & -3.8 & $(32.2)$ \\
\hline & Tschech. Rep. & 20 & (11.4) & 1.34 & $(0.23)$ & 1.1 & (0.8) & -0.25 & $(0.11)$ & 0.75 & $(0.13)$ & -32.5 & (21.9) \\
\hline & Dänemark & 40 & (3.2) & 2.43 & $(0.15)$ & 11.2 & $(1.3)$ & -0.84 & $(0.05)$ & 0.41 & $(0.03)$ & -115.5 & $(10.6)$ \\
\hline & Estland & 30 & (5.2) & 1.53 & $(0.14)$ & 4.1 & (1.1) & -0.38 & $(0.07)$ & 0.65 & $(0.06)$ & -46.5 & (12.0) \\
\hline & Finnland & 65 & (4.6) & 2.65 & $(0.11)$ & 5.2 & $(0.4)$ & -0.99 & $(0.05)$ & 0.38 & $(0.02)$ & -151.5 & (9.7) \\
\hline & Frankreich & 37 & (6.4) & 2.31 & $(0.20)$ & 16.2 & (2.5) & -0.71 & $(0.08)$ & 0.43 & $(0.04)$ & -93.6 & (11.4) \\
\hline & Deutschland & 25 & (5.6) & 1.86 & $(0.16)$ & 10.1 & (1.8) & -0.57 & $(0.06)$ & 0.54 & $(0.05)$ & -66.9 & (11.3) \\
\hline & Griechenland & 28 & (6.4) & 1.93 & $(0.19)$ & 8.9 & (1.8) & -0.61 & $(0.08)$ & 0.52 & $(0.05)$ & -76.1 & (13.9) \\
\hline & Ungarn & -13 & $(13.2)$ & 0.66 & $(0.25)$ & -0.6 & (0.4) & 0.33 & $(0.14)$ & 1.59 & $(0.65)$ & 34.1 & $(24.4)$ \\
\hline & Island & 31 & (8.4) & 1.83 & $(0.22)$ & 2.8 & $(0.8)$ & -0.58 & $(0.10)$ & 0.55 & $(0.07)$ & -78.1 & (19.6) \\
\hline & Irland & 4 & (4.5) & 1.04 & $(0.11)$ & 0.4 & (1.1) & -0.02 & $(0.06)$ & 0.97 & $(0.10)$ & -3.1 & (9.6) \\
\hline & Israel & -16 & (4.8) & 0.81 & $(0.09)$ & -3.5 & (1.9) & 0.07 & $(0.06)$ & 1.23 & $(0.16)$ & 15.8 & $(8.2)$ \\
\hline & Italien & 32 & (3.3) & 1.84 & $(0.09)$ & 5.9 & (0.6) & -0.52 & $(0.04)$ & 0.54 & $(0.03)$ & -73.0 & (7.5) \\
\hline & Japan & c & c & c & c & c & c & c & c & c & c & c & c \\
\hline & Korea & c & c & c & c & c & c & c & c & c & c & c & c \\
\hline & Luxemburg & 10 & (3.3) & 1.98 & $(0.13)$ & 31.2 & (2.9) & -0.44 & $(0.04)$ & 0.51 & $(0.03)$ & -36.1 & (3.3) \\
\hline & Mexiko & 66 & (4.3) & 2.70 & $(0.16)$ & 2.2 & $(0.3)$ & -1.02 & $(0.09)$ & 0.37 & $(0.02)$ & -163.7 & (15.6) \\
\hline & Niederlande & 35 & $(7.2)$ & 1.96 & $(0.19)$ & 9.2 & (1.7) & -0.65 & $(0.07)$ & 0.51 & $(0.04)$ & -77.6 & (13.8) \\
\hline & Neuseeland & -2 & (4.4) & 1.17 & $(0.13)$ & 4.4 & (3.1) & 0.00 & $(0.05)$ & 0.85 & $(0.09)$ & -12.2 & $(8.4)$ \\
\hline & Norwegen & 29 & (6.6) & 1.99 & $(0.15)$ & 8.5 & (1.5) & -0.52 & $(0.08)$ & 0.50 & $(0.04)$ & -82.0 & $(11.0)$ \\
\hline & Polen & c & c & $\mathrm{c}$ & $\mathrm{c}$ & c & c & c & c & c & $\mathrm{C}$ & $\mathrm{C}$ & $\mathrm{c}$ \\
\hline & Portugal & 39 & $(7.8)$ & 1.88 & $(0.19)$ & 5.7 & $(1.2)$ & -0.47 & $(0.08)$ & 0.53 & $(0.05)$ & -76.9 & (15.4) \\
\hline & Slowak. Rep. & -6 & $(18.8)$ & 1.24 & $(0.35)$ & 0.2 & $(0.3)$ & 0.05 & $(0.20)$ & 0.81 & $(0.21)$ & -24.2 & (34.5) \\
\hline & Slowenien & 26 & (4.6) & 1.93 & $(0.16)$ & 7.4 & $(1.2)$ & -0.58 & $(0.06)$ & 0.52 & $(0.04)$ & -78.5 & $(12.2)$ \\
\hline & Spanien & 36 & (4.3) & 2.00 & $(0.12)$ & 9.0 & $(1.2)$ & -0.62 & $(0.05)$ & 0.50 & $(0.03)$ & -82.4 & (9.3) \\
\hline & Schweden & 40 & (4.9) & 2.11 & $(0.16)$ & 13.9 & (2.1) & -0.66 & $(0.06)$ & 0.47 & $(0.04)$ & -81.9 & (9.6) \\
\hline & Schweiz & 42 & (3.0) & 2.30 & $(0.10)$ & 23.9 & (1.5) & -0.70 & $(0.03)$ & 0.43 & $(0.02)$ & -75.1 & (4.6) \\
\hline & Türkei & 5 & $(27.3)$ & 1.48 & $(0.46)$ & 0.4 & $(0.5)$ & 0.03 & $(0.29)$ & 0.69 & $(0.20)$ & -46.9 & $(45.5)$ \\
\hline & Ver. Königreich & 6 & $(6.2)$ & 1.30 & $(0.13)$ & 3.7 & (1.6) & -0.10 & $(0.08)$ & 0.77 & $(0.08)$ & -25.6 & $(10.7)$ \\
\hline & Ver. Staaten & -15 & (4.9) & 1.26 & $(0.12)$ & 5.3 & (2.3) & -0.14 & $(0.07)$ & 0.79 & $(0.08)$ & -19.6 & (8.5) \\
\hline & OECD-Durchschnitt & 21 & (1.5) & 1.71 & $(0.03)$ & 7.0 & $(0.3)$ & -0.39 & $(0.02)$ & 0.67 & $(0.03)$ & -56.1 & (3.0) \\
\hline \multirow{31}{*}{ 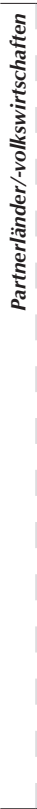 } & Albanien & $\mathrm{m}$ & $m$ & C & c & c & $\mathrm{c}$ & c & c & $\mathrm{c}$ & $\mathrm{C}$ & c & $\mathrm{c}$ \\
\hline & Argentinien & 17 & (6.3) & 1.54 & $(0.23)$ & 2.1 & (0.8) & -0.50 & $(0.08)$ & 0.65 & $(0.10)$ & -51.0 & $(21.2)$ \\
\hline & Brasilien & 63 & (11.7) & 2.27 & $(0.29)$ & 0.9 & $(0.3)$ & -0.71 & $(0.16)$ & 0.44 & $(0.06)$ & -124.5 & $(28.2)$ \\
\hline & Bulgarien & c & C & $\mathrm{C}$ & c & c & c & c & c & c & c & c & c \\
\hline & Kolumbien & 67 & $(13.0)$ & 2.44 & $(0.57)$ & 0.5 & $(0.3)$ & -1.05 & $(0.20)$ & 0.41 & $(0.09)$ & -143.3 & $(56.5)$ \\
\hline & Costa Rica & 14 & (6.7) & 1.79 & $(0.26)$ & 4.2 & (1.6) & -0.42 & $(0.13)$ & 0.56 & $(0.10)$ & -71.4 & $(22.5)$ \\
\hline & Kroatien & 9 & (4.8) & 1.23 & $(0.11)$ & 2.7 & (1.4) & -0.22 & $(0.06)$ & 0.81 & $(0.08)$ & -20.0 & (9.3) \\
\hline & Zypern* & 14 & (4.6) & 1.46 & $(0.12)$ & 3.8 & (1.0) & -0.22 & $(0.05)$ & 0.68 & $(0.05)$ & -40.7 & $(10.1)$ \\
\hline & Hongkong (China) & -11 & (3.8) & 1.16 & $(0.09)$ & 5.2 & (3.0) & -0.08 & $(0.05)$ & 0.86 & $(0.08)$ & -9.8 & (5.8) \\
\hline & Indonesien & c & c & c & c & c & C & C & c & c & c & c & c \\
\hline & Jordanien & -15 & (3.9) & 0.69 & $(0.10)$ & -4.4 & (1.6) & 0.29 & $(0.06)$ & 1.47 & $(0.25)$ & 28.2 & (9.8) \\
\hline & Kasachstan & 2 & (5.3) & 1.13 & $(0.11)$ & 2.0 & (1.7) & -0.09 & $(0.08)$ & 0.89 & $(0.08)$ & -10.6 & (8.5) \\
\hline & Lettland & 10 & $(7.7)$ & 1.16 & $(0.21)$ & 0.7 & $(1.0)$ & -0.07 & $(0.09)$ & 0.87 & $(0.14)$ & -14.8 & $(19.7)$ \\
\hline & Liechtenstein & 40 & (11.9) & 2.20 & $(0.54)$ & 28.3 & (9.6) & -0.55 & $(0.14)$ & 0.46 & $(0.12)$ & -56.7 & $(18.7)$ \\
\hline & Litauen & 2 & (9.3) & 0.99 & $(0.30)$ & 0.0 & $(0.5)$ & -0.01 & $(0.11)$ & 1.06 & $(0.38)$ & 1.0 & (29.6) \\
\hline & Macau (China) & -22 & (2.8) & 0.79 & $(0.05)$ & -16.0 & (4.0) & 0.17 & $(0.03)$ & 1.27 & $(0.07)$ & 8.6 & (2.1) \\
\hline & Malaysia & 2 & (9.9) & 1.27 & $(0.27)$ & 0.5 & $(0.5)$ & -0.28 & $(0.12)$ & 0.79 & $(0.16)$ & -26.6 & $(26.2)$ \\
\hline & Montenegro & -16 & (6.0) & 0.67 & $(0.14)$ & -2.0 & $(0.8)$ & 0.26 & $(0.08)$ & 1.51 & $(0.33)$ & 31.6 & (13.7) \\
\hline & Peru & 86 & (20.8) & 2.89 & $(0.41)$ & 1.0 & $(0.3)$ & -0.97 & $(0.29)$ & 0.35 & $(0.05)$ & -185.8 & $(40.2)$ \\
\hline & Katar & -88 & (1.6) & 0.30 & $(0.01)$ & -56.8 & (1.7) & 1.02 & $(0.02)$ & 3.30 & $(0.14)$ & 52.5 & (1.6) \\
\hline & Rumänien & c & c & c & c & c & c & c & c & c & c & c & c \\
\hline & Russ. Föderation & 19 & (4.4) & 1.32 & $(0.11)$ & 3.3 & (1.0) & -0.26 & $(0.05)$ & 0.76 & $(0.06)$ & -27.3 & (9.0) \\
\hline & Serbien & -14 & (5.6) & 0.85 & $(0.12)$ & -1.3 & $(1.0)$ & 0.16 & $(0.07)$ & 1.18 & $(0.15)$ & 13.8 & $(10.9)$ \\
\hline & Shanghai (China) & 98 & (14.7) & 3.06 & $(0.28)$ & 1.9 & $(0.4)$ & -1.33 & $(0.17)$ & 0.33 & $(0.03)$ & -200.5 & (26.8) \\
\hline & Singapur & -7 & (4.6) & 0.67 & $(0.05)$ & -6.4 & (1.1) & 0.26 & $(0.04)$ & 1.50 & $(0.11)$ & 28.8 & (4.6) \\
\hline & Chinesisch Taipeh & 1 & $(20.1)$ & 1.49 & $(0.39)$ & 0.2 & $(0.2)$ & -0.28 & $(0.21)$ & 0.68 & $(0.26)$ & -48.4 & $(39.0)$ \\
\hline & Thailand & -26 & (53.6) & 1.78 & $(0.68)$ & 0.5 & $(0.7)$ & -0.17 & $(0.57)$ & 0.58 & $(0.24)$ & -77.4 & $(67.0)$ \\
\hline & Tunesien & c & c & c & c & c & c & $\mathrm{c}$ & c & c & c & c & c \\
\hline & Ver. Arab. Emirate & -63 & (3.1) & 0.41 & $(0.03)$ & -48.1 & (3.8) & 0.81 & $(0.04)$ & 2.45 & $(0.16)$ & 39.5 & (2.7) \\
\hline & Uruguay & c & c & c & c & c & c & c & c & c & c & c & c \\
\hline & Vietnam & c & c & c & c & c & c & c & c & c & c & c & c \\
\hline
\end{tabular}

Anmerkung: Diese Tabelle wurde nur für Schüler berechnet, für die Daten zum PISA-Index des wirtschaftlichen, sozialen und kulturellen Status vorliegen. Statistisch signifikante Werte sind durch Fettdruck gekennzeichnet (vgl. Anhang A3).

Schüler mit Migrationshintergrund sind Schüler, deren Eltern in einem anderen Land/einer anderen Volkswirtschaft als dem getesteten Land/der getesteten Volkswirtschaft geboren sind.

*Vgl. die Anmerkungen am Beginn dieses Anhangs.

StatLink inils http://dx.doi.org/10.1787/888932964927 
[Teil 1/3]

Von 2003 bis 2012 festzustellende Veränderung des Zusammenhangs zwischen Mathematikleistungen und Migrationshintergrund

Tabelle II.3.4b Die Ergebnisse basieren auf Schülerangaben

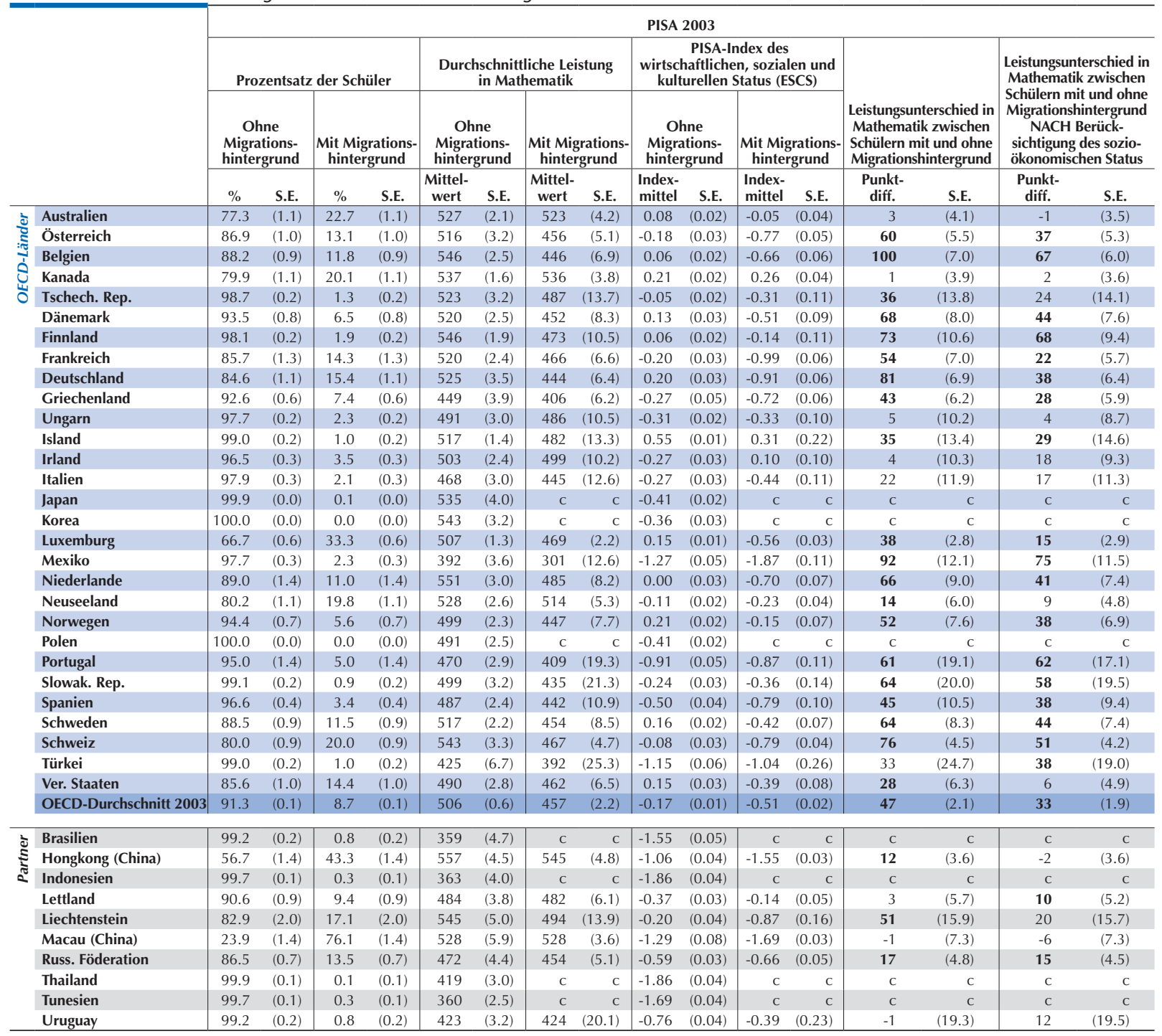

Anmerkung: Diese Tabelle wurde nur für Schüler berechnet, für die Daten zum PISA-Index des wirtschaftlichen, sozialen und kulturellen Status vorliegen. Statistisch signifikante Werte sind durch Fettdruck gekennzeichnet (vgl. Anhang A3).

Schüler mit Migrationshintergrund sind Schüler, deren Eltern in einem anderen Land/einer anderen Volkswirtschaft als dem getesteten Land/der getesteten Volkswirtschaft geboren sind.

Um die Vergleichbarkeit im Zeitverlauf zu gewährleisten, wurden die Werte von PISA 2003 auf dem PISA-Index des wirtschaftlichen, sozialen und kulturellen Status entsprechend der Indexskala von PISA 2012 umskaliert. Die in dieser Abbildung dargestellten Ergebnisse aus PISA 2003 können daher von denen abweichen, die in Lernen für die Welt von morgen: Erste Ergebnisse von PISA 2003 (OECD, 2004) wiedergegeben sind (vgl. Anhang A5 wegen näherer Einzelheiten).

Angegeben sind nur Länder und Volkswirtschaft3en mit vergleichbaren Daten aus PISA 2003 und PISA 2012

StatLink त्ताIs http://dx.doi.org/10.1787/888932964927 
[Teil 2/3]

Von 2003 bis 2012 festzustellende Veränderung des Zusammenhangs zwischen Mathematikleistungen und Migrationshintergrund

Tabelle II.3.4b Die Ergebnisse basieren auf Schülerangaben

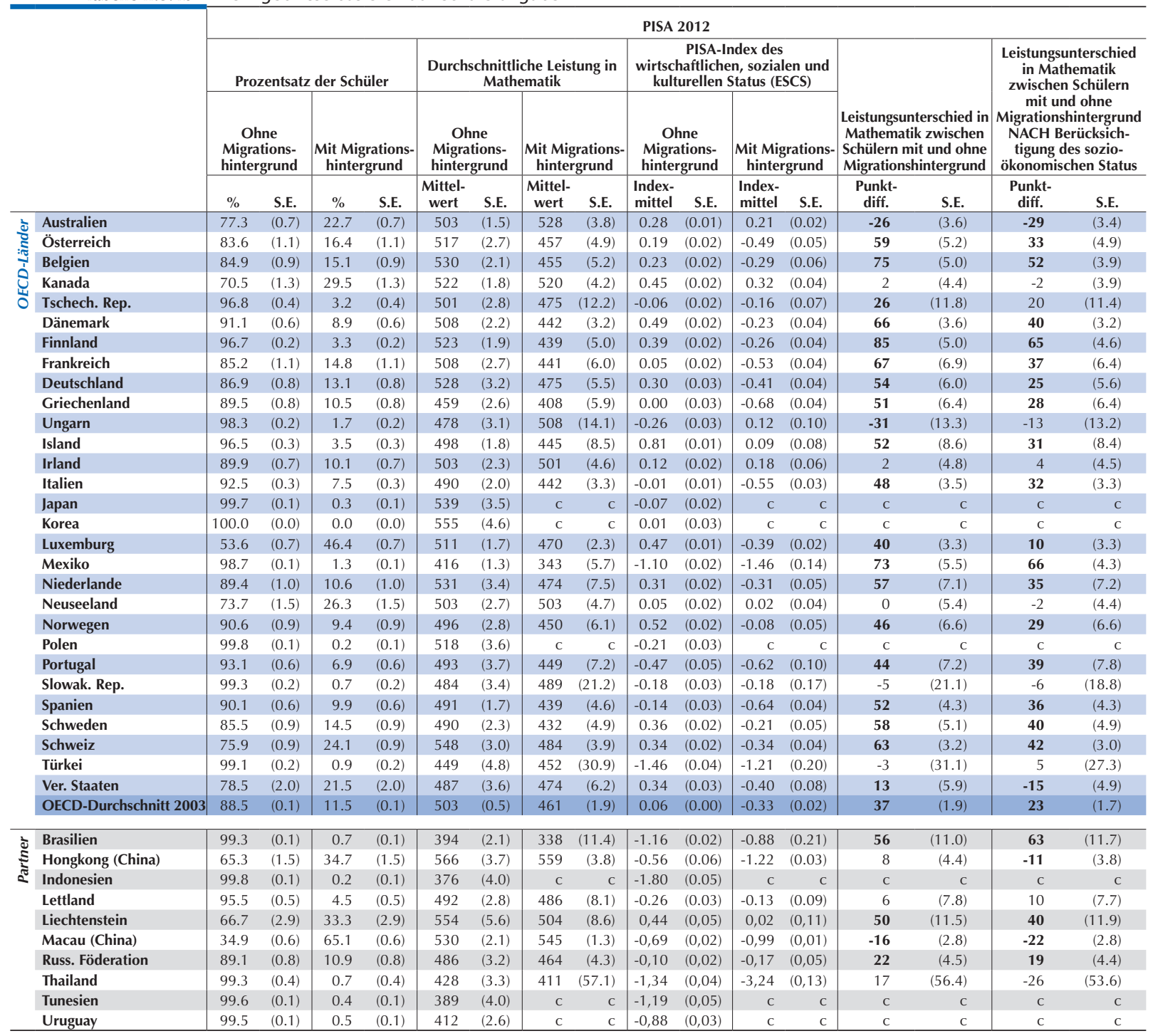

Anmerkung: Diese Tabelle wurde nur für Schüler berechnet, für die Daten zum PISA-Index des wirtschaftlichen, sozialen und kulturellen Status vorliegen. Statistisch signifikante Werte sind durch Fettdruck gekennzeichnet (vgl. Anhang A3).

Schüler mit Migrationshintergrund sind Schüler, deren Eltern in einem anderen Land/einer anderen Volkswirtschaft als dem getesteten Land/der getesteten Volkswirtschaft geboren sind.

Um dieVergleichbarkeit im Zeitverlauf zu gewährleisten, wurden die Werte von PISA 2003 auf dem PISA-Index des wirtschaftlichen, sozialen und kulturellen Status entsprechend der Indexskala von PISA 2012 umskaliert. Die in dieser Abbildung dargestellten Ergebnisse aus PISA 2003 können daher von denen abweichen, die in Lernen für die Welt von morgen: Erste Ergebnisse von PISA 2003 (OECD, 2004) wiedergegeben sind (vgl. Anhang A5 wegen näherer Einzelheiten).

Angegeben sind nur Länder und Volkswirtschaften mit vergleichbaren Daten aus PISA 2003 und PISA 2012

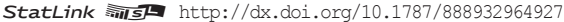


[Teil 3/3]

Von 2003 bis 2012 festzustellende Veränderung des Zusammenhangs zwischen Mathematikleistungen und Migrationshintergrund

Tabelle II.3.4b Die Ergebnisse basieren auf Schülerangaben

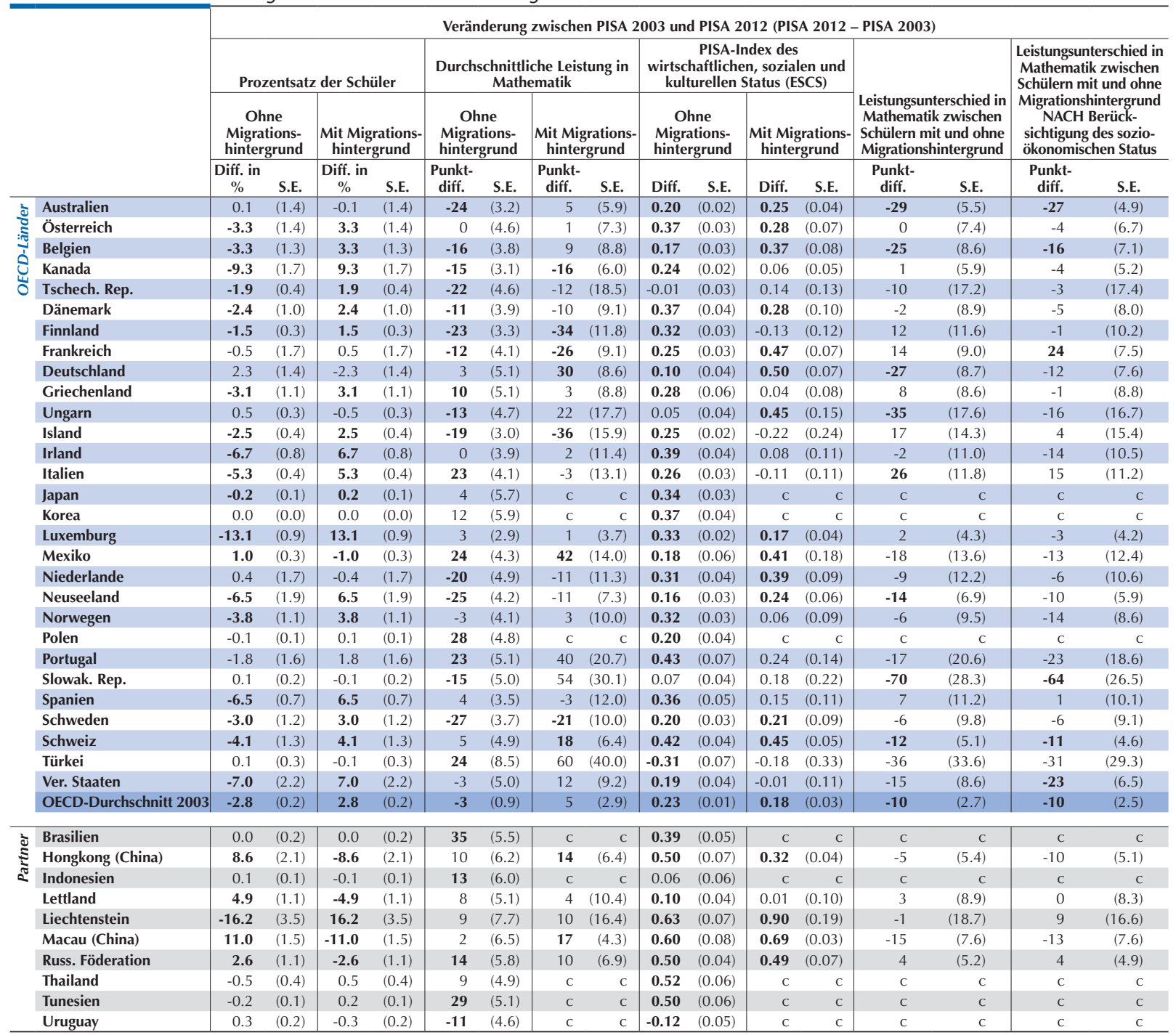

Anmerkung: Diese Tabelle wurde nur für Schüler berechnet, für die Daten zum PISA-Index des wirtschaftlichen, sozialen und kulturellen Status vorliegen. Statistisch signifikante Werte sind durch Fettdruck gekennzeichnet (vgl. Anhang A3).

Schüler mit Migrationshintergrund sind Schüler, deren Eltern in einem anderen Land/einer anderen Volkswirtschaft als dem getesteten Land/der getesteten Volkswirtschaft geboren sind.

Um die Vergleichbarkeit im Zeitverlauf zu gewährleisten, wurden die Werte von PISA 2003 auf dem PISA-Index des wirtschaftlichen, sozialen und kulturellen Status entsprechend der Indexskala von PISA 2012 umskaliert. Die in dieser Abbildung dargestellten Ergebnisse aus PISA 2003 können daher von denen abweichen, die in Lernen für die Welt von morgen: Erste Ergebnisse von PISA 2003 (OECD, 2004) wiedergegeben sind (vgl. Anhang A5 wegen näherer Einzelheiten).

Angegeben sind nur Länder und Volkswirtschaften mit vergleichbaren Daten aus PISA 2003 und PISA 2012.

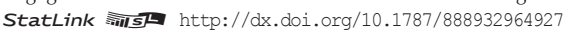


[Teil 1/4]

Mathematikleistungen, Migrationshintergrund und zu Hause gesprochene Sprache Tabelle II.3.5 Die Ergebnisse basieren auf Schülerangaben

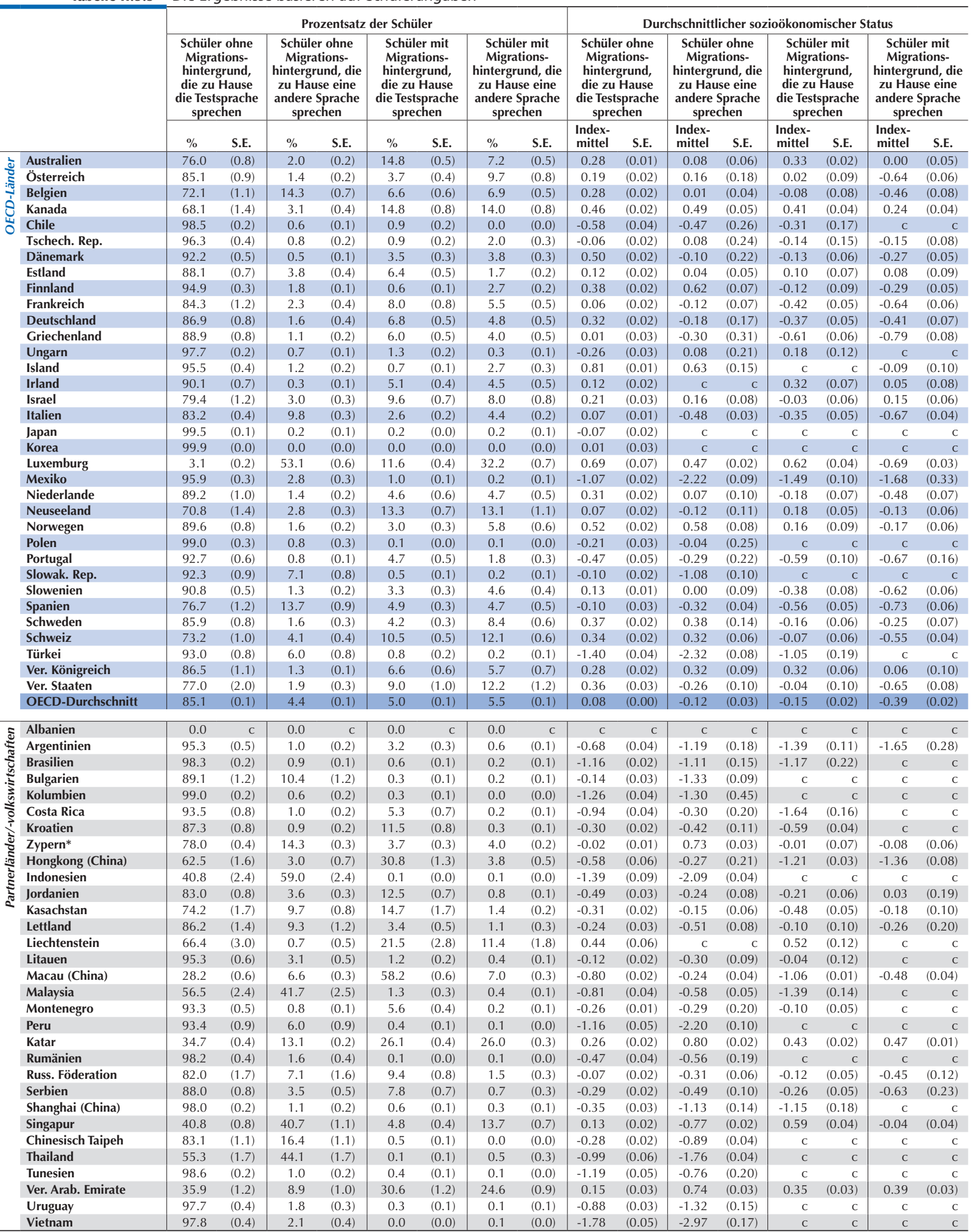

Anmerkung: Diese Tabelle wurde nur für Schüler berechnet, für die Daten zum PISA-Index des wirtschaftlichen, sozialen und kulturellen Status vorliegen. Statistisch signifikante Werte sind durch Fettdruck gekennzeichnet (vgl. Anhang A3).

Die Prozentsätze der Schüler ohne Migrationshintergrund und der Schüler mit Migrationshintergrund, die zu Hause die Testsprache sprechen bzw. zu Hause nicht die Testsprache sprechen, werden für die gesamte Schülerpopulation berechnet. Die Prozentsätze der Schüler der 1. und der 2. Generation, die zu Hause die Testsprache sprechen bzw. nicht die Testsprache sprechen, werden für die gesamte Schülerpopulation mit Migrationshintergrund berechnet.

*Vgl. die Anmerkungen am Beginn dieses Anhangs.

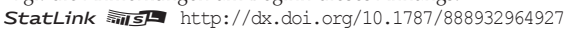


[Teil 2/4]

Mathematikleistungen, Migrationshintergrund und zu Hause gesprochene Sprache Tabelle II.3.5 Die Ergebnisse basieren auf Schülerangaben

\begin{tabular}{|c|c|c|c|c|c|c|c|c|c|c|c|c|c|c|}
\hline & \multicolumn{8}{|c|}{ Durchschnittliche Leistung in Mathematik } & \multicolumn{6}{|c|}{ Leistungsunterschiede vor Berücksichtigung des ESCS } \\
\hline & \multicolumn{2}{|c|}{$\begin{array}{l}\text { Schüler ohne } \\
\text { Migrations- } \\
\text { hintergrund, } \\
\text { die zu Hause } \\
\text { die Testsprache } \\
\text { sprechen }\end{array}$} & \multicolumn{2}{|c|}{\begin{tabular}{|c|} 
Schüler ohne \\
Migrations- \\
hintergrund, die zu \\
Hause eine andere \\
Sprache sprechen
\end{tabular}} & \multicolumn{2}{|c|}{$\begin{array}{l}\text { Schüler mit } \\
\text { Migrations- } \\
\text { hintergrund, } \\
\text { die zu Hause } \\
\text { die Testsprache } \\
\text { sprechen }\end{array}$} & \multicolumn{2}{|c|}{$\begin{array}{c}\text { Schüler mit } \\
\text { Migrations- } \\
\text { hintergrund, die zu } \\
\text { Hause eine andere } \\
\text { Sprache sprechen }\end{array}$} & \multicolumn{2}{|c|}{$\begin{array}{c}\text { Leistungs- } \\
\text { unterschied } \\
\text { zwischen Schülern } \\
\text { ohne Migrations- } \\
\text { hintergrund, } \\
\text { nach zu Hause } \\
\text { gesprochener } \\
\text { Sprache }\end{array}$} & \multicolumn{2}{|c|}{$\begin{array}{c}\text { Leistungs- } \\
\text { unterschied } \\
\text { zwischen Schülern } \\
\text { mit Migrations- } \\
\text { hintergrund, } \\
\text { nach zu Hause } \\
\text { gesprochener } \\
\text { Sprache }\end{array}$} & \multicolumn{2}{|c|}{\begin{tabular}{|c} 
Leistungs- \\
unterschied \\
zwischen Schülern \\
mit Migrations- \\
hintergrund, die \\
zu Hause nicht \\
die Testsprache \\
sprechen, und \\
Schülern ohne \\
Migrations- \\
hintergrund, \\
die zu Hause \\
die Testsprache \\
sprechen
\end{tabular}} \\
\hline & $\begin{array}{c}\text { Mittel- } \\
\text { wert }\end{array}$ & S.E. & $\begin{array}{c}\text { Mittel- } \\
\text { wert }\end{array}$ & S.E. & $\begin{array}{c}\text { Mittel- } \\
\text { wert }\end{array}$ & S.E. & $\begin{array}{c}\text { Mittel- } \\
\text { wert }\end{array}$ & S.E. & $\begin{array}{l}\text { Punkt- } \\
\text { diff. }\end{array}$ & S.E. & $\begin{array}{c}\text { Punkt- } \\
\text { diff. }\end{array}$ & S.E. & $\begin{array}{l}\text { Punkt- } \\
\text { diff. }\end{array}$ & S.E. \\
\hline Australien & 505 & $(1.4)$ & 466 & $(8.8)$ & 528 & $(3.8)$ & 541 & $(6.0)$ & 39 & (8.8) & -13 & (5.5) & -36 & (5.9) \\
\hline : Österreich & 520 & $(2.7)$ & 487 & $(14.5)$ & 498 & (9.4) & 457 & $(6.0)$ & 33 & (14.5) & 41 & (10.6) & 63 & (6.3) \\
\hline Belgien & 535 & $(2.1)$ & 513 & (5.3) & 469 & (7.4) & 448 & $(7.2)$ & 23 & $(5.3)$ & 21 & (10.6) & 88 & (7.4) \\
\hline O Kanada & 523 & $(1.8)$ & 521 & (7.6) & 518 & (5.0) & 530 & (4.4) & 3 & (7.8) & -12 & $(4.3)$ & -7 & $(4.7)$ \\
\hline Chile & 424 & $(3.0)$ & 404 & $(19.0)$ & 417 & (13.8) & C & C & 20 & (18.6) & c & C & c & C \\
\hline Tschech. Rep. & 503 & $(2.7)$ & 461 & $(30.1)$ & 469 & $(22.2)$ & 490 & $(16.3)$ & 42 & (30.1) & -21 & $(27.6)$ & 13 & (15.9) \\
\hline Dänemark & 509 & $(2.3)$ & 456 & (12.8) & 450 & (5.4) & 453 & (3.6) & 53 & (12.8) & -3 & (6.6) & 56 & $(4.2)$ \\
\hline Estland & 526 & $(2.1)$ & 509 & (5.5) & 497 & (6.8) & 488 & (11.5) & 17 & $(5.7)$ & 9 & $(12.7)$ & 38 & (11.6) \\
\hline Finnland & 524 & (1.9) & 518 & (7.6) & 460 & (9.5) & 434 & (5.9) & 5 & (7.9) & 25 & $(11.5)$ & 90 & $(5.7)$ \\
\hline Frankreich & 511 & $(2.8)$ & 465 & $(17.1)$ & 450 & (7.5) & 440 & (8.6) & 46 & $(17.0)$ & 11 & $(9.9)$ & 71 & (9.3) \\
\hline Deutschland & 530 & $(3.2)$ & 504 & $(24.4)$ & 493 & (6.7) & 470 & (8.2) & 26 & $(24.2)$ & 23 & $(10.3)$ & 60 & (8.6) \\
\hline Griechenland & 461 & $(2.6)$ & 408 & (19.7) & 416 & (6.8) & 401 & (8.7) & 53 & (19.8) & 15 & (10.3) & 60 & (9.3) \\
\hline Ungarn & 478 & $(3.1)$ & 454 & $(20.8)$ & 503 & $(16.4)$ & C & C & 24 & $(21.0)$ & C & c & C & $\mathrm{c}$ \\
\hline Island & 499 & (1.7) & 456 & (18.1) & C & C & 443 & (9.9) & 43 & (18.0) & C & c & 56 & (10.0) \\
\hline Irland & 504 & $(2.3)$ & c & C & 508 & $(6.1)$ & 499 & (6.9) & C & C & 9 & (8.9) & 5 & (6.6) \\
\hline Israel & 473 & (4.4) & 453 & (12.1) & 476 & (7.4) & 490 & (7.4) & 20 & (10.3) & -14 & (7.6) & -16 & $(7.3)$ \\
\hline Italien & 499 & $(2.1)$ & 463 & (3.1) & 461 & $(6.2)$ & 442 & (4.1) & 36 & (3.0) & 19 & (7.5) & 57 & $(4.7)$ \\
\hline Japan & 540 & (3.6) & C & C & C & $\mathrm{C}$ & c & c & C & C & C & C & C & C \\
\hline Korea & 555 & $(4.5)$ & c & c & c & C & c & c & c & c & C & C & c & c \\
\hline Luxemburg & 527 & $(7.8)$ & 514 & (1.8) & 536 & (3.9) & 461 & (3.0) & 12 & (8.4) & 75 & (4.7) & 65 & (8.1) \\
\hline Mexiko & 418 & (1.3) & 371 & (4.4) & 342 & (5.3) & 335 & (13.7) & 47 & (4.4) & 7 & (13.4) & 83 & (13.6) \\
\hline Niederlande & 533 & (3.4) & 502 & (13.7) & 490 & (8.7) & 473 & $(9.2)$ & 32 & (13.6) & 16 & (10.3) & 60 & (8.4) \\
\hline Neuseeland & 508 & (2.5) & 421 & (8.7) & 516 & (4.9) & 492 & (8.0) & 87 & $(8.8)$ & 24 & $(9.3)$ & 16 & (8.5) \\
\hline Norwegen & 497 & (2.8) & 482 & (12.1) & 464 & (7.7) & 451 & (6.8) & 15 & (12.3) & 13 & (7.9) & 46 & (7.3) \\
\hline Polen & 519 & (3.6) & 514 & $(20.0)$ & C & $\mathrm{c}$ & c & C & 5 & (18.8) & c & c & c & $\mathrm{C}$ \\
\hline Portugal & 494 & (3.7) & 485 & (19.4) & 445 & (7.4) & 471 & (11.6) & 9 & (19.4) & -26 & (12.1) & 23 & (11.2) \\
\hline Slowak. Rep. & 493 & (3.3) & 394 & (11.5) & C & C & C & $\mathrm{C}$ & 99 & (12.3) & $\mathrm{C}$ & $\mathrm{C}$ & c & c \\
\hline Slowenien & 508 & (1.1) & 448 & (10.5) & 479 & (7.1) & 449 & (6.6) & 61 & (10.5) & 30 & $(10.5)$ & 59 & (6.7) \\
\hline Spanien & 493 & (1.8) & 484 & (2.9) & 442 & (5.5) & 439 & (6.2) & 9 & (3.0) & 3 & (7.0) & 54 & (6.0) \\
\hline Schweden & 494 & $(2.3)$ & 469 & $(23.3)$ & 448 & (6.9) & 438 & (6.7) & 25 & (23.4) & 10 & $(9.2)$ & 56 & (7.1) \\
\hline Schweiz & 551 & $(3.2)$ & 527 & (6.3) & 501 & $(4.7)$ & 478 & (5.0) & 24 & $(6.6)$ & 23 & (5.9) & 73 & (4.4) \\
\hline Türkei & 452 & $(4.9)$ & 400 & $(14.2)$ & 471 & (33.9) & C & $\mathrm{C}$ & 52 & $(14.7)$ & C & c & C & c \\
\hline Ver. Königreich & 500 & $(2.8)$ & 480 & $(10.3)$ & 485 & $(8.2)$ & 501 & $(11.3)$ & 20 & (10.7) & -16 & (10.3) & -1 & (11.3) \\
\hline Ver. Staaten & 489 & $(3.7)$ & 443 & $(8.8)$ & 494 & $(8.5)$ & 461 & $(5.8)$ & 45 & $(8.7)$ & 33 & $(7.6)$ & 28 & $(5.7)$ \\
\hline OECD-Durchschnitt & 503 & $(0.5)$ & 467 & (2.6) & 473 & (2.0) & 462 & (1.6) & 33 & (2.6) & 12 & $(2.1)$ & 43 & (1.6) \\
\hline
\end{tabular}

\begin{tabular}{|c|c|c|c|c|c|c|c|c|c|c|c|c|c|c|}
\hline Albanien & C & C & c & C & C & C & C & C & C & C & C & c & C & C \\
\hline Argentinien & 393 & (3.4) & 348 & (16.3) & 358 & (6.4) & 336 & (26.1) & 45 & $(16.3)$ & 23 & (25.9) & 57 & $(25.2)$ \\
\hline Brasilien & 395 & $(2.1)$ & 397 & (11.0) & 322 & $(11.8)$ & c & C & -2 & (10.8) & $\mathrm{C}$ & $\mathrm{c}$ & c & c \\
\hline Bulgarien & 451 & (3.9) & 374 & $(7.1)$ & c & c & C & c & 77 & (7.5) & c & c & c & c \\
\hline Kolumbien & 379 & (2.9) & 343 & (16.3) & c & c & c & c & 36 & $(15.8)$ & c & c & c & c \\
\hline Costa Rica & 410 & (3.0) & 429 & (15.9) & 377 & (9.8) & c & c & -19 & $(16.0)$ & c & c & c & c \\
\hline \ִ Kroatien & 475 & (3.6) & 470 & (19.3) & 458 & (5.4) & c & c & 4 & (19.1) & c & c & c & c \\
\hline Zypern* & 436 & (1.3) & 497 & (3.4) & 437 & (6.5) & 423 & (6.6) & -61 & (3.6) & 14 & (8.7) & 13 & (6.8) \\
\hline Hongkong (China) & 569 & (3.8) & 529 & (12.7) & 566 & (3.5) & 514 & (10.7) & 41 & (12.9) & 52 & (10.4) & 55 & (11.7) \\
\hline Indonesien & 378 & (6.5) & 374 & (4.1) & c & c & c & c & 4 & (6.8) & c & c & C & c \\
\hline Jordanien & 388 & (2.8) & 387 & (6.8) & 411 & (5.5) & 404 & $(15.6)$ & 1 & $(7.0)$ & 7 & $(16.8)$ & -16 & (15.8) \\
\hline Kasachstan & 432 & (3.0) & 444 & (7.5) & 426 & (6.2) & 433 & $(10.0)$ & -12 & (6.9) & -7 & (11.7) & -1 & (9.8) \\
\hline Lettland & 494 & (3.0) & 476 & (6.0) & 488 & (8.6) & 480 & $(20.4)$ & 18 & (6.5) & 8 & (21.9) & 14 & (20.4) \\
\hline Liechtenstein & 559 & (5.7) & c & c & 524 & (12.0) & C & c & c & c & c & c & c & c \\
\hline Litauen & 484 & (2.8) & 440 & (11.1) & 490 & (10.1) & c & c & 44 & (11.4) & c & c & c & c \\
\hline Macau (China) & 528 & (2.4) & 548 & (4.8) & 548 & (1.6) & 548 & (4.8) & -20 & $(5.3)$ & 1 & (5.3) & -20 & (5.2) \\
\hline Malaysia & 410 & (3.2) & 441 & (4.6) & 408 & (8.5) & c & c & -31 & $(4.8)$ & c & c & c & c \\
\hline Montenegro & 410 & $(1.2)$ & 389 & (15.6) & 433 & (6.0) & c & c & 21 & (15.8) & c & c & c & c \\
\hline Peru & 376 & (3.5) & 300 & (8.1) & c & c & c & c & 76 & $(7.9)$ & c & c & c & c \\
\hline Katar & 323 & (1.5) & 370 & (2.6) & 408 & (2.4) & 444 & (1.8) & -46 & (3.2) & -35 & (3.3) & -120 & (2.4) \\
\hline Rumänien & 446 & (3.8) & 415 & (13.3) & c & c & c & c & 30 & (12.9) & c & c & c & c \\
\hline Russ. Föderation & 487 & (3.4) & 472 & (6.4) & 471 & (4.1) & 426 & (12.6) & 15 & $(6.4)$ & 45 & $(12.0)$ & 61 & $(12.7)$ \\
\hline Serbien & 450 & (3.4) & 442 & (6.8) & 463 & (6.8) & 478 & (25.4) & 7 & (7.0) & -15 & (24.9) & -28 & (25.1) \\
\hline Shanghai (China) & 616 & (3.1) & 502 & (14.9) & 493 & $(21.2)$ & c & c & 115 & $(14.2)$ & c & c & c & c \\
\hline Singapur & 602 & $(2.2)$ & 549 & (2.1) & 609 & (6.3) & 597 & (4.4) & 53 & (3.2) & 12 & (7.4) & 5 & (5.1) \\
\hline Chinesisch Taipeh & 577 & (3.3) & 517 & (5.6) & C & $\mathrm{C}$ & C & C & 60 & (5.8) & C & $\mathrm{C}$ & c & C \\
\hline Thailand & 434 & $(4.7)$ & 422 & (3.8) & c & c & c & c & 12 & (5.6) & c & c & c & c \\
\hline Tunesien & 389 & $(4.0)$ & 378 & $(15.8)$ & c & c & c & c & 12 & (15.5) & c & c & c & c \\
\hline Ver. Arab. Emirate & 395 & (2.6) & 424 & (5.6) & 458 & (3.1) & 479 & (3.8) & -28 & (5.3) & -20 & (4.4) & -83 & $(4.4)$ \\
\hline Uruguay & 413 & (2.6) & 396 & (12.4) & c & c & c & c & 17 & (12.0) & c & c & c & c \\
\hline Vietnam & 513 & $(4.8)$ & 461 & (12.0) & c & c & c & C & 52 & (11.4) & C & c & c & c \\
\hline
\end{tabular}

Anmerkung: Diese Tabelle wurde nur für Schüler berechnet, für die Daten zum PISA-Index des wirtschaftlichen, sozialen und kulturellen Status vorliegen. Statistisch signifikante Werte sind durch Fettdruck gekennzeichnet (vgl. Anhang A3).

Die Prozentsätze der Schüler ohne Migrationshintergrund und der Schüler mit Migrationshintergrund, die zu Hause die Testsprache sprechen bzw. zu Hause nicht die Testsprache sprechen, werden für die gesamte Schülerpopulation berechnet. Die Prozentsätze der Schüler der 1. und der 2. Generation, die zu Hause die Testsprache sprechen bzw. nicht die Testsprache sprechen, werden für die gesamte Schülerpopulation mit Migrationshintergrund berechnet.

*Vgl. die Anmerkungen am Beginn dieses Anhangs.

StatLink 需实 http://dx.doi.org/10.1787/888932964927 
[Teil 3/4]

Mathematikleistungen, Migrationshintergrund und zu Hause gesprochene Sprache Tabelle II.3.5 Die Ergebnisse basieren auf Schülerangaben

\begin{tabular}{|c|c|c|c|c|c|c|c|c|c|c|c|c|}
\hline & \multicolumn{4}{|c|}{ Leistungsunterschiede nach Berücksichtigung des ESCS } & \multirow{2}{*}{\multicolumn{2}{|c|}{ 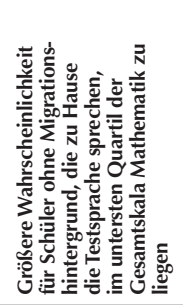 }} & \multirow{2}{*}{\multicolumn{2}{|c|}{ 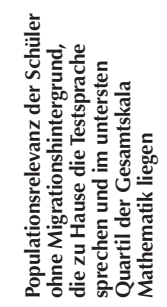 }} & \multirow{2}{*}{\multicolumn{2}{|c|}{ 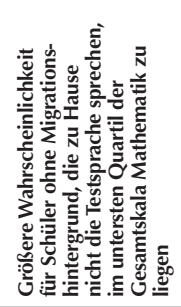 }} & \multirow{2}{*}{\multicolumn{2}{|c|}{ 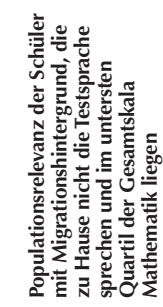 }} \\
\hline & $\begin{array}{c}\text { Leistungs- } \\
\text { unterschied } \\
\text { zwischen Schülern } \\
\text { ohne Migrations- } \\
\text { hintergrund, } \\
\text { nach zu Hause } \\
\text { gesprochener } \\
\text { Sprache }\end{array}$ & $\begin{array}{c}\text { Leistungs- } \\
\text { unterschied } \\
\text { zwischen Schülern } \\
\text { mit Migrations- } \\
\text { hintergrund, } \\
\text { nach zu Hause } \\
\text { gesprochener } \\
\text { Sprache }\end{array}$ & \multicolumn{2}{|c|}{\begin{tabular}{|c|} 
Leistungsunter- \\
schied zwischen \\
Schülern mit \\
Migrationshinter- \\
grund, die zu Hause \\
nicht die Testsprache \\
sprechen, und \\
Schülern ohne \\
Migrationshinter- \\
grund, die zu Hause \\
die Testsprache \\
sprechen
\end{tabular}} & & & & & & & & \\
\hline & Punktdiff. $\quad$ S.E. & Punktdiff. $\quad$ S.E. & Punktdiff. & S.E. & Quotient & S.E. & $\%$ & S.E. & Quotient & S.E. & $\%$ & S.E. \\
\hline ¿ Australien & $(7.7)$ & $(5.1)$ & -48 & $(5.3)$ & 1.17 & $(0.06)$ & 11.4 & (3.6) & 1.75 & $(0.18)$ & 1.5 & $(0.4)$ \\
\hline : Österreich & (13.4) & $(8.7)$ & 31 & $(6.1)$ & 0.52 & $(0.04)$ & -69.6 & (9.5) & 1.33 & $(0.35)$ & 0.5 & $(0.5)$ \\
\hline Belgien & $(4.5)$ & $(9.1)$ & 53 & (5.6) & 0.53 & $(0.04)$ & -51.8 & (5.7) & 1.10 & $(0.09)$ & 1.3 & (1.3) \\
\hline Kanada & (6.6) & (4.4) & -13 & (4.5) & 0.96 & $(0.06)$ & -2.8 & (4.4) & 1.06 & $(0.12)$ & 0.2 & $(0.4)$ \\
\hline ठै Chile & (14.2) & c & c & c & 0.83 & $(0.17)$ & -21.2 & (25.3) & 1.47 & $(0.45)$ & 0.3 & $(0.3)$ \\
\hline Tschech. Rep. & $(23.2)$ & (26.6) & 9 & $(16.0)$ & 0.74 & $(0.14)$ & -33.7 & $(22.0)$ & 1.63 & $(0.53)$ & 0.5 & $(0.4)$ \\
\hline Dänemark & $(12.3)$ & (6.7) & 28 & (4.4) & 0.45 & $(0.03)$ & -103.5 & $(11.8)$ & 2.08 & $(0.50)$ & 0.6 & $(0.3)$ \\
\hline Estland & $(5.7)$ & $(11.7)$ & 37 & (10.7) & 0.71 & $(0.06)$ & -34.0 & $(8.4)$ & 1.10 & $(0.15)$ & 0.4 & $(0.6)$ \\
\hline Finnland & (6.7) & $(9.9)$ & 69 & (5.3) & 0.47 & $(0.02)$ & -102.6 & $(8.4)$ & 1.11 & $(0.13)$ & 0.2 & $(0.2)$ \\
\hline Frankreich & (15.3) & (9.9) & 33 & (9.4) & 0.46 & $(0.04)$ & -84.0 & (11.1) & 1.52 & $(0.32)$ & 1.2 & $(0.6)$ \\
\hline Deutschland & (20.5) & (9.4) & 31 & (7.9) & 0.60 & $(0.05)$ & -52.7 & (11.5) & 1.31 & $(0.46)$ & 0.5 & $(0.6)$ \\
\hline Griechenland & (12.8) & (10.1) & 33 & (9.4) & 0.52 & $(0.04)$ & -73.5 & (12.6) & 1.83 & $(0.44)$ & 0.9 & $(0.6)$ \\
\hline Ungarn & $(18.0)$ & c & c & c & 1.18 & $(0.33)$ & 13.5 & $(24.0)$ & 1.53 & $(0.45)$ & 0.4 & $(0.3)$ \\
\hline Island & (16.5) & c & 30 & (9.3) & 0.55 & $(0.05)$ & -74.1 & (16.6) & 1.69 & $(0.42)$ & 0.8 & $(0.5)$ \\
\hline Irland & c & (8.3) & 2 & (6.3) & 0.98 & $(0.10)$ & -1.6 & $(9.4)$ & c & c & C & c \\
\hline Israel & (9.7) & (6.6) & -19 & (5.6) & 1.15 & $(0.12)$ & 10.8 & (7.1) & 1.41 & $(0.20)$ & 1.2 & $(0.6)$ \\
\hline Italien & (2.6) & $(7.1)$ & 36 & (4.6) & 0.56 & $(0.02)$ & -57.8 & (4.5) & 1.56 & $(0.06)$ & 5.2 & $(0.6)$ \\
\hline Japan & c & C & c & c & 0.48 & $(0.10)$ & -108.5 & $(40.5)$ & c & C & c & C \\
\hline Korea & c & c & c & c & c & c & c & c & c & c & c & c \\
\hline Luxemburg & (7.7) & (5.2) & 32 & (8.2) & 0.49 & $(0.14)$ & -1.6 & (0.5) & 0.58 & $(0.04)$ & -28.5 & (3.3) \\
\hline Mexiko & (4.1) & (11.4) & 71 & (10.9) & 0.44 & $(0.02)$ & -115.6 & (9.2) & 1.97 & $(0.12)$ & 2.7 & $(0.5)$ \\
\hline Niederlande & (13.0) & (10.3) & 29 & $(8.7)$ & 0.55 & $(0.05)$ & -66.1 & (11.8) & 1.27 & $(0.30)$ & 0.4 & $(0.4)$ \\
\hline Neuseeland & $(9.2)$ & (7.0) & 6 & (6.5) & 0.72 & $(0.06)$ & -25.0 & (6.6) & 2.52 & $(0.33)$ & 4.0 & (1.1) \\
\hline Norwegen & (12.1) & (7.9) & 25 & (7.4) & 0.56 & $(0.04)$ & -64.6 & (9.2) & 1.07 & $(0.28)$ & 0.1 & (0.4) \\
\hline Polen & (13.2) & c & c & c & 0.87 & $(0.24)$ & -15.4 & (31.4) & 1.13 & $(0.38)$ & 0.1 & $(0.3)$ \\
\hline Portugal & (14.7) & (11.6) & 16 & $(11.2)$ & 0.59 & $(0.05)$ & -62.2 & (13.6) & 0.82 & (0.35) & -0.1 & $(0.3)$ \\
\hline Slowak. Rep. & (10.2) & C & c & c & 0.38 & $(0.04)$ & -133.7 & (20.8) & 2.75 & $(0.30)$ & 11.0 & $(2.1)$ \\
\hline Slowenien & (9.8) & (11.0) & 30 & (6.5) & 0.53 & $(0.05)$ & -75.2 & (12.6) & 2.05 & (0.35) & 1.3 & $(0.6)$ \\
\hline Spanien & (2.7) & $(6.7)$ & 34 & (5.5) & 0.69 & $(0.03)$ & -30.7 & (4.5) & 0.95 & $(0.06)$ & -0.7 & $(0.8)$ \\
\hline Schweden & (21.4) & (9.0) & 36 & (6.9) & 0.50 & $(0.04)$ & -75.2 & (10.4) & 1.66 & $(0.34)$ & 1.0 & $(0.5)$ \\
\hline Schweiz & (6.3) & (5.5) & 45 & (4.4) & 0.47 & $(0.02)$ & -63.9 & $(4.7)$ & 1.08 & $(0.13)$ & 0.3 & $(0.5)$ \\
\hline Türkei & (13.6) & c & C & C & 0.56 & (0.09) & -69.1 & (25.0) & 1.81 & $(0.32)$ & 4.6 & (1.9) \\
\hline Ver. Königreich & (9.3) & (9.3) & -10 & (8.5) & 0.79 & $(0.07)$ & -22.8 & (9.8) & 1.15 & $(0.19)$ & 0.2 & $(0.2)$ \\
\hline Ver. Staaten & (8.5) & $(6.2)$ & -9 & $(5.2)$ & 0.75 & $(0.08)$ & -23.8 & (8.3) & 1.71 & $(0.23)$ & 1.3 & $(0.4)$ \\
\hline OECD-Durchschnitt & $(2.2)$ & (1.9) & 23 & (1.5) & 0.66 & $(0.02)$ & -50.9 & (2.7) & 1.48 & $(0.06)$ & 0.4 & $(0.2)$ \\
\hline
\end{tabular}

\begin{tabular}{|c|c|c|c|c|c|c|c|c|c|c|c|c|c|c|}
\hline Albanien & $\mathrm{c}$ & c & c & c & c & c & c & c & c & C & c & $\mathrm{C}$ & $\mathrm{c}$ & c \\
\hline Argentinien & 31 & $(13.8)$ & 19 & (30.3) & 31 & $(29.2)$ & 0.62 & $(0.08)$ & -55.9 & $(17.8)$ & 1.80 & $(0.48)$ & 0.8 & $(0.5)$ \\
\hline Brasilien & 0 & (11.1) & c & c & c & c & 0.61 & $(0.07)$ & -63.2 & $(17.9)$ & 1.13 & $(0.19)$ & 0.1 & $(0.2)$ \\
\hline Bulgarien & 31 & $(5.6)$ & c & C & c & c & 0.40 & $(0.04)$ & -114.0 & (16.6) & 2.47 & $(0.24)$ & 13.3 & (2.1) \\
\hline Kolumbien & 35 & (18.2) & C & C & c & c & 0.54 & $(0.12)$ & -85.4 & (39.4) & 1.53 & $(0.63)$ & 0.3 & (0.4) \\
\hline Costa Rica & -4 & (14.3) & c & c & c & c & 0.62 & $(0.11)$ & -55.2 & $(21.2)$ & 0.81 & $(0.32)$ & -0.2 & $(0.3)$ \\
\hline Kroatien & 0 & $(17.8)$ & c & c & c & c & 0.83 & $(0.08)$ & -17.4 & (8.6) & 1.01 & $(0.32)$ & 0.0 & $(0.3)$ \\
\hline Zypern* & -37 & (3.5) & 11 & $(8.0)$ & 11 & (6.4) & 1.36 & $(0.11)$ & 21.9 & (4.9) & 0.43 & $(0.05)$ & -8.8 & (0.9) \\
\hline Hongkong (China) & 50 & (12.0) & 49 & $(9.9)$ & 31 & (10.5) & 0.82 & $(0.07)$ & -12.4 & (5.4) & 1.54 & $(0.22)$ & 1.6 & $(0.8)$ \\
\hline Indonesien & -11 & $(4.5)$ & c & c & c & c & 1.00 & $(0.09)$ & -0.1 & (3.7) & 1.00 & $(0.09)$ & 0.2 & (5.4) \\
\hline Jordanien & 7 & (6.7) & 13 & $(14.7)$ & -4 & $(14.1)$ & 1.35 & $(0.17)$ & 22.2 & $(8.1)$ & 1.03 & $(0.15)$ & 0.1 & $(0.6)$ \\
\hline Kasachstan & -8 & (6.4) & 0 & $(11.2)$ & 2 & (9.9) & 1.00 & $(0.10)$ & 0.0 & $(6.7)$ & 0.81 & $(0.12)$ & -1.9 & (1.2) \\
\hline Lettland & 8 & (6.1) & 4 & $(22.1)$ & 14 & (20.8) & 0.79 & $(0.08)$ & -21.9 & (10.8) & 1.28 & $(0.17)$ & 2.5 & (1.6) \\
\hline Liechtenstein & c & C & c & c & c & c & 0.48 & $(0.12)$ & -52.7 & (18.0) & c & c & C & c \\
\hline Litauen & 37 & $(10.7)$ & c & c & c & c & 0.69 & $(0.09)$ & -41.4 & $(15.1)$ & 1.70 & $(0.21)$ & 2.1 & $(0.7)$ \\
\hline Macau (China) & -8 & $(5.2)$ & 12 & (5.3) & -12 & $(5.2)$ & 1.38 & $(0.09)$ & 9.6 & $(2.0)$ & 0.83 & $(0.09)$ & -1.1 & (0.6) \\
\hline Malaysia & -25 & $(4.2)$ & C & $\mathrm{C}$ & C & C & 1.27 & $(0.11)$ & 13.2 & (4.5) & 0.77 & $(0.07)$ & -10.6 & (3.6) \\
\hline Montenegro & 20 & (14.5) & c & C & c & c & 1.34 & $(0.24)$ & 23.6 & (12.7) & 1.34 & $(0.43)$ & 0.3 & $(0.3)$ \\
\hline Peru & 43 & $(7.2)$ & c & c & c & c & 0.37 & $(0.03)$ & -144.3 & $(16.8)$ & 2.65 & $(0.24)$ & 9.0 & (1.9) \\
\hline Katar & -40 & (3.0) & -34 & $(3.2)$ & -117 & (2.3) & 3.06 & $(0.12)$ & 41.6 & (1.5) & 1.03 & $(0.05)$ & 0.4 & $(0.7)$ \\
\hline Rumänien & 27 & $(10.5)$ & c & c & c & c & 0.69 & $(0.18)$ & -45.6 & $(30.8)$ & 1.61 & $(0.33)$ & 1.0 & $(0.6)$ \\
\hline Russ. Föderation & 6 & $(7.0)$ & 34 & $(14.2)$ & 46 & (14.9) & 0.79 & $(0.06)$ & -21.4 & (6.9) & 1.15 & $(0.15)$ & 1.0 & (1.0) \\
\hline Serbien & 1 & (7.4) & -30 & (20.6) & -40 & (21.2) & 1.14 & $(0.09)$ & 10.7 & $(6.8)$ & 1.00 & $(0.18)$ & 0.0 & (0.6) \\
\hline Shanghai (China) & 84 & (13.7) & c & c & c & c & 0.34 & $(0.02)$ & -184.4 & (18.6) & 2.74 & $(0.30)$ & 1.8 & (0.4) \\
\hline Singapur & 19 & (3.8) & -12 & (7.9) & -2 & (5.5) & 0.61 & $(0.03)$ & -19.0 & $(2.0)$ & 1.91 & $(0.10)$ & 27.0 & (2.2) \\
\hline Chinesisch Taipeh & 27 & $(4.8)$ & c & c & C & c & 0.52 & $(0.03)$ & -67.4 & (7.9) & 1.94 & $(0.13)$ & 13.3 & (1.7) \\
\hline Thailand & -7 & (4.3) & c & c & c & c & 0.98 & $(0.09)$ & -1.0 & (5.0) & 1.00 & $(0.08)$ & 0.0 & (3.7) \\
\hline Tunesien & 21 & (15.7) & c & C & C & c & 0.89 & $(0.26)$ & -12.0 & (28.0) & 1.11 & $(0.37)$ & 0.1 & (0.4) \\
\hline Ver. Arab. Emirate & -17 & $(5.2)$ & -19 & $(4.2)$ & -77 & $(4.1)$ & 2.42 & $(0.14)$ & 33.8 & $(2.3)$ & 1.05 & $(0.08)$ & 0.4 & $(0.7)$ \\
\hline Uruguay & 0 & $(9.2)$ & C & c & c & c & 0.82 & $(0.13)$ & -22.8 & (23.4) & 1.34 & $(0.25)$ & 0.6 & $(0.5)$ \\
\hline Vietnam & 17 & (11.9) & c & C & C & c & 0.54 & $(0.10)$ & -83.6 & (36.6) & 1.82 & $(0.39)$ & 1.7 & $(0.7)$ \\
\hline
\end{tabular}

Anmerkung: Diese Tabelle wurde nur für Schüler berechnet, für die Daten zum PISA-Index des wirtschaftlichen, sozialen und kulturellen Status vorliegen. Statistisch signifikante Werte sind durch Fettdruck gekennzeichnet (vgl. Anhang A3).

Die Prozentsätze der Schüler ohne Migrationshintergrund und der Schüler mit Migrationshintergrund die zu Hause die Testsprache sprechen bzw. zu Hause nicht die Testsprache sprechen, werden für die gesamte Schülerpopulation berechnet. Die Prozentsätze der Schüler der 1. und der 2. Generation, die zu Hause die Testsprache sprechen bzw. nicht die Testsprache sprechen, werden für die gesamte Schülerpopulation mit Migrationshintergrund berechnet.

*Vgl. die Anmerkungen am Beginn dieses Anhangs.

StatLink त्ञाI SL http://dx.doi.org/10.1787/888932964927 
[Teil 4/4]

Mathematikleistungen, Migrationshintergrund und zu Hause gesprochene Sprache Tabelle II.3.5 Die Ergebnisse basieren auf Schülerangaben

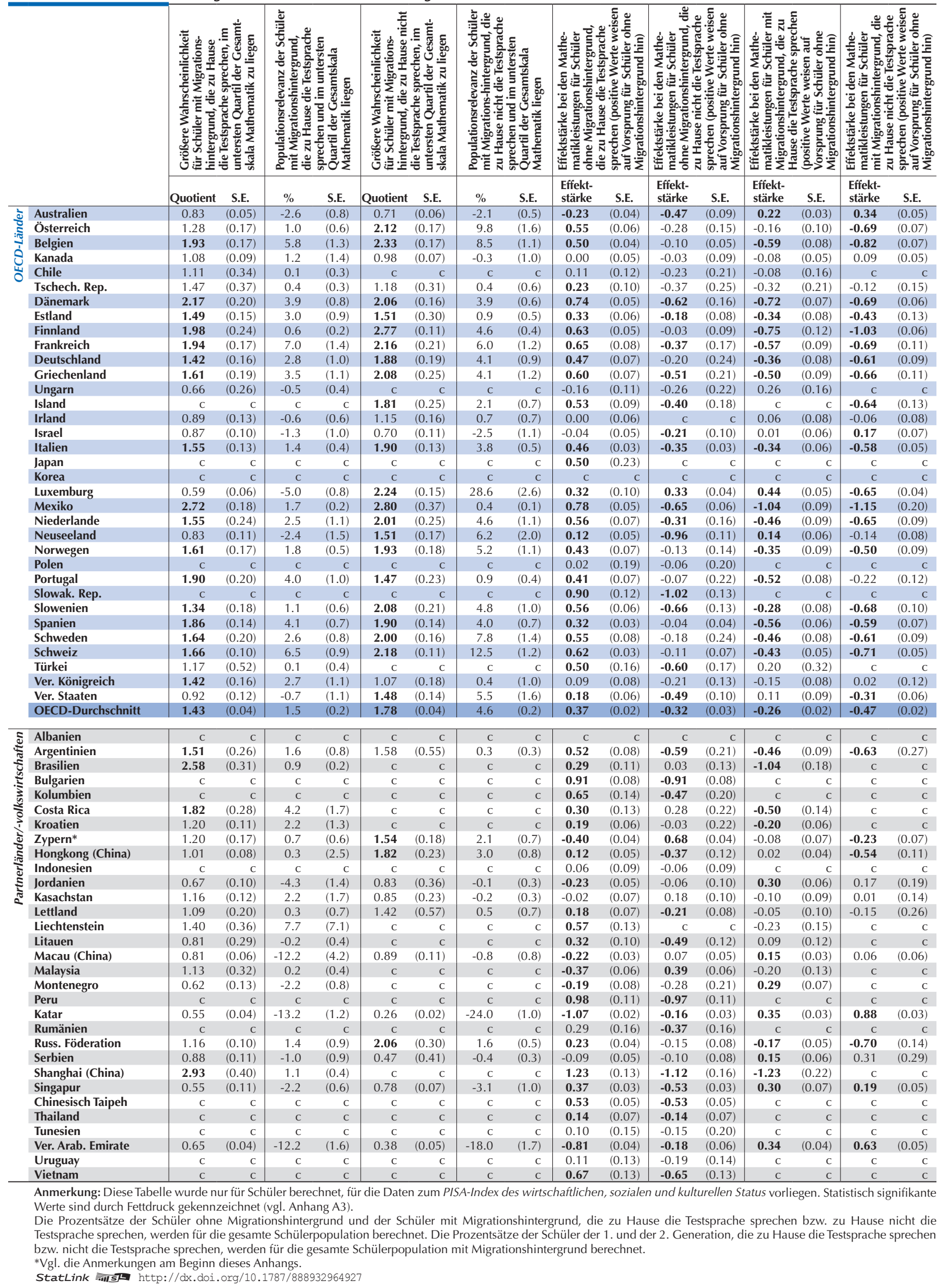


[Teil 1/3]

Mathematikleistungen und Migrationshintergrund für Schüler der 1. und 2. Generation Tabelle II.3.6a Die Ergebnisse basieren auf Schülerangaben

\begin{tabular}{|c|c|c|c|c|c|c|c|c|c|c|c|c|c|c|c|c|c|c|}
\hline & \multicolumn{6}{|c|}{ Prozentsatz der Schüler } & \multicolumn{6}{|c|}{$\begin{array}{l}\text { PISA-Index des wirtschaftlichen, sozialen und } \\
\text { kulturellen Status (ESCS) }\end{array}$} & \multicolumn{6}{|c|}{ Schülerleistungen in Mathematik } \\
\hline & \multicolumn{2}{|c|}{$\begin{array}{c}\text { Ohne } \\
\text { Migrations- } \\
\text { hintergrund } \\
\end{array}$} & \multicolumn{2}{|c|}{ 1. Generation } & \multicolumn{2}{|c|}{ 2. Generation } & \multicolumn{2}{|c|}{$\begin{array}{c}\text { Ohne } \\
\text { Migrations- } \\
\text { hintergrund }\end{array}$} & \multicolumn{2}{|c|}{ 1. Generation } & \multicolumn{2}{|c|}{ 2. Generation } & \multicolumn{2}{|c|}{$\begin{array}{c}\text { Ohne } \\
\text { Migrations- } \\
\text { hintergrund }\end{array}$} & \multicolumn{2}{|c|}{ 1. Generation } & \multicolumn{2}{|c|}{ 2. Generation } \\
\hline & $\%$ & S.E. & $\%$ & S.E. & $\%$ & S.E. & $\begin{array}{l}\text { Index- } \\
\text { mittel }\end{array}$ & S.E. & $\begin{array}{l}\text { Index- } \\
\text { mittel }\end{array}$ & S.E. & $\begin{array}{l}\text { Index- } \\
\text { mittel }\end{array}$ & S.E. & $\begin{array}{c}\text { Mittel- } \\
\text { wert }\end{array}$ & S.E. & $\begin{array}{c}\text { Mittel- } \\
\text { wert }\end{array}$ & S.E. & $\begin{array}{c}\text { Mittel- } \\
\text { wert }\end{array}$ & S.E. \\
\hline Australien & 77.3 & $(0.7)$ & 10.2 & $(0.4)$ & 12.4 & $(0.6)$ & 0.28 & $(0.01)$ & 0.26 & $(0.03)$ & 0.16 & $(0.03)$ & 503 & (1.5) & 516 & $(3.7)$ & 539 & $(5.3)$ \\
\hline : Österreich & 83.6 & (1.1) & 5.5 & $(0.5)$ & 10.8 & $(0.7)$ & 0.19 & $(0.02)$ & -0.50 & $(0.10)$ & -0.49 & $(0.04)$ & 517 & $(2.7)$ & 454 & (8.6) & 458 & (5.3) \\
\hline Belgien & 84.9 & $(0.9)$ & 7.2 & $(0.6)$ & 7.9 & $(0.6)$ & 0.23 & $(0.02)$ & -0.26 & $(0.07)$ & -0.32 & $(0.07)$ & 530 & $(2.1)$ & 448 & (6.5) & 461 & (6.6) \\
\hline Kanada & 70.5 & $(1.3)$ & 13.0 & $(0.7)$ & 16.5 & $(0.8)$ & 0.45 & $(0.02)$ & 0.49 & $(0.04)$ & 0.19 & $(0.04)$ & 522 & $(1.8)$ & 528 & $(5.2)$ & 514 & (4.5) \\
\hline Chile & 99.1 & $(0.2)$ & 0.7 & $(0.1)$ & 0.2 & $(0.1)$ & -0.58 & $(0.04)$ & -0.16 & $(0.19)$ & c & $\mathrm{C}$ & 424 & (3.0) & 423 & (13.3) & c & $\mathrm{c}$ \\
\hline Tschech. Rep. & 96.8 & $(0.4)$ & 1.8 & $(0.2)$ & 1.4 & $(0.3)$ & -0.06 & $(0.02)$ & -0.17 & $(0.09)$ & -0.15 & $(0.09)$ & 501 & $(2.8)$ & 486 & $(12.0)$ & 461 & $(21.0)$ \\
\hline Dänemark & 91.1 & $(0.6)$ & 2.9 & $(0.2)$ & 6.0 & $(0.4)$ & 0.49 & $(0.02)$ & -0.20 & $(0.06)$ & -0.25 & $(0.05)$ & 508 & $(2.2)$ & 430 & $(5.4)$ & 448 & $(4.0)$ \\
\hline Estland & 91.8 & $(0.5)$ & 0.7 & $(0.2)$ & 7.5 & $(0.5)$ & 0.11 & $(0.01)$ & C & C & 0.06 & $(0.06)$ & 524 & $(2.0)$ & c & c & 496 & $(6.2)$ \\
\hline Finnland & 96.7 & $(0.2)$ & 1.9 & $(0.1)$ & 1.5 & $(0.1)$ & 0.39 & $(0.02)$ & -0.38 & $(0.06)$ & -0.12 & $(0.05)$ & 523 & (1.9) & 427 & (8.0) & 454 & (5.0) \\
\hline Frankreich & 85.2 & (1.1) & 4.9 & $(0.5)$ & 9.9 & $(0.8)$ & 0.05 & $(0.02)$ & -0.52 & $(0.08)$ & -0.53 & $(0.05)$ & 508 & $(2.7)$ & 425 & $(10.5)$ & 448 & (6.9) \\
\hline Deutschland & 86.9 & $(0.8)$ & 2.7 & $(0.3)$ & 10.5 & $(0.7)$ & 0.30 & $(0.03)$ & -0.24 & $(0.10)$ & -0.46 & $(0.05)$ & 528 & $(3.2)$ & 462 & $(11.2)$ & 478 & (5.8) \\
\hline Griechenland & 89.5 & $(0.8)$ & 6.3 & $(0.6)$ & 4.3 & $(0.4)$ & 0.00 & $(0.03)$ & -0.83 & $(0.06)$ & $\mid-0.47$ & $(0.07)$ & 459 & (2.6) & 404 & $(7.3)$ & 414 & $(7.7)$ \\
\hline Ungarn & 98.3 & $(0.2)$ & 0.7 & $(0.2)$ & 1.0 & $(0.2)$ & -0.26 & $(0.03)$ & c & c & 0.18 & $(0.12)$ & 478 & $(3.1)$ & c & c & 522 & $(15.2)$ \\
\hline Island & 96.5 & $(0.3)$ & 2.8 & $(0.3)$ & 0.7 & $(0.1)$ & 0.81 & $(0.01)$ & -0.02 & $(0.09)$ & c & C & 498 & $(1.8)$ & 437 & (9.7) & c & c \\
\hline Irland & 89.9 & $(0.7)$ & 8.4 & $(0.7)$ & 1.7 & $(0.2)$ & 0.12 & $(0.02)$ & 0.17 & $(0.07)$ & 0.24 & $(0.11)$ & 503 & $(2.3)$ & 501 & $(4.8)$ & 503 & $(12.1)$ \\
\hline Israel & 81.7 & $(1.2)$ & 5.5 & $(0.6)$ & 12.7 & $(0.8)$ & 0.21 & $(0.03)$ & -0.07 & $(0.08)$ & 0.08 & $(0.06)$ & 470 & $(4.6)$ & 469 & $(7.7)$ & 480 & $(8.3)$ \\
\hline Italien & 92.5 & $(0.3)$ & 5.5 & $(0.3)$ & 2.0 & $(0.2)$ & -0.01 & $(0.01)$ & -0.59 & $(0.03)$ & -0.45 & $(0.06)$ & 490 & $(2.0)$ & 435 & (3.3) & 461 & (7.4) \\
\hline Japan & 99.7 & $(0.1)$ & 0.1 & $(0.0)$ & 0.2 & $(0.1)$ & -0.07 & $(0.02)$ & C & c & c & C & 539 & (3.5) & c & c & c & c \\
\hline Korea & 100.0 & $(0.0)$ & 0.0 & $(0.0)$ & 0.0 & $(0.0)$ & 0.01 & $(0.03)$ & c & c & c & c & 555 & (4.6) & c & c & c & c \\
\hline Luxemburg & 53.6 & $(0.7)$ & 17.4 & $(0.5)$ & 28.9 & $(0.6)$ & 0.47 & $(0.01)$ & -0.27 & $(0.04)$ & -0.46 & $(0.03)$ & 511 & $(1.7)$ & 470 & (4.1) & 470 & (2.5) \\
\hline Mexiko & 98.7 & $(0.1)$ & 0.8 & $(0.1)$ & 0.5 & $(0.1)$ & -1.10 & $(0.02)$ & -1.60 & $(0.16)$ & -1.22 & $(0.17)$ & 416 & (1.3) & 333 & (6.3) & 359 & (9.9) \\
\hline Niederlande & 89.4 & (1.0) & 2.7 & (0.4) & 7.9 & $(0.9)$ & 0.31 & $(0.02)$ & -0.19 & $(0.10)$ & -0.35 & $(0.06)$ & 531 & (3.4) & 471 & (10.1) & 475 & (9.0) \\
\hline Neuseeland & 73.7 & $(1.5)$ & 16.8 & (1.0) & 9.5 & $(0.8)$ & 0.05 & $(0.02)$ & 0.14 & $(0.04)$ & -0.20 & $(0.06)$ & 503 & $(2.7)$ & 509 & $(5.2)$ & 492 & (7.1) \\
\hline Norwegen & 90.6 & $(0.9)$ & 4.7 & (0.5) & 4.7 & $(0.6)$ & 0.52 & $(0.02)$ & -0.17 & $(0.07)$ & 0.00 & $(0.07)$ & 496 & (2.8) & 442 & (6.2) & 457 & (9.2) \\
\hline Polen & 99.8 & $(0.1)$ & 0.0 & $(0.0)$ & 0.2 & $(0.1)$ & -0.21 & $(0.03)$ & c & c & C & c & 518 & (3.6) & c & c & c & c \\
\hline Portugal & 93.1 & $(0.6)$ & 3.6 & $(0.5)$ & 3.3 & $(0.4)$ & -0.47 & $(0.05)$ & -0.67 & $(0.08)$ & -0.57 & $(0.16)$ & 493 & (3.7) & 451 & (8.2) & 445 & (10.1) \\
\hline Slowak. Rep. & 99.3 & $(0.2)$ & 0.3 & $(0.1)$ & 0.4 & $(0.1)$ & -0.18 & $(0.03)$ & c & c & C & c & 484 & (3.4) & c & c & c & c \\
\hline Slowenien & 91.4 & $(0.4)$ & 2.1 & $(0.2)$ & 6.5 & $(0.4)$ & 0.12 & $(0.01)$ & -0.58 & $(0.09)$ & $\mid-0.48$ & $(0.06)$ & 506 & $(1.2)$ & 433 & (10.3) & 463 & (5.3) \\
\hline Spanien & 90.1 & $(0.6)$ & 8.4 & (0.5) & 1.5 & $(0.2)$ & -0.14 & $(0.03)$ & -0.68 & $(0.04)$ & -0.40 & $(0.12)$ & 491 & (1.7) & 436 & (4.8) & 457 & (8.5) \\
\hline Schweden & 85.5 & $(0.9)$ & 5.9 & (0.5) & 8.6 & $(0.7)$ & 0.36 & $(0.02)$ & -0.38 & $(0.08)$ & -0.10 & $(0.06)$ & 490 & $(2.3)$ & 414 & (7.3) & 445 & (5.3) \\
\hline Schweiz & 75.9 & $(0.9)$ & 6.7 & $(0.4)$ & 17.4 & $(0.7)$ & 0.34 & $(0.02)$ & -0.27 & $(0.07)$ & -0.37 & $(0.04)$ & 548 & (3.0) & 472 & (5.8) & 489 & (3.8) \\
\hline Türkei & 99.1 & $(0.2)$ & 0.2 & $(0.1)$ & 0.7 & $(0.2)$ & -1.46 & $(0.04)$ & C & C & -1.03 & $(0.19)$ & 449 & $(4.8)$ & C & C & 476 & (35.4) \\
\hline Ver. Königreich & 87.3 & (1.1) & 7.1 & $(0.8)$ & 5.6 & $(0.5)$ & 0.28 & $(0.02)$ & 0.19 & $(0.09)$ & 0.20 & $(0.06)$ & 499 & (2.8) & 495 & $(11.7)$ & 483 & (7.3) \\
\hline Ver. Staaten & 78.5 & $(2.0)$ & 6.7 & $(0.8)$ & 14.8 & $(1.4)$ & 0.34 & $(0.03)$ & -0.43 & $(0.10)$ & -0.39 & $(0.08)$ & 487 & (3.6) & 463 & (9.3) & 478 & (6.6) \\
\hline OECD-Durchschnitt & 88.8 & $(0.1)$ & 4.8 & $(0.1)$ & 6.4 & $(0.1)$ & 0.06 & $(0.00)$ & $\mid-0.29$ & $(0.02)$ & $\mid-0.27$ & $(0.02)$ & 500 & $(0.5)$ & 453 & (1.6) & 469 & (2.0) \\
\hline
\end{tabular}

\begin{tabular}{|c|c|c|c|c|c|c|c|c|c|c|c|c|c|c|c|c|c|c|}
\hline Albanien & 0.0 & c & 0.0 & C & 0.0 & c & $\mathrm{m}$ & $\mathrm{m}$ & $\mathrm{m}$ & $\mathrm{m}$ & $\mathrm{m}$ & $\mathrm{m}$ & c & C & C & c & C & C \\
\hline Argentinien & 96.1 & $(0.4)$ & 1.5 & $(0.2)$ & 2.4 & $(0.3)$ & -0.68 & $(0.04)$ & -1.54 & $(0.14)$ & -1.39 & $(0.13)$ & 392 & (3.4) & 351 & (9.5) & 358 & (9.4) \\
\hline Brasilien & 99.3 & $(0.1)$ & 0.4 & $(0.1)$ & 0.4 & $(0.1)$ & -1.16 & $(0.02)$ & -1.16 & $(0.21)$ & -0.62 & $(0.29)$ & 394 & $(2.1)$ & 339 & $(18.6)$ & 337 & $(17.4)$ \\
\hline Bulgarien & 99.5 & $(0.2)$ & 0.2 & $(0.1)$ & 0.4 & $(0.1)$ & $\mid-0.27$ & $(0.04)$ & c & c & c & c & 442 & (3.9) & c & c & c & c \\
\hline Kolumbien & 99.7 & $(0.1)$ & 0.1 & $(0.1)$ & 0.2 & $(0.0)$ & -1.26 & $(0.04)$ & c & c & c & c & 378 & (2.9) & c & c & c & c \\
\hline Costa Rica & 94.5 & $(0.7)$ & 2.1 & $(0.3)$ & 3.5 & $(0.7)$ & -0.94 & $(0.04)$ & -1.34 & $(0.17)$ & -1.76 & $(0.20)$ & 409 & (2.9) & 390 & (9.9) & 374 & $(14.1)$ \\
\hline Kroatien & 87.9 & $(0.8)$ & 3.7 & $(0.4)$ & 8.4 & $(0.5)$ & -0.30 & $(0.02)$ & -0.71 & $(0.07)$ & -0.54 & $(0.05)$ & 474 & (3.6) & 453 & $(10.2)$ & 456 & (5.0) \\
\hline Zypern* & 91.5 & $(0.4)$ & 6.7 & $(0.3)$ & 1.8 & $(0.2)$ & 0.10 & $(0.01)$ & -0.18 & $(0.05)$ & 0.32 & $(0.10)$ & 444 & $(1.2)$ & 420 & (5.2) & 439 & (11.0) \\
\hline Hongkong (China) & 65.3 & $(1.5)$ & 14.2 & $(1.0)$ & 20.5 & $(0.8)$ & -0.56 & $(0.06)$ & -1.35 & $(0.04)$ & -1.14 & $(0.03)$ & 566 & (3.7) & 543 & (5.2) & 570 & $(4.2)$ \\
\hline Indonesien & 99.8 & $(0.1)$ & 0.1 & $(0.0)$ & 0.1 & $(0.0)$ & -1.80 & $(0.05)$ & C & c & c & C & 376 & $(4.0)$ & C & c & c & 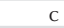 \\
\hline Jordanien & 86.6 & $(0.7)$ & 2.8 & $(0.3)$ & 10.6 & $(0.6)$ & -0.47 & $(0.02)$ & -0.07 & $(0.10)$ & -0.22 & $(0.06)$ & 388 & $(2.8)$ & 416 & (8.9) & 408 & (5.1) \\
\hline Kasachstan & 83.9 & (1.7) & 6.5 & $(1.2)$ & 9.6 & (1.0) & $\mid-0.29$ & $(0.02)$ & -0.67 & $(0.05)$ & -0.31 & $(0.06)$ & 433 & (3.1) & 407 & (5.8) & 440 & (8.2) \\
\hline Lettland & 95.5 & $(0.5)$ & 0.4 & $(0.1)$ & 4.1 & $(0.5)$ & -0.26 & $(0.03)$ & c & $\mathrm{c}$ & -0.15 & $(0.09)$ & 492 & (2.8) & c & $\mathrm{C}$ & 487 & (8.4) \\
\hline Liechtenstein & 66.7 & $(2.9)$ & 13.4 & $(2.2)$ & 19.9 & (2.3) & 0.44 & $(0.05)$ & 0.06 & $(0.19)$ & 0.00 & $(0.13)$ & 554 & (5.6) & 499 & $(14.6)$ & 507 & (11.9) \\
\hline Litauen & 98.3 & $(0.3)$ & 0.2 & $(0.1)$ & 1.4 & $(0.3)$ & -0.13 & $(0.02)$ & c & c & -0.12 & $(0.10)$ & 480 & $(2.7)$ & c & c & 473 & (8.5) \\
\hline Macau (China) & 34.9 & $(0.6)$ & 15.5 & $(0.4)$ & 49.7 & $(0.7)$ & -0.69 & $(0.02)$ & -0.90 & $(0.03)$ & -1.02 & $(0.01)$ & 530 & $(2.1)$ & 541 & (3.0) & 546 & (1.8) \\
\hline Malaysia & 98.3 & $(0.3)$ & 0.1 & $(0.0)$ & 1.7 & $(0.3)$ & -0.71 & $(0.03)$ & c & c & -1.36 & $(0.14)$ & 423 & (3.2) & c & c & 404 & (9.1) \\
\hline Montenegro & 94.2 & $(0.4)$ & 3.1 & $(0.3)$ & 2.7 & $(0.2)$ & -0.26 & $(0.01)$ & -0.21 & $(0.07)$ & -0.01 & $(0.08)$ & 410 & $(1.2)$ & 427 & (8.4) & 436 & (8.1) \\
\hline Peru & 99.5 & $(0.1)$ & 0.2 & $(0.1)$ & 0.3 & $(0.1)$ & -1.22 & $(0.05)$ & c & c & c & c & 370 & (3.6) & c & c & c & c \\
\hline Katar & 48.0 & $(0.4)$ & 34.7 & $(0.4)$ & 17.3 & (0.4) & 0.42 & $(0.01)$ & 0.55 & $(0.01)$ & 0.25 & $(0.02)$ & 335 & (1.1) & 443 & (1.5) & 388 & (2.2) \\
\hline Rumänien & 99.8 & $(0.1)$ & 0.1 & $(0.0)$ & 0.0 & $(0.0)$ & -0.47 & $(0.04)$ & c & c & c & c & 445 & (3.8) & c & c & c & 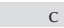 \\
\hline Russ. Föderation & 89.1 & $(0.8)$ & 3.2 & $(0.4)$ & 7.7 & (0.6) & $\mid-0.10$ & $(0.02)$ & -0.18 & $(0.06)$ & -0.17 & $(0.06)$ & 486 & $(3.2)$ & 457 & (7.9) & 467 & (4.9) \\
\hline Serbien & 91.5 & $(0.8)$ & 1.9 & $(0.3)$ & 6.6 & $(0.6)$ & -0.30 & $(0.02)$ & -0.36 & $(0.12)$ & -0.27 & $(0.06)$ & 449 & (3.4) & 439 & $(13.1)$ & 471 & (7.0) \\
\hline Shanghai (China) & 99.1 & $(0.2)$ & 0.6 & $(0.1)$ & 0.3 & $(0.1)$ & -0.35 & $(0.04)$ & -1.03 & $(0.24)$ & c & c & 615 & $(3.2)$ & 510 & $(14.6)$ & c & c \\
\hline Singapur & 81.7 & $(0.8)$ & 12.4 & $(0.7)$ & 5.9 & $(0.3)$ & -0.34 & $(0.01)$ & 0.22 & $(0.04)$ & -0.10 & $(0.05)$ & 570 & (1.6) & 591 & $(4.3)$ & 609 & (6.4) \\
\hline Chinesisch Taipeh & 99.5 & $(0.1)$ & 0.1 & $(0.0)$ & 0.4 & $(0.1)$ & -0.39 & $(0.02)$ & c & c & c & c & 562 & (3.3) & c & c & C & c \\
\hline Thailand & 99.3 & $(0.4)$ & 0.0 & $(0.0)$ & 0.6 & $(0.4)$ & -1.34 & $(0.04)$ & c & c & -3.29 & $(0.16)$ & 428 & (3.3) & c & c & 412 & $(58.0)$ \\
\hline Tunesien & 99.6 & $(0.1)$ & 0.1 & $(0.0)$ & 0.4 & $(0.1)$ & -1.19 & $(0.05)$ & c & c & c & c & 389 & (4.0) & c & c & c & c \\
\hline Ver. Arab. Emirate & 45.1 & (1.4) & 31.6 & $(1.0)$ & 23.3 & $(0.7)$ & 0.27 & $(0.03)$ & 0.47 & $(0.02)$ & 0.23 & $(0.03)$ & 400 & (2.4) & 483 & (2.9) & 443 & (2.9) \\
\hline Uruguay & 99.5 & $(0.1)$ & 0.3 & $(0.1)$ & 0.2 & $(0.1)$ & -0.88 & $(0.03)$ & C & c & c & c & 412 & (2.6) & c & $\mathrm{C}$ & c & c \\
\hline Vietnam & 99.9 & $(0.1)$ & 0.0 & $(0.0)$ & 0.1 & $(0.1)$ & -1.81 & $(0.05)$ & c & c & c & C & 512 & $(4.8)$ & c & c & c & $c$ \\
\hline
\end{tabular}

Anmerkung: Diese Tabelle wurde nur für Schüler berechnet, für die Daten zum PISA-Index des wirtschaftlichen, sozialen und kulturellen Status vorliegen. Statistisch signifikante Werte sind durch Fettdruck gekennzeichnet (vgl. Anhang A3).

Schüler mit Migrationshintergrund sind Schüler, deren Eltern in einem anderen Land/einer anderen Volkswirtschaft als dem getesteten Land/der getesteten Volkswirtschaft geboren sind. Schüler der 2. Generation wurden im getesteten Land/der getesteten Volkswirtschaft geboren, ihre Eltern jedoch nicht. Schüler der 1. Generation wurden nicht im getesteten Land/in der getesteten Volkswirtschaft geboren, und ihre Eltern wurden ebenfalls nicht im getesteten Land/in der getesteten Volkswirtschaft geboren.

*Vgl. die Anmerkungen am Beginn dieses Anhangs.

StatLink त्तils http://dx.doi.org/10.1787/888932964927 
[Teil 2/3]

Mathematikleistungen und Migrationshintergrund für Schüler der 1. und 2. Generation Tabelle II.3.6a Die Ergebnisse basieren auf Schülerangaben

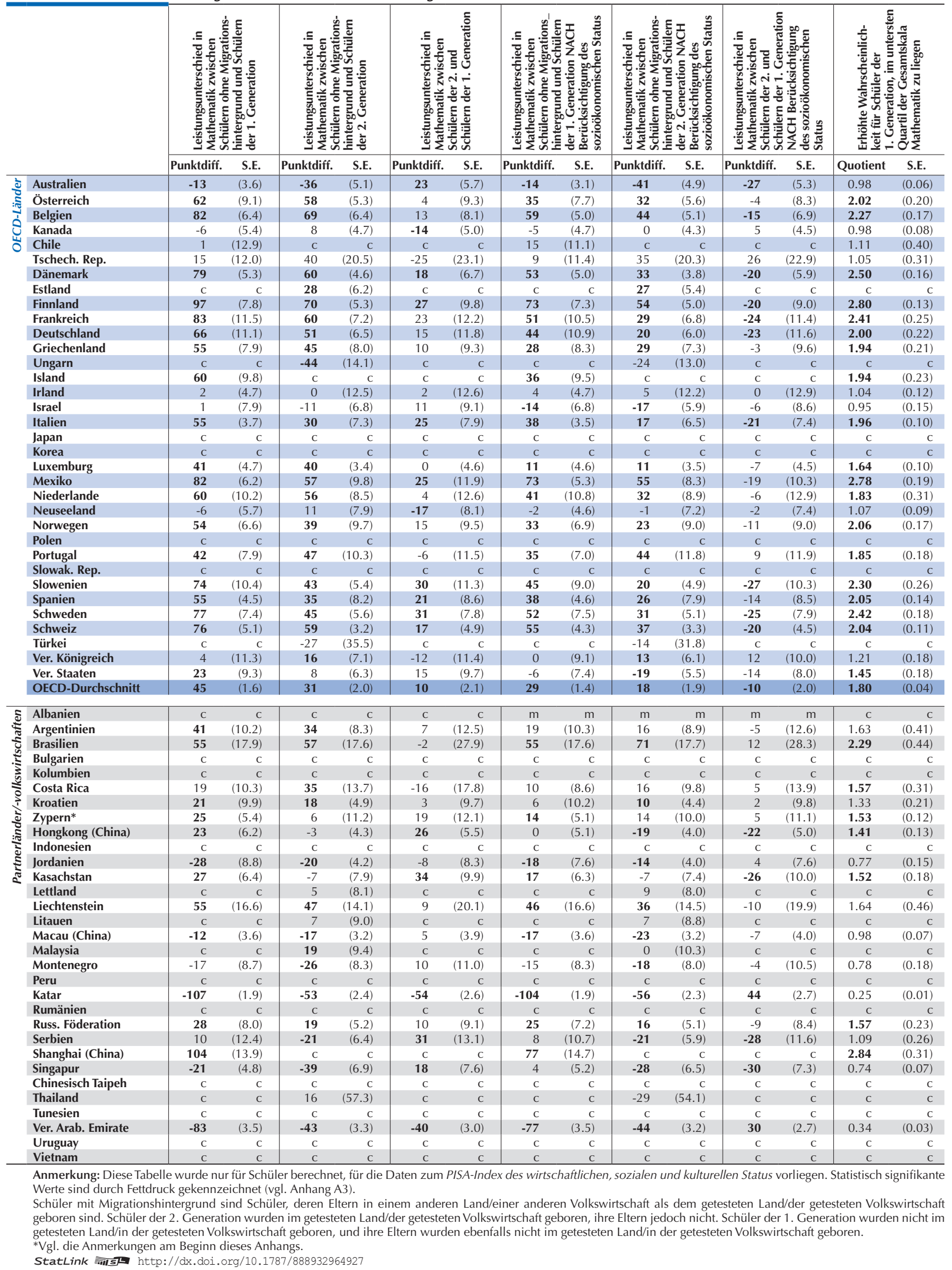


[Teil 3/3]

Mathematikleistungen und Migrationshintergrund für Schüler der 1. und 2. Generation Tabelle II.3.6a Die Ergebnisse basieren auf Schülerangaben

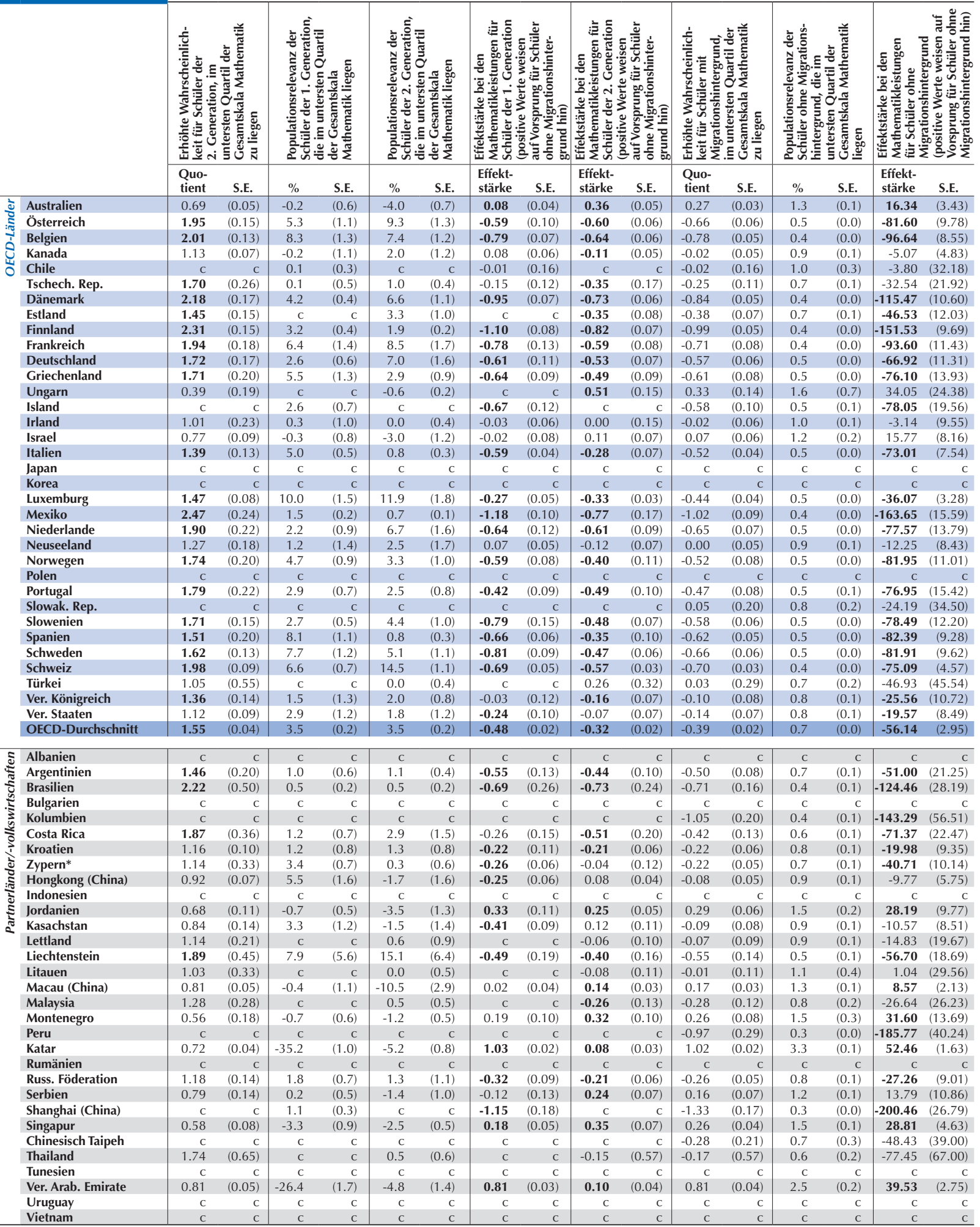

Anmerkung: Diese Tabelle wurde nur für Schüler berechnet, für die Daten zum PISA-Index des wirtschaftlichen, sozialen und kulturellen Status vorliegen. Statistisch signifikante Werte sind durch Fettdruck gekennzeichnet (vgl. Anhang A3).

Schüler mit Migrationshintergrund sind Schüler, deren Eltern in einem anderen Land/einer anderen Volkswirtschaft als dem getesteten Land/der getesteten Volkswirtschaft geboren sind. Schüler der 2. Generation wurden im getesteten Land/der getesteten Volkswirtschaft geboren, ihre Eltern jedoch nicht. Schüler der 1. Generation wurden nicht im getesteten Land/in der getesteten Volkswirtschaft geboren, und ihre Eltern wurden ebenfalls nicht im getesteten Land/in der getesteten Volkswirtschaft geboren.

*Vgl. die Anmerkungen am Beginn dieses Anhangs.

StatLink inis http://dx.doi.org/10.1787/888932964927 
[Teil 1/6]

Von 2003 bis 2012 beobachtete Veränderung des Zusammenhangs zwischen Mathematikleistungen und Migrationshintergrund für Schüler der 1. und 2. Generation

Tabelle II.3.6b Die Ergebnisse basieren auf Schülerangaben

\begin{tabular}{|c|c|c|c|c|c|c|c|c|c|c|c|c|c|c|c|c|c|c|c|}
\hline & & \multicolumn{18}{|c|}{ PISA 2003} \\
\hline & & \multicolumn{6}{|c|}{ Prozentsatz der Schüler } & \multicolumn{6}{|c|}{ Durchschnittliche Leistung in Mathematik } & \multicolumn{6}{|c|}{$\begin{array}{c}\text { PISA-Index des wirtschaftlichen, sozialen und } \\
\text { kulturellen Status (ESCS) }\end{array}$} \\
\hline & & \multicolumn{2}{|c|}{$\begin{array}{c}\text { Ohne } \\
\text { Migrations- } \\
\text { hintergrund }\end{array}$} & \multicolumn{2}{|c|}{ 2. Generation } & \multicolumn{2}{|c|}{ 1. Generation } & \multicolumn{2}{|c|}{$\begin{array}{c}\text { Ohne } \\
\text { Migrations- } \\
\text { hintergrund }\end{array}$} & \multicolumn{2}{|c|}{ 2. Generation } & \multicolumn{2}{|c|}{ 1. Generation } & \multicolumn{2}{|c|}{$\begin{array}{c}\text { Ohne } \\
\text { Migrations- } \\
\text { hintergrund }\end{array}$} & \multicolumn{2}{|c|}{ 2. Generation } & \multicolumn{2}{|c|}{ 1. Generation } \\
\hline & & $\%$ & S.E. & $\%$ & S.E. & $\%$ & S.E. & $\begin{array}{c}\text { Mittel- } \\
\text { wert }\end{array}$ & S.E. & $\begin{array}{c}\text { Mittel- } \\
\text { wert }\end{array}$ & S.E. & $\begin{array}{c}\text { Mittel- } \\
\text { wert }\end{array}$ & S.E. & $\begin{array}{c}\text { Index- } \\
\text { mittel }\end{array}$ & S.E. & $\begin{array}{l}\text { Index- } \\
\text { mittel }\end{array}$ & S.E. & $\begin{array}{l}\text { Index- } \\
\text { mittel }\end{array}$ & S.E. \\
\hline \multirow{30}{*}{ 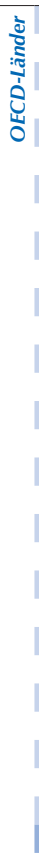 } & Australien & 77.3 & $(1.1)$ & 11.7 & (0.6) & 11.0 & $(0.7)$ & 527 & $(2.1)$ & 522 & $(4.7)$ & 525 & (4.9) & 0.08 & $(0.0)$ & -0.15 & $(0.0)$ & 0.06 & $(0.0)$ \\
\hline & Österreich & 86.9 & $(1.0)$ & 4.0 & $(0.5)$ & 9.1 & $(0.7)$ & 516 & $(3.2)$ & 464 & $(8.3)$ & 453 & (5.9) & -0.18 & $(0.0)$ & -0.81 & $(0.1)$ & -0.76 & $(0.1)$ \\
\hline & Belgien & 88.2 & $(0.9)$ & 6.3 & (0.6) & 5.5 & (0.6) & 546 & (2.5) & 454 & (7.5) & 437 & $(10.8)$ & 0.06 & $(0.0)$ & -0.71 & $(0.1)$ & -0.60 & $(0.1)$ \\
\hline & Kanada & 79.9 & (1.1) & 9.2 & $(0.5)$ & 10.9 & $(0.8)$ & 537 & (1.6) & 543 & $(4.3)$ & 530 & $(4.7)$ & 0.21 & $(0.0)$ & 0.15 & $(0.0)$ & 0.34 & $(0.0)$ \\
\hline & Tschech. Rep. & 98.7 & $(0.2)$ & 0.5 & $(0.1)$ & 0.8 & $(0.1)$ & 523 & (3.2) & c & c & 500 & (14.5) & -0.05 & $(0.0)$ & c & c & -0.30 & $(0.2)$ \\
\hline & Dänemark & 93.5 & $(0.8)$ & 3.5 & (0.6) & 3.0 & $(0.4)$ & 520 & (2.5) & 449 & $(11.2)$ & 455 & $(10.1)$ & 0.13 & $(0.0)$ & -0.61 & $(0.1)$ & -0.39 & $(0.1)$ \\
\hline & Finnland & 98.1 & $(0.2)$ & 0.0 & $(0.0)$ & 1.8 & $(0.2)$ & 546 & (1.9) & c & $\mathrm{c}$ & 474 & (10.6) & 0.06 & $(0.0)$ & c & c & -0.14 & $(0.1)$ \\
\hline & Frankreich & 85.7 & (1.3) & 10.8 & (1.1) & 3.5 & $(0.5)$ & 520 & (2.4) & 472 & (6.1) & 448 & $(15.0)$ & -0.20 & $(0.0)$ & -1.00 & $(0.1)$ & -0.99 & $(0.1)$ \\
\hline & Deutschland & 84.6 & (1.1) & 6.9 & $(0.8)$ & 8.5 & $(0.7)$ & 525 & (3.5) & 432 & (9.1) & 454 & $(7.5)$ & 0.20 & $(0.0)$ & -0.93 & $(0.1)$ & -0.89 & $(0.1)$ \\
\hline & Griechenland & 92.6 & $(0.6)$ & 0.5 & $(0.1)$ & 6.9 & $(0.7)$ & 449 & (3.9) & C & C & 402 & (6.3) & -0.27 & $(0.1)$ & c & C & -0.77 & $(0.1)$ \\
\hline & Ungarn & 97.7 & $(0.2)$ & 0.1 & $(0.0)$ & 2.2 & $(0.2)$ & 491 & (3.0) & c & c & 488 & $(10.8)$ & -0.31 & $(0.0)$ & c & c & -0.35 & $(0.1)$ \\
\hline & Island & 99.0 & $(0.2)$ & 0.2 & $(0.1)$ & 0.8 & $(0.2)$ & 517 & (1.4) & C & C & $\mathrm{C}$ & C & 0.55 & $(0.0)$ & c & c & c & $\mathrm{C}$ \\
\hline & Irland & 96.5 & $(0.3)$ & 1.0 & $(0.2)$ & 2.5 & $(0.3)$ & 503 & (2.4) & 474 & $(19.2)$ & 509 & $(11.8)$ & -0.27 & $(0.0)$ & -0.07 & $(0.2)$ & 0.17 & $(0.1)$ \\
\hline & Italien & 97.9 & $(0.3)$ & 0.4 & $(0.1)$ & 1.7 & $(0.2)$ & 468 & $(3.0)$ & 461 & $(21.2)$ & 441 & $(14.3)$ & -0.27 & $(0.0)$ & -0.06 & $(0.2)$ & -0.54 & $(0.1)$ \\
\hline & Japan & 99.9 & $(0.0)$ & 0.0 & $(0.0)$ & 0.1 & $(0.0)$ & 535 & $(4.0)$ & c & c & C & $\mathrm{C}$ & -0.41 & $(0.0)$ & c & C & c & c \\
\hline & Korea & 100.0 & $(0.0)$ & 0.0 & $(0.0)$ & 0.0 & c & 543 & (3.2) & C & C & c & c & -0.36 & $(0.0)$ & c & $\mathrm{C}$ & c & c \\
\hline & Luxemburg & 66.7 & (0.6) & 15.8 & $(0.6)$ & 17.4 & $(0.5)$ & 507 & (1.3) & 476 & (3.3) & 462 & (3.7) & 0.15 & $(0.0)$ & -0.47 & $(0.1)$ & -0.65 & $(0.0)$ \\
\hline & Mexiko & 97.7 & $(0.3)$ & 0.5 & $(0.1)$ & 1.8 & $(0.2)$ & 392 & (3.6) & 333 & $(29.3)$ & 292 & $(12.7)$ & -1.27 & $(0.1)$ & -1.53 & $(0.3)$ & -1.96 & $(0.1)$ \\
\hline & Niederlande & 89.0 & (1.4) & 7.1 & (1.1) & 3.9 & $(0.4)$ & 551 & (3.0) & 492 & $(10.3)$ & 472 & $(8.4)$ & 0.00 & $(0.0)$ & -0.78 & $(0.1)$ & -0.55 & $(0.1)$ \\
\hline & Neuseeland & 80.2 & (1.1) & 6.6 & $(0.7)$ & 13.3 & $(0.7)$ & 528 & (2.6) & 496 & (8.4) & 523 & (4.9) & -0.11 & $(0.0)$ & -0.47 & $(0.1)$ & -0.10 & $(0.0)$ \\
\hline & Norwegen & 94.4 & $(0.7)$ & 2.3 & $(0.4)$ & 3.4 & $(0.4)$ & 499 & (2.3) & 460 & $(11.7)$ & 438 & (9.3) & 0.21 & $(0.0)$ & -0.06 & $(0.1)$ & -0.21 & $(0.1)$ \\
\hline & Polen & 100.0 & $(0.0)$ & 0.0 & $(0.0)$ & 0.0 & $(0.0)$ & 491 & (2.5) & C & C & C & C & -0.41 & $(0.0)$ & c & C & c & c \\
\hline & Portugal & 95.0 & (1.4) & 2.3 & $(0.4)$ & 2.7 & (1.1) & 470 & (2.9) & 440 & $(14.7)$ & 383 & $(22.0)$ & -0.91 & $(0.0)$ & -0.60 & $(0.2)$ & -1.09 & $(0.1)$ \\
\hline & Slowak. Rep. & 99.1 & $(0.2)$ & 0.6 & $(0.2)$ & 0.3 & $(0.1)$ & 499 & (3.2) & 432 & $(27.2)$ & C & c & -0.24 & $(0.0)$ & -0.38 & $(0.1)$ & c & c \\
\hline & Spanien & 96.6 & $(0.4)$ & 0.6 & $(0.1)$ & 2.8 & $(0.4)$ & 487 & (2.4) & 450 & (18.4) & 440 & (12.4) & -0.50 & $(0.0)$ & -0.81 & $(0.2)$ & -0.78 & $(0.1)$ \\
\hline & Schweden & 88.5 & $(0.9)$ & 5.7 & $(0.5)$ & 5.9 & $(0.7)$ & 517 & $(2.2)$ & 483 & (9.8) & 425 & $(9.6)$ & 0.16 & $(0.0)$ & -0.35 & $(0.1)$ & -0.48 & $(0.1)$ \\
\hline & Schweiz & 80.0 & $(0.9)$ & 8.9 & $(0.5)$ & 11.1 & (0.6) & 543 & (3.3) & 484 & (5.0) & 453 & (6.1) & -0.08 & $(0.0)$ & -0.73 & $(0.0)$ & -0.84 & $(0.1)$ \\
\hline & Türkei & 99.0 & $(0.2)$ & 0.5 & $(0.2)$ & 0.5 & $(0.1)$ & 425 & (6.7) & C & C & 385 & (28.7) & -1.15 & $(0.1)$ & c & c & -0.95 & $(0.2)$ \\
\hline & Ver. Staaten & 85.6 & $(1.0)$ & 8.3 & $(0.7)$ & 6.1 & $(0.4)$ & 490 & $(2.8)$ & 468 & (7.6) & 453 & (7.5) & 0.15 & $(0.0)$ & -0.36 & $(0.1)$ & -0.43 & $(0.1)$ \\
\hline & OECD-Durchschnitt 2003 & 91.3 & $(0.1)$ & 3.9 & $(0.1)$ & 4.7 & $(0.1)$ & 506 & $(0.6)$ & 464 & (3.1) & 452 & $(2.4)$ & -0.17 & $(0.0)$ & -0.54 & $(0.0)$ & -0.55 & $(0.0)$ \\
\hline \multirow{10}{*}{ ఏ } & Brasilien & 99.2 & $(0.2)$ & 0.6 & $(0.2)$ & 0.2 & $(0.1)$ & 359 & $(4.7)$ & c & c & $\mathrm{c}$ & $\mathrm{c}$ & -1.55 & $(0.0)$ & c & c & c & $\mathrm{c}$ \\
\hline & Hongkong (China) & 56.7 & (1.4) & 22.9 & $(0.9)$ & 20.4 & $(1.3)$ & 557 & $(4.5)$ & 570 & (4.6) & 516 & (5.3) & -1.06 & $(0.0)$ & -1.42 & $(0.0)$ & -1.69 & $(0.0)$ \\
\hline & Indonesien & 99.7 & $(0.1)$ & 0.2 & $(0.1)$ & 0.1 & $(0.0)$ & 363 & $(4.0)$ & c & c & c & C & -1.86 & $(0.0)$ & $\mathrm{C}$ & c & c & c \\
\hline & Lettland & 90.6 & $(0.9)$ & 8.3 & $(0.8)$ & 1.1 & $(0.2)$ & 484 & (3.8) & 479 & (6.6) & 498 & (11.8) & -0.37 & $(0.0)$ & -0.18 & $(0.1)$ & 0.15 & $(0.1)$ \\
\hline & Liechtenstein & 82.9 & $(2.0)$ & 7.6 & (1.3) & 9.4 & (1.6) & 545 & (5.0) & $\mathrm{C}$ & c & 482 & (20.9) & -0.20 & $(0.0)$ & c & $\mathrm{c}$ & -0.91 & $(0.2)$ \\
\hline & Macau (China) & 23.9 & (1.4) & 57.9 & (1.5) & 18.2 & (1.4) & 528 & (5.9) & 532 & $(4.1)$ & 517 & $(9.2)$ & -1.29 & $(0.1)$ & -1.67 & $(0.0)$ & -1.75 & $(0.1)$ \\
\hline & Russ. Föderation & 86.5 & $(0.7)$ & 6.4 & $(0.5)$ & 7.0 & $(0.5)$ & 472 & (4.4) & 457 & $(7.2)$ & 452 & (5.9) & -0.59 & $(0.0)$ & -0.63 & $(0.1)$ & -0.68 & $(0.1)$ \\
\hline & Thailand & 99.9 & $(0.1)$ & 0.1 & $(0.1)$ & 0.0 & $(0.0)$ & 419 & (3.0) & C & C & $\mathrm{C}$ & $\mathrm{C}$ & -1.86 & $(0.0)$ & c & C & C & $\mathrm{C}$ \\
\hline & Tunesien & 99.7 & $(0.1)$ & 0.2 & $(0.1)$ & 0.1 & $(0.0)$ & 360 & (2.5) & c & c & c & c & -1.69 & $(0.0)$ & c & c & c & c \\
\hline & Uruguay & 99.2 & $(0.2)$ & 0.4 & $(0.1)$ & 0.4 & $(0.1)$ & 423 & (3.2) & C & $\mathrm{C}$ & $\mathrm{C}$ & c & -0.76 & $(0.0)$ & c & C & C & C \\
\hline
\end{tabular}

Anmerkung: Diese Tabelle wurde nur für Schüler berechnet, für die Daten zum PISA-Index des wirtschaftlichen, sozialen und kulturellen Status vorliegen. Statistisch signifikante Werte sind durch Fettdruck gekennzeichnet (vgl. Anhang A3).

Schüler mit Migrationshintergrund sind Schüler, deren Eltern in einem anderen Land/einer anderen Volkswirtschaft als dem getesteten Land/der getesteten Volkswirtschaft geboren sind. Schüler der 2. Generation wurden im getesteten Land/in der getesteten Volkswirtschaft geboren, ihre Eltern jedoch nicht. Schüler der 1. Generation wurden nicht im getesteten Land/in der getesteten Volkswirtschaft geboren, und ihre Eltern wurden ebenfalls nicht im getesteten Land/in der getesteten Volkswirtschaft geboren.

Um die Vergleichbarkeit im Zeitverlauf zU gewährleisten, wurden die Werte von PISA 2003 auf dem PISA-Index des wirtschaftlichen, sozialen und kulturellen Status entsprechend der Indexskala von PISA 2012 umskaliert. Die in dieser Abbildung dargestellten Ergebnisse aus PISA 2003 können daher von denen abweichen, die in Lernen für die Welt von morgen: Erste Ergebnisse von PISA 2003 (OECD, 2004) wiedergegeben sind (vgl. Anhang A5 wegen näherer Einzelheiten).

Angegeben sind nur Länder und Volkswirtschaften mit vergleichbaren Daten aus PISA 2003 und PISA 2012.

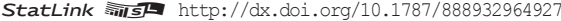


[Teil 2/6]

Von 2003 bis 2012 beobachtete Veränderung des Zusammenhangs zwischen Mathematikleistungen und Migrationshintergrund für Schüler der 1. und 2. Generation

Tabelle II.3.6b Die Ergebnisse basieren auf Schülerangaben

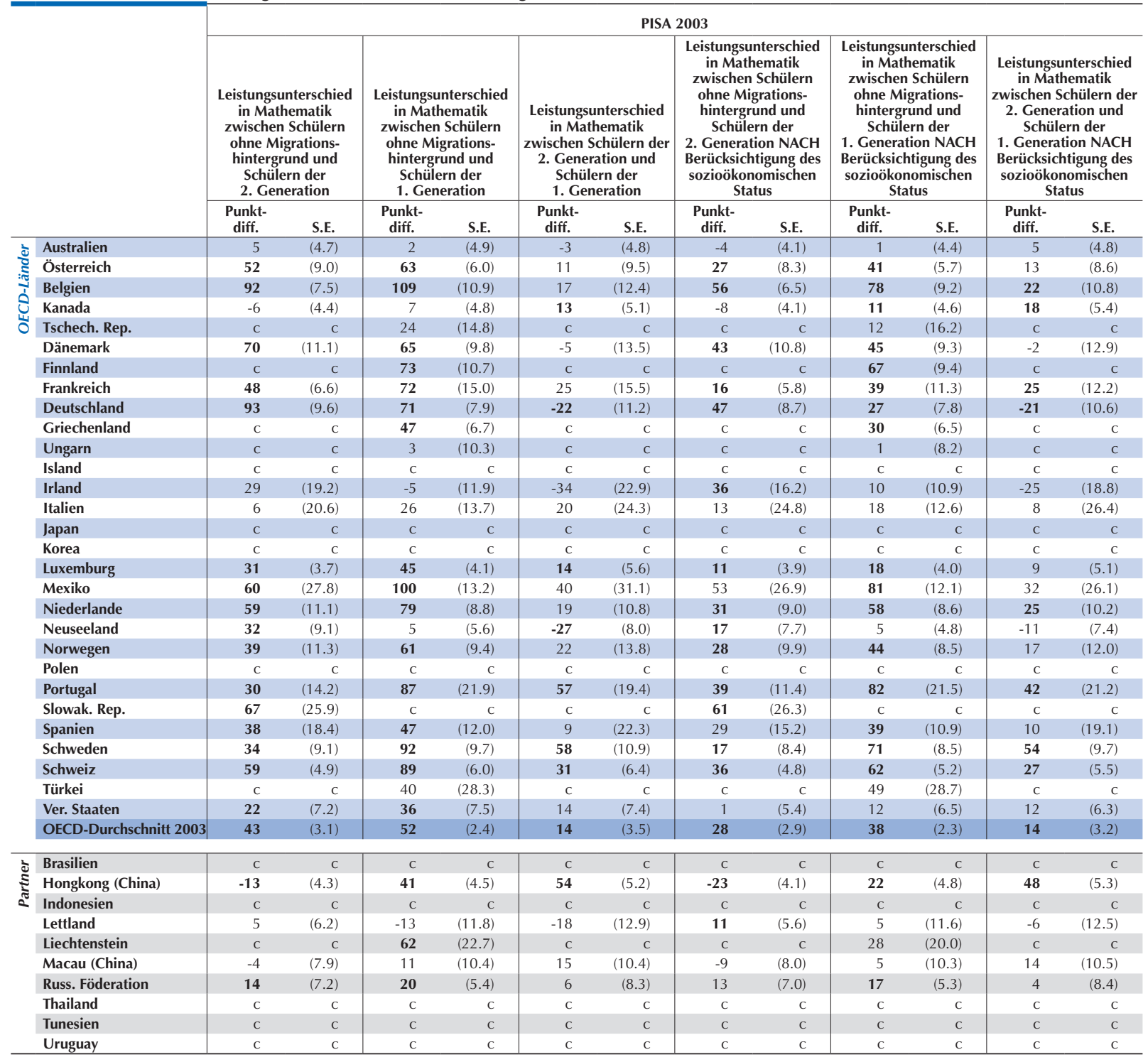

Anmerkung: Diese Tabelle wurde nur für Schüler berechnet, für die Daten zum PISA-Index des wirtschaftlichen, sozialen und kulturellen Status vorliegen. Statistisch signifikante Werte sind durch Fettdruck gekennzeichnet (vgl. Anhang A3).

Schüler mit Migrationshintergrund sind Schüler, deren Eltern in einem anderen Land/einer anderen Volkswirtschaft als dem getesteten Land/der getesteten Volkswirtschaft geboren sind. Schüler der 2. Generation wurden im getesteten Land/in der getesteten Volkswirtschaft geboren, ihre Eltern jedoch nicht. Schüler der 1. Generation wurden nicht im getesteten Land/in der getesteten Volkswirtschaft geboren, und ihre Eltern wurden ebenfalls nicht im getesteten Land/in der getesteten Volkswirtschaft geboren.

Um die Vergleichbarkeit im Zeitverlauf zu gewährleisten, wurden die Werte von PISA 2003 auf dem PISA-Index des wirtschaftlichen, sozialen und kulturellen Status entsprechend der Indexskala von PISA 2012 umskaliert. Die in dieser Abbildung dargestellten Ergebnisse aus PISA 2003 können daher von denen abweichen, die in Lernen für die Welt von morgen: Erste Ergebnisse von PISA 2003 (OECD, 2004) wiedergegeben sind (vgl. Anhang A5 wegen näherer Einzelheiten).

Angegeben sind nur Länder und Volkswirtschaften mit vergleichbaren Daten aus PISA 2003 und PISA 2012.

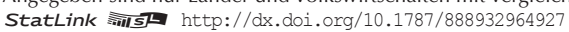


[Teil 3/6]

Von 2003 bis 2012 beobachtete Veränderung des Zusammenhangs zwischen Mathematikleistungen und Migrationshintergrund für Schüler der 1. und 2. Generation

Tabelle II.3.6b Die Ergebnisse basieren auf Schülerangaben

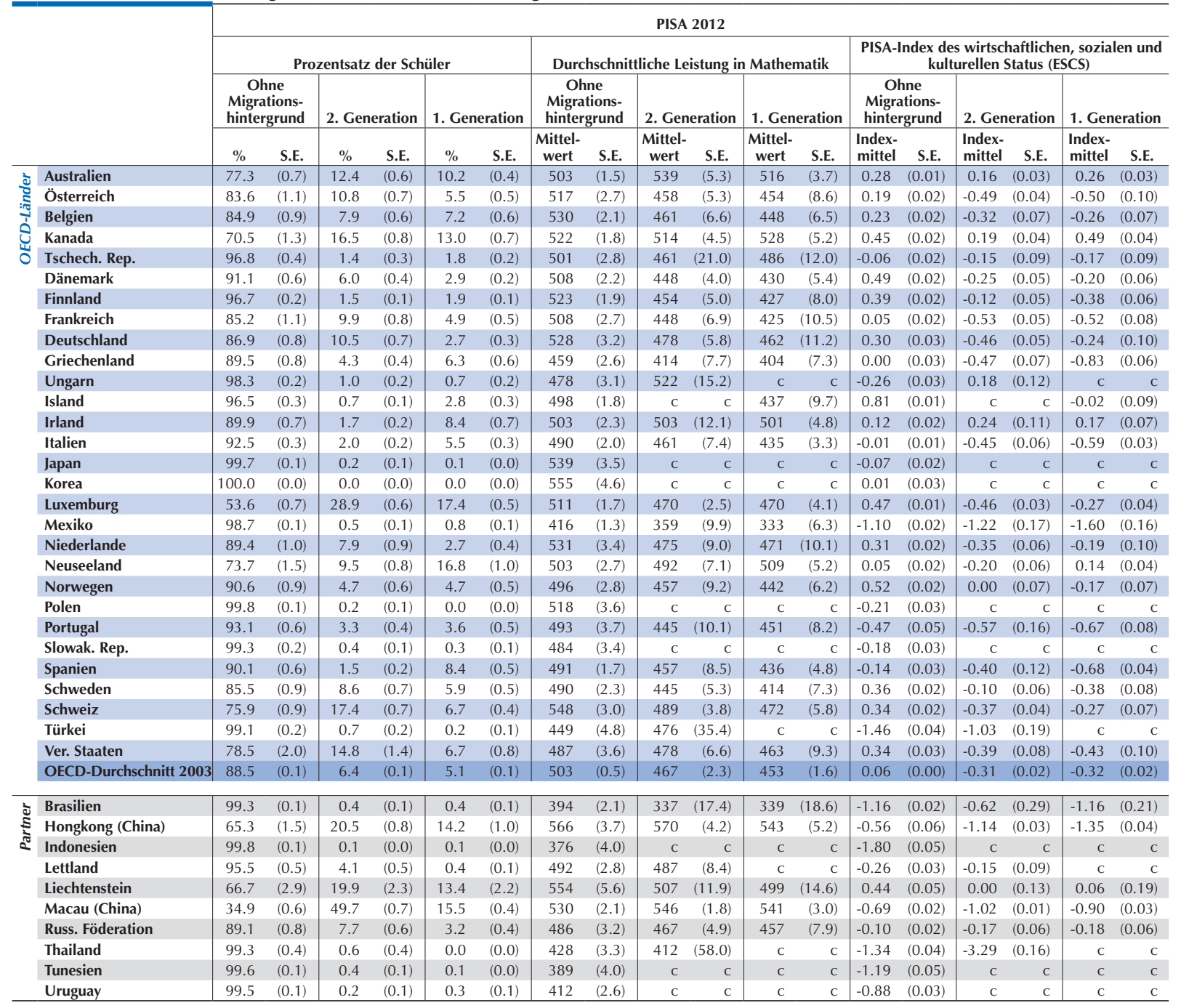

Anmerkung: Diese Tabelle wurde nur für Schüler berechnet, für die Daten zum PISA-Index des wirtschaftlichen, sozialen und kulturellen Status vorliegen. Statistisch signifikante Werte sind durch Fettdruck gekennzeichnet (vgl. Anhang A3).

Schüler mit Migrationshintergrund sind Schüler, deren Eltern in einem anderen Land/einer anderen Volkswirtschaft als dem getesteten Land/der getesteten Volkswirtschaft geboren sind. Schüler der 2. Generation wurden im getesteten Land/in der getesteten Volkswirtschaft geboren, ihre Eltern jedoch nicht. Schüler der 1. Generation wurden nicht im getesteten Land/in der getesteten Volkswirtschaft geboren, und ihre Eltern wurden ebenfalls nicht im getesteten Land/in der getesteten Volkswirtschaft geboren.

Um die Vergleichbarkeit im Zeitverlauf zu gewährleisten, wurden die Werte von PISA 2003 auf dem PISA-Index des wirtschaftlichen, sozialen und kulturellen Status entsprechend der Indexskala von PISA 2012 umskaliert. Die in dieser Abbildung dargestellten Ergebnisse aus PISA 2003 können daher von denen abweichen, die in Lernen für die Welt von morgen: Erste Ergebnisse von PISA 2003 (OECD, 2004) wiedergegeben sind (vgl. Anhang A5 wegen näherer Einzelheiten).

Angegeben sind nur Länder und Volkswirtschaften mit vergleichbaren Daten aus PISA 2003 und PISA 2012.

StatLink त्तils http://dx.doi.org/10.1787/888932964927 
[Teil4/6]

Von 2003 bis 2012 beobachtete Veränderung des Zusammenhangs zwischen Mathematikleistungen und Migrationshintergrund für Schüler der 1. und 2. Generation

Tabelle II.3.6b Die Ergebnisse basieren auf Schülerangaben

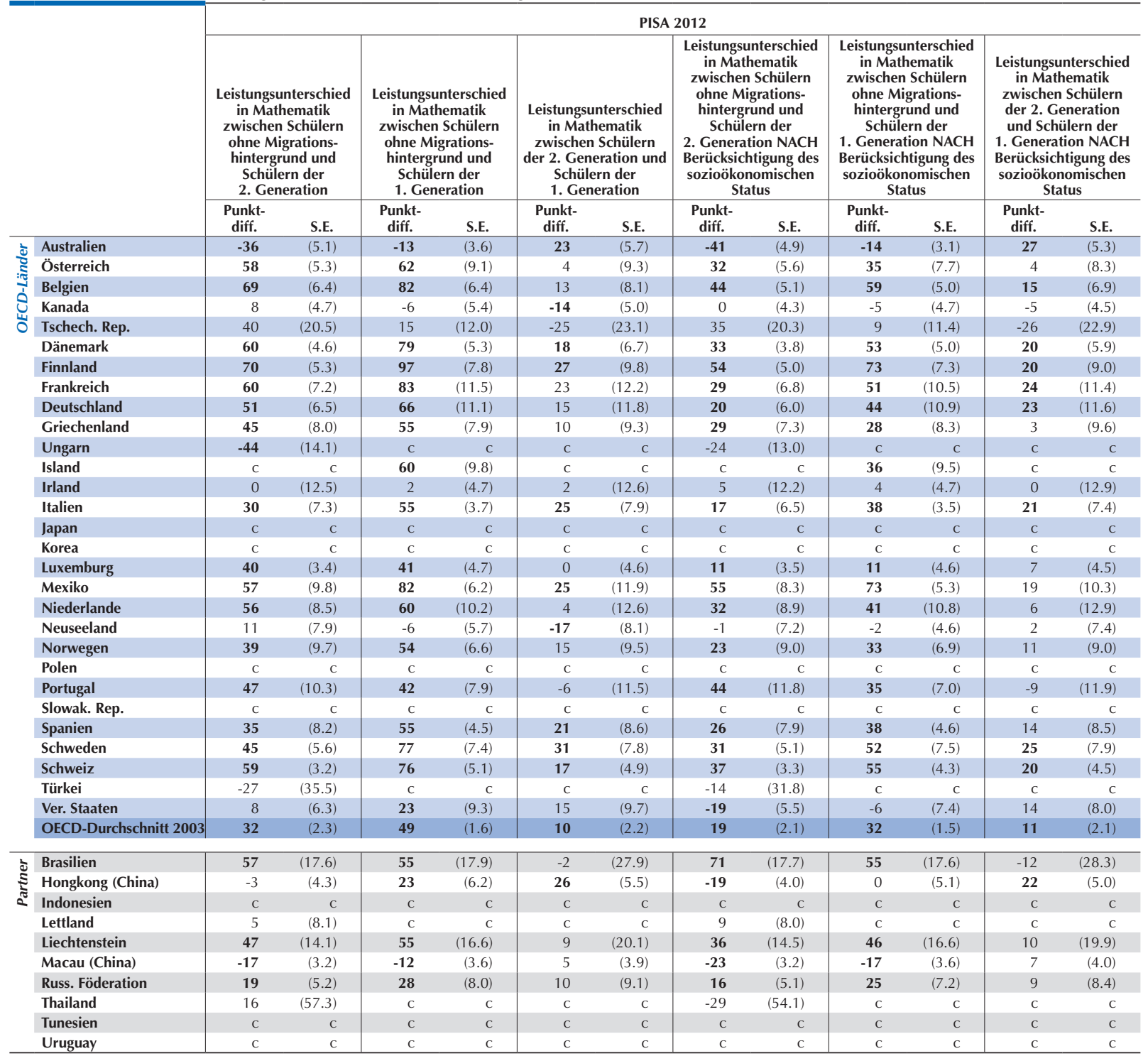

Anmerkung: Diese Tabelle wurde nur für Schüler berechnet, für die Daten zum PISA-Index des wirtschaftlichen, sozialen und kulturellen Status vorliegen. Statistisch signifikante Werte sind durch Fettdruck gekennzeichnet (vgl. Anhang A3).

Schüler mit Migrationshintergrund sind Schüler, deren Eltern in einem anderen Land/einer anderen Volkswirtschaft als dem getesteten Land/der getesteten Volkswirtschaft geboren sind. Schüler der 2. Generation wurden im getesteten Land/in der getesteten Volkswirtschaft geboren, ihre Eltern jedoch nicht. Schüler der 1. Generation wurden nicht im getesteten Land/in der getesteten Volkswirtschaft geboren, und ihre Eltern wurden ebenfalls nicht im getesteten Land/in der getesteten Volkswirtschaft geboren.

Um die Vergleichbarkeit im Zeitverlauf zu gewährleisten, wurden die Werte von PISA 2003 auf dem PISA-Index des wirtschaftlichen, sozialen und kulturellen Status entsprechend der Indexskala von PISA 2012 umskaliert. Die in dieser Abbildung dargestellten Ergebnisse aus PISA 2003 können daher von denen abweichen, die in Lernen für die Welt von morgen: Erste Ergebnisse von PISA 2003 (OECD, 2004) wiedergegeben sind (vgl. Anhang A5 wegen näherer Einzelheiten).

Angegeben sind nur Länder und Volkswirtschaften mit vergleichbaren Daten aus PISA 2003 und PISA 2012

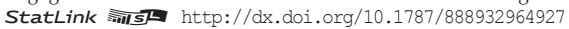


[Teil 5/6]

Von 2003 bis 2012 beobachtete Veränderung des Zusammenhangs zwischen Mathematikleistungen und Migrationshintergrund für Schüler der 1. und 2. Generation

Tabelle II.3.6b Die Ergebnisse basieren auf Schülerangaben

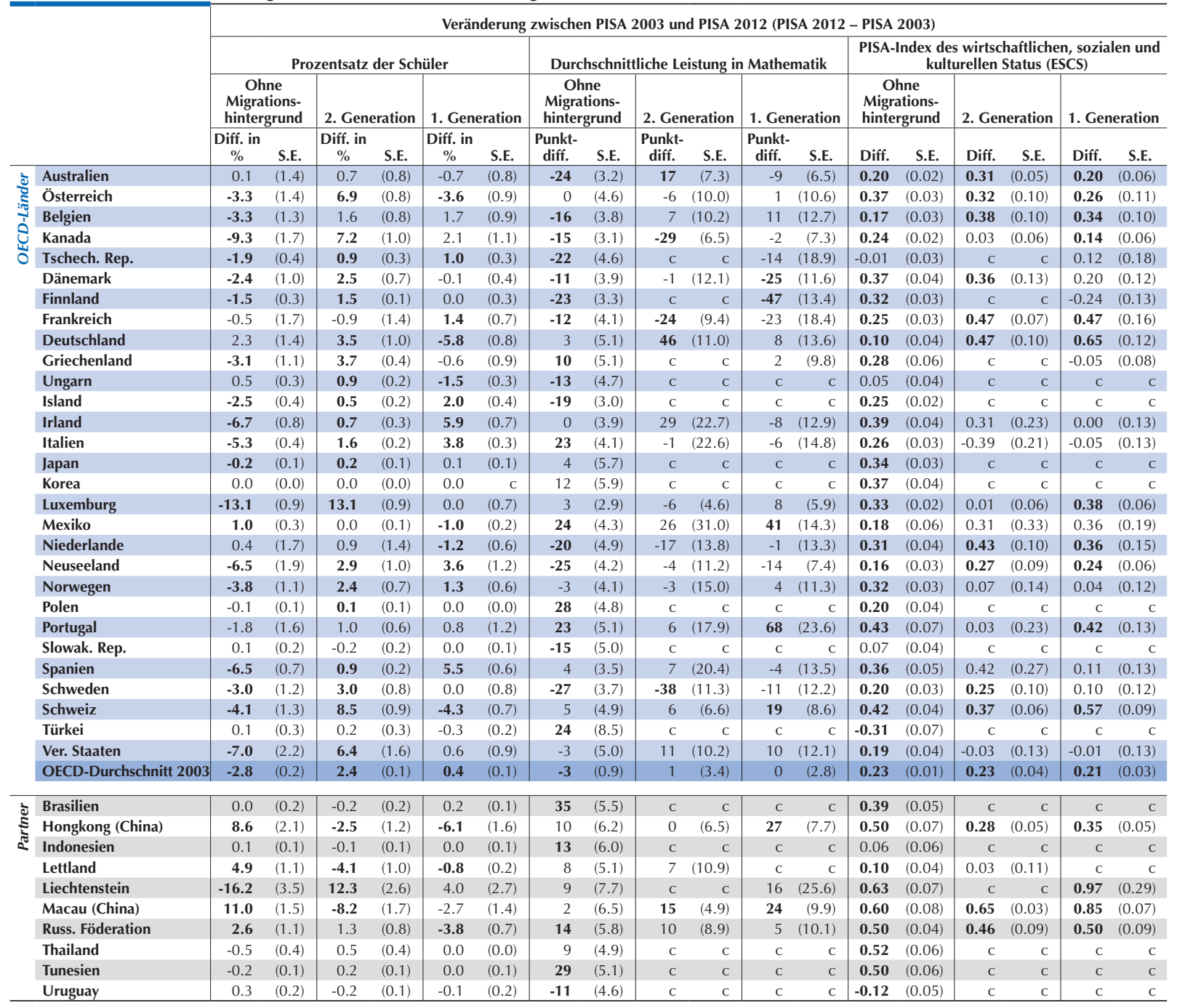

Anmerkung: Diese Tabelle wurde nur für Schüler berechnet, für die Daten zum PISA-Index des wirtschaftlichen, sozialen und kulturellen Status vorliegen. Statistisch signifikante Werte sind durch Fettdruck gekennzeichnet (vgl. Anhang A3).

Schüler mit Migrationshintergrund sind Schüler, deren Eltern in einem anderen Land/einer anderen Volkswirtschaft als dem getesteten Land/der getesteten Volkswirtschaft geboren sind. Schüler der 2. Generation wurden im getesteten Land/in der getesteten Volkswirtschaft geboren, ihre Eltern jedoch nicht. Schüler der 1. Generation wurden nicht im getesteten Land/in der getesteten Volkswirtschaft geboren, und ihre Eltern wurden ebenfalls nicht im getesteten Land/in der getesteten Volkswirtschaft geboren.

Um die Vergleichbarkeit im Zeitverlauf zu gewährleisten, wurden die Werte von PISA 2003 auf dem PISA-Index des wirtschaftlichen, sozialen und kulturellen Status entsprechend der Indexskala von PISA 2012 umskaliert. Die in dieser Abbildung dargestellten Ergebnisse aus PISA 2003 können daher von denen abweichen, die in Lernen für die Welt von morgen: Erste Ergebnisse von PISA 2003 (OECD, 2004) wiedergegeben sind (vgl. Anhang A5 wegen näherer Einzelheiten).

Angegeben sind nur Länder und Volkswirtschaften mit vergleichbaren Daten aus PISA 2003 und PISA 2012.

StatLink 刑 SD http://dx.doi.org/10.1787/888932964927 
[Teil 6/6]

Von 2003 bis 2012 beobachtete Veränderung des Zusammenhangs zwischen Mathematikleistungen und Migrationshintergrund für Schüler der 1. und 2. Generation

Tabelle II.3.6b Die Ergebnisse basieren auf Schülerangaben

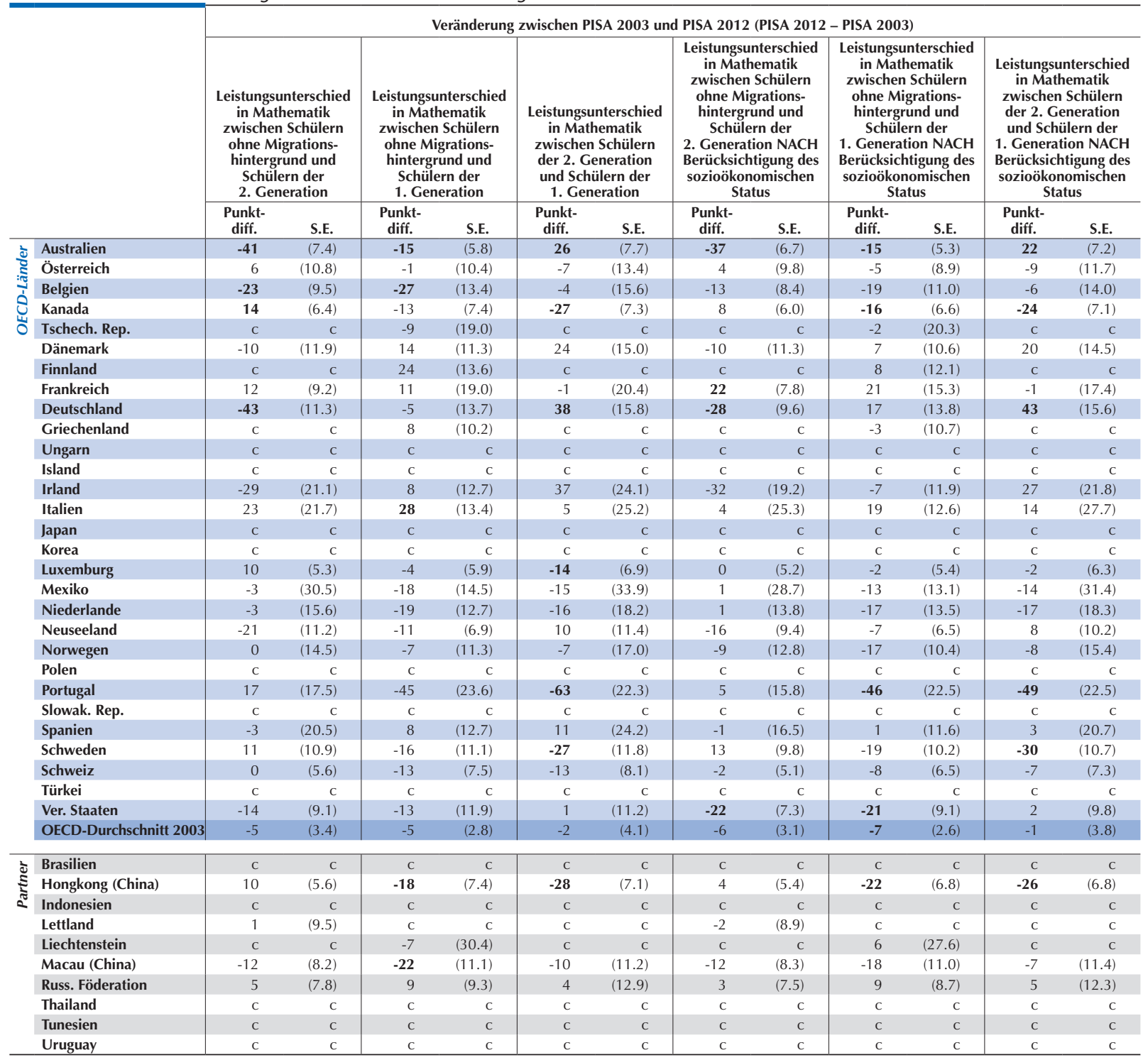

Anmerkung: Diese Tabelle wurde nur für Schüler berechnet, für die Daten zum PISA-Index des wirtschaftlichen, sozialen und kulturellen Status vorliegen. Statistisch signifikante Werte sind durch Fettdruck gekennzeichnet (vgl. Anhang A3).

Schüler mit Migrationshintergrund sind Schüler, deren Eltern in einem anderen Land/einer anderen Volkswirtschaft als dem getesteten Land/der getesteten Volkswirtschaft geboren sind. Schüler der 2. Generation wurden im getesteten Land/in der getesteten Volkswirtschaft geboren, ihre Eltern jedoch nicht. Schüler der 1. Generation wurden nicht im getesteten Land/in der getesteten Volkswirtschaft geboren, und ihre Eltern wurden ebenfalls nicht im getesteten Land/in der getesteten Volkswirtschaft geboren.

Um die Vergleichbarkeit im Zeitverlauf zu gewährleisten, wurden die Werte von PISA 2003 auf dem PISA-Index des wirtschaftlichen, sozialen und kulturellen Status entsprechend der Indexskala von PISA 2012 umskaliert. Die in dieser Abbildung dargestellten Ergebnisse aus PISA 2003 können daher von denen abweichen, die in Lernen für die Welt von morgen: Erste Ergebnisse von PISA 2003 (OECD, 2004) wiedergegeben sind (vgl. Anhang A5 wegen näherer Einzelheiten).

Angegeben sind nur Länder und Volkswirtschaften mit vergleichbaren Daten aus PISA 2003 und PISA 2012.

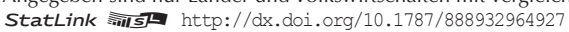


[Teil 1/1]

Kompetenzstufen im Bereich Mathematik, nach Migrationshintergrund für Schüler der

1. und 2. Generation

Tabelle II.3.7 Die Ergebnisse basieren auf Schülerangaben

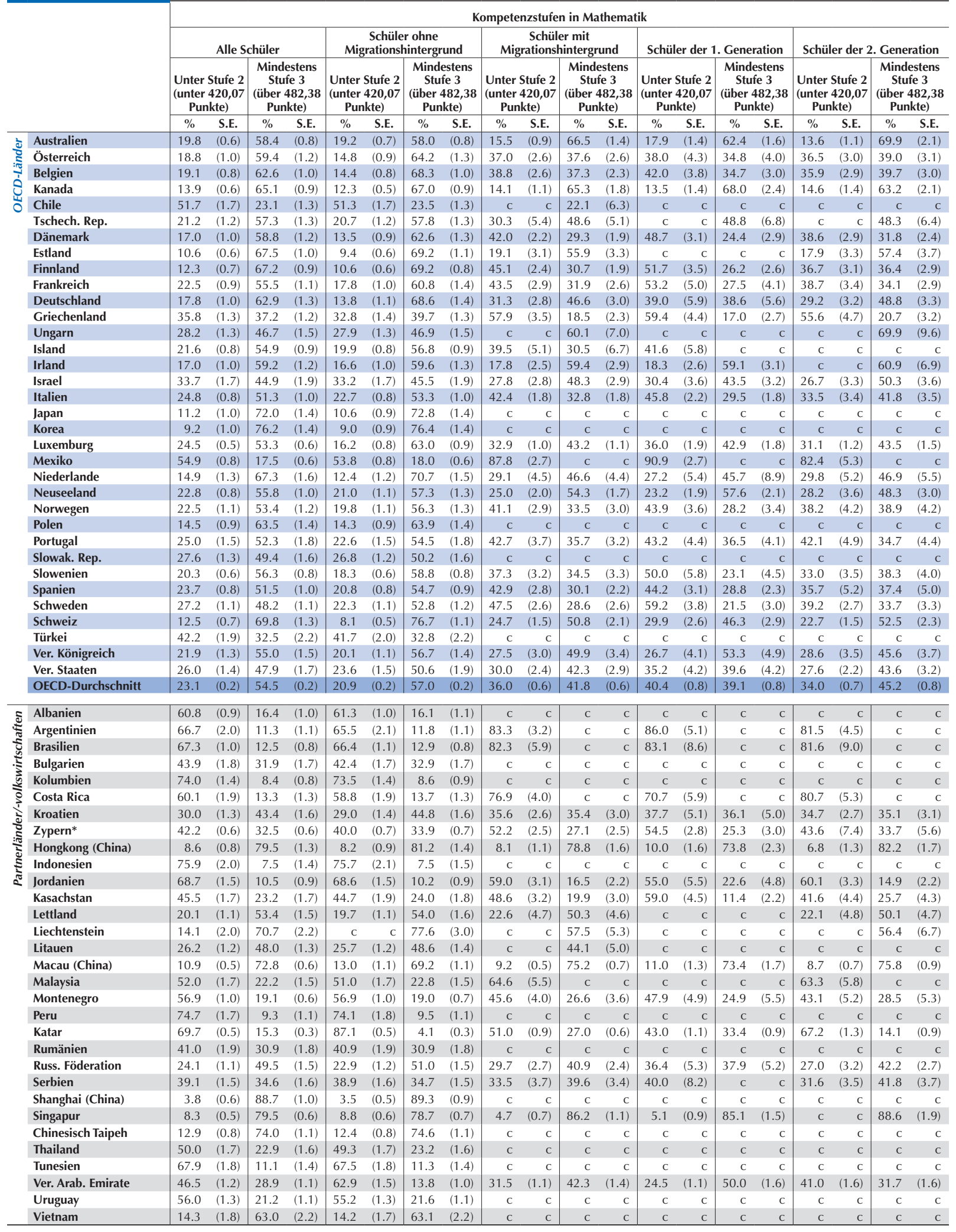

*Vgl. die Anmerkungen am Beginn dieses Anhangs.

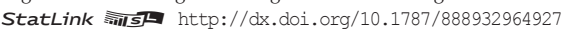


[Teil 1/2]

Mathematikleistungen, Schüler der 1. Generation und Zuwanderungsalter

Tabelle II.3.8 Die Ergebnisse basieren auf Schülerangaben

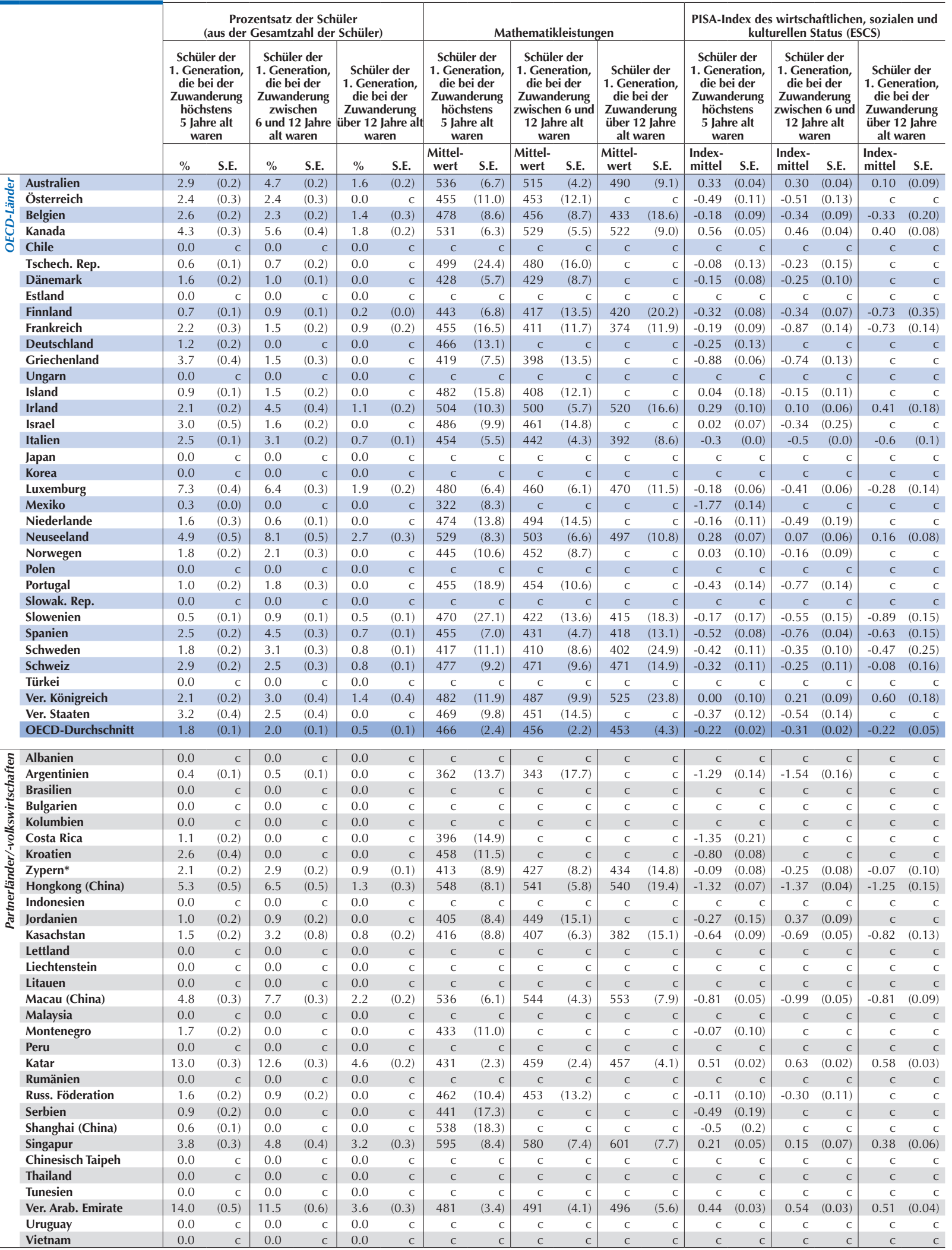

Anmerkung: Statistisch signifikante Werte sind durch Fettdruck gekennzeichnet (vgl. Anhang A3).

*Vgl. die Anmerkungen am Beginn dieses Anhangs.

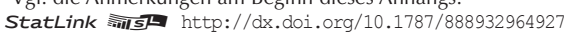


eil $2 / 2$

Mathematikleistungen, Schüler der 1. Generation und Zuwanderungsalter Tabelle II.3.8 Die Ergebnisse basieren auf Schülerangaben

\begin{tabular}{|c|c|c|c|c|c|c|c|c|c|c|c|c|c|c|c|}
\hline & & \multicolumn{14}{|c|}{$\begin{array}{l}\text { Leistungsunterschied in Mathematik zwischen Schülern ohne Migrationshintergrund und Schülern der 1. Generation } \\
\text { (im Modell ist eine Dummy-Variable für Schüler der 2. Generation enthalten, aber nicht aufgeführt) }\end{array}$} \\
\hline & & \multicolumn{2}{|c|}{ Beobachtet } & \multicolumn{2}{|c|}{$\begin{array}{c}\text { Nach Berücksich- } \\
\text { tigung des } \\
\text { Zuwanderungsalters }\end{array}$} & \multicolumn{2}{|c|}{$\begin{array}{c}\text { Nachteil auf } \\
\text { Grund der späten } \\
\text { Zuwanderung } \\
\text { (Alterskoeffizient) }\end{array}$} & \multicolumn{2}{|c|}{\begin{tabular}{|c|} 
Nach Berücksich- \\
tigung des \\
Zuwanderungsalters \\
und des ESCS der \\
Schüler
\end{tabular}} & \multicolumn{2}{|c|}{$\begin{array}{c}\text { Nachteil auf } \\
\text { Grund der späten } \\
\text { Zuwanderung } \\
\text { (Alterskoeffizient) } \\
\text { nach Berücksichti- } \\
\text { gung des ESCS der } \\
\text { Schüler }\end{array}$} & \multicolumn{2}{|c|}{\begin{tabular}{|c|} 
Nach Berücksich- \\
tigung des \\
Zuwanderungsalters \\
und des ESCS der \\
Schüler und der \\
Schule \\
\end{tabular}} & \multicolumn{2}{|c|}{$\begin{array}{c}\text { Nachteil auf } \\
\text { Grund der späten } \\
\text { Zuwanderung } \\
\text { (Alterskoeffizient) } \\
\text { nach Berücksich- } \\
\text { tigung des ESCS der } \\
\text { Schüler und der } \\
\text { Schule }\end{array}$} \\
\hline & & $\begin{array}{l}\text { Punkt- } \\
\text { diff. }\end{array}$ & S.E. & $\begin{array}{l}\text { Punkt- } \\
\text { diff. }\end{array}$ & S.E. & $\begin{array}{c}\text { Punkt- } \\
\text { diff. }\end{array}$ & S.E. & $\begin{array}{l}\text { Punkt- } \\
\text { diff. }\end{array}$ & S.E. & $\begin{array}{c}\text { Punkt- } \\
\text { diff. }\end{array}$ & S.E. & $\begin{array}{l}\text { Punkt- } \\
\text { diff. }\end{array}$ & S.E. & $\begin{array}{l}\text { Punkt- } \\
\text { diff. }\end{array}$ & S.E. \\
\hline ¿ & Australien & 15 & (3.6) & 41 & $(7.4)$ & -3 & $(0.8)$ & 35 & $(7.0)$ & -2 & $(0.7)$ & 30 & (6.9) & -2 & $(0.7)$ \\
\hline & Österreich & -63 & (9.3) & -73 & (14.4) & 2 & (1.8) & -42 & (12.4) & 1 & (1.6) & -41 & $(10.1)$ & 2 & (1.3) \\
\hline ปั & Belgien & -82 & (6.4) & -61 & (9.4) & -3 & (1.4) & -44 & (8.1) & -2 & (1.1) & -40 & (7.5) & -1 & $(0.8)$ \\
\hline U్ & Kanada & 7 & (5.4) & 15 & (8.0) & -1 & $(0.7)$ & 10 & (7.1) & 0 & $(0.7)$ & 8 & (7.0) & -1 & $(0.7)$ \\
\hline & Chile & c & $\mathrm{c}$ & c & c & c & $\mathrm{c}$ & c & c & c & $\mathrm{c}$ & c & c & c & c \\
\hline & Tschech. Rep. & -15 & $(12.1)$ & 10 & $(25.0)$ & -3 & (2.4) & 3 & $(26.1)$ & -1 & (2.6) & -22 & (19.7) & 0 & (2.1) \\
\hline & Dänemark & -79 & (5.4) & -81 & (9.6) & 0 & $(1.8)$ & -59 & (9.6) & 1 & (1.6) & -54 & (9.6) & 1 & (1.6) \\
\hline & Estland & $\mathrm{C}$ & c & c & c & c & c & C & c & c & c & C & c & c & c \\
\hline & Finnland & -96 & (7.9) & -75 & (8.7) & -3 & (1.6) & -57 & (7.3) & -2 & $(1.3)$ & -59 & (7.1) & -2 & $(1.2)$ \\
\hline & Frankreich & -83 & (11.8) & -43 & (19.4) & -6 & (1.8) & -32 & $(17.1)$ & -3 & (1.6) & $w$ & w & w & w \\
\hline & Deutschland & -64 & (11.3) & -62 & (19.6) & 0 & (3.2) & -41 & $(18.2)$ & 0 & (3.0) & -25 & $(15.8)$ & -1 & (2.6) \\
\hline & Griechenland & -55 & $(7.9)$ & -37 & (9.8) & -2 & (1.5) & -8 & (9.8) & -2 & (1.5) & -7 & (8.0) & 0 & (1.4) \\
\hline & Ungarn & $\mathrm{C}$ & c & c & c & $\mathrm{C}$ & c & c & c & c & c & C & c & c & c \\
\hline & Island & -61 & $(10.2)$ & 5 & $(20.0)$ & -9 & $(2.2)$ & 25 & (18.9) & -8 & (2.1) & 27 & (18.6) & -8 & (2.0) \\
\hline & Irland & 0 & (4.9) & 2 & (12.4) & 0 & (1.4) & -5 & (11.6) & 1 & (1.2) & -2 & (11.6) & 1 & (1.2) \\
\hline & Israel & 0 & (8.0) & 29 & $(14.8)$ & -5 & (2.3) & 39 & $(12.5)$ & -4 & (2.0) & 33 & (11.6) & -5 & (2.1) \\
\hline & Italien & -55 & (3.7) & -30 & (8.0) & -3 & $(0.9)$ & -15 & (8.3) & -3 & $(0.9)$ & -12 & (7.8) & -2 & $(0.8)$ \\
\hline & Japan & c & c & c & c & c & c & c & c & c & c & c & c & c & c \\
\hline & Korea & c & c & c & c & c & c & c & c & c & c & c & c & c & c \\
\hline & Luxemburg & -40 & (4.7) & -34 & (7.9) & -1 & $(0.8)$ & -11 & (7.4) & 0 & $(0.7)$ & -19 & (6.9) & 0 & $(0.6)$ \\
\hline & Mexiko & -83 & (6.4) & -98 & (8.5) & 5 & (1.7) & -83 & (8.0) & 2 & (1.6) & -77 & (7.5) & 0 & (1.7) \\
\hline & Niederlande & -60 & (10.6) & -56 & (16.6) & 1 & $(2.2)$ & -37 & $(16.0)$ & 1 & (2.3) & -42 & (13.9) & 1 & (1.9) \\
\hline & Neuseeland & 7 & (5.8) & 37 & (11.3) & -3 & (1.1) & 25 & $(10.0)$ & -3 & (1.0) & 22 & (9.3) & -2 & (1.0) \\
\hline & Norwegen & -54 & (6.7) & -45 & (13.5) & -1 & (1.5) & -34 & (14.4) & 0 & (1.6) & -32 & (13.8) & 0 & (1.5) \\
\hline & Polen & c & c & c & c & c & c & c & c & c & c & c & c & c & c \\
\hline & Portugal & -43 & (8.0) & -14 & $(24.7)$ & -4 & (2.5) & -17 & $(22.3)$ & -2 & $(2.3)$ & -14 & $(22.5)$ & -3 & (2.3) \\
\hline & Slowak. Rep. & c & c & c & c & c & c & c & c & c & c & C & c & c & c \\
\hline & Slowenien & -74 & (10.6) & -31 & $(24.8)$ & -4 & (2.1) & -24 & (21.9) & -2 & (2.1) & -45 & $(21.4)$ & 0 & (2.5) \\
\hline & Spanien & -56 & (4.5) & -24 & (7.3) & -4 & (0.9) & -12 & (7.5) & -3 & $(0.8)$ & -9 & $(7.2)$ & -4 & $(0.8)$ \\
\hline & Schweden & -76 & (7.9) & -60 & (14.6) & -2 & (1.7) & -37 & (14.4) & -2 & (1.5) & -34 & $(14.3)$ & -2 & (1.5) \\
\hline & Schweiz & -77 & (5.1) & -70 & (10.6) & -1 & (1.3) & -48 & (8.9) & -1 & (1.1) & -51 & (7.4) & -1 & (1.0) \\
\hline & Türkei & c & C & c & c & c & c & c & c & c & c & C & c & c & c \\
\hline & Ver. Königreich & -3 & (11.7) & -30 & (11.9) & 4 & (2.0) & -12 & $(12.3)$ & 2 & $(1.7)$ & 2 & (9.8) & 0 & $(1.2)$ \\
\hline & Ver. Staaten & -23 & (9.6) & -27 & $(12.3)$ & 1 & (1.5) & 5 & (10.0) & 0 & (1.3) & 10 & (9.7) & 0 & (1.3) \\
\hline & OECD-Durchschnitt & -47 & (1.6) & -31 & (2.9) & -2 & $(0.3)$ & -18 & (2.7) & -1 & $(0.3)$ & -18 & (2.5) & -1 & $(0.3)$ \\
\hline
\end{tabular}

\begin{tabular}{|c|c|c|c|c|c|c|c|c|c|c|c|c|c|c|}
\hline § Albanien & c & c & c & c & c & C & c & C & c & c & c & c & C & $\mathrm{c}$ \\
\hline$\approx$ Argentinien & -39 & (10.0) & -33 & (15.6) & -1 & (1.9) & -18 & (15.5) & 0 & (2.0) & -17 & (14.5) & 1 & (1.9) \\
\hline Brasilien & c & c & c & c & c & c & c & c & c & c & c & c & c & c \\
\hline Bulgarien & c & C & c & C & c & c & c & c & c & c & c & c & c & c \\
\hline Kolumbien & c & c & c & c & c & c & c & c & c & c & c & c & c & c \\
\hline Costa Rica & -20 & $(10.5)$ & -10 & $(17.0)$ & -2 & $(2.0)$ & -1 & $(15.2)$ & -2 & (1.9) & -7 & (14.5) & -2 & (1.9) \\
\hline Kroatien & -21 & (10.1) & -11 & (13.7) & -2 & (1.7) & 9 & $(13.2)$ & -3 & (1.8) & 7 & (12.0) & -3 & (2.0) \\
\hline Zypern* & -23 & (5.5) & -29 & $(10.6)$ & 1 & (1.1) & -22 & (9.6) & 2 & $(1.0)$ & -29 & (8.9) & 2 & (1.0) \\
\hline Hongkong (China) & -24 & (6.6) & -16 & (9.9) & -1 & $(1.2)$ & 5 & (9.2) & -1 & (1.1) & 6 & (8.8) & -1 & (1.0) \\
\hline Indonesien & c & $\mathrm{C}$ & c & $\mathrm{C}$ & $\mathrm{c}$ & C & c & $\mathrm{C}$ & c & c & c & c & c & C \\
\hline Jordanien & 29 & (8.7) & 21 & (11.6) & 3 & (1.8) & 17 & (10.9) & 1 & (1.6) & 10 & $(10.7)$ & 0 & (1.5) \\
\hline Kasachstan & -26 & (6.3) & -7 & $(10.4)$ & -3 & $(1.2)$ & -2 & $(10.4)$ & -2 & $(1.2)$ & 1 & $(10.4)$ & -1 & $(1.2)$ \\
\hline Lettland & c & $\mathrm{C}$ & c & C & $\mathrm{c}$ & c & c & c & C & c & C & c & c & C \\
\hline Liechtenstein & c & $\mathrm{C}$ & c & C & c & c & c & C & C & c & c & c & c & c \\
\hline Litauen & c & c & c & c & c & c & c & c & c & c & c & c & c & c \\
\hline Macau (China) & 11 & (3.7) & -2 & (7.7) & 2 & $(0.8)$ & 3 & (7.6) & 2 & $(0.8)$ & 8 & (7.7) & 2 & $(0.9)$ \\
\hline Malaysia & c & c & c & c & c & c & c & c & c & c & c & c & c & c \\
\hline Montenegro & 19 & (8.9) & 27 & (13.3) & -1 & (1.8) & 17 & $(12.1)$ & 0 & (1.8) & 12 & (10.6) & 1 & (1.7) \\
\hline Peru & c & c & c & c & c & c & c & c & c & c & c & c & c & c \\
\hline Katar & 109 & (1.9) & 94 & (2.8) & 3 & $(0.3)$ & 92 & $(2.7)$ & 3 & $(0.3)$ & 90 & (2.5) & 2 & $(0.3)$ \\
\hline Rumänien & c & c & c & c & c & c & c & c & c & c & C & C & C & C \\
\hline Russ. Föderation & -29 & (8.2) & -26 & (12.6) & -1 & (1.9) & -27 & $(14.2)$ & 0 & (1.9) & -28 & $(15.2)$ & 0 & (2.0) \\
\hline Serbien & -10 & $(12.4)$ & 1 & $(22.1)$ & -1 & (3.3) & 9 & (18.6) & -2 & (2.9) & 13 & (15.0) & -4 & (2.4) \\
\hline Shanghai (China) & -104 & (13.9) & -141 & $(25.8)$ & 5 & (3.0) & -114 & $(28.6)$ & 5 & (3.3) & -88 & $(22.3)$ & 5 & (3.0) \\
\hline Singapur & 20 & $(4.8)$ & 20 & (8.7) & 0 & $(0.9)$ & -1 & (7.9) & 0 & $(0.9)$ & -12 & (8.0) & 0 & (0.9) \\
\hline Chinesisch Taipeh & c & C & c & C & c & c & c & C & C & c & c & c & C & C \\
\hline Thailand & c & $\mathrm{C}$ & c & $\mathrm{C}$ & c & c & c & C & C & c & c & c & C & c \\
\hline Tunesien & c & C & c & c & c & C & c & c & C & c & c & c & C & C \\
\hline Ver. Arab. Emirate & 84 & (3.5) & 78 & $(4.2)$ & 1 & $(0.4)$ & 74 & $(4.3)$ & 1 & $(0.4)$ & 67 & (4.9) & 1 & $(0.3)$ \\
\hline Uruguay & c & C & c & C & c & c & c & C & c & c & C & c & C & C \\
\hline Vietnam & $\mathrm{C}$ & $\mathrm{C}$ & c & $\mathrm{C}$ & c & $\mathrm{C}$ & c & $\mathrm{C}$ & C & C & $\mathrm{C}$ & $\mathrm{C}$ & $\mathrm{C}$ & $\mathrm{C}$ \\
\hline
\end{tabular}

Anmerkung: Statistisch signifikante Werte sind durch Fettdruck gekennzeichnet (vgl. Anhang A3)

$* V$ gl. die Anmerkungen am Beginn dieses Anhangs.

StatLink inils ht htp://dx.doi.org/10.1787/888932964927 
[Teil 1/2]

Konzentration der Schüler mit Migrationshintergrund in der Schule Tabelle II.3.9 Die Ergebnisse basieren auf Schülerangaben

\begin{tabular}{|c|c|c|c|c|c|c|c|c|c|c|c|}
\hline & \multicolumn{10}{|c|}{ Prozentsatz der Schüler } \\
\hline & & \multicolumn{2}{|c|}{$\begin{array}{c}\text { Schüler mit } \\
\text { Migrationshintergrund }\end{array}$} & \multicolumn{2}{|c|}{$\begin{array}{l}\text { In Schulen, wo der } \\
\text { Prozentsatz der Schüler } \\
\text { mit Migrationshintergrund } \\
\text { gleich null ist }\end{array}$} & \multicolumn{2}{|c|}{$\begin{array}{c}\text { In Schulen, wo der } \\
\text { Prozentsatz der Schüler mit } \\
\text { Migrationshintergrund über } \\
\mathbf{0 \%} \text {, aber unter } 10 \% \text { liegt } \\
\text { (geringe Konzentration) }\end{array}$} & \multicolumn{2}{|c|}{$\begin{array}{l}\text { In Schulen, wo der } \\
\text { Prozentsatz der Schüler } \\
\text { mit Migrationshintergrund } \\
\text { mindestens } 10 \% \text {, aber } \\
\text { weniger als } 25 \% \text { beträgt } \\
\text { (mittlere Konzentration) }\end{array}$} & \multicolumn{2}{|c|}{$\begin{array}{l}\text { In Schulen, wo der } \\
\text { Prozentsatz der Schüler } \\
\text { mit Migrationshintergrund } \\
\text { mindestens } 25 \% \text { beträgt } \\
\text { (hohe Konzentration) }\end{array}$} \\
\hline & & $\%$ & S.E. & $\%$ & S.E. & $\%$ & S.E. & $\%$ & S.E. & $\%$ & S.E. \\
\hline \multirow{6}{*}{ 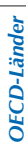 } & Australien & 22.7 & $(0.7)$ & 15.0 & $(1.3)$ & 18.2 & $(1.6)$ & 29.1 & (1.9) & 37.7 & $(1.8)$ \\
\hline & Österreich & 16.4 & (1.1) & 15.1 & (2.9) & 33.6 & (3.9) & 29.1 & $(4.0)$ & 22.2 & (2.9) \\
\hline & Belgien & 15.1 & $(0.9)$ & 17.8 & $(2.2)$ & 36.6 & $(2.9)$ & 24.2 & (2.4) & 21.4 & $(2.2)$ \\
\hline & Kanada & 29.5 & $(1.3)$ & 17.9 & $(1.3)$ & 22.4 & (1.6) & 16.3 & (1.6) & 43.3 & (1.9) \\
\hline & Chile & 0.9 & $(0.2)$ & 78.4 & (3.1) & 21.1 & (3.1) & 0.5 & $(0.3)$ & c & c \\
\hline & Tschech. Rep. & 3.2 & $(0.4)$ & 60.8 & (3.7) & 26.9 & (3.6) & 11.5 & $(2.1)$ & 0.7 & $(0.5)$ \\
\hline & Dänemark & 8.9 & $(0.6)$ & 29.8 & $(2.8)$ & 41.6 & $(2.9)$ & 20.9 & $(2.4)$ & 7.7 & $(1.3)$ \\
\hline & Estland & 8.2 & $(0.5)$ & 39.7 & $(2.2)$ & 34.8 & $(2.1)$ & 12.5 & $(2.1)$ & 13.1 & $(2.1)$ \\
\hline & Finnland & 3.3 & $(0.2)$ & 33.4 & $(3.0)$ & 58.0 & (3.0) & 7.5 & $(0.4)$ & 1.1 & $(0.2)$ \\
\hline & Frankreich & w & w & w & w & w & w & w & w & w & w \\
\hline & Deutschland & 13.1 & $(0.8)$ & 23.3 & $(2.7)$ & 27.8 & $(2.8)$ & 31.3 & $(3.1)$ & 17.6 & $(2.4)$ \\
\hline & Griechenland & 10.5 & $(0.8)$ & 19.9 & $(3.2)$ & 49.8 & (3.9) & 23.3 & (3.0) & 7.0 & $(1.5)$ \\
\hline & Ungarn & 1.7 & $(0.2)$ & 70.7 & (3.5) & 27.1 & (3.4) & 1.8 & $(1.0)$ & 0.4 & $(0.3)$ \\
\hline & Island & 3.5 & $(0.3)$ & 43.2 & $(0.2)$ & 50.7 & $(0.2)$ & 5.5 & $(0.2)$ & 0.6 & $(0.0)$ \\
\hline & Irland & 10.1 & $(0.7)$ & 14.3 & (2.4) & 40.5 & (3.6) & 38.7 & (3.9) & 6.5 & $(2.0)$ \\
\hline & Israel & 18.3 & $(1.2)$ & 17.0 & (1.9) & 25.7 & (3.5) & 29.7 & (3.6) & 27.7 & (3.1) \\
\hline & Italien & 7.5 & $(0.3)$ & 30.3 & $(1.5)$ & 46.1 & (1.6) & 18.0 & (1.3) & 5.6 & (1.1) \\
\hline & Japan & 0.3 & $(0.1)$ & 90.0 & $(2.1)$ & 10.0 & $(2.1)$ & c & c & c & c \\
\hline & Korea & 0.0 & $(0.0)$ & 99.3 & $(0.5)$ & 0.7 & $(0.5)$ & c & c & c & c \\
\hline & Luxemburg & 46.4 & $(0.7)$ & c & c & 0.8 & $(0.0)$ & 16.0 & $(0.1)$ & 83.2 & $(0.1)$ \\
\hline & Mexiko & 1.3 & $(0.1)$ & 77.2 & (1.6) & 20.4 & $(1.5)$ & 2.1 & $(0.5)$ & 0.3 & $(0.2)$ \\
\hline & Niederlande & 10.6 & $(1.0)$ & 29.7 & (3.5) & 35.6 & $(4.0)$ & 22.7 & $(3.7)$ & 12.0 & $(2.1)$ \\
\hline & Neuseeland & 26.3 & (1.5) & 7.4 & (1.9) & 13.9 & (2.5) & 41.3 & (3.5) & 37.3 & (3.4) \\
\hline & Norwegen & 9.4 & $(0.9)$ & 28.9 & (3.5) & 37.6 & (3.6) & 26.2 & (3.0) & 7.2 & $(1.7)$ \\
\hline & Polen & 0.2 & $(0.1)$ & 95.9 & (1.5) & 4.0 & (1.5) & 0.1 & $(0.1)$ & c & c \\
\hline & Portugal & 6.9 & $(0.6)$ & 34.4 & $(4.5)$ & 42.0 & (4.5) & 16.5 & (2.9) & 7.2 & (2.3) \\
\hline & Slowak. Rep. & 0.7 & $(0.2)$ & 85.2 & $(2.7)$ & 13.6 & $(2.7)$ & 1.2 & $(0.7)$ & c & $\mathrm{C}$ \\
\hline & Slowenien & 8.6 & $(0.4)$ & 36.0 & $(0.8)$ & 35.8 & $(0.5)$ & 19.8 & $(0.6)$ & 8.4 & $(0.6)$ \\
\hline & Spanien & 9.9 & $(0.6)$ & 27.7 & $(2.4)$ & 35.7 & $(2.8)$ & 26.6 & $(2.7)$ & 10.0 & $(1.7)$ \\
\hline & Schweden & 14.5 & $(0.9)$ & 17.6 & (2.5) & 34.3 & (3.1) & 28.7 & (2.9) & 19.4 & $(2.2)$ \\
\hline & Schweiz & 24.1 & $(0.9)$ & 3.9 & (1.0) & 14.5 & $(2.1)$ & 41.4 & (3.2) & 40.2 & (3.3) \\
\hline & Türkei & 0.9 & $(0.2)$ & 83.0 & (3.3) & 15.0 & $(3.2)$ & 2.0 & (1.3) & c & C \\
\hline & Ver. Königreich & 12.7 & $(1.1)$ & 33.1 & $(2.9)$ & 29.8 & $(3.1)$ & 20.7 & $(2.9)$ & 16.4 & $(2.2)$ \\
\hline & Ver. Staaten & 21 & $(2.0)$ & 20 & (3.4) & 29 & $(4.1)$ & 17.3 & (3.5) & 34.2 & (3.9) \\
\hline & OECD-Durchschnitt & 11.2 & $(0.1)$ & 40.0 & $(0.4)$ & 28.5 & $(0.5)$ & 19.0 & $(0.4)$ & 18.1 & $(0.4)$ \\
\hline ร & Albanien & 0.0 & c & 0.0 & c & 0.0 & c & 0.0 & c & 0.0 & c \\
\hline 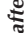 & Argentinien & 3.9 & $(0.4)$ & 56.4 & (3.8) & 33.1 & $(3.7)$ & 7.3 & $(2.2)$ & 3.2 & $(0.8)$ \\
\hline 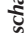 & Brasilien & 0.7 & $(0.1)$ & 86.2 & (1.6) & 12.3 & (1.5) & 1.4 & $(0.5)$ & 0.1 & $(0.1)$ \\
\hline & Bulgarien & 0.5 & $(0.2)$ & 90.3 & (2.3) & 8.6 & $(2.2)$ & 1.0 & $(0.7)$ & 0.1 & $(0.1)$ \\
\hline En & Kolumbien & 0.3 & $(0.1)$ & 91.1 & $(2.0)$ & 8.9 & (2.0) & 0.1 & $(0.1)$ & c & C \\
\hline ริ & Costa Rica & 5.5 & $(0.7)$ & 43.7 & (3.4) & 37.1 & (3.4) & 16.4 & $(2.3)$ & 2.8 & (1.5) \\
\hline${ }^{\top}$ & Kroatien & 12.1 & $(0.8)$ & 11.4 & (2.5) & 34.7 & (3.9) & 43.9 & (3.9) & 10.1 & (2.5) \\
\hline ¿ & Zypern* & 8.5 & $(0.4)$ & 15.9 & $(0.1)$ & 62.5 & $(0.2)$ & 14.2 & $(0.1)$ & 7.4 & $(0.1)$ \\
\hline : & Hongkong (China) & 34.7 & (1.5) & C & c & 7.6 & (2.9) & 18.6 & (3.1) & 73.7 & (3.9) \\
\hline 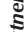 & Indonesien & 0.2 & $(0.1)$ & 96.9 & $(1.2)$ & 2.9 & (1.1) & 0.2 & $(0.1)$ & C & c \\
\hline ปั & Jordanien & 13.4 & $(0.7)$ & 20.8 & $(2.7)$ & 22.8 & $(2.8)$ & 38.8 & $(4.0)$ & 17.6 & (3.1) \\
\hline & Kasachstan & 16.1 & (1.7) & 15.6 & (2.6) & 32.8 & (3.6) & 35.4 & (3.7) & 16.1 & (3.4) \\
\hline & Lettland & 4.5 & $(0.5)$ & 58.9 & (3.5) & 21.7 & (3.2) & 16.1 & (2.5) & 3.2 & (1.3) \\
\hline & Liechtenstein & 33.3 & (2.9) & C & c & c & $\mathrm{C}$ & 53.1 & $(1.0)$ & 46.9 & (1.0) \\
\hline & Litauen & 1.7 & $(0.3)$ & 79.7 & (3.1) & 16.3 & (3.0) & 2.4 & (1.1) & 1.5 & $(0.8)$ \\
\hline & Macau (China) & 65.1 & (0.6) & C & $\mathrm{C}$ & c & $\mathrm{C}$ & C & c & 100.0 & c \\
\hline & Malaysia & 1.7 & $(0.3)$ & 72.8 & $(3.2)$ & 21.6 & (2.9) & 5.6 & (1.3) & c & c \\
\hline & Montenegro & 5.8 & $(0.4)$ & 0.9 & $(0.2)$ & 77.3 & $(0.2)$ & 21.7 & $(0.1)$ & 0.0 & $(0.0)$ \\
\hline & Peru & 0.5 & $(0.1)$ & 88.0 & $(2.1)$ & 11.2 & $(2.1)$ & 0.6 & $(0.4)$ & 0.1 & $(0.1)$ \\
\hline & Katar & 52.0 & $(0.4)$ & C & c & 3.6 & $(0.0)$ & 20.8 & $(0.1)$ & 75.6 & $(0.1)$ \\
\hline & Rumänien & 0.2 & $(0.1)$ & 94.6 & (1.9) & 5.4 & (1.9) & C & c & c & c \\
\hline & Russ. Föderation & 10.9 & $(0.8)$ & 13.8 & $(2.0)$ & 39.3 & $(3.2)$ & 40.5 & $(2.7)$ & 6.3 & $(2.0)$ \\
\hline & Serbien & 8.5 & $(0.8)$ & 27.8 & (3.6) & 42.1 & $(4.2)$ & 23.9 & $(3.0)$ & 6.2 & $(1.8)$ \\
\hline & Shanghai (China) & 0.9 & $(0.2)$ & 80.1 & (3.2) & 18.9 & (3.1) & 0.9 & $(0.7)$ & C & c \\
\hline & Singapur & 18.3 & $(0.8)$ & 2.1 & (0.9) & 21.2 & $(0.1)$ & 58.6 & $(0.2)$ & 18.1 & $(0.9)$ \\
\hline & Chinesisch Taipeh & 0.5 & $(0.1)$ & 84.8 & (2.9) & 15.2 & (2.9) & C & c & C & c \\
\hline & Thailand & 0.7 & $(0.4)$ & 96.8 & (1.4) & 1.5 & (1.0) & 0.7 & $(0.7)$ & 1.0 & $(0.7)$ \\
\hline & Tunesien & 0.4 & $(0.1)$ & 89.2 & (2.6) & 10.8 & (2.6) & c & c & C & c \\
\hline & Ver. Arab. Emirate & 54.9 & (1.4) & 2.3 & $(1.1)$ & 11.1 & (1.7) & 16.5 & (1.9) & 70.1 & $(2.2)$ \\
\hline & Uruguay & 0.5 & $(0.1)$ & 88.6 & (2.4) & 11.0 & (2.5) & 0.5 & $(0.4)$ & c & c \\
\hline & Vietnam & 0.1 & $(0.1)$ & 97.2 & $(1.4)$ & 2.8 & $(1.4)$ & c & C & c & c \\
\hline
\end{tabular}

Anmerkung: Diese Tabelle wurde nur für Schüler berechnet, für die Daten zum PISA-Index des wirtschaftlichen, sozialen und kulturellen Status vorliegen. Statistisch signifikante Werte sind durch Fettdruck gekennzeichnet (vgl. Anhang A3).

1. PISA-Index des wirtschaftlichen, sozialen und kulturellen Status (ESCS).

*Vgl. die Anmerkungen am Beginn dieses Anhangs.

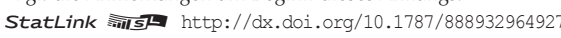


Konzentration der Schüler mit Migrationshintergrund in der Schule Tabelle II.3.9 Die Ergebnisse basieren auf Schülerangaben

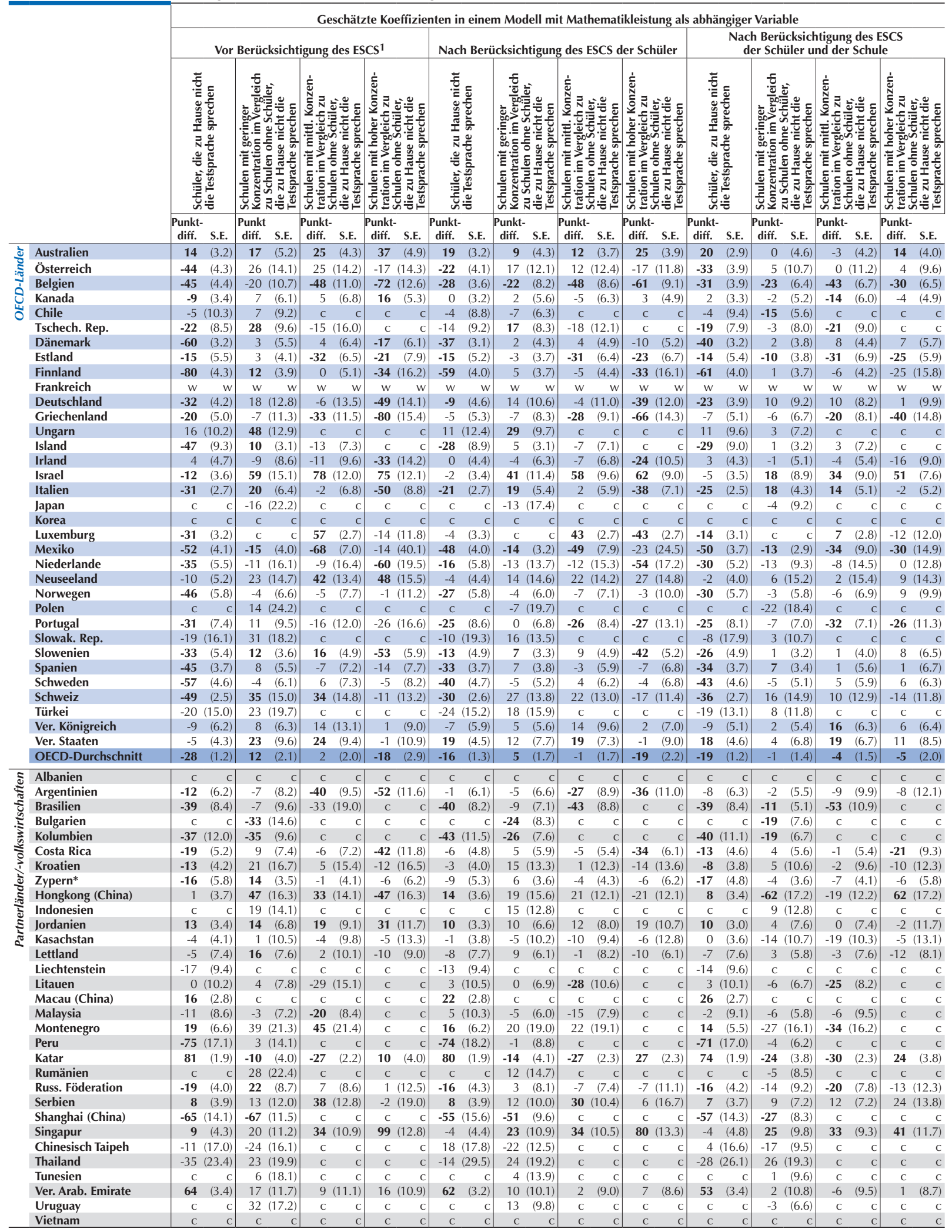

Anmerkung: Diese Tabelle wurde nur für Schüler berechnet, für die Daten zum PISA-Index des wirtschaftlichen, sozialen und kulturellen Status vorliegen. Statistisch signifikante Werte sind durch Fettdruck gekennzeichnet (vgl. Anhang A3).

1. PISA-Index des wirtschaftlichen, sozialen und kulturellen Status (ESCS).

Vgl. die Anmerkungen am Beginn dieses Anhangs.

StatLink तiाs 
[Teil 1/2]

Konzentration von Schülern, die zu Hause nicht die Testsprache sprechen Tabelle II.3.10 Die Ergebnisse basieren auf Schülerangaben

\begin{tabular}{|c|c|c|c|c|c|c|c|c|c|c|c|}
\hline & \multicolumn{10}{|c|}{ Prozentsatz der Schüler } \\
\hline & & \multicolumn{2}{|c|}{$\begin{array}{l}\text { Schüler, die zu Hause nicht } \\
\text { die Testsprache sprechen }\end{array}$} & \multicolumn{2}{|c|}{$\begin{array}{c}\text { In Schulen, wo der } \\
\text { Prozentsatz der Schüler, } \\
\text { die zu Hause nicht die } \\
\text { Testsprache sprechen, } \\
\text { gleich null ist }\end{array}$} & \multicolumn{2}{|c|}{$\begin{array}{l}\text { In Schulen, wo der } \\
\text { Prozentsatz der Schüler, } \\
\text { die zu Hause nicht die } \\
\text { Testsprache sprechen, über } \\
\mathbf{0 \%} \text {, aber unter } 10 \% \text { liegt } \\
\text { (geringe Konzentration) }\end{array}$} & \multicolumn{2}{|c|}{$\begin{array}{l}\text { In Schulen, wo der } \\
\text { Prozentsatz der Schüler, } \\
\text { die zu Hause nicht die } \\
\text { Testsprache sprechen, } \\
\text { mindestens } 10 \% \text {, aber } \\
\text { weniger als } 25 \% \text { beträgt } \\
\text { (mittlere Konzentration) }\end{array}$} & \multicolumn{2}{|c|}{$\begin{array}{l}\text { In Schulen, wo der } \\
\text { Prozentsatz der Schüler, } \\
\text { die zu Hause nicht die } \\
\text { Testsprache sprechen, } \\
\text { mindestens } 25 \% \text { beträgt } \\
\text { (hohe Konzentration) }\end{array}$} \\
\hline & & $\%$ & S.E. & $\%$ & S.E. & $\%$ & S.E. & $\%$ & S.E. & $\%$ & S.E. \\
\hline \multirow{35}{*}{ 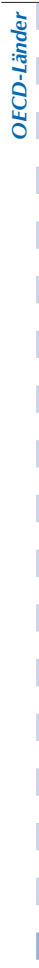 } & Australien & 9.7 & $(0.5)$ & 38.2 & $\begin{array}{l}(1.8) \\
\end{array}$ & 25.9 & (1.6) & 23.7 & (1.4) & 12.1 & $(1.2)$ \\
\hline & Österreich & 11.2 & $(0.8)$ & 26.8 & (3.0) & 38.1 & (3.9) & 18.4 & $(3.2)$ & 16.7 & $(2.3)$ \\
\hline & Belgien & 21.4 & $(0.8)$ & 10.4 & $(1.8)$ & 25.0 & (2.6) & 32.3 & (2.6) & 32.3 & (2.4) \\
\hline & Kanada & 17.3 & $(0.9)$ & 22.8 & (1.8) & 26.6 & $(2.0)$ & 23.3 & $(2.2)$ & 27.2 & (2.3) \\
\hline & Chile & 0.6 & $(0.1)$ & 83.8 & (2.9) & 15.6 & $(2.9)$ & c & c & c & c \\
\hline & Tschech. Rep. & 2.9 & $(0.4)$ & 66.3 & (3.5) & 25.6 & (3.0) & 6.7 & (1.5) & 1.4 & $(0.7)$ \\
\hline & Dänemark & 4.5 & $(0.3)$ & 43.8 & (3.3) & 43.3 & (3.3) & 10.0 & $(1.4)$ & 2.8 & $(0.2)$ \\
\hline & Estland & 5.5 & $(0.5)$ & 50.7 & (2.4) & 34.9 & $(2.3)$ & 10.8 & $(1.7)$ & 3.6 & (1.1) \\
\hline & Finnland & 4.5 & $(0.2)$ & 31.3 & (3.1) & 55.9 & (3.1) & 9.7 & $(0.5)$ & 3.1 & $(0.3)$ \\
\hline & Frankreich & w & w & w & w & w & w & w & w & w & w \\
\hline & Deutschland & 7.0 & $(0.6)$ & 41.0 & (2.9) & 36.9 & $(3.2)$ & 15.4 & (2.4) & 6.7 & (1.6) \\
\hline & Griechenland & 5.1 & $(0.6)$ & 43.2 & (3.6) & 46.0 & (3.7) & 6.8 & (1.7) & 4.0 & (1.1) \\
\hline & Ungarn & 1.0 & $(0.2)$ & 80.6 & (3.2) & 17.9 & $(3.2)$ & c & c & c & $\mathrm{C}$ \\
\hline & Island & 3.9 & $(0.3)$ & 36.3 & $(0.3)$ & 54.8 & $(0.3)$ & 6.3 & $(0.1)$ & c & c \\
\hline & Irland & 4.8 & $(0.5)$ & 43.5 & $(3.7)$ & 38.2 & (3.4) & 17.9 & (3.1) & c & c \\
\hline & Israel & 11.1 & $(0.9)$ & 16.5 & (3.0) & 42.3 & $(4.0)$ & 28.5 & (3.6) & 12.8 & (2.6) \\
\hline & Italien & 14.3 & $(0.4)$ & 11.6 & $(1.2)$ & 39.8 & $(2.2)$ & 31.3 & (2.3) & 17.4 & $(1.1)$ \\
\hline & Japan & c & c & 88.9 & (2.4) & 11.1 & $(2.4)$ & C & C & C & c \\
\hline & Korea & c & c & 98.1 & $(1.0)$ & c & c & C & c & c & c \\
\hline & Luxemburg & 85.3 & $(0.4)$ & C & c & c & c & C & c & 95.2 & $(0.0)$ \\
\hline & Mexiko & 3.2 & $(0.3)$ & 74.0 & (1.6) & 19.3 & (1.6) & 3.0 & $(0.6)$ & 3.7 & $(0.6)$ \\
\hline & Niederlande & 6.3 & $(0.5)$ & 35.7 & (3.9) & 41.2 & $(3.7)$ & 19.1 & (3.4) & 4.0 & (1.5) \\
\hline & Neuseeland & 16.0 & $(1.1)$ & 9.9 & $(1.8)$ & 32.9 & (3.4) & 36.2 & (3.6) & 21.0 & (2.6) \\
\hline & Norwegen & 7.5 & $(0.6)$ & 26.9 & (3.1) & 47.3 & (3.6) & 21.4 & $(2.8)$ & 4.5 & (1.5) \\
\hline & Polen & 0.9 & $(0.3)$ & 86.7 & (2.4) & 11.8 & $(2.2)$ & c & c & c & c \\
\hline & Portugal & 2.6 & $(0.3)$ & 55.0 & (3.6) & 40.5 & $(3.7)$ & 4.0 & (1.3) & c & c \\
\hline & Slowak. Rep. & 7.4 & $(0.9)$ & 55.2 & (3.6) & 26.6 & $(3.2)$ & 9.1 & (1.6) & 9.1 & (1.9) \\
\hline & Slowenien & 5.9 & $(0.4)$ & 51.8 & $(0.7)$ & 30.7 & $(0.5)$ & 11.5 & $(0.6)$ & 6.0 & $(0.5)$ \\
\hline & Spanien & 18.6 & (1.1) & 34.0 & $(2.1)$ & 28.4 & $(2.3)$ & 11.5 & (1.7) & 26.1 & (1.9) \\
\hline & Schweden & 10.1 & $(0.7)$ & 33.1 & (3.0) & 33.3 & $(3.2)$ & 22.3 & $(2.8)$ & 11.3 & (1.6) \\
\hline & Schweiz & 16.4 & $(0.8)$ & 8.6 & (1.6) & 28.0 & (3.3) & 40.7 & (3.3) & 22.7 & $(2.7)$ \\
\hline & Türkei & 6.2 & $(0.8)$ & 64.9 & (3.1) & 21.3 & $(3.1)$ & 4.8 & (1.3) & 9.0 & (1.5) \\
\hline & Ver. Königreich & 6.9 & $(0.7)$ & 43.7 & $(3.2)$ & 39.2 & $(3.2)$ & 7.6 & (1.8) & 9.5 & (1.8) \\
\hline & Ver. Staaten & 14 & $(1.3)$ & 21 & (3.3) & 39 & $(4.3)$ & 17.7 & (3.1) & 21.7 & (3.0) \\
\hline & OECD-Durchschnitt & 10.6 & $(0.1)$ & 44.6 & $(0.5)$ & 32.9 & $(0.5)$ & 16.8 & $(0.4)$ & 15.1 & $(0.3)$ \\
\hline \multirow{31}{*}{ 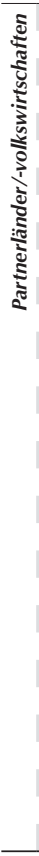 } & Albanien & c & c & c & c & c & c & C & C & c & c \\
\hline & Argentinien & 1.6 & $(0.2)$ & 74.9 & (3.5) & 21.1 & (3.3) & 3.4 & (1.4) & c & c \\
\hline & Brasilien & 1.1 & $(0.1)$ & 77.4 & (2.2) & 21.1 & $(2.2)$ & 1.1 & $(0.5)$ & 0.4 & $(0.2)$ \\
\hline & Bulgarien & 10.8 & $(1.2)$ & 45.3 & (3.3) & 29.5 & (3.3) & 10.4 & $(2.3)$ & 14.8 & $(2.2)$ \\
\hline & Kolumbien & 0.7 & $(0.2)$ & 87.6 & $(2.4)$ & 11.3 & $(2.3)$ & c & c & c & c \\
\hline & Costa Rica & 1.2 & $(0.2)$ & 76.8 & (3.1) & 20.4 & (3.0) & 2.8 & $(1.1)$ & c & c \\
\hline & Kroatien & 1.3 & $(0.3)$ & 75.5 & (3.4) & 22.7 & $(3.4)$ & c & c & c & c \\
\hline & Zypern* & 18.4 & $(0.3)$ & 11.3 & $(0.1)$ & 62.9 & $(0.1)$ & 6.9 & $(0.1)$ & 18.9 & $(0.1)$ \\
\hline & Hongkong (China) & 6.8 & $(0.9)$ & 25.3 & (3.9) & 54.2 & (3.9) & 18.2 & (3.0) & 2.3 & $(1.1)$ \\
\hline & Indonesien & 58.9 & $(2.3)$ & 3.0 & $(1.1)$ & 9.7 & $(2.7)$ & 7.0 & $(2.0)$ & 80.3 & $(2.7)$ \\
\hline & Jordanien & 4.7 & $(0.3)$ & 31.0 & $(3.2)$ & 56.2 & $(3.7)$ & 12.6 & $(2.3)$ & c & c \\
\hline & Kasachstan & 11.1 & $(0.9)$ & 34.3 & (2.6) & 26.5 & $(3.3)$ & 25.8 & (3.5) & 13.3 & (2.0) \\
\hline & Lettland & 10.5 & (1.4) & 40.3 & (3.5) & 29.4 & $(2.8)$ & 19.1 & (2.8) & 11.3 & $(2.2)$ \\
\hline & Liechtenstein & 12.0 & $(1.8)$ & C & c & C & c & 31.9 & (1.1) & C & c \\
\hline & Litauen & 3.5 & $(0.5)$ & 65.2 & (3.2) & 24.3 & $(3.2)$ & 8.7 & (1.7) & 1.8 & $(0.8)$ \\
\hline & Macau (China) & 13.6 & $(0.2)$ & 24.5 & $(0.1)$ & 56.2 & $(0.1)$ & c & c & 16.0 & $(0.1)$ \\
\hline & Malaysia & 42.3 & (2.5) & 13.1 & (2.4) & 16.1 & (3.2) & 18.1 & (3.1) & 52.7 & (3.5) \\
\hline & Montenegro & 1.0 & $(0.1)$ & 41.5 & $(0.2)$ & 58.5 & $(0.2)$ & C & c & $\mathrm{c}$ & c \\
\hline & Peru & 6.4 & $(0.9)$ & 65.5 & (3.3) & 23.0 & (3.3) & 2.5 & $(1.0)$ & 8.9 & $(1.6)$ \\
\hline & Katar & 39.2 & $(0.3)$ & 3.4 & $(0.1)$ & 45.3 & $(0.1)$ & 8.3 & $(0.1)$ & 43.0 & $(0.1)$ \\
\hline & Rumänien & 1.7 & $(0.4)$ & 76.8 & (3.3) & 20.2 & $(3.0)$ & c & C & C & c \\
\hline & Russ. Föderation & 8.7 & (1.8) & 41.9 & (3.7) & 41.9 & $(4.0)$ & 7.2 & (1.8) & 9.1 & (2.9) \\
\hline & Serbien & 4.2 & $(0.6)$ & 52.5 & (3.9) & 38.3 & $(4.0)$ & 5.6 & (1.7) & 3.6 & (1.7) \\
\hline & Shanghai (China) & 1.4 & $(0.2)$ & 66.4 & (3.3) & 33.2 & $(3.2)$ & c & c & c & C \\
\hline & Singapur & 54.4 & $(0.9)$ & c & c & c & c & 10.9 & $(0.2)$ & 86.9 & $(0.7)$ \\
\hline & Chinesisch Taipeh & 16.5 & (1.1) & 7.2 & $(2.0)$ & 37.7 & $(3.6)$ & 31.7 & (3.4) & 23.3 & (3.1) \\
\hline & Thailand & 44.6 & $(1.7)$ & 10.0 & $(1.8)$ & 18.1 & $(2.7)$ & 14.5 & (2.5) & 57.5 & (2.4) \\
\hline & Tunesien & 1.1 & $(0.2)$ & 77.2 & (3.3) & 21.8 & (3.3) & c & c & $\mathrm{C}$ & c \\
\hline & Ver. Arab. Emirate & 33.5 & $(0.9)$ & 16.7 & (1.9) & 32.3 & $(2.5)$ & 11.7 & (1.6) & 39.4 & $(0.9)$ \\
\hline & Uruguay & 2.1 & $(0.4)$ & 70.0 & $(3.1)$ & 25.3 & $(3.0)$ & 3.3 & $(1.1)$ & $\mathrm{C}$ & $\mathrm{C}$ \\
\hline & Vietnam & 2.2 & $(0.4)$ & 81.6 & $(2.8)$ & 12.7 & $(2.5)$ & 3.3 & $(1.5)$ & $\mathrm{c}$ & $\mathrm{C}$ \\
\hline
\end{tabular}

Anmerkung: Diese Tabelle wurde nur für Schüler berechnet, für die Daten zum PISA-Index des wirtschaftlichen, sozialen und kulturellen Status vorliegen. Statistisch signifikante Werte sind durch Fettdruck gekennzeichnet (vgl. Anhang A3).

1. PISA-Index des wirtschaftlichen, sozialen und kulturellen Status (ESCS).

*Vgl. die Anmerkungen am Beginn dieses Anhangs.

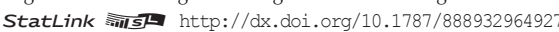


[Teil 2/2]

Konzentration von Schülern, die zu Hause nicht die Testsprache sprechen Tabelle II.3.10 Die Ergebnisse basieren auf Schülerangaben

Geschätzte Koeffizienten in einem Modell mit Mathematikleistung als abhängiger Variable

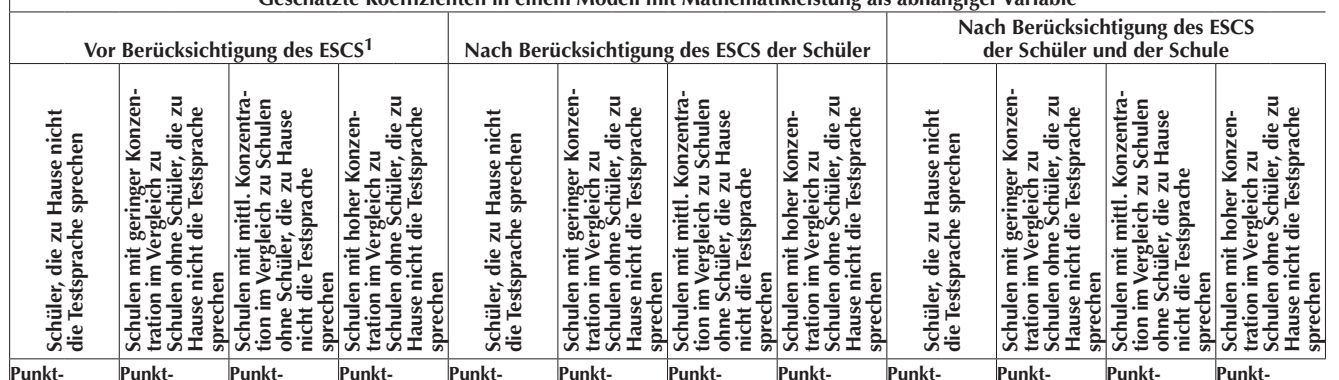

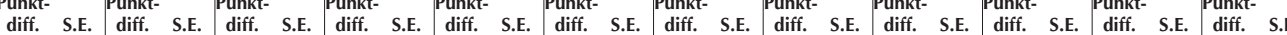

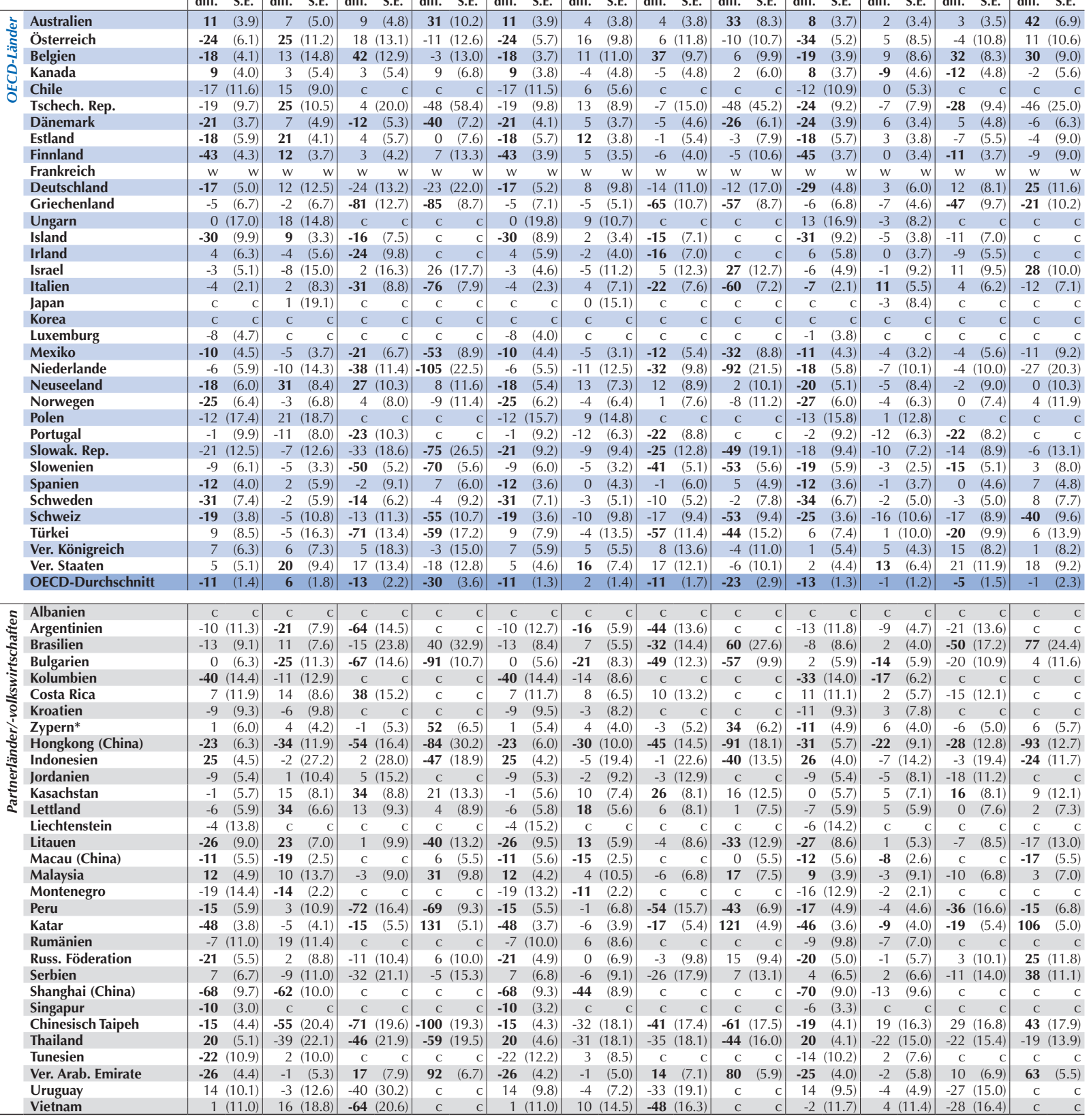

Anmerkung: Diese Tabelle wurde nur für Schüler berechnet, für die Daten zum PISA-Index des wirtschaftlichen, sozialen und kulturellen Status vorliegen. Statistisch signifikante Werte sind durch Fettdruck gekennzeichnet (vgl. Anhang A3).

1. PISA-Index des wirtschaftlichen, sozialen und kulturellen Status (ESCS).

*Vgl. die Anmerkungen am Beginn dieses Anhangs.

StatLink त्ञाI http://dx.doi.org/10.1787/888932964927 
[Teil 1/2]

Aufnahmeland/-volkswirtschaft, Herkunftsland und Mathematikleistungen Tabelle II.3.11 Die Ergebnisse basieren auf Schülerangaben

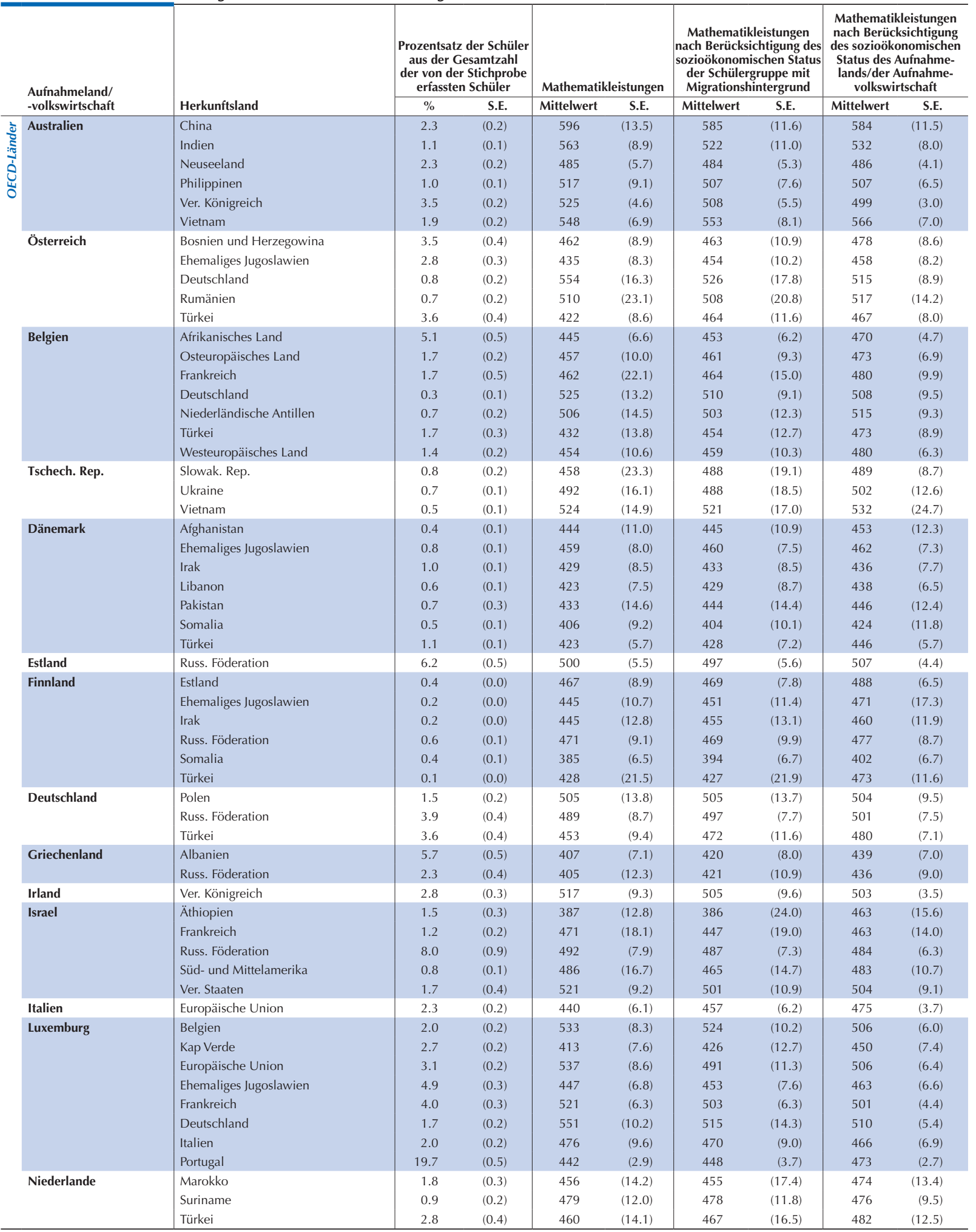

Anmerkung: Das Herkunftsland der Schüler wird bestimmt durch das Geburtsland, das die Schüler sowohl für den Vater als auch für die Mutter angeben (nur Schüler mit gültigen Antworten bei beiden Variablen werden bei dieser Analyse berücksichtigt). Nur Schüler mit Migrationshintergrund (Schüler der 1. und 2. Generation) werden bei dieser Analyse berücksichtigt. Nur Schüler, die ein bestimmtes Herkunftsland angegeben haben (d.h. ein „anderes Land als das Testland“) wurden bei dieser Analyse berücksichtigt. Wenn beide Eltern das gleiche Geburtsland haben (das sich vom Testland unterscheidet), hat der betreffende Schüler das gleiche Geburtsland wie die Eltern. Wenn sie unterschiedlich sind, gilt das Geburtsland des Vaters.

*Vgl. die Anmerkungen am Beginn dieses Anhangs.

StatLink inis ht htp://dx.doi.org/10.1787/888932964927 
[Teil 2/2]

Aufnahmeland/-volkswirtschaft, Herkunftsland und Mathematikleistungen Tabelle II.3.11 Die Ergebnisse basieren auf Schülerangaben

\begin{tabular}{|c|c|c|c|c|c|c|c|c|c|c|}
\hline & \multirow[b]{2}{*}{$\begin{array}{l}\text { Aufnahmeland/ } \\
\text {-volkswirtschaft }\end{array}$} & \multirow[b]{2}{*}{ Herkunftsland } & \multicolumn{2}{|c|}{$\begin{array}{l}\text { Prozentsatz der Schüler } \\
\text { aus der Gesamtzahl } \\
\text { der von der Stichprobe } \\
\text { erfassten Schüler }\end{array}$} & \multicolumn{2}{|c|}{ Mathematikleistungen } & \multicolumn{2}{|c|}{$\begin{array}{c}\text { Mathematikleistungen } \\
\text { nach Berücksichtigung des } \\
\text { sozioökonomischen Status } \\
\text { der Schülergruppe mit } \\
\text { Migrationshintergrund }\end{array}$} & \multicolumn{2}{|c|}{$\begin{array}{c}\text { Mathematikleistungen } \\
\text { nach Berücksichtigung des } \\
\text { sozioökonomischen Status } \\
\text { des Aufnahmelands/der } \\
\text { Aufnahmevolkswirtschaft }\end{array}$} \\
\hline & & & $\%$ & S.E. & $\begin{array}{c}\text { Mittel- } \\
\text { wert }\end{array}$ & S.E. & Mittelwert & S.E. & Mittelwert & S.E. \\
\hline \multirow{18}{*}{ 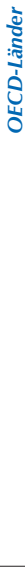 } & \multirow[t]{6}{*}{ Neuseeland } & China & 2.3 & $(0.4)$ & 588 & $(12.2)$ & 582 & $(11.7)$ & 581 & $(10.2)$ \\
\hline & & Fidschi & 1.7 & $(0.2)$ & 465 & (13.8) & 472 & (13.1) & 480 & (11.7) \\
\hline & & Korea & 1.2 & $(0.2)$ & 586 & (14.6) & 569 & $(18.2)$ & 566 & (13.8) \\
\hline & & Samoa & 2.8 & $(0.4)$ & 416 & (7.6) & 433 & (12.5) & 451 & $(7.0)$ \\
\hline & & Südafrika & 1.7 & $(0.2)$ & 507 & $(9.2)$ & 487 & $(11.4)$ & 494 & (9.0) \\
\hline & & Ver. Königreich & 3.5 & $(0.3)$ & 528 & (7.8) & 505 & (7.8) & 515 & (4.4) \\
\hline & \multirow[t]{4}{*}{ Portugal } & Portugiesischspr. afrikanisches Land & 3.4 & $(0.5)$ & 440 & (7.7) & 469 & (8.3) & 493 & (4.7) \\
\hline & & Brasilien & 1.0 & $(0.2)$ & 443 & (11.4) & 444 & (13.3) & 471 & (9.8) \\
\hline & & Osteuropäisches Land (Nicht-EU) & 0.5 & $(0.1)$ & 504 & (16.1) & 507 & $(14.7)$ & 515 & (15.3) \\
\hline & & Europäische Union & 0.7 & $(0.1)$ & 485 & $(16.3)$ & 488 & (16.9) & 511 & (5.9) \\
\hline & \multirow[t]{8}{*}{ Schweiz } & Albanien & 0.5 & $(0.1)$ & 417 & $(17.7)$ & 435 & $(32.8)$ & 448 & $(17.3)$ \\
\hline & & Ehemaliges Jugoslawien & 7.9 & $(0.5)$ & 472 & (5.5) & 481 & $(6.2)$ & 492 & $(5.0)$ \\
\hline & & Frankreich & 0.7 & $(0.1)$ & 524 & (11.3) & 508 & (12.5) & 526 & (6.1) \\
\hline & & Deutschland & 1.6 & $(0.2)$ & 524 & $(10.3)$ & 489 & $(19.3)$ & 513 & (6.6) \\
\hline & & Italien & 1.9 & $(0.2)$ & 476 & $(10.0)$ & 483 & $(8.0)$ & 498 & (6.8) \\
\hline & & Portugal & 3.1 & $(0.2)$ & 487 & (6.9) & 506 & (14.8) & 521 & $(6.2)$ \\
\hline & & Spanien & 0.6 & $(0.1)$ & 494 & $(14.2)$ & 500 & $(16.5)$ & 515 & $(10.4)$ \\
\hline & & Türkei & 1.5 & $(0.2)$ & 462 & $(12.0)$ & 475 & (10.1) & 490 & (10.5) \\
\hline \multirow{26}{*}{ 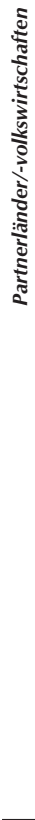 } & \multirow[t]{2}{*}{ Argentinien } & Bolivien & 1.4 & $(0.2)$ & 353 & (12.3) & 368 & (33.4) & 396 & $(10.1)$ \\
\hline & & Paraguay & 1.1 & $(0.3)$ & 360 & (13.1) & 388 & (18.4) & 398 & $(10.9)$ \\
\hline & Costa Rica & Nicaragua & 4.2 & $(0.7)$ & 365 & $(10.2)$ & 393 & (12.9) & 417 & $(6.2)$ \\
\hline & \multirow[t]{2}{*}{ Kroatien } & Bosnien und Herzegowina & 9.6 & $(0.6)$ & 457 & (5.8) & 471 & $(6.6)$ & 481 & (5.1) \\
\hline & & Sonstiges ehemaliges Jugoslawien & 1.1 & $(0.2)$ & 459 & (11.8) & 475 & (14.8) & 498 & (8.6) \\
\hline & \multirow[t]{4}{*}{ Zypern* } & Osteuropäisches Land & 0.8 & $(0.1)$ & 432 & $(15.0)$ & 439 & (15.9) & 445 & $(8.9)$ \\
\hline & & Griechenland & 1.4 & $(0.2)$ & 427 & (11.4) & 429 & $(11.0)$ & 431 & $(7.1)$ \\
\hline & & Russ. Föderation & 1.6 & $(0.2)$ & 461 & $(11.1)$ & 457 & $(9.7)$ & 460 & $(8.2)$ \\
\hline & & Ver. Königreich & 1.0 & $(0.1)$ & 447 & (14.3) & 419 & (15.6) & 438 & (6.1) \\
\hline & \multirow[t]{2}{*}{ Hongkong (China) } & China & 31.7 & $(1.4)$ & 562 & $(3.4)$ & 594 & $(5.2)$ & 596 & $(3.7)$ \\
\hline & & Macau (China) & 0.8 & $(0.1)$ & 554 & (14.5) & 572 & (23.4) & 578 & (12.9) \\
\hline & Kasachstan & Russ. Föderation & 12.1 & (1.5) & 432 & $(7.0)$ & 442 & (6.3) & 448 & (5.4) \\
\hline & \multirow[t]{3}{*}{ Lettland } & Belarus & 0.7 & $(0.1)$ & 507 & (13.7) & 508 & (11.8) & 502 & $(8.7)$ \\
\hline & & Russ. Föderation & 1.8 & $(0.3)$ & 486 & (11.4) & 485 & $(10.9)$ & 494 & $(5.0)$ \\
\hline & & Ukraine & 1.0 & $(0.3)$ & 496 & (20.1) & 508 & (16.7) & 502 & (8.5) \\
\hline & \multirow[t]{3}{*}{ Macau (China) } & China & 57.4 & $(0.7)$ & 548 & $(1.4)$ & 570 & $(2.7)$ & 568 & (1.8) \\
\hline & & Hongkong (China) & 2.5 & $(0.3)$ & 526 & (8.1) & 540 & $(11.0)$ & 543 & (5.8) \\
\hline & & Philippinen & 1.0 & $(0.1)$ & 467 & (13.1) & 478 & (12.4) & 467 & (11.6) \\
\hline & \multirow[t]{2}{*}{ Montenegro } & Bosnien und Herzegowina & 1.3 & $(0.2)$ & 455 & (9.7) & 453 & (9.7) & 442 & $(5.2)$ \\
\hline & & Serbien & 2.9 & $(0.3)$ & 424 & $(8.2)$ & 427 & $(7.5)$ & 426 & (3.7) \\
\hline & \multirow[t]{4}{*}{ Katar } & Ägypten & 7.0 & $(0.2)$ & 416 & (3.7) & 393 & $(4.8)$ & 397 & (3.6) \\
\hline & & Jordanien & 1.6 & $(0.1)$ & 411 & (9.0) & 371 & $(13.2)$ & 388 & $(8.0)$ \\
\hline & & Palästinensische Autonomiegebiete & 2.3 & $(0.1)$ & 396 & $(6.2)$ & 377 & $(8.1)$ & 375 & (5.4) \\
\hline & & Jemen & 2.8 & $(0.2)$ & 349 & $(4.9)$ & 351 & (4.8) & 350 & (4.4) \\
\hline & Russ. Föderation & Sonstige ehemalige Sowjetrepublik & 8.3 & $(0.7)$ & 473 & (4.6) & 477 & (4.1) & 491 & $(3.2)$ \\
\hline & Serbien & Sonstiges ehemaliges Jugoslawien & 7.4 & $(0.7)$ & 468 & $(7.2)$ & 481 & (6.1) & 479 & (4.8) \\
\hline
\end{tabular}

Anmerkung: Das Herkunftsland der Schüler wird bestimmt durch das Geburtsland, das die Schüler sowohl für den Vater als auch für die Mutter angeben (nur Schüler mit gültigen Antworten bei beiden Variablen werden bei dieser Analyse berücksichtigt). Nur Schüler mit Migrationshintergrund (Schüler der 1. und 2. Generation) werden bei dieser Analyse berücksichtigt. Nur Schüler, die ein bestimmtes Herkunftsland angegeben haben (d.h. ein "anderes Land als das Testland") wurden bei dieser Analyse berücksichtigt. Wenn beide Eltern das gleiche Geburtsland haben (das sich vom Testland unterscheidet), hat der betreffende Schüler das gleiche Geburtsland wie die Eltern. Wenn sie unterschiedlich sind, gilt das Geburtsland des Vaters.

*Vgl. die Anmerkungen am Beginn dieses Anhangs.

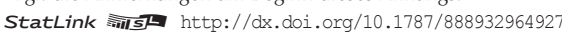


[Teil 1/3]

Chancengerechtigkeit bei den Lernmöglichkeiten: Reine Mathematik

Tabelle II.4.1 Die Ergebnisse basieren auf Schülerangaben

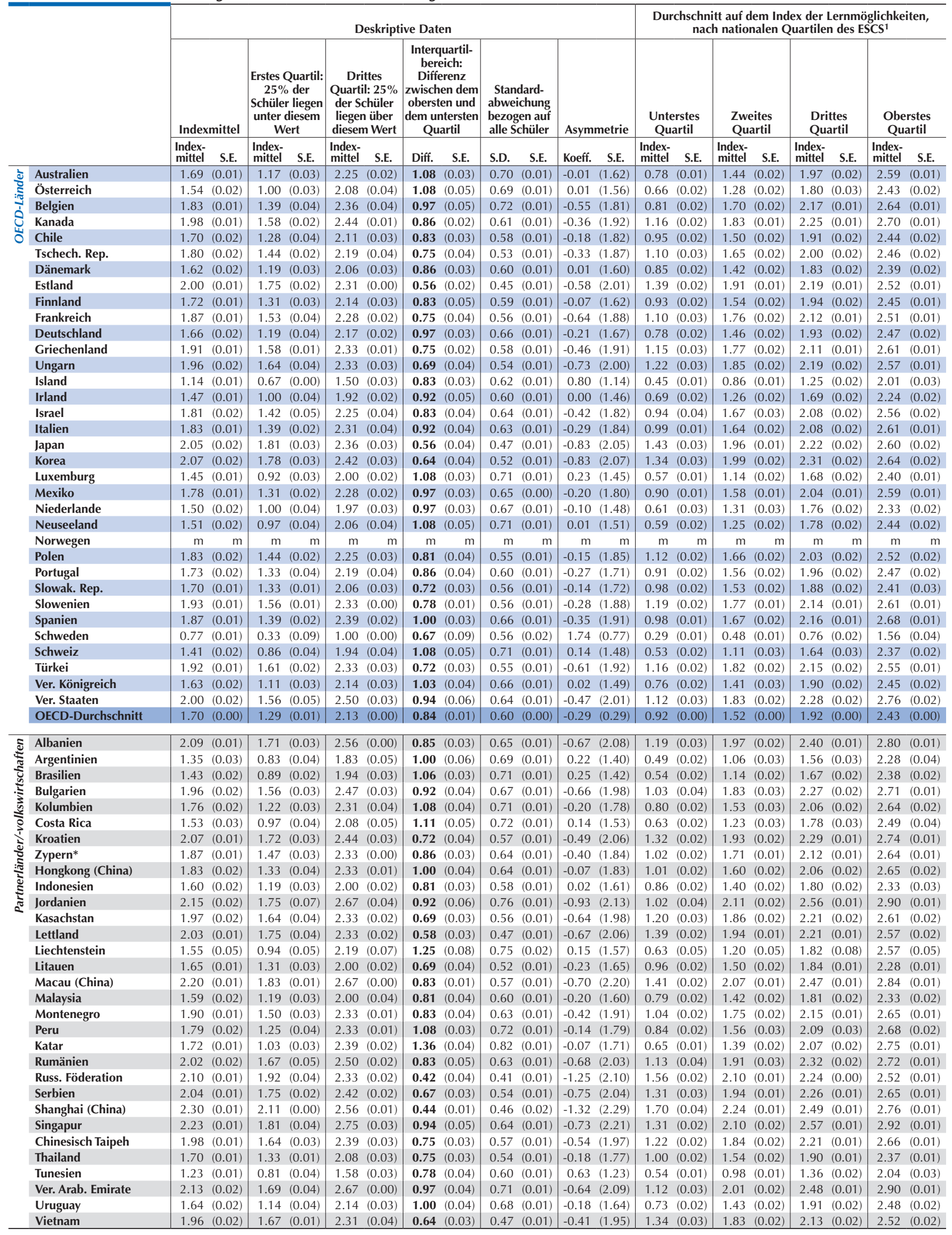

Anmerkung: Statistisch signifikante Werte sind durch Fettdruck gekennzeichnet (vgl. Anhang A3).

1. ESCS bezieht sich auf den PISA-Index des wirtschaftlichen, sozialen und kulturellen Status.

* Vgl. Anmerkungen am Anfang dieses Anhangs.

StatLink 命ISD http://dx.doi.org/10.1787/888932964946 
Teil 2/31

Chancengerechtigkeit bei den Lernmöglichkeiten: Reine Mathematik

Tabelle II.4.1 Die Ergebnisse basieren auf Schülerangaben

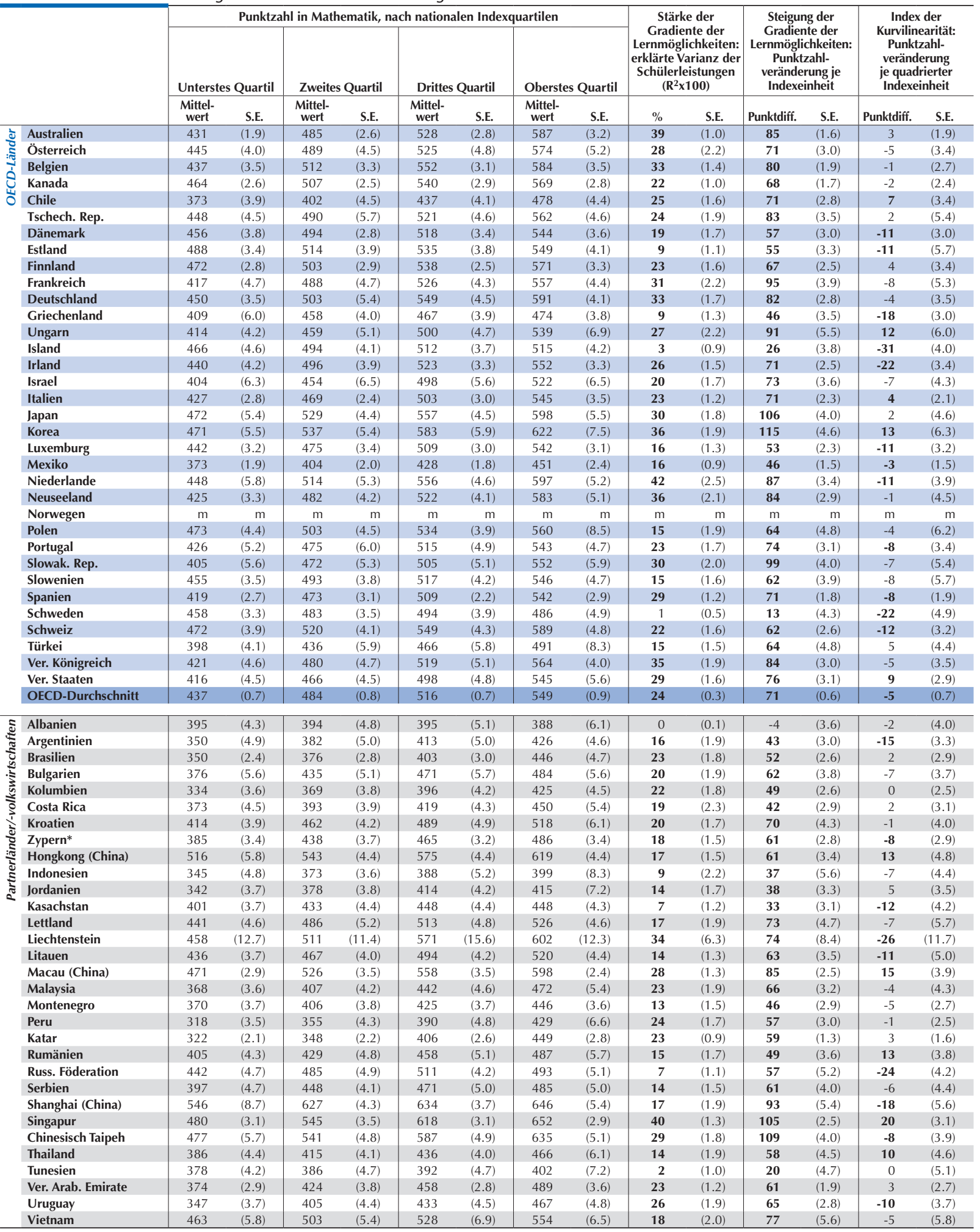

Anmerkung: Statistisch signifikante Werte sind durch Fettdruck gekennzeichnet (vgl. Anhang A3).

1. ESCS bezieht sich auf den PISA-Index des wirtschaftlichen, sozialen und kulturellen Status.

* Vgl. Anmerkungen am Anfang dieses Anhangs.

StatLink 需政 $\mathrm{http}: / / \mathrm{dx}$.doi.org/10.1787/888932964946 
[Teil 3/3]

Chancengerechtigkeit bei den Lernmöglichkeiten: Reine Mathematik

Tabelle II.4.1 Die Ergebnisse basieren auf Schülerangaben

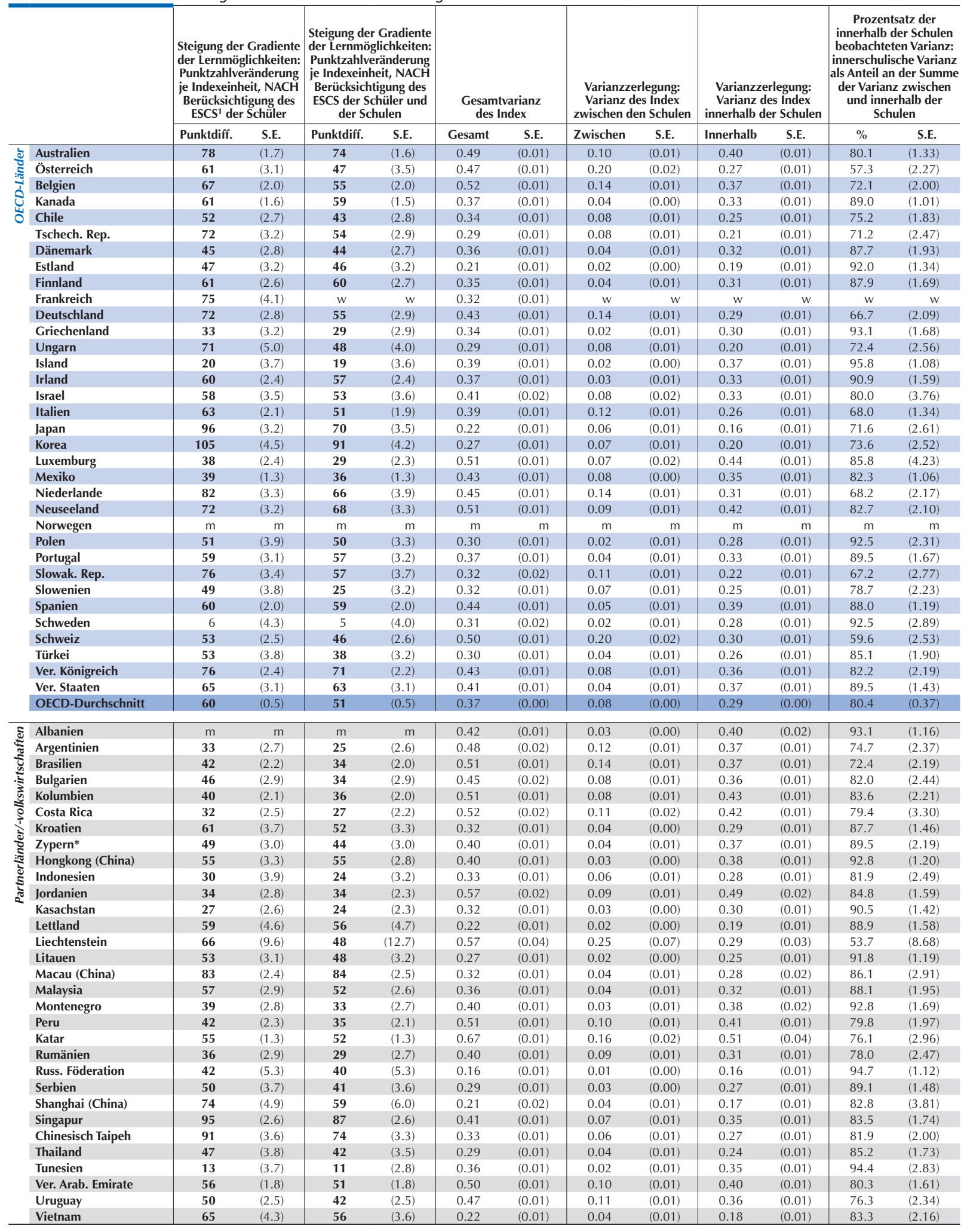

Anmerkung: Statistisch signifikante Werte sind durch Fettdruck gekennzeichnet (vgl. Anhang A3).

1. ESCS bezieht sich auf den PISA-Index des wirtschaftlichen, sozialen und kulturellen Status.

* Vgl. Anmerkungen am Anfang dieses Anhangs.

StatLink तinlst http://dx.doi.org/10.1787/888932964946 
[Teil 1/4]

Mathematikleistungen und Schülerpopulation, nach sozioökonomischem Profil der Schulen Tabelle II.4.2 Die Ergebnisse basieren auf Schülerangaben

\begin{tabular}{|c|c|c|c|c|c|c|c|c|c|c|c|c|c|c|c|c|c|c|}
\hline & \multicolumn{6}{|c|}{ Prozentsatz der Schüler } & \multicolumn{6}{|c|}{ ESCS ${ }^{1}$-Mittelwert } & \multicolumn{6}{|c|}{ Durchschnittsergebnisse in Mathematik } \\
\hline & \multicolumn{2}{|c|}{\begin{tabular}{|c|} 
Sozio- \\
ökonomisch \\
benachteiligte \\
Schulen $^{2}$
\end{tabular}} & \multicolumn{2}{|c|}{$\begin{array}{c}\text { Sozio- } \\
\text { ökonomisch } \\
\text { durchschnittl. } \\
\text { Schulen }^{2}\end{array}$} & \multicolumn{2}{|c|}{$\begin{array}{c}\text { Sozio- } \\
\text { ökonomisch } \\
\text { begünstigte } \\
\text { Schulen }^{2}\end{array}$} & \multicolumn{2}{|c|}{\begin{tabular}{|c|} 
Sozio- \\
ökonomisch \\
benachteiligte \\
Schulen $^{2}$
\end{tabular}} & \multicolumn{2}{|c|}{$\begin{array}{c}\text { Sozio- } \\
\text { ökonomisch } \\
\text { durchschnittl. } \\
\text { Schulen }^{2}\end{array}$} & \multicolumn{2}{|c|}{$\begin{array}{c}\text { Sozio- } \\
\text { ökonomisch } \\
\text { begünstigte } \\
\text { Schulen }^{2}\end{array}$} & \multicolumn{2}{|c|}{\begin{tabular}{|c|}
$\begin{array}{c}\text { Sozio- } \\
\text { okonomisch } \\
\text { benachteiligte } \\
\text { Schulen }\end{array}$ \\
\end{tabular}} & \multicolumn{2}{|c|}{$\begin{array}{c}\text { Sozio- } \\
\text { ökonomisch } \\
\text { durchschnittl. } \\
\text { Schulen }^{2}\end{array}$} & \multicolumn{2}{|c|}{$\begin{array}{c}\text { Sozio- } \\
\text { ökonomisch } \\
\text { begünstigte } \\
\text { Schulen }^{2}\end{array}$} \\
\hline & $\%$ & S.E. & $\%$ & S.E. & $\%$ & S.E. & $\begin{array}{l}\text { Index- } \\
\text { mittel }\end{array}$ & S.E. & $\begin{array}{l}\text { Index- } \\
\text { mittel }\end{array}$ & S.E. & $\begin{array}{l}\text { Index- } \\
\text { mittel }\end{array}$ & S.E. & $\begin{array}{c}\text { Mittel- } \\
\text { wert }\end{array}$ & S.E. & $\begin{array}{c}\text { Mittel- } \\
\text { wert }\end{array}$ & S.E. & $\begin{array}{c}\text { Mittel- } \\
\text { wert }\end{array}$ & S.E. \\
\hline Australien & 23.8 & $(1.2)$ & 51.2 & (1.7) & 25.0 & $(1.4)$ & -0.30 & $(0.02)$ & 0.24 & $(0.01)$ & 0.77 & $(0.01)$ & 459 & $(3.1)$ & 499 & $(2.1)$ & 558 & (3.6) \\
\hline Österreich & 32.5 & $(2.8)$ & 41.4 & (3.5) & 26.1 & $(2.8)$ & -0.41 & $(0.03)$ & 0.07 & $(0.02)$ & 0.68 & $(0.05)$ & 445 & (5.3) & 521 & (5.5) & 558 & (5.5) \\
\hline Belgien & 29.0 & $(2.2)$ & 37.3 & (2.9) & 33.8 & $(2.1)$ & -0.46 & $(0.03)$ & 0.12 & $(0.02)$ & 0.68 & $(0.02)$ & 437 & (4.6) & 512 & (3.6) & 584 & $(4.2)$ \\
\hline Kanada & 21.4 & (2.1) & 52.6 & $(2.9)$ & 26.0 & $(2.0)$ & -0.12 & $(0.02)$ & 0.38 & $(0.01)$ & 0.90 & $(0.02)$ & 489 & $(4.1)$ & 511 & $(2.0)$ & 556 & (3.4) \\
\hline Chile & 42.8 & (3.0) & 22.8 & (3.5) & 34.4 & $(2.4)$ & -1.29 & $(0.04)$ & -0.66 & $(0.02)$ & 0.36 & $(0.05)$ & 387 & (3.8) & 408 & $(6.2)$ & 476 & (5.4) \\
\hline Tschech. Rep. & 21.7 & (2.6) & 57.7 & $(3.0)$ & 20.6 & $(2.0)$ & -0.55 & $(0.03)$ & -0.09 & $(0.01)$ & 0.51 & $(0.02)$ & 434 & (7.5) & 492 & (3.6) & 588 & (4.4) \\
\hline Dänemark & 21.4 & (3.0) & 56.0 & (3.3) & 22.6 & $(2.3)$ & -0.08 & $(0.03)$ & 0.39 & $(0.01)$ & 0.97 & $(0.02)$ & 467 & $(5.0)$ & 497 & $(2.1)$ & 538 & (4.1) \\
\hline Estland & 19.0 & $(2.0)$ & 58.1 & $(2.7)$ & 22.9 & (1.7) & -0.40 & $(0.03)$ & 0.06 & $(0.01)$ & 0.66 & $(0.02)$ & 498 & $(4.1)$ & 513 & $(2.7)$ & 558 & (3.8) \\
\hline Finnland & 16.0 & $(2.4)$ & 67.0 & $(3.0)$ & 17.0 & (1.9) & -0.04 & $(0.03)$ & 0.36 & $(0.01)$ & 0.77 & $(0.02)$ & 499 & $(4.3)$ & 519 & $(2.5)$ & 538 & (4.1) \\
\hline Frankreich & w & w & w & w & w & w & w & w & w & w & w & w & w & w & w & w & w & w \\
\hline Deutschland & 28.0 & $(2.8)$ & 45.2 & (3.3) & 26.8 & $(2.2)$ & -0.44 & $(0.02)$ & 0.19 & $(0.02)$ & 0.82 & $(0.03)$ & 446 & $(5.8)$ & 514 & $(5.0)$ & 586 & (4.5) \\
\hline Griechenland & 25.3 & (3.1) & 48.3 & $(4.0)$ & 26.5 & (3.3) & -0.77 & $(0.04)$ & -0.07 & $(0.02)$ & 0.61 & $(0.03)$ & 397 & $(6.2)$ & 457 & (2.9) & 498 & (4.3) \\
\hline Ungarn & 31.6 & $(2.7)$ & 35.0 & (3.3) & 33.4 & $(2.5)$ & -0.95 & $(0.05)$ & -0.27 & $(0.02)$ & 0.42 & $(0.04)$ & 409 & $(6.1)$ & 465 & (4.9) & 554 & (6.0) \\
\hline Island & 16.3 & $(0.2)$ & 55.9 & $(0.2)$ & 27.8 & $(0.2)$ & 0.27 & $(0.03)$ & 0.74 & $(0.02)$ & 1.16 & $(0.02)$ & 466 & (3.8) & 486 & (2.3) & 522 & (2.8) \\
\hline Irland & 17.2 & $(2.3)$ & 57.0 & (3.6) & 25.8 & $(2.8)$ & -0.51 & $(0.04)$ & 0.09 & $(0.01)$ & 0.63 & $(0.03)$ & 439 & (5.4) & 505 & $(2.2)$ & 536 & (3.3) \\
\hline Israel & 29.7 & $(3.1)$ & 38.4 & (3.8) & 31.9 & (3.2) & -0.39 & $(0.03)$ & 0.18 & $(0.02)$ & 0.67 & $(0.02)$ & 397 & (5.6) & 467 & (7.9) & 529 & (6.8) \\
\hline Italien & 28.8 & $(1.6)$ & 41.2 & $(2.2)$ & 29.9 & (1.7) & -0.65 & $(0.02)$ & -0.08 & $(0.01)$ & 0.55 & $(0.02)$ & 428 & (3.4) & 484 & $(2.7)$ & 543 & (3.4) \\
\hline Japan & 29.5 & $(2.5)$ & 43.1 & (3.3) & 27.4 & $(2.2)$ & -0.49 & $(0.02)$ & -0.08 & $(0.01)$ & 0.37 & $(0.02)$ & 474 & $(6.7)$ & 540 & $(5.2)$ & 599 & (6.2) \\
\hline Korea & 26.7 & $(2.6)$ & 48.6 & $(3.7)$ & 24.8 & $(3.5)$ & -0.46 & $(0.02)$ & 0.03 & $(0.02)$ & 0.48 & $(0.03)$ & 493 & (6.8) & 557 & $(4.7)$ & 613 & (9.1) \\
\hline Luxemburg & 47.9 & $(0.1)$ & 15.0 & $(0.1)$ & 37.1 & $(0.1)$ & -0.45 & $(0.02)$ & 0.17 & $(0.03)$ & 0.71 & $(0.02)$ & 444 & (1.3) & 497 & (2.4) & 546 & (1.8) \\
\hline Mexiko & 34.3 & $(1.4)$ & 35.3 & $(1.8)$ & 30.5 & (1.7) & -1.99 & $(0.02)$ & -1.12 & $(0.01)$ & -0.10 & $(0.04)$ & 384 & $(2.4)$ & 409 & (1.9) & 451 & (2.3) \\
\hline Niederlande & 23.4 & $(2.7)$ & 50.9 & $(4.2)$ & 25.7 & (3.3) & -0.26 & $(0.03)$ & 0.23 & $(0.02)$ & 0.68 & $(0.02)$ & 440 & $(5.7)$ & 527 & (5.9) & 591 & (7.2) \\
\hline Neuseeland & 21.5 & (3.1) & 55.3 & $(4.0)$ & 23.2 & (2.6) & -0.51 & $(0.04)$ & 0.02 & $(0.02)$ & 0.58 & $(0.03)$ & 443 & (4.9) & 497 & (4.4) & 558 & (4.1) \\
\hline Norwegen & 10.0 & (2.2) & 73.3 & $(2.8)$ & 16.6 & (2.6) & 0.03 & $(0.03)$ & 0.42 & $(0.01)$ & 0.91 & $(0.03)$ & 467 & (7.4) & 484 & $(3.2)$ & 527 & (4.6) \\
\hline Polen & 27.5 & (3.1) & 50.2 & (3.9) & 22.3 & (3.1) & -0.74 & $(0.03)$ & -0.22 & $(0.02)$ & 0.47 & $(0.04)$ & 484 & (3.7) & 513 & (3.4) & 566 & (8.4) \\
\hline Portugal & 33.6 & (3.6) & 45.8 & $(4.7)$ & 20.5 & (3.8) & -1.15 & $(0.03)$ & -0.49 & $(0.02)$ & 0.61 & $(0.09)$ & 441 & (7.1) & 496 & (3.9) & 543 & (5.8) \\
\hline Slowak. Rep. & 26.9 & $(2.7)$ & 47.3 & (2.8) & 25.8 & (1.9) & -0.86 & $(0.04)$ & -0.17 & $(0.02)$ & 0.50 & $(0.04)$ & 412 & (6.3) & 477 & (3.7) & 563 & (6.8) \\
\hline Slowenien & 29.5 & (0.6) & 40.2 & $(0.7)$ & 30.3 & $(0.5)$ & -0.47 & $(0.01)$ & 0.03 & $(0.02)$ & 0.66 & $(0.02)$ & 437 & (1.9) & 492 & (1.9) & 579 & (2.2) \\
\hline Spanien & 29.5 & (2.4) & 42.9 & $(3.2)$ & 27.7 & (2.5) & -0.79 & $(0.02)$ & -0.23 & $(0.01)$ & 0.52 & $(0.03)$ & 450 & (3.3) & 484 & $(2.3)$ & 522 & (2.6) \\
\hline Schweden & 18.0 & $(2.7)$ & 58.9 & (3.4) & 23.1 & (2.6) & -0.17 & $(0.03)$ & 0.22 & $(0.01)$ & 0.74 & $(0.02)$ & 453 & (6.0) & 474 & (2.5) & 508 & (5.3) \\
\hline Schweiz & 26.5 & (3.1) & 47.2 & (3.4) & 26.3 & (2.6) & -0.28 & $(0.02)$ & 0.11 & $(0.02)$ & 0.73 & $(0.03)$ & 497 & $(5.3)$ & 516 & (3.4) & 592 & (6.2) \\
\hline Türkei & 32.0 & (3.2) & 43.1 & (4.1) & 24.8 & (2.8) & -2.05 & $(0.03)$ & -1.49 & $(0.02)$ & -0.63 & $(0.07)$ & 402 & (4.5) & 435 & (5.3) & 529 & (11.5) \\
\hline Ver. Königreich & 24.2 & (2.6) & 52.1 & (3.0) & 23.7 & $(2.3)$ & -0.23 & $(0.03)$ & 0.26 & $(0.01)$ & 0.79 & $(0.02)$ & 449 & (7.9) & 489 & (3.5) & 552 & (5.6) \\
\hline Ver. Staaten & 26.6 & (3.5) & 44.4 & (4.3) & 29.0 & (3.3) & -0.48 & $(0.04)$ & 0.17 & $(0.02)$ & 0.78 & $(0.04)$ & 435 & (5.0) & 485 & (4.8) & 519 & (4.8) \\
\hline OECD-Durchschnitt & 26.4 & $(0.4)$ & 47.2 & $(0.6)$ & 26.5 & $(0.4)$ & -0.56 & $(0.01)$ & $\mid-0.02$ & $(0.00)$ & 0.60 & $(0.01)$ & 444 & $(0.9)$ & 492 & $(0.7)$ & 548 & (0.9) \\
\hline
\end{tabular}

\begin{tabular}{|c|c|c|c|c|c|c|c|c|c|c|c|c|c|c|c|c|c|c|c|}
\hline ฐ & Albanien & $\mathrm{m}$ & $\mathrm{m}$ & $\mathrm{m}$ & $\mathrm{m}$ & $\mathrm{m}$ & $\mathrm{m}$ & $\mathrm{m}$ & $\mathrm{m}$ & $\mathrm{m}$ & $\mathrm{m}$ & $\mathrm{m}$ & $\mathrm{m}$ & $\mathrm{m}$ & $\mathrm{m}$ & $\mathrm{m}$ & $\mathrm{m}$ & $\mathrm{m}$ & $\mathrm{m}$ \\
\hline Еँ & Argentinien & 33.4 & (3.0) & 34.6 & (3.6) & 31.9 & (3.4) & -1.43 & $(0.04)$ & -0.75 & $(0.03)$ & 0.05 & $(0.05)$ & 342 & (4.4) & 389 & (6.1) & 436 & (5.3) \\
\hline & Brasilien & 33.1 & (2.0) & 42.6 & $(2.2)$ & 24.3 & (1.6) & -1.87 & $(0.02)$ & -1.23 & $(0.02)$ & -0.11 & $(0.05)$ & 363 & $(2.8)$ & 378 & (1.9) & 454 & $(5.7)$ \\
\hline & Bulgarien & 33.7 & (3.0) & 32.4 & (3.6) & 33.8 & $(2.7)$ & -0.97 & $(0.05)$ & -0.29 & $(0.03)$ & 0.46 & $(0.04)$ & 381 & (5.0) & 425 & (4.9) & 510 & (5.7) \\
\hline $\bar{n}$ & Kolumbien & 28.8 & (3.1) & 41.7 & $(3.8)$ & 29.6 & (3.1) & -2.12 & $(0.04)$ & -1.28 & $(0.02)$ & -0.40 & $(0.06)$ & 339 & $(4.1)$ & 372 & (3.5) & 419 & $(5.8)$ \\
\hline है & Costa Rica & 28.5 & (3.2) & 48.7 & (3.5) & 22.8 & $(2.1)$ & -1.88 & $(0.05)$ & -0.99 & $(0.02)$ & 0.16 & $(0.05)$ & 372 & (3.7) & 402 & (3.7) & 461 & (6.5) \\
\hline & Kroatien & 33.1 & (3.0) & 43.6 & (3.4) & 23.4 & $(1.8)$ & -0.79 & $(0.02)$ & -0.35 & $(0.02)$ & 0.32 & $(0.03)$ & 430 & $(4.1)$ & 463 & (5.2) & 546 & $(8.6)$ \\
\hline & Zypern* & 34.5 & $(0.1)$ & 37.0 & $(0.1)$ & 28.5 & $(0.1)$ & -0.38 & $(0.02)$ & 0.08 & $(0.02)$ & 0.65 & $(0.02)$ & 399 & (1.7) & 442 & $(2.0)$ & 487 & (2.3) \\
\hline & Hongkong (China) & 35.3 & (3.4) & 40.2 & (3.8) & 24.5 & (3.6) & -1.33 & $(0.02)$ & -0.84 & $(0.02)$ & 0.05 & $(0.06)$ & 510 & (6.4) & 573 & (5.8) & 615 & (6.9) \\
\hline & Indonesien & 39.9 & (3.8) & 32.9 & (3.5) & 27.1 & (3.3) & -2.38 & $(0.02)$ & -1.88 & $(0.03)$ & -0.85 & $(0.09)$ & 360 & $(6.0)$ & 358 & (4.5) & 417 & $(8.7)$ \\
\hline & Jordanien & 20.8 & (2.5) & 59.3 & (3.5) & 19.9 & $(2.3)$ & -1.02 & $(0.04)$ & -0.46 & $(0.02)$ & 0.30 & $(0.04)$ & 362 & $(4.7)$ & 376 & (3.9) & 438 & $(9.2)$ \\
\hline & Kasachstan & 22.7 & (3.0) & 45.4 & (3.6) & 31.9 & (3.2) & -0.81 & $(0.02)$ & $\mid-0.37$ & $(0.02)$ & 0.12 & $(0.02)$ & 410 & (7.5) & 423 & $(4.0)$ & 460 & $(6.2)$ \\
\hline & Lettland & 20.2 & (2.5) & 50.3 & (3.4) & 29.5 & $(2.9)$ & -0.95 & $(0.04)$ & -0.32 & $(0.02)$ & 0.32 & $(0.03)$ & 452 & (5.5) & 480 & (2.9) & 534 & (4.9) \\
\hline & Liechtenstein & 14.8 & $(0.9)$ & 51.2 & $(0.9)$ & 34.1 & $(0.4)$ & C & C & 0.24 & $(0.08)$ & C & c & C & c & 506 & (5.3) & C & c \\
\hline & Litauen & 22.8 & $(2.1)$ & 53.1 & $(3.2)$ & 24.1 & (2.6) & -0.76 & $(0.03)$ & -0.13 & $(0.02)$ & 0.45 & $(0.03)$ & 430 & $(4.5)$ & 475 & (3.5) & 534 & $(5.3)$ \\
\hline & Macau (China) & 51.6 & $(0.1)$ & 17.5 & $(0.0)$ & 30.8 & $(0.1)$ & -1.22 & $(0.01)$ & -0.89 & $(0.02)$ & -0.32 & $(0.02)$ & 527 & (1.5) & 535 & (2.4) & 558 & (1.9) \\
\hline & Malaysia & 28.6 & $(2.7)$ & 42.9 & $(4.0)$ & 28.6 & $(3.2)$ & -1.36 & $(0.04)$ & -0.76 & $(0.02)$ & -0.04 & $(0.05)$ & 387 & (3.5) & 408 & (3.9) & 473 & (6.6) \\
\hline & Montenegro & 38.7 & $(0.2)$ & 23.6 & $(0.1)$ & 37.7 & $(0.1)$ & -0.63 & $(0.02)$ & $\mid-0.30$ & $(0.03)$ & 0.17 & $(0.02)$ & 363 & (1.5) & 413 & (2.5) & 455 & (1.9) \\
\hline & Peru & 35.0 & $(2.7)$ & 31.8 & $(3.0)$ & 33.2 & $(2.8)$ & -2.13 & $(0.04)$ & -1.25 & $(0.03)$ & -0.28 & $(0.07)$ & 318 & (3.7) & 359 & (3.6) & 429 & (5.5) \\
\hline & Katar & 33.8 & $(0.1)$ & 22.6 & $(0.1)$ & 43.7 & $(0.1)$ & -0.03 & $(0.01)$ & 0.42 & $(0.02)$ & 0.81 & $(0.01)$ & 353 & (1.3) & 344 & (1.6) & 412 & (1.1) \\
\hline & Rumänien & 30.9 & (3.3) & 40.5 & $(4.2)$ & 28.7 & (3.5) & -1.07 & $(0.05)$ & -0.51 & $(0.02)$ & 0.22 & $(0.05)$ & 403 & (5.5) & 434 & (4.4) & 505 & (6.9) \\
\hline & Russ. Föderation & 21.9 & (2.8) & 50.2 & (3.3) & 27.9 & (2.6) & -0.62 & $(0.03)$ & -0.16 & $(0.02)$ & 0.39 & $(0.03)$ & 450 & $(6.2)$ & 474 & (4.9) & 523 & $(5.2)$ \\
\hline & Serbien & 32.3 & (3.3) & 43.2 & $(4.0)$ & 24.4 & $(2.4)$ & -0.74 & $(0.02)$ & -0.33 & $(0.02)$ & 0.36 & $(0.04)$ & 398 & (5.0) & 444 & (5.1) & 525 & (7.4) \\
\hline & Shanghai (China) & 29.4 & (3.1) & 37.4 & (3.7) & 33.2 & (3.1) & -1.06 & $(0.05)$ & -0.35 & $(0.02)$ & 0.25 & $(0.04)$ & 541 & $(6.2)$ & 611 & (5.2) & 678 & (6.4) \\
\hline & Singapur & 30.5 & $(0.2)$ & 45.3 & $(0.9)$ & 24.2 & $(0.9)$ & -0.71 & $(0.02)$ & -0.33 & $(0.02)$ & 0.45 & $(0.02)$ & 526 & (2.4) & 562 & (2.1) & 655 & (3.4) \\
\hline & Chinesisch Taipeh & 30.0 & (3.1) & 42.2 & (3.9) & 27.7 & $(2.8)$ & -0.86 & $(0.03)$ & -0.44 & $(0.02)$ & 0.15 & $(0.04)$ & 485 & (5.5) & 560 & (4.5) & 641 & $(6.7)$ \\
\hline & Thailand & 39.1 & (2.9) & 31.7 & (3.0) & 29.2 & (2.6) & -2.04 & $(0.03)$ & -1.37 & $(0.02)$ & -0.40 & $(0.05)$ & 404 & (4.5) & 415 & (4.2) & 471 & (6.2) \\
\hline & Tunesien & 29.5 & (2.9) & 44.2 & (3.9) & 26.4 & (3.0) & -2.04 & $(0.05)$ & -1.22 & $(0.03)$ & -0.22 & $(0.06)$ & 353 & $(4.7)$ & 380 & (5.4) & 440 & (8.5) \\
\hline & Ver. Arab. Emirate & 25.4 & (1.5) & 40.0 & $(2.3)$ & 34.6 & $(2.0)$ & -0.26 & $(0.03)$ & 0.29 & $(0.02)$ & 0.80 & $(0.02)$ & 390 & $(2.9)$ & 426 & (3.4) & 476 & $(5.1)$ \\
\hline & Uruguay & 40.4 & (2.9) & 37.4 & (3.0) & 22.2 & $(2.1)$ & -1.51 & $(0.02)$ & -0.89 & $(0.02)$ & 0.26 & $(0.08)$ & 365 & $(4.1)$ & 413 & (4.3) & 483 & (5.5) \\
\hline & Vietnam & 39.4 & (3.3) & 34.4 & $(3.7)$ & 26.2 & $(3.1)$ & -2.46 & $(0.03)$ & -1.85 & $(0.02)$ & -0.78 & $(0.09)$ & 471 & $(6.9)$ & 517 & (4.9) & 565 & $(8.0)$ \\
\hline & $\begin{array}{l}\text { Anmerkung: Statisti } \\
\text { 1. ESCS bezieht sich } \\
\text { 2. Sozioökonomisch } \\
\text { Schule über (unter) } \\
\text { nehmen } 35 \text { als Zufal } \\
\text { Das sozioökonomis } \\
\text { ab, die an den einze } \\
\text { begünstigte, sozioöl } \\
\text { zwischen dem sozi } \\
\text { Landes/der Volkswir } \\
\text { Schule statistisch sig } \\
\text { das Profil der Schul } \\
\text { *Vgl. Anmerkungen } \\
\text { StatLink }\end{array}$ & hisch & $\begin{array}{l}\text { chte } \\
\text { Prof } \\
\text { tistis } \\
\text { nd d } \\
\text { hscl }\end{array}$ & ner & $\begin{array}{l}\text { nozi } \\
\text { nt wen } \\
\text { sCS } \\
\text { SCS }\end{array}$ & $\begin{array}{l}\text { on } \\
\text { den } \\
\text { Nurc } \\
\text { CS d } \\
\text { Lan }\end{array}$ & $\begin{array}{l}\text { d } \\
\text { nd } \\
\text { nd }\end{array}$ & rV & $\begin{array}{l}\text { he } \\
\text { ne } \\
\text { nule } \\
\text { irts }\end{array}$ & $\begin{array}{l}\text { den } \\
\text { durs } \\
\text { t lag }\end{array}$ & $\begin{array}{l}\text { Irde } \\
\text { Innit } \\
\text { tant } \\
\text { und }\end{array}$ & $\begin{array}{l}\text { Fr } \\
\text { er } V \\
\text { em }\end{array}$ & $\begin{array}{l}\text { der } \\
\text { swirt } \\
\text { ioök } \\
\text { als so }\end{array}$ & $\begin{array}{l}\text { auig } \\
t \text { (d } \\
\text { iscl }\end{array}$ & $\begin{array}{l}\text { ber } \\
\text { dur } \\
\text { Pro } \\
\text { ch }\end{array}$ & sicht & $\begin{array}{l}\text { Wer } \\
\text { t. Wen } \\
\text { Schu } \\
\text { Sch }\end{array}$ & no & $\begin{array}{l}\text { rofil de } \\
\text { Schule } \\
\text { nächst) } \\
\text { Schüle } \\
\text { omisch } \\
\text { erschied } \\
\text { ene des } \\
\text { fil eine } \\
\text { t. Wenn }\end{array}$ \\
\hline
\end{tabular}


[Teil 2/4]

Mathematikleistungen und Schülerpopulation, nach sozioökonomischem Profil der Schulen Tabelle II.4.2 Die Ergebnisse basieren auf Schülerangaben

\begin{tabular}{|c|c|c|c|c|c|c|c|c|c|c|c|c|c|c|c|c|c|c|}
\hline & \multicolumn{6}{|c|}{$\begin{array}{l}\text { Prozentsatz der Schüler mit } \\
\text { Migrationshintergrund }\end{array}$} & \multicolumn{6}{|c|}{$\begin{array}{l}\text { Prozentsatz der Schüler mit } \\
\text { Migrationshintergrund, die zu Hause } \\
\text { nicht die Testsprache sprechen }\end{array}$} & \multicolumn{6}{|c|}{$\begin{array}{l}\text { Prozentsatz der Schüler in ländlichen Gebieten } \\
\text { (in Dörfern, Ortschaften oder ländlichen } \\
\text { Gemeinden, unter } 3000 \text { Einwohner) }\end{array}$} \\
\hline & \multicolumn{2}{|c|}{\begin{tabular}{|c|} 
Sozio- \\
ökonomisch \\
benachteiligte \\
Schulen $^{2}$
\end{tabular}} & \multicolumn{2}{|c|}{$\begin{array}{c}\text { Sozio- } \\
\text { ökonomisch } \\
\text { durchschnittl. } \\
\text { Schulen }^{2}\end{array}$} & \multicolumn{2}{|c|}{$\begin{array}{c}\text { Sozio- } \\
\text { ökonomisch } \\
\text { begünstigte } \\
\text { Schulen }\end{array}$} & \multicolumn{2}{|c|}{$\begin{array}{c}\text { Sozio- } \\
\text { ökonomisch } \\
\text { benachteiligte } \\
\text { Schulen }^{2}\end{array}$} & \multicolumn{2}{|c|}{$\begin{array}{c}\text { Sozio- } \\
\text { ökonomisch } \\
\text { durchschnittl. } \\
\text { Schulen }{ }^{2}\end{array}$} & \multicolumn{2}{|c|}{$\begin{array}{c}\text { Sozio- } \\
\text { ökonomisch } \\
\text { begünstigte } \\
\text { Schulen }^{2}\end{array}$} & \multicolumn{2}{|c|}{$\begin{array}{c}\text { Sozio- } \\
\text { ökonomisch } \\
\text { benachteiligte } \\
\text { Schulen }\end{array}$} & \multicolumn{2}{|c|}{$\begin{array}{c}\text { Sozio- } \\
\text { ökonomisch } \\
\text { durchschnittl. } \\
\text { Schulen }^{2}\end{array}$} & \multicolumn{2}{|c|}{$\begin{array}{c}\text { Sozio- } \\
\text { ökonomisch } \\
\text { begünstigte } \\
\text { Schulen }^{2} \\
\end{array}$} \\
\hline & $\%$ & S.E. & $\%$ & S.E. & $\%$ & S.E. & $\%$ & S.E. & $\%$ & S.E. & $\%$ & S.E. & $\%$ & S.E. & $\%$ & S.E. & $\%$ & S.E. \\
\hline Australien & 22.3 & $(2.0)$ & 6.5 & $(0.5)$ & 25.8 & $(1.5)$ & 8.0 & $(1.3)$ & 6.5 & $(0.5)$ & 8.0 & $(1.0)$ & 61.9 & $(7.2)$ & 33.5 & $(7.4)$ & 4.6 & (3.0) \\
\hline Österreich & 24.3 & (2.4) & 8.3 & (1.1) & 11.0 & (1.4) & 15.2 & (1.9) & 8.3 & (1.1) & 6.2 & (1.1) & 35.2 & $(12.0)$ & 41.6 & $(12.2)$ & 23.2 & (11.6) \\
\hline Belgien & 24.9 & $(2.8)$ & 13.1 & (1.5) & 9.7 & $(1.1)$ & 12.9 & (1.5) & 6.1 & $(0.7)$ & 3.4 & $(0.6)$ & 38.1 & $(18.7)$ & 25.9 & (16.6) & 35.9 & (19.6) \\
\hline Kanada & 38.9 & (3.8) & 10.8 & $(0.9)$ & 35.7 & $(2.9)$ & 19.0 & (2.5) & 10.8 & $(0.9)$ & 16.8 & $(1.5)$ & 29.3 & $(5.2)$ & 60.0 & (6.1) & 10.7 & $(5.1)$ \\
\hline Chile & 0.5 & $(0.2)$ & 0.0 & $(0.0)$ & 1.0 & $(0.2)$ & 0.0 & $(0.0)$ & 0.0 & $(0.0)$ & 0.1 & $(0.1)$ & 93.2 & (5.0) & 0.0 & c & 6.8 & (5.0) \\
\hline Tschech. Rep. & 2.5 & $(0.7)$ & 2.0 & $(0.4)$ & 4.1 & $(0.6)$ & 1.1 & $(0.5)$ & 2.0 & $(0.4)$ & 3.3 & $(0.5)$ & 16.6 & (9.4) & 80.0 & (9.6) & 3.3 & (3.3) \\
\hline Dänemark & 17.4 & (2.5) & 3.7 & $(0.3)$ & 3.8 & $(0.7)$ & 7.6 & $(0.9)$ & 3.7 & $(0.3)$ & 1.4 & $(0.4)$ & 24.7 & (7.1) & 67.5 & $(7.7)$ & 7.8 & (4.7) \\
\hline Estland & 8.8 & (1.7) & 1.5 & $(0.3)$ & 5.5 & (1.5) & 1.8 & $(0.9)$ & 1.5 & $(0.3)$ & 1.9 & $(0.5)$ & 36.6 & (6.6) & 61.3 & $(6.7)$ & 2.2 & $(2.2)$ \\
\hline Finnland & 4.8 & $(0.8)$ & 2.5 & $(0.1)$ & 3.4 & $(0.3)$ & 3.9 & $(0.8)$ & 2.5 & $(0.1)$ & 2.6 & $(0.3)$ & 61.2 & (13.5) & 38.7 & $(13.5)$ & 0.2 & $(0.0)$ \\
\hline Frankreich & w & w & w & w & w & w & w & w & w & w & w & w & w & w & w & w & w & w \\
\hline Deutschland & 20.0 & $(2.1)$ & 4.5 & $(0.6)$ & 7.7 & (1.1) & 8.4 & $(1.2)$ & 4.5 & $(0.6)$ & 2.6 & $(0.6)$ & 60.3 & $(27.2)$ & 39.7 & $(27.2)$ & 0.0 & c \\
\hline Griechenland & 18.6 & $(3.2)$ & 2.5 & $(0.4)$ & 5.4 & $(0.7)$ & 9.9 & (2.3) & 2.5 & $(0.4)$ & 1.4 & $(0.3)$ & 57.5 & (13.3) & 32.0 & (12.5) & 10.5 & (9.4) \\
\hline Ungarn & 0.6 & $(0.2)$ & 0.5 & $(0.3)$ & 2.4 & $(0.5)$ & 0.0 & $(0.0)$ & 0.5 & $(0.3)$ & 0.4 & $(0.3)$ & 76.1 & (17.3) & 23.9 & $(17.3)$ & 0.0 & c \\
\hline Island & 6.9 & $(0.8)$ & 2.2 & $(0.4)$ & 2.5 & $(0.5)$ & 6.1 & $(0.7)$ & 2.2 & $(0.4)$ & 1.7 & $(0.5)$ & 37.9 & $(0.7)$ & 57.9 & $(0.6)$ & 4.2 & $(0.2)$ \\
\hline Irland & 13.3 & $(2.1)$ & 4.7 & $(0.7)$ & 8.4 & $(1.1)$ & 7.1 & (1.5) & 4.7 & $(0.7)$ & 2.6 & $(0.6)$ & 8.3 & (4.6) & 83.0 & (6.6) & 8.8 & (5.0) \\
\hline Israel & 16.7 & (2.5) & 9.1 & $(1.3)$ & 16.1 & (1.9) & 6.3 & $(1.3)$ & 9.1 & (1.3) & 8.3 & (1.7) & 21.8 & (8.2) & 15.2 & (7.1) & 63.1 & (9.5) \\
\hline Italien & 10.8 & $(0.7)$ & 4.3 & $(0.4)$ & 4.0 & $(0.3)$ & 7.6 & (0.6) & 4.3 & $(0.4)$ & 1.9 & $(0.2)$ & 45.3 & (13.3) & 54.2 & (13.4) & 0.4 & $(0.4)$ \\
\hline Japan & 0.5 & $(0.1)$ & 0.0 & $(0.0)$ & 0.4 & $(0.2)$ & 0.3 & $(0.1)$ & 0.0 & $(0.0)$ & 0.3 & $(0.1)$ & 0.0 & c & 0.0 & c & 0.0 & c \\
\hline Korea & 0.0 & $(0.0)$ & 0.0 & $(0.0)$ & 0.0 & $(0.0)$ & 0.0 & $(0.0)$ & 0.0 & $(0.0)$ & 0.0 & $(0.0)$ & 73.6 & (26.0) & 0.0 & c & 26.4 & (26.0) \\
\hline Luxemburg & 58.0 & (1.0) & 23.2 & (1.8) & 36.9 & $(0.8)$ & 48.1 & (1.1) & 23.2 & (1.8) & 16.7 & $(0.8)$ & 0.0 & c & 0.0 & c & 100.0 & c \\
\hline Mexiko & 1.7 & $(0.2)$ & 0.0 & $(0.0)$ & 0.9 & $(0.2)$ & 0.5 & $(0.1)$ & 0.0 & $(0.0)$ & 0.2 & $(0.1)$ & 87.0 & (2.2) & 12.0 & $(2.2)$ & 1.0 & (0.6) \\
\hline Niederlande & 17.5 & (3.3) & 5.0 & $(0.9)$ & 5.4 & $(1.0)$ & 7.9 & (1.8) & 5.0 & $(0.9)$ & 1.9 & $(0.5)$ & 0.0 & c & 0.0 & c & 0.0 & c \\
\hline Neuseeland & 29.9 & (3.4) & 11.0 & (1.4) & 31.7 & $(2.7)$ & 17.0 & (2.3) & 11.0 & (1.4) & 15.1 & $(2.4)$ & 63.6 & (13.5) & 36.4 & (13.5) & 0.0 & c \\
\hline Norwegen & 21.4 & (5.6) & 5.5 & (0.5) & 6.7 & (1.7) & 12.8 & (4.0) & 5.5 & $(0.5)$ & 3.8 & $(0.9)$ & 17.0 & $(6.1)$ & 83.0 & (6.1) & 0.0 & c \\
\hline Polen & 0.1 & $(0.1)$ & 0.0 & $(0.0)$ & 0.5 & $(0.2)$ & 0.1 & $(0.1)$ & 0.0 & $(0.0)$ & 0.2 & $(0.1)$ & 54.1 & (7.0) & 43.3 & (7.0) & 2.5 & (2.2) \\
\hline Portugal & 6.7 & (1.3) & 2.4 & $(0.5)$ & 4.6 & $(0.9)$ & 1.7 & $(0.5)$ & 2.4 & $(0.5)$ & 0.9 & $(0.2)$ & 81.2 & (14.5) & 18.8 & (14.5) & 0.0 & c \\
\hline Slowak. Rep. & 0.7 & $(0.3)$ & 0.1 & $(0.1)$ & 0.8 & $(0.2)$ & 0.2 & $(0.1)$ & 0.1 & $(0.1)$ & 0.3 & $(0.2)$ & 51.4 & (8.1) & 48.6 & (8.1) & 0.0 & c \\
\hline Slowenien & 13.2 & (1.1) & 3.8 & $(0.6)$ & 5.7 & $(0.6)$ & 8.2 & (0.8) & 3.8 & $(0.6)$ & 2.3 & $(0.5)$ & 6.1 & (3.4) & 93.9 & (3.4) & 0.0 & c \\
\hline Spanien & 13.3 & (1.4) & 4.7 & $(0.7)$ & 5.0 & $(0.7)$ & 7.1 & $(1.2)$ & 4.7 & $(0.7)$ & 2.1 & $(0.5)$ & 65.9 & (11.5) & 30.4 & (11.5) & 3.7 & (1.7) \\
\hline Schweden & 22.8 & (4.3) & 8.5 & $(0.8)$ & 10.0 & (1.5) & 14.9 & (3.2) & 8.5 & $(0.8)$ & 4.6 & $(1.2)$ & 34.1 & (9.2) & 59.7 & (9.4) & 6.2 & $(4.2)$ \\
\hline Schweiz & 29.8 & $(2.2)$ & 13.1 & $(0.8)$ & 16.8 & (1.2) & 16.6 & (1.8) & 13.1 & $(0.8)$ & 6.6 & $(0.7)$ & 48.4 & $(10.8)$ & 49.4 & (10.8) & 2.3 & (3.3) \\
\hline Türkei & 0.7 & $(0.4)$ & 0.2 & $(0.1)$ & 1.0 & $(0.5)$ & 0.2 & $(0.2)$ & 0.2 & $(0.1)$ & 0.1 & $(0.1)$ & 27.4 & (17.5) & 39.1 & $(23.7)$ & 33.5 & (20.5) \\
\hline Ver. Königreich & 20.9 & $(2.8)$ & 3.2 & $(0.7)$ & 14.6 & $(2.6)$ & 10.2 & (1.6) & 3.2 & $(0.7)$ & 6.9 & $(1.8)$ & 11.6 & $(7.0)$ & 47.6 & $(10.5)$ & 40.8 & $(10.6)$ \\
\hline Ver. Staaten & 40.0 & (3.4) & 8.4 & (1.6) & 12.7 & (2.6) & 25.4 & (2.6) & 8.4 & (1.6) & 6.2 & (1.3) & 22.8 & (12.7) & 77.2 & $(12.7)$ & 0.0 & c \\
\hline OECD-Durchschnitt & 15.7 & $(0.4)$ & 10.1 & $(0.2)$ & 9.1 & $(0.2)$ & 8.7 & $(0.3)$ & 4.8 & $(0.1)$ & 3.9 & $(0.1)$ & 41.2 & (2.2) & 41.2 & (2.3) & 11.7 & (2.1) \\
\hline
\end{tabular}

\begin{tabular}{|c|c|c|c|c|c|c|c|c|c|c|c|c|c|c|c|c|c|c|}
\hline Albanien & $\mathrm{m}$ & $\mathrm{m}$ & $\mathrm{m}$ & $\mathrm{m}$ & $\mathrm{m}$ & $\mathrm{m}$ & $\mathrm{m}$ & $\mathrm{m}$ & $\mathrm{m}$ & $\mathrm{m}$ & $\mathrm{m}$ & $\mathrm{m}$ & $\mathrm{m}$ & $\mathrm{m}$ & $\mathrm{m}$ & $\mathrm{m}$ & $\mathrm{m}$ & $\mathrm{m}$ \\
\hline Argentinien & 6.6 & $(1.0)$ & 0.5 & $(0.3)$ & 1.4 & $(0.4)$ & 1.1 & $(0.3)$ & 0.5 & $(0.3)$ & 0.1 & $(0.1)$ & 51.4 & $(12.8)$ & 48.6 & (12.8) & 0.0 & c \\
\hline Brasilien & 0.5 & $(0.1)$ & 0.1 & $(0.1)$ & 1.0 & $(0.4)$ & 0.0 & $(0.0)$ & 0.1 & $(0.1)$ & 0.4 & $(0.2)$ & 88.8 & (6.6) & 11.2 & (6.6) & 0.0 & c \\
\hline Bulgarien & 0.4 & $(0.2)$ & 0.3 & $(0.2)$ & 0.4 & $(0.2)$ & 0.1 & $(0.1)$ & 0.3 & $(0.2)$ & 0.3 & $(0.2)$ & 88.1 & (8.5) & 11.9 & (8.5) & 0.0 & c \\
\hline Kolumbien & 0.5 & $(0.2)$ & 0.0 & $(0.0)$ & 0.1 & $(0.0)$ & 0.0 & $(0.0)$ & 0.0 & $(0.0)$ & 0.0 & $(0.0)$ & 87.5 & (6.1) & 12.4 & (6.0) & 0.2 & $(0.1)$ \\
\hline Costa Rica & 7.2 & $(2.0)$ & 0.1 & $(0.0)$ & 4.4 & $(0.8)$ & 0.0 & $(0.0)$ & 0.1 & $(0.0)$ & 0.8 & $(0.3)$ & 66.7 & (7.3) & 26.0 & (6.9) & 7.3 & (4.1) \\
\hline Kroatien & 13.9 & (1.5) & 0.2 & $(0.1)$ & 10.3 & $(1.1)$ & 0.4 & $(0.1)$ & 0.2 & $(0.1)$ & 0.1 & $(0.1)$ & 62.6 & (34.5) & 37.4 & (34.5) & 0.0 & c \\
\hline Zypern* & 7.6 & $(0.6)$ & 3.9 & $(0.4)$ & 9.2 & $(0.7)$ & 4.4 & $(0.4)$ & 3.9 & $(0.4)$ & 3.8 & $(0.5)$ & 54.6 & $(0.6)$ & 45.4 & $(0.6)$ & c & c \\
\hline Hongkong (China) & 42.5 & (1.9) & 3.4 & $(0.4)$ & 18.0 & $(2.2)$ & 5.6 & (1.1) & 3.4 & $(0.4)$ & 1.6 & $(0.5)$ & 0.0 & c & 0.0 & c & 0.0 & c \\
\hline Indonesien & 0.1 & $(0.1)$ & 0.1 & $(0.1)$ & 0.2 & $(0.1)$ & 0.0 & $(0.0)$ & 0.1 & $(0.1)$ & 0.1 & $(0.1)$ & 61.7 & (7.3) & 31.8 & (6.8) & 6.4 & (4.7) \\
\hline Jordanien & 8.6 & (1.6) & 0.6 & $(0.1)$ & 19.9 & $(2.4)$ & 0.3 & $(0.1)$ & 0.6 & $(0.1)$ & 2.0 & $(0.5)$ & 52.3 & (11.1) & 34.6 & (10.8) & 13.1 & (8.4) \\
\hline Kasachstan & 28.2 & $(5.0)$ & 1.0 & $(0.2)$ & 13.3 & (1.6) & 0.6 & $(0.3)$ & 1.0 & $(0.2)$ & 2.6 & (0.6) & 45.0 & (5.4) & 52.3 & (5.8) & 2.7 & (2.2) \\
\hline Lettland & 3.8 & $(0.9)$ & 1.1 & $(0.4)$ & 4.2 & $(0.9)$ & 1.5 & $(0.8)$ & 1.1 & $(0.4)$ & 0.8 & $(0.2)$ & 52.9 & (7.0) & 44.1 & (7.1) & 2.9 & (2.1) \\
\hline Liechtenstein & c & C & 12.5 & (2.8) & C & C & c & c & 12.5 & (2.8) & C & C & 0.0 & C & 0.0 & c & 0.0 & c \\
\hline Litauen & 0.8 & $(0.3)$ & 0.5 & $(0.1)$ & 1.6 & $(0.6)$ & 0.4 & $(0.2)$ & 0.5 & $(0.1)$ & 0.3 & $(0.2)$ & 65.2 & (6.6) & 34.8 & (6.6) & 0.0 & c \\
\hline Macau (China) & 74.1 & $(0.8)$ & 4.0 & $(0.5)$ & 50.4 & $(1.3)$ & 1.1 & $(0.2)$ & 4.0 & $(0.5)$ & 18.4 & $(0.9)$ & 0.0 & c & 100.0 & c & 0.0 & c \\
\hline Malaysia & 1.7 & $(0.6)$ & 0.4 & $(0.2)$ & 1.0 & $(0.3)$ & 0.3 & $(0.2)$ & 0.4 & $(0.2)$ & 0.6 & $(0.3)$ & 69.8 & $(10.0)$ & 30.2 & $(10.0)$ & 0.0 & c \\
\hline Montenegro & 4.3 & $(0.5)$ & 0.0 & $(0.0)$ & 7.5 & $(0.7)$ & 0.0 & $(0.0)$ & 0.0 & $(0.0)$ & 0.5 & $(0.2)$ & 0.0 & c & 0.0 & c & 0.0 & c \\
\hline Peru & 0.7 & $(0.2)$ & 0.1 & $(0.1)$ & 0.3 & $(0.1)$ & 0.2 & $(0.1)$ & 0.1 & $(0.1)$ & 0.0 & $(0.0)$ & 80.9 & (5.9) & 16.2 & (5.4) & 2.9 & (2.9) \\
\hline Katar & 52.4 & $(0.7)$ & 11.4 & $(0.4)$ & 53.9 & $(0.5)$ & 27.7 & $(0.5)$ & 11.4 & $(0.4)$ & 32.0 & $(0.5)$ & 35.9 & $(0.3)$ & 18.5 & $(0.2)$ & 45.6 & $(0.3)$ \\
\hline Rumänien & 0.1 & $(0.1)$ & 0.0 & $(0.0)$ & 0.4 & $(0.2)$ & 0.0 & $(0.0)$ & 0.0 & $(0.0)$ & 0.2 & $(0.1)$ & 67.5 & (12.5) & 32.5 & (12.5) & 0.0 & c \\
\hline Russ. Föderation & 12.4 & (1.8) & 1.2 & $(0.2)$ & 10.5 & $(1.0)$ & 3.3 & (1.0) & 1.2 & $(0.2)$ & 0.7 & $(0.2)$ & 47.5 & (5.5) & 52.5 & (5.5) & 0.0 & c \\
\hline Serbien & 8.9 & (1.5) & 0.9 & $(0.6)$ & 8.1 & $(0.9)$ & 0.8 & $(0.3)$ & 0.9 & $(0.6)$ & 0.4 & $(0.2)$ & 100.0 & c & 0.0 & $c$ & 0.0 & c \\
\hline Shanghai (China) & 2.0 & $(0.5)$ & 0.3 & $(0.2)$ & 0.3 & $(0.2)$ & 0.6 & $(0.2)$ & 0.3 & $(0.2)$ & 0.1 & $(0.1)$ & 0.0 & c & 0.0 & c & 0.0 & c \\
\hline Singapur & 15.0 & $(0.8)$ & 12.0 & $(0.7)$ & 28.4 & $(2.1)$ & 13.6 & $(0.7)$ & 12.0 & $(0.7)$ & 17.0 & $(1.7)$ & 0.0 & c & 0.0 & c & 0.0 & c \\
\hline Chinesisch Taipeh & 0.5 & $(0.2)$ & 0.1 & $(0.1)$ & 0.2 & $(0.1)$ & 0.0 & $(0.0)$ & 0.1 & $(0.1)$ & 0.0 & $(0.0)$ & 67.0 & (27.0) & 32.5 & $(27.1)$ & 0.6 & $(0.4)$ \\
\hline Thailand & 1.7 & (1.1) & 0.1 & $(0.1)$ & 0.0 & $(0.0)$ & 1.4 & $(0.9)$ & 0.1 & $(0.1)$ & 0.0 & $(0.0)$ & 74.2 & (7.5) & 21.7 & (7.0) & 4.1 & (4.0) \\
\hline Tunesien & 0.4 & $(0.2)$ & 0.1 & $(0.1)$ & 0.5 & $(0.2)$ & 0.0 & $(0.0)$ & 0.1 & $(0.1)$ & 0.1 & $(0.1)$ & 79.4 & (17.1) & 20.6 & $(17.1)$ & 0.0 & c \\
\hline Ver. Arab. Emirate & 41.5 & $(2.1)$ & 25.1 & $(2.2)$ & 66.9 & (3.3) & 14.9 & (1.8) & 25.1 & $(2.2)$ & 31.0 & $(2.6)$ & 52.4 & $(7.4)$ & 28.7 & $(5.2)$ & 18.9 & $(4.7)$ \\
\hline Uruguay & 0.3 & $(0.1)$ & 0.1 & $(0.1)$ & 0.9 & $(0.3)$ & 0.1 & $(0.1)$ & 0.1 & $(0.1)$ & 0.3 & $(0.2)$ & 70.2 & (10.4) & 29.8 & (10.4) & 0.0 & c \\
\hline Vietnam & 0.1 & $(0.1)$ & 0.0 & $(0.0)$ & 0.2 & $(0.2)$ & 0.0 & $(0.0)$ & 0.0 & $(0.0)$ & 0.2 & $(0.2)$ & 66.0 & (5.8) & 28.2 & (5.4) & 5.8 & (2.9) \\
\hline
\end{tabular}

Anmerkung: Statistisch signifikante Werte sind durch Fettdruck gekennzeichnet (vgl. Anhang A3)

1. ESCS bezieht sich auf den PISA-Index des wirtschaftlichen, sozialen und kulturellen Status.

2. Sozioökonomisch begünstigte (sozioökonomisch benachteiligte) Schulen sind Schulen, in denen der typische Schüler der Schule bzw. das sozioökonomische Profil der Schule über (unter) dem ESCS des typischen Schülers des Landes/der Volkswirtschaft bzw. dem durchschnittlichen ESCS des Landes/der Volkswirtschaft liegt. An jeder Schule nehmen 35 als Zufallsstichprobe ausgewählte Schülerinnen und Schüler an PISA teil (wegen näherer Einzelheiten vgl. PISA 2012 Technical Report, OECD, erscheint demnächst). Das sozioökonomische Profil der Schulen wird ausgehend von den Angaben dieser Schüler ermittelt. Deshalb hängt die Genauigkeit der Schätzung von der Zahl der Schüler $\mathrm{ab}$, die an den einzelnen Schulen tatsächlich an der Erhebung teilnehmen, sowie von der Variationsbreite ihrer Antworten. Bei der Einstufung der Schulen in sozioökonomisch begünstigte, sozioökonomisch benachteiligte oder sozioökonomisch durchschnittliche Schulen wurde diese Frage der Genauigkeit berücksichtigt. Wenn der Unterschied zwischen dem soziö̈konomischen Profil einer Schule und dem ESCS des typischen Schülers des Landes/der Volkswirtschaft (dem durchschnittlichen ESCS auf Ebene des Landes/der Volkswirtschaft) nicht statistisch signifikant war, wurde die Schule als Schule mit durchschnittlichem sozioökonomischem Profil eingestuft. Wenn das Profil einer
Schule statistisch signifikant über dem durchschnittlichen ESCS des Landes/der Volkswirtschaft lag, wurde die Schule als sozioökonomisch begünstigte Schule eingestuft. Wenn das Profil der Schule unter dem durchschnittlichen ESCS des Landes/der Volkswirtschaft lag, wurde die Schule als sozioökonomisch benachteiligte Schule eingestuft.

* Vgl. Anmerkungen am Anfang dieses Anhangs.

StatLink तins http://dx.doi.org/10.1787/888932964946 
[Teil 3/4]

Mathematikleistungen und Schülerpopulation, nach sozioökonomischem Profil der Schulen Tabelle II.4.2 Die Ergebnisse basieren auf Schülerangaben

\begin{tabular}{|c|c|c|c|c|c|c|c|c|c|c|c|c|c|c|}
\hline & \multicolumn{6}{|c|}{$\begin{array}{l}\text { Prozentsatz der Schüler in mittleren Großstädten } \\
\text { oder Großstädten (über } 100000 \text { Einwohner) }\end{array}$} & \multicolumn{4}{|c|}{\begin{tabular}{|c|} 
Leistungsunterschied zwischen \\
Schülern in sozioökonomisch \\
durchschnittlichen und sozioökonomisch \\
benachteiligten Schulen
\end{tabular}} & \multicolumn{4}{|c|}{$\begin{array}{l}\text { Leistungsunterschied zwischen } \\
\text { Schülern in sozioökonomisch } \\
\text { begünstigten und sozioökonomisch } \\
\text { durchschnittlichen Schulen }\end{array}$} \\
\hline & \multicolumn{2}{|c|}{$\begin{array}{c}\text { Sozioökonomisch } \\
\text { benachteiligte } \\
\text { Schulen }^{2}\end{array}$} & \multicolumn{2}{|c|}{$\begin{array}{c}\text { Sozioökonomisch } \\
\text { durchschnittliche } \\
\text { Schulen }^{2}\end{array}$} & \multicolumn{2}{|c|}{$\begin{array}{c}\text { Sozioökonomisch } \\
\text { begünstigte } \\
\text { Schulen }^{2}\end{array}$} & \multicolumn{2}{|c|}{$\begin{array}{l}\text { Vor Berücksich- } \\
\text { tigung des ESCS } \\
\text { der Schüler }\end{array}$} & \multicolumn{2}{|c|}{$\begin{array}{l}\text { Nach Berücksich- } \\
\text { tigung des ESCS } \\
\text { der Schüler }\end{array}$} & \multicolumn{2}{|c|}{$\begin{array}{l}\text { Vor Berücksich- } \\
\text { tigung des ESCS }{ }^{1} \\
\text { der Schüler }\end{array}$} & \multicolumn{2}{|c|}{$\begin{array}{l}\text { Nach Berücksich- } \\
\text { tigung des ESCS } \\
\text { der Schüler }\end{array}$} \\
\hline & $\%$ & S.E. & $\%$ & S.E. & $\%$ & S.E. & Punktdiff. & S.E. & Punktdiff. & S.E. & Punktdiff. & S.E. & Punktdiff. & S.E. \\
\hline ¿ Australien & 18.0 & $(1.5)$ & 49.2 & $(2.2)$ & 32.8 & (1.9) & 41 & $(3.6)$ & 24 & (3.4) & 58 & $(4.1)$ & 42 & $(4.4)$ \\
\hline Österreich & 31.0 & $(4.4)$ & 35.4 & $(7.3)$ & 33.6 & (6.5) & 75 & $(7.8)$ & 63 & (7.7) & 37 & $(7.7)$ & 23 & (7.8) \\
\hline B] Belgien & 35.7 & $(5.8)$ & 24.3 & (5.6) & 40.0 & (7.0) & 75 & (5.9) & 58 & (5.9) & 72 & (6.0) & 57 & (6.1) \\
\hline Kanada & 21.7 & $(3.0)$ & 44.4 & $(3.7)$ & 33.9 & (3.2) & 22 & $(4.7)$ & 9 & $(4.6)$ & 45 & $(4.0)$ & 31 & $(4.0)$ \\
\hline Chile & 29.4 & (3.9) & 29.3 & $(5.0)$ & 41.3 & (4.3) & 21 & $(7.6)$ & 11 & (7.5) & 68 & (8.1) & 46 & (7.9) \\
\hline Tschech. Rep. & 11.4 & $(4.7)$ & 56.2 & $(7.3)$ & 32.4 & (5.6) & 57 & $(8.7)$ & 45 & $(8.3)$ & 96 & (5.2) & 84 & (5.3) \\
\hline Dänemark & 24.9 & (8.0) & 45.0 & $(8.3)$ & 30.1 & $(8.3)$ & 30 & (5.4) & 13 & $(4.2)$ & 41 & (4.6) & 22 & $(4.1)$ \\
\hline Estland & 6.8 & $(2.3)$ & 46.1 & $(5.4)$ & 47.1 & $(4.9)$ & 15 & (5.1) & 5 & (5.1) & 44 & (4.4) & 30 & (4.3) \\
\hline Finnland & 5.3 & $(0.5)$ & 61.0 & $(4.7)$ & 33.7 & $(4.6)$ & 20 & (4.9) & 7 & $(4.5)$ & 19 & (5.1) & 6 & $(4.3)$ \\
\hline Frankreich & w & w & w & w & w & w & w & w & w & w & w & w & w & w \\
\hline Deutschland & 25.2 & $(6.4)$ & 38.1 & $(6.5)$ & 36.6 & $(6.7)$ & 69 & $(7.7)$ & 59 & $(7.9)$ & 72 & (6.9) & 62 & $(6.9)$ \\
\hline Griechenland & 15.7 & $(4.7)$ & 41.0 & $(7.8)$ & 43.3 & (7.1) & 60 & (7.1) & 46 & (6.9) & 41 & (5.1) & 25 & $(4.8)$ \\
\hline Ungarn & 18.9 & (5.2) & 38.2 & (5.4) & 42.8 & (6.1) & 55 & (8.5) & 44 & $(8.1)$ & 90 & (7.5) & 78 & $(6.7)$ \\
\hline Island & 5.9 & $(0.1)$ & 48.4 & $(0.4)$ & 45.7 & $(0.4)$ & 20 & $(4.4)$ & 9 & (4.8) & 36 & (3.2) & 24 & (3.5) \\
\hline Irland & 29.5 & (5.3) & 26.1 & $(6.7)$ & 44.5 & (6.5) & 65 & (5.8) & 49 & (5.6) & 31 & (3.9) & 15 & (3.4) \\
\hline Israel & 21.7 & (5.1) & 53.3 & (6.7) & 25.0 & (5.7) & 69 & (9.7) & 55 & $(9.1)$ & 62 & (9.8) & 43 & (9.5) \\
\hline Italien & 18.2 & (3.1) & 41.4 & $(4.1)$ & 40.4 & (3.4) & 56 & (4.4) & 49 & (4.6) & 59 & (4.6) & 53 & $(4.7)$ \\
\hline Japan & 22.5 & (2.9) & 43.5 & $(4.2)$ & 34.0 & (3.1) & 66 & (8.8) & 60 & (8.3) & 60 & (7.3) & 54 & $(7.1)$ \\
\hline Korea & 24.4 & $(2.5)$ & 50.1 & (3.9) & 25.5 & (3.6) & 65 & (8.3) & 57 & $(8.4)$ & 55 & (9.8) & 45 & (9.9) \\
\hline Luxemburg & 0.0 & c & 0.0 & c & 0.0 & c & 53 & (2.6) & 41 & $(2.9)$ & 48 & (3.1) & 35 & (3.6) \\
\hline Mexiko & 7.9 & (1.5) & 36.5 & (3.1) & 55.6 & $(3.0)$ & 25 & (3.4) & 18 & (3.5) & 42 & (3.0) & 32 & (2.7) \\
\hline Niederlande & 22.8 & (5.5) & 44.2 & $(7.5)$ & 33.0 & (7.1) & 87 & (8.2) & 79 & $(8.1)$ & 65 & (9.9) & 58 & (9.9) \\
\hline Neuseeland & 14.1 & $(3.8)$ & 47.0 & $(5.8)$ & 38.9 & $(4.6)$ & 54 & $(6.6)$ & 32 & (5.6) & 60 & (6.2) & 36 & $(6.2)$ \\
\hline Norwegen & 10.4 & (5.2) & 50.9 & $(7.3)$ & 38.7 & (7.6) & 16 & (8.1) & 5 & (7.9) & 43 & (5.6) & 28 & (5.3) \\
\hline Polen & 6.6 & (4.6) & 33.4 & (9.4) & 60.0 & (9.5) & 29 & (5.1) & 12 & (5.2) & 53 & (8.1) & 31 & (7.5) \\
\hline Portugal & 29.8 & (8.2) & 29.5 & $(9.0)$ & 40.7 & $(8.7)$ & 54 & (8.3) & 36 & (8.0) & 47 & (6.8) & 17 & (5.6) \\
\hline Slowak. Rep. & 6.9 & (4.9) & 33.9 & (6.2) & 59.3 & (6.4) & 65 & $(7.5)$ & 43 & (7.1) & 86 & (7.7) & 66 & $(7.4)$ \\
\hline Slowenien & 22.1 & $(0.8)$ & 38.0 & $(1.6)$ & 39.9 & $(1.3)$ & 54 & (2.7) & 47 & (3.0) & 87 & (3.0) & 80 & (3.0) \\
\hline Spanien & 18.7 & (3.6) & 42.8 & $(4.2)$ & 38.4 & (3.6) & 34 & (3.4) & 19 & (3.7) & 39 & (3.4) & 18 & (3.7) \\
\hline Schweden & 12.6 & (4.4) & 45.8 & $(7.7)$ & 41.6 & (6.9) & 21 & (6.5) & 9 & (5.8) & 34 & (6.2) & 16 & $(6.0)$ \\
\hline Schweiz & 14.1 & (5.7) & 37.1 & (8.4) & 48.8 & (8.5) & 20 & $(6.4)$ & 9 & (6.3) & 76 & (6.7) & 60 & (6.7) \\
\hline Türkei & 32.2 & (5.4) & 42.4 & $(5.5)$ & 25.4 & $(4.4)$ & 33 & $(7.2)$ & 26 & (7.3) & 94 & $(12.8)$ & 81 & $(13.1)$ \\
\hline Ver. Königreich & 29.3 & $(6.3)$ & 42.4 & (6.7) & 28.2 & $(4.8)$ & 39 & (9.1) & 24 & (7.6) & 63 & $(7.0)$ & 45 & $(6.6)$ \\
\hline Ver. Staaten & 35.8 & (6.1) & 31.9 & (7.1) & 32.3 & $(6.2)$ & 50 & $(7.2)$ & 34 & $(7.7)$ & 34 & (6.5) & 14 & (6.0) \\
\hline OECD-Durchschnitt & 19.2 & $(0.8)$ & 40.0 & $(1.1)$ & 37.9 & $(1.0)$ & 47 & $(1.1)$ & 34 & (1.1) & 57 & $(1.1)$ & 41 & $(1.1)$ \\
\hline
\end{tabular}

\begin{tabular}{|l|}
\hline Albanien \\
\hline Argentinien \\
Bulgarien \\
Kolumbien \\
Kroatien \\
\hline Inpern* \\
\hline Letonesien \\
\hline Liechtenstein \\
Litauen \\
Macau (China) \\
Malaysia \\
Montenegro \\
Peru \\
Katar \\
Rumänien \\
Russ. Föderation \\
Serbien \\
Shanghai (China) \\
Singapur \\
Chinesisch Taipeh \\
Thailand \\
Tunesien \\
Ver. Arab. Emirate \\
Uruguay \\
Vietnam \\
\hline
\end{tabular}

\begin{tabular}{rr|rr|rr}
$\mathrm{m}$ & $\mathrm{m}$ & $\mathrm{m}$ & $\mathrm{m}$ & $\mathrm{m}$ & \\
32.8 & $(5.4)$ & 30.5 & $(5.7)$ & 36.6 & $(5.9)$ \\
12.1 & $(2.1)$ & 47.4 & $(3.3)$ & 40.6 & $(3.2)$ \\
14.6 & $(3.8)$ & 32.2 & $(5.8)$ & 53.2 & $(4.8)$ \\
12.5 & $(2.6)$ & 44.1 & $(4.6)$ & 43.4 & $(4.1)$ \\
5.7 & $(4.0)$ & 53.2 & $(8.4)$ & 41.2 & $(8.2)$ \\
12.4 & $(3.9)$ & 47.4 & $(4.3)$ & 40.3 & $(3.4)$ \\
18.2 & $(0.2)$ & 24.0 & $(0.1)$ & 57.8 & $(0.2)$ \\
35.3 & $(3.4)$ & 40.2 & $(3.8)$ & 24.5 & $(3.6)$ \\
6.1 & $(3.8)$ & 28.5 & $(8.6)$ & 65.4 & $(9.0)$ \\
11.9 & $(3.6)$ & 55.7 & $(5.6)$ & 32.4 & $(4.3)$ \\
8.9 & $(3.7)$ & 39.5 & $(5.9)$ & 51.6 & $(6.0)$ \\
4.5 & $(2.6)$ & 38.2 & $(6.5)$ & 57.2 & $(6.5)$ \\
0.0 & $\mathrm{c}$ & 0.0 & $\mathrm{c}$ & 0.0 & \\
5.0 & $(2.4)$ & 48.2 & $(5.6)$ & 46.8 & $(5.2)$ \\
51.8 & $(0.1)$ & 17.3 & $(0.0)$ & 30.9 & $(0.1)$ \\
6.9 & $(3.8)$ & 40.3 & $(7.9)$ & 52.8 & $(8.0 .3$ \\
30.9 & $(0.2)$ & 26.3 & $(0.3)$ & 42.8 & $(0.2)$ \\
5.8 & $(2.4)$ & 32.2 & $(5.1)$ & 62.0 & $(5.3)$ \\
22.1 & $(0.1)$ & 17.1 & $(0.1)$ & 60.7 & $(0.1)$ \\
15.2 & $(4.7)$ & 39.7 & $(6.1)$ & 45.1 & $(6.2)$ \\
8.6 & $(2.8)$ & 41.6 & $(4.8)$ & 49.8 & $(4.7)$ \\
22.4 & $(4.7)$ & 37.2 & $(5.4)$ & 40.4 & $(5.1)$ \\
29.4 & $(3.1)$ & 37.4 & $(3.7)$ & 33.2 & $(3.1)$ \\
30.5 & $(0.3)$ & 45.5 & $(0.6)$ & 24.1 & $(0.7)$ \\
23.2 & $(3.6)$ & 40.1 & $(5.3)$ & 36.7 & $(4.0)$ \\
12.2 & $(3.4)$ & 29.6 & $(4.9)$ & 58.2 & $(5.7)$ \\
14.4 & $(6.3)$ & 36.9 & $(8.4)$ & 48.7 & $(7.7)$ \\
13.1 & $(2.0)$ & 39.1 & $(2.8)$ & 47.8 & $(2.9)$ \\
22.8 & $(4.4)$ & 35.2 & $(3.9)$ & 41.9 & $(4.6)$ \\
3.5 & $(3.5)$ & 15.4 & $(5.8)$ & 81.1 & $(6.4)$
\end{tabular}

\begin{tabular}{r|r}
$\mathrm{m}$ & $\mathrm{m}$ \\
$(5.9)$ & $\mathbf{4}$ \\
$(3.2)$ & $\mathbf{1}$ \\
$(4.8)$ & $\mathbf{4}$ \\
$(4.1)$ & $\mathbf{3}$ \\
$(8.2)$ & $\mathbf{3}$ \\
$(3.4)$ & $\mathbf{3}$ \\
$(0.2)$ & $\mathbf{4}$ \\
$(3.6)$ & $\mathbf{6}$ \\
$(9.0)$ & -2 \\
$(4.3)$ & $\mathbf{1}$ \\
$(6.0)$ & 1 \\
$(6.5)$ & $\mathbf{2}$ \\
$\mathrm{C}$ & \\
$(5.2)$ & $\mathbf{4}$ \\
$(0.1)$ & \\
$(8.0)$ & $\mathbf{2}$ \\
$(0.2)$ & $\mathbf{5}$ \\
$(5.3)$ & $\mathbf{4}$ \\
$(0.1)$ & -9 \\
$(6.2)$ & $\mathbf{3}$ \\
$(4.7)$ & $\mathbf{2}$ \\
$(5.1)$ & $\mathbf{4}$ \\
$(3.1)$ & $\mathbf{7}$ \\
$(0.7)$ & $\mathbf{3}$ \\
$(4.0)$ & $\mathbf{7}$ \\
$(5.7)$ & 1 \\
$(7.7)$ & $\mathbf{2 7}$ \\
$(2.9)$ & $\mathbf{3 7}$ \\
$(4.6)$ & $\mathbf{4}$ \\
$(6.4)$ & $\mathbf{4}$ \\
\hline & \\
\hline
\end{tabular}

\begin{tabular}{rr|r}
$\mathbf{m}$ & $\mathrm{m}$ & \\
$\mathbf{4 6}$ & $(7.8)$ & 39 \\
$\mathbf{1 5}$ & $(3.4)$ & \\
$\mathbf{4 4}$ & $(7.2)$ & 32 \\
$\mathbf{3 2}$ & $(5.3)$ & 23 \\
$\mathbf{3 0}$ & $(5.1)$ & \\
$\mathbf{3 3}$ & $(6.9)$ & 26 \\
$\mathbf{4 3}$ & $(2.8)$ & 32 \\
$\mathbf{6 3}$ & $(8.5)$ & 59 \\
-2 & $(7.9)$ & \\
$\mathbf{1 4}$ & $(6.5)$ & \\
$\mathbf{1 3}$ & $(9.3)$ & \\
$\mathbf{2 9}$ & $(6.1)$ & \\
$\mathrm{C}$ & $\mathrm{c}$ & \\
$\mathbf{4 5}$ & $(5.9)$ & 30 \\
$\mathbf{8}$ & $(3.1)$ & \\
$\mathbf{2 1}$ & $(5.3)$ & \\
$\mathbf{5 0}$ & $(3.2)$ & \\
$\mathbf{4 0}$ & $(5.3)$ & 2 \\
$\mathbf{- 9}$ & $(1.8)$ & -14 \\
$\mathbf{3 1}$ & $(6.8)$ & 21 \\
$\mathbf{2 3}$ & $(8.3)$ & \\
$\mathbf{4 6}$ & $(7.7)$ & $\mathbf{4 1}$ \\
$\mathbf{7 0}$ & $(8.0)$ & 58 \\
$\mathbf{3 5}$ & $(3.4)$ & 25 \\
$\mathbf{7 4}$ & $(7.3)$ & 60 \\
$\mathbf{1 1}$ & $(6.5)$ & \\
$\mathbf{2 7}$ & $(7.6)$ & 22 \\
$\mathbf{3 7}$ & $(4.7)$ & $\mathbf{2 8}$ \\
$\mathbf{4 9}$ & $(6.1)$ & 36 \\
$\mathbf{4 6}$ & $(8.7)$ & 38 \\
& &
\end{tabular}

\begin{tabular}{rr|rr|rr}
$\mathrm{m}$ & $\mathrm{m}$ & $\mathrm{m}$ & $\mathrm{m}$ & $\mathrm{m}$ & $\mathrm{m}$ \\
$\mathbf{3 9}$ & $(7.5)$ & $\mathbf{4 7}$ & $(8.1)$ & $\mathbf{3 5}$ & $(7.9)$ \\
$\mathbf{8}$ & $(3.4)$ & $\mathbf{7 6}$ & $(6.0)$ & $\mathbf{5 8}$ & $(5.1)$ \\
$\mathbf{3 2}$ & $(6.8)$ & $\mathbf{8 5}$ & $(7.0)$ & $\mathbf{7 0}$ & $(6.5)$ \\
$\mathbf{2 3}$ & $(5.2)$ & $\mathbf{4 8}$ & $(6.2)$ & $\mathbf{3 3}$ & $(6.0)$ \\
$\mathbf{1 9}$ & $(5.0)$ & $\mathbf{5 9}$ & $(7.5)$ & $\mathbf{4 1}$ & $(7.1)$ \\
$\mathbf{2 6}$ & $(6.8)$ & $\mathbf{8 3}$ & $(10.3)$ & $\mathbf{7 3}$ & $(10.3)$ \\
$\mathbf{3 2}$ & $(2.8)$ & $\mathbf{4 5}$ & $(3.0)$ & $\mathbf{2 7}$ & $(3.2)$ \\
$\mathbf{5 9}$ & $(8.4)$ & $\mathbf{4 2}$ & $(9.5)$ & $\mathbf{3 5}$ & $(9.1)$ \\
-5 & $(8.1)$ & $\mathbf{5 9}$ & $(9.9)$ & $\mathbf{4 4}$ & $(8.9)$ \\
8 & $(6.1)$ & $\mathbf{6 2}$ & $(9.9)$ & $\mathbf{4 8}$ & $(9.5)$ \\
5 & $(9.2)$ & $\mathbf{3 8}$ & $(7.1)$ & $\mathbf{2 8}$ & $(6.9)$ \\
$\mathbf{1 3}$ & $(5.7)$ & $\mathbf{5 4}$ & $(5.4)$ & $\mathbf{3 7}$ & $(5.3)$ \\
$\mathrm{C}$ & $\mathrm{C}$ & $\mathrm{c}$ & $\mathrm{C}$ & $\mathrm{c}$ & $\mathrm{C}$ \\
$\mathbf{3 0}$ & $(5.9)$ & $\mathbf{5 9}$ & $(6.7)$ & $\mathbf{4 7}$ & $(6.4)$ \\
5 & $(3.3)$ & $\mathbf{2 4}$ & $(3.0)$ & $\mathbf{1 9}$ & $(3.0)$ \\
$\mathbf{1 2}$ & $(5.1)$ & $\mathbf{6 5}$ & $(7.7)$ & $\mathbf{5 0}$ & $(7.4)$ \\
$\mathbf{4 5}$ & $(3.3)$ & $\mathbf{4 1}$ & $(3.0)$ & $\mathbf{3 2}$ & $(3.1)$ \\
$\mathbf{2 8}$ & $(5.1)$ & $\mathbf{7 1}$ & $(6.9)$ & $\mathbf{5 3}$ & $(5.7)$ \\
$\mathbf{- 1 4}$ & $(2.1)$ & $\mathbf{6 8}$ & $(2.0)$ & $\mathbf{5 9}$ & $(2.2)$ \\
$\mathbf{2 1}$ & $(6.8)$ & $\mathbf{7 1}$ & $(7.8)$ & $\mathbf{5 1}$ & $(6.6)$ \\
10 & $(8.2)$ & $\mathbf{4 9}$ & $(6.6)$ & $\mathbf{3 3}$ & $(6.1)$ \\
$\mathbf{4 1}$ & $(7.6)$ & $\mathbf{8 0}$ & $(9.8)$ & $\mathbf{7 2}$ & $(9.6)$ \\
$\mathbf{5 8}$ & $(7.8)$ & $\mathbf{6 8}$ & $(8.2)$ & $\mathbf{6 0}$ & $(8.1)$ \\
$\mathbf{2 5}$ & $(3.3)$ & $\mathbf{9 3}$ & $(4.4)$ & $\mathbf{7 3}$ & $(4.6)$ \\
$\mathbf{6 0}$ & $(6.7)$ & $\mathbf{8 2}$ & $(8.6)$ & $\mathbf{6 1}$ & $(8.6)$ \\
9 & $(6.4)$ & $\mathbf{5 6}$ & $(7.0)$ & $\mathbf{3 7}$ & $(6.5)$ \\
$\mathbf{2 2}$ & $(7.7)$ & $\mathbf{6 0}$ & $(10.3)$ & $\mathbf{4 6}$ & $(9.1)$ \\
$\mathbf{2 8}$ & $(4.5)$ & $\mathbf{5 0}$ & $(6.5)$ & $\mathbf{3 9}$ & $(6.5)$ \\
$\mathbf{3 6}$ & $(6.1)$ & $\mathbf{7 0}$ & $(6.6)$ & $\mathbf{4 5}$ & $(5.5)$ \\
$\mathbf{3 8}$ & $(9.0)$ & $\mathbf{4 9}$ & $(9.9)$ & $\mathbf{3 3}$ & $(9.8)$ \\
\hline & & & & & \\
\hline
\end{tabular}

Anmerkung: Statistisch signifikante Werte sind durch Fettdruck gekennzeichnet (vgl. Anhang A3).

1. ESCS bezieht sich auf den PISA-Index des wirtschaftlichen, sozialen und kulturellen Status.

2. Sozioökonomisch begünstigte (sozioökonomisch benachteiligte) Schulen sind Schulen, in denen der typische Schüler der Schule bzw. das sozioökonomische Profil der nehmen 35 als Zufallsstichprobe ausgewählte Schülerinnen und Schüler an PISA teil (wegen näherer Einzelheiten vgl.PISA 2012 Technical Repswirtschaft liegt. An jeder Schule nehmen 35 als Zufallsstichprobe ausgewählte Schülerinnen und Schüler an PISA teil (wegen näherer Einzelheiten vgl. PISA 2012 Technical Report, OECD, erscheint demnächst). Das sozon den Angaben dieser Schuler ermittelt. Deshalb hangt die Genauigkeit der Schatzung von der Zahl der Schüler ab, die an den einzelnen Schulen tatsächlich an der Erhebung teilnehmen, sowie von der Variationsbreite ihrer Antworten. Bei der Einstufung der Schulen in sozioökonomisch begünstigte, sozioökonomisch benachteiligte oder sozioökonomisch durchschnittliche Schulen wurde diese Frage der Genauigkeit berücksichtigt. Wenn der Unterschied zwischen dem sozioökonomischen Profil einer Schule und dem ESCS des typischen Schülers des Landes/der Volkswirtschaft (dem durchschnittlichen ESCS auf Ebene des
Landes/der Volkswirtschaft) nicht statistisch signifikant war, wurde die Schule als Schule mit durchschnittlichem sozioökonomischem Profil eingestuft. Wenn das Profil einer Landes/der Volkswirtschaft) nicht statistisch signifikant war, wurde die Schule als Schule mit durchschnittlichem sozioökonomischem Profil eingestuft. Wenn das Profil einer
Schule statistisch signifikant über dem durchschnittlichen ESCS des Landes/der Volkswirtschaft lag, wurde die Schule als sozioökonomisch begünstigte Schule eingestuft. Wenn das Profil der Schule unter dem durchschnittlichen ESCS des Landes/der Volkswirtschaft lag, wurde die Schule als sozioökonomisch benachteiligte Schule eingestuft. $*$ Vgl. Anmerkungen am Anfang dieses Anhangs.

StatLink ints http://dx.doi.org/10.1787/888932964946 
[Part4/4]

Mathematikleistungen und Schülerpopulation, nach sozioökonomischem Profil der Schulen Tabelle II.4.2 Die Ergebnisse basieren auf Schülerangaben

\begin{tabular}{|c|c|c|c|c|c|c|c|c|c|c|c|c|c|c|c|c|c|c|c|}
\hline & \multicolumn{12}{|c|}{$\begin{array}{l}\text { Relatives Risiko und Populationsrelevanz von Ergebnissen } \\
\text { im untersten Quartil der Leistungsverteilung }\end{array}$} & \multicolumn{6}{|c|}{ Effektstärke } \\
\hline & & \multicolumn{4}{|c|}{$\begin{array}{l}\text { Schüler in sozioökonomisch } \\
\text { benachteiligten Schulen }{ }^{2}\end{array}$} & \multicolumn{4}{|c|}{$\begin{array}{l}\text { Schüler in sozioökonomisch } \\
\text { durchschnittlichen Schulen }{ }^{2}\end{array}$} & $\begin{array}{r}\begin{array}{r}\text { Schüle } \\
\text { beg }\end{array}\end{array}$ & $\begin{array}{l}\text { er in so } \\
\text { zünstig }\end{array}$ & $\begin{array}{l}\text { zioökonom } \\
\text { ten Schuler }\end{array}$ & sch & $\begin{array}{r}\text { Mittel } \\
\text { der Sch } \\
\text { sozioök } \\
\text { benacht } \\
\text { Schu }\end{array}$ & $\begin{array}{l}\text { vert } \\
\text { iler in } \\
\text { onom. } \\
\text { iligten } \\
\text { en }\end{array}$ & \begin{tabular}{|r} 
Mittel \\
der Sch \\
sozioök \\
durchsc \\
Schu
\end{tabular} & $\begin{array}{l}\text { wert } \\
\text { üler in } \\
\text { onom. } \\
\text { chnittl. } \\
\text { len }\end{array}$ & $\begin{array}{r}\text { Mittel } \\
\text { der Sch } \\
\text { sozioök } \\
\text { begüns } \\
\text { Schu }\end{array}$ & $\begin{array}{l}\text { wert } \\
\text { üler in } \\
\text { onom. } \\
\text { tigten } \\
\text { len }\end{array}$ \\
\hline & & $\begin{array}{l}\text { Relativ. } \\
\text { Risiko }\end{array}$ & S.E. & $\begin{array}{l}\text { Populat.- } \\
\text { relevanz }\end{array}$ & S.E. & $\begin{array}{l}\text { Relativ. } \\
\text { Risiko }\end{array}$ & S.E. & $\begin{array}{l}\text { Populat.- } \\
\text { relevanz }\end{array}$ & S.E. & $\begin{array}{l}\text { Relativ. } \\
\text { Risiko }\end{array}$ & S.E. & $\begin{array}{l}\text { Populat.- } \\
\text { relevanz }\end{array}$ & S.E. & $\begin{array}{l}\text { Effekt- } \\
\text { stärke }\end{array}$ & S.E. & $\begin{array}{l}\text { Effekt- } \\
\text { stärke }\end{array}$ & S.E. & $\begin{array}{l}\text { Effekt- } \\
\text { stärke }\end{array}$ & S.E. \\
\hline ¿ & Australien & 2.1 & $(0.1)$ & 20.6 & $(1.7)$ & 1.0 & $(0.1)$ & 0.1 & (2.6) & 0.3 & $(0.0)$ & -21.0 & (1.8) & -0.65 & $(0.04)$ & -0.10 & $(0.04)$ & 0.79 & $(0.04)$ \\
\hline & Österreich & 3.4 & $(0.4)$ & 43.5 & (5.1) & 0.6 & $(0.1)$ & -17.9 & (6.4) & 0.2 & $(0.1)$ & -25.5 & (3.7) & -1.10 & $(0.09)$ & 0.28 & $(0.09)$ & 0.84 & $(0.08)$ \\
\hline & Belgien & 4.0 & $(0.3)$ & 46.4 & (3.2) & 0.8 & $(0.1)$ & -8.8 & (3.2) & 0.1 & $(0.0)$ & -41.4 & (3.5) & -1.22 & $(0.06)$ & -0.04 & $(0.06)$ & 1.19 & $(0.07)$ \\
\hline 8 & Kanada & 1.6 & $(0.1)$ & 12.1 & $(2.0)$ & 1.1 & $(0.1)$ & 6.7 & (3.1) & 0.4 & $(0.0)$ & -17.2 & (2.1) & -0.42 & $(0.06)$ & -0.17 & $(0.04)$ & 0.61 & $(0.05)$ \\
\hline 㫕 & Chile & 2.5 & $(0.3)$ & 38.3 & (5.5) & 1.1 & $(0.2)$ & 3.3 & (3.5) & 0.2 & $(0.0)$ & -37.3 & (3.5) & -0.84 & $(0.09)$ & -0.25 & $(0.09)$ & 1.14 & $(0.09)$ \\
\hline & Tschech. Rep. & 2.6 & $(0.3)$ & 25.6 & (3.9) & 1.0 & $(0.1)$ & -2.5 & (7.1) & 0.1 & $(0.0)$ & -23.9 & (2.9) & -0.94 & $(0.10)$ & -0.18 & $(0.08)$ & 1.40 & $(0.08)$ \\
\hline & Dänemark & 1.9 & $(0.2)$ & 16.4 & (3.5) & 1.0 & $(0.1)$ & 1.6 & (5.9) & 0.3 & $(0.1)$ & -17.5 & (2.6) & -0.53 & $(0.07)$ & -0.08 & $(0.06)$ & 0.64 & $(0.06)$ \\
\hline & Estland & 1.5 & $(0.1)$ & 8.0 & (2.1) & 1.2 & $(0.1)$ & 12.1 & (4.6) & 0.4 & $(0.0)$ & -15.0 & (1.9) & -0.36 & $(0.06)$ & -0.21 & $(0.05)$ & 0.61 & $(0.05)$ \\
\hline & Finnland & 1.4 & $(0.1)$ & 6.1 & (1.6) & 1.0 & $(0.1)$ & -1.7 & (4.5) & 0.7 & $(0.1)$ & -5.5 & (1.5) & -0.28 & $(0.05)$ & 0.00 & $(0.05)$ & 0.27 & $(0.06)$ \\
\hline & Frankreich & w & w & w & w & w & w & w & w & w & w & w & $\mathrm{w}$ & w & w & w & $\mathrm{w}$ & w & w \\
\hline & Deutschland & 3.5 & $(0.4)$ & 41.3 & (4.4) & 0.7 & $(0.1)$ & -13.4 & (5.7) & 0.1 & $(0.0)$ & -30.6 & (3.4) & -1.12 & $(0.09)$ & 0.00 & $(0.09)$ & 1.19 & $(0.09)$ \\
\hline & Griechenland & 3.1 & $(0.3)$ & 34.4 & (4.1) & 0.7 & $(0.1)$ & -16.4 & (6.0) & 0.3 & $(0.0)$ & -23.4 & (4.0) & -0.93 & $(0.09)$ & 0.10 & $(0.07)$ & 0.75 & $(0.07)$ \\
\hline & Ungarn & 4.0 & (0.6) & 48.6 & $(4.7)$ & 0.8 & $(0.1)$ & -6.0 & (5.3) & 0.1 & $(0.0)$ & -44.0 & (4.8) & -1.26 & $(0.11)$ & -0.22 & $(0.08)$ & 1.53 & $(0.09)$ \\
\hline & Island & 1.6 & $(0.1)$ & 9.1 & (1.7) & 1.2 & $(0.1)$ & 10.9 & (3.1) & 0.5 & $(0.0)$ & -17.2 & (1.7) & -0.35 & $(0.05)$ & -0.17 & $(0.03)$ & 0.46 & $(0.04)$ \\
\hline & Irland & 2.8 & $(0.2)$ & 23.7 & (3.1) & 0.8 & $(0.1)$ & -13.8 & (5.1) & 0.4 & $(0.0)$ & -18.5 & (2.7) & -0.93 & $(0.08)$ & 0.09 & $(0.06)$ & 0.57 & $(0.05)$ \\
\hline & Israel & 3.2 & $(0.4)$ & 40.0 & (5.0) & 0.8 & $(0.1)$ & -7.7 & (5.5) & 0.2 & $(0.0)$ & -34.3 & (5.4) & -1.04 & $(0.09)$ & 0.01 & $(0.10)$ & 0.98 & $(0.10)$ \\
\hline & Italien & 3.0 & $(0.2)$ & 36.7 & (2.4) & 0.8 & $(0.1)$ & -7.0 & (2.5) & 0.2 & (0.0) & -31.4 & (2.9) & -0.96 & $(0.06)$ & -0.02 & $(0.05)$ & 0.98 & $(0.05)$ \\
\hline & Japan & 3.2 & $(0.5)$ & 39.5 & (5.6) & 0.7 & $(0.1)$ & -15.6 & (5.0) & 0.2 & $(0.1)$ & -26.1 & (3.5) & -1.06 & $(0.10)$ & 0.06 & $(0.08)$ & 1.03 & $(0.09)$ \\
\hline & Korea & 2.8 & $(0.3)$ & 32.1 & (4.1) & 0.7 & $(0.1)$ & -16.3 & (6.5) & 0.3 & $(0.1)$ & -20.2 & (4.2) & -0.93 & $(0.10)$ & 0.07 & $(0.09)$ & 0.84 & $(0.11)$ \\
\hline & Luxemburg & 3.8 & $(0.2)$ & 57.5 & (1.8) & 0.8 & $(0.1)$ & -3.6 & $(0.8)$ & 0.2 & $(0.0)$ & -42.9 & (1.2) & -1.04 & $(0.02)$ & 0.10 & $(0.03)$ & 1.05 & $(0.03)$ \\
\hline & Mexiko & 2.2 & $(0.1)$ & 29.2 & (2.5) & 0.9 & $(0.1)$ & -2.4 & (2.3) & 0.3 & $(0.0)$ & -25.4 & (2.1) & -0.62 & $(0.04)$ & -0.09 & $(0.04)$ & 0.77 & $(0.04)$ \\
\hline & Niederlande & 4.2 & $(0.5)$ & 42.7 & (5.3) & 0.6 & (0.1) & -23.5 & (9.1) & 0.1 & (0.1) & -28.5 & (6.0) & $\mid-1.42$ & $(0.10)$ & 0.08 & $(0.13)$ & 1.16 & $(0.17)$ \\
\hline & Neuseeland & 2.5 & $(0.2)$ & 23.9 & $(4.0)$ & 0.9 & $(0.1)$ & -8.9 & (6.3) & 0.3 & $(0.0)$ & -19.2 & (3.0) & -0.77 & $(0.07)$ & -0.05 & $(0.08)$ & 0.80 & $(0.06)$ \\
\hline & Norwegen & 1.4 & $(0.2)$ & 3.8 & (1.6) & 1.3 & $(0.1)$ & 19.3 & (7.0) & 0.4 & (0.1) & -10.2 & (2.1) & -0.27 & $(0.09)$ & -0.23 & $(0.07)$ & 0.52 & $(0.06)$ \\
\hline & Polen & 1.8 & $(0.2)$ & 18.8 & (3.5) & 1.0 & $(0.1)$ & -2.3 & (5.1) & 0.4 & $(0.1)$ & -16.1 & (3.1) & -0.52 & $(0.06)$ & -0.09 & $(0.07)$ & 0.72 & $(0.09)$ \\
\hline & Portugal & 2.7 & $(0.3)$ & 36.3 & (4.6) & 0.7 & (0.1) & -17.2 & (6.2) & 0.2 & (0.1) & -18.6 & (4.1) & \begin{tabular}{|l|}
-0.78 \\
\end{tabular} & $(0.10)$ & 0.17 & $(0.08)$ & 0.81 & $(0.08)$ \\
\hline & Slowak. Rep & 3.0 & $(0.3)$ & 35.3 & $(4.2)$ & 0.9 & $(0.1)$ & -6.7 & (5.2) & 0.1 & $(0.0)$ & -30.0 & (2.9) & -1.05 & $(0.08)$ & -0.09 & $(0.08)$ & 1.26 & $(0.10)$ \\
\hline & Slowenien & 3.5 & $(0.2)$ & 42.1 & (2.3) & 0.9 & $(0.1)$ & -3.8 & (2.6) & 0.1 & (0.0) & -39.3 & (1.2) & -1.17 & $(0.04)$ & -0.20 & $(0.03)$ & 1.47 & $(0.04)$ \\
\hline & Spanien & 2.0 & $(0.1)$ & 23.1 & (2.8) & 0.9 & $(0.1)$ & -2.2 & (3.0) & 0.4 & (0.0) & -20.8 & (2.6) & -0.58 & $(0.04)$ & -0.02 & $(0.04)$ & 0.63 & $(0.04)$ \\
\hline & Schweden & 1.5 & $(0.2)$ & 8.0 & (2.6) & 1.1 & $(0.1)$ & 6.3 & (5.6) & 0.5 & $(0.1)$ & -11.9 & (2.7) & -0.34 & $(0.07)$ & -0.11 & $(0.06)$ & 0.44 & $(0.07)$ \\
\hline & Schweiz & 1.8 & $(0.1)$ & 17.3 & (3.0) & 1.2 & $(0.1)$ & 10.3 & (4.8) & 0.2 & (0.0) & -24.6 & (3.2) & -0.51 & $(0.06)$ & -0.30 & $(0.07)$ & 0.96 & $(0.08)$ \\
\hline & Türkei & 2.0 & $(0.2)$ & 24.3 & (4.6) & 1.1 & (0.1) & 3.6 & (4.8) & 0.2 & $(0.1)$ & -24.7 & (3.8) & -0.83 & $(0.09)$ & -0.26 & $(0.09)$ & 1.32 & $(0.17)$ \\
\hline & Ver. Königreich & 2.1 & $(0.2)$ & 21.1 & (4.5) & 1.0 & $(0.1)$ & 1.1 & (6.6) & 0.3 & $(0.0)$ & -21.6 & (2.7) & -0.66 & $(0.10)$ & -0.12 & $(0.09)$ & 0.87 & $(0.08)$ \\
\hline & Ver. Staaten & 2.4 & $(0.3)$ & 26.9 & (4.9) & 0.8 & $(0.1)$ & -9.0 & (5.1) & 0.4 & $(0.1)$ & -20.8 & (3.9) & -0.76 & $(0.08)$ & 0.07 & $(0.08)$ & 0.61 & $(0.07)$ \\
\hline & OECD-Durchschnitt & 2.6 & $(0.0)$ & 28.8 & (0.6) & 0.9 & (0.0) & -4.4 & (0.9) & 0.3 & $(0.0)$ & -24.8 & (0.6) & \begin{tabular}{|l|}
-0.81 \\
\end{tabular} & $(0.01)$ & \begin{tabular}{|l|}
-0.06 \\
\end{tabular} & $(0.01)$ & 0.89 & $(0.01)$ \\
\hline & Albanien & $\mathrm{m}$ & $\mathrm{m}$ & $\mathrm{m}$ & $\mathrm{m}$ & $\mathrm{m}$ & $\mathrm{m}$ & $\mathrm{m}$ & $\mathrm{m}$ & $\mathrm{m}$ & $\mathrm{m}$ & $\mathrm{m}$ & $\mathrm{m}$ & $\mathrm{m}$ & $\mathrm{m}$ & $\mathrm{m}$ & $\mathrm{m}$ & $\mathrm{m}$ & $\bar{m}$ \\
\hline & Argentinien & 3.2 & (0.4) & 42.1 & (5.1) & 0.8 & (0.1) & -6.1 & (5.1) & 0.2 & (0.0) & -35.4 & (5.2) & -1.01 & $(0.09)$ & 0.01 & $(0.11)$ & 1.02 & $(0.10)$ \\
\hline & Brasilien & 1.8 & $(0.1)$ & 20.8 & (2.9) & 1.2 & $(0.1)$ & 7.4 & (3.1) & 0.2 & $(0.0)$ & -24.0 & (2.3) & -0.58 & $(0.05)$ & -0.31 & $(0.05)$ & 1.14 & $(0.07)$ \\
\hline İ & Bulgarien & 3.3 & $(0.4)$ & 43.6 & (5.1) & 1.0 & (0.1) & -1.0 & (3.9) & 0.1 & (0.0) & -42.6 & (5.2) & -1.07 & $(0.09)$ & -0.23 & $(0.08)$ & 1.36 & (0.09) \\
\hline & Kolumbien & 2.3 & $(0.3)$ & 27.0 & (4.4) & 1.0 & $(0.1)$ & -0.3 & (4.0) & 0.3 & $(0.0)$ & -27.0 & (3.7) & -0.76 & $(0.08)$ & -0.11 & $(0.07)$ & 0.87 & $(0.09)$ \\
\hline & Costa Rica & 2.4 & $(0.3)$ & 27.9 & (4.6) & 0.9 & $(0.1)$ & -4.1 & (5.8) & 0.2 & $(0.1)$ & -23.1 & (3.5) & -0.78 & $(0.08)$ & -0.14 & $(0.09)$ & 1.09 & $(0.11)$ \\
\hline & Kroatien & 2.3 & $(0.3)$ & 30.2 & (5.1) & 1.0 & $(0.1)$ & -0.2 & (5.4) & 0.1 & $(0.0)$ & -26.2 & (2.7) & -0.76 & $(0.08)$ & -0.17 & $(0.10)$ & 1.26 & $(0.11)$ \\
\hline 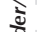 & Zypern* & 2.4 & $(0.1)$ & 32.3 & (2.0) & 0.8 & $(0.0)$ & -6.9 & (1.9) & 0.3 & $(0.0)$ & -23.5 & (1.5) & -0.71 & $(0.03)$ & 0.04 & $(0.03)$ & 0.74 & $(0.03)$ \\
\hline & Hongkong (China) & 3.0 & $(0.4)$ & 41.7 & (5.6) & 0.6 & $(0.1)$ & -17.9 & (6.4) & 0.3 & $(0.1)$ & -21.6 & (5.1) & -0.88 & $(0.10)$ & 0.21 & $(0.10)$ & 0.81 & $(0.12)$ \\
\hline & Indonesien & 1.5 & $(0.2)$ & 16.0 & (6.4) & 1.3 & $(0.2)$ & 8.7 & (5.1) & 0.4 & $(0.1)$ & -21.2 & (4.2) & -0.35 & $(0.12)$ & -0.37 & $(0.10)$ & 0.83 & $(0.13)$ \\
\hline & Jordanien & 1.5 & $(0.2)$ & 8.8 & $(2.7)$ & 1.3 & $(0.1)$ & 14.4 & (5.5) & 0.3 & $(0.1)$ & -16.0 & (2.3) & -0.40 & $(0.08)$ & -0.30 & $(0.07)$ & 0.87 & $(0.10)$ \\
\hline & Kasachstan & 1.7 & $(0.2)$ & 13.8 & (4.3) & 1.2 & $(0.2)$ & 8.5 & (5.8) & 0.4 & $(0.1)$ & -22.4 & (4.0) & -0.40 & $(0.14)$ & -0.24 & $(0.10)$ & 0.61 & $(0.10)$ \\
\hline & Lettland & 2.0 & $(0.2)$ & 16.8 & (3.0) & 1.3 & $(0.1)$ & 11.3 & (4.7) & 0.3 & (0.0) & -26.9 & (3.9) & -0.62 & $(0.08)$ & -0.25 & $(0.05)$ & 0.81 & $(0.08)$ \\
\hline & Liechtenstein & c & c & c & c & c & c & c & C & c & c & c & c & c & c & c & c & c & c \\
\hline & Litauen & 2.3 & $(0.2)$ & 22.8 & (3.6) & 1.0 & $(0.1)$ & -0.9 & (6.0) & 0.2 & $(0.0)$ & -22.7 & (3.2) & -0.76 & $(0.07)$ & -0.10 & $(0.07)$ & 0.89 & $(0.08)$ \\
\hline & Macau (China) & 1.4 & $(0.1)$ & 16.0 & (2.4) & 1.1 & $(0.1)$ & 1.8 & (1.1) & 0.6 & $(0.0)$ & -13.4 & (1.2) & -0.24 & $(0.03)$ & -0.04 & $(0.03)$ & 0.32 & $(0.03)$ \\
\hline & Malaysia & 1.8 & $(0.2)$ & 18.0 & (3.6) & 1.3 & $(0.1)$ & 9.8 & (4.4) & 0.3 & $(0.1)$ & -25.9 & (4.0) & -0.62 & $(0.07)$ & -0.28 & $(0.08)$ & 0.97 & $(0.10)$ \\
\hline & Montenegro & 3.2 & $(0.3)$ & 46.3 & (3.1) & 0.8 & $(0.1)$ & -6.2 & (2.4) & 0.3 & $(0.0)$ & -38.0 & (1.9) & -1.04 & $(0.04)$ & 0.06 & $(0.04)$ & 0.96 & $(0.03)$ \\
\hline & Peru & 3.2 & $(0.3)$ & 43.5 & (3.5) & 0.9 & $(0.1)$ & -2.1 & (3.3) & 0.1 & $(0.0)$ & -40.2 & (5.0) & -1.03 & $(0.07)$ & -0.17 & $(0.08)$ & 1.25 & $(0.08)$ \\
\hline & Katar & 1.4 & $(0.0)$ & 11.9 & (1.1) & 1.5 & $(0.1)$ & 10.6 & (1.0) & 0.5 & $(0.0)$ & -28.6 & (1.6) & -0.37 & $(0.02)$ & -0.45 & $(0.02)$ & 0.65 & $(0.02)$ \\
\hline & Rumänien & 2.5 & $(0.3)$ & 30.9 & (5.5) & 1.1 & $(0.2)$ & 2.9 & (5.9) & 0.1 & $(0.0)$ & -32.3 & (5.4) & -0.83 & $(0.10)$ & -0.23 & $(0.10)$ & 1.16 & $(0.10)$ \\
\hline & Russ. Föderation & 1.7 & $(0.2)$ & 13.1 & (3.3) & 1.2 & $(0.1)$ & 9.2 & (5.5) & 0.4 & $(0.1)$ & -20.5 & (3.3) & -0.49 & $(0.09)$ & -0.20 & $(0.08)$ & 0.68 & $(0.08)$ \\
\hline & Serbien & 2.7 & $(0.3)$ & 35.9 & (5.3) & 0.9 & $(0.1)$ & -6.4 & (5.7) & 0.1 & (0.0) & -27.4 & (3.2) & -0.93 & $(0.09)$ & -0.09 & $(0.10)$ & 1.26 & $(0.11)$ \\
\hline & Shanghai (China) & 3.5 & $(0.4)$ & 42.3 & (5.3) & 0.8 & $(0.1)$ & -8.1 & (5.0) & 0.2 & $(0.1)$ & -37.2 & (5.1) & -1.13 & $(0.08)$ & -0.03 & $(0.09)$ & 1.12 & $(0.11)$ \\
\hline & Singapur & 1.9 & $(0.1)$ & 21.9 & (1.7) & 1.2 & $(0.1)$ & 6.6 & (2.1) & 0.2 & $(0.0)$ & -24.8 & $(1.2)$ & -0.69 & $(0.03)$ & -0.20 & $(0.03)$ & 1.16 & $(0.05)$ \\
\hline & Chinesisch Taipeh & 3.0 & $(0.3)$ & 38.0 & (3.7) & 0.8 & $(0.1)$ & -9.0 & $(4.2)$ & 0.2 & $(0.0)$ & -29.6 & (3.6) & -1.03 & $(0.06)$ & 0.00 & $(0.07)$ & 1.11 & $(0.08)$ \\
\hline & Thailand & 1.7 & $(0.2)$ & 20.3 & (4.4) & 1.1 & $(0.1)$ & 4.3 & (3.5) & 0.4 & $(0.1)$ & -21.6 & (3.8) & -0.48 & $(0.08)$ & -0.22 & $(0.08)$ & 0.77 & $(0.09)$ \\
\hline & Tunesien & 2.0 & $(0.3)$ & 22.1 & (5.5) & 1.1 & $(0.2)$ & 3.1 & (6.8) & 0.3 & $(0.1)$ & -23.5 & (4.3) & -0.68 & $(0.10)$ & -0.18 & $(0.11)$ & 0.94 & $(0.11)$ \\
\hline & Ver. Arab. Emirate & 2.1 & $(0.2)$ & 22.4 & $(2.8)$ & 1.1 & $(0.1)$ & 3.2 & (3.4) & 0.4 & $(0.0)$ & -28.6 & (3.1) & -0.73 & $(0.05)$ & -0.15 & $(0.06)$ & 0.75 & $(0.07)$ \\
\hline & Uruguay & 3.0 & $(0.4)$ & 44.2 & $(5.2)$ & 0.7 & $(0.1)$ & 11.2 & (4.5) & 0.1 & $(0.0)$ & -24.9 & (3.1) & -0.94 & $(0.08)$ & 0.08 & $(0.08)$ & 1.23 & $(0.07)$ \\
\hline & Vietnam & 2.8 & $(0.4)$ & 41.3 & (5.6) & 0.7 & $(0.1)$ & -10.2 & (5.5) & 0.2 & $(0.1)$ & -24.8 & (4.8) & -0.84 & $(0.10)$ & 0.10 & $(0.11)$ & 0.93 & $(0.12)$ \\
\hline & $\begin{array}{l}\text { 2. Sozioökonomisch } \\
\text { über (unter) dem ESC } \\
35 \text { als Zufallsstichpro } \\
\text { sozioökonomische Pr } \\
\text { die an den einzelnen } \\
\text { begünstigte, sozioöko } \\
\text { dem sozioökonomise } \\
\text { Volkswirtschaft) nicht } \\
\text { signifikant über dem } \\
\text { unter dem durchschni }\end{array}$ & $\begin{array}{l}\text { isch } \\
\text { innit }\end{array}$ & onifik & ESCS des & 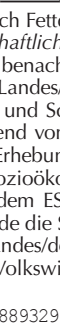 & $\begin{array}{l}\text { Schule } \\
\text { er Volk } \\
\text { irtscha }\end{array}$ & 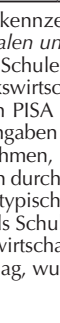 & $\begin{array}{l}\text { Schü } \\
\text { hnittlid } \\
\text { mit du } \\
\text { lag, w } \\
\text { e die S }\end{array}$ & chnitt & $\begin{array}{l}\text { ttlichem s } \\
\text { Schule al }\end{array}$ & 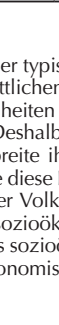 & $\begin{array}{l}\text { rage de } \\
\text { wirtsch } \\
\text { onomis } \\
\text { konom } \\
\text { ch bena }\end{array}$ & Prof 1 the & $\begin{array}{l}\text { eit be } \\
\text { urchs } \\
\text { eing } \\
\text { tigte }\end{array}$ & $\begin{array}{l}\text { ft. Wen } \\
\text { ule ein }\end{array}$ & $\begin{array}{l}\text { zung vo } \\
\text { der So } \\
\text { t. Wen } \\
\text { n ESCS } \\
\text { an das } \\
\text { gestuft. }\end{array}$ & $\begin{array}{l}\text { I einer } \\
\text { enn das }\end{array}$ & $\begin{array}{l}\text { s lofil } \\
\text { chule } \\
\text { rofil }\end{array}$ & $\begin{array}{l}\text { istisch } \\
\text { Schule }\end{array}$ \\
\hline
\end{tabular}


[Teil 1/2]

Ungleichheit beim Zugang zu Unterrichtsinhalten: Reine Mathematik

Tabelle II.4.3 Die Ergebnisse basieren auf Schülerangaben

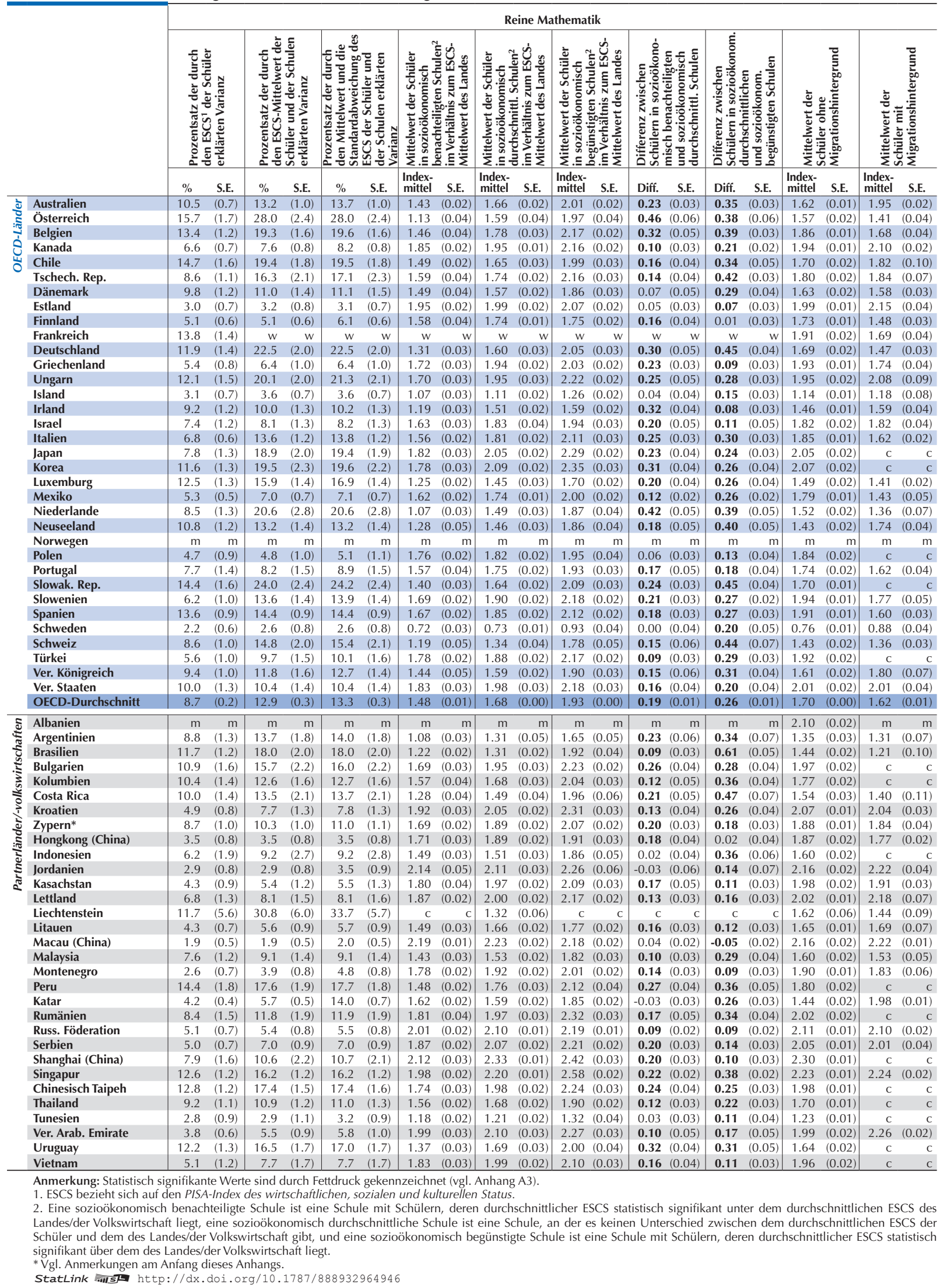

EXZELLENZ DURCH CHANCENGERECHTIGKETT: ALLEN SCHÜLERINNEN UND SCHÜLERN DIE VORAUSSETZUNGEN ZUM ERFOLG SICHERN - BAND II @ O OECD 2014 275 
[Teil 2/2]

Ungleichheit beim Zugang zu Unterrichtsinhalten: Reine Mathematik

Tabelle II.4.3 Die Ergebnisse basieren auf Schülerangaben

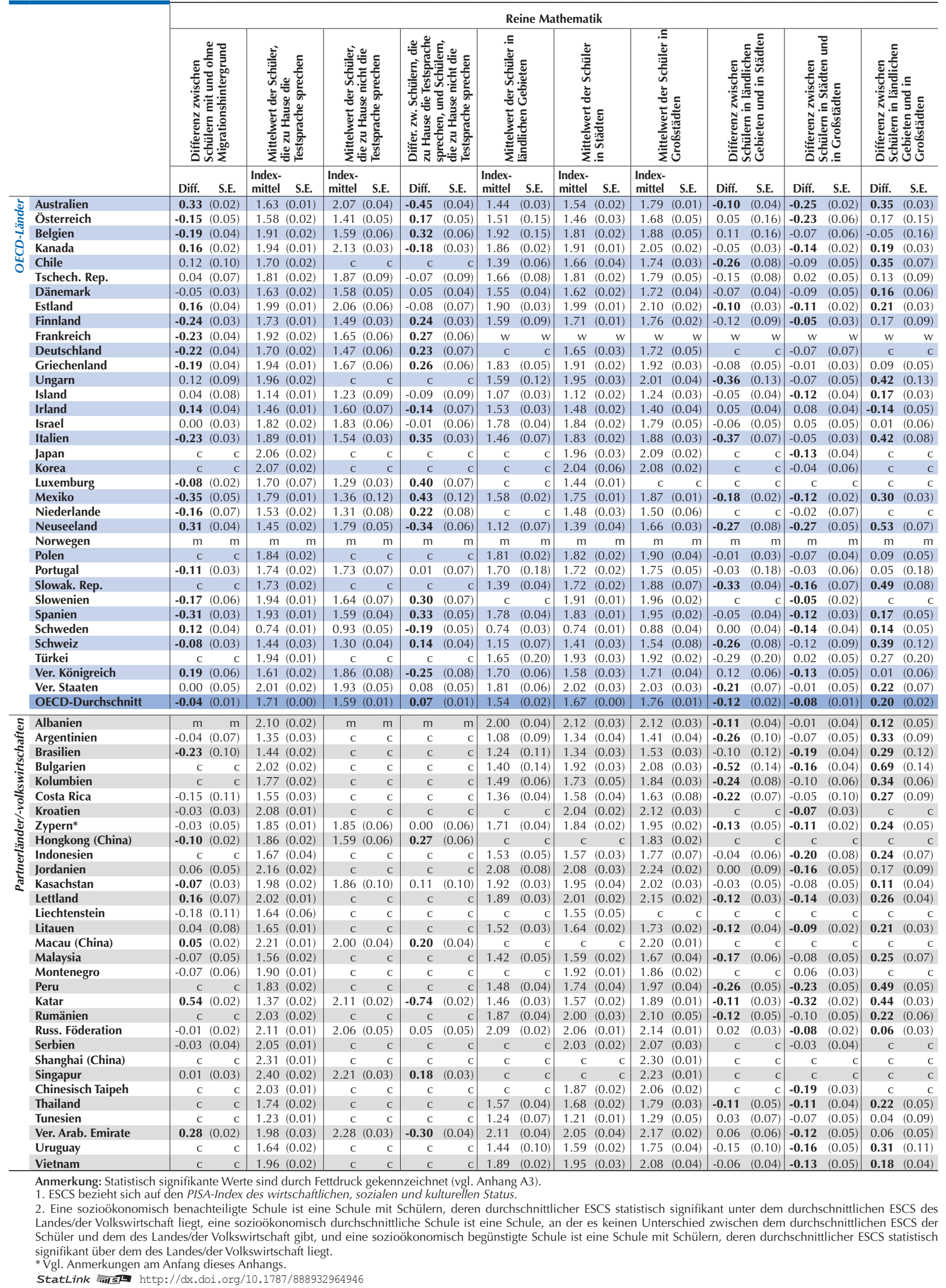


[Teil 1/1]

Korrelation zwischen den Schülerleistungen und ausgewählten Schüler- und Schulmerkmalen Tabelle II.4.4 Die Ergebnisse basieren auf Schülerangaben

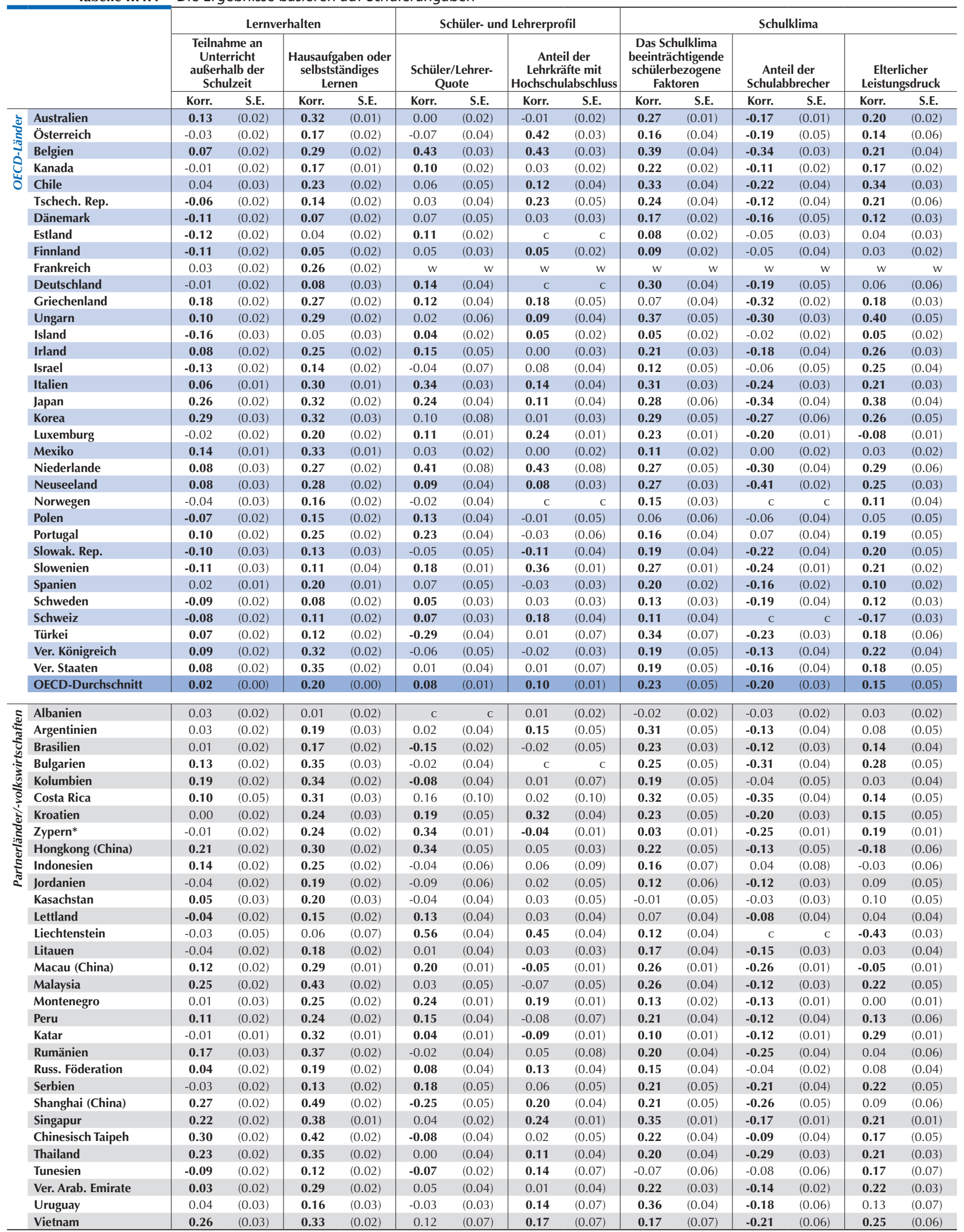

Anmerkung: Statistisch signifikante Werte sind durch Fettdruck gekennzeichnet (vgl. Anhang A3)

* Vgl. Anmerkungen am Anfang dieses Anhangs.

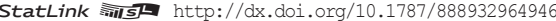


[Teil 1/1]

Korrelation zwischen dem sozioökonomischen Status der Schüler und ausgewählten Schüler- und Schulmerkmalen

Tabelle II.4.5 Die Ergebnisse basieren auf Schülerangaben

\begin{tabular}{|c|c|c|c|c|c|c|c|c|c|c|c|c|c|c|}
\hline & \multicolumn{4}{|c|}{ Lernverhalten } & \multicolumn{4}{|c|}{ Schüler- und Lehrerprofil } & \multicolumn{6}{|c|}{ Schulklima } \\
\hline & \multicolumn{2}{|c|}{$\begin{array}{l}\text { Teilnahme an } \\
\text { Unterricht } \\
\text { außerhalb der } \\
\text { Schulzeit }\end{array}$} & \multicolumn{2}{|c|}{$\begin{array}{c}\text { Hausaufgaben oder } \\
\text { selbstständiges } \\
\text { Lernen }\end{array}$} & \multicolumn{2}{|c|}{$\begin{array}{c}\text { Schüler/Lehrer- } \\
\text { Quote }\end{array}$} & \multicolumn{2}{|c|}{$\begin{array}{c}\text { Anteil der } \\
\text { Lehrkräfte mit } \\
\text { Hochschulabschluss }\end{array}$} & \multicolumn{2}{|c|}{$\begin{array}{c}\text { Das Schulklima } \\
\text { beeinträchtigende } \\
\text { schülerbezogene } \\
\text { Faktoren }\end{array}$} & \multicolumn{2}{|c|}{$\begin{array}{c}\text { Anteil der } \\
\text { Schulabbrecher }\end{array}$} & \multicolumn{2}{|c|}{$\begin{array}{c}\text { Elterlicher } \\
\text { Leistungsdruck }\end{array}$} \\
\hline & Korr. & S.E. & Korr. & S.E. & Korr. & S.E. & Korr. & S.E. & Korr. & S.E. & Korr. & S.E. & Korr. & S.E. \\
\hline ¿ Australien & 0.14 & $(0.01)$ & 0.19 & $(0.01)$ & -0.03 & $(0.02)$ & 0.01 & $(0.02)$ & 0.28 & $(0.01)$ & -0.16 & $(0.01)$ & 0.19 & $(0.02)$ \\
\hline Österreich & 0.11 & $(0.02)$ & 0.13 & $(0.02)$ & -0.06 & $(0.03)$ & 0.35 & $(0.03)$ & 0.13 & $(0.04)$ & -0.12 & $(0.03)$ & 0.14 & $(0.06)$ \\
\hline Belgien & 0.13 & $(0.02)$ & 0.20 & $(0.02)$ & 0.33 & $(0.03)$ & 0.33 & $(0.03)$ & 0.30 & $(0.02)$ & -0.19 & $(0.03)$ & 0.16 & $(0.03)$ \\
\hline Kanada & 0.12 & $(0.01)$ & 0.15 & $(0.01)$ & 0.09 & $(0.02)$ & 0.01 & $(0.01)$ & 0.17 & $(0.02)$ & -0.14 & $(0.03)$ & 0.19 & $(0.02)$ \\
\hline Chile & 0.08 & $(0.02)$ & 0.13 & $(0.02)$ & -0.03 & $(0.04)$ & 0.14 & $(0.07)$ & 0.33 & $(0.04)$ & -0.25 & $(0.03)$ & 0.33 & $(0.04)$ \\
\hline Tschech. Rep. & 0.09 & $(0.02)$ & 0.12 & $(0.02)$ & 0.03 & $(0.03)$ & 0.15 & $(0.03)$ & 0.17 & $(0.03)$ & -0.10 & $(0.03)$ & 0.15 & $(0.04)$ \\
\hline Dänemark & 0.03 & $(0.03)$ & 0.08 & $(0.02)$ & 0.09 & $(0.04)$ & 0.04 & $(0.02)$ & 0.17 & $(0.02)$ & -0.13 & $(0.03)$ & 0.17 & $(0.03)$ \\
\hline Estland & 0.06 & $(0.02)$ & 0.06 & $(0.02)$ & 0.22 & $(0.02)$ & c & c & 0.05 & $(0.03)$ & -0.06 & $(0.02)$ & 0.07 & $(0.03)$ \\
\hline Finnland & 0.06 & $(0.01)$ & 0.08 & $(0.02)$ & 0.13 & $(0.02)$ & 0.01 & $(0.03)$ & 0.00 & $(0.02)$ & 0.00 & $(0.02)$ & 0.05 & $(0.02)$ \\
\hline Frankreich & 0.11 & $(0.02)$ & 0.21 & $(0.02)$ & w & w & w & w & w & w & w & w & w & w \\
\hline Deutschland & 0.11 & $(0.02)$ & 0.08 & $(0.02)$ & 0.11 & $(0.03)$ & $\mathrm{m}$ & $\mathrm{m}$ & 0.17 & $(0.03)$ & -0.10 & $(0.02)$ & 0.07 & $(0.04)$ \\
\hline Griechenland & 0.23 & $(0.02)$ & 0.17 & $(0.02)$ & 0.10 & $(0.04)$ & 0.11 & $(0.05)$ & 0.08 & $(0.03)$ & -0.21 & $(0.03)$ & 0.19 & $(0.03)$ \\
\hline Ungarn & 0.17 & $(0.02)$ & 0.22 & $(0.03)$ & -0.03 & $(0.04)$ & 0.11 & $(0.03)$ & 0.30 & $(0.04)$ & -0.28 & $(0.03)$ & 0.32 & $(0.04)$ \\
\hline Island & 0.03 & $(0.02)$ & 0.05 & $(0.02)$ & 0.18 & $(0.02)$ & 0.07 & $(0.02)$ & 0.00 & $(0.02)$ & -0.03 & $(0.01)$ & 0.10 & $(0.02)$ \\
\hline Irland & 0.15 & $(0.02)$ & 0.18 & $(0.02)$ & 0.15 & $(0.04)$ & -0.04 & $(0.06)$ & 0.20 & $(0.03)$ & -0.16 & $(0.04)$ & 0.27 & $(0.03)$ \\
\hline Israel & 0.01 & $(0.02)$ & 0.07 & $(0.01)$ & -0.02 & $(0.05)$ & 0.11 & $(0.04)$ & 0.07 & $(0.04)$ & -0.11 & $(0.04)$ & 0.20 & $(0.04)$ \\
\hline Italien & 0.18 & $(0.01)$ & 0.22 & $(0.01)$ & 0.22 & $(0.02)$ & 0.16 & $(0.02)$ & 0.22 & $(0.02)$ & -0.19 & $(0.02)$ & 0.16 & $(0.02)$ \\
\hline Japan & 0.22 & $(0.02)$ & 0.19 & $(0.02)$ & 0.15 & $(0.03)$ & 0.08 & $(0.03)$ & 0.16 & $(0.04)$ & -0.19 & $(0.03)$ & 0.22 & $(0.03)$ \\
\hline Korea & 0.33 & $(0.02)$ & 0.21 & $(0.02)$ & 0.13 & $(0.05)$ & 0.01 & $(0.02)$ & 0.12 & $(0.03)$ & -0.12 & $(0.05)$ & 0.21 & $(0.03)$ \\
\hline Luxemburg & 0.14 & $(0.02)$ & 0.15 & $(0.02)$ & 0.09 & $(0.01)$ & 0.25 & $(0.01)$ & 0.25 & $(0.01)$ & -0.21 & $(0.01)$ & -0.03 & $(0.01)$ \\
\hline Mexiko & 0.10 & $(0.01)$ & 0.11 & $(0.01)$ & 0.02 & $(0.01)$ & 0.00 & $(0.03)$ & 0.09 & $(0.02)$ & -0.02 & $(0.02)$ & 0.07 & $(0.02)$ \\
\hline Niederlande & 0.08 & $(0.02)$ & 0.10 & $(0.02)$ & 0.20 & (0.05) & 0.24 & $(0.05)$ & 0.10 & $(0.03)$ & -0.15 & $(0.03)$ & 0.18 & (0.03) \\
\hline Neuseeland & 0.17 & $(0.03)$ & 0.20 & $(0.02)$ & 0.07 & $(0.04)$ & 0.11 & $(0.03)$ & 0.28 & $(0.03)$ & -0.42 & $(0.02)$ & 0.23 & $(0.03)$ \\
\hline Norwegen & 0.08 & $(0.02)$ & 0.12 & $(0.02)$ & 0.10 & $(0.03)$ & $\mathrm{m}$ & $\mathrm{m}$ & 0.10 & $(0.02)$ & c & C & 0.17 & $(0.03)$ \\
\hline Polen & 0.06 & $(0.02)$ & 0.07 & $(0.02)$ & 0.03 & $(0.03)$ & -0.03 & $(0.04)$ & 0.02 & $(0.05)$ & -0.02 & $(0.03)$ & 0.03 & $(0.05)$ \\
\hline Portugal & 0.18 & $(0.02)$ & 0.19 & $(0.02)$ & 0.23 & $(0.05)$ & -0.09 & $(0.06)$ & 0.10 & $(0.05)$ & 0.05 & $(0.05)$ & 0.22 & $(0.05)$ \\
\hline Slowak. Rep. & 0.04 & $(0.02)$ & 0.11 & $(0.03)$ & 0.03 & $(0.05)$ & -0.09 & $(0.03)$ & 0.15 & $(0.03)$ & -0.18 & $(0.03)$ & 0.19 & $(0.05)$ \\
\hline Slowenien & 0.04 & $(0.02)$ & 0.09 & $(0.02)$ & 0.14 & $(0.01)$ & 0.24 & $(0.01)$ & 0.15 & $(0.01)$ & -0.13 & $(0.01)$ & 0.15 & $(0.02)$ \\
\hline Spanien & 0.10 & $(0.01)$ & 0.12 & $(0.02)$ & 0.09 & $(0.06)$ & -0.02 & $(0.03)$ & 0.23 & $(0.03)$ & -0.16 & $(0.03)$ & 0.14 & $(0.03)$ \\
\hline Schweden & 0.12 & $(0.02)$ & 0.13 & $(0.02)$ & 0.11 & $(0.03)$ & 0.05 & $(0.03)$ & 0.18 & $(0.03)$ & -0.21 & $(0.03)$ & 0.17 & $(0.03)$ \\
\hline Schweiz & 0.07 & $(0.02)$ & 0.09 & $(0.02)$ & -0.03 & $(0.03)$ & 0.09 & $(0.04)$ & 0.04 & $(0.03)$ & c & c & -0.04 & $(0.03)$ \\
\hline Türkei & 0.11 & $(0.02)$ & 0.00 & $(0.02)$ & -0.21 & $(0.04)$ & 0.02 & $(0.04)$ & 0.17 & $(0.04)$ & -0.11 & $(0.03)$ & 0.12 & $(0.04)$ \\
\hline Ver. Königreich & 0.15 & $(0.02)$ & 0.24 & $(0.02)$ & -0.09 & $(0.04)$ & 0.00 & $(0.03)$ & 0.17 & $(0.03)$ & -0.15 & $(0.02)$ & 0.24 & $(0.03)$ \\
\hline Ver. Staaten & 0.15 & $(0.02)$ & 0.22 & $(0.02)$ & 0.01 & $(0.03)$ & -0.01 & $(0.07)$ & 0.22 & $(0.04)$ & -0.17 & $(0.05)$ & 0.25 & $(0.04)$ \\
\hline OECD-Durchschnitt & 0.12 & $(0.00)$ & 0.14 & $(0.00)$ & 0.07 & $(0.01)$ & 0.08 & $(0.01)$ & 0.16 & $(0.01)$ & -0.15 & $(0.01)$ & 0.16 & $(0.01)$ \\
\hline
\end{tabular}

\begin{tabular}{|c|c|c|c|c|c|c|c|c|c|c|c|c|c|c|}
\hline Albanien & $\mathrm{m}$ & $\mathrm{m}$ & $\mathrm{m}$ & $\mathrm{m}$ & $\mathrm{m}$ & $\mathrm{m}$ & $\mathrm{m}$ & $\mathrm{m}$ & $\mathrm{m}$ & $\mathrm{m}$ & $\mathrm{m}$ & $\mathrm{m}$ & $\mathrm{m}$ & $\mathrm{m}$ \\
\hline Argentinien & 0.08 & $(0.02)$ & 0.10 & $(0.02)$ & 0.03 & $(0.03)$ & 0.10 & $(0.05)$ & 0.20 & $(0.05)$ & -0.15 & $(0.04)$ & 0.09 & $(0.05)$ \\
\hline Brasilien & 0.09 & $(0.02)$ & 0.09 & $(0.01)$ & -0.13 & $(0.03)$ & -0.01 & $(0.04)$ & 0.24 & $(0.03)$ & -0.14 & $(0.03)$ & 0.20 & $(0.03)$ \\
\hline Bulgarien & 0.20 & $(0.02)$ & 0.26 & $(0.03)$ & -0.02 & $(0.03)$ & c & c & 0.15 & $(0.04)$ & -0.25 & $(0.04)$ & 0.27 & $(0.03)$ \\
\hline Kolumbien & 0.16 & $(0.02)$ & 0.17 & $(0.02)$ & -0.04 & $(0.04)$ & -0.02 & $(0.06)$ & 0.16 & $(0.04)$ & -0.04 & $(0.04)$ & 0.05 & $(0.05)$ \\
\hline Costa Rica & 0.15 & $(0.03)$ & 0.14 & $(0.02)$ & 0.12 & $(0.07)$ & 0.10 & $(0.10)$ & 0.28 & $(0.04)$ & -0.27 & $(0.04)$ & 0.14 & $(0.05)$ \\
\hline Kroatien & 0.11 & $(0.02)$ & 0.15 & $(0.02)$ & 0.12 & $(0.05)$ & 0.21 & $(0.03)$ & 0.11 & $(0.04)$ & -0.11 & $(0.04)$ & 0.10 & $(0.04)$ \\
\hline Zypern* & 0.09 & $(0.02)$ & 0.19 & $(0.02)$ & 0.26 & $(0.01)$ & 0.03 & $(0.02)$ & 0.08 & $(0.01)$ & -0.14 & $(0.01)$ & 0.17 & $(0.01)$ \\
\hline Hongkong (China) & 0.19 & $(0.02)$ & 0.10 & $(0.02)$ & 0.02 & $(0.05)$ & 0.02 & $(0.03)$ & 0.12 & $(0.05)$ & 0.01 & $(0.06)$ & -0.04 & $(0.05)$ \\
\hline Indonesien & 0.14 & $(0.02)$ & 0.11 & $(0.03)$ & -0.07 & $(0.05)$ & 0.12 & $(0.04)$ & 0.11 & $(0.05)$ & -0.12 & $(0.04)$ & -0.04 & $(0.05)$ \\
\hline Jordanien & 0.05 & $(0.02)$ & 0.05 & $(0.02)$ & -0.04 & $(0.03)$ & 0.00 & $(0.03)$ & 0.03 & $(0.04)$ & -0.09 & $(0.04)$ & 0.09 & $(0.04)$ \\
\hline Kasachstan & 0.14 & $(0.02)$ & 0.14 & $(0.03)$ & 0.12 & $(0.04)$ & 0.11 & $(0.04)$ & -0.02 & $(0.04)$ & -0.02 & $(0.01)$ & 0.10 & $(0.04)$ \\
\hline Lettland & 0.12 & $(0.02)$ & 0.14 & $(0.02)$ & 0.21 & $(0.04)$ & 0.10 & $(0.04)$ & 0.00 & $(0.04)$ & -0.08 & $(0.04)$ & 0.07 & $(0.04)$ \\
\hline Liechtenstein & 0.21 & $(0.07)$ & 0.16 & $(0.08)$ & 0.19 & $(0.06)$ & 0.17 & $(0.07)$ & 0.18 & $(0.06)$ & c & c & -0.22 & $(0.06)$ \\
\hline Litauen & 0.08 & $(0.02)$ & 0.12 & $(0.02)$ & 0.02 & $(0.04)$ & 0.03 & (0.03) & 0.12 & $(0.03)$ & -0.09 & $(0.03)$ & 0.08 & $(0.04)$ \\
\hline Macau (China) & 0.15 & $(0.01)$ & 0.13 & $(0.02)$ & -0.03 & $(0.01)$ & -0.05 & $(0.01)$ & 0.13 & $(0.01)$ & -0.12 & $(0.01)$ & 0.08 & $(0.01)$ \\
\hline Malaysia & 0.17 & $(0.02)$ & 0.18 & $(0.02)$ & 0.05 & $(0.03)$ & -0.05 & $(0.04)$ & 0.23 & $(0.04)$ & -0.12 & $(0.03)$ & 0.17 & $(0.05)$ \\
\hline Montenegro & 0.07 & $(0.02)$ & 0.07 & $(0.02)$ & 0.18 & $(0.01)$ & 0.12 & $(0.01)$ & 0.09 & $(0.01)$ & -0.11 & $(0.01)$ & -0.03 & $(0.01)$ \\
\hline Peru & 0.15 & $(0.02)$ & 0.11 & $(0.02)$ & 0.13 & $(0.03)$ & -0.04 & $(0.07)$ & 0.20 & $(0.04)$ & -0.10 & $(0.04)$ & 0.13 & $(0.05)$ \\
\hline Katar & 0.04 & $(0.01)$ & 0.11 & $(0.01)$ & 0.03 & $(0.01)$ & -0.04 & $(0.01)$ & -0.01 & $(0.01)$ & -0.03 & $(0.01)$ & 0.09 & $(0.01)$ \\
\hline Rumänien & 0.18 & $(0.02)$ & 0.21 & $(0.02)$ & -0.11 & $(0.04)$ & 0.15 & $(0.03)$ & 0.17 & $(0.04)$ & -0.15 & $(0.03)$ & 0.04 & $(0.05)$ \\
\hline Russ. Föderation & 0.11 & $(0.02)$ & 0.10 & $(0.02)$ & 0.19 & $(0.03)$ & 0.15 & $(0.03)$ & 0.11 & $(0.05)$ & -0.04 & $(0.02)$ & 0.14 & $(0.04)$ \\
\hline Serbien & 0.02 & $(0.02)$ & 0.02 & $(0.02)$ & 0.14 & $(0.04)$ & 0.03 & $(0.03)$ & 0.12 & $(0.04)$ & -0.10 & $(0.03)$ & 0.15 & $(0.04)$ \\
\hline Shanghai (China) & 0.21 & $(0.02)$ & 0.26 & $(0.02)$ & -0.16 & $(0.05)$ & 0.15 & $(0.05)$ & 0.10 & $(0.04)$ & -0.21 & $(0.05)$ & 0.12 & $(0.04)$ \\
\hline Singapur & 0.18 & $(0.02)$ & 0.16 & $(0.02)$ & 0.05 & $(0.05)$ & 0.18 & $(0.02)$ & 0.23 & $(0.01)$ & -0.09 & $(0.04)$ & 0.19 & $(0.02)$ \\
\hline Chinesisch Taipeh & 0.28 & $(0.02)$ & 0.26 & $(0.02)$ & -0.01 & $(0.03)$ & 0.01 & $(0.03)$ & 0.18 & $(0.04)$ & -0.10 & $(0.03)$ & 0.15 & $(0.04)$ \\
\hline Thailand & 0.20 & $(0.02)$ & 0.16 & $(0.02)$ & 0.07 & $(0.04)$ & 0.02 & $(0.03)$ & 0.08 & $(0.04)$ & -0.19 & $(0.03)$ & 0.19 & $(0.04)$ \\
\hline Tunesien & 0.13 & $(0.02)$ & 0.06 & $(0.02)$ & 0.03 & $(0.02)$ & 0.02 & $(0.03)$ & -0.05 & $(0.05)$ & -0.11 & $(0.05)$ & 0.14 & $(0.06)$ \\
\hline Ver. Arab. Emirate & 0.03 & $(0.02)$ & 0.09 & $(0.02)$ & -0.03 & $(0.03)$ & -0.03 & $(0.02)$ & 0.06 & $(0.02)$ & -0.11 & $(0.02)$ & 0.14 & $(0.03)$ \\
\hline Uruguay & 0.11 & $(0.02)$ & 0.07 & $(0.02)$ & -0.05 & $(0.04)$ & 0.15 & $(0.06)$ & 0.35 & $(0.04)$ & -0.23 & $(0.04)$ & 0.16 & $(0.07)$ \\
\hline Vietnam & 0.23 & $(0.02)$ & 0.16 & $(0.03)$ & 0.08 & $(0.06)$ & 0.07 & $(0.06)$ & 0.13 & $(0.05)$ & -0.17 & $(0.05)$ & 0.16 & $(0.05)$ \\
\hline
\end{tabular}

Anmerkung: Statistisch signifikante Werte sind durch Fettdruck gekennzeichnet (vgl. Anhang A3).

$* \mathrm{Vgl}$. Anmerkungen am Anfang dieses Anhangs.

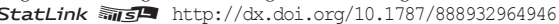


[Teil 1/1]

Korrelation zwischen dem sozioökonomischen Profil der Schulen und ausgewählten Schüler- und Schulmerkmalen

Tabelle II.4.6 Die Ergebnisse basieren auf Schülerangaben

\begin{tabular}{|c|c|c|c|c|c|c|c|c|c|c|c|c|c|c|c|}
\hline & & \multicolumn{4}{|c|}{ Lernverhalten } & \multicolumn{4}{|c|}{ Schüler- und Lehrerprofil } & \multicolumn{6}{|c|}{ Schulklima } \\
\hline & & \multicolumn{2}{|c|}{$\begin{array}{c}\text { Teilnahme an } \\
\text { Unterricht } \\
\text { außerhalb der } \\
\text { Schulzeit }\end{array}$} & \multicolumn{2}{|c|}{$\begin{array}{c}\text { Hausaufgaben oder } \\
\text { selbstständiges } \\
\text { Lernen }\end{array}$} & \multicolumn{2}{|c|}{$\begin{array}{c}\text { Schüler/Lehrer- } \\
\text { Quote }\end{array}$} & \multicolumn{2}{|c|}{$\begin{array}{c}\text { Anteil der } \\
\text { Lehrkräfte mit } \\
\text { Hochschulabschluss }\end{array}$} & $\begin{array}{r}\text { Das S } \\
\text { beeintr } \\
\text { schüle } \\
\text { Fa }\end{array}$ & $\begin{array}{l}\text { ulklima } \\
\text { htigende } \\
\text { ezogene } \\
\text { oren }\end{array}$ & $\begin{array}{r}\text { An } \\
\text { Schul }\end{array}$ & $\begin{array}{l}\text { der } \\
\text { orecher }\end{array}$ & $\begin{array}{r}\text { Elte } \\
\text { Leistu }\end{array}$ & $\begin{array}{l}\text { cher } \\
\text { sdruck }\end{array}$ \\
\hline & & Korr. & S.E. & Korr. & S.E. & Korr. & S.E. & Korr. & S.E. & Korr. & S.E. & Korr. & S.E. & Korr. & S.E. \\
\hline ذ & Australien & 0.14 & $(0.01)$ & 0.25 & $(0.01)$ & -0.05 & $(0.04)$ & 0.02 & $(0.04)$ & 0.52 & $(0.02)$ & -0.31 & $(0.02)$ & 0.36 & $(0.04)$ \\
\hline$\tau$ & Österreich & 0.12 & $(0.03)$ & 0.23 & $(0.03)$ & -0.11 & $(0.05)$ & 0.60 & $(0.05)$ & 0.23 & $(0.07)$ & -0.22 & $(0.06)$ & 0.25 & $(0.10)$ \\
\hline : & Belgien & 0.17 & $(0.02)$ & 0.31 & $(0.02)$ & 0.59 & $(0.05)$ & 0.61 & $(0.04)$ & 0.56 & $(0.04)$ & -0.36 & $(0.05)$ & 0.30 & $(0.05)$ \\
\hline తి & Kanada & 0.10 & $(0.02)$ & 0.18 & $(0.02)$ & 0.20 & $(0.04)$ & 0.02 & $(0.03)$ & 0.36 & $(0.05)$ & -0.31 & $(0.06)$ & 0.41 & $(0.03)$ \\
\hline & Chile & 0.08 & $(0.02)$ & 0.16 & $(0.02)$ & -0.03 & $(0.05)$ & 0.19 & $(0.09)$ & 0.45 & $(0.05)$ & -0.34 & $(0.04)$ & 0.44 & (0.05) \\
\hline & Tschech. Rep. & 0.02 & $(0.02)$ & 0.14 & $(0.02)$ & 0.05 & $(0.06)$ & 0.28 & $(0.06)$ & 0.31 & $(0.06)$ & -0.18 & $(0.06)$ & 0.28 & $(0.08)$ \\
\hline & Dänemark & 0.00 & $(0.02)$ & 0.05 & $(0.03)$ & 0.20 & $(0.08)$ & 0.09 & $(0.05)$ & 0.35 & $(0.05)$ & -0.30 & $(0.07)$ & 0.35 & $(0.07)$ \\
\hline & Estland & 0.02 & $(0.02)$ & 0.04 & $(0.02)$ & 0.45 & $(0.04)$ & c & C & 0.09 & $(0.05)$ & -0.12 & $(0.05)$ & 0.13 & $(0.05)$ \\
\hline & Finnland & 0.05 & $(0.02)$ & 0.05 & $(0.02)$ & 0.36 & $(0.05)$ & 0.01 & $(0.07)$ & 0.01 & $(0.06)$ & 0.02 & $(0.06)$ & 0.14 & $(0.05)$ \\
\hline & Frankreich & 0.13 & $(0.03)$ & 0.29 & $(0.03)$ & w & w & w & w & w & w & w & w & w & w \\
\hline & Deutschland & 0.08 & $(0.03)$ & 0.14 & $(0.03)$ & 0.19 & $(0.05)$ & $\mathrm{m}$ & $\mathrm{m}$ & 0.29 & $(0.06)$ & -0.18 & $(0.04)$ & 0.13 & $(0.07)$ \\
\hline & Griechenland & 0.21 & $(0.02)$ & 0.20 & $(0.02)$ & 0.18 & $(0.07)$ & 0.19 & $(0.09)$ & 0.14 & $(0.05)$ & -0.37 & $(0.04)$ & 0.35 & $(0.06)$ \\
\hline & Ungarn & 0.20 & $(0.03)$ & 0.32 & $(0.03)$ & -0.04 & $(0.07)$ & 0.16 & $(0.05)$ & 0.47 & $(0.06)$ & -0.43 & $(0.04)$ & 0.49 & (0.06) \\
\hline & Island & 0.05 & $(0.02)$ & 0.11 & $(0.02)$ & 0.42 & $(0.01)$ & 0.18 & $(0.01)$ & -0.01 & $(0.01)$ & -0.07 & $(0.00)$ & 0.24 & $(0.01)$ \\
\hline & Irland & 0.10 & $(0.03)$ & 0.15 & $(0.03)$ & 0.32 & $(0.09)$ & -0.08 & $(0.14)$ & 0.42 & $(0.06)$ & -0.33 & $(0.07)$ & 0.56 & $(0.04)$ \\
\hline & Israel & -0.06 & $(0.02)$ & 0.07 & $(0.02)$ & -0.03 & $(0.09)$ & 0.21 & $(0.07)$ & 0.14 & $(0.07)$ & -0.20 & $(0.08)$ & 0.37 & $(0.07)$ \\
\hline & Italien & 0.24 & $(0.02)$ & 0.38 & $(0.02)$ & 0.40 & $(0.04)$ & 0.30 & $(0.03)$ & 0.41 & $(0.04)$ & -0.35 & $(0.03)$ & 0.30 & $(0.04)$ \\
\hline & Japan & 0.31 & $(0.02)$ & 0.33 & $(0.02)$ & 0.30 & $(0.05)$ & 0.18 & $(0.07)$ & 0.34 & $(0.08)$ & -0.39 & $(0.06)$ & 0.44 & $(0.06)$ \\
\hline & Korea & 0.36 & $(0.03)$ & 0.28 & $(0.03)$ & 0.27 & $(0.11)$ & 0.02 & $(0.05)$ & 0.25 & $(0.07)$ & -0.24 & $(0.11)$ & 0.42 & $(0.06)$ \\
\hline & Luxemburg & 0.06 & $(0.02)$ & 0.16 & $(0.02)$ & 0.17 & $(0.00)$ & 0.46 & $(0.00)$ & 0.47 & $(0.00)$ & -0.38 & $(0.00)$ & -0.06 & $(0.00)$ \\
\hline & Mexiko & 0.09 & $(0.01)$ & 0.16 & $(0.01)$ & 0.02 & $(0.02)$ & 0.01 & $(0.04)$ & 0.12 & $(0.03)$ & -0.02 & $(0.03)$ & 0.10 & $(0.04)$ \\
\hline & Niederlande & 0.12 & $(0.03)$ & 0.22 & $(0.02)$ & 0.43 & $(0.09)$ & 0.51 & $(0.11)$ & 0.21 & $(0.07)$ & -0.34 & $(0.06)$ & 0.39 & $(0.07)$ \\
\hline & Neuseeland & 0.14 & $(0.04)$ & 0.24 & $(0.03)$ & 0.15 & $(0.08)$ & 0.21 & $(0.07)$ & 0.53 & $(0.05)$ & -0.80 & $(0.02)$ & 0.44 & $(0.06)$ \\
\hline & Norwegen & 0.09 & $(0.02)$ & 0.12 & $(0.02)$ & 0.27 & $(0.07)$ & c & c & 0.28 & $(0.06)$ & c & C & 0.47 & $(0.06)$ \\
\hline & Polen & 0.01 & $(0.02)$ & 0.03 & $(0.02)$ & 0.07 & $(0.07)$ & -0.07 & $(0.08)$ & 0.04 & $(0.09)$ & -0.05 & $(0.05)$ & 0.07 & $(0.09)$ \\
\hline & Portugal & 0.12 & $(0.03)$ & 0.17 & $(0.03)$ & 0.41 & $(0.07)$ & -0.15 & $(0.11)$ & 0.17 & $(0.09)$ & 0.08 & $(0.08)$ & 0.38 & $(0.07)$ \\
\hline & Slowak. Rep. & -0.01 & $(0.03)$ & 0.16 & $(0.04)$ & 0.04 & $(0.08)$ & -0.15 & $(0.05)$ & 0.25 & $(0.05)$ & -0.28 & $(0.04)$ & 0.30 & $(0.08)$ \\
\hline & Slowenien & 0.04 & $(0.03)$ & 0.16 & $(0.03)$ & 0.25 & $(0.01)$ & 0.43 & $(0.01)$ & 0.27 & $(0.01)$ & -0.23 & $(0.01)$ & 0.27 & $(0.02)$ \\
\hline & Spanien & 0.04 & $(0.01)$ & 0.08 & $(0.02)$ & 0.17 & $(0.11)$ & -0.04 & $(0.05)$ & 0.45 & $(0.04)$ & -0.31 & $(0.05)$ & 0.27 & $(0.05)$ \\
\hline & Schweden & 0.11 & $(0.03)$ & 0.17 & $(0.03)$ & 0.26 & $(0.06)$ & 0.12 & $(0.06)$ & 0.43 & $(0.06)$ & -0.49 & $(0.06)$ & 0.40 & $(0.06)$ \\
\hline & Schweiz & 0.06 & $(0.02)$ & 0.12 & $(0.03)$ & -0.07 & $(0.06)$ & 0.18 & $(0.08)$ & 0.08 & $(0.07)$ & c & c & -0.10 & $(0.07)$ \\
\hline & Türkei & 0.05 & $(0.03)$ & 0.04 & $(0.02)$ & -0.37 & $(0.06)$ & 0.04 & $(0.08)$ & 0.31 & $(0.07)$ & -0.19 & $(0.05)$ & 0.21 & $(0.07)$ \\
\hline & Ver. Königreich & 0.16 & $(0.03)$ & 0.31 & $(0.03)$ & -0.18 & $(0.10)$ & 0.00 & $(0.06)$ & 0.35 & $(0.06)$ & -0.29 & $(0.05)$ & 0.48 & $(0.05)$ \\
\hline & Ver. Staaten & 0.14 & $(0.02)$ & 0.25 & $(0.03)$ & 0.02 & $(0.05)$ & -0.02 & $(0.13)$ & 0.42 & $(0.06)$ & -0.31 & $(0.09)$ & 0.47 & $(0.07)$ \\
\hline & OECD-Durchschnitt & 0.10 & $(0.00)$ & 0.18 & $(0.00)$ & 0.16 & $(0.01)$ & 0.14 & $(0.01)$ & 0.30 & $(0.01)$ & -0.28 & $(0.01)$ & 0.31 & $(0.01)$ \\
\hline & Albanien & $\mathrm{m}$ & $\mathrm{m}$ & $\mathrm{m}$ & $\mathrm{m}$ & $\mathrm{m}$ & $\mathrm{m}$ & $\mathrm{m}$ & $\mathrm{m}$ & $\mathrm{m}$ & $\mathrm{m}$ & $\mathrm{m}$ & $\mathrm{m}$ & $\mathrm{m}$ & $\mathrm{m}$ \\
\hline હ્ટ & Argentinien & 0.04 & $(0.02)$ & 0.10 & $(0.03)$ & 0.05 & $(0.05)$ & 0.17 & $(0.08)$ & 0.33 & $(0.08)$ & -0.24 & $(0.07)$ & 0.15 & (0.08) \\
\hline క్ & Brasilien & 0.05 & $(0.02)$ & 0.13 & $(0.02)$ & -0.21 & $(0.05)$ & -0.01 & $(0.06)$ & 0.38 & $(0.04)$ & -0.21 & $(0.05)$ & 0.31 & $(0.04)$ \\
\hline$\stackrel{\Sigma}{=}$ & Bulgarien & 0.17 & $(0.02)$ & 0.33 & $(0.03)$ & -0.02 & $(0.05)$ & C & C & 0.23 & $(0.06)$ & -0.39 & $(0.06)$ & 0.40 & (0.05) \\
\hline 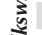 & Kolumbien & 0.12 & $(0.03)$ & 0.18 & $(0.03)$ & -0.07 & $(0.06)$ & -0.04 & $(0.10)$ & 0.25 & $(0.06)$ & -0.06 & $(0.06)$ & 0.07 & $(0.08)$ \\
\hline 3) & Costa Rica & 0.13 & $(0.04)$ & 0.22 & $(0.03)$ & 0.18 & $(0.11)$ & 0.15 & $(0.15)$ & 0.43 & $(0.07)$ & -0.41 & $(0.05)$ & 0.22 & $(0.07)$ \\
\hline$\frac{1}{2}$ & Kroatien & 0.10 & $(0.02)$ & 0.24 & $(0.03)$ & 0.22 & $(0.09)$ & 0.42 & $(0.04)$ & 0.20 & $(0.08)$ & -0.22 & $(0.07)$ & 0.19 & $(0.08)$ \\
\hline$\stackrel{\Xi}{\Xi}$ & Zypern* & 0.06 & $(0.02)$ & 0.28 & $(0.02)$ & 0.53 & $(0.00)$ & 0.07 & $(0.00)$ & 0.15 & $(0.00)$ & -0.29 & $(0.00)$ & 0.35 & $(0.00)$ \\
\hline : & Hongkong (China) & 0.20 & $(0.02)$ & 0.14 & $(0.03)$ & 0.04 & $(0.09)$ & 0.04 & $(0.05)$ & 0.21 & $(0.09)$ & 0.02 & $(0.10)$ & -0.07 & $(0.09)$ \\
\hline ఏ & Indonesien & 0.14 & $(0.02)$ & 0.16 & $(0.03)$ & -0.11 & $(0.08)$ & 0.20 & $(0.06)$ & 0.17 & $(0.08)$ & -0.19 & $(0.06)$ & -0.06 & $(0.08)$ \\
\hline ฐั & Jordanien & -0.03 & $(0.02)$ & 0.04 & $(0.03)$ & -0.07 & $(0.07)$ & -0.01 & $(0.06)$ & 0.06 & $(0.08)$ & -0.18 & $(0.07)$ & 0.19 & $(0.08)$ \\
\hline & Kasachstan & 0.08 & $(0.03)$ & 0.13 & $(0.03)$ & 0.22 & $(0.08)$ & 0.21 & $(0.08)$ & -0.04 & $(0.07)$ & -0.04 & $(0.03)$ & 0.20 & $(0.07)$ \\
\hline & Lettland & 0.11 & $(0.03)$ & 0.17 & $(0.03)$ & 0.37 & $(0.07)$ & 0.16 & $(0.07)$ & 0.01 & $(0.08)$ & -0.14 & $(0.07)$ & 0.13 & $(0.07)$ \\
\hline & Liechtenstein & 0.01 & $(0.06)$ & 0.12 & $(0.06)$ & 0.50 & $(0.01)$ & 0.46 & $(0.02)$ & 0.45 & $(0.02)$ & c & c & -0.56 & $(0.01)$ \\
\hline & Litauen & 0.04 & $(0.02)$ & 0.16 & $(0.02)$ & 0.05 & $(0.07)$ & 0.05 & $(0.05)$ & 0.24 & $(0.06)$ & -0.17 & $(0.06)$ & 0.15 & $(0.07)$ \\
\hline & Macau (China) & 0.15 & $(0.02)$ & 0.16 & $(0.02)$ & -0.05 & $(0.00)$ & -0.09 & $(0.00)$ & 0.26 & $(0.00)$ & -0.23 & $(0.00)$ & 0.16 & $(0.00)$ \\
\hline & Malaysia & 0.11 & $(0.02)$ & 0.18 & $(0.02)$ & 0.08 & $(0.06)$ & -0.10 & $(0.07)$ & 0.41 & $(0.06)$ & -0.23 & $(0.05)$ & 0.30 & (0.09) \\
\hline & Montenegro & 0.05 & $(0.02)$ & 0.16 & $(0.03)$ & 0.40 & $(0.01)$ & 0.27 & $(0.01)$ & 0.20 & $(0.01)$ & -0.25 & $(0.00)$ & -0.07 & $(0.00)$ \\
\hline & Peru & 0.08 & $(0.02)$ & 0.13 & $(0.02)$ & 0.20 & $(0.05)$ & -0.05 & $(0.11)$ & 0.29 & $(0.06)$ & -0.14 & $(0.06)$ & 0.18 & $(0.08)$ \\
\hline & Katar & -0.03 & $(0.01)$ & 0.13 & $(0.01)$ & 0.07 & $(0.00)$ & -0.09 & $(0.00)$ & -0.02 & $(0.00)$ & -0.06 & $(0.00)$ & 0.19 & $(0.00)$ \\
\hline & Rumänien & 0.16 & $(0.03)$ & 0.25 & $(0.03)$ & -0.19 & $(0.06)$ & 0.24 & $(0.04)$ & 0.27 & $(0.06)$ & -0.24 & $(0.05)$ & 0.06 & $(0.07)$ \\
\hline & Russ. Föderation & 0.06 & $(0.03)$ & 0.09 & $(0.03)$ & 0.35 & $(0.07)$ & 0.27 & $(0.07)$ & 0.21 & $(0.09)$ & -0.07 & $(0.04)$ & 0.26 & $(0.07)$ \\
\hline & Serbien & 0.03 & $(0.02)$ & 0.10 & $(0.02)$ & 0.29 & $(0.08)$ & 0.07 & $(0.06)$ & 0.24 & $(0.08)$ & -0.21 & $(0.05)$ & 0.31 & $(0.08)$ \\
\hline & Shanghai (China) & 0.24 & $(0.02)$ & 0.35 & $(0.03)$ & -0.26 & $(0.07)$ & 0.26 & $(0.08)$ & 0.17 & $(0.07)$ & -0.35 & $(0.08)$ & 0.19 & $(0.07)$ \\
\hline & Singapur & 0.13 & $(0.02)$ & 0.18 & $(0.02)$ & 0.11 & $(0.10)$ & 0.36 & $(0.02)$ & 0.47 & $(0.01)$ & -0.17 & $(0.07)$ & 0.38 & $(0.02)$ \\
\hline & Chinesisch Taipeh & 0.29 & $(0.02)$ & 0.36 & $(0.03)$ & -0.01 & $(0.07)$ & 0.02 & $(0.07)$ & 0.36 & $(0.07)$ & -0.20 & $(0.06)$ & 0.29 & $(0.07)$ \\
\hline & Thailand & 0.22 & $(0.02)$ & 0.24 & $(0.02)$ & 0.11 & $(0.07)$ & 0.03 & $(0.04)$ & 0.12 & $(0.06)$ & -0.28 & $(0.04)$ & 0.30 & $(0.07)$ \\
\hline & Tunesien & 0.03 & $(0.03)$ & 0.07 & $(0.03)$ & 0.05 & $(0.04)$ & 0.03 & $(0.06)$ & -0.08 & $(0.08)$ & -0.19 & $(0.08)$ & 0.23 & $(0.10)$ \\
\hline & Ver. Arab. Emirate & -0.03 & $(0.02)$ & 0.11 & $(0.02)$ & -0.05 & $(0.05)$ & -0.05 & $(0.04)$ & 0.11 & $(0.04)$ & -0.22 & $(0.04)$ & 0.26 & (0.05) \\
\hline & Uruguay & 0.09 & $(0.02)$ & 0.10 & $(0.02)$ & -0.08 & $(0.05)$ & 0.23 & $(0.09)$ & 0.54 & $(0.05)$ & -0.35 & $(0.05)$ & 0.25 & $(0.10)$ \\
\hline & Vietnam & 0.21 & $(0.03)$ & 0.20 & $(0.03)$ & 0.12 & $(0.08)$ & 0.10 & $(0.10)$ & 0.20 & $(0.08)$ & -0.26 & $(0.08)$ & 0.24 & $(0.08)$ \\
\hline
\end{tabular}

Anmerkung: Statistisch signifikante Werte sind durch Fettdruck gekennzeichnet (vgl. Anhang A3).

* Vgl. Anmerkungen am Anfang dieses Anhangs.

StatLink 部西 http://dx.doi.org/10.1787/888932964946 
[Teil 1/1]

Korrelation zwischen der Varianz des sozioökonomischen Status der Schüler innerhalb einer Schule und ausgewählten Schüler- und Schulmerkmalen

Tabelle II.4.7 Die Ergebnisse basieren auf Schülerangaben

\begin{tabular}{|c|c|c|c|c|c|c|c|c|c|c|c|c|c|c|c|}
\hline & \multicolumn{4}{|c|}{ Lernverhalten } & \multicolumn{4}{|c|}{ Schüler- und Lehrerprofil } & \multicolumn{6}{|c|}{ Schulklima } \\
\hline & & \multicolumn{2}{|c|}{$\begin{array}{l}\text { Teilnahme an } \\
\text { Unterricht } \\
\text { außerhalb der } \\
\text { Schulzeit }\end{array}$} & \multicolumn{2}{|c|}{$\begin{array}{l}\text { Hausaufgaben oder } \\
\text { selbstständiges } \\
\text { Lernen }\end{array}$} & \multicolumn{2}{|c|}{$\begin{array}{c}\text { Schüler/Lehrer- } \\
\text { Quote }\end{array}$} & $\begin{array}{r}\text { An } \\
\text { Lehrl } \\
\text { Hochscl }\end{array}$ & $\begin{array}{l}\text { der } \\
\text { te mit } \\
\text { abschluss }\end{array}$ & $\begin{array}{r}\text { Das S } \\
\text { beeintr } \\
\text { schüle } \\
\text { Fa }\end{array}$ & $\begin{array}{l}\text { alklima } \\
\text { htigende } \\
\text { ezogene } \\
\text { ren }\end{array}$ & $\begin{array}{r}\text { An } \\
\text { Schula }\end{array}$ & $\begin{array}{l}\text { der } \\
\text { recher }\end{array}$ & $\begin{array}{r}\text { Elte } \\
\text { Leistu }\end{array}$ & $\begin{array}{l}\text { cher } \\
\text { sdruck }\end{array}$ \\
\hline & & Korr. & S.E. & Korr. & S.E. & Korr. & S.E. & Korr. & S.E. & Korr. & S.E. & Korr. & S.E. & Korr. & S.E. \\
\hline j) & Australien & 0.00 & $(0.02)$ & -0.03 & $(0.02)$ & -0.01 & $(0.04)$ & -0.02 & $(0.03)$ & -0.12 & $(0.04)$ & 0.05 & $(0.03)$ & -0.05 & $(0.05)$ \\
\hline : & Österreich & -0.05 & $(0.03)$ & -0.05 & $(0.03)$ & -0.10 & $(0.05)$ & -0.02 & $(0.06)$ & 0.03 & $(0.09)$ & 0.00 & $(0.04)$ & 0.01 & $(0.07)$ \\
\hline 年 & Belgien & -0.03 & $(0.02)$ & -0.10 & $(0.02)$ & -0.06 & $(0.06)$ & -0.14 & $(0.09)$ & -0.09 & $(0.06)$ & 0.10 & $(0.09)$ & -0.14 & $(0.05)$ \\
\hline O & Kanada & 0.10 & $(0.02)$ & 0.08 & $(0.02)$ & -0.19 & $(0.05)$ & 0.03 & $(0.03)$ & -0.17 & $(0.04)$ & 0.03 & $(0.06)$ & -0.01 & $(0.06)$ \\
\hline ठّ & Chile & -0.01 & $(0.02)$ & -0.05 & $(0.01)$ & 0.13 & $(0.08)$ & 0.04 & $(0.02)$ & -0.13 & $(0.06)$ & 0.03 & $(0.06)$ & -0.01 & $(0.04)$ \\
\hline & Tschech. Rep. & 0.01 & $(0.03)$ & -0.04 & $(0.02)$ & 0.12 & $(0.06)$ & 0.02 & $(0.05)$ & -0.06 & $(0.05)$ & -0.17 & $(0.04)$ & -0.10 & $(0.07)$ \\
\hline & Dänemark & -0.03 & $(0.02)$ & -0.03 & $(0.01)$ & 0.13 & $(0.11)$ & -0.18 & $(0.08)$ & 0.11 & $(0.14)$ & 0.01 & $(0.05)$ & -0.17 & $(0.05)$ \\
\hline & Estland & -0.03 & $(0.02)$ & -0.01 & $(0.04)$ & -0.42 & $(0.06)$ & c & c & 0.13 & $(0.07)$ & -0.09 & $(0.02)$ & -0.13 & $(0.05)$ \\
\hline & Finnland & 0.00 & $(0.02)$ & 0.02 & $(0.02)$ & -0.30 & $(0.11)$ & 0.16 & $(0.06)$ & -0.05 & $(0.05)$ & -0.09 & $(0.05)$ & 0.13 & $(0.11)$ \\
\hline & Frankreich & -0.04 & $(0.03)$ & -0.11 & $(0.03)$ & w & w & w & w & w & w & w & w & w & w \\
\hline & Deutschland & -0.05 & $(0.03)$ & -0.07 & $(0.03)$ & -0.08 & $(0.07)$ & $\mathrm{m}$ & $\mathrm{m}$ & -0.11 & $(0.09)$ & 0.43 & $(0.19)$ & -0.07 & $(0.07)$ \\
\hline & Griechenland & -0.03 & $(0.03)$ & -0.06 & $(0.03)$ & 0.00 & $(0.07)$ & -0.01 & $(0.12)$ & 0.01 & $(0.08)$ & 0.00 & $(0.09)$ & -0.11 & $(0.07)$ \\
\hline & Ungarn & -0.01 & $(0.02)$ & -0.07 & $(0.02)$ & -0.17 & $(0.05)$ & 0.07 & $(0.03)$ & -0.01 & $(0.07)$ & -0.14 & $(0.04)$ & -0.03 & $(0.06)$ \\
\hline & Island & -0.01 & $(0.02)$ & -0.05 & $(0.02)$ & -0.11 & $(0.01)$ & -0.01 & $(0.01)$ & -0.05 & $(0.01)$ & 0.02 & $(0.01)$ & -0.17 & $(0.00)$ \\
\hline & Irland & 0.01 & $(0.03)$ & 0.00 & $(0.03)$ & -0.12 & $(0.09)$ & -0.09 & $(0.08)$ & -0.15 & $(0.08)$ & 0.02 & $(0.10)$ & -0.13 & $(0.07)$ \\
\hline & Israel & 0.02 & $(0.02)$ & -0.09 & $(0.03)$ & -0.10 & $(0.09)$ & 0.01 & $(0.09)$ & -0.12 & $(0.06)$ & 0.10 & $(0.07)$ & -0.13 & $(0.06)$ \\
\hline & Italien & 0.01 & $(0.03)$ & 0.00 & $(0.04)$ & -0.02 & $(0.03)$ & -0.09 & $(0.06)$ & 0.00 & $(0.07)$ & 0.02 & $(0.08)$ & 0.00 & $(0.04)$ \\
\hline & Japan & -0.01 & $(0.04)$ & -0.02 & $(0.04)$ & -0.01 & $(0.09)$ & -0.02 & $(0.06)$ & -0.07 & $(0.07)$ & 0.12 & $(0.10)$ & -0.06 & (0.08) \\
\hline & Korea & -0.10 & $(0.04)$ & -0.08 & $(0.04)$ & -0.17 & $(0.09)$ & 0.03 & $(0.02)$ & -0.17 & $(0.07)$ & 0.01 & $(0.08)$ & -0.12 & $(0.07)$ \\
\hline & Luxemburg & -0.05 & $(0.02)$ & -0.16 & $(0.02)$ & -0.11 & $(0.00)$ & -0.16 & $(0.00)$ & -0.40 & $(0.00)$ & 0.14 & $(0.00)$ & -0.02 & $(0.00)$ \\
\hline & Mexiko & 0.01 & $(0.01)$ & 0.01 & $(0.01)$ & -0.01 & (0.03) & 0.01 & $(0.04)$ & -0.07 & $(0.06)$ & -0.03 & $(0.03)$ & 0.08 & (0.04) \\
\hline & Niederlande & 0.03 & $(0.03)$ & -0.01 & $(0.04)$ & 0.06 & $(0.10)$ & -0.06 & $(0.08)$ & -0.10 & $(0.04)$ & 0.26 & (0.15) & 0.08 & (0.05) \\
\hline & Neuseeland & -0.01 & $(0.04)$ & -0.08 & $(0.02)$ & 0.08 & $(0.07)$ & -0.05 & $(0.06)$ & -0.32 & $(0.05)$ & 0.36 & $(0.05)$ & -0.18 & $(0.07)$ \\
\hline & Norwegen & -0.02 & $(0.02)$ & -0.04 & $(0.02)$ & -0.15 & $(0.07)$ & C & c & -0.15 & $(0.07)$ & C & c & -0.09 & $(0.07)$ \\
\hline & Polen & 0.01 & $(0.02)$ & 0.00 & $(0.02)$ & 0.17 & $(0.08)$ & 0.06 & $(0.06)$ & -0.09 & $(0.07)$ & -0.12 & $(0.06)$ & -0.04 & $(0.08)$ \\
\hline & Portugal & 0.01 & (0.03) & 0.04 & $(0.04)$ & -0.09 & $(0.09)$ & -0.02 & (0.14) & -0.16 & $(0.08)$ & 0.16 & $(0.06)$ & -0.08 & (0.13) \\
\hline & Slowak. Rep. & 0.02 & $(0.03)$ & -0.03 & $(0.03)$ & -0.08 & $(0.07)$ & -0.25 & $(0.04)$ & 0.12 & $(0.05)$ & -0.14 & $(0.04)$ & -0.06 & $(0.06)$ \\
\hline & Slowenien & 0.02 & $(0.02)$ & 0.04 & $(0.02)$ & 0.15 & $(0.05)$ & -0.01 & $(0.03)$ & 0.07 & $(0.02)$ & -0.15 & $(0.02)$ & 0.05 & (0.05) \\
\hline & Spanien & 0.02 & $(0.02)$ & 0.04 & $(0.02)$ & -0.03 & $(0.02)$ & 0.16 & $(0.02)$ & -0.10 & $(0.04)$ & -0.03 & $(0.08)$ & -0.20 & (0.05) \\
\hline & Schweden & -0.01 & $(0.02)$ & -0.04 & $(0.03)$ & -0.24 & $(0.08)$ & -0.04 & $(0.07)$ & -0.35 & $(0.06)$ & 0.30 & $(0.10)$ & -0.15 & $(0.06)$ \\
\hline & Schweiz & -0.03 & $(0.02)$ & -0.02 & $(0.02)$ & -0.04 & $(0.10)$ & -0.13 & $(0.08)$ & -0.10 & $(0.04)$ & C & c & 0.03 & $(0.09)$ \\
\hline & Türkei & 0.06 & $(0.02)$ & 0.06 & $(0.02)$ & -0.14 & $(0.08)$ & -0.13 & $(0.06)$ & 0.32 & $(0.07)$ & -0.25 & $(0.06)$ & 0.09 & $(0.07)$ \\
\hline & Ver. Königreich & 0.00 & $(0.04)$ & -0.05 & $(0.04)$ & -0.07 & $(0.09)$ & 0.05 & $(0.03)$ & -0.02 & $(0.04)$ & -0.06 & $(0.13)$ & 0.03 & $(0.07)$ \\
\hline & Ver. Staaten & 0.02 & $(0.03)$ & -0.01 & $(0.05)$ & 0.14 & $(0.13)$ & 0.08 & $(0.04)$ & 0.05 & $(0.14)$ & 0.01 & $(0.12)$ & -0.28 & $(0.13)$ \\
\hline & OECD-Durchschnitt & 0.00 & $(0.00)$ & -0.03 & $(0.00)$ & -0.06 & $(0.01)$ & -0.02 & $(0.01)$ & -0.08 & $(0.01)$ & 0.04 & $(0.01)$ & -0.06 & $(0.01)$ \\
\hline & Albanien & $\mathrm{m}$ & $\mathrm{m}$ & $\mathrm{m}$ & $\mathrm{m}$ & $\mathrm{m}$ & $\mathrm{m}$ & $\mathrm{m}$ & $\mathrm{m}$ & $\mathrm{m}$ & $\mathrm{m}$ & $\mathrm{m}$ & $\mathrm{m}$ & $\mathrm{m}$ & $\mathrm{m}$ \\
\hline 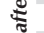 & Argentinien & 0.07 & $(0.06)$ & 0.07 & $(0.05)$ & -0.11 & $(0.07)$ & -0.05 & (0.09) & 0.16 & $(0.14)$ & 0.05 & $(0.07)$ & 0.06 & $(0.10)$ \\
\hline క్ర & Brasilien & -0.01 & (0.02) & -0.02 & $(0.02)$ & -0.02 & $(0.06)$ & -0.06 & $(0.06)$ & -0.08 & $(0.06)$ & 0.08 & $(0.07)$ & -0.08 & $(0.06)$ \\
\hline$\stackrel{5}{\Sigma}$ & Bulgarien & -0.07 & $(0.02)$ & -0.12 & $(0.02)$ & 0.19 & $(0.20)$ & C & C & -0.17 & $(0.06)$ & 0.21 & $(0.07)$ & -0.14 & (0.05) \\
\hline 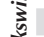 & Kolumbien & -0.01 & $(0.03)$ & 0.00 & $(0.04)$ & -0.03 & $(0.05)$ & 0.05 & $(0.09)$ & -0.06 & $(0.04)$ & 0.36 & $(0.17)$ & 0.03 & $(0.11)$ \\
\hline ๘ & Costa Rica & 0.00 & $(0.05)$ & -0.06 & $(0.04)$ & -0.09 & $(0.06)$ & 0.04 & (0.08) & -0.16 & $(0.06)$ & 0.01 & $(0.10)$ & -0.04 & $(0.10)$ \\
\hline$\frac{5}{1}$ & Kroatien & 0.03 & $(0.02)$ & 0.08 & $(0.04)$ & -0.01 & $(0.07)$ & 0.17 & $(0.07)$ & 0.06 & $(0.09)$ & -0.14 & $(0.06)$ & 0.03 & $(0.08)$ \\
\hline 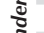 & Zypern* & 0.05 & $(0.02)$ & -0.12 & $(0.02)$ & 0.06 & $(0.00)$ & -0.05 & $(0.00)$ & -0.33 & $(0.00)$ & -0.10 & $(0.00)$ & -0.20 & $(0.00)$ \\
\hline : & Hongkong (China) & 0.01 & $(0.03)$ & 0.02 & $(0.03)$ & 0.22 & $(0.08)$ & -0.03 & $(0.08)$ & 0.01 & $(0.08)$ & -0.06 & $(0.08)$ & -0.07 & $(0.08)$ \\
\hline 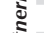 & Indonesien & 0.02 & $(0.03)$ & -0.02 & $(0.03)$ & 0.03 & $(0.07)$ & 0.05 & $(0.12)$ & -0.08 & $(0.06)$ & 0.05 & $(0.09)$ & -0.09 & $(0.08)$ \\
\hline స & Jordanien & 0.02 & $(0.02)$ & -0.04 & $(0.02)$ & 0.10 & $(0.06)$ & -0.03 & $(0.07)$ & -0.06 & $(0.09)$ & 0.08 & $(0.05)$ & -0.10 & $(0.08)$ \\
\hline & Kasachstan & -0.05 & $(0.02)$ & -0.06 & $(0.03)$ & -0.24 & $(0.07)$ & -0.14 & $(0.06)$ & -0.01 & $(0.08)$ & -0.03 & $(0.03)$ & -0.08 & $(0.06)$ \\
\hline & Lettland & -0.09 & $(0.02)$ & -0.07 & $(0.04)$ & -0.29 & $(0.08)$ & -0.19 & $(0.07)$ & 0.11 & $(0.06)$ & 0.07 & $(0.10)$ & -0.01 & $(0.06)$ \\
\hline & Liechtenstein & -0.03 & $(0.06)$ & -0.08 & $(0.07)$ & -0.14 & $(0.01)$ & -0.48 & $(0.03)$ & -0.12 & $(0.04)$ & c & c & 0.19 & $(0.02)$ \\
\hline & Litauen & 0.02 & $(0.02)$ & -0.03 & $(0.02)$ & 0.03 & $(0.07)$ & -0.04 & $(0.04)$ & -0.11 & $(0.05)$ & -0.03 & $(0.07)$ & -0.07 & $(0.05)$ \\
\hline & Macau (China) & -0.04 & $(0.02)$ & -0.07 & $(0.02)$ & -0.14 & $(0.00)$ & -0.12 & $(0.00)$ & -0.15 & $(0.00)$ & 0.10 & $(0.00)$ & -0.06 & $(0.00)$ \\
\hline & Malaysia & -0.03 & $(0.02)$ & -0.07 & $(0.02)$ & 0.07 & $(0.08)$ & 0.07 & $(0.05)$ & -0.16 & $(0.08)$ & 0.17 & $(0.08)$ & -0.18 & $(0.08)$ \\
\hline & Montenegro & 0.01 & $(0.02)$ & 0.00 & $(0.02)$ & 0.27 & $(0.00)$ & -0.09 & $(0.01)$ & -0.24 & $(0.00)$ & 0.07 & $(0.00)$ & 0.22 & $(0.00)$ \\
\hline & Peru & 0.01 & $(0.03)$ & -0.03 & $(0.03)$ & 0.05 & $(0.11)$ & -0.21 & $(0.15)$ & -0.16 & $(0.07)$ & 0.08 & $(0.07)$ & -0.03 & $(0.04)$ \\
\hline & Katar & -0.06 & $(0.01)$ & -0.23 & $(0.01)$ & -0.12 & $(0.00)$ & 0.20 & $(0.00)$ & -0.10 & $(0.00)$ & 0.19 & $(0.00)$ & -0.30 & $(0.00)$ \\
\hline & Rumänien & 0.04 & $(0.03)$ & -0.03 & $(0.03)$ & -0.01 & $(0.04)$ & -0.16 & $(0.07)$ & 0.00 & $(0.05)$ & -0.07 & $(0.06)$ & -0.01 & $(0.05)$ \\
\hline & Russ. Föderation & 0.00 & $(0.04)$ & 0.01 & $(0.04)$ & -0.40 & $(0.05)$ & -0.14 & $(0.05)$ & -0.04 & $(0.07)$ & 0.07 & $(0.06)$ & -0.08 & $(0.05)$ \\
\hline & Serbien & 0.06 & $(0.03)$ & 0.08 & $(0.03)$ & 0.07 & $(0.08)$ & 0.02 & $(0.06)$ & 0.08 & $(0.08)$ & -0.14 & $(0.05)$ & 0.20 & $(0.08)$ \\
\hline & Shanghai (China) & -0.03 & $(0.04)$ & -0.04 & $(0.06)$ & -0.05 & $(0.07)$ & -0.01 & $(0.04)$ & 0.12 & $(0.08)$ & -0.10 & $(0.04)$ & -0.22 & $(0.11)$ \\
\hline & Singapur & -0.05 & $(0.01)$ & -0.08 & $(0.02)$ & -0.08 & $(0.06)$ & -0.08 & $(0.01)$ & -0.12 & $(0.01)$ & 0.10 & $(0.04)$ & -0.04 & $(0.01)$ \\
\hline & Chinesisch Taipeh & 0.00 & $(0.03)$ & -0.03 & $(0.03)$ & -0.41 & $(0.05)$ & 0.01 & $(0.06)$ & -0.06 & $(0.08)$ & 0.01 & $(0.05)$ & 0.05 & $(0.07)$ \\
\hline & Thailand & 0.04 & $(0.02)$ & 0.04 & $(0.03)$ & 0.10 & $(0.08)$ & -0.07 & $(0.08)$ & -0.07 & $(0.07)$ & 0.04 & $(0.06)$ & -0.06 & $(0.09)$ \\
\hline & Tunesien & -0.02 & $(0.03)$ & -0.06 & $(0.02)$ & -0.01 & $(0.02)$ & 0.09 & $(0.09)$ & 0.02 & $(0.09)$ & 0.15 & $(0.10)$ & -0.23 & $(0.08)$ \\
\hline & Ver. Arab. Emirate & 0.00 & $(0.03)$ & -0.07 & $(0.03)$ & 0.04 & $(0.05)$ & 0.08 & $(0.04)$ & -0.10 & $(0.08)$ & -0.01 & $(0.05)$ & -0.20 & $(0.06)$ \\
\hline & Uruguay & 0.05 & $(0.03)$ & 0.06 & $(0.03)$ & -0.02 & $(0.06)$ & -0.04 & $(0.07)$ & -0.05 & $(0.04)$ & -0.05 & $(0.06)$ & -0.09 & $(0.07)$ \\
\hline & Vietnam & 0.03 & $(0.03)$ & 0.02 & $(0.03)$ & -0.14 & $(0.09)$ & -0.05 & $(0.10)$ & 0.07 & $(0.08)$ & -0.06 & $(0.09)$ & 0.15 & $(0.07)$ \\
\hline
\end{tabular}

Anmerkung: Statistisch signifikante Werte sind durch Fettdruck gekennzeichnet (vgl. Anhang A3).

* Vgl. Anmerkungen am Anfang dieses Anhangs.

StatLink ints http://dx.doi.org/10.1787/888932964946 
[Teil 1/2]

Ungleichheit beim Zugang zu Bildungsressourcen: Schüler/Lehrer-Quote

Tabelle II.4.8 Die Ergebnisse basieren auf Schülerangaben

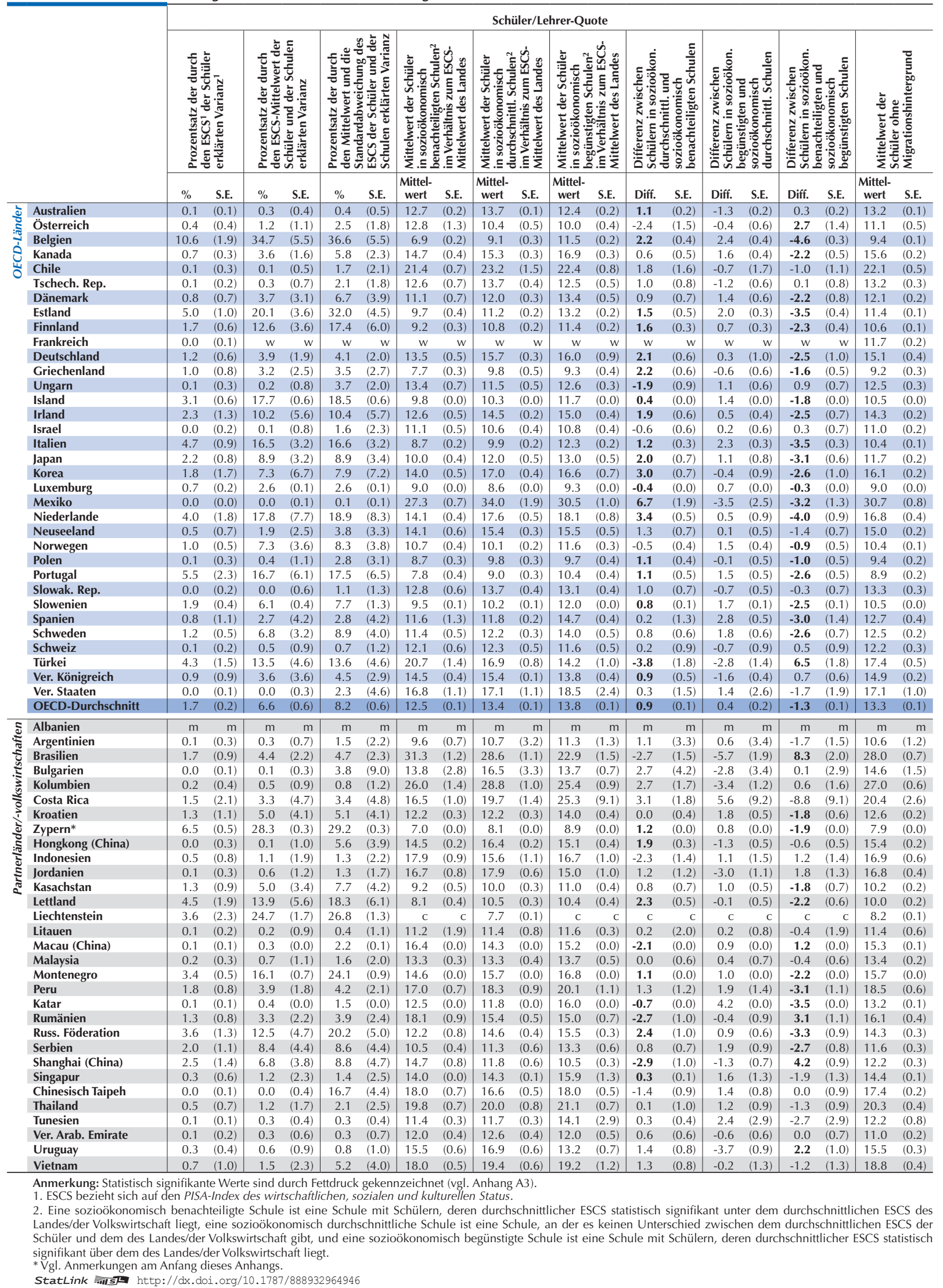


[Teil 2/2]

Ungleichheit beim Zugang zu Bildungsressourcen: Schüler/Lehrer-Quote Tabelle II.4.8 Die Ergebnisse basieren auf Schülerangaben

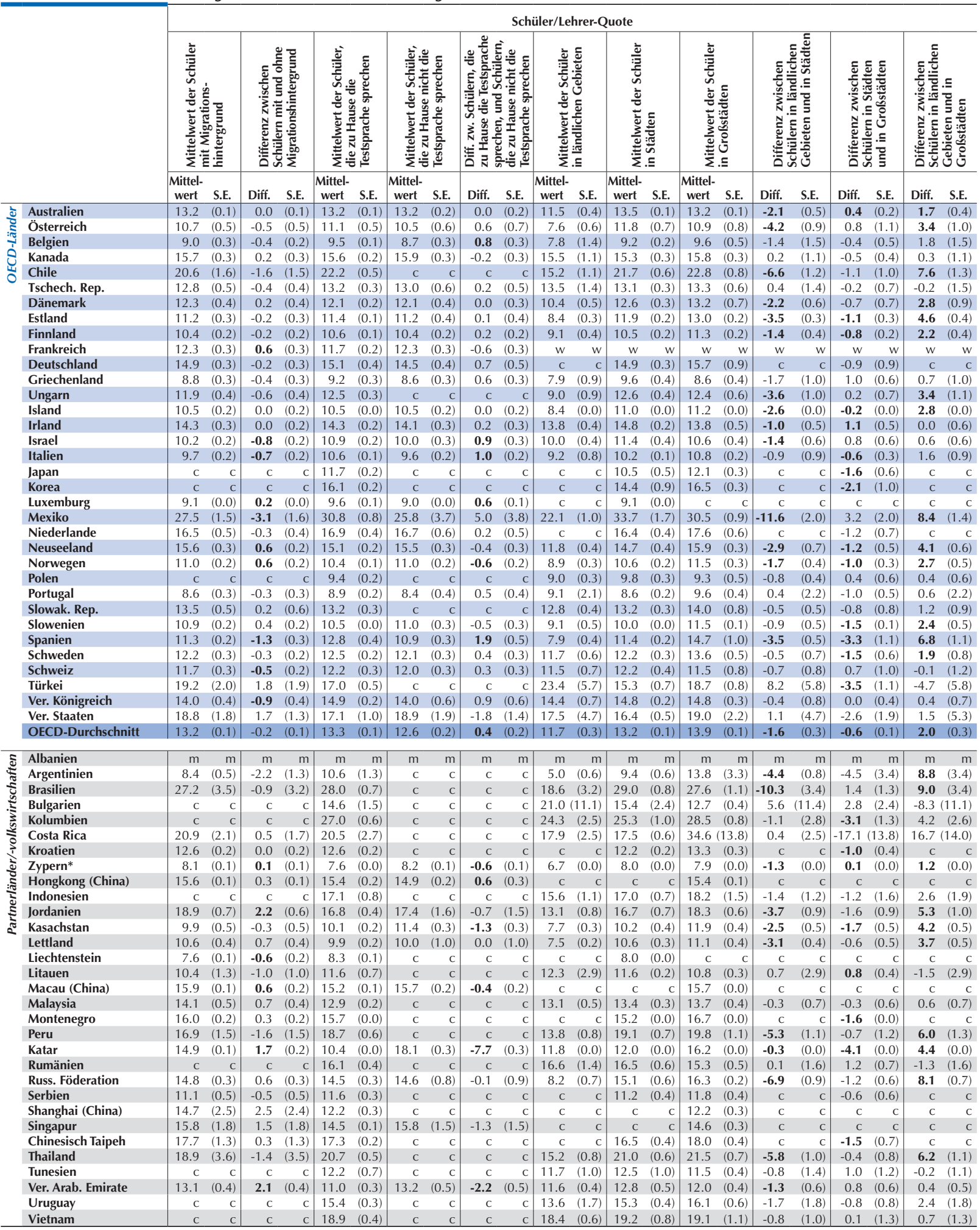

\section{Anmerkung: Statistisch signifikante Werte sind durch Fettdruck gekennzeichnet (vgl. Anhang A3).}

1. ESCS bezieht sich auf den PISA-Index des wirtschaftlichen, sozialen und kulturellen Status.

2. Eine sozioökonomisch benachteiligte Schule ist eine Schule mit Schülern, deren durchschnittlicher ESCS statistisch signifikant unter dem durchschnittlichen ESCS des Landes/ der Volkswirtschaft liegt, eine sozioökonomisch durchschnittliche Schule ist eine Schule, an der es keinen Unterschied zwischen dem durchschnittlichen ESCS der Schüler und dem des Landes/der Volkswirtschaft gibt, und eine sozioökonomisch begünstigte Schule ist eine Schule mit Schülern, deren durchschnittlicher ESCS statistisch signifikant über dem des Landes/der Volkswirtschaft liegt.

* Vgl. Anmerkungen am Anfang dieses Anhangs.

StatLink त्राज़ http://dx.doi.org/10.1787/888932964946 
[Teil 1/2]

Ungleichheit beim Zugang zu Bildungsressourcen: Prozentsatz der Lehrkräfte mit Hochschulabschluss Tabelle II.4.9 Die Ergebnisse basieren auf Schülerangaben

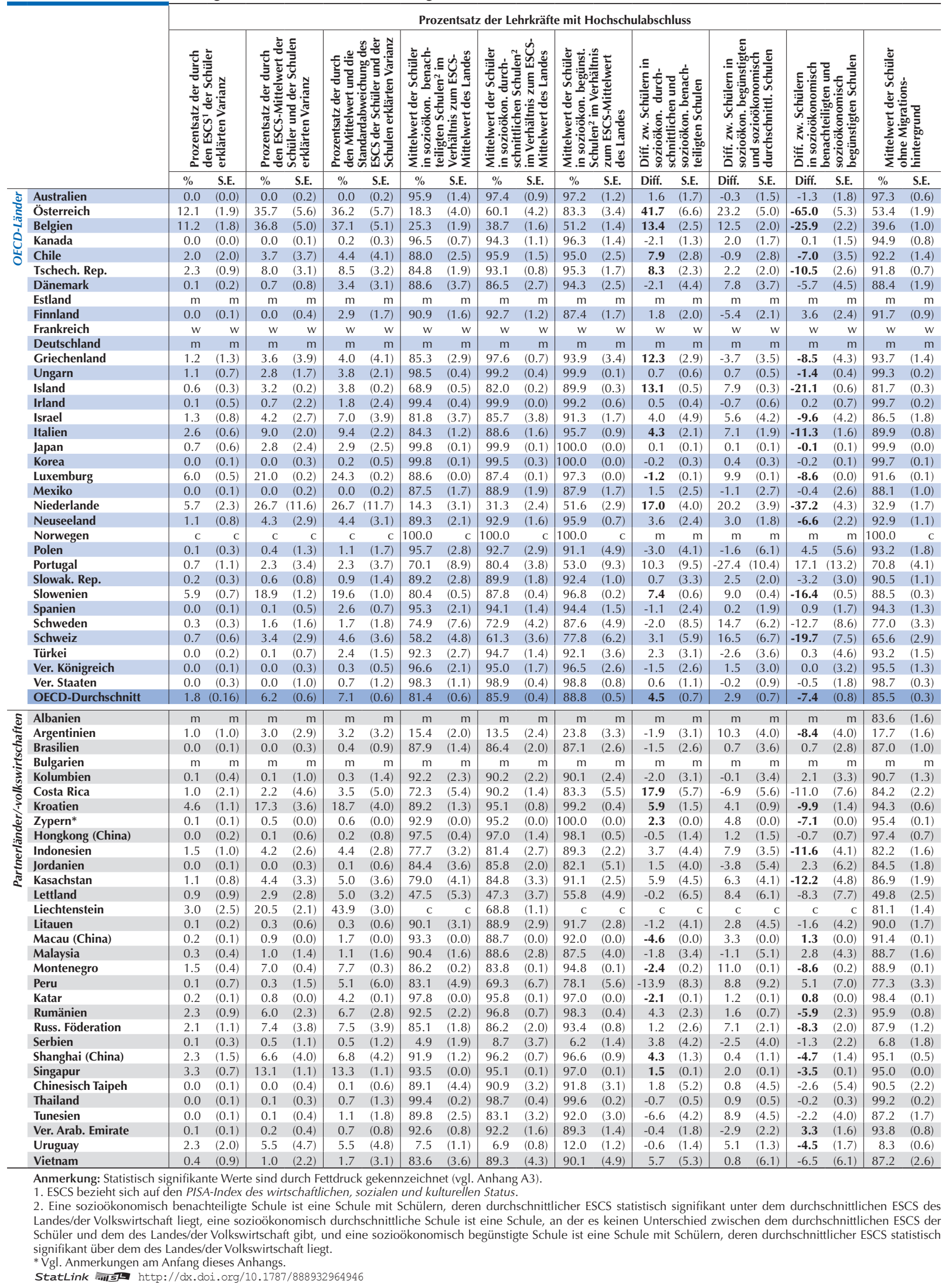


[Teil 2/2]

Ungleichheit beim Zugang zu Bildungsressourcen: Prozentsatz der Lehrkräfte mit Hochschulabschluss Tabelle II.4.9 Die Ergebnisse basieren auf Schülerangaben

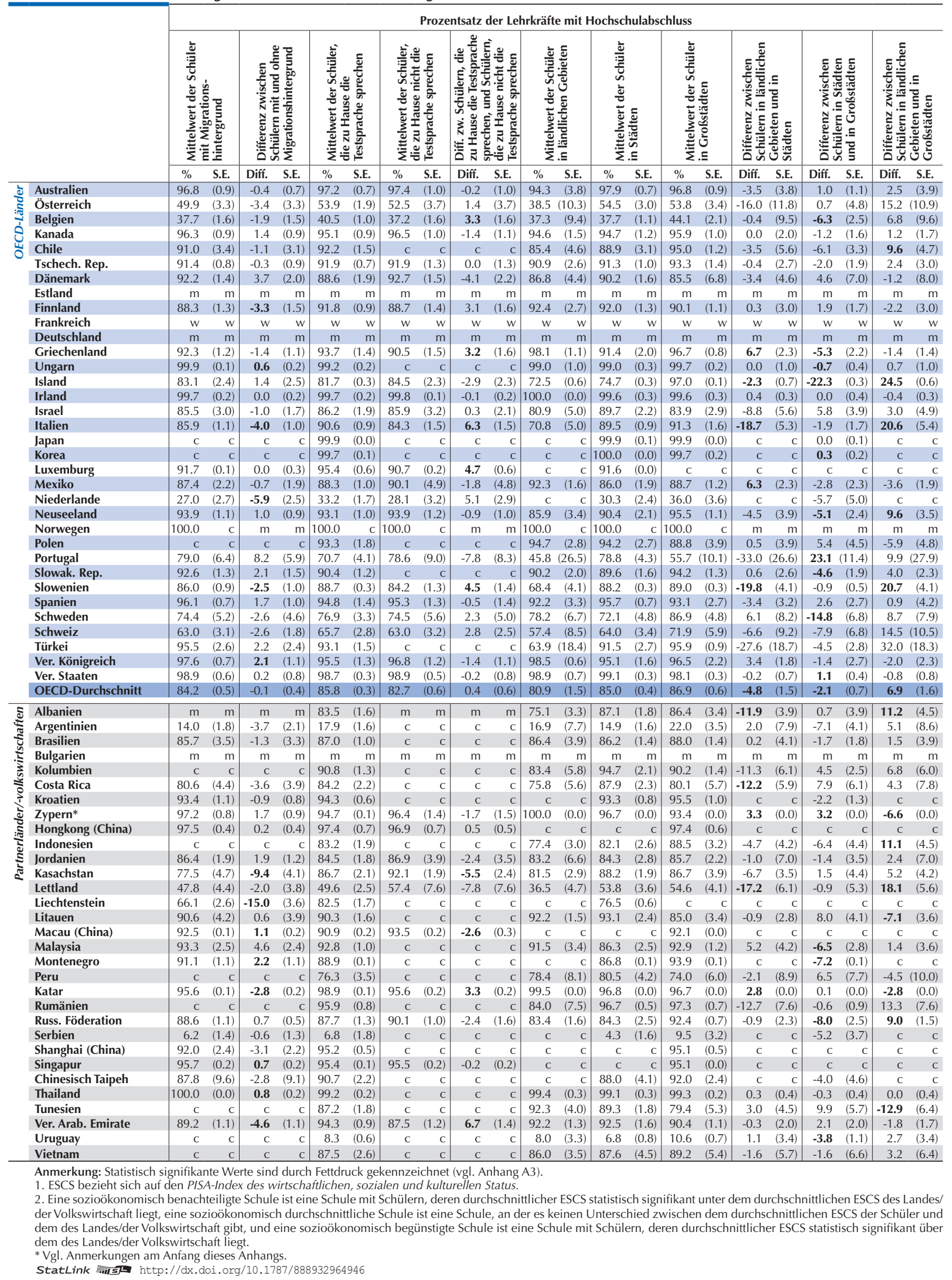


[Teil 1/2]

Ungleichheit beim Zugang zu Bildungsressourcen: Schuldisziplin Tabelle II.4.10 Die Ergebnisse basieren auf Schülerangaben

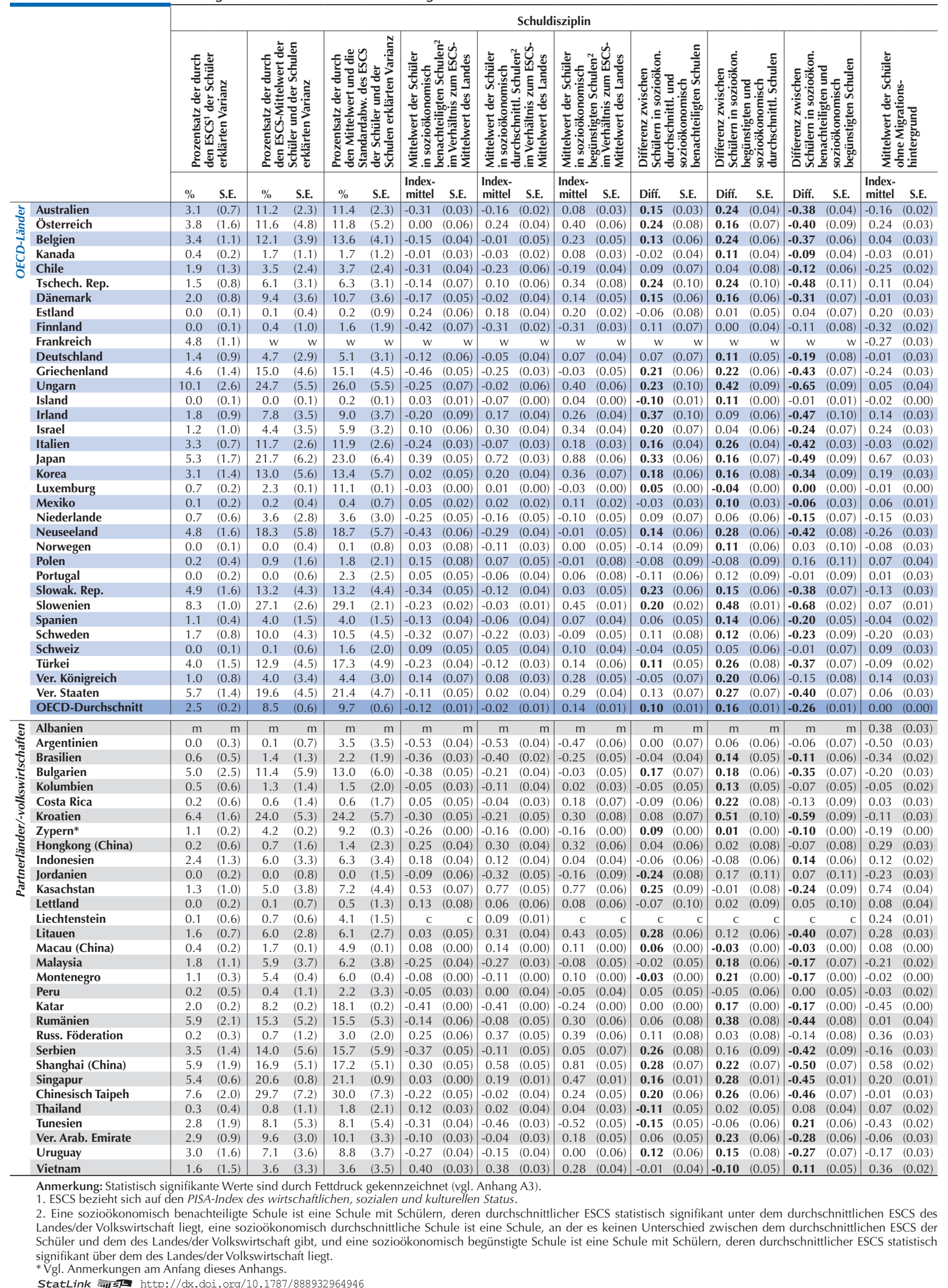

StatLink 需宁L http://dx.doi.org/10.1787/888932964946 
[Teil 2/2]

Ungleichheit beim Zugang zu Bildungsressourcen: Schuldisziplin Tabelle II.4.10 Die Ergebnisse basieren auf Schülerangaben

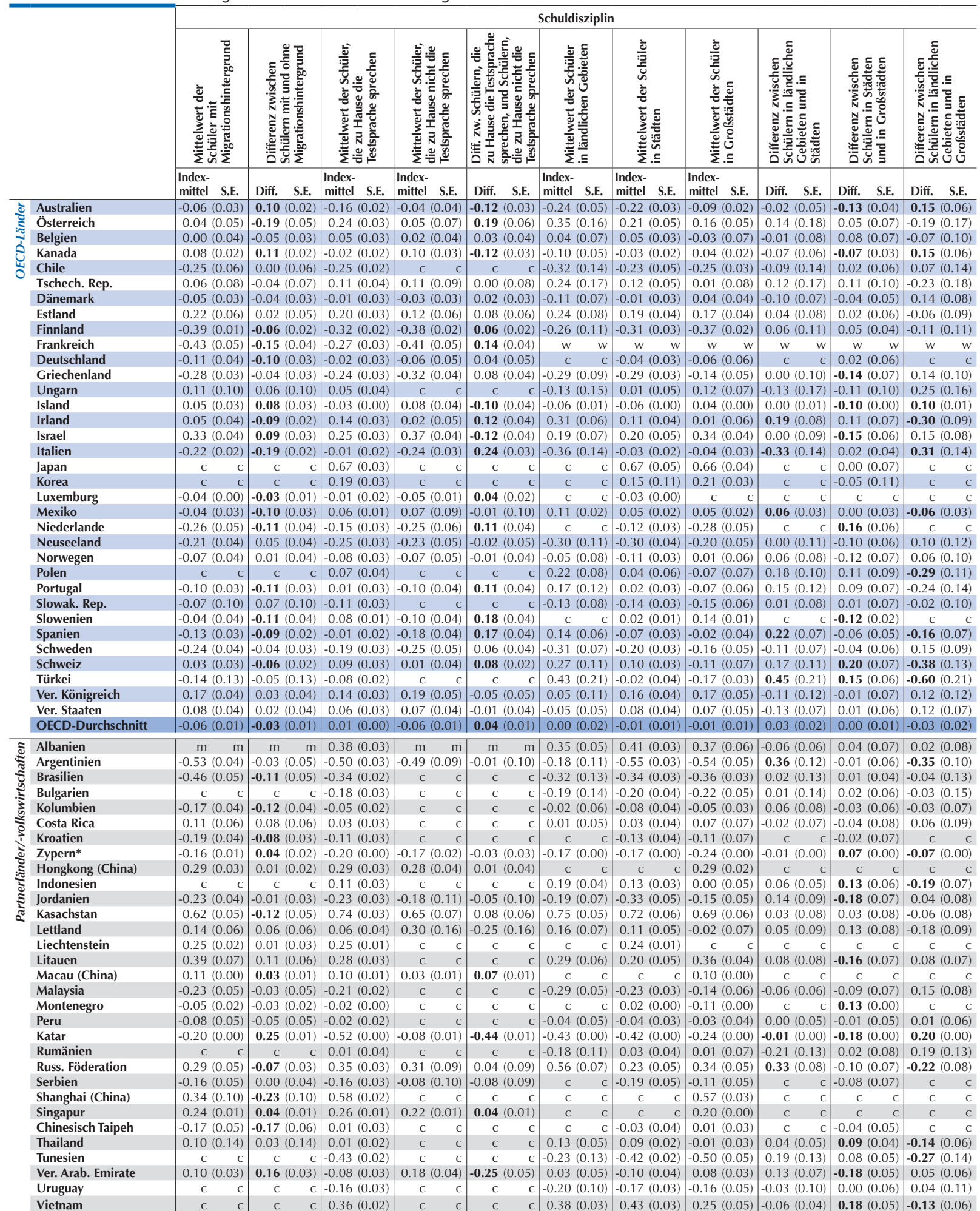

Anmerkung: Statistisch signifikante Werte sind durch Fettdruck gekennzeichnet (vgl. Anhang A3).

1. ESCS bezieht sich auf den PISA-Index des wirtschaftlichen, sozialen und kulturellen Status.

2. Eine sozioökonomisch benachteiligte Schule ist eine Schule mit Schülern, deren durchschnittlicher ESCS statistisch signifikant unter dem durchschnittlichen ESCS des Landes/ der Volkswirtschaft liegt, eine sozioökonomisch durchschnittliche Schule ist eine Schule, an der es keinen Unterschied zwischen dem durchschnittlichen ESCS der Schüler und dem des Landes/der Volkswirtschaft gibt, und eine sozioökonomisch begünstigte Schule ist eine Schule mit Schülern, deren durchschnittlicher ESCS statistisch signifikant über dem des Landes/der Volkswirtschaft liegt.

* Vgl. Anmerkungen am Anfang dieses Anhangs.

StatLink तinाs http://dx.doi.org/10.1787/888932964946 
[Teil 1/3]

Effekt des sozioökonomischen Status nach Berücksichtigung der Schülermerkmale und der Bildungsressourcen der Schulen

Tabelle II.4.11 Die Ergebnisse basieren auf Schülerangaben

\begin{tabular}{|c|c|c|c|c|c|c|c|c|c|c|c|c|c|c|c|c|c|}
\hline & \multicolumn{4}{|c|}{$\begin{array}{l}\text { Beobachteter Effekt des ESCS }{ }^{1} \\
\text { auf die Mathematikleistungen }\end{array}$} & \multicolumn{4}{|c|}{$\begin{array}{c}\text {... nach Berücksichtigung des } \\
\text { Geschlechts, des Migrationsstatus, } \\
\text { der zu Hause gesprochenen } \\
\text { Sprache sowie des Schulstandorts }\end{array}$} & \multicolumn{4}{|c|}{$\begin{array}{c}\ldots \text { und des Schulklimas: } \\
\text { Elterlicher Leistungsdruck }\end{array}$} & \multicolumn{4}{|c|}{... und der Schuldisziplin ${ }^{3}$} \\
\hline & & \multicolumn{2}{|c|}{$\begin{array}{l}\text { Sozioökonom. } \\
\text { Status der } \\
\text { Schüler }\end{array}$} & $\begin{array}{r}\text { Sozio } \\
\text { Pro } \\
\text { Sc }\end{array}$ & $\begin{array}{l}\text { konom. } \\
\text { il der } \\
\text { ulen }\end{array}$ & $\begin{array}{r}\text { Sozioo } \\
\text { Stat } \\
\text { Scl }\end{array}$ & $\begin{array}{l}\text { onom. } \\
\text { der } \\
\text { ler }\end{array}$ & $\begin{array}{r}\text { Sozioō } \\
\text { Prof } \\
\text { Sch }\end{array}$ & $\begin{array}{l}\text { konom. } \\
\text { il der } \\
\text { ulen }\end{array}$ & $\begin{array}{r}\text { Sozioö } \\
\text { Statu } \\
\text { Sch }\end{array}$ & $\begin{array}{l}\text { onom. } \\
\text { der } \\
\text { ler }\end{array}$ & $\begin{array}{r}\text { Sozioö } \\
\text { Prof } \\
\text { Sch }\end{array}$ & $\begin{array}{l}\text { konom. } \\
\text { il der } \\
\text { ulen }\end{array}$ & $\begin{array}{r}\text { Sozioc } \\
\text { Stat } \\
\text { Scl }\end{array}$ & $\begin{array}{l}\text { onom. } \\
\text { der } \\
\text { ler }\end{array}$ & $\begin{array}{r}\text { Sozioö } \\
\text { Proo } \\
\text { Sch }\end{array}$ & $\begin{array}{l}\text { konom. } \\
\text { Il der } \\
\text { ulen }\end{array}$ \\
\hline & & Koeff. & S.E. & Koeff. & S.E. & Koeff. & S.E. & Koeff. & S.E. & Koeff. & S.E. & Koeff. & S.E. & Koeff. & S.E. & Koeff. & S.E. \\
\hline ¿ & Australien & 24 & $(1.2)$ & 64 & (3.8) & 24 & $(1.1)$ & 58 & (3.8) & 24 & $(1.1)$ & 53 & (3.8) & 24 & $(1.1)$ & 43 & (4.3) \\
\hline$\sigma$ & Österreich & 15 & (1.6) & 86 & (5.5) & 10 & (2.0) & 87 & $(5.0)$ & 10 & $(2.0)$ & 86 & $(5.7)$ & 11 & (1.9) & 72 & (5.3) \\
\hline & Belgien & 18 & (1.4) & 101 & (5.4) & 16 & (1.5) & 99 & (5.9) & 16 & (1.5) & 97 & $(6.2)$ & 16 & (1.5) & 76 & $(5.8)$ \\
\hline i & Kanada & 23 & $(1.1)$ & 41 & $(4.8)$ & 23 & (1.1) & 38 & $(4.7)$ & 23 & (1.1) & 32 & (5.2) & 23 & (1.1) & 27 & $(4.5)$ \\
\hline u & Chile & 9 & $(1.2)$ & 46 & (2.4) & 9 & $(1.2)$ & 49 & (2.6) & 9 & $(1.2)$ & 44 & (2.8) & 9 & (1.3) & 42 & (3.3) \\
\hline ठ & Tschech. Rep. & 14 & $(2.0)$ & 127 & (6.2) & 14 & $(2.0)$ & 133 & (6.4) & 14 & $(2.0)$ & 129 & $(6.7)$ & 14 & $(2.0)$ & 112 & (6.7) \\
\hline & Dänemark & 31 & (1.6) & 39 & $(4.3)$ & 29 & (1.6) & 35 & (4.9) & 29 & $(1.7)$ & 36 & (4.9) & 30 & $(1.7)$ & 25 & (5.1) \\
\hline & Estland & 20 & (1.6) & 39 & $(5.2)$ & 20 & (1.6) & 45 & (5.8) & 20 & (1.6) & 45 & (5.8) & 20 & (1.8) & 45 & $(6.0)$ \\
\hline & Finnland & 30 & (1.6) & 28 & $(6.7)$ & 27 & $(1.7)$ & 38 & (6.7) & 27 & $(1.7)$ & 38 & $(6.7)$ & 28 & (1.7) & 24 & (5.9) \\
\hline & Frankreich & w & w & w & w & w & w & w & w & w & w & w & w & w & w & w & w \\
\hline & Deutschland & 11 & (1.3) & 104 & (5.5) & 8 & (1.5) & 108 & (6.1) & 8 & (1.5) & 111 & (6.5) & 8 & (1.5) & 94 & $(6.2)$ \\
\hline & Griechenland & 18 & (1.4) & 54 & $(4.1)$ & 17 & (1.5) & 56 & (4.6) & 17 & (1.5) & 54 & (5.0) & 17 & (1.5) & 48 & (5.0) \\
\hline & Ungarn & 7 & (1.4) & 96 & (4.9) & 6 & $(1.3)$ & 101 & (5.1) & 6 & (1.3) & 94 & (5.7) & 6 & (1.3) & 86 & (6.0) \\
\hline & Island & 24 & (2.5) & 37 & (5.1) & 23 & (2.6) & 33 & (6.0) & 23 & (2.6) & 34 & (6.5) & 22 & (2.6) & 31 & (6.3) \\
\hline & Irland & 26 & (1.6) & 53 & (4.6) & 26 & (1.6) & 54 & $(4.2)$ & 25 & $(1.7)$ & 50 & (5.4) & 25 & $(1.7)$ & 53 & (4.6) \\
\hline & Israel & 23 & (1.9) & 98 & (7.5) & 25 & (1.8) & 92 & (7.1) & 25 & (1.9) & 91 & $(7.2)$ & 25 & (1.9) & 85 & (6.4) \\
\hline & Italien & 7 & $(0.7)$ & 83 & (3.8) & 5 & $(0.8)$ & 86 & (3.9) & 5 & $(0.9)$ & 82 & $(4.3)$ & 5 & $(0.8)$ & 67 & $(4.9)$ \\
\hline & Japan & 4 & (1.8) & 149 & (6.8) & 4 & $(1.7)$ & 147 & (7.6) & 4 & (1.7) & 129 & (9.5) & 4 & (1.7) & 117 & (10.0) \\
\hline & Korea & 15 & $(2.0)$ & 113 & (10.5) & 15 & $(2.0)$ & 111 & (10.4) & 15 & (2.0) & 102 & (11.1) & 14 & $(2.1)$ & 81 & (8.3) \\
\hline & Luxemburg & 18 & (1.4) & 69 & (2.0) & 14 & (1.6) & 66 & (2.2) & 14 & (1.6) & 67 & $(2.3)$ & 14 & (1.6) & 63 & (2.4) \\
\hline & Mexiko & 5 & $(0.5)$ & 29 & (1.3) & 5 & $(0.5)$ & 28 & (1.6) & 5 & $(0.5)$ & 28 & (1.6) & 5 & $(0.5)$ & 26 & (1.6) \\
\hline & Niederlande & 8 & (1.7) & 146 & (10.1) & 6 & (1.8) & 148 & (11.0) & 6 & (1.8) & 141 & (14.8) & 7 & (1.9) & 124 & (14.1) \\
\hline & Neuseeland & 35 & (2.0) & 66 & $(5.2)$ & 33 & (2.3) & 65 & (5.1) & 33 & (2.3) & 58 & $(5.7)$ & 33 & (2.3) & 53 & (6.3) \\
\hline & Norwegen & 26 & (2.3) & 46 & (9.9) & 24 & (2.2) & 40 & (10.9) & 24 & $(2.2)$ & 41 & (11.8) & 24 & $(2.2)$ & 38 & (9.2) \\
\hline & Polen & 31 & (1.9) & 38 & (7.1) & 31 & (1.9) & 41 & (10.0) & 31 & (1.9) & 41 & (10.1) & 31 & (1.9) & 37 & (8.0) \\
\hline & Portugal & 23 & (1.4) & 33 & $(4.1)$ & 23 & (1.4) & 39 & (3.9) & 23 & (1.4) & 41 & (4.3) & 23 & (1.4) & 39 & $(4.0)$ \\
\hline & Slowak. Rep. & 23 & (1.9) & 81 & (6.4) & 21 & (2.0) & 81 & (6.5) & 21 & $(2.0)$ & 80 & (6.8) & 21 & $(2.0)$ & 75 & (7.8) \\
\hline & Slowenien & 4 & (1.6) & 121 & (3.6) & 1 & $(1.5)$ & 125 & (3.7) & 1 & (1.5) & 124 & $(4.0)$ & 1 & (1.6) & 108 & $(4.6)$ \\
\hline & Spanien & 26 & (1.0) & 27 & (3.0) & 25 & (1.1) & 25 & $(3.2)$ & 25 & (1.1) & 24 & (3.5) & 25 & (1.1) & 20 & (3.4) \\
\hline & Schweden & 28 & (2.0) & 43 & (6.9) & 24 & (2.0) & 32 & (7.1) & 24 & $(2.0)$ & 32 & (7.6) & 24 & $(2.0)$ & 27 & (7.2) \\
\hline & Schweiz & 24 & (1.7) & 66 & (6.5) & 17 & (1.8) & 68 & (6.5) & 18 & (1.9) & 65 & (6.6) & 17 & (1.9) & 65 & (6.3) \\
\hline & Türkei & 6 & $(1.0)$ & 83 & (6.8) & 6 & (1.1) & 90 & (6.1) & 6 & (1.1) & 89 & (6.7) & 6 & (1.1) & 72 & (5.3) \\
\hline & Ver. Königreich & 23 & (1.6) & 73 & (6.8) & 24 & $(1.7)$ & 75 & (7.1) & 24 & (1.7) & 73 & $(8.1)$ & 24 & (1.7) & 66 & $(9.2)$ \\
\hline & Ver. Staaten & 24 & (1.8) & 41 & $(5.0)$ & 25 & (1.9) & 42 & $(5.2)$ & 26 & $(2.0)$ & 39 & (6.5) & 25 & $(2.0)$ & 34 & $(6.2)$ \\
\hline & OECD-Durchschnitt & 19 & $(0.3)$ & 72 & (1.0) & 18 & $(0.3)$ & 72 & (1.1) & 18 & $(0.3)$ & 70 & $(1.2)$ & 18 & $(0.3)$ & 61 & (1.1) \\
\hline 5 & Albanien & $\mathrm{m}$ & $\mathrm{m}$ & $\mathrm{m}$ & $\mathrm{m}$ & $\mathrm{m}$ & $\mathrm{m}$ & $\mathrm{m}$ & $\mathrm{m}$ & $\mathrm{m}$ & $\mathrm{m}$ & $\mathrm{m}$ & $\mathrm{m}$ & $\mathrm{m}$ & $\mathrm{m}$ & $\mathrm{m}$ & $\mathrm{m}$ \\
\hline$=$ & Argentinien & 9 & (1.1) & 49 & (5.7) & 9 & (1.2) & 49 & (5.3) & 9 & $(1.2)$ & 47 & (5.1) & 9 & $(1.2)$ & 41 & (5.4) \\
\hline อี & Brasilien & 8 & $(0.7)$ & 46 & (3.2) & 8 & $(0.7)$ & 50 & (3.3) & 8 & $(0.7)$ & 51 & (3.4) & 8 & $(0.7)$ & 47 & (3.0) \\
\hline$\underline{2}$ & Bulgarien & 11 & (1.5) & 70 & (6.0) & 12 & (1.5) & 71 & (6.9) & 12 & (1.5) & 67 & (8.4) & 11 & (1.5) & 57 & (7.2) \\
\hline$\$$ & Kolumbien & 11 & $(1.2)$ & 35 & (3.4) & 10 & $(1.2)$ & 40 & (3.7) & 10 & (1.2) & 40 & (3.7) & 10 & (1.2) & 37 & (4.1) \\
\hline$\stackrel{\vec{n}}{\approx}$ & Costa Rica & 10 & $(0.9)$ & 34 & (3.0) & 9 & $(0.9)$ & 36 & (3.7) & 9 & $(0.9)$ & 36 & (3.8) & 9 & $(0.9)$ & 31 & (3.6) \\
\hline$\pi$ & Kroatien & 12 & (1.5) & 89 & (8.0) & 10 & (1.5) & 97 & $(8.2)$ & 10 & (1.5) & 95 & (8.9) & 10 & (1.5) & 67 & $(8.0)$ \\
\hline$\stackrel{T}{=}$ & Zypern* & 20 & (1.8) & 72 & (3.1) & 20 & (1.7) & 80 & $(4.0)$ & 20 & (1.8) & 78 & $(4.2)$ & 20 & (1.8) & 73 & (4.4) \\
\hline 气 & Hongkong (China) & 5 & (1.4) & 65 & $(7.0)$ & 5 & (1.4) & 66 & (6.6) & 5 & (1.4) & 64 & (6.1) & 5 & (1.4) & 59 & (7.1) \\
\hline : & Indonesien & 5 & (1.0) & 37 & (6.1) & 6 & (1.0) & 38 & $(8.3)$ & 6 & (1.0) & 38 & $(8.3)$ & 6 & (1.1) & 39 & $(8.0)$ \\
\hline & Jordanien & 11 & (1.3) & 46 & (9.0) & 13 & $(1.2)$ & 40 & (9.6) & 13 & (1.3) & 40 & (9.9) & 13 & (1.3) & 39 & (7.4) \\
\hline$\tau$ & Kasachstan & 15 & (1.7) & 44 & (8.8) & 15 & $(1.7)$ & 50 & (9.3) & 15 & (1.7) & 49 & (9.5) & 15 & (1.7) & 41 & $(9.8)$ \\
\hline & Lettland & 21 & (1.8) & 42 & $(4.5)$ & 22 & (1.8) & 46 & (6.0) & 22 & (1.8) & 42 & $(6.1)$ & 22 & (1.8) & 43 & $(5.7)$ \\
\hline & Liechtenstein & 8 & (6.3) & 129 & (12.6) & 4 & $(7.2)$ & 120 & (12.9) & 4 & $(7.0)$ & 94 & (15.5) & 2 & $(6.8)$ & 118 & (13.2) \\
\hline & Litauen & 19 & (1.5) & 65 & $(4.6)$ & 19 & (1.5) & 74 & (6.6) & 18 & (1.5) & 75 & (6.5) & 18 & (1.5) & 67 & $(6.0)$ \\
\hline & Macau (China) & 7 & (1.8) & 38 & (3.1) & 9 & (1.9) & 48 & (3.5) & 9 & (1.9) & 49 & (3.5) & 9 & (1.7) & 34 & (3.5) \\
\hline & Malaysia & 15 & (1.4) & 49 & $(5.2)$ & 15 & (1.4) & 41 & $(5.8)$ & 15 & (1.4) & 38 & (5.6) & 15 & (1.5) & 30 & (5.6) \\
\hline & Montenegro & 12 & (1.3) & 100 & (3.5) & 12 & (1.3) & 101 & (3.6) & 12 & (1.3) & 102 & (3.6) & 12 & (1.3) & 99 & (3.7) \\
\hline & Peru & 10 & $(1.3)$ & 48 & (2.5) & 10 & $(1.2)$ & 46 & $(4.0)$ & 10 & $(1.2)$ & 45 & $(4.1)$ & 9 & $(1.2)$ & 45 & $(4.0)$ \\
\hline & Katar & 8 & (1.4) & 75 & (2.3) & 9 & (1.3) & 50 & $(2.2)$ & 10 & (1.3) & 52 & $(2.3)$ & 9 & (1.3) & 42 & $(2.2)$ \\
\hline & Rumänien & 16 & (1.8) & 57 & (6.3) & 15 & $(1.8)$ & 63 & $(7.2)$ & 16 & (1.8) & 64 & (7.3) & 15 & (1.8) & 49 & $(8.2)$ \\
\hline & Russ. Föderation & 26 & $(2.2)$ & 42 & (7.3) & 26 & $(2.1)$ & 41 & (7.6) & 26 & $(2.2)$ & 43 & $(7.7)$ & 26 & $(2.2)$ & 28 & (7.9) \\
\hline & Serbien & 9 & $(1.3)$ & 101 & (6.3) & 9 & $(1.2)$ & 103 & (7.7) & 8 & $(1.2)$ & 95 & (7.4) & 9 & $(1.2)$ & 86 & (7.9) \\
\hline & Shanghai (China) & 10 & (1.6) & 88 & (5.3) & 9 & (1.6) & 87 & (5.3) & 9 & (1.6) & 88 & (5.5) & 9 & (1.6) & 67 & (5.9) \\
\hline & Singapur & 21 & (1.7) & 85 & (5.8) & 20 & (2.0) & 91 & (5.7) & 20 & $(2.0)$ & 89 & (6.6) & 21 & $(2.0)$ & 60 & (6.5) \\
\hline & Chinesisch Taipeh & 27 & (1.7) & 124 & (7.1) & 26 & $(1.8)$ & 118 & $(8.2)$ & 26 & (1.8) & 116 & (8.4) & 25 & $(2.0)$ & 91 & (10.4) \\
\hline & Thailand & 8 & $(1.2)$ & 33 & $(4.3)$ & 10 & $(1.2)$ & 44 & $(5.1)$ & 10 & $(1.2)$ & 42 & (5.4) & 10 & $(1.2)$ & 39 & $(5.2)$ \\
\hline & Tunesien & 6 & $(0.9)$ & 45 & (6.1) & 6 & $(0.9)$ & 47 & $(6.0)$ & 5 & $(0.9)$ & 46 & (5.7) & 6 & $(0.9)$ & 47 & (5.5) \\
\hline & Ver. Arab. Emirate & 12 & (1.3) & 71 & (5.8) & 14 & $(1.2)$ & 56 & (5.1) & 14 & $(1.2)$ & 55 & (5.1) & 14 & $(1.2)$ & 50 & $(4.8)$ \\
\hline & Uruguay & 15 & $(1.2)$ & 52 & (3.0) & 15 & $(1.3)$ & 51 & (3.2) & 15 & $(1.3)$ & 51 & (3.3) & 15 & (1.3) & 43 & $(4.2)$ \\
\hline & Vietnam & 8 & (1.3) & 50 & (5.2) & 7 & $(1.3)$ & 65 & (7.9) & 7 & (1.3) & 60 & (7.6) & 7 & (1.3) & 66 & $(6.2)$ \\
\hline
\end{tabular}

Anmerkung: Statistisch signifikante Werte sind durch Fettdruck gekennzeichnet (vgl. Anhang A3).

Alle hier gezeigten Modelle berücksichtigen den sozioökonomischen Status auf Schüler- und Schulebene. Dann werden individuelle Schülermerkmale (Geschlecht, Migrationsstatus und zu Hause gesprochene Sprache) sowie der Schulstandort einbezogen. Diese Variablen werden in allen Einzelmodellen verwendet. Anschließend kommen nacheinander Variablen auf Schul- und Schülerebene hinzu, die die Verfügbarkeit und die Qualität der Ressourcen messen. Am Ende werden alle Variablen in einem einzigen Modell kombiniert. In einigen Fällen sind auf Ebene der Schüler, der Schulen und der Länder keine Daten für manche dieser Variablen verfügbar, deshalb sind sie im kombinierten Modell nicht enthalten. Im Anschluss daran wird ein Basismodell präsentiert, in dem nur der sozioökonomische Status auf Schüler- und Schulebene berücksichtigt wird und in dem alle Schüler, Schulen und Länder, für die keine Daten vorliegen, ausgeklammert wurden, was einen direkteren Vergleich mit den Schätzwerten des kombinierten Modells ermöglicht.

1. ESCS bezieht sich auf den PISA-Index des wirtschaftlichen, sozialen und kulturellen Status.

2. Elterlicher Leistungsdruck basiert auf dem entsprechenden Index auf Schulebene (PARPRE).

3. Schuldisziplin umfasst die folgenden Variablen: xdisclim (aggregiert aus dem Schülerfragebogen auf die Schulebene) sowie studclim und teacclim, wobei es sich bei beiden Variablen um Indizes auf Schulebene handelt, die schüler- bzw. lehrerbezogene Faktoren im Zusammenhang mit der Schuldisziplin messen.

4. Lernmöglichkeiten umfassen die folgenden Variablen (jeweils den einfachen und den quadrierten Index): Index des Kontakts mit reiner Mathematik, Index des Kontakts mit Textaufgaben, Index des Kontakts mit angewandter Mathematik.

5. Lernmöglichkeiten auf Schulebene umfassen dieselben Indizes der Lernmöglichkeiten auf Schüler- und auf Schulebene (mit Ausnahme des quadrierten Index des Kontakts mit angewandter Mathematik).

6. Lehrerprofil umfasst die folgenden Variablen: Schüler/Lehrer-Quote, Zusammensetzung und Qualifikationen des Mathematik-Lehrkörpers, Anteil der Lehrkräfte mit Lehrbefähigung und Lehrermangel.

7. Bildungsressourcen und räumliche Bedingungen der Schulen umfassen: fehlende Infrastruktur und Mangel an Bildungsressourcen in den Schulen.

8. Anteil der Schulabbrecher in einer Schule umfasst: Verhaltenseffekt - Schulabbruch.

$*$ Vgl. Anmerkungen am Anfang dieses Anhangs.

StatLink त्तils http://dx.doi.org/10.1787/888932964946 
[Teil 2/3]

Effekt des sozioökonomischen Status nach Berücksichtigung der Schülermerkmale und der Bildungsressourcen der Schulenl

Tabelle II.4.11 Die Ergebnisse basieren auf Schülerangaben

\begin{tabular}{|c|c|c|c|c|c|c|c|c|c|c|c|c|c|c|c|c|}
\hline & $\ldots$ un & $\begin{array}{l}\text { der Ler } \\
\text { (Schüle }\end{array}$ & $\begin{array}{l}\text { Imöglich } \\
\text { ebene) }{ }^{4}\end{array}$ & keiten & $\ldots$ un & $\begin{array}{r}\text { der Ler } \\
\text { (Schul }\end{array}$ & $\begin{array}{l}\text { möglicl } \\
\text { bene })^{5}\end{array}$ & keiten & & id des I & hrerpro & fils ${ }^{6}$ & $\begin{array}{c}\ldots \text { un } \\
\text { und de }\end{array}$ & $\begin{array}{r}\text { der Bild } \\
\text { räumlic } \\
\text { der } S\end{array}$ & $\begin{array}{l}\text { ungsress } \\
\text { en Bedi } \\
\text { hule }\end{array}$ & $\begin{array}{l}\text { urcen } \\
\text { gungen }\end{array}$ \\
\hline & $\begin{array}{r}\text { Sozioö } \\
\text { Stat } \\
\text { Sch } \\
\end{array}$ & $\begin{array}{l}\text { conom. } \\
\text { s der } \\
\text { uler }\end{array}$ & $\begin{array}{r}\text { Sozioö } \\
\text { Prof } \\
\text { Sch } \\
\end{array}$ & $\begin{array}{l}\text { konom. } \\
\text { Il der } \\
\text { ulen }\end{array}$ & $\begin{array}{r}\text { Sozioö } \\
\text { Staty } \\
\text { Sch }\end{array}$ & $\begin{array}{l}\text { onom. } \\
\text { der } \\
\text { ler }\end{array}$ & $\begin{array}{r}\text { Sozio } \\
\text { Pro } \\
\text { Scl }\end{array}$ & $\begin{array}{l}\text { konom. } \\
\text { I der } \\
\text { ulen }\end{array}$ & $\begin{array}{r}\text { Sozioö } \\
\text { Stat } \\
\text { Sch }\end{array}$ & $\begin{array}{l}\text { onom. } \\
\text { der } \\
\text { ler }\end{array}$ & $\begin{array}{r}\text { Sozioo } \\
\text { Pro } \\
\text { Sch }\end{array}$ & $\begin{array}{l}\text { konom. } \\
\text { Il der } \\
\text { ulen }\end{array}$ & $\begin{array}{r}\text { Sozioö } \\
\text { Stat } \\
\text { Sch }\end{array}$ & $\begin{array}{l}\text { onom. } \\
\text { der } \\
\text { iler }\end{array}$ & $\begin{array}{r}\text { Sozioo } \\
\text { Pro } \\
\text { Sch } \\
\end{array}$ & $\begin{array}{l}\text { xonom. } \\
\text { I der } \\
\text { Ilen }\end{array}$ \\
\hline & Koeff. & S.E. & Koeff. & S.E. & Koeff. & S.E. & Koeff. & S.E. & Koeff. & S.E. & Koeff. & S.E. & Koeff. & S.E. & Koeff. & S.E. \\
\hline Australien & 8 & (1.5) & 38 & (3.8) & 9 & $(1.4)$ & 26 & (4.3) & 24 & $(1.2)$ & 56 & (4.5) & 24 & $(1.1)$ & 58 & (4.5) \\
\hline Österreich & 4 & (2.1) & 60 & (5.6) & 5 & $(2.1)$ & 37 & (8.2) & 9 & (2.4) & 79 & (7.8) & 9 & (2.1) & 88 & (6.1) \\
\hline Belgien & 6 & (1.5) & 72 & (5.0) & 7 & (1.5) & 66 & (7.6) & 17 & (1.8) & 75 & (8.6) & 16 & $(1.5)$ & 98 & (5.8) \\
\hline Kanada & 12 & (1.3) & 30 & (4.4) & 12 & $(1.3)$ & 22 & $(4.8)$ & 24 & (1.4) & 36 & (5.4) & 23 & $(1.1)$ & 38 & (4.7) \\
\hline Chile & 5 & (1.7) & 37 & (2.9) & 6 & (1.6) & 19 & $(4.2)$ & 9 & (1.4) & 48 & (2.9) & 9 & $(1.2)$ & 48 & (2.8) \\
\hline Tschech. Rep. & 7 & (2.5) & 97 & (7.6) & 7 & (2.4) & 83 & $(10.1)$ & 14 & (2.1) & 118 & $(7.3)$ & 14 & (2.0) & 134 & (6.5) \\
\hline Dänemark & 20 & (1.9) & 18 & (4.8) & 20 & (1.9) & 19 & (5.3) & $\mathrm{m}$ & $\mathrm{m}$ & $\mathrm{m}$ & $\mathrm{m}$ & 30 & $(1.7)$ & 34 & (5.3) \\
\hline Estland & 13 & (2.0) & 46 & (5.8) & 13 & $(2.0)$ & 41 & (6.1) & 20 & $(1.7)$ & 48 & (5.8) & 20 & (1.7) & 44 & (5.9) \\
\hline Finnland & 17 & $(1.7)$ & 27 & (6.2) & 17 & (1.7) & 30 & (5.9) & 27 & (1.6) & 30 & (5.9) & 28 & (1.6) & 39 & (6.9) \\
\hline Frankreich & w & w & w & w & w & w & w & w & w & w & w & w & w & w & w & w \\
\hline Deutschland & 2 & (2.1) & 74 & (5.6) & 4 & $(2.1)$ & 45 & (6.9) & 8 & $(1.9)$ & 103 & (8.1) & 8 & $(1.5)$ & 109 & (6.1) \\
\hline Griechenland & 14 & (1.8) & 49 & (5.2) & 15 & (1.8) & 34 & $(6.2)$ & 16 & (2.0) & 57 & (6.1) & 17 & $(1.5)$ & 57 & (4.7) \\
\hline Ungarn & 2 & (1.6) & 84 & (4.9) & 2 & (1.6) & 60 & $(6.2)$ & $\mathrm{m}$ & $\mathrm{m}$ & $\mathrm{m}$ & $\mathrm{m}$ & 6 & $(1.3)$ & 102 & (5.1) \\
\hline Island & 20 & (2.8) & 19 & (6.6) & 20 & $(2.8)$ & 16 & (7.5) & 24 & (3.0) & 39 & (7.0) & 22 & $(2.7)$ & 34 & (6.3) \\
\hline Irland & 16 & (1.7) & 42 & (4.4) & 16 & (1.7) & 40 & (5.3) & 25 & (1.9) & 58 & (5.3) & 25 & $(1.8)$ & 61 & (4.6) \\
\hline Israel & 18 & (2.3) & 82 & (6.8) & 18 & $(2.3)$ & 68 & (8.4) & 25 & (2.2) & 101 & (8.3) & 25 & $(2.0)$ & 96 & (6.8) \\
\hline Italien & 2 & (1.1) & 66 & (3.6) & 3 & $(1.0)$ & 40 & (4.4) & 5 & $(0.9)$ & 71 & (5.1) & 5 & $(0.8)$ & 85 & (4.0) \\
\hline Japan & 0 & (2.1) & 112 & (8.3) & 1 & $(2.1)$ & 59 & (11.5) & $\mathrm{m}$ & $\mathrm{m}$ & $\mathrm{m}$ & $\mathrm{m}$ & 4 & $(1.7)$ & 148 & (7.8) \\
\hline Korea & 5 & (2.0) & 71 & (8.1) & 7 & (2.0) & 12 & (9.6) & 15 & (2.1) & 120 & (11.9) & 14 & (2.1) & 109 & (10.1) \\
\hline Luxemburg & 8 & (1.9) & 56 & (3.0) & 9 & (1.8) & 41 & (5.3) & 14 & (1.6) & 66 & (2.6) & 14 & (1.6) & 68 & (2.2) \\
\hline Mexiko & 3 & $(0.7)$ & 22 & (1.7) & 3 & $(0.7)$ & 15 & (2.0) & 5 & (0.8) & 26 & (2.7) & 5 & $(0.5)$ & 27 & (1.7) \\
\hline Niederlande & -3 & (2.2) & 91 & (11.8) & 0 & $(2.2)$ & 29 & (15.2) & 7 & (2.5) & 113 & (19.9) & 6 & (1.8) & 148 & (10.9) \\
\hline Neuseeland & 17 & (2.4) & 44 & $(4.5)$ & 17 & (2.4) & 40 & $(5.7)$ & 32 & (2.4) & 68 & $(6.7)$ & 33 & (2.3) & 65 & (5.0) \\
\hline Norwegen & 25 & (2.8) & 32 & (11.5) & 25 & $(2.8)$ & 28 & (11.3) & 24 & (2.4) & 42 & (12.3) & 24 & (2.3) & 46 & (10.6) \\
\hline Polen & 24 & (1.8) & 35 & $(8.8)$ & 24 & (1.9) & 28 & $(8.9)$ & 32 & (2.0) & 40 & (11.7) & 31 & (1.9) & 40 & (9.6) \\
\hline Portugal & 17 & (1.5) & 35 & (4.2) & 17 & (1.5) & 26 & (4.5) & 24 & (1.6) & 37 & (5.6) & 23 & (1.4) & 39 & (4.3) \\
\hline Slowak. Rep. & 17 & (2.3) & 69 & (6.8) & 17 & $(2.2)$ & 50 & (8.9) & 23 & (2.5) & 83 & (7.7) & 21 & (2.0) & 81 & (6.5) \\
\hline Slowenien & 0 & (2.1) & 113 & (4.3) & 0 & $(2.0)$ & 90 & (5.4) & 1 & (1.6) & 126 & $(4.2)$ & 1 & $(1.6)$ & 126 & (4.1) \\
\hline Spanien & 13 & (1.2) & 20 & (2.9) & 13 & $(1.2)$ & 18 & (3.8) & 24 & $(1.2)$ & 22 & (4.1) & 25 & $(1.1)$ & 25 & (3.4) \\
\hline Schweden & 22 & (2.5) & 26 & (7.4) & 22 & (2.5) & 22 & (8.0) & 25 & (2.4) & 29 & (7.3) & 24 & (2.0) & 32 & (7.2) \\
\hline Schweiz & 12 & (1.9) & 46 & (5.4) & 12 & $(1.8)$ & 45 & (5.7) & 16 & (1.9) & 61 & (7.0) & 17 & $(1.9)$ & 68 & (6.5) \\
\hline Türkei & 3 & (1.5) & 82 & (6.1) & 4 & (1.4) & 57 & (6.3) & 6 & $(1.2)$ & 93 & (7.1) & 6 & $(1.1)$ & 90 & (6.9) \\
\hline Ver. Königreich & 9 & (1.8) & 51 & (5.4) & 10 & (1.8) & 45 & (6.5) & 25 & (1.9) & 70 & $(8.2)$ & 24 & $(1.7)$ & 75 & (6.9) \\
\hline Ver. Staaten & 14 & (2.1) & 32 & (4.7) & 14 & $(2.1)$ & 24 & (6.1) & 25 & (2.2) & 41 & (5.6) & 25 & $(2.0)$ & 40 & (5.4) \\
\hline OECD-Durchschnitt & 11 & $(0.3)$ & 55 & (1.0) & 11 & $(0.3)$ & 39 & $(1.3)$ & 18 & $(0.3)$ & 67 & (1.4) & 18 & $(0.3)$ & 73 & $(1.1)$ \\
\hline
\end{tabular}

\begin{tabular}{|c|c|c|c|c|c|c|c|c|c|c|c|c|c|c|c|c|}
\hline Albanien & $\mathrm{m}$ & $\mathrm{m}$ & $\mathrm{m}$ & $\mathrm{m}$ & $\mathrm{m}$ & $\mathrm{m}$ & $\mathrm{m}$ & $\mathrm{m}$ & $\mathrm{m}$ & $\mathrm{m}$ & $\mathrm{m}$ & $\mathrm{m}$ & $\mathrm{m}$ & $\mathrm{m}$ & $\mathrm{m}$ & $\mathrm{m}$ \\
\hline Argentinien & 6 & (1.4) & 39 & (5.4) & 6 & (1.4) & 34 & (6.6) & 9 & (1.4) & 56 & (5.4) & 9 & $(1.2)$ & 47 & (6.2) \\
\hline Brasilien & 4 & $(0.9)$ & 40 & (3.0) & 5 & $(0.9)$ & 21 & (3.6) & $\mathrm{m}$ & $\mathrm{m}$ & $\mathrm{m}$ & $\mathrm{m}$ & 8 & $(0.7)$ & 49 & (3.6) \\
\hline Bulgarien & 7 & (1.7) & 64 & $(7.2)$ & 7 & (1.8) & 46 & (10.1) & $\mathrm{m}$ & $\mathrm{m}$ & $\mathrm{m}$ & $\mathrm{m}$ & 12 & (1.5) & 70 & $(7.1)$ \\
\hline Kolumbien & 3 & (1.5) & 31 & (3.2) & 3 & (1.5) & 26 & (3.9) & 9 & (1.8) & 40 & (4.6) & 10 & (1.2) & 39 & (3.7) \\
\hline Costa Rica & 7 & (1.4) & 29 & (3.5) & 7 & (1.3) & 19 & $(4.0)$ & 8 & (1.3) & 32 & (5.4) & 9 & $(0.9)$ & 33 & (3.7) \\
\hline Kroatien & 7 & (1.8) & 81 & (7.9) & 7 & (1.8) & 51 & (7.3) & 12 & (1.8) & 112 & (11.5) & 11 & (1.5) & 98 & (8.1) \\
\hline Zypern* & 15 & $(2.0)$ & 59 & (4.6) & 15 & $(2.0)$ & 22 & (5.6) & 21 & (1.9) & 64 & $(5.0)$ & 21 & (1.8) & 78 & $(4.2)$ \\
\hline Hongkong (China) & 0 & (1.8) & 65 & (5.9) & 1 & (1.8) & 52 & (5.9) & 5 & (1.4) & 61 & (7.0) & 5 & (1.4) & 67 & (6.7) \\
\hline Indonesien & 5 & (1.6) & 32 & (7.3) & 6 & (1.5) & 13 & (6.8) & 5 & (1.1) & 21 & $(8.1)$ & 6 & (1.0) & 35 & (7.1) \\
\hline Jordanien & 9 & (1.4) & 39 & (9.2) & 9 & (1.4) & 33 & $(8.4)$ & 12 & (1.4) & 49 & (7.1) & 13 & $(1.2)$ & 40 & (9.9) \\
\hline Kasachstan & 11 & $(2.1)$ & 47 & (9.2) & 11 & $(2.1)$ & 35 & (9.4) & 14 & (1.9) & 52 & $(9.2)$ & 15 & (1.7) & 50 & (9.3) \\
\hline Lettland & 15 & (2.3) & 43 & (6.1) & 16 & $(2.3)$ & 40 & (6.6) & 20 & $(2.3)$ & 47 & (8.4) & 22 & (1.8) & 47 & (5.8) \\
\hline Liechtenstein & 0 & (7.9) & 64 & (25.6) & 5 & (7.0) & -8 & (26.7) & 4 & $(6.0)$ & 32 & (16.5) & 3 & (6.9) & 87 & (13.5) \\
\hline Litauen & 14 & (1.7) & 62 & (6.6) & 14 & (1.7) & 52 & $(7.3)$ & 17 & (1.8) & 76 & (7.9) & 19 & (1.5) & 74 & (6.7) \\
\hline Macau (China) & 2 & $(2.0)$ & 41 & (4.5) & 4 & (1.8) & 19 & (4.5) & 9 & (1.8) & 43 & (3.7) & 9 & (1.9) & 47 & (3.5) \\
\hline Malaysia & 9 & (1.7) & 33 & (5.6) & 10 & (1.7) & 16 & (6.0) & 15 & (1.7) & 37 & (6.6) & 15 & (1.4) & 41 & (5.7) \\
\hline Montenegro & 8 & (1.6) & 94 & (4.1) & 8 & (1.6) & 67 & (5.5) & 11 & (1.8) & 90 & (6.9) & 12 & (1.3) & 101 & (3.6) \\
\hline Peru & 6 & (1.4) & 35 & (3.7) & 6 & (1.4) & 23 & $(4.3)$ & 9 & (1.4) & 44 & (5.0) & 10 & $(1.2)$ & 46 & (4.0) \\
\hline Katar & 4 & (1.5) & 49 & (3.1) & 5 & (1.5) & 40 & $(3.2)$ & 8 & (1.4) & 47 & $(2.7)$ & 9 & (1.3) & 52 & (2.3) \\
\hline Rumänien & 12 & (1.9) & 53 & $(7.2)$ & 12 & (1.9) & 40 & (8.6) & 15 & (1.8) & 66 & (6.7) & 15 & (1.8) & 63 & $(7.2)$ \\
\hline Russ. Föderation & 23 & (2.5) & 35 & (8.0) & 23 & (2.5) & 31 & (9.3) & 25 & $(2.3)$ & 39 & (7.9) & 26 & $(2.1)$ & 41 & $(7.8)$ \\
\hline Serbien & 7 & (1.7) & 86 & $(7.8)$ & 7 & (1.6) & 68 & (8.6) & 7 & (2.0) & 103 & (16.6) & 9 & (1.3) & 102 & (7.7) \\
\hline Shanghai (China) & 7 & (1.8) & 73 & (5.1) & 8 & (1.8) & 53 & (6.3) & 9 & (1.6) & 79 & $(5.7)$ & 9 & (1.6) & 87 & (5.5) \\
\hline Singapur & 4 & $(2.0)$ & 65 & (4.5) & 5 & (2.0) & 27 & (5.5) & 20 & $(2.0)$ & 87 & (5.2) & 22 & (2.1) & 84 & (5.8) \\
\hline Chinesisch Taipeh & 14 & (1.9) & 98 & (6.7) & 15 & (1.9) & 59 & (9.8) & 26 & $(2.1)$ & 90 & (11.5) & 25 & (2.0) & 118 & (8.6) \\
\hline Thailand & 6 & (1.4) & 37 & (5.5) & 6 & (1.4) & 23 & (6.5) & 10 & (1.2) & 43 & $(5.8)$ & 10 & $(1.2)$ & 44 & (5.2) \\
\hline Tunesien & 5 & (1.3) & 47 & (5.7) & 5 & (1.3) & 38 & (5.3) & 6 & (1.0) & 43 & (6.7) & 6 & $(0.9)$ & 47 & (6.1) \\
\hline Ver. Arab. Emirate & 9 & (1.4) & 44 & (4.6) & 9 & (1.4) & 36 & $(4.8)$ & $\mathrm{m}$ & $\mathrm{m}$ & $\mathrm{m}$ & $\mathrm{m}$ & 14 & (1.2) & 54 & (5.1) \\
\hline Uruguay & 10 & (1.5) & 35 & (3.6) & 11 & (1.5) & 22 & (4.6) & 15 & (1.3) & 49 & $(4.0)$ & 15 & (1.3) & 47 & (3.6) \\
\hline Vietnam & 5 & (1.6) & 52 & (7.1) & 5 & (1.6) & 22 & (7.7) & 8 & (1.4) & 65 & (8.3) & 7 & (1.3) & 65 & $(7.9)$ \\
\hline
\end{tabular}

Notes: Statistisch signifikante Werte sind durch Fettdruck gekennzeichnet (vgl. Anhang A3).

Alle hier gezeigten Modelle berücksichtigen den sozioökonomischen Status auf Schüler- und Schulebene. Dann werden individuelle Schülermerkmale (Geschlecht, Migrationsstatus und zu Hause gesprochene Sprache) sowie der Schulstandort einbezogen. Diese Variablen werden in allen Einzelmodellen verwendet. Anschließend kommen nacheinander Variablen auf Schul- und Schülerebene hinzu, die die Verfügbarkeit und die Qualität der Ressourcen messen. Am Ende werden alle Variablen in einem einzigen Modell kombiniert. In einigen Fällen sind auf Ebene der Schüler, der Schulen und der Länder keine Daten für manche dieser Variablen verfügbar, deshalb sind sie im kombinierten Modell nicht enthalten. Im Anschluss daran wird ein Basismodell präsentiert, in dem nur der sozioökonomische Status auf Schüler- und Schulebene berücksichtigt wird und in dem alle Schüler, Schulen und Länder, für die keine Daten vorliegen, ausgeklammert wurden, was einen direkteren Vergleich mit den Schätzwerten des kombinierten Modells ermöglicht.

1. ESCS bezieht sich auf den PISA-Index des wirtschaftlichen, sozialen und kulturellen Status.

2. Elterlicher Leistungsdruck basiert auf dem entsprechenden Index auf Schulebene (PARPRES).

3. Schuldisziplin umfasst die folgenden Variablen: xdisclim (aggregiert aus dem Schülerfragebogen auf die Schulebene) sowie studclim und teacclim, wobei es sich bei beiden Variablen um Indizes auf Schulebene handelt, die schüler- bzw. lehrerbezogene Faktoren im Zusammenhang mit der Schuldisziplin messen.

4. Lernmöglichkeiten umfassen die folgenden Variablen (jeweils den einfachen und den quadrierten Index): Index des Kontakts mit reiner Mathematik, Index des Kontakts mit Textaufgaben, Index des Kontakts mit angewandter Mathematik.

5. Lernmöglichkeiten auf Schulebene umfassen dieselben Indizes der Lernmöglichkeiten auf Schüler- und auf Schulebene (mit Ausnahme des quadrierten Index des Kontakts mit angewandter Mathematik).

6. Lehrerprofil umfasst die folgenden Variablen: Schüler/Lehrer-Quote, Zusammensetzung und Qualifikationen des Mathematik-Lehrkörpers, Anteil der Lehrkräfte mit Lehrbefähigung und Lehrermangel.

7. Bildungsressourcen und räumliche Bedingungen der Schulen umfassen: fehlende Infrastruktur und Mangel an Bildungsressourcen in den Schulen.

8. Anteil der Schulabbrecher in einer Schule umfasst: Verhaltenseffekt - Schulabbruch.

$*$ Vgl. Anmerkungen am Anfang dieses Anhangs.

StatLink त्ञाI 
[Teil 3/3]

Effekt des sozioökonomischen Status nach Berücksichtigung der Schülermerkmale und der Bildungsressourcen der Schulen

Tabelle II.4.11 Die Ergebnisse basieren auf Schülerangaben

\begin{tabular}{|c|c|c|c|c|c|c|c|c|c|c|c|c|}
\hline & \multicolumn{4}{|c|}{... und des Anteils der Schulabbrecher ${ }^{8}$} & \multicolumn{4}{|c|}{ Kombiniertes Modell } & \multicolumn{4}{|c|}{$\begin{array}{c}\text { Basismodell unter Ausklammerung } \\
\text { der Beobachtungen mit fehlenden Werten } \\
\text { zu den einzelnen Variablen }\end{array}$} \\
\hline & \multicolumn{2}{|c|}{$\begin{array}{l}\text { Sozioökonomischer } \\
\text { Status der Schüler }\end{array}$} & \multicolumn{2}{|c|}{$\begin{array}{l}\text { Sozioökonomisches } \\
\text { Profil der Schulen }\end{array}$} & \multicolumn{2}{|c|}{$\begin{array}{l}\text { Sozioökonomischer } \\
\text { Status der Schüler }\end{array}$} & \multicolumn{2}{|c|}{$\begin{array}{l}\text { Sozioökonomisches } \\
\text { Profil der Schulen }\end{array}$} & \multicolumn{2}{|c|}{$\begin{array}{l}\text { Sozioökonomischer } \\
\text { Status der Schüler }\end{array}$} & \multicolumn{2}{|c|}{$\begin{array}{l}\text { Sozioökonomisches } \\
\text { Profil der Schulen }\end{array}$} \\
\hline & Koeff. & S.E. & Koeff. & S.E. & Koeff. & S.E. & Koeff. & S.E. & Koeff. & S.E. & Koeff. & S.E. \\
\hline ¿ Australien & 24 & $(1.2)$ & 56 & $(4.0)$ & 9 & (1.6) & 22 & (5.6) & 22 & (1.8) & 66 & $(5.2)$ \\
\hline Österreich & 11 & $(2.0)$ & 85 & $(5.5)$ & 4 & $(2.7)$ & 28 & $(14.9)$ & 12 & $(2.6)$ & 90 & (10.5) \\
\hline : Belgien & 16 & (1.7) & 93 & (7.4) & 7 & $(2.2)$ & 48 & (11.3) & 19 & (2.1) & 102 & $(7.4)$ \\
\hline Kanada & 24 & $(1.2)$ & 36 & (5.6) & 13 & (1.8) & 20 & $(6.7)$ & 22 & $(2.0)$ & 41 & $(6.2)$ \\
\hline Chile & 9 & $(1.3)$ & 48 & $(2.7)$ & 6 & $(1.8)$ & 18 & (5.4) & 10 & $(2.0)$ & 45 & (3.0) \\
\hline Ō Tschech. Rep. & 14 & $(2.1)$ & 134 & (7.4) & 7 & $(2.8)$ & 66 & $(10.3)$ & 13 & $(3.0)$ & 120 & $(7.4)$ \\
\hline Dänemark & 29 & $(1.7)$ & 33 & (4.9) & C & c & c & c & c & c & c & c \\
\hline Estland & 20 & $(1.7)$ & 45 & $(6.0)$ & 13 & $(2.2)$ & 43 & (7.6) & 18 & $(2.4)$ & 42 & (6.3) \\
\hline Finnland & 27 & $(1.7)$ & 36 & $(6.8)$ & 17 & (1.8) & 28 & (5.8) & 31 & $(2.0)$ & 16 & $(7.5)$ \\
\hline Frankreich & w & w & w & w & w & w & w & w & w & w & w & w \\
\hline Deutschland & 9 & $(1.5)$ & 103 & $(6.1)$ & 4 & $(2.5)$ & 44 & $(8.8)$ & 12 & $(2.6)$ & 101 & $(7.3)$ \\
\hline Griechenland & 17 & $(1.5)$ & 44 & $(4.7)$ & 16 & $(2.4)$ & 33 & (7.8) & 19 & $(2.6)$ & 53 & (5.8) \\
\hline Ungarn & 6 & $(1.3)$ & 100 & (5.1) & c & c & c & $\mathrm{c}$ & c & c & c & c \\
\hline Island & 23 & $(2.6)$ & 34 & (6.1) & 17 & (3.1) & 12 & $(9.7)$ & 23 & $(3.2)$ & 32 & (6.8) \\
\hline Irland & 26 & $(1.7)$ & 51 & $(4.2)$ & 14 & $(2.0)$ & 37 & $(8.0)$ & 24 & $(2.3)$ & 57 & (6.4) \\
\hline Israel & 25 & $(1.8)$ & 94 & (7.5) & 18 & (2.6) & 62 & (10.5) & 27 & $(2.8)$ & 99 & (9.3) \\
\hline Italien & 5 & $(0.9)$ & 78 & $(4.6)$ & 3 & $(1.2)$ & 33 & (5.5) & 7 & (1.3) & 76 & (5.0) \\
\hline Japan & 4 & $(1.7)$ & 132 & $(9.1)$ & c & c & c & c & c & C & C & c \\
\hline Korea & 14 & $(2.0)$ & 102 & (10.1) & 6 & $(2.1)$ & 14 & $(10.2)$ & 16 & $(2.4)$ & 114 & (10.3) \\
\hline Luxemburg & 15 & (1.8) & 64 & $(2.5)$ & 11 & (1.9) & 63 & (12.1) & 18 & $(2.0)$ & 62 & $(3.0)$ \\
\hline Mexiko & 5 & (0.5) & 28 & (1.6) & 3 & (1.1) & 15 & (3.1) & 5 & $(1.2)$ & 27 & (2.6) \\
\hline Niederlande & 6 & (1.9) & 143 & (12.9) & 2 & (2.9) & 20 & $(17.2)$ & 10 & (3.4) & 137 & $(20.8)$ \\
\hline Neuseeland & 33 & $(2.3)$ & 35 & (8.4) & 18 & (2.3) & 24 & $(8.1)$ & 32 & $(2.7)$ & 75 & $(6.2)$ \\
\hline Norwegen & $\mathrm{m}$ & $\mathrm{m}$ & $\mathrm{m}$ & $\mathrm{m}$ & c & C & c & C & C & C & c & C \\
\hline Polen & 32 & (1.9) & 38 & (10.7) & 25 & $(2.1)$ & 27 & $(9.2)$ & 33 & (2.3) & 33 & $(9.0)$ \\
\hline Portugal & 23 & (1.4) & 41 & $(4.0)$ & 19 & (1.8) & 27 & (5.6) & 25 & $(2.0)$ & 35 & (5.5) \\
\hline Slowak. Rep. & 21 & (2.3) & 76 & (7.8) & 19 & (2.9) & 45 & (10.9) & 25 & (3.1) & 90 & (7.8) \\
\hline Slowenien & 1 & (1.5) & 122 & (4.0) & 0 & (2.3) & 85 & (5.9) & 4 & $(2.5)$ & 125 & (4.8) \\
\hline Spanien & 25 & (1.1) & 21 & (3.6) & 12 & (1.5) & 15 & (5.3) & 24 & (1.7) & 31 & $(4.2)$ \\
\hline Schweden & 24 & $(2.1)$ & 24 & $(8.7)$ & 22 & (2.9) & 18 & (11.7) & 27 & $(2.8)$ & 38 & (7.9) \\
\hline Schweiz & $\mathrm{m}$ & $\mathrm{m}$ & $\mathrm{m}$ & $\mathrm{m}$ & C & C & c & c & c & C & c & c \\
\hline Türkei & 6 & (1.1) & 89 & (6.7) & 4 & (1.5) & 53 & (7.7) & 5 & (1.6) & 87 & (7.5) \\
\hline Ver. Königreich & 24 & $(1.8)$ & 75 & (7.8) & 10 & (1.9) & 44 & $(9.2)$ & 23 & $(2.5)$ & 71 & (8.7) \\
\hline Ver. Staaten & 25 & $(2.0)$ & 37 & (5.9) & 14 & $(2.3)$ & 25 & (7.5) & 26 & $(2.4)$ & 35 & (5.8) \\
\hline OECD-Durchschnitt & 18 & $(0.3)$ & 69 & $(1.2)$ & 11 & $(0.4)$ & 35 & $(1.7)$ & 19 & $(0.5)$ & 69 & (1.5) \\
\hline
\end{tabular}

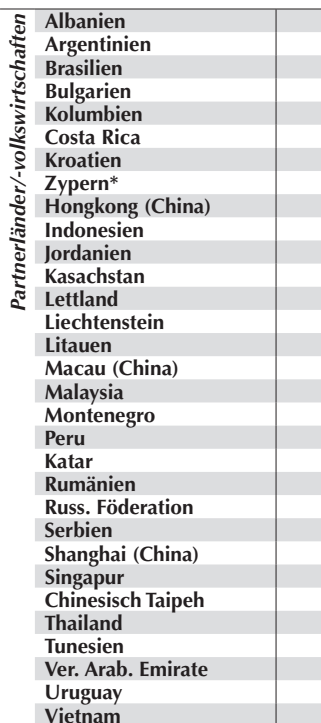

\begin{tabular}{rr|r}
$\mathbf{m}$ & $\mathrm{m}$ & $\mathrm{m}$ \\
$\mathbf{9}$ & $(1.2)$ & $\mathbf{4 7}$ \\
$\mathbf{8}$ & $(0.8)$ & $\mathbf{4 7}$ \\
$\mathbf{1 2}$ & $(1.5)$ & $\mathbf{6 6}$ \\
$\mathbf{1 0}$ & $(1.2)$ & $\mathbf{3 9}$ \\
$\mathbf{1 0}$ & $(1.2)$ & $\mathbf{2 9}$ \\
$\mathbf{1 0}$ & $(1.6)$ & $\mathbf{9 2}$ \\
$\mathbf{2 0}$ & $(1.8)$ & $\mathbf{6 9}$ \\
$\mathbf{5}$ & $(1.4)$ & $\mathbf{7 1}$ \\
$\mathbf{7}$ & $(1.1)$ & $\mathbf{4 2}$ \\
$\mathbf{1 4}$ & $(1.3)$ & 39 \\
$\mathbf{1 5}$ & $(1.7)$ & $\mathbf{5 0}$ \\
$\mathbf{2 2}$ & $(1.9)$ & $\mathbf{4 0}$ \\
$\mathrm{m}$ & $\mathrm{m}$ & $\mathrm{m}$ \\
$\mathbf{1 8}$ & $(1.5)$ & $\mathbf{7 2}$ \\
$\mathbf{9}$ & $(1.9)$ & $\mathbf{3 8}$ \\
$\mathbf{1 5}$ & $(1.5)$ & $\mathbf{4}$ \\
$\mathbf{1 2}$ & $(1.3)$ & $\mathbf{1 0 2}$ \\
$\mathbf{1 0}$ & $(1.1)$ & $\mathbf{4}$ \\
$\mathbf{1 0}$ & $(1.3)$ & $\mathbf{4 9}$ \\
$\mathbf{1 5}$ & $(1.8)$ & $\mathbf{5 7}$ \\
$\mathbf{2 6}$ & $(2.2)$ & $\mathbf{4}$ \\
$\mathbf{9}$ & $(1.4)$ & $\mathbf{1 0}$ \\
$\mathbf{9}$ & $(1.7)$ & $\mathbf{8 3}$ \\
$\mathbf{2 0}$ & $(2.0)$ & $\mathbf{8} 8$ \\
$\mathbf{2 6}$ & $(1.8)$ & $\mathbf{1 1 9}$ \\
$\mathbf{1 0}$ & $(1.3)$ & $\mathbf{3 7}$ \\
$\mathbf{5}$ & $(1.1)$ & $\mathbf{5 0}$ \\
$\mathbf{1 5}$ & $(1.3)$ & $\mathbf{4 9}$ \\
$\mathbf{1 5}$ & $(1.4)$ & $\mathbf{4 9}$ \\
$\mathbf{8}$ & $(1.4)$ & $\mathbf{6} 2$ \\
\hline & &
\end{tabular}

\begin{tabular}{rr|r}
$\mathbf{m}$ & $\mathrm{m}$ & $\mathrm{m}$ \\
$\mathbf{4 7}$ & $(6.5)$ & \\
$\mathbf{4 7}$ & $(3.4)$ & \\
$\mathbf{6 6}$ & $(7.5)$ & \\
$\mathbf{3 9}$ & $(3.9)$ & \\
$\mathbf{2 9}$ & $(4.6)$ & \\
$\mathbf{9 2}$ & $(8.5)$ & \\
$\mathbf{6 9}$ & $(4.5)$ & $\mathbf{1}$ \\
$\mathbf{7 1}$ & $(7.1)$ & \\
$\mathbf{4 2}$ & $(8.2)$ & \\
$\mathbf{3 9}$ & $(10.0)$ & \\
$\mathbf{5 0}$ & $(9.4)$ & $\mathbf{1}$ \\
$\mathbf{4 0}$ & $(6.5)$ & $\mathbf{1}$ \\
$\mathrm{m}$ & $\mathrm{m}$ & \\
$\mathbf{7 2}$ & $(6.7)$ & $\mathbf{1}$ \\
$\mathbf{3 8}$ & $(3.7)$ & \\
$\mathbf{4 4}$ & $(6.4)$ & $\mathbf{1}$ \\
$\mathbf{1 0 2}$ & $(3.3)$ & \\
$\mathbf{4 4}$ & $(4.2)$ & \\
$\mathbf{4 9}$ & $(2.6)$ & \\
$\mathbf{5 7}$ & $(7.0)$ & $\mathbf{1}$ \\
$\mathbf{4 1}$ & $(7.8)$ & $\mathbf{2}$ \\
$\mathbf{1 0 7}$ & $(8.4)$ & \\
$\mathbf{8 3}$ & $(5.8)$ & \\
$\mathbf{8 8}$ & $(5.4)$ & \\
$\mathbf{1 1 9}$ & $(8.3)$ & $\mathbf{1}$ \\
$\mathbf{3 7}$ & $(5.6)$ & \\
$\mathbf{5 0}$ & $(7.4)$ & \\
$\mathbf{4 9}$ & $(5.2)$ & \\
$\mathbf{4 9}$ & $(3.8)$ & $\mathbf{1 1}$ \\
$\mathbf{6 2}$ & $(8.6)$ & \\
\hline $\mathbf{2}$ & &
\end{tabular}

\begin{tabular}{rr|r}
$\mathrm{m}$ & $\mathrm{m}$ & \\
$\mathbf{8}$ & $(2.1)$ & 3 \\
$\mathrm{c}$ & $\mathrm{c}$ & \\
$\mathrm{c}$ & $\mathrm{c}$ & \\
3 & $(2.2)$ & 22 \\
$\mathbf{6}$ & $(2.7)$ & 14 \\
$\mathbf{8}$ & $(2.2)$ & 5 \\
$\mathbf{1 7}$ & $(2.3)$ & 23 \\
0 & $(1.8)$ & 4 \\
3 & $(1.8)$ & \\
$\mathbf{8}$ & $(1.9)$ & 4 \\
$\mathbf{1 0}$ & $(2.2)$ & 34 \\
$\mathbf{1 5}$ & $(2.9)$ & 39 \\
$\mathrm{C}$ & $\mathrm{c}$ & \\
$\mathbf{1 4}$ & $(1.8)$ & 48 \\
3 & $(1.7)$ & \\
$\mathbf{1 0}$ & $(2.1)$ & 1 \\
$\mathbf{8}$ & $(2.1)$ & 78 \\
$\mathbf{6}$ & $(1.7)$ & 23 \\
$\mathbf{5}$ & $(1.7)$ & 27 \\
$\mathbf{1 2}$ & $(2.0)$ & 39 \\
$\mathbf{2 2}$ & $(3.0)$ & 23 \\
$\mathbf{6}$ & $(2.6)$ & 29 \\
$\mathbf{6}$ & $(1.6)$ & 42 \\
$\mathbf{6}$ & $(2.1)$ & 18 \\
$\mathbf{1 5}$ & $(2.3)$ & 38 \\
$\mathbf{6}$ & $(1.4)$ & 26 \\
$\mathbf{7}$ & $(1.7)$ & 39 \\
$\mathrm{C}$ & $\mathrm{c}$ & \\
$\mathbf{1 1}$ & $(1.6)$ & 10 \\
$\mathbf{8}$ & $(1.7)$ & 23 \\
\hline
\end{tabular}

\begin{tabular}{rr|rr|rr} 
m & m & m & m & m & m \\
$\mathbf{3 5}$ & $(8.7)$ & $\mathbf{1 1}$ & $(2.3)$ & $\mathbf{5 1}$ & $(4.9)$ \\
$\mathrm{C}$ & $\mathrm{C}$ & $\mathrm{C}$ & $\mathrm{C}$ & $\mathrm{C}$ & $\mathrm{C}$ \\
$\mathrm{C}$ & $\mathrm{C}$ & $\mathrm{C}$ & $\mathrm{C}$ & $\mathrm{C}$ & $\mathrm{C}$ \\
$\mathbf{2 2}$ & $(5.2)$ & $\mathbf{8}$ & $(2.5)$ & $\mathbf{3 8}$ & $(4.5)$ \\
$\mathbf{1 4}$ & $(7.0)$ & $\mathbf{1 0}$ & $(2.8)$ & $\mathbf{3 2}$ & $(5.5)$ \\
$\mathbf{5 0}$ & $(9.4)$ & $\mathbf{1 3}$ & $(2.3)$ & $\mathbf{1 0 4}$ & $(11.2)$ \\
$\mathbf{2 3}$ & $(7.7)$ & $\mathbf{2 3}$ & $(2.4)$ & $\mathbf{6 1}$ & $(4.7)$ \\
$\mathbf{4 8}$ & $(5.7)$ & $\mathbf{5}$ & $(2.1)$ & $\mathbf{6 9}$ & $(8.1)$ \\
8 & $(8.4)$ & 3 & $(1.9)$ & $\mathbf{3 2}$ & $(9.3)$ \\
$\mathbf{4 6}$ & $(6.3)$ & $\mathbf{1 3}$ & $(1.9)$ & $\mathbf{6 3}$ & $(10.1)$ \\
$\mathbf{3 4}$ & $(9.1)$ & $\mathbf{1 2}$ & $(2.3)$ & $\mathbf{5 2}$ & $(10.0)$ \\
$\mathbf{3 9}$ & $(9.7)$ & $\mathbf{2 1}$ & $(3.3)$ & $\mathbf{3 4}$ & $(7.6)$ \\
$\mathrm{C}$ & $\mathrm{C}$ & $\mathrm{C}$ & $\mathrm{C}$ & $\mathrm{C}$ & $\mathrm{C}$ \\
$\mathbf{4 8}$ & $(9.1)$ & $\mathbf{1 7}$ & $(2.0)$ & $\mathbf{6 4}$ & $(5.6)$ \\
9 & $(5.3)$ & $\mathbf{9}$ & $(2.2)$ & $\mathbf{3 6}$ & $(5.0)$ \\
11 & $(8.1)$ & $\mathbf{1 5}$ & $(2.3)$ & $\mathbf{5 1}$ & $(7.2)$ \\
$\mathbf{7 8}$ & $(12.0)$ & $\mathbf{1 1}$ & $(2.3)$ & $\mathbf{9 0}$ & $(6.5)$ \\
$\mathbf{2 3}$ & $(5.5)$ & $\mathbf{1 3}$ & $(1.9)$ & $\mathbf{4 3}$ & $(3.9)$ \\
$\mathbf{2 7}$ & $(4.5)$ & $\mathbf{9}$ & $(2.1)$ & $\mathbf{6 5}$ & $(4.7)$ \\
$\mathbf{3 9}$ & $(9.1)$ & $\mathbf{1 6}$ & $(2.2)$ & $\mathbf{5 7}$ & $(6.5)$ \\
$\mathbf{2 3}$ & $(11.4)$ & $\mathbf{2 7}$ & $(3.0)$ & $\mathbf{4 8}$ & $(8.4)$ \\
29 & $(15.0)$ & $\mathbf{1 1}$ & $(2.8)$ & $\mathbf{9 4}$ & $(12.9)$ \\
$\mathbf{4 2}$ & $(6.6)$ & $\mathbf{1 0}$ & $(2.0)$ & $\mathbf{8 8}$ & $(5.6)$ \\
$\mathbf{1 8}$ & $(6.2)$ & $\mathbf{2 0}$ & $(2.4)$ & $\mathbf{8 7}$ & $(6.4)$ \\
$\mathbf{3 8}$ & $(11.3)$ & $\mathbf{2 7}$ & $(2.5)$ & $\mathbf{1 1 9}$ & $(8.8)$ \\
$\mathbf{2 6}$ & $(6.8)$ & $\mathbf{9}$ & $(1.5)$ & $\mathbf{3 5}$ & $(4.7)$ \\
$\mathbf{3 9}$ & $(7.0)$ & $\mathbf{8}$ & $(1.7)$ & $\mathbf{3 0}$ & $(6.2)$ \\
$\mathrm{C}$ & $\mathrm{C}$ & $\mathrm{C}$ & $\mathrm{C}$ & $\mathrm{C}$ & $\mathrm{C}$ \\
$\mathbf{1 0}$ & $(4.8)$ & $\mathbf{1 6}$ & $(1.7)$ & $\mathbf{4 4}$ & $(3.7)$ \\
$\mathbf{2 3}$ & $(9.4)$ & $\mathbf{1 0}$ & $(1.9)$ & $\mathbf{5 0}$ & $(6.4)$ \\
& & & & & \\
& & & & &
\end{tabular}

Notes: Statistisch signifikante Werte sind durch Fettdruck gekennzeichnet (vgl. Anhang A3).

Alle hier gezeigten Modelle berücksichtigen den sozioökonomischen Status auf Schüler- und Schulebene. Dann werden individuelle Schülermerkmale (Geschlecht, Migrationsstatus und zu Hause gesprochene Sprache) sowie der Schulstandort einbezogen. Diese Variablen werden in allen Einzelmodellen verwendet. Anschließend kommen nacheinander Variablen auf Schul- und Schülerebene hinzu, die die Verfügbarkeit und die Qualität der Ressourcen messen. Am Ende werden alle Variablen in einem einzigen Modell kombiniert. In einigen Fällen sind auf Ebene der Schüler, der Schulen und der Länder keine Daten für manche dieser Variablen verfügbar, deshalb sind sie im kombinierten Modell nicht enthalten. Im Anschluss daran wird ein Basismodell präsentiert, in dem nur der sozioökonomische Status auf Schüler- und Schulebene berücksichtigt wird und in dem alle Schüler Schulen und Länder, für die keine Daten vorliegen, ausgeklammert wurden, was einen direkteren Vergleich mit den Schätzwerten des kombinierten Modells ermöglicht.

1. ESCS bezieht sich auf den PISA-Index des wirtschaftlichen, sozialen und kulturellen Status.

2. Elterlicher Leistungsdruck basiert auf dem entsprechenden Index auf Schulebene (PARPRES).

3. Schuldisziplin umfasst die folgenden Variablen: xdisclim (aggregiert aus dem Schülerfragebogen auf die Schulebene) sowie studclim und teacclim, wobei es sich bei beiden

Variablen um Indizes auf Schulebene handelt, die schüler- bzw. lehrerbezogene Faktoren im Zusammenhang mit der Schuldisziplin messen.

4. Lernmöglichkeiten umfassen die folgenden Variablen (jeweils den einfachen und den quadrierten Index): Index des Kontakts mit reiner Mathematik, Index des Kontakts mit Textaufgaben, Index des Kontakts mit angewandter Mathematik.

5. Lernmöglichkeiten auf Schulebene umfassen dieselben Indizes der Lernmöglichkeiten auf Schüler- und auf Schulebene (mit Ausnahme des quadrierten Index des Kontakts mit angewandter Mathematik.

6. Lehrerprofil umfasst die folgenden Variablen: Schüler/Lehrer-Quote, Zusammensetzung und Qualifikationen des Mathematik-Lehrkörpers, Anteil der Lehrkräfte mit Lehrbefähigung und Lehrermangel.

7. Bildungsressourcen und räumliche Bedingungen der Schulen umfassen: fehlende Infrastruktur und Mangel an Bildungsressourcen in den Schulen.

8. Anteil der Schulabbrecher in einer Schule umfasst: Verhaltenseffekt - Schulabbruch.

StatLink 需s http://dx.doi.org/10.1787/888932964946 
[Teil 1/3]

Teilnahme an Vorschulbildung, Mathematikleistungen und sozioökonomischer Status der Schüler Tabelle II.4.12 Die Ergebnisse basieren auf Schülerangaben

\begin{tabular}{|c|c|c|c|c|c|c|c|c|c|c|c|c|c|c|c|c|c|c|c|}
\hline & \multicolumn{6}{|c|}{ Prozentsatz der Schüler, die } & \multicolumn{6}{|c|}{ ESCS ${ }^{1}$-Mittelwert der Schüler, die } & \multicolumn{6}{|c|}{$\begin{array}{l}\text { Durchschnittliche Mathematikleistungen } \\
\text { der Schüler, die }\end{array}$} \\
\hline & & \multicolumn{2}{|c|}{$\begin{array}{c}\text { nicht an } \\
\text { Vorschul- } \\
\text { bildung teil- } \\
\text { genommen } \\
\text { haben }\end{array}$} & $\begin{array}{r}\text { ein Ja } \\
\text { weni } \\
\text { Vor } \\
\text { bildu } \\
\text { geno } \\
\text { ha } \\
\end{array}$ & $\begin{array}{l}\text { roder } \\
\text { er an } \\
\text { chul- } \\
\text { g teil- } \\
\text { nmen } \\
\text { en }\end{array}$ & $\begin{array}{c}\text { über } \\
\text { an Vo } \\
\text { bildu } \\
\text { genc } \\
\text { h }\end{array}$ & $\begin{array}{l}\text { n Jahr } \\
\text { chul- } \\
\text { g teil- } \\
\text { imen } \\
\text { en }\end{array}$ & $\begin{array}{r}\text { nich } \\
\text { Vors } \\
\text { bild } \\
\text { teilgen } \\
\text { hal }\end{array}$ & $\begin{array}{l}\text { tan } \\
\text { hul- } \\
\text { ung } \\
\text { ommen } \\
\text { en }\end{array}$ & $\begin{array}{r}\text { ein Jah } \\
\text { wenig } \\
\text { Vors } \\
\text { bildun } \\
\text { genor } \\
\text { hal }\end{array}$ & $\begin{array}{l}\text { roder } \\
\text { er an } \\
\text { hul- } \\
\text { g teil- } \\
\text { imen } \\
\text { en }\end{array}$ & $\begin{array}{c}\text { über ei } \\
\text { an Vors } \\
\text { bildung } \\
\text { genom } \\
\text { hab }\end{array}$ & $\begin{array}{l}\text { in Jahr } \\
\text { schul- } \\
\text { g teil- } \\
\text { nmen } \\
\text { en }\end{array}$ & $\begin{array}{r}\text { nich } \\
\text { Vors } \\
\text { bildur } \\
\text { geno } \\
\text { ha }\end{array}$ & $\begin{array}{l}\text { ht an } \\
\text { schul- } \\
\text { ng teil- } \\
\text { mmen } \\
\text { ben }\end{array}$ & $\begin{array}{r}\text { ein Jah } \\
\text { wenig } \\
\text { Vors } \\
\text { bildun } \\
\text { genon } \\
\text { hal } \\
\end{array}$ & $\begin{array}{l}\text { hr oder } \\
\text { ger an } \\
\text { chul- } \\
\text { ng teil- } \\
\text { mmen } \\
\text { ben }\end{array}$ & $\begin{array}{c}\text { über e } \\
\text { an Vor } \\
\text { bildur } \\
\text { genor } \\
\text { hal }\end{array}$ & $\begin{array}{l}\text { in Jahr } \\
\text { rschul- } \\
\text { ng teil- } \\
\text { mmen } \\
\text { ben }\end{array}$ \\
\hline & & $\%$ & S.E. & $\%$ & S.E. & $\%$ & S.E. & $\begin{array}{c}\text { Mittel- } \\
\text { wert }\end{array}$ & S.E. & $\begin{array}{c}\text { Mittel- } \\
\text { wert }\end{array}$ & S.E. & $\begin{array}{c}\text { Mittel- } \\
\text { wert }\end{array}$ & S.E. & $\begin{array}{l}\text { Index- } \\
\text { mittel }\end{array}$ & S.E. & $\begin{array}{l}\text { Index- } \\
\text { mittel }\end{array}$ & S.E. & $\begin{array}{l}\text { Index- } \\
\text { mittel }\end{array}$ & S.E. \\
\hline ¿ & Australien & 4.5 & $(0.2)$ & 43.6 & $(0.6)$ & 51.9 & $(0.6)$ & -0.1 & $(0.0)$ & 0.2 & $(0.0)$ & 0.4 & $(0.0)$ & 463 & (5.3) & 500 & (1.8) & 516 & $(2.0)$ \\
\hline & Österreich & 1.8 & $(0.3)$ & 10.5 & $(0.6)$ & 87.7 & $(0.7)$ & -0.8 & $(0.2)$ & -0.2 & $(0.0)$ & 0.1 & $(0.0)$ & 447 & (14.4) & 482 & (5.9) & 510 & (2.6) \\
\hline & Belgien & 2.3 & $(0.2)$ & 4.5 & $(0.3)$ & 93.2 & $(0.4)$ & -0.4 & $(0.1)$ & -0.2 & $(0.1)$ & 0.2 & $(0.0)$ & 437 & (8.1) & 443 & (7.1) & 523 & (2.2) \\
\hline & Kanada & 9.0 & $(0.3)$ & 40.4 & $(0.7)$ & 50.6 & $(0.6)$ & 0.1 & $(0.0)$ & 0.3 & $(0.0)$ & 0.5 & $(0.0)$ & 500 & (3.2) & 512 & (1.8) & 532 & (2.6) \\
\hline & Chile & 9.2 & $(0.7)$ & 56.4 & $(0.9)$ & 34.4 & $(0.8)$ & -1.4 & $(0.1)$ & -0.6 & $(0.0)$ & -0.3 & $(0.0)$ & 382 & (5.4) & 423 & (3.0) & 436 & (3.6) \\
\hline & Tschech. Rep. & 3.2 & $(0.5)$ & 8.7 & $(0.6)$ & 88.1 & $(0.8)$ & -0.4 & $(0.1)$ & -0.2 & $(0.0)$ & 0.0 & $(0.0)$ & 435 & $(15.3)$ & 483 & (6.8) & 504 & $(2.7)$ \\
\hline & Dänemark & 1.0 & $(0.1)$ & 20.0 & $(0.6)$ & 79.0 & $(0.6)$ & 0.0 & $(0.1)$ & 0.3 & $(0.0)$ & 0.5 & $(0.0)$ & 445 & (11.4) & 469 & (3.3) & 511 & (2.2) \\
\hline & Estland & 7.3 & $(0.6)$ & 8.7 & $(0.5)$ & 84.0 & $(0.8)$ & -0.2 & $(0.0)$ & -0.1 & $(0.0)$ & 0.2 & $(0.0)$ & 525 & (6.4) & 508 & (5.4) & 522 & $(2.0)$ \\
\hline & Finnland & 2.4 & $(0.2)$ & 34.9 & $(1.0)$ & 62.7 & $(1.0)$ & -0.1 & $(0.1)$ & 0.2 & $(0.0)$ & 0.5 & $(0.0)$ & 474 & $(10.7)$ & 512 & (2.6) & 527 & (2.2) \\
\hline & Frankreich & 1.7 & $(0.2)$ & 6.3 & $(0.3)$ & 92.0 & $(0.4)$ & -0.7 & $(0.1)$ & -0.3 & $(0.1)$ & 0.0 & $(0.0)$ & 404 & $(13.6)$ & 440 & (5.6) & 504 & (2.5) \\
\hline & Deutschland & 3.2 & $(0.3)$ & 11.0 & $(0.6)$ & 85.7 & $(0.7)$ & -0.1 & $(0.1)$ & -0.1 & $(0.1)$ & 0.3 & $(0.0)$ & 472 & (8.6) & 466 & (5.2) & 529 & (3.2) \\
\hline & Griechenland & 4.6 & $(0.5)$ & 27.4 & $(0.9)$ & 68.0 & $(1.0)$ & -0.8 & $(0.1)$ & -0.2 & $(0.0)$ & 0.0 & $(0.0)$ & 395 & $(7.8)$ & 439 & (3.9) & 463 & (2.5) \\
\hline & Ungarn & 0.5 & $(0.1)$ & 3.9 & $(0.4)$ & 95.6 & $(0.4)$ & c & c & -0.5 & $(0.1)$ & -0.2 & $(0.0)$ & c & c & 433 & $(10.1)$ & 480 & (3.2) \\
\hline & Island & 2.0 & $(0.2)$ & 3.2 & $(0.3)$ & 94.7 & $(0.4)$ & 0.1 & $(0.1)$ & 0.5 & $(0.1)$ & 0.8 & $(0.0)$ & 450 & (12.1) & 463 & (9.4) & 497 & (1.7) \\
\hline & Irland & 13.6 & $(0.7)$ & 43.6 & $(0.9)$ & 42.8 & $(0.9)$ & -0.2 & $(0.0)$ & 0.1 & $(0.0)$ & 0.3 & $(0.0)$ & 491 & $(4.2)$ & 506 & (2.8) & 503 & $(2.7)$ \\
\hline & Israel & 2.1 & $(0.2)$ & 16.4 & $(0.8)$ & 81.5 & $(0.8)$ & -0.7 & $(0.1)$ & 0.0 & $(0.0)$ & 0.2 & $(0.0)$ & 383 & (10.5) & 425 & (6.0) & 481 & (4.8) \\
\hline & Italien & 4.3 & $(0.2)$ & 8.0 & $(0.2)$ & 87.7 & $(0.3)$ & -0.4 & $(0.0)$ & -0.1 & $(0.0)$ & 0.0 & $(0.0)$ & 429 & (4.4) & 454 & (3.3) & 492 & $(2.1)$ \\
\hline & Japan & 0.8 & $(0.1)$ & 2.2 & $(0.2)$ & 96.9 & $(0.2)$ & -0.1 & $(0.1)$ & -0.3 & $(0.1)$ & -0.1 & $(0.0)$ & 506 & (18.9) & 489 & (8.0) & 540 & (3.5) \\
\hline & Korea & 4.5 & $(0.4)$ & 12.6 & $(0.7)$ & 82.8 & $(0.9)$ & -0.2 & $(0.1)$ & -0.1 & $(0.0)$ & 0.0 & $(0.0)$ & 533 & (8.6) & 542 & (6.9) & 557 & (4.5) \\
\hline & Luxemburg & 4.6 & $(0.3)$ & 12.8 & $(0.4)$ & 82.6 & $(0.5)$ & -0.4 & $(0.1)$ & -0.1 & $(0.0)$ & 0.1 & $(0.0)$ & 454 & (6.4) & 455 & (4.0) & 499 & (1.4) \\
\hline & Mexiko & 9.4 & $(0.3)$ & 18.7 & $(0.3)$ & 71.8 & $(0.5)$ & -1.8 & $(0.0)$ & -1.3 & $(0.0)$ & -1.0 & $(0.0)$ & 378 & (2.5) & 411 & (1.8) & 419 & (1.4) \\
\hline & Niederlande & 2.3 & $(0.3)$ & 2.7 & $(0.3)$ & 95.0 & $(0.3)$ & -0.1 & $(0.2)$ & 0.1 & $(0.1)$ & 0.2 & $(0.0)$ & 484 & (12.6) & 522 & $(10.1)$ & 526 & (3.4) \\
\hline & Neuseeland & 9.1 & $(0.6)$ & 19.4 & $(0.7)$ & 71.5 & $(0.9)$ & -0.4 & $(0.0)$ & -0.1 & $(0.0)$ & 0.1 & $(0.0)$ & 454 & (6.9) & 491 & (4.1) & 513 & (2.5) \\
\hline & Norwegen & 7.9 & $(0.4)$ & 5.7 & $(0.4)$ & 86.4 & $(0.6)$ & 0.1 & $(0.0)$ & 0.1 & $(0.0)$ & 0.5 & $(0.0)$ & 465 & (5.2) & 461 & (5.8) & 496 & (2.7) \\
\hline & Polen & 2.5 & $(0.3)$ & 46.4 & (1.5) & 51.1 & (1.5) & -0.6 & $(0.1)$ & -0.5 & $(0.0)$ & 0.1 & $(0.0)$ & 471 & (9.3) & 504 & (3.0) & 532 & (4.8) \\
\hline & Portugal & 14.9 & $(0.8)$ & 20.7 & $(0.8)$ & 64.4 & (1.1) & -0.9 & $(0.0)$ & -0.7 & $(0.1)$ & -0.3 & $(0.1)$ & 461 & (4.9) & 465 & (5.0) & 505 & $(4.0)$ \\
\hline & Slowak. Rep. & 6.8 & $(0.7)$ & 13.1 & $(0.8)$ & 80.1 & (1.1) & -1.0 & $(0.1)$ & -0.5 & $(0.0)$ & -0.1 & $(0.0)$ & 391 & (8.0) & 463 & (6.3) & 494 & (3.5) \\
\hline & Slowenien & 14.6 & $(0.5)$ & 12.8 & $(0.6)$ & 72.6 & $(0.7)$ & -0.3 & $(0.0)$ & -0.1 & $(0.0)$ & 0.2 & $(0.0)$ & 484 & $(4.2)$ & 484 & (4.4) & 509 & (1.6) \\
\hline & Spanien & 5.9 & $(0.3)$ & 8.3 & $(0.2)$ & 85.9 & $(0.4)$ & -0.6 & $(0.0)$ & -0.4 & $(0.0)$ & -0.1 & $(0.0)$ & 436 & (3.2) & 455 & (3.9) & 492 & (1.8) \\
\hline & Schweden & 8.1 & $(0.5)$ & 20.4 & $(0.8)$ & 71.5 & $(0.8)$ & -0.1 & $(0.1)$ & 0.2 & $(0.0)$ & 0.3 & $(0.0)$ & 442 & (6.3) & 472 & (3.0) & 489 & (2.4) \\
\hline & Schweiz & 1.8 & $(0.2)$ & 25.0 & (1.8) & 73.1 & (1.9) & -0.4 & $(0.1)$ & 0.1 & $(0.0)$ & 0.2 & $(0.0)$ & 458 & (13.9) & 537 & (5.2) & 532 & (3.2) \\
\hline & Türkei & 70.3 & (1.4) & 21.0 & (1.0) & 8.7 & $(0.8)$ & -1.8 & $(0.0)$ & -0.9 & $(0.0)$ & -0.3 & $(0.1)$ & 433 & (4.3) & 480 & (6.0) & 495 & (10.0) \\
\hline & Ver. Königreich & 4.7 & $(0.4)$ & 26.0 & $(0.6)$ & 69.3 & $(0.6)$ & -0.1 & $(0.1)$ & 0.2 & $(0.0)$ & 0.3 & $(0.0)$ & 448 & (7.8) & 481 & (3.7) & 507 & (2.9) \\
\hline & Ver. Staaten & 1.4 & $(0.2)$ & 24.0 & $(0.9)$ & 74.6 & $(0.9)$ & -0.3 & $(0.2)$ & -0.2 & $(0.0)$ & 0.3 & $(0.0)$ & 454 & (11.9) & 472 & (3.6) & 487 & (4.1) \\
\hline & OECD-Durchschnitt & 7.1 & $(0.1)$ & 18.8 & $(0.1)$ & 74.1 & $(0.1)$ & -0.5 & $(0.0)$ & -0.2 & $(0.0)$ & 0.1 & $(0.0)$ & 451 & (1.6) & 475 & $(0.9)$ & 504 & (0.6) \\
\hline$\Phi$ & Albanien & $\mathrm{m}$ & $\mathrm{m}$ & $\mathrm{m}$ & $\mathrm{m}$ & $\mathrm{m}$ & $\mathrm{m}$ & $\mathrm{m}$ & $\mathrm{m}$ & $\mathrm{m}$ & $\mathrm{m}$ & $\mathrm{m}$ & $\mathrm{m}$ & $\mathrm{m}$ & $\mathrm{m}$ & $\mathrm{m}$ & $\mathrm{m}$ & $\mathrm{m}$ & $\mathrm{m}$ \\
\hline స్心 & Argentinien & 6.1 & $(0.9)$ & 22.6 & $(1.0)$ & 71.4 & (1.4) & -1.3 & $(0.1)$ & -1.1 & $(0.1)$ & -0.5 & $(0.0)$ & 338 & (6.3) & 367 & $(4.2)$ & 403 & (3.4) \\
\hline 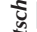 & Brasilien & 18.8 & $(0.6)$ & 33.4 & $(0.7)$ & 47.8 & $(0.8)$ & -1.6 & $(0.0)$ & -1.2 & $(0.0)$ & -0.9 & $(0.0)$ & 368 & (2.4) & 386 & (2.0) & 408 & $(2.8)$ \\
\hline 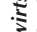 & Bulgarien & 10.2 & $(0.7)$ & 12.9 & $(0.6)$ & 76.9 & $(1.0)$ & -0.8 & $(0.1)$ & -0.4 & $(0.1)$ & -0.2 & $(0.0)$ & 399 & (6.6) & 427 & (6.2) & 448 & (3.7) \\
\hline$=$ & Kolumbien & 14.2 & $(0.8)$ & 52.5 & $(0.8)$ & 33.3 & (1.1) & -1.9 & $(0.1)$ & -1.3 & $(0.0)$ & -0.9 & $(0.1)$ & 351 & (3.9) & 379 & (3.0) & 385 & $(4.1)$ \\
\hline$y$ & Costa Rica & 15.4 & $(0.9)$ & 39.6 & $(1.1)$ & 45.0 & $(1.2)$ & -1.5 & $(0.1)$ & -1.1 & $(0.1)$ & -0.7 & $(0.1)$ & 384 & (4.4) & 408 & (3.2) & 416 & $(4.0)$ \\
\hline & Kroatien & 26.8 & (1.1) & 22.4 & $(0.8)$ & 50.8 & (1.1) & -0.7 & $(0.0)$ & -0.5 & $(0.0)$ & 0.0 & $(0.0)$ & 457 & (3.6) & 456 & (4.0) & 487 & $(5.0)$ \\
\hline & Zypern* & 3.5 & $(0.3)$ & 23.4 & $(0.6)$ & 73.1 & $(0.7)$ & -0.2 & $(0.1)$ & 0.0 & $(0.0)$ & 0.1 & $(0.0)$ & 423 & (8.0) & 427 & (2.5) & 448 & (1.5) \\
\hline 5 & Hongkong (China) & 1.6 & $(0.2)$ & 3.3 & $(0.3)$ & 95.1 & $(0.4)$ & -1.3 & $(0.1)$ & -1.2 & $(0.1)$ & -0.8 & $(0.1)$ & 483 & (15.5) & 503 & (8.3) & 567 & (3.1) \\
\hline & Indonesien & 46.1 & $(2.2)$ & 31.4 & $(2.0)$ & 22.5 & (1.5) & -2.2 & $(0.0)$ & -1.6 & $(0.1)$ & -1.3 & $(0.1)$ & 351 & (3.7) & 390 & (4.5) & 405 & (9.2) \\
\hline 8 & Jordanien & 24.1 & (1.0) & 49.4 & $(0.9)$ & 26.5 & $(1.0)$ & -0.8 & $(0.0)$ & -0.4 & $(0.0)$ & -0.1 & $(0.0)$ & 370 & (3.1) & 393 & (2.8) & 410 & (5.6) \\
\hline & Kasachstan & 65.0 & $(1.7)$ & 11.2 & $(0.6)$ & 23.8 & (1.4) & -0.5 & $(0.0)$ & -0.1 & $(0.0)$ & 0.0 & $(0.0)$ & 425 & (3.1) & 434 & (5.2) & 449 & $(4.8)$ \\
\hline & Lettland & 11.5 & $(0.8)$ & 13.3 & $(0.7)$ & 75.2 & $(0.9)$ & -0.6 & $(0.1)$ & -0.5 & $(0.0)$ & -0.2 & $(0.0)$ & 485 & (6.2) & 482 & (5.3) & 494 & (2.9) \\
\hline & Liechtenstein & 0.7 & $(0.5)$ & 8.6 & $(1.7)$ & 90.7 & (1.8) & $\mathrm{C}$ & $\mathrm{C}$ & c & c & 0.3 & $(0.1)$ & c & C & c & c & 541 & (5.0) \\
\hline & Litauen & 30.4 & (1.0) & 13.3 & $(0.6)$ & 56.3 & $(1.0)$ & -0.5 & $(0.0)$ & -0.2 & $(0.0)$ & 0.1 & $(0.0)$ & 457 & (3.3) & 472 & (4.3) & 493 & (3.0) \\
\hline & Macau (China) & 2.4 & $(0.2)$ & 11.9 & $(0.4)$ & 85.7 & (0.5) & -0.8 & $(0.1)$ & -1.0 & $(0.0)$ & -0.9 & $(0.0)$ & 495 & (8.6) & 492 & (4.0) & 547 & (1.1) \\
\hline & Malaysia & 23.8 & (1.3) & 28.7 & (1.0) & 47.6 & (1.4) & -1.0 & $(0.0)$ & -0.9 & $(0.0)$ & -0.5 & $(0.0)$ & 399 & (3.7) & 407 & (3.0) & 441 & $(4.2)$ \\
\hline & Montenegro & 32.8 & $(0.6)$ & 24.8 & $(0.6)$ & 42.3 & $(0.7)$ & -0.6 & $(0.0)$ & -0.3 & $(0.0)$ & 0.1 & $(0.0)$ & 393 & (2.0) & 408 & (2.8) & 426 & (1.9) \\
\hline & Peru & 13.8 & $(0.7)$ & 25.0 & $(0.7)$ & 61.1 & (1.1) & -2.0 & $(0.1)$ & -1.4 & $(0.0)$ & -1.0 & $(0.1)$ & 327 & (3.8) & 360 & $(4.0)$ & 384 & $(4.4)$ \\
\hline & Katar & 30.5 & $(0.5)$ & 41.6 & $(0.5)$ & 27.9 & (0.4) & 0.0 & $(0.0)$ & 0.6 & $(0.0)$ & 0.7 & $(0.0)$ & 347 & (1.6) & 382 & (1.4) & 410 & $(2.1)$ \\
\hline & Rumänien & 4.5 & $(0.5)$ & 9.0 & $(0.5)$ & 86.6 & $(0.8)$ & -1.2 & $(0.2)$ & -0.7 & $(0.1)$ & -0.4 & $(0.0)$ & 400 & $(7.7)$ & 419 & (5.5) & 450 & (3.8) \\
\hline & Russ. Föderation & 18.8 & (1.1) & 10.1 & $(0.6)$ & 71.1 & (1.4) & -0.5 & $(0.0)$ & -0.2 & $(0.0)$ & 0.0 & $(0.0)$ & 462 & $(4.7)$ & 464 & (5.0) & 491 & (3.0) \\
\hline & Serbien & 20.4 & $(0.9)$ & 28.8 & (1.1) & 50.8 & $(1.2)$ & -0.6 & $(0.0)$ & -0.5 & $(0.0)$ & -0.1 & $(0.0)$ & 433 & (5.1) & 433 & (3.3) & 465 & (4.3) \\
\hline & Shanghai (China) & 3.6 & $(0.6)$ & 8.6 & $(0.6)$ & 87.8 & (1.0) & -1.6 & $(0.1)$ & -0.9 & $(0.1)$ & -0.3 & $(0.0)$ & 505 & (9.1) & 555 & (8.5) & 623 & (2.7) \\
\hline & Singapur & 2.3 & $(0.2)$ & 7.1 & $(0.4)$ & 90.6 & $(0.4)$ & -0.7 & $(0.1)$ & -0.3 & $(0.1)$ & -0.2 & $(0.0)$ & 524 & (9.3) & 530 & (5.5) & 579 & (1.4) \\
\hline & Chinesisch Taipeh & 1.5 & $(0.2)$ & 14.7 & $(0.6)$ & 83.8 & (0.6) & -0.7 & $(0.1)$ & -0.6 & $(0.0)$ & -0.4 & $(0.0)$ & 508 & (19.4) & 530 & (5.6) & 566 & (3.3) \\
\hline & Thailand & 1.7 & $(0.2)$ & 10.5 & $(0.6)$ & 87.8 & $(0.6)$ & -1.8 & $(0.1)$ & -1.7 & $(0.1)$ & -1.3 & $(0.0)$ & 375 & (11.5) & 395 & (4.8) & 432 & (3.5) \\
\hline & Tunesien & 37.5 & (1.5) & 39.3 & (1.1) & 23.2 & $(1.0)$ & -1.8 & $(0.0)$ & -0.9 & $(0.1)$ & -0.7 & $(0.1)$ & 373 & (3.8) & 394 & (4.8) & 408 & (6.0) \\
\hline & Ver. Arab. Emirate & 23.5 & $(0.7)$ & 26.7 & $(0.6)$ & 49.8 & (0.9) & 0.0 & $(0.0)$ & 0.4 & $(0.0)$ & 0.5 & $(0.0)$ & 399 & (3.0) & 438 & (3.4) & 454 & (2.8) \\
\hline & Uruguay & 16.2 & $(0.8)$ & 14.2 & $(0.7)$ & 69.7 & (1.0) & -1.3 & $(0.0)$ & -1.2 & $(0.0)$ & -0.7 & $(0.0)$ & 370 & $(3.2)$ & 390 & (4.7) & 426 & (3.2) \\
\hline & Vietnam & 9.3 & $(1.0)$ & 22.5 & $(1.2)$ & 68.2 & (1.5) & -2.4 & $(0.1)$ & -2.1 & $(0.0)$ & -1.6 & $(0.1)$ & 457 & $(12.0)$ & 499 & $(4.4)$ & 523 & (4.8) \\
\hline
\end{tabular}

Anmerkung: Diese Tabelle wurde nur für Schüler berechnet, für die Daten zum PISA-Index des wirtschaftlichen, sozialen und kulturellen Status vorliegen. Statistisch signifikante Werte sind durch Fettdruck gekennzeichnet (vgl. Anhang A3).

1. ESCS bezieht sich auf den PISA-Index des wirtschaftlichen, sozialen und kulturellen Status.

$* \mathrm{Vgl}$. Anmerkungen am Anfang dieses Anhangs.

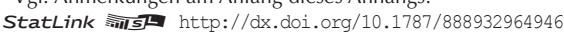


[Teil 2/3]

Teilnahme an Vorschulbildung, Mathematikleistungen und sozioökonomischer Status der Schüler Tabelle II.4.12 Die Ergebnisse basieren auf Schülerangaben

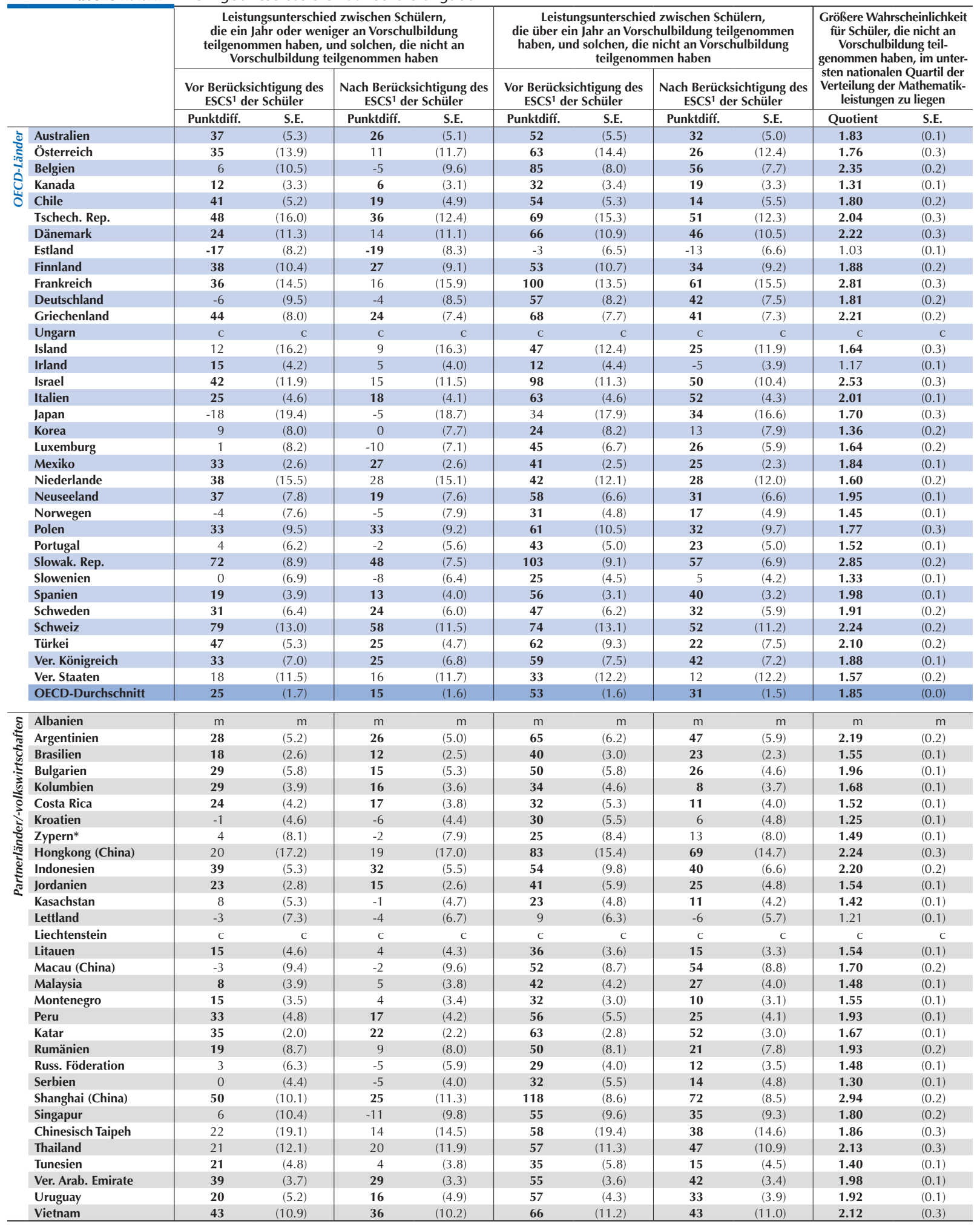

Anmerkung: Diese Tabelle wurde nur für Schüler berechnet, für die Daten zum PISA-Index des wirtschaftlichen, sozialen und kulturellen Status vorliegen. Statistisch signifikante Werte sind durch Fettdruck gekennzeichnet (vgl. Anhang A3).

1. ESCS bezieht sich auf den PISA-Index des wirtschaftlichen, sozialen und kulturellen Status.

$*$ Vgl. Anmerkungen am Anfang dieses Anhangs.

StatLink त्ञाज http://dx.doi.org/10.1787/888932964946 
[Teil 3/3]

Teilnahme an Vorschulbildung, Mathematikleistungen und sozioökonomischer Status der Schüler Tabelle II.4.12 Die Ergebnisse basieren auf Schülerangaben

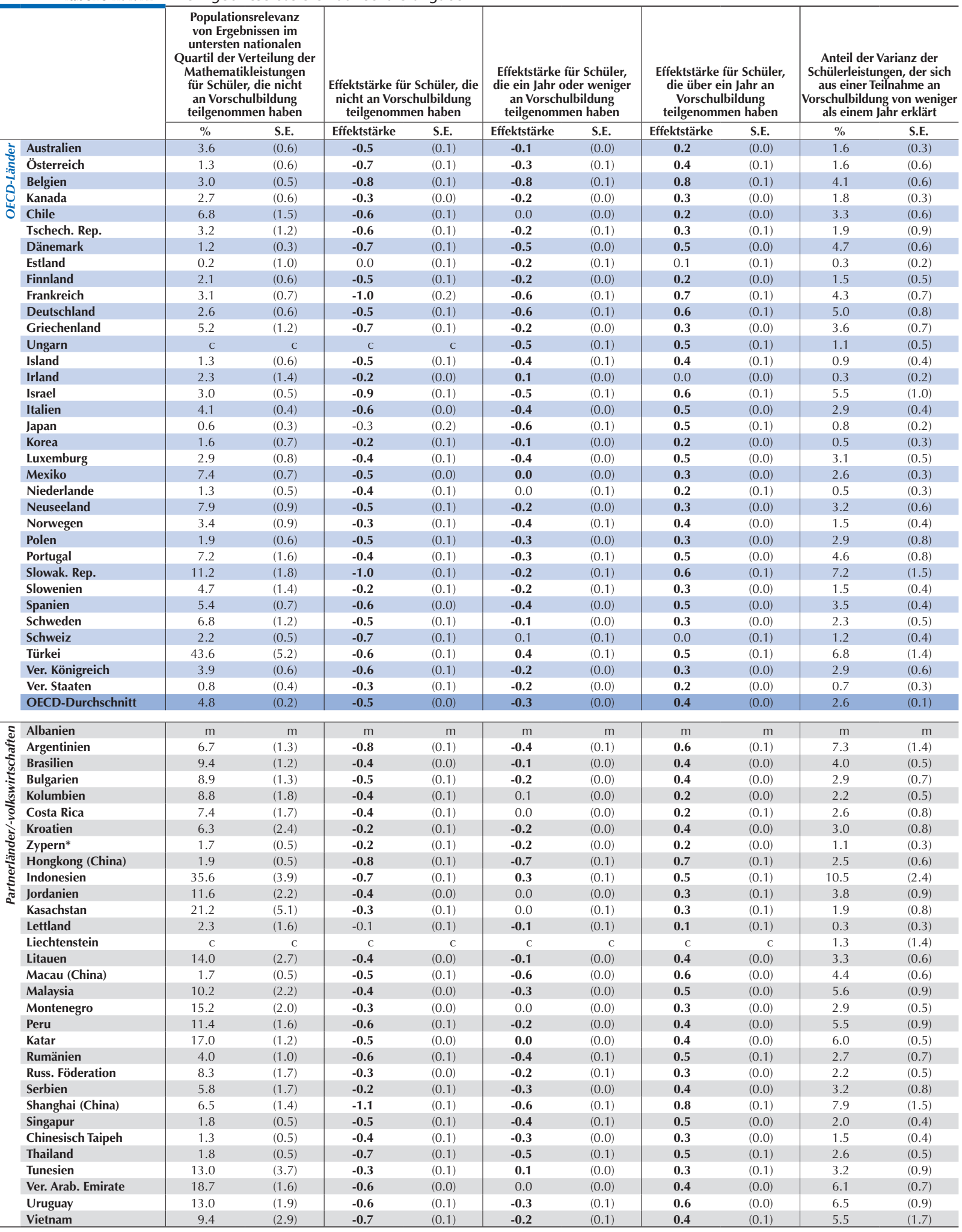

Anmerkung: Diese Tabelle wurde nur für Schüler berechnet, für die Daten zum PISA-Index des wirtschaftlichen, sozialen und kulturellen Status vorliegen. Statistisch signifikante Werte sind durch Fettdruck gekennzeichnet (vgl. Anhang A3).

1. ESCS bezieht sich auf den PISA-Index des wirtschaftlichen, sozialen und kulturellen Status.

$* \mathrm{Vgl}$. Anmerkungen am Anfang dieses Anhangs.

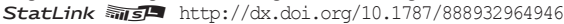


[Teil 1/1]

Tabelle II.4.13 Zusammenhang zwischen Leistungen, Teilnahme an Vorschulbildung und sozioökonomischem Status ${ }^{1}$

\begin{tabular}{|c|c|c|c|c|c|c|c|}
\hline & & \multicolumn{2}{|c|}{$\begin{array}{l}\text { Leistungsabstand in Mathematik für Schüler, } \\
\text { die an Vorschulbildung teilgenommen haben }\end{array}$} & \multicolumn{2}{|c|}{$\begin{array}{c}\text { Leistungsabstand für sozioökonomisch } \\
\text { begünstigte Schüler }{ }^{2}\end{array}$} & \multicolumn{2}{|c|}{$\begin{array}{l}\text { Zusätzlicher Leistungsabstand für } \\
\text { sozioökonomisch begünstigte Schüler², } \\
\text { die an Vorschulbildung teilgenommen haben }\end{array}$} \\
\hline & & Punktdiff. & S.E. & Punktdiff. & S.E. & Punktdiff. & S.E. \\
\hline \multirow{35}{*}{ 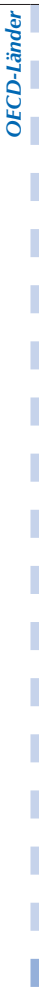 } & Australien & 28 & (5.6) & 20 & (6.0) & 4 & (6.1) \\
\hline & Österreich & 13 & $(9.2)$ & -1 & (9.3) & 12 & $(9.2)$ \\
\hline & Belgien & 23 & (8.8) & 14 & (8.4) & 1 & $(8.7)$ \\
\hline & Kanada & 15 & (3.1) & 21 & (3.4) & 1 & (3.5) \\
\hline & Chile & 14 & (6.1) & 3 & (3.3) & 6 & (3.1) \\
\hline & Tschech. Rep. & 19 & (9.7) & 6 & $(8.1)$ & 7 & (7.9) \\
\hline & Dänemark & 14 & $(9.0)$ & 15 & (8.3) & 12 & (8.6) \\
\hline & Estland & -14 & (6.7) & 10 & (6.5) & 10 & (6.9) \\
\hline & Finnland & 20 & (8.0) & 38 & (6.6) & -13 & $(7.0)$ \\
\hline & Frankreich & 46 & $(14.6)$ & -7 & (13.5) & 26 & (13.4) \\
\hline & Deutschland & 11 & (7.8) & -2 & $(8.7)$ & 11 & $(9.1)$ \\
\hline & Griechenland & 22 & (8.1) & 17 & $(7.2)$ & 0 & $(7.2)$ \\
\hline & Ungarn & c & c & c & c & c & c \\
\hline & Island & 17 & (11.4) & 25 & (11.4) & -4 & $(12.0)$ \\
\hline & Irland & 0 & (3.6) & 27 & $(4.0)$ & -2 & $(4.4)$ \\
\hline & Israel & 46 & (12.3) & 15 & $(7.2)$ & 10 & (6.6) \\
\hline & Italien & 34 & (3.8) & -1 & (3.8) & 6 & $(4.0)$ \\
\hline & Japan & 11 & $(14.6)$ & 19 & $(13.7)$ & -15 & (13.9) \\
\hline & Korea & 5 & (6.8) & 22 & $(9.2)$ & -7 & $(9.7)$ \\
\hline & Luxemburg & 12 & (6.0) & 20 & (4.3) & -6 & (4.3) \\
\hline & Mexiko & 27 & $(4.2)$ & 5 & $(2.2)$ & 4 & (1.9) \\
\hline & Niederlande & 11 & (8.6) & 3 & (9.8) & 2 & $(10.2)$ \\
\hline & Neuseeland & 35 & (6.7) & 30 & (8.6) & 2 & (9.6) \\
\hline & Norwegen & 12 & (5.3) & 13 & (5.9) & 11 & (6.2) \\
\hline & Polen & 28 & (12.3) & 36 & $(10.1)$ & -5 & $(10.5)$ \\
\hline & Portugal & 24 & (6.5) & 11 & (4.6) & 12 & (4.8) \\
\hline & Slowak. Rep. & 41 & $(8.7)$ & 12 & (8.7) & 10 & $(9.2)$ \\
\hline & Slowenien & -1 & (3.6) & -2 & $(4.7)$ & 3 & (5.1) \\
\hline & Spanien & 33 & $(4.0)$ & 20 & (3.6) & 4 & (3.5) \\
\hline & Schweden & 21 & (5.5) & 27 & (5.5) & -4 & (5.5) \\
\hline & Schweiz & 40 & (9.4) & 27 & (6.7) & -11 & (6.6) \\
\hline & Türkei & 8 & (4.5) & 8 & (3.7) & 0 & $(2.7)$ \\
\hline & Ver. Königreich & 36 & (6.9) & 20 & $(5.4)$ & 3 & $(6.0)$ \\
\hline & Ver. Staaten & 9 & (11.5) & 22 & (13.5) & 4 & $(13.2)$ \\
\hline & OECD-Durchschnitt & 20 & (1.4) & 15 & $(1.4)$ & 3 & (1.5) \\
\hline \multirow{31}{*}{ 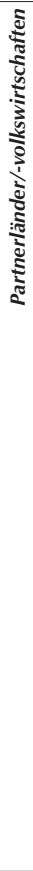 } & Albanien & $\mathrm{m}$ & $\mathrm{m}$ & $\mathrm{m}$ & $\mathrm{m}$ & $\mathrm{m}$ & $\mathrm{m}$ \\
\hline & Argentinien & 42 & (8.3) & 4 & $(4.3)$ & 7 & $(4.1)$ \\
\hline & Brasilien & 21 & (3.1) & 9 & (2.3) & 4 & (1.8) \\
\hline & Bulgarien & 9 & (4.8) & 15 & (5.3) & -2 & (5.4) \\
\hline & Kolumbien & 6 & (5.9) & 14 & (4.3) & -2 & (3.0) \\
\hline & Costa Rica & 14 & (4.8) & 11 & (2.9) & 4 & (2.5) \\
\hline & Kroatien & -6 & $(4.0)$ & 9 & (3.3) & 3 & (3.6) \\
\hline & Zypern* & 2 & (7.8) & 22 & (6.8) & -2 & (6.5) \\
\hline & Hongkong (China) & 49 & (23.5) & 2 & $(14.4)$ & 1 & $(14.4)$ \\
\hline & Indonesien & 30 & (7.8) & 11 & (4.3) & 3 & $(3.2)$ \\
\hline & Jordanien & 15 & $(3.2)$ & 10 & (2.8) & 6 & $(2.7)$ \\
\hline & Kasachstan & 0 & (3.2) & 15 & (2.6) & -1 & (3.0) \\
\hline & Lettland & -9 & (5.7) & 13 & (5.4) & 10 & $(6.1)$ \\
\hline & Liechtenstein & c & c & c & c & c & c \\
\hline & Litauen & 1 & (3.4) & 14 & (3.0) & 6 & (3.4) \\
\hline & Macau (China) & 48 & (9.0) & 0 & (7.4) & 11 & (7.5) \\
\hline & Malaysia & 18 & $(4.1)$ & 17 & (3.4) & 6 & (3.1) \\
\hline & Montenegro & -3 & $(2.7)$ & 11 & $(2.7)$ & 2 & (3.0) \\
\hline & Peru & 25 & (5.9) & 7 & (3.5) & 7 & $(2.7)$ \\
\hline & Katar & -6 & $(2.0)$ & -2 & (1.8) & 17 & (2.8) \\
\hline & Rumänien & 31 & (9.0) & 0 & (7.5) & 24 & (7.3) \\
\hline & Russ. Föderation & 6 & (3.7) & 16 & (4.8) & 11 & (5.0) \\
\hline & Serbien & 0 & (4.8) & 8 & (3.7) & 2 & (3.9) \\
\hline & Shanghai (China) & 57 & (17.1) & -11 & $(10.1)$ & 17 & (9.5) \\
\hline & Singapur & 22 & (11.5) & 29 & (7.8) & -11 & (7.9) \\
\hline & Chinesisch Taipeh & 27 & (17.5) & 41 & (15.6) & -18 & $(15.1)$ \\
\hline & Thailand & 37 & $(17.7)$ & 25 & (10.5) & -2 & (9.8) \\
\hline & Tunesien & 4 & $(4.7)$ & 12 & (3.6) & 6 & $(2.7)$ \\
\hline & Ver. Arab. Emirate & 26 & (2.6) & 8 & (2.5) & 5 & (3.1) \\
\hline & Uruguay & 35 & (5.3) & 7 & (3.8) & 9 & (3.2) \\
\hline & Vietnam & 37 & (13.9) & 9 & (5.9) & 4 & (6.1) \\
\hline
\end{tabular}

Anmerkung: Statistisch signifikante Werte sind durch Fettdruck gekennzeichnet (vgl. Anhang A3).

1. Die Leistungen im Bereich Mathematik sind auf die folgenden Variablen regressiert: Teilnahme an Vorschulbildung, sozioökonomischer Status der Schüler (Teilnahme an Vorschulbildung)*(sozioökonomischer Status der Schüler), Quadrat des sozioökonomischen Status der Schüler, sozioökonomisches Profil der Schule, Geschlecht, Migrationshintergrund, Schule im ländlichen Raum, Schule in einer Großstadt, Schulgröße, Quadrat der Schulgröße sowie Privatschule.

2. Sozioökonomisch begünstigte Schüler entsprechen Schülern, deren PISA-Index des wirtschaftlichen, sozialen und kulturellen Status um eine Einheit über dem OECDDurchschnitt liegt.

*Vgl. Anmerkungen am Anfang dieses Anhangs.

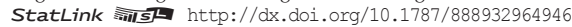


[Teil 1/1]

Tabelle II.4.14 Zusammenhang zwischen Leistungen, Teilnahme an Vorschulbildung und Migrationshintergrund ${ }^{1}$

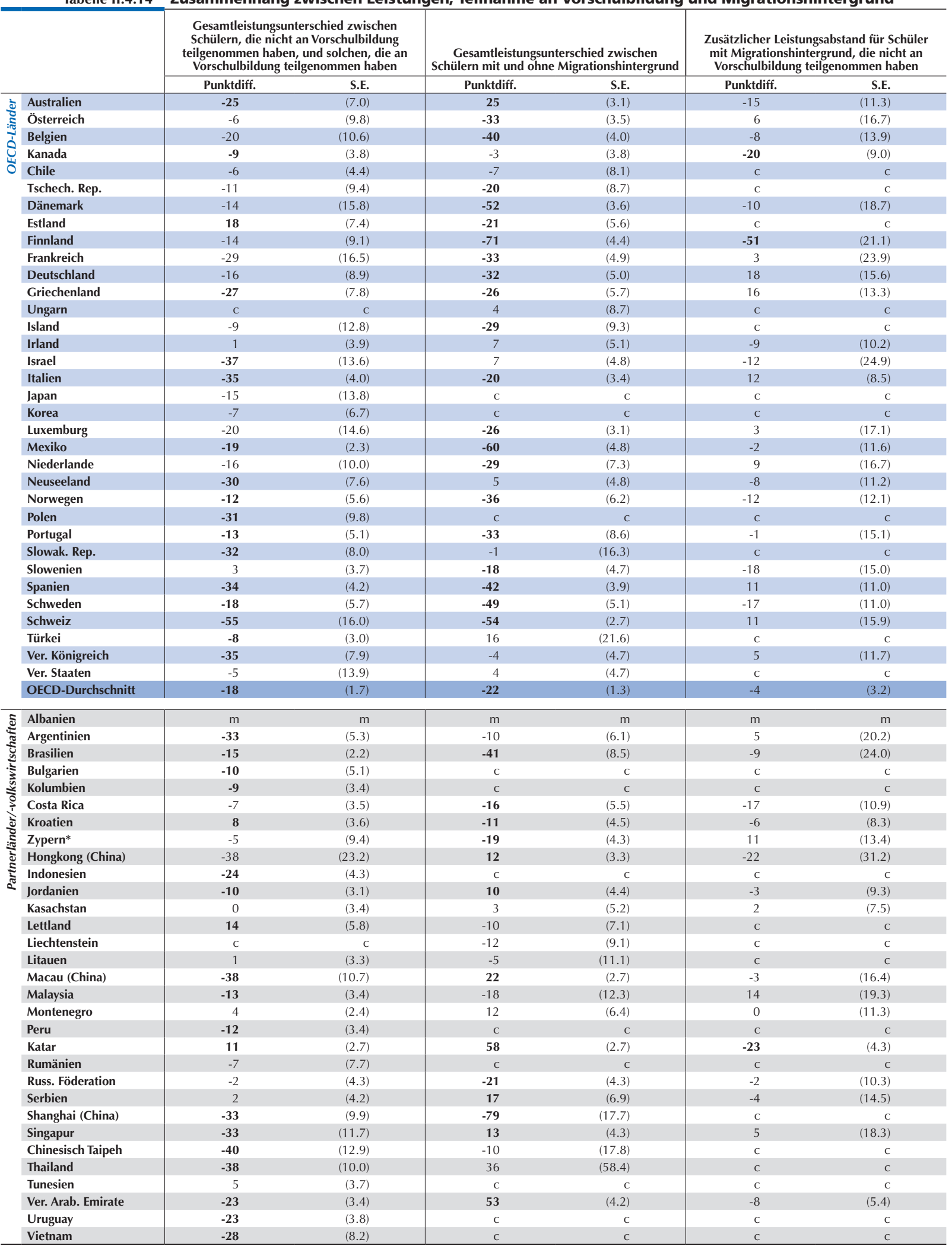

Anmerkung: Statistisch signifikante Werte sind durch Fettdruck gekennzeichnet (vgl. Anhang A3).

1. Die Leistungen im Bereich Mathematik sind auf die folgenden Variablen regressiert: Nichtteilnahme an Vorschulbildung, Schüler mit Migrationshintergrund (Nichtteilnahme an Vorschulbildung)*(Schüler mit Migrationshintergrund), sozioökonomischer Status der Schüler, Quadrat des sozioökonomischen Status der Schüler, sozioökonomisches Profil der Schule, Geschlecht, Schule im ländlichen Raum, Schule in einer Großstadt, Schulgröße, Quadrat der Schulgröße sowie Privatschule.

StatLink त्गाडs http://dx.doi.org/10.1787/888932964946 


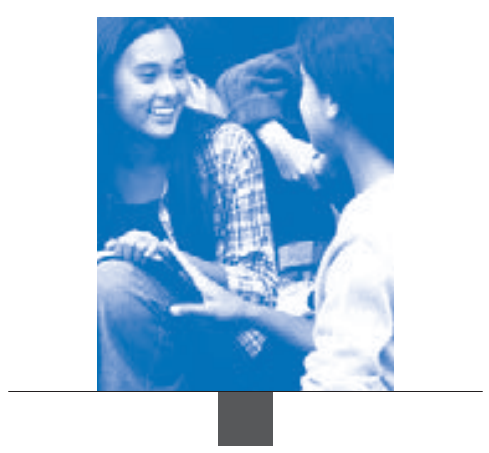

Anhang C

ENTWICKLUNG UND UMSETZUNG VON PISA EIN KOOPERATIONSPROJEKT 
PISA ist ein Kooperationsprojekt, bei dem Experten aus allen Teilnehmerländern zusammenkommen und die Orientierungen von den Regierungen der Teilnehmerländer gemeinsam auf der Basis gemeinsamer bildungspolitischer Interessen vorgegeben werden.

Der PISA-Verwaltungsrat, in dem jedes Land vertreten ist, definiert im Rahmen der OECD-Zielsetzungen die Politikprioritäten für PISA und überwacht die Einhaltung dieser Prioritäten während der Programmumsetzung. Diese Aufgabe umfasst die Festlegung von Prioritäten für die Ausarbeitung der Indikatoren, die Erstellung der Instrumente zur Leistungsmessung und die Berichterstattung über die Ergebnisse.

Es wurden Arbeitsgruppen mit Experten aus den Teilnehmerstaaten gebildet, die gewährleisten sollen, dass die Politikziele mit der größtmöglichen international verfügbaren verfahrenstechnischen Kompetenz verknüpft werden. Durch ihre Beteiligung an diesen Expertengruppen stellen die Länder sicher, dass die eingesetzten Instrumente international valide sind und zugleich dem kulturellen und bildungspolitischen Kontext der OECD-Mitgliedsländer sowie der Partnerländer und -volkswirtschaften Rechnung tragen, die eingesetzten Beurteilungsinstrumente über sehr gute messtechnische Eigenschaften verfügen und diese Instrumente sowohl authentisch als auch bildungspolitisch relevant sind.

Über die nationalen Projektmanager setzen die Teilnehmer das PISA-Programm gemäß den vereinbarten administrativen Verfahren auf nationaler Ebene um. Die nationalen Projektmanager spielen eine entscheidende Rolle, indem sie gewährleisten, dass die Umsetzung hohen qualitativen Ansprüchen genügt, und indem sie die Ergebnisse, Analysen, Berichte und Veröffentlichungen überprüfen und evaluieren.

Zuständig für Design und Implementierung der Erhebungen innerhalb des vom PISA-Verwaltungsrat festgelegten Rahmens sind externe Vertragspartner. Die Ausarbeitung und Umsetzung der kognitiven Beurteilung und der Fragebogen sowie der internationalen Optionen für PISA 2012 wurde von einem Konsortium unter der Leitung des Australian Council for Educational Research (ACER) durchgeführt. Zu den weiteren Partnern in diesem Konsortium zählen: cApStAn Linguistic Quality Control in Belgien, das Centre de Recherche Public Henri Tudor (CRP-HT) in Luxemburg, das Department of Teacher Education and School Research (ILS) an der Universität Oslo in Norwegen, das Deutsche Institut für Internationale Pädagogische Forschung (DIPF) in Deutschland, der Educational Testing Service (ETS) in den Vereinigten Staaten, das Leibniz-Institut für die Pädagogik der Naturwissenschaften und Mathematik (IPN) in Deutschland, das National Institute for Educational Policy Research (NIER) in Japan, die Unité d'analyse des systèmes et des pratiques d'enseignement (aSPe) an der Universität Lüttich in Belgien und WESTAT in den Vereinigten Staaten, ebenso wie einzelne Consultants aus verschiedenen Ländern. Das ACER kooperierte darüber hinaus mit Achieve, Inc. in den Vereinigten Staaten, um das Mathematik-Rahmenkonzept für PISA 2012 auszuarbeiten.

Das OECD-Sekretariat hat die Gesamtmanagementverantwortung für das Programm, verfolgt dessen praktische Umsetzung, fungiert als Sekretariat für den PISA-Verwaltungsrat, bemüht sich um Konsensbildung zwischen den Ländern und dient als Ansprechpartner zwischen dem PISA-Verwaltungsrat und dem internationalen Konsortium, das mit der Implementierung der Aktivitäten beauftragt ist. Das OECD-Sekretariat erstellt auch die Indikatoren und Analysen und arbeitet die internationalen Berichte und Veröffentlichungen in Zusammenarbeit mit dem PISA-Konsortium sowie in enger Abstimmung mit den Mitglieds- und Partnerländern und -volkswirtschaften sowohl auf Politikebene (PISA-Verwaltungsrat) als auch auf Implementierungsebene (nationale Projektmanager) aus.

\section{PISA-Verwaltungsrat}

Vorsitz des PISA-Verwaltungsrats: Lorna Bertrand

\section{OECD-Länder}

Australien: Tony Zanderigo

Österreich: Mark Német

Belgien: Christiane Blondin und Isabelle Erauw

Kanada: Pierre Brochu, Patrick Bussiere und Tomasz Gluszynski

Chile: Leonor Cariola Huerta

Tschech. Rep.: Jana Paleckova

Dänemark: Tine Bak und Elsebeth Aller

Estland: Maie Kitsing

Finnland: Tommi Karjalainen

Frankreich: Bruno Trosseille

Deutschland: Elfriede Ohrnberger und Susanne von Below

Griechenland: Vassilia Hatzinikita und Chryssa Sofianopoulou

Ungarn: Benõ Csapó

Island: Júlíus Björnsson

Irland: Jude Cosgrove und Gerry Shiel

Israel: Michal Beller und Hagit Glickman

Italien: Paolo Sestito
Japan: Ryo Watanabe

Korea: Sungsook Kim und Keunwoo Lee

Luxemburg: Amina Kafai

Mexiko: Francisco Ciscomani und Eduardo Backhoff Escudero

Niederlande: Paul van Oijen

Neuseeland: Lynne Whitney

Norwegen : Anne-Berit Kavli und Alette Schreiner

Polen: Stanislaw Drzazdzewski und Hania Bouacid

Portugal: Luisa Canto und Castro Loura

Slowak. Rep.: Romana Kanovska und Paulina Korsnakova

Slowenien: Andreja Barle Lakota

Spanien: Ismael Sanz Labrador

Schweden: Anita Wester

Schweiz: Vera Husfeldt und Claudia Zahner Rossier

Türkei: Nurcan Devici und Mustafa Nadir Çalis

Ver. Königreich: Lorna Bertrand und Jonathan Wright

Ver. Staaten: Jack Buckley, Dana Kelly und Daniel McGrath

Beobachter

Albanien: Ermal Elezi

Argentinien: Liliana Pascual 
Brasilien: Luiz Claudio Costa

Bulgarien: Neda Kristanova

Chinesisch Taipeh: Gwo-Dong Chen und Chih-Wei Hue

Kolumbien: Adriana Molina

Costa Rica: Leonardo Garnier Rimolo

Croatia: Michelle Bras Roth

Hongkong (China): Esther Sui-chu Ho

Indonesien: Khairil Anwar Notodiputro

Jordanien: Khattab Mohammad Abulibdeh

Kasachstan: Almagul Kultumanova

Lettland: Andris Kangro, Ennata Kivrina und Dita Traidas

Litauen: Rita Dukynaite

Macau (China): Leong Lai

Montenegro: Zeljko Jacimovic

Panama: Arturo Rivera

Peru: Liliana Miranda Molina

Katar: Hamda Al Sulaiti

Rumänien: Roxana Mihail

Russ. Föderation: Isak Froumin und Galina Kovaleva

Serbien: Dragica Pavlovic-Babic

Shanghai (China): Minxuan Zhang

Singapur: Khah Gek Low

Thailand: Precharn Dechsri

Ver. Arab. Emirate: Moza al Ghufly und Ayesha G. Khalfan Almerri

Uruguay: Andrés Peri und Maria Helvecia Sanchez Nunez

Vietnam: Le Thi My Ha

Nationale Projektmanager für PISA 2012

Albanien: Alfonso Harizaj

Argentinien: Liliana Pascual

Australien: Sue Thomson

Österreich: Ursula Schwantner

Belgien: Inge De Meyer und Ariane Baye

Brasilien: João Galvão Bacchetto

Bulgarien: Svetla Petrova

Kanada: Pierre Brochu und Tamara Knighton

Chile: Ema Lagos Campos

Kolumbien: Francisco Reyes

Costa Rica: Lilliam Mora

Croatia: Michelle Bras Roth

Tschech. Rep.: Jana Paleckova

Dänemark: Niels Egelund

Estland: Gunda Tire

Finnland: Jouni Välijärvi

Frankreich: Ginette Bourny

Deutschland: Christine Sälzer und Manfred Prenzel

Griechenland: Vassilia Hatzinikita

Hongkong (China): Esther Sui-chu Ho

Ungarn: Ildikó Balazsi

Island: Almar Midvik Halldorsson

Indonesien: Yulia Wardhani Nugaan und Hari Setiadi
Irland: Gerry Shiel und Rachel Perkins

Israel: Joel Rapp und Inbal Ron-Kaplan

Italien: Carlo Di Chiacchio

Japan: Ryo Watanabe

Jordanien: Khattab Mohammad Abulibdeh

Kasachstan: Gulmira Berdibayeva und Zhannur Azmagambetova

Korea: Ji-Min Cho und Mi-Young Song

Lettland: Andris Kangro

Liechtenstein: Christian Nidegger

Litauen: Mindaugas Stundza

Luxemburg: Bettina Boehm

Macau (China): Kwok Cheung Cheung

Malaysia: Ihsan Ismail und Muhamad Zaini Md Zain

Mexiko: María Antonieta Díaz Gutierrez

Montenegro: Divna Paljevic Sturm

Niederlande: Jesse Koops

Neuseeland: Kate Lang und Steven May

Norwegen: Marit Kjaernsli

Peru: Liliana Miranda Molina

Polen: Michal Federowicz

Portugal: Ana Sousa Ferreira

Katar: Aysha Al-Hashemi und Assad Tounakti

Rumänien: Silviu Cristian Mirescu

Russ. Föderation: Galina Kovaleva

Schottland: Rebecca Wheater

Serbien: Dragica Pavlovic-Babic

Shanghai (China): Jing Lu und Minxuan Zhang

Singapur: Chew Leng Poon und Sean Tan

Slowak. Rep.: Julia Miklovicova und Jana Ferencova

Slowenien: Mojca Straus

Spanien: Lis Cercadillo Pérez

Schweden: Magnus Oskarsson

Schweiz: Christian Nidegger

Chinesisch Taipeh: Pi-Hsia Hung

Thailand: Sunee Klainin

Tunesien: Mohamed Kamel Essid

Türkei: Serdar Aztekin

Ver. Arab. Emirate: Moza al Ghufly

Ver. Königreich: Rebecca Wheater

Ver. Staaten: Dana Kelly und Holly Xie

Uruguay: Maria Helvecia Sánchez Nunez

Vietnam: Thi My Ha Le

\section{OECD-Sekretariat}

Andreas Schleicher (Strategische Ausarbeitung)

Marilyn Achiron (Redaktionelle Unterstützung)

Francesco Avvisati (Analytische Unterstützung)

Brigitte Beyeler (Administrative Unterstützung)

Simone Bloem (Analytische Unterstützung)

Marika Boiron (Unterstützung im Bereich Übersetzung)

Francesca Borgonovi (Analytische Unterstützung)

Jenny Bradshaw (Projektmanagement)

Célia Braga-Schich (Unterstützung im Bereich Produktion) 
Claire Chetcuti (Administrative Unterstützung)

Michael Davidson (Projektmanagement und analytische Unterstützung)

Cassandra Davis (Koordination Verbreitung)

Elizabeth del Bourgo (Unterstützung im Bereich Produktion)

Juliet Evans (Administrative Unterstützung und Kontakte zu den Partnerländern/-volkswirtschaften)

Tue Halgreen (Projektmanagement)

Miyako Ikeda (Analytische Unterstützung)

Tadakazu Miki (Analytische Unterstützung)

Guillermo Montt (Analytische Unterstützung)

Giannina Rech (Analytische Unterstützung)

Diana Tramontano (Administrative Unterstützung)

Sophie Vayssettes (Analytische Unterstützung)

Elisabeth Villoutreix (Produktionskoordination)

Pablo Zoido (Analytische Unterstützung)

\section{Deutsche Übersetzung}

Thomas Krischer (Revision)

Ira Haugk (Revision)

Martina Dzierzawski (Übersetzung)

Daniela Herzog (Übersetzung)

Hubertus Hesse (Übersetzung)

Verena Holler (Übersetzung)

Bettina Peche (Übersetzung)

Judith Schmid (Übersetzung)

Susanne Gniech (Terminologische Unterstützung)

Gabriele Gwinner (Produktionskoordination)

Geert Gruben (Administrative Unterstützung)

Gabriele Speer (Administrative Unterstützung)

Ilse Ferrario (Administrative Unterstützung)

\section{Expertengruppe Mathematik, PISA 2012}

Kaye Stacey (Vorsitz) (University of Melbourne, Australien)

Caroline Bardini (University of Melbourne, Australien)

Werner Blum (Universität Kassel, Deutschland)

Joan Ferrini-Mundy (Michigan State University, Vereinigte

Staaten)

Solomon Garfunkel (COMAP, Vereinigte Staaten)

Toshikazu Ikeda (Yokohama National University, Japan)

Zbigniew Marciniak (Universität Warschau, Polen)

Mogens Niss (Universität Roskilde, Dänemark)

Martin Ripley (World Class Arena Limited, Vereinigtes

Königreich)

William Schmidt (Michigan State University, Vereinigte Staaten)

\section{Expertengruppe Problemlösekompetenz, PISA}

\section{2}

Joachim Funke (Vorsitz) (Universität Heidelberg, Deutschland) Benő Csapó (Universität Szeged, Ungarn)

John Dossey (Illinois State University, Vereinigte Staaten)

Arthur Graesser (The University of Memphis, Vereinigte Staaten)

Detlev Leutner (Universität Duisburg-Essen, Deutschland)

Romain Martin (Université de Luxembourg FLSHASE,

Luxemburg)
Richard Mayer (University of California, Vereinigte Staaten)

Ming Ming Tan (Ministry of Education, Singapur)

\section{Expertengruppe Finanzielle Allgemeinbildung, PISA 2012}

Annamaria Lusardi (Vorsitz) (The George Washington University School of Business, Vereinigte Staaten)

Jean-Pierre Boisivon (Université de Paris II Panthéon-Assas, Frankreich)

Diana Crossan (Commission for Financial Literacy und Retirement Income, Neuseeland)

Peter Cuzner (Australian Securities und Investments Commission, Australien)

Jeanne Hogarth (Federal Reserve System, Vereinigte Staaten)

Dušan Hradil (Ministry of Finance, Tschechische Republik)

Stan Jones (Consultant, Kanada)

Sue Lewis (Consultant, Vereinigtes Königreich)

Expertengruppe Fragebogen, PISA 2012

Eckhard Klieme (Vorsitz) (Deutsches Institut für Internationale Pädagogische Forschung - DIPF, Deutschland)

Eduardo Backhoff (University of Baja California am Institute of Educational Research und Development, Mexiko)

Ying-yi Hong (Nanyang Business School der Nanyang Technological University, Singapur)

David Kaplan (University of Wisconsin - Madison, Vereinigte Staaten)

Henry Levin (Columbia University, Vereinigte Staaten)

Jaap Scheerens (Universität Twente, Niederlande)

William Schmidt (Michigan State University, Vereinigte Staaten) Fons van de Vijver (Universität Tilburg, Niederlande)

\section{Technische Beratergruppe}

Keith Rust (Vorsitz) (Westat, Vereinigte Staaten)

Ray Adams (ACER, Australien)

Cees Glas (Universität Twente, Niederlande)

John de Jong (Language Testing Services, Niederlande)

David Kaplan (University of Wisconsin - Madison, Vereinigte Staaten)

Christian Monseur (Universität Lüttich, Belgien)

Sophia Rabe-Hesketh (University of California - Berkeley, Vereinigte Staaten)

Thierry Rocher (Ministère de l'éducation nationale, Frankreich)

Norman Verhelst (CITO, Niederlande)

Kentaro Yamamoto (ETS, Vereinigte Staaten)

Rebecca Zwick (University of California, Vereinigte Staaten)

\section{PISA-Konsortium 2012}

\section{Australian Council for Educational Research}

Ray Adams (Internationale Projektleitung)

Susan Bates (Projektverwaltung)

Alla Berezner (Datenmanagement und -analyse)

Yan Bibby (Datenverarbeitung und -analyse)

Phillipe Bickham (IT-Dienste)

Esther Brakey (Administrative Unterstützung)

Robin Buckley (IT-Dienste) 
Mark Butler (Erhebungsinstrumente Finanzielle Allgemeinbildung und Testausarbeitung)

Wei Buttress (Projektverwaltung und Qualitätskontrolle)

Renee Chow (Datenverarbeitung und -analyse)

John Cresswell (Berichterstattung und Verbreitung)

Alex Daraganov (Datenverarbeitung und -analyse)

Jorge Fallas (Datenverarbeitung und -analyse)

Kate Fitzgerald (Datenverarbeitung und Stichprobenauswahl)

Kim Fitzgerald (IT-Dienste)

Paul Golden (IT- und Helpdesk-Support)

Jennifer Hong (Datenverarbeitung und Stichprobenauswahl)

Nora Kovarcikova (Erhebungsverfahren)

Winson Lam (IT-Dienste)

Petra Lietz (Fragebogenausarbeitung)

Tom Lumley (Erhebungsinstrumente Lesekompetenz und Testausarbeitung)

Greg Macaskill (Datenmanagement und -verarbeitung, Stichprobenauswahl)

Ron Martin (Erhebungsinstrumente Naturwissenschaften und Testausarbeitung)

Barry McCrae (Erhebungsinstrumente Problemlösekompetenz und Naturwissenschaften, Testausarbeitung)

Louise McDonald (Grafische Gestaltung)

Juliette Mendelovits (Erhebungsinstrumente Lesekompetenz und finanzielle Allgemeinbildung, Testausarbeitung)

Martin Murphy (Feldoperationen und Stichprobenauswahl)

Thoa Nguyen (Datenmanagement und -analyse)

Stephen Oakes (IT-Management und -Support)

Elizabeth O'Grady (Fragebogenausarbeitung und

Projektunterstützung)

Penny Pearson (Administrative Unterstützung)

Ray Peck (Erhebungsinstrumente Mathematik und finanzielle

Allgemeinbildung, Testausarbeitung)

Fei Peng (Qualitätskontrolle und Projektunterstützung)

Ray Philpot (Erhebungsinstrumente Problemlösekompetenz und Testausarbeitung)

Anna Plotka (Grafische Gestaltung)

Dara Ramalingam (Erhebungsinstrumente Lesekompetenz und Testausarbeitung)

Sima Rodrigues (Datenverarbeitung und -analyse)

Alla Routitsky (Datenmanagement und -verarbeitung)

James Spithill (Erhebungsinstrumente Mathematik und

Testausarbeitung)

Rachel Stanyon (Projektunterstützung)

Naoko Tabata (Erhebungsverfahren)

Stephanie Templeton (Projektverwaltung und -unterstützung)

Mollie Tobin (Fragebogenausarbeitung und Projektunterstützung)

David Tout (Erhebungsinstrumente Mathematik und

Testausarbeitung)

Ross Turner (Management, Erhebungsinstrumente Mathematik und Testausarbeitung)

Maryanne Van Grunsven (Projektunterstützung)

Charlotte Waters (Projektverwaltung, Datenverarbeitung und

-analyse)
Maurice Walker (Management, computergestützte Erhebung)

Louise Wenn (Datenmanagement und -analyse)

Yan Wiwecka (IT-Dienste)

cApStAn Linguistic Quality Control (BELGIEN)

Raphael Choppinet (Computergestütztes

Überprüfungsmanagement)

Steve Dept (Übersetzungs- und Überprüfungsdienste)

Andrea Ferrari (Gestaltung der sprachlichen Qualitätssicherung und Qualitätskontrolle)

Musab Hayatli (Linksläufige Schriften, kulturelle Adaptationen)

Elica Krajceva (Koordination Fragebogenüberprüfung)

Shinoh Lee (Koordination Überprüfung der kognitiven Tests)

Irene Liberati (Koordination Handbuchüberprüfung)

Laura Wayrynen (Überprüferschulung und

Überprüfungsverfahren)

\section{Educational Testing Service (ETS)}

Jonas Bertling (Erhebungsinstrumente und Testausarbeitung)

Irwin Kirsch (Lesekomponenten)

Patricia Klag (Erhebungsinstrumente Problemlösekompetenz und Testausarbeitung)

Patrick Kyllonen (Erhebungsinstrumente und Testausarbeitung) Marylou Lennon (Erhebungsinstrumente und Testausarbeitung)

Richard Roberts (Erhebungsinstrumente und Testausarbeitung)

Matthias von Davier (Erhebungsinstrumente und

Testausarbeitung)

Kentaro Yamamoto (Mitglied der technischen Beratergruppe, Erhebungsinstrumente Problemlösekompetenz und Testausarbeitung)

Deutsches Institut für Internationale Pädagogische Forschung (DIPF, DEUTSCHLAND)

Frank Goldhammer (Testentwicklung, Problemlösekompetenz)

Eckhard Klieme (Vorsitz Expertengruppe Fragebogen)

Silke Hertel (Fragebogenausarbeitung)

Jean-Paul Reeff (International Consultant)

Heiko Rolke (Management Software-Design und Software-

Entwicklung [Erhebungssystem, Übersetzungssystem])

Brigitte Steinert (Fragebogenausarbeitung)

Svenja Vieluf (Fragebogenausarbeitung)

\section{Institutt for Lærerutdanning Og Skoleutvikling (ILS, NORWEGEN)}

Bjornar Alseth (Erhebungsinstrumente Mathematik und Testausarbeitung)

Ole Kristian Bergem (Erhebungsinstrumente Mathematik und Testausarbeitung)

Knut Skrindo (Erhebungsinstrumente Mathematik und Testausarbeitung)

Rolf V. Olsen (Erhebungsinstrumente Mathematik und Testausarbeitung)

Arne Hole (Erhebungsinstrumente Mathematik und Testausarbeitung)

Therese Hopfenbeck (Erhebungsinstrumente

Problemlösekompetenz und Testausarbeitung) 
Leibniz-Institut für die Pädagogik der Naturwissenschaften und Mathematik (IPN, DEUTSCHLAND)

Christoph Duchhardt (Erhebungsinstrumente Mathematik und Testausarbeitung)

Aiso Heinze (Erhebungsinstrumente Mathematik und Testausarbeitung)

Eva Knopp (Erhebungsinstrumente Mathematik und Testausarbeitung)

Martin Senkbeil (Erhebungsinstrumente Mathematik und Testausarbeitung)

\section{National Institute for Educational Policy Research (NIER, JAPAN)}

Keiichi Nishimura (Erhebungsinstrumente Mathematik und Testausarbeitung)

Yuji Surata (Erhebungsinstrumente Mathematik und Testausarbeitung)

Die TAO-Initiative: Henry Tudor Public Research Centre, Universität Luxemburg (LUXEMBURG)

Joel Billard (Softwareingenieur, Schulfragebogen)

Marilyn Binkley (Projekt-Consultant, Expertin für Leistungsmessung)

Jerome Bogaerts (Softwareingenieur, TAO-Plattform)

Gilbert Busana (Elektronische Erhebungsinstrumente, Benutzerfreundlichkeit)

Christophe Henry (Systemingenieur, Schulfragebogen und Hosting)

Raynald Jadoul (Technische Leitung, Schulfragebogen und elektronische Erhebungsinstrumente)

Isabelle Jars (Projektmanagement)

Vincent Koenig (Elektronische Erhebungsinstrumente, Benutzerfreundlichkeit)

Thibaud Latour (Projektleitung, TAO-Plattform)

Lionel Lecaque (Softwareingenieur, Qualität)

Primael Lorbat (Softwareingenieur, elektronische Erhebungsinstrumente)

Romain Martin (Mitglied der Expertengruppe Problemlösekompetenz)

Matteo Melis (Softwareingenieur, Schulfragebogen)

Patrick Plichart (Softwarearchitektur, TAO-Plattform)

Vincent Porro (Softwareingenieur, elektronische

Erhebungsinstrumente)

Igor Ribassin (Softwareingenieur, elektronische

Erhebungsinstrumente)

Somsack Sipasseuth (Softwareingenieur, elektronische Erhebungsinstrumente)

Unité d'analyse des Systèmes et des Pratiques d'enseignement (ASPE, BELGIEN)

Isabelle Demonty (Erhebungsinstrumente Mathematik und Testausarbeitung

Annick Fagnant (Erhebungsinstrumente Mathematik und Testausarbeitung)

Anne Matoul (Ausarbeitung französische Quellen)

Christian Monseur (Mitglied der technischen Beratergruppe)

\section{WESTAT}

Susan Fuss (Stichprobenauswahl und Gewichtung)

Amita Gopinath (Gewichtung)

Jing Kang (Stichprobenauswahl und Gewichtung)
Sheila Krawchuk (Stichprobenauswahl, Gewichtung und Qualitätskontrolle)

Thanh Le (Stichprobenauswahl, Gewichtung und Qualitätskontrolle)

John Lopdell (Stichprobenauswahl und Gewichtung)

Keith Rust (Leitung des PISA-Konsortiums für

Stichprobenauswahl und Gewichtung)

Erin Willey (Stichprobenauswahl und Gewichtung)

Shawn Lu (Gewichtung)

Teresa Strickler (Gewichtung)

Yumiko Sugawara (Gewichtung)

Joel Wakesberg (Stichprobenauswahl und Gewichtung)

Sergey Yagodin (Gewichtung)

Achieve Inc.

Michael Cohen (Ausarbeitung des Mathematik-

Rahmenkonzepts)

Kaye Forgione (Ausarbeitung des Mathematik-Rahmenkonzepts)

Morgan Saxby (Ausarbeitung des Mathematik-Rahmenkonzepts)

Laura Slover (Ausarbeitung des Mathematik-Rahmenkonzepts)

Bonnie Verrico (Projektunterstützung)

\section{HallStat SPRL}

Béatrice Halleux (Consultant, Übersetzung/Überprüfung, Ausarbeitung französische Quellen)

\section{Universität Heidelberg}

Joachim Funke (Vorsitz, Expertengruppe Problemlösekompetenz) Samuel Greiff (Erhebungsinstrumente Problemlösekompetenz und Testausarbeitung)

\section{University of Melbourne}

Caroline Bardini (Mitglied der Expertengruppe Mathematik)

John Dowsey (Erhebungsinstrumente Mathematik und

Testausarbeitung)

Derek Holton (Erhebungsinstrumente Mathematik und

Testausarbeitung)

Kaye Stacey (Vorsitz, Expertengruppe Mathematik)

\section{Sonstige Experten}

Michael Besser (Erhebungsinstrumente Mathematik und Testausarbeitung, Universität Kassel, Deutschland)

Khurrem Jehangir (Datenanalyse für die technische Beratergruppe, Universität Twente, Niederlande)

Kees Lagerwaard (Erhebungsinstrumente Mathematik und Testausarbeitung, Institute for Educational Measurement of Netherlands, Niederlande)

Dominik Leiss (Erhebungsinstrumente Mathematik und Testausarbeitung, Universität Kassel, Deutschland)

Anne-Laure Monnier (Consultant Ausarbeitung französische Quellen, Frankreich)

Hanako Senuma (Erhebungsinstrumente Mathematik und Testausarbeitung, Tamagawa University, Japan)

\section{Publikationslayout}

Fung-Kwan Tam

Geert Gruben

Gabriele Gwinner

Gabriele Speer 


\section{ORGANISATION FÜR WIRTSCHAFTLICHE ZUSAMMENARBEIT UND ENTWICKLUNG}

Die OECD ist ein in seiner Art einzigartiges Forum, in dem die Regierungen gemeinsam an der Bewältigung von Herausforderungen der Globalisierung im Wirtschafts-, Sozial- und Umweltbereich arbeiten. Die OECD steht auch in vorderster Linie bei den Bemühungen um ein besseres Verständnis der neuen Entwicklungen und durch sie ausgelöster Befürchtungen, indem sie Untersuchungen zu Themen wie Corporate Governance, Informationswirtschaft oder Bevölkerungsalterung durchführt. Die Organisation bietet den Regierungen einen Rahmen, der es ihnen ermöglicht, ihre Politikerfahrungen auszutauschen, nach Lösungsansätzen für gemeinsame Probleme zu suchen, empfehlenswerte Praktiken aufzuzeigen und auf eine Koordinierung nationaler und internationaler Politiken hinzuarbeiten.

Die OECD-Mitgliedstaaten sind: Australien, Belgien, Chile, Dänemark, Deutschland, Estland, Finnland, Frankreich, Griechenland, Irland, Island, Israel, Italien, Japan, Kanada, Korea, Luxemburg, Mexiko, Neuseeland, die Niederlande, Norwegen, Österreich, Polen, Portugal, Schweden, Schweiz, die Slowakische Republik, Slowenien, Spanien, die Tschechische Republik, Türkei, Ungarn, das Vereinigte Königreich und die Vereinigten Staaten. Die Europäische Union nimmt an den Arbeiten der OECD teil.

OECD Publishing sorgt dafür, dass die Ergebnisse der statistischen Analysen und der Untersuchungen der Organisation zu wirtschaftlichen, sozialen und umweltpolitischen Themen sowie die von den Mitgliedstaaten vereinbarten Übereinkommen, Leitlinien und Standards weite Verbreitung finden. 


\section{PISA 2012 Ergebnisse:}

\section{Exzellenz durch Chancengerechtigkeit \\ ALLEN SCHÜLERINNEN UND SCHÜLERN DIE VORAUSSETZUNGEN \\ ZUM ERFOLG SICHERN \\ BAND II}

Die Internationale Schulleistungsstudie der OECD (PISA) untersucht nicht nur, was Schülerinnen und Schüler in den Bereichen Mathematik, Lesekompetenz und Naturwissenschaften wissen, sondern auch, inwieweit sie dieses Wissen anwenden können. Dies ist einer von insgesamt sechs Bänden, in denen die Ergebnisse von PISA 2012, der fünften Runde dieser im Dreijahresturnus durchgeführten Erhebung, präsentiert werden.

Band I, Was Schülerinnen und Schüler wissen und können: Schülerleistungen in Mathematik, Lesekompetenz und Naturwissenschaften, gibt einen Überblick über die Schülerleistungen bei der PISA-Erhebung 2012.

Band II, Exzellenz durch Chancengerechtigkeit: Allen Schülerinnen und Schülern die Voraussetzungen zum Erfolg sichern, definiert und misst die Chancengerechtigkeit in der Bildung und analysiert, wie sie sich von PISA 2003 bis PISA 2012 in den verschiedenen Ländern entwickelt hat.

Band III, Lernbereitschaft: Engagement, Motivation und Selbsteinschätzung von Schülerinnen und Schülern, untersucht das schulische Engagement, die Einsatz- und Leistungsbereitschaft sowie das Vertrauen der Schülerinnen und Schüler in die eigenen Lernfähigkeiten im Bereich der Mathematik.

Band IV, Was macht Schulen erfolgreich? Lernumfeld und schulische Organisation in PISA, untersucht den Zusammenhang zwischen den Schülerleistungen und verschiedenen Merkmalen der Schulen und der Schulsysteme.

Band V, Lernen für das Leben: Schülerleistungen im Bereich Problemlösekompetenz, befasst sich mit den Schülerleistungen beim Problemlösekompetenztest der PISA-Erhebung 2012, in dem die Fähigkeit der Schülerinnen und Schüler gemessen wurde, nicht routinemäßigen Situationen gerecht zu werden.

Band VI, Schülerinnen und Schüler und ihr Bezug zum Geld: Finanzielle Allgemeinbildung für das 21. Jahrhundert, beschäftigt sich mit den Erfahrungen der Schülerinnen und Schüler mit und ihrem Wissen über Geld.

Die Bände III bis VI sind nicht auf Deutsch erhältlich.

Inhalt

Kapitel 1 Definition und Messung der Bildungsgerechtigkeit

Kapitel 2 Verteilungsgerechtigkeit der Bildungserträge

Kapitel 3 Die Herausforderung der Vielfalt

Kapitel 4 Chancengerechtigkeit bei den Lernmöglichkeiten und der Ressourcenverteilung

Kapitel 5 Politikimplikationen der Frage der Bildungsgerechtigkeit

Diese Publikation kann online eingesehen werden unter: http://dx.doi.org/10.1787/9789264207486-de.

Diese Studie ist in der OECD iLibrary veröffentlicht, die alle Bücher, periodisch erscheinenden Publikationen und statistischen Datenbanken der OECD enthält.

Weitere Informationen finden Sie unter: www.oecd-ilibrary.org. 